
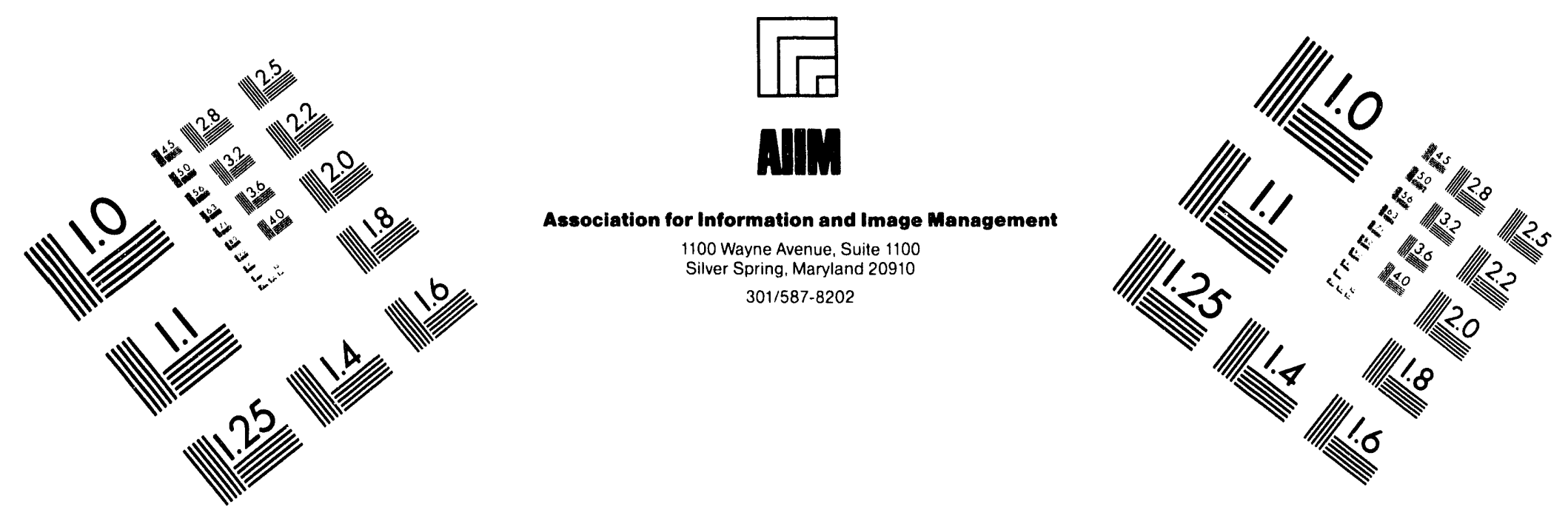

\title{
Centimeter
}

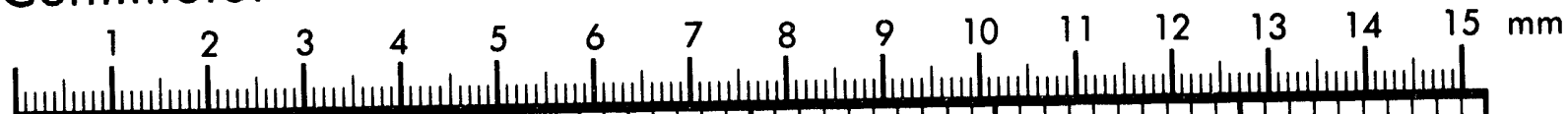
作 Inches
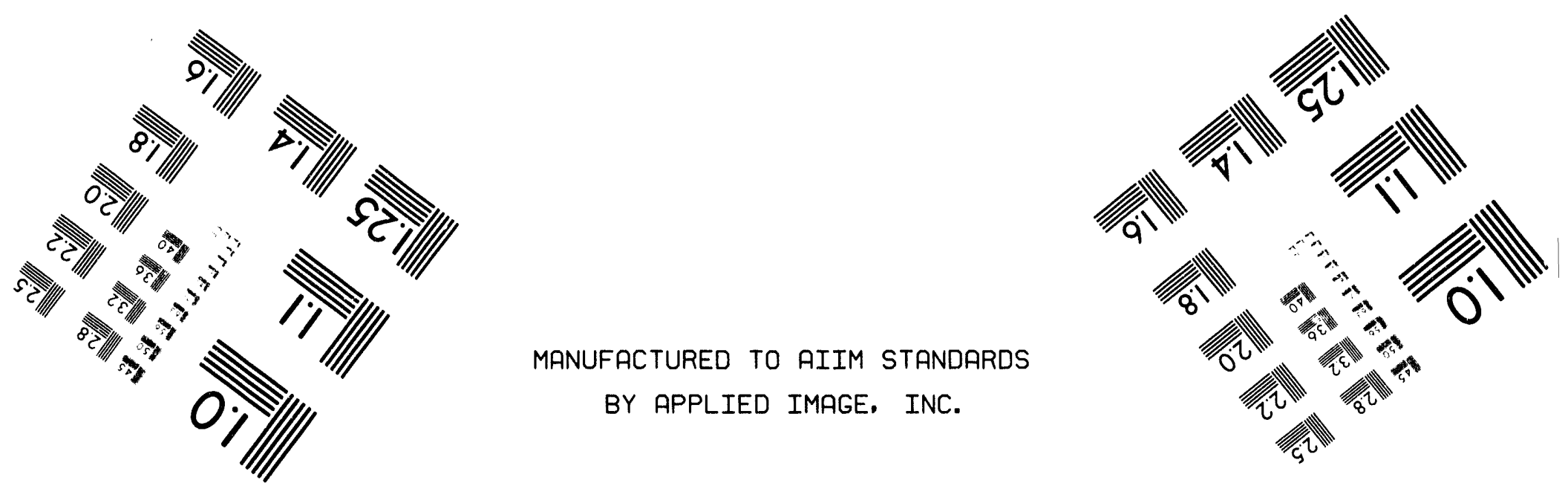

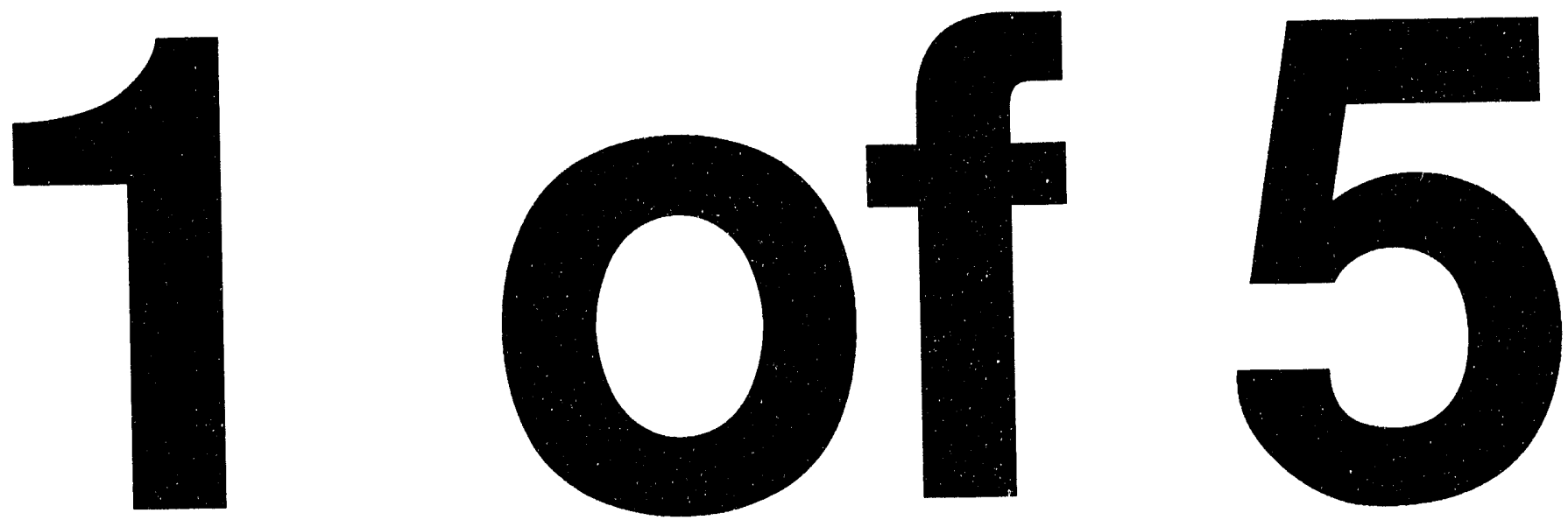
NUREG/CR-5973

PNL-8462

Rev. 1

\section{Codes and Standards and Other Guidance Cited in Regulatory Documents}

Manuscript Completed: July 1994

Date Published: August 1994

Prepared by

A. Ankrum, J. Nickolaus, R. Vinther, N. Maguire-Moffitt,

J. Hammer, L. Sherfey, R. Warner

Pacific Northwest Laboratory

Battelle Boulevard

Richland, WA 99352

\section{Prepared for}

Program Management, Policy Development, and Analysis Stafi

Office of Nuclear Reactor Regulation

U.S. Nuclear Regulatory Commission

Washington, DC 20555-0001

NRC JCN L2013 


\begin{abstract}
As part of the U.S. Nuclear Regulatory Commission (NRC) Standard Review Plan Update and Development Program, Pacific Northwest Laboratory developed a listing of industry consensus codes and standards and other government and industry guidance referred to in regulatory documents. In addition to updating previous information, Revision 1 adds citations from the NRC Inspection Manual and the Improved Standard Technical Specifications. This listing identifies the version of the cocie or standard cited in the regulatory document, the regulatory document, and the current version of the crede or standard. It also provides a summary characterization of the nature of the citation. This listing was developed from electronic searches of the Code of Federal Regulations and the NRC's Bulletins, Information Notices, Circulars, Generic Letters, Policy Statements, Regulatory Guides, and the Standard Review Plan (NUREG-0800).
\end{abstract}




\section{Contents}

Section Page

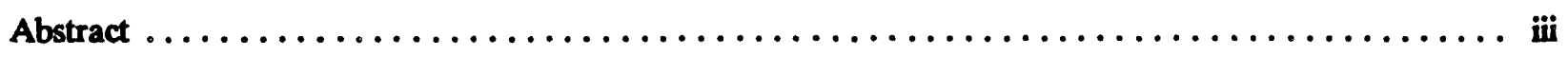

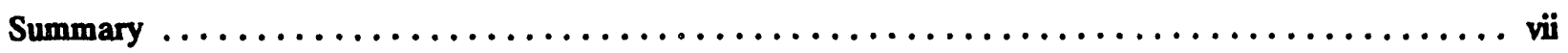

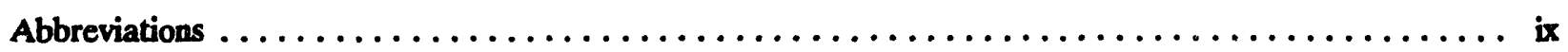

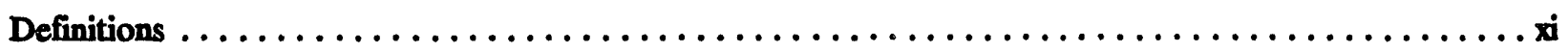

Part A - Industry Consensus Codes and Standards $\ldots \ldots \ldots \ldots \ldots \ldots \ldots \ldots$

Part B - Other Guidance. . . . . . . . . . . . . . . . . . . . . . . . . . . 343

\section{Appendices}

A Summation of Codes and Standards Citations in Regulatory Documents $\ldots \ldots \ldots \ldots \ldots$ A-1

B Summation of Other Government and Industry Guidance Citations in Regulatory Documents .. B-1 


\section{Summary}

For a study sponsored by the U.S. Nuclear Regulatory Commission (NRC), Pacific Northwest Laboratory ${ }^{1}$ developed a listing of industry consensus codes and standards and other government and industry guidance referred to in regulatory documents, identified the most current version, and developed a summary characterization of the reference. This listing was developed from electronic searches of the Code of Federal Regulations and the NRC's Bulletins, Information Notices, Circulars, Generic Letters, Policy Statements, Regulatory Guides, and the Standard Review Plan (NUREG-0800). Therefore, this is not an exhaustive listing of all code and standard citations in all NRC documents.

This work was conducted for the Standard Review Plan Update and Development Program (SRP-UDP) under JCN L-2013 (formerly FNN L-2013). The users of this listing may find errors or omissions. These items should be referred to Gene Suh, the NRC Lead Engineer for the SRP-UDP. He may be contacted by telephone at (301)504-1263 or by mail at the following address:

\section{U.S. Nuclear Regulatory Commission \\ Office of Nuclear Reactor Regulation \\ Mailstop 0-12 E4 \\ Washington, DC 20555}

In addition to updating previous information, Revision 1 adds citations from the NRC Inspection Manual and the Improved Standard Technical Specifications. This listing comprises over $\mathbf{4 4 0 0}$ records divided into two parts. Part A lists industry consensus codes and standards cited in NRC regulatory documents. Part B lists other government and industry guidance and requirements found in NRC regulatory documents that are not codes or standards but which provide information.

The listings are organized alphabetically by code group and include such information as a unique record number, code group and code or standard number, title, date of referenced code or standard edition, and the current version and version date. The listings also include a comment section and the NRC document in which the citation was found and its date. A column is also provided that indicates the degree to which the NRC document finds the specific code or standard acceptable for the specific application. 


\begin{tabular}{|c|c|}
\hline ACI & American Concrete Institute \\
\hline AEC & Atomic Energy Commission \\
\hline AISC & American Institute of Steel Construction \\
\hline ANS & American Nuclear Society \\
\hline ANSI & American National Standards Institute \\
\hline API & American Petroleum Institute \\
\hline ASA & Acoustical Society of America \\
\hline ASCE & American Society of Civil Engineers \\
\hline ASME & American Society of Mechanical Engineers \\
\hline ASNT & American Society for Nondestructive Testing \\
\hline ASTM & American Society for Testing and Materials \\
\hline AWS & American Welding Society \\
\hline AWWA & American Water Works Association \\
\hline bul & NRC Bulletin \\
\hline cfir & Code of Federal Regulations \\
\hline cir & NRC Circular \\
\hline CMAA & Crane Manufacturers Association of America \\
\hline DEMA & Diesel Engine Manufacturers Association \\
\hline DOE & Department of Energy \\
\hline drg & Draft Regulatory Guide \\
\hline DOT & Department of Transportation \\
\hline EPA. & Environmental Protection Agency \\
\hline ERDA & Energy Research and Development Administration \\
\hline FFPR & Federal Specification \\
\hline FWPCA & Federal Water Pollution Control Act \\
\hline $\mathbf{F M}$ & Factory Mutual \\
\hline git & NRC Generic Letter \\
\hline HPS & Health Physics Society \\
\hline HPSSC & Health Physics Society Standards Committee \\
\hline ICRP & International Commission on Radiological Protection \\
\hline IEEE & Institute of Electrical and Electronics Engineers \\
\hline inm-toc & NRC Inspection Manual Table of Contents \\
\hline inm.... & NRC Inspection Manual with Procedure Reference \\
\hline ISA & Instrument Society of America \\
\hline ISO & International Standards Organization \\
\hline MSS & Manufacturers Standards Society \\
\hline NBS & National Bureau of Standards \\
\hline NCMA & National Concrete and Masonry Association \\
\hline NCRP & National Council on Radiation Protection \\
\hline NEMA & National Electrical Manufacturers Association \\
\hline NETA & National Electrical Testing Association \\
\hline NIOSH & National Institute for Occupational Health and Safety \\
\hline NFPA & National Fire Protection Association \\
\hline not & NRC Information Notice \\
\hline nureg & A formal NRC Staff Publication \\
\hline
\end{tabular}




\section{Acronymns and tbbreviations (continued)}

\begin{tabular}{|c|c|}
\hline $\begin{array}{l}\text { NRA } \\
\text { N/S }\end{array}$ & $\begin{array}{l}\text { National Rifle Association } \\
\text { in the "Standard Version" column indicates the version date was not specified in the citation. }\end{array}$ \\
\hline pol & NRC Policy Statement \\
\hline $\mathbf{R}$ & $\begin{array}{l}\text { in the "Current Version Date" column indicates the date the Code or Standard was reaffirmed by } \\
\text { the issuing body }\end{array}$ \\
\hline reg & NRC Regulatory Guide \\
\hline srp & Standard Review Plan (NUREG-0800) \\
\hline sts & Standard Technical Specifications \\
\hline sts4-ge... & $\begin{array}{l}\text { Standard Technical Specifications for the General Electric Model } 4 \text { (NUREG-1433) with } \\
\text { paragraph reference }\end{array}$ \\
\hline sts6-ge... & $\begin{array}{l}\text { Standard Technical Specifications for the General Electric Model } 6 \text { (NUREG-1434) with } \\
\text { paragraph reference }\end{array}$ \\
\hline stsb\&w.... & $\begin{array}{l}\text { Standard Technical Specifications for Babcock \& Wilcox (NUREG-1430) with paragraph } \\
\text { reference }\end{array}$ \\
\hline stsce...... & $\begin{array}{l}\text { Standard Technical Specifications for Combustion Engineering (NUREG-1432) with paragraph } \\
\text { reference }\end{array}$ \\
\hline stswst.... & $\begin{array}{l}\text { Standard Technical Specifications for Westinghouse Electric (NUREG-1431) with paragraph } \\
\text { reference }\end{array}$ \\
\hline $\mathbf{L}$ & \\
\hline
\end{tabular}




\section{Definitions}

Disclaimer: These definitions were developed for, and only pertain to, the Standard Review Plan Update and Development Program that is being conducted by the Pacific Northwest Laboratory for the NRC.

The following acceptability codes are used in the 'Acceptability' column.

A - denotes that the document is acceptable to the NRC. The regulatory document does not state the need for any limitations, exceptions, or modifications to the provisions of the code or standard.

P - denotes partial acceptance; this means that the regulatory document indicated acceptability of the code or standard but also stated the need for limitations, exceptions, or modifications to the provisions of the code or standard. If the regulatory document indicates acceptability of only a portion of the code or standard (e.g., Section 18.18 of ACI 318-77), the acceptability code is "P" instead of "A."

R - denotes that the citation is used as a reference for definition purposes or to provide background information. The regulatory document does not provide an indication of acceptability or unacceptability of the code or standard.

N - denotes that the regulatory document indicated that the code or standard was unacceptable for a given application and has no indication of acceptability of any portion of the code or standard.

Standard: A set of rules that is based upon proven practices that are mutually agreed upon through an industry-consensus process.

Industry-consensus process: The process by which the participating members of a standards writing group, who represent the various interests of that industry (e.g., product manufacturers, material manufacturers, product users, utilities, insurers, designers, constructors, consultants, and regulators, whether local, regional, or national), develop a standard. An industry-consensus process does not approve, recommend, or endorse any specific or proprietary design or manufacturing process. The standards writing group members meet regularly on a formal basis to consider revisions of the current requirements, requests for interpretation of current requirements, and new requirements as dictated by technological development. The industryconsensus process satisfies the separate and distinct needs, requirements, and interest of its participants through a process of arriving at mutually agreed upon rules.

Code: A standard that is mandated by a regulatory organization or a governing body, for example, a State. 


\section{Part A}

Industry Consensus Codes and Standards 


\section{Codes and Standards and Other Guidance Clted in Regulatory Documents}

Part A - Industry Consensus Codes and Standards

\begin{tabular}{|c|c|c|c|c|c|c|c|c|c|c|}
\hline P.oord & Codo & Standurd & 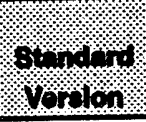 & 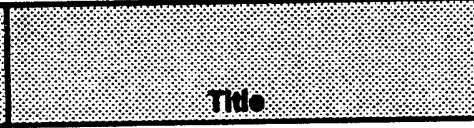 & boounhn: & $100 \%$ & \%or.tity & Commont & Cunont Vorou & opom \\
\hline 0001 & $\mathrm{ACl}$ & $\mathrm{ACl}$ & N/S & $\begin{array}{l}\text { Bullding Code Requirements for } \\
\text { Masonry Structures }\end{array}$ & glt80-032 & 1980 & $\mathbf{R}$ & Proposed Standard & $\mathrm{ACl} 530$ & 1992 \\
\hline 0002 & $\mathrm{ACl}$ & $\mathrm{ACl} 201$ & $\mathbf{N} / \mathbf{S}$ & $\begin{array}{l}\text { Guide for Making a Condition } \\
\text { Survey of Concreto in Senvice }\end{array}$ & reg1.127.r01 & 1978 & $\mathbf{P}$ & & $\mathrm{ACl} 201.1 \mathrm{R}$ & 1992 \\
\hline 0003 & $\mathrm{ACl}$ & $\mathrm{ACl} 201$ & 1968 & $\begin{array}{l}\text { Guide for Making a Condition } \\
\text { Survey of Concrote in Service }\end{array}$ & reg1.35.r03 & 1990 & $\mathbf{R}$ & Cited for definitions & $\mathrm{ACl} 201.1 \mathrm{R}$ & 1992 \\
\hline 0004 & $\mathrm{ACl}$ & $\begin{array}{l}\mathrm{ACl} 201 \\
\text { Committeo } \\
\end{array}$ & $\mathbf{N} / \mathbf{S}$ & $\begin{array}{l}\text { Guide for Making a Condition } \\
\text { Survey of Concrete in Service }\end{array}$ & reg1.90.ro1 & 1977 & $\mathbf{R}$ & & $\mathrm{ACl} 201.1 \mathrm{R}$ & 1992 \\
\hline 0005 & $\mathrm{ACt}$ & $\begin{array}{l}\mathrm{ACl} 212 \\
\text { Committee } \\
\end{array}$ & $\mathbf{N} / \mathbf{S}$ & Chemical Admixtures for Concreto & reg1.107.ro1 & 1977 & R & & $\mathrm{ACl} 212.3 \mathrm{R}$ & 1991 \\
\hline 0006 & $\mathrm{ACl}$ & ACl 212.1 & $\mathrm{~N} / \mathrm{S}$ & Admixtures for Concrete & inm46051 & 1983 & $\mathbf{R}$ & & $\mathrm{ACl}$ 212.1R & 1986 \\
\hline 0007 & $\mathrm{ACl}$ & $\mathrm{ACl} 212.2$ & $\mathbf{N} / \mathbf{S}$ & $\begin{array}{l}\text { Guide for Use of Admixtures in } \\
\text { Concrete }\end{array}$ & inm46051 & 1983 & $\mathbf{R}$ & & $\mathrm{ACl} 212.2 \mathrm{~A}$ & 1981 R86 \\
\hline 0008 & $\mathrm{ACl}$ & $\mathrm{ACl} 214$ & $N / S$ & $\begin{array}{l}\text { Rocommended Practice for } \\
\text { Evaluation of Strength Test Results } \\
\text { of Concrete }\end{array}$ & inm46051 & 1983 & $\mathbf{R}$ & & $\mathrm{ACl} 214$ & 1977 R89 \\
\hline 0009 & $\mathrm{ACl}$ & $\widehat{A C l} 214$ & $\mathbf{N} / \mathbf{S}$ & $\begin{array}{l}\text { Pecommended Practice for } \\
\text { Evaluation of Strength Test Results } \\
\text { of Concrete }\end{array}$ & inm46055 & 1983 & $\mathbf{R}$ & & $\mathrm{ACl} 214$ & 1977 RBg \\
\hline 0010 & $\mathrm{ACl}$ & $A C I 221$ & $N / S$ & $\begin{array}{l}\text { Guide for Use of Normal Weight } \\
\text { Aggregates in Concrete }\end{array}$ & inm46051 & 1983 & $\mathbf{R}$ & & $\mathrm{ACl} 221 \mathrm{R}$ & 1989 \\
\hline 0011 & $\mathrm{ACl}$ & $\mathrm{ACl} 301$ & $\mathbf{N} / \mathbf{S}$ & $\begin{array}{l}\text { Specifications for Structural } \\
\text { Concrete for Bulldings }\end{array}$ & inm46051 & 1983 & $\mathbf{R}$ & & ACl 301 & 1989 \\
\hline 0012 & $\mathrm{ACl}$ & ACl 301 & $\mathrm{~N} / \mathrm{S}$ & $\begin{array}{l}\text { Specifications for Structural } \\
\text { Concrete for Buildings }\end{array}$ & inm46053 & 1983 & $\mathbf{R}$ & & ACI 301 & 1989 \\
\hline 0013 & $\mathrm{ACl}$ & $\begin{array}{l}\mathrm{ACl} 301 \\
\text { Committee } \\
\end{array}$ & 1972 & $\begin{array}{l}\text { Specifications for Structural } \\
\text { Concrete for Buildings }\end{array}$ & reg1.107.ro1 & 1977 & $\mathbf{R}$ & & $\mathrm{ACl} 301$ & 1989 \\
\hline 0014 & $\mathrm{ACl}$ & $\mathrm{ACl} 304$ & N/S & $\begin{array}{l}\text { Guide for Measuring, Mixing, } \\
\text { Transporting, and Placing Concrete }\end{array}$ & inm46051 & 1983 & $\mathbf{R}$ & & $\mathrm{ACl} 304 \mathrm{R}$ & 1989 \\
\hline 0015 & $\mathrm{ACl}$ & $\mathrm{ACl} 304.2$ & $\mathbf{N} / \mathbf{S}$ & $\begin{array}{l}\text { Placing Concrete by Pumping } \\
\text { Mothods }\end{array}$ & inm46051 & 1983 & $\mathbf{R}$ & & ACI 304.2R & 1991 \\
\hline 0016 & $\mathrm{ACl}$ & ACl 305 & $N / S$ & Hot Woather Concreting & inm46051 & 1983 & $R$ & & ACl 305R & 1991 \\
\hline 0017 & $\mathrm{ACl}$ & $\mathrm{ACl} 306$ & $N / S$ & Cold Weather Concreting & inm46051 & 1983 & $\mathbf{R}$ & & ACl 306R & 1988 \\
\hline
\end{tabular}


Codes and Standards and Other Guidance Cited in Regulatory Documents

Part A - Industry Consensus Codes and Standards (continued)

\begin{tabular}{|c|c|c|c|c|c|c|c|c|c|c|}
\hline Rocord & Codo & Standard & $\begin{array}{l}\text { Standard } \\
\text { veralon }\end{array}$ & 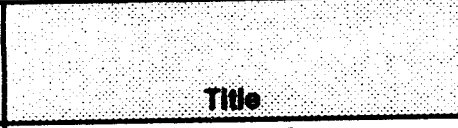 & Documont & Doo, & Aooppts & Comments & Curront Vorelon & $\begin{array}{l}\text { crront } \\
\text { volson } \\
\text { D } 10\end{array}$ \\
\hline 0018 & $\mathrm{ACl}$ & $\mathrm{ACl} 308$ & N/S & $\begin{array}{l}\text { Standard Practice for Curing } \\
\text { Concrete }\end{array}$ & inm46051 & 1983 & $\mathbf{R}$ & & $\mathrm{ACl} 308$ & 1992 \\
\hline 0019 & $\mathrm{ACl}$ & $\mathrm{ACl} 308$ & 1971 & $\begin{array}{l}\text { Standard Practice for Curing } \\
\text { Concrete }\end{array}$ & reg1.136.r02 & 1981 & $\mathbf{P}$ & & $\mathrm{ACl} 3 \mathrm{SQ} 9$ & 1992 \\
\hline 0020 & $\mathrm{ACI}$ & $\mathrm{ACI} 309$ & $\mathrm{~N} / \mathrm{S}$ & Guide for Consolidation of & inm46051 & 1983 & $\mathbf{R}$ & & $\mathrm{ACl} 309 \mathrm{R}$ & 1987 \\
\hline 0021 & $\mathrm{ACl}$ & $\mathrm{ACl} 309$ & 1972 & Guide for Consolidation of & reg1.94.ro1 & 1976 & $R$ & & $\mathrm{ACl} 309 \mathrm{R}$ & 1987 \\
\hline 0022 & $\mathrm{ACl}$ & $\mathrm{ACl} 311$ & $\mathrm{~N} / \mathrm{S}$ & Guide for Concrete Inspection & inm46051 & 1983 & $R$ & & $\mathrm{ACl} 311.4 \mathrm{R}$ & 1988 \\
\hline 0023 & $\mathrm{ACl}$ & $\mathrm{ACl} 311.1$ & $\mathrm{~N} / \mathrm{S}$ & ACI Manual of Concrete Inspection & inm46051 & 1983 & $\mathbf{R}$ & & $\mathrm{ACl}$ SP-2 & 1992 \\
\hline 0024 & $\mathrm{ACl}$ & $\mathrm{ACl} 311.2$ & $N / S$ & Guide for Concrete Inspection & inm46051 & 1983 & $\mathbf{R}$ & & $\mathrm{ACl} 311.4 \mathrm{R}$ & 1988 \\
\hline 0025 & $\mathrm{ACl}$ & $\mathrm{ACl} 311.3$ & $N / S$ & Guide for Concrete Inspection & inm46051 & 1983 & $R$ & & $\mathrm{ACl} 311.4 \mathrm{R}$ & 1988 \\
\hline 0026 & $\mathrm{ACl}$ & AC! 315 & $\mathrm{~N} / \mathrm{S}$ & $\begin{array}{l}\text { Detalls and Detalling of Concrete } \\
\text { Reinforcement }\end{array}$ & inm46051 & 1983 & $\mathbf{R}$ & & $\mathrm{ACl} 315$ & $1980 \mathrm{R} 86$ \\
\hline 0027 & $\mathrm{ACl}$ & $\mathrm{ACl} 318$ & 1989 & $\begin{array}{l}\text { Building Code Requirements for } \\
\text { Reinforced Concrete }\end{array}$ & 10 ctr36 & 1993 & $\bar{R}$ & & $\mathrm{ACl} 318$ & 1992 \\
\hline 0028 & $\mathrm{ACl}$ & $A C l 318$ & 1971 & $\begin{array}{l}\text { Building Code Requirements for } \\
\text { Reinforced Concrete }\end{array}$ & git79-005 & 1979 & $\mathbf{R}$ & & $\mathrm{ACl} 318$ & 1992 \\
\hline 0029 & $\mathrm{ACl}$ & $\mathrm{ACl} 318$ & $\mathrm{~N} / \mathrm{S}$ & $\begin{array}{l}\text { Building Code Requirements for } \\
\text { Peinforced Concrete }\end{array}$ & inm46051 & 1983 & $\mathbf{R}$ & & $\mathrm{ACl} 318$ & 1992 \\
\hline 0030 & $\overline{\mathrm{ACl}}$ & $\mathrm{ACl} 318$ & $\mathrm{~N} / \mathrm{S}$ & $\begin{array}{l}\text { Building Code Requirements for } \\
\text { Reinforced Concrete }\end{array}$ & inm46053 & 1983 & $R$ & & ACl 318 & 1992 \\
\hline 0031 & $\mathrm{ACl}$ & $\mathrm{ACl} 318$ & $\mathrm{~N} / \mathrm{S}$ & $\begin{array}{l}\text { Building Code Requirements for } \\
\text { Reinforced Concrete }\end{array}$ & inm47051 & 1983 & $\mathbf{R}$ & & $\mathrm{ACl} 318$ & 1992 \\
\hline 0032 & $\mathrm{ACl}$ & $\mathrm{ACl} 318$ & $\mathrm{~N} / \mathrm{S}$ & $\begin{array}{l}\text { Building Code Requirements for } \\
\text { Reinforced Concrete }\end{array}$ & inm47053 & $19 \overline{83}$ & $\mathbf{R}$ & & $\mathrm{ACl} 318$ & 1992 \\
\hline 0033 & $\mathrm{ACl}$ & $A C I 318$ & 1977 & $\begin{array}{l}\text { Building Code Requirements for } \\
\text { Reinforced Concrete }\end{array}$ & reg1.136.r02 & 1981 & $P$ & & $\mathrm{ACl} 318$ & 1992 \\
\hline 0034 & $\mathrm{ACl}$ & $\mathrm{ACl} 318$ & 1971 & $\begin{array}{l}\text { Building Code Requirements for } \\
\text { Reinforced Concrete }\end{array}$ & reg 1.142.r00 & 1978 & $\mathbf{P}$ & & $\mathrm{ACl} 318$ & 1992 \\
\hline 0035 & $\mathrm{ACl}$ & $\mathrm{ACl} 318$ & 1977 & $\begin{array}{l}\text { Building Code Requirements for } \\
\text { Reinforced Concrete }\end{array}$ & reg1.142.r00 & 1978 & $\mathbf{R}$ & & $\mathrm{ACl} 318$ & 1992 \\
\hline 0036 & $\overline{\mathrm{ACl}}$ & $\mathrm{ACl} 318$ & 1977 & $\begin{array}{l}\text { Building Code Requirements for } \\
\text { Reinforced Concrete }\end{array}$ & reg1.143.r00 & 1978 & $P$ & & $\mathrm{ACl} 318$ & 1992 \\
\hline 0037 & $\overline{\mathrm{ACl}}$ & $\mathrm{ACl} 318$ & 1977 & $\begin{array}{l}\text { Building Code Requirements for } \\
\text { Reinforced Concrete }\end{array}$ & reg1.143.ro1 & 1979 & A & & $\mathrm{ACl} 318$ & 1992 \\
\hline
\end{tabular}




\section{Codes and Standards and Other Guidance Cited in Regulatory Documents}

Part A - Industry Consensus Codes and Standards (continued)

en

\begin{tabular}{|c|c|c|c|c|c|c|c|c|c|c|}
\hline Record & Codo & Standard & Standard & $\mathrm{TH}$ o & Dooumont & Doo: & $\begin{array}{l}\text { Accept } \\
\text { Ablinty }\end{array}$ & Comments & Current Voralon & $\begin{array}{l}\text { Curront } \\
\text { Version } \\
\text { Dato: }\end{array}$ \\
\hline 0038 & $\mathrm{ACl}$ & $\mathrm{ACl} 318$ & N/S & $\begin{array}{l}\text { Building Code Requirements for } \\
\text { Reinforced Concrete }\end{array}$ & reg1.35.1.r00 & 1990 & $R$ & & $\mathrm{ACl} 318$ & 1992 \\
\hline 0039 & $\mathrm{ACl}$ & $\mathrm{ACl} 318$ & $\mathrm{~N} / \mathrm{S}$ & $\begin{array}{l}\text { Bullding Code Requirements for } \\
\text { Reinforced Concrete }\end{array}$ & $\operatorname{srp3.8.3}$ & 1981 & $\mathbf{P}$ & & $\mathrm{ACl} 318$ & 1992 \\
\hline 0040 & $\overline{\mathrm{ACl}}$ & $\mathrm{ACl} 318$ & $N / S$ & $\begin{array}{l}\text { Bullding Code Requirements for } \\
\text { Reinforced Concrete }\end{array}$ & $\operatorname{srp3.8.4}$ & 1981 & $P$ & & $\mathrm{ACl} 318$ & 1992 \\
\hline 0041 & $\mathrm{ACl}$ & $\mathrm{ACl} 347$ & $\mathrm{~N} / \mathrm{S}$ & Formwork for Concrete & inm46051 & 1983 & $\mathbf{R}$ & & $\mathrm{ACl} 347$ & 1989 \\
\hline 0042 & $\mathrm{ACl}$ & $\mathrm{ACl} 349$ & 1976 & $\begin{array}{l}\text { Code Requirements for Nuclear } \\
\text { Safety Related Concrete Structures }\end{array}$ & bui79-02.r02 & 1979 & $\mathbf{N}$ & $\begin{array}{l}\text { The use of reduced } \\
\text { factors of safety in the } \\
\text { factored load } \\
\text { approach of } \mathrm{ACl} 349 \text { - } \\
76 \text { has not yet been } \\
\text { accepted by the NRC. }\end{array}$ & ACI 349 & 1990 \\
\hline 0043 & $\overline{\mathrm{ACl}}$ & $\overline{\mathrm{ACl}} 349$ & 1976 & $\begin{array}{l}\text { Code Requirements for Nuclear } \\
\text { Safety Related Concrete Structures }\end{array}$ & bul79-2.ro2 & 1979 & $\mathbf{R}$ & $\begin{array}{l}\text { Use of reduced factors } \\
\text { not accepted }\end{array}$ & $\mathrm{ACI} 349$ & 1990 \\
\hline 0044 & $\mathrm{ACl}$ & $\overline{\mathrm{ACl}} 349$ & $N / S$ & $\begin{array}{l}\text { Code Requirements for Nuclear } \\
\text { Safety Related Concrete Structures }\end{array}$ & glt79-005 & 1979 & $\mathbf{P}$ & & $\mathrm{ACl} 349$ & 1990 \\
\hline 0045 & $\overline{A C l}$ & $\widehat{A C l} 349$ & $N / S$ & $\begin{array}{l}\text { Code Requirements for Nuclear } \\
\text { Safety Related Concrete Structures }\end{array}$ & glt87-002.s01 & 1992 & $\mathbf{N}$ & $\begin{array}{l}\text { Anchorage outliers is } \\
\text { not acceptable to the } \\
\text { staff. }\end{array}$ & $\mathrm{ACl} 349$ & 1990 \\
\hline 0046 & $\overline{\mathrm{ACl}}$ & $\overline{\mathrm{ACl}} 349$ & $N / S$ & $\begin{array}{l}\text { Code Requirements for Nuclear } \\
\text { Safety Related Concrete Structures }\end{array}$ & inm46051 & 1983 & R & & $\mathrm{ACl} 349$ & 1990 \\
\hline 0047 & $\overline{\mathrm{ACl}}$ & $\mathrm{ACl} 349$ & $N / S$ & $\begin{array}{l}\text { Code Requirements for Nuclear } \\
\text { Safety Related Concrete Structures }\end{array}$ & inm47051 & 1983 & $\mathbf{R}$ & & ACI 349 & 1990 \\
\hline 0048 & $\overline{\mathrm{ACl}}$ & $\overline{\mathrm{ACl}} 349$ & $N / S$ & $\begin{array}{l}\text { Code Requirements for Nuclear } \\
\text { Safety Related Concrete Structures }\end{array}$ & inm48051 & 1986 & R & & ACl 349 & 1990 \\
\hline 0049 & $\overline{\mathrm{ACl}}$ & $\mathrm{ACl} 349$ & $N / S$ & $\begin{array}{l}\text { Code Requirements for Nuclear } \\
\text { Safety Related Concrete Structures }\end{array}$ & inm48053 & 1986 & $R$ & & ACI 349 & 1990 \\
\hline 0050 & $\overline{\mathrm{ACl}}$ & $\mathrm{ACl} 349$ & $N / S$ & $\begin{array}{l}\text { Code Requirements for Nuclear } \\
\text { Safety Related Concrete Structures }\end{array}$ & reg1.70.r03 & 1978 & $\mathbf{P}$ & & $\mathrm{ACl} 349$ & 1990 \\
\hline 0051 & $\mathrm{ACl}$ & $\mathrm{ACl} 349$ & 1976 & $\begin{array}{l}\text { Code Requirements for Nuclear } \\
\text { Safety Related Concrete Structures }\end{array}$ & reg1.136.r02 & 1981 & $\mathbf{P}$ & & $\mathrm{ACl} 349$ & 1990 \\
\hline 0052 & $\overline{\mathrm{ACl}}$ & $\mathrm{ACl} 349$ & 1976 & $\begin{array}{l}\text { Code Requirements for Nuclear } \\
\text { Safety Related Concrete Structures }\end{array}$ & reg1.142.r00 & 1978 & $\mathbf{P}$ & Based on $\mathrm{ACl} 318$ & ACl 349 & 1990 \\
\hline
\end{tabular}


Codes and Standards and Other Guidance Cited in Regulatory Documents

Part A - Industry Consensus Codes and Standards (continued)

\begin{tabular}{|c|c|c|c|c|c|c|c|c|c|c|}
\hline $\begin{array}{l}\text { Hocord } \\
\text { No. }\end{array}$ & codos & stindord & striddard & L & boocument & Dor. & poppos & (Commento & conront vor hon & vorinon \\
\hline 0053 & $\mathrm{ACl}$ & $\mathrm{ACl} 349$ & 1979 & $\begin{array}{l}\text { Code Requirements for Nuclear } \\
\text { Safety Related Concrete Structures }\end{array}$ & reg1.142.ro0 & 1978 & $\mathbf{P}$ & Except Appendix B & $\mathrm{ACl} 349$ & 1990 \\
\hline 0054 & $\mathrm{ACl}$ & $\mathrm{ACl} 349$ & 1976 & $\begin{array}{l}\text { Code Requirements for Nuclear } \\
\text { Safety Rolated Concrete Structures }\end{array}$ & srp3.8.3 & 1981 & $\mathbf{P}$ & & $\mathrm{ACl} 349$ & 1990 \\
\hline 0055 & $\mathrm{ACl}$ & $\mathrm{ACl} 349$ & $\mathrm{~N} / \mathrm{S}$ & $\begin{array}{l}\text { Code Requirements for Nuclear } \\
\text { Safety Relarad Concrete Structures }\end{array}$ & srp3.8.4 & 1981 & $\mathbf{P}$ & & ACI 349 & 1990 \\
\hline 0056 & $\mathrm{ACl}$ & ACI 349 & $\mathrm{~N} / \mathrm{S}$ & $\begin{array}{l}\text { Code Requirements for Nuclear } \\
\text { Safoty Related Concrete Structures }\end{array}$ & sip3.8.5 & 1981 & $\mathbf{P}$ & & $\mathrm{ACl} 349$ & 1990 \\
\hline 0057 & $\mathrm{ACl}$ & $\mathrm{ACl} 359$ & $\mathrm{~N} / \mathrm{S}$ & $\begin{array}{l}\text { Code for Concrete Reactor Vessels } \\
\text { and Contalnments }\end{array}$ & glt79-005 & 1979 & $\mathbf{R}$ & $\begin{array}{l}\text { Same as ASME } \\
\text { Section III, Division } 5\end{array}$ & $\mathrm{ACl} 359$ & 1992 \\
\hline 0058 & $\mathbf{A C l}$ & ACI 359 & $\mathrm{~N} / \mathrm{S}$ & $\begin{array}{l}\text { Code for Concrete Reactor Vessels } \\
\text { and Containments }\end{array}$ & inm46051 & 1983 & $\mathbf{R}$ & $\begin{array}{l}\text { Same as ASME } \\
\text { Section III, Division } 4 \\
\end{array}$ & ACI 359 & 1992 \\
\hline 0059 & $\mathrm{ACl}$ & $\mathrm{ACl} 359$ & $N / S$ & $\begin{array}{l}\text { Code for Concrete Reactor Vessels } \\
\text { and Containments }\end{array}$ & inm47051 & 1983 & $\mathbf{A}$ & $\begin{array}{l}\text { Same as ASME } \\
\text { Section III, Division } 3 \\
\end{array}$ & ACI 359 & $19 S 2$ \\
\hline 0060 & $\mathrm{ACl}$ & $\mathrm{ACl} 359$ & $N / S$ & $\begin{array}{l}\text { Code for Concrete Reactor Vessels } \\
\text { and Containments }\end{array}$ & inm47053 & 1983 & $\mathbf{R}$ & $\begin{array}{l}\text { Same as ASME } \\
\text { Section III, Division } 2 \\
\end{array}$ & ACI 359 & 1992 \\
\hline 0061 & $\mathrm{ACl}$ & AC1 359 & $N / S$ & $\begin{array}{l}\text { Code for Concrete Reactor Vessels } \\
\text { and Containments }\end{array}$ & inm48051 & 1986 & $R$ & $\begin{array}{l}\text { Same as ASME } \\
\text { Section III, Division } 1 \\
\end{array}$ & ACI 359 & 1992 \\
\hline 0062 & $\mathrm{ACl}$ & $\mathrm{ACl} 359$ & N/S & $\begin{array}{l}\text { Code for Concrete Reactor Vessols } \\
\text { and Containments }\end{array}$ & inm48053 & 1986 & $\mathbf{R}$ & \begin{tabular}{|l|} 
Same as ASME \\
Section III, Division 0 \\
\end{tabular} & ACI 359 & 1992 \\
\hline 0063 & $\mathrm{ACl}$ & ACl 359 & $N / S$ & $\begin{array}{l}\text { Code for Concrete Reactor Vessels } \\
\text { and Containments }\end{array}$ & inm63050 & 1983 & $\mathbf{R}$ & $\begin{array}{l}\text { Same as ASME } \\
\text { Section lif, Division } 1 \\
\end{array}$ & ACl 359 & 1992 \\
\hline 0064 & $\mathrm{ACl}$ & $\mathrm{ACl} 359$ & 1980 & $\begin{array}{l}\text { Code for Concrete Reactor Vessels } \\
\text { and Containments }\end{array}$ & reg1.136.r02 & 1981 & $\mathbf{P}$ & $\begin{array}{l}\text { Same as ASME } \\
\text { Section III, Division } 2\end{array}$ & $\mathrm{ACl} 359$ & 1992 \\
\hline 0065 & $\mathrm{ACl}$ & ACI 359 & 1980 & $\begin{array}{l}\text { Code for Concrete Reactor Vessels } \\
\text { and Containments }\end{array}$ & reg1.142.ro1 & 1981 & $P$ & $\begin{array}{l}\text { Same as ASME } \\
\text { Section III, Division } 2 \\
\end{array}$ & $\mathrm{ACl} 359$ & 1992 \\
\hline 0066 & $\mathrm{ACl}$ & ACI 359 & 1986 & $\begin{array}{l}\text { Code for Concrete Reactor Vessels } \\
\text { and Containments }\end{array}$ & reg1.35.1.r00 & 1990 & $\mathbf{P}$ & $\begin{array}{l}\text { Same as ASME } \\
\text { Section III, Division } 2 \\
\end{array}$ & ACl 359 & 1992 \\
\hline 0067 & $\mathrm{ACl}$ & $\mathrm{ACl} 359$ & $\mathrm{~N} / \mathrm{S}$ & $\begin{array}{l}\text { Code for Concrete Reactor Vessels } \\
\text { and Containments }\end{array}$ & $\operatorname{sip} 3.8 .3$ & 1981 & $\mathbf{P}$ & $\begin{array}{l}\text { Same as ASME } \\
\text { Section III, Division } 2 \\
\end{array}$ & $\mathrm{ACl} 359$ & 1992 \\
\hline 0068 & $\mathrm{ACl}$ & $\begin{array}{l}\mathrm{ACl} 359 \\
\text { Committee } \\
\end{array}$ & 1975 & $\begin{array}{l}\text { Code for Concrete Reactor Vessels } \\
\text { and Containments }\end{array}$ & reg1.107.ro1 & 1977 & $R$ & $\begin{array}{l}\text { Same as ASME } \\
\text { Section III, Division } 2 \\
\end{array}$ & $\mathrm{ACl} 359$ & 1992 \\
\hline 0069 & F.בI & $\begin{array}{l}\mathrm{ACl} 359 \\
\text { Committee }\end{array}$ & 1980 & $\begin{array}{l}\text { Code for Concrete Resctor Vessels } \\
\text { and Containments }\end{array}$ & reg1.142.r01 & 1981 & $\mathbf{R}$ & $\begin{array}{l}\text { Same as ASME } \\
\text { Section III, Division } 2\end{array}$ & ACI 359 & 1992 \\
\hline
\end{tabular}


Codes and Standards and Other Guidance Cited in Regulatory Documents

Part A - Industry Consensus Codes and Standards (continued)

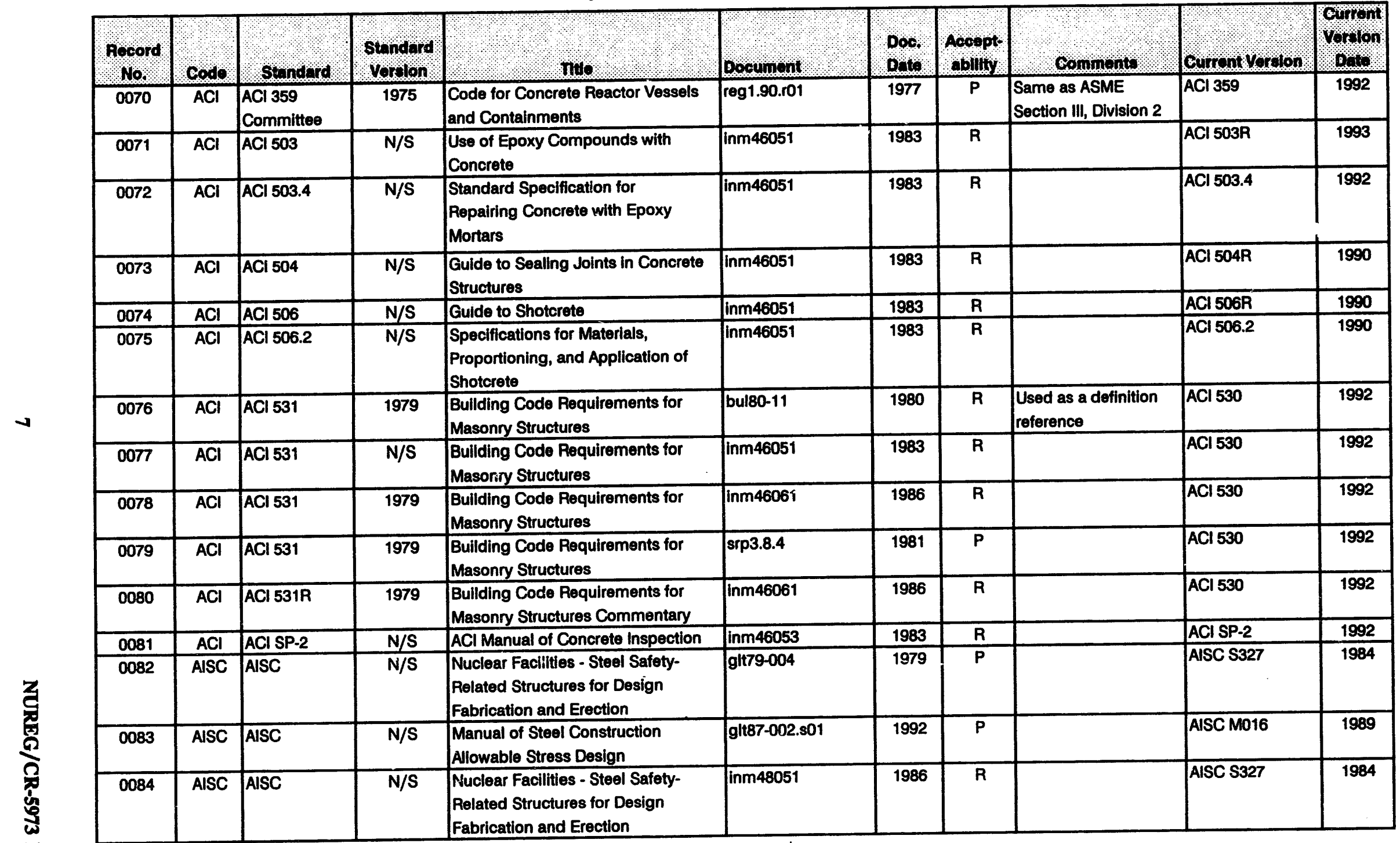




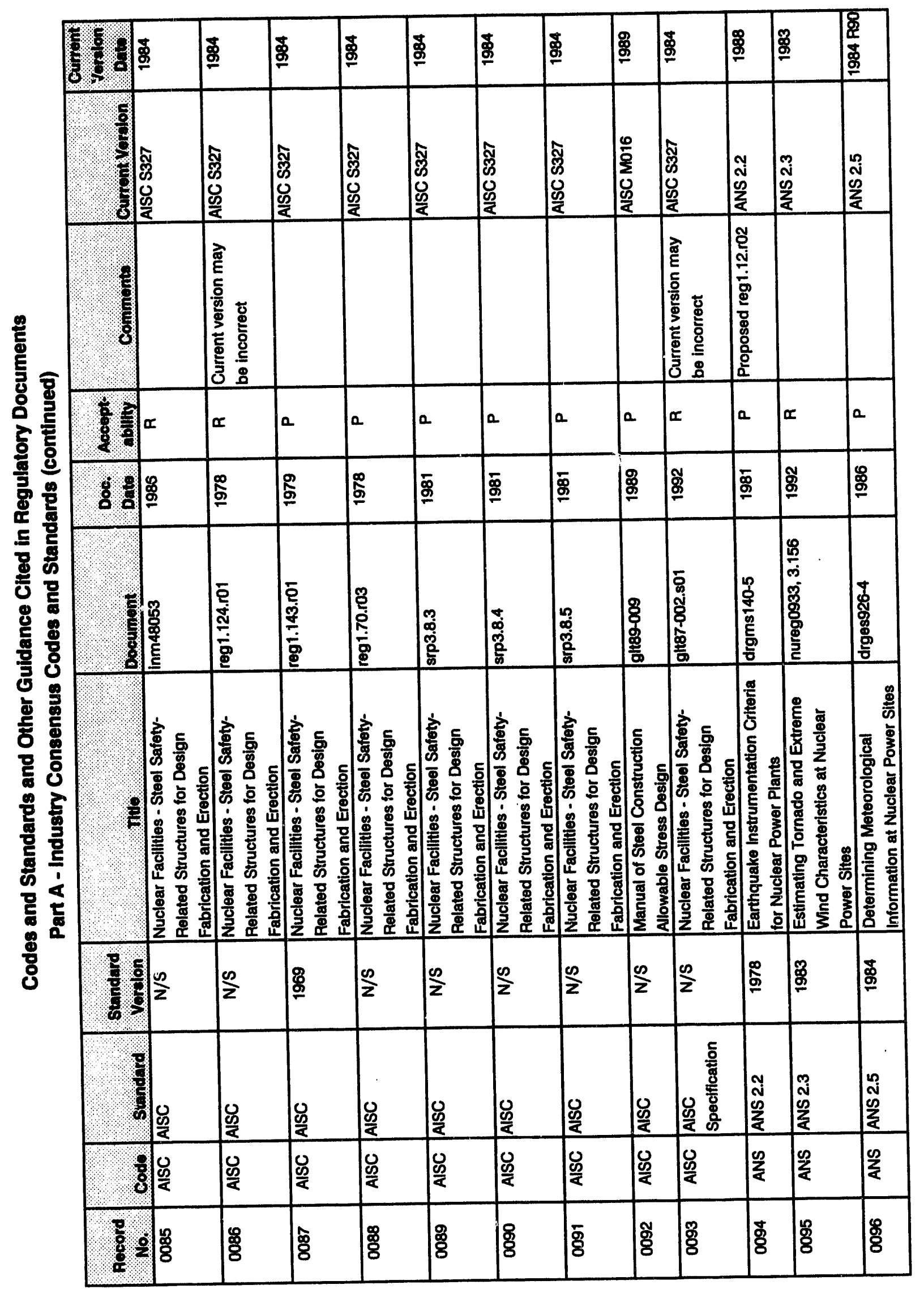


Codes and Standards and Other Guidance Cited in Regulatory Documents

Part A - Industry Consensus Codes and Standards (continued)

\begin{tabular}{|c|c|c|c|c|c|c|c|c|c|c|}
\hline hocord & 0040 & standard & Standard & nor & Dooumont & $\begin{array}{l}\text { Dor. } \\
\text { Dots. }\end{array}$ & accept & Comnonts & Cunrent Vorlon & Qnotiol \\
\hline 0097 & ANS & ANS 2.5 & 1984 & $\begin{array}{l}\text { Determining Meteorological } \\
\text { Information at Nuclear Power Sites }\end{array}$ & inm82207 & 1993 & $\mathbf{R}$ & & ANS 2.5 & 1984 R90 \\
\hline 0098 & ANS & ANS 2.5 & 1984 & $\begin{array}{l}\text { Determining Meteorological } \\
\text { Information at Nuclear Power Sites }\end{array}$ & inm82207 & 1993 & $\mathbf{R}$ & & ANS 2.5 & 1984 R90 \\
\hline 0099 & ANS & ANS 2.5 & 1984 & $\begin{array}{l}\text { Determining Meteorological } \\
\text { Information at Nuclear Power Sites }\end{array}$ & reg3.63.r00 & 1988 & $\mathbf{R}$ & & ANS 2.5 & 1984 R9O \\
\hline 0100 & ANS & ANS 2.8 & 1981 & $\begin{array}{l}\text { Determining Design Basis Flooding } \\
\text { at Power Reactor Sites }\end{array}$ & drgce 406-4 & 1986 & $\mathbf{P}$ & Proposed reg3.48.r01 & ANS 2.8 & 1992 \\
\hline 0101 & ANS & ANS 2.8 & 1981 & $\begin{array}{l}\text { Determining Design Basis Flooding } \\
\text { at Pcwer Reactor Sites }\end{array}$ & reg3.44.r02 & 1989 & $\mathbf{P}$ & $\begin{array}{l}\text { Implied endorsement. } \\
\text { Guidance should be } \\
\text { followed. }\end{array}$ & ANS 2.8 & 1992 \\
\hline 0102 & ANS & ANS 2.8 & 1981 & $\begin{array}{l}\text { Determining Design Basis Flooding } \\
\text { at Power Reactor Sites }\end{array}$ & reg3.48.ro1 & 1989 & $\mathbf{P}$ & $\begin{array}{l}\text { Implied endorsement. } \\
\text { Procedures can be } \\
\text { used. }\end{array}$ & ANS 2.8 & 1992 \\
\hline 0103 & ANS & ANS 2.8 & 1981 & $\begin{array}{l}\text { Determining Design Basis Flooding } \\
\text { at Power Reactor Sites }\end{array}$ & reg4.17.ro1 & 1987 & $\mathbf{R}$ & $\begin{array}{l}\text { Referred to as } \\
\text { ANSI/ANS } 2.8 \text { N170 }\end{array}$ & ANS 2.8 & 1992 \\
\hline 0104 & ANS & ANS 2.19 & 1981 & $\begin{array}{l}\text { Guidelines for Establishing Site- } \\
\text { Related Parameters for Site } \\
\text { Selection and Design of an } \\
\text { Independent Spent Fuel Storage } \\
\text { Installation (Water-Pool Type) } \\
\end{array}$ & reg3.49.ro0 & 1981 & $\mathbf{R}$ & & ANS 2.19 & $1981 \mathrm{R} 90$ \\
\hline 0105 & ANS & ANS 2.19 & 1981 & $\begin{array}{l}\text { Guidelines for Establishing Site- } \\
\text { Related Parameters for Site } \\
\text { Selection and Design of an } \\
\text { Independent Spent Fuel Storage } \\
\text { Installation (Water-Pool Type) }\end{array}$ & reg3.60.r00 & 1987 & $\mathbf{R}$ & & ANS 2.19 & $1981 \mathrm{R} 90$ \\
\hline 0106 & ANS & ANS 3.1 & 1978 & $\begin{array}{l}\text { Selection, Qualification, and } \\
\text { Training of Persorinel for Nuclear } \\
\text { Power Plants }\end{array}$ & cir80-21 & 1980 & $\mathbf{P}$ & $\begin{array}{l}\text { Must meet training and } \\
\text { qualification } \\
\text { requirements }\end{array}$ & ANS 3.1 & 1993 \\
\hline 0107 & ANS & ANS 3.1 & $\mathrm{~N} / \mathrm{S}$ & $\begin{array}{l}\text { Selection, Qualification and } \\
\text { Training of Personnel for Nuclear } \\
\text { Power Plants }\end{array}$ & ght83-012.A & 1983 & $\mathbf{R}$ & $\begin{array}{l}\text { Must meet training and } \\
\text { qualification } \\
\text { requirements }\end{array}$ & ANS 3.1 & 1993 \\
\hline
\end{tabular}


Codes and Standards and Other Guidance Cited in Regulatory Documents

Part A - Industry Consensus Codes and Standards (continued)

\section{Part A - Industry Consensus Codes and standards (continued}

\begin{tabular}{|c|c|c|c|c|c|c|c|c|c|c|}
\hline $\begin{array}{l}\text { Rocord } \\
10 .\end{array}$ & Codo & Stondards & $\begin{array}{l}\text { Standard } \\
\text { Youlon }\end{array}$ & ( & Dorumont & $\begin{array}{l}000 \\
0,1 \%\end{array}$ & Acoopts & Comments & Curront Vordon & Corront \\
\hline 0108 & ANS & ANS 3.1 & 1981 & $\begin{array}{l}\text { Selection, Qualification, and } \\
\text { Training of Personnel for Nucle.s. } \\
\text { Power Plants }\end{array}$ & inm2525/117 & 1992 & $\mathbf{R}$ & & ANS 3.1 & 1993 \\
\hline 0109 & ANS & ANS 3.1 & 1978 & $\begin{array}{l}\text { Solection, Qualification, and } \\
\text { Training of Personnel for Nuclear } \\
\text { Power Plants }\end{array}$ & inm35701 & 1992 & $\mathbf{R}$ & & ANS 3.1 & 1993 \\
\hline 0110 & ANS & ANS 3.1 & 1978 & $\begin{array}{l}\text { Selection, Qualification, and } \\
\text { Training of Personnel for Nuclear } \\
\text { Power Plants }\end{array}$ & inm37001 & 1992 & $\mathbf{R}$ & & ANS 3.1 & 1993 \\
\hline 0111 & ANS & ANS 3.1 & 1978 & $\begin{array}{l}\text { Selection, Qualification, and } \\
\text { Training of Personnel for Nuclear } \\
\text { Power Plants }\end{array}$ & inm40703 & 1992 & $\mathbf{R}$ & & ANS 3.1 & 1993 \\
\hline 0112 & ANS & ANS 3.1 & 1987 & $\begin{array}{l}\text { Selection, Qualification, and } \\
\text { Training of Personnel for Nuclear } \\
\text { Power Plants }\end{array}$ & inm40703 & 1992 & $\mathbf{R}$ & & ANS 3.1 & 1993 \\
\hline 0113 & ANS & ANS 3.1 & 1978 & $\begin{array}{l}\text { Selection, Qualification, and } \\
\text { Training of Personnel for Nuclear } \\
\text { Power Plants }\end{array}$ & inm61700 & 1983 & $\mathbf{R}$ & & ANS 3.1 & 1993 \\
\hline 0114 & ANS & ANS 3.1 & 1978 & $\begin{array}{l}\text { Selection, Qualification, and } \\
\text { Training of Personnel for Nuclear } \\
\text { Power Plants }\end{array}$ & inm61701 & 1985 & $\mathbf{R}$ & & ANS 3.1 & 1993 \\
\hline 0115 & ANS & ANS 3.1 & 1981 & $\begin{array}{l}\text { Selection, Qualification, and } \\
\text { Training of Personnel for Nuclear } \\
\text { Power Plants }\end{array}$ & inm70323 & 1985 & $\mathbf{R}$ & & ANS 3.1 & 1993 \\
\hline 0116 & ANS & ANS 3.1 & 1981 & $\begin{array}{l}\text { Selection, Qualification, and } \\
\text { Training of Personnel for Nuclear } \\
\text { Power Plants }\end{array}$ & inm82102 & 1993 & $\mathbf{R}$ & & ANS 3.1 & 1993 \\
\hline 0117 & ANS & ANS 3.1 & 1981 & $\begin{array}{l}\text { Selection, Qualification, and } \\
\text { Training of Personnel for Nuclear } \\
\text { Power Plants }\end{array}$ & inm82301 & 1993 & $\mathbf{R}$ & & ANS 3.1 & 1993 \\
\hline 0118 & ANS & ANS 3.1 & 1981 & $\begin{array}{l}\text { Selection, Qualification, and } \\
\text { Training of Personnel for Nuclear } \\
\text { Power Piants }\end{array}$ & inm82701 & 1993 & $\mathbf{R}$ & & ANS 3.1 & 1993 \\
\hline
\end{tabular}


Codes and Standards and Other Guidance Cited in Regulatory Documents

Part A - Industry Consensus Codes and Standards (continued)

\begin{tabular}{|c|c|c|c|c|c|c|c|c|c|c|}
\hline $\begin{array}{l}\text { Hocord } \\
\text { ro. }\end{array}$ & Codo & Strandard & $\begin{array}{l}\text { Standard } \\
\text { vorolon }\end{array}$ & Tlolo, & Dooumont & Doc. & $\begin{array}{l}\text { Aocepts } \\
\text { ablilly }\end{array}$ & Comments & Cunrent Vordon & $\begin{array}{l}\text { oproth } \\
\text { proton }\end{array}$ \\
\hline 0119 & ANS & ANS 3.1 & 1981 & $\begin{array}{l}\text { Selection, Qualification and } \\
\text { Training of Personnel for Nuclear } \\
\text { Power Plants }\end{array}$ & inm83522 & 1991 & $R$ & & ANS 3.1 & 1993 \\
\hline 0120 & ANS & ANS 3.1 & 1981 & $\begin{array}{l}\text { Selection, Qualification and } \\
\text { Training of Personnel for Nuclear } \\
\text { Power Plants }\end{array}$ & inm83523 & 1984 & $\mathbf{R}$ & & ANS 3.1 & 1993 \\
\hline 0121 & ANS & ANS 3.1 & 1981 & $\begin{array}{l}\text { Selection, Qualification and } \\
\text { Training of Personnel for Nuclear } \\
\text { Power Plants }\end{array}$ & inm83722 & 1984 & $\mathbf{R}$ & & ANS 3.1 & 1993 \\
\hline 0122 & ANS & ANS 3.1 & 1981 & $\begin{array}{l}\text { Selection, Qualification and } \\
\text { Training of Personnel for Nuclear } \\
\text { Power Plants }\end{array}$ & inm83723 & 1991 & $\vec{R}$ & & ANS 3.1 & 1993 \\
\hline 0123 & ANS & ANS 3.1 & 1987 & $\begin{array}{l}\text { Selection, Qualification and } \\
\text { Training of Personnel for Nuclear } \\
\text { Power Plants }\end{array}$ & inm83750 & 1988 & $\mathbf{R}$ & & ANS 3.1 & 1993 \\
\hline 0124 & ANS & ANS 3.1 & 1981 & $\begin{array}{l}\text { Selection, Qualification and } \\
\text { Training of Personnel for Nuclear } \\
\text { Power Plants }\end{array}$ & nureg0933, 1.I.A.2 & 1987 & $\mathbf{R}$ & & ANS 3.1 & 1993 \\
\hline 0125 & ANS & ANS 3.1 & 1978 & $\begin{array}{l}\text { Selection, Qualification and } \\
\text { Training of Personnel for Nuclear } \\
\text { Power Plants }\end{array}$ & reg1.149.r00 & 1981 & $\bar{R}$ & & ANS 3.1 & 1993 \\
\hline 0126 & ANS & ANS 3.1 & 1978 & $\begin{array}{l}\text { Selection, Qualification and } \\
\text { Training of Personnel for Nuclear } \\
\text { Power Plants } \\
\end{array}$ & reg1.8.r02 & 1987 & $\mathbf{R}$ & & ANS 3.1 & 1993 \\
\hline 录 & ANS & ANS 3.1 & 1981 & $\begin{array}{l}\text { Selection, Qualification and } \\
\text { Training of Personnel for Nuclear } \\
\text { Power Plants }\end{array}$ & reg1.8.r02 & 1987 & $\mathbf{P}$ & $\begin{array}{l}\text { With exceptions and } \\
\text { conditions }\end{array}$ & ANS 3.1 & 1993 \\
\hline$\frac{\pi}{2}$ & ANS & ANS 3.1 & 1978 & $\begin{array}{l}\text { Selection, Qualification and } \\
\text { Training of Personnel for Nuclear } \\
\text { Power Plants }\end{array}$ & $\operatorname{sip} 12.5$ & 1981 & $\mathbf{P}$ & $\begin{array}{l}\text { As related to selection, } \\
\text { qualification, and } \\
\text { training criteria }\end{array}$ & ANS 3.1 & 1993 \\
\hline $\begin{array}{l}0 \\
\omega \\
0\end{array}$ & ANS & ANS 3.1 & 1978 & $\begin{array}{l}\text { Selection, Qualification and } \\
\text { Training of Personnel for Nuclear } \\
\text { Power Plants }\end{array}$ & $\operatorname{srp} 13.2 .1$ & 1981 & $\mathbf{R}$ & & ANS 3.1 & 1993 \\
\hline
\end{tabular}


Codes and Standards and Other Guidance Cited in Regulatory Documents

Part A - Industry Consensus Codes and Standards (continued)

T. Th

\begin{tabular}{|c|c|c|c|c|c|c|c|c|c|c|}
\hline $\begin{array}{l}\text { Rocord } \\
\text { No. }\end{array}$ & codo & Standard & $\begin{array}{l}\text { Standard } \\
\text { Yorlon }\end{array}$ & २ै, THo & Bocument & $\begin{array}{l}000 . \\
\text { poto }\end{array}$ & $\begin{array}{l}\text { Acopt } \\
\text { Nollity }\end{array}$ & Comments & Gurront Vordon & $\begin{array}{l}\text { Current } \\
\text { vorplon } \\
\text { Deto }\end{array}$ \\
\hline 0130 & ANS & ANS 3.1 & $\mathrm{~N} / \mathrm{S}$ & $\begin{array}{l}\text { Selection, Qualification and } \\
\text { Training of Personnel for Nuclear } \\
\text { Power Plants }\end{array}$ & sip 13.2.2 & 1981 & $P$ & $\begin{array}{l}\text { Sections 5.1, 5.3, 5.4, } \\
\text { and } 5.5\end{array}$ & ANS 3.1 & 1993 \\
\hline 0131 & ANS & ANS 3.1 & 1978 & $\begin{array}{l}\text { Selection, Qualification and } \\
\text { Training of Personnel for Nuclear } \\
\text { Power Plants }\end{array}$ & srp13.4 & 1981 & $\mathbf{P}$ & Section 4.7 & ANS 3.1 & 1993 \\
\hline 0132 & ANS & ANS 3.1 & 1979 & $\begin{array}{l}\text { Selection, Qualification and } \\
\text { Training of Personnel for Nuclear } \\
\text { Power Plants }\end{array}$ & $\operatorname{srp13.5.1}$ & 1981 & $\mathbf{P}$ & $\begin{array}{l}\text { Section 4.4.6 of ANS } \\
\text { 3.1 Draft Revision }\end{array}$ & ANS 3.1 & 1993 \\
\hline 0133 & ANS & ANS 3.1 & 1978 & $\begin{array}{l}\text { Selection, Qualification and } \\
\text { Training of Personnel for Nuclear } \\
\text { Power Plants }\end{array}$ & $\operatorname{srp} 17.1$ & 1981 & $\mathbf{P}$ & $\begin{array}{l}\text { Scction } 4.4 .5 \text { as } \\
\text { endorsed by Reg. } \\
\text { Guide } 1.8\end{array}$ & ANS 3.1 & 1993 \\
\hline 0134 & ANS & ANS 3.1 & 1981 & $\begin{array}{l}\text { Selection, Qualification and } \\
\text { Training of Personnel for Nuclear } \\
\text { Power Plants }\end{array}$ & sts4-ge5.5 & 1991 & $\mathbf{P}$ & & ANS 3.1 & 1993 \\
\hline 0135 & ANS & ANS 3.1 & 1981 & $\begin{array}{l}\text { Selection, Quallication, and } \\
\text { Training of Personnel for Nuclear } \\
\text { Power Plants }\end{array}$ & stsb\&wB5.5 & 1991 & A & & ANS 3.1 & 1993 \\
\hline 0136 & ANS & ANS 3.1 & 1981 & $\begin{array}{l}\text { Selection, Qualification, and } \\
\text { Training of Personnel for Nuclear } \\
\text { Power Plants }\end{array}$ & stsceB5.5 & 1991 & $A$ & & ANS 3.1 & 1993 \\
\hline 0137 & ANS & ANS 3.1 & 1981 & $\begin{array}{l}\text { Selection, Qualification, and } \\
\text { Training of Personnel for Nuclear } \\
\text { Power Plants }\end{array}$ & sts4-geB3.8.7 & 1991 & A & & ANS 3.1 & 1993 \\
\hline 0138 & ANS & ANS 3.1 & 1981 & $\begin{array}{l}\text { Selection, Qualification, and } \\
\text { Training of Personnel for Nuclear } \\
\text { Power Plants }\end{array}$ & sts6-geB5.5 & 1991 & $\bar{A}$ & & ANS 3.1 & 1993 \\
\hline 0139 & ANS & ANS 3.1 & 1981 & $\begin{array}{l}\text { Selection, Qualification, and } \\
\text { Training of Personnel for Nuclear } \\
\text { Power Plants }\end{array}$ & stswstB5.5 & 1991 & A & & ANS 3.1 & 1993 \\
\hline 0140 & ANS & ANS 3.1 & $N / S$ & $\begin{array}{l}\text { Solection, Qualification and } \\
\text { Training of Personnel for Nuclear } \\
\text { Power Plants }\end{array}$ & inm99100 & 1992 & $\mathbf{R}$ & & ANS 3.1 & 1993 \\
\hline
\end{tabular}

Power Plants 
Codes and Standards and Other Guidance Cited in Regulatory Documents

Part A - Industry Consensus Codes and Standards (continued)

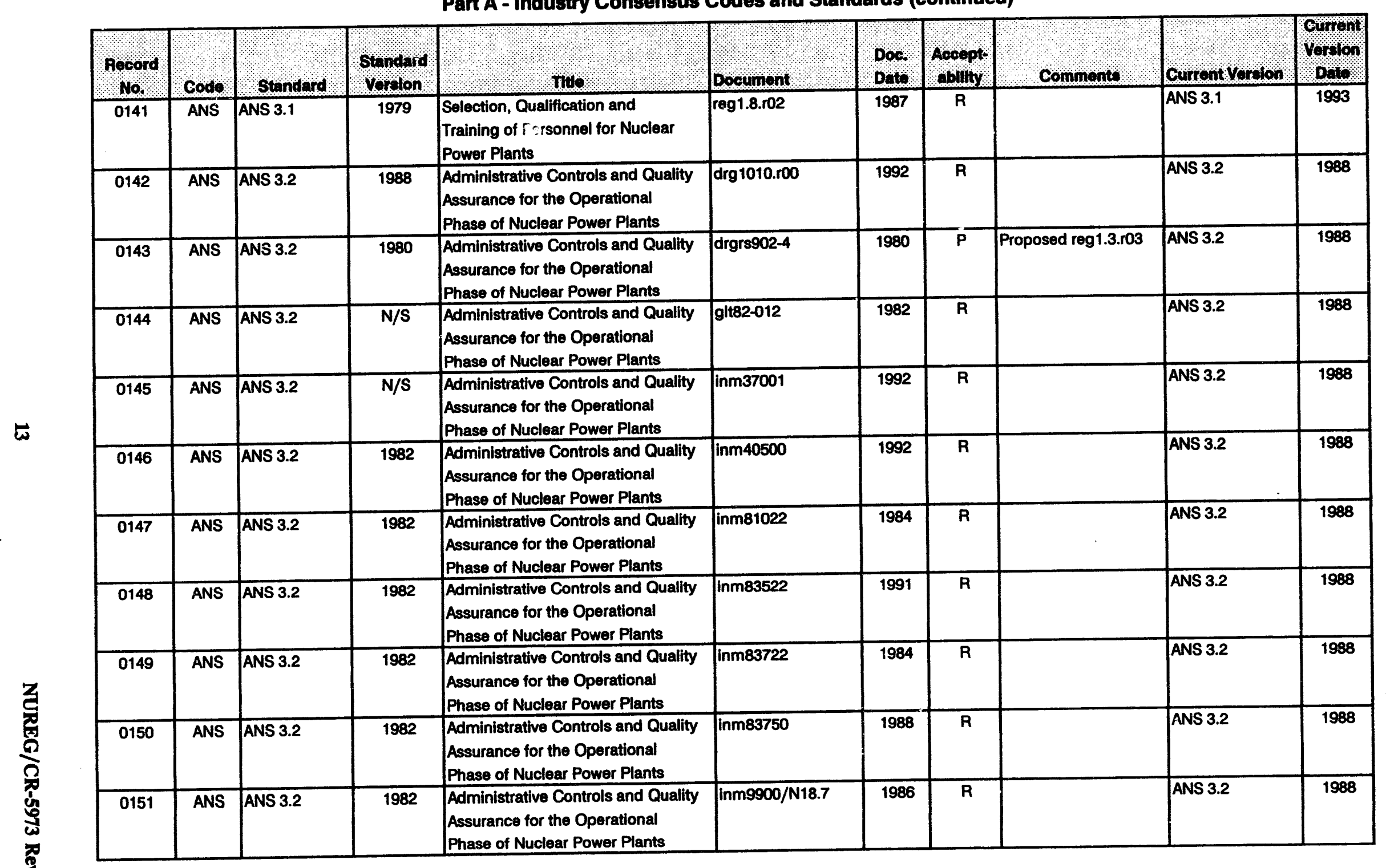


Codes and Standards and Other Guidance Cited in Regulatory Documents

Part A - Industry Consensus Codes and Standards (continued)

\begin{tabular}{|c|c|c|c|c|c|c|c|c|c|c|}
\hline $\begin{array}{l}\text { Hocord } \\
\text { No. }\end{array}$ & Codo & Standard & Stundand & In_. & Dooumont & Dos. & $\begin{array}{l}\text { Aocepte } \\
\text { ablity }\end{array}$ & Comments. & Curront Vorolon & Qunent \\
\hline 0152 & ANS & ANS 3.2 & 1982 & $\begin{array}{l}\text { Administrative Controls and Quality } \\
\text { Assurance for the Operational } \\
\text { Phase of Nuclear Power Plants }\end{array}$ & inm9900/OPS & 1991 & $\mathbf{R}$ & & ANS 3.2 & 1988 \\
\hline 0153 & ANS & ANS 3.2 & 1988 & $\begin{array}{l}\text { Administrative Controls and Quality } \\
\text { Assurance for the Operational } \\
\text { Phase of Nuclear Power Plants }\end{array}$ & inm9900/OPS & 1991 & $\mathbf{R}$ & & ANS 3.2 & 1988 \\
\hline 0154 & ANS & ANS 3.2 & $N / S$ & $\begin{array}{l}\text { Administrative Controls and Quality } \\
\text { Assurance for the Operational } \\
\text { Phase of Nuclear Power Plants }\end{array}$ & not82-03 & 1982 & $\mathbf{R}$ & & ANS 3.2 & 1988 \\
\hline 0155 & ANS & ANS 3.2 & $N / S$ & $\begin{array}{l}\text { Administrative Controls and Quality } \\
\text { Assurance for the Operational } \\
\text { Phase of Nuclear Power Plants }\end{array}$ & not84-51 & 1984 & $\mathbf{R}$ & $\begin{array}{l}\text { Reg. } 1.33 \text { Rev. } 2 \\
\text { Endorses draft revision }\end{array}$ & ANS 3.2 & 1988 \\
\hline 0156 & ANS & ANS 3.2 & 1982 & $\begin{array}{l}\text { Administrative Controls and Quality } \\
\text { Assurance for the Operational } \\
\text { Phase of Nuclear Power Plants }\end{array}$ & not85-90 & 1985 & $\mathbf{R}$ & & ANS 3.2 & 1988 \\
\hline 0157 & ANS & ANS 3.2 & $\mathrm{~N} / \mathrm{S}$ & $\begin{array}{l}\text { Administrative Controls and Quality } \\
\text { Assurance for the Operational } \\
\text { Phase of Nuclear Power Plants }\end{array}$ & not86-64.s01 & 1987 & $\mathbf{R}$ & & ANS 3.2 & 1988 \\
\hline 0158 & ANS & ANS 3.2 & 1976 & $\begin{array}{l}\text { Administrative Controls and Quality } \\
\text { Assurance for the Operational } \\
\text { Phase of Nuclear Power Plants }\end{array}$ & not86-75 & 1986 & $\mathbf{R}$ & & ANS 3.2 & 1988 \\
\hline 0159 & ANS & ANS 3.2 & 1982 & $\begin{array}{l}\text { Administrative Controls and Quality } \\
\text { Assurance for the Operational } \\
\text { Phase of Nuclear Power Plants }\end{array}$ & not86-75 & 1986 & $\mathbf{R}$ & & ANS 3.2 & 1988 \\
\hline 0160 & ANS & ANS 3.2 & $N / S$ & $\begin{array}{l}\text { Administrative Controls and Quality } \\
\text { Assurance for the Operational } \\
\text { Phase of Nuclear Power Plants }\end{array}$ & nureg0737 & 1980 & $\mathbf{P}$ & & ANS 3.2 & 1988 \\
\hline 0161 & ANS & ANS 3.2 & $N / S$ & $\begin{array}{l}\text { Administrative Controls and Quality } \\
\text { Assurance for the Operational } \\
\text { Phase of Nuclear Power Plants }\end{array}$ & nureg0933, 3.75 & 1990 & R & & ANS 3.2 & 1988 \\
\hline 0162 & ANS & ANS 3.2 & 1982 & $\begin{array}{l}\text { Administrative Controls and Quality } \\
\text { Assurance for the Operational } \\
\text { Phase of Nuclear Power Plants }\end{array}$ & reg1.28.r03 & 1985 & $\mathbf{R}$ & $\begin{array}{l}\text { Expected to be } \\
\text { endorsed by Reg. } \\
\text { Guide } 1.33 \text {, Rev. } 3\end{array}$ & ANS 3.2 & 1988 \\
\hline
\end{tabular}


Codes and Standards and Other Guidance Cited in Regulatory Documents

Part A - Industry Consensus Codes and Standards (continued)

\begin{tabular}{|c|c|c|c|c|c|c|c|c|c|c|}
\hline $\begin{array}{l}\text { Rocord } \\
\text { No. }\end{array}$ & Code & Standerd & $\begin{array}{l}\text { Standard } \\
\text { Vorolon }\end{array}$ & nuo,, & Booumont & $\begin{array}{l}\text { Doo. } \\
\text { Dota }\end{array}$ & $\begin{array}{l}\text { Aocopts } \\
\text { ability }\end{array}$ & Comments. & Cunont vorion & vorton \\
\hline 0163 & ANS & ANS 3.2 & 1976 & $\begin{array}{l}\text { Administrative Controls and Quality } \\
\text { Assurance for the Operational } \\
\text { Phase of Nuclear Power Plants }\end{array}$ & reg1.33.ro2 & 1978 & $P$ & Subject to conditions & ANS 3.2 & 1988 \\
\hline 0164 & ANS & ANS 3.2 & N/S & $\begin{array}{l}\text { Administrative Controls and Quality } \\
\text { Assurance for the Operational } \\
\text { Phase of Nuclear Power Piants }\end{array}$ & $\operatorname{sip} 13.4$ & 1981 & $\mathbf{P}$ & $\begin{array}{l}\text { Implied endorsement - } \\
\text { cited in SRP as both } \\
\text { ANSI and ANS } 3.2\end{array}$ & ANS 3.2 & 1988 \\
\hline 0165 & ANS & ANS 3.2 & $\mathrm{~N} / \mathrm{S}$ & $\begin{array}{l}\text { Administrative Controls and Quality } \\
\text { Assurance for the Operational } \\
\text { Phase of Nuclear Power Plants }\end{array}$ & $\operatorname{srp} 13.5 .1$ & 1981 & $\bar{P}$ & Section 5.2 & ANS 3.2 & 1988 \\
\hline 0166 & ANS & ANS 3.2 & 1982 & $\begin{array}{l}\text { Administrative Controls and Quality } \\
\text { Assurance for the Operational } \\
\text { Phase of Nuclear Power Plants }\end{array}$ & $\operatorname{sip} 13.5 .2$ & 1985 & $\mathbf{P}$ & Section 5.3 & ANS 3.2 & 1988 \\
\hline 0167 & ANS & ANS 3.3 & 1982 & Security for Nuclear Power Plants & inm81018 & 1984 & $\bar{R}$ & $\begin{array}{l}\text { ANS } 3.3 \text { withdrawn } \\
1992\end{array}$ & Withdrawn & $n / a$ \\
\hline 0168 & ANS & ANS 3.3 & 1982 & Security for Nuclear Power Plants & inm81501 & 1984 & $\mathbf{R}$ & $\begin{array}{l}\text { ANS } 3.3 \text { withdrawn } \\
1992\end{array}$ & Withdrawn & $n / a$ \\
\hline 0169 & ANS & ANS 3.3 & $\mathrm{~N} / \mathrm{S}$ & Security for Nuclear Power Plants & not86-83 & 1986 & $\mathbf{R}$ & $\begin{array}{l}\text { ANS } 3.3 \text { withdrawn } \\
1992\end{array}$ & Withdrawn & $n / a$ \\
\hline 0170 & ANS & ANS 3.3 & 1982 & Security for Nuciear Power Plants & not88-91 & 1988 & $\mathbf{R}$ & $\begin{array}{l}\text { ANS } 3.3 \text { withdrawn } \\
1992\end{array}$ & Withdrawn & $n / a$ \\
\hline 0171 & ANS & ANS 3.4 & N/S & $\begin{array}{l}\text { Medical Certification and } \\
\text { Monitoring of Personnel Requiring } \\
\text { Operator Licenses for Nuclear } \\
\text { Power Plants } \\
\end{array}$ & git82-013 & 1982 & $\bar{P}$ & $\begin{array}{l}\text { Incorrectly typed as } \\
\text { ANS } 546\end{array}$ & ANS 3.4 & $1983 R 88$ \\
\hline 0172 & ANS & ANS 3.4 & 1983 & $\begin{array}{l}\text { Medical Certification and } \\
\text { Monitoring of Personnel Requiring } \\
\text { Operator Licenses for Nuclear }\end{array}$ & not84-03 & 1984 & $\mathbf{R}$ & & ANS 3.4 & 1983 R88 \\
\hline 0173 & ANS & ANS 3.4 & 1983 & $\begin{array}{l}\text { Medical Certification and } \\
\text { Monitoring of Personnel Requiring } \\
\text { Operator Licenses for Nuclear } \\
\text { Power Plants }\end{array}$ & not91-08 & 1991 & R & & ANS 3.4 & $1983 \mathrm{R} 88$ \\
\hline
\end{tabular}


Codes and Standards and Other Guidance Cited in Regulatory Documents

Part A - Industry Consensus Codes and Standards (continued)

\begin{tabular}{|c|c|c|c|c|c|c|c|c|c|c|}
\hline $\begin{array}{l}\text { Fecord } \\
\text { No. }\end{array}$ & Code & Standerd & $\begin{array}{l}\text { Standand } \\
\text { Verdon }\end{array}$ & The, & Docoument. & $\begin{array}{l}\text { Doo. } \\
\text { Date. }\end{array}$ & Aooppe & Comments & Gurrent Voran & Quntert \\
\hline 0174 & ANS & ANS 3.4 & 1983 & $\begin{array}{l}\text { Medical Certification and } \\
\text { Monitoring of Personnel Requiring } \\
\text { Operator Licenses for Nuclear } \\
\text { Power Plants }\end{array}$ & reg1.134.r02 & 1987 & $\mathbf{R}$ & & ANS 3.4 & 1983 P88 \\
\hline 0175 & ANS & ANS 3.5 & 1979 & $\begin{array}{l}\text { Nuclear Power Plant Simulators for } \\
\text { Use in Operator Training }\end{array}$ & nureg0933, 1.I.A.4 & 1987 & $\mathbf{R}$ & & ANS 3.5 & 1985 \\
\hline 0176 & ANS & ANS 3.5 & 1981 & $\begin{array}{l}\text { Nuclear Power Piant Simulators for } \\
\text { Use in Operator Training }\end{array}$ & nureg0933, 1.I.A.4 & 1987 & $\mathbf{R}$ & & ANS 3.5 & 1985 \\
\hline 0177 & ANS & ANS 3.5 & 1985 & $\begin{array}{l}\text { Nuclear Power Plant Simulators for } \\
\text { Use in Operator Training }\end{array}$ & reg1.149.ro1 & 1987 & $\mathbf{P}$ & Subject to conditions & ANS 3.5 & 1985 \\
\hline 0178 & ANS & ANS 4.5 & 1980 & $\begin{array}{l}\text { Criteria for Accident Monitoring } \\
\text { Functions in Light Water Cooled } \\
\text { Reactors }\end{array}$ & reg1.97.ro3 & 1983 & $\mathbf{P}$ & Subject to conditions & ANS 4.5 & $1980 \mathrm{PB8}$ \\
\hline 0179 & ANS & ANS 4.5 & 1980 & $\begin{array}{l}\text { Criteria for Accident Monitoring } \\
\text { Functions in Light Water Cooled } \\
\text { Reactors }\end{array}$ & $\operatorname{sip} 7.5$ & 1984 & $\mathbf{P}$ & & ANS 4.5 & $1980 \mathrm{R} 88$ \\
\hline 0180 & ANS & ANS 5.1 & 1971 & $\begin{array}{l}\text { Docay Hoat Power in Light Water } \\
\text { Reactors }\end{array}$ & 10 ctr50 App. K & 1993 & $\mathbf{P}$ & Proposed standard & ANS 5.1 & 1979 R85 \\
\hline 0181 & ANS & ANS 5.1 & 1979 & $\begin{array}{l}\text { Decay Hoat Power in Light Water } \\
\text { Peactors }\end{array}$ & nureg0933, 2.C-5 & 1986 & $\mathbf{A}$ & & ANS 5.1 & 1979 R85 \\
\hline 0182 & ANS & ANS 5.1 & 1979 & $\begin{array}{l}\text { Decay Heat Power in Light Water } \\
\text { Reactors }\end{array}$ & reg1.157.roo & 1989 & $\mathbf{R}$ & & ANS 5.1 & 1979 R85 \\
\hline 0183 & ANS & ANS 5.1 & 1979 & $\begin{array}{l}\text { Decay Hoat Power in Light Water } \\
\text { Reactors }\end{array}$ & reg3.54.100 & 1984 & $\mathbf{R}$ & & ANS 5.1 & 1979 P85 \\
\hline 0184 & ANS & ANS 5.1 & 1979 & $\begin{array}{l}\text { Decay Hoat Power in Light Water } \\
\text { Reactors }\end{array}$ & reg3.60.ro0 & 1987 & $\mathbf{R}$ & & ANS 5.1 & 1979 R85 \\
\hline 0185 & ANS & ANS 6.3.1 & 1980 & $\begin{array}{l}\text { Program for Testing Radiation } \\
\text { Shiolds in Light Water Roactors } \\
\text { (LWR) }\end{array}$ & inm83521 & 1984 & $\mathbf{R}$ & & ANS 6.3.1 & 1987 \\
\hline 0186 & ANS & ANS 6.4 & 1977 & $\begin{array}{l}\text { Guidelines on the Nuctear Analysis } \\
\text { and Design of Concrete Radiation } \\
\text { Shielding for Nuclear Power Plants }\end{array}$ & drgem805-5 & 1979 & $\mathbf{P}$ & & ANS 6.4 & 1985 \\
\hline
\end{tabular}


Codes and Standards and Other Guidance Cited in Regulatory Documents

Part A - Industry Consensus Codes and Standards (continued)

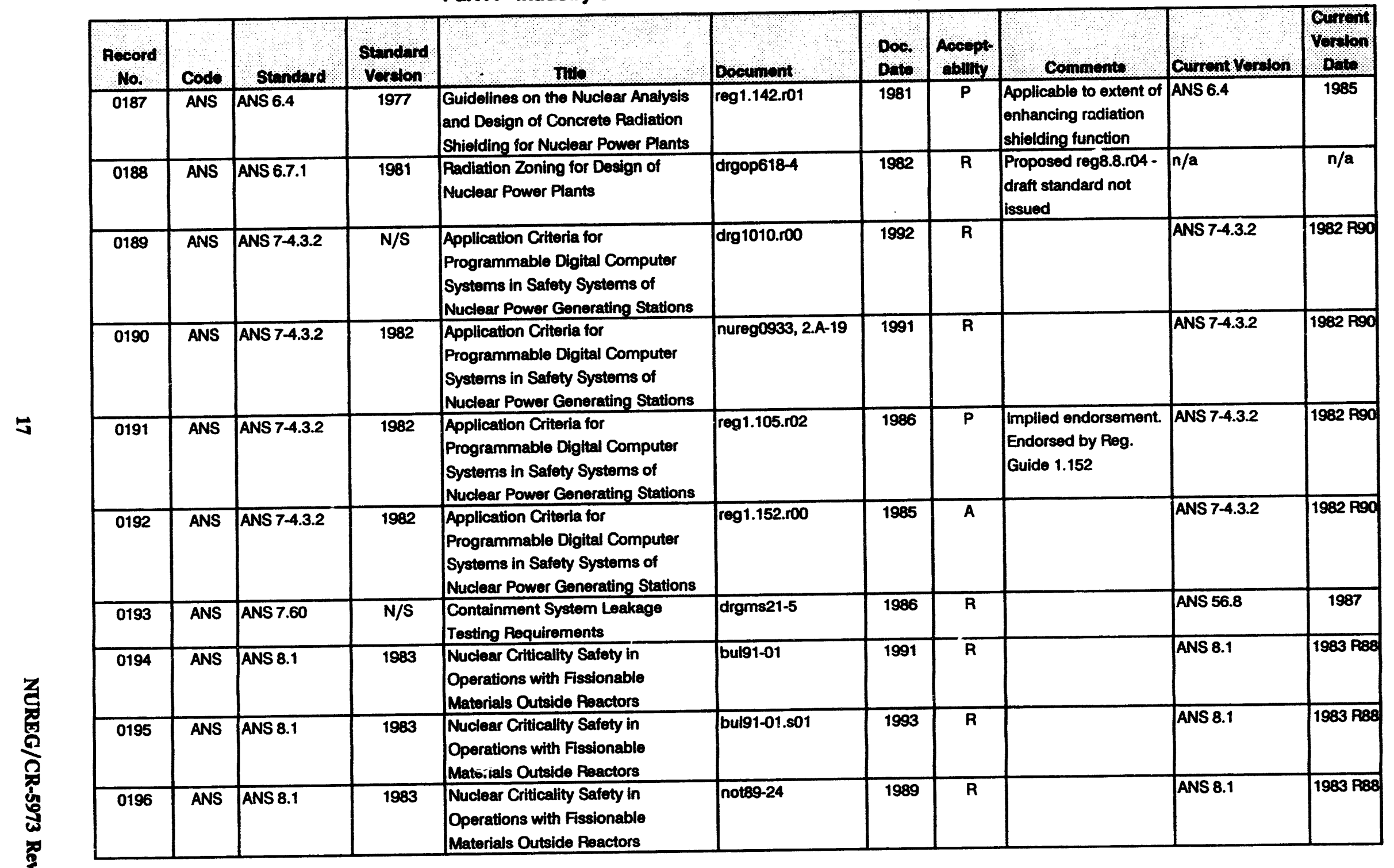


Codes and Standards and Other Guidance Cited in Regulatory Documents

Part A - Industry Consensus Codes and Standards (continued)

\begin{tabular}{|c|c|c|c|c|c|c|c|c|c|c|}
\hline Hocord & Code & Sthendard & $\begin{array}{l}\text { Standard } \\
\text { Vordion }\end{array}$ & This & Document & $\begin{array}{l}\text { Doc. } \\
\text { Date }\end{array}$ & $\begin{array}{l}\text { Aocopt } \\
\text { ebiuty }\end{array}$ & Comments & Current Vorolon & Quntents \\
\hline 0197 & ANS & ANS 8.1 & 1983 & $\begin{array}{l}\text { Nuclear Criticality Safety in } \\
\text { Operations with Fissionable } \\
\text { Materials Outside Reactors }\end{array}$ & drg3004.r00 & 1990 & $\mathbf{R}$ & Proposed reg3.58.ro1 & ANS 8.1 & 1983 RB8 \\
\hline 0198 & ANS & ANS 8.1 & 1983 & $\begin{array}{l}\text { Nuclear Criticality Safety in } \\
\text { Operations with Fissionable } \\
\text { Materials Outside Reactors }\end{array}$ & reg3.4.ro2 & 1986 & $\mathbf{P}$ & $\begin{array}{l}\text { Certain procedures } \\
\text { acceptable to NRC }\end{array}$ & ANS 8.1 & $1983 \mathrm{PB8}$ \\
\hline 0199 & ANS & ANS 8.1 & N/S & $\begin{array}{l}\text { Nuclear Criticality Safety in } \\
\text { Operations with Fissionable } \\
\text { Materials Outside Reactors }\end{array}$ & reg3.4.ro2 & 1986 & $\mathbf{R}$ & & ANS 8.1 & $1983 \mathrm{PB8}$ \\
\hline 0200 & ANS & ANS 8.1 & 1983 & $\begin{array}{l}\text { Nuclear Criticality Safety in } \\
\text { Operations with Fissionable } \\
\text { Materials Outside Reactors }\end{array}$ & reg3.58.ro1 & 1990 & $\mathbf{R}$ & & ANS 8.1 & $1983 \mathrm{RB8}$ \\
\hline 0201 & ANS & ANS 8.3 & 1986 & Criticality Accident Alarm System & drg3004.100 & 1990 & $\mathbf{R}$ & Proposed reg3.58.ro1 & ANS 8.3 & 1986 \\
\hline 0202 & ANS & ANS 8.3 & 1979 & Criticality Accident Alarm System & inm83100 & 1991 & $\mathbf{R}$ & & ANS 8.3 & 1986 \\
\hline 0203 & ANS & ANS 8.3 & 1979 & Criticality Accident Alarm System & inm83526 & 1934 & $\mathbf{R}$ & & ANS 8.3 & 1986 \\
\hline 0204 & ANS & ANS 8.3 & 1979 & Criticality Accident Alarm System & inm83726 & 1991 & $\mathbf{R}$ & & ANS 8.3 & 1986 \\
\hline 0205 & ANS & ANS 8.3 & 1979 & Criticality Accident Alarm System & inm83750 & 1988 & $\mathbf{R}$ & & ANS 8.3 & 1986 \\
\hline 0206 & ANS & ANS 8.3 & 1979 & Criticality Ascident Alarm System & reg3.58.roo & 1986 & $\mathbf{R}$ & & ANS 8.3 & 1986 \\
\hline 0207 & ANS & ANS 8.3 & 1986 & Criticality Accident Alarm System. & reg3.58.ro1 & 1990 & $R$ & & ANS 8.3 & 1986 \\
\hline 0208 & ANS & ANS 8.3 & 1986 & Criticality Accident Alarm System & reg8.12.r02 & 1988 & $\mathbf{P}$ & With conditions & ANS 8.3 & 1986 \\
\hline 0209 & ANS & ANS 8.5 & 1986 & $\begin{array}{l}\text { Use of Borosilicate Glass Paschig } \\
\text { Rings as a Noutron Absorber in } \\
\text { Solutions of Fissile Material }\end{array}$ & reg3.1.ro2 & 1987 & $\bar{A}$ & & ANS 8.5 & 1986 \\
\hline 0210 & ANS & ANS 8.9 & 1987 & $\begin{array}{l}\text { Nuclear Criticality Safety Criteria for } \\
\text { Steel-Pipe Intersections Containing } \\
\text { Aqueous Solutions of Fissile } \\
\text { Material }\end{array}$ & reg3.45.r01 & 1989 & A & & ANS 8.9 & 1987 \\
\hline 0211 & ANS & ANS 8.11 & $\mathrm{N} / \mathrm{S}$ & $\begin{array}{l}\text { Validation of Calculation Mothods } \\
\text { for Nuclear Criticality Safety }\end{array}$ & reg3.4.r02 & 1986 & $R$ & $\begin{array}{l}\text { Withdrawn and } \\
\text { incorporated into } \\
\text { another ANS standard }\end{array}$ & Withdrawn & $n / a$ \\
\hline 0212 & ANS & ANS 8.12 & 1978 & $\begin{array}{l}\text { Nuclear Criticality Control and } \\
\text { Safety of Homogeneous Plutonium- } \\
\text { Uranium Fuel Mixtures Outside } \\
\text { Reactors }\end{array}$ & reg3.47.r00 & 1981 & $\mathbf{P}$ & & ANS 8.12 & 1987 \\
\hline
\end{tabular}


Codes and Standards and Other Guidance Cited in Regulatory Documents

Part A - Industry Consensus Codes and Standards (continued)

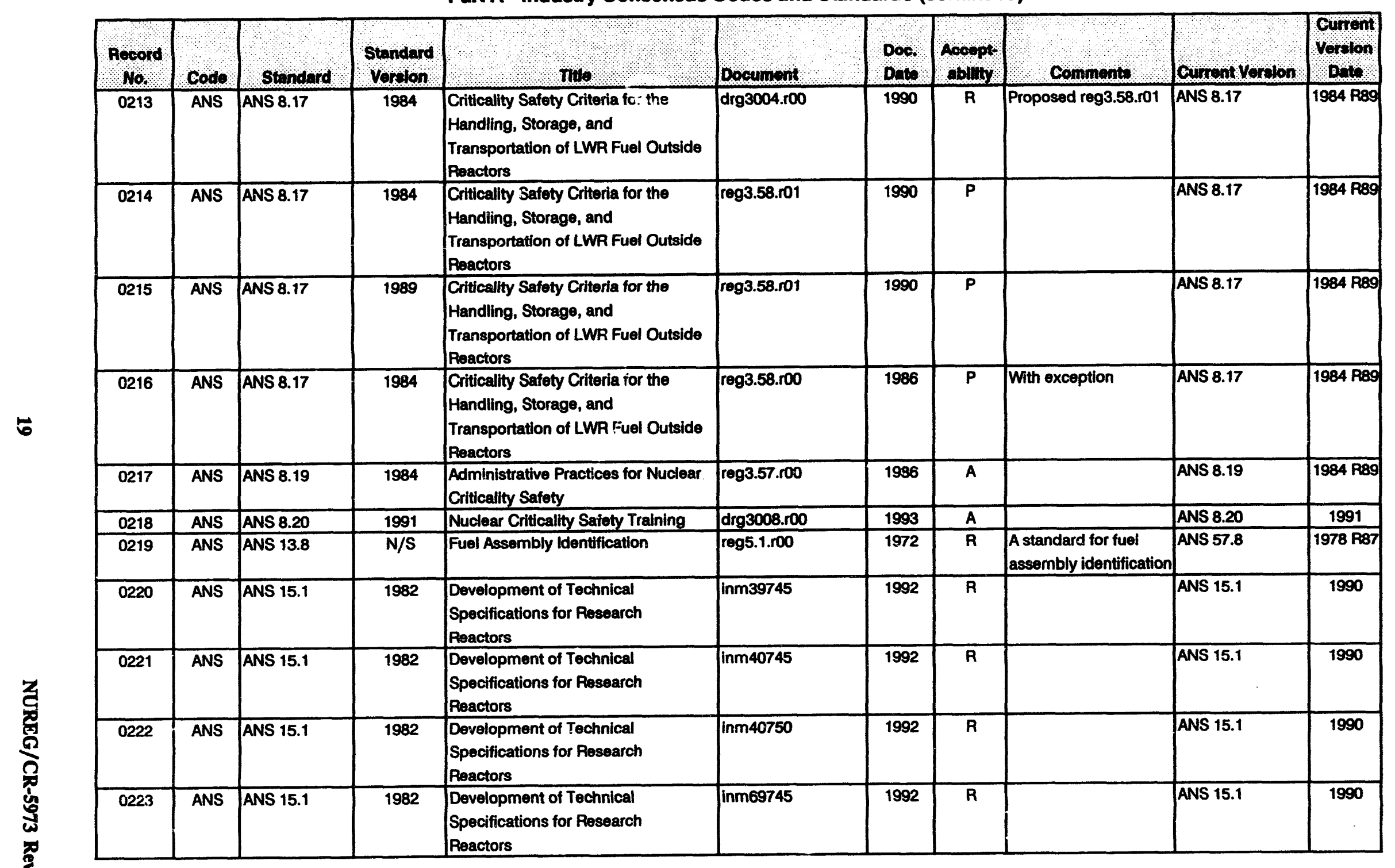


Codes and Standards and Other Guldance Clted in Regulatory Documents

Part A - Industry Conse iasus Codes and Standards (continued)

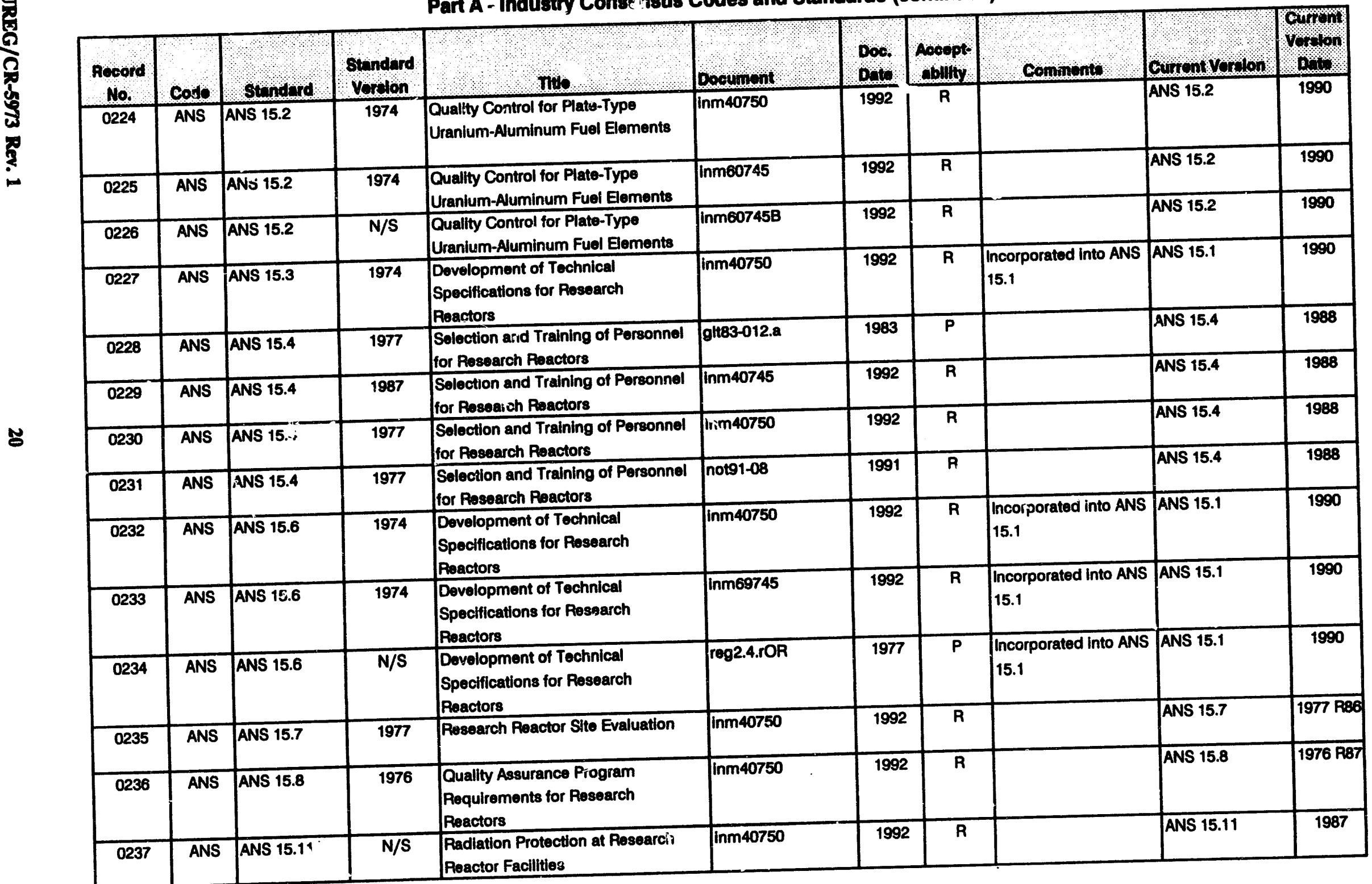


Codes and Standards and Other Guidance Cited in Regulatory Documents

Part A - Industry Consensus Codes and Standards (continued)

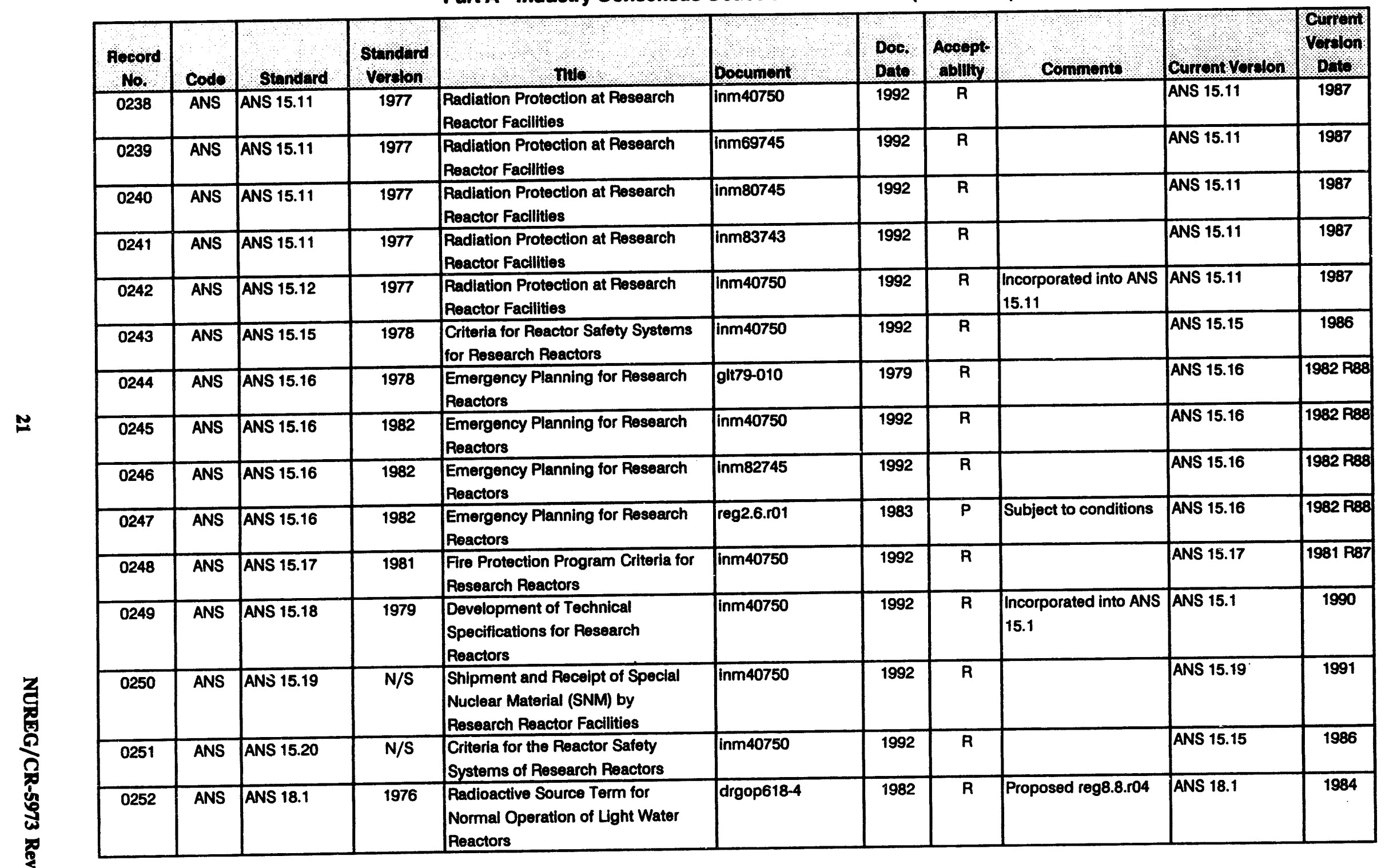


Codes and Standards and Other Guidance Cited in Regulatory Documents

Part A - Industry Consensus Codes and Standards (continued)

\begin{tabular}{|c|c|c|c|c|c|c|c|c|c|c|}
\hline $\begin{array}{l}\text { Rocord } \\
\text { No. }\end{array}$ & codo & Standerd & $\begin{array}{l}\text { Standard } \\
\text { Yoralon }\end{array}$ & THo, & Doounont & Dor & oforoply & Gomnonth & Cunout Vorolon & Gorion \\
\hline 0253 & ANS & ANS 19.6.1 & 1984 & $\begin{array}{l}\text { Reload Startup Physics Tests for } \\
\text { Pressurized Water Reactors }\end{array}$ & Inm61708 & N/S & $\mathbf{R}$ & & ANS 19.6 .1 & 1985 \\
\hline 0254 & ANS & ANS 19.6.1 & 1985 & $\begin{array}{l}\text { Reload Startup Physics Tests for } \\
\text { Pressurized Water Reactors }\end{array}$ & inm61709 & 1986 & $\mathbf{R}$ & & ANS 19.6.1 & 1985 \\
\hline 0255 & ANS & ANS 19.6.1 & 1985 & $\begin{array}{l}\text { Reload Startup Physics Tests for } \\
\text { Pressurized Water Reactors }\end{array}$ & inm61710 & 1985 & $\mathbf{R}$ & & ANS 19.6.1 & 1985 \\
\hline 0256 & ANS & ANS 19.6.1 & 1985 & $\begin{array}{l}\text { Reload Startup Physics Tests for } \\
\text { Pressurized Water Reactors }\end{array}$ & stsb\&wB3.1.8 & 1991 & $\mathbf{P}$ & & ANS 19.6.1 & 1985 \\
\hline 0257 & ANS & ANS 19.6.1 & 1985 & $\begin{array}{l}\text { Reload Startup Physics Tests for } \\
\text { Pressurized Water Reactors }\end{array}$ & stsb\&wB3.1.9 & 1991 & $\mathbf{P}$ & & ANS 19.6.1 & 1985 \\
\hline 0258 & ANS & ANS 19.6.1 & 1985 & $\begin{array}{l}\text { Reload Startup Physics Tests for } \\
\text { Pressurized Water Reactors }\end{array}$ & stsceB3.1.10 & 1991 & $\mathbf{P}$ & & ANS 19.6.1 & 1985 \\
\hline 0259 & ANS & ANS 19.6.1 & 1985 & $\begin{array}{l}\text { Reload Startup Physics Tests for } \\
\text { Pressurized Water Reactors }\end{array}$ & stsceB B3.1.8 & 1991 & $\mathbf{P}$ & & ANS 19.6.1 & 1985 \\
\hline 0260 & ANS & ANS 19.6.1 & 1985 & $\begin{array}{l}\text { Reload Startup Physics Tests for } \\
\text { Pressurized Water Reactors }\end{array}$ & stsceB3.1.9(a) & 1991 & $\mathbf{P}$ & & ANS 19.6.1 & 1985 \\
\hline 0261 & ANS & ANS 19.6.1 & 1985 & $\begin{array}{l}\text { Reload Startup Physics Tests for } \\
\text { Pressurized Water Roactors }\end{array}$ & stsceB3.1.9(d) & 1991 & $\mathbf{P}$ & & ANS 19.6.1 & 1985 \\
\hline 0262 & ANS & ANS 19.6.1 & 1985 & $\begin{array}{l}\text { Reload Startup Physics Tests for } \\
\text { Pressurized Water Reactors }\end{array}$ & stswstB3.1.10 & 1991 & $\mathbf{P}$ & & ANS 19.6.1 & 1985 \\
\hline 0263 & ANS & ANS 19.6.1 & 1985 & $\begin{array}{l}\text { Reload Startup Physics Tests for } \\
\text { Pressurized Water Reactors }\end{array}$ & stswstB3.1.11 & 1991 & $\mathbf{P}$ & & ANS 19.6.1 & 1985 \\
\hline 0264 & ANS & ANS 19.6.1 & 1985 & $\begin{array}{l}\text { Reload Startup Physics Tests for } \\
\text { Pressurized Water Reactors }\end{array}$ & stswstB3.1.9 & 1991 & $P$ & & ANS 19.6.1 & 1985 \\
\hline 0265 & ANS & ANS 51.10 & N/S & $\begin{array}{l}\text { Auxiliary Feedwater System for } \\
\text { Pressurized Water Reactors }\end{array}$ & nureg0933, 1.II.E.1 & 1986 & $\bar{R}$ & $\begin{array}{l}\text { ANS } 51.10 \text { is assumed } \\
\text { vice ANS } 51.00\end{array}$ & ANS 51.10 & 1991 \\
\hline 0266 & ANS & ANS 52.00 & $\mathrm{N} / \mathrm{S}$ & Not Found & nureg0933, 1.ll.E.1 & 1986 & $\mathbf{R}$ & & Not Found & $n / a$ \\
\hline 0267 & ANS & ANS 55.1 & 1979 & $\begin{array}{l}\text { Solid Radioactive Waste Processing } \\
\text { System for Light Water Cooled } \\
\text { Reactor Plants }\end{array}$ & drgop618-4 & 1982 & $\bar{R}$ & & ANS 55.1 & 1992 \\
\hline 0268 & ANS & ANS 55.1 & 1979 & $\begin{array}{l}\text { Solid Radioactive Waste Processing } \\
\text { System for Light Water Cooled } \\
\text { Reactor Plants }\end{array}$ & glt80-009 & 1980 & $\mathbf{R}$ & & ANS 55.1 & 1992 \\
\hline
\end{tabular}


Codes and Standards and Other Guidance Clted in Regulatory Documents

Part A - Industry Consensus Codes and Standards (continued)

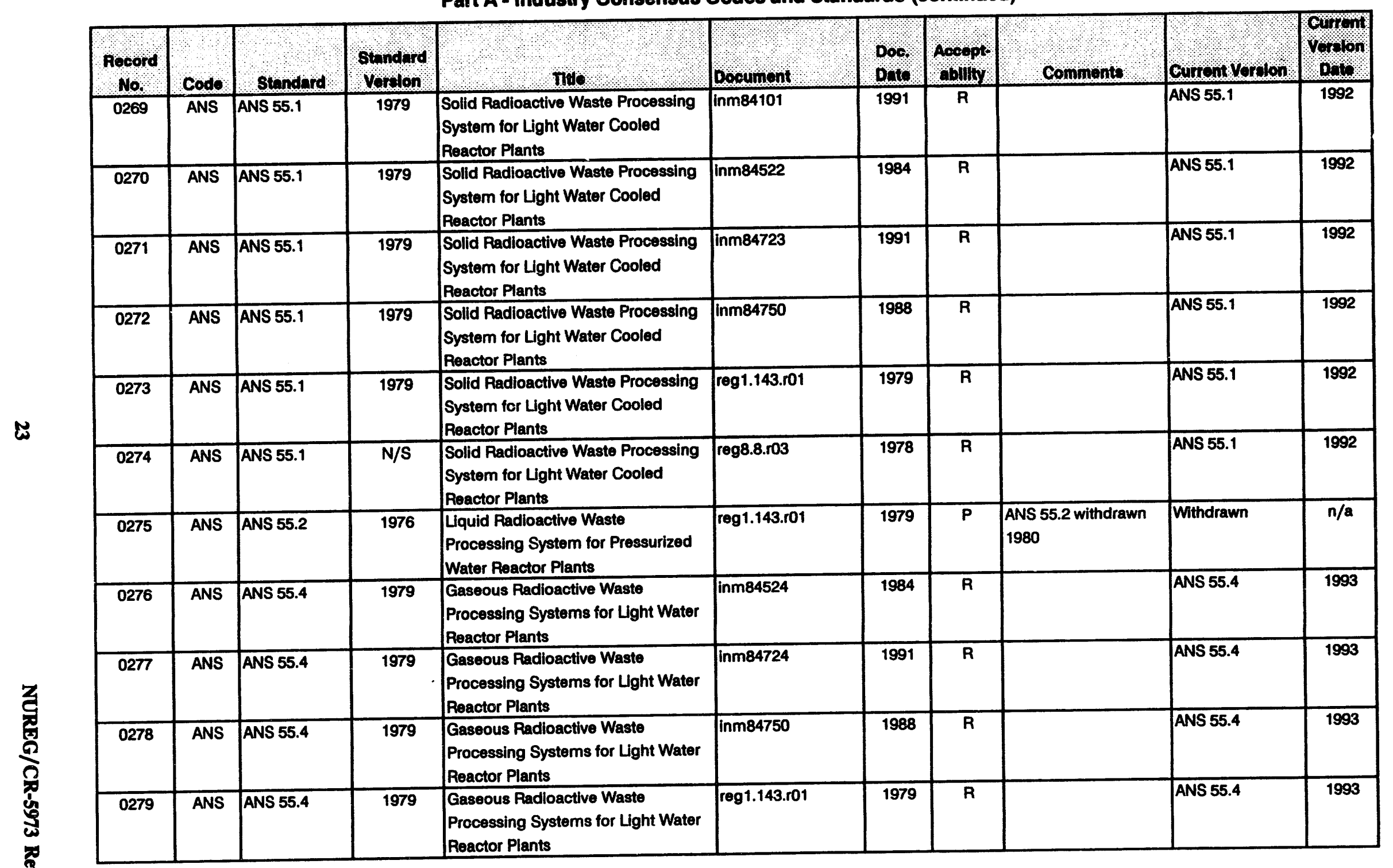


Codes and Standards and Other Guidance Cited in Regulatory Documents

Part A - Industry Consensus Codes and Standards (continued)

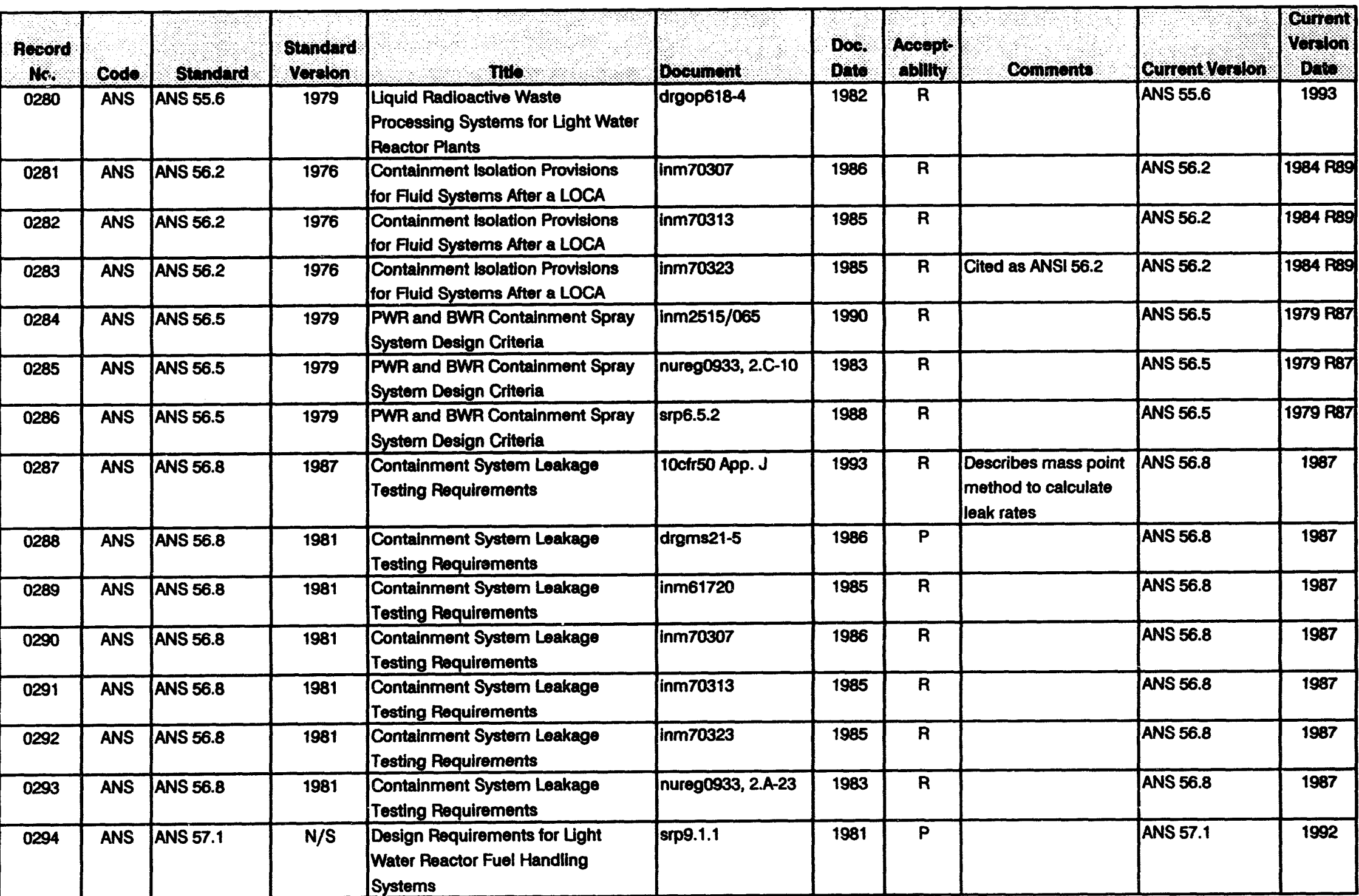


Codes and Standards and Other Guidance Clted in Regulatory Documents

Part A - Industry Consensus Codes and Standards (continued)

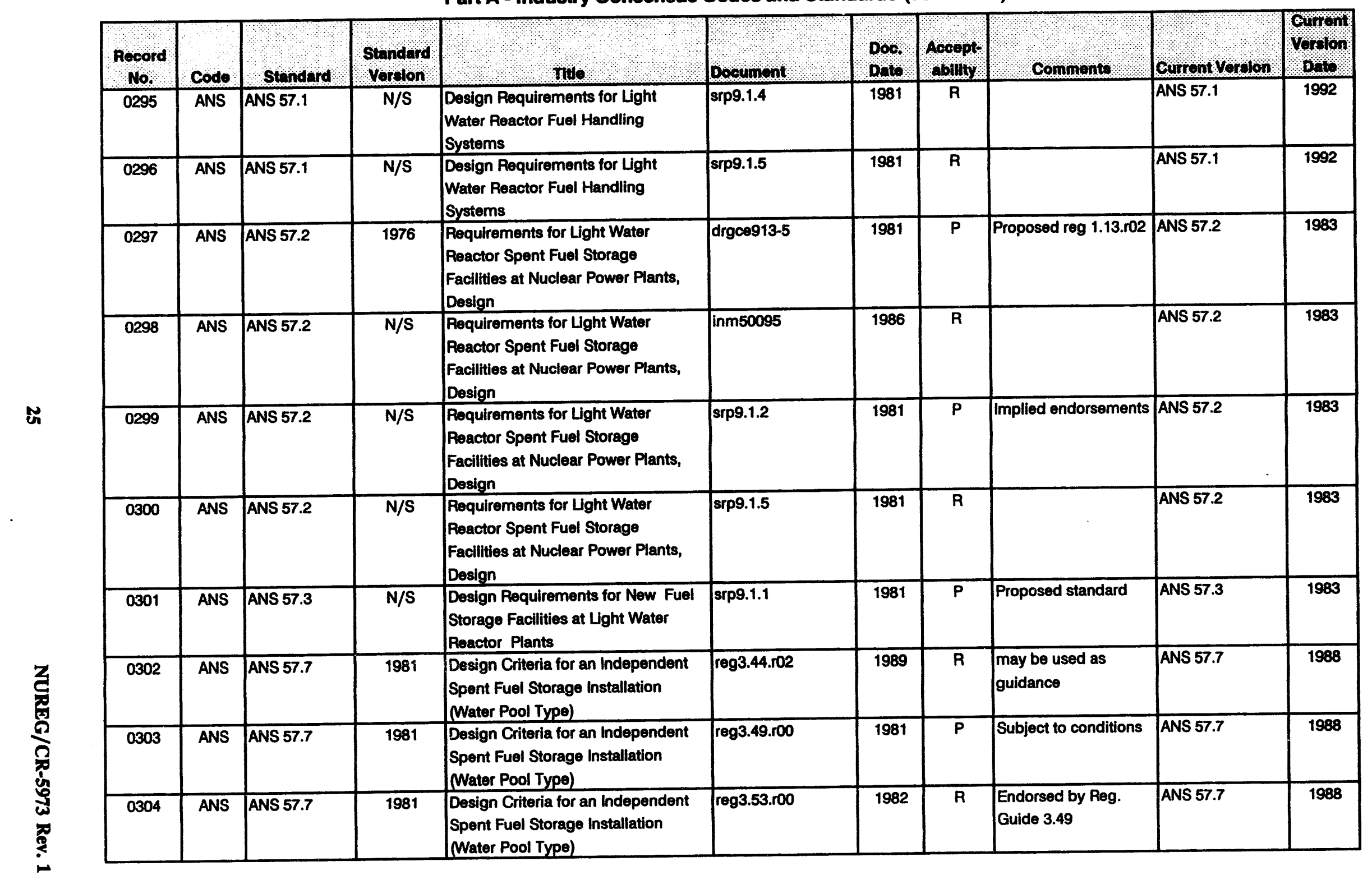


Codes and Standards and Other Guidance Cited in Regulatory Documents

Part A - Industry Consensus Codes and Standards (continued)

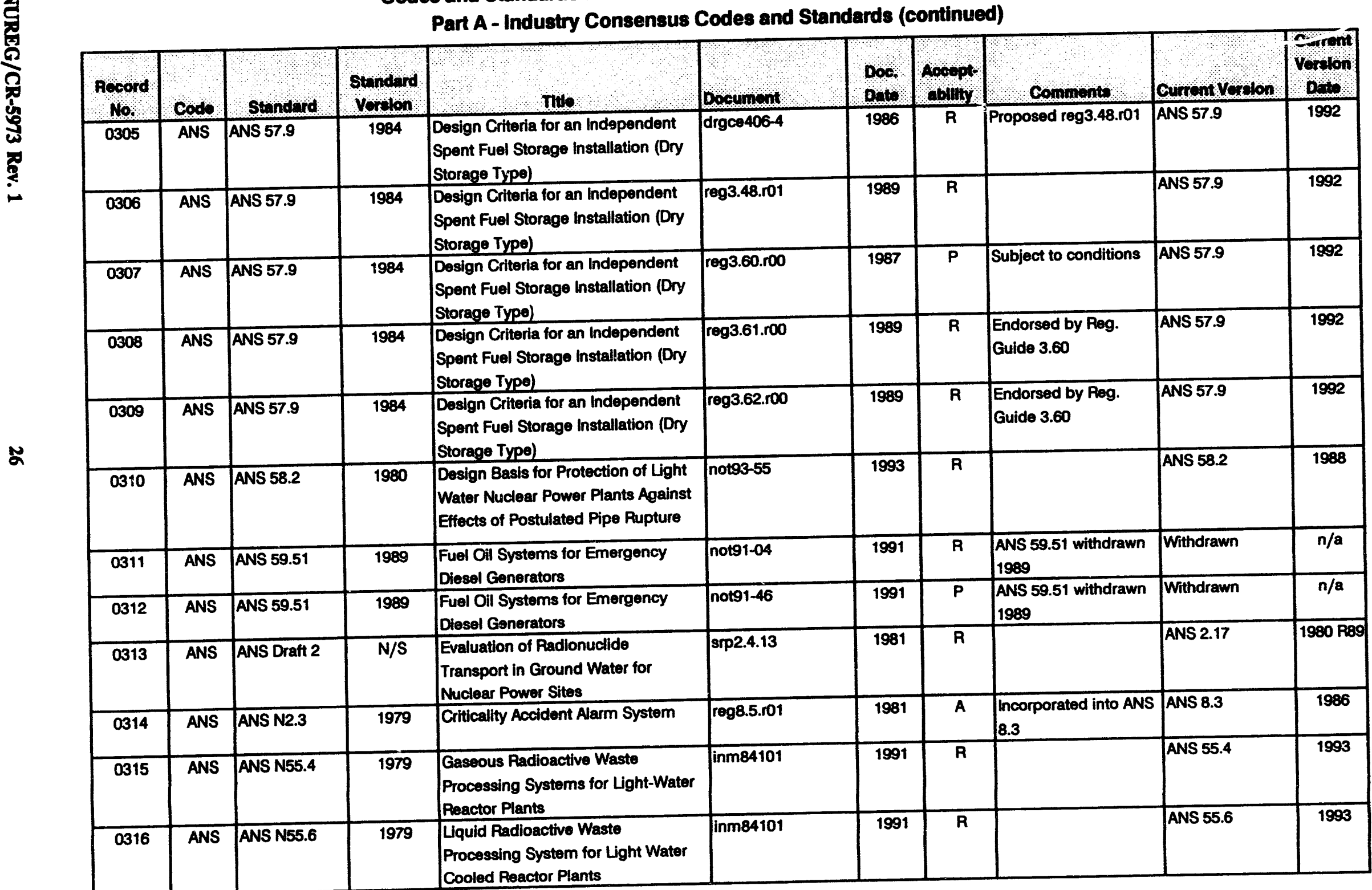

Cooled Reactor Plants 
Codes and Standards and Other Guidance Cited in Regulatory Documents

Part A - Industry Consensus Codes and Standards (continued)

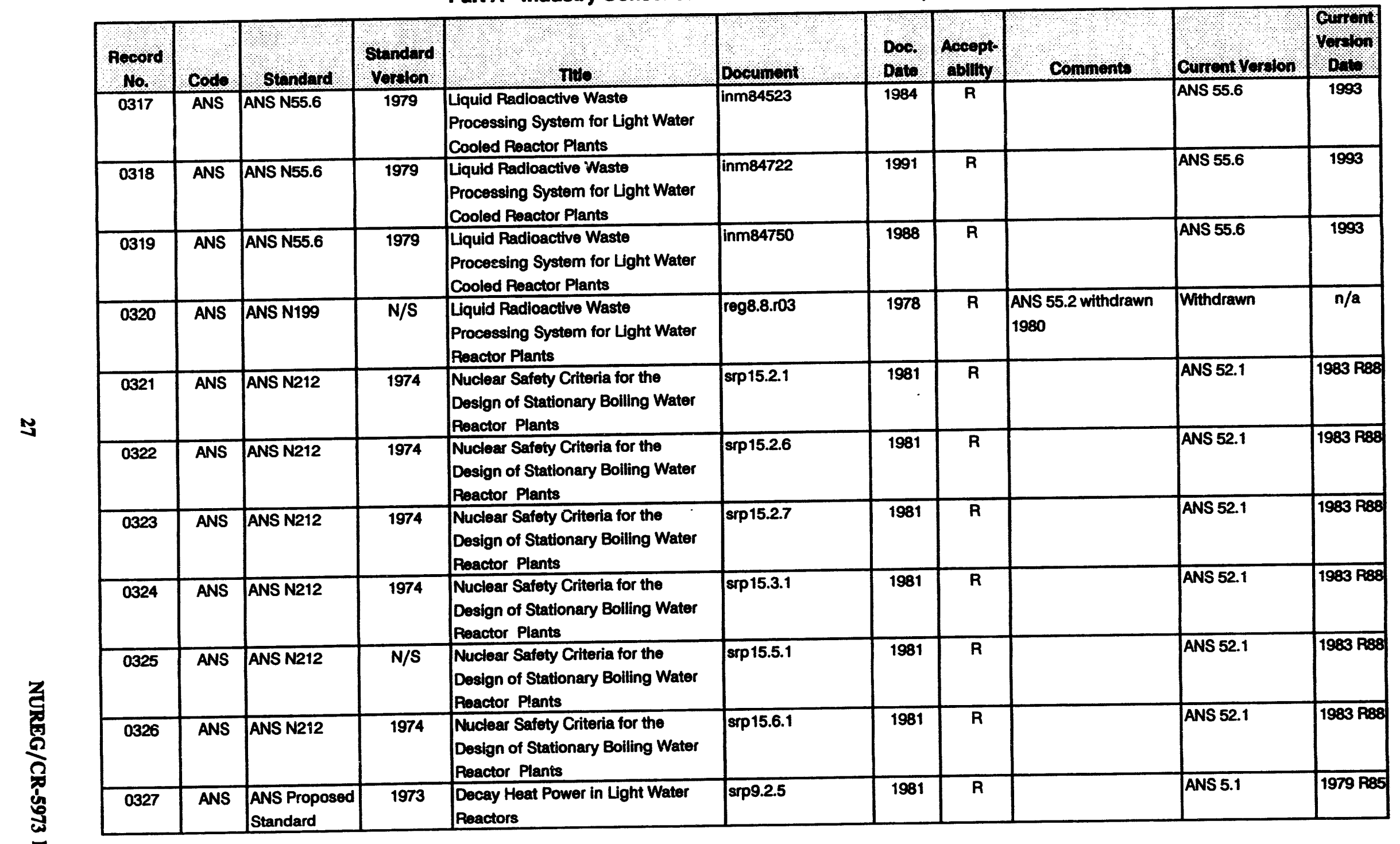


Codes and Standards and Other Guidance Cited in Regulatory Documents

Part A - Industry Consensus Codes and Standards (continued)

\begin{tabular}{|c|c|c|c|c|c|c|c|c|c|c|}
\hline $\begin{array}{l}\text { Pocond } \\
\text { No. }\end{array}$ & Cods: & Standard & $\begin{array}{l}\text { Standand } \\
\text { Vordion }\end{array}$ & The & Document. & $\begin{array}{l}\text { Doc. } \\
\text { Date }\end{array}$ & $\begin{array}{l}\text { Aocopt } \\
\text { abmy }\end{array}$ & Comments: & Current verston & 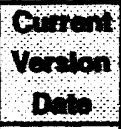 \\
\hline 0328 & ANSI & ANSI A2.1 & 1972 & $\begin{array}{l}\text { Standard Test Mothods for Fire } \\
\text { Tests of Bullding Construction and } \\
\text { Materials }\end{array}$ & drgrse09-5 & 1979 & $\mathbf{R}$ & & ASTM E119 & 1988 \\
\hline 0329 & ANSI & ANSI A2.1 & 1972 & $\begin{array}{l}\text { Standard Test Mothods for Fire } \\
\text { Tests of Building Construction and } \\
\text { Materials }\end{array}$ & reg3.16.roo & 1974 & $\mathbf{R}$ & & ASTME119 & 1968 \\
\hline 0330 & ANSI & ANSI A58.1 & 1972 & $\begin{array}{l}\text { Minimum Design Loads in } \\
\text { Buildings and Other Structures }\end{array}$ & $\operatorname{srp2.3.1}$ & 1981 & $\mathbf{R}$ & & ASCE 7 & 1988 \\
\hline 0331 & ANSI & ANSI A58.1 & $\mathbf{N} / \mathbf{S}$ & $\begin{array}{l}\text { Minimum Design Loads in } \\
\text { Buildings and Other Structures }\end{array}$ & $\operatorname{sip} 3.3 .1$ & 1981 & $\mathbf{P}$ & & ASCE 7 & 1988 \\
\hline 0332 & ANSI & ANSI A58.1 & N/S & $\begin{array}{l}\text { Minimum Design Loads in } \\
\text { Buildings and Other Structures }\end{array}$ & $\operatorname{sip} 3.3 .2$ & 1981 & $\mathbf{P}$ & & ASCE 7 & 1988 \\
\hline 0333 & ANSI & ANSI A89.1 & 1972 & $\begin{array}{l}\text { Building Code Pequirements for } \\
\text { Peinforced Concrete }\end{array}$ & reg1.142.ro1 & 1981 & $\mathbf{R}$ & & ACI 318 & 1992 \\
\hline 0334 & ANSI & ANSI B16.5 & $\mathbf{N} / \mathbf{S}$ & Pipe Flanges and Flanged Fittings & not $92-68$ & 1992 & $\mathbf{R}$ & Flanges & ASME B16.5 & 1988 \\
\hline 0335 & ANSI & ANSI B16.5 & $\mathrm{N} / \mathrm{S}$ & Pipe Fanges and Flanged Fittings & sip3.9.4 & 1984 & $\mathbf{A}$ & & ASME B16.5 & 1988 \\
\hline 0336 & ANSI & ANSI B16.9 & $\mathrm{N} / \mathrm{S}$ & $\begin{array}{l}\text { Factory-Made Wrought Steel } \\
\text { Buttwelding Fittings }\end{array}$ & ssp3.9.4 & 1984 & A & & ASME B16.9 & 1993 \\
\hline 0337 & ANSI & ANSI B16.11 & $\mathbf{N} / \mathbf{S}$ & $\begin{array}{l}\text { Forged Steel Fittings, Socket- } \\
\text { Wolding and Threaded }\end{array}$ & srp3.9.4 & 1984 & A & & ASME B16.11 & 1980 \\
\hline 0338 & ANSI & ANSI B16.25 & $\mathbf{N} / \mathrm{S}$ & Buttwelding Ends & sp3.9.4 & 1984 & A & & ASME B16.25 & 1992 \\
\hline 0339 & ANSI & ANSI B16.34 & $\mathrm{N} / \mathrm{S}$ & $\begin{array}{l}\text { Valves-Flanged, Threaded and } \\
\text { Welding Ends }\end{array}$ & $\operatorname{srp3.2.2}$ & 1981 & $\mathbf{R}$ & & ASME B16.34 & 1988 \\
\hline 0340 & ANSI & ANSI B16.34 & $\mathbf{N} / \mathbf{S}$ & $\begin{array}{l}\text { Valves-Flanged, Threaded and } \\
\text { Welding Ends }\end{array}$ & $\operatorname{sip} 3.9 .4$ & 1984 & $\mathbf{A}$ & & ASME B16.34 & 1988 \\
\hline 0341 & ANSI & $\begin{array}{l}\text { ANSI B16.41 } \\
\text { Draft }\end{array}$ & $\mathrm{N} / \mathrm{S}$ & $\begin{array}{l}\text { Functional Qualification } \\
\text { Requirements for Power Operated } \\
\text { Active Valve Assemblies for Nuclear } \\
\text { Power Plants }\end{array}$ & sip3.10 & 1981 & $\mathbf{R}$ & & ANSI B16.41 & 1983 Fed \\
\hline 0342 & ANSI & ANSI B30.2 & 1976 & $\begin{array}{l}\text { Overhead and Gantry Cranes (Top } \\
\text { Punning Bridge, Single or Multiple } \\
\text { Girder Top Running Hoist) }\end{array}$ & glt81-007 & 1981 & $\mathbf{P}$ & Chapters 2-1 and 2-2 & ASME B30.2B & 1992 \\
\hline
\end{tabular}


Codes and Standards and Other Guidance Cited in Regulatory Documents

Part A - Industry Consensus Codes and Standards (continued)

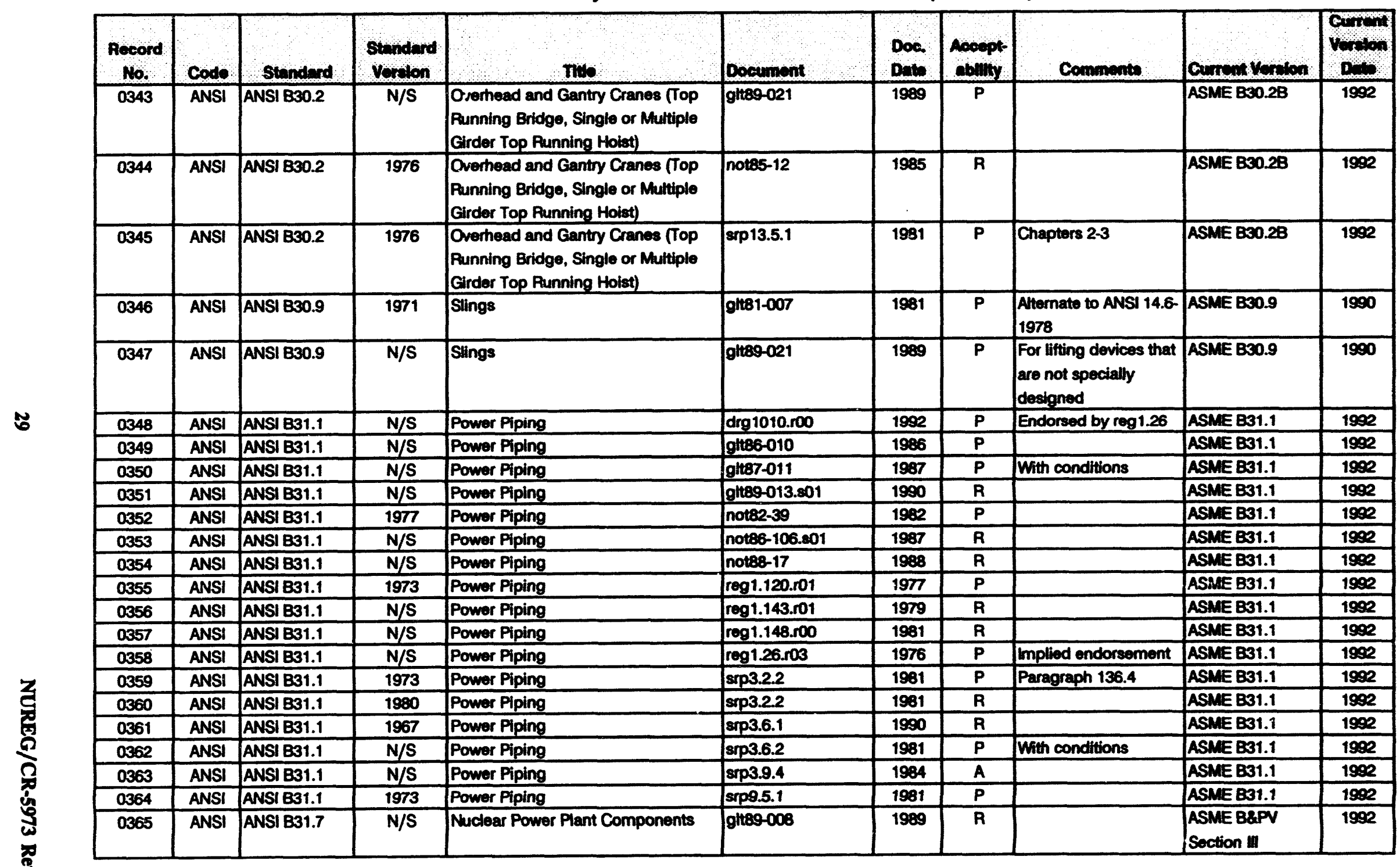




\begin{tabular}{|c|c|c|c|c|c|c|c|c|c|c|}
\hline \multicolumn{11}{|c|}{$\begin{array}{c}\text { Codes and Standards and Other Guidance Cited in Regubatory Documents } \\
\text { Part A - Industry Consensus Codes and Standards (continued) }\end{array}$} \\
\hline $\begin{array}{l}\text { Pecord } \\
\text { No. }\end{array}$ & Cods & stondnd & $\begin{array}{l}\text { Standerd } \\
\text { Verelen }\end{array}$ & Tmo & Document & $\begin{array}{l}\text { Dos. } \\
\text { Dete }\end{array}$ & Aocesply & Commente & Conson Voulon & Dim \\
\hline 0366 & ANSI & ANSI B31.7 & $\mathrm{N} / \mathrm{S}$ & Nuclear Power Plant Components & inm49055 & 1982 & $\mathbf{R}$ & & $\begin{array}{l}\text { ASME BBPV } \\
\text { Section II }\end{array}$ & 1992 \\
\hline 0367 & ANSI & ANSI B31.7 & $\mathrm{N} / \mathrm{S}$ & Nuclear Powor Plant Compononts & not87-36 & 1987 & $\overline{\mathbf{R}}$ & & $\begin{array}{l}\text { ASME BAPV } \\
\text { Section } \mathrm{II}\end{array}$ & 1982 \\
\hline 0368 & ANSI & ANSI B31.7 & $\mathrm{N} / \mathrm{S}$ & Nucloar Power Plant Components & not88-17 & 1988 & $\mathbf{R}$ & & $\begin{array}{l}\text { ASME BSPV } \\
\text { Soction in }\end{array}$ & 1992 \\
\hline 0369 & $\overline{\text { ANSI }}$ & ANS1 B31.7 & $\mathrm{N} / \mathrm{S}$ & Nuclear Power Plant Components & reg1.70.r01 & 1972 & A & & $\begin{array}{l}\text { ASME BEPV } \\
\text { Section in }\end{array}$ & 1992 \\
\hline 0370 & ANSI & ANSI B31.7 & 1970 & Nuclear Power Plant Compononts & $\log 1.84 .120$ & 1993 & $\mathbf{R}$ & $\begin{array}{l}\text { Use of } 1970 \text { Addenda } \\
\text { of B31.7 Section it } \\
\text { annullod } 1978\end{array}$ & $\begin{array}{l}\text { ASME BRPV } \\
\text { Section } 1 \text { il }\end{array}$ & 1992 \\
\hline 0371 & ANSI & ANSI B95.1 & 1972 & $\begin{array}{l}\text { Torminology for Prossure Roliof } \\
\text { Dovices }\end{array}$ & $\operatorname{sip} 15.6 .1$ & 1981 & A & & MUSI BO5.1 & 1977 \\
\hline 0372 & ANSI & ANS1 B96.1 & $\mathrm{N} / \mathrm{S}$ & $\begin{array}{l}\text { Weldod Aluminum-Alloy Storago } \\
\text { Tanks }\end{array}$ & drg1010.ro0 & 1992 & $\bar{P}$ & Endorsed by reg 1.26 & ASUE BS6.1 & 1993 \\
\hline 0373 & $\overline{\text { ANSI }}$ & ANSI B96.1 & N/S & $\begin{array}{l}\text { Welded Aluminum-Alloy Storege } \\
\text { Tanks }\end{array}$ & rog1.26.103 & 1976 & $\mathbf{P}$ & $\begin{array}{l}\text { mplied endorsement } \\
\text { (Table 1, Quality } \\
\text { Standards) }\end{array}$ & ASME BO6.1 & 1993 \\
\hline 0374 & ANSI & ANS1 B96.1 & 1980 & $\begin{array}{l}\text { Welded Aluminum-Alloy Storaoe } \\
\text { Tanks }\end{array}$ & $\operatorname{arp3.22}$ & 1981 & $\bar{R}$ & & ASWE BO6.1 & 1903 \\
\hline 0375 & ANSI & ANSI B124.1 & 1971 & $\begin{array}{l}\text { UL Standard for Safoty Test } \\
\text { Performanco of Av Finor Units }\end{array}$ & reg1.52.ro2 & 1978 & $\mathbf{R}$ & & $\longdiv { U L . 9 0 0 }$ & 1987 \\
\hline 0376 & ANSI & ANS1 B132.1 & $\mathrm{N} / \mathrm{S}$ & $\begin{array}{l}\text { UL Standard for Safoty High- } \\
\text { Etficiency, Panticulate. Air Filter } \\
\text { Units }\end{array}$ & inm88055 & 1991 & $\mathbf{R}$ & & UL 586 & 1990 \\
\hline 037 & ANSI & ANS1 B132.1 & 1971 & $\begin{array}{l}\text { UL Standard for Satoty High- } \\
\text { Efficiency, Particulato, Air Filtor } \\
\text { Units }\end{array}$ & regas.12.roo & 1973 & $\mathbf{R}$ & & UL 586 & 1990 \\
\hline 0378 & ANSI & ANS1 B132.1 & 1971 & $\begin{array}{l}\text { UL. Standard for Saffoty High- } \\
\text { Efficiency, Particulate, Air Fittor } \\
\text { Units }\end{array}$ & 10093.32 .100 & 1975 & $\bar{A}$ & & UL 586 & 1990 \\
\hline 0379 & ANSI & ANSi C37.04 & $\mathrm{N} / \mathrm{S}$ & $\begin{array}{l}\text { Standard Pating Structure for AC } \\
\text { High-Voltage Crrount Broakers } \\
\text { Patod on a Symmotrical Basis }\end{array}$ & $\operatorname{sip} 8.2$ & 1993 & $\bar{A}$ & & EEEC37.04 & 1979 PEO \\
\hline
\end{tabular}


Codes and Standards and Other Guldance Ched in Regulatory Documents

Part A - Industry Consensus Codes and Standards (continued)

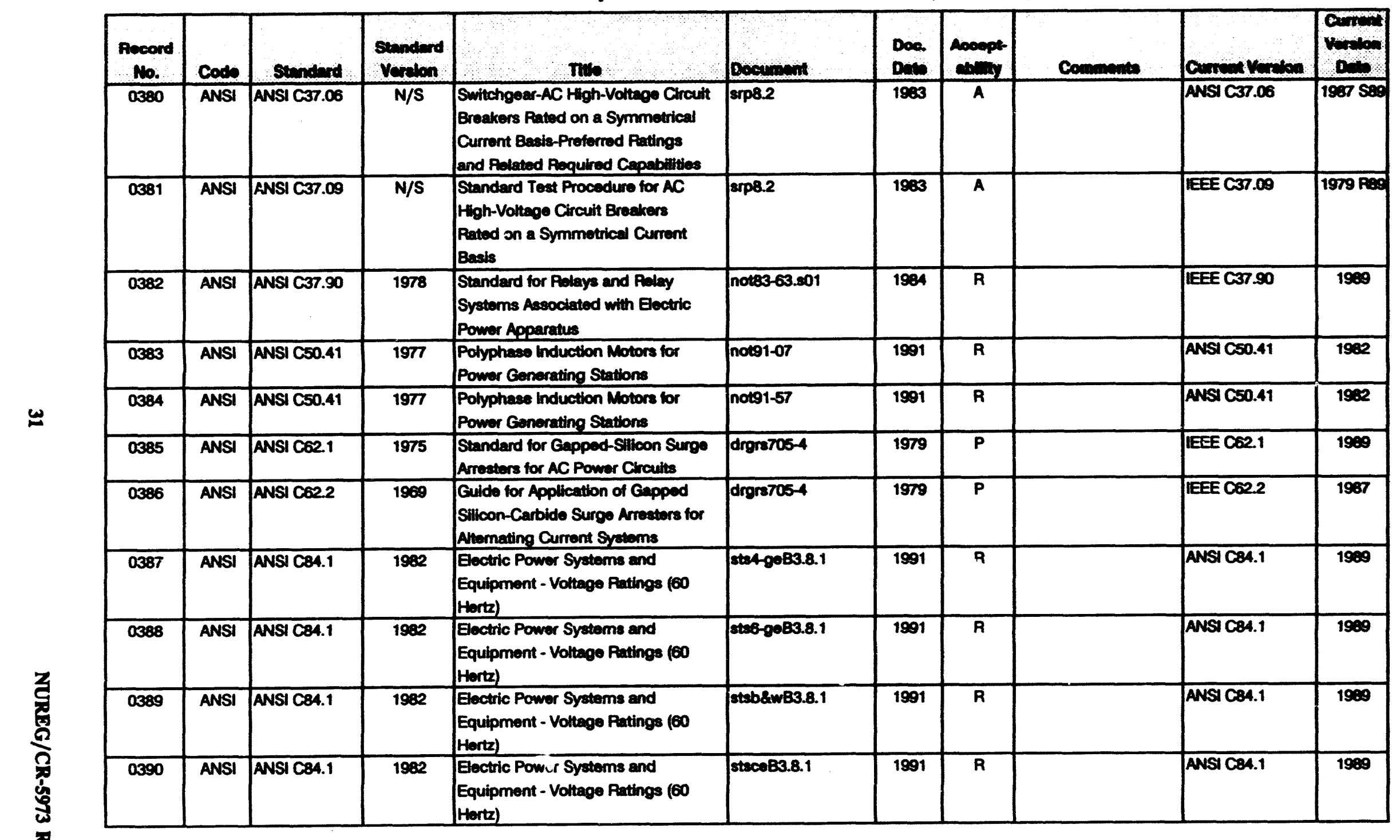


Codes and Standards and Other Guidance Ched in Regulatory Documents

Part A - Industry Consensus Codes and Standards (continued)

\begin{tabular}{|c|c|c|c|c|c|c|c|c|c|c|}
\hline $\begin{array}{l}\text { Aecond } \\
\text { No. }\end{array}$ & Cods & Stundund & $\begin{array}{l}\text { Standard } \\
\text { Vorelon }\end{array}$ & The & Doovinent & Don & Aocept & Comments & Gunent Vouton & Crintion \\
\hline 0391 & ANSI & ANSI C84.1 & 1982 & $\begin{array}{l}\text { Electric Power Systems and } \\
\text { Equipment - Voltage Ratings (60 } \\
\text { Hertz) }\end{array}$ & stawstB3.8.1 & 1991 & $\mathbf{R}$ & & ANSI CB4.1 & 1989 \\
\hline 0392 & ANSI & ANSI MC 11.1 & 1976 & Quality Standard's for instrument Air & sip9.3.1 & 1981 & $\bar{P}$ & $\begin{array}{l}\text { Supplements Reg. } \\
\text { Guide 1.68.3 }\end{array}$ & ISA S7.3 & $1975 \mathrm{RB1}$ \\
\hline 0393 & ANSI & ANSI MH5.1 & 1971 & $\begin{array}{l}\text { Cargo Container Chassis - Basic } \\
\text { Interface Requirements }\end{array}$ & $10 \mathrm{ctr} 73$ & 1993 & $\bar{A}$ & & ANSI MH5.6 & 1990 \\
\hline 0394 & ANSI & ANSI MH 5.1 & $\mathrm{~N} / \mathrm{S}$ & $\begin{array}{l}\text { Cargo Contriner Chassis - Basic } \\
\text { Interface Requirements }\end{array}$ & inm81370 & 1986 & $\bar{R}$ & & ANSI MHL5.6 & 1990 \\
\hline 0395 & ANSI & ANSI N2.1 & 1969 & $\begin{array}{l}\text { Waming Symbols - Radiation } \\
\text { Symbol }\end{array}$ & reg8.1.r00 & 1973 & $\bar{P}$ & & ANSIN2.1 & 1989 \\
\hline 0396 & ANSI & ANSI N4.1 & 1973 & $\begin{array}{l}\text { Classification System for Polymeric } \\
\text { Materials for Services in lonizing } \\
\text { Padiation }\end{array}$ & reg1.70.r03 & 1978 & $\overline{\mathbf{P}}$ & $\begin{array}{l}\text { ASTM D2953 } \\
\text { discontinued }\end{array}$ & discontinued & $n / a$ \\
\hline 0397 & ANSI & ANSI N8.1 & 1967 & $\begin{array}{l}\text { Quality Control for Plate-Type } \\
\text { Uranium-Aluminum Fuel Elements }\end{array}$ & reg2.3.ro1 & 1976 & $\mathbf{R}$ & & ANS 15.2 & 1990 \\
\hline 0398 & ANSI & ANSI N13.1 & 1969 & $\begin{array}{l}\text { Guide for Sampling Airborne } \\
\text { Padioactive Materials in Nuclear } \\
\text { Faciltities }\end{array}$ & drg3003.r00 & 1990 & $\bar{A}$ & & ANSI N13.1 & $1969 \mathrm{R} 93$ \\
\hline 0399 & ANSI & ANSI N13.1 & 1969 & $\begin{array}{l}\text { Guide for Sampling Airborne } \\
\text { Radioactive Materials in Nuclear } \\
\text { Facilities }\end{array}$ & drg8013.r00 & 1992 & $\bar{A}$ & & ANSI N13.1 & $1969 \mathrm{R98}$ \\
\hline 0450 & ANSI & ANSI N13.1 & 1969 & $\begin{array}{l}\text { Guide for Sampling Airborne } \\
\text { Redioactive Materials in Nuclear } \\
\text { Facilities }\end{array}$ & drgop618-4 & 1982 & $\mathbf{R}$ & Proposed reg8.8.ro4 & ANSI N13.1 & $1969 \mathrm{R93}$ \\
\hline 0401 & ANSI & ANSI N13.1 & 1969 & $\begin{array}{l}\text { Guide for Sampling Airborne } \\
\text { Radioactive Materials in Nuclear } \\
\text { Facilities }\end{array}$ & inm2515/065 & 1990 & $\overline{\mathbf{R}}$ & & ANSI N13.1 & $1969 \mathrm{PQ3}$ \\
\hline 0402 & ANSI & ANSI N13.1 & 1982 & $\begin{array}{l}\text { Guide for Sampling Airborne } \\
\text { Padioactive Materials in Nuclear } \\
\text { Facilities }\end{array}$ & inm2515/065 & 1990 & $\mathbf{R}$ & & ANSI N13.1 & 1969 RQO \\
\hline 0403 & ANSI & ANSI N13.1 & 1969 & $\begin{array}{l}\text { Guide for Sampling Airborne } \\
\text { Radioactive Materials in Nuclear } \\
\text { Facilities }\end{array}$ & inm83100 & 1991 & $\mathbf{R}$ & & ANSI N13.1 & 1969 R99: \\
\hline
\end{tabular}


Codes and Standards and Other Guidance Cited in Regulatory Documents

Part A - Industry Consensus Codes and Standards (continued)

జ

\begin{tabular}{|c|c|c|c|c|c|c|c|c|c|c|}
\hline $\begin{array}{l}\text { Record } \\
\text { No. }\end{array}$ & Codo & Standurd & $\begin{array}{l}\text { stundard } \\
\text { verlon }\end{array}$ & Thes: & Dooumont & Dos. & $\begin{array}{l}\text { Accepts } \\
\text { ebilly }\end{array}$ & Comments & Gunent Vorolon & ontent \\
\hline 0404 & ANSI & ANSI N13.1 & 1969 & $\begin{array}{l}\text { Guide for Sampling Airborne } \\
\text { Radioactive Materials in Nuclear } \\
\text { Facilities }\end{array}$ & inm83525 & 1984 & R & & ANSI N13.1 & 1969 R93 \\
\hline 0405 & ANSI & ANSI N13.1 & 1981 & $\begin{array}{l}\text { Guide for Sampling Airborne } \\
\text { Radioactive Materials in Nuclear } \\
\text { Facilities }\end{array}$ & inm83525 & 1984 & $\mathbf{R}$ & & ANSI N13.1 & $1969 \mathrm{RQ3}$ \\
\hline 0406 & ANSI & ANSI N13.1 & 1982 & $\begin{array}{l}\text { Guide for Sampling Airborne } \\
\text { Radioactive Materials in Nuclear } \\
\text { Facilities }\end{array}$ & inm83525 & 1984 & $\mathbf{R}$ & & ANSI N13.1 & 1969 R93 \\
\hline 0407 & ANSI & ANSI N13.1 & 1969 & $\begin{array}{l}\text { Guide for Sampling Airborne } \\
\text { Radioactive Materials in Nuclear } \\
\text { Facilities }\end{array}$ & inm83526 & 1984 & $\mathbf{R}$ & & ANSI N13.1 & 1969 P93: \\
\hline 0408 & ANSI & ANSI N13.1 & 1982 & $\begin{array}{l}\text { Guide for Sampling Airborne } \\
\text { Radioactive Materials in Nuclear } \\
\text { Facilities }\end{array}$ & inm83526 & 1984 & $\mathbf{R}$ & & ANSI N13.1 & 1969 P93 \\
\hline 0409 & ANSI & ANSI N13.1 & 1969 & $\begin{array}{l}\text { Guide for Sampling Airborne } \\
\text { Radioactive Materials in Nuclear } \\
\text { Facilities }\end{array}$ & inm83725 & 1991 & $\mathbf{A}$ & & ANSI N13.1 & 1969 PQ3 \\
\hline 0410 & ANS! & ANSI N13.1 & 1982 & $\begin{array}{l}\text { Guide for Sampling Airborne } \\
\text { Padioactive Materials in Nuclear } \\
\text { Facilities }\end{array}$ & inm83725 & 1991 & $\mathbf{R}$ & & ANSI N13.1 & 1969 R93 \\
\hline 0411 & ANSI & ANSI N13.1 & 1969 & $\begin{array}{l}\text { Guide for Sampling Airborne } \\
\text { Radioactive Materials in ivislear } \\
\text { Facilities }\end{array}$ & inm83726 & 1991 & $\mathbf{R}$ & & ANSI N13.1 & 1969 R93 \\
\hline 0412 & ANSI & ANSI N13.1 & 1982 & $\begin{array}{l}\text { Guide for Sampling Airborne } \\
\text { Radioactive Materials in Nuclear } \\
\text { Facilties }\end{array}$ & inm83726 & 1991 & $R$ & & ANSI N13.1 & 1969 PQ3 \\
\hline 0413 & ANSI & ANSI N13.1 & $N / S$ & $\begin{array}{l}\text { Guide for Sampling Airborne } \\
\text { Radioactive Materials in Nuclear } \\
\text { Facilities }\end{array}$ & inm83729 & 1991 & R & & ANSI N13.1 & $1969 \mathrm{R93}$ \\
\hline 0414 & ANSI & ANSI N13.1 & 1969 & $\begin{array}{l}\text { Guide for Sampling Airborne } \\
\text { Radioactive Materials in Nuclear } \\
\text { Facilities }\end{array}$ & inm 83750 & 1988 & $\mathbf{R}$ & & ANSI N13.1 & $1969 \mathrm{R93}$ \\
\hline
\end{tabular}


Codes and Standards and Other Guidance Cited in Regulatory Documents

Part A - Industry Consensus Codes and Standards (cortinued)

\begin{tabular}{|c|c|c|c|c|c|c|c|c|c|c|}
\hline $\begin{array}{l}\text { Record } \\
\text { No. }\end{array}$ & Code & Standard & $\begin{array}{l}\text { Standard } \\
\text { Vorelon }\end{array}$ & Tilo & Dooumont & $\begin{array}{l}\text { Doc. } \\
\text { Dats. }\end{array}$ & $\begin{array}{l}\text { Aooupt } \\
\text { abllity }\end{array}$ & Comments & Eurront Vordon & orton \\
\hline 0415 & ANSI & ANSIN13.1 & 1982 & $\begin{array}{l}\text { Guide for Sampling Airborne } \\
\text { Radioactive Materials in Nuclear } \\
\text { Facilities }\end{array}$ & inm83750 & 1988 & $\mathbf{R}$ & & ANSIN13.1 & 1969 PQ3 \\
\hline 0416 & ANSI & ANSI N13.1 & 1969 & $\begin{array}{l}\text { Guide for Sampling Airborne } \\
\text { Padioactive Materials in Nuclear } \\
\text { Facillties }\end{array}$ & inm84101 & 1991 & $\mathbf{A}$ & & ANSI N13.1 & $1969 \mathrm{R93}$ \\
\hline 0417 & ANSI & ANSI N13.1 & 1982 & $\begin{array}{l}\text { Guide for Sampling Airborne } \\
\text { Radloactive Materials in Nuclear } \\
\text { Facllities }\end{array}$ & inm84101 & 1991 & $\mathbf{R}$ & & ANSI N13.1 & 1909 R93 \\
\hline 0418 & ANSI & ANSI N13.1 & 1969 & $\begin{array}{l}\text { Guide for Sampling Arborne } \\
\text { Radioactive Materials in Nuclear } \\
\text { Facilities }\end{array}$ & inm84524 & 1984 & $\mathbf{R}$ & & ANSIN13.1 & $1969 \mathrm{APB}$ \\
\hline 0419 & ANSI & ANSI N13.1 & 1982 & $\begin{array}{l}\text { Guide for Sampling Airbome } \\
\text { Radioactive Materials in Nuclear } \\
\text { Facilities }\end{array}$ & inm84524 & 1984 & $\mathbf{R}$ & & ANSI N13.1 & $1969 \mathrm{R} 93$ \\
\hline 0420 & ANSI & ANSI N13.1 & 1969 & $\begin{array}{l}\text { Guide for Sampling Airborne } \\
\text { Radioactive Materials in Nuclear } \\
\text { Facilities }\end{array}$ & inm84724 & 1991 & $\mathbf{R}$ & & ANSI N13.1 & 1969 R9S \\
\hline 0421 & ANSI & ANSI N13.1 & 1982 & $\begin{array}{l}\text { Guide for Sampling Airborne } \\
\text { Radioactive Materials in Nuclear } \\
\text { Facilities }\end{array}$ & inm84724 & 1991 & $\mathbf{R}$ & & ANSI N13.1 & 1969 R93 \\
\hline 0422 & ANSI & ANSI N13.1 & 1969 & $\begin{array}{l}\text { Guide for Sampling Airborne } \\
\text { Radioactive Materials in Nuclear } \\
\text { Facilities }\end{array}$ & inm84750 & 1988 & $\mathbf{R}$ & & ANSI N13.1 & 1969 R93 \\
\hline 0423 & ANSI & ANSI N13.1 & 1982 & $\begin{array}{l}\text { Guide for Sampling Airborne } \\
\text { Radioactive Materials in Nuclear } \\
\text { Facilities }\end{array}$ & inm84750 & 1988 & $R$ & & ANSI N13.1 & $1969 \mathrm{R} 93$ \\
\hline 0424 & ANSI & ANSI N13.1 & 1969 & $\begin{array}{l}\text { Guide for Sampling Airborne } \\
\text { Radioactive Materials in Nuclear } \\
\text { Facilities }\end{array}$ & nureg0737 & 1980 & $\mathbf{P}$ & Typo N131 & ANSI N13.1 & $1969 \mathrm{R93}$ \\
\hline 0425 & ANSI & ANSI N13.1 & 1969 & $\begin{array}{l}\text { Guide for Sampling Airborne } \\
\text { Radioactive Materials in Nuclear } \\
\text { Facilities }\end{array}$ & $\mathrm{reg} 1.21 . \mathrm{r01}$ & 1976 & $\mathbf{P}$ & & ANSIN13.1 & $1969 \mathrm{R93}$ \\
\hline
\end{tabular}


Codes and Standards and Other Guidance Cited in Regulatory Documents

Part A - Industry Consensus Codes and Standards (continued)

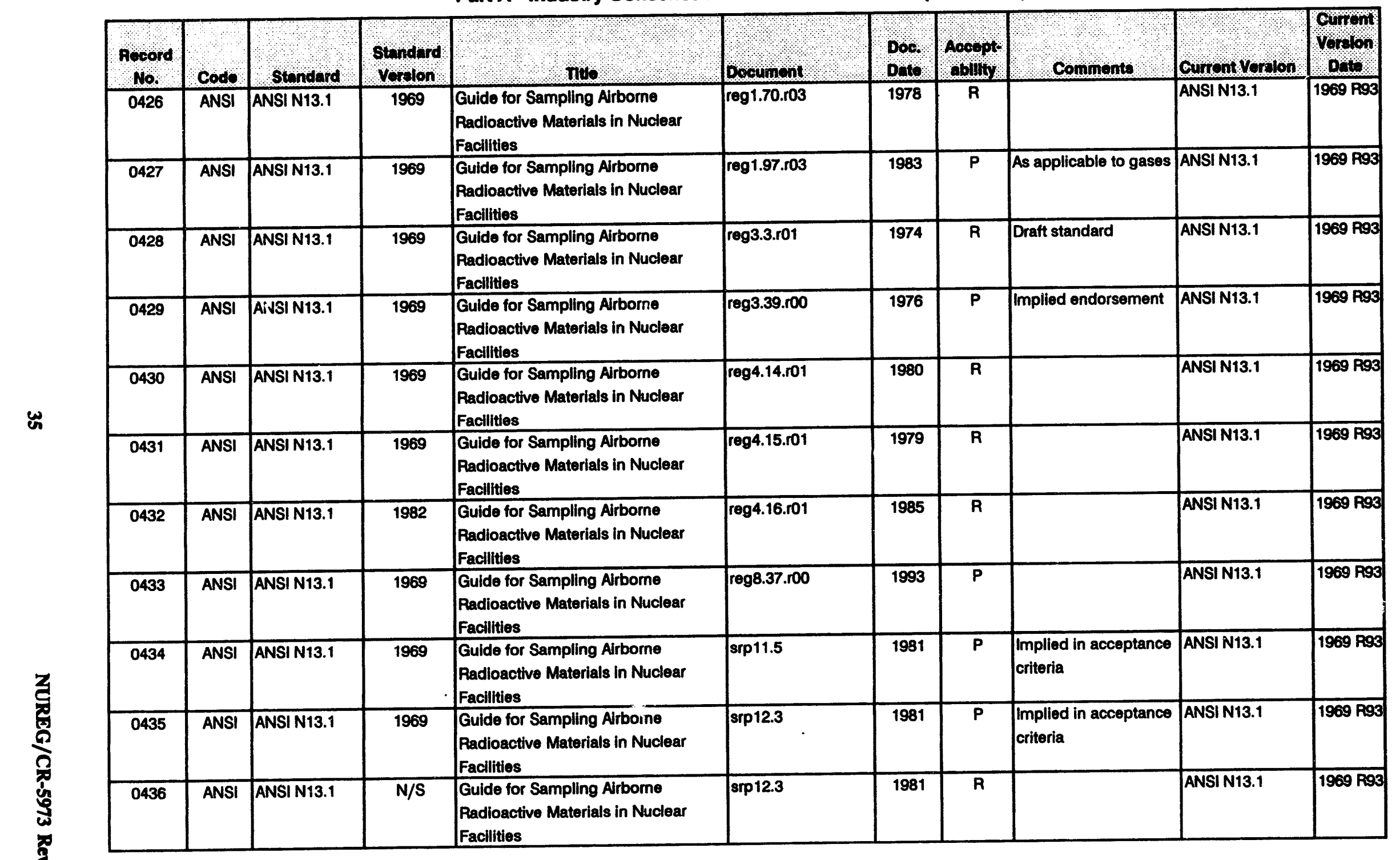


Codes and Standards and Other Guidance Chted in Regulatory Documents

Part A - Industry Consensus Codes and Standards (continued)

\begin{tabular}{|c|c|c|c|c|c|c|c|c|c|c|}
\hline $\begin{array}{l}\text { Hocord } \\
\text { No. }\end{array}$ & Codo & Stondurd & $\begin{array}{l}\text { Stondord } \\
\text { Vordlon }\end{array}$ & \&ै। THLO & Doorousont & Dor & Acoepts & Comments: & Cunront Vorolon & Qnof \\
\hline 0437 & ANSI & ANSI N13.1 & 1969 & $\begin{array}{l}\text { Guide for Sampling Airborne } \\
\text { Radioactive Materials in Nuclear } \\
\text { Facilities }\end{array}$ & sip9.3.2 & 1981 & $\mathbf{P}$ & & ANSI N13.1 & $1969 \mathrm{Rg3}$ \\
\hline 0438 & ANSI & ANSI N13.2 & 1969 & $\begin{array}{l}\text { Guide to Administrative Practices in } \\
\text { Radiation Monitoring }\end{array}$ & inm83100 & 1991 & $\mathbf{R}$ & & ANSI N13.2 & 1969 R88 \\
\hline 0439 & ANSI & ANSI N13.2 & 1969 & $\begin{array}{l}\text { Guide to Administrative Practices in } \\
\text { Radiation Monitoring }\end{array}$ & inm83524 & 1984 & $\mathbf{R}$ & & ANSI N13.2 & $1969 \mathrm{PB8}$ \\
\hline 0440 & ANSI & ANSI N13.2 & 1969 & $\begin{array}{l}\text { Guide to Administrative Practices in } \\
\text { Radiation Monitoring }\end{array}$ & inm83525 & 1984 & $\mathbf{R}$ & & ANSI N13.2 & $1969 \mathrm{PB8}$ \\
\hline 0441 & ANSI & ANSI N13.2 & 1969 & $\begin{array}{l}\text { Guide to Administrative Practices in } \\
\text { Radiation Monitoring }\end{array}$ & inm83725 & 1991 & $\mathbf{R}$ & & ANSI N13.2 & $1969 \mathrm{RBB}$ \\
\hline 0442 & ANSI & ANSI N13.2 & 1982 & $\begin{array}{l}\text { Guide to Administrative Practices in } \\
\text { Radiation Monitoring }\end{array}$ & inm83725 & 1991 & $\mathbf{R}$ & & ANSI N13.2 & 1969 R88 \\
\hline 0443 & ANSI & ANSI N13.2 & 1982 & $\begin{array}{l}\text { Guide to Administrative Practices in } \\
\text { Radiation Monitoring }\end{array}$ & inm83750 & 1988 & $\mathbf{R}$ & & ANSI N13.2 & $1969 \mathrm{PB8}$ \\
\hline 0444 & ANSI & ANSI N13.2 & 1969 & $\begin{array}{l}\text { Gulde to Administrative Practices in } \\
\text { Radiation Monitoring }\end{array}$ & inm83750 & 1988 & $\mathbf{R}$ & & ANSI N13.2 & $1969 \mathrm{RB8}$ \\
\hline 0445 & ANSI & ANSI N13.2 & 1969 & $\begin{array}{l}\text { Guide to Administrative Practices in } \\
\text { Radiation Monitoring }\end{array}$ & reg10.8.ro2 & 1987 & $\mathbf{R}$ & & ANSI N13.2 & $1969 \mathrm{R} 88$ \\
\hline 0446 & ANSI & ANSI N13.2 & 1969 & $\begin{array}{l}\text { Guide to Administrative Practices in } \\
\text { Radiation Monitoring }\end{array}$ & reg8.2.ro0 & 1973 & $\mathbf{P}$ & Subject to conditions & ANSI N13.2 & 1969 R88 \\
\hline 0447 & ANSI & ANSI N13.2 & 1969 & $\begin{array}{l}\text { Guide to Administrative Practices in } \\
\text { Radiation Monitoring }\end{array}$ & srp12.5 & 1981 & $\mathbf{P}$ & $\begin{array}{l}\text { As relates to } \\
\text { administrative }\end{array}$ & ANSI N13.2 & 1969 R88 \\
\hline 0448 & ANSI & ANSI N13.4 & 1971 & $\begin{array}{l}\text { Performance Specifications for } \\
\text { Health Physics Instrumentation }\end{array}$ & reg10.8.r02 & 1987 & $\mathbf{R}$ & & $\begin{array}{l}\text { ANSI N42.17a, } \\
\text { N42.17b, \& } \\
\text { N42.17c } \\
\end{array}$ & 1989 \\
\hline 0449 & ANSI & ANSI N13.5 & 1972 & $\begin{array}{l}\text { Performance Specifications for } \\
\text { Direct-Reading and Indirect- } \\
\text { Reading Pocket Dosimeters for X- } \\
\text { and Gamma Radiation }\end{array}$ & inm83100 & 1991 & $\mathbf{R}$ & & ANSI N13.5 & 1972 R89 \\
\hline 0450 & ANSI & ANSI N13.5 & 1972 & $\begin{array}{l}\text { Performance Specifications for } \\
\text { Direct-Reading and Indirect- } \\
\text { Reading Pocket Dosimeters for X- } \\
\text { and Gamma Radiation }\end{array}$ & inm83524 & 1984 & $\mathbf{R}$ & & ANSI N13.5 & $1972 \mathrm{R89}$ \\
\hline
\end{tabular}


Codes and Standards and Other Guidance Clted in Regulatory Documents

Part A - Industry Consensus Codes and Standards (continued)

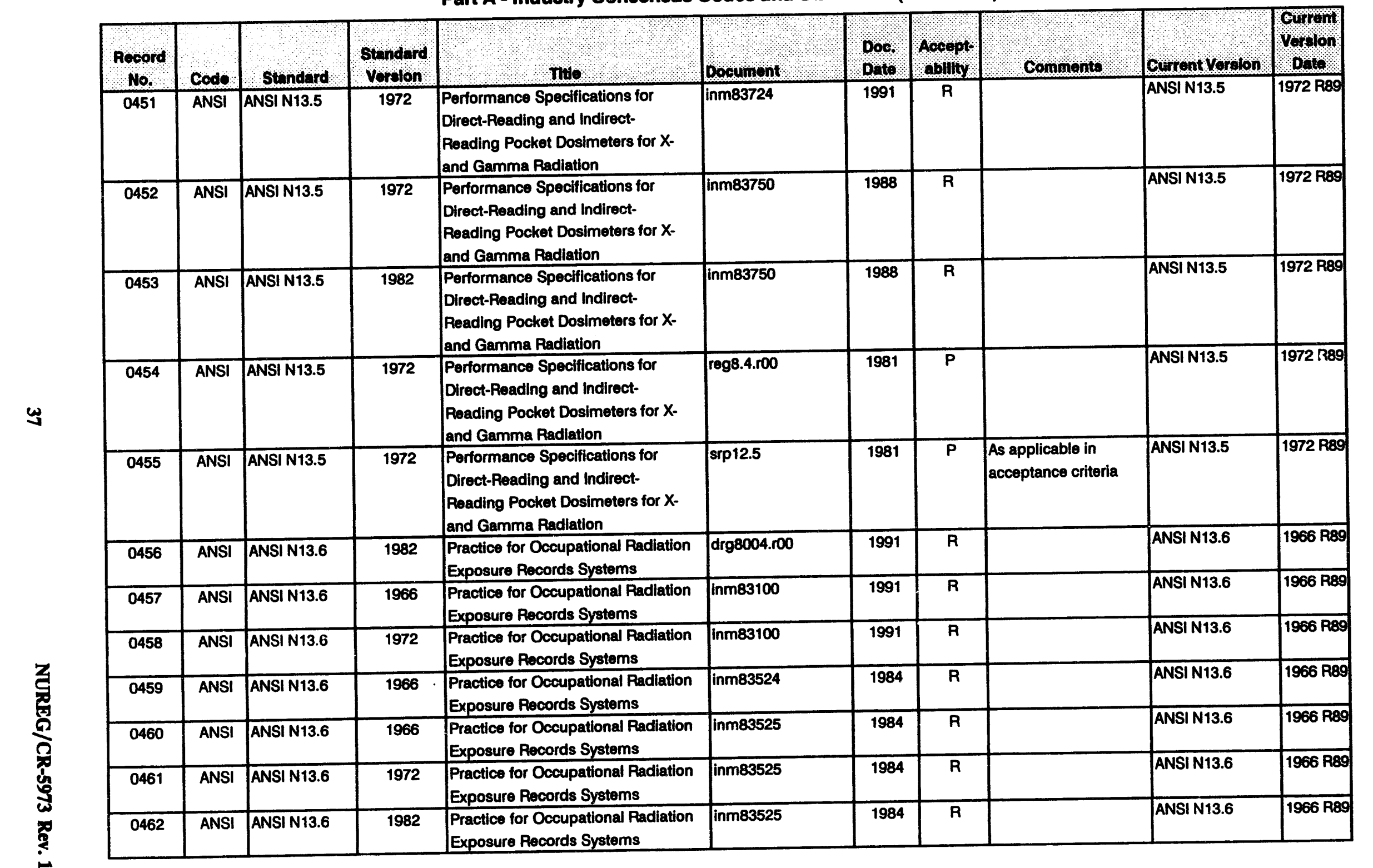




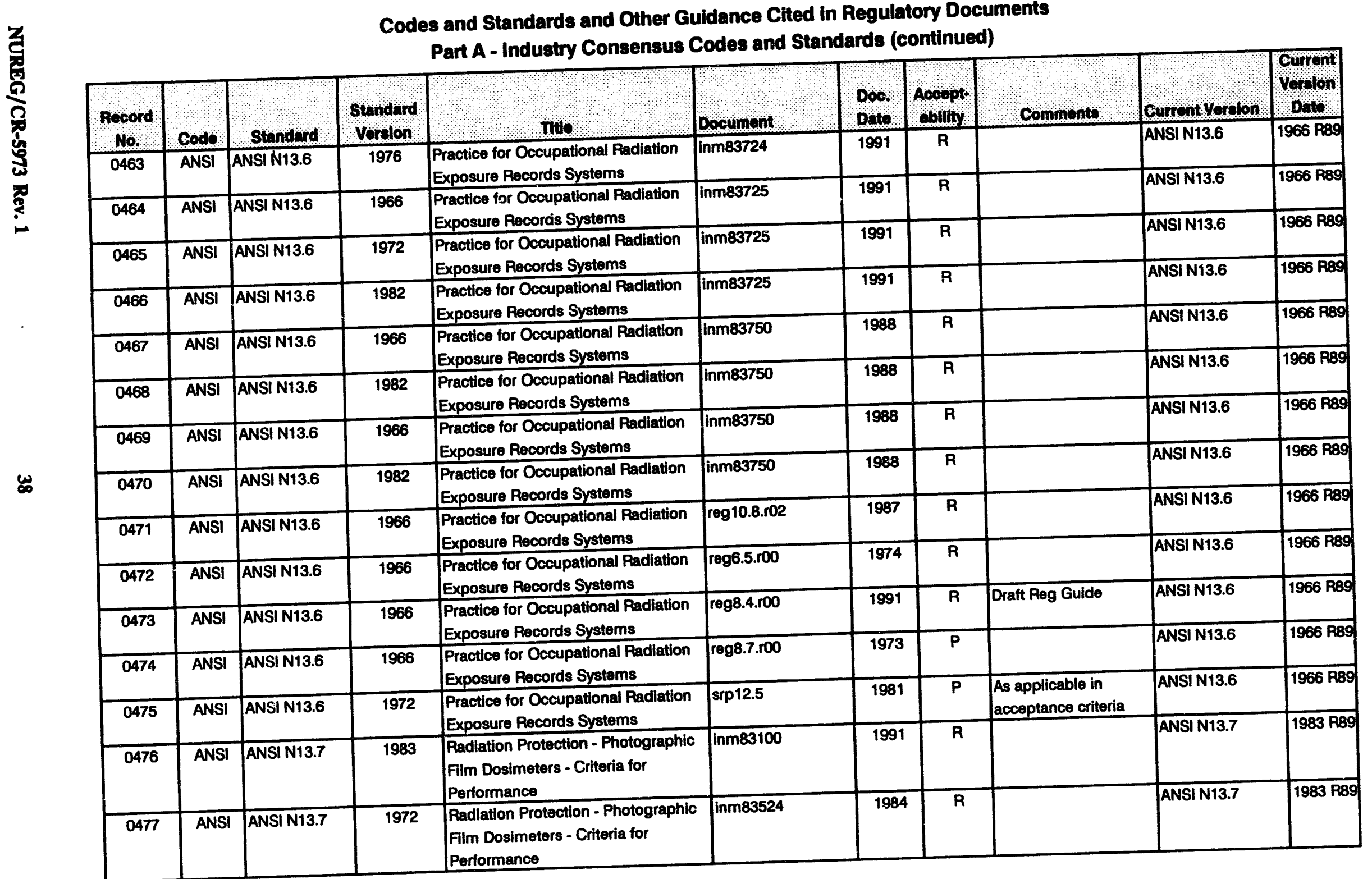


Codes and Standards and Other Guidance Cited in Regulatory Documents

Part A - Industry Consensus Codes and Standards (continued)

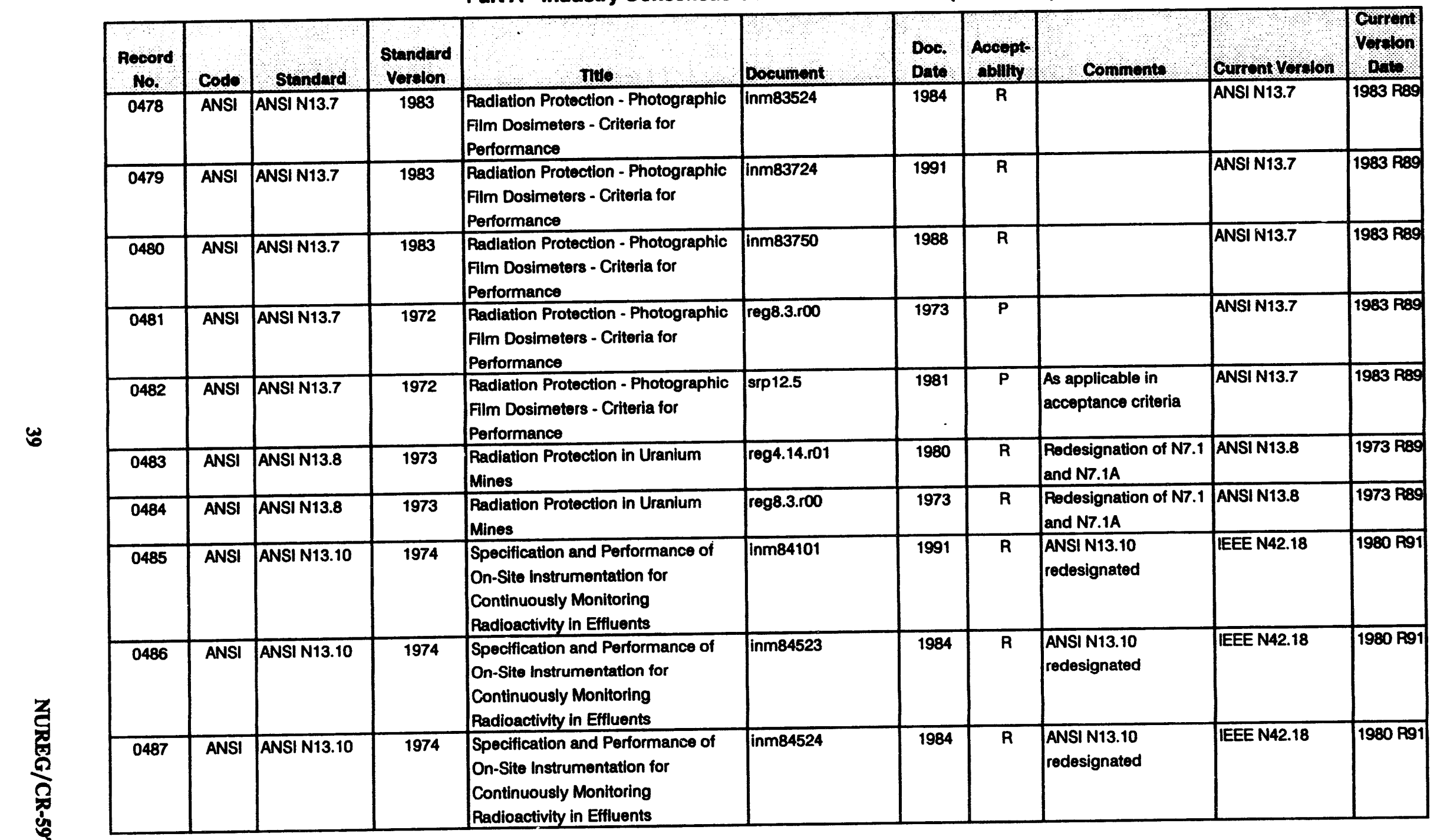


Codes and Standards and Other Guidance Cited in Regulatory Documents

Part A - Industry Consensus Codes and Standards (continued)

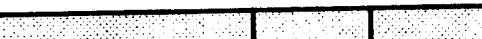

\begin{tabular}{|c|c|c|c|c|c|c|c|c|c|c|}
\hline Ropord & Code & Standard & $\begin{array}{l}\text { Standard } \\
\text { Vordon }\end{array}$ & NIILO, & Dooument & $\begin{array}{l}\text { Doc. } \\
\text { Dote. }\end{array}$ & $\begin{array}{l}\text { Nooppts } \\
\text { Nollity }\end{array}$ & Comments & Cumont Vorolon & ourtent \\
\hline 0488 & ANSI & ANSI N13.10 & 1974 & $\begin{array}{l}\text { Specification and Performance of } \\
\text { On-Site Instrumentation for } \\
\text { Continuously Monitoring } \\
\text { Radioactivity in Effluents }\end{array}$ & inm84723 & 1991 & $\mathbf{R}$ & $\begin{array}{l}\text { ANSI N13.10 } \\
\text { redesignated }\end{array}$ & IEEE N42.18 & $1980 \mathrm{PQ1}$ \\
\hline 0489 & ANSI & ANSI N13.10 & 1974 & $\begin{array}{l}\text { Specification and Performance of } \\
\text { On-Site instrumentation for } \\
\text { Continuously Monitoring } \\
\text { Radioactivity in Effluents }\end{array}$ & inm84724 & 1991 & $\mathbf{R}$ & $\begin{array}{l}\text { ANSI N13.10 } \\
\text { redesignated }\end{array}$ & IEEE N42.18 & $1980 \mathrm{R91}$ \\
\hline 0490 & ANSI & ANSI N13.10 & 1974 & $\begin{array}{l}\text { Specification and Performance of } \\
\text { On-Site Instrumentation for } \\
\text { Continuously Monitoring } \\
\text { Radioactivity in Effluents }\end{array}$ & inm84750 & 1988 & $\mathbf{R}$ & \begin{tabular}{|l|} 
ANSI N13.10 \\
redesignated
\end{tabular} & IEEE N42.18 & $1980 \mathrm{R91}$ \\
\hline 0491 & ANSI & ANSI N13.10 & 1974 & $\begin{array}{l}\text { Specification and Performance of } \\
\text { On-site instrumentation for } \\
\text { Continuously Monitoring } \\
\text { Radioactivity in Effluents }\end{array}$ & reg4.14.001 & 1980 & $\mathbf{R}$ & \begin{tabular}{|l|} 
Typo in reg4.14.ro1 - \\
ANSI N14.10 not a \\
valid designation. Title \\
cited is for ANSI \\
N13.10 \\
\end{tabular} & IEEE N42.18 & $1980 \mathrm{R} 91$ \\
\hline 0492 & ANSI & ANSI N13.10 & 1974 & $\begin{array}{l}\text { Specification and Performance of } \\
\text { On-Site Instrumentation for } \\
\text { Continuously Monitoring } \\
\text { Radioactivity in Effluents }\end{array}$ & reg4.15.ro1 & 1979 & $\mathbf{R}$ & $\begin{array}{l}\text { ANSI N13.10 } \\
\text { redesignated }\end{array}$ & IEEE N42.18 & $1980 \mathrm{R} 91$ \\
\hline 0493 & ANSI & ANSI N13.10 & 1974 & $\begin{array}{l}\text { Specification and Performance of } \\
\text { On-Site Instrumentation for } \\
\text { Continuously Monitoring } \\
\text { Radioactivity in Effluents }\end{array}$ & $\operatorname{srp} 11.5$ & 1981 & $\mathbf{P}$ & $\begin{array}{l}\text { ANSI N13.10 } \\
\text { redesignated }\end{array}$ & IEEE N42.18 & $1980 \mathrm{RQ1}$ \\
\hline 0494 & ANSI & ANSI N13.11 & $\mathrm{N} / \mathrm{S}$ & $\begin{array}{l}\text { Dosimetry - Personnel Dosimetry } \\
\text { Performance, Criteria for Testing }\end{array}$ & drg8007.r00 & 1991 & $\bar{P}$ & & ANSI N13.11 & 1983 \\
\hline 0495 & ANSI & ANSI N13.11 & 1983 & $\begin{array}{l}\text { Dosimetry - Personnel Dosimetry } \\
\text { Performance, Criteria for Testing }\end{array}$ & inm83100 & 1991 & $\bar{R}$ & & ANSI N13.11 & 1983 \\
\hline 0496 & ANSI & ANSI N13.11 & 1983 & $\begin{array}{l}\text { Dosimetry - Personnel Dosimetry } \\
\text { Performance, Criteria for Testing }\end{array}$ & inm83524 & 1984 & $R$ & & ANSI N13.11 & 1983 \\
\hline 0497 & ANSI & ANSI N13.11 & 1983 & $\begin{array}{l}\text { Dosimetry - Personnel Dosimetry } \\
\text { Performance, Criteria for Testing }\end{array}$ & inm83724 & 1991 & $\mathbf{R}$ & & ANSI N13.11 & 1983 \\
\hline
\end{tabular}


Codes and Standards and Other Guidance Cited in Regulatory Documents

Part A - Industry Consensus Codes and Standards (continued)

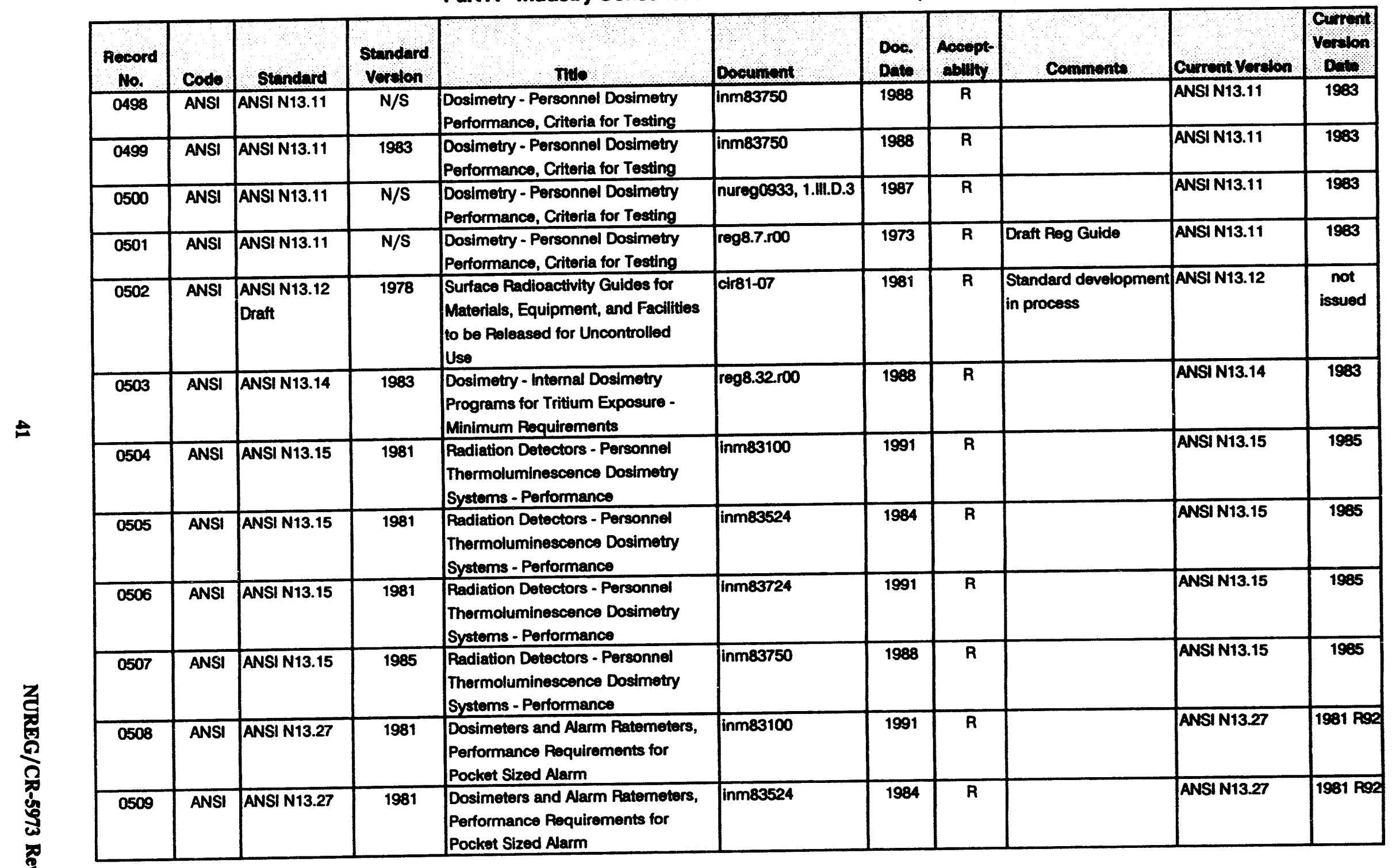


Codes and Standards and Other Guidance Chted in Regulatory Documents

Part A - Industry Consensus Codes and Standards (continued)

\begin{tabular}{|c|c|c|c|c|c|c|c|c|c|c|}
\hline $\begin{array}{l}\text { Aocord } \\
\text { No. }\end{array}$ & codes & Stondard & $\begin{array}{l}\text { Stinderd } \\
\text { voralon }\end{array}$ & Illo, & Doroument & $\begin{array}{l}\text { Doo. } \\
\text { Dote }\end{array}$ & $\begin{array}{l}\text { Aocept } \\
\text { chllhy }\end{array}$ & Connenents & Gunowlomon & Qn, \\
\hline 0510 & ANSI & ANSI N13.27 & 1981 & $\begin{array}{l}\text { Dosimeters and Alarm Ratemeters, } \\
\text { Performance Requirements for } \\
\text { Pocket Sized Alarm }\end{array}$ & inm83724 & 1991 & $\mathbf{R}$ & & ANSI N13.27 & $1981 \mathrm{RPC}$ \\
\hline 0511 & ANSI & ANSI N13.27 & 1981 & $\begin{array}{l}\text { Dosimeters and Alarm Ratemeters, } \\
\text { Performance Requirements for } \\
\text { Pocket Sized Alarm }\end{array}$ & inm83750 & 1988 & $\mathbf{R}$ & & ANSI N13.27 & $1981 \mathrm{PQ2}$ \\
\hline 0512 & ANSI & ANSI N13.27 & 1981 & $\begin{array}{l}\text { Dosimeters and Alarm Ratemeters, } \\
\text { Performance Requirements for } \\
\text { Pocket Sized Alarm }\end{array}$ & reg8.28.roo & 1981 & $\mathbf{P}$ & One exception & ANSI N13.27 & 1981 Ro? \\
\hline 0513 & ANSI & ANSI N14.1 & 1982 & $\begin{array}{l}\text { Nuclear Materials-Uranium } \\
\text { Hexafluoride-Packaging for } \\
\text { Transport } \\
\end{array}$ & not87-26 & 1987 & $\mathbf{A}$ & \begin{tabular}{|l|} 
DOT requires UFG \\
shipping containers to \\
meot ANSI \\
\end{tabular} & ANSIN14.1 & 1990 \\
\hline 0514 & ANSI & ANSIN14.1 & 1987 & $\begin{array}{l}\text { Nuclear Materials-Uranium } \\
\text { Hexafluoride-Packaging for } \\
\text { Transport }\end{array}$ & not89-78 & 1989 & $\mathbf{R}$ & $\begin{array}{l}\text { Provides criteria for } \\
\text { transport }\end{array}$ & ANSI N14.1 & 1990 \\
\hline 0515 & ANSI & ANSI N14.1 & 1987 & $\begin{array}{l}\text { Nuclear Materials-Uranium } \\
\text { Hoxafluoride-Packaging for } \\
\text { Transport }\end{array}$ & not90-27 & 1990 & $\mathbf{R}$ & & ANSI N14.1 & 1900 \\
\hline 0516 & ANSI & ANSI N14.1 & 1971 & $\begin{array}{l}\text { Nuclear Materials-Uranium } \\
\text { Hexafluoride-Packaging for } \\
\text { Transport }\end{array}$ & not92-58 & 1992 & $\mathbf{R}$ & & ANSI N14.1 & 1990 \\
\hline 0517 & ANSI & ANSI N14.2 & 1986 & $\begin{array}{l}\text { Tiedowns for Transport of Fissile } \\
\text { and Radioactive Containers Greater } \\
\text { than One-Ton Truck Transport }\end{array}$ & not87-31 & 1987 & $\mathbf{R}$ & $\begin{array}{l}\text { Standard under } \\
\text { development - } \\
\text { standard has not been } \\
\text { published }\end{array}$ & $n / a$ & $n / a$ \\
\hline 0518 & ANSI & ANSI N14.3 & 1973 & $\begin{array}{l}\text { Packaging and Transportation of } \\
\text { Radioactively Contaminated } \\
\text { Biological Materials }\end{array}$ & reg7.2.r00 & 1974 & $\mathbf{P}$ & Subject to conditions & Withdrawn & $n / a$ \\
\hline 0519 & ANSI & ANSI N14.4 & $\mathbf{N} / \mathbf{S}$ & ANSI N14.4 Subcommittee & reg7.10.ro1 & 1986 & $\mathbf{R}$ & \begin{tabular}{|l|} 
Pofers to the ANSI \\
N14.4 Subcommittee
\end{tabular} & $n / a$ & $n / a$ \\
\hline 0520 & ANSI & ANSI N14.5 & 1977 & $\begin{array}{l}\text { Radioactive Materials - Leakage } \\
\text { Tests on Packages for Shipment }\end{array}$ & reg 10.8.r02 & 1987 & $\mathbf{R}$ & & ANSI N14.5 & 1987 \\
\hline 0521 & ANSI & ANSI N14.5 & $N / S$ & $\begin{array}{l}\text { Radioactive Materials - Leakage } \\
\text { Tests on Packages for Shipment }\end{array}$ & reg7.4.ro0 & 1975 & A & & ANSI N14.5 & 1987 \\
\hline
\end{tabular}




\section{Codes and Standards and Other Guidance Cited in Regulatory Documents}

Part A - Industry Consensus Codes and Standards (continued)

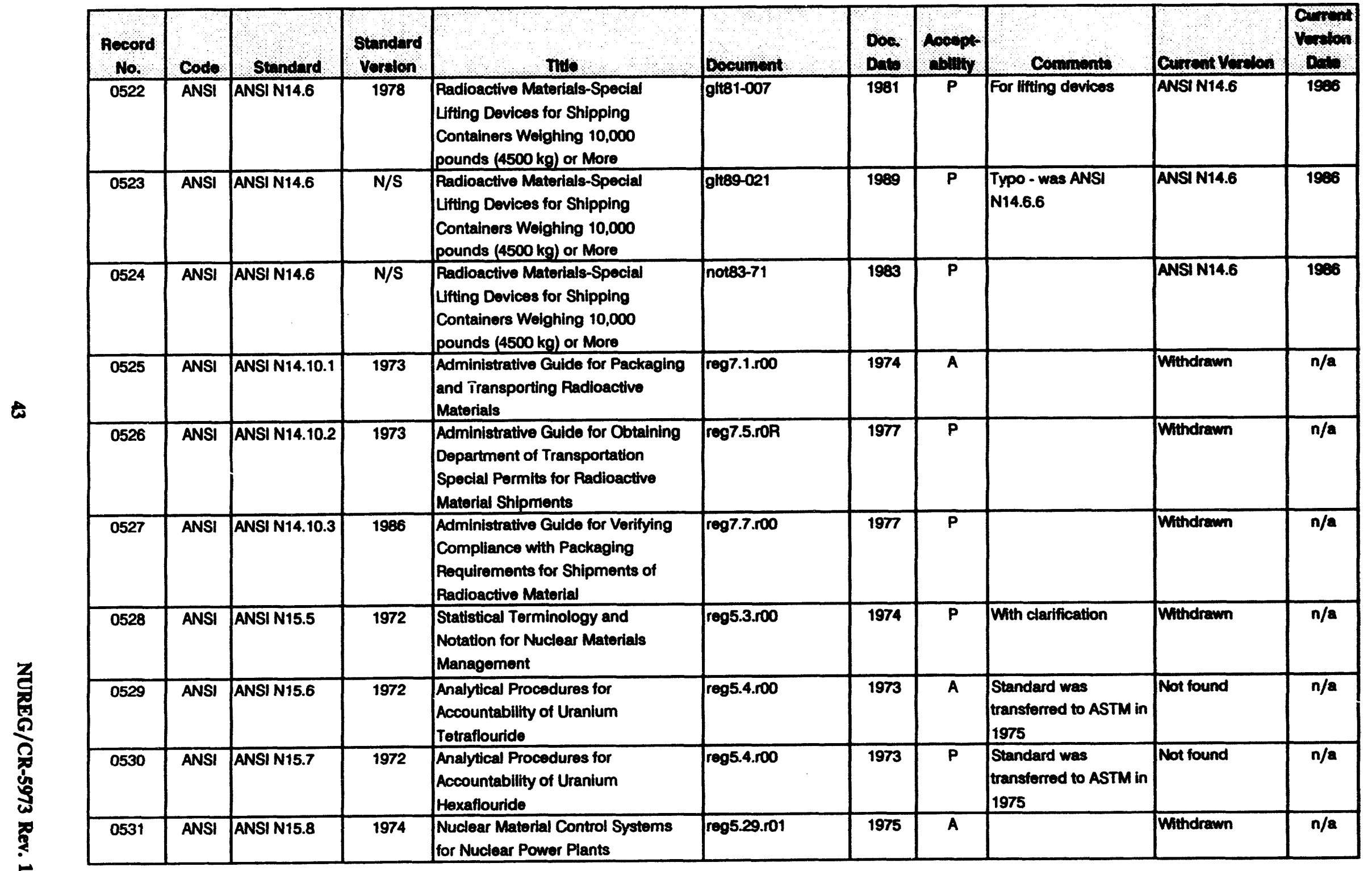


Codes and Standards and Other Guidance Cited in Regulatory Documents

Part A - Industry Consensus Codes and Standards (continued)

\begin{tabular}{|c|c|c|c|c|c|c|c|c|c|c|}
\hline $\begin{array}{l}\text { Rocord } \\
\text { No. }\end{array}$ & Cods & Standend & $\begin{array}{l}\text { Stundard } \\
\text { vorlon }\end{array}$ & $\mathbf{n} \mathbf{l}$, & Downons & $\begin{array}{l}\text { Doo. } \\
\text { Dote. }\end{array}$ & Anoeph- & Commente & Ounnew yous & $e^{2+1, n}$ \\
\hline 0532 & ANSI & ANSI N15.15 & 1974 & $\begin{array}{l}\text { Assessment of the Assumption of } \\
\text { Normality (Employing individual } \\
\text { Observed Values) }\end{array}$ & reg1.126.ro1 & 1978 & $R$ & & ANSIN15.15 & $1974 \mathrm{RB1}$ \\
\hline 0533 & ANSI & ANSI N15.15 & 1973 & $\begin{array}{l}\text { Assessment of the Assumption of } \\
\text { Normality (Employing Individual } \\
\text { Observed Values) }\end{array}$ & reg5.22.100 & 1974 & $\mathbf{P}$ & & ANSIN15.15 & $1974 \mathrm{RB1}$ \\
\hline 0.534 & ANSI & ANSI N15.16 & $\mathrm{N} / \mathrm{S}$ & $\begin{array}{l}\text { Limit of Error Concepts and } \\
\text { Principles of Calculation in Nuclear } \\
\text { Materials Control }\end{array}$ & reg5.18.100 & 1974 & $\mathbf{P}$ & Whth exceptions & Wathdram & $n / a$ \\
\hline 0535 & ANSI & ANSI N15.16 & 1974 & $\begin{array}{l}\text { Limit of Error Concepts and } \\
\text { Principles of Calculation in Nuclear } \\
\text { Materials Control }\end{array}$ & reg5.28.r00 & 1974 & $\mathbf{R}$ & & Withdram & $n / a$ \\
\hline 0536 & ANSI & ANSI N15.17 & 1974 & $\begin{array}{l}\text { Concepts and Principles for the } \\
\text { Statistical Evaluation of Shipper- } \\
\text { Recelver Differences in the Transfer } \\
\text { of Special Nuclear Materials }\end{array}$ & reg5.28.roo & 1974 & $\mathbf{P}$ & With exceptions & Windrawn & $n / a$ \\
\hline 0537 & ANSI & ANSI N15.18 & N/S & $\begin{array}{l}\text { Nuclear Materials - Mass } \\
\text { Calibration Techniques for Control }\end{array}$ & reg5.48.100 & 1975 & $\mathbf{R}$ & & ANSI N15.18 & 1988 \\
\hline 0538 & ANSI & ANSI N15.18 & 1975 & $\begin{array}{l}\text { Nuclear Materials - Mass } \\
\text { Calibration Techniques for Control }\end{array}$ & reg5.58.r01 & 1980 & $\mathbf{A}$ & & ANSIN15.18 & 1988 \\
\hline 0539 & ANSI & ANSI N15.19 & $N / S$ & $\begin{array}{l}\text { Nuclear Materials Control - Volume } \\
\text { Calibration Techniques }\end{array}$ & reg5.48.100 & 1975 & $\mathbf{R}$ & & ANSI N15.19 & 1900 \\
\hline 0540 & ANSI & ANSI N15.19 & 1975 & $\begin{array}{l}\text { Nuclear Materials Control - Volume } \\
\text { Callbration Techniques }\end{array}$ & reg5.58.101 & 1980 & $\mathbf{R}$ & & ANGIN15.19 & 1900 \\
\hline 0541 & ANSI & ANSI N15.20 & 1975 & $\begin{array}{l}\text { Guide to Callbrating Nondestructive } \\
\text { Assay Systems }\end{array}$ & reg5.11.01 & 1984 & $\mathbf{R}$ & $\begin{array}{l}\text { Endoreed by Reg } \\
\text { Guide } 5.53\end{array}$ & AMSI N15.20 & 1975 RES \\
\hline 0542 & ANSI & ANSI N15.20 & 1975 & $\begin{array}{l}\text { Guide to Callbrating Nondestructive } \\
\text { Assay Systems }\end{array}$ & reg5.34.101 & 1984 & $\mathbf{R}$ & $\begin{array}{l}\text { Endorsed by Reg } \\
\text { Guide } 5.53\end{array}$ & AisS1 N15.20 & $1975 \mathrm{RB7}$ \\
\hline 0543 & ANSI & ANSI N15.20 & 1975 & $\begin{array}{l}\text { Guido to Callibrating Nondestructive } \\
\text { Assay Systems }\end{array}$ & reg5.38.ro1 & 1983 & $\bar{R}$ & & ANS1 N1520 & $1975 \mathrm{REF}$ \\
\hline 0544 & ANSI & ANSI N15.20 & 1975 & $\begin{array}{l}\text { Guide to Calibrating Nondestructivo } \\
\text { Assay Systems }\end{array}$ & reg5.53.01 & 1984 & $\mathbf{P}$ & $\begin{array}{l}\text { Whe supplomemtal } \\
\text { condilions }\end{array}$ & ANSIN1520 & 1975 Reg \\
\hline 0545 & ANSI & ANSI N15.20 & 1975 & $\begin{array}{l}\text { Guido to Calibrating Nondeatructivo } \\
\text { Assay Systems }\end{array}$ & reg5.58.r01 & 1980 & $\mathbf{A}$ & & ANSTN15.20 & $1975 \mathrm{RBg}$ \\
\hline
\end{tabular}


Codes and Standands and Other Guidance Ched in Regulatory Documents

Part A - Industry Consonsus Codes and Standands (continued)

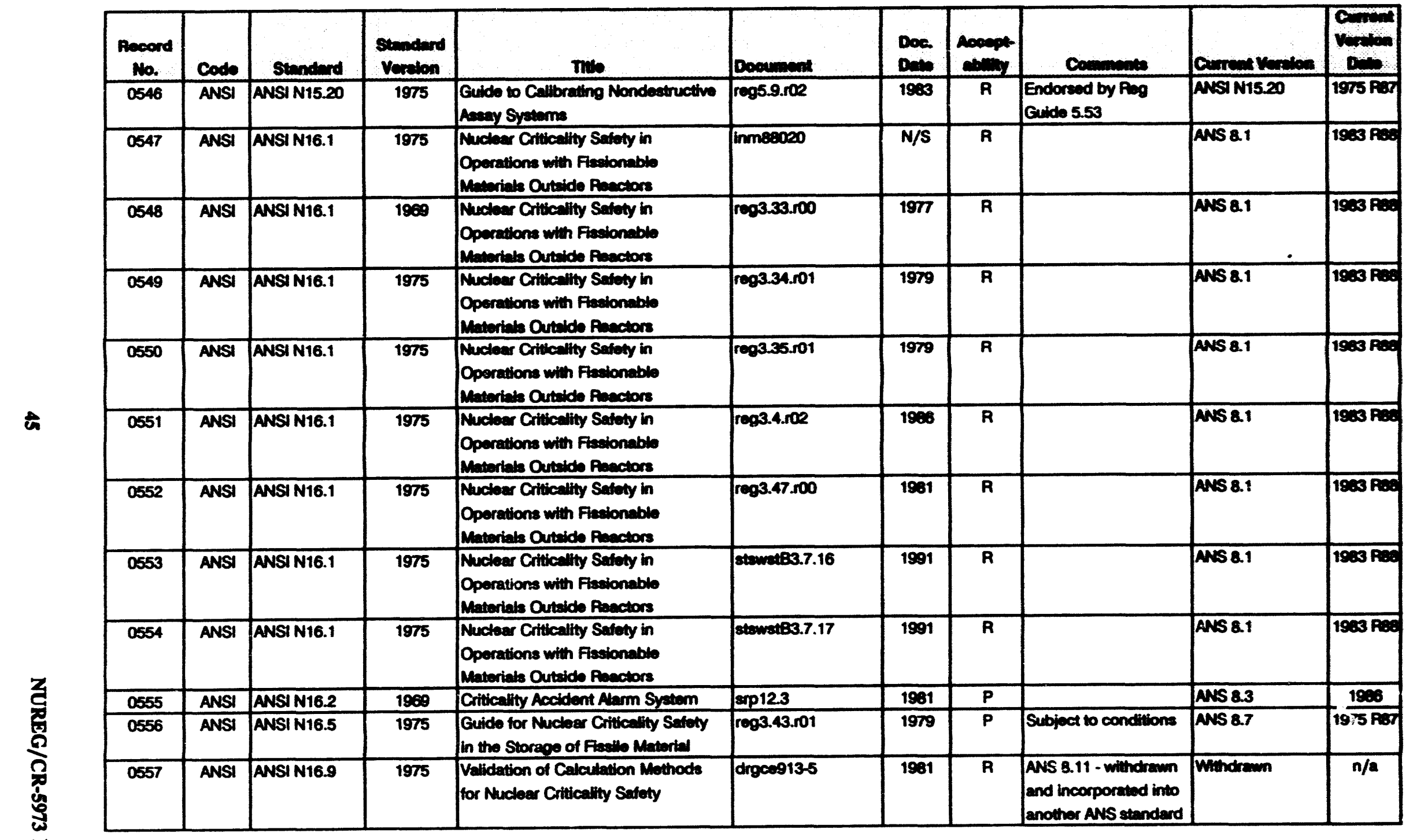




\begin{tabular}{|c|c|c|c|c|c|c|c|c|c|c|}
\hline & & & Cod & $\begin{array}{l}\text { and Standards and Other } \\
\text { Part A - Indusetry Consenst }\end{array}$ & $\begin{array}{l}\text { uidance C } \\
\text { Codes anc }\end{array}$ & andis ( & $\begin{array}{l}\text { tory Do } \\
\text { sontinus }\end{array}$ & $\begin{array}{l}\text { cuments } \\
\text { d) }\end{array}$ & & \\
\hline $\begin{array}{l}\text { Record } \\
\text { No. }\end{array}$ & Cods & sundard & $\begin{array}{l}\text { Standard } \\
\text { Vereion }\end{array}$ & the & Docounent & Doe. & Aconpe & conous. & 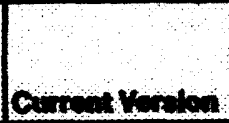 & Vomion \\
\hline 0558 & ANSI & ANSIN16.9 & 1975 & $\begin{array}{l}\text { Validation of Calculation Mothods } \\
\text { for Nuctoar Criticallty Satety }\end{array}$ & inm88015 & $N / S$ & R & $\begin{array}{l}\text { ANS } 8.11 \text { - withdreawn } \\
\text { and incorporated into } \\
\text { another ANS stenderd }\end{array}$ & Withotrawn & $n / a$ \\
\hline 0559 & ANSI & ANSI N16.9 & 1975 & $\begin{array}{l}\text { Validation of Calculation Methods } \\
\text { for Nuclear Criticallty Safoty }\end{array}$ & rog3.4.ro2 & 1986 & $\mathbf{R}$ & $\begin{array}{l}\text { ANS } 8.11 \text { - withdrawn } \\
\text { and incorporated into } \\
\text { another ANS standard }\end{array}$ & Withdrawn & $n / a$ \\
\hline 0560 & ANSI & ANSIN16.9 & 1975 & $\begin{array}{l}\text { Validation of Calculation Mothods } \\
\text { for Nuclear Criticality Safoty }\end{array}$ & rog3.47.100 & 1981 & $\mathbf{R}$ & $\begin{array}{l}\text { ANS } 8.11 \text { - withdrawn } \\
\text { and incorporated into } \\
\text { another ANS standard }\end{array}$ & Withdrawn & $n / a$ \\
\hline 0561 & ANSI & ANSI N16.9 & 1975 & $\begin{array}{l}\text { Validation of Calculation Mothods } \\
\text { for Nuclear Criticality Safoty }\end{array}$ & reg3.53.roo & 1982 & $\mathbf{R}$ & $\begin{array}{l}\text { ANS 8.11 - withdrawn } \\
\text { and incorporatod into } \\
\text { another ANS standerd }\end{array}$ & Withdrawn & $n / a$ \\
\hline 0562 & ANSI & ANSI N18.1 & 1971 & $\begin{array}{l}\text { Solection, Qualfication, and } \\
\text { Training of Porsonnel for Nuctear } \\
\text { Power Plants }\end{array}$ & ctr80-21 & 1960 & $\mathbf{P}$ & General ondorsement & ANS 3.1 & 1993 \\
\hline 0563 & ANSI & ANSI N18.1 & 1971 & $\begin{array}{l}\text { Soloction, Qualfication and } \\
\text { Training of Personnel for Nuclear } \\
\text { Powor Ptants }\end{array}$ & cir81-07 & 1981 & A & $\begin{array}{l}\text { Endorsed by Reg } \\
\text { Guide } 1.8 \text { Rov } 1\end{array}$ & ANS 3.1 & 1993 \\
\hline 0564 & ANSI & ANSI N18.1 & $N / S$ & $\begin{array}{l}\text { Soloction, Quallfication and } \\
\text { Training of Porsonnel for Nuclear } \\
\text { Power Plants }\end{array}$ & drgrs $902-4$ & 1960 & $\mathbf{R}$ & Proposed rog1.3.ro3 & ANS 3.1 & 1993 \\
\hline 0565 & ANSI & ANSI N18.1 & $N / S$ & $\begin{array}{l}\text { Soloction, Quallfication and } \\
\text { Training of Personnel for Nuclear } \\
\text { Power Plants }\end{array}$ & glt79-025 & 1979 & A & & ANS 3.1 & 1993 \\
\hline 0566 & ANSI & ANSI N18.1 & 1971 & $\begin{array}{l}\text { Solection, Qualfication and } \\
\text { Tralning of Personnel for Nuclear } \\
\text { Power Plants }\end{array}$ & inm35701 & 1992 & R & & ANS 3.1 & 1993 \\
\hline 0567 & ANSI & ANSI N18.1 & N/S & $\begin{array}{l}\text { Solvotion, Qualification and } \\
\text { Training of Porsonnel for Nuclear } \\
\text { Power Plants }\end{array}$ & inm36301B & 1976 & R & & ANS 3.1 & 1993 \\
\hline 0568 & ANSI & ANSI N18.1 & 1971 & $\begin{array}{l}\text { Seloction, Qualification and } \\
\text { Training of Porsonnol for Nuclear } \\
\text { Power Plants }\end{array}$ & inm37001 & 1992 & $\mathbf{R}$ & & ANS 3.1 & 1993 \\
\hline
\end{tabular}


Codes and Standards and Other Guidance Cited in Regulatory Documents

Part A - Industry Consensus Codes and Standards (continued)

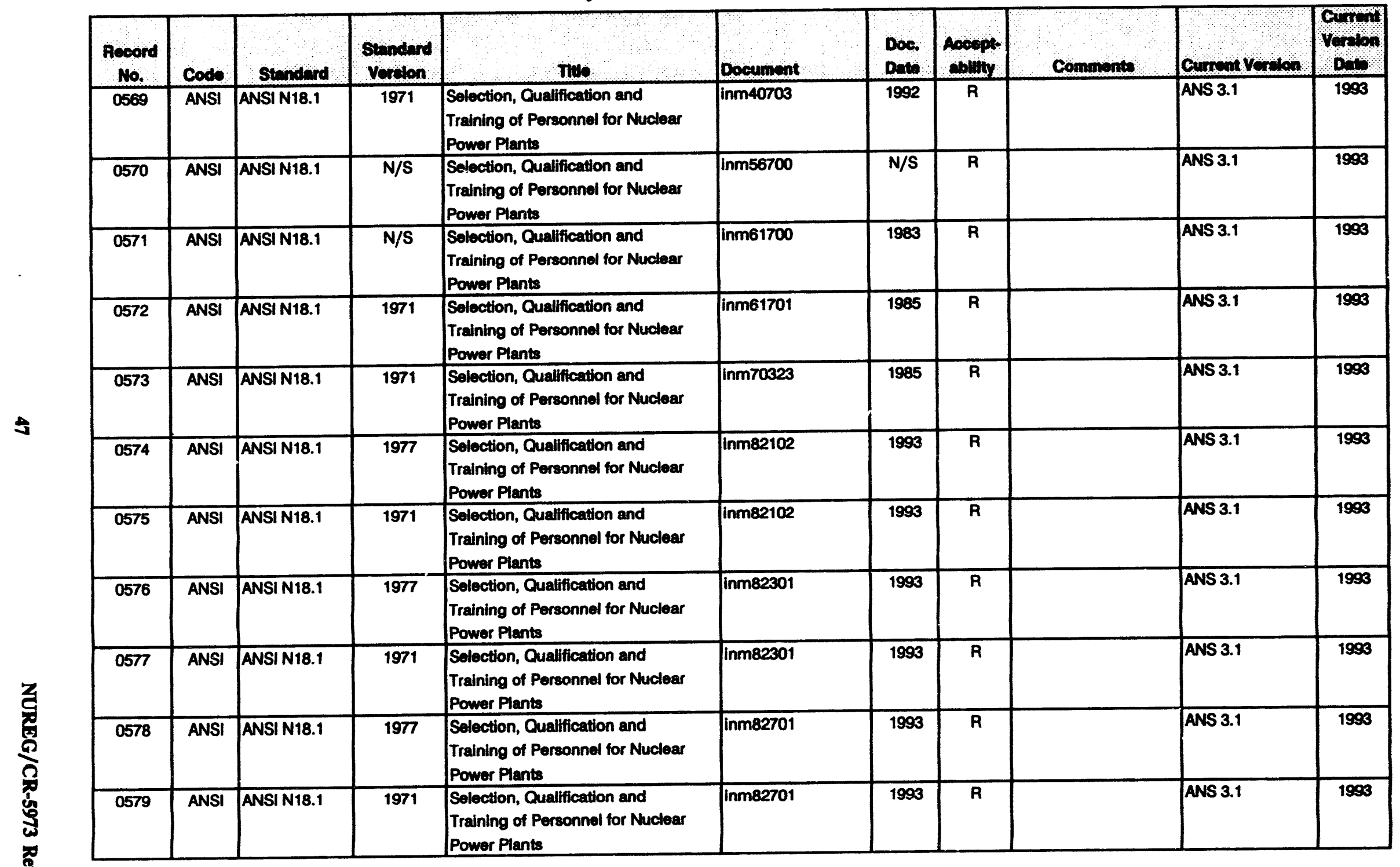


Codes and Standards and Other Guidance Clted in Regulatory Documents

Part A - Industry Consensus Codes and Standards (continued)

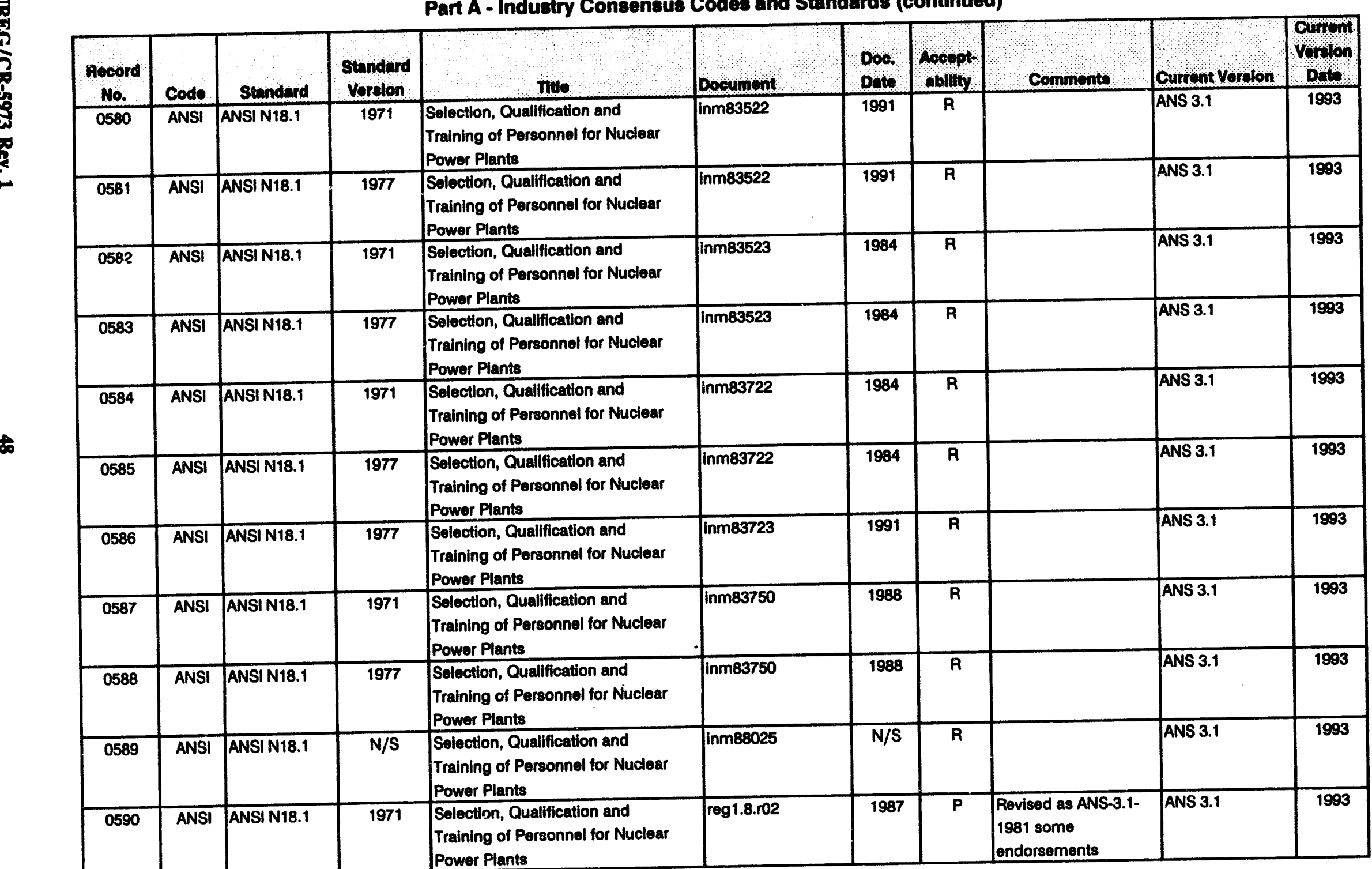


Codes and Standards and Other Guidance Cited in Regulatory Documents

Part A - Industry Consensus Codes and Standards (continued)

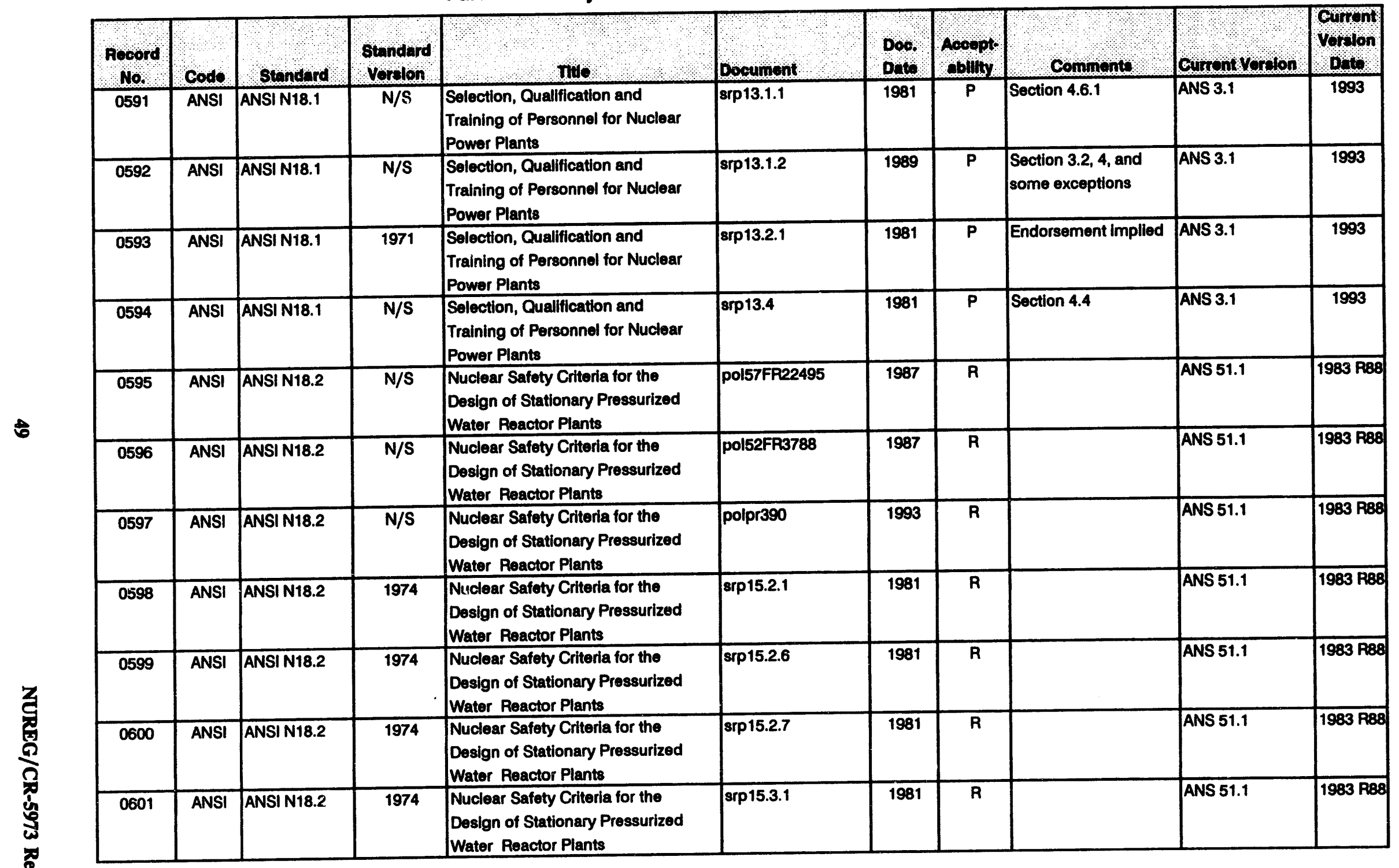


Codes and Standards and Other Guidance Ched in Regulatory Documents

Part A - Industry Consensus Codes and Standards (continued)

\begin{tabular}{|c|c|c|c|c|c|c|c|c|c|c|}
\hline $\begin{array}{l}\text { Rocord } \\
\text { No. }\end{array}$ & codo & shondurd & $\begin{array}{l}\text { Sandard } \\
\text { Vordon }\end{array}$ & 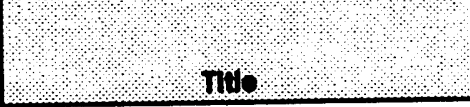 & Documont & $\begin{array}{l}\text { Doo. } \\
\text { Dow }\end{array}$ & $\begin{array}{l}\text { Aecept } \\
\text { abilly }\end{array}$ & Comments & Cunonivorolon & onph \\
\hline 0602 & ANSI & ANSI N18.2 & 1974 & $\begin{array}{l}\text { Nuclear Safety Criteria for the } \\
\text { Design of Stationary Pressurized } \\
\text { Water Reactor Plants }\end{array}$ & $\operatorname{srp15.5.1}$ & 1981 & $\mathbf{R}$ & & ANS 51.1 & $1983 \mathrm{PB8}$ \\
\hline 0603 & ANSI & ANSI N18.2 & 1974 & $\begin{array}{l}\text { Nuclear Safety Criteria for the } \\
\text { Design of Stationary Pressurized } \\
\text { Water Reactor Plants }\end{array}$ & srp15.6.1 & 1981 & $\mathbf{A}$ & & ANS 51.1 & $1983 \mathrm{RB8}$ \\
\hline 0604 & ANSI & ANSI N18.2 & 1973 & \begin{tabular}{|l|} 
Nuclear Safety Criteria for the \\
Design of Stationary Pressurized \\
Water Reactor Plants \\
\end{tabular} & stsb\&wB3.2.4 & 1991 & $\mathbf{R}$ & & ANS 51.1 & 1983 R88 \\
\hline 0605 & ANSI & ANSI N18.3 & 1972 & Fuel Assembly Identification & reg5.1.roo & 1972 & $\mathbf{P}$ & $\begin{array}{l}\text { Special conditions } \\
\text { require further } \\
\text { identification } \\
\text { measures } \\
\end{array}$ & ANS 57.8 & $1978 \mathrm{RB7}$ \\
\hline 0606 & ANSI & ANSI N18.5 & 1974 & $\begin{array}{l}\text { Earthquake Instrumentation Criteria } \\
\text { for Nuclear Power Piants }\end{array}$ & drgms 140-5 & 1981 & $\mathbf{P}$ & Proposed reg1.12.ro2 & ANS 2.2 & 1988 \\
\hline 0607 & ANSI & ANSI N18.5 & N/S & $\begin{array}{l}\text { Earthquake Instrumentation Criteria } \\
\text { for Nuclear Power Plants }\end{array}$ & reg1.12.r01 & 1976 & $\mathbf{P}$ & Subject to conditions & ANS 2.2 & 1988 \\
\hline 0608 & ANSI & ANSIN18.7 & $\mathrm{N} / \mathrm{S}$ & $\begin{array}{l}\text { Administrative Controls and Quality } \\
\text { Assurance for the Operational } \\
\text { Phase of Nuclear Power Plants }\end{array}$ & cir80-02 & 1980 & $\mathbf{R}$ & & ANS 3.2 & 1988 \\
\hline 0609 & ANSI & ANSI N18.7 & 1976 & $\begin{array}{l}\text { Administrative Controls and Quality } \\
\text { Assurance for the Operational } \\
\text { Phase of Nuclear Power Piants }\end{array}$ & drgrs902-4 & 1980 & $\mathbf{P}$ & Proposed reg1.3.r03 & ANS 3.2 & 1988 \\
\hline 0610 & ANSI & ANSI N18.7 & 1972 & $\begin{array}{l}\text { Administrative Controls and Quality } \\
\text { Assurance for the Operational } \\
\text { Phase of Nuclear Power Plants }\end{array}$ & drgrs902-4 & 1980 & $\mathbf{R}$ & Proposed reg1.3.r03 & ANS 3.2 & 1988 \\
\hline 0611 & ANSI & ANSI N18.7 & 1976 & $\begin{array}{l}\text { Administrative Controls and Quality } \\
\text { Assurance for the Operational } \\
\text { Phase of Nuclear Power Plants }\end{array}$ & glt83-028 & 1983 & $\mathbf{R}$ & & ANS 3.2 & 1988 \\
\hline 0612 & ANSI & ANSI N18.7 & 1972 & $\begin{array}{l}\text { Administrative Controls and Quality } \\
\text { Assurance for the Operational } \\
\text { Phase of Nuclear Power Plants }\end{array}$ & inm-toc & 1993 & $\mathbf{R}$ & & ANS 3.2 & 1988 \\
\hline
\end{tabular}


Codes and Standards and Other Guidance Cited in Regulatory Documents

Part A - Industry Consensus Codes and Standards (continued)

\begin{tabular}{|c|c|c|c|c|c|c|c|c|c|c|}
\hline $\begin{array}{c}\text { Rocord } \\
\text { No. }\end{array}$ & codo & Standard & $\begin{array}{l}\text { Standerd } \\
\text { vortlon }\end{array}$ & ২ $\quad$ THo, & Doconont & $\begin{array}{l}\text { Doo. } \\
\text { Dote }\end{array}$ & $\begin{array}{l}\text { Accopt } \\
\text { abulty }\end{array}$ & Commento & Cunont vorolon & otrots \\
\hline 0613 & ANSI & ANSI N18.7 & 1972 & $\begin{array}{l}\text { Administrative Controls and Quality } \\
\text { Assurance for the Operational } \\
\text { Phase of Nuclear Power Plants }\end{array}$ & inm2515/065 & 1990 & $\mathbf{R}$ & & ANS 3.2 & 1988 \\
\hline 0614 & ANSI & ANSI N18.7 & 1976 & $\begin{array}{l}\text { Administrative Controls and Quality } \\
\text { Assurance for the Operational } \\
\text { Phase of Nuclear Power Plants }\end{array}$ & inm35701 & 1992 & $\mathbf{R}$ & & ANS 3.2 & 1988 \\
\hline 0615 & ANSI & ANSI N18.7 & 1976 & $\begin{array}{l}\text { Administrative Controls and Quality } \\
\text { Assurance for the Operational } \\
\text { Phase of Nuclear Power Plants }\end{array}$ & inm35740B & 1979 & $\mathbf{R}$ & & ANS 3.2 & 1988 \\
\hline 0616 & ANSI & ANSI N18.7 & 1976 & $\begin{array}{l}\text { Administrative Controls and Quality } \\
\text { Assurance for the Operational } \\
\text { Phase of Nuclear Power Piants }\end{array}$ & inm35741B & 1979 & $\bar{R}$ & & ANS 3.2 & 1988 \\
\hline 0617 & ANSI & ANSI N18.7 & 1976 & $\begin{array}{l}\text { Administrative Controls and Quality } \\
\text { Assurance for the Operational } \\
\text { Phase of Nuclear Power Plants }\end{array}$ & inm35742B & 1976 & $\mathbf{R}$ & & ANS 3.2 & 1988 \\
\hline 0618 & ANSI & ANSI N18.7 & 1976 & $\begin{array}{l}\text { Administrative Controls and Quality } \\
\text { Assurance for the Operational } \\
\text { Phase of Nuclear Power Plants }\end{array}$ & inm35743B & 1982 & $\mathbf{R}$ & & ANS 3.2 & 1988 \\
\hline 0619 & ANSI & ANSI N18.7 & 1976 & $\begin{array}{l}\text { Administrative Controls and Quality } \\
\text { Assurance for the Operational } \\
\text { Phase of Nuclear Power Plants }\end{array}$ & inm35744B & 1979 & $R$ & & ANS 3.2 & 1988 \\
\hline 0620 & ANSI & ANSI N18.7 & 1976 & $\begin{array}{l}\text { Administrative Controls and Quality } \\
\text { Assurance for the Operational } \\
\text { Phase of Nuclear Power Plants }\end{array}$ & inm35745B & 1976 & $\mathbf{R}$ & & ANS 3.2 & 1988 \\
\hline 0621 & ANSI & ANSI N18.7 & 1976 & $\begin{array}{l}\text { Administrative Controls and Quality } \\
\text { Assurance for the Operational } \\
\text { Phase of Nuclear Power Plants }\end{array}$ & inm35746B & 1979 & $\mathbf{R}$ & & ANS 3.2 & 1988 \\
\hline 0622 & ANSi & ANSI N18.7 & 1976 & $\begin{array}{l}\text { Administrative Controls and Quality } \\
\text { Assurance for the Operational } \\
\text { Phase of Nuclear Power Plants }\end{array}$ & inm35749B & 1976 & R & & ANS 3.2 & 1988 \\
\hline 0623 & ANSI & ANSI N18.7 & $\mathrm{N} / \mathrm{S}$ & $\begin{array}{l}\text { Administrative Controls and Quality } \\
\text { Assurance for the Operational } \\
\text { Phase of Nuclear Power Plants }\end{array}$ & inm37001 & 1992 & $\mathbf{R}$ & & ANS 3.2 & 1988 \\
\hline
\end{tabular}


Codes and Standards and Other Guidance Cited in Regulatory Documents

Part A - Industry Consensus Codes and Standards (continued)

\begin{tabular}{|c|c|c|c|c|c|c|c|c|c|c|}
\hline $\begin{array}{c}\text { Bocord } \\
\text { No. }\end{array}$ & Codo & Standard & $\begin{array}{l}\text { Standard } \\
\text { Yorslon }\end{array}$ & (3) & Document & $\begin{array}{l}\text { Dos. } \\
\text { Date }\end{array}$ & $\begin{array}{l}\text { Acceppt. } \\
\text { obully }\end{array}$ & Comments & Current Vorglon & $\begin{array}{l}\text { Current } \\
\text { Vorolon } \\
\text { Bow }\end{array}$ \\
\hline 0624 & ANSI & ANSI N18.7 & 1976 & $\begin{array}{l}\text { Administrative Controls and Quality } \\
\text { Assurance for the Operational } \\
\text { Phase of Nuclear Power Plants }\end{array}$ & inm37700 & 1990 & $R$ & & ANS 3.2 & 1988 \\
\hline 0625 & ANSI & ANSI N18.7 & 1976 & $\begin{array}{l}\text { Administrative Controls and Quality } \\
\text { Assurance for the Operational } \\
\text { Phase of Nuclear Power Plants }\end{array}$ & inm37702 & 1987 & $\mathbf{R}$ & & ANS 3.2 & 1988 \\
\hline 0626 & ANSI & ANSI N18.7 & 1976 & $\begin{array}{l}\text { Administrative Controls and Quality } \\
\text { Assurance for the Operational } \\
\text { Phase of Nuclear Power Plants }\end{array}$ & inm37703 & 1992 & $\mathbf{R}$ & & ANS 3.2 & 1988 \\
\hline 0627 & ANSI & ANSI N18.7 & 1976 & $\begin{array}{l}\text { Administrative Controls and Quality } \\
\text { Assurance for the Operational } \\
\text { Phase of Nuclear Power Plants }\end{array}$ & inm37828 & 1988 & $\mathbf{R}$ & & ANS 3.2 & 1988 \\
\hline 0628 & ANSI & ANSI N18.7 & 1976 & $\begin{array}{l}\text { Administrative Controls and Quality } \\
\text { Assurance for the Operational } \\
\text { Phase of Nuclear Power Plants }\end{array}$ & inm38701 & 1992 & $\overline{\mathbf{R}}$ & & ANS 3.2 & 1988 \\
\hline 0629 & ANSI & ANSI N18.7 & 1976 & $\begin{array}{l}\text { Administrative Controls and Quality } \\
\text { Assurance for the Operational } \\
\text { Phase of Nuclear Power Plants }\end{array}$ & inm40500 & 1992 & $\mathbf{R}$ & & ANS 3.2 & 1988 \\
\hline 0630 & ANSI & ANSI N18.7 & 1976 & $\begin{array}{l}\text { Administrative Controls and Quality } \\
\text { Assurance for the Operational } \\
\text { Phase of Nuctear Power Plants }\end{array}$ & inm40702 & 1992 & $\bar{R}$ & & ANS 3.2 & 1988 \\
\hline 0631 & ANSI & ANSI N18.7 & 1976 & $\begin{array}{l}\text { Administrative Controls and Quality } \\
\text { Assurance for the Operational } \\
\text { Phase of Nuclear Power Plants }\end{array}$ & inm40703 & 1992 & $\mathbf{R}$ & & ANS 3.2 & 1988 \\
\hline$\overline{0632}$ & ANSI & ANSI N18.7 & 1976 & $\begin{array}{l}\text { Administrative Controls and Quality } \\
\text { Assurance for the Operational } \\
\text { Phase of Nuclear Power Plants }\end{array}$ & inm40704 & 1992 & $\mathbf{R}$ & & ANS 3.2 & 1988 \\
\hline 0633 & ANSI & ANSI N18.7 & 1976 & $\begin{array}{l}\text { Administrative Controls and Quality } \\
\text { Assurance for the Operational } \\
\text { Phase of Nuclear Power Plants }\end{array}$ & inm $42400 \mathrm{~B}$ & 1976 & $\bar{R}$ & & ANS 3.2 & 1988 \\
\hline 0634 & ANSI & ANSI N18.7 & 1972 & $\begin{array}{l}\text { Administrative Controls and Quality } \\
\text { Assurance for the Operational } \\
\text { Phase of Nuclear Power Plants }\end{array}$ & $\operatorname{linm} 42400 B$ & 1976 & $\mathbf{R}$ & & ANS 3.2 & 1988 \\
\hline
\end{tabular}


Codes and Standards and Other Guidance Cited in Regulatory Documents

Part A - Industry Consensus Codes and Standards (continued)

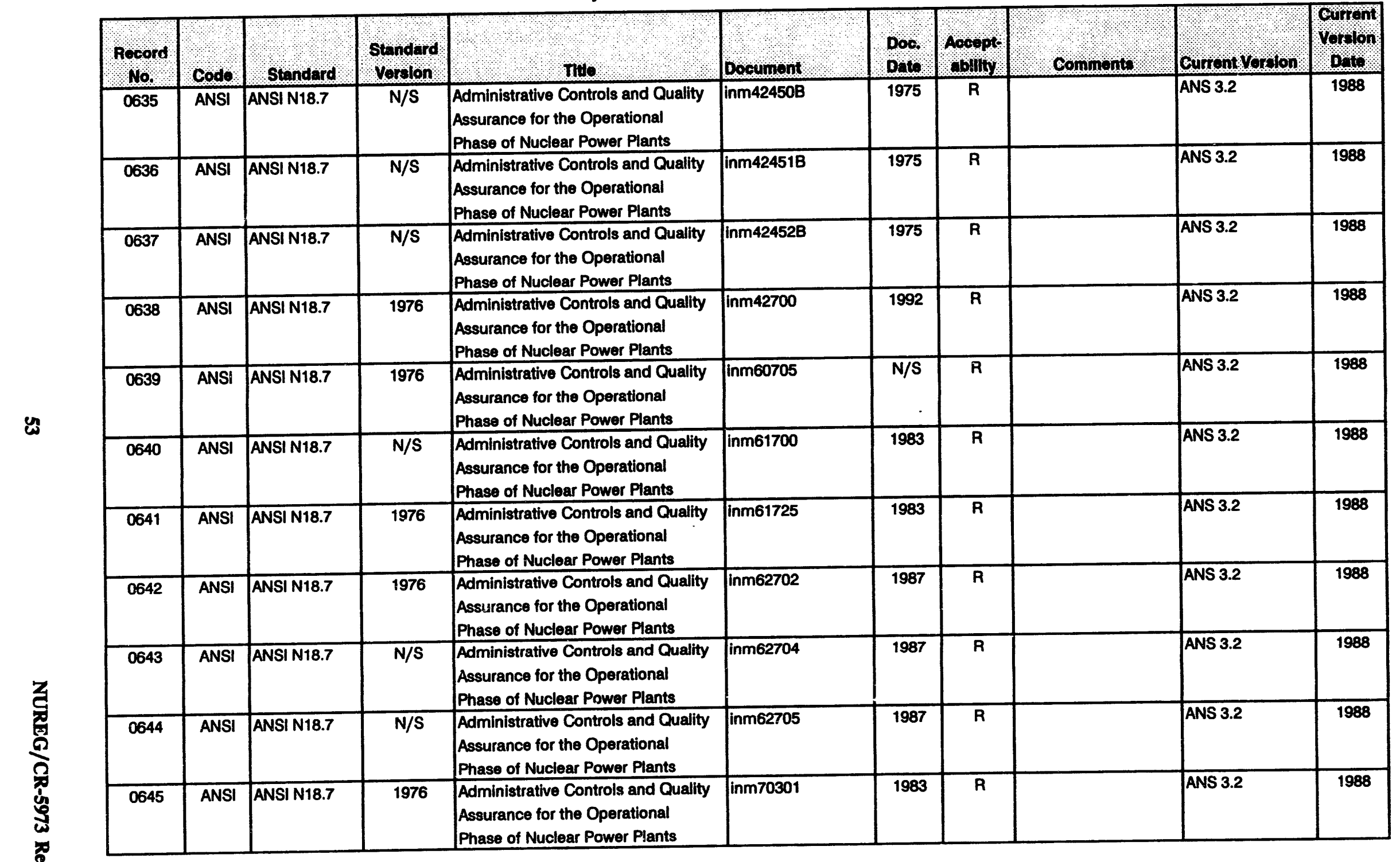


Codes and Standards and Other Guidance Cited in Regulatory Documents

Part A - Industry Consensus Codes and Standards (continued)

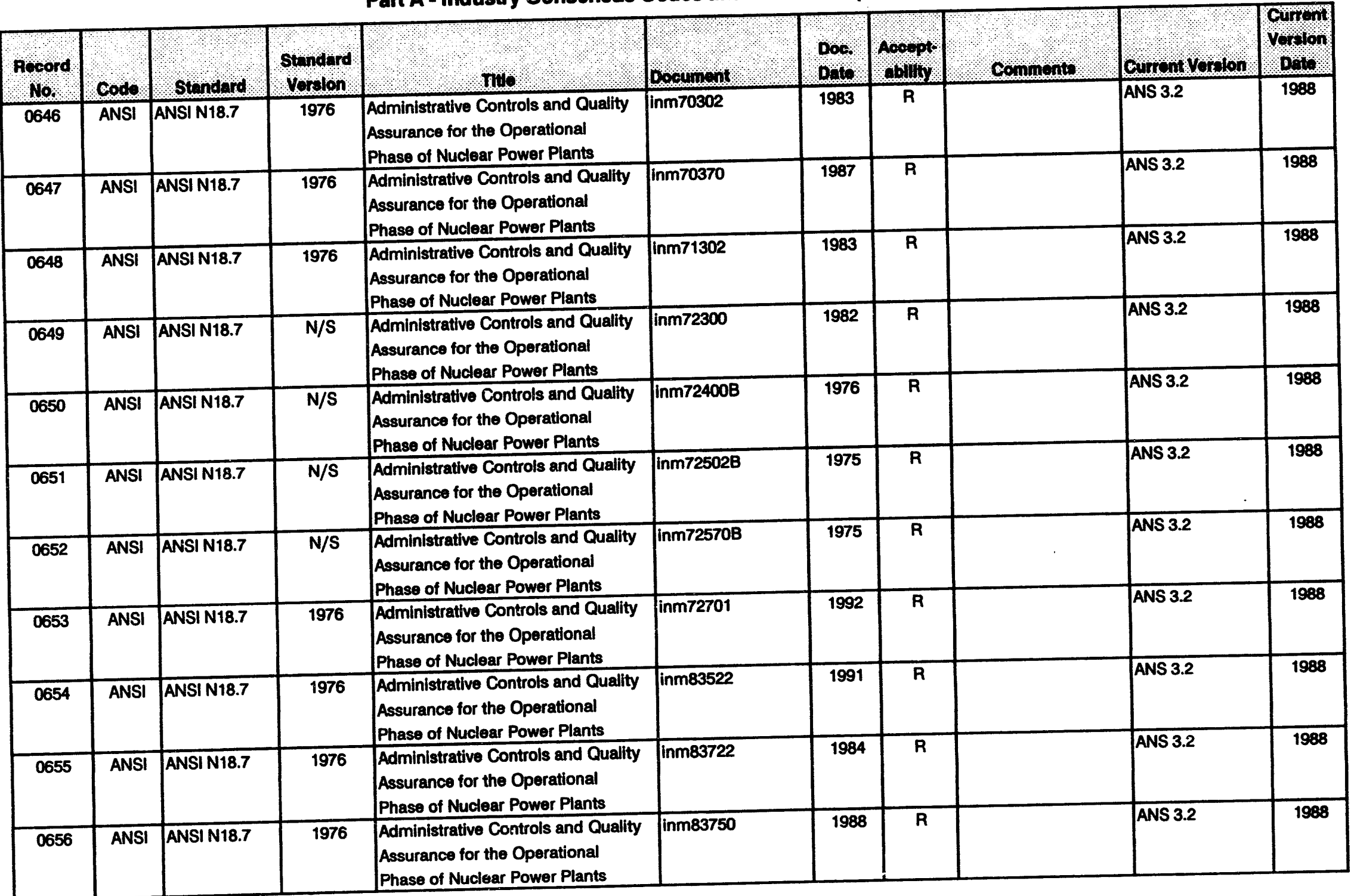


Codes and Standards and Other Guidance Cited in Regulatory Documents

Part A - Industry Consensus Codes and Standards (continued)

\begin{tabular}{|c|c|c|c|c|c|c|c|c|c|c|}
\hline $\begin{array}{l}\text { Record } \\
\text { No. }\end{array}$ & Code & Stonderd & $\begin{array}{l}\text { Standard } \\
\text { Vorslon }\end{array}$ & , Tha & Dooument. & $\begin{array}{l}\text { Doo. } \\
\text { Dows. }\end{array}$ & $\begin{array}{l}\text { Aosepts } \\
\text { ablly }\end{array}$ & Commonts & Cunomvorion. & Qring \\
\hline 0657 & ANSI & ANSI N18.7 & 1976 & $\begin{array}{l}\text { Administrative Controls and Quality } \\
\text { Assurance for the Operational } \\
\text { Phase of Nuclear Power Plants }\end{array}$ & inm92720 & 1992 & $\mathbf{R}$ & & ANS 3.2 & 1988 \\
\hline 0658 & ANSI & ANSI N18.7 & 1972 & $\begin{array}{l}\text { Administrative Controls and Quality } \\
\text { Assurance for the Operational } \\
\text { Phase of Nuclear Power Plants }\end{array}$ & inm9900/5.1.5 & 1986 & $\mathbf{R}$ & & ANS 3.2 & 1988 \\
\hline 0659 & ANSI & ANSI N18.7 & 1976 & $\begin{array}{l}\text { Administrative Controls and Quality } \\
\text { Assurance for the Operational } \\
\text { Phase of Nuclear Power Plants }\end{array}$ & inm9900/5.2.7 & 1977 & $\mathbf{R}$ & & ANS 3.2 & 1988 \\
\hline 0660 & ANSI & ANSI N18.7 & 1976 & $\begin{array}{l}\text { Administrative Controls and Quality } \\
\text { Assurance for the Operational } \\
\text { Phase of Nuclear Power Plants }\end{array}$ & inm9900/6.5.1 & 1980 & $\mathbf{R}$ & & ANS 3.2 & 1988 \\
\hline 0661 & ANSI & ANSI N18.7 & 1972 & $\begin{array}{l}\text { Administrative Controls and Quality } \\
\text { Assurance for the Operational } \\
\text { Phase of Nuclear Power Plants }\end{array}$ & inm9900/B & 1977 & $\mathbf{R}$ & & ANS 3.2 & 1988 \\
\hline 0662 & ANSI & ANSI N18.7 & 1976 & $\begin{array}{l}\text { Administrative Controls and Quality } \\
\text { Assurance for the Operational } \\
\text { Phase of Nuclear Power Plants }\end{array}$ & inm9900/B & 1977 & $\mathbf{P}$ & & ANS 3.2 & 1988 \\
\hline 0663 & ANSI & ANSI N18.7 & 1976 & $\begin{array}{l}\text { Administrative Controls and Quality } \\
\text { Assurance for the Operational } \\
\text { Phase of Nuclear Power Plants }\end{array}$ & inm9900/OPS & 1991 & $\mathbf{R}$ & & ANS 3.2 & 1988 \\
\hline 0664 & ANSI & ANSI N18.7 & 1976 & $\begin{array}{l}\text { Administrative Controls and Quality } \\
\text { Assurance for the Operational } \\
\text { Phase of Nuclear Power Plants }\end{array}$ & not79-15 & 1979 & $\mathbf{R}$ & $\begin{array}{l}\text { Endorsed by Reg. } \\
\text { Guide } 1.33\end{array}$ & ANS 3.2 & 1988 \\
\hline 0665 & ANSI & ANSI N18.7 & 1972 & $\begin{array}{l}\text { Administrative controls and Quality } \\
\text { Assurance for the Operational } \\
\text { Phase of Nuclear Power Plants }\end{array}$ & not84-51 & 1984 & $\mathbf{R}$ & $\begin{array}{l}\text { Partial endorsement } \\
\text { by Reg Guide } 1.33\end{array}$ & ANS 3.2 & 1988 \\
\hline 0666 & ANSI & ANSI N18.7 & 1976 & $\begin{array}{l}\text { Administrative Controls and Quality } \\
\text { Assurance for the Operational } \\
\text { Phase of Nuclear Power Plants }\end{array}$ & not85-66 & 1985 & $\mathbf{P}$ & $\begin{array}{l}\text { Typo - ANSI N8.7 is not } \\
\text { a valid ANSI standard. } \\
\text { ANSI N18.7 assumed. }\end{array}$ & ANS 3.2 & 1988 \\
\hline 0667 & ANSI & ANSI N18.7 & $\mathrm{N} / \mathrm{S}$ & $\begin{array}{l}\text { Administrative Controls and Quality } \\
\text { Assurance for the Operational } \\
\text { Phase of Nuclear Power Plants }\end{array}$ & nureg0737 & 1980 & $\bar{R}$ & & ANS 3.2 & 1988 \\
\hline
\end{tabular}


Codes and Standards and Other Guidance Cited in Regulatory Documents

Part A - Industry Consensus Codes and Standards (continued)

\begin{tabular}{|c|c|c|c|c|c|c|c|c|c|c|}
\hline Nocord & Cods & Standard & $\begin{array}{l}\text { Standard } \\
\text { Vordion }\end{array}$ & . & Bosonent & $\begin{array}{l}\text { Dos } \\
\text { Doto }\end{array}$ & $\begin{array}{l}\text { Aooept. } \\
\text { abilty }\end{array}$ & Componts & Gurront Votolon & onnt \\
\hline 0668 & ANSI & ANSI N18.7 & 1972 & $\begin{array}{l}\text { Administrative Controls and Quality } \\
\text { Assurance for the Operational } \\
\text { Phase of Nuclear Power Plants }\end{array}$ & reg1.33.ro1 & 1977 & $\mathbf{R}$ & & ANS 3.2 & 1988 \\
\hline 0669 & ANSI & ANSI N18.7 & 1976 & $\begin{array}{l}\text { Administrative Controls and Quality } \\
\text { Assurance for the Operational } \\
\text { Phase of Nuclear Power Plants }\end{array}$ & reg1.33.ro1 & 1977 & $\mathbf{P}$ & Subject to conditions & ANS 3.2 & 1988 \\
\hline 0670 & ANSI & ANSI N18.7 & 1972 & $\begin{array}{l}\text { Administrative Controls and Quality } \\
\text { Assurance for the Operational } \\
\text { Phase of Nuclear Power Plants }\end{array}$ & reg1.33.ro2 & 1978 & $\mathbf{R}$ & & ANS 3.2 & 1988 \\
\hline 0671 & ANSI & ANSI N18.7 & 1976 & $\begin{array}{l}\text { Administrative Controls and Quality } \\
\text { Assurance for the Operational } \\
\text { Phase of Nuclear Power Plants }\end{array}$ & reg1.33.ro2 & 1978 & $\mathbf{P}$ & Subject to conditions & ANS 3.2 & 1988 \\
\hline 0672 & ANSI & ANSI N18.7 & N/S & $\begin{array}{l}\text { Administrative Controls and Quality } \\
\text { Assurance for the Operational } \\
\text { Phase of Nuclear Power Plants }\end{array}$ & $\operatorname{sip} 13.1 .2$ & 1989 & $\mathbf{P}$ & $\begin{array}{l}\text { Sections } 3.4,4.4 \text {, and } \\
5.2 \text { endorsed by Reg } \\
\text { Guide } 1.33\end{array}$ & ANS 3.2 & 1988 \\
\hline 0673 & ANSI & ANSI N18.7 & $N / S$ & $\begin{array}{l}\text { Administrative Controls and Qualty } \\
\text { Assurance for the Operational } \\
\text { Phase of Nuclear Power Plants }\end{array}$ & srp13.4 & 1981 & $\mathbf{P}$ & Section $4.3,4.4$ & ANS 3.2 & 1988 \\
\hline 0674 & ANSI & ANSI N18.9 & 1972 & $\begin{array}{l}\text { Program of Testing Blological } \\
\text { Shiolding in Nuclear Reactor Plants }\end{array}$ & reg2.1.roo & 1973 & $\mathbf{P}$ & Subject to conditions & ANS 6.3.1 & 1987 \\
\hline 0675 & ANSI & ANSI N18.17 & $N / S$ & Security for Nuclear Power Plants & bul79-16 & 1979 & $\mathbf{P}$ & $\begin{array}{l}\text { ANSI N18.17 } \\
\text { withdrawn } 1992\end{array}$ & Withdrawn & $n / a$ \\
\hline 0676 & ANSI & ANSI N18.17 & 1973 & Security for Nuclear Power Plants & cir78-17 & 1978 & $\mathbf{R}$ & \begin{tabular}{|l|} 
ANSI N18.17 \\
withdrawn 1992 \\
\end{tabular} & Withdrawn & $n / a$ \\
\hline 0677 & ANSI & ANSI N18.17 & 1973 & Security for Nuclear Power Plants & cir79-03 & 1979 & $\mathbf{R}$ & $\begin{array}{l}\text { ANSI N18.17 } \\
\text { withdrawn } 1992 \\
\end{array}$ & Withdrawn & $n / a$ \\
\hline 0678 & ANSI & ANSI N18.17 & N/S & Security for Nuclear Power Plants & drgrs902-4 & 1980 & $\mathbf{P}$ & $\begin{array}{l}\text { ANSI N18.17 } \\
\text { withdrawn } 1992 \\
\end{array}$ & Withdrawn & $n / a$ \\
\hline 0679 & ANSI & ANSI N18.17 & $N / S$ & Security for Nuclear Power Plants & glt79-016 & 1979 & $\mathbf{R}$ & $\begin{array}{l}\text { ANSI N18.17 } \\
\text { withdrawn } 1992 \\
\end{array}$ & Withdrawn & $n / a$ \\
\hline 0680 & ANSI & ANSI N18.17 & N/S & Security for Nuclear Power Plants & not83-15 & 1983 & $\mathbf{R}$ & $\begin{array}{l}\text { ANSI N18.17 } \\
\text { withdrawn } 1992 \\
\end{array}$ & Withdrawn & $n / a$ \\
\hline 0681 & ANSI & ANSI N18.17 & N/S & Security for Nuclear Power Plants & not88-91 & 1988 & $\mathbf{R}$ & $\begin{array}{l}\text { ANSI N18.17 } \\
\text { withdrawn } 1992\end{array}$ & Withdrawn & $n / a$ \\
\hline
\end{tabular}


Codes and Standards and Other Guidance Clted in Regulatory Documents

Part A - Industry Consensus Codes and Standards (continued)

\begin{tabular}{|c|c|c|c|c|c|c|c|c|c|c|}
\hline $\begin{array}{l}\text { Record } \\
\text { No. }\end{array}$ & Codo & Stendard & Standard & Nito & boounent & Doo. & Aooppt: & Comments & Cunrent Voulon & 8010 \\
\hline 0682 & ANSI & ANSI N18.17 & 1973 & Security for Nuclear Power Plants & $\operatorname{reg} 1.70 .103$ & 1978 & $\mathbf{P}$ & $\begin{array}{l}\text { ANSI N18.17 } \\
\text { withdrawn } 1992 \\
\end{array}$ & Withdrawn & $n / a$ \\
\hline 0683 & ANSI & ANSI N18.17 & N/S & Security for Nuclear Power Plants & sip13.6 & 1981 & $\mathbf{P}$ & $\begin{array}{l}\text { ANSI N18.17 } \\
\text { withdrawn } 1992 \\
\end{array}$ & Withdrawn & $n / a$ \\
\hline 0684 & ANSI & ANSI N41.2 & N/S & $\begin{array}{l}\text { Standard Application of the Single } \\
\text { Failure Criterion to Nuclear Power } \\
\text { Generating Station Safety Systems }\end{array}$ & reg1.53.r00 & 1973 & $\mathbf{R}$ & $\begin{array}{l}\text { Alternate title for IEEE } \\
379\end{array}$ & IEEE 379 & 1988 \\
\hline 0685 & ANSI & ANSI N41.6 & N/S & $\begin{array}{l}\text { Standard for Qualification of } \\
\text { Actuators for Power Operated Valve } \\
\text { Assemblies With Safety-Pelated } \\
\text { Functions for Nuclear Power Plants }\end{array}$ & reg1.73.roo & 1974 & $\mathbf{R}$ & Subject to conditions & IEEE 382 & 1985 \\
\hline 0686 & ANSI & $\begin{array}{l}\text { ANSI N41.6 } \\
\text { Draft }\end{array}$ & N/S & $\begin{array}{l}\text { Standard for Qualification of } \\
\text { Actuators for Power Operated Valve } \\
\text { Assemblies With Safety-Prelated } \\
\text { Functions for Nuclear Power Plants }\end{array}$ & sip3.10 & 1981 & $\mathbf{R}$ & & IEEE 382 & 1985 \\
\hline 0687 & ANSI & ANSI N41.10 & 1975 & $\begin{array}{l}\text { Standard for Type Test of Class IE } \\
\text { Electric Cables, Field Splices, and } \\
\text { Connections for Nuclear Power } \\
\text { Generating Stations }\end{array}$ & drgrs50-2 & 1979 & $\mathbf{R}$ & Proposed reg1.131.r01 & IEEE 383 & $1974 \mathrm{Rg2}$ \\
\hline 0688 & ANSI & ANSI N41.10 & 1975 & $\begin{array}{l}\text { Standard for Type Test of Class IE } \\
\text { Electric Cables, Field Splices, and } \\
\text { Connections for Nuclear Power } \\
\text { Generating Stations } \\
\end{array}$ & reg1.131.ro0 & 1977 & $\mathbf{R}$ & $\begin{array}{l}\text { Historical development } \\
\text { cited }\end{array}$ & IEEE 383 & 1974 R92 \\
\hline 0689 & ANSI & ANSI N41.14 & $N / S$ & $\begin{array}{l}\text { Standard Criteria for Independence } \\
\text { of Class IE Equipment and Circuits }\end{array}$ & reg1.75.ro2 & 1978 & $\mathbf{P}$ & With exceptions & IEEE 384 & 1992 \\
\hline 0690 & ANSI & ANSI N41.15 & 1976 & $\begin{array}{l}\text { Recommended Practice for } \\
\text { Maintenance, Testing, and } \\
\text { Replacement of Large Lead } \\
\text { Storage Batteries for Generating } \\
\text { Stations and Substations }\end{array}$ & reg1.129.ro1 & 1978 & $\mathbf{R}$ & $\begin{array}{l}\text { Historical development } \\
\text { cited }\end{array}$ & IEEE 450 & 1987 \\
\hline
\end{tabular}


Codes and Standards and Other Guidance Clted in Regulatory Documents

Part A - Industry Consensus Codes and Standards (continued)

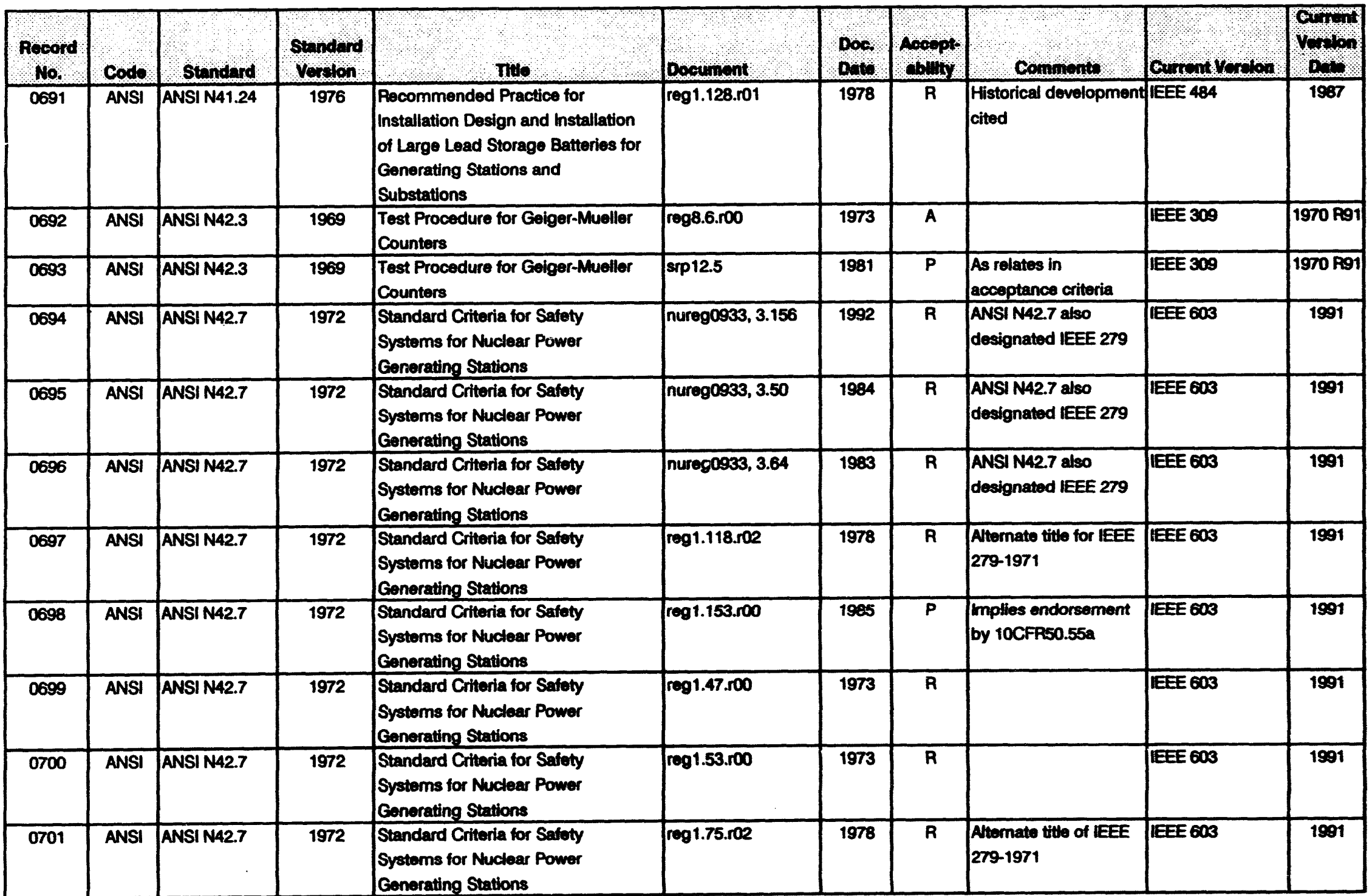


Codes and Standards and Other Guldance Clied in Regulatory Documents

Part A - Industry Consensus Codes and Standards (continued)

\begin{tabular}{|c|c|c|c|c|c|c|c|c|c|c|}
\hline $\begin{array}{l}\text { Asoord } \\
\text { No. }\end{array}$ & Code & Standind & $\begin{array}{l}\text { Sthuiderd } \\
\text { Vhrition }\end{array}$ & The & Doounent & $\begin{array}{l}\text { Doc } \\
\text { Dow }\end{array}$ & Acospt: & Commensen & Connat Variag & onsh \\
\hline 0702 & ANSI & ANSI N12.7 & $N / S$ & $\begin{array}{l}\text { Standard Critoria for Safoty } \\
\text { Systoms for Nuctoar Power } \\
\text { Generating Stations }\end{array}$ & reg2.2.00 & 1973 & R & & EEE COS & 1991 \\
\hline 0703 & ANSI & ANSI N42.7 & 1972 & $\begin{array}{l}\text { Standard Criteria for Saloty } \\
\text { Systems for Nuclear Power } \\
\text { Generating Stations }\end{array}$ & $\operatorname{arp3.11}$ & 1981 & $\overline{\mathbf{R}}$ & & EEE 600 & 1991 \\
\hline 0704 & ANSI & ANSI N42.12 & 1980 & $\begin{array}{l}\text { Callbration and Usege of Sodlum } \\
\text { lodide Detector Syctems }\end{array}$ & reg10.8.02 & 1987 & $\mathbf{R}$ & & EEE N12.12 & 1900 R91 \\
\hline 0705 & ANSI & ANSI N42.13 & 1978 & $\begin{array}{l}\text { Callbration and Uaege of Dose } \\
\text { Calibrator lonization Chambers for } \\
\text { the Acsay of Radionuclides }\end{array}$ & drgic410-4 & 1985 & $\overline{\mathbf{R}}$ & & EEENA2.13 & 1986 \\
\hline 0706 & ANSI & ANSI N42.13 & 1985 & $\begin{array}{l}\text { Calibration and Usage of Dose } \\
\text { Calibrator lonization Chambers for } \\
\text { the Aseay of Radionuclides }\end{array}$ & reg10.8.r02 & 1987 & $\mathbf{R}$ & & IEEEN12.13 & 1906 \\
\hline 0707 & ANSI & ANSI N2.14 & 1978 & $\begin{array}{l}\text { Calibration and Use of Germantum } \\
\text { Spectrometers for the } \\
\text { Mosasurement of Gamma-Pay } \\
\text { Emisaion Pates of Radionuclides }\end{array}$ & reg4.15.rot & 1979 & $\mathbf{R}$ & & EEEN12.14 & 1991 \\
\hline 0708 & ANSI & ANSI N42.15 & 1980 & $\begin{array}{l}\text { Porformanco Vorffication of Lquid } \\
\text { Scintillation Counting Syetems }\end{array}$ & reg10.8.02 & 1987 & $\mathbf{R}$ & & EEENA215 & 1990 \\
\hline 0709 & ANSI & ANSI N42.17 & $N / S$ & $\begin{array}{l}\text { Porformance Specifications for } \\
\text { Hoalth Phyaics instrumentation- } \\
\text { Portable instrumentation for Ube in } \\
\text { Normal Environmental Conditions }\end{array}$ & nuregos33, 1.MI.D.3 & 1937 & R & & MSTIME17A & 1900 \\
\hline 0710 & ANSI & ANSI N42.17A & $\mathbf{N} / \mathbf{S}$ & $\begin{array}{l}\text { Porformance Specifications for } \\
\text { Hoalth Physics Instrumentation- } \\
\text { Portablo instrumentation for Use in } \\
\text { Normal Environmental Conditions }\end{array}$ & notso-44 & 1900 & R & & KEEEM2.17A & 1900 \\
\hline 0711 & ANSI & ANSI N42.18 & $N / S$ & $\begin{array}{l}\text { Spectification and Porformance of } \\
\text { On-site Instrumentation for } \\
\text { Continuously Monitoring } \\
\text { Radioactivity in Eflituents }\end{array}$ & drg8013.100 & 1992 & $\mathbf{A}$ & $\begin{array}{l}\text { ANS1 N13.10 } \\
\text { rededignated }\end{array}$ & EEE N2.18 & $1980 \mathrm{PQ1}$ \\
\hline
\end{tabular}


Codes and Standards and Other Guidance Ched in Regubtory Documents

Part A - Industry Consensus Codes and Standards (continued) 
Codes and Standards and Other Guidance Clted in Regulatory Documents

Part A - Industry Consensus Codes and Standards (continued)

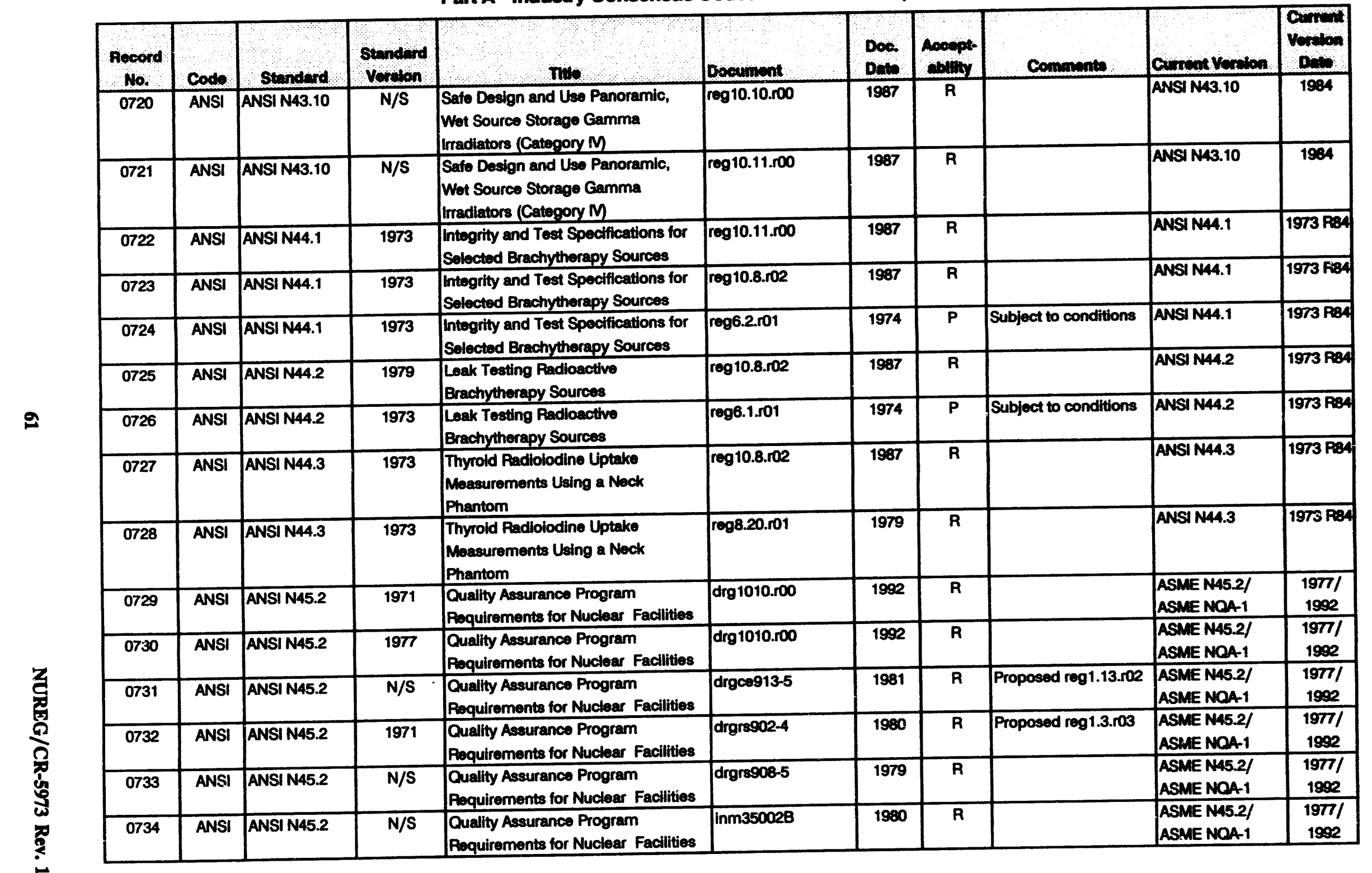


Codes and Standards and Other Guidance Cited in Regulatory Documents

Part A - Industry Consensus Codes and Standards (continued)

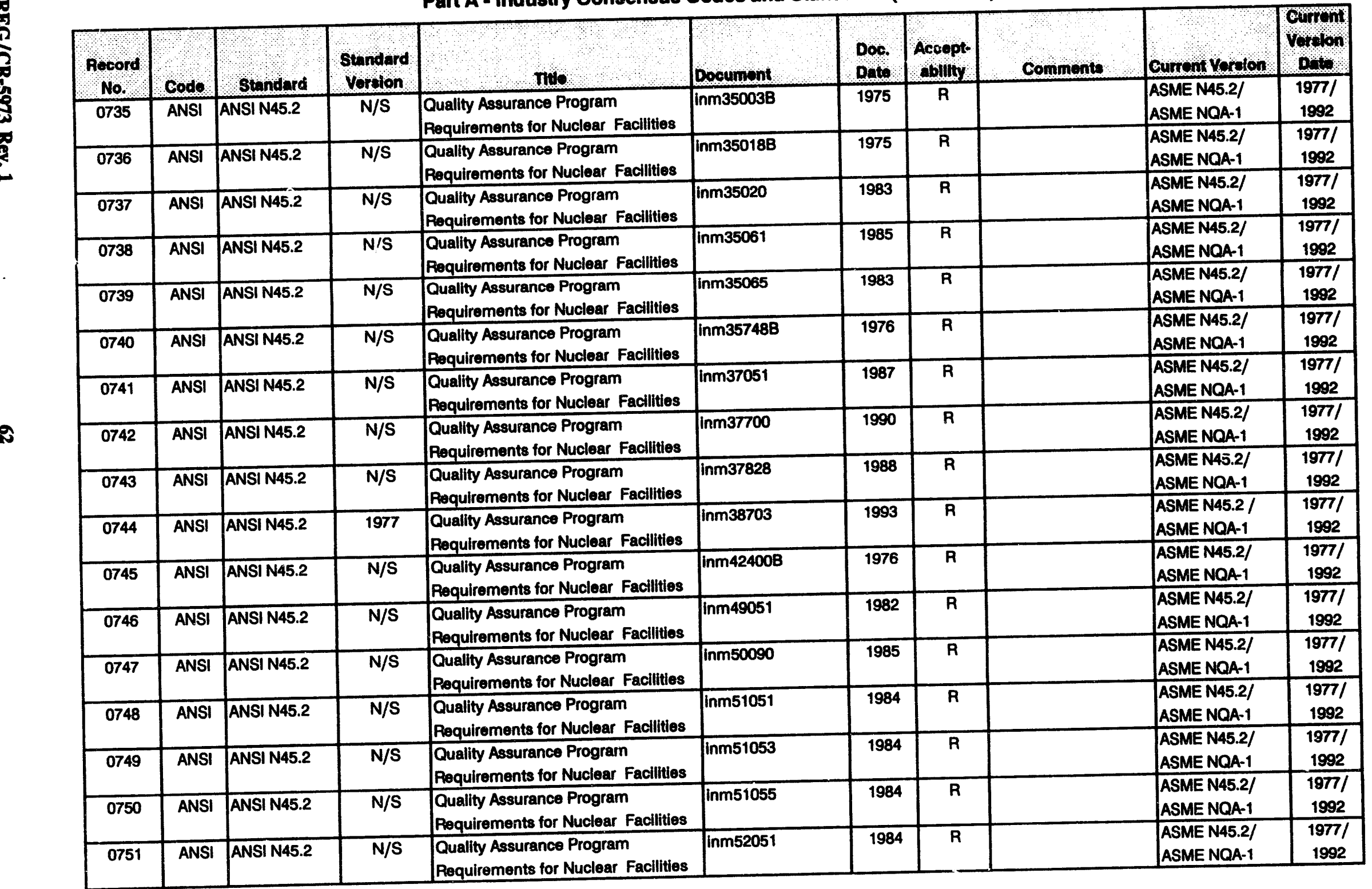


Codes and Standards and Other Guidance Clted in Regulatory Documents

Part A - Industry Consensus Codes and Standards (continued)

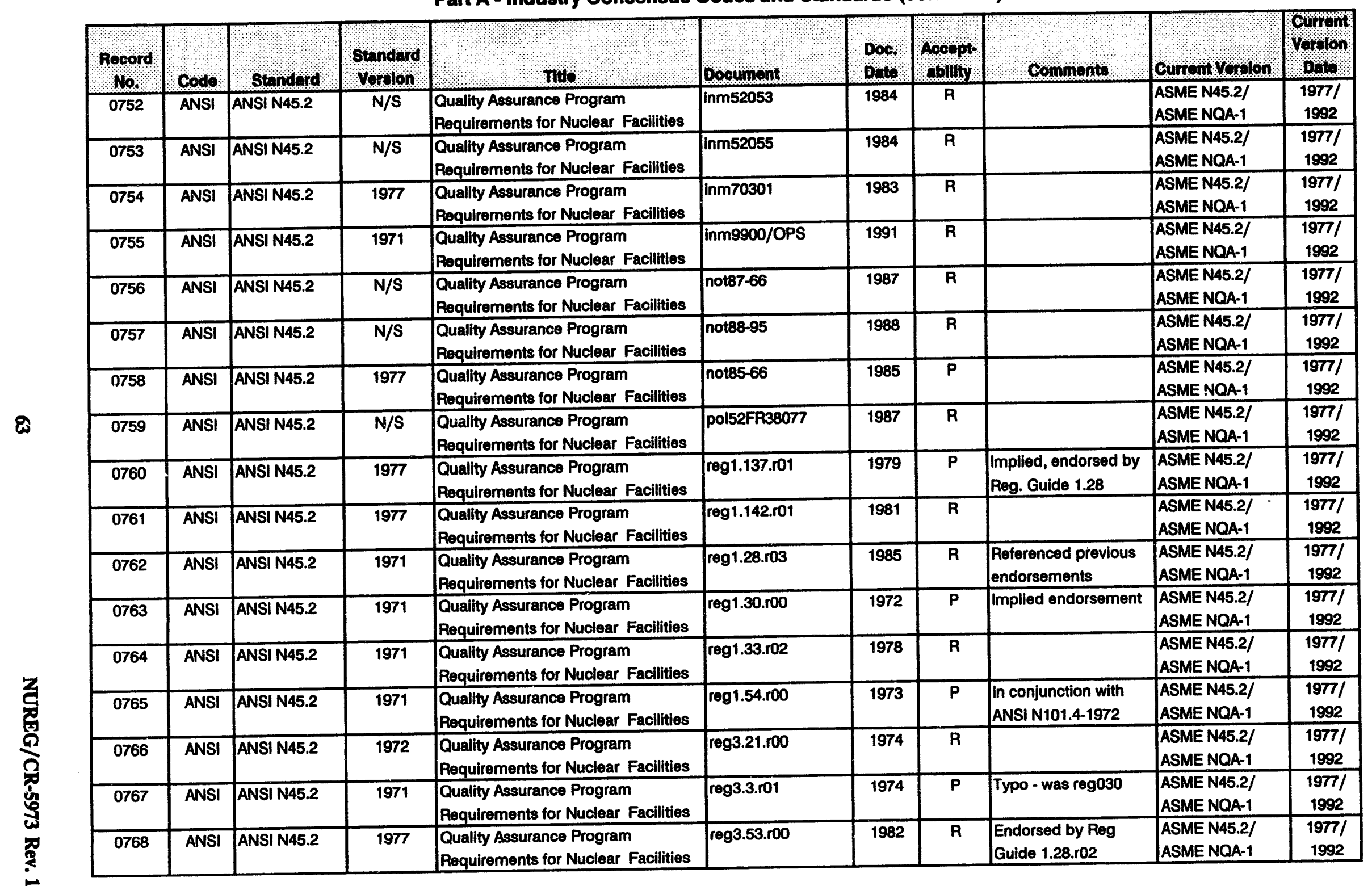


Codes and Standards and Other Guidance Cited in Regulatory Documents

Part A - Industry Consensus Codes and Standards (continued)

\begin{tabular}{|c|c|c|c|c|c|c|c|c|c|c|}
\hline Pocord & cods & Standard & Standrol & ribo & Documont & $\begin{array}{l}\text { Doo. } \\
\text { Dote. }\end{array}$ & $\begin{array}{l}\text { Aocept } \\
\text { Abilty }\end{array}$ & Comments & Cunent Vor lon & Vorron: \\
\hline 0769 & ANSI & $\begin{array}{l}\text { ANSI N45.2 } \\
\text { Series } \\
\end{array}$ & N/S & Misc. & drg1010.r00 & 1992 & $P$ & & $\begin{array}{l}\text { ASME N45.2/ } \\
\text { ASME NQA-1 } \\
\end{array}$ & 1992 \\
\hline 0770 & ANSI & $\begin{array}{l}\text { ANSI N45.2 } \\
\text { Series } \\
\end{array}$ & N/S & Misc. & inm2512/13 & 1984 & $\mathbf{R}$ & & $\begin{array}{l}\text { ASME N45.2/ } \\
\text { ASME NQA-1 }\end{array}$ & 1992 \\
\hline 0771 & ANSI & \begin{tabular}{|l} 
ANSI N45.2 \\
Series \\
\end{tabular} & $\mathrm{N} / \mathrm{S}$ & Misc. & inm35002B & 1980 & $\mathbf{R}$ & & $\begin{array}{l}\text { ASME N45.2/ } \\
\text { ASME NQA-1 }\end{array}$ & 1992 \\
\hline 0772 & ANSI & $\begin{array}{l}\text { ANSI N45.2 } \\
\text { series }\end{array}$ & $N / S$ & Misc. & inm92720 & 1992 & $\mathbf{R}$ & & $\begin{array}{l}\text { ANSI N45.2 } \\
\text { series/ ASME } \\
\text { NQA-1 \&2 } \\
\end{array}$ & $\begin{array}{c}\text { Misc./ } \\
1992\end{array}$ \\
\hline 0773 & ANSI & ANSI N45.2.1 & N/S & $\begin{array}{l}\text { Cleaning of Fuld Systems and } \\
\text { Associated Components for } \\
\text { Nuclear Power Plants }\end{array}$ & drg1010.r00 & 1992 & $P$ & Endorsed by reg1.37 & $\begin{array}{l}\text { ASME N45.2.1/ } \\
\text { ASME NQA-2 }\end{array}$ & $\begin{array}{l}1980 / \\
1992\end{array}$ \\
\hline 0774 & ANSI & ANSI N45.2.1 & $N / S$ & $\begin{array}{l}\text { Cleaning of Fluid Systems and } \\
\text { Associated Components for } \\
\text { Nuclear Power Plants }\end{array}$ & inm35016B & 1975 & $\mathbf{R}$ & & $\begin{array}{l}\text { ASME N45.2.1/ } \\
\text { ASME NQA-2 }\end{array}$ & $\begin{array}{c}1980 / \\
1992\end{array}$ \\
\hline 0775 & ANSI & ANSI N45.2.1 & $\mathrm{N} / \mathrm{S}$ & $\begin{array}{l}\text { Cleaning of Fuid Systems and } \\
\text { Associated Components for } \\
\text { Nuclear Power Plants }\end{array}$ & inm35018B & 1975 & $\mathbf{R}$ & & $\begin{array}{l}\text { ASME N45.2.1/ } \\
\text { ASME NQA-2 }\end{array}$ & $\begin{array}{l}1980 / \\
1992\end{array}$ \\
\hline 0776 & ANS! & ANSI N45.2.1 & N/S & $\begin{array}{l}\text { Cleaning of Fluid Systems and } \\
\text { Associated Components for } \\
\text { Nuclear Power Plants }\end{array}$ & inm35028B & 1975 & $\mathbf{R}$ & & $\begin{array}{l}\text { ASME N45.2.1/ } \\
\text { ASME NQA-2 }\end{array}$ & $\begin{array}{c}1980 / \\
1992\end{array}$ \\
\hline 0777 & ANSI & ANSI N45.2.1 & 1973 & $\begin{array}{l}\text { Cleaning of Fluid Systems and } \\
\text { Associated Components for } \\
\text { Nuclear Power Plants }\end{array}$ & inm35743B & 1982 & $\mathbf{R}$ & & $\begin{array}{l}\text { ASME N45.2.1/ } \\
\text { ASME NQA-2 }\end{array}$ & $\begin{array}{l}1980 / \\
1992\end{array}$ \\
\hline 0778 & ANSI & ANSI N45.2.1 & $N / S$ & $\begin{array}{l}\text { Cleaning of Fluid Systems and } \\
\text { Associated Components for } \\
\text { Nuclear Power Plants }\end{array}$ & inm49051 & 1982 & $\mathbf{R}$ & & $\begin{array}{l}\text { ASME N45.2.1/ } \\
\text { ASME NQA-2 }\end{array}$ & $\begin{array}{r}1980 / \\
1992\end{array}$ \\
\hline 0779 & ANSI & ANSI N45.2.1 & $\mathrm{N} / \mathrm{S}$ & $\begin{array}{l}\text { Cleaning of Fluid Systems and } \\
\text { Associated Components for } \\
\text { Nuclear Power Plants }\end{array}$ & inm49053 & 1985 & $\mathbf{R}$ & & $\begin{array}{l}\text { ASME N45.2.1/ } \\
\text { ASME NQA-2 }\end{array}$ & $\begin{array}{l}1980 / \\
1992\end{array}$ \\
\hline 0780 & ANSI & ANSI N45.2.1 & 1976 & $\begin{array}{l}\text { Cleaning of Fluid Systems and } \\
\text { Associated Components for } \\
\text { Nuclear Power Plants }\end{array}$ & inm62702 & 1987 & $\mathbf{R}$ & & $\begin{array}{l}\text { ASME N45.2.1/ } \\
\text { ASME NQA-2 }\end{array}$ & $\begin{array}{r}1980 / \\
1992\end{array}$ \\
\hline
\end{tabular}


Codes and Standards and Other Guidance Cited in Regulatory Documents

Part A - Industry Consensus Codes and Standards (continued)

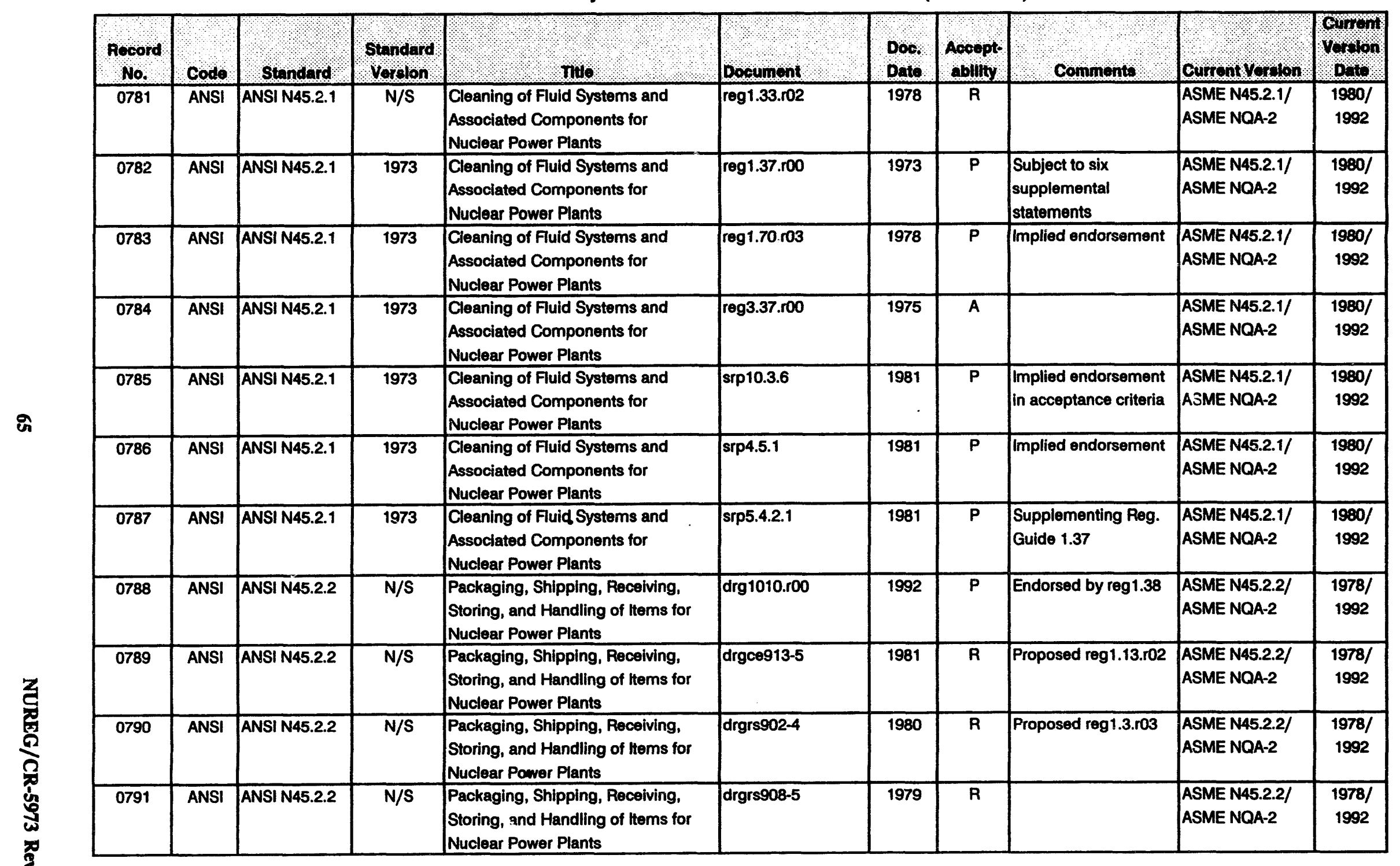


Codes and Standards and Other Guidance Cited in Regulatory Documents

Part A - Industry Consensus Codes and Standards (continued)

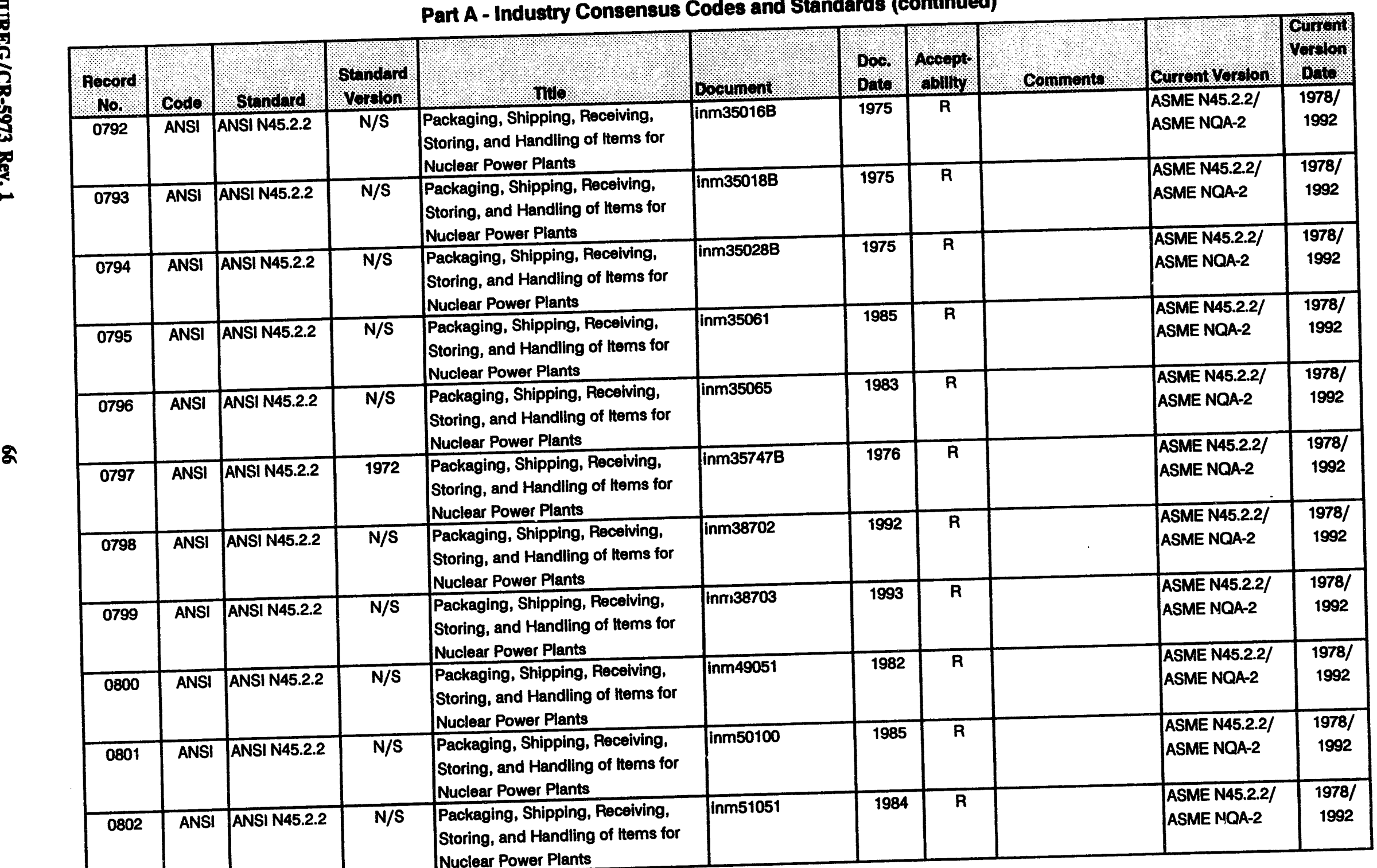


Codes and Standards and Other Guidance Clted in Regulatory Documents

Part A - Industry Consensus Codes and Standards (continued)

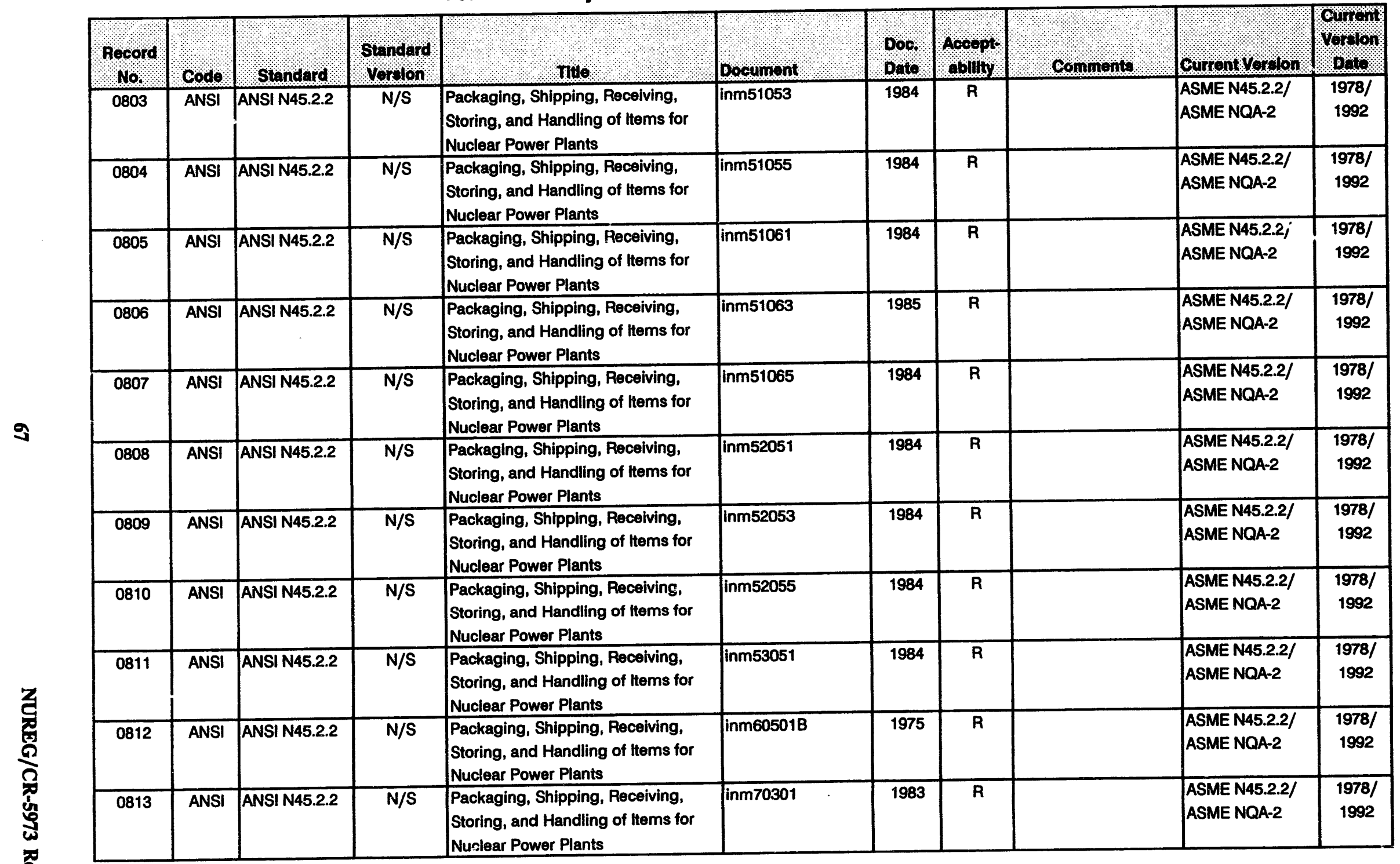


Codes and Standards and Other Guidance Cited in Regulatory Documents

Part A - Industry Consensus Codes and Standards (continued)

\begin{tabular}{|c|c|c|c|c|c|c|c|c|c|c|}
\hline Record & codo & Standard & Sthidard & (1) & Booumont & 000 & $\begin{array}{l}\text { noppt } \\
101161\end{array}$ & Commonts & Gunrent Vorolon & onsplon \\
\hline 0814 & ANSI & ANSI N45.2.2 & $\mathrm{N} / \mathrm{S}$ & $\begin{array}{l}\text { Packaging, Shipping, Receiving, } \\
\text { Storing, and Handling of ltems for } \\
\text { Nuclear Power Plants }\end{array}$ & inm9900/5.5 & 1982 & $\mathbf{R}$ & & $\begin{array}{l}\text { ASME N45.2.2/ } \\
\text { ASME NQA-2 }\end{array}$ & $\begin{array}{r}1978 / \\
1992\end{array}$ \\
\hline 0815 & ANSI & ANSI N45.2.2 & 1972 & $\begin{array}{l}\text { Packaging, Shipping, Receiving, } \\
\text { Storing, and Handling of ttems for } \\
\text { Nuclear Power Plants }\end{array}$ & reg1.142.ro1 & 1981 & $\mathbf{R}$ & & $\begin{array}{l}\text { ASME N45.2.2/ } \\
\text { ASME NQA-2 }\end{array}$ & $\begin{array}{c}1978 / \\
1992\end{array}$ \\
\hline 0816 & ANSI & ANSI N45.2.2 & $\mathrm{N} / \mathrm{S}$ & $\begin{array}{l}\text { Packaging, Shipping, Receiving, } \\
\text { Storing, and Handling of Hems for } \\
\text { Nuclear Power Plants }\end{array}$ & reg1.33.ro2 & 1978 & $\bar{R}$ & & $\begin{array}{l}\text { ASME N45.2.2/ } \\
\text { ASME NQA-2 }\end{array}$ & $\begin{array}{c}1978 / \\
1992\end{array}$ \\
\hline 0817 & ANSI & ANSI N45.2.2 & 1972 & $\begin{array}{l}\text { Packaging, Shipping, Receiving, } \\
\text { Storing, and Handling of lems for } \\
\text { Nuclear Power Plants }\end{array}$ & reg1.38.ro0 & 1973 & $\mathbf{P}$ & Subject to conditions & $\begin{array}{l}\text { ASME N45.2.2/ } \\
\text { ASME NQA-2 }\end{array}$ & $\begin{array}{c}1978 / \\
1992\end{array}$ \\
\hline 0818 & ANSI & ANSI N45.2.3 & N/S & $\begin{array}{l}\text { Housekeeping During the } \\
\text { Construction Phase of Nuclear } \\
\text { Power Plants }\end{array}$ & drg1010.r00 & 1992 & $\mathbf{P}$ & Endorsed by reg1.39 & $\begin{array}{l}\text { ASME N45.2.3/ } \\
\text { ASME NQA-2 }\end{array}$ & $\begin{array}{c}1978 / \\
1992\end{array}$ \\
\hline 0819 & ANSI & ANSI N45.2.3 & $\mathrm{N} / \mathrm{S}$ & $\begin{array}{l}\text { Housekeeping During the } \\
\text { Construction Phase of Nuclear } \\
\text { Power Plants } \\
\end{array}$ & drgrs902-4 & 1980 & $\mathbf{R}$ & Proposed reg1.3.r03 & $\begin{array}{l}\text { ASME N45.2.3/ } \\
\text { ASME NQA-2 }\end{array}$ & $\begin{array}{c}1978 / \\
1992\end{array}$ \\
\hline 0820 & ANSI & ANSI N45.2.3 & $\mathrm{N} / \mathrm{S}$ & $\begin{array}{l}\text { Housekeeping During the } \\
\text { Construction Phase of Nuclear } \\
\text { Power Plants }\end{array}$ & drgrs908-5 & 1979 & $\bar{R}$ & & $\begin{array}{l}\text { ASME N45.2.3/ } \\
\text { ASME NQA-2 }\end{array}$ & $\begin{array}{c}1978 / \\
1992\end{array}$ \\
\hline 0821 & ANSI & ANSI N45.2.3 & $N / S$ & $\begin{array}{l}\text { Housekeeping During the } \\
\text { Construction Phase of Nuclear } \\
\text { Power Plants }\end{array}$ & inm350168 & 1975 & $\overline{\mathbf{R}}$ & & $\begin{array}{l}\text { ASME N45.2.3/ } \\
\text { ASME NQA-2 }\end{array}$ & $\begin{array}{c}1978 / \\
1992\end{array}$ \\
\hline 0822 & ANSI & ANSI N45.2.3 & $\mathrm{N} / \mathrm{S}$ & $\begin{array}{l}\text { Housekeeping During the } \\
\text { Construction Phase of Nuclear } \\
\text { Power Plants }\end{array}$ & inm35018B & 1975 & $\mathbf{R}$ & & $\begin{array}{l}\text { ASME N45.2.3/ } \\
\text { ASME NQA-2 }\end{array}$ & $\begin{array}{l}1978 / \\
1992\end{array}$ \\
\hline 0823 & ANSI & ANSI N45.2.3 & $\mathrm{N} / \mathrm{S}$ & $\begin{array}{l}\text { Housekeeping During the } \\
\text { Construction Phase of Nuclear } \\
\text { Power Plants }\end{array}$ & inm35028B & 1975 & $\mathbf{R}$ & & $\begin{array}{l}\text { ASME N45.2.3/ } \\
\text { ASME NQA-2 }\end{array}$ & $\begin{array}{c}1978 / \\
1992\end{array}$ \\
\hline 0824 & ANSI & ANSI N45.2.3 & $\mathrm{N} / \mathrm{S}$ & $\begin{array}{l}\text { Housekeeping During the } \\
\text { Construction Phase of Nuclear } \\
\text { Power Plants }\end{array}$ & inm35061 & 1985 & $\bar{R}$ & . & $\begin{array}{l}\text { ASME N45.2.3/ } \\
\text { ASME NQA-2 }\end{array}$ & $\begin{array}{l}1978 / \\
1992\end{array}$ \\
\hline
\end{tabular}


Codes and Standards and Other Guidance Cited in Regulatory Documents

Part A - Industry Consensus Codes and Standards (continued)

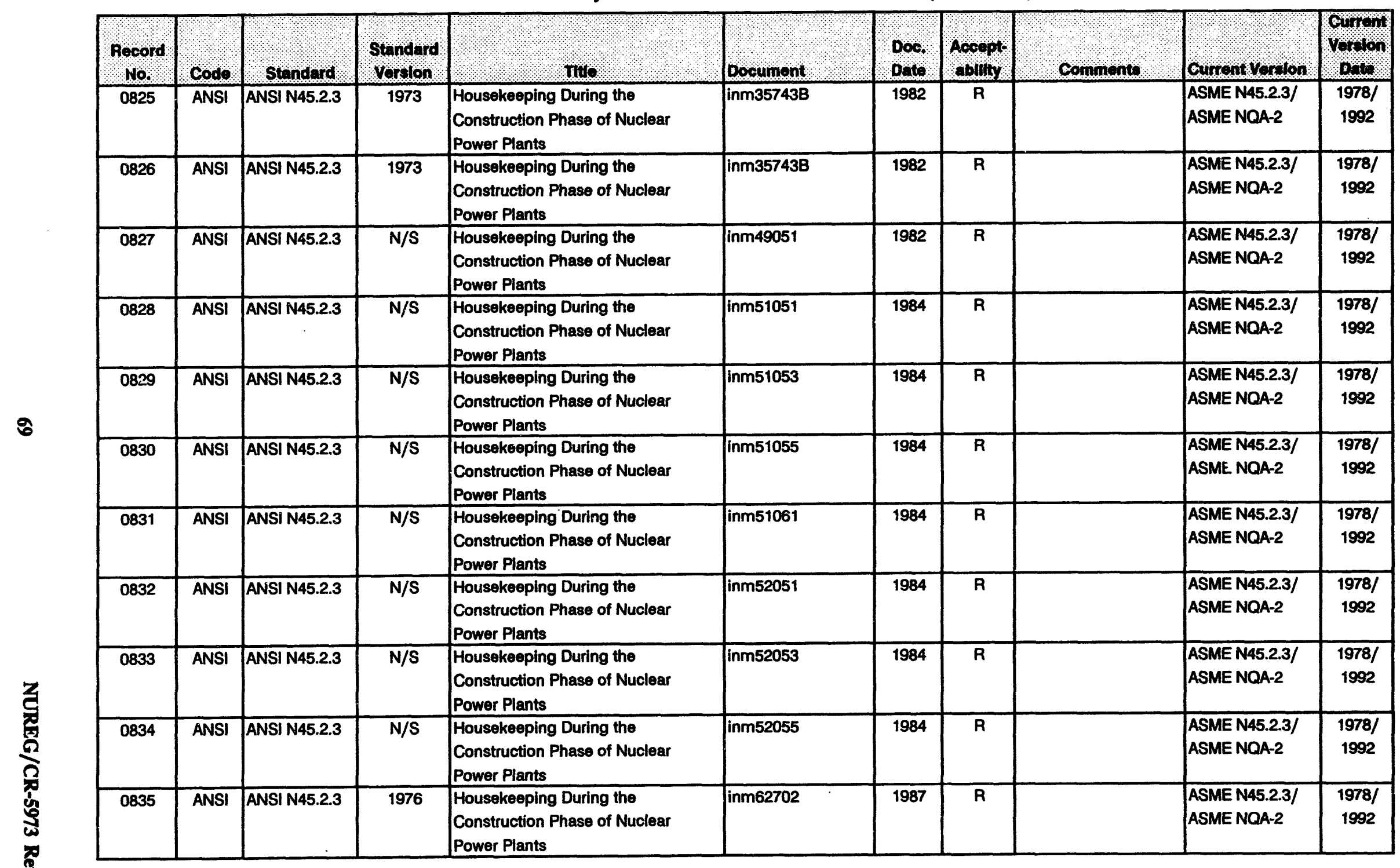


Codes and Standards and Other Guidance Cited in Regulatory Documents

Part A - Industry Consensus Codes and Standards (continued)

\begin{tabular}{|c|c|c|c|c|c|c|c|c|c|c|}
\hline hocord & $00 d 0$ & Sthondard & Standard & 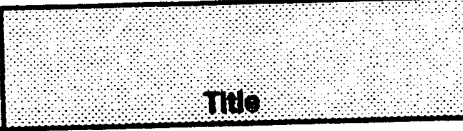 & Documont & $\begin{array}{l}\text { Dor. } \\
\text { Dete }\end{array}$ & Aooppts & Comments & Current vorolon & Qurpon \\
\hline 0836 & ANSI & ANSI N45.2.3 & $\mathrm{N} / \mathrm{S}$ & $\begin{array}{l}\text { Housekeeping During the } \\
\text { Construction Phase of Nuclear } \\
\text { Power Plants }\end{array}$ & inm70301 & 1983 & $\mathbf{R}$ & & $\begin{array}{l}\text { ASME N45.2.3/ } \\
\text { ASME NQA-2 }\end{array}$ & $\begin{array}{c}1978 / \\
1992\end{array}$ \\
\hline 0837 & ANSI & ANSI N45.2.3 & N/S & $\begin{array}{l}\text { Housekeeping During the } \\
\text { Construction Phase of Nuclear } \\
\text { Power Plants }\end{array}$ & not82-03 & 1982 & $\mathbf{R}$ & & $\begin{array}{l}\text { ASME N45.2.3/ } \\
\text { ASME NQA-2 }\end{array}$ & $\begin{array}{r}1978 / \\
1992\end{array}$ \\
\hline 0838 & ANSI & ANSI N45.2.3 & 1973 & $\begin{array}{l}\text { Housekeeping During the } \\
\text { Construction Phase of Nuclear } \\
\text { Power Plants }\end{array}$ & reg1.142.ro1 & 1981 & $\mathbf{R}$ & & $\begin{array}{l}\text { ASME N45.2.3/ } \\
\text { ASME NQA-2 }\end{array}$ & $\begin{array}{c}1978 / \\
1992\end{array}$ \\
\hline 0839 & ANSI & ANSI N45.2.3 & $\mathbf{N} / \mathrm{S}$ & $\begin{array}{l}\text { Housekeeping During the } \\
\text { Construction Phase of Nuclear } \\
\text { Power Plants }\end{array}$ & reg1.33.ro2 & 1978 & $\mathbf{R}$ & & $\begin{array}{l}\text { ASME N45.2.3/ } \\
\text { ASME NQA-2 }\end{array}$ & $\begin{array}{c}1978 / \\
1992\end{array}$ \\
\hline 0840 & ANSI & ANSI N45.2.3 & 1973 & $\begin{array}{l}\text { Housekeeping During the } \\
\text { Construction Phase of Nuclear } \\
\text { Power Plants }\end{array}$ & reg1.39.ro2 & 1977 & $\mathbf{P}$ & Subject to conditions & $\begin{array}{l}\text { ASME N45.2.3/ } \\
\text { ASME NQA-2 }\end{array}$ & $\begin{array}{c}1978 / \\
1992\end{array}$ \\
\hline 0841 & ANSI & ANSI N45.2.4 & $\mathbf{N} / \mathbf{S}$ & $\begin{array}{l}\text { Standard Installation, Inspection, } \\
\text { and Testing Requirements for } \\
\text { Power Instrumentation and Control } \\
\text { Equipment at Nuclear Facilities }\end{array}$ & drgce913-5 & 1981 & $\mathbf{R}$ & Proposed reg1.13.r02 & IEEE 336 & 1985 P91 \\
\hline 0842 & ANSI & ANSI N45.2.4 & $\mathrm{N} / \mathrm{S}$ & $\begin{array}{l}\text { Standard Installation, Inspection, } \\
\text { and Testing Requirements for } \\
\text { Power instrumentation and Control } \\
\text { Equipment at Nuclear Facilities }\end{array}$ & drgrs902-4 & 1980 & $\mathbf{R}$ & Proposed reg1.3.r03 & IEEE 336 & $1985 \mathrm{R} 91$ \\
\hline 0843 & ANSI & ANSI N45.2.4 & $\mathrm{N} / \mathrm{S}$ & $\begin{array}{l}\text { Standard Installation, Inspection, } \\
\text { and Testing Requirements for } \\
\text { Power Instrumentation and Control } \\
\text { Equipment at Nuclear Facilities }\end{array}$ & inm35016B & 1975 & $\mathbf{R}$ & & IEEE 336 & $1985 \mathrm{RQ1}$ \\
\hline 0844 & ANSI & ANSI N45.2.4 & $\mathrm{N} / \mathrm{S}$ & $\begin{array}{l}\text { Standard Installation, Inspection, } \\
\text { and Testing Requirements for } \\
\text { Power Instrumentation and Control } \\
\text { Equipment at Nuclear Facilities }\end{array}$ & inm35018B & 1975 & $\mathbf{R}$ & & IEEE 336 & $1985 \mathrm{R} 91$ \\
\hline
\end{tabular}


Codes and Standards and Other Guidance Cited in Regulatory Documents Part A - Industry Consensus Codes and Standards (continued)

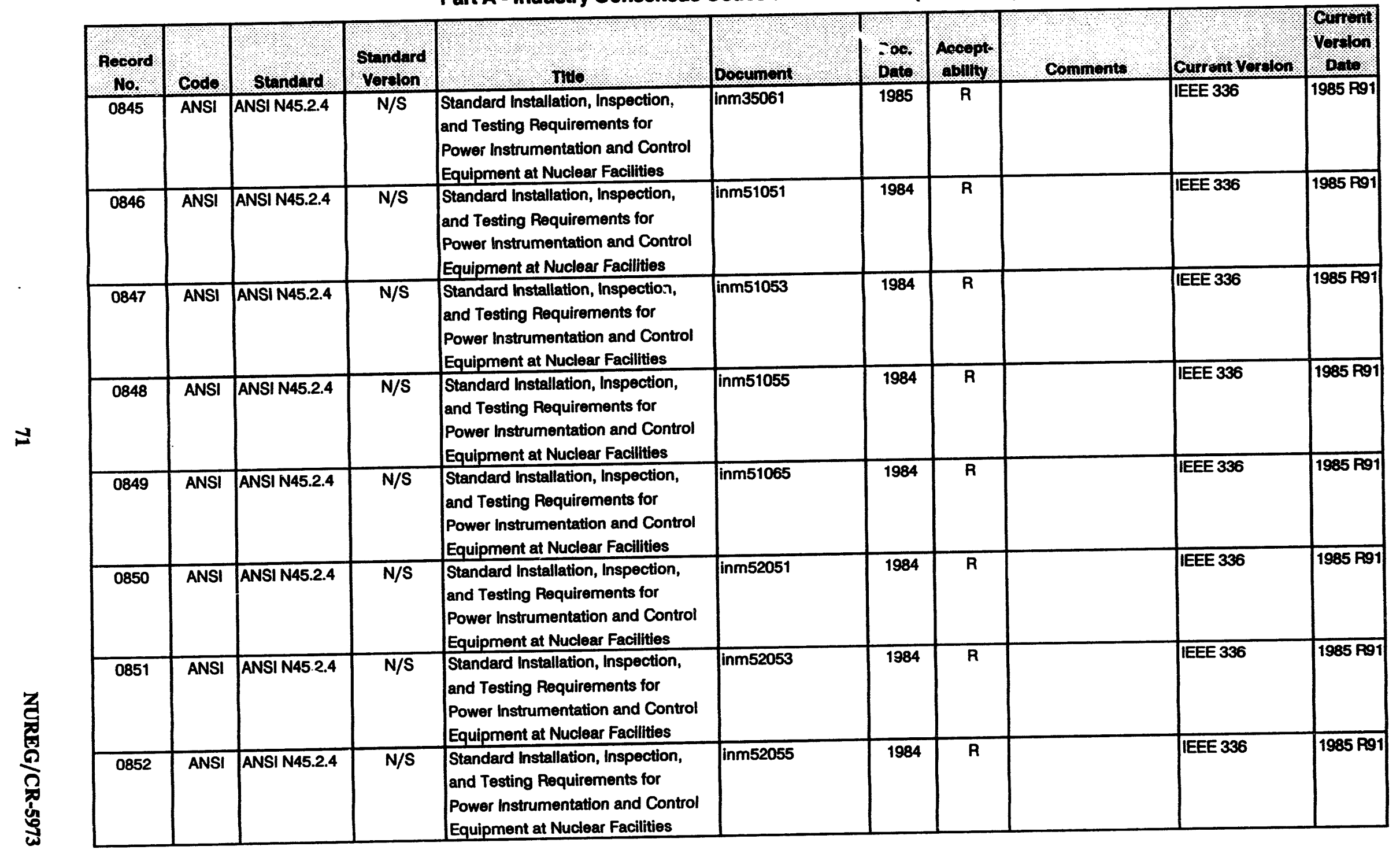


Codes and Standards and Other Guidance Cited in Regulatory Documents

Part A - Industry Consensus Codes and Standards (continued)

\begin{tabular}{|c|c|c|c|c|c|c|c|c|c|c|}
\hline $\begin{array}{l}\text { Pecord } \\
\text { No. }\end{array}$ & codo & standind & $\begin{array}{l}\text { Standard } \\
\text { verlon }\end{array}$ & 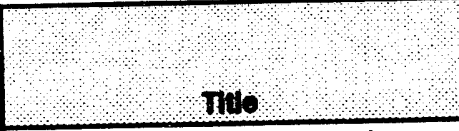 & Doroumont & $\begin{array}{l}\text { Dos } \\
\text { Dows }\end{array}$ & $\begin{array}{l}\text { noppt } \\
\text { ablltyl }\end{array}$ & Commont & Gunent verolon & Qning \\
\hline 0853 & ANSI & ANSI N45.2.4 & 1972 & $\begin{array}{l}\text { Standard Installation, Inspection, } \\
\text { and Testing Requirements for } \\
\text { Power instrumentation and Control } \\
\text { Equipment at Nuclear Facilities }\end{array}$ & inm72701 & 1992 & $\mathbf{R}$ & & IEEE 336 & $1985 \mathrm{RO1}$ \\
\hline 0854 & ANSI & ANSI N45.2.4 & 1972 & $\begin{array}{l}\text { Standard Installation, Inspection, } \\
\text { and Testing Requirements for } \\
\text { Power Instrumentation and Control } \\
\text { Equipment at Nuclear Facilities }\end{array}$ & reg1.30.r00 & 1972 & $\mathbf{P}$ & With exceptions & IEEE 336 & $1985 \mathrm{P} 91$ \\
\hline 0855 & ANSI & ANSI N45.2.4 & $\mathrm{N} / \mathrm{S}$ & $\begin{array}{l}\text { Standard Installation, Inspection, } \\
\text { and Testing Requirements for } \\
\text { Power Instrumentation and Cortrol } \\
\text { Equipment at Nuclear Facilities }\end{array}$ & reg1.33.r02 & 1978 & $\mathbf{R}$ & & IEEE 336 & $1985 \mathrm{R91}$ \\
\hline 0856 & ANSI & ANSI N45.2.5 & $\mathbf{N} / \mathbf{S}$ & $\begin{array}{l}\text { Supplementary Quality Assurance } \\
\text { Requirements for Installation, } \\
\text { inspection, and Testing of } \\
\text { Structural Concrete, Structural } \\
\text { Steel, Soils, and Foundations } \\
\text { During the Construction Phase of } \\
\text { Nuclear Power Plants }\end{array}$ & drg1010.r00 & 1992 & $\mathbf{P}$ & Endorsed by reg 1.94 & $\begin{array}{l}\text { ASME N45.2.5/ } \\
\text { ASME NQA-2 }\end{array}$ & $\begin{array}{l}1978 / \\
1992\end{array}$ \\
\hline 0857 & ANSI & ANSI N45.2.5 & $\mathrm{N} / \mathrm{S}$ & $\begin{array}{l}\text { Supplementary Quality Assurance } \\
\text { Requirements for Installation, } \\
\text { Inspection, and Testing of } \\
\text { Structural Concrete, Structural } \\
\text { Steel, Solls, and Foundations } \\
\text { During the Construction Phase of } \\
\text { Nuclear Power Plants }\end{array}$ & drgce913-5 & 1981 & $\mathbf{R}$ & Proposed reg1.13.r02 & $\begin{array}{l}\text { ASME N45.2.5/ } \\
\text { ASME NOA-2 }\end{array}$ & $\begin{array}{l}1978 / \\
1992\end{array}$ \\
\hline 0858 & ANSI & ANSI N45.2.5 & $\mathrm{N} / \mathrm{S}$ & $\begin{array}{l}\text { Supplementary Quality Assurance } \\
\text { Requirements for Installation, } \\
\text { Inspection, and Testing of } \\
\text { Structural Concrete, Structural } \\
\text { Steel, Solls, and Foundations } \\
\text { During the Construction Phase of } \\
\text { Nuclear Power Plants }\end{array}$ & drgrs902-4 & 1980 & $\mathbf{R}$ & Proposed reg1.3.ro3 & $\begin{array}{l}\text { ASME N45.2.5/ } \\
\text { ASME NOA-2 }\end{array}$ & $\begin{array}{l}1978 / \\
1992\end{array}$ \\
\hline
\end{tabular}


Codes and Standards and Other Guidance Cited in Regulatory Documents

Part A - Industry Consensus Codes and Standards (continued)

\begin{tabular}{|c|c|c|c|c|c|c|c|c|c|c|}
\hline Rocord & Cods & Stendard & $\begin{array}{l}\text { Standind } \\
\text { Vordon }\end{array}$ & nw. & 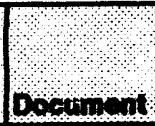 & $\begin{array}{l}\text { Dos: } \\
\text { Dads }\end{array}$ & $\begin{array}{l}\text { Aocept } \\
\text { soulty }\end{array}$ & Comments & Cunner Yordion & $\mathrm{O}_{0}=\mathrm{s}$ \\
\hline 0859 & ANSI & ANSI N45.2.5 & 1974 & $\begin{array}{l}\text { Supplomentary Quality Assurance } \\
\text { Requirements for Installation, } \\
\text { Inspection, and Testing of } \\
\text { Structural Concrete, Structural } \\
\text { Stoel, Soils, and Foundations } \\
\text { During the Construction Phase of } \\
\text { Nuclear Power Plants }\end{array}$ & drgrs908-5 & 1979 & $\mathbf{R}$ & & $\begin{array}{l}\text { ASME N45.2.5/ } \\
\text { ASME NOA2 }\end{array}$ & $\begin{array}{l}1978 / \\
1992\end{array}$ \\
\hline 0860 & ANSI & ANSI N45.2.5 & 1978 & $\begin{array}{l}\text { Supplementary Ouality Assurance } \\
\text { Roquirements for Installation, } \\
\text { Inspection, and Testing of } \\
\text { Structural Concrete, Structural } \\
\text { Stoel, Soils, and Foundations } \\
\text { During the Construction Phase of } \\
\text { Nuclear Power Plants }\end{array}$ & drgras08-5 & 1979 & $\mathbf{R}$ & & $\begin{array}{l}\text { ASME NA5.25/ } \\
\text { ASME NOA2 }\end{array}$ & $\begin{array}{l}1978 / \\
1992\end{array}$ \\
\hline 0861 & ANSI & ANSI N45.2.5 & N/S & $\begin{array}{l}\text { Supplementary Quality Assurance } \\
\text { Pequirements for Installation, } \\
\text { Inspection, and Testing of } \\
\text { Structural Concrote, Structural } \\
\text { Steel, Soils, and Foundations } \\
\text { During the Construction Phase of } \\
\text { Nuclear Power Plants }\end{array}$ & drgra908-5 & 1979 & $\mathbf{R}$ & & $\begin{array}{l}\text { ASME N45.2.5/ } \\
\text { ASME NOA-2 }\end{array}$ & $\begin{array}{l}1978 / \\
1992\end{array}$ \\
\hline 0862 & ANSI & ANSI N45.2.5 & $N / S$ & $\begin{array}{l}\text { Supplementary Quality Assurance } \\
\text { Pequirements for Installation, } \\
\text { Inspection, and Testing of } \\
\text { Structural Concrete, Structural } \\
\text { Steel, Soils, and Foundations } \\
\text { During the Construction Phase of } \\
\text { Nuclear Power Plants }\end{array}$ & drgrs908-5 & 1979 & $\mathbf{R}$ & & $\begin{array}{l}\text { ASME N45.2.5/ } \\
\text { ASME NOA.2 }\end{array}$ & $\begin{array}{l}1978 / \\
1992\end{array}$ \\
\hline
\end{tabular}


Codes and Standards and Other Guidance Cited in Regulatory Documents

Part A - Industry Consensus Codes and Standards (continued)

\begin{tabular}{|c|c|c|c|c|c|c|c|c|c|c|}
\hline $\begin{array}{l}\text { Hocord } \\
\text { No. }\end{array}$ & codo & Sthodad & $\begin{array}{l}\text { Stundand } \\
\text { Verton }\end{array}$ & Tus & Borophout & Doos & asoppt. & Cononents & Camentyonolon & $\mathrm{C}_{\mathrm{N}} \mathrm{H}$ \\
\hline 0863 & ANSI & ANSI N45.2.5 & $N / S$ & $\begin{array}{l}\text { Supplementary Quality Assurance } \\
\text { Requirements for Installation, } \\
\text { Inspection, and Testing of } \\
\text { Structural Concrete, Structural } \\
\text { Steel, Solls, and Foundations } \\
\text { During the Construction Phase of } \\
\text { Nuclear Power Plants }\end{array}$ & drgrso08-5 & 1979 & R & & $\begin{array}{l}\text { ASME NA5.2.5/ } \\
\text { ASME NOA-2 }\end{array}$ & $\begin{array}{l}1978 / \\
1982\end{array}$ \\
\hline 0864 & ANSI & ANSI N45.2.5 & $N / S$ & $\begin{array}{l}\text { Supplementary Quality Assurance } \\
\text { Requirements for Installation, } \\
\text { Inspection, and Testing of } \\
\text { Structural Concrete, Structural } \\
\text { Steel, Solls, and Foundations } \\
\text { During the Construction Phase of } \\
\text { Nuclear Power Plants }\end{array}$ & drgre908-5 & 1979 & $\bar{R}$ & & $\begin{array}{l}\text { ASME N45.2.5/ } \\
\text { ASME NOA-2 }\end{array}$ & $\begin{array}{l}1978 / \\
1992\end{array}$ \\
\hline 0865 & ANSI & ANSI N45.2.5 & $N / S$ & $\begin{array}{l}\text { Supplementary Cuality Assurance } \\
\text { Requirements for Installation, } \\
\text { Inspection, and Testing of } \\
\text { Structural Concrete, Structural } \\
\text { Steol, Soils, and Foundations } \\
\text { During the Construction Phase of } \\
\text { Nuclear Power Plants }\end{array}$ & drgrs:008-5 & 1979 & $\mathbf{R}$ & & $\begin{array}{l}\text { ASME N45.2.5/ } \\
\text { ASME NOA-2 }\end{array}$ & $\begin{array}{l}1978 / \\
1992\end{array}$ \\
\hline 0866 & ANSI & ANSI N45.2.5 & 1974 & $\begin{array}{l}\text { Supplementary Quality Assurance } \\
\text { Requirements for Installation, } \\
\text { Inspection, and Testing of } \\
\text { Structural Concrete, Structural } \\
\text { Steel, Solls, and Foundations } \\
\text { During the Construction Phase of } \\
\text { Nuclear Power Plants }\end{array}$ & drgrse08-5 & 1979 & $\mathbf{R}$ & & $\begin{array}{l}\text { ASME NA5.2.5/ } \\
\text { ASME NOA-2 }\end{array}$ & $\begin{array}{l}1978 / \\
1992\end{array}$ \\
\hline
\end{tabular}


Codes and Standards and Other Guidance Ched in Regulatory Documents

Part A - Industry Consensus Codes and Standards (continued)

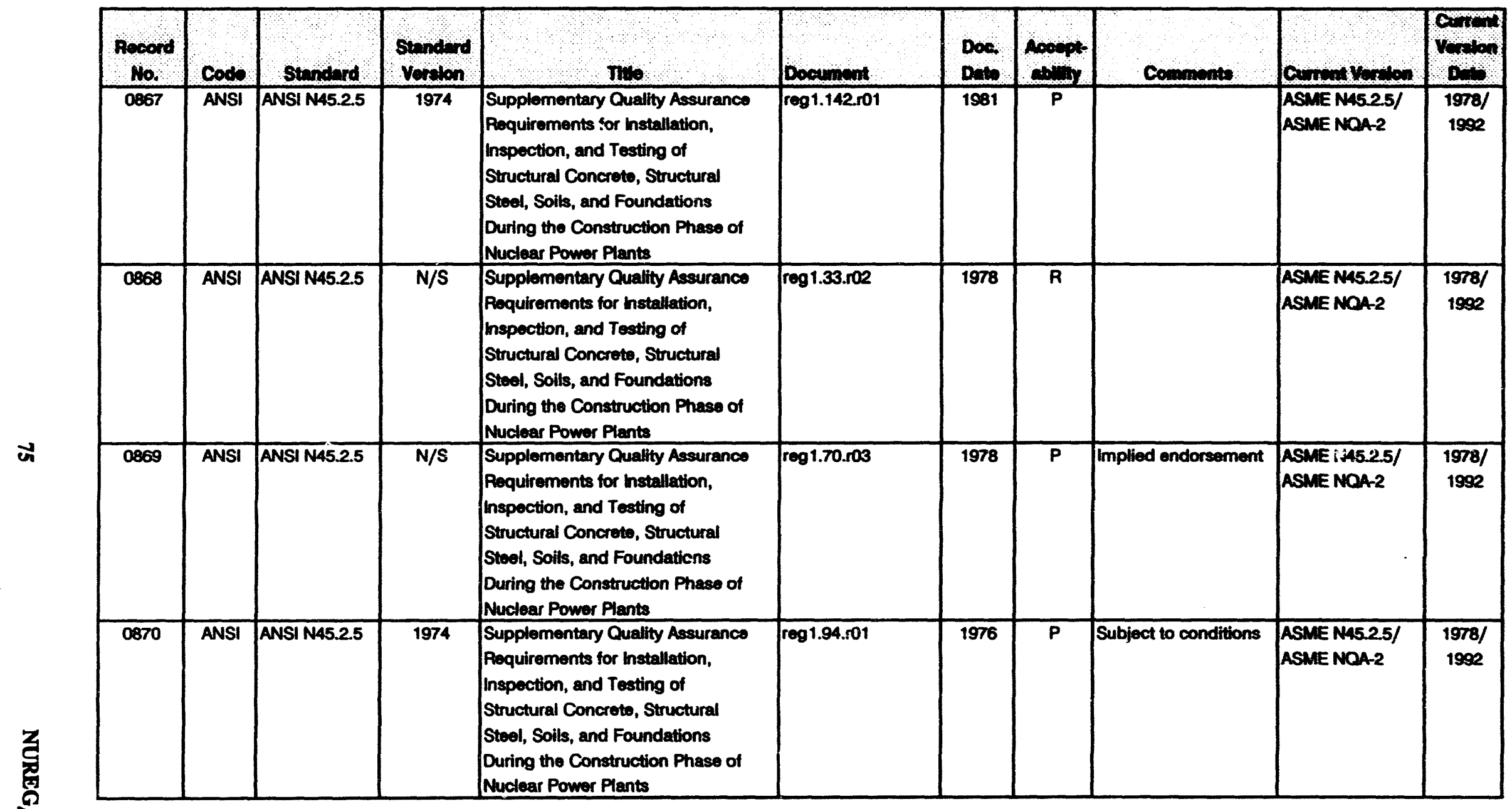


Codes and Standards and Other Guidance Cited in Regulatory Documents

Part A - Industry Consensus Codes and Standards (continued)

\begin{tabular}{|c|c|c|c|c|c|c|c|c|c|c|}
\hline Hecord & eodo & stond ard & Standard & (1) & Documont & Doo & Accept & comments & Cunont vorolon & vorion \\
\hline 0871 & ANSI & ANSI N45.2.5 & N/S & $\begin{array}{l}\text { Supplementary Quality Assurance } \\
\text { Requirements for installation, } \\
\text { Inspection, and Testing of } \\
\text { Structural Concrete, Structural } \\
\text { Steel, Soils, and Foundations } \\
\text { During the Construction Phase of } \\
\text { Nuclear Power Plants }\end{array}$ & $\operatorname{sip} 3.8 .3$ & 1981 & $P$ & & $\begin{array}{l}\text { ASME N45.2.5/ } \\
\text { ASME NQA-2 }\end{array}$ & $\begin{array}{c}1978 / \\
1992\end{array}$ \\
\hline 0872 & ANSI & ANSI N45.2.6 & $\mathrm{N} / \mathrm{S}$ & $\begin{array}{l}\text { Qualifications of Inspection, } \\
\text { Examination, and Testing } \\
\text { Personnel for Nuclear Power Plants }\end{array}$ & drg 1010.r00 & 1992 & $\mathbf{P}$ & Endorsed by reg 1.58 & $\begin{array}{l}\text { ASME N45.2.6/ } \\
\text { ASME NQA-1 }\end{array}$ & $\begin{array}{r}1978 / \\
1992\end{array}$ \\
\hline 0873 & ANSI & ANSI N45.2.6 & $N / S$ & $\begin{array}{l}\text { Qualifications of Inspection, } \\
\text { Examination, and Testing } \\
\text { Personnel for Nuclear Power Plants }\end{array}$ & drgce913-5 & 1981 & $\mathbf{R}$ & Proposed reg1.13.r02 & $\begin{array}{l}\text { ASME N45.2.6/ } \\
\text { ASME NQA-1 }\end{array}$ & $\begin{array}{r}1978 / \\
1992\end{array}$ \\
\hline 0874 & ANSI & ANSI N45.2.6 & $\mathrm{N} / \mathrm{S}$ & $\begin{array}{l}\text { Qualifications of Inspeciirn, } \\
\text { Examination, and Testing } \\
\text { Personnel for Nuclear Power Plants }\end{array}$ & drgrs902-4 & 1980 & $\mathbf{R}$ & Proposed reg1.3.r03 & $\begin{array}{l}\text { ASME N45.2.6/ } \\
\text { ASME NQA-1 }\end{array}$ & $\begin{array}{r}1978 / \\
1992\end{array}$ \\
\hline 0875 & ANSI & ANSI N45.2.6 & $\mathrm{N} / \mathrm{S}$ & $\begin{array}{l}\text { Qualifications of Inspection, } \\
\text { Examination, and Testing } \\
\text { Personnel for Nuclear Power Plants }\end{array}$ & drgrs908-5 & 1979 & $\mathbf{R}$ & & $\begin{array}{l}\text { ASME N45.2.6/ } \\
\text { ASME NQA-1 }\end{array}$ & $\begin{array}{l}1978 / \\
1992\end{array}$ \\
\hline 0876 & ANSI & ANSI N45.2.6 & $N / S$ & $\begin{array}{l}\text { Qualifications of Inspection, } \\
\text { Examination, and Testing } \\
\text { Personnel for Nuclear Power Plants }\end{array}$ & gltB1-001 & 1981 & $\mathbf{P}$ & $\begin{array}{l}\text { Reg. Guide } 1.58 \\
\text { endorses with } \\
\text { exceptions }\end{array}$ & $\begin{array}{l}\text { ASME N45.2.6/ } \\
\text { ASME NQA-1 }\end{array}$ & $\begin{array}{r}1978 / \\
1992\end{array}$ \\
\hline 0877 & ANSI & ANSI N45.2.6 & $N / S$ & $\begin{array}{l}\text { Quallfications of Inspection, } \\
\text { Examination, and Testing } \\
\text { Personnel for Nuclear Power Plants }\end{array}$ & inm35016B & 1975 & $\mathbf{R}$ & & $\begin{array}{l}\text { ASME N45.2.6/ } \\
\text { ASME NQA-1 }\end{array}$ & $\begin{array}{r}1978 / \\
1992\end{array}$ \\
\hline 0878 & ANSI & ANSI N45.2.6 & $N / S$ & $\begin{array}{l}\text { Qualifications of Inspection, } \\
\text { Examination, and Testing } \\
\text { Personnel for Nuclear Power Plants }\end{array}$ & inm35018B & 1975 & $\mathbf{R}$ & & $\begin{array}{l}\text { ASME N45.2.6/ } \\
\text { ASME NQA-1 }\end{array}$ & $\begin{array}{r}1978 / \\
1992\end{array}$ \\
\hline 0879 & ANSI & ANSI N45.2.6 & $\mathrm{N} / \mathrm{S}$ & $\begin{array}{l}\text { Qualifications of Inspection, } \\
\text { Examination, and Testing } \\
\text { Personnel for Nuclear Power Plants }\end{array}$ & inm35028B & 1975 & $\mathbf{R}$ & & $\begin{array}{l}\text { ASME N45.2.6/ } \\
\text { ASME NQA-1 }\end{array}$ & $\begin{array}{r}1978 / \\
1992\end{array}$ \\
\hline 0880 & ANSI & ANSI N45.2.6 & $\mathrm{N} / \mathrm{S}$ & $\begin{array}{l}\text { Qualifications of Inspection, } \\
\text { Examination, and Testing } \\
\text { Personnel for Nuclear Power Plants }\end{array}$ & inm35061 & 1985 & $\mathbf{R}$ & & $\begin{array}{l}\text { ASME N45.2.6/ } \\
\text { ASME NQA-1 }\end{array}$ & $\begin{array}{r}1978 / \\
1992\end{array}$ \\
\hline
\end{tabular}

Personnel for Nuclear Power Plants 
Codes and Standards and Other Guidance Cited in Regulatory Documents

Part A - Industry Consensus Codes and Standards (continued)

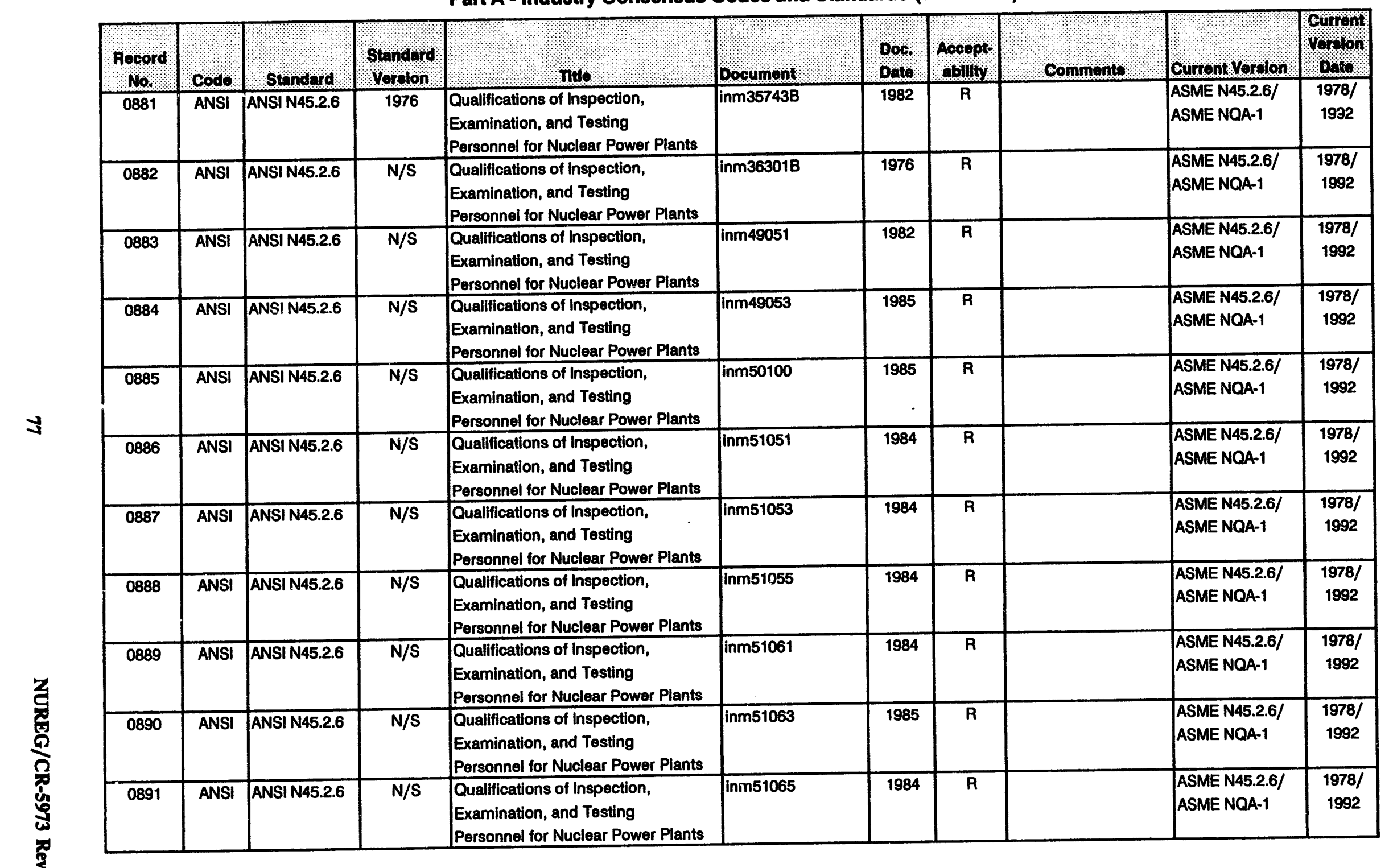


Codes and Standards and Other Guidance Cited in Regulatory Documents

Part A - Industry Consensus Codes and Standards (continued)

\begin{tabular}{|c|c|c|c|c|c|c|c|c|c|c|}
\hline Pocord & 00.0 & standord & Strondror & 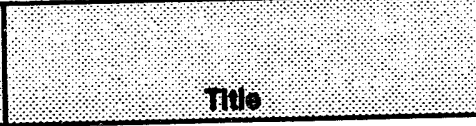 & Dooumont & $\frac{000}{0.16}$ & Accepts & Comments & C.1. & quntom \\
\hline 0892 & ANSI & ANSI N45.2.6 & $\mathrm{N} / \mathrm{S}$ & $\begin{array}{l}\text { Qualifications of Inspection, } \\
\text { Examination, and Testing } \\
\text { Personnel for Nuclear Power Plants }\end{array}$ & inm52051 & 1984 & $\mathbf{R}$ & & $\begin{array}{l}\text { ASME N45.2.6/ } \\
\text { ASME NQA-1 }\end{array}$ & $\begin{array}{c}1978 / \\
1992\end{array}$ \\
\hline 0,393 & ANSI & ANSI N45.2.6 & $\mathrm{N} / \mathrm{S}$ & $\begin{array}{l}\text { Qualifications of Inspection, } \\
\text { Examination, and Testing } \\
\text { Personnel for Nuclear Power Plants }\end{array}$ & inm52053 & 1984 & $\mathbf{R}$ & & $\begin{array}{l}\text { ASME N45.2.6/ } \\
\text { ASME NQA-1 }\end{array}$ & $\begin{array}{l}1978 / \\
1992\end{array}$ \\
\hline 0894 & ANSI & ANSI N45.2.6 & $\mathrm{N} / \mathrm{S}$ & $\begin{array}{l}\text { Qualifications of Inspection, } \\
\text { Examination, and Testing } \\
\text { Personnel for Nuclear Power Plants }\end{array}$ & inm52055 & 1984 & $\mathbf{R}$ & & $\begin{array}{l}\text { ASME N45.2.6/ } \\
\text { ASME NQA-1 }\end{array}$ & $\begin{array}{c}1978 / \\
1992\end{array}$ \\
\hline 0895 & ANSI & ANSI N45.2.6 & $\mathrm{N} / \mathrm{S}$ & $\begin{array}{l}\text { Qualifications of Inspection, } \\
\text { Examination, and Testing } \\
\text { Personnel for Nuclear Power Plants }\end{array}$ & inm57050 & 1983 & $\mathbf{R}$ & & $\begin{array}{l}\text { ASME N45.2.6/ } \\
\text { ASME NQA-1 }\end{array}$ & $\begin{array}{c}1978 / \\
1992\end{array}$ \\
\hline 0896 & ANSI & ANSI N45.2.6 & N/S & $\begin{array}{l}\text { Qualifications of Inspection, } \\
\text { Examination, and Testing } \\
\text { Personnel for Nuclear Power Plants }\end{array}$ & inm57060 & 1983 & $\mathbf{R}$ & & $\begin{array}{l}\text { ASME N45.2.6/ } \\
\text { ASME NQA-1 }\end{array}$ & $\begin{array}{l}1978 / \\
1992\end{array}$ \\
\hline 0897 & ANSI & ANSI N45.2.6 & $\mathrm{N} / \mathrm{S}$ & $\begin{array}{l}\text { Qualifications of Inspection, } \\
\text { Examination, and Testing } \\
\text { Personne! for Nuclear Power Plants }\end{array}$ & inm57070 & 1983 & $\mathbf{R}$ & & $\begin{array}{l}\text { ASME N45.2.6/ } \\
\text { ASME NQA-1 }\end{array}$ & $\begin{array}{c}1978 / \\
1992\end{array}$ \\
\hline 0898 & ANSI & ANSI N45.2.6 & $\mathrm{N} / \mathrm{S}$ & $\begin{array}{l}\text { Qualifications of inspection, } \\
\text { Examination, and Testing } \\
\text { Personnel for Nuclear Power Plants }\end{array}$ & inm57080 & 1983 & $\mathbf{R}$ & & $\begin{array}{l}\text { ASME N45.2.6/ } \\
\text { ASME NQA-1 }\end{array}$ & $\begin{array}{c}1978 / \\
1992\end{array}$ \\
\hline 0899 & ANSI & ANSI N45.2.6 & N/S & $\begin{array}{l}\text { Qualifications of Inspection, } \\
\text { Examination, and Testing } \\
\text { Personnel for Nuclear Power Plants }\end{array}$ & inm57090 & 1993 & $\mathbf{R}$ & & $\begin{array}{l}\text { ASME N45.2.6/ } \\
\text { ASME NQA-1 }\end{array}$ & $\begin{array}{l}1978 / \\
1992\end{array}$ \\
\hline 0900 & ANSI & ANSI N45.2.6 & 1976 & $\begin{array}{l}\text { Qualifications of Inspection, } \\
\text { Examination, and Testing } \\
\text { Personnel for Nuclear Power Plants }\end{array}$ & inm62702 & 1987 & $\mathbf{R}$ & & $\begin{array}{l}\text { ASME N45.2.6/ } \\
\text { ASME NQA-1 }\end{array}$ & $\begin{array}{r}1978 / \\
1992\end{array}$ \\
\hline 0901 & ANSI & ANSI N45.2.6 & $N / S$ & $\begin{array}{l}\text { Qualifications of Inspection, } \\
\text { Examination, and Testing } \\
\text { Personnel for Nuclear Power Plants }\end{array}$ & inm62704 & 1987 & $\mathbf{A}$ & & $\begin{array}{l}\text { ASME N45.2.6/ } \\
\text { ASME NQA-1 }\end{array}$ & $\begin{array}{l}1978 / \\
1992\end{array}$ \\
\hline 0902 & ANSI & ANSI N45.2.6 & N/S & $\begin{array}{l}\text { Qualifications of Inspection, } \\
\text { Examination, and Testing } \\
\text { Personnel for Nuclear Power Plants }\end{array}$ & inm62705 & 1987 & $\mathbf{R}$ & & $\begin{array}{l}\text { ASME N45.2.6/ } \\
\text { ASME NQA-1 }\end{array}$ & $\begin{array}{r}1978 / \\
1992\end{array}$ \\
\hline
\end{tabular}


Codes and Standards and Other Guidance Cited in Regulatory Documents

Part A - Industry Consensus Codes and Standards (continued)

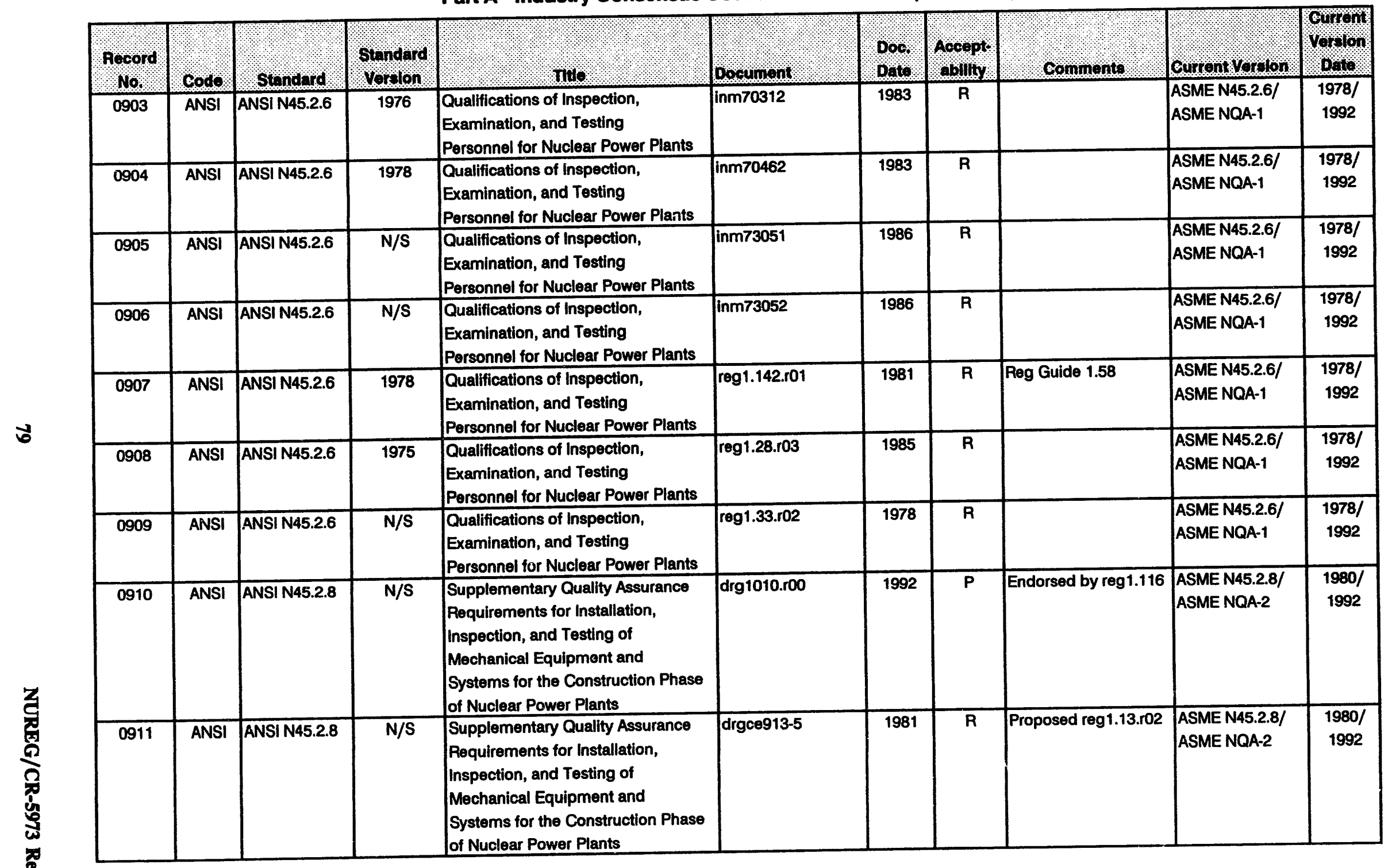


Codes and Standards and Other Guidance Clted in Regulatory Documents

Part A - Industry Consensus Codes and Standards (continued)

\begin{tabular}{|c|c|c|c|c|c|c|c|c|c|c|}
\hline Rocord & Codo & Standald & Sthorord & 1. & Dooveront & pool & 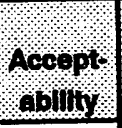 & (6omment & (4) & 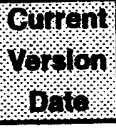 \\
\hline 0912 & ANSI & ANSI N45.2.8 & N/S & $\begin{array}{l}\text { Supplementary Quality Assurance } \\
\text { Requirements for Installation, } \\
\text { Inspection, and Testing of } \\
\text { Mechanical Equipment and } \\
\text { Systems for the Construction Phase } \\
\text { of Nuclear Power Plants }\end{array}$ & drgrs902-4 & 1980 & $\mathbf{R}$ & Proposed reg1.3.r03 & $\begin{array}{l}\text { ASME N45.2.8/ } \\
\text { ASME NQA-2 }\end{array}$ & $\begin{array}{c}1980 / \\
1992\end{array}$ \\
\hline 0913 & ANSI & ANSI N45.2.8 & $\mathrm{N} / \mathrm{S}$ & $\begin{array}{l}\text { Supplementary Quality Assurance } \\
\text { Requirements for Installation, } \\
\text { Inspection, and Testing of } \\
\text { Mechanical Equipment and } \\
\text { Systems for the Construction Phase } \\
\text { of Nuclear Power Plants }\end{array}$ & inm35018B & 1975 & $\mathbf{R}$ & & $\begin{array}{l}\text { ASME N45.2.8/ } \\
\text { ASME NQA-2 }\end{array}$ & $\begin{array}{l}1980 / \\
1992\end{array}$ \\
\hline 0914 & ANSI & ANSI N45.2.8 & $\mathrm{N} / \mathrm{S}$ & $\begin{array}{l}\text { Supplementary Quality Assurance } \\
\text { Requirements for Installation, } \\
\text { Inspection, and Testing of } \\
\text { Mechanical Equipment and } \\
\text { Systems for the Construction Phase } \\
\text { of Nuclear Power Plants }\end{array}$ & inm35028B & 1975 & $\mathbf{R}$ & & $\begin{array}{l}\text { ASME N45.2.8/ } \\
\text { ASME NQA-2 }\end{array}$ & $\begin{array}{l}1980 / \\
1992\end{array}$ \\
\hline 0915 & ANSI & ANSI N45.2.8 & 1975 & $\begin{array}{l}\text { Supplementary Quality Assurance } \\
\text { Requirements for Installation, } \\
\text { Inspection, and Testing of } \\
\text { Mechanical Equipment and } \\
\text { Systems for the Construction Phase } \\
\text { of Nuclear Power Plants }\end{array}$ & inm35749B & 1976 & $\mathbf{R}$ & & $\begin{array}{l}\text { ASME N45.2.8/ } \\
\text { ASME NQA-2 }\end{array}$ & $\begin{array}{l}1980 / \\
1992\end{array}$ \\
\hline 0916 & ANSI & ANSI N45.2.8 & $\mathrm{N} / \mathrm{S}$ & $\begin{array}{l}\text { Supplementary Quality Assurance } \\
\text { Requirements for Installation, } \\
\text { Inspection, and Testing of } \\
\text { Mechanical Equipment and } \\
\text { Systems for the Construction Phase } \\
\text { of Nuclear Power Plants }\end{array}$ & inm49053 & 1985 & $\mathbf{R}$ & & $\begin{array}{l}\text { ASME N45.2.8/ } \\
\text { ASME NQA-2 }\end{array}$ & $\begin{array}{l}1980 / \\
1992\end{array}$ \\
\hline
\end{tabular}


Codes and Standards and Other Guidance Cited in Regulatory Documents

Part A - Industry Consensus Codes and Standards (continued)

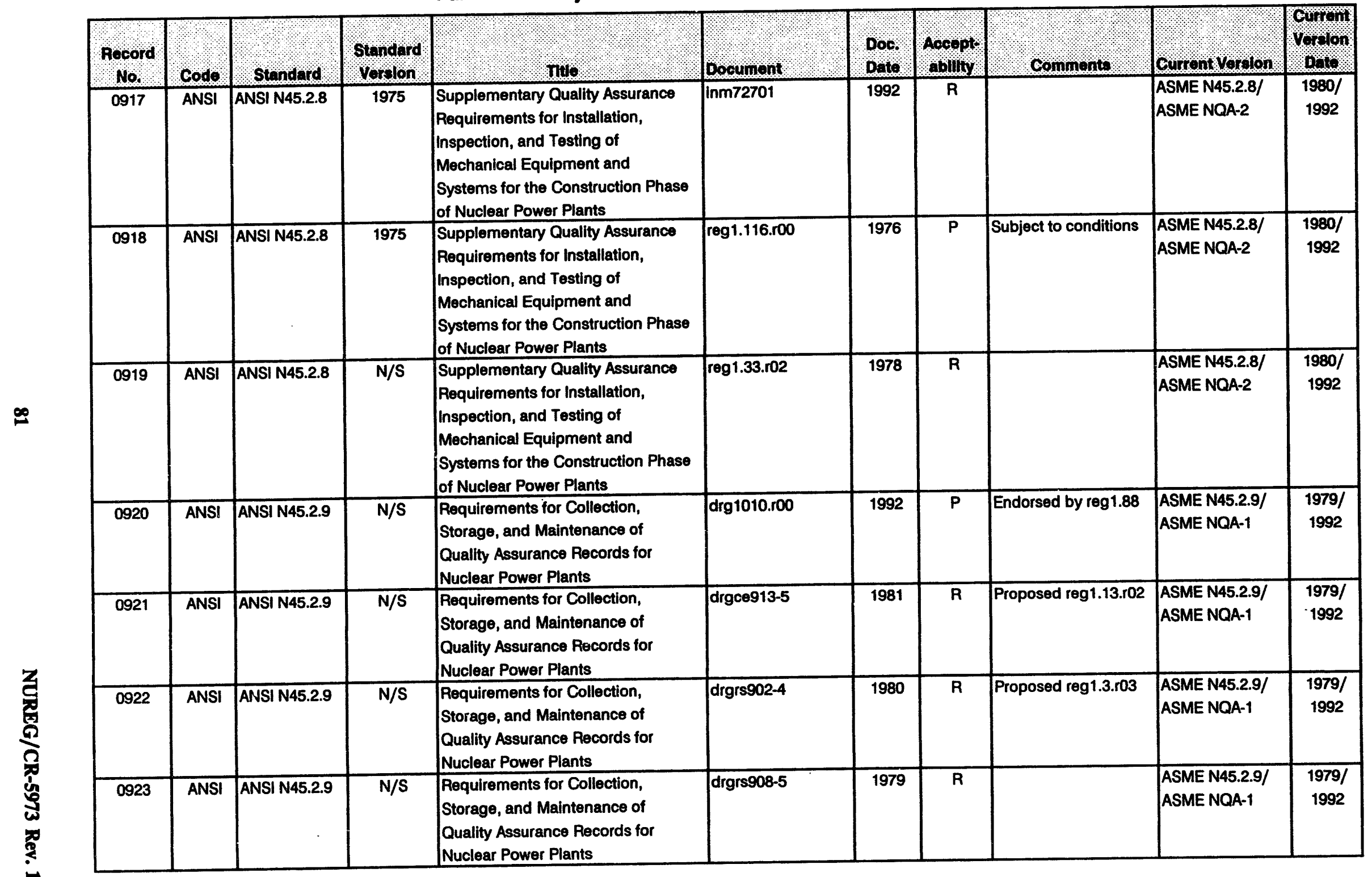


Codes and Standards and Other Guidance Cited in Regulatory Documents Part A - Industry Consensus Codes and Standards (continued)

\begin{tabular}{|c|c|c|c|c|c|c|c|c|c|c|}
\hline horord & $00 d 0$ & stindurd & standord & (1, & boovonent & Door & acoepl & Comnents: & current vorton & onf \\
\hline 0924 & ANSI & ANSI N45.2.9 & 1974 & $\begin{array}{l}\text { Requirements for Collection, } \\
\text { Storage, and Maintenance of } \\
\text { Quality Assurance Records for } \\
\text { Nuclear Power Plants }\end{array}$ & glt88-018 & 1988 & $\mathbf{P}$ & $\begin{array}{l}\text { Endorsed by Reg. } \\
\text { Guide } 1.88\end{array}$ & $\begin{array}{l}\text { ASME N45.2.9/ } \\
\text { ASME NQA-1 }\end{array}$ & $\begin{array}{c}1979 / \\
1992\end{array}$ \\
\hline 0925 & ANSI & ANSI N45.2.9 & $\mathrm{N} / \mathrm{S}$ & $\begin{array}{l}\text { Requirements for Collection, } \\
\text { Storage, and Maintenance of } \\
\text { Quality Assurance Records for } \\
\text { Nuclear Power Plants }\end{array}$ & inm-toc & 1993 & $\mathbf{R}$ & & $\begin{array}{l}\text { ASME N45.2.9/ } \\
\text { ASME NQA-1 }\end{array}$ & $\begin{array}{c}1979 / \\
1992\end{array}$ \\
\hline 0926 & ANSI & ANSI N45.2.9 & $\mathrm{N} / \mathrm{S}$ & $\begin{array}{l}\text { Requirements for Collection, } \\
\text { Storage, and Maintenance of } \\
\text { Quality Assurance Records for } \\
\text { Nuclear Power Plants }\end{array}$ & inm35002B & 1980 & $\mathbf{R}$ & & $\begin{array}{l}\text { ASME N45.2.9/ } \\
\text { ASME NQA-1 }\end{array}$ & $\begin{array}{r}1979 / \\
1992\end{array}$ \\
\hline 0927 & ANSI & ANSI N45.2.9 & $N / S$ & $\begin{array}{l}\text { Requirements for Collection, } \\
\text { Storage, and Maintenance of } \\
\text { Quality Assurance Records for } \\
\text { Nuclear Power Plants }\end{array}$ & inm35016B & 1975 & $R$ & & $\begin{array}{l}\text { ASME N45.2.9/ } \\
\text { ASME NQA-1 }\end{array}$ & $\begin{array}{r}1979 / \\
1992\end{array}$ \\
\hline 0928 & ANSI & ANSI N45.2.9 & $\mathrm{N} / \mathrm{S}$ & $\begin{array}{l}\text { Requirements for Collection, } \\
\text { Storage, and Maintenance of } \\
\text { Quality Assurance Records for } \\
\text { Nuclear Power Plants }\end{array}$ & inm35018B & 1975 & $\mathbf{R}$ & & $\begin{array}{l}\text { ASME N45.2.9/ } \\
\text { ASME NQA-1 }\end{array}$ & $\begin{array}{r}1979 / \\
1992\end{array}$ \\
\hline 0929 & ANSI & ANSI N45.2.9 & N/S & $\begin{array}{l}\text { Requirements for Collection, } \\
\text { Storage, and Maintenance of } \\
\text { Quality Assurance Records for } \\
\text { Nuclear Power Plants }\end{array}$ & inm35028B & 1975 & $\mathbf{R}$ & & $\begin{array}{l}\text { ASME N45.2.9/ } \\
\text { ASME NQA-1 }\end{array}$ & $\begin{array}{r}1979 / \\
1992\end{array}$ \\
\hline 0930 & ANSI & ANSI N45.2.9 & $\mathrm{N} / \mathrm{S}$ & $\begin{array}{l}\text { Requirements for Collection, } \\
\text { Storage, and Maintenance of } \\
\text { Quality Assurance Records for } \\
\text { Nuclear Power Plants }\end{array}$ & inm35061 & 1985 & $\mathbf{R}$ & & $\begin{array}{l}\text { ASME N45.2.9/ } \\
\text { ASME NQA-1 }\end{array}$ & $\begin{array}{r}1979 / \\
1992\end{array}$ \\
\hline 0931 & ANSI & ANSI N45.2.9 & $\mathrm{N} / \mathrm{S}$ & $\begin{array}{l}\text { Requirements for Collection, } \\
\text { Storage, and Maintenance of } \\
\text { Quality Assurance Records for } \\
\text { Nuclear Power Plants }\end{array}$ & inm35100 & 1983 & R & & $\begin{array}{l}\text { ASME N45.2.9/ } \\
\text { ASME NQA-1 }\end{array}$ & $\begin{array}{r}1979 / \\
1992\end{array}$ \\
\hline
\end{tabular}


Codes and Standards and Other Guidance Cited in Regulatory Documents

Part A - Industry Consensus Codes and Standards (continued)

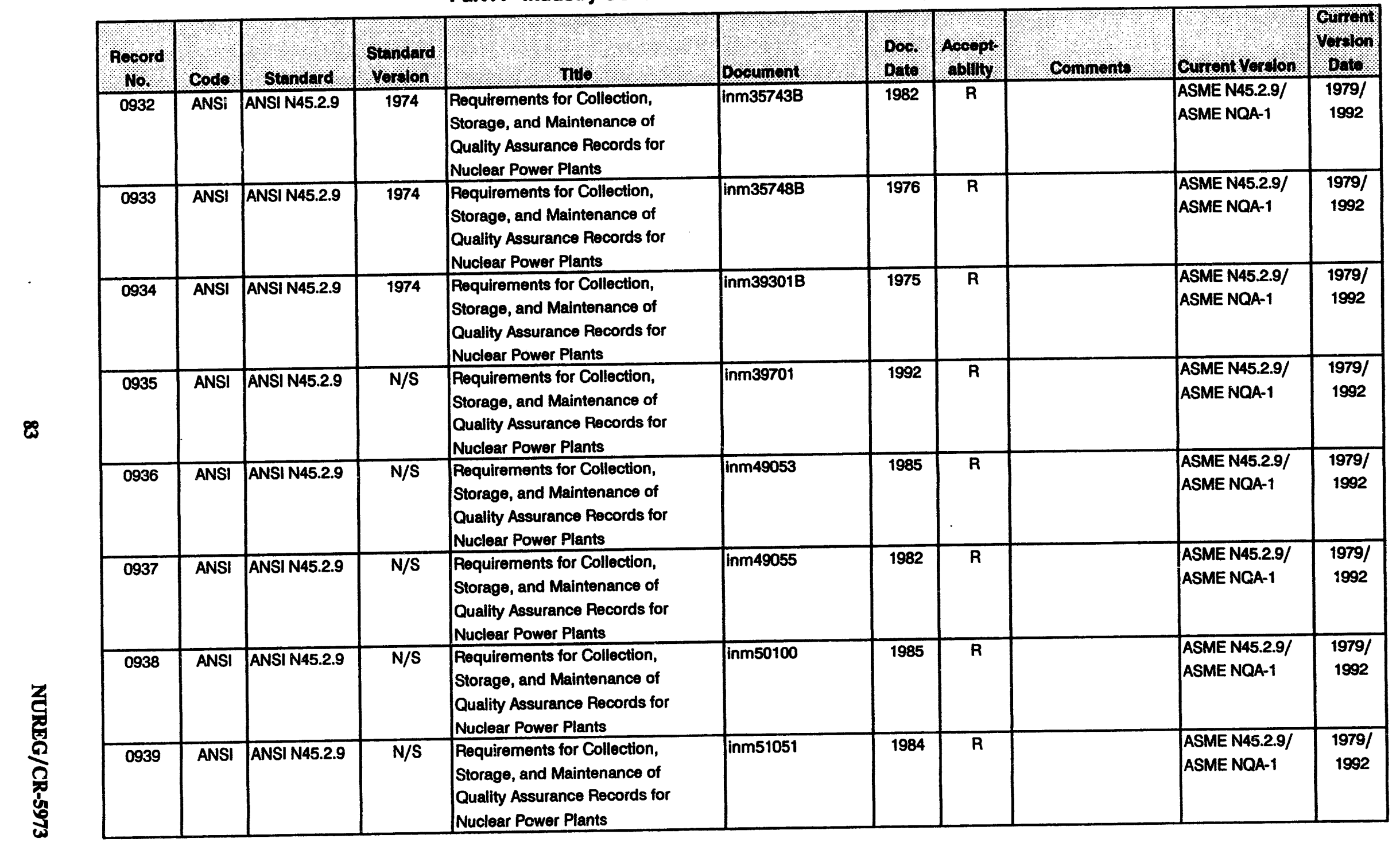


Codes and Standards and Other Guidance Cited in Regulatory Documents

Part A - Industry Consensus Codes and Standards (continued)

\begin{tabular}{|c|c|c|c|c|c|c|c|c|c|c|}
\hline $\begin{array}{l}\text { Rocord } \\
\text { No. }\end{array}$ & cods & Standard & $\begin{array}{l}\text { standard } \\
\text { vorolon }\end{array}$ & 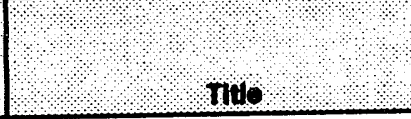 & bocumont & Doo. & Aocopt & Comments & Current Vorglon & onfont \\
\hline 0940 & ANSI & ANSI N45.2.9 & N/S & $\begin{array}{l}\text { Requirements for Collection, } \\
\text { Storage, and Maintenance of } \\
\text { Quality Assurance Records for } \\
\text { Nuclear Power Plants } \\
\end{array}$ & inm51055 & 1984 & $\mathbf{R}$ & & $\begin{array}{l}\text { ASME N45.2.9/ } \\
\text { ASME NQA-1 }\end{array}$ & $\begin{array}{r}1979 / \\
1992\end{array}$ \\
\hline 0941 & ANSI & ANSI N45.2.9 & $\mathrm{N} / \mathrm{S}$ & $\begin{array}{l}\text { Requirements for Collection, } \\
\text { Storage, and Maintenance of } \\
\text { Quality Assurance Records for } \\
\text { Nuclear Power Plants }\end{array}$ & inm51061 & 1984 & $\mathbf{R}$ & & $\begin{array}{l}\text { ASME N45.2.9/ } \\
\text { ASME NQA-1 }\end{array}$ & $\begin{array}{c}1979 / \\
1992\end{array}$ \\
\hline 0942 & ANSI & ANSI N45.2.9 & $\mathrm{N} / \mathrm{S}$ & $\begin{array}{l}\text { Requirements for Collection, } \\
\text { Storage, and Maintenance of } \\
\text { Quality Assurance Records for } \\
\text { Nuclear Power Plants }\end{array}$ & inm51063 & 1985 & $\mathbf{R}$ & & $\begin{array}{l}\text { ASME N45.2.9/ } \\
\text { ASME NQA-1 }\end{array}$ & $\begin{array}{l}1979 / \\
1992\end{array}$ \\
\hline 0943 & ANSI & ANSI N45.2.9 & $\mathbf{N} / \mathbf{S}$ & $\begin{array}{l}\text { Requirements for Collection, } \\
\text { Storage, and Maintenance of } \\
\text { Quality Assurance Records for } \\
\text { Nuclear Power Plants }\end{array}$ & inm51065 & 1984 & $\mathbf{R}$ & & $\begin{array}{l}\text { ASME N45.2.9/ } \\
\text { ASME NQA-1 }\end{array}$ & $\begin{array}{c}1979 / \\
1992\end{array}$ \\
\hline 0944 & ANSI & ANSI N45.2.9 & $\mathrm{N} / \mathrm{S}$ & $\begin{array}{l}\text { Requirements for Collection, } \\
\text { Storage, and Maintenance of } \\
\text { Quality Assurance Records for } \\
\text { Nuclear Power Plants }\end{array}$ & inm52055 & 1984 & $\mathbf{R}$ & & $\begin{array}{l}\text { ASME N45.2.9/ } \\
\text { ASME NQA-1 }\end{array}$ & $\begin{array}{c}1979 / \\
1992\end{array}$ \\
\hline 0945 & ANSI & ANSI N45.2.9 & $\mathrm{N} / \mathrm{S}$ & $\begin{array}{l}\text { Requirements for Collection, } \\
\text { Storage, and Maintenance of } \\
\text { Quality Assurance Records for } \\
\text { Nuclear Power Plants }\end{array}$ & inm57050 & 1983 & $\mathbf{R}$ & & $\begin{array}{l}\text { ASME N45.2.9/ } \\
\text { ASME NQA-1 }\end{array}$ & $\begin{array}{r}1979 / \\
1992\end{array}$ \\
\hline 0946 & ANSI & ANSI N45.2.9 & $\mathrm{N} / \mathrm{S}$ & $\begin{array}{l}\text { Requirements for Collection, } \\
\text { Storage, and Maintenance of } \\
\text { Quality Assurance Records for } \\
\text { Nuclear Power Plants } \\
\end{array}$ & inm57060 & 1983 & $\mathbf{R}$ & & $\begin{array}{l}\text { ASME N45.2.9/ } \\
\text { ASME NQA-1 }\end{array}$ & $\begin{array}{l}1979 / \\
1992\end{array}$ \\
\hline 0947 & ANSI & ANSI N45.2.9 & $\mathrm{N} / \mathrm{S}$ & $\begin{array}{l}\text { Requirements for Collection, } \\
\text { Storage, and Maintenance of } \\
\text { Quality Assurance Records for } \\
\text { Nuclear Power Plants }\end{array}$ & inm57070 & 1983 & $\mathbf{R}$ & & $\begin{array}{l}\text { ASME N45.2.9/ } \\
\text { ASME NQA-1 }\end{array}$ & $\begin{array}{r}1979 / \\
1992\end{array}$ \\
\hline
\end{tabular}

Nuclear Power Plants 
Codes and Standards and Other Guidance Cited in Regulatory Documents

Part A - Industry Consensus Codes and Standards (continued)

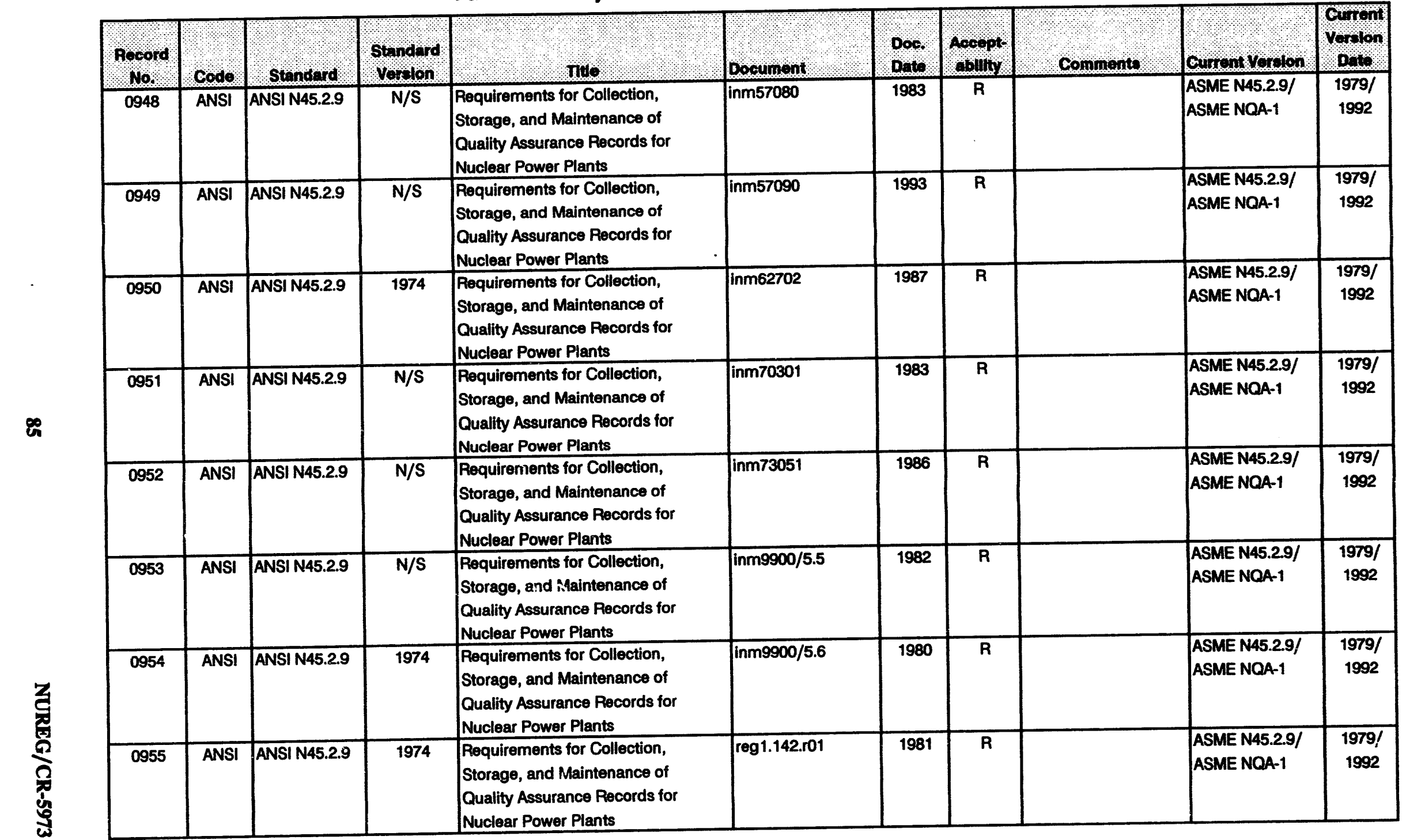


Codes and Standards and Other Guidance Clted in Regulatory Documents

Part A - Industry Consensus Codes and Standards (continued)

\begin{tabular}{|c|c|c|c|c|c|c|c|c|c|c|}
\hline Horord & codo & Standord & Stondard & ( & Doroument & Doo: & Aocept. & Connents & Connost veroton & orion \\
\hline 0956 & ANSI & ANSI N45.2.9 & 1975 & $\begin{array}{l}\text { Faquirements for Collection, } \\
\text { Storage, and Maintenance of } \\
\text { Quality Assurance Records for } \\
\text { Nuclear Power Plants }\end{array}$ & reg1.28.r03 & 1985 & $\mathbf{R}$ & & $\begin{array}{l}\text { ASME N45.2.9/ } \\
\text { ASME NOA-1 }\end{array}$ & $\begin{array}{c}1979 / \\
1992\end{array}$ \\
\hline 0957 & ANSI & ANSI N45.2.9 & $\mathrm{N} / \mathrm{S}$ & $\begin{array}{l}\text { Requirements for Collection, } \\
\text { Storage, and Maintenance of } \\
\text { Quality Assurance Records for } \\
\text { Nuclear Power Plants }\end{array}$ & reg1.33.r02 & 1978 & $\mathbf{R}$ & & $\begin{array}{l}\text { ASME N45.2.9/ } \\
\text { ASME NQA-1 }\end{array}$ & $\begin{array}{r}1979 / \\
1992\end{array}$ \\
\hline 0958 & ANSI & ANSI N45.2.10 & $N / S$ & $\begin{array}{l}\text { Quality Assurance Terms and } \\
\text { Definitions }\end{array}$ & $\operatorname{drg} 1010.100$ & 1992 & $\mathbf{R}$ & & $\begin{array}{l}\text { ASME N45.2.10/ } \\
\text { ASME NQA-1 }\end{array}$ & $\begin{array}{l}1973 / \\
1992 \\
\end{array}$ \\
\hline 0959 & ANSI & ANSI N45.2.10 & $N / S$ & $\begin{array}{l}\text { Quality Assurance Terms and } \\
\text { Definitions }\end{array}$ & drgce913-5 & 1981 & $\mathbf{R}$ & Proposed reg1.13.r02 & $\begin{array}{l}\text { ASME N45.2.10/ } \\
\text { ASME NOA-1 }\end{array}$ & $\begin{array}{c}1973 / \\
1992 \\
\end{array}$ \\
\hline 0960 & ANSI & ANSI N45.2.10 & $\mathrm{N} / \mathrm{S}$ & $\begin{array}{l}\text { Quality Assurance Terms and } \\
\text { Definitions }\end{array}$ & drgrs902-4 & 1980 & $\mathbf{R}$ & Proposed reg1.3.ro3 & $\begin{array}{l}\text { ASME N45.2.10/ } \\
\text { ASME NOA-1 }\end{array}$ & $\begin{array}{l}1973 / \\
1992\end{array}$ \\
\hline 0961 & ANSI & ANSI N45.2.10 & $N / S$ & $\begin{array}{l}\text { Quality Assurance Terms and } \\
\text { Definitions }\end{array}$ & drgrs908-5 & 1979 & $\mathbf{R}$ & & $\begin{array}{l}\text { ASME N45.2.10/ } \\
\text { ASME NOA-1 }\end{array}$ & $\begin{array}{r}1973 / \\
1992 \\
\end{array}$ \\
\hline 0962 & ANSI & ANSI N45.2.10 & N/S & $\begin{array}{l}\text { Quality Assurance Terms and } \\
\text { Definitions }\end{array}$ & inm38703 & 1993 & $\mathbf{R}$ & & $\begin{array}{l}\text { ASME N45.2.10/ } \\
\text { ASME NQA-1 }\end{array}$ & $\begin{array}{r}1973 / \\
1992 \\
\end{array}$ \\
\hline 0963 & ANSI & ANSI N45.2.10 & 1975 & $\begin{array}{l}\text { Quality Assurance Terms and } \\
\text { Definitions }\end{array}$ & reg1.28.ro3 & 1985 & $\mathbf{R}$ & & $\begin{array}{l}\text { ASME N45.2.10/ } \\
\text { ASME NOA-1 }\end{array}$ & $\begin{array}{r}1973 / \\
1992 \\
\end{array}$ \\
\hline 0964 & ANSI & ANSI N45.2.10 & $N / S$ & $\begin{array}{l}\text { Quality Assurance Terms and } \\
\text { Definitions }\end{array}$ & reg1.33.r02 & 1978 & $\mathbf{R}$ & & $\begin{array}{l}\text { ASME N45.2.10/ } \\
\text { ASME NOA-1 }\end{array}$ & $\begin{array}{c}1973 / \\
1992 \\
\end{array}$ \\
\hline 0965 & ANSI & ANSI N45.2.11 & $\mathrm{N} / \mathrm{S}$ & $\begin{array}{l}\text { Quality Assurance Requirements } \\
\text { for the Design of Nuclear Power } \\
\text { Plants }\end{array}$ & drg 1010.ro0 & 1992 & $\bar{P}$ & Endorsed by reg1.64 & $\begin{array}{l}\text { ASME N45.2.11/ } \\
\text { ASME NOA-1 }\end{array}$ & $\begin{array}{c}1974 / \\
1992\end{array}$ \\
\hline 0966 & ANSI & ANSI N45.2.11 & $\mathrm{N} / \mathrm{S}$ & $\begin{array}{l}\text { Quality Assurance Requirements } \\
\text { for the Design of Nuclear Power } \\
\text { Plants }\end{array}$ & drgce913-5 & 1981 & R & Proposed reg1.13.ro2 & $\begin{array}{l}\text { ASME N45:2.11/ } \\
\text { ASME NOA-1 }\end{array}$ & $\begin{array}{r}1974 / \\
1992\end{array}$ \\
\hline 0967 & ANSI & ANSI N45.2.11 & $\mathrm{N} / \mathrm{S}$ & $\begin{array}{l}\text { Quality Assurance Requirements } \\
\text { for the Design of Nuclear Power } \\
\text { Plants }\end{array}$ & drgrs902-4 & 1980 & $\bar{R}$ & Proposed reg1.3.r03 & $\begin{array}{l}\text { ASME N45.2.11/ } \\
\text { ASME NOA-1 }\end{array}$ & $\begin{array}{c}1974 / \\
1992\end{array}$ \\
\hline
\end{tabular}


Codes and Standards and Other Guidance Cited in Regulatory Documents

Part A - Industry Consensus Codes and Standards (continued)

\begin{tabular}{|c|c|c|c|c|c|c|c|c|c|c|}
\hline Record & Codo & Standhrd & $\begin{array}{l}\text { stnderd } \\
\text { yorolon }\end{array}$ & (ै) & Dosulnent & $\begin{array}{l}\text { Dor. } \\
\text { Date. }\end{array}$ & Accopt & Comments. & Gunrent Vorton & 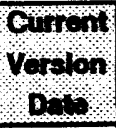 \\
\hline 0968 & ANSI & ANSI N45.2.11 & $\mathrm{N} / \mathrm{S}$ & $\begin{array}{l}\text { Quality Assurance Requirements } \\
\text { for the Design of Nuclear Power } \\
\text { Plants }\end{array}$ & inm35002B & 1980 & $\mathbf{R}$ & & $\begin{array}{l}\text { ASME N45.2.11/ } \\
\text { ASME NQA-1 }\end{array}$ & $\begin{array}{c}1974 / \\
1992\end{array}$ \\
\hline 0969 & ANSI & ANSI N45.2.11 & $N / S$ & $\begin{array}{l}\text { Quality Assurance Requirements } \\
\text { for the Design of Nuclear Power } \\
\text { Plants }\end{array}$ & inm35018B & 1975 & $\mathbf{R}$ & & $\begin{array}{l}\text { ASME N45.2.11/ } \\
\text { ASME NQA-1 }\end{array}$ & $\begin{array}{c}1974 / \\
1992\end{array}$ \\
\hline 0970 & ANSI & ANSI N45.2.11 & 1974 & $\begin{array}{l}\text { Quality Assurance Requirements } \\
\text { for the Design of Nuclear Power } \\
\text { Plants }\end{array}$ & inm35060 & 1983 & $\mathbf{R}$ & & $\begin{array}{l}\text { ASME N45.2.11/ } \\
\text { ASME NOA-1 }\end{array}$ & $\begin{array}{c}1974 / \\
1992\end{array}$ \\
\hline c971 & ANSI & ANSI N45.2.11 & 1974 & $\begin{array}{l}\text { Quality Assurance Requirements } \\
\text { for the Design of Nuclear Power } \\
\text { Flants }\end{array}$ & inm35744B & 1979 & $\mathbf{R}$ & & $\begin{array}{l}\text { ASME N45.2.11/ } \\
\text { ASME NQA-1 }\end{array}$ & $\begin{array}{c}1974 / \\
1992\end{array}$ \\
\hline 0972 & ANSI. & ANSI N45.2.11 & $\mathbf{N} / \mathbf{S}$ & $\begin{array}{l}\text { Quality Assurance Requirements } \\
\text { for the Design of Nuclear Power } \\
\text { Plants }\end{array}$ & inm37051 & 1987 & $\mathbf{R}$ & & $\begin{array}{l}\text { ASME N45.2.11/ } \\
\text { ASME NOA-1 }\end{array}$ & $\begin{array}{c}1974 / \\
1992\end{array}$ \\
\hline 0973 & ANSI & ANSI N45.2.11 & $N / S$ & $\begin{array}{l}\text { Quality Assurance Requirements } \\
\text { for the Design of Nuclear Power } \\
\text { Plants }\end{array}$ & inm37055 & 1983 & $\mathbf{R}$ & & $\begin{array}{l}\text { ASME N45.2.11/ } \\
\text { ASME NOA-1 }\end{array}$ & $\begin{array}{l}1974 / \\
1992\end{array}$ \\
\hline 0974 & ANSI & ANSI N45.2.11 & 1974 & $\begin{array}{l}\text { Quality Assurance Requirements } \\
\text { for the Design of Nuclear Power } \\
\text { Plants }\end{array}$ & inm37702 & 1987 & $\mathbf{R}$ & $\begin{array}{l}\text { Typo - was ANSI } \\
\text { N45.11 }\end{array}$ & $\begin{array}{l}\text { ASME N45.2.11/ } \\
\text { ASME NQA-1 }\end{array}$ & $\begin{array}{c}1974 / \\
1992\end{array}$ \\
\hline 0975 & ANSI & ANSI N45.2.11 & $\mathrm{N} / \mathrm{S}$ & $\begin{array}{l}\text { Quality Assurance Requirements } \\
\text { for the Design of Nuclear Power } \\
\text { Plants }\end{array}$ & inm49053 & 1985 & $\mathbf{R}$ & & $\begin{array}{l}\text { ASME N45.2.11/ } \\
\text { ASME NOA-1 }\end{array}$ & $\begin{array}{c}1974 / \\
1992\end{array}$ \\
\hline 0976 & ANSI & ANSI N45.2.11 & $N / S$ & $\begin{array}{l}\text { Quality Assurance Requirements } \\
\text { for the Design of Nuclear Power } \\
\text { Plants }\end{array}$ & inm49063 & 1985 & $\mathbf{R}$ & & $\begin{array}{l}\text { ASME N45.2.11/ } \\
\text { ASME NOA-1 }\end{array}$ & $\begin{array}{l}1974 / \\
1992\end{array}$ \\
\hline 0977 & ANSI & ANSI N45.2.11 & $N / S$ & $\begin{array}{l}\text { Quality Assurance Requirements } \\
\text { for the Design of Nuclear Power } \\
\text { Plants }\end{array}$ & inm50090 & 1985 & $\mathbf{R}$ & & $\begin{array}{l}\text { ASME N45.2.11/ } \\
\text { ASME NQA-1 }\end{array}$ & $\begin{array}{c}1974 / \\
1992\end{array}$ \\
\hline
\end{tabular}


Codes and Standards and Other Guidance Clted in Regulatory Documents

Part A - Industry Consensus Codes and Standards (continued)

\begin{tabular}{|c|c|c|c|c|c|c|c|c|c|c|}
\hline Pocord & codo & Standord & stondard & 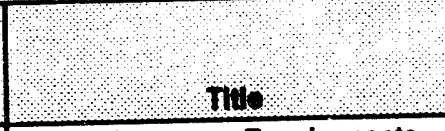 & Boovonst & Doa. & $\begin{array}{l}\text { Acospt: } \\
\text { onduly }\end{array}$ & Connonosis & Guncul Yerotion & 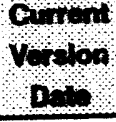 \\
\hline 0978 & ANSI & ANSI N45.2.11 & N/S & $\begin{array}{l}\text { Quality Assurance Requirements } \\
\text { for the Design of Nuclear Power } \\
\text { Plants }\end{array}$ & inm50100 & 1985 & $\mathbf{R}$ & & $\begin{array}{l}\text { ASME N45.2.11/ } \\
\text { ASME NOA-1 }\end{array}$ & $\begin{array}{l}1974 / \\
1992\end{array}$ \\
\hline 0979 & ANSI & ANSI N45.2.11 & $N / S$ & $\begin{array}{l}\text { Quality Assurance Requirements } \\
\text { for the Design of Nuclear Power } \\
\text { Plants }\end{array}$ & inm51053 & 1984 & $\mathbf{R}$ & & $\begin{array}{l}\text { ASME N45.2.11/ } \\
\text { ASME NOA-1 }\end{array}$ & $\begin{array}{l}1974 / \\
1992\end{array}$ \\
\hline 0980 & ANSI & ANSI N45.2.11 & $N / S$ & $\begin{array}{l}\text { Quality Assurance Requirements } \\
\text { for the Design of Nuclear Power } \\
\text { Plants }\end{array}$ & inm51063 & 1985 & A & & $\begin{array}{l}\text { ASME N45.2.11/ } \\
\text { ASME NOA-1 }\end{array}$ & $\begin{array}{l}1974 / \\
1992\end{array}$ \\
\hline 0981 & ANSI & ANSI N45.2.11 & $N / S$ & $\begin{array}{l}\text { Quality Assurance Requirements } \\
\text { for the Design of Nuclear Power } \\
\text { Plants }\end{array}$ & inm52053 & 1984 & $\mathbf{R}$ & & $\begin{array}{l}\text { ASME N45.2.11/ } \\
\text { ASME NOA-1 }\end{array}$ & $\begin{array}{l}1974 / \\
1992\end{array}$ \\
\hline 0982 & ANSI & ANSI N45.2.11 & N/S & $\begin{array}{l}\text { Quality Assurance Requirements } \\
\text { for the Design of Nuclear Power } \\
\text { Plants }\end{array}$ & inm70301 & 1983 & $\mathbf{R}$ & & $\begin{array}{l}\text { ASME N45.2.11/ } \\
\text { ASME NOA-1 }\end{array}$ & $\begin{array}{l}1974 / \\
1992\end{array}$ \\
\hline 0983 & ANSI & ANSI N45.2.11 & $N / S$ & $\begin{array}{l}\text { Quality Assurance Requirements } \\
\text { for the Design of Nuclear Power } \\
\text { Plants }\end{array}$ & inm93801 & 1990 & $\mathbf{R}$ & & $\begin{array}{l}\text { ASME N45.2.11/ } \\
\text { ASME NOA-1 }\end{array}$ & $\begin{array}{r}1974 / \\
1992\end{array}$ \\
\hline 0984 & ANSI & ANSI N45.2.11 & 1974 & $\begin{array}{l}\text { Quality Assurance Requirements } \\
\text { for the Destgn of Nuclear Power } \\
\text { Plants }\end{array}$ & not84-54 & 1984 & $\mathbf{P}$ & $\begin{array}{l}\text { Endorsed by Reg. } \\
\text { Guide } 1.64\end{array}$ & $\begin{array}{l}\text { ASME N45.2.11/ } \\
\text { ASME NOA-1 }\end{array}$ & $\begin{array}{r}1974 / \\
1992\end{array}$ \\
\hline 0985 & ANSI & ANSI N45.2.11 & 1974 & $\begin{array}{l}\text { Quality Assurance Requirements } \\
\text { for the Design of Nuclear Power } \\
\text { Plants }\end{array}$ & reg1.142.ro1 & 1981 & $\mathbf{R}$ & Reg Guide 1.64 & $\begin{array}{l}\text { ASME N45.2.11/ } \\
\text { ASME NOA-1 }\end{array}$ & $\begin{array}{l}1974 / \\
1992\end{array}$ \\
\hline 0986 & ANSI & ANSI N45.2.11 & 1975 & $\begin{array}{l}\text { Quality Assurance Requirements } \\
\text { for the Design of Nuclear Power } \\
\text { Plants }\end{array}$ & reg 1.28.r03 & 1985 & $\mathbf{R}$ & & $\begin{array}{l}\text { ASME N45.2.11/ } \\
\text { ASME NOA-1 }\end{array}$ & $\begin{array}{r}1974 / \\
1992\end{array}$ \\
\hline 0987 & ANSI & ANSI N45.2.11 & $\mathrm{N} / \mathrm{S}$ & $\begin{array}{l}\text { Quality Assurance Roquirements } \\
\text { for the Design of Nuclear Power } \\
\text { Plants }\end{array}$ & reg1.33.102 & 1978 & $\mathbf{R}$ & & $\begin{array}{l}\text { ASME N45.2.11/ } \\
\text { ASME NOA-1 }\end{array}$ & $\begin{array}{r}1974 / \\
1992\end{array}$ \\
\hline
\end{tabular}


Codes and Standards and Other Guidance Cited in Regulatory Documents

Part A - Industry Consensus Codes and Standards (continued)

\begin{tabular}{|c|c|c|c|c|c|c|c|c|c|c|}
\hline $\begin{array}{l}\text { Rocord } \\
\text { No. }\end{array}$ & Code & Standard & $\begin{array}{l}\text { Stendard } \\
\text { Verolon }\end{array}$ & (1) & Documents & $\begin{array}{l}\text { Dor. } \\
\text { Date }\end{array}$ & $\begin{array}{l}\text { Acoopt- } \\
\text { abliny }\end{array}$ & Comments & Current Veritos & Q volom \\
\hline 0988 & ANSI & ANSI N45.2.12 & $N / S$ & $\begin{array}{l}\text { Requirements for Auditing of } \\
\text { Quality Assurance Programs for } \\
\text { Nuclear Power Plants }\end{array}$ & $\operatorname{drg} 1010.100$ & 1992 & $P$ & Endorsed by reg1.144 & $\begin{array}{l}\text { ASME N45.2.12/ } \\
\text { ASME NCA-1 }\end{array}$ & $\begin{array}{l}1977 / \\
1992\end{array}$ \\
\hline 0989 & ANSI & ANSI N45.2.12 & N/S & $\begin{array}{l}\text { Requirements for Auditing of } \\
\text { Quality Assurance Programs for } \\
\text { Nuclear Power Plants }\end{array}$ & drgrs902-4 & 1980 & $\mathbf{R}$ & Proposed reg1.3.r03 & $\begin{array}{l}\text { ASME N45.2.12/ } \\
\text { ASME NOA-1 }\end{array}$ & $\begin{array}{l}1977 / \\
1992\end{array}$ \\
\hline 0990 & ANSI & ANSI N45.2.12 & N/S & $\begin{array}{l}\text { Requirements for Auditing of } \\
\text { Quality Assurance Programs for } \\
\text { Nuclear Power Plants }\end{array}$ & inm35002B & 1980 & $\mathbf{R}$ & & $\begin{array}{l}\text { ASMEE N45.2.12 } \\
\text { ASME NOA-1 }\end{array}$ & $\begin{array}{l}197 / 1 \\
1992\end{array}$ \\
\hline 0991 & ANSI & ANSI N45.2.12 & N/S & $\begin{array}{l}\text { Requirements for Auditing of } \\
\text { Quality Assurance Programs for } \\
\text { Nuclear Power Plants }\end{array}$ & inm35016B & 1975 & $\mathbf{R}$ & & $\begin{array}{l}\text { ASME N45.2.12/ } \\
\text { ASME NOA-1 }\end{array}$ & $\begin{array}{l}1977 / \\
1992\end{array}$ \\
\hline 0992 & ANSI & $\overline{\text { ANSI N45.2.12 }}$ & $\mathrm{N} / \mathrm{S}$ & $\begin{array}{l}\text { Requiroments for Auditing of } \\
\text { Quality Assurance Programs for } \\
\text { Nuclear Power Plants }\end{array}$ & inm35018B & 1975 & $\mathbf{R}$ & & $\begin{array}{l}\text { ASME N45.2.12/ } \\
\text { ASME NOA-1 }\end{array}$ & $\begin{array}{l}1977 / \\
1992\end{array}$ \\
\hline 0993 & ANOI & ANSI N45.2.12 & $N / S$ & $\begin{array}{l}\text { Requirements for Auditing of } \\
\text { Quality Assurance Programs for } \\
\text { Nuclear Power Plants }\end{array}$ & inm35020 & 1983 & $\mathbf{R}$ & & $\begin{array}{l}\text { ASME N45.2.12/ } \\
\text { ASME NOA-1 }\end{array}$ & $\begin{array}{l}1977 / \\
1992\end{array}$ \\
\hline 0994 & ANSI & ANSI N45.2.12 & $\mathrm{N} / \mathrm{S}$ & $\begin{array}{l}\text { Requirements for Auditing of } \\
\text { Quality Assurance Programs for } \\
\text { Nuclear Power Plants }\end{array}$ & inm35060 & 1983 & $\mathbf{R}$ & & $\begin{array}{l}\text { ASME N45.2.1E/ } \\
\text { ASME NOA-1 }\end{array}$ & $\begin{array}{l}197 / 1 \\
1992\end{array}$ \\
\hline 0995 & ANSI & ANSI N45.2.12 & 1973 & $\begin{array}{l}\text { Requirements for Auditing of } \\
\text { Quality Assurance Programs for } \\
\text { Nuclear Power Plants }\end{array}$ & inm35741B & 1979 & $\mathbf{R}$ & $\begin{array}{l}\text { Typo - was ANSI } \\
\text { N45.12 }\end{array}$ & $\begin{array}{l}\text { ASME N45.2.12/ } \\
\text { ASME NOA-1 }\end{array}$ & $\begin{array}{l}1977 / \\
1992\end{array}$ \\
\hline 0996 & ANSI & ANSI N45.2.12 & $\mathrm{N} / \mathrm{S}$ & $\begin{array}{l}\text { Requirements for Auditing of } \\
\text { Quality Assurance Programs for } \\
\text { Nuclear Power Plants }\end{array}$ & inm37055 & 1983 & $\mathbf{R}$ & & $\begin{array}{l}\text { ASME N45.2.12/ } \\
\text { ASME NOA-1 }\end{array}$ & $\begin{array}{l}1977 / \\
1992\end{array}$ \\
\hline 0997 & ANSI & ANSI N45.2.12 & 1977 & $\begin{array}{l}\text { Requirements for Auditing of } \\
\text { Quality Assurance Programs for } \\
\text { Nuclear Power Plants }\end{array}$ & inm38703 & 1993 & $\bar{R}$ & & $\begin{array}{l}\text { ASME N45.2.12/ } \\
\text { ASME NOA-1 }\end{array}$ & $\begin{array}{l}1977 / \\
1992\end{array}$ \\
\hline 0998 & ANSI & ANSI N45.2.12 & 1977 & $\begin{array}{l}\text { Requirements for Auditing of } \\
\text { Quality Assurance Programs for } \\
\text { Nuclear Power Plants }\end{array}$ & inm40702 & 1992 & $\mathbf{R}$ & & $\begin{array}{l}\text { ASME N45.2.12/ } \\
\text { ASME NOA-1 }\end{array}$ & $\begin{array}{l}1977 / \\
1992\end{array}$ \\
\hline
\end{tabular}



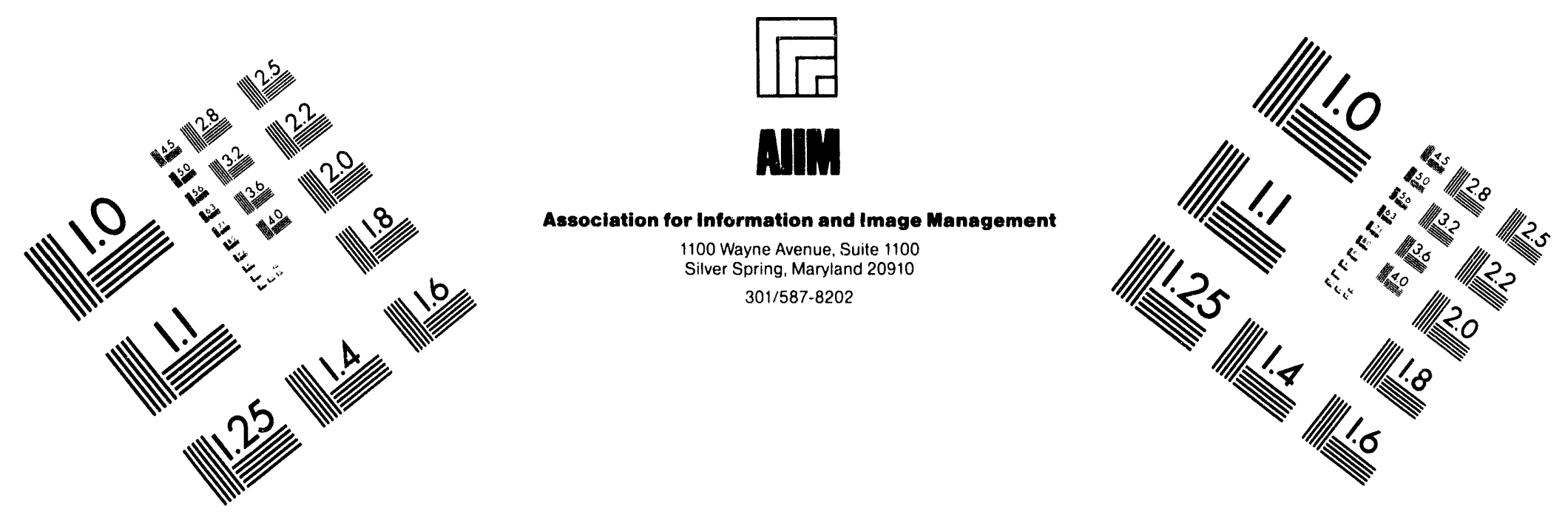

\section{Centimeter}

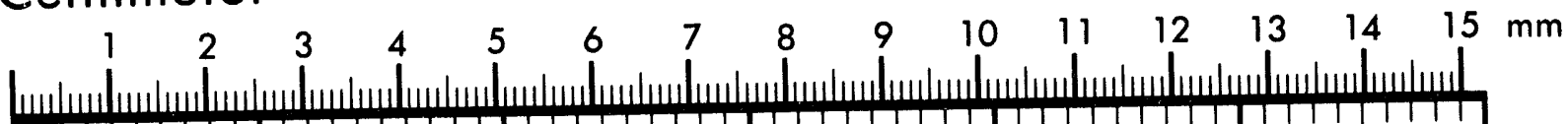

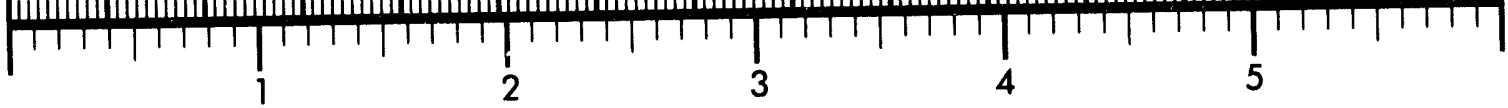
Inches

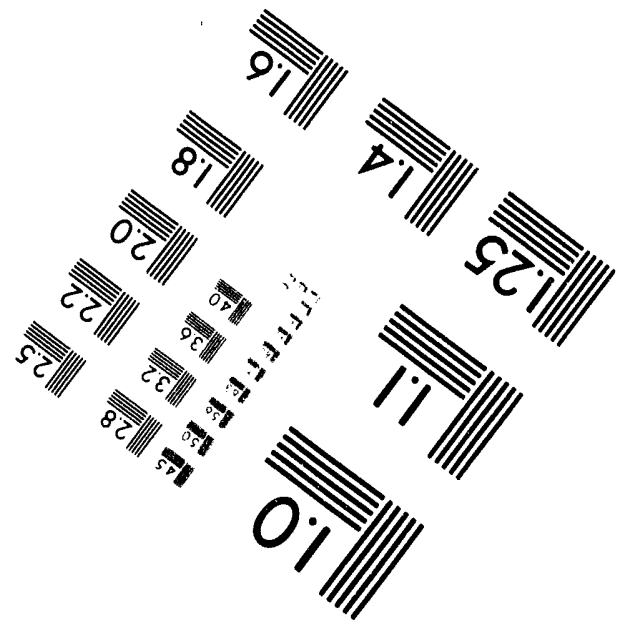

MANUFACTURED TO AIIM STANDARDS

BY APPLIED IMAGE, INC.

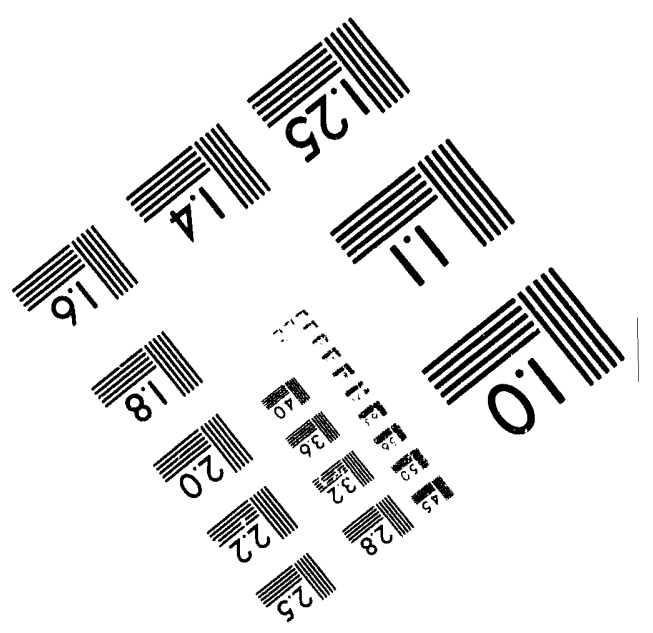



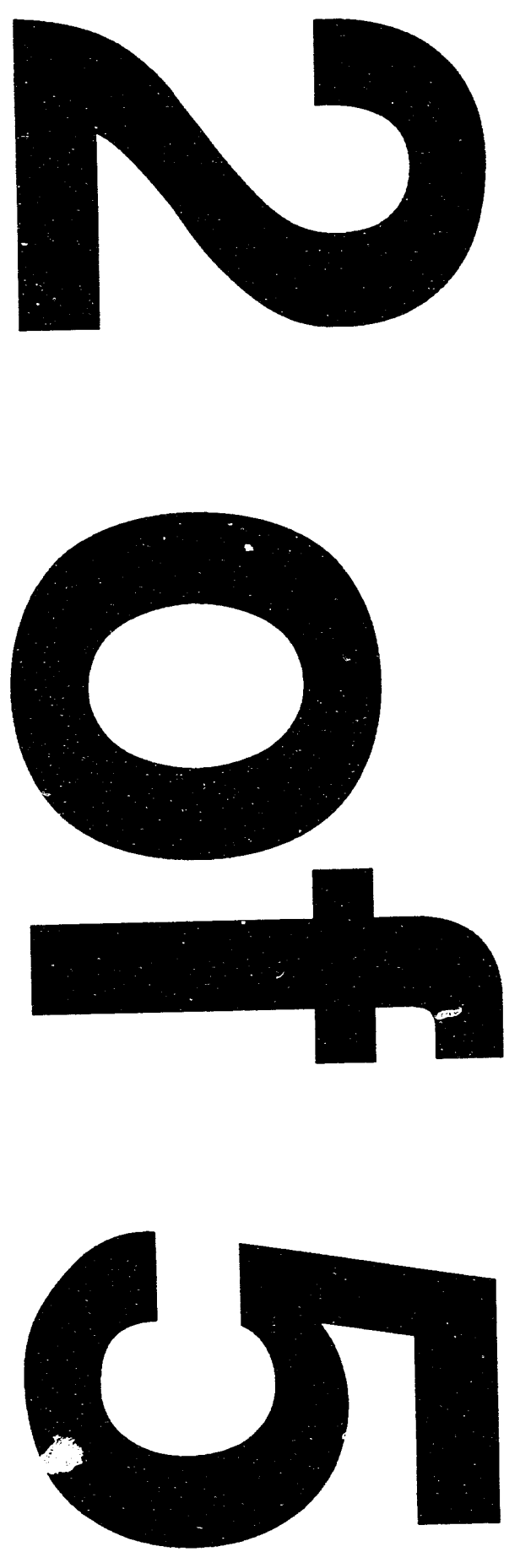
Codes and Standards and Other Guidance Cited in Regulatory Documents

Part A - Industry Consensus Codes and Standards (continued)

\begin{tabular}{|c|c|c|c|c|c|c|c|c|c|c|}
\hline Rocord & codo & Standard & stondord & , & Document & Doce & Accepts & Comnonts & Current Korrlon & vonolot \\
\hline 0999 & ANSI & ANSI N45.2.12 & 1977 & $\begin{array}{l}\text { Requirements for Auditing of } \\
\text { Quality Assurance Programs for } \\
\text { Nuclear Power Plants }\end{array}$ & inm40704 & 1992 & R & & $\begin{array}{l}\text { ASME N45.2.12/ } \\
\text { ASME NQA-1 }\end{array}$ & $\begin{array}{r}1977 / \\
1992\end{array}$ \\
\hline 1000 & ANSI & ANSI N45.2.12 & $\mathrm{N} / \mathrm{S}$ & $\begin{array}{l}\text { Requirements for Auditing of } \\
\text { Quality Assurance Programs for } \\
\text { Nuclear Power Plants }\end{array}$ & inm45051 & 1984 & $\mathbf{R}$ & & $\begin{array}{l}\text { ASME N45.2.12/ } \\
\text { ASME NQA-1 }\end{array}$ & $\begin{array}{r}1977 / \\
1992\end{array}$ \\
\hline 1001 & ANSI & ANSI N45.2.12 & $\mathrm{N} / \mathrm{S}$ & $\begin{array}{l}\text { Requirements for Auditing of } \\
\text { Quality Assurance Programs for } \\
\text { Nuclear Power Plants }\end{array}$ & inm49051 & 1982 & $\mathbf{R}$ & & $\begin{array}{l}\text { ASME N45.2.12/ } \\
\text { ASME NQA-1 }\end{array}$ & $\begin{array}{l}1977 / \\
1992\end{array}$ \\
\hline 1002 & ANSI & ANSI N45.2.12 & $\mathrm{N} / \mathrm{S}$ & $\begin{array}{l}\text { Requirements for Auditing of } \\
\text { Quality Assurance Programs for } \\
\text { Nuclear Power Plants }\end{array}$ & inm50075 & 1983 & $\mathbf{R}$ & & $\begin{array}{l}\text { ASME N45.2.12/ } \\
\text { ASME NQA-1 }\end{array}$ & $\begin{array}{r}1977 / \\
1992\end{array}$ \\
\hline 1003 & ANSI & ANSI N45.2.12 & $\mathrm{N} / \mathrm{S}$ & $\begin{array}{l}\text { Requirements for Auditing of } \\
\text { Quality Assurance Programs for } \\
\text { Nuclear Power Plants }\end{array}$ & inm51061 & 1984 & $\mathbf{R}$ & & $\begin{array}{l}\text { ASME N45.2.12/ } \\
\text { ASME NQA-1 }\end{array}$ & $\begin{array}{r}1977 / \\
1992\end{array}$ \\
\hline 1004 & ANSI & ANSI N45.2.12 & $\mathrm{N} / \mathrm{S}$ & $\begin{array}{l}\text { Requirements for Auditing of } \\
\text { Quality Assurance Programs for } \\
\text { Nuclear Power Plants }\end{array}$ & inm51065 & 1984 & $\mathbf{R}$ & & $\begin{array}{l}\text { ASME N45.2.12/ } \\
\text { ASME NQA-1 }\end{array}$ & $\begin{array}{r}1977 / \\
1992\end{array}$ \\
\hline 1005 & ANSI & ANSI N45.2.12 & $\mathrm{N} / \mathrm{S}$ & $\begin{array}{l}\text { Requirements for Auditing of } \\
\text { Quality Assurance Prograrns for } \\
\text { Nuclear Power Plants }\end{array}$ & inm52055 & 1984 & $\mathbf{R}$ & 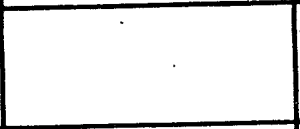 & $\begin{array}{l}\text { ASME N45.2.12/ } \\
\text { ASME NQA-1 }\end{array}$ & $\begin{array}{r}1977 / \\
1992\end{array}$ \\
\hline 1006 & ANSI & ANSI N45.2.12 & 1977 & $\begin{array}{l}\text { Requirements for Auditing of } \\
\text { Quality Assurance Programs for } \\
\text { Nuclear Power Plants }\end{array}$ & not86-21 & 1986 & $\mathbf{R}$ & $\begin{array}{l}\text { States endorsement by } \\
\text { RG } 1.44\end{array}$ & $\begin{array}{l}\text { ASf.:E N45.2.12/ } \\
\text { ASME NQA-1 }\end{array}$ & $\begin{array}{r}1977 / \\
1992\end{array}$ \\
\hline 1007 & ANSI & ANSI N45.2.12 & 1975 & $\begin{array}{l}\text { Requirements for Auditing of } \\
\text { Quality Assurance Programs for } \\
\text { Nuclear Power Plants }\end{array}$ & reg1.28.r03 & 1985 & $\mathbf{R}$ & & $\begin{array}{l}\text { ASME N45.2.12/ } \\
\text { ASME NQA-1 }\end{array}$ & $\begin{array}{r}1977 / \\
1992\end{array}$ \\
\hline 1008 & ANSI & ANSI N45.2.12 & $N / S$ & $\begin{array}{l}\text { Requiliuments for Auditing of } \\
\text { Quality Assurance Programs for } \\
\text { Nuclear Power Plants }\end{array}$ & sip17.2 & 1981 & $\mathbf{P}$ & Implied endorsement & $\begin{array}{l}\text { ASME N45.2.12/ } \\
\text { ASME NQA-1 }\end{array}$ & $\begin{array}{r}1977 / \\
1992\end{array}$ \\
\hline 1009 & ANSI & ANSI N45.2.13 & $\mathrm{N} / \mathrm{S}$ & $\begin{array}{l}\text { Quality Assurance Requirements } \\
\text { for Control of Prcsurement of Hems } \\
\text { and Services for Nuclear Power } \\
\text { Plants }\end{array}$ & drg1010.r00 & 1992 & $\mathbf{P}$ & Endorsed by reg1.123 & $\begin{array}{l}\text { ASME N45.2.13/ } \\
\text { ASME NQA-1 }\end{array}$ & $\begin{array}{r}1976 / \\
1992\end{array}$ \\
\hline
\end{tabular}


Codes and Standards and Other Guidance Cited in Regulatory Documents

Part A - Industry Consensus Codes and Standards (continued)

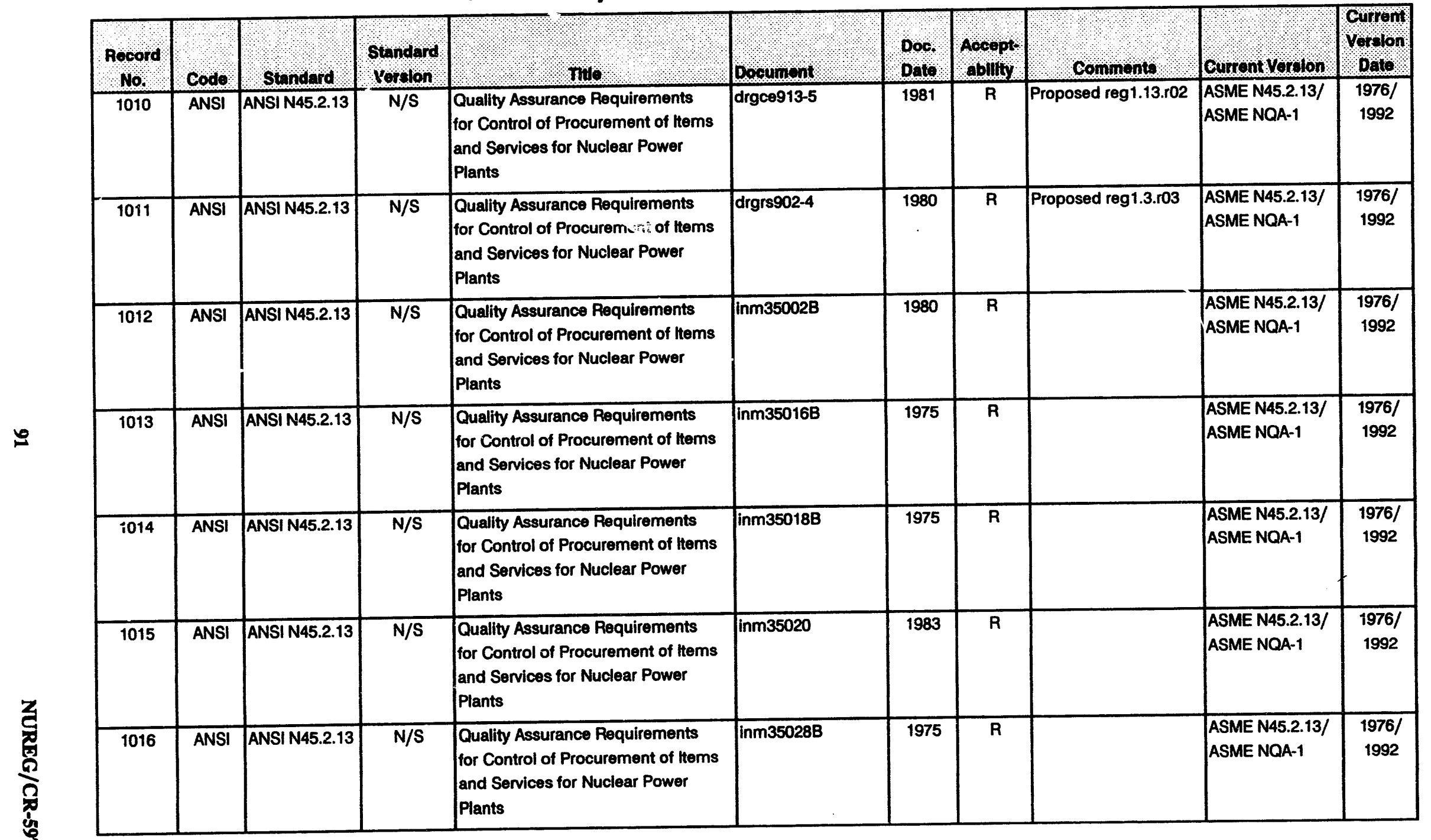


Codes and Standards and Other Guidance Cited in Regulatory Documents

Part A - Industry Consensus Codes and Standards (continued)

\begin{tabular}{|c|c|c|c|c|c|c|c|c|c|c|}
\hline $\begin{array}{l}\text { Rocord } \\
\text { No. }\end{array}$ & Code & Standard & $\begin{array}{l}\text { Standard } \\
\text { Vorjlon }\end{array}$ & $\mathrm{T}$ & Document: & $\begin{array}{l}\text { Doo. } \\
\text { Dat6. }\end{array}$ & $\begin{array}{l}\text { Accopt } \\
\text { ability }\end{array}$ & Comments & Curront Vorolon & $\begin{array}{l}\text { Current } \\
\text { Vorron } \\
\text { paro }\end{array}$ \\
\hline 1017 & ANSI & ANSI N45.2.13 & 1976 & $\begin{array}{l}\text { Quality Assurance Requirements } \\
\text { for Control of Procurement of Items } \\
\text { and Services for Nuclear Power } \\
\text { Plants }\end{array}$ & inm35060 & 1983 & $\mathbf{R}$ & & $\begin{array}{l}\text { ASME N45.2.13/ } \\
\text { ASME NQA-1 }\end{array}$ & $\begin{array}{l}1976 / \\
1992\end{array}$ \\
\hline 1018 & ANSI & ANSI N45.2.13 & $\mathrm{N} / \mathrm{S}$ & $\begin{array}{l}\text { Quality Assurance Requirements } \\
\text { for Control of Procuremient of tiems } \\
\text { and Services for Nuclear Power } \\
\text { Plants }\end{array}$ & inm35061 & 1985 & $\mathbf{R}$ & & $\begin{array}{l}\text { ASME N45.2.13/ } \\
\text { ASME NQA-1 }\end{array}$ & $\begin{array}{l}1976 / \\
1992\end{array}$ \\
\hline 1019 & ANSI & ANSI N45.2.13 & $N / S$ & $\begin{array}{l}\text { Quality Assurance Requirements } \\
\text { for Control of Procurement of ltems } \\
\text { and Services for Nuclear Power } \\
\text { Plants }\end{array}$ & inm35065 & 1983 & $\mathbf{R}$ & & $\begin{array}{l}\text { ASME N45.2.13/ } \\
\text { ASME NQA-1 }\end{array}$ & $\begin{array}{l}1976 / \\
1992\end{array}$ \\
\hline 1020 & ANSI & ANSI N45.2.13 & 1976 & $\begin{array}{l}\text { Quality Assurance Requirements } \\
\text { for Control of Procurement of ltems } \\
\text { and Services for Nuclear Power } \\
\text { Plants }\end{array}$ & inm35746B & 1979 & A & & $\begin{array}{l}\text { ASME N45.2.13/ } \\
\text { ASME NQA-1 }\end{array}$ & $\begin{array}{l}1976 / \\
1992\end{array}$ \\
\hline 1021 & ANSI & ANSI N45.2.13 & 1976 & $\begin{array}{l}\text { Quality Assurance Requirements - } \\
\text { for Control of Procurement of Items } \\
\text { and Services for Nuclear Power } \\
\text { Plants }\end{array}$ & inm35747B & 1976 & $\mathbf{R}$ & & $\begin{array}{l}\text { ASME N45.2.13/ } \\
\text { ASME NQA-1 }\end{array}$ & $\begin{array}{l}1976 / \\
1992\end{array}$ \\
\hline 1022 & ANSI & ANSI N45.2.13 & $N / S$ & $\begin{array}{l}\text { Quality Assurance Requirements } \\
\text { for Control of Procurement of ltems } \\
\text { and Services for Nuclear Power } \\
\text { Plants }\end{array}$ & inm37055 & 1983 & $\mathbf{R}$ & & $\begin{array}{l}\text { ASME N45.2.13/ } \\
\text { ASME NQA-1 }\end{array}$ & $\begin{array}{l}1976 / \\
1992\end{array}$ \\
\hline 1023 & ANSI & ANSI N45.2.13 & $N / S$ & $\begin{array}{l}\text { Quality Assurance Requirements } \\
\text { for Control of Procurement of ltems } \\
\text { and Services for Nuclear Power } \\
\text { Plants }\end{array}$ & inm38701 & 1992 & $\mathbf{R}$ & & $\begin{array}{l}\text { ASME N45.2.13/ } \\
\text { ASME NQA-1 }\end{array}$ & $\begin{array}{l}1976 / \\
1992\end{array}$ \\
\hline
\end{tabular}


Codes and Standards and Other Guidance Cited in Regulatory Documents

Part A - Industry Consensus Codes and Standards (continued)

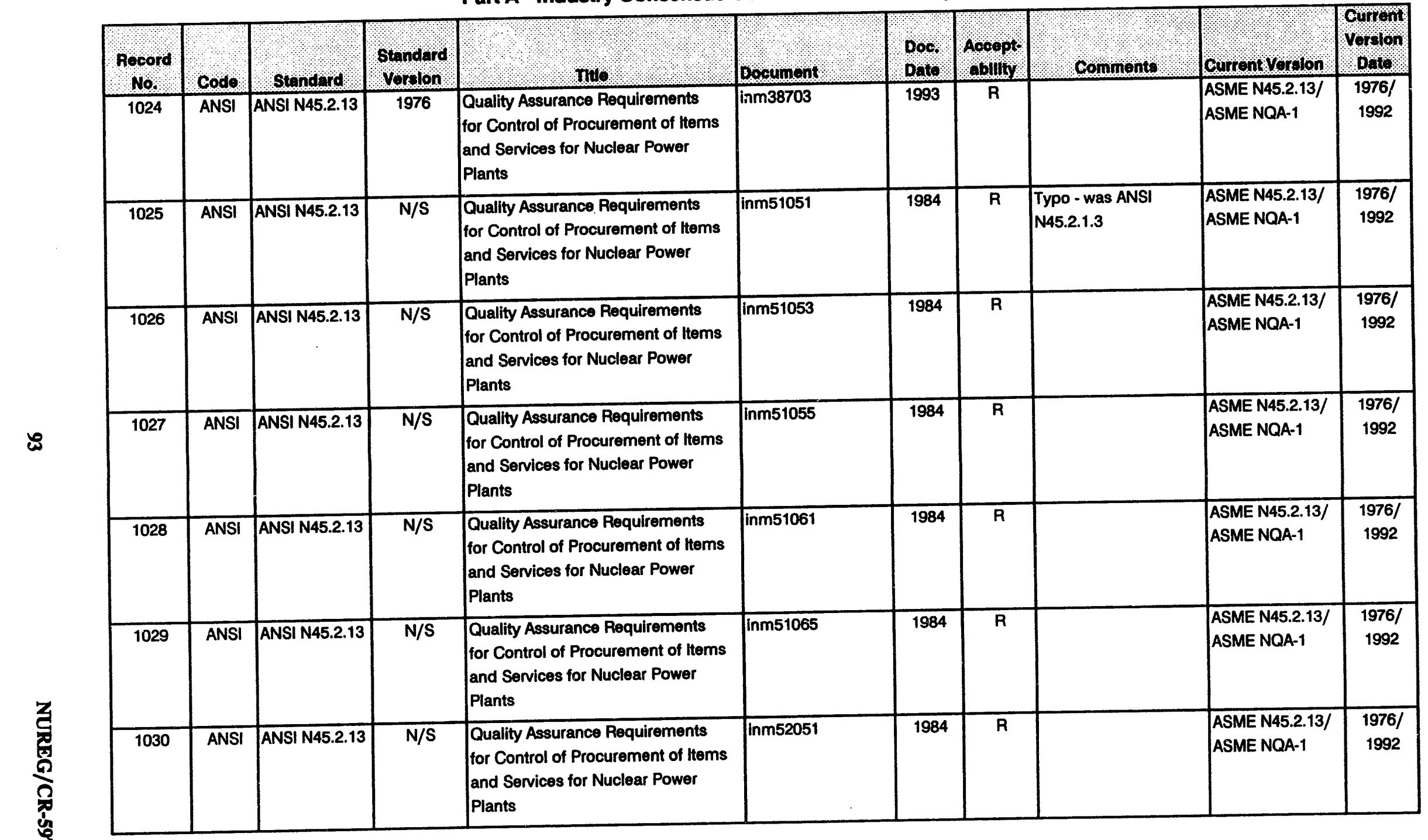




\begin{tabular}{|c|c|c|c|c|c|c|c|c|c|c|}
\hline & & & Code & $\begin{array}{l}\text { is and Standards and Other G } \\
\text { Part A - Industry Consensus }\end{array}$ & $\begin{array}{l}\text { uidance Cite } \\
\text { Codes and S }\end{array}$ & $\begin{array}{l}\text { Regula } \\
\text { ards (c }\end{array}$ & ontinue & d) & & \\
\hline Rocord: & codo & Standard & $\begin{array}{l}\text { Stindard } \\
\text { Vorsion }\end{array}$ & Tho & bocument. & Doc. & Accept: & Comments: & Curront Vorsion & Vurrsion \\
\hline 1031 & ANSI & ANSI N45.2.13 & $\mathrm{N} / \mathrm{S}$ & $\begin{array}{l}\text { Quality Assurance Requirements } \\
\text { for Control of Procurement of thems } \\
\text { and Services for Nuclear Power } \\
\text { Plants }\end{array}$ & inm52055 & 1984 & $R$ & & $\begin{array}{l}\text { ASME N45.2.13/ } \\
\text { ASME NQA-1 }\end{array}$ & $\begin{array}{l}1976 / \\
1992\end{array}$ \\
\hline 1032 & ANSI & ANSI N45.2.13 & 1976 & $\begin{array}{l}\text { Quality Assurance Requirements } \\
\text { for Control of Procurement of ttems } \\
\text { and Services for Nuclear Power } \\
\text { Plants }\end{array}$ & inm9900/BVII & 1980 & $\mathbf{R}$ & & $\begin{array}{l}\text { ASME N45.2.13/ } \\
\text { ASME NQA-1 }\end{array}$ & $\begin{array}{l}1976 / \\
1992\end{array}$ \\
\hline 1033 & ANSI & ANSI N45.2.13 & 1976 & $\begin{array}{l}\text { Quality Assurance Requirements } \\
\text { for Control of Procurement of thems } \\
\text { and Services for Nuclear Power } \\
\text { Plants }\end{array}$ & reg1.142.ro1 & 1981 & $\mathbf{R}$ & & $\begin{array}{l}\text { ASME N45.2.13/ } \\
\text { ASME NQA-1 }\end{array}$ & $\begin{array}{l}1976 / \\
1992\end{array}$ \\
\hline 1034 & ANSI & ANSI N45.2.13 & 1975 & $\begin{array}{l}\text { Quality Assurance Requirements } \\
\text { for Control of Procurement of thems } \\
\text { and Services for Nuclear Power } \\
\text { Plants }\end{array}$ & reg1.28.ro3 & 1985 & $\mathbf{R}$ & & $\begin{array}{l}\text { ASME N45.2.13/ } \\
\text { ASME NQA-1 }\end{array}$ & $\begin{array}{l}1976 / \\
1992\end{array}$ \\
\hline 1035 & ANSI & ANSI N45.2.13 & $\mathrm{N} / \mathrm{S}$ & $\begin{array}{l}\text { Quality Assurance Requirements } \\
\text { for Control of Procurement of tems } \\
\text { and Services for Nuclear Power } \\
\text { Plants }\end{array}$ & reg1.33.ro2 & 1978 & $\mathbf{R}$ & & $\begin{array}{l}\text { ASME N45.2.13/ } \\
\text { ASME NQA-1 }\end{array}$ & $\begin{array}{l}1976 / \\
1992\end{array}$ \\
\hline 1036 & ANSI & ANSI N45.2.23 & $\mathrm{N} / \mathrm{S}$ & $\begin{array}{l}\text { Qualification of Quality Assurance } \\
\text { Program Audit Personnel for } \\
\text { Nuclear Power Plants }\end{array}$ & drg1010.r00 & 1992 & $\mathbf{P}$ & Endorsed by reg1.146 & $\begin{array}{l}\text { ASME N45.2.23/ } \\
\text { ASME NQA-1 }\end{array}$ & $\begin{array}{l}1978 / \\
1992\end{array}$ \\
\hline 1037 & ANS! & ANSI N45.2.23 & $\mathrm{N} / \mathrm{S}$ & $\begin{array}{l}\text { Qualification of Quality Assurance } \\
\text { Program Audit Personnel for } \\
\text { Nuclear Power Plants }\end{array}$ & glt82-020 & 1982 & $\mathbf{P}$ & Implied endorsement & $\begin{array}{l}\text { ASME N45.2.23/ } \\
\text { ASME NQA-1 }\end{array}$ & $\begin{array}{l}1978 / \\
1992\end{array}$ \\
\hline 1038 & ANSI & ANSI N45.2.23 & $N / S$ & $\begin{array}{l}\text { Qualification of Quality Assurance } \\
\text { Program Audit Personnel for } \\
\text { Nuclear Power Plants }\end{array}$ & glt82-021 & 1982 & $\bar{P}$ & & $\begin{array}{l}\text { ASME N45.2.23/ } \\
\text { ASME NQA-1 }\end{array}$ & $\begin{array}{l}1978 / \\
1992\end{array}$ \\
\hline
\end{tabular}


Codes and Standards and Other Guidance Cited in Regulatory Documents

Part A - Industry Consensus Codes and Standards (continued)

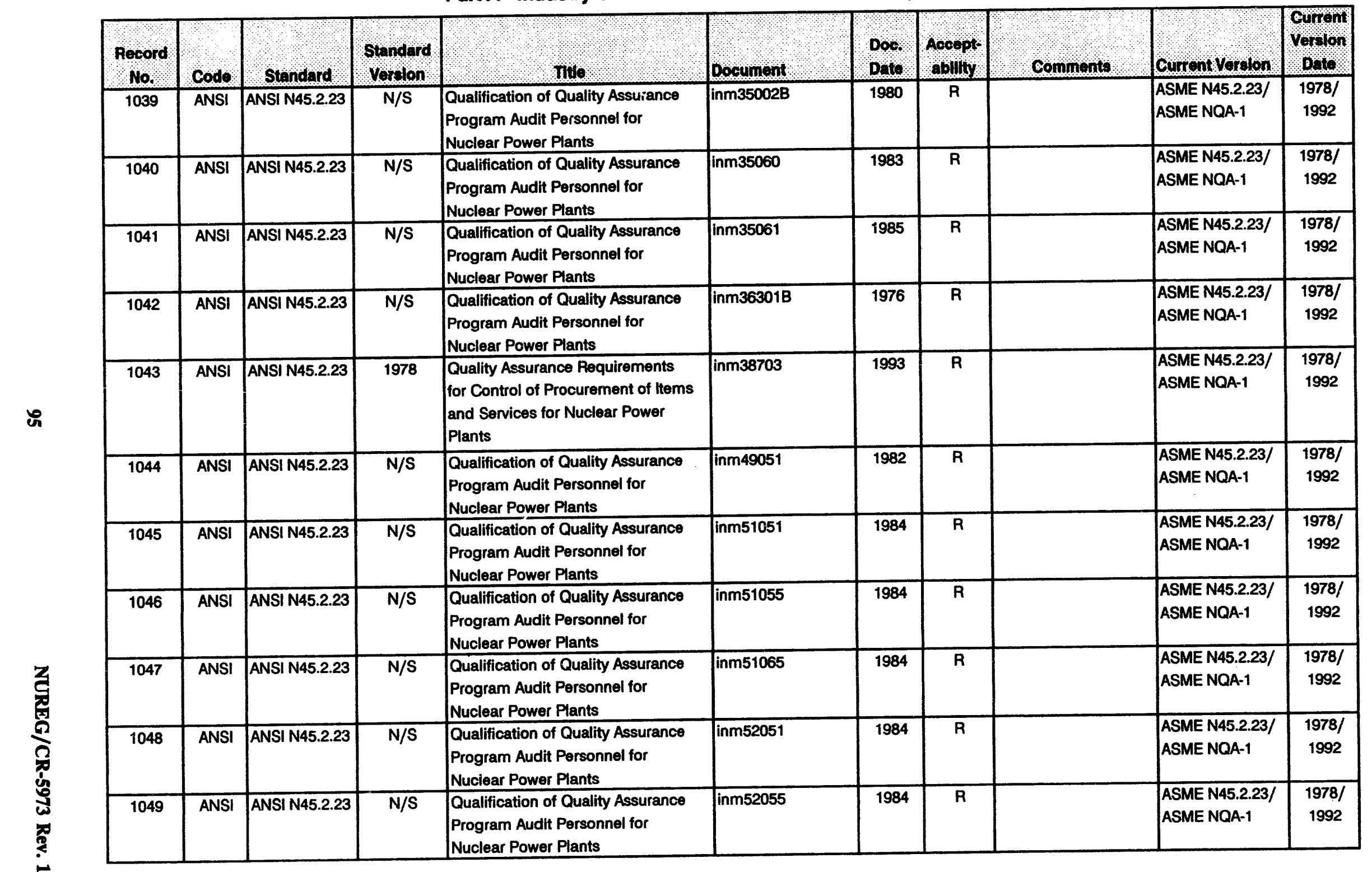


Codes and Standards and Other Guidance Cited in Regulatory Documents

Part A - Industry Consensus Codes and Standards (continued)

\begin{tabular}{|c|c|c|c|c|c|c|c|c|c|c|}
\hline $\begin{array}{l}\text { Aecord } \\
\text { No. }\end{array}$ & Codo & standhord & Sthrondard & (1) & Bocument & $\begin{array}{l}\text { Dor } \\
\text { pato }\end{array}$ & Acospl & Commonts & Curront Vordon & Qupons \\
\hline 1050 & ANSI & ANSI N45.2.23 & 1975 & $\begin{array}{l}\text { Qualification of Quality Assurance } \\
\text { Program Audit Personnel for } \\
\text { Nuclear Power Plants }\end{array}$ & reg1.28.r03 & 1985 & $\mathbf{R}$ & & $\begin{array}{l}\text { ASME N45.2.23/ } \\
\text { ASME NQA-1 }\end{array}$ & $\begin{array}{l}1978 / \\
1992\end{array}$ \\
\hline 1051 & ANSI & ANSI N45.4 & 1972 & $\begin{array}{l}\text { Containment System Leakage } \\
\text { Testing Requirements }\end{array}$ & 10 cfr50 App. J & 1993 & A & $\begin{array}{l}\text { In conjunction with } \\
\text { ANS } 56.8\end{array}$ & ANS 56.8 & 1987 \\
\hline 1052 & ANSI & ANSI N45.4 & 1972 & $\begin{array}{l}\text { Containment System Leakage } \\
\text { Testing Requirements }\end{array}$ & drgms21-5 & 1986 & $\mathbf{R}$ & & ANS 56.8 & 1987 \\
\hline 1053 & ANSI & ANSI N45.4 & 1972 & $\begin{array}{l}\text { Containment System Leakage } \\
\text { Testing Requirements }\end{array}$ & inm61720 & 1985 & $\mathbf{R}$ & & ANS 56.8 & 1987 \\
\hline 1054 & ANSI & ANSI N45.4 & 1972 & $\begin{array}{l}\text { Containment System Leakage } \\
\text { Testing Requirements }\end{array}$ & inm70307 & 1986 & $\mathbf{R}$ & & ANS 56.8 & 1987 \\
\hline 1055 & ANSI & ANSI N45.4 & 1972 & $\begin{array}{l}\text { Containment System Leakage } \\
\text { Testing Requirements } \\
\end{array}$ & inm70313 & 1985 & $\mathbf{R}$ & & ANS 56.8 & 1987 \\
\hline 1056 & ANSI & ANSI N45.4 & 1972 & $\begin{array}{l}\text { Containment System Leakage } \\
\text { Testing Requirements }\end{array}$ & inm70323 & 1985 & $\mathbf{R}$ & & ANS 56.8 & 1987 \\
\hline 1057 & ANSI & ANSI N45.4 & 1972 & $\begin{array}{l}\text { Containment System Leakage } \\
\text { Testing Requirements } \\
\end{array}$ & not85-71 & 1985 & $\mathbf{R}$ & Endorsed by 10CFR.J & ANS 56.8 & 1987 \\
\hline 1058 & ANSI & ANSI N46.2 & 1978 & $\begin{array}{l}\text { Quality Assurance Program } \\
\text { Requirements for Post Reactor } \\
\text { Nuclear Fuel Cycle Facilities }\end{array}$ & drg1010.r00 & 1992 & $\bar{R}$ & & AICHE A27 & 1978 \\
\hline 1059 & ANSI & ANSI N46.2 & 1978 & $\begin{array}{l}\text { Quality Assurance Program } \\
\text { Requirements for Post Reactor } \\
\text { Nuclear Fuel Cycle Facilities }\end{array}$ & reg1.28.ro3 & 1985 & $\mathbf{R}$ & & AICHE A27 & 1978 \\
\hline 1060 & ANSI & ANSI N55.1 & 1979 & $\begin{array}{l}\text { Solid Radioactive Waste Processing } \\
\text { System for Light-Water-Cooled } \\
\text { Reactor Plants }\end{array}$ & inm84522 & 1984 & $\bar{R}$ & & ANS 55.1 & 1992 \\
\hline 1061 & ANSI & ANSI N101.1 & 1972 & $\begin{array}{l}\text { Nuclear Power Plant Air-Cleaning } \\
\text { Units and Components }\end{array}$ & reg3.2.r00 & 1973 & $\bar{A}$ & & ASME N509 & 1989 \\
\hline 1062 & ANSI & ANSI N101.2 & 1972 & $\begin{array}{l}\text { Protective Coatings (Paints) for } \\
\text { Light Water Nuclear Reactor } \\
\text { Containment Facilities }\end{array}$ & drgop618-4 & 1982 & $\mathbf{R}$ & $\begin{array}{l}\text { Proposed reg8.8.104 - } \\
\text { ANSI N101.2 } \\
\text { withdrawn } \\
\end{array}$ & $n / a$ & $n / a$ \\
\hline 1063 & ANSI & ANSI N101.2 & 1972 & $\begin{array}{l}\text { Protective Coatings (Paints) for } \\
\text { Light Water Nuclear Reactor } \\
\text { Containment Facilities }\end{array}$ & reg8.8.r03 & 1978 & $\mathbf{R}$ & $\begin{array}{l}\text { ANSI N101.2 } \\
\text { withdrawn }\end{array}$ & Withdrawn & $n / a$ \\
\hline
\end{tabular}


Codes and Standards and Other Guidance Clted in Regulatory Documents

Part A - Industry Consensus Cocies and Standards (continued)

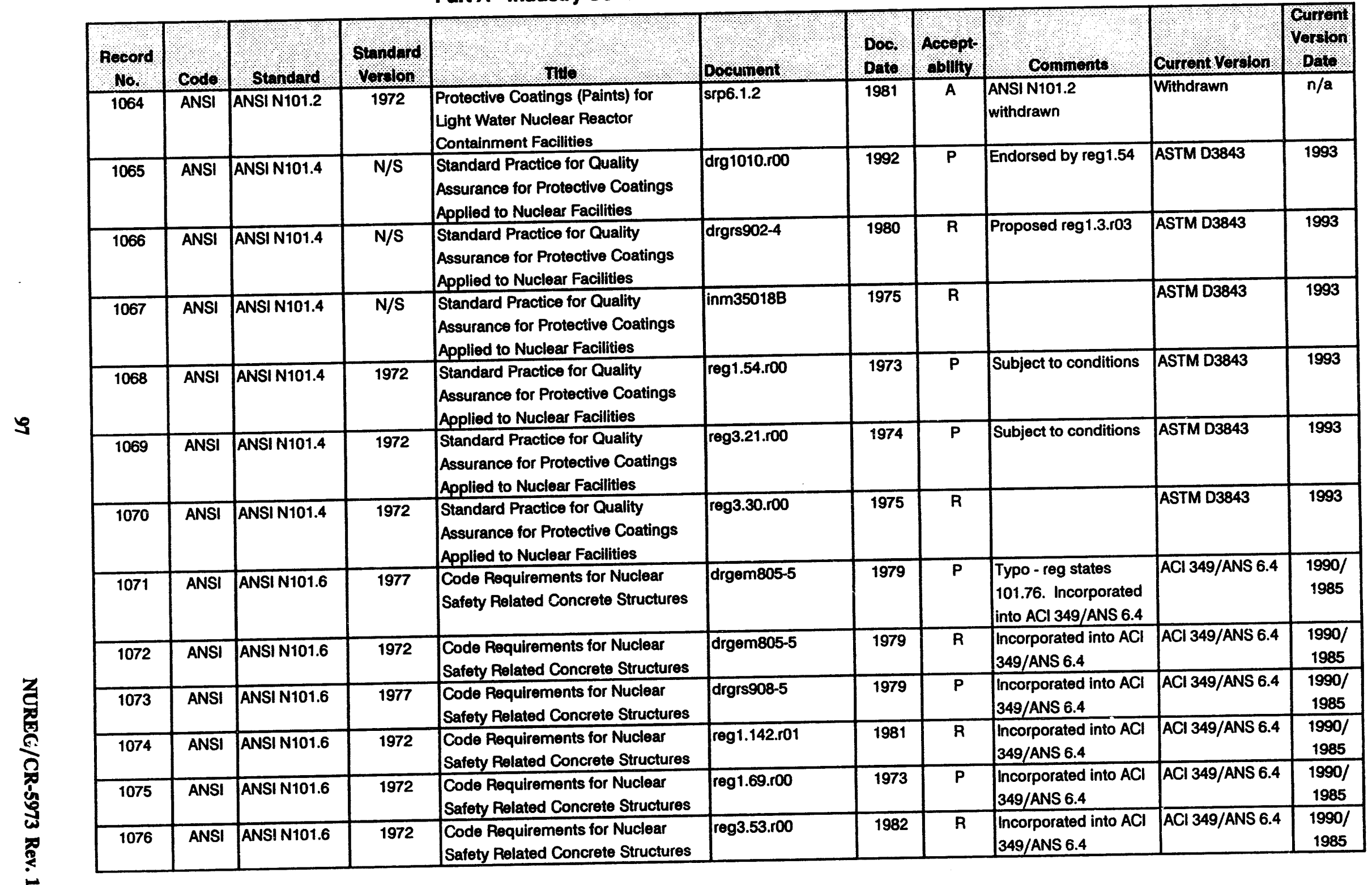


Codes and Standards and Other Guidance Cited in Regulatory Documents

Part A - Industry Consensus Codes and Standards (continued)

\begin{tabular}{|c|c|c|c|c|c|c|c|c|c|c|}
\hline $\begin{array}{l}\text { Pecord } \\
\text { No. }\end{array}$ & Cods & Standard & $\begin{array}{l}\text { Standard } \\
\text { Verslon }\end{array}$ & ( & Bocument & $\begin{array}{l}\text { Doc. } \\
\text { Date }\end{array}$ & $\begin{array}{l}\text { Accept } \\
\text { ebilty }\end{array}$ & Commonts, & Current Vorrion & $\begin{array}{l}\text { Ourrent } \\
\text { verslon } \\
\text { bale }\end{array}$ \\
\hline 1077 & ANSI & ANSI N101.6 & 1972 & $\begin{array}{l}\text { Code Requirements for Nuclear } \\
\text { Safety Related Concrete Structures }\end{array}$ & reg3.9.100 & 1973 & $\mathbf{P}$ & $\begin{array}{l}\text { Incorporated into ACI } \\
\text { 349/ANS } 6.4\end{array}$ & ACl 349/ANS 6.4 & $\begin{array}{r}1990 / \\
1985 \\
\end{array}$ \\
\hline 1078 & ANSI & ANSI N101.6 & 1972 & $\begin{array}{l}\text { Code Requirements for Nuclear } \\
\text { Safety Related Concrete Structures }\end{array}$ & $\operatorname{srp} 12.3$ & 1981 & $\mathbf{P}$ & $\begin{array}{l}\text { Incorporated into ACI } \\
\text { 349/ANS } 6.4\end{array}$ & ACl 349/ANS 6.4 & $\begin{array}{r}1990 / \\
1985 \\
\end{array}$ \\
\hline 1079 & ANSI & ANSI N170 & 1976 & $\begin{array}{l}\text { Determining Design Basis Flooding } \\
\text { at Power Reactor Sites }\end{array}$ & nureg0933, 3.103 & 1989 & $\mathbf{R}$ & & ANS 2.8 & 1992 \\
\hline 1080 & ANSI & ANSI N170 & 1976 & $\begin{array}{l}\text { Determining Design Basis Flooding } \\
\text { at Power Reactor Sites }\end{array}$ & reg1.59.r02 & 1977 & $\vdash$ & $\begin{array}{l}\text { One exception in App. } \\
\text { A of Reg. Guide } 1.59\end{array}$ & ANS 2.8 & 1992 \\
\hline 1081 & ANSI & ANSI N170 & 1976 & $\begin{array}{l}\text { Determining Design Basis Flooding } \\
\text { at Power Reactor Sites }\end{array}$ & reg3.40.ro1 & 1977 & $\mathbf{P}$ & Subject to conditions & ANS 2.8 & 1692 \\
\hline 1082 & ANSI & ANSI N170 & $N / S$ & $\begin{array}{l}\text { Determining Design Basis Flooding } \\
\text { at Power Reactor Sites }\end{array}$ & $\operatorname{sip} 2.4 .10$ & 1981 & $\mathbf{R}$ & & ANS 2.8 & 1992 \\
\hline 1083 & ANSI & ANSI N170 & 1976 & $\begin{array}{l}\text { Determining Design Basis Flooding } \\
\text { at Power Reactor Sites }\end{array}$ & srp2.4.14 & 1981 & $\mathbf{R}$ & & ANS 2.8 & 1992 \\
\hline 1084 & ANSI & ANSI N170 & $\mathrm{N} / \mathrm{S}$ & $\begin{array}{l}\text { Determining Design Basis Flooding } \\
\text { at Power Reactor Sites }\end{array}$ & srp2.4.2 & 1989 & $\mathbf{R}$ & & ANS 2.8 & 1992 \\
\hline 1085 & ANSI & ANSI N170 & N/S & $\begin{array}{l}\text { Determining Design Basis Flooding } \\
\text { at Power Reactor Sites }\end{array}$ & srp2.4.3 & 1989 & $R$ & & ANS 2.8 & 1992 \\
\hline 1086 & ANSI & ANSI N170 & $N / S$ & $\begin{array}{l}\text { Determining Design Basis Flooding } \\
\text { at Power Reactor Sites }\end{array}$ & $\operatorname{srp2.4.4}$ & 1981 & $\mathbf{R}$ & & ANS 2.8 & 1992 \\
\hline 1087 & ANSI & ANSI N170 & 1976 & $\begin{array}{l}\text { Determining Design Basis Flooding } \\
\text { at Power Reactor Sites }\end{array}$ & srp2.4.5 & 1981 & $\mathbf{R}$ & & ANS 2.8 & 1992 \\
\hline 1088 & ANSI & ANSI N170 & $\mathrm{N} / \mathrm{S}$ & $\begin{array}{l}\text { Determining Design Basis Flooding } \\
\text { at Power Reactor Sites }\end{array}$ & srp2.4.8 & 1981 & $\mathbf{R}$ & & ANS 2.8 & 1992 \\
\hline 1089 & ANSI & ANSI N170 & 1976 & $\begin{array}{l}\text { Determining Design Basis Flooding } \\
\text { at Power Reactor Sites }\end{array}$ & srp2.4.7 & 1981 & $\mathbf{R}$ & & ANS 2.8 & 1992 \\
\hline 1090 & ANSI & ANSI N195 & $N / S$ & $\begin{array}{l}\text { Fuel Oil Systems for Emergency } \\
\text { Diesel Generators }\end{array}$ & glt83-026 & 1983 & $\mathbf{R}$ & & ANS 59.51 & 1989 \\
\hline 1091 & ANSI & ANSI N195 & 1976 & $\begin{array}{l}\text { Fuel Oil Systems for Emergency } \\
\text { Diesel Generators }\end{array}$ & reg1.137.501 & 1979 & $\mathbf{P}$ & & ANS 59.51 & 1989 \\
\hline 1092 & ANSI & ANSI N195 & $N / S$ & $\begin{array}{l}\text { Fuel Oil Systems for Emergency } \\
\text { Diesel Generators }\end{array}$ & srp9.5.4 & 1981 & $\mathbf{P}$ & & ANS 59.51 & 1989 \\
\hline 1093 & ANSI & ANSI N195 & 1976 & $\begin{array}{l}\text { Fuel Oll Systems for Emergency } \\
\text { Diesel Generators }\end{array}$ & sts4-geB3.8.3 & 1991 & $\mathbf{P}$ & & ANS 59.51 & 1989 \\
\hline
\end{tabular}


Codes and Standards and Other Guidance Cited in Regulatory Documents

Part A - Industry Consensus Codes and Standards (continued)

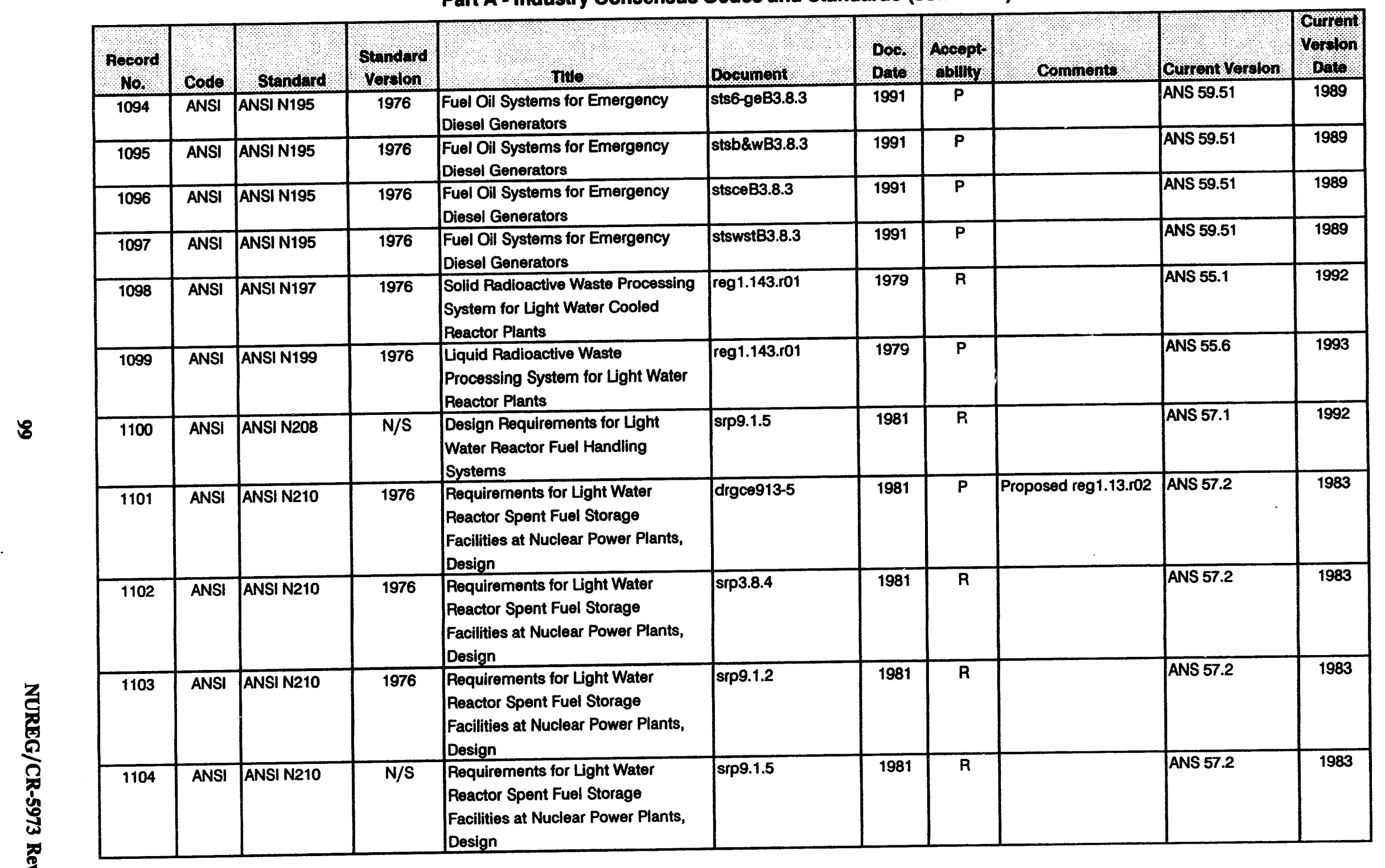




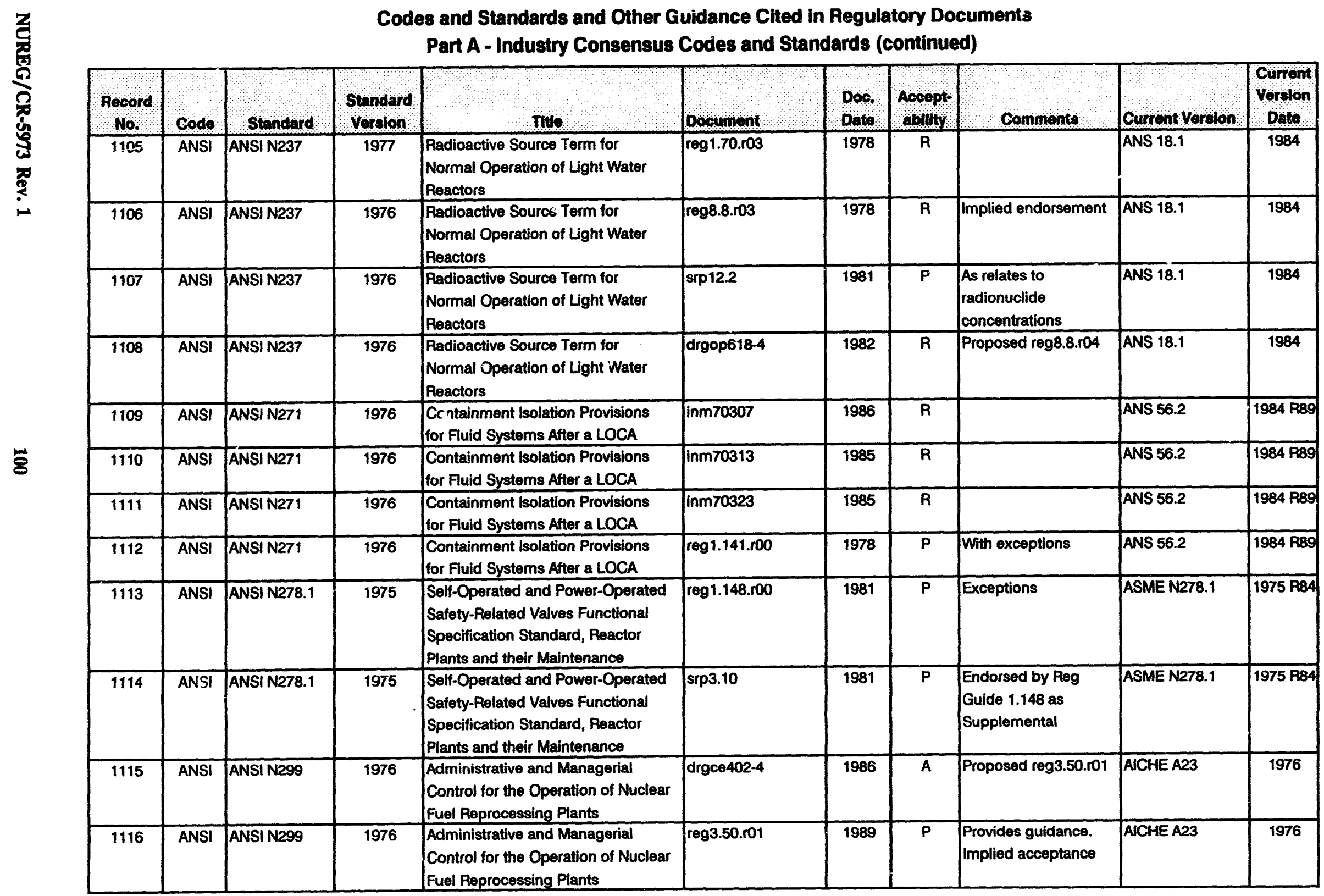


Codes and Standards and Other Guidance Cited in Regulatory Documents

Part A - Industry Consensus Codes and Standards (continued)

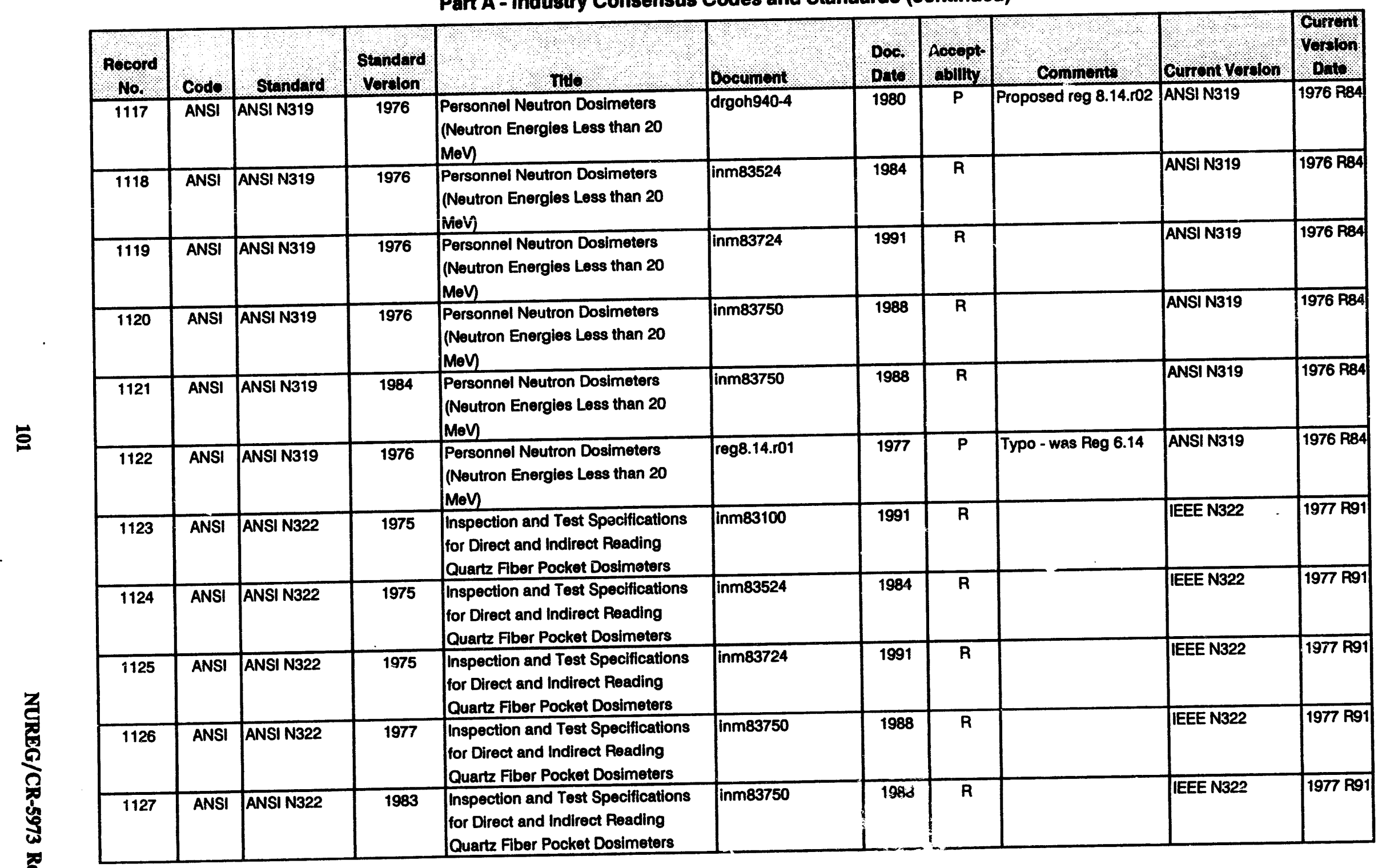




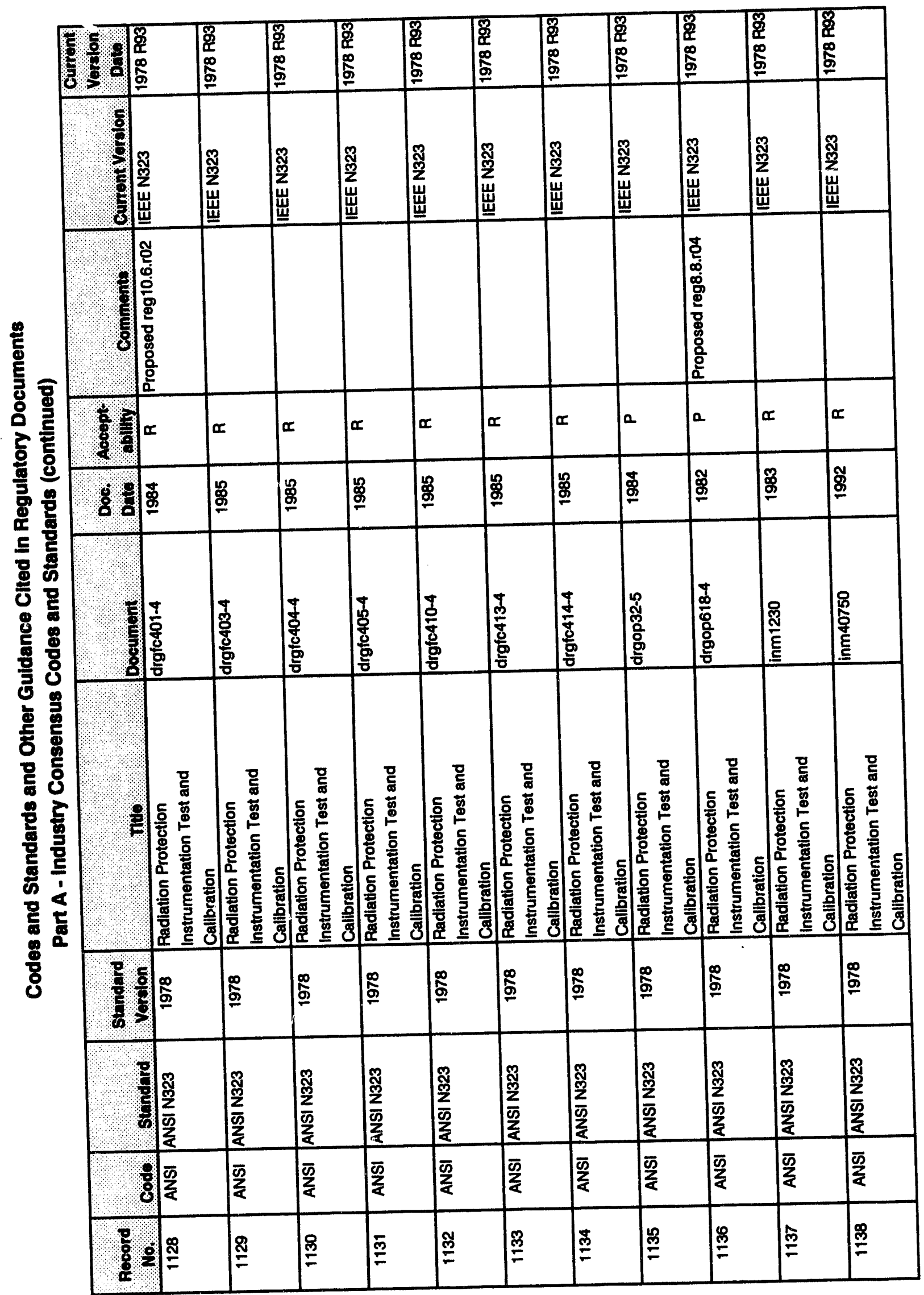


Codes and Standards and Other Guidance Cited in Regulatory Documents

Part A - Industry Consensus Codes and Standards (continued)

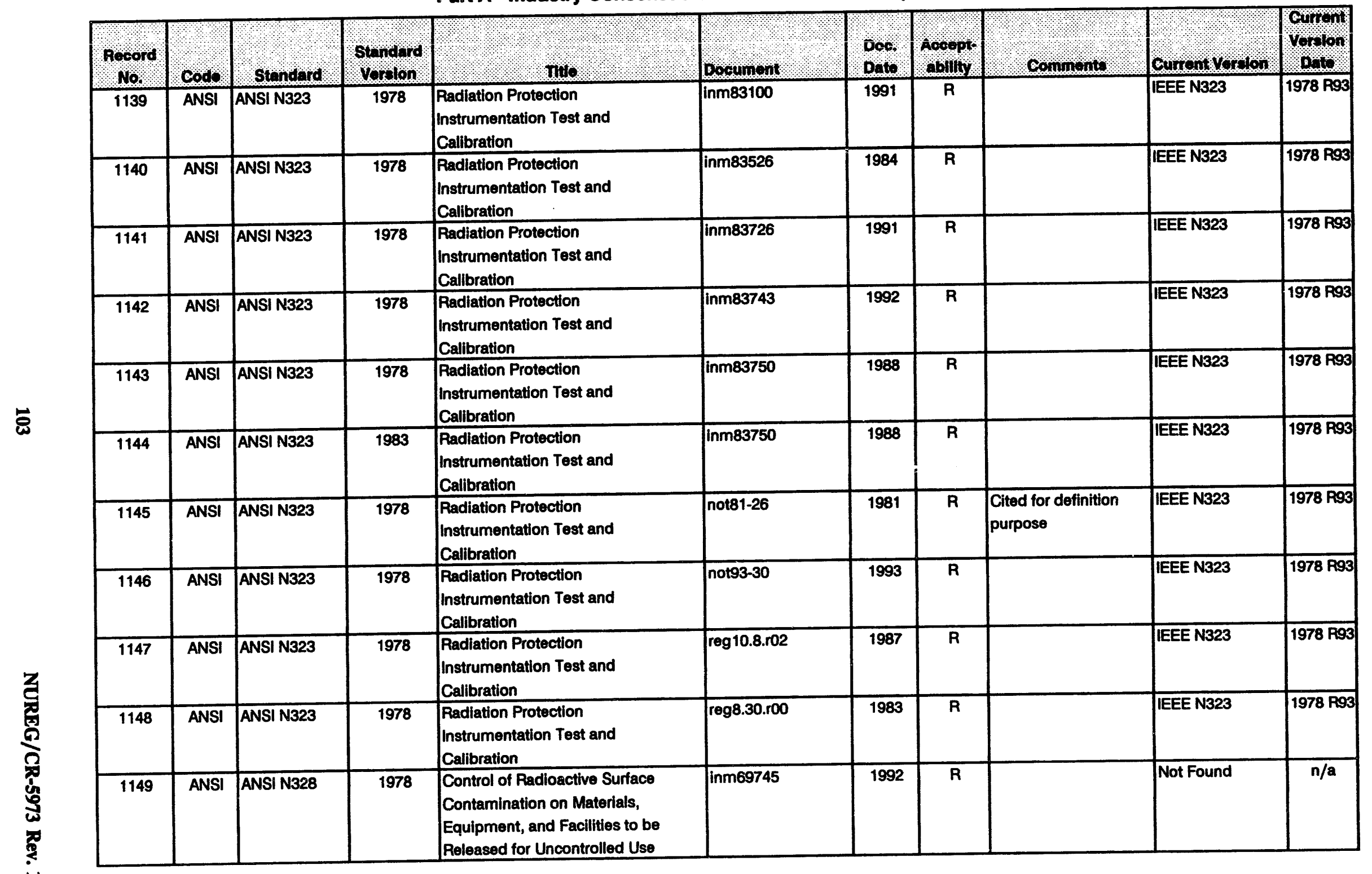


Codes and Standards and Other Guidance Cited in Regulatory Documents

Part A - Industry Consensus Codes and Standards (Continued)

Tेong

\begin{tabular}{|c|c|c|c|c|c|c|c|c|c|c|}
\hline $\begin{array}{l}\text { Rocord } \\
\text { No. }\end{array}$ & cods & Standard & strondard & ४ & Dosunont. & $\begin{array}{l}\text { Doo. } \\
\text { Deto. }\end{array}$ & $\begin{array}{l}\text { Acoept } \\
\text { aloulty }\end{array}$ & Connents & Current Voralon & vorpols \\
\hline 1150 & ANSI & ANSI N343 & 1978 & $\begin{array}{l}\text { Internal Dosimetry for Mixed Fission } \\
\text { and Activation Products }\end{array}$ & inm83100 & 1991 & $\mathbf{R}$ & & ANSI N343 & 1978 R84 \\
\hline 1151 & ANSI & ANSI N343 & 1978 & $\begin{array}{l}\text { Internal Dosimetry for Mixed Fission } \\
\text { and Activation Products }\end{array}$ & inm83525 & 1984 & $\mathbf{R}$ & & ANSI N343 & 1978 R84 \\
\hline 1152 & ANSI & ANSI N343 & 1978 & $\begin{array}{l}\text { Internal Dosimetry for Mixed Fission } \\
\text { and Activation Products }\end{array}$ & inm83725 & 1991 & $\mathbf{R}$ & & ANSI N343 & 1978 R84 \\
\hline 1153 & ANSI & ANSI N343 & 1978 & $\begin{array}{l}\text { Internal Dosimetry for Mixed Fission } \\
\text { and Activation Products }\end{array}$ & inm83750 & 1988 & $\mathbf{R}$ & & ANSI N343 & $1978 \mathrm{RB4}$ \\
\hline 1154 & ANSI & ANSI N343 & 1984 & $\begin{array}{l}\text { Internal Dosimetry for Mixed Fission } \\
\text { and Activation Products }\end{array}$ & inm83750 & 1988 & $\mathbf{R}$ & & ANSI N343 & 1978 R84 \\
\hline 1155 & ANSI & ANSI N343 & 1978 & $\begin{array}{l}\text { Internal Dosimetry for Mixed Fission } \\
\text { and Activation Products }\end{array}$ & reg3.53.r00 & 1982 & $\mathbf{R}$ & $\begin{array}{l}\text { Endorsed by Reg. } \\
\text { Guide } 8.26\end{array}$ & ANSI N343 & 1978 R84 \\
\hline 1156 & ANSI & ANSI N343 & 1978 & $\begin{array}{l}\text { Internal Dosimetry for Mixed Fission } \\
\text { and Activation Products }\end{array}$ & reg8.26.r00 & 1980 & $\mathbf{P}$ & With exceptions & ANSI N343 & $1978 \mathrm{RBA}$ \\
\hline 1157 & ANSI & ANSI N398 & $N / S$ & $\begin{array}{l}\text { Quality Control for Plate-Type } \\
\text { Uranium-Aluminum Fuel Elements }\end{array}$ & inm60745B & 1992 & $\mathbf{R}$ & & ANS 15.2 & 1990 \\
\hline 1158 & ANSI & ANSI N398 & 1974 & $\begin{array}{l}\text { Quality Control for Plate-Type } \\
\text { Uranium-Aluminum Fuel Elements }\end{array}$ & reg2.3.r01 & 1976 & $\mathbf{P}$ & Subject to conditions & ANS 15.2 & 1990 \\
\hline 1159 & ANSI & ANSI N401 & 1974 & $\begin{array}{l}\text { Development of Technical } \\
\text { Specifications for Research } \\
\text { Reactors }\end{array}$ & reg2.4.100 & 1977 & $\mathbf{P}$ & $\begin{array}{l}\text { ANS } 15.6 \text { - withdrawn } \\
1983 \text { - incorporated } \\
\text { into ANS } 15.1 \\
\end{array}$ & ANS 15.1 & 1990 \\
\hline 1160 & ANSI & ANSI N402 & $\mathrm{N} / \mathrm{S}$ & $\begin{array}{l}\text { Quality Assurance Program } \\
\text { Requirements for Research } \\
\text { Reactors }\end{array}$ & reg2.3.ro1 & 1976 & $\mathbf{R}$ & & ANS 15.8 & $1976 \mathrm{RB7}$ \\
\hline 1161 & ANSI & ANSI N402 & 1976 & $\begin{array}{l}\text { Quality Assurance Program } \\
\text { Requirements for Research } \\
\text { Reactors }\end{array}$ & reg2.4.rOR & 1977 & A & $\begin{array}{l}\text { Acceptable means of } \\
\text { complying with } \\
10 \text { CFR50.34 } \\
\end{array}$ & ANS 15.8 & $1976 \mathrm{RB7}$ \\
\hline 1162 & ANSI & ANSI N403 & 1977 & $\begin{array}{l}\text { Guidelines on the Nuclear Analysis } \\
\text { and Design of Concrete Radiation } \\
\text { Shielding for Nuclear Power Plants }\end{array}$ & drgem805-5 & 1979 & $\mathbf{P}$ & & ANS 6.4 & 1985 \\
\hline 1163 & ANSI & ANSI N413 & 1974 & $\begin{array}{l}\text { Guidelines for the Documentation } \\
\text { of Digital Computer Programs }\end{array}$ & reg4.15.r01 & 1979 & $\mathbf{R}$ & & ANS 10.3 & 1986 \\
\hline
\end{tabular}


Codes and Standards and Other Guidance Cited in Regulatory Documents

Part A - Industry Consensus Codes and Standards (continue J)

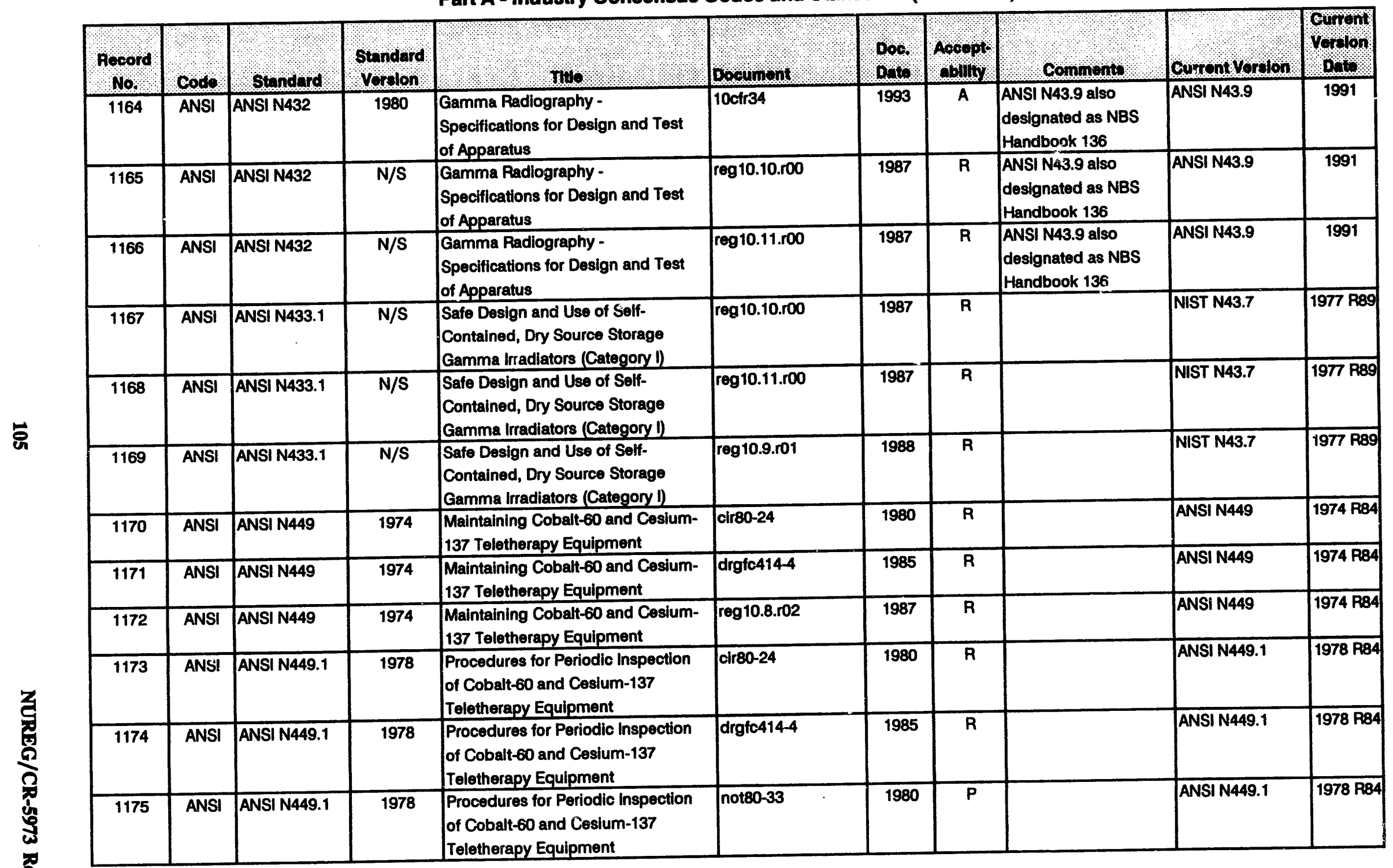




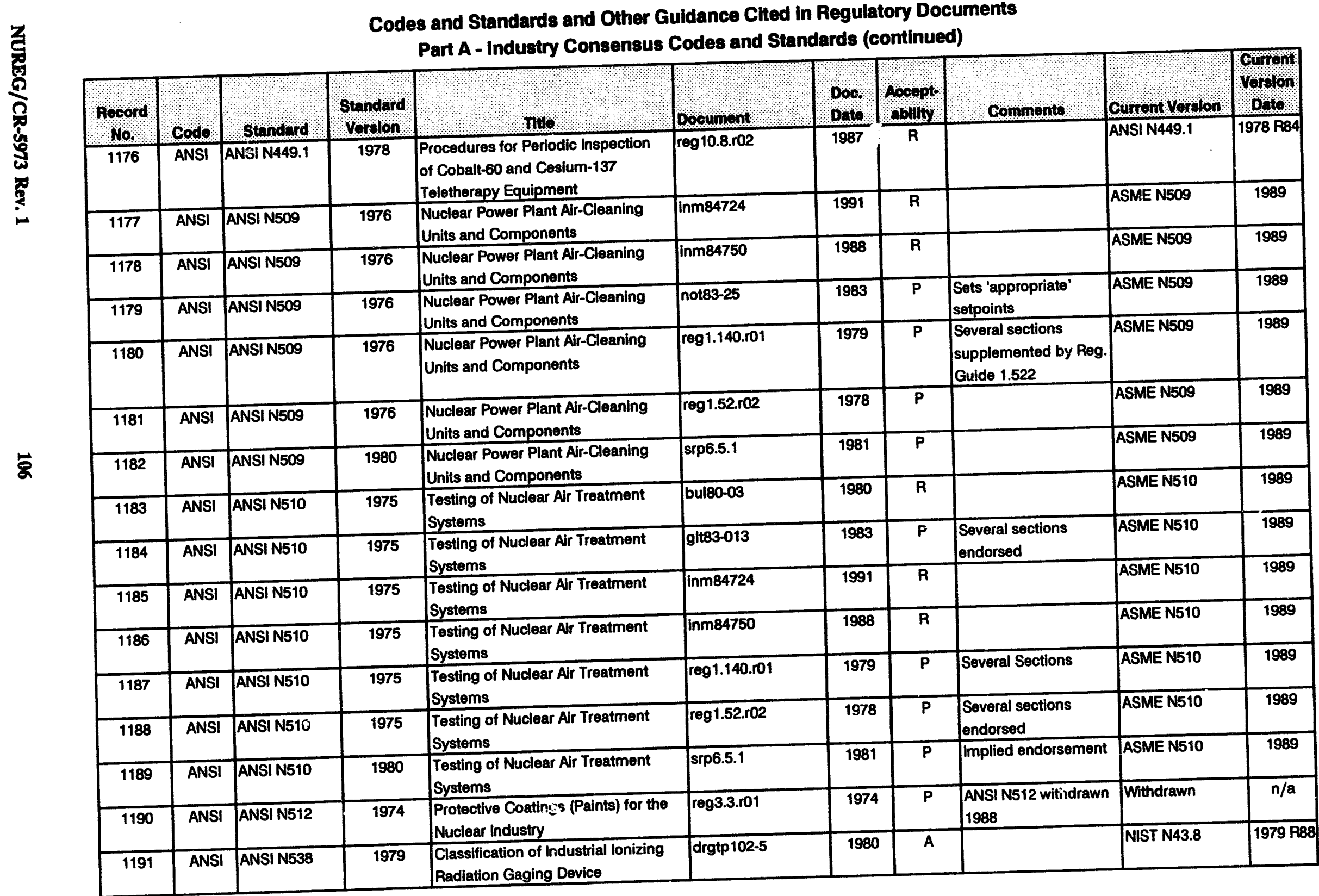


Codes and Standards and Other Guidance Cited in Regulatory Documents

Part A - Industry Consensus Codes and Standards (continued)

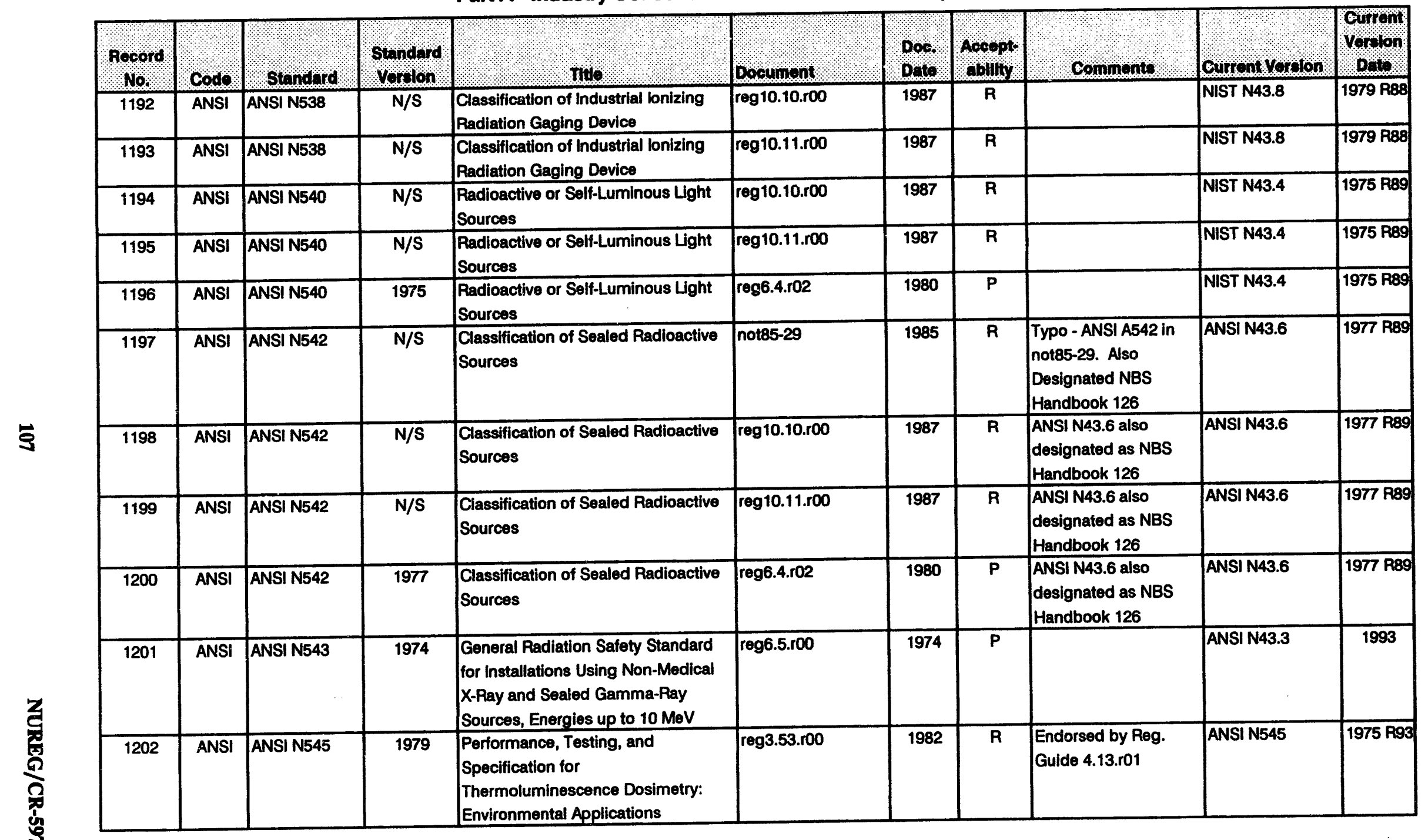


Codes and Standards and Other Guidance Cited in Regulatory Documents

Part A - Industry Consensus Codes and Standards (continued)

\begin{tabular}{|c|c|c|c|c|c|c|c|c|c|c|}
\hline $\begin{array}{l}\text { Aecord } \\
\text { No. }\end{array}$ & Codo & Standard & Standard & , Tilo, & Document & Doo. & $\begin{array}{l}\text { Acospt? } \\
\text { oblithy }\end{array}$ & comments & Gurront Vordon & Currom \\
\hline 1203 & ANSI & ANSI N545 & 1975 & $\begin{array}{l}\text { Performance, Testing, and } \\
\text { Specification for } \\
\text { Thermoluminescence Dosimetry: } \\
\text { Environmental Applications }\end{array}$ & reg4.13.ro1 & 1977 & i & $\begin{array}{l}\text { Additional provision } \\
\text { and qualifications }\end{array}$ & ANSI N545 & $1975 \mathrm{R} 93$ \\
\hline 1204 & ANSI & ANSI N546 & 1976 & $\begin{array}{l}\text { Medical Certification and } \\
\text { Monitoring of Personnel Requiring } \\
\text { Operator Licenses for Nuclear } \\
\text { Power Plants }\end{array}$ & not84-03 & 1984 & $\mathbf{R}$ & & ANS 3.4 & $1983 \mathrm{R} 88$ \\
\hline 1205 & ANSI & ANSI N719 & 1979 & $\begin{array}{l}\text { Setpoints for Nuclear Safety- } \\
\text { Related Instrumentation Used in } \\
\text { Nuclear Power Plants }\end{array}$ & nureg0933, 3.3 & 1985 & $\mathbf{R}$ & & ISA S67.04 & 1988 \\
\hline 1206 & ANSI & ANSI N546 & 1976 & $\begin{array}{l}\text { Medical Certification and } \\
\text { Monitoring of Personnel Requiring } \\
\text { Operator Licenses for Nuclear } \\
\text { Power Plants } \\
\end{array}$ & drghf608.r04 & 1982 & $\mathbf{P}$ & & ANS 3.4 & 1983 R88 \\
\hline 1207 & ANSI & ANSI N581 & $\mathrm{N} / \mathrm{S}$ & $\begin{array}{l}\text { PWR and BWR Containment Spray } \\
\text { System Design Criteria }\end{array}$ & nureg0933, 2.C-10 & 1983 & $\mathbf{R}$ & & ANS 56.5 & 1979 R87 \\
\hline 1208 & ANSI & ANSI N658 & N/S & $\begin{array}{l}\text { Single Failure Criteria for Light } \\
\text { Water Reactor Safety-Related Fluid } \\
\text { Systems }\end{array}$ & srp6.3 & 1984 & $\mathbf{R}$ & & ANS 58.9 & $1981 \mathrm{RB7}$ \\
\hline 1209 & ANSI & ANSI N660 & $\mathrm{N} / \mathrm{S}$ & $\begin{array}{l}\text { Nuclear Power Plants-Time } \\
\text { Response Design Criteria for Safety } \\
\text { Related Operator Actions }\end{array}$ & nureg0933, 1.ll.E.6 & 1984 & $\mathbf{R}$ & & ANS 58.8 & 1984 \\
\hline 1210 & ANSI & $\begin{array}{l}\text { ANSI N660 } \\
\text { Draft }\end{array}$ & $\mathrm{N} / \mathrm{S}$ & $\begin{array}{l}\text { Time Response Design Criteria for } \\
\text { Nuclear Safety Related Operator } \\
\text { Actions }\end{array}$ & glt83-010.a & 1983 & $\mathbf{R}$ & & ANS 58.8 & 1984 \\
\hline 1211 & ANSI & $\begin{array}{l}\text { ANSI N660 } \\
\text { Draft }\end{array}$ & $\mathrm{N} / \mathrm{S}$ & $\begin{array}{l}\text { Time Response Design Criteria for } \\
\text { Nuclear Safety Related Operator } \\
\text { Actions }\end{array}$ & glt83-010.b & 1983 & $\mathbf{R}$ & & ANS 58.8 & 1984 \\
\hline 1212 & ANSI & $\begin{array}{l}\text { ANSI N660 } \\
\text { Draft }\end{array}$ & $\mathrm{N} / \mathrm{S}$ & $\begin{array}{l}\text { Time Response Design Criteria for } \\
\text { Nuclear Safety Related Operator } \\
\text { Actions }\end{array}$ & glt83-010.c & 1983 & $R$ & & ANS 58.8 & 1984 \\
\hline
\end{tabular}




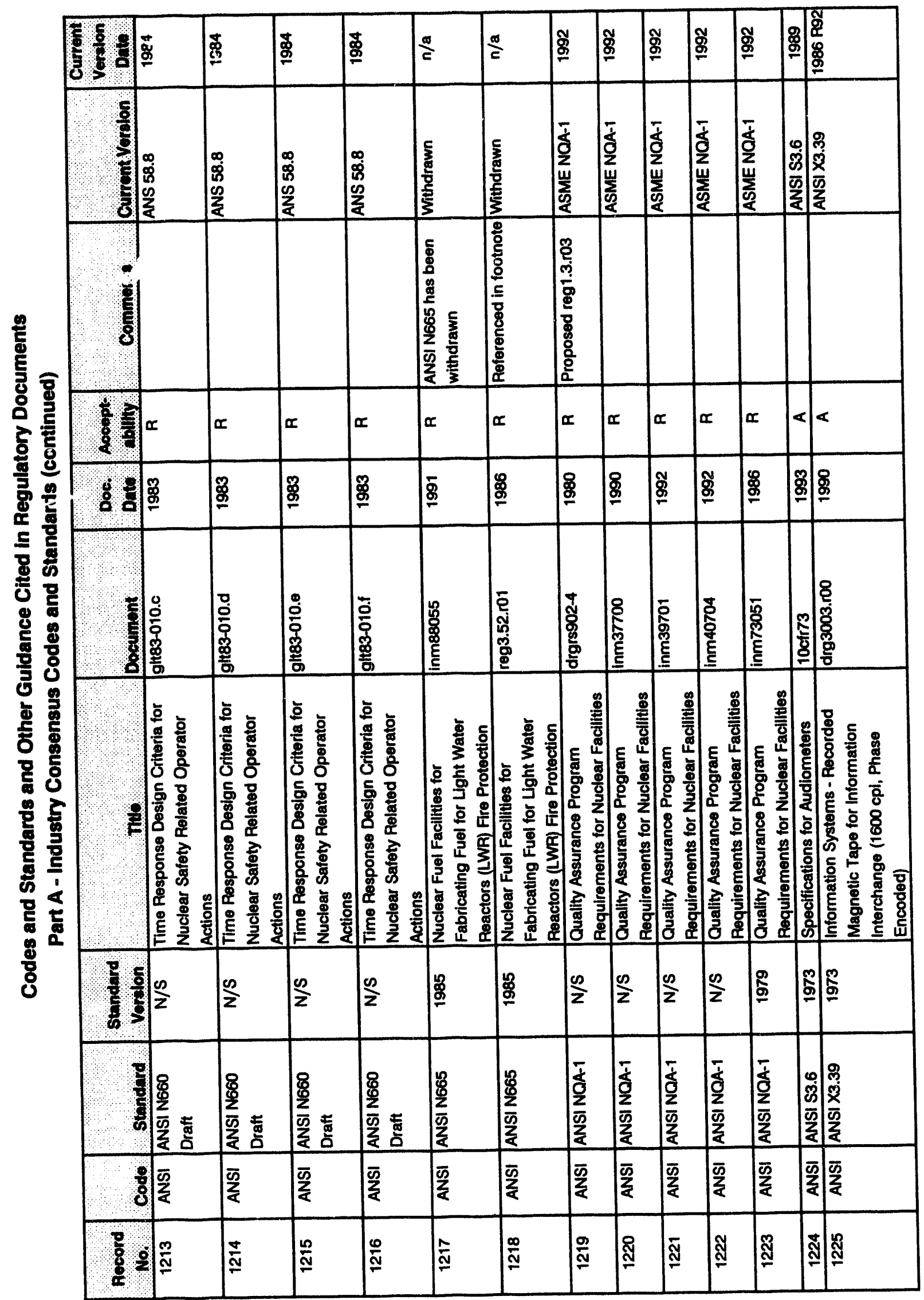




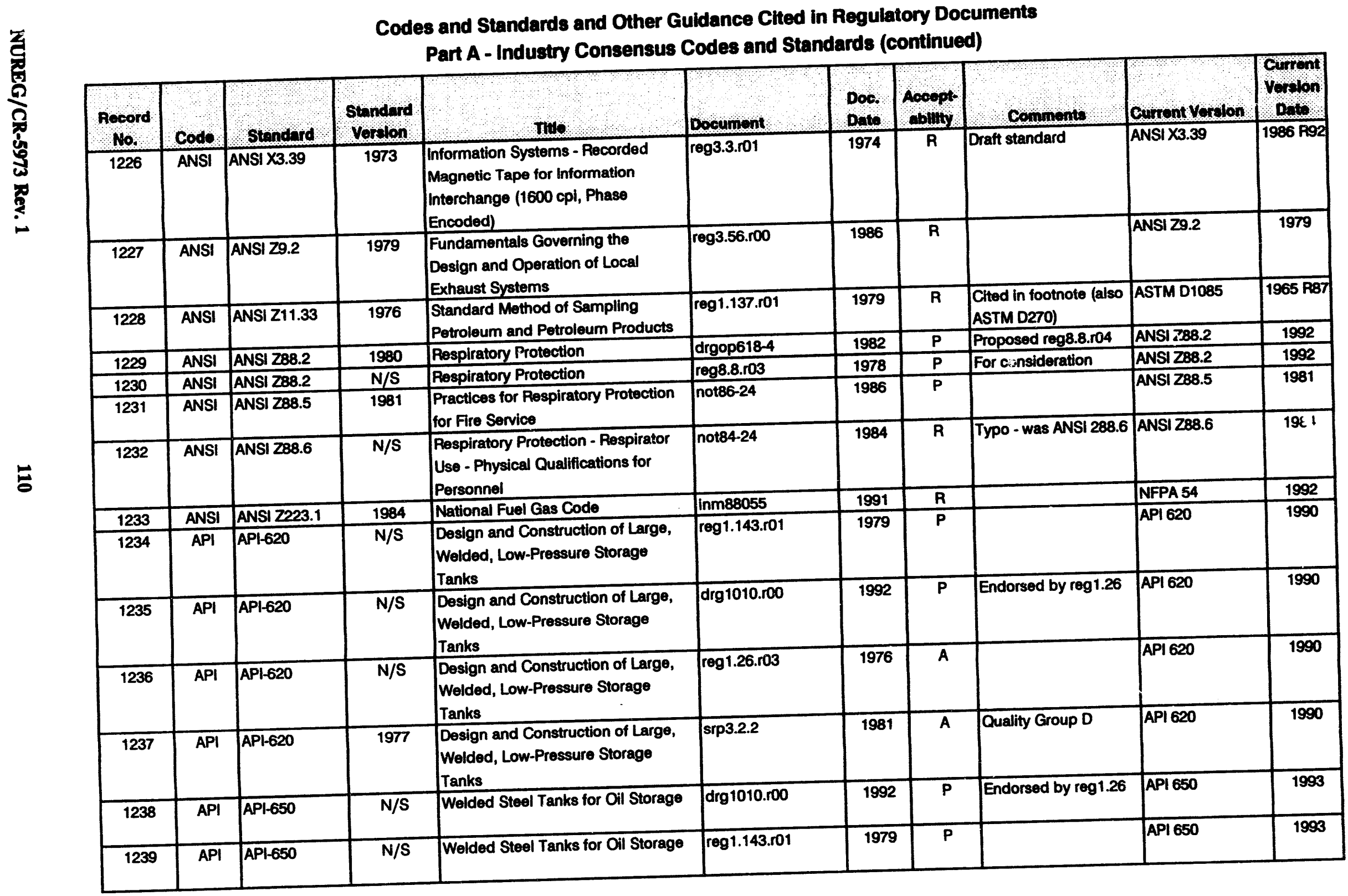


Codes and Standards and Other Guidance Clted in Regulatory Documents

Part A - Industry Consensus Codes and Standards (continued)

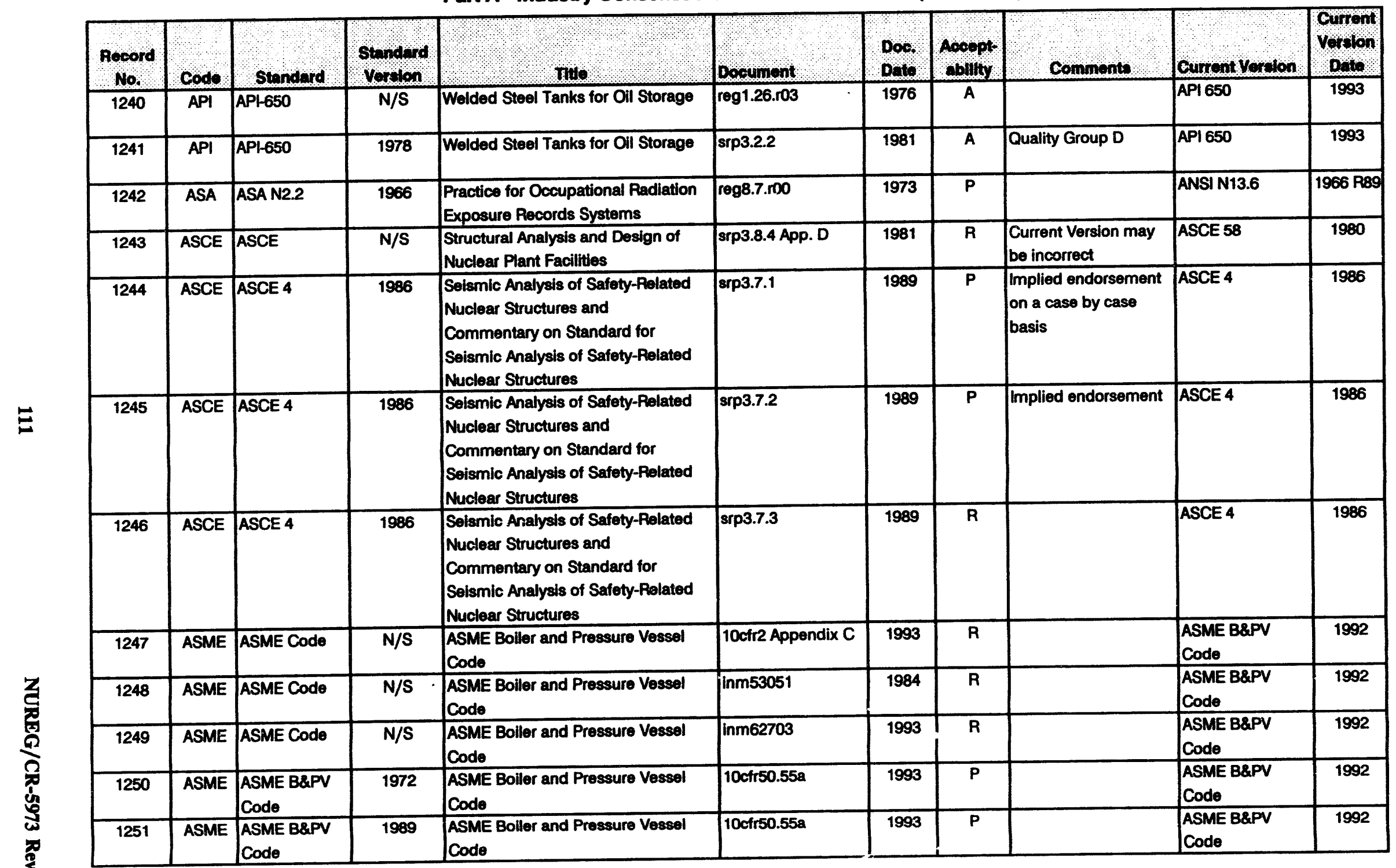




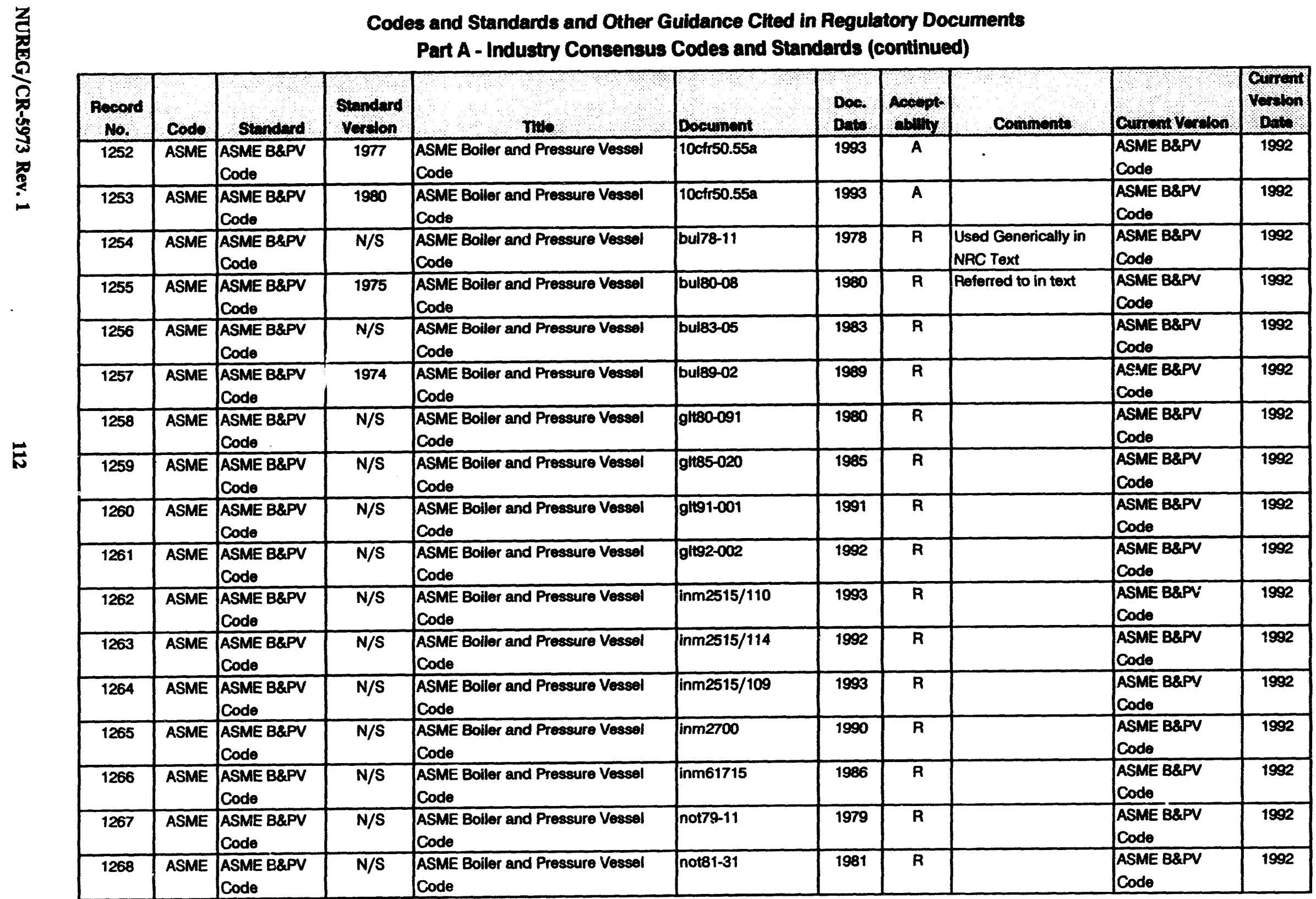


Codes and Standards and Other Guidance Cited in Regulatory Documents

Part A - Industry Consensus Codes and Standards (continued)

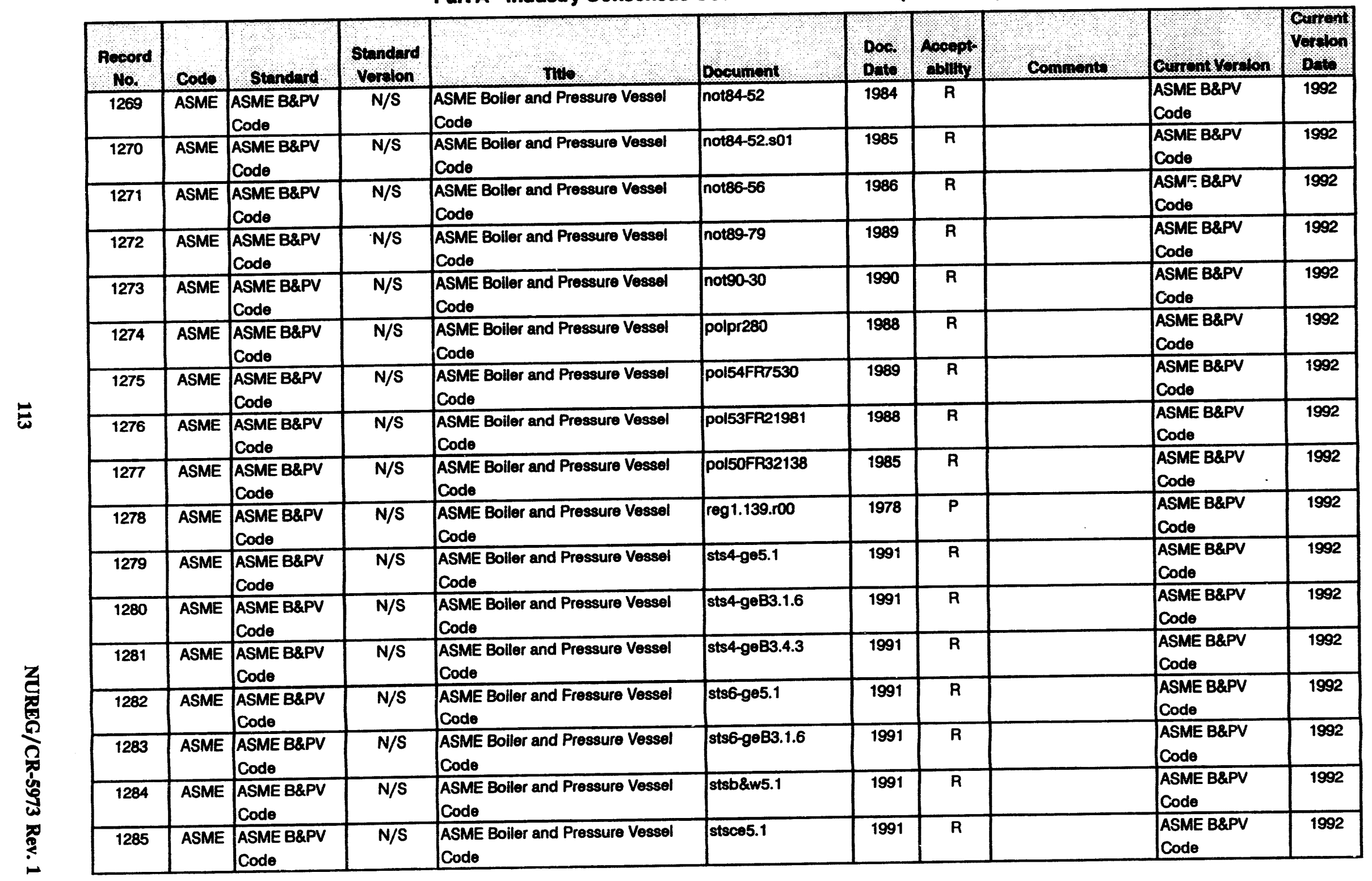


Codes and Standards and Other Guldance Cited in Regulatory Documents

Part A - Industry Consensus Codes and Standards (continued)

\begin{tabular}{|c|c|c|c|c|c|c|c|c|c|c|}
\hline $\begin{array}{l}\text { Rocord } \\
\text { No. }\end{array}$ & Cods & Standard & $\begin{array}{l}\text { Stundard } \\
\text { verston. }\end{array}$ & TWo $\ldots$ & Document: & Doo. & $\begin{array}{l}\text { Acoept } \\
\text { abuluy }\end{array}$ & Comments & Curnont Vorolon & $\begin{array}{l}\text { Corrent } \\
\text { Vorlon } \\
\text { Date }\end{array}$ \\
\hline 1286 & ASME & \begin{tabular}{|l|} 
ASME B\&PV \\
Code
\end{tabular} & N/S & $\begin{array}{l}\text { ASME Boiler and Pressure Vessel } \\
\text { Code }\end{array}$ & stswst5.1 & 1991 & $\mathbf{R}$ & & $\begin{array}{l}\text { ASME B\&PV } \\
\text { Code } \\
\end{array}$ & 1992 \\
\hline 1287 & ASME & $\begin{array}{l}\text { ASME B\&PV } \\
\text { Code Addenda }\end{array}$ & 1972 & $\begin{array}{l}\text { ASME Boller and Pressure Vessel } \\
\text { Code }\end{array}$ & 10 cfr50 App. G & 1993 & $\mathbf{R}$ & & $\begin{array}{l}\text { ASME B\&PV } \\
\text { Code }\end{array}$ & 1992 \\
\hline 1288 & ASME & $\begin{array}{l}\text { ASME B\&PV } \\
\text { Code Addenda }\end{array}$ & $N / S$ & $\begin{array}{l}\text { ASME Boiler and Pressure Vessel } \\
\text { Code }\end{array}$ & 10 cfr50.55a & 1993 & $\mathbf{R}$ & $\begin{array}{l}\text { Footnotes to CFR } \\
50.55(\mathrm{a})\end{array}$ & $\begin{array}{l}\text { ASME B\&PV } \\
\text { Code }\end{array}$ & 1992 \\
\hline 1289 & ASME & \begin{tabular}{|l|} 
ASME Code \\
Case 1141-1
\end{tabular} & 1976 & Foreign Produced Steel & reg1.85.r29 & 1993 & $\mathbf{N}$ & Annulled by ASME & $n / a$ & $n / a$ \\
\hline 1290 & ASME & $\begin{array}{l}\text { ASME Code } \\
\text { Case 1332-6 }\end{array}$ & 1979 & $\begin{array}{l}\text { Requirements for Steel Forgings, } \\
\text { Section III, Requirements for } \\
\text { Corrosion-Resisting Steel Bars and } \\
\text { Shapes, Section III } \\
\end{array}$ & $\operatorname{reg} 1.85 . r 29$ & 1993 & $\mathbf{N}$ & Annulled by ASME & $n / a$ & $n / a$ \\
\hline 1291 & ASME & $\begin{array}{l}\text { ASME Code } \\
\text { Case 1335-9 } \\
\end{array}$ & 1978 & $\begin{array}{l}\text { Poquirements for Bolting Materials, } \\
\text { Section III }\end{array}$ & reg1.85.r29 & 1993 & $\mathbf{N}$ & Annulled by ASME & $n / a$ & $n / a$ \\
\hline 1292 & ASME & $\begin{array}{l}\text { ASME Code } \\
\text { Case 1337-10 }\end{array}$ & 1978 & $\begin{array}{l}\text { Special Type } 403 \text { Modified } \\
\text { Forgings or Bars, Section III, } \\
\text { Division 1, Class } 1 \text { and CS }\end{array}$ & reg1.85.r29 & 1993 & $\mathbf{N}$ & Annulled by ASME & $\begin{array}{l}\text { ASME Code Case } \\
\mathrm{N}-4-11\end{array}$ & 1993 \\
\hline 1293 & ASME & $\begin{array}{l}\text { ASME Code } \\
\text { Case 1337-9 }\end{array}$ & 1975 & $\begin{array}{l}\text { Special Type } 403 \text { Modified } \\
\text { Forgings or Bars, Section III, } \\
\text { Division 1, Class } 1 \text { and CS }\end{array}$ & reg1.85.r29 & 1993 & $\mathbf{N}$ & Annulled by ASME & $\begin{array}{l}\text { ASME Code Case } \\
\mathrm{N}-4-11\end{array}$ & 1993 \\
\hline 1294 & ASME & $\begin{array}{l}\text { ASME Code } \\
\text { Case 1355-3 } \\
\end{array}$ & $\mathrm{N} / \mathrm{S}$ & $\begin{array}{l}\text { Electroslag Welding, Sections I, III } \\
\text { and VIII, Divisions } 1 \text { and } 2\end{array}$ & reg1.34.ro0 & 1972 & $\mathbf{N}$ & Annulled by ASME & $n / a$ & $n / a$ \\
\hline 1295 & ASME & $\begin{array}{l}\text { ASME Code } \\
\text { Case 1361-2 } \\
\end{array}$ & 1979 & Socket Welds, Section III & reg1.84.r29 & 1993 & $\mathbf{N}$ & Annulled by ASME & $n / a$ & $n / a$ \\
\hline 1296 & ASME & $\begin{array}{l}\text { ASME Code } \\
\text { Case 1395-3 }\end{array}$ & 1979 & $\begin{array}{l}\text { SA-508, Class } 2 \text { Forgings with } \\
\text { Modified Manganese Content, } \\
\text { Section III or Section VIII, Division } 2\end{array}$ & reg1.85.r29 & 1993 & $\mathbf{N}$ & Annulled by ASME & $n / a$ & $n / a$ \\
\hline 1297 & ASME & $\begin{array}{l}\text { ASME Code } \\
\text { Case 1407-2 } \\
\end{array}$ & 1974 & $\begin{array}{l}\text { Time of Examination for Class 1, 2, } \\
\text { and 3, Section III Vessels }\end{array}$ & reg1.85.r29 & 1993 & $\mathbf{N}$ & Annulled by ASME & $n / a$ & $n / a$ \\
\hline 1298 & ASME & $\begin{array}{l}\text { ASME Code } \\
\text { Case 1412-4 }\end{array}$ & 1977 & $\begin{array}{l}\text { Modified High Yield Strength Steel } \\
\text { for Section III, Division 1, Class } 1 \\
\text { Vessals }\end{array}$ & reg1.85.r29 & 1993 & $\mathbf{N}$ & Annullod by ASME & $n / a$ & $n / a$ \\
\hline
\end{tabular}


Codes and Standards and Other Guldance Clted in Regulatory Documents

Part A - Industry Consensus Codes and Standards (continued)

\begin{tabular}{|c|c|c|c|c|c|c|c|c|c|c|}
\hline $\begin{array}{l}\text { Rooord } \\
\mathrm{Non}\end{array}$ & Codo & sinndard & Standurd & 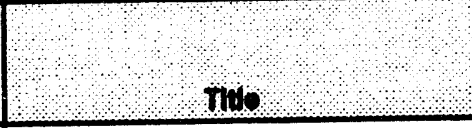 & Dooumont: & Doc. & noppls & Q & curnonvorolon & Orinon: \\
\hline 1299 & ASME & $\begin{array}{l}\text { ASME Code } \\
\text { Case 1414-3 }\end{array}$ & 1976 & $\begin{array}{l}\text { High Vield Strength Cr-Mo Steel for } \\
\text { Section III, Division 1, Class } 1 \\
\text { Vessels }\end{array}$ & reg1.85.r29 & 1993 & $N$ & Annulled by ASME & $n / a$ & $n / a$ \\
\hline 1300 & ASME & $\begin{array}{l}\text { ASME Code } \\
\text { Case 1414-4 }\end{array}$ & 1977 & $\begin{array}{l}\text { High Viold Strength Cr-Mo Steel for } \\
\text { Section III, Division 1, Class } 1 \\
\text { Vessels }\end{array}$ & reg1.85.r29 & 1993 & $\mathbf{N}$ & Annulled by ASME & $n / a$ & $n / a$ \\
\hline 1301 & ASME & $\begin{array}{l}\text { ASME Code } \\
\text { Case 1423-2 }\end{array}$ & 1977 & $\begin{array}{l}\text { Wrought Type } 304 \text { and } 316 \text { with } \\
\text { Nitrogen Added, Sections 1, III, VIII, } \\
\text { Dlvision } 1 \text { end } 2\end{array}$ & reg1.85.r29 & 1993 & $\mathbf{N}$ & Annulled by ASME & $n / a$ & $n / a$ \\
\hline 1302 & ASME & $\begin{array}{l}\text { ASME Code } \\
\text { Case 1434-1 }\end{array}$ & 1978 & $\begin{array}{l}\text { Postweld Heat Treatment of SA-487 } \\
\text { Class 8N Steel Castings, Section ill }\end{array}$ & reg1.85.r29 & 1993 & $\mathbf{N}$ & Annulled by ASME & $n / a$ & $n / a$ \\
\hline 1303 & ASME & \begin{tabular}{|l|} 
ASME Code \\
Case 1461-1 \\
\end{tabular} & 1977 & $\begin{array}{l}\text { Electron Beam Wolding, Section } 1 \text {, } \\
\text { III, and Vill, Division } 1 \text { and } 2\end{array}$ & reg1.84.r29 & 1993 & $\bar{N}$ & Annulled by ASME & $n / a$ & $n / a$ \\
\hline 1304 & ASME & $\begin{array}{l}\text { ASME Code } \\
\text { Case 1470-2 }\end{array}$ & 1974 & $\begin{array}{l}\text { Exterrial Pressure Charts for High- } \\
\text { Strength Carbon Steels and for } \\
\text { Low-Alioy Steels, Section Vill, } \\
\text { Division } 1 \text { and 2, and Section ill } \\
\end{array}$ & reg1.84.r29 & 1993 & $\mathbf{N}$ & Annullod by ASME & $n / a$ & $n / a$ \\
\hline 1305 & ASME & $\begin{array}{l}\text { ASME Code } \\
\text { Case 1471-1 } \\
\end{array}$ & 1978 & $\begin{array}{l}\text { Vacuum Electron Beam Wolding of } \\
\text { Tube Sheot Joints, Section lil }\end{array}$ & reg1.84.r29 & 1993 & $\mathbf{N}$ & Annulled by ASME & $n / a$ & $n / a$ \\
\hline 1306 & ASME & $\begin{array}{l}\text { ASME Code } \\
\text { Case 1475-1 }\end{array}$ & 1975 & $\begin{array}{l}\text { Ferritic-Austenitic Stainloss Steel } \\
\text { Seamless Tubes for Section ill, } \\
\text { Class } 2 \text { and } 3 \text { Construction }\end{array}$ & reg1.85.r29 & 1993 & $\mathbf{N}$ & Annulled by ASME & $n / a$ & $n / a$ \\
\hline 1307 & ASME & $\begin{array}{l}\text { ASME Code } \\
\text { Case 1477-1 }\end{array}$ & 1978 & $\begin{array}{l}\text { Use of } 1970 \text { Addenda of ANSI } \\
\text { B31.7, Section III }\end{array}$ & reg1.84.r29 & 1993 & $\mathbf{N}$ & Annulled by ASME & $n / a$ & $n / a$ \\
\hline 1308 & ASME & $\begin{array}{l}\text { ASME Code } \\
\text { Case 1484-1 }\end{array}$ & 1974 & $\begin{array}{l}\text { SB-163 Nickel-Chromilm Iron } \\
\text { Tubing (Alloy 600) at a Specified } \\
\text { Minimum Vield Strength of } 40.0 \\
\text { Ksl, Section III, Class } 1 \\
\end{array}$ & reg1.85.r29 & 1993 & $\mathbf{N}$ & Annulled by ASME & $\begin{array}{l}\text { ASME Code Case } \\
\text { N-20-3 }\end{array}$ & 1991 \\
\hline 1309 & ASME & $\begin{array}{l}\text { ASME Code } \\
\text { Case 1484-2 }\end{array}$ & 1976 & $\begin{array}{l}\text { SB-163 Nickel-Chromlum(N-20) } \\
\text { Iron Tubing (Alloy } 600 \text { and } 690 \text { ) and } \\
\text { Nickel-lron Chrom!um Alloy } 800 \text { at } \\
\text { a Specified Misimum Yield } \\
\text { Strength of } 40.0 \text { Ksi, Section III, } \\
\text { Division 1, Class } 1\end{array}$ & reg1.85.r29 & 1993 & $\mathbf{N}$ & Annulied by ASME & $\begin{array}{l}\text { ASME Code Case } \\
N-20-3\end{array}$ & 1991 \\
\hline
\end{tabular}




\begin{tabular}{|c|c|c|c|c|c|c|c|c|c|c|}
\hline \multicolumn{11}{|c|}{$\begin{array}{c}\text { Codes and Standards and Other Guldance Ched in Regulatory Documents } \\
\text { Part A - Industry Consensus Codes and Standards (continued) }\end{array}$} \\
\hline $\begin{array}{l}\text { nocord } \\
\text { No. }\end{array}$ & codo & stand ord & Standard & (ै., Ino & boounont & $\begin{array}{l}\text { Doo. } \\
\text { Doto }\end{array}$ & Aocept: & (6ommonth & cuncunt vorolon & $100.0 \%$ \\
\hline 1310 & ASME & $\begin{array}{l}\text { ASME Code } \\
\text { Case } 1492\end{array}$ & 1975 & $\begin{array}{l}\text { Post Wold Heat Treatment, Section } \\
\text { I, III and VIII, Division } 1 \text { and } 2\end{array}$ & reg1.85.r29 & 1993 & $N$ & Annulled by ASME & $n / a$ & $n / a$ \\
\hline 1311 & ASME & $\begin{array}{l}\text { ASME Code } \\
\text { Case 1494-1 }\end{array}$ & 1973 & $\begin{array}{l}\text { Wold Procedure Qualification Test, } \\
\text { Section III }\end{array}$ & reg1.84.r29 & 1993 & $\mathbf{N}$ & Annulled by ASME & $n / a$ & $n / a$ \\
\hline 1312 & ASME & $\begin{array}{l}\text { ASME Codo } \\
\text { Case } 1506\end{array}$ & 1978 & $\begin{array}{l}\text { Stress Intensification Factors, } \\
\text { Section III, Class } 2 \text { and } 3 \text { Piping }\end{array}$ & reg1.84.r29 & 1993 & $\mathbf{N}$ & Annulled by ASME & $n / a$ & $n / a$ \\
\hline 1313 & ASME & $\begin{array}{l}\text { ASME Code } \\
\text { Case } 1508\end{array}$ & 1975 & $\begin{array}{l}\text { Allowable Stresses, Design Intensity } \\
\text { and/or Yield Strength Values, } \\
\text { Section I, III, and VIII, Dlvisions } 1 \\
\text { and } 2\end{array}$ & reg1.84.r29 & 1993 & $\mathbf{N}$ & Annulled by ASME & $n / a$ & $n / a$ \\
\hline 1314 & ASME & $\begin{array}{l}\text { ASME Code } \\
\text { Case } 1515\end{array}$ & 1977 & $\begin{array}{l}\text { Uitrasonic Examination of Ring } \\
\text { Forgings for Shell Sections, Section } \\
\text { III, Class } 1 \text { Vessels }\end{array}$ & reg1.85.r29 & 1993 & $\mathbf{N}$ & Annulled by ASME & $n / a$ & $n / a$ \\
\hline 1315 & ASME & $\begin{array}{l}\text { ASME Code } \\
\text { Case 1516-1 }\end{array}$ & 1975 & $\begin{array}{l}\text { Welding of Seats in Valves for } \\
\text { Section III Applications }\end{array}$ & $\operatorname{reg} 1.84 .129$ & 1993 & $\mathbf{N}$ & Annulled by ASME & $n / a$ & $n / a$ \\
\hline 1316 & ASME & $\begin{array}{l}\text { ASME Cods } \\
\text { Case 1521-1 }\end{array}$ & 1978 & $\begin{array}{l}\text { Use of H-Grades of SA-240, SA-479, } \\
\text { SA-336, and SA-358, Section III }\end{array}$ & $\operatorname{reg} 1.85 .129$ & 1993 & $\mathbf{N}$ & Annulled by ASME & $n / a$ & $n / a$ \\
\hline 1317 & ASME & $\begin{array}{l}\text { ASME Code } \\
\text { Case 1528-3 }\end{array}$ & 1978 & $\begin{array}{l}\text { High Strength Steel SA-508, Class } \\
2 \text { and SA-541, Class } 2 \text { Forgings, } \\
\text { Section III, Class } 1 \text { Components }\end{array}$ & $\operatorname{reg} 1.85 .129$ & 1993 & $\mathbf{N}$ & Annulled by ASME & $n / a$ & $n / a$ \\
\hline 1318 & ASME & $\begin{array}{l}\text { ASME Code } \\
\text { Case } 1529\end{array}$ & 1973 & $\begin{array}{l}\text { Materials for Instrument Line } \\
\text { Fittings, Section III }\end{array}$ & reg1.85.r29 & 1993 & $\bar{N}$ & Annulled by ASME & $n / a$ & $n / a$ \\
\hline 1319 & ASME & $\begin{array}{l}\text { ASME Code } \\
\text { Case } 1531\end{array}$ & 1977 & $\begin{array}{l}\text { Electrical Penetrations, Special } \\
\text { Alloys for Electrical Penetration } \\
\text { Seals, Section ill }\end{array}$ & reg1.85.r29 & 1993 & $N$ & Annulled by ASME & $n / a$ & $n / a$ \\
\hline 1320 & ASME & $\begin{array}{l}\text { ASME Codo } \\
\text { Case } 1532\end{array}$ & 1978 & $\begin{array}{l}\text { Section III, Class } 3 \text { Components } \\
\text { Made of } 8 \text { Percent and } 9 \text { Percent } \\
\text { Nickel Steol }\end{array}$ & reg1.85.r29 & 1993 & $\bar{N}$ & Annulled by ASME & $n / a$ & $n / a$ \\
\hline 1321 & ASME & $\begin{array}{l}\text { ASME Code } \\
\text { Case } 1533\end{array}$ & 1975 & $\begin{array}{l}\text { Pressure Temperature Ratings of } \\
\text { SA-35 of SA-351 Grades CF8A, CF3, } \\
\text { and CF3M, Section III }\end{array}$ & reg1.84.r29 & 1993 & $N$ & Annulled by ASME & $n / a$ & $n / a$ \\
\hline 1322 & ASME & $\begin{array}{l}\text { ASME Code } \\
\text { Case 1535-2 }\end{array}$ & 1977 & $\begin{array}{l}\text { Hydrostatic Testing of Section III, } \\
\text { Class } 1 \text { Valves }\end{array}$ & reg1.84.r29 & 1993 & $\bar{N}$ & Annulled by ASME & $n / a$ & $n / a$ \\
\hline
\end{tabular}


Codes and Standards and Other Guidance Cited in Regulatory Documents

Part A - Industry Consensus Codes and Standards (continued)

\begin{tabular}{|c|c|c|c|c|c|c|c|c|c|c|}
\hline Hecord & Codo & Standard & stondon & 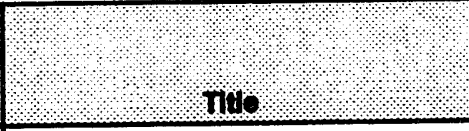 & $6,0,0$ & Dock & acopots & (commonte & cuncoms Yorolon & 6,10 \\
\hline 1323 & ASME & $\begin{array}{l}\text { ASME Code } \\
\text { Case } 1536\end{array}$ & 1977 & $\begin{array}{l}\text { Closing Seam for Electrical } \\
\text { Penetrations for Section III, Class 2, } \\
3 \text {, and MC }\end{array}$ & reg1.84.r29 & 1993 & $\mathbf{N}$ & Annulled by ASME & $n / a$ & $n / a$ \\
\hline 1324 & ASME & $\begin{array}{l}\text { ASME Code } \\
\text { Case 1540-1 }\end{array}$ & 1977 & $\begin{array}{l}\text { Elastomer Diaphragm Valves, } \\
\text { Section III Class } 2 \text { and } 3\end{array}$ & reg1.84.r29 & 1993 & $\mathbf{N}$ & Annulled by ASME & \begin{tabular}{|l|} 
ASME Code Case \\
$\mathrm{N}-31-1$
\end{tabular} & 1991 \\
\hline 1325 & ASME & $\begin{array}{l}\text { ASME Code } \\
\text { Case 1541-1 }\end{array}$ & 1976 & $\begin{array}{l}\text { Hydrostatic Testing of Embedded } \\
\text { Class } 2 \text { and Class } 3 \text { Piping for } \\
\text { Section III Construction }\end{array}$ & reg1.84.r29 & 1993 & $\mathbf{N}$ & Annulled by ASME & $n / a$ & $n / a$ \\
\hline 1326 & ASME & $\begin{array}{l}\text { ASME Code } \\
\text { Case 1541-2 }\end{array}$ & 1978 & $\begin{array}{l}\text { Hydrostatic Testing of Embedded } \\
\text { Class } 2 \text { and Class } 3 \text { Plping for } \\
\text { Section III Construction }\end{array}$ & reg1.84.r29 & 1993 & $\mathbf{N}$ & Annulled by ASME & $n / a$ & $n / a$ \\
\hline 1327 & ASME & $\begin{array}{l}\text { ASME Code } \\
\text { Case } 1552 \\
\end{array}$ & 1977 & $\begin{array}{l}\text { Design by Analysis of Section III, } \\
\text { Class } 1 \text { Valves }\end{array}$ & reg1.84.r29 & 1993 & $\mathbf{N}$ & Annulled by ASME & $n / a$ & $n / a$ \\
\hline 1328 & ASME & $\begin{array}{l}\text { ASME Code } \\
\text { Case } 1553 \\
\end{array}$ & 1975 & $\begin{array}{l}\text { Upset Heading and Roll Threading } \\
\text { of SA-453 for Bolting in Section III }\end{array}$ & reg1.84.r29 & 1993 & $\mathbf{N}$ & Annulled by ASME & $n / a$ & $n / a$ \\
\hline 1329 & ASME & $\begin{array}{l}\text { ASME Code } \\
\text { Case 1553-1 } \\
\end{array}$ & 1976 & $\begin{array}{l}\text { Upset Heading and Roll Threading } \\
\text { of SA-453 for Bolting in Section III }\end{array}$ & reg1.84.r29 & 1993 & $N$ & Annulled by ASME & $n / a$ & $n / a$ \\
\hline 1330 & ASME & $\begin{array}{l}\text { ASME Code } \\
\text { Case } 1555 \\
\end{array}$ & 1977 & $\begin{array}{l}\text { Certification of Safety Rellef Valves } \\
\text { on Liquids }\end{array}$ & reg1.84.r29 & 1993 & $\mathbf{N}$ & Annulled by ASME & $n / a$ & $n / a$ \\
\hline 1331 & ASME & $\begin{array}{l}\text { ASME Code } \\
\text { Case 1555-1 } \\
\end{array}$ & 1978 & $\begin{array}{l}\text { Certification of Safety Relief Valves, } \\
\text { Section III, Division } 1\end{array}$ & $\operatorname{reg1.84.r29}$ & 1993 & $\bar{N}$ & Annulled by ASME & $n / a$ & $n / a$ \\
\hline 1332 & ASME & $\begin{array}{l}\text { ASME C de } \\
\text { Case 1557-2 }\end{array}$ & 1979 & $\begin{array}{l}\text { Steel Products Refined by } \\
\text { Secondary Remelting, Section III } \\
\text { and Vill, Division } 1 \text { and } 2\end{array}$ & reg1.85.r29 & 1993 & $\mathbf{N}$ & Annulled by ASME & $n / a$ & $n / a$ \\
\hline 1333 & ASME & $\begin{array}{l}\text { ASME Code } \\
\text { Case } 1567\end{array}$ & 1978 & $\begin{array}{l}\text { Testing Lots of Carbon and Low } \\
\text { Alloy Steel Covered Electrodes, } \\
\text { Section III }\end{array}$ & $\operatorname{reg} 1.85 .129$ & 1993 & $\mathbf{N}$ & Annulled by ASME & $n / a$ & $n / a$ \\
\hline 1334 & ASME & $\begin{array}{l}\text { ASME Code } \\
\text { Case } 1568\end{array}$ & 1978 & $\begin{array}{l}\text { Testing Lots of Flux Cored and } \\
\text { Fabricated Carbon and Low Alioy } \\
\text { Steel Welding Electrodes, Section } \\
\text { III }\end{array}$ & reg1.85.r29 & 1993 & $\mathbf{N}$ & Annulled by ASME & $n / a$ & $n / a$ \\
\hline 1335 & ASME & $\begin{array}{l}\text { ASME Code } \\
\text { Case } 1569\end{array}$ & 1979 & $\begin{array}{l}\text { Design of Piping for Pressure Relief } \\
\text { Valve Station, Section III }\end{array}$ & reg1.84.r29 & 1993 & $\mathbf{N}$ & Annulled by ASME & $n / a$ & $n / a$ \\
\hline
\end{tabular}




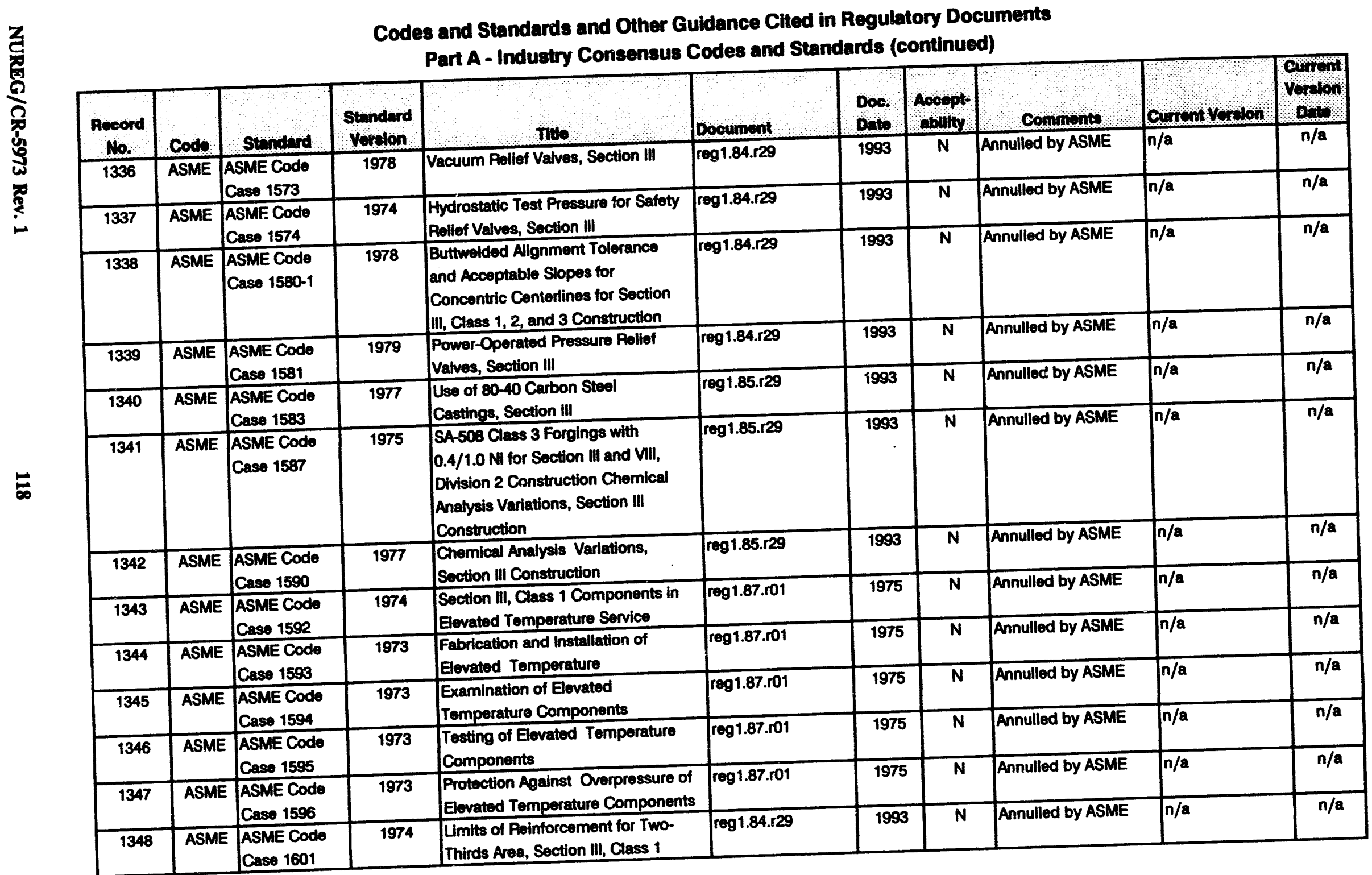


Codes and Standards and Other Guidance Cited in Regulatory Documents

Part A - Industry Consensus Codes and Standards (continued)

\begin{tabular}{|c|c|c|c|c|c|c|c|c|c|c|}
\hline Rocord & Codes & Standard & Standard & $1, \quad$ nIdo & Documont & $\begin{array}{l}\text { Doo. } \\
\text { Dollo }\end{array}$ & Aosepts & Commonts, & Curront Yordow & ontopton \\
\hline 1349 & ASME & $\begin{array}{l}\text { ASME Code } \\
\text { Case 1602-1 }\end{array}$ & 1974 & $\begin{array}{l}\text { Use of SB-42 Alloy 122, SB-111 } \\
\text { Alloys } 122,715 \text { and 706, SB-171 } \\
\text { Alloys } 715 \text { and } 706 \text { and SB-466 } \\
\text { Alloys } 706 \text { and 715, Section III, } \\
\text { Class } 2 \text { and } 3 \text { Components } \\
\end{array}$ & reg1.85.r29 & 1993 & $\mathbf{N}$ & Annulled by ASME & $n / a$ & $n / a$ \\
\hline 1350 & ASME & $\begin{array}{l}\text { ASME Code } \\
\text { Case } 1603\end{array}$ & 1974 & $\begin{array}{l}\text { Toughness Tests When Cross- } \\
\text { Section Limits Orientation and } \\
\text { Location of Specimens }\end{array}$ & reg1.85.r29 & 1993 & $\mathbf{N}$ & Annulled by ASME & $n / a$ & $n / a$ \\
\hline 1351 & ASME & $\begin{array}{l}\text { ASME Code } \\
\text { Case } 1605 \\
\end{array}$ & 1980 & $\begin{array}{l}\text { Cr-Ni-Mo-V Bolting Material for } \\
\text { Soction III, Class } 1 \text { Components }\end{array}$ & reg1.85.r29 & 1993 & $\mathbf{N}$ & Annulled by ASME & $n / a$ & $n / a$ \\
\hline 1352 & ASME & $\begin{array}{l}\text { ASME Code } \\
\text { Case } 1606\end{array}$ & 1974 & $\begin{array}{l}\text { Stress Criteria for Section III, Class } \\
2 \text { and } 3 \text { Piping Subjected to Upset, } \\
\text { Emergency, and Faulted Operating } \\
\text { Conditions }\end{array}$ & $\operatorname{reg} 1.84 .129$ & 1993 & $\mathbf{N}$ & Annulled by ASME & $n / a$ & $n / a$ \\
\hline 1353 & ASME & $\begin{array}{l}\text { ASME Code } \\
\text { Case 1606-1 }\end{array}$ & 1977 & $\begin{array}{l}\text { Stress Criteria Section III, Classes } 2 \\
\text { and } 3 \text { Piping Subject to Upset, } \\
\text { Emergency, and Faulted Operating } \\
\text { Conditions }\end{array}$ & reg1.84.r29 & 1993 & $\mathbf{N}$ & Annulled by ASME & $n / a$ & $n / a$ \\
\hline 1354 & ASME & $\begin{array}{l}\text { ASME Code } \\
\text { Case } 1607\end{array}$ & 1974 & $\begin{array}{l}\text { Stress Criteria for Section III, } \\
\text { Classes } 2 \text { and } 3 \text { Vessels Subjected } \\
\text { to Upset, Emergency, and Faulted } \\
\text { Operating Conditions }\end{array}$ & reg1.84.r29 & 1993 & $\mathbf{N}$ & Annulled by ASME & $n / a$ & $n / a$ \\
\hline 1355 & ASME & $\begin{array}{l}\text { ASME Code } \\
\text { Case 1607-1 }\end{array}$ & 1977 & $\begin{array}{l}\text { Stress Criteria for Section III, } \\
\text { Classes } 2 \text { and } 3 \text { Vessels Designed } \\
\text { to NC/ND-3300 Excluding the NC- } \\
3200 \text { Alternate }\end{array}$ & reg1.84.r29 & 1993 & $\mathbf{N}$ & Annulled by ASME & $n / a$ & $n / a$ \\
\hline 1356 & ASME & $\begin{array}{l}\text { ASME Code } \\
\text { Case 1608-1 }\end{array}$ & 1977 & $\begin{array}{l}\text { Use of ASME SB-265, SB-337, SB- } \\
\text { 338, SB-348, and SB-381, Grades 1, } \\
\text { 2, 3, and } 7 \text { Unalloyed Titanium and } \\
\text { ASTM B-363 Titanium Welding } \\
\text { Fittings, Section III Class } 2 \text { and } 3 \\
\text { Components }\end{array}$ & reg1.85.r29 & 1993 & $\mathbf{N}$ & Annulled by ASME & $n / a$ & $n / a$ \\
\hline 1357 & ASME & $\begin{array}{l}\text { ASME Code } \\
\text { Case } 1613 \\
\end{array}$ & 1978 & $\begin{array}{l}\text { Use of SA-372 Class IV Forgings, } \\
\text { Section III Construction }\end{array}$ & reg1.85.r29 & 1993 & $N$ & Annulled by ASME & $n / a$ & $n / a$ \\
\hline
\end{tabular}




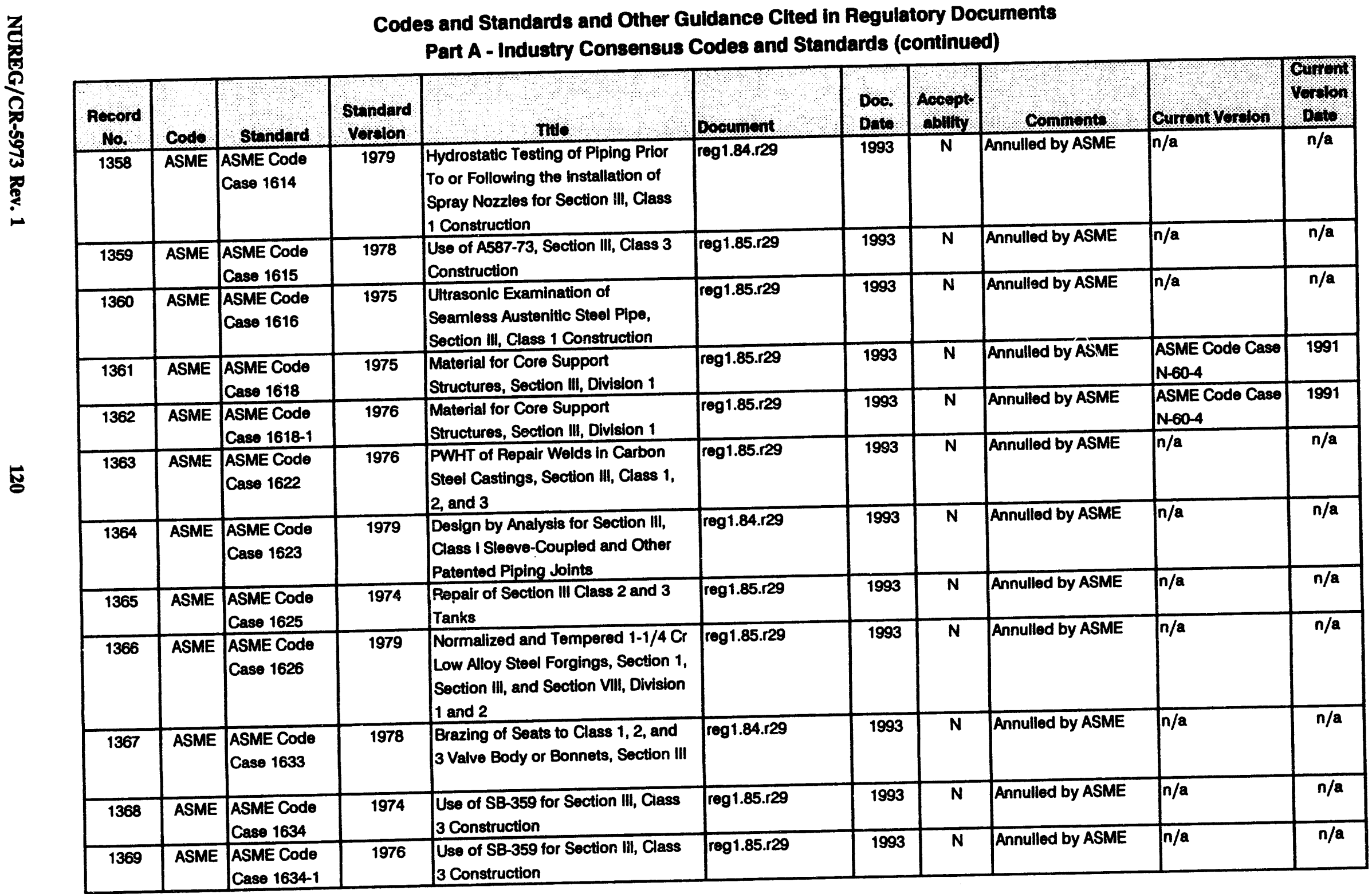


Codes and Standards and Other Guidance Cited in Reguiatory Documents

Part A - Industry Consensus Codes and Standards (continued)

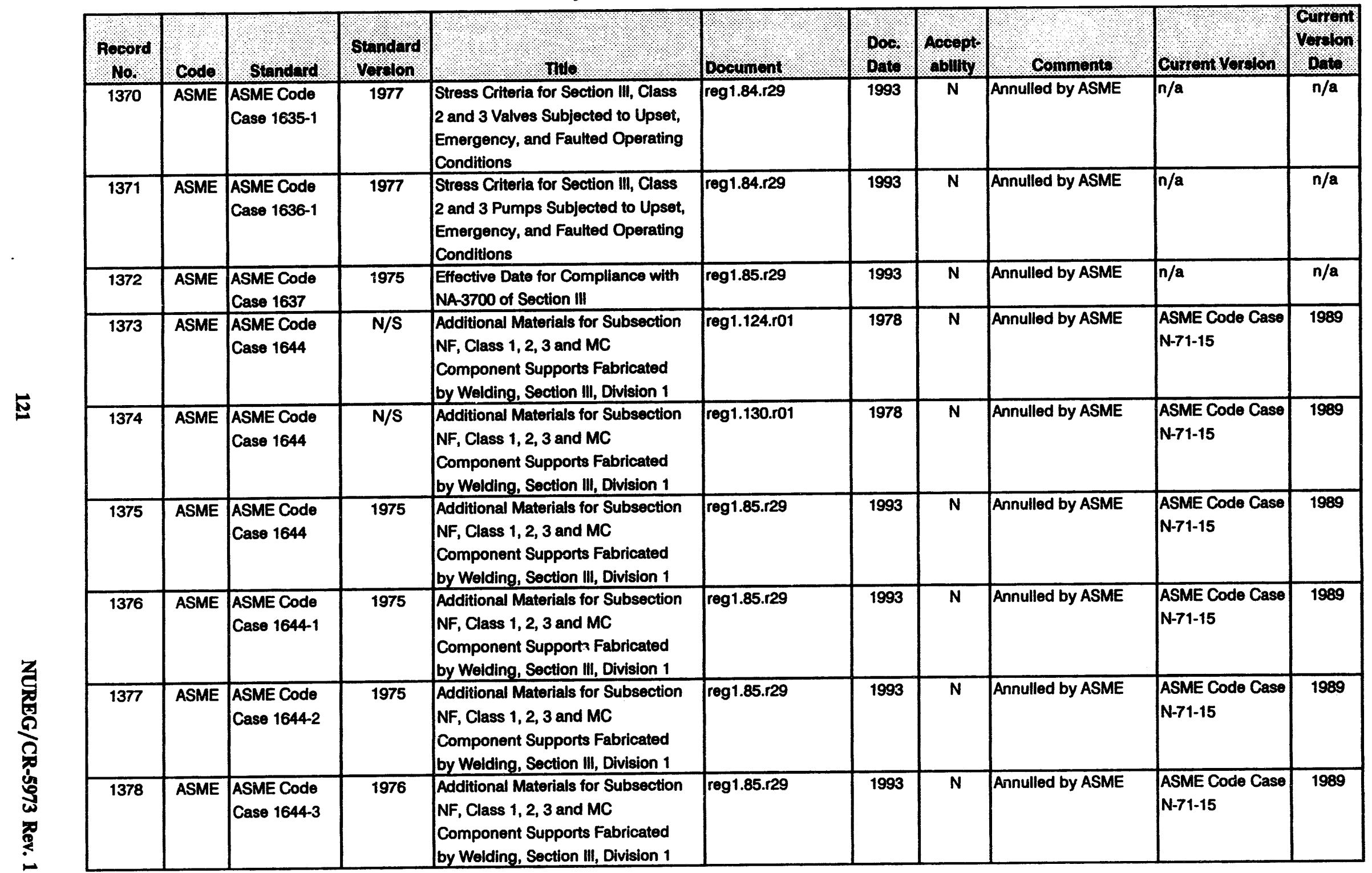




\begin{tabular}{|c|c|c|c|c|c|c|c|c|c|c|}
\hline \multicolumn{11}{|c|}{$\begin{array}{l}\text { Codes and Standards and Other Guidance Cited in Regulatory Documents } \\
\text { Part A - Industry Consensus Codes and Standards (continued) }\end{array}$} \\
\hline $\begin{array}{c}\text { Rocord } \\
\text { No. }\end{array}$ & Code & Stendard & $\begin{array}{l}\text { Standard } \\
\text { Vordlon }\end{array}$ & , nos: & Document & $\begin{array}{l}\text { Door. } \\
\text { Dater }\end{array}$ & Aocopt & Commento & Cursent Vorelon & Conring \\
\hline 1379 & ASME & $\begin{array}{l}\text { ASME Code } \\
\text { Case 1644-4 }\end{array}$ & 1976 & $\begin{array}{l}\text { Additional Materials for Subsection } \\
\text { NF, Class 1, 2, } 3 \text { and MC } \\
\text { Component Supports Fabricated } \\
\text { by Welding, Section III, Division } 1 \\
\end{array}$ & reg1.85.r29 & 1993 & $\mathbf{N}$ & Annulled by ASME & \begin{tabular}{|l} 
ASME Code Case \\
N-71-15
\end{tabular} & 1989 \\
\hline 1380 & ASME & $\begin{array}{l}\text { ASME Code } \\
\text { Case } 1644-5\end{array}$ & 1977 & $\begin{array}{l}\text { Additional Materials for Subsection } \\
\text { NF, Class 1, 2, } 3 \text { and MC } \\
\text { Component Supports Fabricated } \\
\text { by Welding, Section III, Division } 1 \\
\end{array}$ & reg1.85.r29 & 1993 & $\mathbf{N}$ & Annulled by ASME & \begin{tabular}{|l|} 
ASME Code Case \\
N-71-15
\end{tabular} & 1989 \\
\hline 1381 & ASME & $\begin{array}{l}\text { ASME Code } \\
\text { Case 1644-6 }\end{array}$ & 1977 & $\begin{array}{l}\text { Additional Materials for Subsection } \\
\text { NF, Class 1, 2, } 3 \text { and MC } \\
\text { Component Supports Fabricated } \\
\text { by Welding, Section III, Division } 1\end{array}$ & reg1.85.r29 & 1993 & $\mathbf{N}$ & Annulled by ASME & \begin{tabular}{|l|} 
ASME Code Case \\
N-71-15
\end{tabular} & 1989 \\
\hline 1382 & ASME & $\begin{array}{l}\text { ASME Code } \\
\text { Case } 1645\end{array}$ & 1976 & $\begin{array}{l}\text { Use of DeLong Diagram for } \\
\text { Calculating the Delta Ferrite } \\
\text { Content of Welds in Section III, } \\
\text { Class 1, 2, and CS Construction }\end{array}$ & $\operatorname{reg} 1.85 . r 29$ & 1993 & $\mathbf{N}$ & Annulled by ASME & $n / a$ & $n / a$ \\
\hline 1383 & ASME & \begin{tabular}{|l} 
ASME Code \\
Case 1648 \\
\end{tabular} & 1976 & $\begin{array}{l}\text { SA-537 Plates for Section III, Class } \\
1,2,3 \text {, and MC Components }\end{array}$ & reg1.85.r29 & 1993 & $\mathbf{N}$ & Annulled by ASME & $n / a$ & $n / a$ \\
\hline 1384 & ASME & \begin{tabular}{|l|} 
ASME Code \\
Case 1649 \\
\end{tabular} & 1976 & $\begin{array}{l}\text { Modified SA 453-GR } 660 \text { for Class } \\
1,2,3 \text {, and CS Construction } \\
\end{array}$ & reg1.85.r29 & 1993 & $\bar{N}$ & Annulled by ASME & $n / a$ & $n / a$ \\
\hline 1385 & ASME & $\begin{array}{l}\text { ASME Code } \\
\text { Case } 1650\end{array}$ & 1974 & $\begin{array}{l}\text { Use of SA-4 } 14 \text { Grade C for Class } 2 \\
\text { and } 3 \text { Components, Section III, } \\
\text { Division } 1\end{array}$ & reg1.85.r29 & 1993 & $\mathbf{N}$ & Annulled by ASME & $n / a$ & $\mathrm{n} / \mathbf{a}$ \\
\hline 1386 & ASME & $\begin{array}{l}\text { ASME Code } \\
\text { Case } 1651\end{array}$ & 1974 & $\begin{array}{l}\text { Interim Requirements for } \\
\text { Certification of Component } \\
\text { Supports, Section III, Subsection } \\
\text { NF }\end{array}$ & $\operatorname{reg} 1.84 .129$ & 1993 & $N$ & Annulled by ASME & $n / a$ & $n / a$ \\
\hline 1387 & ASME & $\begin{array}{l}\text { ASME Code } \\
\text { Case } 1657\end{array}$ & 1977 & $\begin{array}{l}\text { Stress Criteria for Class } 2 \text { and } 3 \\
\text { Atmospheric and Low Pressure (0- } \\
15 \text { psig) Steel Storage Tanks } \\
\end{array}$ & $\operatorname{reg} 1.84 .129$ & 1993 & $\mathbf{N}$ & Annulled by ASME & $n / a$ & $n / a$ \\
\hline 1388 & ASME & $\begin{array}{l}\text { ASME Code } \\
\text { Case } 1659\end{array}$ & 1977 & $\begin{array}{l}\text { Interconnection of Two Piping } \\
\text { Systems for Section III, Class 1, 2, } \\
\text { and } 3 \text { Construction }\end{array}$ & $\operatorname{rog} 1.84 .129$ & 1993 & $\bar{N}$ & Annulled by ASME & $n / a$ & $n / a$ \\
\hline
\end{tabular}


Codes and Standards and Other Guidance Cited in Regulatory Documents

Part A - Industry Consensus Codes and Standards (continued)

\begin{tabular}{|c|c|c|c|c|c|c|c|c|c|c|}
\hline $\begin{array}{l}\text { Rocord } \\
\text { No. }\end{array}$ & Codo & Stendard & Siroulard & Tue & Documsent & Dor & $\begin{array}{l}\text { Aocopts } \\
\text { collity }\end{array}$ & Comments & Curnont Vorolon & Qnift, \\
\hline 1389 & ASME & $\begin{array}{l}\text { ASME Code } \\
\text { Case } 1661\end{array}$ & 1978 & $\begin{array}{l}\text { Postweld Heat Treatment P-No. } 1 \\
\text { Materials for Section III, Class I } \\
\text { Vessels }\end{array}$ & reg1.84.r29 & 1993 & $\mathbf{N}$ & Annulled by ASME & $n / a$ & $n / a$ \\
\hline 1390 & ASME & $\begin{array}{l}\text { ASME Code } \\
\text { Case } 1662\end{array}$ & 1978 & $\begin{array}{l}\text { Shop Assembly of Components, } \\
\text { Appurtenances and Piping } \\
\text { Subassemblies for Section III, Class } \\
1.2,3 \text { and MC Construction }\end{array}$ & reg1.84.r29 & 1993 & $\mathbf{N}$ & Annulled by ASME & $n / a$ & $n / a$ \\
\hline 1391 & ASME & $\begin{array}{l}\text { ASME Code } \\
\text { Case } 1664\end{array}$ & 1977 & $\begin{array}{l}\text { Use of Cr-Ni-Fe-Mo-Cu-Cb } \\
\text { Stabilized Alloy Cb-3 for Section III } \\
\text { Class } 2 \text { and } 3 \text { Construction }\end{array}$ & reg1.85.r29 & 1993 & $\mathbf{N}$ & Annulled by ASME & $n / a$ & $n / a$ \\
\hline 1392 & ASME & \begin{tabular}{|l|} 
ASME Code \\
Case 1666 \\
\end{tabular} & 1975 & $\begin{array}{l}\text { Use of SB-12, Alloy } 122 \text { for Section } \\
\text { iil, Class } 2 \text { and } 3 \text { Construction }\end{array}$ & $\operatorname{reg} 1.85 . r 29$ & 1993 & $\mathbf{N}$ & Annulled by ASME & $n / a$ & $n / a$ \\
\hline 1393 & ASME & \begin{tabular}{|l} 
ASME Code \\
Case 1672 \\
\end{tabular} & 1977 & $\begin{array}{l}\text { Nuclear Valves for Section III, } \\
\text { Division } 1 \text { Class 1, 2, } 3 \text { Construction }\end{array}$ & reg1.84.r29 & 1993 & $\mathbf{N}$ & Annulled by ASME & $n / a$ & $n / a$ \\
\hline 1394 & ASME & $\begin{array}{l}\text { ASME Code } \\
\text { Case } 1675\end{array}$ & 1976 & $\begin{array}{l}\text { Tubesheet to Shell or Formed Head } \\
\text { Weld Joints, Section III, Class I } \\
\text { Vessels }\end{array}$ & reg1.84.r29 & 1993 & $\mathbf{N}$ & Annulled by ASME & $n / a$ & $n / a$ \\
\hline 1395 & ASME & \begin{tabular}{|l} 
ASME Code \\
Case 1676
\end{tabular} & 1976 & $\begin{array}{l}\text { Clarification of Stress Intensities in } \\
\text { Curved Pipe or Welded Elbows, } \\
\text { Section III }\end{array}$ & reg1.84.r29 & 1993 & $\mathbf{N}$ & Annulled by ASME & $n / a$ & $n / a$ \\
\hline 1396 & ASME & \begin{tabular}{|l} 
ASME Code \\
Case 1678
\end{tabular} & 1980 & $\begin{array}{l}\text { Butterfly Valves of Circular Cross } \\
\text { Section Larger than } 24 \text { in. NPS for } \\
\text { Section III, Class } 2 \text { and } 3 \\
\text { Construction } \\
\end{array}$ & reg1.84.r29 & 1993 & $\mathbf{N}$ & Annulled by ASME & $n / a$ & $n / a$ \\
\hline 1397 & ASME & $\begin{array}{l}\text { ASME Code } \\
\text { Case } 1682\end{array}$ & 1975 & $\begin{array}{l}\text { Avternate Rules for Material } \\
\text { Manutacturers and Suppliers, } \\
\text { Section III, Subarticle NA-3700 }\end{array}$ & reg1.85.r29 & 1993 & $\mathbf{N}$ & Annulled by ASME & $n / a$ & $n / a$ \\
\hline 1398 & ASME & $\begin{array}{l}\text { ASME Code } \\
\text { Case 1682-1 }\end{array}$ & 1975 & $\begin{array}{l}\text { Alternate Rules for Material } \\
\text { Manufacturers and Suppliers, } \\
\text { Section III, Subarticle NA-3700 } \\
\end{array}$ & reg1.85.r2o & 1993 & $\mathbf{N}$ & Annulled by ASME & $n / a$ & $n / a$ \\
\hline 1399 & ASME & $\begin{array}{l}\text { ASME Code } \\
\text { Case } 1683\end{array}$ & 1976 & $\begin{array}{l}\text { Bolt Holes for Section II, Division } 1, \\
\text { Class 1, } 2,3 \text { and MC Component } \\
\text { Supports }\end{array}$ & reg1.84.r29 & 1993 & $\mathbf{N}$ & Annulled by ASME & $n / a$ & $n / a$ \\
\hline
\end{tabular}




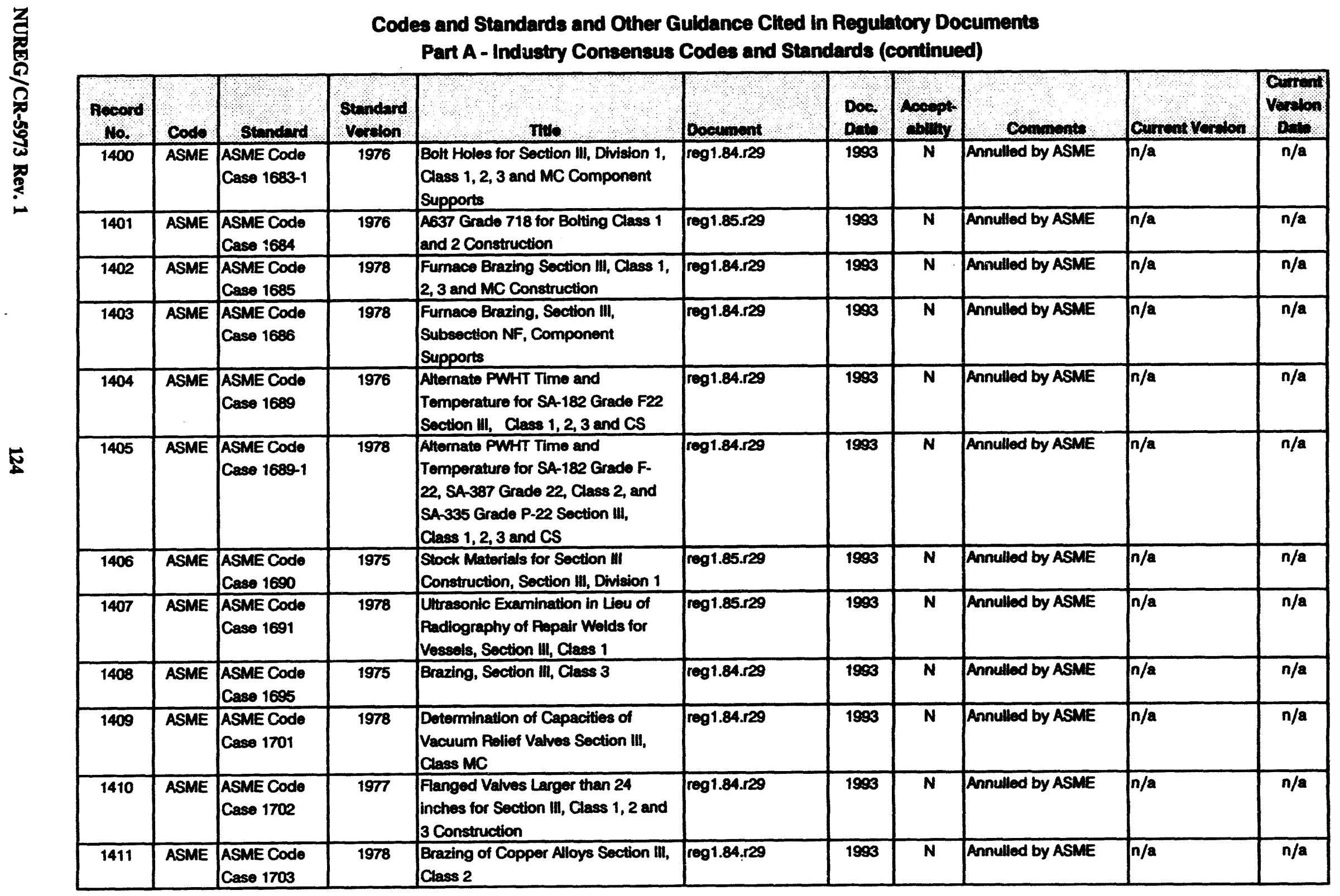


Codes and Standards and Other Guidance Cited in Regulatory Documents

Part A - Industry Consensus Codes and Standards (continued)

\begin{tabular}{|c|c|c|c|c|c|c|c|c|c|c|}
\hline $\begin{array}{l}\text { Hecord } \\
\text { No. }\end{array}$ & Cods & Stand and & $\begin{array}{l}\text { Stenderd } \\
\text { Vordien }\end{array}$ & L $\quad$ nte $\quad$ & Docennens: & Does & And & 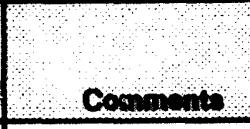 & 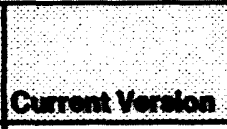 & 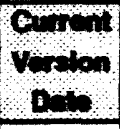 \\
\hline 1412 & ASME & $\begin{array}{l}\text { ASME Code } \\
\text { Case } 1706\end{array}$ & 1975 & $\begin{array}{l}\text { Data Roport Forms for Component } \\
\text { Supports, Section HI, Class 1,2 and } \\
3\end{array}$ & reg1.84.r29 & 1993 & $\mathbf{N}$ & Annulled by ASME & $n / a$ & $n / a$ \\
\hline 1413 & ASME & $\begin{array}{l}\text { ASME Code } \\
\text { Case } 1713\end{array}$ & 1975 & $\begin{array}{l}\text { Small Material Moms, Section HI, } \\
\text { Division 1, Class 1, 2, 3, CS and MC }\end{array}$ & reg1.85.r29 & 1993 & $\mathbf{N}$ & Annulled by ASME & $n / a$ & $n / a$ \\
\hline 1414 & ASME & $\begin{array}{l}\text { ASME Code } \\
\text { Case } 1714 \\
\end{array}$ & 1977 & $\begin{array}{l}\text { Postweld Hoat Troatment of P-1 } \\
\text { Material, Section ill, Class MC }\end{array}$ & $\operatorname{reg} 1.85 .229$ & 1993 & $\mathbf{N}$ & Annulled by ASME & $n / a$ & $n / a$ \\
\hline 1415 & ASME & \begin{tabular}{|l|} 
ASME Code \\
Case 1718
\end{tabular} & 1976 & $\begin{array}{l}\text { Design of Structural Connections } \\
\text { for Linear Type Component } \\
\text { Supports, Section II. Division 1. } \\
\text { Class 1, } 2 \text { and } 3 \text { and MC }\end{array}$ & reg1.84.r29 & 1993 & $\mathbf{N}$ & Annulled by ASME & $n / a$ & $n / a$ \\
\hline 1416 & ASME & $\begin{array}{l}\text { ASME Code } \\
\text { Case } 1719\end{array}$ & 1976 & $\begin{array}{l}\text { Single-Welded, Full-Penetration } \\
\text { Sidewall Butt Joints in Atmospheric } \\
\text { Storage Tanks, Section III, Diviaion } \\
\text { 1, Class } 2\end{array}$ & reg1.84.529 & 1993 & $\bar{N}$ & Annulled by ASME & $n / a$ & $n / a$ \\
\hline 1417 & ASME & $\begin{array}{l}\text { ASME Code } \\
\text { Cese } 1720 \\
\end{array}$ & 1976 & $\begin{array}{l}\text { Wold End Preparation for Section } \\
\text { III, Division } 1 \text { Construction }\end{array}$ & reg1.84.r29 & 1993 & $\mathbf{N}$ & Annulled by ASME & $n / a$ & $n / \mathbf{a}$ \\
\hline 1418 & ASME & $\begin{array}{l}\text { ASME Code } \\
\text { Case 1720-1 }\end{array}$ & 1978 & $\begin{array}{l}\text { Wold End Preperation for Section } \\
\text { Iil, Division } 1 \text { Construction }\end{array}$ & reg1.84.r29 & 1993 & $\mathbf{N}$ & Annulled by ASME & $n / a$ & $n / a$ \\
\hline 1419 & ASME & $\begin{array}{l}\text { ASME Code } \\
\text { Case } 1722\end{array}$ & 1979 & $\begin{array}{l}\text { Vacuum, Carbon Deoxidized SA } \\
508 \text { Forgings, Section III, Diviaion } 1 \text {, } \\
\text { and Vil, Division } 1 \text { and } 2\end{array}$ & reg1.85.529 & 1993 & $\mathbf{N}$ & Annulled by ASME & $n / a$ & $n / a$ \\
\hline 1420 & ASME & $\begin{array}{l}\text { ASME Codo } \\
\text { Case } 1728\end{array}$ & 1977 & $\begin{array}{l}\text { Steel Structural Shapes and Small } \\
\text { Material Products for Component } \\
\text { Supports, Section III, Division } 1 \\
\text { Construction }\end{array}$ & $\operatorname{reg} 1.85 .129$ & 1993 & $\mathbf{N}$ & Annulled by ASME & $n / a$ & $n / a$ \\
\hline 1421 & ASME & $\begin{array}{l}\text { ASME Code } \\
\text { Case } 1740 \\
\end{array}$ & 1976 & $\begin{array}{l}\text { Weld Motal Test, Section M, Class } \\
1,2,3, \text { MC and CS }\end{array}$ & $\operatorname{reg} 1.85 . \mathrm{r} 29$ & 1993 & $\mathbf{N}$ & Annulled by ASME & $n / a$ & $n / a$ \\
\hline 1422 & ASME & $\begin{array}{l}\text { ASME Code } \\
\text { Case } 1741\end{array}$ & 1977 & $\begin{array}{l}\text { Interim Pules for the Required } \\
\text { Number of impact Tests for Polled } \\
\text { Shapes, Section III, Division 1, } \\
\text { Subsection NF, Component } \\
\text { Supports }\end{array}$ & reg1.85.r29 & 1993 & $\mathbf{N}$ & Annulled by ASME & $n / a$ & $n / a$ \\
\hline
\end{tabular}




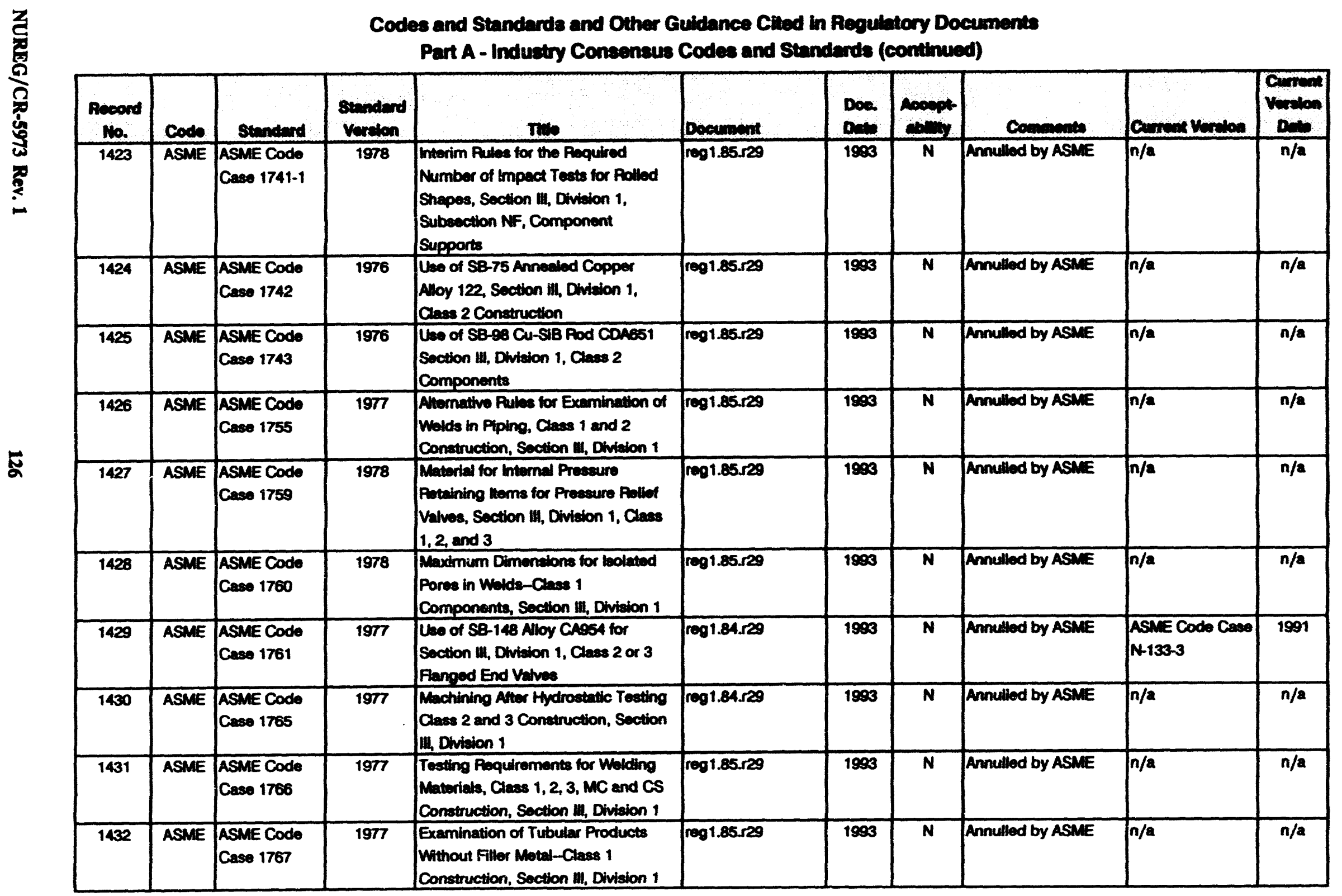




\section{Codes and Standards and Other Guidance Chted in Regulatory Documents}

Part A - Industry Consensus Codes and Standards (continued)

\begin{tabular}{|c|c|c|c|c|c|c|c|c|c|c|}
\hline $\begin{array}{l}\text { Hocord } \\
\text { No. }\end{array}$ & Code & Standerd: & $\begin{array}{l}\text { Stendard } \\
\text { verslon }\end{array}$ & Ino $\quad \ldots$ & Boovenent & $\begin{array}{l}\text { Dos. } \\
\text { Dete }\end{array}$ & Aosopt. & Counesents & Ounchl Voulon & $\operatorname{loph}_{n+1}$ \\
\hline 1433 & ASME & $\begin{array}{l}\text { ASME Code } \\
\text { Case } 1768\end{array}$ & 1978 & $\begin{array}{l}\text { Permanent Attachments to } \\
\text { Containment Vessels-Class MC, } \\
\text { Section III, Division } 1\end{array}$ & rog1.84.r29 & 1993 & $\mathbf{N}$ & Annulled by ASME & $n / a$ & $n / a$ \\
\hline 1434 & ASME & $\begin{array}{l}\text { ASME Code } \\
\text { Case } 1769 \\
\end{array}$ & 1977 & $\begin{array}{l}\text { Qualification of NDE Level III } \\
\text { Personnel, Section III, Division } 1\end{array}$ & reg1.84.r29 & 1993 & $\mathbf{N}$ & Annulled by ASME & $n / a$ & $n / a$ \\
\hline 1435 & ASME & $\begin{array}{l}\text { ASME Code } \\
\text { Case 1769-1 } \\
\end{array}$ & 1977 & $\begin{array}{l}\text { Qualificaticn of NDE Level III } \\
\text { Personnel, Section III, Division } 1\end{array}$ & reg1.84.r29 & 1993 & $\mathbf{N}$ & Annulled by ASME & $n / a$ & $n / a$ \\
\hline 1436 & ASME & $\begin{array}{l}\text { ASME Code } \\
\text { Case } 1773\end{array}$ & 1977 & $\begin{array}{l}\text { Use of Other Product Forms of } \\
\text { Materials for Valves, Section II., } \\
\text { Division } 1\end{array}$ & reg1.85.r29 & 1993 & $\mathbf{N}$ & Annulled by ASME & $n / a$ & $n / a$ \\
\hline 1437 & ASME & $\begin{array}{l}\text { ASME Code } \\
\text { Case } 1774\end{array}$ & 1977 & $\begin{array}{l}\text { Minimum Wall Thickness for Class } \\
2 \text { and } 3 \text { Valves, Section III, Division } \\
1\end{array}$ & reg1.84.r29 & 1993 & $\mathbf{N}$ & Annulled by ASNE & $n / a$ & $n / a$ \\
\hline 1438 & ASME & $\begin{array}{l}\text { ASME Code } \\
\text { Case } 1775\end{array}$ & 1979 & $\begin{array}{l}\text { Data Report Forms for Core } \\
\text { Support Structures, Class CS, } \\
\text { Section III, Division } 1\end{array}$ & reg1.84.r29 & 1998 & $\mathbf{N}$ & Annulled by ASME & $n / a$ & $n / a$ \\
\hline 1439 & ASME & $\begin{array}{l}\text { ASME Code } \\
\text { Case } 1777\end{array}$ & 1977 & $\begin{array}{l}\text { Use of SA-106, Grade C in Class } \\
\text { MC Construction, Section III, } \\
\text { Division } 1\end{array}$ & $\operatorname{reg} 1.85 . r 29$ & 1993 & $\mathbf{N}$ & Annulled by ASME & $n / a$ & $n / a$ \\
\hline 1440 & ASME & $\begin{array}{l}\text { ASME Code } \\
\text { Case } 1780\end{array}$ & 1978 & $\begin{array}{l}\text { Hydrostatic Testing and Stamping } \\
\text { of Pumps for Class } 1 \text { Construction, } \\
\text { Section III, Division } 1\end{array}$ & reg1.84.r29 & 1993 & $\mathbf{N}$ & Annulled by ASME & $n / a$ & $n / a$ \\
\hline 1441 & ASME & \begin{tabular}{|l} 
ASME Code \\
Case 1783
\end{tabular} & 1977 & $\begin{array}{l}\text { Qualification of Nondestructive } \\
\text { Examination Personnel, Section III, } \\
\text { Dlvision } 1\end{array}$ & reg1.84.r29 & 1993 & $\mathbf{N}$ & Annulled by ASME & $n / a$ & $n / a$ \\
\hline 1442 & ASME & $\begin{array}{l}\text { ASME Code } \\
\text { Case 1783-1 }\end{array}$ & 1979 & $\begin{array}{l}\text { Qualification of Nondestructive } \\
\text { Examination Personnel, Section III, } \\
\text { Division } 1\end{array}$ & reg1.84.r29 & 1993 & $\mathbf{N}$ & Annulled by ASME & $n / a$ & $n / a$ \\
\hline 1443 & ASME & $\begin{array}{l}\text { ASME Code } \\
\text { Case } 1787\end{array}$ & 1978 & $\begin{array}{l}\text { Depth of Weld Repairs for Forgings, } \\
\text { Section III Division 1, Class 1, 2, 3, } \\
\text { MC and CS Construction Structural } \\
\text { Steel Rolled Shapes, Section III, } \\
\text { Division 1, Class 2, 3, and MC }\end{array}$ & reg1.85.r29 & 1993 & $\mathbf{N}$ & Annulled by ASME & $n / a$ & $n / a$ \\
\hline
\end{tabular}




\begin{tabular}{|c|c|c|c|c|c|c|c|c|c|c|}
\hline \multicolumn{11}{|c|}{$\begin{array}{c}\text { Codes and Standards and Other Guidance Cited in Regulatory Documents } \\
\text { Part A - Industry Consensus Codes and Standards (continued) }\end{array}$} \\
\hline $\begin{array}{l}\text { Hecord } \\
\text { Ne. }\end{array}$ & Code & standard & Standard & L_ _ $\quad$ ntwo & Document & $\begin{array}{l}\text { Doc. } \\
\text { Date }\end{array}$ & Acoopt & Comments & Current Verolon & $\begin{array}{l}\text { Corrent } \\
\text { verion } \\
\text { pous: }\end{array}$ \\
\hline 1444 & ASME & $\begin{array}{l}\text { ASME Code } \\
\text { Case } 1798\end{array}$ & 1978 & $\begin{array}{l}\text { Use of ASTM A352-75, Grades LCA } \\
\text { and LCC, Section III, Division 1, } \\
\text { Class 1, 2, and 3 }\end{array}$ & reg1.85.r29 & 1993 & $N$ & Annulled by ASME & $n / a$ & $n / a$ \\
\hline 1445 & ASME & $\begin{array}{l}\text { ASME Code } \\
\text { Case } 1808\end{array}$ & 1978 & $\begin{array}{l}\text { F-Number Classification of Low } \\
\text { Allow and Carbon Steel Bare Rod } \\
\text { Electrodes Sections I, II, III, N, V, } \\
\text { VIII, and XX }\end{array}$ & $\operatorname{reg} 1.84 .229$ & 1993 & $\mathbf{N}$ & Annulled by ASME & $n / a$ & $n / a$ \\
\hline 1446 & $\overline{A S M E}$ & $\begin{array}{l}\text { ASME Code } \\
\text { Case } 1810\end{array}$ & 1980 & $\begin{array}{l}\text { Testing Lots of Carbon Stoel Solid, } \\
\text { Bare Welding Electrode or Wire, } \\
\text { Section III, Division 1, Class 1, 2, 3, } \\
\text { MC, and CS }\end{array}$ & $\operatorname{reg} 1.85 .129$ & 1993 & $\mathbf{N}$ & Annulled by ASME & $n / a$ & $n / a$ \\
\hline 1447 & $\overline{\text { ASME }}$ & $\begin{array}{l}\text { ASME Code } \\
\text { Case } 1819\end{array}$ & 1978 & $\begin{array}{l}\text { Use of Type XM-19 for } \\
\text { Construction, Section III Division } 1 \text {, } \\
\text { Class } 1,2 \text {, and } 3\end{array}$ & $\operatorname{rog} 1.85 .129$ & 1993 & $\mathbf{N}$ & Annulled by ASME & $n / a$ & $n / a$ \\
\hline 1448 & ASME & \begin{tabular}{|l} 
ASME Code \\
Case N-1-7 \\
$(1332-7)$
\end{tabular} & 1982 & $\begin{array}{l}\text { Requirements for Steel Forgings, } \\
\text { Section III, Division } 1\end{array}$ & $\operatorname{rog} 1.85 .129$ & 1993 & $\mathbf{N}$ & Annullod by ASME & $n / a$ & $n / a$ \\
\hline 1449 & ASME & $\begin{array}{l}\text { ASME Code } \\
\text { Case N-2 } \\
(1334-3)\end{array}$ & 1981 & Steel Bars and Shapes, Section III & reg1.85.r29 & 1993 & $\bar{N}$ & Annulled by ASME & $n / a$ & $n / a$ \\
\hline 1450 & $\widehat{\text { ASME }}$ & $\begin{array}{l}\text { ASME Code } \\
\text { Case N-3-10 } \\
(1335-10)\end{array}$ & 1984 & $\begin{array}{l}\text { Requirements for Bolting Materials, } \\
\text { Section III }\end{array}$ & $\operatorname{reg} 1.85 .129$ & 1993 & $\mathbf{N}$ & Annulled by ASME & $n / a$ & $n / a$ \\
\hline 1451 & $\overline{\text { ASME }}$ & $\begin{array}{l}\text { ASME Code } \\
\text { Case N-4-11 } \\
(1337-11)\end{array}$ & 1990 & $\begin{array}{l}\text { Special Type } 403 \text { Modified } \\
\text { Forgings or Bars, Section III, } \\
\text { Division 1, Class } 1 \text { and CS }\end{array}$ & reg1.85.r29 & 1993 & $\bar{A}$ & & $\begin{array}{l}\text { ASME Code Case } \\
\mathrm{N}-4-11\end{array}$ & 1993 \\
\hline 1452 & ASME & $\begin{array}{l}\text { ASME Code } \\
\text { Case N-5 } \\
(\mathbf{1 3 4 4 5 )}\end{array}$ & 1982 & $\begin{array}{l}\text { Nickel-Chromium, Age Hardenable } \\
\text { Alloys (Alloy X750), Section IIi }\end{array}$ & reg1.85.r29 & 1993 & $\bar{N}$ & Annulled by ASME & $n / 2$ & $n / a$ \\
\hline 1453 & ASME & $\begin{array}{l}\text { ASME Code } \\
\text { Case N-5-1 }\end{array}$ & 1985 & $\begin{array}{l}\text { Nickel Chromium Age Hardenable } \\
\text { Alloys (Alloy X750), Section III, } \\
\text { Division 1, Classes 1, 2, 3, MC, and } \\
\text { CS }\end{array}$ & reg1.85.r29 & 1993 & $\bar{N}$ & Annulled by ASME & $n / a$ & $n / a$ \\
\hline
\end{tabular}


Codes and Standards and Other Guldance Clted in Regulatory Documents
Part A - Industry Consensus Codes and Standards (continued)

\begin{tabular}{|c|c|c|c|c|c|c|c|c|c|c|}
\hline $\begin{array}{l}\text { Aocord } \\
\text { No. }\end{array}$ & Code & Standand & $\begin{array}{l}\text { Btindard } \\
\text { Vorelon }\end{array}$ & $\pi$ Tlo & Dooument & $\begin{array}{l}\text { Doo. } \\
\text { Date. }\end{array}$ & $\begin{array}{l}\text { Acoept- } \\
\text { bellity }\end{array}$ & Commonta & Curront Vordon & owton \\
\hline 1454 & ASME & $\begin{array}{l}\text { ASME Code } \\
\text { Case N-6 } \\
(1345-2) \\
\end{array}$ & 1979 & $\begin{array}{l}\text { Requirements for Nickel- } \\
\text { Molybdenum-Chromium-Iron } \\
\text { Alloys, Section III }\end{array}$ & reg1.85.r29 & 1993 & $\mathbf{N}$ & Annulled by ASME & $n / a$ & $n / a$ \\
\hline 1455 & ASME & $\begin{array}{l}\text { ASME Code } \\
\text { Case N-7 } \\
(1358-5)\end{array}$ & 1982 & $\begin{array}{l}\text { High Vield Strength Steol, Section } \\
\text { II!, Division 1, Class } 1 \text { Vessels }\end{array}$ & $\operatorname{reg} 1.85 .129$ & 1993 & $\bar{N}$ & Annulled by ASME & $\begin{array}{l}\text { ASME Code Case } \\
\text { N-7-1 }\end{array}$ & 1992 \\
\hline 1456 & ASME & $\begin{array}{l}\text { ASME Code } \\
\text { Case N-7-1 }\end{array}$ & 1989 & $\begin{array}{l}\text { High Yield Strength Steel, Section } \\
\text { III, Diviaion 1, Class I Vessols }\end{array}$ & reg1.85.r29 & 1993 & $\bar{A}$ & & $\begin{array}{l}\text { ASME Code Case } \\
N-7-1\end{array}$ & 1992 \\
\hline 1457 & ASME & $\begin{array}{l}\text { ASME Code } \\
\text { Case N-9-4 } \\
(1395-4) \\
\end{array}$ & 1982 & $\begin{array}{l}\text { SA-508, Class } 2 \text { Forgings with } \\
\text { Modified Manganese Content, } \\
\text { Section III }\end{array}$ & reg1.85.r29 & 1993 & $\mathbf{N}$ & Annulled by ASME & 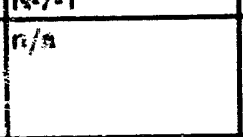 & $n / a$ \\
\hline 1458 & ASME & $\begin{array}{l}\text { ASME Code } \\
\text { Case N-10 } \\
(1407-3)\end{array}$ & 1987 & $\begin{array}{l}\text { Time of Examination for Classes 1, } \\
2 \text {, and } 3 \text { Section III Vessels }\end{array}$ & reg1.85.r29 & 1993 & $\mathbf{N}$ & Annulled by ASME & $n / a$ & $n / a$ \\
\hline 1459 & ASME & $\begin{array}{l}\text { ASME Code } \\
\text { Case N-11-5 } \\
(1414-5)\end{array}$ & 1980 & $\begin{array}{l}\text { High Vield Strength Cr-Mo Steel for } \\
\text { Section III, Division 1, Class } 1 \\
\text { Vessels }\end{array}$ & reg1.85.r29 & 1993 & $\mathbf{N}$ & Annulled by ASME & $n / a$ & $n / a$ \\
\hline 1460 & ASME & $\begin{array}{l}\text { ASME Code } \\
\text { Case N-15 } \\
(1456-2)\end{array}$ & 1979 & $\begin{array}{l}\text { Substitution of Ultrasonic } \\
\text { Examination for Progressive } \\
\text { Penetrant or Magnetic Particle } \\
\text { Examinations of Partial Penetration } \\
\text { and Oblique Nozzle Attachment } \\
\text { Welds, Section III } \\
\end{array}$ & reg1.85.r29 & 1993 & $\mathbf{N}$ & Annulled by ASME & $n / a$ & $n / a$ \\
\hline 1461 & ASME & $\begin{array}{l}\text { ASME Code } \\
\text { Case N-17 } \\
(1474-1) \\
\end{array}$ & 1991 & $\begin{array}{l}\text { Integrally Finned Tubes for Section } \\
\text { III }\end{array}$ & reg1.85.r29 & 1993 & $\mathbf{N}$ & Annulled by ASME & $n / a$ & $n / a$ \\
\hline 1462 & ASME & $\begin{array}{l}\text { ASME Code } \\
\text { Case N-20 } \\
(1484-3)\end{array}$ & 1985 & $\begin{array}{l}\text { SB-163 Nickel-Chromium Iron } \\
\text { Tubing (Alloy } 600 \text { and 690) and } \\
\text { Nickel-Iron-Chromium Alloy } 800 \text { at } \\
\text { a Specified Minimum Yield } \\
\text { Strength of } 40.0 \mathrm{Ksl} \text {, and Cold } \\
\text { Worked Alloy } 800 \text { at a Yield } \\
\text { Strength of } 47.0 \mathrm{Ksi} \text {, Section III, } \\
\text { Division 1, Class } 1\end{array}$ & reg1.85.r29 & 1993 & $\mathbf{N}$ & Annulled by ASME & $\begin{array}{l}\text { ASME Code Case } \\
\text { N-20-3 }\end{array}$ & 1991 \\
\hline
\end{tabular}


Codes and Standards and Other Guidance Cited in Regulatory Documents

Part A - Industry Consensus Codes and Standards (continued)

\begin{tabular}{|c|c|c|c|c|c|c|c|c|c|c|}
\hline $\begin{array}{l}\text { Reoord } \\
\text { No. }\end{array}$ & code & Standerd & Standard & 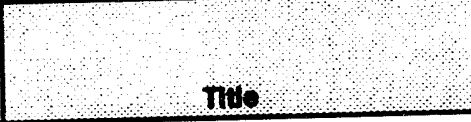 & Document. & $\frac{006 .}{1993}$ & poopt & Qomunnto & Gunour vorous & on, \\
\hline 1463 & ASME & $\begin{array}{l}\text { ASME Code } \\
\text { Case N-20-1 }\end{array}$ & $\frac{190101}{1997}$ & $\begin{array}{l}\text { SB-163 Nickel-Chromium Iron } \\
\text { Tubing (Alloy } 600 \text { and } 690 \text { ) and } \\
\text { Nickel-Iron-Chromium Alloy } 800 \text { at } \\
\text { a Specified Minimum Yield } \\
\text { Strength of } 40.0 \mathrm{Ksi} \text {, and Cold } \\
\text { Worked Alloy } 800 \text { at a Yield } \\
\text { Strength of } 47.0 \mathrm{Ksi} \text {, Section III, } \\
\text { Division 1, Class } 1\end{array}$ & reg1.85.r29 & & $\frac{N}{N}$ & Annulled by ASME & 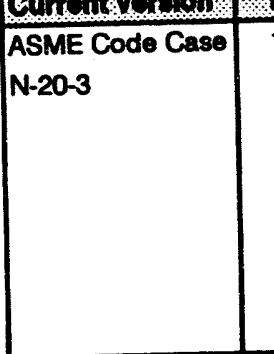 & 1991 \\
\hline 1464 & ASME & $\begin{array}{l}\text { ASME Code } \\
\text { Case N-20-2 }\end{array}$ & 1988 & $\begin{array}{l}\text { SB-163 Nickel-Chromium Iron } \\
\text { Tubing (Alloy } 600 \text { and } 690 \text { ) and } \\
\text { Nickel-Iron-Chromium Alloy } 800 \text { at } \\
\text { a Spectiled Minimum Yield } \\
\text { Strength of } 40.0 \mathrm{Ksi} \text {, and Cold } \\
\text { Worked Alloy } 800 \text { at a Viold } \\
\text { Strength of } 47.0 \mathrm{Ksi} \text {, Section III, } \\
\text { Division 1, Class } 1\end{array}$ & reg1.85.r29 & 1993 & $\mathbf{N}$ & Annulled by ASME & $\begin{array}{l}\text { ASME Code Case } \\
\text { N-2O-3 }\end{array}$ & 1991 \\
\hline 1465 & ASME & $\begin{array}{l}\text { ASME Code } \\
\text { Case N-20-3 }\end{array}$ & 1988 & $\begin{array}{l}\text { SB-163 Nickel-Chromium lron } \\
\text { Tubing (Alloy } 600 \text { and } 690 \text { ) and } \\
\text { Nickel-Iron-Chromium Alloy } 800 \text { at } \\
\text { a Specified Minimum Yilir" } \\
\text { Strength of } 40.0 \mathrm{Ksi} \text {, and Cold } \\
\text { Worked Alloy } 800 \text { at a Yield } \\
\text { Strength of } 47.0 \mathrm{Ksi} \text {, Section III, } \\
\text { Division 1, Class } 1\end{array}$ & reg1.85.r29 & 1993 & $\bar{A}$ & & $\begin{array}{l}\text { ASME Code Case } \\
N-20-3\end{array}$ & 1991 \\
\hline 1466 & ASME & $\begin{array}{l}\text { ASME Code } \\
\text { Case N-22 } \\
(1498-1)\end{array}$ & 1982 & $\begin{array}{l}\text { SA-508-Class } 2 \text { and 3, Minimum } \\
\text { Tempering Temperature, Section III }\end{array}$ & reg1.85.r29 & 1993 & $N$ & Annulled by ASME & $n / a$ & $n / a$ \\
\hline 1467 & ASME & $\begin{array}{l}\text { ASME Code } \\
\text { Case N-24 } \\
(1516-2)\end{array}$ & 1980 & $\begin{array}{l}\text { Welding of Seats or Minor Internal } \\
\text { Permanent Attachments in Valves } \\
\text { for Section III Applications }\end{array}$ & reg1.84.r29 & 1993 & $N$ & Annulled by ASME & $n / a$ & $n / a$ \\
\hline 1468 & ASME & $\begin{array}{l}\text { ASME Code } \\
\text { Case N-26 } \\
(1527)\end{array}$ & 1991 & Integrally Finned Tubes, Section III & $\operatorname{reg} 1.85 .129$ & 1993 & $\mathbf{N}$ & Annulled by ASME & $n / a$ & $n / a$ \\
\hline
\end{tabular}


Codes and Standards and Other Guidance Clted in Regulatory Documents

Part A - Industry Consensus Codes and Standards (continued)

\begin{tabular}{|c|c|c|c|c|c|c|c|c|c|c|}
\hline $\begin{array}{l}\text { Aocord } \\
\text { No. }\end{array}$ & codo & Stundard & stondard & (ै. & Dooumont & $\begin{array}{l}\text { Doo. } \\
0.16\end{array}$ & Aopepts & Comments: & Curront vorton & roonon \\
\hline 1469 & ASME & $\begin{array}{l}\text { ASME Code } \\
\text { Case N-30-1 } \\
\text { (1539) }\end{array}$ & 1977 & $\begin{array}{l}\text { Metal Bellows and Metal } \\
\text { Diaphragm Stem Sealed Valves, } \\
\text { Section III, Classes 1,2, and } 3 \\
\end{array}$ & reg1.84.r29 & 1993 & $N$ & Annulled by ASME & $n / a$ & $n / a$ \\
\hline 1470 & ASME & $\begin{array}{l}\text { ASME Code } \\
\text { Case N-30-1 } \\
(1539-1)\end{array}$ & 1981 & $\begin{array}{l}\text { Metal Bellows and Metal } \\
\text { Diaphragm Stem Sealed Valves, } \\
\text { Section III, Classes 1, 2, and 3 }\end{array}$ & reg1.84.r29 & 1993 & $\mathbf{N}$ & Annulled by ASME & $n / a$ & $n / a$ \\
\hline 1471 & ASME & $\begin{array}{l}\text { ASME Code } \\
\text { Case N-31 } \\
(1540-2)\end{array}$ & 1985 & $\begin{array}{l}\text { Elastomer Diaphragm Valves, } \\
\text { Section III Class } 2 \text { and } 3\end{array}$ & reg1.84.r29 & 1993 & $\bar{N}$ & Annulled by ASME & $n / a$ & $n / a$ \\
\hline 1472 & ASME & $\begin{array}{l}\text { ASME Code } \\
\text { Case N-31-1 }\end{array}$ & 1991 & $\begin{array}{l}\text { Elastomer Diaphragm Valves, } \\
\text { Section III, Class } 2 \text { and } 3 \\
\end{array}$ & reg1.84.r29 & 1993 & $\mathbf{P}$ & Annulled by ASME & $n / a$ & $n / a$ \\
\hline 1473 & ASME & $\begin{array}{l}\text { ASME Code } \\
\text { Case N-32-3 } \\
(1541-3)\end{array}$ & 1979 & $\begin{array}{l}\text { Hydrostatic Testing of Embedded } \\
\text { Class } 2 \text { and Class } 3 \text { Piping for } \\
\text { Section III Construction }\end{array}$ & reg1.84.r29 & 1993 & $\mathbf{N}$ & Annulled by ASME & $n / a$ & $n / a$ \\
\hline 1474 & ASME & $\begin{array}{l}\text { ASME Code } \\
\text { Case N-32-4 }\end{array}$ & 1984 & $\begin{array}{l}\text { Hydrostatic Testing of Embedded } \\
\text { Class } 2 \text { and } 3 \text { Piping for Section III, } \\
\text { Division } 1 \text { Construction }\end{array}$ & reg1.84.r29 & 1993 & $\mathbf{N}$ & Annulled by ASME & $n / a$ & $n / a$ \\
\hline 1475 & ASME & $\begin{array}{l}\text { ASME Code } \\
\text { Case N-33 } \\
(1542-1)\end{array}$ & 1985 & $\begin{array}{l}\text { Type } 403 \text { Forgings or Bars for } \\
\text { Bolting Material, Section III }\end{array}$ & reg1.85.r29 & 1993 & $\mathbf{N}$ & Annulled by ASME & $n / a$ & $n / a$ \\
\hline 1476 & ASME & $\begin{array}{l}\text { ASME Code } \\
\text { Case N-34 } \\
(1551)\end{array}$ & 1972 & $\begin{array}{l}\text { Inservice Inspection of Wolds on } \\
\text { Nuclear Components, Section XI }\end{array}$ & reg1.147.r10 & 1993 & $\mathbf{N}$ & Annulled by ASME & $n / a$ & $n / a$ \\
\hline 1477 & ASME & $\begin{array}{l}\text { ASME Code } \\
\text { Case N-35-1 } \\
(1552-1)\end{array}$ & 1979 & $\begin{array}{l}\text { Design by Analysis of Section III, } \\
\text { Class } 1 \text { Valves }\end{array}$ & reg1.84.r29 & 1993 & $\mathbf{N}$ & Annulied by ASME & $n / a$ & $n / a$ \\
\hline 1478 & ASME & $\begin{array}{l}\text { ASME Code } \\
\text { Case N-37-3 } \\
(1557-3) \\
\end{array}$ & 1982 & $\begin{array}{l}\text { Steel Products Refined by } \\
\text { Secondary Remelting, Section III } \\
\text { and VIII, Division } 1 \text { and } 2\end{array}$ & $\operatorname{reg} 1.85 . r 29$ & 1993 & $\mathbf{N}$ & Annulled by ASME & $n / a$ & $n / a$ \\
\hline 1479 & ASME & $\begin{array}{l}\text { ASME Code } \\
\text { Case N-41 } \\
(1571)\end{array}$ & 1985 & $\begin{array}{l}\text { Additional Material for SA-234 } \\
\text { Carbon Steel Fittings, Section III }\end{array}$ & reg1.85.r29 & 1993 & $\bar{N}$ & Annulled by ASME & $n / a$ & $n / a$ \\
\hline
\end{tabular}


Codes and Standards and Other Guidance Cited in Regulatory Documents

Part A - Industry Consensus Codes and Standards (continued)

\begin{tabular}{|c|c|c|c|c|c|c|c|c|c|c|}
\hline & & & & & & & & & & \\
\hline $\begin{array}{l}\text { Pocord } \\
\text { No. }\end{array}$ & codo & Stondurd & $\begin{array}{l}\text { Standard } \\
\text { Yorton }\end{array}$ & ( nol, & Dorounoul & $\begin{array}{l}\text { Dor. } \\
\text { D.to }\end{array}$ & Acoopts & Qownonts & 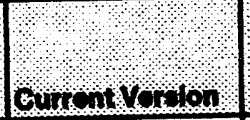 & Yoron \\
\hline 1480 & ASME & $\begin{array}{l}\text { ASME Code } \\
\text { Case N-43 } \\
\text { (1578) }\end{array}$ & 1982 & $\begin{array}{l}\text { SB-167 Nickel-Chromium-Iron } \\
\text { (Alloy 600) Pipe or Tube, Section III }\end{array}$ & reg1.85.r29 & 1993 & $\mathbf{N}$ & Annulled by ASME & $n / a$ & $n / a$ \\
\hline 1481 & ASME & $\begin{array}{l}\text { ASME Code } \\
\text { Case N-46 } \\
(1588)\end{array}$ & 1982 & $\begin{array}{l}\text { Electro-Etching of Section III Code } \\
\text { Symbols }\end{array}$ & reg1.84.r29 & 1993 & $\bar{N}$ & Annulled by ASME & $n / a$ & $n / a$ \\
\hline 1482 & ASME & $\begin{array}{l}\text { ASME Code } \\
\text { Case N-55 } \\
(1609-1) \\
\end{array}$ & 1984 & $\begin{array}{l}\text { Inertia and Continuous Drive (N-55) } \\
\text { Friction Welding, Section I, III, IV, } \\
\text { VIII, Division } 1 \text { and 2, and IX }\end{array}$ & reg1.84.r29 & 1993 & $\mathbf{N}$ & Annulled by ASME & $n / a$ & $n / a$ \\
\hline 1483 & ASME & $\begin{array}{l}\text { ASME Code } \\
\text { Case N-55-1 } \\
(1609)\end{array}$ & 1987 & $\begin{array}{l}\text { Inertia and Continuous Drive } \\
\text { Friction Welding, Section III, } \\
\text { Division } 1\end{array}$ & $\operatorname{reg1.84.129}$ & 1993 & $\mathbf{N}$ & Annulled by ASME & $n / a$ & $n / a$ \\
\hline 1484 & ASME & $\begin{array}{l}\text { ASME Code } \\
\text { Case N-56 } \\
(1612)\end{array}$ & 1973 & $\begin{array}{l}\text { Use of Type } 308 \text { Stainless Steol } \\
\text { Rod and Bar for Section III, Class 1, } \\
2,3 \text {, and CS Construction }\end{array}$ & $\operatorname{reg} 1.85 . r 29$ & 1993 & $\mathbf{N}$ & Annulled by ASME & $n / a$ & $n / a$ \\
\hline 1485 & ASME & $\begin{array}{l}\text { ASME Code } \\
\text { Case } N-60 \\
(16 \text { i8-2) }\end{array}$ & 1983 & $\begin{array}{l}\text { Material for Core Support } \\
\text { Structures, Section III, Division } 1\end{array}$ & reg1.85.r29 & 1993 & $\mathbf{N}$ & Annulled by ASME & $\begin{array}{l}\text { ASME Code Case } \\
\text { N-60-4 }\end{array}$ & 1991 \\
\hline 1486 & ASME & $\begin{array}{l}\text { ASME Code } \\
\text { Case N-60-1 }\end{array}$ & 1984 & $\begin{array}{l}\text { Material for Core Support } \\
\text { Structures, Section III, Division } 1\end{array}$ & reg1.85.r29 & 1993 & $\bar{N}$ & Annulled by ASME & $\begin{array}{l}\text { ASME Code Case } \\
N-60-4\end{array}$ & 1991 \\
\hline 1487 & ASME & $\begin{array}{l}\text { ASME Code } \\
\text { Case N-60-2 }\end{array}$ & 1985 & $\begin{array}{l}\text { Material for Core Support } \\
\text { Structures, Section III, Division } 1\end{array}$ & $\operatorname{reg} 1.85 . r 29$ & 1993 & $\mathbf{N}$ & Annulled by ASME & $\begin{array}{l}\text { ASME Code Case } \\
N-60-4\end{array}$ & 1991 \\
\hline 1488 & ASME & $\begin{array}{l}\text { ASME Code } \\
\text { Case N-60-3 }\end{array}$ & 1988 & $\begin{array}{l}\text { Material for Core Support } \\
\text { Structures, Section III, Division } 1\end{array}$ & reg1.85.r29 & 1993 & $\mathbf{N}$ & Annulled by ASME & $\begin{array}{l}\text { ASME Code Case } \\
\text { N-60-4 }\end{array}$ & 1991 \\
\hline 1489 & ASME & $\begin{array}{l}\text { ASME Code } \\
\text { Case N-60-4 }\end{array}$ & 1991 & $\begin{array}{l}\text { Material for Core Support } \\
\text { Structures, Section III, Division } 1\end{array}$ & reg1.85.r29 & 1993 & $\mathbf{P}$ & & $\begin{array}{l}\text { ASME Code Case } \\
\text { N-60-4 }\end{array}$ & 1991 \\
\hline 1490 & ASME & $\begin{array}{l}\text { ASME Code } \\
\text { Case N-61 } \\
(1620)\end{array}$ & 1982 & $\begin{array}{l}\text { Stress Category for Partial } \\
\text { Penetration Welded Penetrations, } \\
\text { Section III, Class } 1 \text { Construction }\end{array}$ & reg1.84.r29 & 1993 & $\mathbf{N}$ & Annulled by ASME & $n / a$ & $n / a$ \\
\hline 1491 & ASME & $\begin{array}{l}\text { ASME Code } \\
\text { Case N-62-2 }\end{array}$ & $\mathrm{N} / \mathrm{S}$ & $\begin{array}{l}\text { Internal and External Valve Items, } \\
\text { Section III, Division 1, Classes 1, } 2 \\
\text { and } 3\end{array}$ & reg1.148.roo & 1981 & $\bar{N}$ & Annulled by ASME & $\begin{array}{l}\text { ASME Code Case } \\
\text { N-62-6 }\end{array}$ & 1989 \\
\hline
\end{tabular}


Codes and Standards and Other Guldance Clted in Regulatory Documents

Part A - Industry Consersisus Codes and Standards (continued)

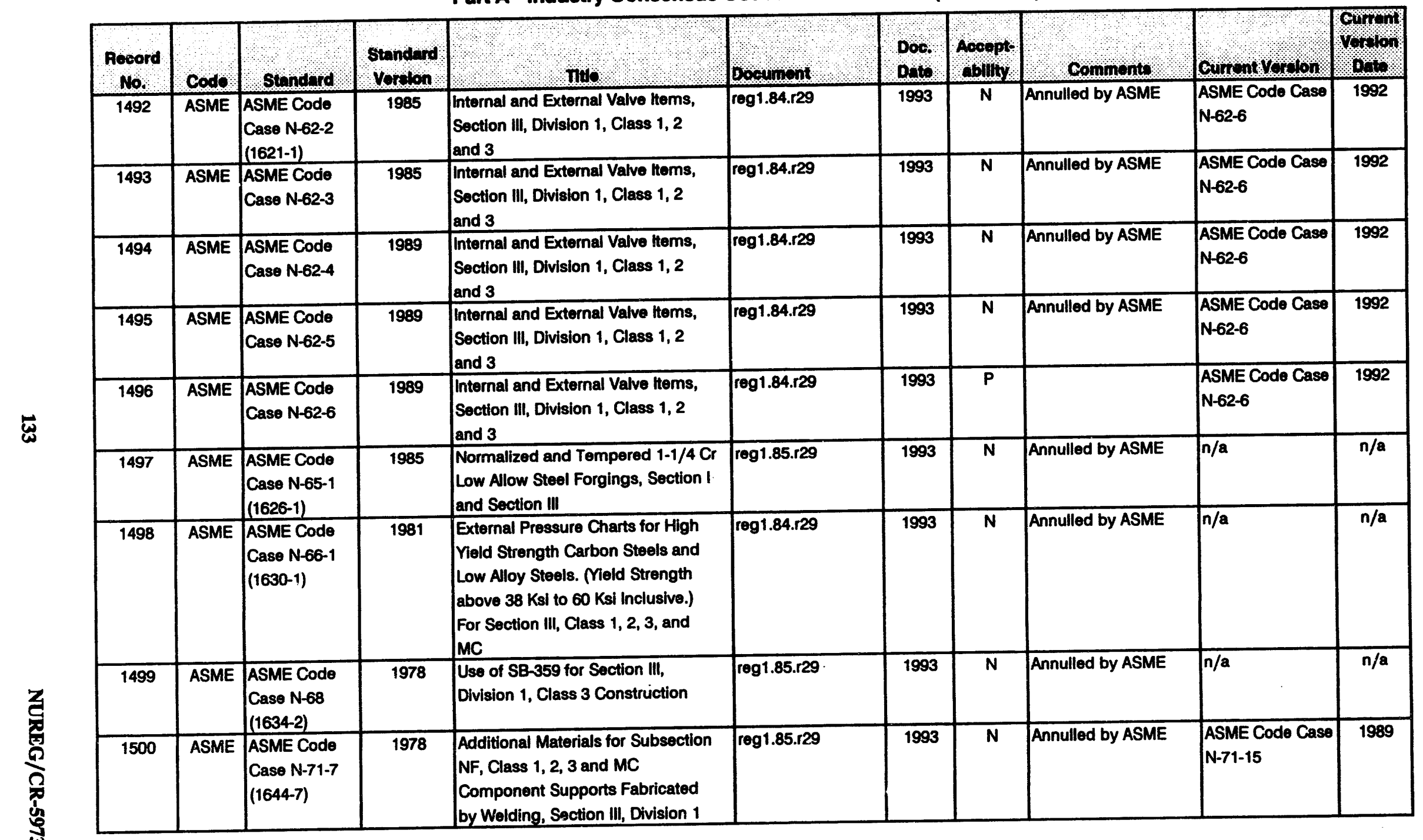




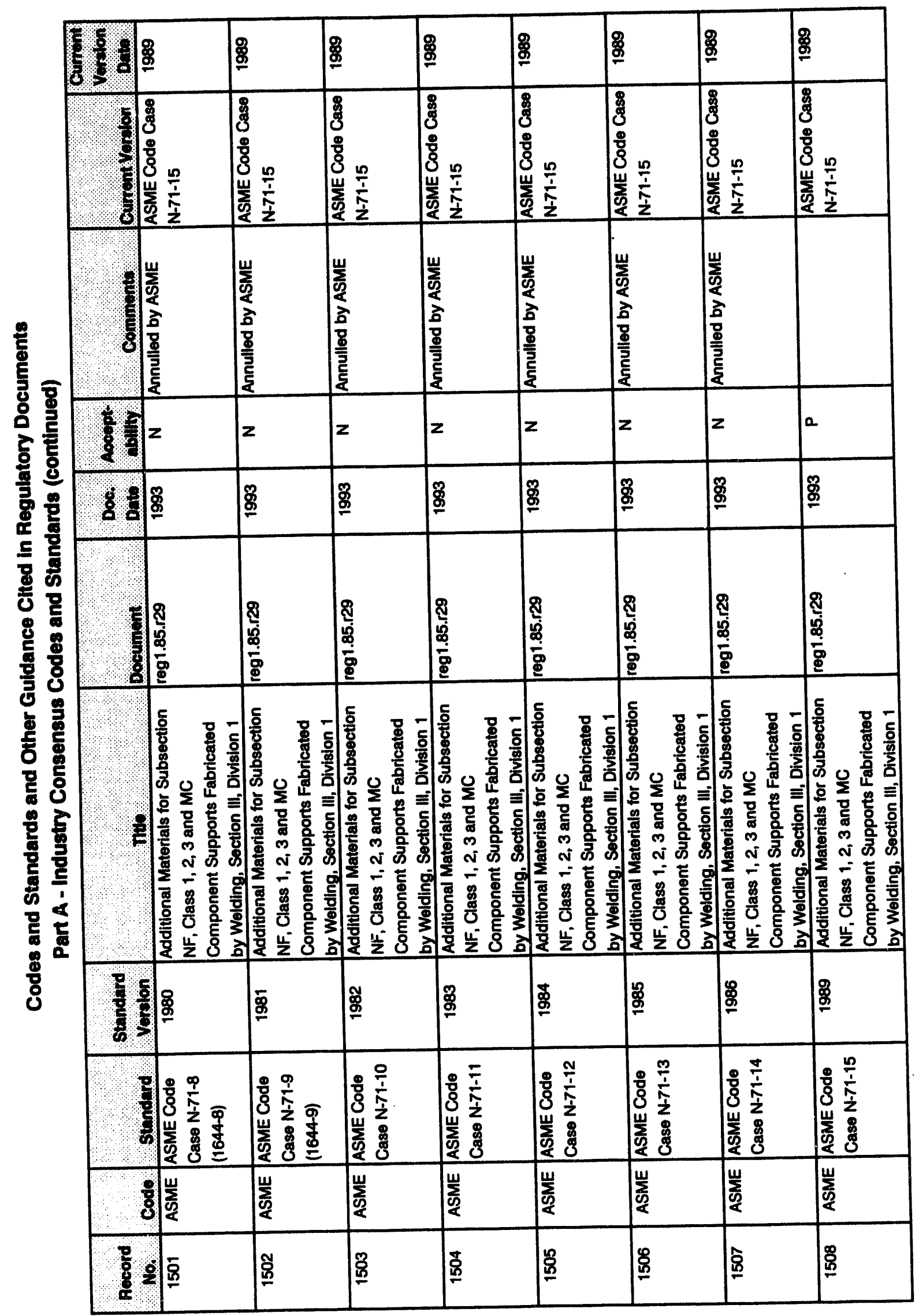


Codes and Standards and Other Guidance Cited in Regulatory Documents

Part A - Industry Consensus Codes and Standards (continued)

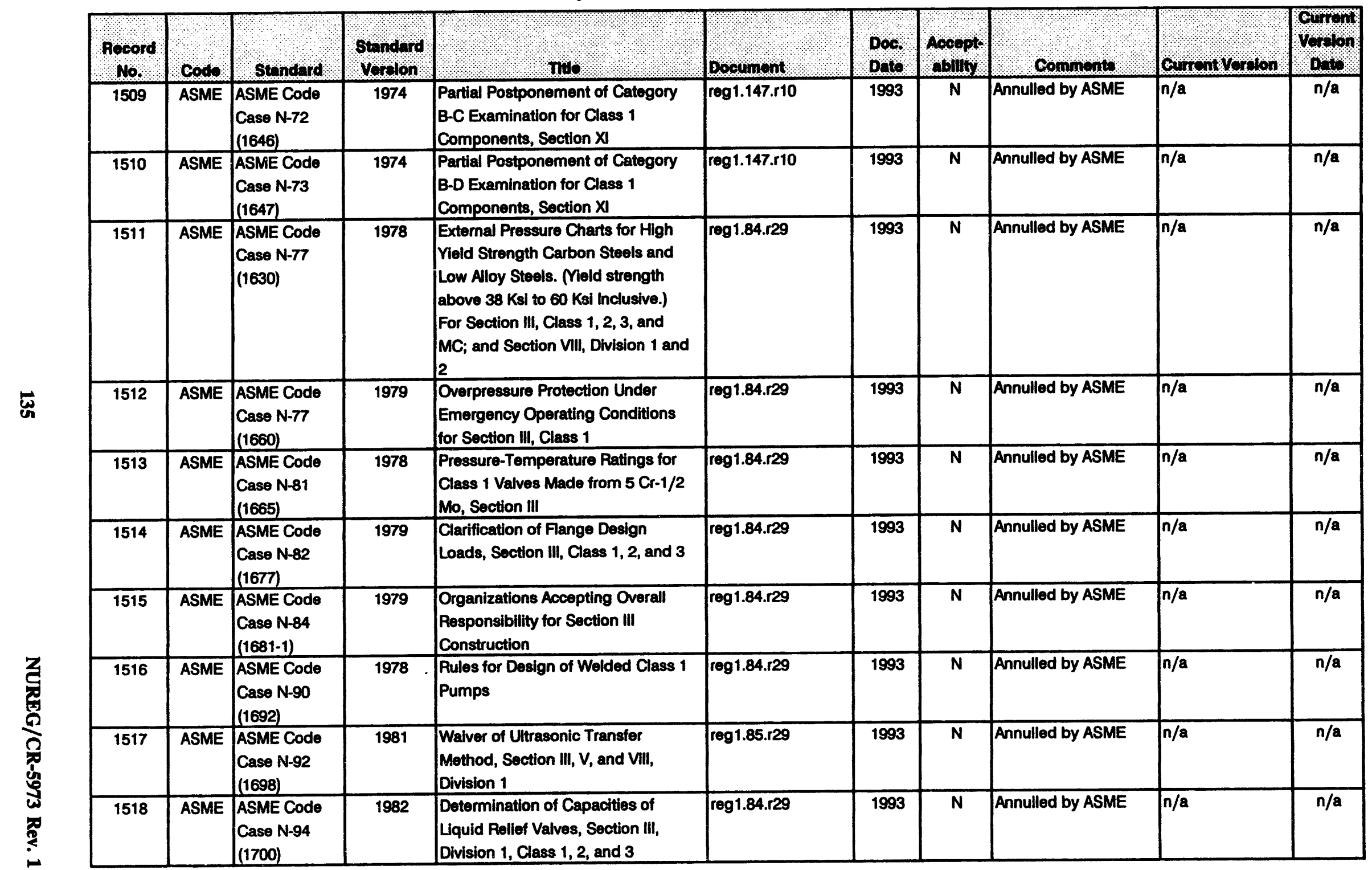




\begin{tabular}{|c|c|c|c|c|c|c|c|c|c|c|}
\hline \multicolumn{11}{|c|}{$\begin{array}{l}\text { Codes and Standards and Other Guidance Cited in Regulatory Documents } \\
\text { Part A - Industry Consensus Codes and Standards (continued) }\end{array}$} \\
\hline $\begin{array}{l}\text { Rocord } \\
\text { No: }\end{array}$ & Codo & stinndard & $\begin{array}{l}\text { Standard } \\
\text { Vordion }\end{array}$ & (n) & Document & $\begin{array}{l}\text { Doo. } \\
\text { Date. }\end{array}$ & $\begin{array}{c}\text { Accopt } \\
\text { ablity }\end{array}$ & Commonts & Curnont Verion & Vormonton \\
\hline 1519 & ASME & $\begin{array}{l}\text { ASME Code } \\
\text { Case N-95-1 } \\
(1701-1)\end{array}$ & 1979 & $\begin{array}{l}\text { Determination of Capacities of } \\
\text { Vacuum Relief Valves, Section III, } \\
\text { Division } 1 \text { and 2, Class MC } \\
\end{array}$ & reg1.84.r29 & 1993 & $N$ & Annulled by ASME & $n / a$ & $n / a$ \\
\hline 1520 & ASME & $\begin{array}{l}\text { ASME Code } \\
\text { Case N-95-2 } \\
(1701-2)\end{array}$ & 1982 & $\begin{array}{l}\text { Determination of Capacities of } \\
\text { Vacuum Relief Valves, Section III, } \\
\text { Division 1, Classes 2, 3, and MC } \\
\text { and Dlvision } 2 \text { Concrete } \\
\text { Containments } \\
\end{array}$ & reg1.84.r29 & 1993 & $\bar{N}$ & Annulled by ASME & $n / a$ & $n / a$ \\
\hline 1521 & ASME & $\begin{array}{l}\text { ASME Code } \\
\text { Case N-96-1 } \\
(1702-1)\end{array}$ & 1980 & $\begin{array}{l}\text { Flanged Valves Larger than } 24 \\
\text { inches for Section III, Division } 1 \text {, } \\
\text { Class } 1,2 \text { and } 3 \text { Construction }\end{array}$ & reg1.84.r29 & 1993 & $\mathbf{N}$ & Annulled by ASME & $n / a$ & $n / a$ \\
\hline 1522 & ASME & $\begin{array}{l}\text { ASME Code } \\
\text { Case N-98 } \\
(1705-1) \\
\end{array}$ & 1990 & $\begin{array}{l}\text { Uitrasonic Examination - } \\
\text { Calibration Block Tolerances, } \\
\text { Section XI }\end{array}$ & reg1.147.r10 & 1993 & $\bar{A}$ & & \begin{tabular}{|l|} 
ASME Code Case \\
N-98
\end{tabular} & 1993 \\
\hline 1523 & ASME & $\begin{array}{l}\text { ASME Code } \\
\text { Case N-100 } \\
\text { (1711) }\end{array}$ & $\mathrm{N} / \mathrm{S}$ & $\begin{array}{l}\text { Pressure Relief Valve Design Rules, } \\
\text { Section III, Division 1, Classes 1, 2, } \\
\text { and 3 }\end{array}$ & srp3.9.3 & 1981 & $\bar{N}$ & Annulled by ASME & $n / a$ & $n / a$ \\
\hline 1524 & T.MME & $\begin{array}{l}\text { ASME Code } \\
\text { Case N-100 } \\
\text { (1711) }\end{array}$ & 1983 & $\begin{array}{l}\text { Pressure Rellef Valve Design Rules, } \\
\text { Section III, Division 1, Class 1, } 2 \\
\text { and 3 }\end{array}$ & reg1.84.r29 & $\overline{1993}$ & $\mathbf{N}$ & Annulled by ASME & $n / a$ & $n / a$ \\
\hline 1525 & ASME & $\begin{array}{l}\text { ASME Code } \\
\text { Case N-101 } \\
\text { (1712) }\end{array}$ & 1979 & $\begin{array}{l}\text { Nameplates and Stamping for } \\
\text { Section III, Division 1, Class 1, 2, } 3 \\
\text { and MC Construction as } \\
\text { Referenced in NA-8300 }\end{array}$ & reg1.84.r29 & 1993 & $\bar{N}$ & Annulled by ASME & $n / a$ & $n / a$ \\
\hline 1526 & ASME & \begin{tabular}{|l} 
ASME Code \\
Case N-102-1 \\
$(1714-1)$ \\
\end{tabular} & 1978 & $\begin{array}{l}\text { Postwold Heat Treatment of P-1 } \\
\text { Material, Section III, Class MC }\end{array}$ & reg1.85.r29 & 1993 & $\mathbf{N}$ & Annulled by ASME & $n / a$ & $n / a$ \\
\hline 1527 & ASME & $\begin{array}{l}\text { ASME Code } \\
\text { Case N-102-2 } \\
(1714-2)\end{array}$ & 1981 & $\begin{array}{l}\text { Postweld Heat Treatment of P-1 } \\
\text { Material, Section III, Class MC }\end{array}$ & reg1.85.r29 & 1993 & $\mathbf{N}$ & Annulled by ASME & $n / a$ & $n / a$ \\
\hline 1528 & ASME & $\begin{array}{l}\text { ASME Code } \\
\text { Case N-106-2 } \\
\text { (1720-2) }\end{array}$ & 1990 & $\begin{array}{l}\text { Weld End Preparation for Section } \\
\text { III, Division } 1 \text { Construction }\end{array}$ & reg 1.84.r29 & 1993 & $\bar{P}$ & Annulled by ASME & $n / a$ & $n / a$ \\
\hline
\end{tabular}


Codes and Standards and Other Guidance Cited in Regulatory Documents

Part A - Industry Consensus Codes and Standards (continued)

\begin{tabular}{|c|c|c|c|c|c|c|c|c|c|c|}
\hline $\begin{array}{l}\text { Feoord } \\
\text { No. }\end{array}$ & Codo & Stundhal & $\begin{array}{l}\text { Standard } \\
\text { Vordion }\end{array}$ & 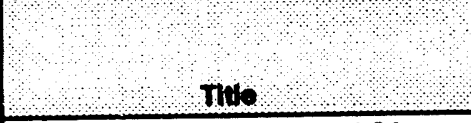 & Dosoumont & $\begin{array}{l}\text { Doo. } \\
\text { Dold }\end{array}$ & Aocopt & Connment, & Cunent Voulon & Qton \\
\hline 1529 & ASME & $\begin{array}{l}\text { ASME Code } \\
\text { Case N-107-1 } \\
(1722-1)\end{array}$ & 1982 & $\begin{array}{l}\text { Vacuum, Carbon Deoxidized SA- } \\
508 \text { Forgings, Section III, Division 1, } \\
\text { and VIII, Division } 1 \text { and } 2\end{array}$ & reg1.85.r29 & 1993 & $\mathbf{N}$ & Annulled by ASME & $n / a$ & $n / a$ \\
\hline 1530 & ASME & $\begin{array}{l}\text { ASME Code } \\
\text { Case N-108 } \\
(1724)\end{array}$ & 1978 & $\begin{array}{l}\text { Deviation from the Specified Silicon } \\
\text { Ranges in ASME Material } \\
\text { Specifications, Section III, Division } \\
\text { 1, and VIII, Division } 1 \text { and } 2\end{array}$ & $\operatorname{reg} 1.85 . r 29$ & 1993 & $\mathbf{N}$ & Annulled by ASME & $n / a$ & $n / a$ \\
\hline 1531 & ASME & $\begin{array}{l}\text { ASME Code } \\
\text { Case N-109 } \\
(1726)\end{array}$ & 1979 & $\begin{array}{l}\text { Refinement of Low Alloy Steel Heat } \\
\text { Affected Zone Under Overlay } \\
\text { Cladding, Section III, Division 1, } \\
\text { Class } 1 \text { Components }\end{array}$ & $\operatorname{reg} 1.84 .129$ & 1993 & $\mathbf{N}$ & Annulled by ASME & $n / a$ & $n / a$ \\
\hline 1532 & ASME & $\begin{array}{l}\text { ASME Code } \\
\text { Case } \mathrm{N}-110 \\
(1727)\end{array}$ & 1979 & $\begin{array}{l}\text { Alternate Test Fluids, Section III, } \\
\text { Division } 1\end{array}$ & reg1.84.r29 & 1993 & $\mathbf{N}$ & Annulled by ASME & $n / a$ & $n / a$ \\
\hline 1533 & ASME & $\begin{array}{l}\text { ASME Code } \\
\text { Case N-111 } \\
(1729)\end{array}$ & 1979 & $\begin{array}{l}\text { Minimum Edge Distance-Bolting for } \\
\text { Section III, Division 1, Class 1, 2, } \\
\text { and } 3 \text { and MC Construction of } \\
\text { Component Supports }\end{array}$ & reg1.84.r29 & 1993 & $\mathbf{N}$ & Annulled by ASME & $n / a$ & $n / a$ \\
\hline 1534 & ASME & $\begin{array}{l}\text { ASME Code } \\
\text { Case N-112 } \\
(1730) \\
\end{array}$ & 1975 & $\begin{array}{l}\text { Acceptance Standards for Class } 2 \\
\text { and } 3 \text { Components, Section XI, } \\
\text { Division } 1\end{array}$ & reg1.147.r10 & 1993 & $\mathbf{N}$ & Annulled by ASME & $n / a$ & $n / a$ \\
\hline 1535 & ASME & $\begin{array}{l}\text { ASME Code } \\
\text { Case } \mathrm{N}-113 \\
(1731)\end{array}$ & 1982 & $\begin{array}{l}\text { Basic Calibration Block for } \\
\text { Uitrasonic Examination of Wolds } 10 \\
\text { in. to } 13 \text { in. Thick, Section XI, } \\
\text { Division } 1\end{array}$ & reg1.147.r10 & 1993 & $\mathbf{N}$ & Annulled by ASME & $\begin{array}{l}\text { ASME Code Case } \\
\text { N-113-1 }\end{array}$ & 1993 \\
\hline 1536 & ASME & $\begin{array}{l}\text { ASME Code } \\
\text { Case N-113-1 }\end{array}$ & 1990 & $\begin{array}{l}\text { Basic Callbration Block for } \\
\text { Uitrasonic Examination of Welds } 10 \\
\text { in. to } 14 \text { in. Thick, Section XI, } \\
\text { Division } 1\end{array}$ & reg1.147.r10 & 1993 & $\mathbf{A}$ & & $\begin{array}{l}\text { ASME Code Caso } \\
\text { N-113-1 }\end{array}$ & 1993 \\
\hline 1537 & ASME & $\begin{array}{l}\text { ASME Code } \\
\text { Case N-114 } \\
(1732)\end{array}$ & 1979 & $\begin{array}{l}\text { Hard-surfaced Valves with Inlet } \\
\text { Connections less than 2-in. } \\
\text { Nominal Pipe Size for Section III, } \\
\text { Division } 1 \text { Class } 1 \text { and } 2 \\
\text { Construction }\end{array}$ & $\operatorname{reg} 1.84 .129$ & 1993 & $\mathbf{N}$ & Annulled by ASME & $n / a$ & $n / a$ \\
\hline
\end{tabular}




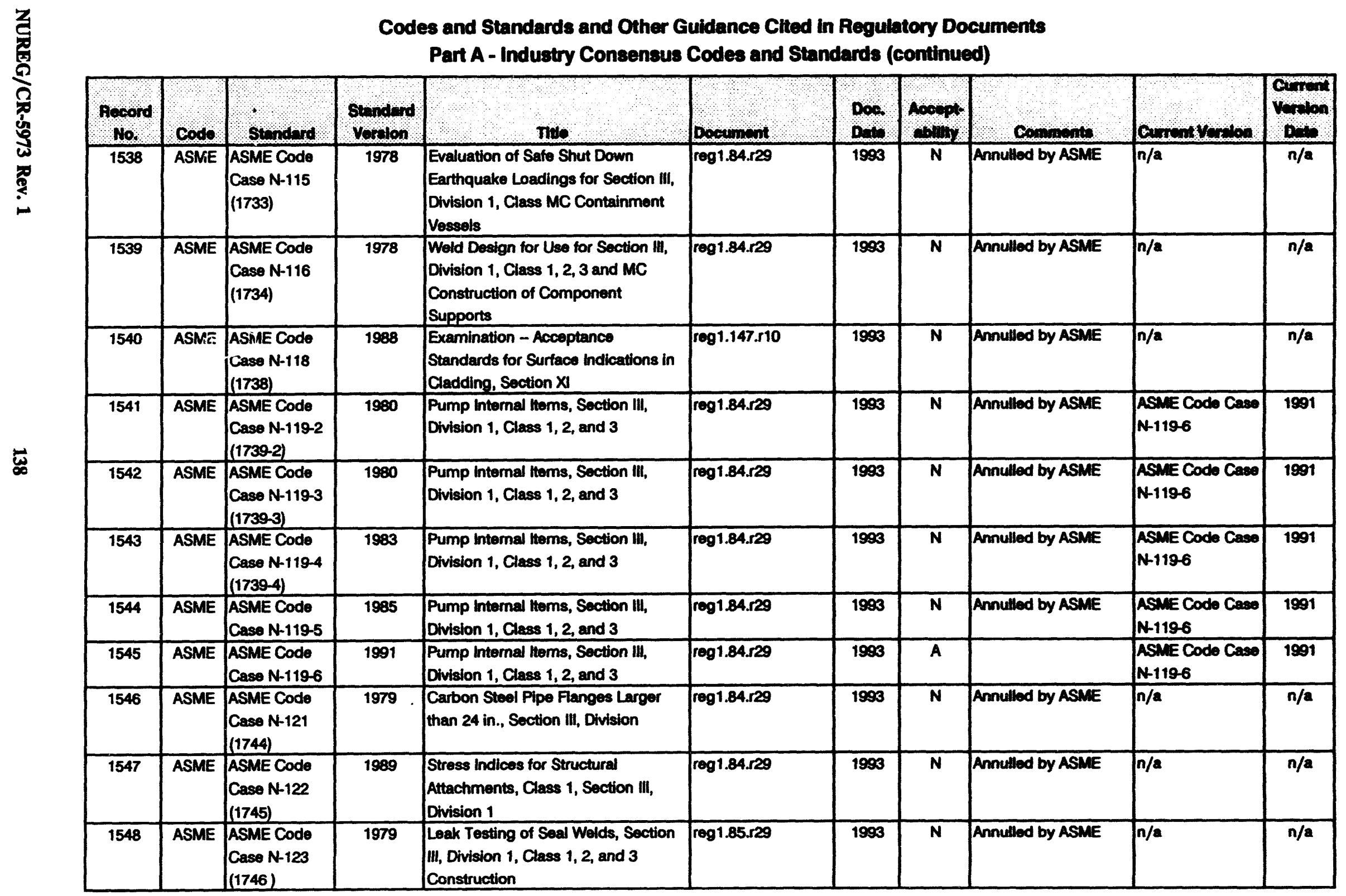


Codes and Standards and Other Guidance Chted in Regulatory Documents

Part A - Industry Consensus Codes and Standards (continued)

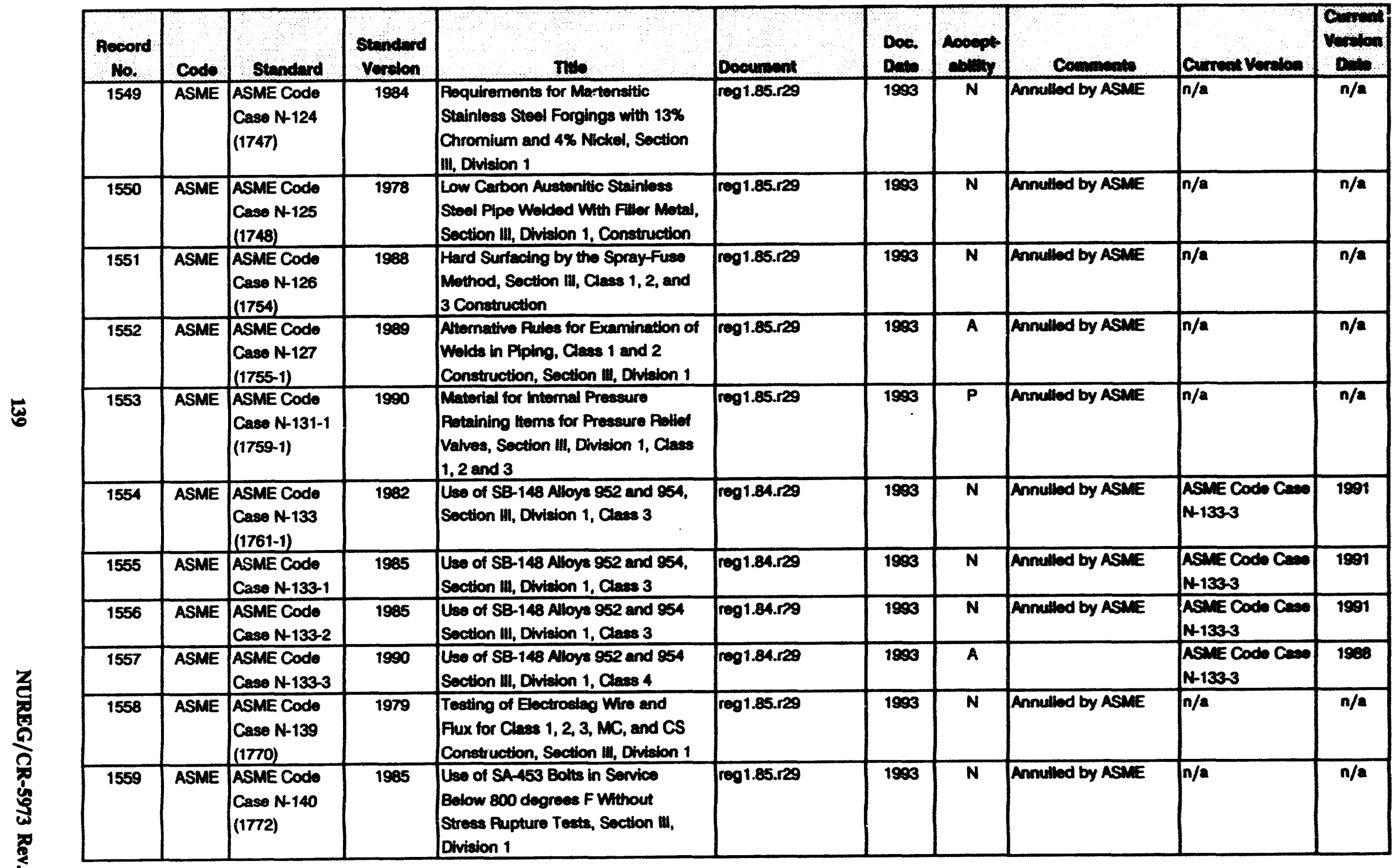


Codes and Standards and Other Guldance Ched in Regulatory Documents Part A - Induatry Consensus Codes and Standards (continued)

\begin{tabular}{|c|c|c|c|c|c|c|c|c|c|c|}
\hline $\begin{array}{l}\text { Pecond } \\
\text { No. }\end{array}$ & code & Stondend & $\begin{array}{l}\text { Sundard } \\
\text { Vonelon }\end{array}$ & mo & Dovernonst & $\begin{array}{l}\text { Dos. } \\
\text { Dene }\end{array}$ & Doper & Conneses & ondentrengen & $\operatorname{lom}_{\operatorname{lom}}$ \\
\hline 1560 & ASME & $\begin{array}{l}\text { ASME Code } \\
\text { Case N-142-1 } \\
(1774-1)\end{array}$ & 1980 & $\begin{array}{l}\text { Minimum Wall Thickneas for Clases } \\
2 \text { and } 3 \text { Valves, Section it, Diviaion. } \\
1\end{array}$ & 1001.84 .529 & 1893 & $\mathbf{N}$ & Annulled by ASME & $n / a$ & $n / a$ \\
\hline 1561 & ASME & $\begin{array}{l}\text { ASME Code } \\
\text { Case N-146-1 } \\
(1780-1)\end{array}$ & 1982 & $\begin{array}{l}\text { Hydrostatic Teating and Stamping } \\
\text { of Pumps for Class } 1 \text { Construction, } \\
\text { Section Ifl, Dhvilon } 2\end{array}$ & reg1.84.529 & 1998 & $\mathbf{N}$ & Annulliad by ASYE & $n / a$ & $n / a$ \\
\hline 1562 & ASME & $\begin{array}{l}\text { ASME Code } \\
\text { Case N-147 } \\
(1781)\end{array}$ & 1978 & $\begin{array}{l}\text { Uee of Modilied SA } 487 \text { Grade } \\
\text { CAsAMM, Section M, Diviaion 1, } \\
\text { Class 1, 2, 3, MC or CS }\end{array}$ & reg1.85.r29 & 1900 & $\mathbf{N}$ & Annulled by ASUE & $n / a$ & $n / \mathbf{a}$ \\
\hline 1563 & ASME & $\begin{array}{l}\text { ASME Code } \\
\text { Cese N-148 } \\
(1782)\end{array}$ & 1983 & $\begin{array}{l}\text { Use of Copper-Nickel Alloy } 982 \text { for } \\
\text { Castings, Section II, Diviaion } 1 \text {. } \\
\text { Class } 3 \text { Construction }\end{array}$ & reg1.85.129 & 1900 & $\mathbf{N}$ & Anmillod by ASME & $n / a$ & $n / a$ \\
\hline 1564 & ASME & $\begin{array}{l}\text { ASME Code } \\
\text { Case N-154 } \\
(1791)\end{array}$ & 1985 & $\begin{array}{l}\text { Projection Rosiatance Welding of } \\
\text { Valve Seats. Section II., Divlaion 1, } \\
\text { Clase } 1,2 \text { and } 3 \text { Valves }\end{array}$ & reg1.84.r29 & 1993 & $\mathbf{N}$ & Annullod by ASSE & $\begin{array}{l}\text { ASUE Codo Ceseo } \\
\text { N-154-1 }\end{array}$ & 1992 \\
\hline 1565 & ASME & $\begin{array}{l}\text { ASME Code } \\
\text { Case N-154-1 }\end{array}$ & 1988 & 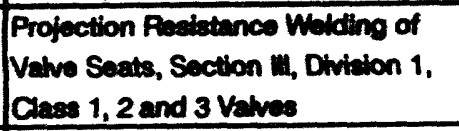 & 1001.84 .29 & 1980 & $\mathbf{A}$ & & $\begin{array}{l}\text { ASWE Cods Caseo } \\
\text { N-154-1 }\end{array}$ & 1992 \\
\hline 1566 & ASME & $\begin{array}{l}\text { ASwE Code } \\
\text { Case N-155-1 } \\
(1792-1)\end{array}$ & $N / S$ & $\begin{array}{l}\text { Fiberglases Painforced } \\
\text { Thermosetting Posin Pipe, Section } \\
\text { II, Division } 1\end{array}$ & $\operatorname{reg} 1.72 .502$ & 1978 & $\mathbf{N}$ & Anoutled by ASTE & $\begin{array}{l}\text { AsNE Cods Caseo } \\
N+155-2\end{array}$ & 1991 \\
\hline 1567 & ASME & $\begin{array}{l}\text { ASNE Code } \\
\text { Cose N-155-2 } \\
(1792-2)\end{array}$ & 1991 & $\begin{array}{l}\text { Fiberglase Pwinforced } \\
\text { Thermosetting Presin Pipo, Section } \\
\text { the, Division } 1\end{array}$ & $\operatorname{reg} 1.84 .520$ & 1998 & $\mathbf{P}$ & & $\begin{array}{l}\text { ASNEC Cods Case } \\
N-155-2\end{array}$ & 1991 \\
\hline 1568 & ASME & $\begin{array}{l}\text { ASME Code } \\
\text { Case N-156 } \\
(1793)\end{array}$ & 1986 & $\begin{array}{l}\text { Structurad Sied Aollod Shapes, } \\
\text { Section II, Divition 1, Cless 2, 3, } \\
\text { and MC }\end{array}$ & reg1.85.r29 & 1993 & $\mathbf{N}$ & Annulied by ASTE & $n / a$ & $n / a$ \\
\hline 1569 & ASME & $\begin{array}{l}\text { ASME Code } \\
\text { Case N-157 } \\
\text { (1794) }\end{array}$ & 1983 & $\begin{array}{l}\text { Uee of Seamless A-Br, Alloy CDA } \\
614 \text { Pipe, Section If, Division 1, } \\
\text { Class } 3\end{array}$ & reg1.85.r29 & 1993 & $\mathbf{N}$ & Annullad by ASME & $n / \mathbf{a}$ & $n / a$ \\
\hline 1570 & ASME & $\begin{array}{l}\text { ASME Codo } \\
\text { Case N-158 } \\
(1795)\end{array}$ & 1978 & $\begin{array}{l}\text { Examination of Weld Repairs in } \\
\text { Forgings, Section ill, Division 1, } \\
\text { Class 1,2,3, MC and CS }\end{array}$ & reg1.85.r29 & 1998 & $\mathbf{N}$ & Annulled by ASME & $n / a$ & $n / a$ \\
\hline
\end{tabular}


Codes and Standards and Other Guidance Ched in Regulatory Documents

Part A - Industry Consensus Codes and Standards (continued)

\begin{tabular}{|c|c|c|c|c|c|c|c|c|c|c|}
\hline $\begin{array}{l}\text { Pecond } \\
\text { No. }\end{array}$ & Code: & Standend & $\begin{array}{l}\text { Stondard } \\
\text { Verten }\end{array}$ & $\mathrm{min}$ & Docaminas & Doe. & soopt & Connoneste & 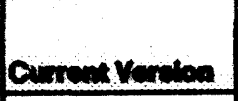 & Voridon \\
\hline 1571 & ASME & $\begin{array}{l}\text { ASME Code } \\
\text { Case N-159 } \\
(1796)\end{array}$ & 1978 & $\begin{array}{l}\text { Body Neck Thiokness } \\
\text { Dotermination for Valves with inlet } \\
\text { Connections } 4 \text { inch Nominal Pipe } \\
\text { Size and Smaller, Section III, } \\
\text { Division 1, Class 1, 2, and } 3\end{array}$ & reg1.84.r29 & 1993 & $\mathbf{N}$ & Annulled by ASME & $n / a$ & $n / a$ \\
\hline 1572 & ASME & $\begin{array}{l}\text { ASME Code } \\
\text { Case N-160 } \\
(1797)\end{array}$ & 1985 & $\begin{array}{l}\text { Finned Tubling for Construction, } \\
\text { Section II, Division } 1\end{array}$ & reg1.84.r29 & 1993 & $\mathbf{N}$ & Annulled by ASME & $\begin{array}{l}\text { ASNE Code Case } \\
\text { N-160-1 }\end{array}$ & 1991 \\
\hline 1573 & ASME & $\begin{array}{l}\text { ASME Code } \\
\text { Case N-160-1 }\end{array}$ & 1991 & $\begin{array}{l}\text { Finned Tubing for Construction, } \\
\text { Section ill, Division } 1\end{array}$ & reg1.84.r29 & 1993 & A & & $\begin{array}{l}\text { ASME Code Case } \\
\text { N-160-1 }\end{array}$ & 1991 \\
\hline 1574 & ASME & $\begin{array}{l}\text { ASME Code } \\
\text { Case N-167 } \\
(1804) \\
\end{array}$ & 1977 & $\begin{array}{l}\text { Ninimum Section Thickness } \\
\text { Requirements for Repair of } \\
\text { Nozzles, Section XI, Division } 1 \\
\end{array}$ & reg1.147.r10 & 1993 & $\mathbf{N}$ & Annulled by ASME & $n / a$ & $n / a$ \\
\hline 1575 & ASME & $\begin{array}{l}\text { ASME Code } \\
\text { Case N-174 } \\
\text { (1812) }\end{array}$ & 1981 & $\begin{array}{l}\text { Size of Fillet Wolds for Socket } \\
\text { Woiding of Piping. Section III, } \\
\text { Division } 1\end{array}$ & $\operatorname{rog} 1.84 .529$ & 1993 & $\mathbf{N}$ & Annulled by ASME & $n / a$ & $n / a$ \\
\hline 1576 & ASME & $\begin{array}{l}\text { ASME Code } \\
\text { Case N-175 } \\
(1818)\end{array}$ & 1979 & $\begin{array}{l}\text { Wolded Joints in Component } \\
\text { Standard Supports, Section III, } \\
\text { Division } 1\end{array}$ & reg1.84.r29 & 1993 & $\mathbf{N}$ & Annulled by ASME & $n / a$ & $n / a$ \\
\hline 1577 & ASME & $\begin{array}{l}\text { ASME Code } \\
\text { Case N-176-1 } \\
(1819-1)\end{array}$ & 1983 & $\begin{array}{l}\text { Use of Type XM-19 for } \\
\text { Construction, Section ill Division } 1 \text {, } \\
\text { Class 1, 2, and } 3\end{array}$ & reg1.85.r29 & 1993 & $\mathbf{N}$ & Annulled by ASME & $n / a$ & $n / a$ \\
\hline 1578 & ASME & $\begin{array}{l}\text { ASME Code } \\
\text { Case N-177 } \\
(1820)\end{array}$ & 1984 & $\begin{array}{l}\text { Alternative Uitrasonic Examination } \\
\text { Technique, Section III, Division } 1\end{array}$ & reg1.85.r29 & 1993 & $\mathbf{N}$ & Annulled by ASME & $n / a$ & $n / a$ \\
\hline 1579 & ASME & $\begin{array}{l}\text { ASME Code } \\
\text { Case N-178 }\end{array}$ & 1980 & $\begin{array}{l}\text { Use of ASTM B271, CDA 954, Alloy } \\
9 \mathrm{C} \text { for Class } 3 \text { Construction, } \\
\text { Section III, Division } 1\end{array}$ & reg1.85.r29 & 1993 & $\mathbf{N}$ & Annulled by ASME & $n / a$ & $n / a$ \\
\hline 1580 & ASME & $\begin{array}{l}\text { ASME Code } \\
\text { Case N-179 }\end{array}$ & 1980 & $\begin{array}{l}\text { Openings in Valves for Section III, } \\
\text { Division 1, Class 1, } 2 \text { and } 3 \\
\text { Construction }\end{array}$ & reg1.84.r29 & 1993 & $\mathbf{N}$ & Annulled by ASME & $n / a$ & $n / a$ \\
\hline 1581 & ASME & $\begin{array}{l}\text { ASME Code } \\
\text { Case N-180 }\end{array}$ & 1978 & $\begin{array}{l}\text { Examination of Springs for Class } 1 \\
\text { Component Standard Supports, } \\
\text { Section III, Division } 1\end{array}$ & reg1.85.r29 & 1993 & $\mathbf{N}$ & Annulled by ASME & $n / a$ & $n / a$ \\
\hline
\end{tabular}




\begin{tabular}{|c|c|c|c|c|c|c|c|c|c|c|}
\hline \multicolumn{11}{|c|}{$\begin{array}{l}\text { Codes and Standards and Other Guldance Cited in Regulatory Documeits } \\
\text { Part A - Industry Consensus Codes and Standards (continued) }\end{array}$} \\
\hline $\begin{array}{l}\text { Aocord } \\
\text { No. }\end{array}$ & Code & Standard & Standard & Tllo & Document & $\begin{array}{l}\text { Dor. } \\
\text { Dote }\end{array}$ & Acoept & Commente & Curropt Yoraten & Vorron \\
\hline 1582 & ASME & $\begin{array}{l}\text { ASME Code } \\
\text { Case N-181 }\end{array}$ & 1980 & $\begin{array}{l}\text { Steel Castings Pefined by the } \\
\text { Argon Decarburization Process, } \\
\text { Section III, Division 1, Construction }\end{array}$ & reg1.85.r29 & 1993 & $\mathbf{N}$ & Annulled by ASME & $n / a$ & $n / a$ \\
\hline 1583 & ASME & $\begin{array}{l}\text { ASME Code } \\
\text { Case N-182 }\end{array}$ & 1981 & $\begin{array}{l}\text { Alternate Rules for Procedure } \\
\text { Qualification Base Material } \\
\text { Orientation, Section III, Division 1, } \\
\text { Class } 2 \text { and } 3 \text { Construction } \\
\end{array}$ & $\operatorname{rog} 1.84 .129$ & 1993 & $\mathbf{N}$ & Annulled by ASME & $n / a$ & $n / \mathbf{a}$ \\
\hline 1584 & ASME & $\begin{array}{l}\text { ASME Code } \\
\text { Case N-183 }\end{array}$ & 1980 & $\begin{array}{l}\mathrm{N}-183 \text { Use of Modified SA-182 } \\
\text { Grade F22 for Section III, Division 1, } \\
\text { Class 1, } 2 \text { and } 3 \text { Construction }\end{array}$ & 1091.85 .129 & 1993 & $\mathbf{N}$ & Annulled by ASME & $n / a$ & $n / a$ \\
\hline 1585 & ASME & $\begin{array}{l}\text { ASME Code } \\
\text { Case N-184 }\end{array}$ & 1979 & $\begin{array}{l}\text { Roll Threading of SA-453 Bolting for } \\
\text { Section II, Division 1, Class 1, 2,3 } \\
\text { or CS Construction }\end{array}$ & rog 1.84.r29 & 1993 & $\bar{N}$ & Annullod by ASME & $n / a$ & $\mathbf{n / a}$ \\
\hline 1586 & ASME & $\begin{array}{l}\text { ASME Code } \\
\text { Case N-188 }\end{array}$ & 1977 & $\begin{array}{l}\text { Use of Wolded Ni-Fo-Cr-Mo-3 Cu } \\
\text { (Alloy 825) and Ni-Cr-Mo-Cb (Alloy } \\
\text { 625) Tubing, Section III, Division 1, } \\
\text { Class } 3\end{array}$ & reg1.85.r29 & 1993 & $\bar{N}$ & Annulled by ASME & \begin{tabular}{|l|} 
ASME Code Case \\
N-188-1
\end{tabular} & 1993 \\
\hline$i 587$ & ASME & $\begin{array}{l}\text { ASME Code } \\
\text { Case N-188-1 }\end{array}$ & 1990 & $\begin{array}{l}\text { Use of Welded Ni-Fe-Cr-Mo-Cu } \\
\text { (Alloy } 825 \text { ) and Ni-Cr-Mo-Cb (Alloy } \\
625 \text { ) Tubing, Section III, Division 1, } \\
\text { Class } 2 \text { and } 3 \text {. }\end{array}$ & $\log 1.85 .829$ & 1993 & A & & \begin{tabular}{|l|} 
ASME Codo Caso \\
N-188-1
\end{tabular} & 1993 \\
\hline 1588 & ASME & $\begin{array}{l}\text { ASME Code } \\
\text { Case N-189 }\end{array}$ & 1979 & $\begin{array}{l}\text { Primary Membrane Plus Primary } \\
\text { Bending Stress Intenstty Limits for } \\
\text { Other Than Solid Pectangular } \\
\text { Soctions for Section III, Division 1, } \\
\text { Class MC Construction } \\
\end{array}$ & $\operatorname{rog} 1.84 .229$ & 1993 & $\mathbf{N}$ & Annulled by ASME & $n / a$ & $\mathrm{n} / \mathbf{a}$ \\
\hline 1589 & ASME & $\begin{array}{l}\text { ASME Code } \\
\text { Case N-190 }\end{array}$ & 1978 & $\begin{array}{l}\text { Use of SA-455 for Class } 3 \\
\text { Components, Soction III, Division } 1\end{array}$ & reg1.85.r29 & 1993 & $\mathbf{N}$ & Annulled by ASME & $n / a$ & $n / a$ \\
\hline 1590 & ASME & $\begin{array}{l}\text { ASME Code } \\
\text { Case } \mathrm{N}-192\end{array}$ & 1979 & $\begin{array}{l}\text { Use of Bralded Flexible Connoctors } \\
\text { for Section lil, Division 1, Class 1, 2, } \\
\text { and } 3 \text { Construction }\end{array}$ & $\operatorname{reg} 1.84 . r 29$ & 1993 & $\mathbf{N}$ & Annulled by ASME & \begin{tabular}{|l|} 
ASME Code Cese \\
$N-192-2$
\end{tabular} & 1993 \\
\hline 1591 & ASME & $\begin{array}{l}\text { ASME Code } \\
\text { Case N-192-1 }\end{array}$ & 1981 & $\begin{array}{l}\text { Use of Bralded Flexible Connectors } \\
\text { for Section III, Division 1, Class 1, 2, } \\
\text { and } 3 \text { Construction }\end{array}$ & reg1.84.r29 & 1993 & $\bar{N}$ & Annulled by ASME & $\begin{array}{l}\text { ASME Code Ceso } \\
N-192-2\end{array}$ & 1993 \\
\hline
\end{tabular}


Codes and Standards and Other Guldance Clted in Regulatory Documents

Part A - Industry Consensus Codes and Standards (continued)

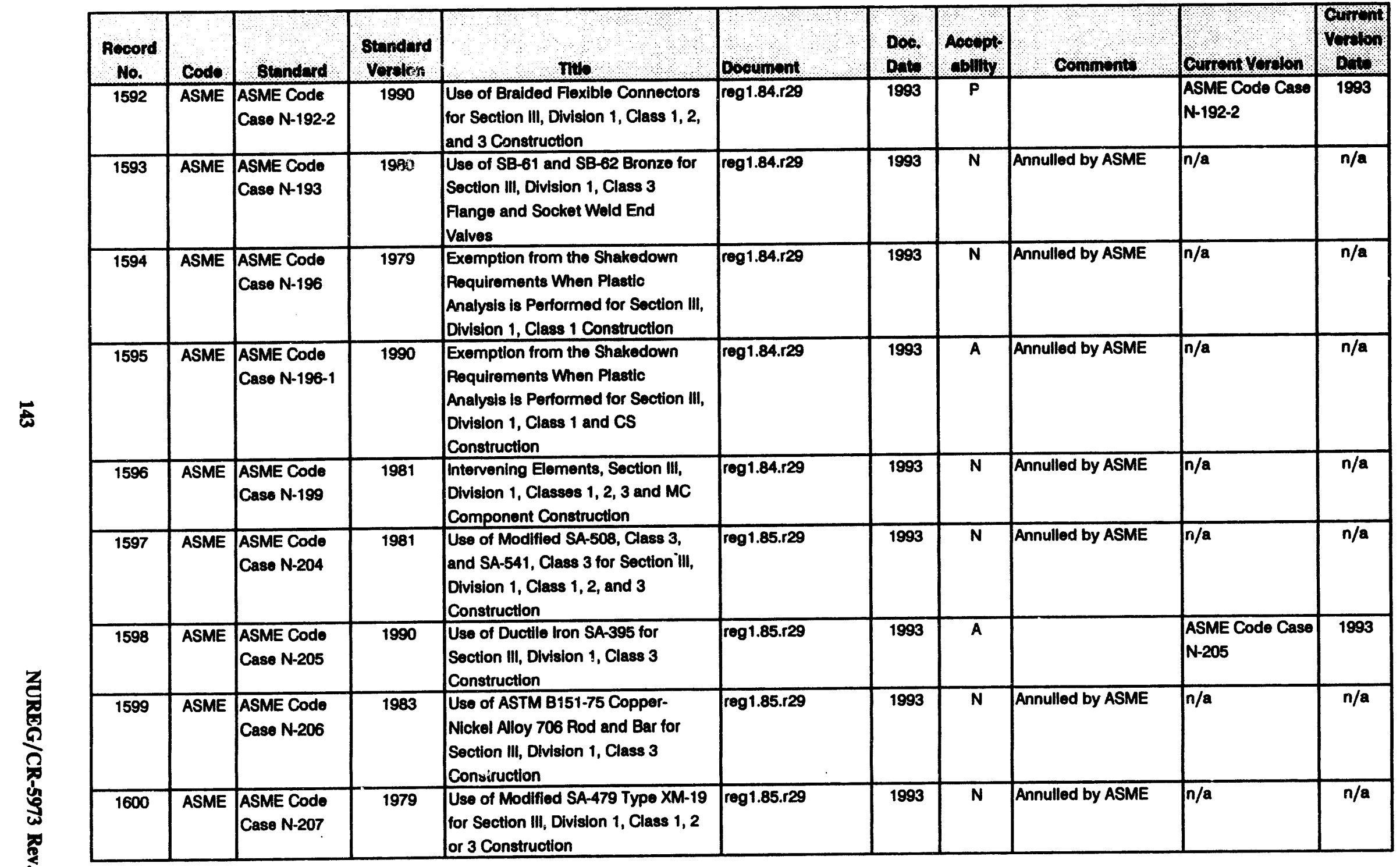


Codes and Standards and Other Guidance Clted in Regulatory Documents

Part A - Industry Consensus Codes and Standards (continued)

\begin{tabular}{|c|c|c|c|c|c|c|c|c|c|c|}
\hline & & & & Part A - Indusiry Consensus & Lo, & & 政 & & & \\
\hline $\begin{array}{l}\text { Rocord } \\
\text { No. }\end{array}$ & Codo & Standard & $\begin{array}{l}\text { Stundard } \\
\text { vorlon }\end{array}$ & nul & Doounoul & Doo, & Aocopt & Commuto & Curront vordon & vorion \\
\hline 1601 & ASME & $\begin{array}{l}\text { ASME Code } \\
\text { Case N-207-1 }\end{array}$ & 1985 & $\begin{array}{l}\text { Use of Modified SA-479 Type XM-19 } \\
\text { for Section III, Division 1, Class 1, 2, } \\
3, \text { or CS Construction }\end{array}$ & reg1.85.r29 & 1993 & \begin{tabular}{l|l}
$\mathrm{N}$ & $\mathrm{A}$ \\
\end{tabular} & Annulled by ASME & $n / a$ & $n / a$ \\
\hline 1602 & ASME & $\begin{array}{l}\text { ASME Code } \\
\text { Case N-210 }\end{array}$ & 1978 & $\begin{array}{l}\text { Exemption to Hydrostatic Tests } \\
\text { After Repairs, Section X, Division } 1\end{array}$ & reg1.147.r10 & 1993 & $\mathbf{N}$ & Annulled by ASME & $n / a$ & $n / a$ \\
\hline 1603 & ASME & $\begin{array}{l}\text { ASME Code } \\
\text { Case N-211 }\end{array}$ & 1990 & $\begin{array}{l}\text { Recalibration of Uitrasonic } \\
\text { Equipment Upon Change of } \\
\text { Personnel, Section XI, Division } 1 \\
\end{array}$ & reg1.147.r10 & 1993 & $\bar{A}$ & & $\begin{array}{l}\text { ASME Code Case } \\
\text { N-211 }\end{array}$ & 1993 \\
\hline 1604 & ASME & $\begin{array}{l}\text { ASME Code } \\
\text { Case N-212 } \\
(1693)\end{array}$ & 1981 & $\begin{array}{l}\text { Wolding Procedure Qualification of } \\
\text { Dissimilar Motal Wolds When } \\
\text { 'Buttering' with Alloy Wold Motal } \\
\text { and Heat Treatment May Be } \\
\text { Involved, Section III, Division 1, and } \\
\text { Section IX }\end{array}$ & reg1.84.r29 & 1993 & $\mathbf{N}$ & Annulled by ASME & $n / a$ & $n / a$ \\
\hline 1605 & ASME & $\begin{array}{l}\text { ASME Code } \\
\text { Case N-214 }\end{array}$ & 1982 & $\begin{array}{l}\text { Use of SA-351, Grade CN7M, for } \\
\text { Valves for Section III, Division 1, } \\
\text { Construction }\end{array}$ & reg1.84.r29 & 1993 & $\mathbf{N}$ & Annulled by ASME & $n / a$ & $n / a$ \\
\hline 1606 & ASME & $\begin{array}{l}\text { ASME Code } \\
\text { Case N-214-1 }\end{array}$ & 1983 & $\begin{array}{l}\text { Use of SA-351 Grade CN7M, for } \\
\text { Valves, Section III, Division } 1\end{array}$ & reg1.84.r29 & 1993 & $\mathbf{N}$ & Annulled by ASME & $n / a$ & $n / a$ \\
\hline 1607 & ASME & $\begin{array}{l}\text { ASME Code } \\
\text { Case N-214-2 }\end{array}$ & 1983 & $\begin{array}{l}\text { Use of SA-351 Grade CN7M, for } \\
\text { Valves, Section III, Division } 1\end{array}$ & reg1.84.r29 & 1993 & $\mathbf{N}$ & Annulled by ASME & $n / a$ & $n / a$ \\
\hline 1608 & ASME & $\begin{array}{l}\text { ASME Code } \\
\text { Case N-215 }\end{array}$ & 1981 & $\begin{array}{l}\text { Integrally Finned Titanium Tubes, } \\
\text { Section III, Dlvision 1, Class } 3 \\
\text { Construction }\end{array}$ & reg1.84.r29 & 1993 & $\mathbf{N}$ & Annulled by ASME & $n / a$ & $n / a$ \\
\hline 1609 & ASME & $\begin{array}{l}\text { ASME Code } \\
\text { Case N-216 }\end{array}$ & 1987 & $\begin{array}{l}\text { Alternative Rules for Reactor Vessel } \\
\text { Closure Stud Examination, Section } \\
X \text {, Division } 1 \\
\end{array}$ & reg1.147.r10 & 1993 & $\mathbf{N}$ & Annulled by ASME & $n / a$ & $n / a$ \\
\hline 1610 & ASME & $\begin{array}{l}\text { ASME Code } \\
\text { Case N-217-1 }\end{array}$ & 1987 & $\begin{array}{l}\text { Postwold Heat Treatment of Weld } \\
\text { Deposit Cladding on Classes 1, 2, } \\
\text { 3, MC, and CS items, Section III, } \\
\text { Division } 1\end{array}$ & reg1.84.r29 & 1993 & $\mathbf{N}$ & Annulled by ASME & $n / a$ & $n / a$ \\
\hline 1611 & ASME & $\begin{array}{l}\text { ASME Code } \\
\text { Case N-220 }\end{array}$ & 1984 & $\begin{array}{l}\text { Code Effective Date for Component } \\
\text { Supports, Section III, Division } 1\end{array}$ & reg1.84.r29 & 1993 & $\mathbf{N}$ & Annulied by ASME & $n / a$ & $n / a$ \\
\hline
\end{tabular}


Codes and Standards and Other Guidance Ched in Regulatory Documents

Part A - Industry Consensus Codes and Standerds (continued)

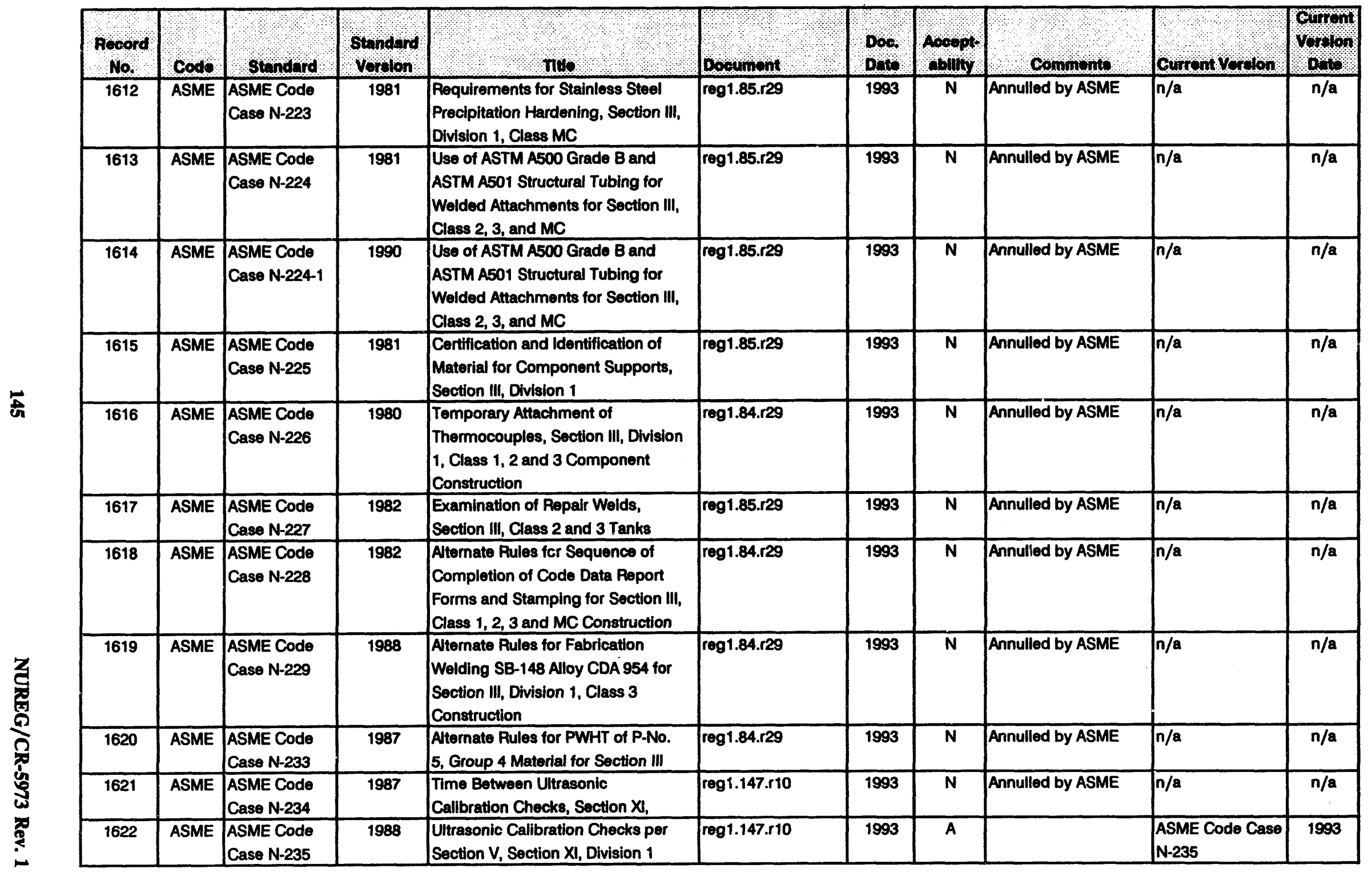




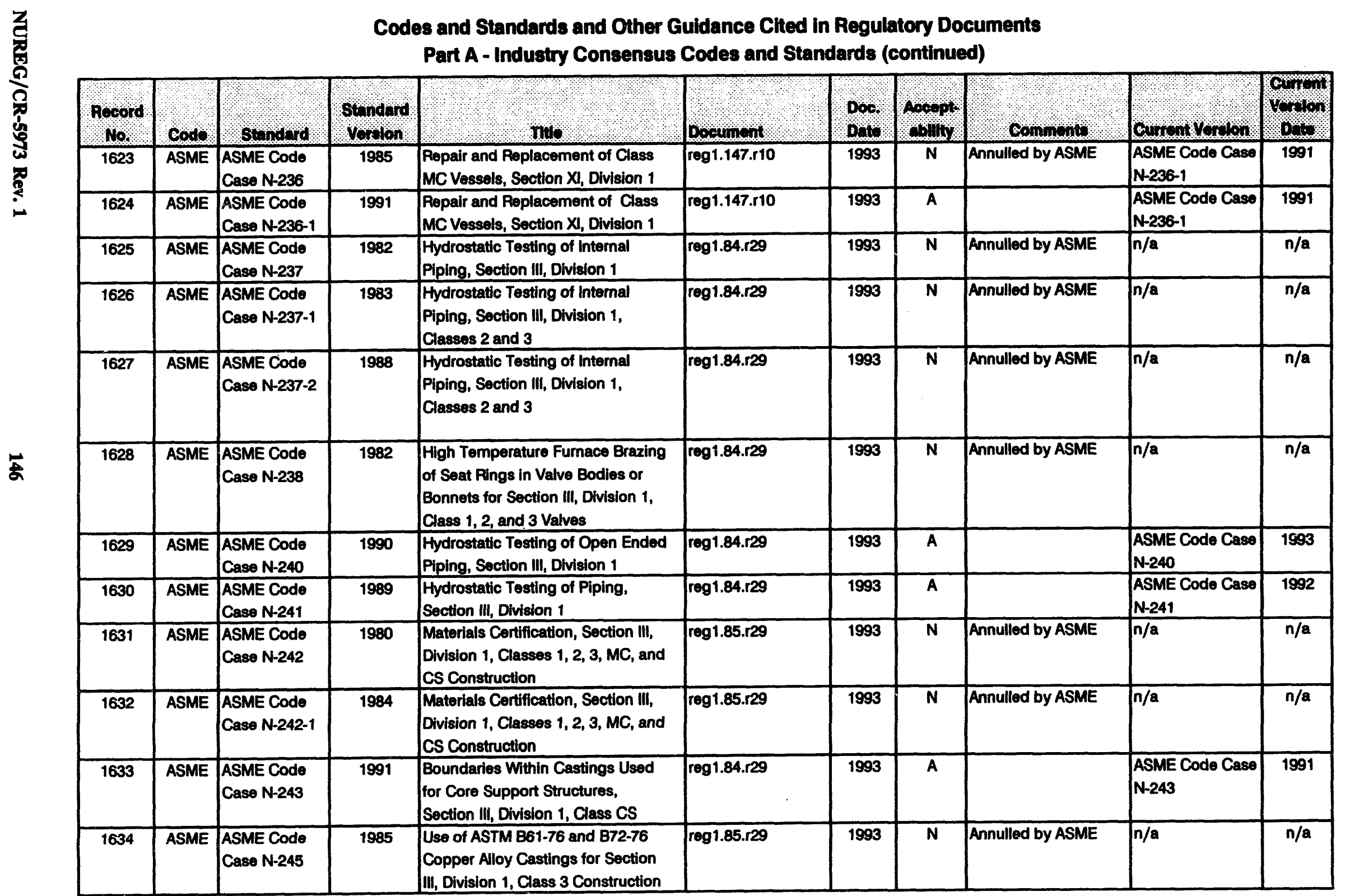


Codes and Standards and Other Guidance Cited in Regulatory Documents

Part A - Industry Consensus Codes and Standards (continued)

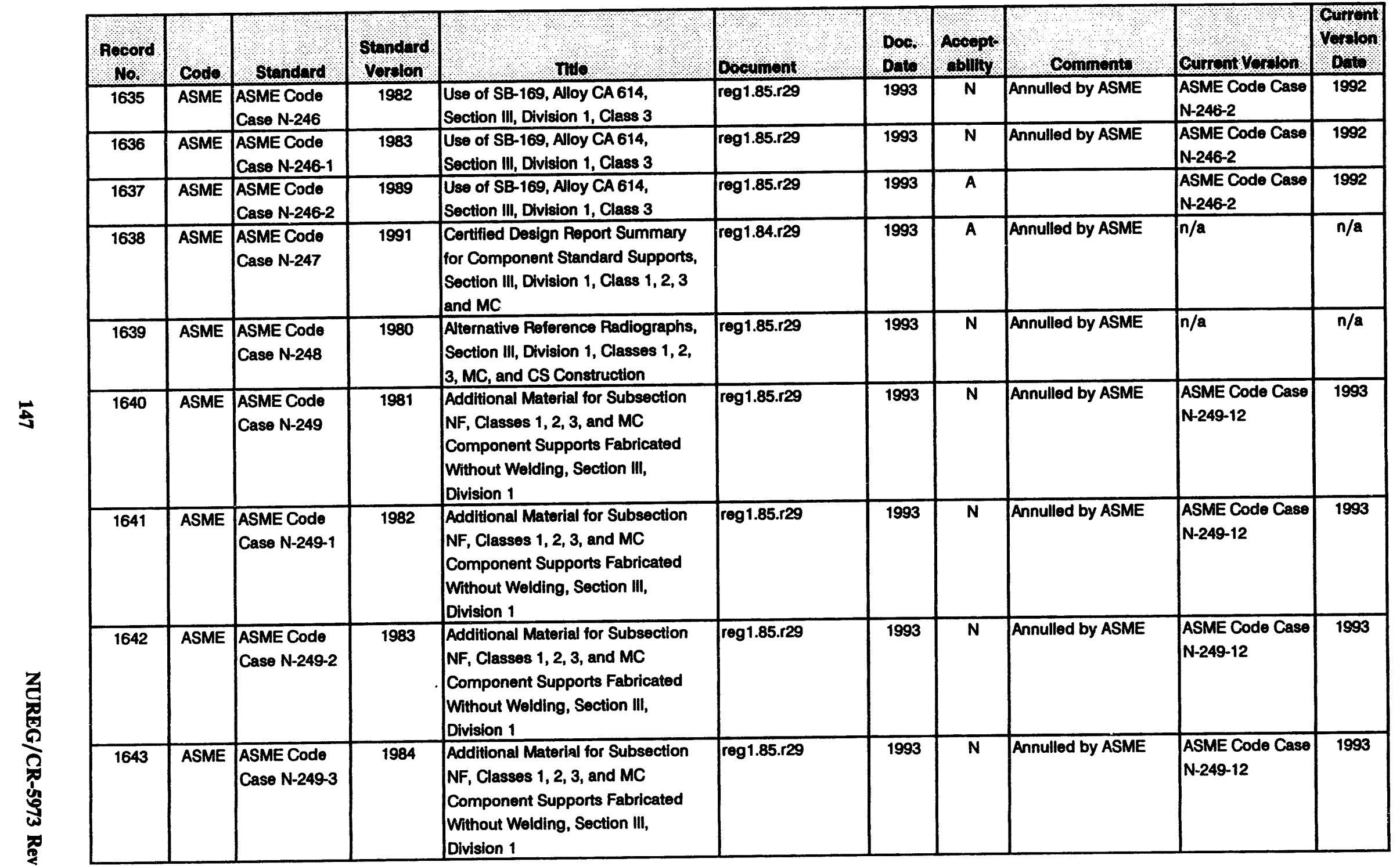




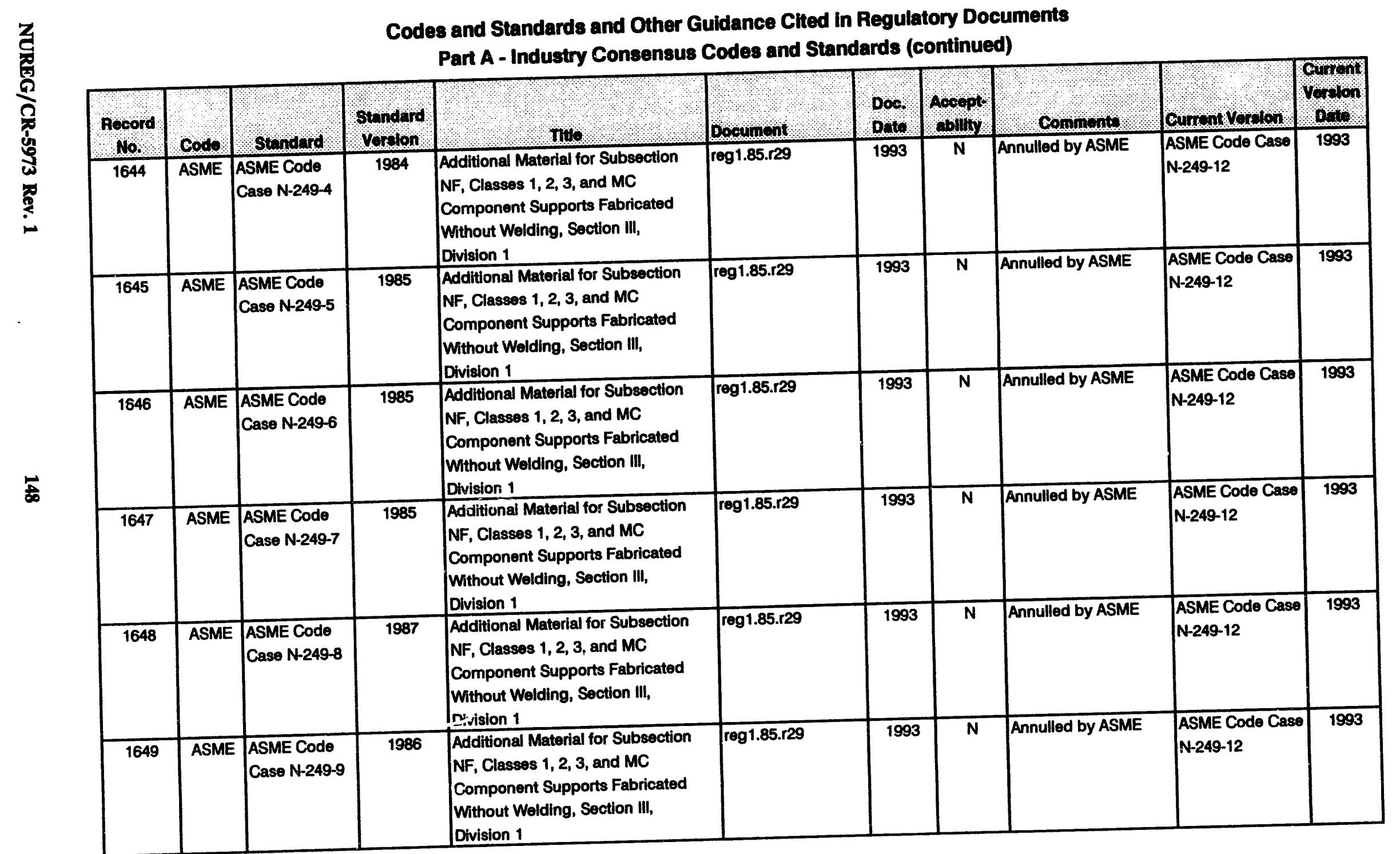


Codes and Standards and Other Guidance Cited in Regulatory Documents

Part A - Industry Consensus Codes and Standards (continued)

\begin{tabular}{|c|c|c|c|c|c|c|c|c|c|c|}
\hline Pecord & Code & standard & $\begin{array}{l}\text { Standard } \\
\text { vordon }\end{array}$ & nono & Doovnent & $\begin{array}{l}\text { Dor. } \\
\text { Dalo }\end{array}$ & Aocept & Connent & Cumont Vomolon & Corion \\
\hline 1650 & ASME & \begin{tabular}{|l|} 
ASME Code \\
Case N-249-10
\end{tabular} & 1989 & $\begin{array}{l}\text { Additional Material for Subsection } \\
\text { NF, Classes 1, 2, 3, and MC } \\
\text { Component Supports Fabricated } \\
\text { Without Welding, Section III, } \\
\text { Division } 1\end{array}$ & reg1.85.r29 & 1993 & $\mathbf{N}$ & & $\begin{array}{l}\text { ASME Code Case } \\
\text { N-249-12 }\end{array}$ & 1993 \\
\hline 1651 & ASME & \begin{tabular}{|l|} 
ASME Code \\
Case N-249-11
\end{tabular} & 1991 & $\begin{array}{l}\text { Additional Material for Subsection } \\
\text { NF, Classes 1, 2, 3, and MC } \\
\text { Component Supports Fabricated } \\
\text { Without Welding, Section III, } \\
\text { Division } 1\end{array}$ & reg1.85.r29 & 1993 & $\mathbf{P}$ & & $\begin{array}{l}\text { ASME Code Case } \\
\text { N-249-12 }\end{array}$ & 1993 \\
\hline 1652 & ASME & $\begin{array}{l}\text { ASME Code } \\
\text { Case N-252 }\end{array}$ & 1979 & $\begin{array}{l}\text { Low Energy Capacitive Discharge } \\
\text { Welding Method for Temporary or } \\
\text { Permanent Attachments to } \\
\text { Components and Supports, Section } \\
\text { III, Division } 1 \text {, and XI }\end{array}$ & reg1.147.r10 & 1993 & $\mathbf{N}$ & Annulled by ASME & $n / a$ & $n / a$ \\
\hline 1653 & ASME & $\begin{array}{l}\text { ASME Code } \\
\text { Case N-252 }\end{array}$ & 1982 & $\begin{array}{l}\text { Low Energy Capacitive Discharge } \\
\text { Welding Method for Temporary or } \\
\text { Permanent Attachments to } \\
\text { Components and Supports, Section } \\
\text { III, Division } 1 \text {, and XI } \\
\end{array}$ & reg1.84.r29 & 1993 & $\mathbf{N}$ & Annulled by ASME & $n / a$ & $n / a$ \\
\hline 1654 & ASME & $\begin{array}{l}\text { ASME Code } \\
\text { Case N-259 }\end{array}$ & 1984 & $\begin{array}{l}\text { Ni-Cu-Al Bolting Material SB } 164 \\
\text { Modified, Section III, Division 1, } \\
\text { Class } 3\end{array}$ & reg1.85.r29 & 1993 & $\mathbf{N}$ & Annulled by ASME & $n / a$ & $n / a$ \\
\hline 1655 & ASME & $\begin{array}{l}\text { ASME Code } \\
\text { Case N-260 }\end{array}$ & 1985 & $\begin{array}{l}\text { Weld Repair of SA-182 Type } 316 \\
\text { Forgirgs Section III, Division 1, } \\
\text { Classes 1, 2, 3, and MC }\end{array}$ & reg1.84.r29 & 1993 & $\mathbf{N}$ & Annulled by ASME & $n / a$ & $n / a$ \\
\hline 1656 & ASME & $\begin{array}{l}\text { ASME Code } \\
\text { Case N-260-1 }\end{array}$ & 1985 & $\begin{array}{l}\text { Weld Repair of SA-182 Type } 316 \\
\text { Forgings, Section III, Division 1, } \\
\text { Classes 1, 2, 3, and MC } \\
\end{array}$ & $\operatorname{rog} 1.84 .129$ & 1993 & $\mathbf{N}$ & Annulled by ASME & $n / a$ & $n / a$ \\
\hline 1657 & ASME & $\begin{array}{l}\text { ASME Code } \\
\text { Case N-260-2 }\end{array}$ & 1988 & $\begin{array}{l}\text { Weld Repair of SA-182 Type } 316 \\
\text { Forgings, Section III, Division 1, } \\
\text { Classes 1, 2, 3, and MC }\end{array}$ & reg1.84.r29 & 1993 & $\mathbf{N}$ & Annulled by ASME & $n / a$ & $n / a$ \\
\hline
\end{tabular}


Codes and Standaró and Other Guidance Clted in Regulatory Documents

Part A - Industry Consensus Codes and Standards (continued)

\begin{tabular}{|c|c|c|c|c|c|c|c|c|c|c|}
\hline & & & & Part A - Inousiry Consensus & Gula andu & 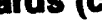 & & & & \\
\hline $\begin{array}{l}\text { Record } \\
\text { No. }\end{array}$ & & Standerd & Strondard & 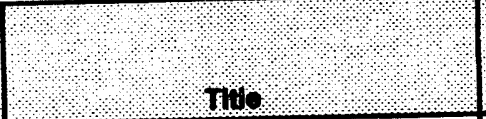 & Boounont & Dos: & Aocopts & Commonts & Gunout vorolon & ploptol \\
\hline 1658 & ASME & $\begin{array}{l}\text { ASME Code } \\
\text { Case N-261 }\end{array}$ & 1982 & $\begin{array}{l}\text { Wold Procedure Qualification for } \\
\text { Materials with Impact Requirements } \\
\text { for Section III, Division 1, Class } 3 \\
\text { Construction }\end{array}$ & reg1.84.r29 & 1993 & $\mathbf{N}$ & Annulled by ASME & $n / a$ & $n / a$ \\
\hline 1659 & ASME & $\begin{array}{l}\text { ASME Code } \\
\text { Case N-262 }\end{array}$ & 1991 & $\begin{array}{l}\text { Electric Resistance Spot Wolding } \\
\text { for Structural Use in Component } \\
\text { Supports, Section III, Division } 1\end{array}$ & reg1.84.r29 & 1993 & A & & $\begin{array}{l}\text { ASME Code Case } \\
\text { N-262 }\end{array}$ & 1991 \\
\hline 1660 & ASME & $\begin{array}{l}\text { ASME Code } \\
\text { Case N-263 }\end{array}$ & 1983 & $\begin{array}{l}\text { Alternate Thread Forms, Series and } \\
\text { Fits for Component Supports, } \\
\text { Section III, Division } 1\end{array}$ & reg1.84.r29 & 1993 & $\mathbf{N}$ & Annulled by ASME & $n / a$ & $n / a$ \\
\hline 1661 & ASME & $\begin{array}{l}\text { ASME Code } \\
\text { Case N-265 }\end{array}$ & 1985 & $\begin{array}{l}\text { Modified SA-487 Castings, Section } \\
\text { III, Division 1, Class } 1\end{array}$ & reg1.85.r29 & 1993 & $\mathbf{N}$ & Annulled by ASME & $\begin{array}{l}\text { ASME Code Case } \\
\text { N-265-1 }\end{array}$ & 1991 \\
\hline 1662 & ASME & $\begin{array}{l}\text { ASME Code } \\
\text { Case N-265-1 }\end{array}$ & 1988 & $\begin{array}{l}\text { Modified SA-487 Castings, Section } \\
\text { III, Division 1, Class } 1\end{array}$ & reg1.85.r29 & 1993 & $\bar{A}$ & & $\begin{array}{l}\text { ASME Code Case } \\
\text { N-265-1 }\end{array}$ & 1991 \\
\hline 1663 & ASME & $\begin{array}{l}\text { ASME Code } \\
\text { Case N-267 }\end{array}$ & 1981 & $\begin{array}{l}\text { Double-Wall Radiography, Section } \\
\text { III, Division 1, Class } 1 \text { and } 2\end{array}$ & reg1.85.r29 & 1993 & $\mathbf{N}$ & Annulled by ASME & $n / a$ & $n / a$ \\
\hline 1664 & ASME & $\begin{array}{l}\text { ASME Code } \\
\text { Case N-271 }\end{array}$ & 1986 & $\begin{array}{l}\text { Simplified Mothod for Analyzing } \\
\text { Flat Face Flanges with Motal to } \\
\text { Metal Contact Outside the Bolt } \\
\text { Circle for Section III, Class 2, 3, and } \\
\text { MC Construction }\end{array}$ & reg1.84.r29 & 1993 & $\mathbf{N}$ & Annulled by ASME & $n / a$ & $n / a$ \\
\hline 1665 & ASME & $\begin{array}{l}\text { ASME Code } \\
\text { Case N-272 }\end{array}$ & 1982 & $\begin{array}{l}\text { Compling Data Report Forms, } \\
\text { Section III, Division } 1\end{array}$ & reg1.84.r29 & 1993 & $\mathbf{N}$ & Annulled by ASME & $n / a$ & $n / a$ \\
\hline 1666 & ASME & $\begin{array}{l}\text { ASME Code } \\
\text { Case N-274 }\end{array}$ & 1986 & $\begin{array}{l}\text { Alternative Rules for Examination of } \\
\text { Weld Repairs for Section III, } \\
\text { Division } 1 \text { Construction }\end{array}$ & reg1.85.r29 & 1993 & $\mathbf{N}$ & Annulled by ASME & $n / a$ & $n / a$ \\
\hline 1667 & ASME & $\begin{array}{l}\text { ASME Code } \\
\text { Case N-275 }\end{array}$ & 1983 & $\begin{array}{l}\text { Repair of Welds, Section III, Division } \\
1\end{array}$ & reg1.84.r29 & 1993 & $\mathbf{N}$ & Annulled by ASME & $n / a$ & $n / a$ \\
\hline 1668 & ASME & $\begin{array}{l}\text { ASME Code } \\
\text { Case N-276 }\end{array}$ & 1986 & $\begin{array}{l}\text { Wolding of SA-358 Pipe, Soction III, } \\
\text { Division } 1\end{array}$ & reg1.84.r29 & 1993 & $N$ & Annulled by ASME & $n / a$ & $n / a$ \\
\hline 1669 & ASME & $\begin{array}{l}\text { ASME Code } \\
\text { Case N-277 }\end{array}$ & 1980 & $\begin{array}{l}\text { Use of Type XM-19 Austenitic } \\
\text { Stainless Steel for Section III, } \\
\text { Division 1, Class MC Construction }\end{array}$ & reg1.85.r29 & 1993 & $\mathbf{N}$ & Annulled by ASME & $n / a$ & $n / a$ \\
\hline
\end{tabular}

Division 1, Class MC Construction 
Codes and Standards and Other Guidance Cited in Regulatory Documents

Part A - Industry Consensus Codes and Standards (continued)

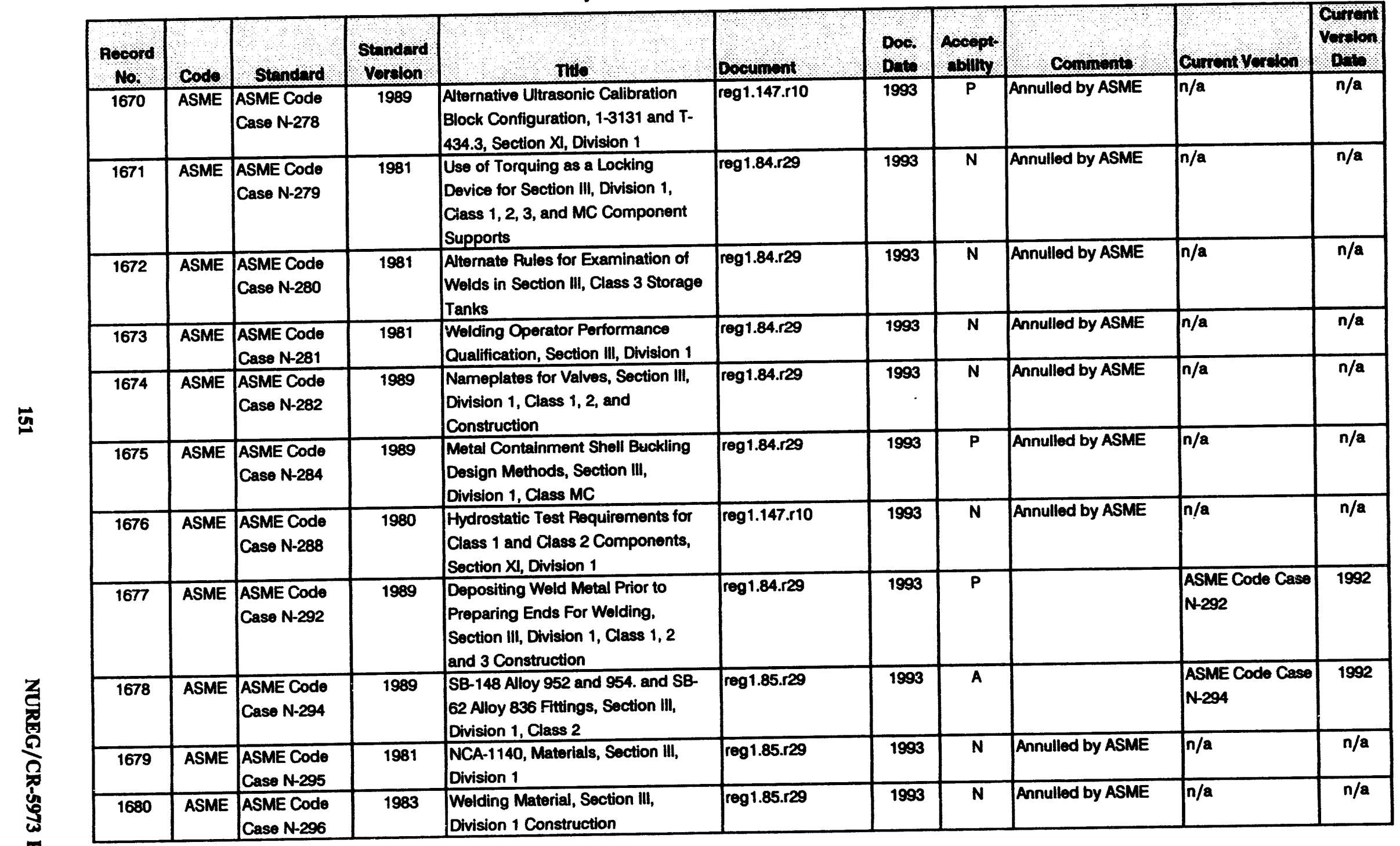




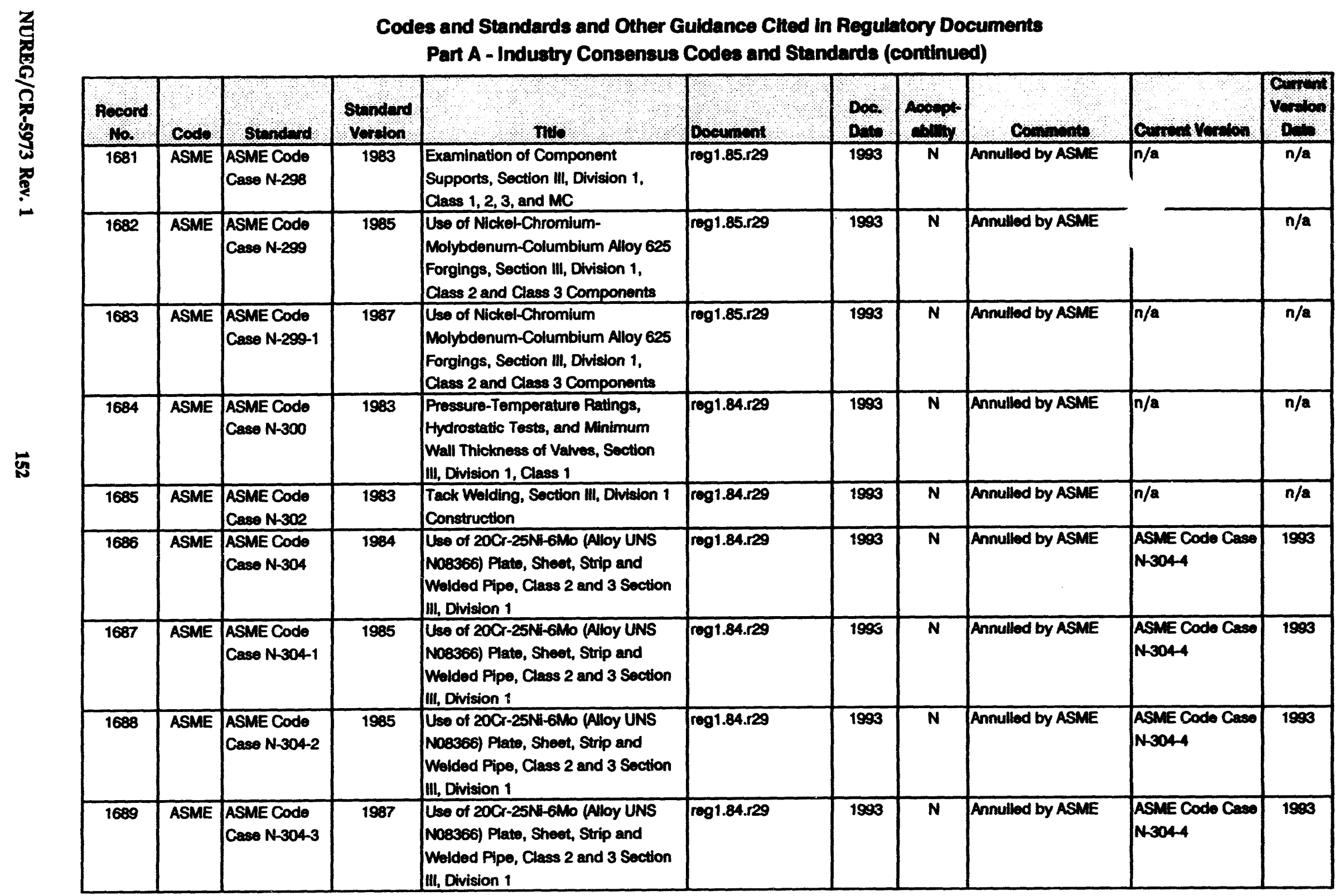


Codes and Standards and Other Guldance Ched in Regulatory Documents

Part A - Industry Consensus Codes and Standards (continued)

\begin{tabular}{|c|c|c|c|c|c|c|c|c|c|c|}
\hline $\begin{array}{l}\text { Recond } \\
\text { No. }\end{array}$ & Code & Standard & $\begin{array}{l}\text { Standand } \\
\text { Veralon }\end{array}$ & $\mathbf{m}_{\mathbf{l}}$ & Doeunens: & $\begin{array}{l}\text { Don. } \\
\text { Dom }\end{array}$ & Anespet & Comanome & 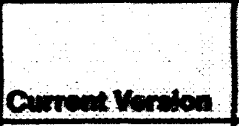 & opin \\
\hline 1690 & ASME & $\begin{array}{l}\text { ASME Code } \\
\text { Case N-304-4 }\end{array}$ & 1989 & $\begin{array}{l}\text { Use of 20CT-25Ni-6iNo (Aloy UNS } \\
\text { No8366) Plate, Sheet, Strip and } \\
\text { Wolded Pipe, Cleses } 2 \text { and } 3 \text { Section } \\
\text { III, Division } 1\end{array}$ & reg1.84.r29 & 1993 & A & & $\begin{array}{l}\text { ASIE Cods Caso } \\
\text { N-3O4-4 }\end{array}$ & 1993 \\
\hline 1691 & ASME & $\begin{array}{l}\text { ASME Code } \\
\text { Case N-306 }\end{array}$ & 1987 & $\begin{array}{l}\text { Calibration Block Matorial } \\
\text { Solection, Appendix 1, 1-3121, } \\
\text { Soction X, Division } 1\end{array}$ & reg1.147.r10 & 1993 & $\mathbf{N}$ & Arinullod by ASME & $n / a$ & $n / a$ \\
\hline 1692 & ASME & $\begin{array}{l}\text { ASME Code } \\
\text { Case N-307 }\end{array}$ & 1984 & $\begin{array}{l}\text { Rovised Utrasonic Examination } \\
\text { Volume for Class } 1 \text { Bolting, Table } \\
\text { mB-2500-1, Examination Catiegory } \\
\text { B-G-1. When the Examinations Are } \\
\text { Conducted from the Center-Drilled } \\
\text { Hole, Section X, Diviaton } 1 \\
\end{array}$ & $\operatorname{rog} 1.147 .110$ & 1993 & $\mathbf{N}$ & Annulled by ASME & $\begin{array}{l}\text { ASME Code Case } \\
\text { N307-1 }\end{array}$ & 1993 \\
\hline 1693 & ASME & $\begin{array}{l}\text { ASME Code } \\
\text { Case N-307-1 }\end{array}$ & 1990 & $\begin{array}{l}\text { Rovised Uitrasonic Examination } \\
\text { Volume for Class } 1 \text { Bolting, Table } \\
\text { WB-2500-1, Examination Category } \\
\text { B-G-1. When the Examinations Are } \\
\text { Conducted from the Center-Drilled } \\
\text { Hole, Section XI, Divieion } 1\end{array}$ & reg1.147.r10 & 1993 & $\bar{A}$ & & $\begin{array}{l}\text { ASNE Codo Caso } \\
\text { N307-1 }\end{array}$ & 1993 \\
\hline 1694 & ASME & $\begin{array}{l}\text { ASME Code } \\
\text { Case N-308 }\end{array}$ & 1990 & $\begin{array}{l}\text { Documentation of Pepairs and } \\
\text { Poplacements of Components in } \\
\text { Nuclear Power Plants, Section XI, } \\
\text { Division } 1 \\
\end{array}$ & $\operatorname{reg} 1.147 .110$ & 1993 & $\mathbf{N}$ & Annullod by ASME & $n / a$ & $n / a$ \\
\hline 1695 & ASME & $\begin{array}{l}\text { ASME Code } \\
\text { Case N-309 }\end{array}$ & 1985 & $\begin{array}{l}\text { Identfication of Materials for } \\
\text { Component Supports, Section if, } \\
\text { Division } 1\end{array}$ & $\operatorname{reg} 1.84 .129$ & 1993 & $\mathbf{N}$ & Annullad by ASNE & $\begin{array}{l}\text { ASIE Code Cane } \\
\text { N309-1 }\end{array}$ & 1991 \\
\hline 1696 & ASME & $\begin{array}{l}\text { ASME Code } \\
\text { Case N-309-1 }\end{array}$ & 1991 & $\begin{array}{l}\text { Identification of Materials for } \\
\text { Component Supports Soction III, } \\
\text { Division } 1\end{array}$ & 1091.84 .129 & 1993 & $\mathbf{A}$ & & $\begin{array}{l}\text { ASME Cods Case } \\
\text { M309-1 }\end{array}$ & 1991 \\
\hline 1697 & ASME & $\begin{array}{l}\text { ASME Code } \\
\text { Case N-310-1 }\end{array}$ & 1982 & $\begin{array}{l}\text { Cortification of Bolting Matoriats, } \\
\text { Soction III, Division 1, Class 1, 2,3, } \\
\text { MC and CS }\end{array}$ & $\operatorname{reg} 1.85 .529$ & 1993 & $N$ & Annullod by ASME & $n / a$ & $n / a$ \\
\hline
\end{tabular}




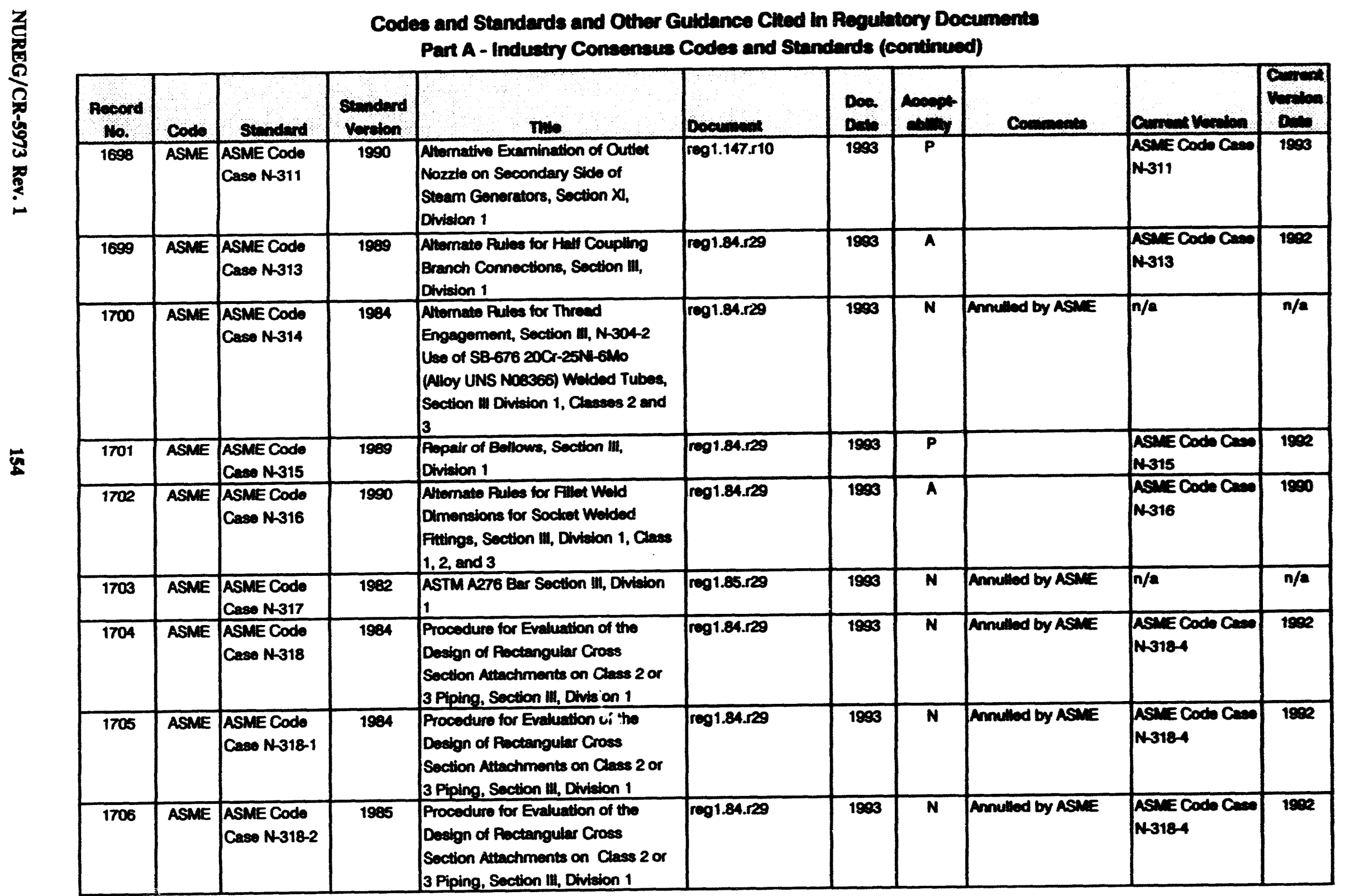


Codes and Standards and Other Guidance Ched in Regulatory Documents

Part A - Industry Consensus Codes and Standands (continued)

\begin{tabular}{|c|c|c|c|c|c|c|c|c|c|c|}
\hline $\begin{array}{c}\text { Pocond } \\
\text { No. }\end{array}$ & Code & Standend & $\begin{array}{l}\text { Stendind } \\
\text { Veraton }\end{array}$ & Ine & Doconnest & $\begin{array}{l}\text { Doen } \\
\text { Dene }\end{array}$ & Aosept. & Commenth & Curvent Vuraten & $\mathrm{C}_{\mathrm{b}, \mathrm{m}}$ \\
\hline 1707 & ASME & $\begin{array}{l}\text { ASME Code } \\
\text { Case N-318-3 }\end{array}$ & 1989 & $\begin{array}{l}\text { Procedure for Evaluation of the } \\
\text { Design of Pectangular Cross } \\
\text { Section Attachments on Class } 2 \text { or } \\
3 \text { Piping, Section II, Division } 1\end{array}$ & reg1.84.r29 & 1993 & $\mathbf{N}$ & Annullod by ASME & $\begin{array}{l}\text { ASNE Code Case } \\
\text { N318 } 4\end{array}$ & 1992 \\
\hline 1708 & ASME & $\begin{array}{l}\text { ASME Code } \\
\text { Case N-318-4 }\end{array}$ & 1989 & $\begin{array}{l}\text { Procedure for Evaluation of the } \\
\text { Design of Pectangular Cross } \\
\text { Section Attachments on Class } 2 \text { or } \\
3 \text { Piping, Section III, Division } 1\end{array}$ & reg1.84.r29 & 1993 & $\mathbf{P}$ & Annullod by ASNE & $\begin{array}{l}\text { ASIE Cods Case } \\
\text { N318-4 }\end{array}$ & 1992 \\
\hline 1709 & ASME & $\begin{array}{l}\text { ASME Code } \\
\text { Case N-319 }\end{array}$ & 1989 & $\begin{array}{l}\text { Atternate Procedure for Evaluation } \\
\text { of Stresses in Butt Wold Elbows in } \\
\text { Class } 1 \text { Piping, Section III Division I }\end{array}$ & $\operatorname{reg} 1.84 .529$ & 1993 & $\mathbf{N}$ & Annulled by ASME & $\begin{array}{l}\text { ASINE Codo Caso } \\
\text { N-319-2 }\end{array}$ & 1992 \\
\hline 1710 & ASME & $\begin{array}{l}\text { ASME Code } \\
\text { Case N-319-1 }\end{array}$ & 1989 & $\begin{array}{l}\text { Alternate Procedure for Evaluation } \\
\text { of Stresses in Butt Wold Elbows in } \\
\text { Class } 1 \text { Piping, Section III Division I }\end{array}$ & reg1.84.r29 & 1993 & $\mathbf{N}$ & Annulled by ASME & $\begin{array}{l}\text { ASNE Code Case } \\
\text { N-319-2 }\end{array}$ & 1998 \\
\hline 1711 & ASME & $\begin{array}{l}\text { ASME Code } \\
\text { Case N-320 } \\
\end{array}$ & 1983 & $\begin{array}{l}\text { Aternate PWHT for SA-487, Grade } \\
\text { CA6NM, Section III, Division } 1 \\
\end{array}$ & reg1.84.r29 & 1993 & $\mathbf{N}$ & Annullod by ASME & $n / a$ & $n / a$ \\
\hline 1712 & ASME & $\begin{array}{l}\text { ASME Code } \\
\text { Case N-321 }\end{array}$ & 1985 & $\begin{array}{l}\text { Use of Modified SA-249, Type } 304 \\
\text { for Section III, Division 1, Class } 1 \\
\text { Construction }\end{array}$ & reg1.85.r29 & 1993 & $\mathbf{N}$ & Annulled by ASME & $n / a$ & $n / a$ \\
\hline 1713 & ASME & $\begin{array}{l}\text { ASME Code } \\
\text { Case N-321-1 }\end{array}$ & 1988 & $\begin{array}{l}\text { Use of Modified SA-249, Grade TP } \\
304 \text {, Section III, Division 1, Class } 1\end{array}$ & reg1.85.r29 & 1993 & $\mathbf{N}$ & Annulled by ASME & $n / a$ & $n / a$ \\
\hline 1714 & ASME & $\begin{array}{l}\text { ASME Code } \\
\text { Case N-328 }\end{array}$ & 1987 & $\begin{array}{l}\text { Thermit Brazing or Wolding of } \\
\text { Nonstructural Attachments, Section } \\
\text { Iili, Diviaion } 1\end{array}$ & reg1.84.r29 & 1993 & $\mathbf{N}$ & Annulled by ASME & $n / a$ & $n / a$ \\
\hline 1715 & ASME & $\begin{array}{l}\text { ASME Code } \\
\text { Case N-329 } \\
\end{array}$ & 1990 & $\begin{array}{l}\text { Examination of Bar Material, } \\
\text { Section III, Division 1, Class } 1\end{array}$ & reg1.85.r29 & 1993 & $\bar{A}$ & & $\begin{array}{l}\text { ASNE Code Case } \\
\text { N3es }\end{array}$ & 1990 \\
\hline 1716 & ASME & $\begin{array}{l}\text { ASME Code } \\
\text { Case N-335 }\end{array}$ & 1985 & $\begin{array}{l}\text { Rules for Uttrasonic Examination of } \\
\text { Similar and Dissimilar Motal Piping } \\
\text { Wolds, Section XI, Division } 1 \\
\end{array}$ & reg1.147.s10 & 1993 & $\mathbf{N}$ & Annulled by ASME & $\begin{array}{l}\text { ASME Code Caso } \\
\text { N-335-1 }\end{array}$ & 1991 \\
\hline 1717 & ASME & $\begin{array}{l}\text { ASME Code } \\
\text { Case N-335-1 }\end{array}$ & 1991 & $\begin{array}{l}\text { Rules for Ultrasonic Examination of } \\
\text { Similar and Dissimilar Niotal Piping } \\
\text { Welds, Section XI, Division } 1\end{array}$ & reg1.147.r10 & 1993 & A & & $\begin{array}{l}\text { ASME Code Case } \\
\text { N-335-1 }\end{array}$ & 1991 \\
\hline
\end{tabular}


Codes and Standards and Other Guidance Clted in Regulatory Documents

Part A - Industry Consensus Codes and Standards (continued)

\begin{tabular}{|c|c|c|c|c|c|c|c|c|c|c|}
\hline Rocord & Cods & Stundind & $\begin{array}{l}\text { Standard } \\
\text { Vorolon }\end{array}$ & $m_{b}$ & Boounont & Dos & socept & Comments & Cunent verilon & Qimon \\
\hline 1718 & ASME & $\begin{array}{l}\text { ASME Code } \\
\text { Case N-336 }\end{array}$ & 1983 & $\begin{array}{l}\text { Examination of Welds Inaccessible } \\
\text { During Pressure Test, Section III, } \\
\text { Division 1, Class MC }\end{array}$ & reg1.84.r29 & 1993 & $\mathbf{N}$ & Annulled by ASME & $n / a$ & $n / a$ \\
\hline 1719 & ASME & $\begin{array}{l}\text { ASME Code } \\
\text { Case N-337 }\end{array}$ & 1985 & $\begin{array}{l}\text { Use of ASTM B525-70 Grade II, } \\
\text { Type II, Sintered Austenitic } \\
\text { Stainless Steel for Class 2, 3, and } \\
\text { MC Component Standard } \\
\text { Supports, Section III, Division } 1\end{array}$ & $\operatorname{reg} 1.85 .129$ & 1993 & $\mathbf{N}$ & Annulled by ASME & $\begin{array}{l}\text { ASME Code Case } \\
\text { N-337-1 }\end{array}$ & 1991 \\
\hline 1720 & ASME & $\begin{array}{l}\text { ASME Code } \\
\text { Case N-337-1 }\end{array}$ & 1991 & $\begin{array}{l}\text { Use of ASTM B525-70 Grade II, } \\
\text { Type II, Sintered Austenitic } \\
\text { Stainless Steel for Class 2, 3, and } \\
\text { MC Component Standard } \\
\text { Supports, Section III, Division } 1\end{array}$ & reg1.85.r29 & 1993 & A & & $\begin{array}{l}\text { ASME Code Case } \\
\text { N-337-1 }\end{array}$ & 1991 \\
\hline 1721 & ASME & $\begin{array}{l}\text { ASME Code } \\
\text { Case N-339 }\end{array}$ & 1984 & $\begin{array}{l}\text { Examination of Ends of Fillot } \\
\text { Weids, Section III, Division 1, } \\
\text { Classes 1, 2, and MC }\end{array}$ & reg1.84.r29 & 1993 & $\mathbf{N}$ & Annulled by ASME & $n / a$ & $n / a$ \\
\hline 1722 & ASME & $\begin{array}{l}\text { ASME Code } \\
\text { Case N-341 }\end{array}$ & 1988 & $\begin{array}{l}\text { Certification of Lovol III NDE } \\
\text { Examiner, Section III, Division } 1 \text { and } \\
2\end{array}$ & reg1.84.r29 & 1993 & $\mathbf{N}$ & Annulled by ASME & $n / a$ & $n / a$ \\
\hline 1723 & ASME & $\begin{array}{l}\text { ASME Code } \\
\text { Case N-342 }\end{array}$ & 1985 & $\begin{array}{l}\text { Use of SA-249 and SA-312 Type } 317 \\
\text { Stainless Steel, Section III, Division } \\
1, \text { Classes 1, 2, and } 3\end{array}$ & reg1.85.r29 & 1993 & $\mathbf{N}$ & Annulled by ASME & $n / a$ & $n / a$ \\
\hline 1724 & ASME & $\begin{array}{l}\text { ASME Code } \\
\text { Case N-343 }\end{array}$ & 1988 & $\begin{array}{l}\text { Alternative Scope of Examination of } \\
\text { Attachment Wolds for Examination } \\
\text { Categories B-H, B-K-1, and C-C, } \\
\text { Section XI, Division } 1\end{array}$ & reg1.147.r10 & 1893 & $\mathbf{N}$ & Annulled by ASME & $n / a$ & $n / a$ \\
\hline 1725 & ASME & $\begin{array}{l}\text { ASME Code } \\
\text { Case N-345 }\end{array}$ & 1982 & $\begin{array}{l}\text { Attachment of AMS } 5382 \text { Alloy } 31 \\
\text { Seat Rings by Friction Wolding, } \\
\text { Section III, Division 1, Classes 1, 2, } \\
\text { and } 3\end{array}$ & reg1.84.r29 & 1993 & $\mathbf{N}$ & Annulled by ASME & $\begin{array}{l}\text { ASME Code Case } \\
\text { N-345-1 }\end{array}$ & 1991 \\
\hline 1726 & ASME & $\begin{array}{l}\text { ASME Code } \\
\text { Case N-345-1 }\end{array}$ & 1991 & $\begin{array}{l}\text { Attachment of AMS } 5382 \text { Alloy } 31 \\
\text { Seat Pings by Friction Wolding, } \\
\text { Section III, Division 1, Classes 1,2, } \\
\text { and } 3\end{array}$ & reg1.84.r29 & 1993 & A & & $\begin{array}{l}\text { ASME Code Case } \\
\text { N-345-1 }\end{array}$ & 1991 \\
\hline
\end{tabular}


Codes and Standards and Other Guidance Cited in Regulatory Documents

Part A - Industry Consensus Codes and Standards (continued)

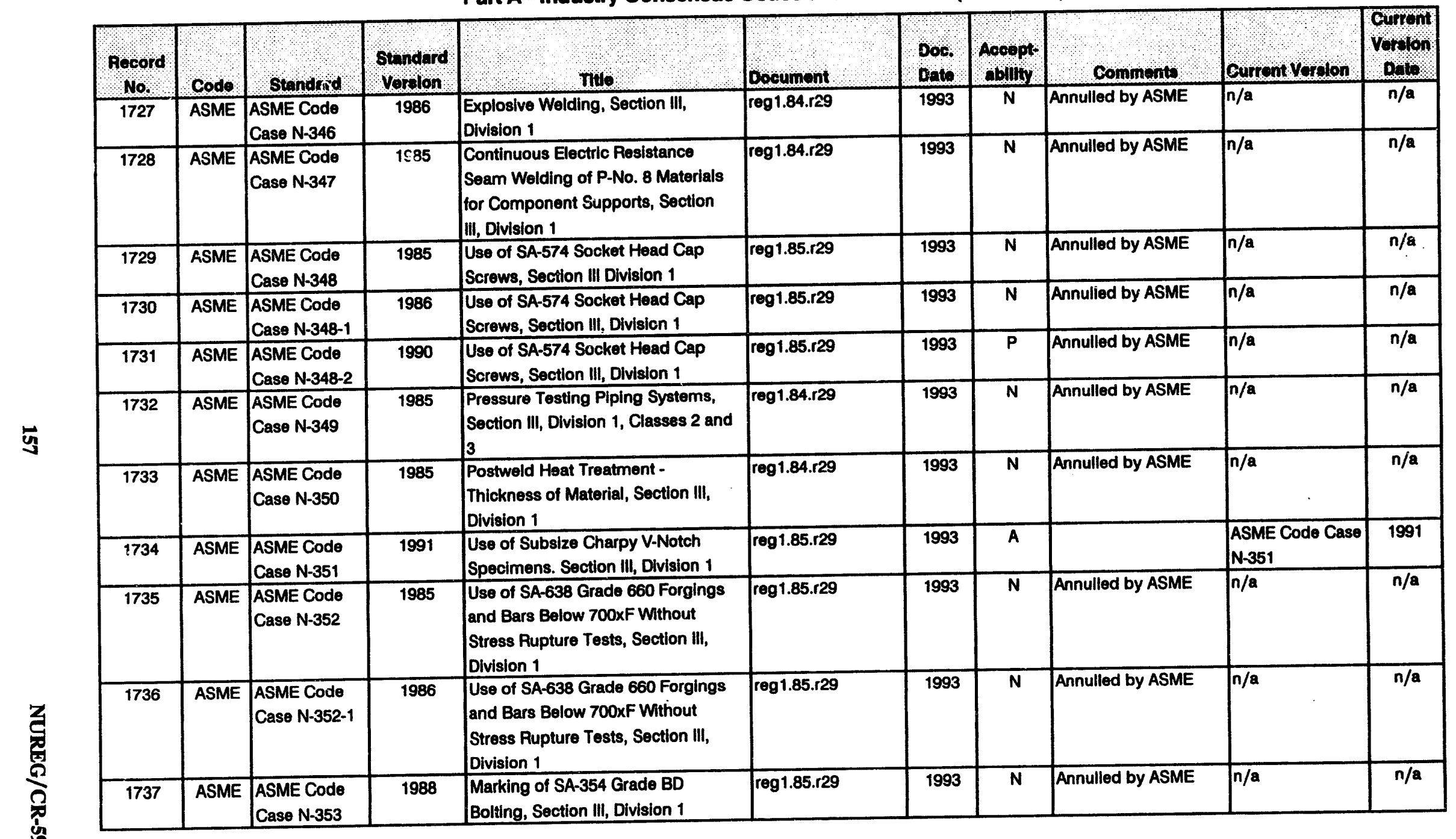


Codes and Standards and Other Guidance Cited in Regulatory Documents

Part A - Industry Consensus Codes and Standards (continued)

\begin{tabular}{|c|c|c|c|c|c|c|c|c|c|c|}
\hline Hocord & Codo & Standard & Standard & ( & borounont & Dow & opopt & Comments & Gurront Vorolon & opton \\
\hline 1738 & ASME & $\begin{array}{l}\text { ASME Code } \\
\text { Case N-355 }\end{array}$ & 1990 & $\begin{array}{l}\text { Calibration Block for Angle Beam } \\
\text { Ultrasonic Examination of Large } \\
\text { Fittings in Accordance with } \\
\text { Appendix III-3410, Section XI, } \\
\text { Division } 1\end{array}$ & reg1.147.r10 & 1993 & A & & $\begin{array}{l}\text { ASME Code Case } \\
\text { N-355 }\end{array}$ & 1993 \\
\hline 1739 & ASME & $\begin{array}{l}\text { ASME Code } \\
\text { Case N-356 }\end{array}$ & 1988 & $\begin{array}{l}\text { Certification Period for Level III NDE } \\
\text { Personnel, Section XI, Division 1, } 2 \\
\text { and } 3\end{array}$ & reg1.147.r10 & 1993 & $\bar{A}$ & & $\begin{array}{l}\text { ASME Code Case } \\
\text { N-356 }\end{array}$ & 1991 \\
\hline 1740 & ASME & $\begin{array}{l}\text { ASME Code } \\
\text { Case N-357 }\end{array}$ & 1988 & $\begin{array}{l}\text { Certification of Material for } \\
\text { Component Supports, Section III, } \\
\text { Division } 1 \text { Class } 1\end{array}$ & reg1.84.r29 & 1993 & $\mathbf{N}$ & Annulled by ASME & $n / a$ & $n / a$ \\
\hline 1741 & ASME & $\begin{array}{l}\text { ASME Code } \\
\text { Case N-359 } \\
\end{array}$ & 1984 & $\begin{array}{l}\text { Weld Connection for Coaxial } \\
\text { Cylinders, Section III, Dlvision } 1\end{array}$ & $\operatorname{reg} 1.84 . r 29$ & 1993 & $\mathbf{N}$ & Annulled by ASME & $n / a$ & $n / a$ \\
\hline 1742 & ASME & $\begin{array}{l}\text { ASME Code } \\
\text { Case N-362 }\end{array}$ & 1983 & $\begin{array}{l}\text { Alternate Rules for Pressure Testing } \\
\text { of Containment ltems, Section III, } \\
\text { Division } 1 \\
\end{array}$ & $\operatorname{rog} 1.84 . r 29$ & 1993 & $\mathbf{N}$ & Annulled by ASME & $n / a$ & $n / a$ \\
\hline 1743 & ASME & $\begin{array}{l}\text { ASME Code } \\
\text { Case N-362-1 }\end{array}$ & 1984 & $\begin{array}{l}\text { Pressure Testing of Containment } \\
\text { Items, Section.lli, Division 1, } \\
\text { Classes 1, 2, and MC }\end{array}$ & reg 1.84.r29 & 1993 & $\mathbf{N}$ & Annulled by ASME & $n / a$ & $n / a$ \\
\hline 1744 & ASME & $\begin{array}{l}\text { ASME Code } \\
\text { Case N-362-2 }\end{array}$ & 1987 & $\begin{array}{l}\text { Pressure Testing of Containment } \\
\text { Items, Section III, Division 1, } \\
\text { Classes 1, 2, and MC }\end{array}$ & reg1.84.r29 & 1993 & $\mathbf{N}$ & Annulled by ASME & $n / a$ & $n / a$ \\
\hline 1745 & ASME & $\begin{array}{l}\text { ASME Code } \\
\text { Case N-367 }\end{array}$ & 1989 & $\begin{array}{l}\text { SA-372 Type V, Grade 1, Class B, } \\
\text { Section III, Division } 1\end{array}$ & reg1.85.r29 & 1993 & $\mathbf{N}$ & Annulied by ASME & $n / a$ & $n / a$ \\
\hline 1746 & ASME & $\begin{array}{l}\text { ASME Code } \\
\text { Case N-368 }\end{array}$ & 1990 & $\begin{array}{l}\text { Pressure Testing of Pump } \\
\text { Discharge, Section III, Division 1, } \\
\text { Classes } 2 \text { and } 3\end{array}$ & reg1.84.r29 & 1993 & $\bar{P}$ & & $\begin{array}{l}\text { ASME Code Case } \\
\text { N-368 }\end{array}$ & 1993 \\
\hline 1747 & ASME & $\begin{array}{l}\text { ASME Code } \\
\text { Case N-369 }\end{array}$ & 1989 & $\begin{array}{l}\text { Resistance Welding of Bellows, } \\
\text { Section III, Division } 1\end{array}$ & reg1.84.r29 & 1993 & $\bar{A}$ & Annulled by ASME & \begin{tabular}{|l|} 
ASME Code Case \\
N-369
\end{tabular} & 1992 \\
\hline 1748 & ASME & $\begin{array}{l}\text { ASME Code } \\
\text { Case N-370 }\end{array}$ & 1985 & $\begin{array}{l}\text { Modified SA-705 Grade XM-13 } \\
\text { Forgings, Section III, Division } 1 \\
\end{array}$ & reg1.85.r29 & 1993 & $\bar{N}$ & Annulled by ASME & \begin{tabular}{|l|} 
ASME Code Case \\
N-370-2 \\
\end{tabular} & 1991 \\
\hline 1749 & ASME & $\begin{array}{l}\text { ASME Code } \\
\text { Case N-370-1 }\end{array}$ & 1985 & $\begin{array}{l}\text { Modified SA-705 Grade XM-13 } \\
\text { Forgings, Section III, Division } 1\end{array}$ & reg1.85.r29 & 1993 & $\mathbf{N}$ & Annulled by ASME & $\begin{array}{l}\text { ASME Code Case } \\
\text { N-370-2 }\end{array}$ & 1991 \\
\hline
\end{tabular}




\section{Codes and Standards and Other Guidance Cited in Regulatory Documents}

Part A - Industry Consensus Codes and Standards (continued)

\begin{tabular}{|c|c|c|c|c|c|c|c|c|c|c|}
\hline $\begin{array}{l}\text { Rocord } \\
\text { No. }\end{array}$ & codes & standnerd & Stundard & (1) nno & Dooumont & $\begin{array}{l}\text { Dor. } \\
\text { Dota. }\end{array}$ & $\begin{array}{l}\text { Accopt } \\
\text { abillty }\end{array}$ & Comments: & Curremt Vorion & owront \\
\hline 1750 & ASME & $\begin{array}{l}\text { ASME Code } \\
\text { Case N-370-2 }\end{array}$ & 1988 & $\begin{array}{l}\text { Modified SA-705 Grade XM-13 } \\
\text { Forgings, Section III, Division } 1\end{array}$ & reg1.85.r29 & 1993 & A & & \begin{tabular}{|l|} 
ASME Code Case \\
N-370-2
\end{tabular} & 1991 \\
\hline 1751 & ASME & $\begin{array}{l}\text { ASME Code } \\
\text { Case N-371 }\end{array}$ & 1985 & $\begin{array}{l}12 \text { Cr-IW-IMo-1/4V Martensitic } \\
\text { Stainless Steol Valve Internals, } \\
\text { Section III, Division } 1\end{array}$ & reg1.85.r29 & 1993 & $\mathbf{N}$ & Annulled by ASME & $n / a$ & $n / a$ \\
\hline 1752 & ASME & $\begin{array}{l}\text { ASME Code } \\
\text { Case N-371-1 }\end{array}$ & 1988 & $\begin{array}{l}\text { 12CR-1W-IMo-1/4V Martensitic } \\
\text { Stainless Steel Valve Internals, } \\
\text { Section III, Division } 1 \\
\end{array}$ & $\operatorname{reg} 1.85 .129$ & 1993 & $\mathbf{N}$ & Annulled by ASME & $n / a$ & $n / a$ \\
\hline 1753 & ASME & $\begin{array}{l}\text { ASME Code } \\
\text { Case N-372 }\end{array}$ & 1983 & $\begin{array}{l}\text { SB-163 Ni-Fe-Cr-Mo-Cu Alloy } 825 \\
\text { (UNS N08825) Tubing, Section III, } \\
\text { Division } 1\end{array}$ & reg1.85.r29 & 1993 & $\mathbf{N}$ & Annulled by ASME & $n / a$ & $n / a$ \\
\hline 1754 & ASME & $\begin{array}{l}\text { ASME Code } \\
\text { Case N-375 }\end{array}$ & 1983 & $\begin{array}{l}\text { Rules for Ultrasonic Examination of } \\
\text { Bolts and Studs, Section XI, } \\
\text { Division } 1\end{array}$ & reg1.147.r10 & 1993 & $\mathbf{N}$ & Annulled by ASME & $n / a$ & $n / a$ \\
\hline 1755 & ASME & $\begin{array}{l}\text { ASME Code } \\
\text { Case N-375-1 }\end{array}$ & 1984 & $\begin{array}{l}\text { Rules for Ultrasonic Examination of } \\
\text { Bolts and Studs, Section XI, } \\
\text { Division } 1\end{array}$ & reg1.147.r10 & 1993 & $\mathbf{N}$ & Annulled by ASME & $n / a$ & $n / 2$ \\
\hline 1756 & ASME & $\begin{array}{l}\text { ASME Code } \\
\text { Case N-375-2 }\end{array}$ & 1987 & $\begin{array}{l}\text { Rules for Ultrasonic Examination of } \\
\text { Bolts and Studs, Section XI, } \\
\text { Division } 1\end{array}$ & reg1.147.r10 & 1993 & $\mathbf{N}$ & Annulled by ASME & $n / a$ & $n / a$ \\
\hline 1757 & ASME & $\begin{array}{l}\text { ASME Code } \\
\text { Case N-376 }\end{array}$ & 1986 & $\begin{array}{l}\text { Pressure Testing of Embedded } \\
\text { Class } 2 \text { and } 3 \text { Piping, Section III, } \\
\text { Division } 1\end{array}$ & reg1.84.r29 & 1993 & $\mathbf{N}$ & Annulled by ASME & $n / a$ & $n / a$ \\
\hline 1758 & ASME & $\begin{array}{l}\text { ASME Code } \\
\text { Case N-377 }\end{array}$ & 1983 & $\begin{array}{l}\text { Effective Throat Thickness of Partial } \\
\text { Penetration Groove Welds, Section } \\
\text { III, Division 1, Classes 1, 2, and } 3\end{array}$ & reg1.84.r29 & 1993 & $\mathbf{N}$ & Annulled by ASME & $n / a$ & $n / a$ \\
\hline 1759 & ASME & $\begin{array}{l}\text { ASME Code } \\
\text { Case N-378 }\end{array}$ & 1986 & $\begin{array}{l}\text { Examination of Piping Support } \\
\text { Material, Section III, Division 1, } \\
\text { Class } 1\end{array}$ & reg1.85.r29 & 1993 & $\mathbf{N}$ & Annuilled by ASME & $n / a$ & $n / a$ \\
\hline 1760 & ASME & \begin{tabular}{|l|} 
ASME Code \\
Case N-379 \\
\end{tabular} & 1985 & \begin{tabular}{|l|} 
Bimetallic Tubing Section III, \\
Division 1, Class 1 \\
\end{tabular} & reg1.85.r29 & 1993 & $N$ & Annulled by ASME & $n / a$ & $n / a$ \\
\hline 1761 & ASME & $\begin{array}{l}\text { ASME Code } \\
\text { Case N-379-1 }\end{array}$ & 1991 & $\begin{array}{l}\text { Bimetallic Tubing Section III, } \\
\text { Division 1, Class } 1\end{array}$ & reg1.85.r29 & 1993 & A & Annulled by ASME & $n / a$ & $n / a$ \\
\hline
\end{tabular}


Codes and Standards and Other Guidance Cited in Regulatory Documents

Part A - Industry Consensus Codes and Standards (continued)

\begin{tabular}{|c|c|c|c|c|c|c|c|c|c|c|}
\hline & & & & Part A - Indusury Consentsus & 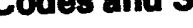 & & & & & \\
\hline $\begin{array}{l}\text { hocord } \\
\mathrm{No}\end{array}$ & & & Shondard & (1) & Docum & Dor. & Aocopt & Comments & Curront Vorglon & voron \\
\hline 1762 & ASME & $\begin{array}{l}\text { ASME Code } \\
\text { Case N-383 }\end{array}$ & 1985 & $\begin{array}{l}\text { Weld Repair of SA-182 Austenitic } \\
\text { Forgings, Section III, Division 1, } \\
\text { Classes 1, 2, and } 3\end{array}$ & reg1.84.r29 & 1993 & $N$ & Annulled by ASME & $n / a$ & $n / a$ \\
\hline 1763 & ASME & $\begin{array}{l}\text { ASME Ccde } \\
\text { Case N-383-1 }\end{array}$ & 1985 & $\begin{array}{l}\text { Weld Repair of SA-182 Austenitic } \\
\text { Forgings, Section III, Division 1, } \\
\text { Classes } 1,2 \text {, and } 3\end{array}$ & $\operatorname{reg} 1.84 . r 29$ & 1993 & $\mathbf{N}$ & Annulled by ASME & $n / a$ & $n / a$ \\
\hline 1764 & ASME & $\begin{array}{l}\text { ASME Code } \\
\text { Case N-388 }\end{array}$ & 1987 & $\begin{array}{l}\text { Component Support Bolting, } \\
\text { Section Iil, Division 1, Classes 2, 3, } \\
\text { and MC }\end{array}$ & reg1.85.r29 & 1993 & $\mathbf{N}$ & Annulled by ASME & $n / a$ & $n / a$ \\
\hline 1765 & ASME & $\begin{array}{l}\text { ASME Code } \\
\text { Case N-389 }\end{array}$ & 1989 & $\begin{array}{l}\text { Alternative Rules for Repairs, } \\
\text { Replacements, or Modifications, } \\
\text { Section XI, Division } 1\end{array}$ & reg1.147.r10 & 1993 & $\mathbf{P}$ & & $\begin{array}{l}\text { ASME Code Case } \\
\text { N-389 }\end{array}$ & 1992 \\
\hline 1766 & ASME & $\begin{array}{l}\text { ASME Code } \\
\text { Case N-391 }\end{array}$ & 1989 & $\begin{array}{l}\text { Procedure for Evaluation of the } \\
\text { Design of Hollow Circular Cross } \\
\text { Section Weided Attachments on } \\
\text { Class } 1 \text { Piping, Section III, Division. } \\
1\end{array}$ & reg1.84.r29 & 1993 & $\mathbf{N}$ & Annulled by ASME & $n / a$ & $n / a$ \\
\hline 1767 & ASME & $\begin{array}{l}\text { ASME Code } \\
\text { Case N-391-1 }\end{array}$ & 1989 & $\begin{array}{l}\text { Procedure for Evaluation of the } \\
\text { Design of Hollow Circular Cross } \\
\text { Section Welded Attachments on } \\
\text { Class } 1 \text { Piping, Section III, Division } \\
1\end{array}$ & reg1.84.r29 & 1993 & $\mathbf{P}$ & & $\begin{array}{l}\text { ASME Code Case } \\
\text { N-391-1 }\end{array}$ & 1992 \\
\hline 1768 & ASME & $\begin{array}{l}\text { ASME Code } \\
\text { Case N-392 }\end{array}$ & 1989 & $\begin{array}{l}\text { Procedure for Evaluation of the } \\
\text { Design of Hollow Circular Cross } \\
\text { Section Welded Attachments on } \\
\text { Classes } 2 \text { and } 3 \text { Piping, Section III, } \\
\text { Division } 1\end{array}$ & reg1.84.r29 & 1993 & $\mathbf{N}$ & Annulled by ASME & $n / a$ & $n / a$ \\
\hline 1769 & ASME & $\begin{array}{l}\text { ASME Code } \\
\text { Case N-392-1 }\end{array}$ & 1986 & $\begin{array}{l}\text { Procedure for Evaluation of the } \\
\text { Design of Hollow Circular Cross } \\
\text { Section Welded Attachments on } \\
\text { Classes } 2 \text { and } 3 \text { Piping, Section III, } \\
\text { Division } 1\end{array}$ & reg1.84.r29 & 1993 & $\mathbf{P}$ & Annulled by ASME & $n / a$ & $n / a$ \\
\hline
\end{tabular}

Division 1 
Codes and Standards and Other Guidance Clited in Regulatory Documents

Part A - Industry Consensus Codes and Standards (continued)

\begin{tabular}{|c|c|c|c|c|c|c|c|c|c|c|}
\hline $\begin{array}{l}\text { Pecord } \\
\text { No. }\end{array}$ & Codo & standard & Suandard & HIto & Dowumont & Dor & Accopt & Commonts & ४ & Curvont \\
\hline 1770 & ASME & $\begin{array}{l}\text { ASME Code } \\
\text { Case N-393 }\end{array}$ & 1989 & $\begin{array}{l}\text { Repair Welding Structural Steel } \\
\text { Rolled Shapes and Plates for } \\
\text { Component Supports, Section III, } \\
\text { Division } 1\end{array}$ & reg1.84.r29 & 1993 & A & & $\begin{array}{l}\text { ASME Code Case } \\
\text { N-393 }\end{array}$ & 1992 \\
\hline 1771 & ASME & $\begin{array}{l}\text { ASME Code } \\
\text { Case N-394 }\end{array}$ & 1989 & $\begin{array}{l}\text { Restricting Lift to Achieve Reduced } \\
\text { Relieving Capacities of Full Lift, } \\
\text { Nozzle Type, and Flat Seated } \\
\text { Safety and Safety Relief Valves for } \\
\text { Compressible Fluid Applications, } \\
\text { Section III, Division 1, Classes } 2 \text { and } \\
3\end{array}$ & reg1.84.r29 & 1993 & $\bar{A}$ & & $\begin{array}{l}\text { ASME Code Case } \\
\text { N-394 }\end{array}$ & 1992 \\
\hline 1772 & ASME & $\begin{array}{l}\text { ASME Code } \\
\text { Case N-395 }\end{array}$ & 1989 & $\begin{array}{l}\text { Laser Wolding, Section III, Division } \\
1\end{array}$ & reg1.84.r29 & 1993 & $\bar{A}$ & & $\begin{array}{l}\text { ASME Code Case } \\
\text { N-395 }\end{array}$ & 1992 \\
\hline 1773 & ASME & $\begin{array}{l}\text { ASME Code } \\
\text { Case N-397 }\end{array}$ & 1987 & $\begin{array}{l}\text { Alternative Rules to the Spectral } \\
\text { Broadening Procedures of N-1226.3 } \\
\text { for Classes 1, 2, and } 3 \text { Piping, } \\
\text { Section III, Division } 1 \\
\end{array}$ & reg1.84.r29 & 1993 & $\mathbf{N}$ & Annulled by ASME & $n / a$ & $n / a$ \\
\hline 1774 & ASME & $\begin{array}{l}\text { ASME Code } \\
\text { Case N-398 } \\
\end{array}$ & 1987 & $\begin{array}{l}\text { Reporting of Charpy-V Notch Test } \\
\text { Results, Section III, Division } 1\end{array}$ & reg1.85.r29 & 1993 & $\mathbf{N}$ & Annulled by ASME & $n / a$ & $n / a$ \\
\hline 1775 & ASME & $\begin{array}{l}\text { ASME Code } \\
\text { Case N-401 }\end{array}$ & 1988 & $\begin{array}{l}\text { Eddy Current Examination, Section } \\
\mathrm{XI} \text {, Division } 1\end{array}$ & reg1.147.r10 & 1993 & $\mathbf{N}$ & Annulled by ASME & $\begin{array}{l}\text { ASME Code Case } \\
N-401-1\end{array}$ & 1991 \\
\hline 1776 & ASME & $\begin{array}{l}\text { ASME Code } \\
\text { Case } \mathrm{N}-401-1\end{array}$ & 1991 & $\begin{array}{l}\text { Eddy Current Examination, Section } \\
\mathrm{XI} \text {, Division } 1\end{array}$ & reg1.147.r10 & 1993 & $\bar{A}$ & & $\begin{array}{l}\text { ASME Code Case } \\
\text { N-401-1 }\end{array}$ & 1991 \\
\hline 1777 & ASME & $\begin{array}{l}\text { ASME Code } \\
\text { Case N-402 }\end{array}$ & 1990 & $\begin{array}{l}\text { Eddy Current Calibration Standard } \\
\text { Material, Section XI, Division } 1\end{array}$ & reg1.147.r10 & 1993 & $\mathbf{N}$ & Annulled by ASME & $\begin{array}{l}\text { ASME Code Case } \\
\mathrm{N}-402-1\end{array}$ & 1991 \\
\hline 1778 & ASME & $\begin{array}{l}\text { ASME Code } \\
\text { Case N-402-1 } \\
\end{array}$ & 1991 & $\begin{array}{l}\text { Eddy Current Calibration Standard } \\
\text { Material, Section XI, Division } 1\end{array}$ & reg1.147.r10 & 1993 & A & & $\begin{array}{l}\text { ASME Code Case } \\
\text { N-402-1 } \\
\end{array}$ & 1991 \\
\hline 1779 & ASME & $\begin{array}{l}\text { ASlivE Code } \\
\text { Case N-404 }\end{array}$ & 1989 & $\begin{array}{l}\text { Annealed Alloy UNS N06625 Over } \\
\text { UNS N06600 Bimetallic Tubing, } \\
\text { Section III, Division 1, Class } 1 \\
\end{array}$ & reg1.85.r29 & 1993 & $\bar{P}$ & & $\begin{array}{l}\text { ASME Code Case } \\
\text { N-404 }\end{array}$ & 1992 \\
\hline 1780 & ASME & $\begin{array}{l}\text { ASME Code } \\
\text { Case N-406 }\end{array}$ & 1987 & $\begin{array}{l}\text { Alternative Rules for Replacement, } \\
\text { Section XI, Division } 1\end{array}$ & reg1.147.r10 & 1993 & $\mathbf{N}$ & Annulled by ASME & $n / a$ & $n / a$ \\
\hline
\end{tabular}




\begin{tabular}{|c|c|c|c|c|c|c|c|c|c|c|}
\hline \multicolumn{11}{|c|}{$\begin{array}{l}\text { Codes and Standards and Other Guidance Clted in Regulatory Documents } \\
\text { Part A - Industry Consensus Codes and Standards (continued) }\end{array}$} \\
\hline Pocord & Codo. & Standird & $\begin{array}{l}\text { Siandird } \\
\text { Vorulon }\end{array}$ & (.) & Document: & Dor. & Aoeppt & commont: & Current Vorlons & Gorron: \\
\hline 1781 & ASME & \begin{tabular}{|l} 
ASME Code \\
Case N-408
\end{tabular} & 1989 & $\begin{array}{l}\text { Alternative Rules for Examination of } \\
\text { Class } 2 \text { Piping, Section XI, Division } \\
1\end{array}$ & reg1.147.r10 & 1993 & $\mathbf{N}$ & Annulled by ASME & $n / a$ & $n / a$ \\
\hline 1782 & ASME & $\begin{array}{l}\text { ASME Code } \\
\text { Case N-408-1 }\end{array}$ & 1989 & $\begin{array}{l}\text { Alternative Rules for Examination of } \\
\text { Class } 2 \text { Piping, Section XI, Division } \\
1\end{array}$ & reg1.147.r10 & 1993 & $\mathbf{N}$ & Annulled by ASME & $n / a$ & $n / a$ \\
\hline 1783 & ASME & $\begin{array}{l}\text { ASME Code } \\
\text { Case N-408-2 }\end{array}$ & 1989 & $\begin{array}{l}\text { Alternative Rules for Examination of } \\
\text { Class } 2 \text { Piping, Section XI, Division } \\
1\end{array}$ & reg1.147.r10 & 1993 & $\bar{P}$ & Annulled by ASME & $n / a$ & $n / \mathbf{a}$ \\
\hline 1784 & ASME & $\begin{array}{l}\text { ASME Code } \\
\text { Case N-409 }\end{array}$ & 1987 & $\begin{array}{l}\text { Procedure and Personnel } \\
\text { Qualification Requirements for } \\
\text { Ultrasonic Detection and Sizing of } \\
\text { Flaws in Piping Welds, Section XI, } \\
\text { Division } 1\end{array}$ & reg1.147.r10 & 1993 & $\mathbf{N}$ & Annulled by ASME & \begin{tabular}{|l|} 
ASME Code Case \\
N-409-2
\end{tabular} & 1991 \\
\hline 1785 & ASME & $\begin{array}{l}\text { ASME Code } \\
\text { Case N-409-1 }\end{array}$ & 1988 & $\begin{array}{l}\text { Procedure and Personnel } \\
\text { Qualification Requirements for } \\
\text { Ultrasonic Detection and Sizing of } \\
\text { Flaws in Piping Welds, Section XI, } \\
\text { Division } 1\end{array}$ & reg1.147.r10 & 1993 & $\mathbf{N}$ & Annulled by ASME & $\begin{array}{l}\text { ASME Code Case } \\
\text { N-409-2 }\end{array}$ & 1991 \\
\hline 1786 & ASME & $\begin{array}{l}\text { ASME Code } \\
\text { Case N-409-2 }\end{array}$ & 1991 & $\begin{array}{l}\text { Procedure and Personnel } \\
\text { Qualification Requirements for } \\
\text { Ultrasonic Detection and Sizing of } \\
\text { Flaws in Piping Welds, Section XI, } \\
\text { Division } 1\end{array}$ & reg1.147.r10 & 1993 & $\bar{P}$ & & \begin{tabular}{|l|} 
ASME Code Case \\
N-409-2
\end{tabular} & 1991 \\
\hline 1787 & ASME & $\begin{array}{l}\text { ASME Code } \\
\text { Case N-410 }\end{array}$ & 1991 & $\begin{array}{l}\text { Certified Relieving Capacities of } \\
\text { Pressure Relief Valves Having Set } \\
\text { Pressure of } 3 \text { psig up to but Not } \\
\text { including } 15 \text { psig installed for } \\
\text { Overpressure of Compressible } \\
\text { Fluid Systems, Section III, Division } \\
1, \text { Classes } 2 \text { and } 3\end{array}$ & reg1.84.r29 & 1993 & $\bar{A}$ & & \begin{tabular}{|l|} 
ASME Code Case \\
N-410
\end{tabular} & 1991 \\
\hline
\end{tabular}


Codes and Standards and Other Guidance Cited in Regulatory Documents

Part A - Industry Consensus Codes and Standards (continued)

\begin{tabular}{|c|c|c|c|c|c|c|c|c|c|c|}
\hline $\begin{array}{l}\text { Bocord } \\
\text { No. }\end{array}$ & Code & Standerd & $\begin{array}{l}\text { stmond } \\
\text { yordon }\end{array}$ & 1,4 mos, & Document & $\begin{array}{l}\text { Doo. } \\
\text { Doter }\end{array}$ & $\begin{array}{l}\text { Acoppt. } \\
\text { Abllily }\end{array}$ & Commonts & Gurront torolon & Corron \\
\hline 1788 & ASME & $\begin{array}{l}\text { ASME Code } \\
\text { Case N-411 }\end{array}$ & $\mathrm{N} / \mathrm{S}$ & $\begin{array}{l}\text { Alternative Damping Values for } \\
\text { Seismic Analysis of Classes 1, 2, } \\
\text { and } 3 \text { Piping Sections, Section III, } \\
\text { Division } 1\end{array}$ & nureg(1033, 3.119 & 1991 & $\mathbf{R}$ & & $\begin{array}{l}\text { ASME Code Case } \\
\text { N-411 }\end{array}$ & 1992 \\
\hline 1789 & ASME & $\begin{array}{l}\text { ASME Code } \\
\text { Case N-411 }\end{array}$ & 1986 & $\begin{array}{l}\text { Alternative Damping Values for } \\
\text { Seismic Analysis of Classes } 1,2 \text {, } \\
\text { and } 3 \text { Piping Sections, Section III, } \\
\text { Division } 1\end{array}$ & reg1.84.r29 & 1993 & $\mathbf{N}$ & Annulled by ASME & $\begin{array}{l}\text { ASME Code Case } \\
\text { N-411-1 }\end{array}$ & 1992 \\
\hline 1790 & ASME & $\begin{array}{l}\text { ASME Code } \\
\text { Case N-411-1 }\end{array}$ & 1989 & $\begin{array}{l}\text { Alternative Damping Values for } \\
\text { Response Spectra Analysis of } \\
\text { Classes 1, 2, and } 3 \text { Piping, Section } \\
\text { III, Division } 1\end{array}$ & reg1.84.r29 & 1993 & $\mathbf{P}$ & & $\begin{array}{l}\text { ASME Code Case } \\
\text { N-411-1 }\end{array}$ & 1992 \\
\hline 1791 & ASME & $\begin{array}{l}\text { ASME Code } \\
\text { Case N-412 }\end{array}$ & 1991 & $\begin{array}{l}\text { Alternative Rules for Witnessing the } \\
\text { Piping System Pressure Tests of } \\
\text { Classes 1, 2, and } 3 \text { Piping Systems, } \\
\text { Section III, Division } 1\end{array}$ & reg1.84.r29 & 1993 & $\mathbf{N}$ & Annulled by ASME & $n / a$ & $n / a$ \\
\hline 1792 & ASME & $\begin{array}{l}\text { ASME Code } \\
\text { Case N-413 }\end{array}$ & 1988 & $\begin{array}{l}\text { Minimum Size of Fillet Welds for } \\
\text { Subsection NF Linear Type } \\
\text { Supports, Section III, Division } 1\end{array}$ & reg1.84.r29 & 1993 & $\mathbf{N}$ & Annulled by ASME & $n / a$ & $n / a$ \\
\hline 1793 & ASME & $\begin{array}{l}\text { ASME Code } \\
\text { Case } \mathrm{N}-414\end{array}$ & 1986 & $\begin{array}{l}\text { Tack Welds for Class 1, 2, } 3 \text { and } \\
\text { MC Components and Piping } \\
\text { Supports, Section III, Division } 1 \\
\end{array}$ & reg1.84.r29 & 1993 & A & Annulled by ASME & $n / a$ & $n / a$ \\
\hline 1794 & ASME & $\begin{array}{l}\text { ASME Code } \\
\text { Case N-415 }\end{array}$ & 1991 & $\begin{array}{l}\text { Alternate Rules for Testing Pressure } \\
\text { Relief Devices, Section XI, Division } \\
1\end{array}$ & reg1.147.r10 & 1993 & A & & $\begin{array}{l}\text { ASME Code Case } \\
\text { N-415 }\end{array}$ & 1991 \\
\hline 1795 & ASME & $\begin{array}{l}\text { ASME Code } \\
\text { Case N-416 }\end{array}$ & 1990 & $\begin{array}{l}\text { Alternative Rules for Hydrostatic } \\
\text { Testing of Repair or Replacement } \\
\text { of Class } 2 \text { Piping, Section XI, } \\
\text { Division } 1 \\
\end{array}$ & reg1.147.r10 & 1993 & $\bar{A}$ & & $\begin{array}{l}\text { ASME Code Case } \\
\text { N-416 }\end{array}$ & 1990 \\
\hline 1796 & ASME & $\begin{array}{l}\text { ASME Code } \\
\text { Case N-418 }\end{array}$ & 1986 & $\begin{array}{l}\text { Use of Seamless Ni-Fe-Cr-Mo-Cu } \\
\text { Low Carbon (UNS N08028 and UNS } \\
\text { NO8904) Tubing, Section III, } \\
\text { Division 1, Classes } 2 \text { and } 3 \\
\end{array}$ & reg1.85.r29 & 1993 & $N$ & Annulled by ASME & $\begin{array}{l}\text { ASME Code Case } \\
\text { N-418-1 }\end{array}$ & 1992 \\
\hline
\end{tabular}


Codes and Standards and Other Guidance Cited in Regulatory Documents

Part A - Industry Consensus Codes and Standards (continued)

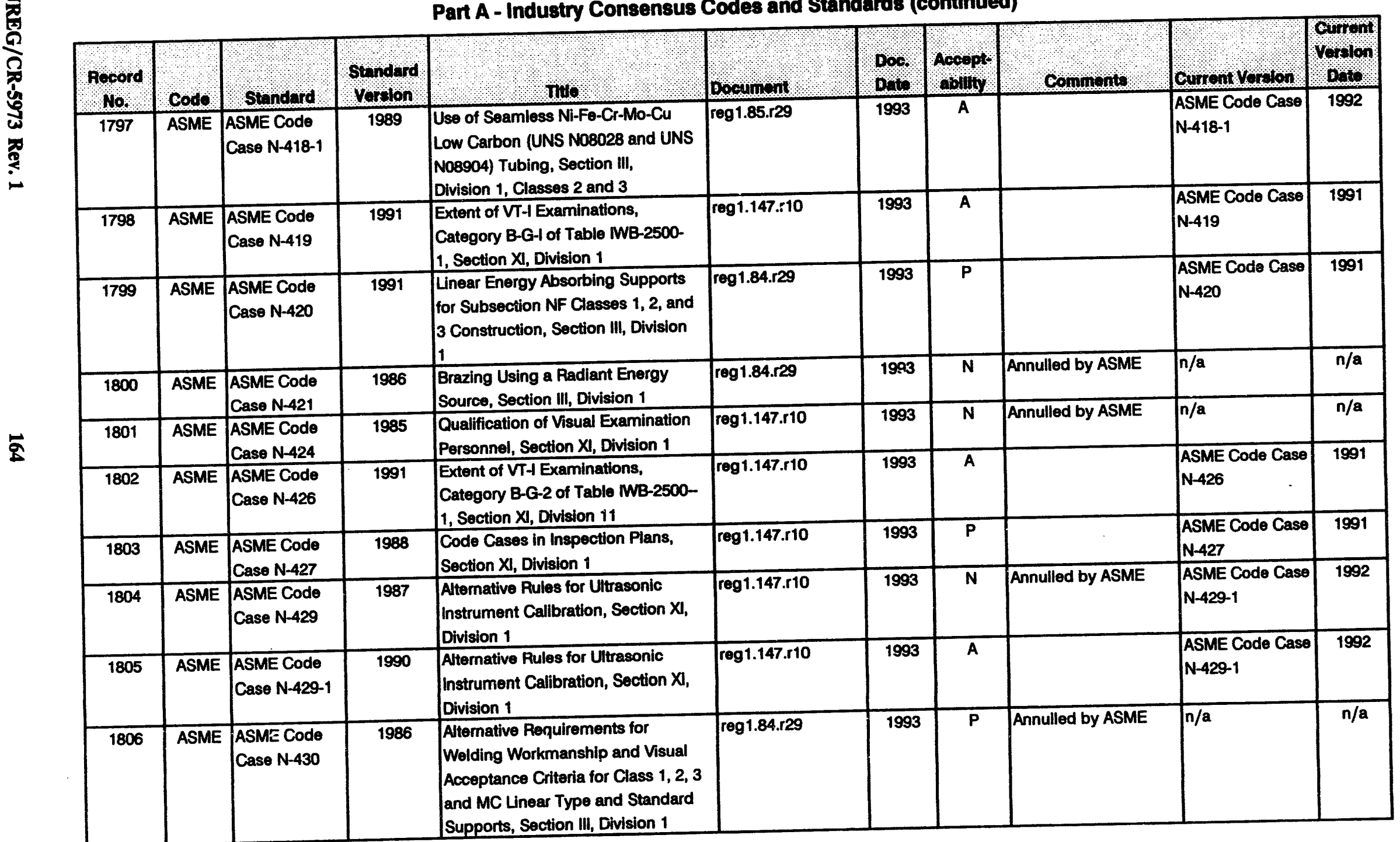


Codes and Standards and Other Guidance Cited in Regulatory Documents

Part A - Industry Consensus Codes and Standards (continued)

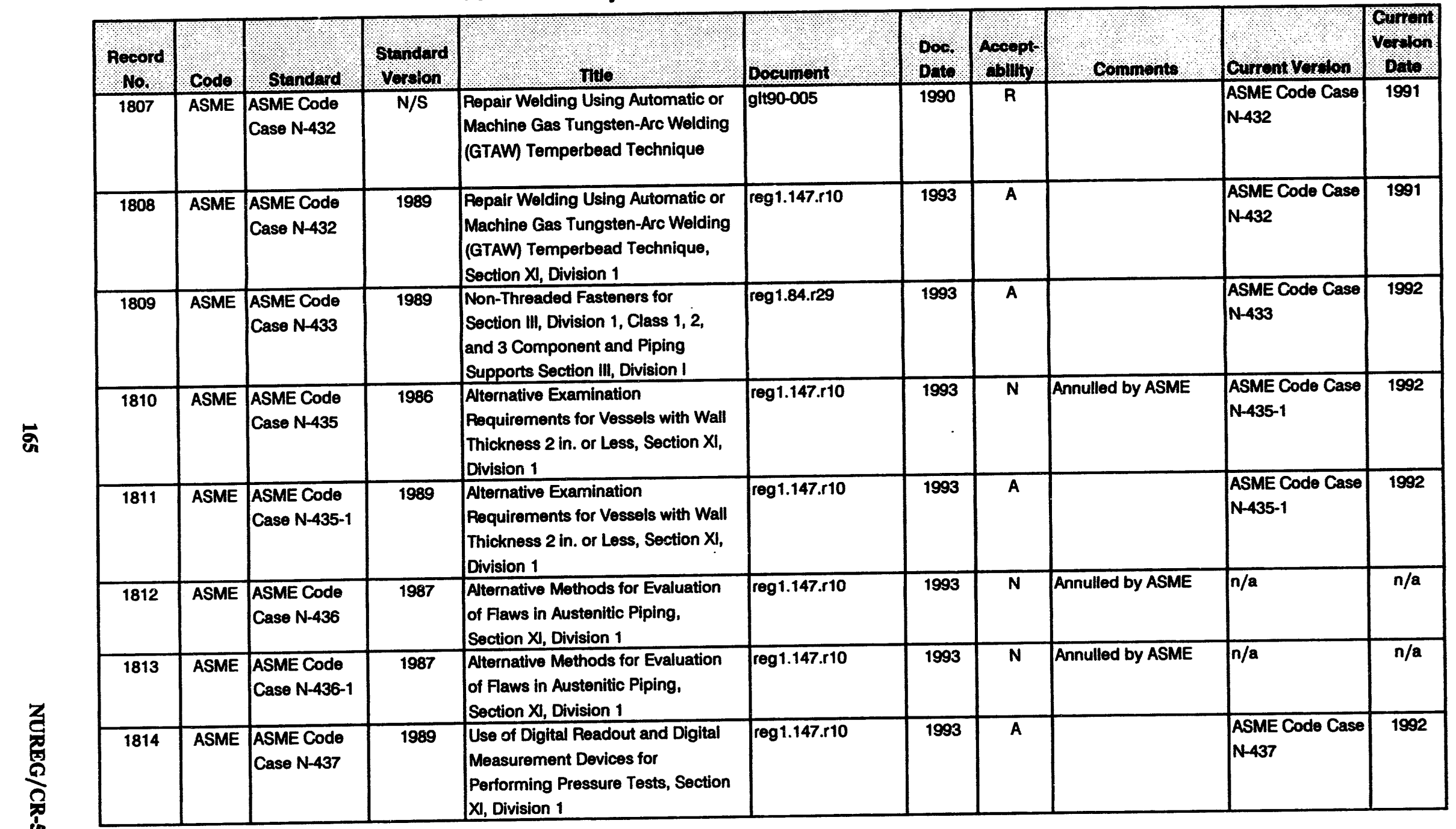




\begin{tabular}{|c|c|c|c|c|c|c|c|c|c|c|}
\hline \multicolumn{11}{|c|}{$\begin{array}{c}\text { Codes and Standards and Other Guidance Clted in Regulatory Documents } \\
\text { Part A - Industry Consensus Codes and Standards (continued) }\end{array}$} \\
\hline $\begin{array}{c}\text { Record } \\
\text { No. }\end{array}$ & Code. & Stendard & $\begin{array}{l}\text { Standard } \\
\text { Vorslon }\end{array}$ & noe & Documont. & Doo. & Aocopt: & Corvenents & Curront Voulon & Crrom \\
\hline 1815 & ASME & $\begin{array}{l}\text { ASME Code } \\
\text { Case N-438 }\end{array}$ & 1989 & $\begin{array}{l}\text { Fe-24.5Ni-21Cr-6.5 Mo-0.2N (Alloy } \\
\text { UNS N08367) Seamless and } \\
\text { Welded Pipe, Tube, Plate, Bar, } \\
\text { Fittings, and Forgings, Class } 2 \text { and } \\
3 \text { Construction, Section III, Division } \\
1\end{array}$ & reg1.85.r29 & 1993 & $\mathbf{N}$ & Annulled by ASME & $n / a$ & $n / a$ \\
\hline 1816 & ASME & $\begin{array}{l}\text { ASME Code } \\
\text { Case N-438-1 }\end{array}$ & 1990 & $\begin{array}{l}\text { Fe-24.5Ni-21Cr-6.5 Mo-0.2N (Alloy } \\
\text { UNS N08367) Seamloss and } \\
\text { Welded Pipe, Tube, Plate, Bar, } \\
\text { Fittings, and Forgings, Class } 2 \text { and } \\
3 \text { Construction, Section III, Division } \\
1\end{array}$ & reg1.85.r29 & 1993 & $\mathbf{N}$ & & $n / \mathbf{a}$ & $n / a$ \\
\hline 1817 & ASME & $\begin{array}{l}\text { ASME Code } \\
\text { Case N-438-2 }\end{array}$ & 1990 & $\begin{array}{l}\text { Fe-24.5Ni-21Cr-6.5Mo-0.2N (Alloy } \\
\text { UNS N08367) Seamless and } \\
\text { Welded Pipe, Tube, Plate, Bar, } \\
\text { Fittings, and Forgings, Class } 2 \text { and } \\
3 \text { Construction, Section III, Division } \\
1\end{array}$ & reg1.85.r29 & 1993 & $\mathbf{A}$ & Annulled by ASME & $n / a$ & $n / a$ \\
\hline 1818 & ASME & $\begin{array}{l}\text { ASME Code } \\
\text { Case N-439 }\end{array}$ & 1989 & $\begin{array}{l}\text { Use of 20Cr-18Ni-6Mo (Alloy UNS } \\
\text { S31254) Forgings, Plate, Seamless } \\
\text { and Wolded Pipe, and Welded } \\
\text { Tube, Class } 2 \text { and } 3 \text { Construction, } \\
\text { Section III, Division } 1\end{array}$ & $\log 1.85 .122$ & 1993 & A & & $\begin{array}{l}\text { ASME Code Case } \\
\text { N-439 }\end{array}$ & 1992 \\
\hline 1819 & ASME & $\begin{array}{l}\text { ASME Code } \\
\text { Case N-440 }\end{array}$ & 1987 & $\begin{array}{l}\text { Use of } 20 \mathrm{Cr}-18 \mathrm{Ni}-6 \mathrm{Mo} \text { (Alloy UNS } \\
\text { j93254) Castings, Class } 2 \text { and } 3 \\
\text { Construction, Section III, Division } 1 \\
\end{array}$ & $\operatorname{reg} 1.85 .129$ & 1993 & A & & $\begin{array}{l}\text { ASME Code Case } \\
\text { N-440 }\end{array}$ & 1992 \\
\hline 1820 & ASME & $\begin{array}{l}\text { ASME Code } \\
\text { Case N-441 }\end{array}$ & 1989 & $\begin{array}{l}\text { Use of 20Cr-18Ni-6Mo (Alloy UNS } \\
\text { S31254) Fittings, Class } 2 \text { and } 3 \\
\text { Construction, Section III, Division } 1\end{array}$ & reg1.85.r29 & 1993 & $\bar{A}$ & & $\begin{array}{l}\text { ASME Code Case } \\
\text { N-441 }\end{array}$ & 1992 \\
\hline 1821 & ASME & $\begin{array}{l}\text { ASME Code } \\
\text { Case N-442 }\end{array}$ & 1990 & $\begin{array}{l}1977 \text { Addendum to ANSI/ASME } \\
\text { PTC 25.3-1976, Safety and Safety } \\
\text { Relief Valves, Class 1, 2, 3, and MC, } \\
\text { Section III, Division } 1\end{array}$ & reg1.84.r29 & 1993 & A & Annulled by ASME & $n / a$ & $n / a$ \\
\hline
\end{tabular}




\section{Codes and Standards and Other Guidance Chted in Regulatory Documents}

Part A - Industry Consensus Codes and Standards (continued)

\begin{tabular}{|c|c|c|c|c|c|c|c|c|c|c|}
\hline $\begin{array}{l}\text { Pocord } \\
\text { No. }\end{array}$ & codo & Sthndurd & $\begin{array}{l}\text { Standard } \\
\text { Vorolon }\end{array}$ & nuts & Doconount & $\begin{array}{l}\text { Dos. } \\
\text { Date }\end{array}$ & Acoeps & Comnnenth. & Cornont Voreson & $w_{n+1}$ \\
\hline 1822 & ASME & $\begin{array}{l}\text { ASME Code } \\
\text { Case N-443 }\end{array}$ & 1989 & $\begin{array}{l}\text { High Yield Strength Cr-Mo Steel, } \\
\text { Class } 1 \text { Components, Section III, } \\
\text { Division } 1\end{array}$ & reg1.85.r29 & 1993 & $N$ & Annulled by ASME & $\begin{array}{l}\text { ASME Codo Case } \\
\text { N-443-1 }\end{array}$ & 1992 \\
\hline 1823 & ASME & $\begin{array}{l}\text { ASME Code } \\
\text { Case N-443-1 }\end{array}$ & 1989 & $\begin{array}{l}\text { High Yield Strength Cr-Mo Steel, } \\
\text { Class } 1 \text { Components, Section III, } \\
\text { Division } 1\end{array}$ & reg1.85.r29 & 1993 & $\bar{A}$ & & $\begin{array}{l}\text { ASME Codo Caso } \\
N-443-1\end{array}$ & 1992 \\
\hline 1824 & ASME & $\begin{array}{l}\text { ASME Code } \\
\text { Case N-444 }\end{array}$ & 1987 & $\begin{array}{l}\text { Preparation of Inspection Plans, } \\
\text { Section XI, Division } 1\end{array}$ & reg1.147.r10 & 1993 & $\mathbf{N}$ & Annulled by ASME & $n / a$ & $n / a$ \\
\hline 1825 & ASME & $\begin{array}{l}\text { ASME Codo } \\
\text { Case N-445 }\end{array}$ & 1987 & $\begin{array}{l}\text { Use of Later Editions of SNT-TC-1A } \\
\text { for Qualification of Nondestructive } \\
\text { Examination Personnel, Section XI, } \\
\text { Division } 1,2, \text { and } 3\end{array}$ & reg1.147.r10 & 1993 & $\mathbf{N}$ & Annulled by ASME & $n / a$ & $n / a$ \\
\hline 1826 & ASME & $\begin{array}{l}\text { ASME Code } \\
\text { Case N-446 }\end{array}$ & 1987 & $\begin{array}{l}\text { Recertification of Visual } \\
\text { Examination Personnel, Section XI, } \\
\text { Division } 1\end{array}$ & reg1.147.r10 & 1993 & $\mathbf{N}$ & Annulled by ASME & $n / a$ & $n / a$ \\
\hline 1827 & ASME & $\begin{array}{l}\text { ASME Code } \\
\text { Case N-448 }\end{array}$ & 1990 & $\begin{array}{l}\text { Qualification of VT-2 and VT-3 } \\
\text { Visual Examination Personnel, } \\
\text { Section XI, Division } 1\end{array}$ & reg1.147.r10 & 1993 & $\bar{A}$ & & $\begin{array}{l}\text { ASME Code Caso } \\
\text { N448 }\end{array}$ & 1993 \\
\hline 1828 & ASME & $\begin{array}{l}\text { ASME Code } \\
\text { Case N-449 }\end{array}$ & 1990 & $\begin{array}{l}\text { Qualification of VT-4 Visual } \\
\text { Examination Personnel, Section XI, } \\
\text { Olvision } 1\end{array}$ & rog1.147.r10 & 1993 & $\bar{A}$ & & $\begin{array}{l}\text { ASME Codo Case } \\
\mathrm{N}-449\end{array}$ & 1993 \\
\hline 1829 & ASME & $\begin{array}{l}\text { ASME Code } \\
\text { Case N-452 }\end{array}$ & 1989 & $\begin{array}{l}\text { Specialized Subcontracted Wolding } \\
\text { Process (Electron Beam Welding), } \\
\text { Section III, Division 1, Class } 2 \text { and } 3 \\
\text { Construction }\end{array}$ & reg1.84.r29 & 1993 & $\bar{A}$ & & $\begin{array}{l}\text { ASME Code Case } \\
\text { N-452 }\end{array}$ & 1992 \\
\hline 1830 & ASME & $\begin{array}{l}\text { ASME Code } \\
\text { Case N-453 }\end{array}$ & 1988 & $\begin{array}{l}\text { Nickel-Chromium-Molybdenum- } \\
\text { Copper Stainless Steel (UNS } \\
\text { N08925) Seamless and Welded } \\
\text { Pipe for Class } 2 \text { and } 3 \text { Construction } \\
\text { Section III, Division } 1\end{array}$ & reg1.84.r29 & 1993 & $\mathbf{N}$ & Annulled by ASME & $\begin{array}{l}\text { ASME Code Casso } \\
N-453-1\end{array}$ & 1991 \\
\hline
\end{tabular}




\begin{tabular}{|c|c|c|c|c|c|c|c|c|c|c|}
\hline & & & Cod & $\begin{array}{l}\text { es and Standards and Other } \\
\text { Part A - Industry Consensus }\end{array}$ & $\begin{array}{l}\text { Culdance } \mathrm{Cl} \\
\text { Codes and }\end{array}$ & $\begin{array}{l}\text { Regut } \\
\text { ands ( }\end{array}$ & tory Do & $\begin{array}{l}\text { cuments } \\
\text { d) }\end{array}$ & & \\
\hline $\begin{array}{l}\text { Rocord } \\
\text { No. }\end{array}$ & Codo & standerd & $\begin{array}{l}\text { Stundard } \\
\text { Verciton }\end{array}$ & $n_{n} \quad n_{0}$ & Docoument & $\begin{array}{l}\text { Doe. } \\
\text { Dons }\end{array}$ & $\begin{array}{l}\text { Acoespt. } \\
\text { semply }\end{array}$ & Commentis & Comsent Vurion & Qnom \\
\hline 1831 & ASME & $\begin{array}{l}\text { ASME Code } \\
\text { Case N-453-1 }\end{array}$ & 1988 & $\begin{array}{l}\text { Nickel-Chromium-Molybdonum- } \\
\text { Copper Stainloss Stoel (UNS } \\
\text { No8925) Seamless and Wolded } \\
\text { Pipe for Class } 2 \text { and } 3 \text { Constriction } \\
\text { Section III, Division } 1 \\
\end{array}$ & $\operatorname{reg} 1.84 . r 29$ & 1993 & A & & $\begin{array}{l}\text { ASME Code Case } \\
\text { N-453-1 }\end{array}$ & 1991 \\
\hline 1832 & ASME & $\begin{array}{l}\text { ASME Code } \\
\text { Case N-454 }\end{array}$ & 1990 & $\begin{array}{l}\text { Nickel-Chromium-Molybdenum- } \\
\text { Copper Stainless Steel (UNS } \\
\text { No8925) Wrought Fittings for Class } \\
2 \text { and } 3 \text { Construction, Soction III, } \\
\text { Division } 1\end{array}$ & $\operatorname{rog} 1.84 .229$ & 1993 & A & & $\begin{array}{l}\text { ASME Code Caso } \\
\text { NA54 }\end{array}$ & 1990 \\
\hline 1833 & ASME & $\begin{array}{l}\text { ASME Code } \\
\text { Case N-455 }\end{array}$ & 1990 & $\begin{array}{l}\text { Nickel-Chromium-Molybdenum- } \\
\text { Copper Stainloss Stoel (UNS } \\
\text { No8925) Forgod Flanges and } \\
\text { Fittings for Class } 2 \text { and } 3 \\
\text { Construction, Section III, Division } 1 \\
\end{array}$ & $\operatorname{rog} 1.84 .129$ & 1993 & A & & $\begin{array}{l}\text { ASME Code Case } \\
\text { N-455 }\end{array}$ & 1990 \\
\hline 1834 & $\overline{A S M E}$ & $\begin{array}{l}\text { ASME Code } \\
\text { Case N-457 }\end{array}$ & 1990 & $\begin{array}{l}\text { Qualification Specimen Notch } \\
\text { Location for Ultrasonic } \\
\text { Examination of Bolts and Studs, } \\
\text { Section X, Division } 1\end{array}$ & reg1.147.J10 & 1993 & A & & $\begin{array}{l}\text { ASUE Codo Casso } \\
\text { N-457 }\end{array}$ & 1993 \\
\hline 1835 & ASME & $\begin{array}{l}\text { ASME Code } \\
\text { Case N-459 }\end{array}$ & 1991 & $\begin{array}{l}\text { SA-508 Basic Oxygen-Ladlo } \\
\text { Furnace Process for Code } \\
\text { Construction, Section III, Division } 1 \\
\end{array}$ & $\log 1.85 .122$ & 1993 & A & & $\begin{array}{l}\text { ASME Code Caso } \\
\text { N-459 }\end{array}$ & 1991 \\
\hline 1836 & ASME & $\begin{array}{l}\text { ASME Code } \\
\text { Case N-460 }\end{array}$ & 1991 & $\begin{array}{l}\text { Alternative Examination Coverage } \\
\text { for Class } 1 \text { and Class } 2 \text { Wolds, } \\
\text { Section X1. Division } 1\end{array}$ & reg1.147.r10 & 1993 & A & & $\begin{array}{l}\text { ASME Code Cases } \\
N-460\end{array}$ & 1991 \\
\hline 1837 & ASME & $\begin{array}{l}\text { ASME Code } \\
\text { Case N-461 }\end{array}$ & 1991 & $\begin{array}{l}\text { Alternativo Pulos for Plping } \\
\text { Calibration Block Thickness, } \\
\text { Soction XI, Dlvision I }\end{array}$ & $\operatorname{rog} 1.147 .110$ & 1993 & $\mathbf{P}$ & & $\begin{array}{l}\text { ASME Codo Caso } \\
\text { N461 }\end{array}$ & 1991 \\
\hline 1838 & $\overline{\text { ASME }}$ & $\begin{array}{l}\text { ASME Code } \\
\text { Case N-463 }\end{array}$ & $N / S$ & $\begin{array}{l}\text { Evaluation Procedures and } \\
\text { Acceptance Criteria for Flaws in } \\
\text { Class I Ferritic Piping that excoed } \\
\text { the Acceptance Standards of MB- } \\
3514.2 \text {, Section XI, Division } 1\end{array}$ & gitt50-005 & 1990 & $\bar{A}$ & Annulled by ASME & $n / a$ & $n / a$ \\
\hline
\end{tabular}


Codes and Standards and Other Guidance CHed in Regulatory Documents

Part A - Industry Consensus Codes and Standards (continued)

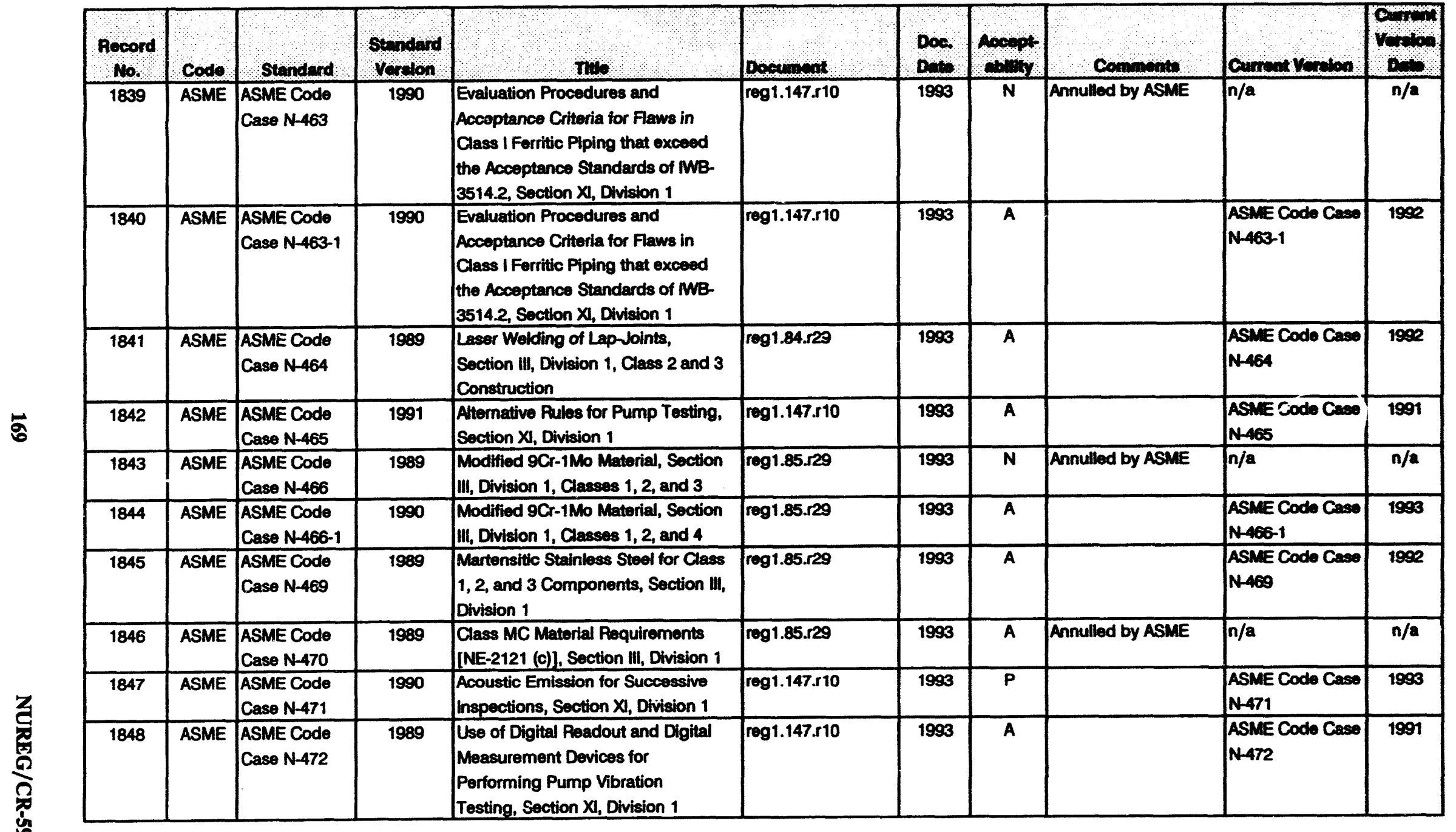




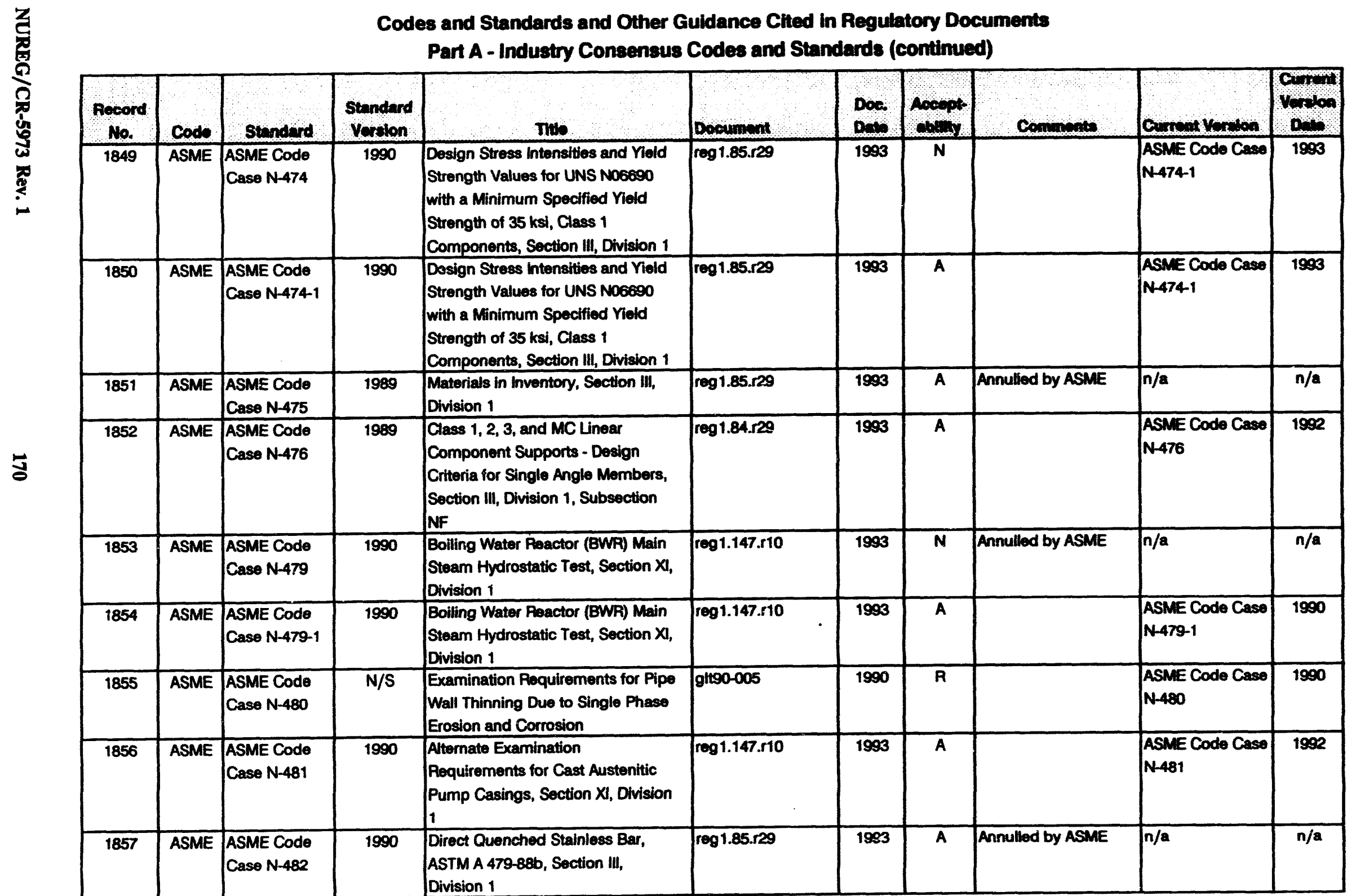




\section{Codes and Standards and Other Guidance Cited in Regulatory Documents}

Part A - Industry Consensus Codes and Standards (continued)

\begin{tabular}{|c|c|c|c|c|c|c|c|c|c|c|}
\hline Rocord & 000 & standard & Strondard & , & Doodinont & $\begin{array}{l}\text { Dor. } \\
\text { Dots }\end{array}$ & Pooppto & Comnonts & curront vorton & $\frac{601 \%}{100110}$ \\
\hline 1858 & ASME & $\begin{array}{l}\text { ASME Code } \\
\text { Case N-485 }\end{array}$ & 1990 & $\begin{array}{l}\text { Eddy Current Examination of } \\
\text { Coated Ferritic Surfaces as an } \\
\text { Alternative to Surface Examination, } \\
\text { Section Xl, Division } 1\end{array}$ & reg1.147.r10 & 1993 & $\mathbf{N}$ & & $\begin{array}{l}\text { ASME Code Case } \\
\text { N-485- } 1\end{array}$ & 1991 \\
\hline 1859 & ASME & $\begin{array}{l}\text { ASME Code } \\
\text { Case N-485-1 }\end{array}$ & 1991 & $\begin{array}{l}\text { Eddy Current Examination of } \\
\text { Coated Ferritic Surfaces as an } \\
\text { Alternative to Surface Examination, } \\
\text { Section Xl, Division } 1 \\
\end{array}$ & reg1.147.r10 & 1993 & $\bar{A}$ & & $\begin{array}{l}\text { ASME Code Case } \\
\text { N-485-1 }\end{array}$ & 1991 \\
\hline 1860 & ASME & $\begin{array}{l}\text { ASME Code } \\
\text { Case N-489 }\end{array}$ & 1990 & $\begin{array}{l}\text { Alternative Rules for Level ill NDE } \\
\text { Qualification Examinaticns, Section } \\
\mathrm{Xl} \text {, Division } 1,2 \text { and } 3\end{array}$ & reg1.147.r10 & 1993 & A & & $\begin{array}{l}\text { ASME Code Case } \\
\text { N-489 }\end{array}$ & 1993 \\
\hline 1861 & ASME & $\begin{array}{l}\text { ASME Code } \\
\text { Case N-490 }\end{array}$ & 1990 & $\begin{array}{l}\text { Alternative Vision Test } \\
\text { Requirements for Nondestructive } \\
\text { Examiners, Section XI, Divisions } 1 \text {, } \\
2, \text { and } 3\end{array}$ & reg1.147.r10 & 1993 & $\mathbf{N}$ & & $\begin{array}{l}\text { ASME Code Case } \\
\text { N-490 }\end{array}$ & 1990 \\
\hline 1362 & ASME & $\begin{array}{l}\text { ASME Code } \\
\text { Case N-490-1 }\end{array}$ & 1991 & $\begin{array}{l}\text { Alternaílve Vision Test } \\
\text { Requirements for Nondestructive } \\
\text { Examiners, Section XI, Divisions 1, } \\
2 \text {, and } 3\end{array}$ & reg1.147.r10 & 1993 & $\bar{A}$ & & $\begin{array}{l}\text { ASME Code Case } \\
\text { N-490-1 }\end{array}$ & 1991 \\
\hline 1863 & ASME & $\begin{array}{l}\text { ASME Code } \\
\text { Case } \mathrm{N}-491\end{array}$ & 1991 & $\begin{array}{l}\text { Alternative Rules for Examination of } \\
\text { Class } 1,2, \text { and } 3 \text { and MC } \\
\text { Component Supports of Light- } \\
\text { Water Cooled Power Plants, } \\
\text { Section XI, Division } 1 \\
\end{array}$ & reg1.147.r10 & 1993 & $\bar{A}$ & & $\begin{array}{l}\text { ASME Code Case } \\
\text { N-491-1 }\end{array}$ & 1993 \\
\hline 1864 & ASME & $\begin{array}{l}\text { ASME Code } \\
\text { Case N-492 }\end{array}$ & 1990 & $\begin{array}{l}\text { Grade } 9 \text { Titanium Alloy Section III, } \\
\text { Division 1, Class 1, 2, and } 3\end{array}$ & reg1.85.r29 & 1993 & $\bar{A}$ & & $\begin{array}{l}\text { ASME Code Case } \\
\text { N-492 }\end{array}$ & 1990 \\
\hline 1865 & ASME & $\begin{array}{l}\text { ASME Code } \\
\text { Case N-494 }\end{array}$ & 1990 & $\begin{array}{l}\text { Pipe Specific Evaluation } \\
\text { Procedures and Acceptance } \\
\text { Criteria for Flaws in Class } 1 \text { Ferritic } \\
\text { Piping that Exceeds the } \\
\text { Acceptance Standards of IWB- } \\
3514.2 \text {, Section XI, Division } 1 \\
\end{array}$ & reg1.147.r10 & 1993 & $\bar{A}$ & & $\begin{array}{l}\text { ASME Code Case } \\
\text { N-494-1 }\end{array}$ & 1992 \\
\hline 1866 & ASME & $\begin{array}{l}\text { ASME Code } \\
\text { Case N-495 }\end{array}$ & 1990 & $\begin{array}{l}\text { Hydrostatic Testing of Relief Valves, } \\
\text { Section XI, Division } 1\end{array}$ & reg1.147.r10 & 1993 & $\bar{A}$ & & \begin{tabular}{|l|} 
ASME Code Case \\
N-495
\end{tabular} & 1990 \\
\hline
\end{tabular}




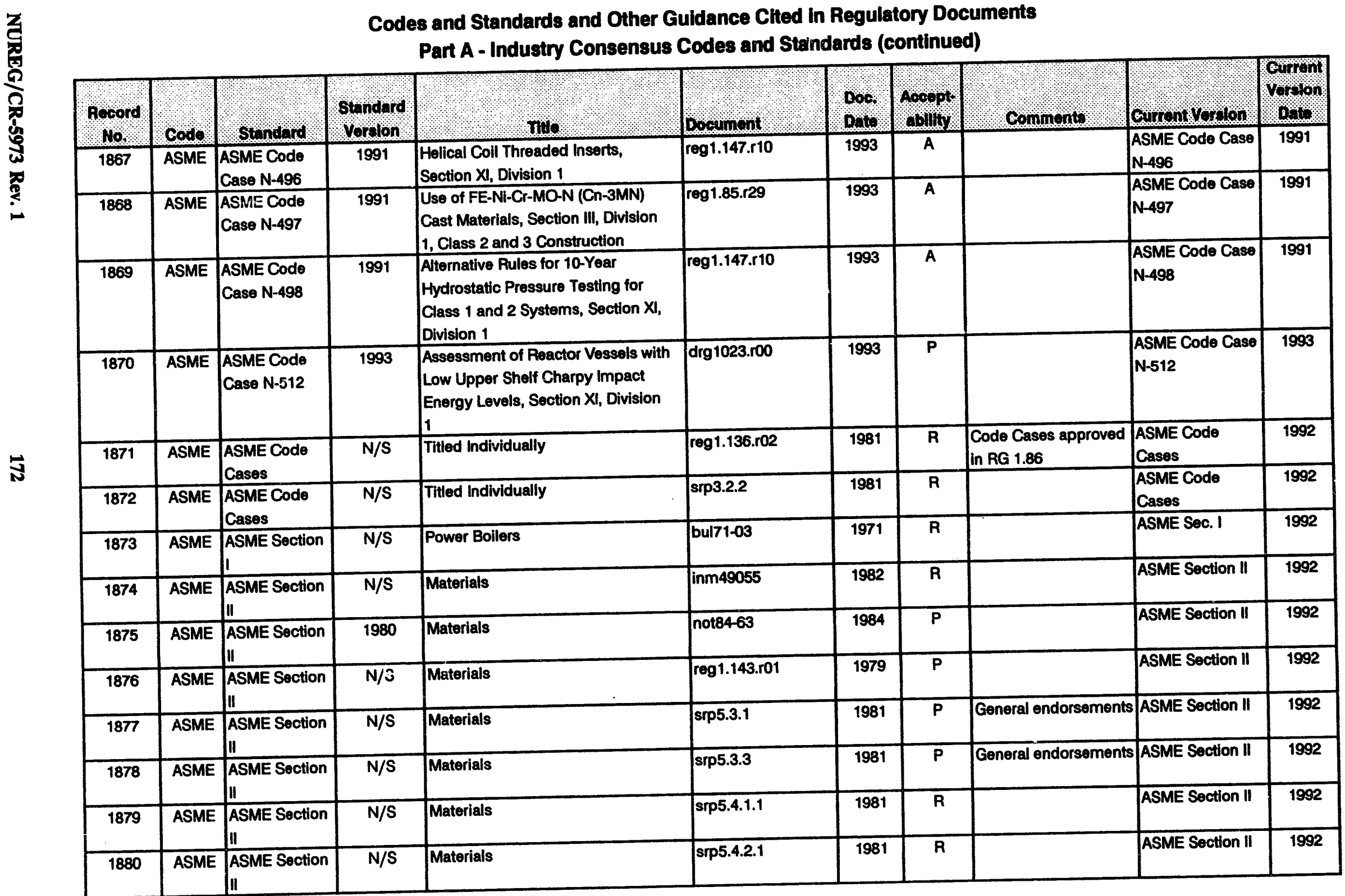


Codes and Standards and Other Guidance Clted in Regulatory Documents

Part A - Industry Consensus Codes and Standards (continued)

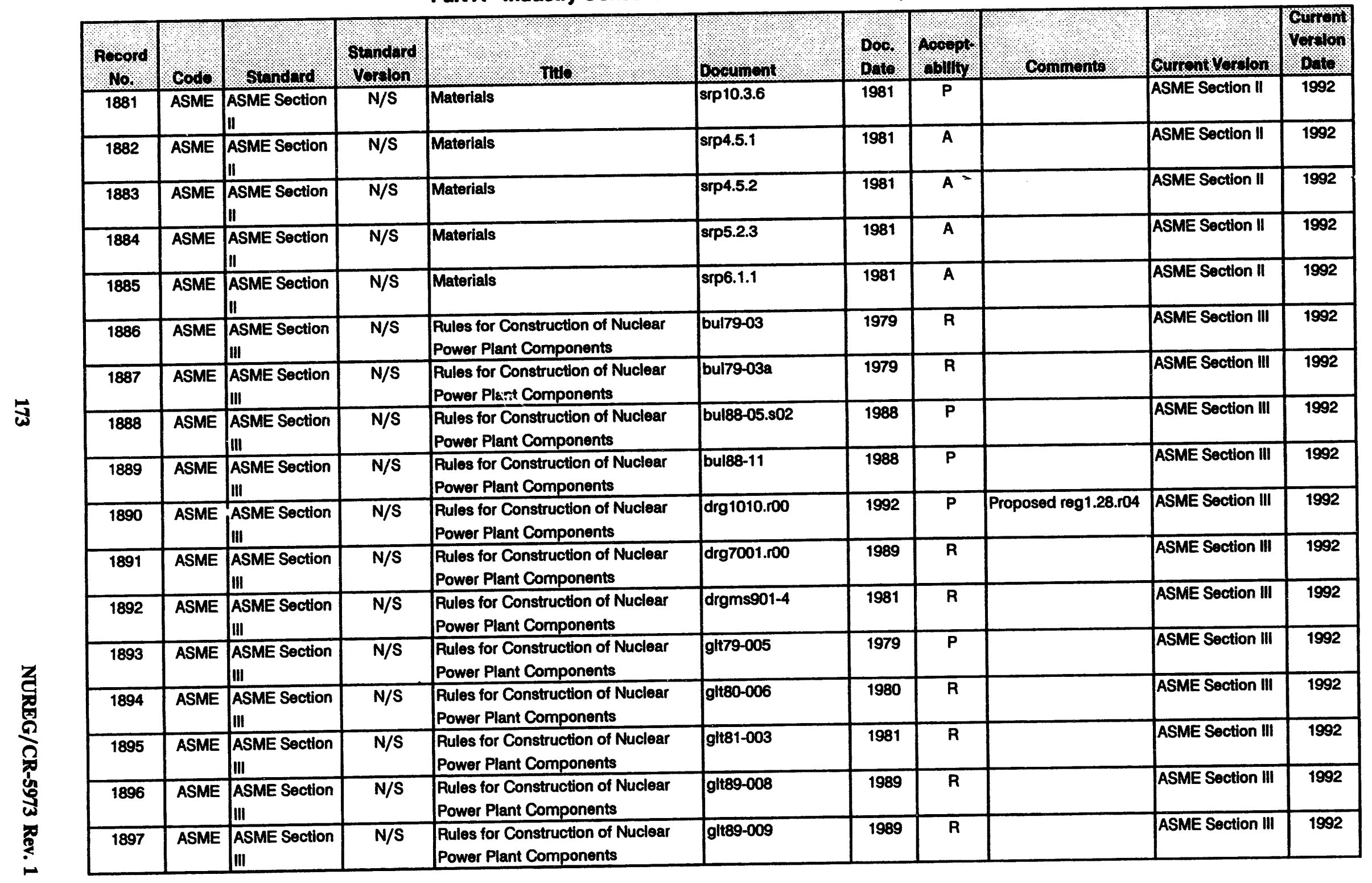


Codes and Standards and Other Guidance Clted in Regulatory Documents

Part A - Industry Consensus Codes and Standards (continued)

\begin{tabular}{|c|c|c|c|c|c|c|c|c|c|c|}
\hline Pocord & 000 & Shandord & $\begin{array}{l}\text { Stundard } \\
\text { Yorion }\end{array}$ & $1,1,6$ orior & Boovinont & Doo: & Acoeptl & 1. & cunnow Yorolons & $\begin{array}{l}6,1041 \\
16+101\end{array}$ \\
\hline 1898 & ASME & $\begin{array}{l}\text { ASME Section } \\
\text { III }\end{array}$ & $\mathbf{N} / \mathbf{S}$ & $\begin{array}{l}\text { Rules for Construction of Nuclear } \\
\text { Power Piant Components }\end{array}$ & glt89-010.s03 & 1990 & $\mathbf{R}$ & & ASME Section III & 1992 \\
\hline 1899 & ASME & $\begin{array}{l}\text { ASME Section } \\
\text { III }\end{array}$ & $\mathrm{N} / \mathrm{S}$ & $\begin{array}{l}\text { Rules for Construction of Nuclear } \\
\text { Power Plant Components }\end{array}$ & glt89-013 & 1989 & $\mathbf{R}$ & $\begin{array}{l}\text { Before } 1971 \text { B31.1, } \\
\text { After, ASME Code } \\
\text { Section III }\end{array}$ & ASME Section III & 1992 \\
\hline 1900 & ASME & \begin{tabular}{|l|} 
ASME Section \\
IIi
\end{tabular} & $N / S$ & $\begin{array}{l}\text { Rules for Construction of Nuclear } \\
\text { Power Plant Components }\end{array}$ & git89-013.s01 & 1990 & $\mathbf{R}$ & & ASME Section III & 1992 \\
\hline 1901 & ASME & $\begin{array}{l}\text { ASME Section } \\
\text { III }\end{array}$ & 1972 & $\begin{array}{l}\text { Rules for Construction of Nuclear } \\
\text { Power Plant Components }\end{array}$ & glt92-001.ro1 & 1992 & $\mathbf{R}$ & & ASME Section III & 1992 \\
\hline 1902 & ASME & $\begin{array}{l}\text { ASME Section } \\
\text { III }\end{array}$ & 1989 & $\begin{array}{l}\text { Rules for Construction of Nuclear } \\
\text { Power Plant Components }\end{array}$ & inm-toc & 1993 & $\mathbf{R}$ & & ASME Section III & 1992 \\
\hline 1903 & ASME & \begin{tabular}{|l|} 
ASME Section \\
IIII
\end{tabular} & $\mathrm{N} / \mathrm{S}$ & $\begin{array}{l}\text { Rules for Construction of Nuclear } \\
\text { Power Plant Components }\end{array}$ & inm2500.027 & 1989 & $\mathbf{R}$ & & ASME Section III & 1992 \\
\hline 1904 & ASME & \begin{tabular}{|l|} 
ASME Section \\
IIII \\
\end{tabular} & $\mathrm{N} / \mathrm{S}$ & $\begin{array}{l}\text { Rules for Construction of Nuclear } \\
\text { Power Plant Components }\end{array}$ & inm35016B & 1975 & $\mathbf{R}$ & & ASME Section III & 1992 \\
\hline 1905 & ASME & $\begin{array}{l}\text { ASME Section } \\
\text { III }\end{array}$ & $\mathrm{N} / \mathrm{S}$ & $\begin{array}{l}\text { Rules for Construction of Nuclear } \\
\text { Power Plant Components }\end{array}$ & inm35018B & 1975 & $\mathbf{R}$ & & ASME Section III & 1992 \\
\hline 1906 & ASME & \begin{tabular}{|l|} 
ASME Section \\
ilI
\end{tabular} & $\mathbf{N} / \mathrm{S}$ & $\begin{array}{l}\text { Rules for Construction of Nuclear } \\
\text { Power Plant Components }\end{array}$ & inm35028B & 1975 & $\mathbf{R}$ & & ASME Section III & 1992 \\
\hline 1907 & ASME & \begin{tabular}{|l|} 
ASME Section \\
IIII
\end{tabular} & $N / S$ & $\begin{array}{l}\text { Rules for Construction of Nuclear } \\
\text { Power Plant Components }\end{array}$ & inm38701 & 1992 & $\mathbf{R}$ & & ASME Section III & 1992 \\
\hline 1908 & ASME & \begin{tabular}{|l|} 
ASME Section \\
IIII \\
\end{tabular} & $N / S$ & $\begin{array}{l}\text { Rules for Construction of Nuclear } \\
\text { Power Plant Components }\end{array}$ & inm38702 & 1992 & $\mathbf{R}$ & & ASME Section III & 1992 \\
\hline 1909 & ASME & $\begin{array}{l}\text { ASME Section } \\
\text { III }\end{array}$ & N/S & $\begin{array}{l}\text { Rules for Construction of Nuclear } \\
\text { Power Plant Components }\end{array}$ & inm49001 & 1992 & $\mathbf{R}$ & & ASME Section III & 1992 \\
\hline 1910 & ASME & \begin{tabular}{|l|} 
ASME Section \\
ill
\end{tabular} & $N / S$ & $\begin{array}{l}\text { Rules for Construction of Nuclear } \\
\text { Power Plant Components }\end{array}$ & inm49051 & 1982 & $\mathbf{R}$ & & ASME Section III & 1992 \\
\hline 1911 & ASME & $\begin{array}{l}\text { ASME Soction } \\
\text { III }\end{array}$ & $N / S$ & $\begin{array}{l}\text { Rules for Construction of Nuclear } \\
\text { Power Plant Components }\end{array}$ & inm49053 & 1985 & $\mathbf{R}$ & & ASME Section III & 1992 \\
\hline 1912 & ASME & $\begin{array}{l}\text { ASME Section } \\
\text { ill }\end{array}$ & $\mathrm{N} / \mathrm{S}$ & $\begin{array}{l}\text { Rules for Construction of Nuclear } \\
\text { Power Plant Components }\end{array}$ & inm49055 & 1982 & $\mathbf{R}$ & & ASME Section III & 1992 \\
\hline 1913 & ASME & $\begin{array}{l}\text { ASME Section } \\
\text { III }\end{array}$ & $N / S$ & $\begin{array}{l}\text { Rules for Construction of Nuclear } \\
\text { Power Plant Components }\end{array}$ & inm49061 & 1982 & $\mathbf{R}$ & & ASME Section III & 1992 \\
\hline
\end{tabular}


Codes and Standards and Other Guidance Cited in Regulatory Documents

Part A - Industry Consensus Codes and Standards (continued)

\begin{tabular}{|c|c|c|c|c|c|c|c|c|c|c|}
\hline $\begin{array}{l}\text { Rocord } \\
\text { No }\end{array}$ & 0.0 & Stondord & Standord & (1, & Documont & Dovo & popept & Commonth & Gument Yorron & Yorion \\
\hline 1914 & ASME & $\begin{array}{l}\text { ASME Section } \\
\text { III }\end{array}$ & $\mathrm{N} / \mathrm{S}$ & $\begin{array}{l}\text { Rules for Construction of Nuclear } \\
\text { Power Plant Components }\end{array}$ & inm50082B & 1976 & $\mathbf{R}$ & & ASME Section III & 1992 \\
\hline 1915 & ASME & $\begin{array}{l}\text { ASME Section } \\
\text { III }\end{array}$ & $\mathrm{N} / \mathrm{S}$ & $\begin{array}{l}\text { Rules for Construction of Nuclear } \\
\text { Power Plant Components }\end{array}$ & inm50085B & 1975 & $\bar{R}$ & & ASME Section III & 1992 \\
\hline 1916 & ASME & $\begin{array}{l}\text { ASME Soction } \\
\text { IIII }\end{array}$ & N/S & $\begin{array}{l}\text { Rules for Construction of Nuclear } \\
\text { Power Plant Components }\end{array}$ & inm52053 & 1984 & $\mathbf{R}$ & & ASME Section III & 1992 \\
\hline 1917 & ASME & $\begin{array}{l}\text { ASME Section } \\
\text { III }\end{array}$ & $\mathrm{N} / \mathrm{S}$ & $\begin{array}{l}\text { Rules for Construction of Nuclear } \\
\text { Power Plant Components }\end{array}$ & inm55050 & 1983 & $\mathbf{R}$ & & ASME Section III & 1992 \\
\hline 1918 & ASME & $\begin{array}{l}\text { ASME Section } \\
\text { Iii }\end{array}$ & $\mathrm{N} / \mathrm{S}$ & $\begin{array}{l}\text { Rules for Construction of Nuclear } \\
\text { Power Plant Components }\end{array}$ & inm55092B & 1976 & $\mathbf{R}$ & & ASME Section III & 1992 \\
\hline 1919 & ASME & $\begin{array}{l}\text { ASME Soction } \\
\text { III }\end{array}$ & $\mathrm{N} / \mathrm{S}$ & $\begin{array}{l}\text { Rules for Construction of Nuclear } \\
\text { Power Plant Components }\end{array}$ & inm550938 & 1977 & $\mathbf{R}$ & & ASME Section III & 1992 \\
\hline 1920 & ASME & $\begin{array}{l}\text { ASME Section } \\
\text { III }\end{array}$ & $N / S$ & $\begin{array}{l}\text { Rules for Construction of Nuclear } \\
\text { Power Plant Components }\end{array}$ & inm57050 & 1983 & $\mathbf{R}$ & & ASME Section III & 1992 \\
\hline 1921 & ASME & $\begin{array}{l}\text { ASME Section } \\
\text { III }\end{array}$ & $N / S$ & $\begin{array}{l}\text { Rules for Construction of Nuclear } \\
\text { Power Plant Components }\end{array}$ & inm57060 & 1983 & $\mathbf{R}$ & & ASME Section III & 1992 \\
\hline 1922 & ASME & $\begin{array}{l}\text { ASME Section } \\
\text { IIII }\end{array}$ & $N / S$ & $\begin{array}{l}\text { Rules for Construction of Nuclear } \\
\text { Power Plant Components }\end{array}$ & inm57070 & 1983 & $R$ & & ASME Section III & 1992 \\
\hline 1923 & ASME & $\begin{array}{l}\text { ASME Section } \\
\text { IIII }\end{array}$ & $N / S$ & $\begin{array}{l}\text { Rules for Construction of Nuclear } \\
\text { Power Plant Components }\end{array}$ & inm57080 & 1983 & $\mathbf{R}$ & & ASME Section III & 1992 \\
\hline 1924 & ASME & $\begin{array}{l}\text { ASME Section } \\
\text { IIII }\end{array}$ & $\mathrm{N} / \mathrm{S}$ & $\begin{array}{l}\text { Rules for Construction of Nuclear } \\
\text { Power Plant Components }\end{array}$ & inm57090 & 1993 & $\mathbf{R}$ & & ASME Section ill & 1992 \\
\hline 1925 & ASME & $\begin{array}{l}\text { ASME Section } \\
\text { IIII }\end{array}$ & $\mathrm{N} / \mathrm{S}$ & $\begin{array}{l}\text { Rules for Construction of Nuclear } \\
\text { Power Plant Components }\end{array}$ & $\operatorname{lnm} 70370$ & 1987 & $\mathbf{R}$ & & ASME Section III & 1992 \\
\hline 1926 & ASME & $\begin{array}{l}\text { ASME Section } \\
\text { IIII }\end{array}$ & $\mathrm{N} / \mathrm{S}$ & $\begin{array}{l}\text { Rules for Construction of Nuclear } \\
\text { Power Plant Components }\end{array}$ & inm73051 & 1986 & $\mathbf{R}$ & & ASME Section III & 1992 \\
\hline 1927 & ASME & $\begin{array}{l}\text { ASMiE Section } \\
\text { III }\end{array}$ & $\mathrm{N} / \mathrm{S}$ & $\begin{array}{l}\text { Rules for Construction of Nuclear } \\
\text { Power Plant Components }\end{array}$ & inm73052 & 1986 & $\mathbf{R}$ & & ASME Section III & 1992 \\
\hline 1928 & ASME & $\begin{array}{l}\text { ASME Section } \\
\text { IIII }\end{array}$ & $\mathrm{N} / \mathrm{S}$ & $\begin{array}{l}\text { Rules for Construction of Nuclear } \\
\text { Power Plant Components }\end{array}$ & inm9900/ASME III & 1989 & $\mathbf{R}$ & & ASME Section III & 1992 \\
\hline 1929 & ASME & $\begin{array}{l}\text { ASME Section } \\
\text { III }\end{array}$ & $N / S$ & $\begin{array}{l}\text { Rules for Construction of Nuclear } \\
\text { Power Plant Components }\end{array}$ & not80-38 & 1980 & $\mathbf{R}$ & & ASME Section III & 1992 \\
\hline 1930 & ASME & $\begin{array}{l}\text { ASME Section } \\
\text { III }\end{array}$ & 1972 & $\begin{array}{l}\text { Rules for Construction of Nuclear } \\
\text { Power Plant Components }\end{array}$ & not83-31 & 1983 & $\mathbf{R}$ & & ASME Section III & 1992 \\
\hline
\end{tabular}


Codes and Standards and Other Guidance Cited in Regulatory Documents

Part A - Industry Consensus Codes and Standards (continued)

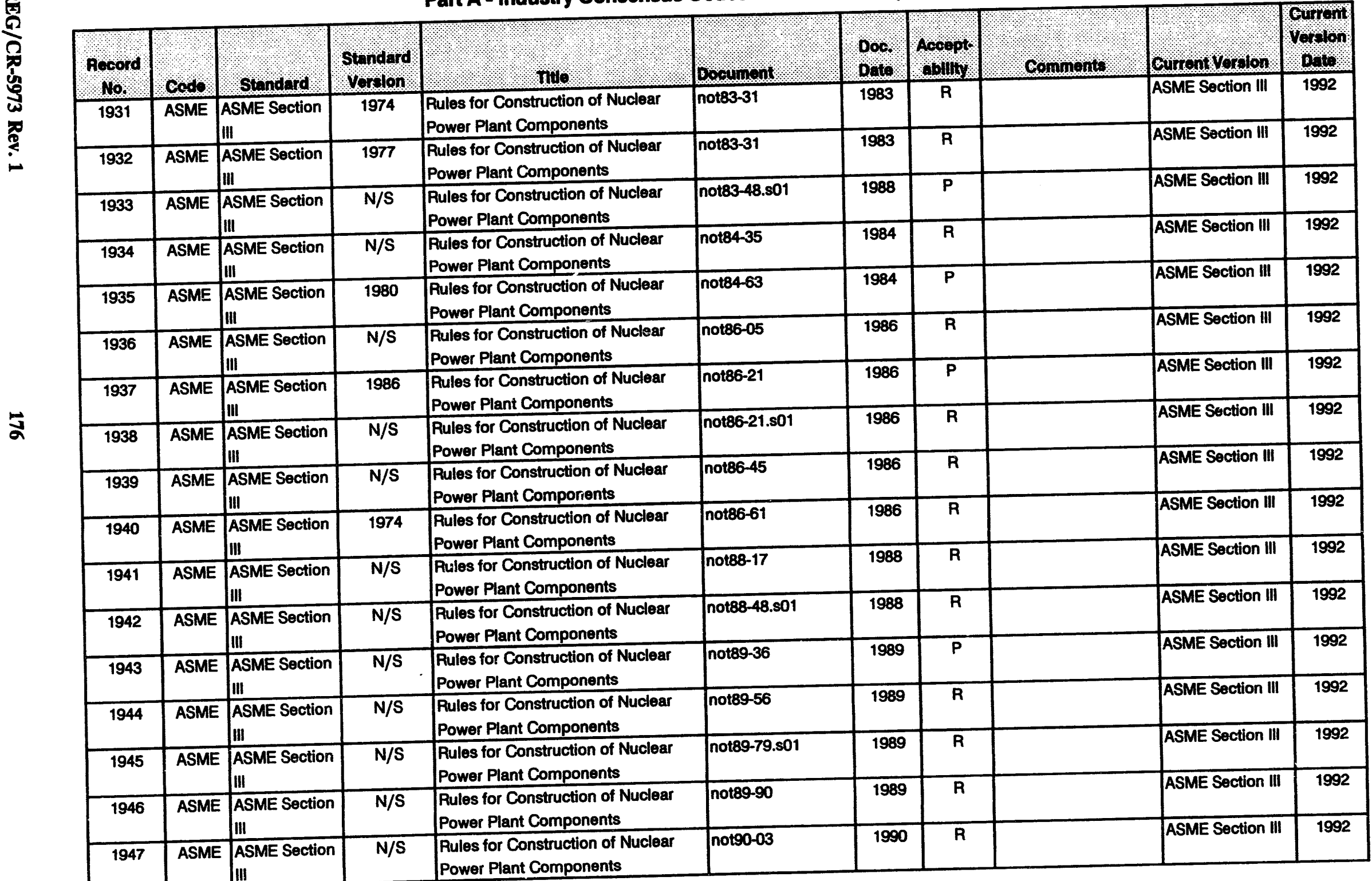


Codes and Standards and Other Guidance Clted in Regulatory Documents

Part A - Industry Consensus Codes and Standards (continued)

\begin{tabular}{|c|c|c|c|c|c|c|c|c|c|c|}
\hline Roporo & eodes & stond nd & Sthndard & 1 & Bowounght & Dor. & Acoppts & Comments & Currout Vorolon & owont \\
\hline 1948 & ASME & $\begin{array}{l}\text { ASME Section } \\
\text { lii }\end{array}$ & $\mathrm{N} / \mathrm{S}$ & $\begin{array}{l}\text { Rules for Construction of Nuclear } \\
\text { Power Plant Components }\end{array}$ & not92-50 & 1992 & $\mathbf{R}$ & & ASME Section III & 1992 \\
\hline 1949 & ASME & $\begin{array}{l}\text { ASME Section } \\
\text { III }\end{array}$ & N/S & $\begin{array}{l}\text { Rules for Construction of Nuclear } \\
\text { Power Plant Components }\end{array}$ & nureg0933, 2.B-27 & 1983 & $\mathbf{R}$ & & ASME Section III & 1992 \\
\hline 1950 & ASME & \begin{tabular}{|l|} 
ASME Section \\
III \\
\end{tabular} & $\mathrm{N} / \mathrm{S}$ & $\begin{array}{l}\text { Rules for Construction of Nuclear } \\
\text { Power Plant Components }\end{array}$ & nureg0933, 2.B-5 & 1988 & $\mathbf{R}$ & & ASME Section III & 1992 \\
\hline 1951 & ASME & $\begin{array}{l}\text { ASME Section } \\
\text { III }\end{array}$ & $\mathrm{N} / \mathrm{S}$ & $\begin{array}{l}\text { Rules for Construction of Nuclear } \\
\text { Power Plant Components }\end{array}$ & nureg0933, 3.109 & 1990 & $\mathbf{R}$ & & ASME Section III & 1992 \\
\hline 1952 & ASME & $\begin{array}{l}\text { ASME Section } \\
\text { III }\end{array}$ & $\mathrm{N} / \mathrm{S}$ & $\begin{array}{l}\text { Rules for Construction of Nuclear } \\
\text { Power Plant Components }\end{array}$ & nureg0933, 3.111 & 1991 & $\mathbf{R}$ & & ASME Section III & 1992 \\
\hline 1953 & ASME & $\begin{array}{l}\text { ASME Section } \\
\text { IIII }\end{array}$ & $N / S$ & $\begin{array}{l}\text { Rules for Construction of Nuclear } \\
\text { Power Plant Components }\end{array}$ & nureg0933, 3.127 & 1987 & $\mathbf{R}$ & & ASME Section III & 1992 \\
\hline 1954 & ASME & $\begin{array}{l}\text { ASME Section } \\
\text { III }\end{array}$ & $\mathrm{N} / \mathrm{S}$ & $\begin{array}{l}\text { Rules for Construction of Nuclear } \\
\text { Power Plant Components }\end{array}$ & nureg0933, 3.135 & 1991 & $\mathbf{R}$ & & ASME Soction III & 1992 \\
\hline 1955 & ASME & $\begin{array}{l}\text { ASME Section } \\
\text { IIII }\end{array}$ & $\mathrm{N} / \mathrm{S}$ & $\begin{array}{l}\text { Rules for Construction of Nuclear } \\
\text { Power Plant Components }\end{array}$ & nureg0933, 3.78 & 1992 & $\mathbf{R}$ & & ASME Section III & 1992 \\
\hline 1956 & ASME & $\begin{array}{l}\text { ASME Section } \\
\text { III }\end{array}$ & N/S & $\begin{array}{l}\text { Rules for Construction of Nuclear } \\
\text { Power Plant Components }\end{array}$ & nureg0933, 3.89 & 1992 & $\mathbf{R}$ & & ASME Soction III & 1992 \\
\hline 1957 & ASME & $\begin{array}{l}\text { ASME Section } \\
\text { IiI }\end{array}$ & $\mathrm{N} / \mathrm{S}$ & $\begin{array}{l}\text { Rules for Construction of Nuclear. } \\
\text { Power Plant Components }\end{array}$ & reg1.116.ro0 & 1976 & $\bar{R}$ & & ASME Section III & 1992 \\
\hline 1958 & ASME & $\begin{array}{l}\text { ASME Section } \\
\text { III }\end{array}$ & $\mathrm{N} / \mathrm{S}$ & $\begin{array}{l}\text { Rules for Construction of Nuclear } \\
\text { Power Plant Components }\end{array}$ & reg1.121.ro0 & 1976 & $\mathbf{P}$ & & ASME Section III & 1992 \\
\hline 1959 & ASME & $\begin{array}{l}\text { ASME Soction } \\
\text { IIII }\end{array}$ & 1976 & $\begin{array}{l}\text { Rules for Construction of Nuclear } \\
\text { Power Plant Components }\end{array}$ & reg 1.124.r01 & 1978 & $\mathbf{P}$ & & ASME Section III & 1992 \\
\hline 1960 & ASME & $\begin{array}{l}\text { ASME Section } \\
\text { IIII }\end{array}$ & 1977 & $\begin{array}{l}\text { Rules for Construction of Nuclear } \\
\text { Power Plant Components }\end{array}$ & reg1.130.ro1 & 1978 & $\mathbf{R}$ & & ASME Section III & 1992 \\
\hline 1961 & ASME & $\begin{array}{l}\text { ASME Section } \\
\text { III }\end{array}$ & $\mathrm{N} / \mathrm{S}$ & $\begin{array}{l}\text { Rules for Construction of Nuclear } \\
\text { Power Plant Components }\end{array}$ & reg1.143.ro1 & 1979 & $\mathbf{P}$ & & ASME Section III & 1992 \\
\hline 1962 & ASME & $\begin{array}{l}\text { ASME Section } \\
\text { IIII }\end{array}$ & 1977 & $\begin{array}{l}\text { Rules for Construction of Nuclear } \\
\text { Power Plant Components }\end{array}$ & reg1.148.r00 & 1981 & $\mathbf{R}$ & General endorsements & ASME Section III & 1992 \\
\hline 1963 & ASME & $\begin{array}{l}\text { ASME Section } \\
\text { IIII }\end{array}$ & 1981 & $\begin{array}{l}\text { Rules for Construction of Nuclear } \\
\text { Power Plant Components }\end{array}$ & reg1.151.r00 & 1983 & $\mathbf{R}$ & & ASME Section III & 1992 \\
\hline 1964 & ASME & $\begin{array}{l}\text { ASME Section } \\
\text { III }\end{array}$ & 1971 & $\begin{array}{l}\text { Rules for Construction of Nuclear } \\
\text { Power Plant Components }\end{array}$ & reg1.26.r03 & 1976 & $\bar{A}$ & & ASME Section III & 1992 \\
\hline
\end{tabular}


Codes and Standards and Other Guidance Cited in Regulatory Documents

Part A - Industry Consensus Codes and Standards (continued)

\begin{tabular}{|c|c|c|c|c|c|c|c|c|c|c|}
\hline $\begin{array}{l}\text { Recoro } \\
\text { No. }\end{array}$ & codo & Standerd & $\begin{array}{l}\text { Standard } \\
\text { Vorston }\end{array}$ & n nuo, & Documont & Doot & Aocept: & Comments: & Curront toroson & $\begin{array}{l}\text { Giment } \\
\text { boron }\end{array}$ \\
\hline 1965 & ASME & $\begin{array}{l}\text { ASME Section } \\
\text { III }\end{array}$ & 1976 & $\begin{array}{l}\text { Rules for Construction of Nuclear } \\
\text { Power Plant Components }\end{array}$ & reg1.31.r03 & ;978 & $\mathbf{R}$ & & ASME Soction III & 1992 \\
\hline 1966 & ASME & $\begin{array}{l}\text { ASME Section } \\
\text { III }\end{array}$ & 1972 & $\begin{array}{l}\text { Rules for Construction of Nuclear } \\
\text { Power Plant Components }\end{array}$ & reg1.34.ro0 & 1972 & $R$ & & ASME Section III & 1992 \\
\hline 1967 & ASME & $\begin{array}{l}\text { ASME Section } \\
\text { IiI }\end{array}$ & $N / S$ & $\begin{array}{l}\text { Rules for Construction of Nuclear } \\
\text { Power Plant Components }\end{array}$ & reg1.43.ro0 & 1973 & $\mathbf{P}$ & & ASME Section III & 1992 \\
\hline 1968 & ASME & $\begin{array}{l}\text { ASME Section } \\
\text { IIII }\end{array}$ & N/S & $\begin{array}{l}\text { Rules for Construction of Nuclear } \\
\text { Power Plant Components }\end{array}$ & reg1.50.r00 & 1973 & $\mathbf{P}$ & With supplements & ASME Section III & 1992 \\
\hline 1969 & ASME & $\begin{array}{l}\text { ASME : action } \\
\text { IIII }\end{array}$ & 1973 & $\begin{array}{l}\text { Rules for Construction of Nuclear } \\
\text { Power Plant Components }\end{array}$ & reg1.57.ro0 & 1973 & $\mathbf{R}$ & Referenced in Note 1 & ASME Section III & 1992 \\
\hline 1970 & ASME & $\begin{array}{l}\text { ASME Section } \\
\text { III }\end{array}$ & N/S & $\begin{array}{l}\text { Rules for Construction of Nuclear } \\
\text { Power Plant Components }\end{array}$ & reg1.65.r00 & 1973 & $\mathbf{P}$ & & ASME Section III & 1992 \\
\hline 1971 & ASME & $\begin{array}{l}\text { ASME Section } \\
\text { III }\end{array}$ & $N / S$ & $\begin{array}{l}\text { Rules for Construction of Nuclear } \\
\text { Power Plant Components }\end{array}$ & reg1.68.r02 & 1978 & $\mathbf{P}$ & & ASME Section III & 1992 \\
\hline 1972 & ASME & $\begin{array}{l}\text { ASME Section } \\
\text { III }\end{array}$ & $\mathrm{N} / \mathrm{S}$ & $\begin{array}{l}\text { Rules for Construction of Nuclear } \\
\text { Power Plant Components }\end{array}$ & reg1.70.ro3 & 1978 & $\mathbf{P}$ & & ASME Section III & 1992 \\
\hline 1973 & ASME & $\begin{array}{l}\text { ASME Section } \\
\text { ili }\end{array}$ & N/S & $\begin{array}{l}\text { Rules for Construction of Nuclear } \\
\text { Power Plant Components }\end{array}$ & reg1.71.r00 & 1973 & $\mathbf{P}$ & & ASME Soction III & 1992 \\
\hline 1974 & ASME & $\begin{array}{l}\text { ASME Section } \\
\text { IIII }\end{array}$ & $\mathrm{N} / \mathrm{S}$ & $\begin{array}{l}\text { Rules for Construction of Nuclear } \\
\text { Power Plant Components }\end{array}$ & reg1.77.r00 & 1974 & $\mathbf{P}$ & & ASME Section III & 1992 \\
\hline 1975 & ASME & $\begin{array}{l}\text { ASME Section } \\
\text { IIII }\end{array}$ & $N / S$ & $\begin{array}{l}\text { Rules for Construction of Nuclear } \\
\text { Power Plant Components }\end{array}$ & reg1.83.r01 & 1975 & $\mathbf{P}$ & & ASME Section III & 1992 \\
\hline 1976 & ASME & $\begin{array}{l}\text { ASME Section } \\
\text { III }\end{array}$ & N/S & $\begin{array}{l}\text { Rules for Construction of Nuclear } \\
\text { Power Plant Components }\end{array}$ & reg1.87.ro1 & 1975 & $\mathbf{R}$ & & ASME Section III & 1992 \\
\hline 1977 & ASME & $\begin{array}{l}\text { ASME Section } \\
\text { IIII }\end{array}$ & $\mathrm{N} / \mathrm{S}$ & $\begin{array}{l}\text { Rules for Construction of Nuclear } \\
\text { Power Plant Components }\end{array}$ & reg1.99.r02 & 1988 & $\mathbf{R}$ & & ASME Section III & 1992 \\
\hline 1978 & ASME & $\begin{array}{l}\text { ASME Section } \\
\text { IIII }\end{array}$ & 1974 & $\begin{array}{l}\text { Rules for Construction of Nuclear } \\
\text { Power Plant Components }\end{array}$ & reg3.28.r00 & 1975 & $\mathbf{P}$ & & ASME Section III & 1992 \\
\hline 1979 & ASME & $\begin{array}{l}\text { ASME Section } \\
\text { IIII }\end{array}$ & 1971 & $\begin{array}{l}\text { Rules for Construction of Nuclear } \\
\text { Power Plant Components }\end{array}$ & reg3.29.ro0 & 1975 & $\mathbf{P}$ & & ASME Section III & 1992 \\
\hline 1980 & ASME & $\begin{array}{l}\text { ASME Section } \\
\text { IIII }\end{array}$ & $\mathrm{N} / \mathrm{S}$ & $\begin{array}{l}\text { Rules for Construction of Nuclear } \\
\text { Power Plant Components }\end{array}$ & reg3.60.ro0 & 1987 & $\mathbf{P}$ & & ASME Section III & 1992 \\
\hline 1981 & ASME & $\begin{array}{l}\text { ASME Section } \\
\text { IIII }\end{array}$ & N/S & $\begin{array}{l}\text { Rules for Construction of Nuclear } \\
\text { Power Plant Components }\end{array}$ & reg7.11.r00 & 1991 & $\mathbf{R}$ & & ASME Section III & 1992 \\
\hline
\end{tabular}


Codes and Standards and Other Guidance Cited in Regulatory Documents

Part A - Industry Consensus Codes and Standards (continued)

\begin{tabular}{|c|c|c|c|c|c|c|c|c|c|c|}
\hline Rerord & codos & Standard & $\begin{array}{l}\text { Standard } \\
\text { Yorlon }\end{array}$ & ( & Document & $\begin{array}{l}\text { Doc. } \\
\text { Date }\end{array}$ & nopept & Componte & cuncontvorolos & olph \\
\hline 1982 & ASME & $\begin{array}{l}\text { ASME Section } \\
\text { III }\end{array}$ & $\mathrm{N} / \mathrm{S}$ & $\begin{array}{l}\text { Rules for Construction of Nuclear } \\
\text { Power Plant Components }\end{array}$ & reg7.12.100 & 1991 & $\mathrm{R}$ & & ASME Section ill & 1992 \\
\hline 1983 & ASME & $\begin{array}{l}\text { ASME Section } \\
\text { III }\end{array}$ & 1977 & $\begin{array}{l}\text { Rules for Construction of Nuclear } \\
\text { Power Plant Components } \\
\end{array}$ & reg7.6.01 & 1978 & $\mathbf{P}$ & & ASME Section III & 1992 \\
\hline 1984 & ASME & $\begin{array}{l}\text { ASME Soction } \\
\text { III } \\
\end{array}$ & N/S & $\begin{array}{l}\text { Rules for Construction of Nuclear } \\
\text { Power Plant Components }\end{array}$ & $\operatorname{sip} 10.2 .3$ & 1981 & $\mathbf{P}$ & & ASME Section III & 1992 \\
\hline 1985 & ASME & $\begin{array}{l}\text { ASME Section } \\
\text { III }\end{array}$ & $\mathrm{N} / \mathrm{S}$ & $\begin{array}{l}\text { Rules for Construction of Nuclear } \\
\text { Power Plant Components } \\
\end{array}$ & $\operatorname{srp} 10.3 .6$ & 1981 & $\mathbf{P}$ & & ASME Section III & 1992 \\
\hline 1986 & ASME & \begin{tabular}{|l|} 
ASME Section \\
III
\end{tabular} & $\mathrm{N} / \mathrm{S}$ & $\begin{array}{l}\text { Rules for Construction of Nuclear } \\
\text { Power Plant Components }\end{array}$ & srp15.0 & 1981 & $\mathbf{P}$ & & ASME Section III & 1992 \\
\hline 1987 & ASME & \begin{tabular}{|l|} 
ASME Section \\
IIII
\end{tabular} & $\mathrm{N} / \mathrm{S}$ & $\begin{array}{l}\text { Rules for Construction of Nuclear } \\
\text { Power Plant Components }\end{array}$ & $\operatorname{srp} 15.1 .1$ & 1981 & $\bar{R}$ & & ASME Section III & 1992 \\
\hline 1988 & ASME & $\begin{array}{l}\text { ASME Section } \\
\text { III }\end{array}$ & $\mathrm{N} / \mathrm{S}$ & $\begin{array}{l}\text { Rules for Construction of Nuclear } \\
\text { Power Plant Components }\end{array}$ & $\operatorname{srp} 15.2 .1$ & 1981 & $\mathbf{R}$ & & ASME Section III & 1992 \\
\hline 1989 & ASME & $\begin{array}{l}\text { ASME Section } \\
\text { IIII }\end{array}$ & $\mathrm{N} / \mathrm{S}$ & $\begin{array}{l}\text { Rules for Construction of Nuclear } \\
\text { Power Plant Components }\end{array}$ & $\operatorname{srp} 15.4 .4$ & 1981 & $\mathbf{R}$ & & ASME Section III & 1992 \\
\hline 1990 & ASME & $\begin{array}{l}\text { ASME Section } \\
\text { IIII }\end{array}$ & N/S & $\begin{array}{l}\text { Rules for Construction of Nuclear } \\
\text { Power Plant Components }\end{array}$ & $\operatorname{srp} 15.4 .8$ & 1981 & $P$ & & ASME Section III & 1992 \\
\hline 1991 & $\widehat{A S M E}$ & \begin{tabular}{|l|} 
ASME Section \\
IIII
\end{tabular} & $N / S$ & $\begin{array}{l}\text { Rules for Construction of Nuclear } \\
\text { Power Plant Components }\end{array}$ & $\operatorname{sip} 15.4 .9$ & 1981 & $\mathbf{P}$ & & ASME Section III & 1992 \\
\hline 1992 & ASME & $\begin{array}{l}\text { ASME Section } \\
\text { IIII }\end{array}$ & $N / S$ & $\begin{array}{l}\text { Rules for Construction of Nuclear } \\
\text { Power Plant Components }\end{array}$ & srp15.5.1 & 1981 & R & & ASME Soction III & 1992 \\
\hline 1993 & ASME & $\begin{array}{l}\text { ASME Section } \\
\text { lii }\end{array}$ & $N / S$ & $\begin{array}{l}\text { Rules for Construction of Nuclear } \\
\text { Power Plant Components }\end{array}$ & $\operatorname{srp15.6.1}$ & 1981 & $\mathbf{R}$ & & ASME Section III & 1992 \\
\hline 1994 & ASME & \begin{tabular}{|l|} 
ASME Section \\
III
\end{tabular} & $\mathrm{N} / \mathrm{S}$ & $\begin{array}{l}\text { Rules for Construction of Nuclear } \\
\text { Power Plant Components }\end{array}$ & srp17.1 & 1981 & $\bar{P}$ & & ASME Section III & 1992 \\
\hline 1995 & ASME & \begin{tabular}{|l|} 
ASME Section \\
IIII
\end{tabular} & $\mathrm{N} / \mathrm{S}$ & $\begin{array}{l}\text { Rules for Construction of Nuclear } \\
\text { Power Plant Components }\end{array}$ & srp17.3 & 1990 & $\overline{\mathbf{P}}$ & & ASME Section III & 1992 \\
\hline 1996 & ASME & $\begin{array}{l}\text { ASME Section } \\
\text { IIII }\end{array}$ & 1972 & $\begin{array}{l}\text { Rules for Construction of Nuclear } \\
\text { Power Plant Components }\end{array}$ & srp3.6.1 & 1990 & $P$ & & ASME Soction III & 1992 \\
\hline 1997 & ASME & \begin{tabular}{|l|} 
ASME Section \\
IIII
\end{tabular} & 1977 & $\begin{array}{l}\text { Rules for Construction of Nuclear } \\
\text { Power Plant Components }\end{array}$ & $\operatorname{srp4.2}$ & 1981 & $R$ & & ASME Section III & 1992 \\
\hline 1998 & ASME & $\begin{array}{l}\text { ASME Section } \\
\text { IIII }\end{array}$ & $\mathrm{N} / \mathrm{S}$ & $\begin{array}{l}\text { Rules for Construction of Nuclear } \\
\text { Power Plant Components }\end{array}$ & srp4.5.1 & 1981 & $\mathbf{P}$ & & ASME Section III & 1992 \\
\hline
\end{tabular}


Codes and Standards and Other Guidance Cited in Regulatory Documents

Part A - Industry Consensus Codes and Standards (continued)

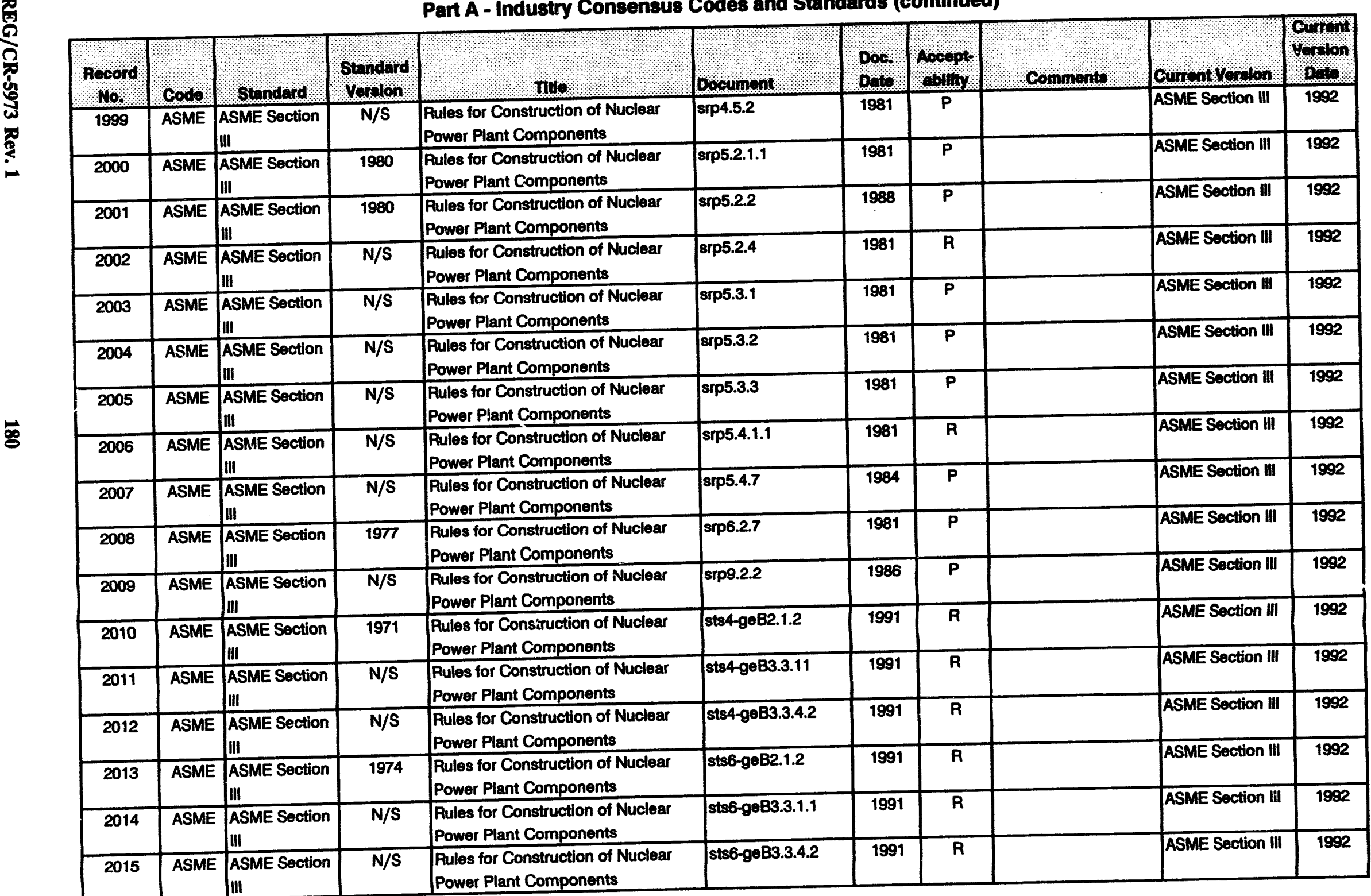


Codes and Standards and Other Guidance Cited in Regulatory Documents

Part A - Industry Consensus Codes and Standards (continued)

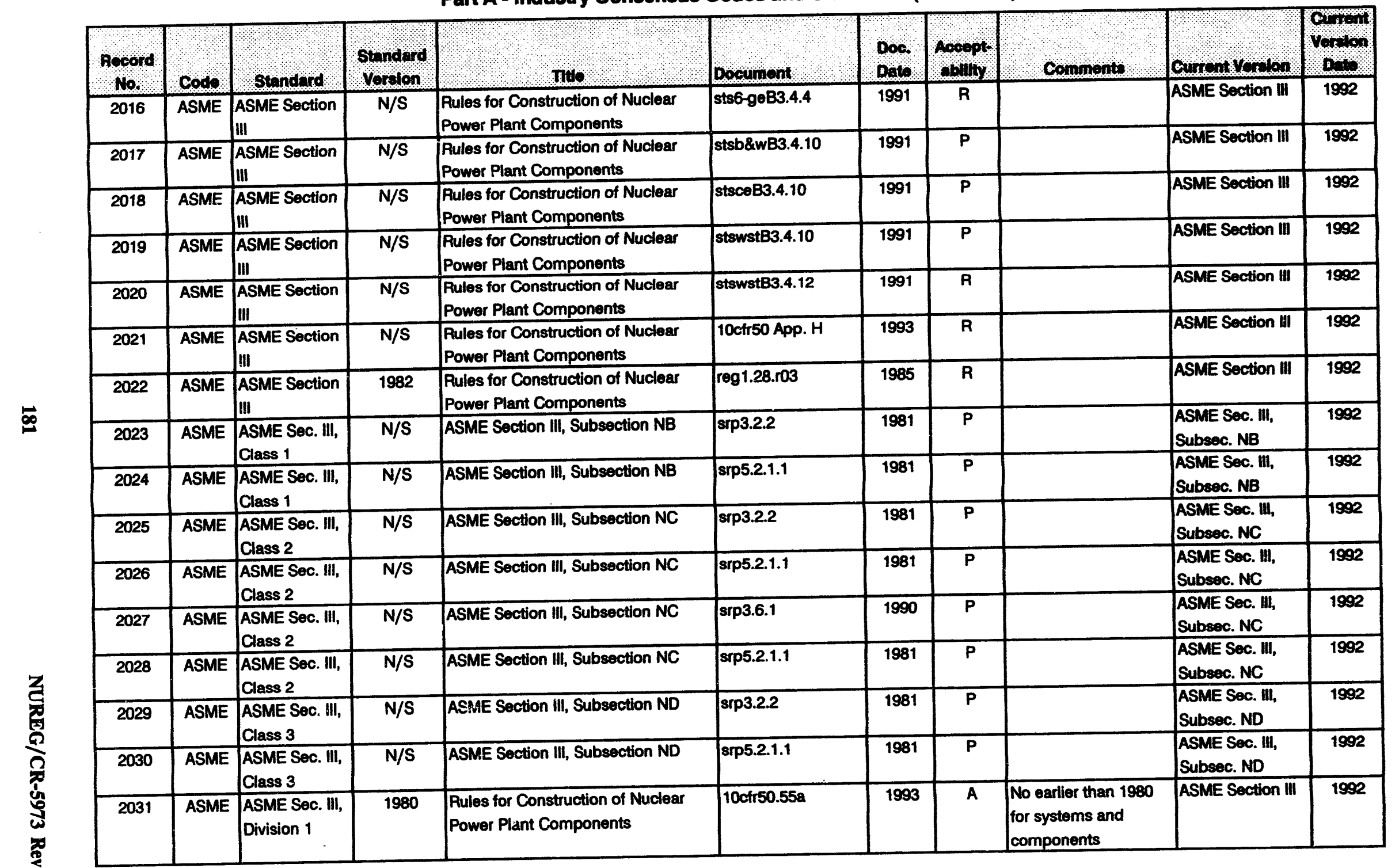


Codes and Standards and Other Guidance Cited in Regulatory Documents

Part A - Industry Consensus Codes and Standards (continued)

\begin{tabular}{|c|c|c|c|c|c|c|c|c|c|c|}
\hline $\begin{array}{l}\text { Record } \\
\text { No. }\end{array}$ & cods & Standard & $\begin{array}{l}\text { Standard } \\
\text { Yorelon }\end{array}$ & \& rilo, & Bocumont & $\begin{array}{l}\text { Dor. } \\
\text { Dont }\end{array}$ & Acoopt & Cormmente & 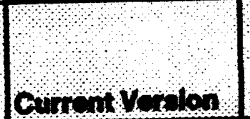 & 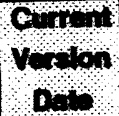 \\
\hline 2032 & ASME & $\begin{array}{l}\text { ASME Sec. III, } \\
\text { Division } 1\end{array}$ & 1989 & $\begin{array}{l}\text { Rules for Construction of Nuclear } \\
\text { Power Plant Components }\end{array}$ & 10 ctr50.55a & 1993 & $P$ & $\begin{array}{l}\text { Except }(c)(2),(c)(3), \\
\text { and }(c)(4) \text { in Reg Pos }\end{array}$ & $\begin{array}{l}\text { ASME Sec. III, Div. } \\
1\end{array}$ & 1992 \\
\hline 2033 & ASME & $\begin{array}{l}\text { ASME Sec. III, } \\
\text { Division } 1 \\
\end{array}$ & $N / S$ & $\begin{array}{l}\text { Rules for Construction of Nuclear } \\
\text { Power Plant Components }\end{array}$ & not90-27 & 1990 & $\mathbf{P}$ & & $\begin{array}{l}\text { ASME Sec. III, Div. } \\
1\end{array}$ & 1992 \\
\hline 2034 & ASME & $\begin{array}{l}\text { ASME Sec. III, } \\
\text { Division } 1\end{array}$ & $\mathrm{~N} / \mathrm{S}$ & $\begin{array}{l}\text { Rules for Construction of Nuclear } \\
\text { Power Plant Components }\end{array}$ & reg1.72.ro2 & 1978 & $\mathbf{P}$ & & $\begin{array}{l}\text { ASME Soc. III, Din. } \\
1\end{array}$ & 1992 \\
\hline 2035 & ASME & $\begin{array}{l}\text { ASME Sec. III, } \\
\text { Division } 1 \\
\end{array}$ & N/S & $\begin{array}{l}\text { Rules for Construction of Nuclear } \\
\text { Power Plant Components }\end{array}$ & $\operatorname{sip3.8.3}$ & 1981 & $\mathbf{P}$ & & $\begin{array}{l}\text { ASME Soc. III, Div. } \\
1\end{array}$ & 1992 \\
\hline 2036 & ASME & $\begin{array}{l}\text { ASME Sec. III, } \\
\text { Division } 1 \\
\end{array}$ & $\mathrm{~N} / \mathrm{S}$ & $\begin{array}{l}\text { Pules for Construction of Nuclear } \\
\text { Power Plant Components }\end{array}$ & sip3.8.4 & 1981 & $\mathbf{P}$ & & $\begin{array}{l}\text { ASME Sec. III, Div. } \\
1\end{array}$ & 1992 \\
\hline 2037 & ASME & $\begin{array}{l}\text { ASME Sec. III, } \\
\text { Division } 1 \\
\end{array}$ & N/S & $\begin{array}{l}\text { Rules for Conistruction of Nuclear } \\
\text { Power Plant Components }\end{array}$ & srp3.9.1 & 1981 & $\mathbf{A}$ & & $\begin{array}{l}\text { ASME Sec. III, Div. } \\
1\end{array}$ & 1992 \\
\hline 2038 & ASME & $\begin{array}{l}\text { ASME Sec. III, } \\
\text { Division } 1 \\
\end{array}$ & $\mathrm{~N} / \mathrm{S}$ & $\begin{array}{l}\text { Rules for Construction of Nuclear } \\
\text { Power Plant Components }\end{array}$ & 8rp3.9.2 & 1981 & $\mathbf{P}$ & & $\begin{array}{l}\text { ASME Sec. III, Div. } \\
1\end{array}$ & 1992 \\
\hline 2039 & ASME & $\begin{array}{l}\text { ASME Sec. III, } \\
\text { Division } 1 \\
\end{array}$ & $\mathrm{~N} / \mathrm{S}$ & $\begin{array}{l}\text { Rules for Construction of Nuclear } \\
\text { Power Plant Components }\end{array}$ & srp3.9.3 & 1981 & $\mathbf{p}$ & & $\begin{array}{l}\text { ASME SOC. III, Div. } \\
1\end{array}$ & 1992 \\
\hline 2040 & ASME & $\begin{array}{l}\text { ASME Sec. III, } \\
\text { Division } 1 \\
\end{array}$ & $\mathrm{~N} / \mathrm{S}$ & $\begin{array}{l}\text { Rules for Construction of Nuclear } \\
\text { Power Plant Components }\end{array}$ & $\operatorname{sip3.9.4}$ & 1984 & $\bar{P}$ & & $\begin{array}{l}\text { ASME Sec. III, Div. } \\
1\end{array}$ & 1992 \\
\hline 2041 & ASME & $\begin{array}{l}\text { ASME Sec. III, } \\
\text { Division } 1 \\
\end{array}$ & $N / S$ & $\begin{array}{l}\text { Rules for Construction of Nuctear } \\
\text { Power Plant Components }\end{array}$ & sip3.9.5 & 1981 & $\mathbf{P}$ & & $\begin{array}{l}\text { ASME Sec. In, Div. } \\
1\end{array}$ & 1992 \\
\hline 2042 & ASME & $\begin{array}{l}\text { ASME Sec. III, } \\
\text { Division } 1 \\
\end{array}$ & $\mathrm{~N} / \mathrm{S}$ & $\begin{array}{l}\text { Rules for Construction of Nuclear } \\
\text { Power Plant Components }\end{array}$ & sip6.1.1 & 1981 & $\mathbf{P}$ & & $\begin{array}{l}\text { ASME Sec. HI, Div. } \\
1\end{array}$ & 1992 \\
\hline 2043 & ASME & $\begin{array}{l}\text { ASME Sec. III, } \\
\text { Division } 1 \\
\end{array}$ & $\mathrm{~N} / \mathrm{S}$ & $\begin{array}{l}\text { Rules for Construction of Nuclear } \\
\text { Power Plant Components }\end{array}$ & reg1.38.r02 & 1977 & $\bar{R}$ & & $\begin{array}{l}\text { ASME Sec. III, Div. } \\
1\end{array}$ & 1992 \\
\hline 2044 & ASME & $\begin{array}{l}\text { ASME Sec. III, } \\
\text { Subsec. NA }\end{array}$ & $N / S$ & $\begin{array}{l}\text { General Requirements for Division } \\
1 \text { and Divisic? } 2\end{array}$ & inm55093B & 1977 & $\mathbf{R}$ & & $\begin{array}{l}\text { ASME Soc. III, } \\
\text { Subsec. NCA }\end{array}$ & 1992 \\
\hline 2045 & ASME & $\begin{array}{l}\text { ASME Sec. III, } \\
\text { N-322.1 }\end{array}$ & 1969 & Not found & not86-19 & 1986 & $\bar{R}$ & $\begin{array}{l}\text { Typo in not86-19 - N- } \\
322.1 \text { is not a valid } \\
\text { ASME reference }\end{array}$ & $n / a$ & $n / a$ \\
\hline 2046 & ASME & $\begin{array}{l}\text { ASME SeC. III, } \\
\text { NA-2000 }\end{array}$ & $\mathrm{N} / \mathrm{S}$ & Classification of Components & srp6.6 & 1981 & $\mathbf{P}$ & & $\begin{array}{l}\text { ASME SeC. III, } \\
\text { NCA-2000 }\end{array}$ & 1992 \\
\hline 2047 & ASME & $\begin{array}{l}\text { ASME Sec. III, } \\
\text { NA-3700 }\end{array}$ & $N / S$ & $\begin{array}{l}\text { Responsibilities of an NA Cortificate } \\
\text { Holder }\end{array}$ & not85-15 & 1985 & $\mathbf{R}$ & & $\begin{array}{l}\text { ASME Sec. III, } \\
\text { NCA-3700 }\end{array}$ & 1992 \\
\hline
\end{tabular}


Codes and Standards and Other Guidance Clted in Regulatory Documents

Part A - Industry Consensus Codes and Standards (continued)

\begin{tabular}{|c|c|c|c|c|c|c|c|c|c|c|}
\hline Rocord & 000 & Stand no & Stonderd & mo & Docoument & Doc. & Aoopt & componts & Cuncot rosolon & phof \\
\hline 2048 & ASME & $\begin{array}{l}\text { ASME Sec. III, } \\
\text { NA-4000 }\end{array}$ & $N / S$ & Quality Assurance & inm35051B & 1976 & $\mathbf{R}$ & & $\begin{array}{l}\text { ASME SeC. III, } \\
\text { NCA-4000 }\end{array}$ & 1992 \\
\hline 2049 & ASME & $\begin{array}{l}\text { ASME Sec. III, } \\
\text { NA-4000 }\end{array}$ & $\mathrm{N} / \mathrm{S}$ & Quality Assurance & inm55092B & 1976 & $\mathbf{R}$ & & $\begin{array}{l}\text { ASME Sec. II, } \\
\text { NCA-4000 }\end{array}$ & 1992 \\
\hline 2050 & ASME & $\begin{array}{l}\text { ASME Sec. III, } \\
\text { NA-4COO }\end{array}$ & $\mathbf{N} / \mathbf{S}$ & Quality Assurance & inm55093B & 1977 & $\mathbf{R}$ & & $\begin{array}{l}\text { ASME SeC. UI, } \\
\text { NCA-4000 } \\
\end{array}$ & 1992 \\
\hline 2051 & ASME & $\begin{array}{l}\text { ASME Sec. III, } \\
\text { NA-8000 }\end{array}$ & N/S & $\begin{array}{l}\text { Certificates of Authorization, } \\
\text { Nameplates, Code Symbol } \\
\text { Stamping, and Data Peports }\end{array}$ & $\operatorname{srp5.3.1}$ & 1981 & $\mathbf{P}$ & & $\begin{array}{l}\text { ASME Sec. III, } \\
\text { NCA-8000 }\end{array}$ & 1992 \\
\hline 2052 & ASME & $\begin{array}{l}\text { ASME Sec. III, } \\
\text { NA-8400 }\end{array}$ & $\mathbf{N} / \mathbf{S}$ & Data Reports & reg1.87.ro1 & 1975 & $\mathbf{R}$ & & $\begin{array}{l}\text { ASME Soc. III, } \\
\text { NCA } 8400\end{array}$ & 1992 \\
\hline 2053 & ASME & $\begin{array}{l}\text { ASME Sec. III, } \\
\text { NCA-1110 }\end{array}$ & $\mathrm{N} / \mathrm{S}$ & $\begin{array}{l}\text { Nature of These Rules and } \\
\text { Components to Which They are } \\
\text { Applicable }\end{array}$ & srp3.2.2 & 1981 & $\mathbf{R}$ & Cited for definition & $\begin{array}{l}\text { ASME SoC. } 111 \\
\text { NCA-1110 }\end{array}$ & 1992 \\
\hline 2054 & ASME & $\begin{array}{l}\text { ASME Sec. III, } \\
\text { NCA-1110 }\end{array}$ & $\mathbf{N} / \mathbf{S}$ & $\begin{array}{l}\text { Nature of These Rules and } \\
\text { Components to Which They are } \\
\text { Applicable }\end{array}$ & srp3.9.4 & 1984 & $\mathbf{R}$ & & $\begin{array}{l}\text { ASME Sec. IIf, } \\
\text { NCA-1110 }\end{array}$ & 1992 \\
\hline 2055 & ASME & $\begin{array}{l}\text { ASME Sec. III, } \\
\text { NCA-1140 }\end{array}$ & $\mathrm{N} / \mathrm{S}$ & $\begin{array}{l}\text { Nature of These Rules and } \\
\text { Components to Which They are } \\
\text { Applicable }\end{array}$ & 10 cfr $50.55 a$ & 1993 & $\mathbf{P}$ & & $\begin{array}{l}\text { ASME Soc. Hil, } \\
\text { NCA-1140 }\end{array}$ & 1992 \\
\hline 2056 & ASME & $\begin{array}{l}\text { ASME Sec. III, } \\
\text { NCA-2142(b) }\end{array}$ & 1977 & $\begin{array}{l}\text { Establishment of Design, Service, } \\
\text { and Test Loadings and Limits }\end{array}$ & reg1.148.rco & 1981 & $\mathbf{R}$ & Referred to in text & $\begin{array}{l}\text { ASME Soc. III, } \\
\text { NCA-2142(b) }\end{array}$ & 1992 \\
\hline 2057 & ASME & $\begin{array}{l}\text { ASME SOC. III, } \\
\text { NCA-2142.2(b) }\end{array}$ & $N / S$ & $\begin{array}{l}\text { Establishment of Design, Service, } \\
\text { and Test Loadings and Limits }\end{array}$ & reg1.130.r01 & 1978 & $\mathbf{A}$ & Cited for definition & $\begin{array}{l}\text { ASME Soc. 11, } \\
\text { NCA-2142.2(b) }\end{array}$ & 1992 \\
\hline 2058 & ASME & $\begin{array}{l}\text { ASME SeC. III, } \\
\text { NCA-3250 } \\
\end{array}$ & $\mathrm{N} / \mathrm{S}$ & Provision of Design Specification & reg1.148.roo & 1981 & $\mathbf{P}$ & & $\begin{array}{l}\text { ASME SeC. III, } \\
\text { NCA-3250 }\end{array}$ & 1992 \\
\hline 2059 & ASME & $\begin{array}{l}\text { ASME Sec. III, } \\
\text { NCA-3252(a) }\end{array}$ & N/S & Provision of Design Specification & reg1.148.roo & 1981 & $\mathbf{P}$ & & $\begin{array}{l}\text { ASNE Sec. II, } \\
\text { NCA-3252(a) }\end{array}$ & 1992 \\
\hline 2060 & ASME & $\begin{array}{l}\text { ASME Sec. III, } \\
\text { NCA-3256 }\end{array}$ & $\mathbf{N} / \mathrm{S}$ & Filing of Design Spectfications & reg 1.148.r00 & 1981 & $\mathbf{R}$ & & $\begin{array}{l}\text { ASME Sec. Hit, } \\
\text { NCA-3256 }\end{array}$ & 1992 \\
\hline 2061 & ASME & $\begin{array}{l}\text { ASME Sec. III, } \\
\text { NCA-3554 }\end{array}$ & $\mathbf{N} / \mathbf{S}$ & $\begin{array}{l}\text { Modification of Documents and } \\
\text { Reconciliation With Design Report }\end{array}$ & inm37051 & 1987 & $\mathbf{R}$ & & $\begin{array}{l}\text { ASME SOC. III, } \\
\text { NCA } 3554\end{array}$ & 1992 \\
\hline
\end{tabular}


Codes and Standards and Other Guidance Ched in Regulatory Documents Part A - Industry Consensus Codes and Standards (continued)

\begin{tabular}{|c|c|c|c|c|c|c|c|c|c|c|}
\hline Rocord & Codo & Standord & $\begin{array}{l}\text { Stendard } \\
\text { veralon }\end{array}$ & no, 1, & boconow & Doo. & Acoop & Connesonts & Conom Vorblos & Gomant \\
\hline 2062 & ASME & $\begin{array}{l}\text { ASME SoC. III, } \\
\text { NCA-3800 }\end{array}$ & N/S & $\begin{array}{l}\text { Motallic Material Manufacturer's } \\
\text { and Material Supplier's Quality } \\
\text { Systom Program }\end{array}$ & bulB3-06 & 1983 & $\mathbf{A}$ & & $\begin{array}{l}\text { ASME Soc. III, } \\
\text { NCA-3800 }\end{array}$ & 1992 \\
\hline 2063 & ASME & $\begin{array}{l}\text { ASME Sec. III, } \\
\text { NCA-3800 }\end{array}$ & $\mathrm{N} / \mathrm{S}$ & $\begin{array}{l}\text { Metallic Material Manufacturer's } \\
\text { and Material Supplier's Quality } \\
\text { System Program }\end{array}$ & bul88-05 & 1988 & $\mathbf{P}$ & & $\begin{array}{l}\text { ASME SOC. III, } \\
\text { NCA-3800 }\end{array}$ & 1992 \\
\hline 2064 & ASME & $\begin{array}{l}\text { ASME SeC. III, } \\
\text { NCA-3800 }\end{array}$ & 1977 & $\begin{array}{l}\text { Metallic Material Manufacturer's } \\
\text { and Material Supplier's Quality } \\
\text { System Program }\end{array}$ & inm36100 & 1983 & $\mathbf{R}$ & & $\begin{array}{l}\text { ASME SOC. III, } \\
\text { NCA3800 }\end{array}$ & 1992 \\
\hline 2065 & ASME & $\begin{array}{l}\text { ASME Sec. III, } \\
\text { NCA-3800 }\end{array}$ & N/S & $\begin{array}{l}\text { Motallic Material Manufacturer's } \\
\text { and Material Supplier's Quality } \\
\text { System Program }\end{array}$ & inm50090 & 1985 & $\boldsymbol{R}$ & & $\begin{array}{l}\text { ASME Sec. III, } \\
\text { NCA } 3800\end{array}$ & 1992 \\
\hline 2066 & ASME & $\begin{array}{l}\text { ASME SeC. III, } \\
\text { NCA-3800 }\end{array}$ & $N / S$ & $\begin{array}{l}\text { Metallic Material Manufacturer's } \\
\text { and Material Suppilier's Quality } \\
\text { System Program }\end{array}$ & not83-07 & 1983 & $\mathbf{P}$ & Implied endorsement & $\begin{array}{l}\text { ASME SeC. III, } \\
\text { NCA } 3800\end{array}$ & 1992 \\
\hline 2067 & ASME & $\begin{array}{l}\text { ASME SeC. III, } \\
\text { NCA-3800 }\end{array}$ & $N / S$ & $\begin{array}{l}\text { Metallic Material Manufacturer's } \\
\text { and Material Supplier's Quality } \\
\text { System Program }\end{array}$ & not88-95 & 1988 & $\bar{P}$ & & $\begin{array}{l}\text { ASME Sec. III, } \\
\text { NCA-3800 }\end{array}$ & 1992 \\
\hline 2068 & ASME & $\begin{array}{l}\text { ASME Sec. III, } \\
\text { NCA-4137.7 }\end{array}$ & $\mathrm{N} / \mathrm{S}$ & Not Found & inm37051 & 1987 & $\mathbf{R}$ & $\begin{array}{l}\text { NCA-4137.7 not in } \\
\text { ASME B\&PV Code }\end{array}$ & $n / a$ & $n / a$ \\
\hline 2069 & ASME & $\begin{array}{l}\text { ASME Sec. III, } \\
\text { Subsec. NB }\end{array}$ & $N / S$ & Class I Components & inm48051 & 1986 & $\mathbf{R}$ & & $\begin{array}{l}\text { ASME Soc. III, } \\
\text { Subsec. NB }\end{array}$ & 1992 \\
\hline 2070 & ASME & $\begin{array}{l}\text { ASME Sec. III, } \\
\text { Subsec. NB }\end{array}$ & $\mathrm{N} / \mathrm{S}$ & Class I Components & inm48053 & 1986 & $\mathbf{R}$ & & $\begin{array}{l}\text { ASME Sec. III, } \\
\text { Subsec. NB }\end{array}$ & 1992 \\
\hline 2071 & ASME & $\begin{array}{l}\text { ASME Sec. III, } \\
\text { Subsec. NB }\end{array}$ & $N / S$ & Class I Components & inm55050 & 1983 & $\mathbf{R}$ & & $\begin{array}{l}\text { ASME Sec. IIt, } \\
\text { Subsec. NB }\end{array}$ & 1992 \\
\hline 2072 & ASME & $\begin{array}{l}\text { ASME Sec. III, } \\
\text { Subsec.JB }\end{array}$ & $N / S$ & Class I Components & inm55092B & 1976 & $\mathbf{R}$ & & $\begin{array}{l}\text { ASME Sec. III, } \\
\text { Subsec. NB }\end{array}$ & 1992 \\
\hline 2073 & ASME & $\begin{array}{l}\text { ASME Sec. III, } \\
\text { Subsec. NB }\end{array}$ & $N / S$ & Class I Components & inm55093B & 1977 & $\mathbf{R}$ & & $\begin{array}{l}\text { ASME Sec. III, } \\
\text { Subsec. NB }\end{array}$ & 1992 \\
\hline 2074 & ASME & $\begin{array}{l}\text { ASME Sec. III, } \\
\text { Subsec. NB }\end{array}$ & $N / S$ & Class I Components & inm70462 & 1983 & $\mathbf{R}$ & & $\begin{array}{l}\text { ASME Sec. III, } \\
\text { Subsec. NB }\end{array}$ & 1992 \\
\hline 2075 & ASME & $\begin{array}{l}\text { ASME Sec. III, } \\
\text { Subsec. NB }\end{array}$ & $N / S$ & Class I Components & inm70562 & 1983 & $\mathbf{R}$ & & $\begin{array}{l}\text { ASHE Sec. III, } \\
\text { Subsec. NB }\end{array}$ & 1992 \\
\hline
\end{tabular}



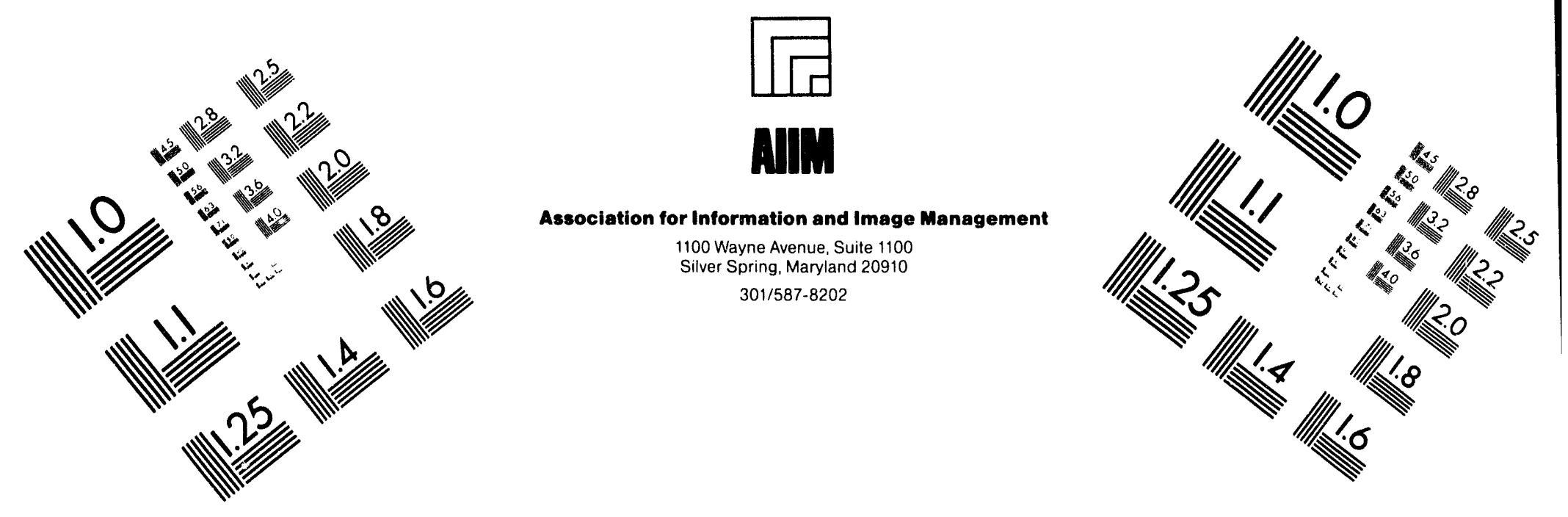

\section{Centimeter}

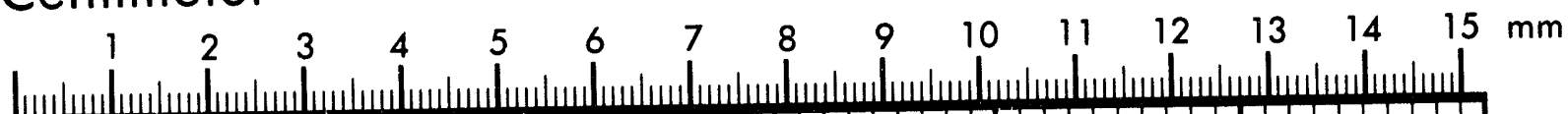

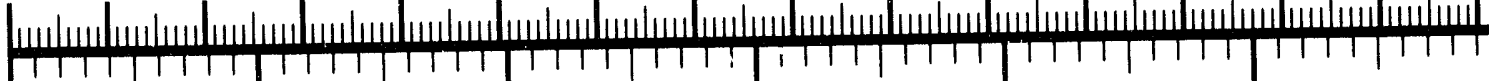

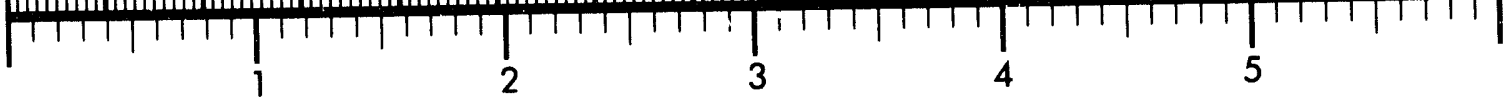
Inches
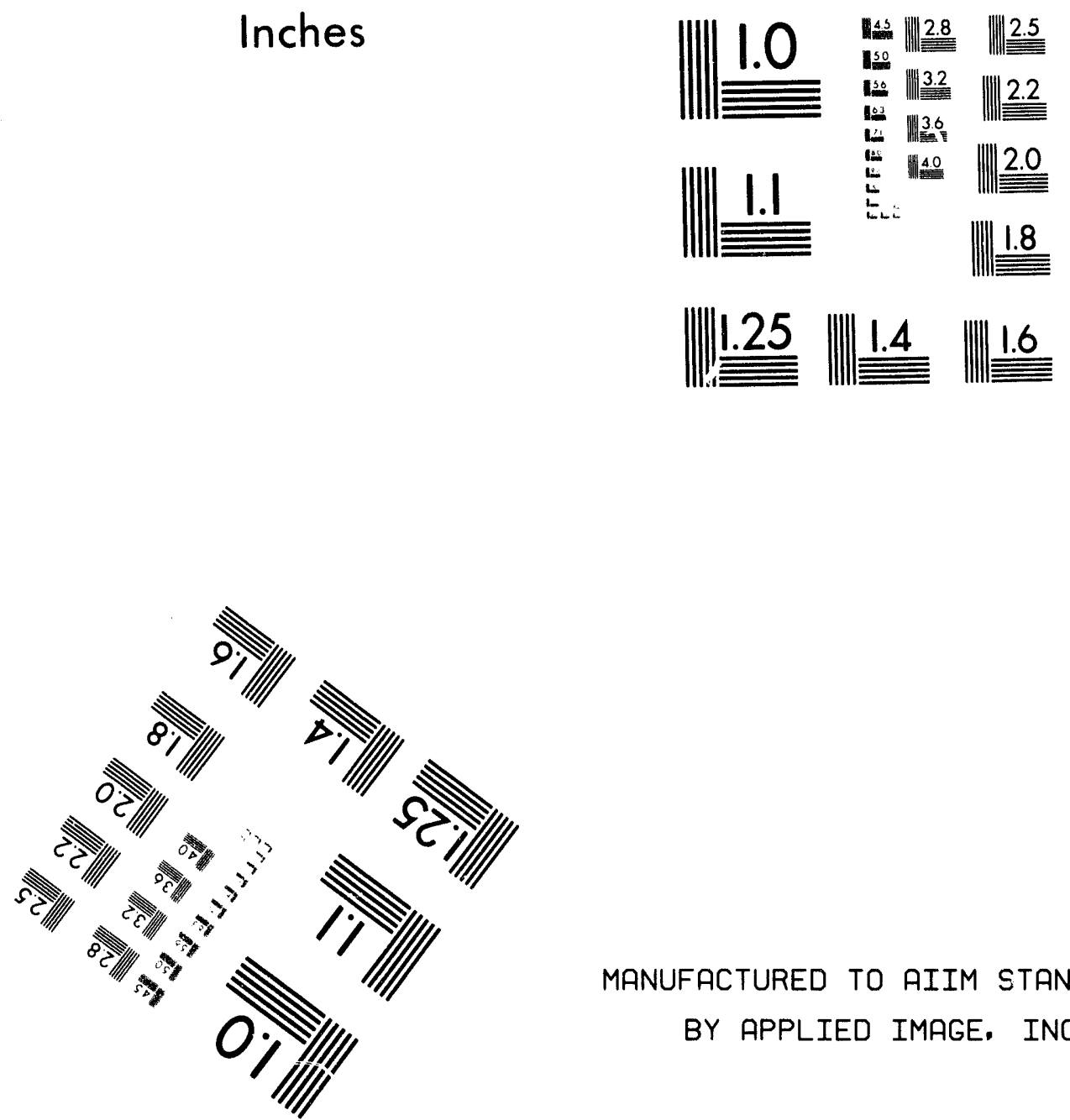

MANUFACTURED TO AIIM STANDARDS

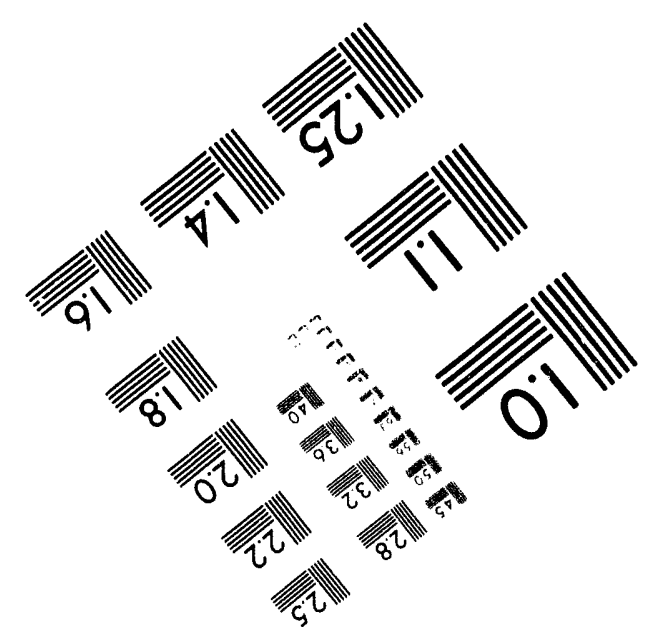



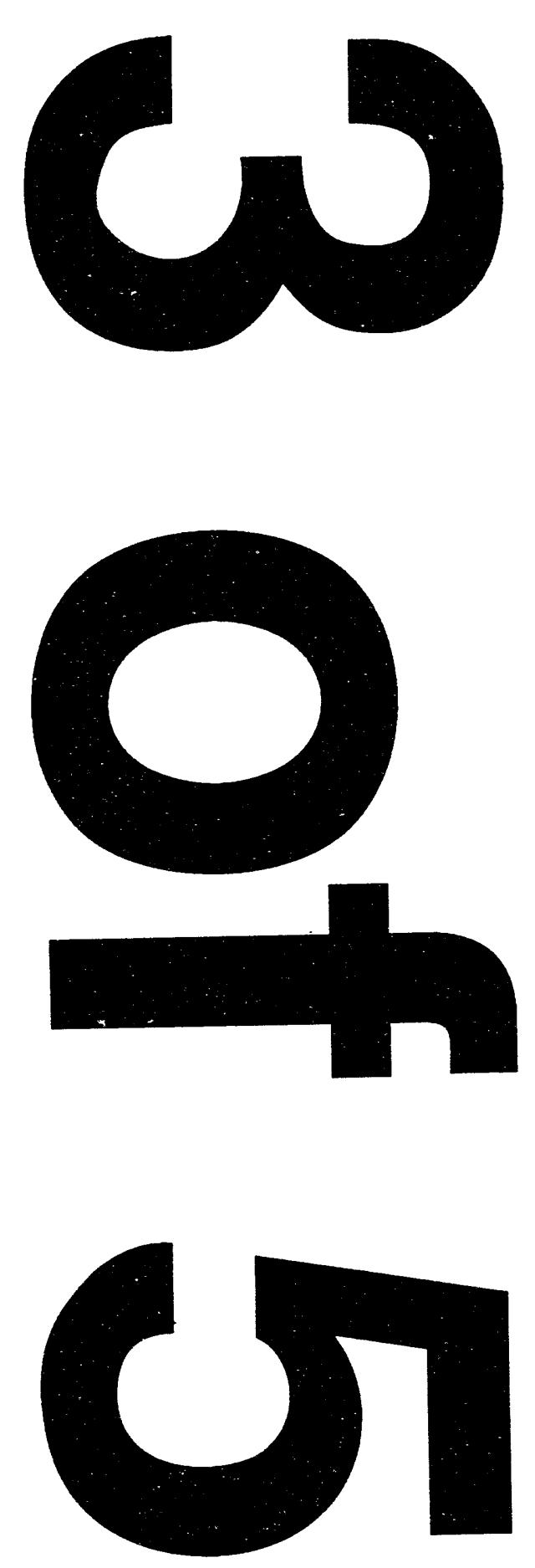


\section{Codes and Standards and Other Guidance Cited in Regulatory Documents}

Part A - Industry Consensus Codes and Standards (continsied)

\begin{tabular}{|c|c|c|c|c|c|c|c|c|c|c|}
\hline hocord & Codo & Standurd & Strondard & (ै) & Docunont & $\begin{array}{l}\text { Dor } \\
\text { Dato }\end{array}$ & Apeppls & Commonts & curront Vorolon & Vorront \\
\hline 2076 & ASME & $\begin{array}{l}\text { ASME Sec. III, } \\
\text { Subsec. NB }\end{array}$ & N/S & Class I Components & reg1.121.r00 & 1976 & $P$ & & $\begin{array}{l}\text { ASME Sec. III, } \\
\text { Subsec. NB }\end{array}$ & 1992 \\
\hline 2077 & ASME & $\begin{array}{l}\text { ASME Sec. III, } \\
\text { Subsec. NB }\end{array}$ & $\mathrm{N} / \mathrm{S}$ & Class I Components & reg1.65.ro0 & 1973 & $\mathbf{P}$ & & $\begin{array}{l}\text { ASME Sec. III, } \\
\text { Subsec. NB }\end{array}$ & 1992 \\
\hline 2078 & ASME & $\begin{array}{l}\text { ASME Sec. III, } \\
\text { Subsec. NB }\end{array}$ & $\mathrm{N} / \mathrm{S}$ & Class I Components & reg1.70.r03 & 1978 & $\mathbf{P}$ & & $\begin{array}{l}\text { ASME Sec. III, } \\
\text { Subsec. NB }\end{array}$ & 1992 \\
\hline 2079 & ASME & $\begin{array}{l}\text { ASME Sec. III, } \\
\text { Subsec. NB }\end{array}$ & $\mathrm{N} / \mathrm{S}$ & Class I Components & reg1.87.ro1 & 1975 & $\mathbf{R}$ & & $\begin{array}{l}\text { ASME Sec. III, } \\
\text { Subsec. NB }\end{array}$ & 1992 \\
\hline 2080 & ASME & $\begin{array}{l}\text { ASME Sec. III, } \\
\text { Subsec. NB } \\
\end{array}$ & 1975 & Class I Components & reg3.27.ro1 & $197 i$ & $\bar{P}$ & & $\begin{array}{l}\text { ASME Sec. III, } \\
\text { Subsec. NB }\end{array}$ & 1992 \\
\hline 2081 & ASME & $\begin{array}{l}\text { ASME Sec. III, } \\
\text { Subsec. NB }\end{array}$ & $N / S$ & Class I Components & srp3.2.2 & 1981 & $\bar{P}$ & & $\begin{array}{l}\text { ASME Sec. III, } \\
\text { Subsec. NB }\end{array}$ & 1992 \\
\hline 2082 & ASME & $\begin{array}{l}\text { ASME Sec. III, } \\
\text { Subsec. NB }\end{array}$ & $\mathrm{N} / \mathrm{S}$ & Class I Components & $\operatorname{sip5.2.1.2}$ & 1981 & R & & $\begin{array}{l}\text { ASME Sec. III, } \\
\text { Subsec. NB }\end{array}$ & 1992 \\
\hline 2083 & ASME & $\begin{array}{l}\text { ASME Sec. III, } \\
\text { Subsec. NB }\end{array}$ & $N / S$ & Class I Components & srp5.3.1 & 1981 & $P$ & & $\begin{array}{l}\text { ASME Sec. III, } \\
\text { Subsec. NB }\end{array}$ & 1992 \\
\hline 2084 & ASME & $\begin{array}{l}\text { ASME Sec. III, } \\
\text { NB-1000 }\end{array}$ & $\mathrm{N} / \mathrm{S}$ & Introduction & sip15.1.1 & 1981 & $\mathbf{R}$ & & \begin{tabular}{|l|} 
ASME Sec. III, NB- \\
1000
\end{tabular} & 1992 \\
\hline 2085 & ASME & $\begin{array}{l}\text { ASME Sec. III, } \\
\text { NB-2121 }\end{array}$ & $N / S$ & Permitted Material Specifications & inm9900 & 1991 & $\mathbf{R}$ & & \begin{tabular}{|l|} 
ASME Sec. III, NB- \\
2121
\end{tabular} & 1992 \\
\hline 2086 & ASME & $\begin{array}{l}\text { ASME Sec. III, } \\
\text { NB-2121(b) }\end{array}$ & $\mathrm{N} / \mathrm{S}$ & Permitted Material Specifications & drg1008.r00 & 1991 & $\mathbf{R}$ & & \begin{tabular}{|l|} 
ASME Sec. III, NB- \\
2121 (b)
\end{tabular} & 1992 \\
\hline 2087 & ASME & $\begin{array}{l}\text { ASME Sec. III, } \\
\text { NB-2121(b) }\end{array}$ & $\mathrm{N} / \mathrm{S}$ & Permitted Material Specifications & not93-61 & 1993 & $\mathbf{R}$ & & \begin{tabular}{|l|} 
ASME Sec. III, NB- \\
2121 (b)
\end{tabular} & 1992 \\
\hline 2088 & ASME & $\begin{array}{l}\text { ASME Sec. III, } \\
\text { NB-2300 }\end{array}$ & $\mathrm{N} / \mathrm{S}$ & $\begin{array}{l}\text { Fracture Toughness Requirements } \\
\text { for Material }\end{array}$ & $\operatorname{srp5.4.2.1}$ & 1981 & $P$ & & $\begin{array}{l}\text { ASME Sec. III, NB- } \\
2300\end{array}$ & 1992 \\
\hline 2089 & ASME & $\begin{array}{l}\text { ASME Sec. III, } \\
\text { NB-2311 }\end{array}$ & 1979 & $\begin{array}{l}\text { Material for Which Impact Testing is } \\
\text { Required }\end{array}$ & not89-22 & 1989 & $\mathbf{R}$ & & \begin{tabular}{|l|} 
ASME Sec. III, NB- \\
2311
\end{tabular} & 1992 \\
\hline 2090 & ASME & $\begin{array}{l}\text { ASME Sec. III, } \\
\text { NB-2331 } \\
\end{array}$ & $\mathrm{N} / \mathrm{S}$ & Material for Vessels & 10 cfr50.61 & 1993 & $\mathbf{R}$ & Cited for definition & \begin{tabular}{|l|} 
ASME Sec. III, NB- \\
2331
\end{tabular} & 1992 \\
\hline 2091 & ASME & $\begin{array}{l}\text { ASME Sec. III, } \\
\text { NB-2331 }\end{array}$ & $\mathrm{N} / \mathrm{S}$ & Material for Vessels & reg1.99.ro0 & 1988 & $\mathbf{R}$ & Used for definition & $\begin{array}{l}\text { ASME Sec. III, NB- } \\
2331\end{array}$ & 1992 \\
\hline 2092 & ASME & $\begin{array}{l}\text { ASME Sec. III, } \\
\text { NB-2331 (a) }\end{array}$ & $N / S$ & Material for Vessels & reg1.14.r01 & 1975 & $\mathbf{P}$ & & $\begin{array}{l}\text { ASME Sec. III, NB- } \\
2331 \text { (a) }\end{array}$ & 1992 \\
\hline
\end{tabular}


Codes and Standards and Other Guidance Cited in Regulatory Documents

Part A - Industry Consensus Codes and Standards (continued)

\begin{tabular}{|c|c|c|c|c|c|c|c|c|c|c|}
\hline Hocord & codo & Stondind & stmondord & Iitio & Bocoluon & Don. & & Complomt: & cumentyorolon & Vorron \\
\hline 2093 & ASME & $\begin{array}{l}\text { ASME Sec. III, } \\
\text { NB-2350 }\end{array}$ & N/S & Retests & reg1.65.r00 & 1973 & $\mathbf{P}$ & & $\begin{array}{l}\text { ASME Sec. III, NB- } \\
2350\end{array}$ & 1992 \\
\hline 2094 & ASME & $\begin{array}{l}\text { ASME Sec. III, } \\
\text { NB-2500 }\end{array}$ & $N / S$ & $\begin{array}{l}\text { Examination and Repair of } \\
\text { Pressure Retaining Material }\end{array}$ & reg1.14.ro1 & 1975 & $\mathbf{P}$ & & $\begin{array}{l}\text { ASME Sec. III, NB- } \\
2500\end{array}$ & 1992 \\
\hline 2095 & ASME & $\begin{array}{l}\text { ASME Sec. III, } \\
\text { NB-2530 }\end{array}$ & $\mathrm{N} / \mathrm{S}$ & Examination and Repair of Plate & srp5.4.1.1 & 1981 & $P$ & & \begin{tabular}{|l|} 
ASME Sec. III, \\
NB-2530
\end{tabular} & 1992 \\
\hline 2096 & ASME & $\begin{array}{l}\text { ASME Sec. III, } \\
\text { NB-2530 }\end{array}$ & $\mathrm{N} / \mathrm{S}$ & Examination and Repair of Plate & sip10.2.3 & 1981 & $\mathbf{P}$ & & $\begin{array}{l}\text { ASME Sec. III, NB- } \\
2530\end{array}$ & 1992 \\
\hline 2097 & ASME & $\begin{array}{l}\text { ASME Sec. III, } \\
\text { NB-2540 }\end{array}$ & $N / S$ & $\begin{array}{l}\text { Examination and Repair } 0 . \\
\text { Forgings and Bars }\end{array}$ & sip10.2.3 & 1981 & $\mathbf{P}$ & & $\begin{array}{l}\text { ASME Sec. III, NB- } \\
2540\end{array}$ & 1992 \\
\hline 2098 & ASME & $\begin{array}{l}\text { ASME Sec. III, } \\
\text { NB-2545 }\end{array}$ & $\mathrm{N} / \mathrm{S}$ & Magnetic Particle Examination & reg1.65.r00 & 1973 & $\mathbf{P}$ & & $\begin{array}{l}\text { ASME Sec. III, NB- } \\
2545\end{array}$ & 1992 \\
\hline 2099 & ASME & $\begin{array}{l}\text { ASME Sec. III, } \\
\text { NB-2546 }\end{array}$ & $\mathrm{N} / \mathrm{S}$ & Liquid Penetrant Examination & reg1.65.ro0 & 1973 & $\mathbf{P}$ & & $\begin{array}{l}\text { ASME Sec. III, NB- } \\
2546\end{array}$ & 1992 \\
\hline 2100 & ASME & $\begin{array}{l}\text { ASME Sec. III, } \\
\text { NB/NC/ND } \\
2550\end{array}$ & $\mathrm{~N} / \mathrm{S}$ & $\begin{array}{l}\text { Examination and Repair of } \\
\text { Seamless and Welded Without } \\
\text { Filler Metal) Tubular Products and } \\
\text { Fittings }\end{array}$ & $\operatorname{srp} 10.3 .6$ & 1981 & $\mathbf{P}$ & & $\begin{array}{l}\text { ASME SeC. III, } \\
\text { NB/NC/ND-2550 }\end{array}$ & 1992 \\
\hline 2101 & ASME & $\begin{array}{l}\text { ASME Sec. III, } \\
\text { NB-2550 }\end{array}$ & $\mathrm{N} / \mathrm{S}$ & $\begin{array}{l}\text { Examination and Repair of } \\
\text { Seamless and Welded (Without } \\
\text { Filler Metal) Tubular Products and } \\
\text { Fittings }\end{array}$ & not84-63 & 1984 & $\mathbf{P}$ & & $\begin{array}{l}\text { ASME SeC. III, NB- } \\
2550\end{array}$ & 1992 \\
\hline 2102 & ASME & $\begin{array}{l}\text { ASME Sec. IH, } \\
\text { NB-2550 }\end{array}$ & $\mathrm{N} / \mathrm{S}$ & $\begin{array}{l}\text { Examination and Repair of } \\
\text { Seamless and Welded Without } \\
\text { Filler Metal) Tubular Products and } \\
\text { Fittings }\end{array}$ & sip5.2.3 & 1981 & $P$ & & $\begin{array}{l}\text { ASME Sec. III, NB- } \\
2550\end{array}$ & 1992 \\
\hline 2103 & ASME & $\begin{array}{l}\text { ASME Sec. III, } \\
\text { NB-2560 }\end{array}$ & $N / S$ & $\begin{array}{l}\text { Examination and Repair of Tubular } \\
\text { Products and Fittings Welded with } \\
\text { Filler Metal }\end{array}$ & srp5.2.3 & 1981 & $\mathbf{P}$ & & $\begin{array}{l}\text { ASME Sec. III, NB- } \\
2560\end{array}$ & 1992 \\
\hline 2104 & ASME & $\begin{array}{l}\text { ASME Sec. III, } \\
\text { NB- } 2570\end{array}$ & $\mathrm{~N} / \mathrm{S}$ & $\begin{array}{l}\text { Examination and Repair of } \\
\text { Statically and Centrifugally Cast } \\
\text { Products }\end{array}$ & srp5.2.3 & 1981 & $\mathbf{P}$ & & $\begin{array}{l}\text { ASME Sec. III, NB- } \\
2570\end{array}$ & 1992 \\
\hline 2105 & ASME & $\begin{array}{l}\text { ASME Sec. III, } \\
\text { NB-2580 }\end{array}$ & $\mathrm{N} / \mathrm{S}$ & $\begin{array}{l}\text { Examination of Bolts, Studs, and } \\
\text { Nuts }\end{array}$ & reg1.65.ro0 & 1973 & $P$ & & $\begin{array}{l}\text { ASME Sec. III, NB- } \\
2580\end{array}$ & 1992 \\
\hline
\end{tabular}


Codes and Standards and Other Guidance Clted in Regulatory Documents

Part A - Industry Consensus Codes and Standards (continued)

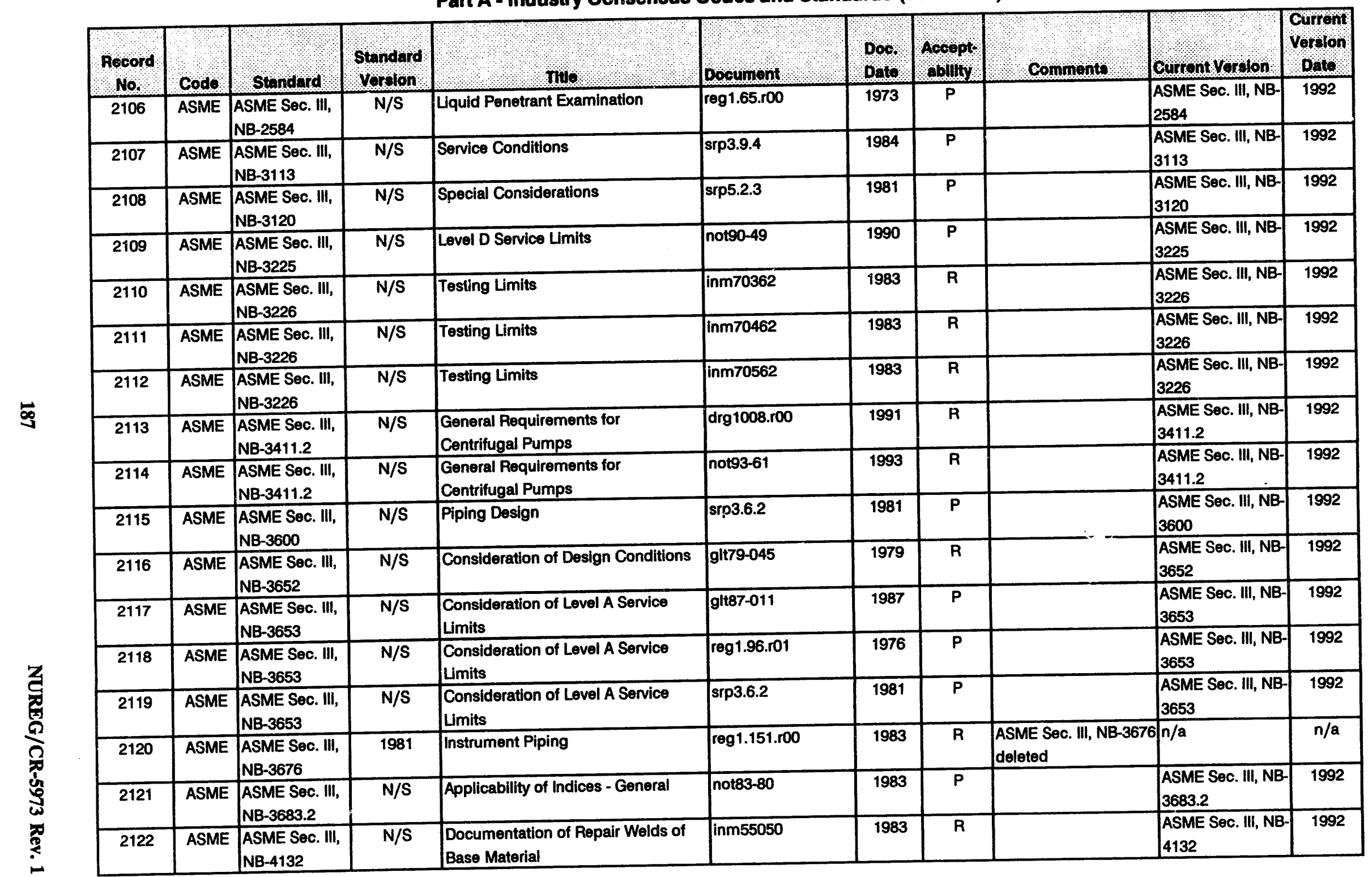


Codes and Standards and Other Guidance Cited in Regulatory Documents

Part A - Industry Consensus Codes and Standards (continued)

\begin{tabular}{|c|c|c|c|c|c|c|c|c|c|c|}
\hline $\begin{array}{l}\text { Rocord } \\
\text { No. }\end{array}$ & codo & Standard & Standard & . & Documont & 00 pats & Aocopt & Comment: & Current Vordion & $\begin{array}{l}\text { curtont } \\
\text { votiont } \\
\text { oflat }\end{array}$ \\
\hline 2123 & ASME & $\begin{array}{l}\text { ASME Sec. III, } \\
\text { NB-4300 }\end{array}$ & $N / S$ & Welding Qualifications & inm50082B & 1976 & $R$ & & $\begin{array}{l}\text { ASME Sec. III, NB- } \\
4300\end{array}$ & 1992 \\
\hline 2124 & ASME & $\begin{array}{l}\text { ASME Sec. III, } \\
\text { NB-4600 }\end{array}$ & $\mathrm{N} / \mathrm{S}$ & Heat Treatment & inm55092B & 1976 & $\bar{R}$ & & $\begin{array}{l}\text { ASME Sec. III, NB- } \\
4600\end{array}$ & 1992 \\
\hline 2125 & ASME & $\begin{array}{l}\text { ASME Sec. III, } \\
\text { NB-4622 }\end{array}$ & $\mathrm{N} / \mathrm{S}$ & $\begin{array}{l}\text { PWHT Time and Temperature } \\
\text { Requirements }\end{array}$ & glt90-005 & 1990 & $\mathbf{R}$ & & $\begin{array}{l}\text { ASME Sec. III, NB- } \\
4622\end{array}$ & 1992 \\
\hline 2126 & ASME & $\begin{array}{l}\text { ASME SeC. III, } \\
\text { NB-4650 }\end{array}$ & $\mathrm{N} / \mathrm{S}$ & $\begin{array}{l}\text { Heat Treatment After Bending or } \\
\text { Forming for Pipes, Pumps, and } \\
\text { Valves }\end{array}$ & inm55092B & 1976 & $\mathbf{R}$ & & $\begin{array}{l}\text { ASME Sec. III, NB- } \\
4650\end{array}$ & 1992 \\
\hline 2127 & ASME & $\begin{array}{l}\text { ASME Sec. III, } \\
\text { NB-5000 }\end{array}$ & $\mathrm{N} / \mathrm{S}$ & Examination & bul82-01 & 1981 & $\mathbf{R}$ & & $\begin{array}{l}\text { ASME Sec. III, NB- } \\
5000\end{array}$ & 1992 \\
\hline 2128 & ASME & $\begin{array}{l}\text { ASME Sec. III, } \\
\text { NB-5000 }\end{array}$ & $\mathrm{N} / \mathrm{S}$ & Examination & bul82-01.sr1 & 1982 & $\mathbf{R}$ & & \begin{tabular}{|l|} 
ASME Sec. III, NB- \\
5000
\end{tabular} & 1992 \\
\hline 2129 & ASME & $\begin{array}{l}\text { ASME Sec. III, } \\
\text { NB-5000 }\end{array}$ & $\mathrm{N} / \mathrm{S}$ & Examination & bul82-01.r01 & 1982 & $\mathbf{R}$ & & $\begin{array}{l}\text { ASME Sec. III, NB- } \\
5000\end{array}$ & 1992 \\
\hline 2130 & ASME & $\begin{array}{l}\text { ASME Sec. III, } \\
\text { NB-5320 } \\
\end{array}$ & 1977 & $\begin{array}{l}\text { Radiographic Accoptance } \\
\text { Standards }\end{array}$ & reg1.150.r01 & 1983 & $\mathbf{P}$ & Implied endorsement & \begin{tabular}{|l|} 
ASME Sec. III, NB- \\
5320
\end{tabular} & 1992 \\
\hline 2131 & ASME & $\begin{array}{l}\text { ASME Sec. III, } \\
\text { NB-7000 }\end{array}$ & $N / S$ & Overpressure Protection & $\operatorname{srp} 15.2 .1$ & 1981 & $\mathbf{R}$ & & $\begin{array}{l}\text { ASME Sec. III, NB- } \\
7000\end{array}$ & 1992 \\
\hline 2132 & ASME & $\begin{array}{l}\text { ASME Sec. III, } \\
\text { NB-7000 } \\
\end{array}$ & $\mathrm{N} / \mathrm{S}$ & Overpressure Protection & srp15.2.6 & 1981 & $\mathbf{R}$ & & \begin{tabular}{|l|} 
ASME Sec. III, NB- \\
7000
\end{tabular} & 1992 \\
\hline 2133 & ASME & $\begin{array}{l}\text { ASME Sec. III, } \\
\text { NB-7000 }\end{array}$ & $\mathrm{N} / \mathrm{S}$ & Overpressure Protection & srp15.2.7 & 1981 & $\mathrm{R}$ & & $\begin{array}{l}\text { ASME Sec. III, NB- } \\
7000\end{array}$ & 1992 \\
\hline 2134 & ASME & $\begin{array}{l}\text { ASME Sec. III, } \\
\text { NB-7000 }\end{array}$ & N/S & Overpressure Protection & srp15.2.8 & 1981 & $\bar{R}$ & & $\begin{array}{l}\text { ASME Sec. III, NB- } \\
7000\end{array}$ & 1992 \\
\hline 2135 & ASME & $\begin{array}{l}\text { ASME Sec. III, } \\
\text { NB-7000 }\end{array}$ & $\mathrm{N} / \mathrm{S}$ & Overpressure Protection & sip15.3.1 & 1981 & $\bar{R}$ & & $\begin{array}{l}\text { ASME Sec. III, NB- } \\
7000\end{array}$ & 1992 \\
\hline 2136 & ASME & $\begin{array}{l}\text { ASME Sec. III, } \\
\text { NB-7000 }\end{array}$ & $\mathrm{N} / \mathrm{S}$ & Overpressure Protection & srp15.3.3 & 1981 & $R$ & & \begin{tabular}{|l|} 
ASME Sec. III, NB- \\
7000
\end{tabular} & 1992 \\
\hline 2137 & ASME & $\begin{array}{l}\text { ASME Sec. III, } \\
\text { NB-7000 }\end{array}$ & $\mathrm{N} / \mathrm{S}$ & Overpressure Protection & srp15.4.4 & 1981 & $\bar{R}$ & & $\begin{array}{l}\text { ASME Sec. III, NB- } \\
7000\end{array}$ & 1992 \\
\hline 2138 & ASME & $\begin{array}{l}\text { ASME Sec. III, } \\
\text { NB-7000 }\end{array}$ & $\mathrm{N} / \mathrm{S}$ & Overpressure Protection & srp15.4.6 & 1981 & $\mathrm{R}$ & & $\begin{array}{l}\text { ASME Sec. III, NB- } \\
7000\end{array}$ & 1992 \\
\hline
\end{tabular}


Codes and Standards and Other Guidance Cited in Regulatory Documents

Part A - Industry Consensus Codes and Standards (continued)

\begin{tabular}{|c|c|c|c|c|c|c|c|c|c|c|}
\hline $\begin{array}{l}\text { Rocord } \\
\text { No. }\end{array}$ & Code & Standard & $\begin{array}{l}\text { Standard } \\
\text { Vorston }\end{array}$ & (, 5 rno & Dooument & $\begin{array}{l}\text { Doo. } \\
\text { Date }\end{array}$ & $\begin{array}{l}\text { Accept. } \\
\text { ability }\end{array}$ & Commonts & Curront Vorolon & $\begin{array}{l}\text { Chront } \\
\text { Vorton } \\
\text { phlo }\end{array}$ \\
\hline 2139 & ASME & $\begin{array}{l}\text { ASME Sec. III, } \\
\text { NB-7000 }\end{array}$ & N/S & Overpressure Protection & sip15.5.1 & 1981 & $\mathbf{R}$ & & $\begin{array}{l}\text { ASME Sec. III, NB- } \\
7000\end{array}$ & 1992 \\
\hline 2140 & ASME & $\begin{array}{l}\text { ASME SeC. III, } \\
\text { NB-7000 }\end{array}$ & $\mathrm{N} / \mathrm{S}$ & Overpressure Protection & $\operatorname{srp15.6.1}$ & 1981 & R & & $\begin{array}{l}\text { ASME Soc. III, NB- } \\
7000\end{array}$ & 1992 \\
\hline 2141 & ASME & $\begin{array}{l}\text { ASME Sec. III, } \\
\text { NB-7000. }\end{array}$ & $\mathrm{N} / \mathrm{S}$ & Overpressure Protection & sts4-geB2.1.2 & 1991 & $\mathbf{P}$ & & $\begin{array}{l}\text { ASME SoC. III, NB- } \\
7000\end{array}$ & 1992 \\
\hline 2142 & ASME & $\begin{array}{l}\text { ASME Sec. III, } \\
\text { NB-7000. }\end{array}$ & $\mathrm{N} / \mathrm{S}$ & Overpressure Protection & sts6-geB2.1.2 & 1991 & $\mathbf{P}$ & & $\begin{array}{l}\text { ASME Sec. III, NB- } \\
7000\end{array}$ & 1992 \\
\hline 2143 & ASME & $\begin{array}{l}\text { ASME Sec. III, } \\
\text { NB-7000. }\end{array}$ & $N / S$ & Overpressure Protection & stsb\&wB2.1.2 & 1991 & $\mathbf{P}$ & & $\begin{array}{l}\text { ASME Soc. III, NB- } \\
7000\end{array}$ & 1992 \\
\hline 2144 & ASME & $\begin{array}{l}\text { ASME Sec. III, } \\
\text { NB-7000. }\end{array}$ & $\mathrm{N} / \mathrm{S}$ & Overpressure Protection & stsceB2.1.2(a) & 1991 & $\mathbf{P}$ & & $\begin{array}{l}\text { ASME Sec. III, NB- } \\
7000\end{array}$ & 1992 \\
\hline 2145 & ASME & $\begin{array}{l}\text { ASME Sec. III, } \\
\text { NB-7000. }\end{array}$ & $\mathrm{N} / \mathrm{S}$ & Overpressure Protection & stsceB2.1.2(d) & 1991 & $\mathbf{P}$ & & $\begin{array}{l}\text { ASME Sec. III, NB- } \\
7000\end{array}$ & 1992 \\
\hline 2146 & ASME & $\begin{array}{l}\text { ASME Sec. III, } \\
\text { NB-7000. } \\
\end{array}$ & $N / S$ & Overpressure Protection & stswstB2.1.2 & 1991 & $\mathbf{P}$ & & $\begin{array}{l}\text { ASME Sec. III, NB- } \\
7000\end{array}$ & 1992 \\
\hline 2147 & ASME & $\begin{array}{l}\text { ASME Sec. III, } \\
\text { NB-7311(b) }\end{array}$ & 1989 & $\begin{array}{l}\text { Reliefing Capacity of Pressure } \\
\text { Relief Devices }\end{array}$ & drg1023.r00 & 1993 & $\mathbf{R}$ & & $\begin{array}{l}\text { ASME Sec. III, NB- } \\
7311 \text { (b) }\end{array}$ & 1992 \\
\hline 2148 & ASME & $\begin{array}{l}\text { ASME Sec. III, } \\
\text { NB-7611 }\end{array}$ & $N / S$ & Burst Pressure Tolerance & srp5.2.2 & 1988 & $\mathbf{R}$ & & $\begin{array}{l}\text { ASME Sec. III, NB- } \\
7611\end{array}$ & 1992 \\
\hline 2149 & ASME & $\begin{array}{l}\text { ASME Sec. III, } \\
\text { Subsec. NC }\end{array}$ & $\mathrm{N} / \mathrm{S}$ & Class 2 Components & inm48051 & 1986 & $\mathbf{R}$ & & $\begin{array}{l}\text { ASME Sec. III, } \\
\text { Subsec. NC }\end{array}$ & 1992 \\
\hline 2150 & ASME & $\begin{array}{l}\text { ASME Sec. III, } \\
\text { Subsec. NC }\end{array}$ & $\mathrm{N} / \mathrm{S}$ & Class 2 Components & inm48053 & 1986 & $\mathbf{R}$ & & $\begin{array}{l}\text { ASME Sec. III, } \\
\text { Subsec. NC }\end{array}$ & 1992 \\
\hline 2151 & ASME & $\begin{array}{l}\text { ASME Sec. III, } \\
\text { Subsec. NC }\end{array}$ & $N / S$ & Class 2 Components & inm55050 & 1983 & $\mathrm{R}$ & & $\begin{array}{l}\text { ASME Sec. III, } \\
\text { Subsec. NC }\end{array}$ & 1992 \\
\hline 2152 & ASME & $\begin{array}{l}\text { ASME Sec. III, } \\
\text { Subsec. NC }\end{array}$ & $\mathrm{N} / \mathrm{S}$ & Class 2 Components & not87-36 & 1987 & $\mathbf{R}$ & & $\begin{array}{l}\text { ASME Sec. III, } \\
\text { Subsec. NC }\end{array}$ & 1992 \\
\hline 2153 & ASME & $\begin{array}{l}\text { ASME Sec. III, } \\
\text { Subsec. NC }\end{array}$ & $N / S$ & Class 2 Components & reg1.70.r03 & 1978 & $\bar{P}$ & & $\begin{array}{l}\text { ASME Sec. III, } \\
\text { Subsec. NC }\end{array}$ & 1992 \\
\hline 2154 & ASME & $\begin{array}{l}\text { ASME Sec. III, } \\
\text { Subsec. NC }\end{array}$ & $N / S$ & Class 2 Components & $\operatorname{sip3.2.2}$ & 1981 & $\bar{P}$ & & $\begin{array}{l}\text { ASME Sec. III, } \\
\text { Subsec. NC } \\
\end{array}$ & 1992 \\
\hline 2155 & ASME & $\begin{array}{l}\text { ASME Sec. III, } \\
\text { Subsec. NC }\end{array}$ & $\mathrm{N} / \mathrm{S}$ & Class 2 Components & $\operatorname{sip5.2.1.2}$ & 1981 & $\bar{R}$ & & $\begin{array}{l}\text { ASME Sec. III, } \\
\text { Subsec. NC }\end{array}$ & 1992 \\
\hline
\end{tabular}


Codes and Standards and Other Guidance Cited in Regulatory Documents

Part A - Industry Consensus Codes and Standards (continued)

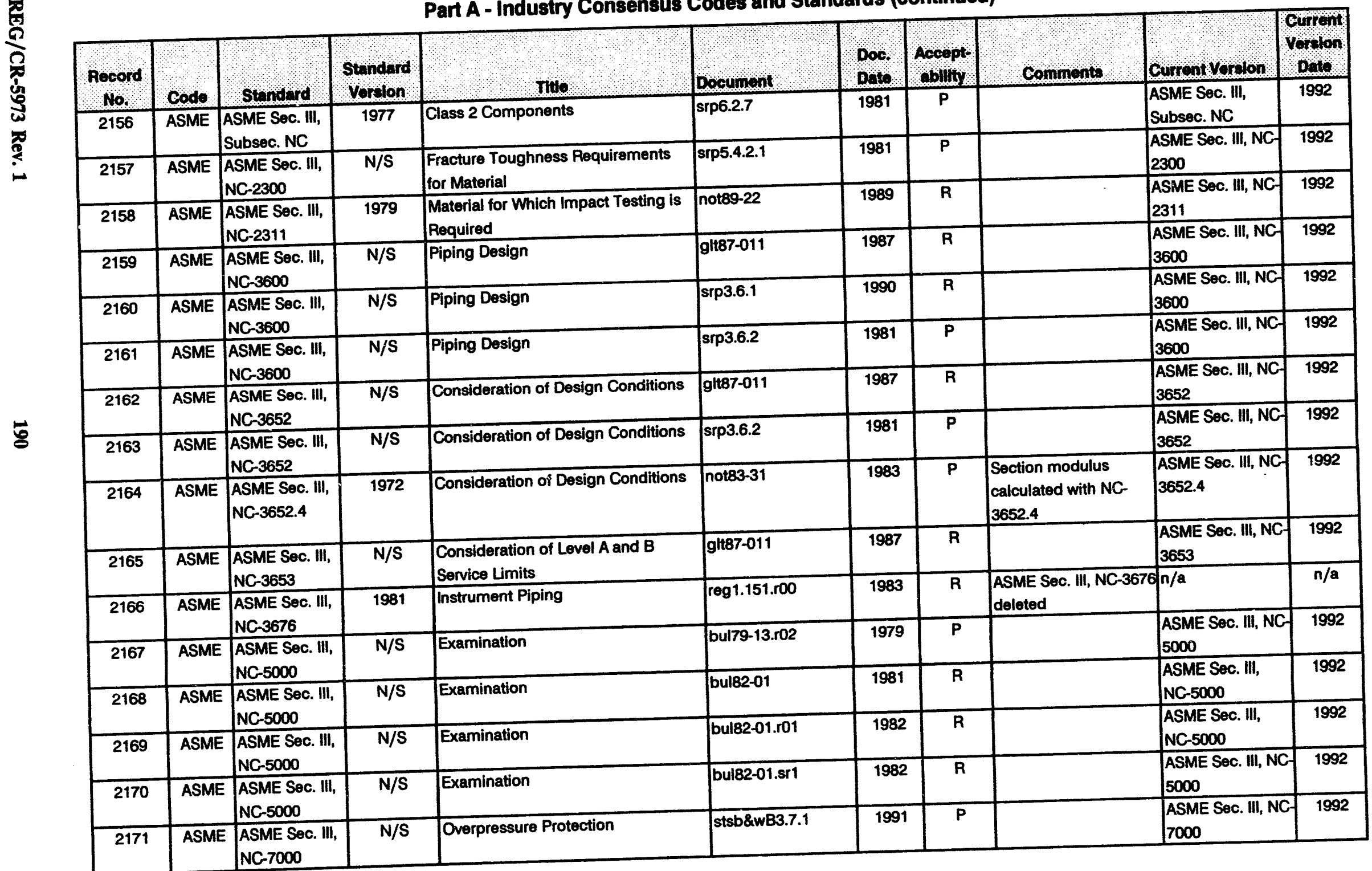


Codes and Standards and Other Guidance Cited in Regulatory Documents

Part A - Industry Consensus Codes and Standards (continued)

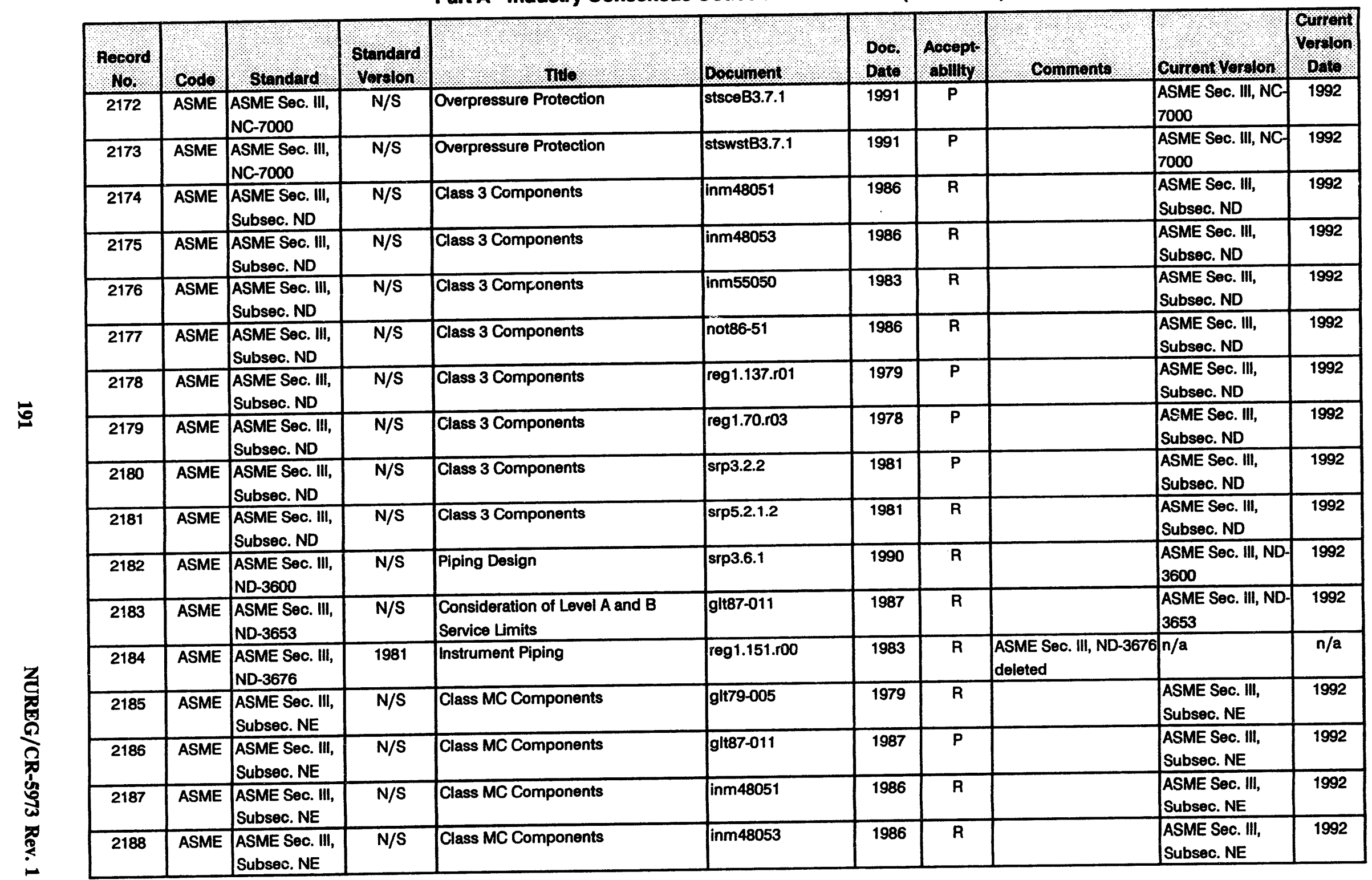


Codes and Standards and Other Guidance Cited in Regulatory Documents

Part A - Industry Consensus Codes and Standards (continued)

\begin{tabular}{|c|c|c|c|c|c|c|c|c|c|c|}
\hline & & & & 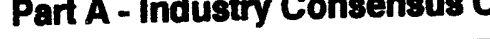 & - & & & & & \\
\hline \multirow{2}{*}{$\begin{array}{l}\text { Rocord } \\
\text { No. }\end{array}$} & \multirow[b]{2}{*}{ codo } & \multirow[b]{2}{*}{ Standard } & \multirow{2}{*}{$\begin{array}{l}\text { Standard } \\
\text { vordon }\end{array}$} & & & poo, & $\begin{array}{l}\text { Aocept } \\
\text { obllity }\end{array}$ & Comments & Current Voralon & $\begin{array}{l}\text { omoll } \\
\text { verton } \\
\text { pls }\end{array}$ \\
\hline & & & & Class MC Components & $\frac{\text { Docelument }}{\text { inm63050 }}$ & 1983 & $\mathbf{R}$ & & ASME Sec. III, & 1992 \\
\hline & ASME & $\begin{array}{l}\text { ASME Sec. ill, } \\
\text { Subsec. NE }\end{array}$ & & & & & & & Subsec. NE & \\
\hline 2190 & ASME & $\begin{array}{l}\text { ASME Sec. III, } \\
\text { Subsec. NE }\end{array}$ & $\mathrm{N} / \mathrm{S}$ & Class MC Components & reg1.57.r00 & 1973 & $\mathbf{P}$ & & $\begin{array}{l}\text { ASME Sec. III, } \\
\text { Subsec. NE }\end{array}$ & 1992 \\
\hline 2191 & ASME & $\begin{array}{l}\text { ASME Sec. III, } \\
\text { Subsec. NE }\end{array}$ & $\mathrm{N} / \mathrm{S}$ & Class MC Components & reg 1.70.r03 & 1978 & $P$ & & $\begin{array}{l}\text { ASME Sec. III, } \\
\text { Subsec. NE }\end{array}$ & 1992 \\
\hline 2192 & ASME & $\begin{array}{l}\text { ASME Sec. III, } \\
\text { Subsec. NE }\end{array}$ & 1975 & Class MC Components & reg3.27.rot & 1977 & $\mathbf{P}$ & & $\begin{array}{l}\text { ASME Sec. III, } \\
\text { Subsec. NE }\end{array}$ & 1992 \\
\hline 2193 & ASME & $\begin{array}{l}\text { ASME Sec. III, } \\
\text { Subsec. NE }\end{array}$ & $\mathrm{N} / \mathrm{S}$ & Class MC Components & $\operatorname{srp3.6.2}$ & 1981 & $P$ & & $\begin{array}{l}\text { ASME Sec. IfI, } \\
\text { Subsec. NE }\end{array}$ & 1992 \\
\hline 2194 & ASME & $\begin{array}{l}\text { ASME Sec. III, } \\
\text { Subsec. NE }\end{array}$ & $\mathrm{N} / \mathrm{S}$ & Class MC Components & srp3.8.2 & 1981 & $\mathbf{P}$ & & $\begin{array}{l}\text { ASME Sec. III, } \\
\text { Subsec. NE }\end{array}$ & 1992 \\
\hline 2195 & ASME & $\begin{array}{l}\text { ASME Sec. III, } \\
\text { Subsec. NE } \\
\end{array}$ & $\mathrm{N} / \mathrm{S}$ & Class MC Components & $\operatorname{srp3.8.3}$ & 1981 & $\mathbf{P}$ & & $\begin{array}{l}\text { ASME Sec. III, } \\
\text { Subsec. NE }\end{array}$ & 1992 \\
\hline 2196 & ASME & $\begin{array}{l}\text { ASME Sec. III, } \\
\text { Subsec. NE }\end{array}$ & $\mathrm{N} / \mathrm{S}$ & Class MC Components & srp5.2.1.2 & 1981 & $\mathbf{R}$ & & $\begin{array}{l}\text { ASME Sec. III, } \\
\text { Subsec. NE }\end{array}$ & 1992 \\
\hline 2197 & ASME & $\begin{array}{l}\text { ASME Sec. III, } \\
\text { Subsec. NE }\end{array}$ & $N / S$ & Class MC Components & srp6.2.1.1.C & 1984 & $\mathbf{P}$ & & $\begin{array}{l}\text { ASME Sec. III, } \\
\text { Subsec. NE }\end{array}$ & 1992 \\
\hline 2198 & ASME & $\begin{array}{l}\text { ASME Sec. III, } \\
\text { Subsec. NE }\end{array}$ & N/S & Class MC Components & sip6.2.7 & 1981 & $\mathbf{P}$ & & $\begin{array}{l}\text { ASME Sec. III, } \\
\text { Subsec. NE } \\
\end{array}$ & 1992 \\
\hline 2199 & ASME & $\begin{array}{l}\text { ASME Sec. III, } \\
\text { NE-1120 }\end{array}$ & $\mathrm{N} / \mathrm{S}$ & $\begin{array}{l}\text { Rules for Class MC Containment } \\
\text { Vessels }\end{array}$ & glt87-011 & 1987 & $\mathbf{P}$ & $\begin{array}{l}\text { With additional listed } \\
\text { design requirements }\end{array}$ & \begin{tabular}{|l} 
ASME Sec. III, NE- \\
1120
\end{tabular} & 1992 \\
\hline 2200 & ASME & $\begin{array}{l}\text { ASME Soc. III, } \\
\text { NE-1120 }\end{array}$ & $\mathrm{N} / \mathrm{S}$ & $\begin{array}{l}\text { Rules for Class MC Containment } \\
\text { Vessels }\end{array}$ & reg1.98.r01 & 1976 & $\mathbf{P}$ & & \begin{tabular}{|l|} 
ASME Soc. III, NE- \\
1120
\end{tabular} & 1992 \\
\hline 2201 & ASME & $\begin{array}{l}\text { ASME Søc. III, } \\
\text { NE-1120 }\end{array}$ & N/S & $\begin{array}{l}\text { Rules for Class MC Containment } \\
\text { Vessels }\end{array}$ & srp3.6.2 & 1981 & $\mathbf{P}$ & & $\begin{array}{l}\text { ASME Sec. III, NE- } \\
1120\end{array}$ & 1992 \\
\hline 2202 & ASME & $\begin{array}{l}\text { ASME Soc. III, } \\
\text { NE-3000 }\end{array}$ & $\mathrm{N} / \mathrm{S}$ & Design & srp3.8.3 & 1981 & $\mathbf{P}$ & & $\begin{array}{l}\text { ASME SoC. III, NE- } \\
3000\end{array}$ & 1992 \\
\hline 2203 & ASME & $\begin{array}{l}\text { ASME Sec. III, } \\
\text { NE-3220 }\end{array}$ & 1980 & $\begin{array}{l}\text { Stress Intensity and Buckling Stress } \\
\text { Values for Other Than Bolts }\end{array}$ & $10 \mathrm{cfr} 50.34$ & 1993 & A & $\begin{array}{l}\text { Except evaluation of } \\
\text { instability not required }\end{array}$ & \begin{tabular}{|l|} 
ASME Sec. III, NE- \\
3220
\end{tabular} & 1992 \\
\hline 2204 & ASME & $\begin{array}{l}\text { ASME Sec. Iil, } \\
\text { NE-3220 }\end{array}$ & 1980 & $\begin{array}{l}\text { Stress Intensity and Buckling Stress } \\
\text { Values for Other Than Bolts }\end{array}$ & $10 \mathrm{cfr} 50.44$ & 1993 & A & & $\begin{array}{l}\text { ASME Sec. III, NE- } \\
3220\end{array}$ & 1992 \\
\hline 2205 & ASME & \begin{tabular}{|l} 
ASME Søc. III, \\
NE-3220 \\
\end{tabular} & N/S & $\begin{array}{l}\text { Stress Intensity and Buckling Stress } \\
\text { Values for Other Than Bolts }\end{array}$ & glte7-011 & 1987 & $\mathbf{R}$ & & $\begin{array}{l}\text { ASME Søc. III, NE- } \\
3220\end{array}$ & 1992 \\
\hline
\end{tabular}


Codes and Standards and Other Guidance Cited in Regulatory Documents

Part A - Industry Consensus Codes and Standards (continued)

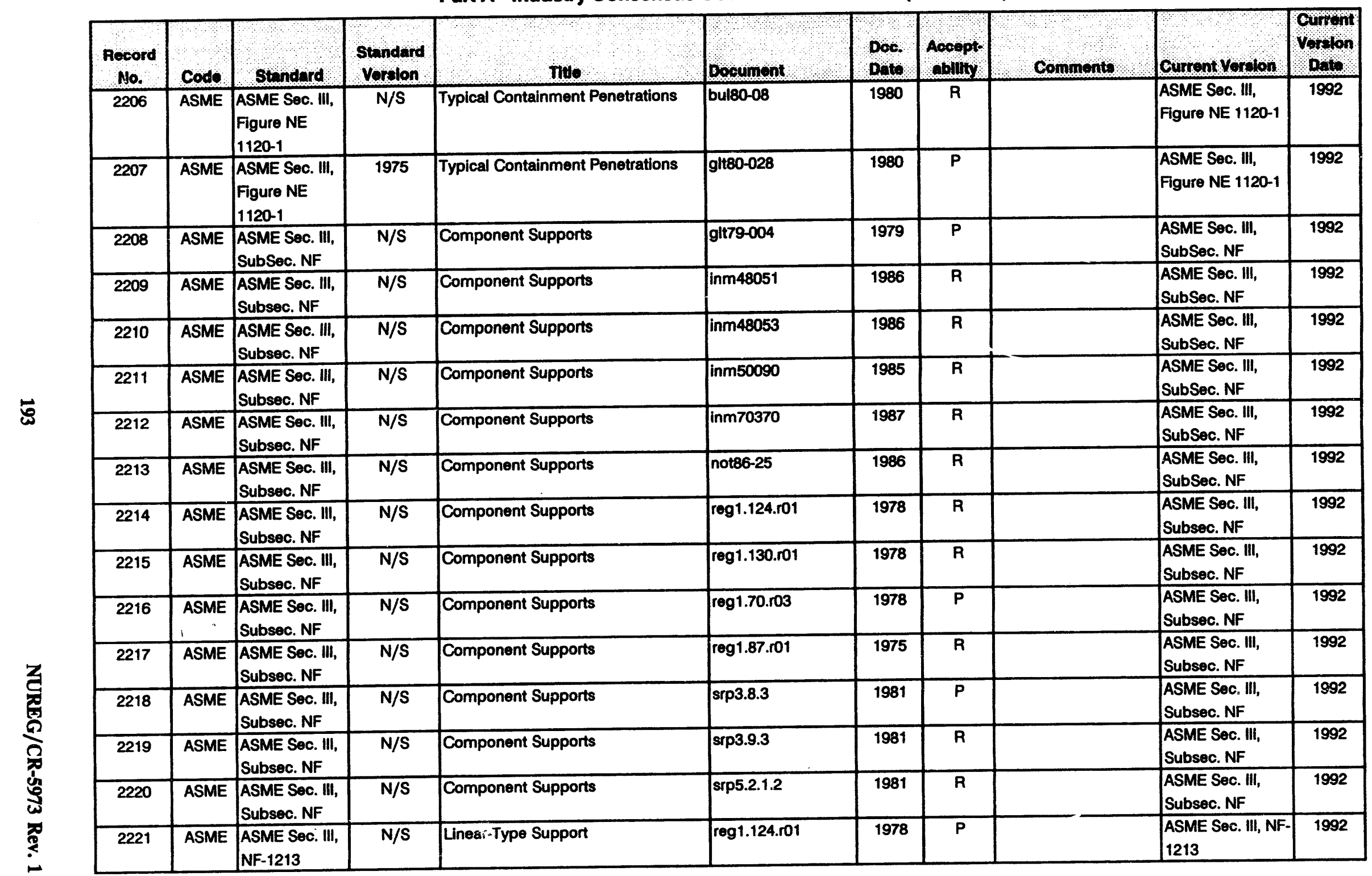


Codes and Standards and Other Guidance Cited in Regulatory Documents

Part A - Industry Consensus Codes and Standards (continued)

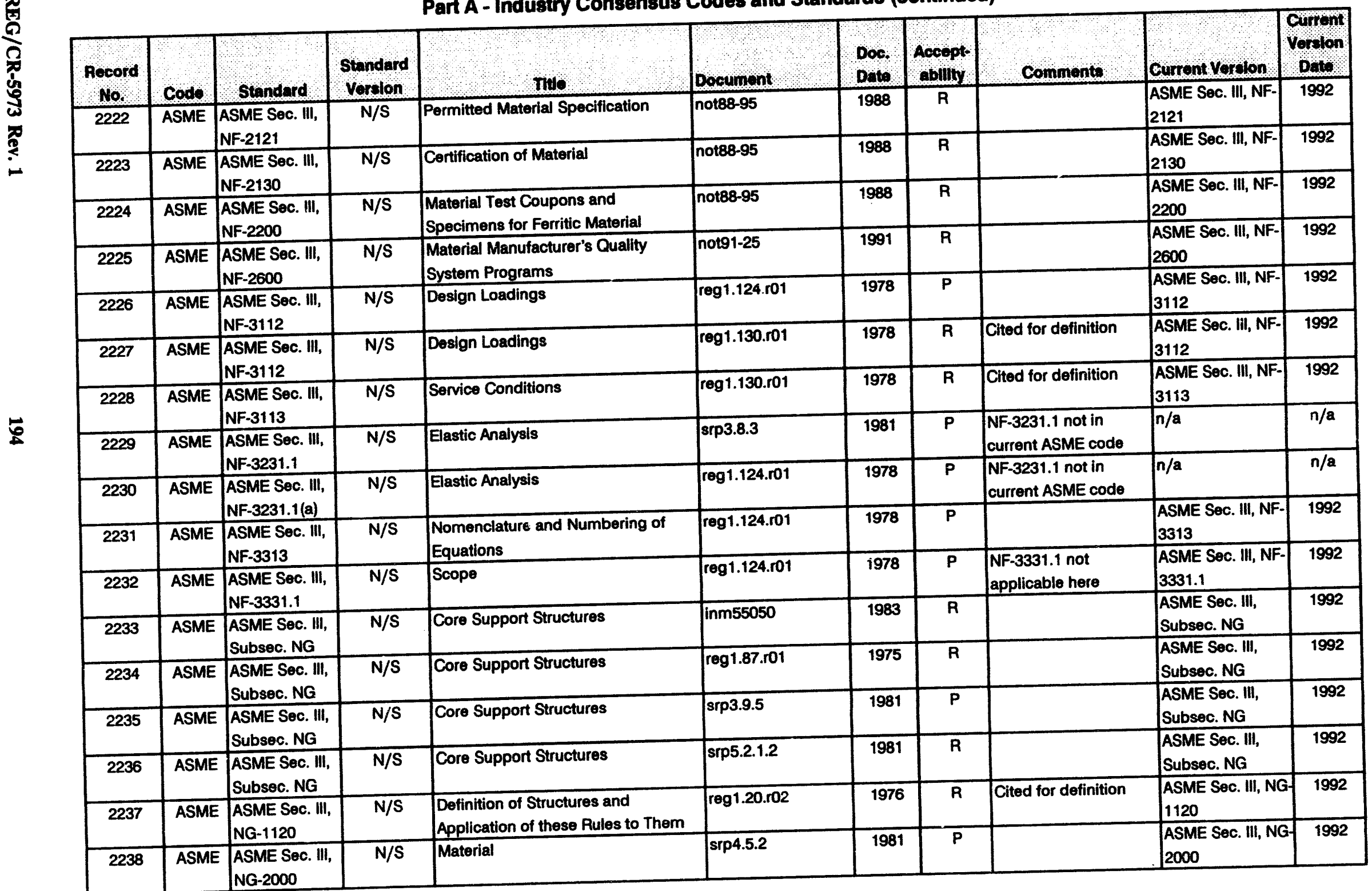


Codes and Standards and Other Guidance Cited in Regulatory Documents

Part A - Industry Consensus Codes and Standards (continued)

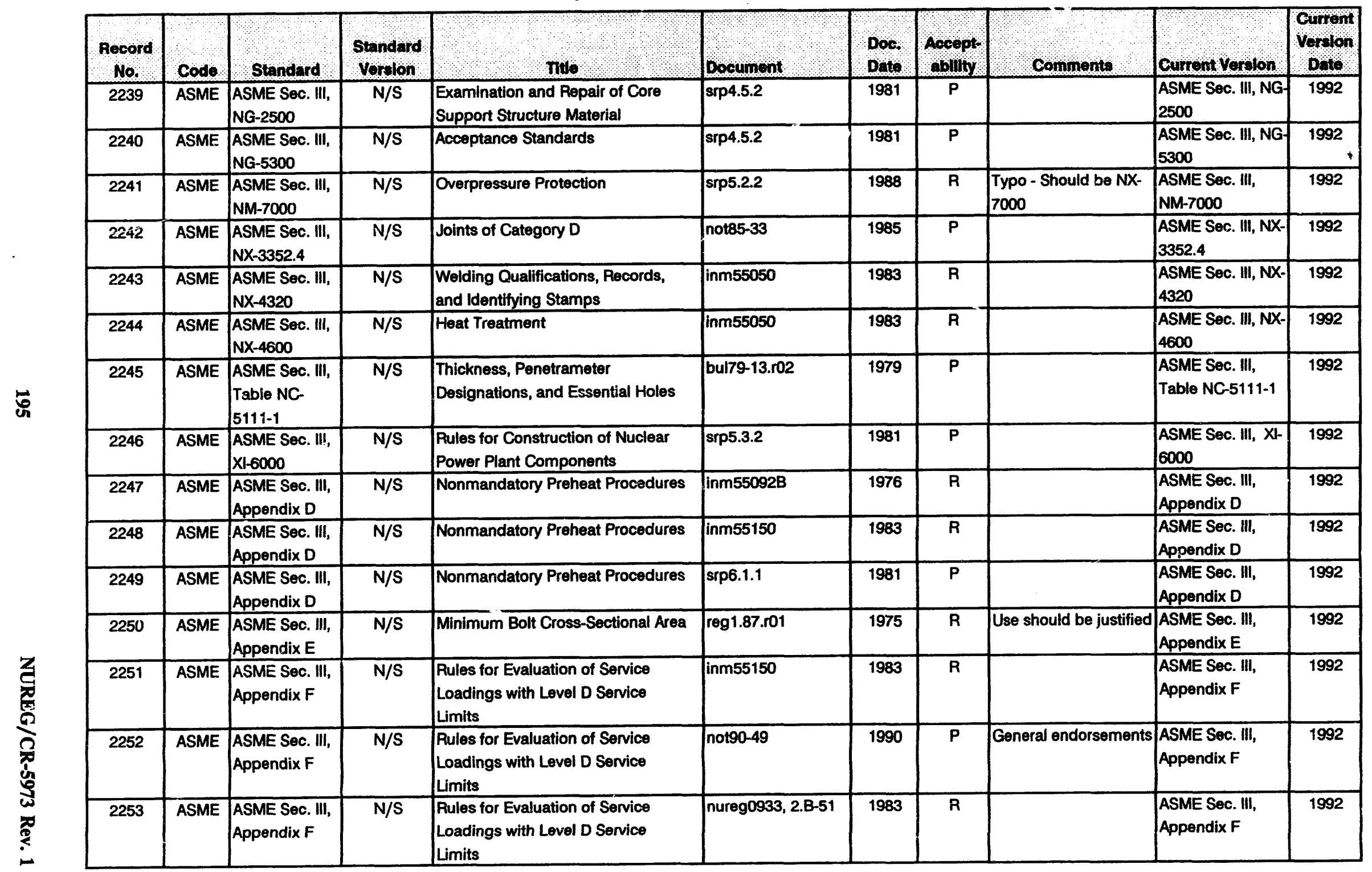




\begin{tabular}{|c|c|c|c|c|c|c|c|c|c|c|}
\hline & & & Cod & $\begin{array}{l}8 \text { and Standards and Othe } \\
\text { Part A - Industry Consens }\end{array}$ & 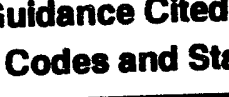 & rds (c & ontinue & & & \\
\hline $\begin{array}{l}\text { Pocord } \\
\text { No. }\end{array}$ & & Standud & $\begin{array}{l}\text { Standard } \\
\text { Vordion }\end{array}$ & \% nulo & Document' & Dor: & Accept: & Commonts: & cuncont Yorolon & Gorron \\
\hline 2254 & ASME & $\begin{array}{l}\text { ASME Sec. III, } \\
\text { Appendix F }\end{array}$ & $\mathrm{N} / \mathrm{S}$ & $\begin{array}{l}\text { Rules for Evaluatlon of Service } \\
\text { Loadings with Level D Service } \\
\text { Limits }\end{array}$ & reg1.124.ro1 & 1978 & $P$ & & $\begin{array}{l}\text { ASME Sec. III, } \\
\text { Appendix F }\end{array}$ & 1992 \\
\hline 2255 & ASME & $\begin{array}{l}\text { ASME Sec. III, } \\
\text { Appendix F }\end{array}$ & $\mathrm{N} / \mathrm{S}$ & $\begin{array}{l}\text { Rules for Evaluation of Service } \\
\text { Loadings with Level D Service } \\
\text { Limite }\end{array}$ & reg1.87.ro1 & 1975 & $\bar{R}$ & Use shoc:d be justified & $\begin{array}{l}\text { ASME Sec. III, } \\
\text { Appendix F }\end{array}$ & 1992 \\
\hline 2256 & ASME & $\begin{array}{l}\text { ASME Sec. III, } \\
\text { Appendix G }\end{array}$ & N/S & \begin{tabular}{|l} 
Protection Against Non-Ductile \\
Failure \\
\end{tabular} & 10 cfr50 App. G & 1993 & $\mathbf{P}$ & $\begin{array}{l}\text { With supplemental } \\
\text { requirements }\end{array}$ & $\begin{array}{l}\text { ASME Gec. III, } \\
\text { Appendix G }\end{array}$ & 1992 \\
\hline 2257 & ASME & $\begin{array}{l}\text { ASME Sec. III, } \\
\text { Appendix G } \\
\end{array}$ & 1389 & $\begin{array}{l}\text { Protection Against Non-Ductile } \\
\text { Failure } \\
\end{array}$ & drg1023.r00 & 1993 & $\bar{A}$ & & $\begin{array}{l}\text { ASME Sec. III, } \\
\text { Appendix G }\end{array}$ & 1992 \\
\hline 2258 & ASME & $\begin{array}{l}\text { ASME Sec. III, } \\
\text { Appendix G }\end{array}$ & $\mathrm{N} / \mathrm{S}$ & $\begin{array}{l}\text { Protection Against Non-Ductile } \\
\text { Failure }\end{array}$ & glt89-021 & 1989 & $\overline{\mathbf{P}}$ & & $\begin{array}{l}\text { ASME Sec. III, } \\
\text { Appendix G }\end{array}$ & 1992 \\
\hline 2259 & ASME & $\begin{array}{l}\text { ASME Sec. III, } \\
\text { Appendix G }\end{array}$ & $\mathrm{N} / \mathrm{S}$ & $\begin{array}{l}\text { Protection Against Non-Ductile } \\
\text { Failure }\end{array}$ & not82-17 & 1982 & $\overline{\mathbf{R}}$ & & $\begin{array}{l}\text { ASME Soc. III, } \\
\text { Appendix G }\end{array}$ & 1992 \\
\hline 2260 & ASME & $\begin{array}{l}\text { ASME Sec. III, } \\
\text { Appendix G }\end{array}$ & $\mathrm{N} / \mathrm{S}$ & $\begin{array}{l}\text { Protection Against Non-Ductile } \\
\text { Fallure }\end{array}$ & reg1.65.ro0 & 1973 & $\mathbf{P}$ & & $\begin{array}{l}\text { ASME Soc. III, } \\
\text { Appendix G }\end{array}$ & 1992 \\
\hline 2261 & ASME & $\begin{array}{l}\text { ASME Sec. III, } \\
\text { Appendix G }\end{array}$ & $\mathrm{N} / \mathrm{S}$ & $\begin{array}{l}\text { Protection Against Non-Ductile } \\
\text { Fallure } \\
\end{array}$ & $\log 1.70 .103$ & 1978 & $P$ & & $\begin{array}{l}\text { ASME Soc. III, } \\
\text { Appendix G }\end{array}$ & 1992 \\
\hline 2262 & ASME & $\begin{array}{l}\text { ASME Sec. III, } \\
\text { Appendix G }\end{array}$ & $\mathrm{N} / \mathrm{S}$ & $\begin{array}{l}\text { Protection Against Non-Ductile } \\
\text { Fallure }\end{array}$ & srp5.2.3 & 1981 & $\bar{P}$ & & $\begin{array}{l}\text { ASME Soc. III, } \\
\text { Appendix G }\end{array}$ & 1992 \\
\hline 2263 & ASME & $\begin{array}{l}\text { ASME Sec. III, } \\
\text { Appendix G } \\
\end{array}$ & $N / S$ & $\begin{array}{l}\text { Protection Against Non-Ductile } \\
\text { Failure }\end{array}$ & srp5.3.2 & 1981 & $\bar{P}$ & & $\begin{array}{l}\text { ASME Sec. III, } \\
\text { Appendix G }\end{array}$ & 1992 \\
\hline 2264 & ASME & $\begin{array}{l}\text { ASME Sec. III, } \\
\text { Appendix G }\end{array}$ & $\mathrm{N} / \mathrm{S}$ & $\begin{array}{l}\text { Protecticn Against Non-Ductile } \\
\text { Fallure } \\
\end{array}$ & srp5.3.3 & 1981 & $\bar{P}$ & & $\begin{array}{l}\text { ASME Sec. III, } \\
\text { Appendix G }\end{array}$ & 1992 \\
\hline 2265 & ASME & $\begin{array}{l}\text { ASME Sec. III, } \\
\text { Appendix G }\end{array}$ & N/S & $\begin{array}{l}\text { Protectlon Against Non-Ductile } \\
\text { Fallure }\end{array}$ & sip5.4.1.1 & 1981 & $\bar{R}$ & & $\begin{array}{l}\text { ASME Sec. III, } \\
\text { Appendix G }\end{array}$ & 1992 \\
\hline 2266 & ASME & $\begin{array}{l}\text { ASME Sec. III, } \\
\text { Appendlix G } \\
\end{array}$ & $\mathrm{N} / \mathrm{S}$ & $\begin{array}{l}\text { Protection Against Non-Ductile } \\
\text { Failure }\end{array}$ & st34-geB3.4.10 & 1991 & $\bar{A}$ & & $\begin{array}{l}\text { ASME Soc. III, } \\
\text { Appendix G }\end{array}$ & 1992 \\
\hline 2267 & ASME & $\begin{array}{l}\text { ASME Sec. III, } \\
\text { Appondix G }\end{array}$ & $\mathrm{N} / \mathrm{S}$ & $\begin{array}{l}\text { Protection Against Non-Ductile } \\
\text { Failure } \\
\end{array}$ & sts6-geB3.4.11 & 1991 & A & & $\begin{array}{l}\text { ASME Sec. III, } \\
\text { Appendix G }\end{array}$ & 1992 \\
\hline 2268 & ASME & $\begin{array}{l}\text { ASME Sec. III, } \\
\text { Appendix G }\end{array}$ & $\mathrm{N} / \mathrm{S}$ & $\begin{array}{l}\text { Protection Against Non-Ductlle } \\
\text { Fallure }\end{array}$ & stsb\&wB3.4.3 & 1991 & $\bar{A}$ & & $\begin{array}{l}\text { ASME Sec. III, } \\
\text { Appendix G }\end{array}$ & 1992 \\
\hline 2269 & ASME & $\begin{array}{l}\text { ASPME Sec. III, } \\
\text { Appendix G }\end{array}$ & $\mathrm{N} / \mathrm{S}$ & $\begin{array}{l}\text { Fallure } \\
\text { Protection Against Non-Ductile } \\
\text { Failure } \\
\end{array}$ & stsceB3.4.3 & 1931 & $\bar{A}$ & & $\begin{array}{l}\text { ASME Sec. III, } \\
\text { Appendix G }\end{array}$ & 1992 \\
\hline
\end{tabular}


Codes and Standards and Other Guidance Cited in Regulatory Documents

Part A - Industry Consensus Codes and Standards (continued)

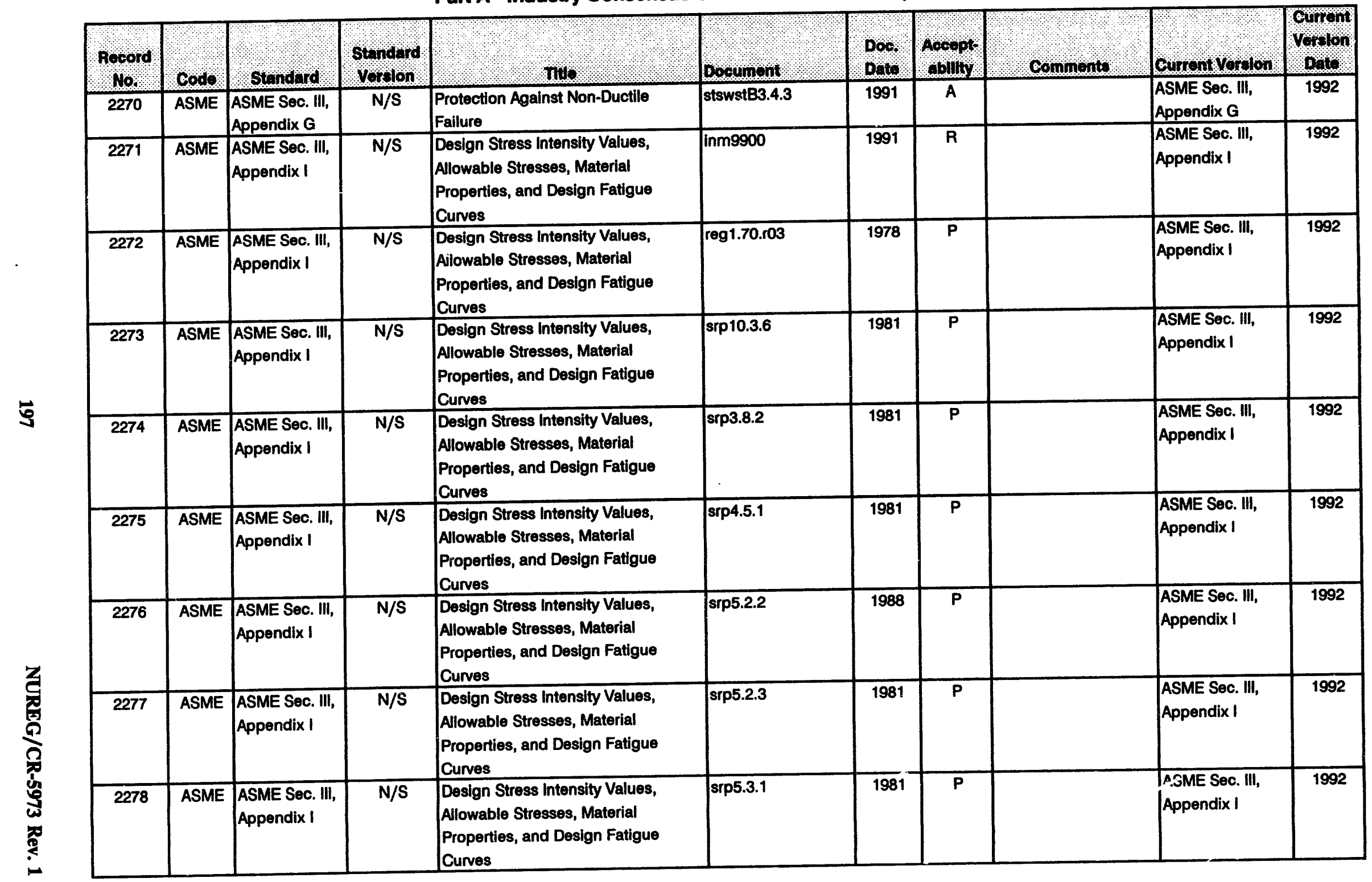




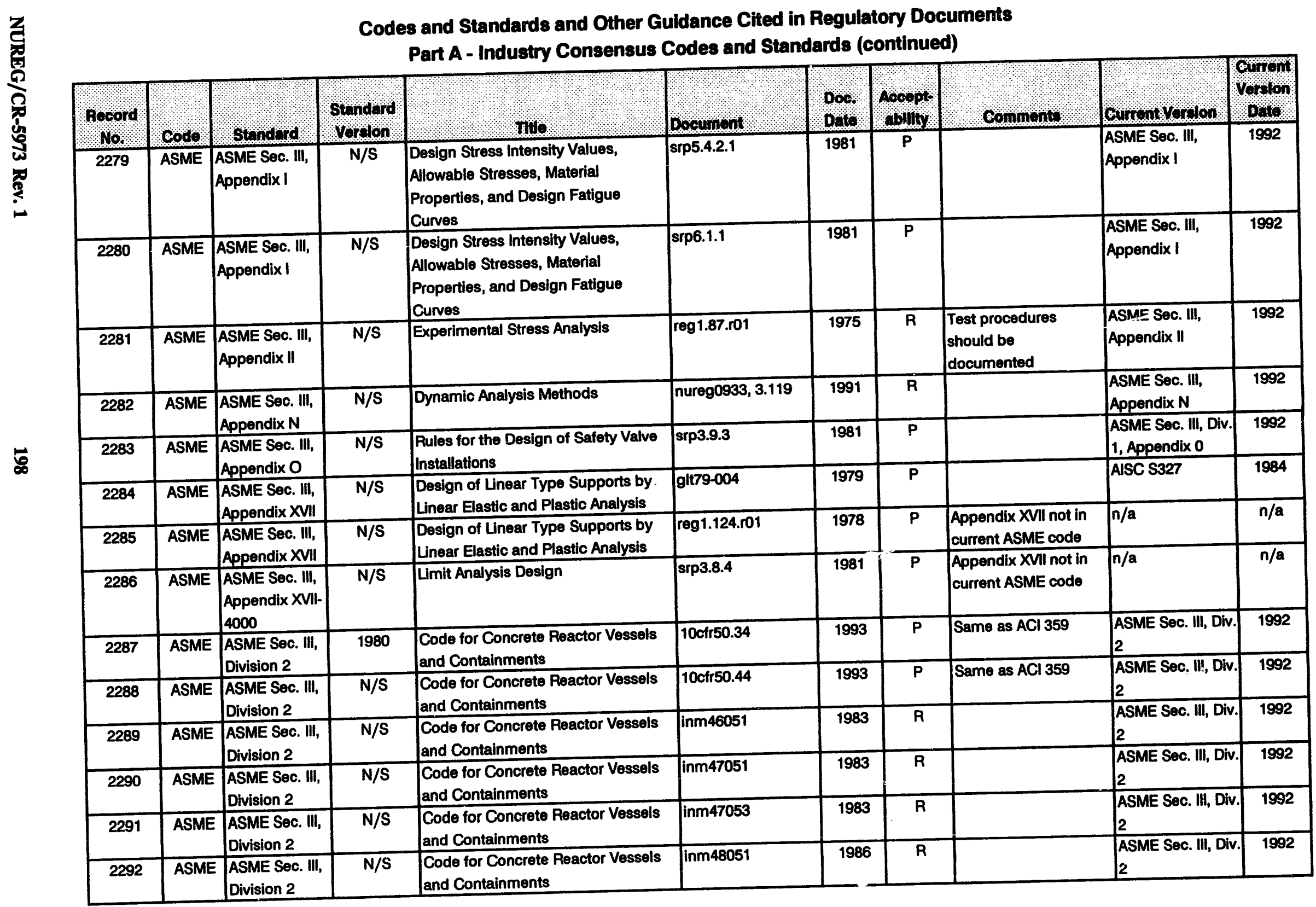




\section{Codes and Standards and Other Guidance Cited in Regulatory Documents}

Part A - Industry Consensus Codes and Standards (continued)

\begin{tabular}{|c|c|c|c|c|c|c|c|c|c|c|}
\hline $\begin{array}{l}\text { Hocord } \\
\text { No. }\end{array}$ & Codo & Standand & Standard & ४ & Dooumont & $\begin{array}{l}\text { Dor } \\
\text { Date }\end{array}$ & Aopopts & Comments & Curront voralon & Gorron \\
\hline 2293 & ASME & $\begin{array}{l}\text { ASME Sec. III, } \\
\text { Division } 2\end{array}$ & $N / S$ & $\begin{array}{l}\text { Code for Concrete Reactor Vessels } \\
\text { and Containments }\end{array}$ & inm48053 & 1986 & $\mathbf{R}$ & & $\begin{array}{l}\text { ASME Sec. III, Div. } \\
2\end{array}$ & 1992 \\
\hline 2294 & ASME & $\begin{array}{l}\text { ASME Sec. III, } \\
\text { Division } 2 \\
\end{array}$ & $\mathrm{~N} / \mathrm{S}$ & $\begin{array}{l}\text { Code for Concrete Reactor Vessels } \\
\text { and Containments }\end{array}$ & inm63050 & 1983 & $\bar{R}$ & & $\begin{array}{l}\text { ASME Sec. III, Div. } \\
2\end{array}$ & 1992 \\
\hline 2295 & ASME & $\begin{array}{l}\text { ASME Sec. III, } \\
\text { Division } 2 \\
\end{array}$ & $\mathrm{~N} / \mathrm{S}$ & $\begin{array}{l}\text { Code for Concrete Reactor Vessels } \\
\text { and Containments }\end{array}$ & inm70462 & 1983 & $\mathbf{R}$ & & $\begin{array}{l}\text { ASME Sec. III, Div. } \\
2\end{array}$ & 1992 \\
\hline 2296 & ASME & \begin{tabular}{|l} 
ASME Sec. III, \\
Division 2 \\
\end{tabular} & 1975 & $\begin{array}{l}\text { Code for Concrete Reactor Vessels } \\
\text { and Containments }\end{array}$ & reg1.107.ro1 & 1977 & $\mathbf{P}$ & Same as ACI 359 & $\begin{array}{l}\text { ASME Sec. III, Div. } \\
2\end{array}$ & 1992 \\
\hline 2297 & ASME & $\begin{array}{l}\text { ASME Sec. III, } \\
\text { Division } 2\end{array}$ & 1980 & $\begin{array}{l}\text { Code for Concrete Reactor Vessels } \\
\text { and Containments }\end{array}$ & reg1.136.r02 & 1981 & $\mathbf{P}$ & Same as ACI 359 & $\begin{array}{l}\text { ASME Sec. III, Div. } \\
2\end{array}$ & 1992 \\
\hline 2298 & ASME & $\begin{array}{l}\text { ASME Sec. III, } \\
\text { Division } 2 \\
\end{array}$ & 1980 & $\begin{array}{l}\text { Code for Concrete Reactor Vessels } \\
\text { and Containments }\end{array}$ & reg1.142.r01 & 1981 & $\mathbf{P}$ & Same as ACI 359 & $\begin{array}{l}\text { ASME Sec. III, Div. } \\
2\end{array}$ & 1992 \\
\hline 2299 & ASME & $\begin{array}{l}\text { ASME Sec. III, } \\
\text { Division } 2 \\
\end{array}$ & 1982 & $\begin{array}{l}\text { Code for Concrete Reactor Vessels } \\
\text { and Containments }\end{array}$ & reg1.35.r03 & 1990 & $\mathbf{R}$ & Same as ACl 359 & $\begin{array}{l}\text { ASME Sec. III, Div. } \\
2\end{array}$ & 1992 \\
\hline 2300 & ASME & $\begin{array}{l}\text { ASME Sec. III, } \\
\text { Division } 2 \\
\end{array}$ & $N / S$ & $\begin{array}{l}\text { Code for Concrete Reactor Vessels } \\
\text { and Containments }\end{array}$ & reg1.38.r02 & 1977 & $\mathbf{R}$ & Same as $\mathrm{ACl} 359$ & $\begin{array}{l}\text { ASME Sec. III, Div. } \\
2\end{array}$ & 1992 \\
\hline 2301 & ASME & $\begin{array}{l}\text { ASME Sec. III, } \\
\text { Division } 2 \\
\end{array}$ & $\mathrm{~N} / \mathrm{S}$ & $\begin{array}{l}\text { Code for Concrete Reactor Vessels } \\
\text { and Containments }\end{array}$ & reg1.70.r03 & 1978 & $\mathbf{P}$ & Same as ACI 359 & $\begin{array}{l}\text { ASME Sec. III, Div. } \\
2\end{array}$ & 1992 \\
\hline 2302 & ASME & $\begin{array}{l}\text { ASME Sec. III, } \\
\text { Division } 2 \\
\end{array}$ & 1975 & $\begin{array}{l}\text { Code for Concrete Reactor Vessels } \\
\text { and Containments }\end{array}$ & reg1.90.ro1 & 1977 & $\mathbf{P}$ & Same as ACl 359 & $\begin{array}{l}\text { ASME Sec. III, Div. } \\
2\end{array}$ & 1992 \\
\hline 2303 & ASME & $\begin{array}{l}\text { ASME Sec. III, } \\
\text { Division } 2 \\
\end{array}$ & 1975 & $\begin{array}{l}\text { Code for Concrete Reactor Vessels } \\
\text { and Containments }\end{array}$ & reg1.94.ro1 & 1976 & $\mathbf{P}$ & Same as ACl 359 & $\begin{array}{l}\text { ASME Sec. III, Div. } \\
2\end{array}$ & 1992 \\
\hline 2304 & ASME & $\begin{array}{l}\text { ASME Sec. III, } \\
\text { Division } 2 \\
\end{array}$ & 1975 & $\begin{array}{l}\text { Code for Concrete Reactor Vessels } \\
\text { and Containments }\end{array}$ & reg3.27.101 & 1977 & $\mathbf{P}$ & Same as ACl 359 & $\begin{array}{l}\text { ASME Sec. III, Div. } \\
2\end{array}$ & 1992 \\
\hline 2305 & ASME & $\begin{array}{l}\text { ASME Sec. III, } \\
\text { Division } 2 \\
\end{array}$ & $N / S$ & $\begin{array}{l}\text { Code for Concrete Reactor Vessels } \\
\text { and Containments }\end{array}$ & $\operatorname{srp3.8.1}$ & 1981 & $\mathbf{P}$ & Same as ACl 359 & \begin{tabular}{|l} 
ASME SOc. III, Div. \\
2
\end{tabular} & 1992 \\
\hline 2306 & ASME & $\begin{array}{l}\text { ASME Sec. III, } \\
\text { Division } 2 \\
\end{array}$ & $N / S$ & $\begin{array}{l}\text { Code for Concrete Reactor Vessels } \\
\text { and Containments }\end{array}$ & $\operatorname{sip3.8.3}$ & 1981 & $\bar{P}$ & Same as ACl 359 & $\begin{array}{l}\text { ASME Sec. III, Div. } \\
2\end{array}$ & 1992 \\
\hline 2307 & ASME & $\begin{array}{l}\text { ASME Sec. III, } \\
\text { Division } 2 \\
\end{array}$ & $N / S$ & $\begin{array}{l}\text { Code for Concreie Reactor Vessels } \\
\text { and Containments }\end{array}$ & $\operatorname{sip5.2.1.2}$ & 1981 & $\mathbf{R}$ & Same as ACl 359 & $\begin{array}{l}\text { ASME Sec. III, Div. } \\
2\end{array}$ & 1992 \\
\hline 2308 & ASME & $\begin{array}{l}\text { ASME Sec. III, } \\
\text { Division } 2 \\
\end{array}$ & $N / S$ & $\begin{array}{l}\text { Code for Concrete Reactor Vessels } \\
\text { and Containments }\end{array}$ & srp6.1.1 & 1981 & $\mathbf{P}$ & Same as $\mathrm{ACl} 359$ & ASME Sec. III, Div. & 1992 \\
\hline
\end{tabular}


Codes and Standards and Other Guidance Cited in Regulatory Documents

Part A - Industry Consensus Codes and Standards (continued)

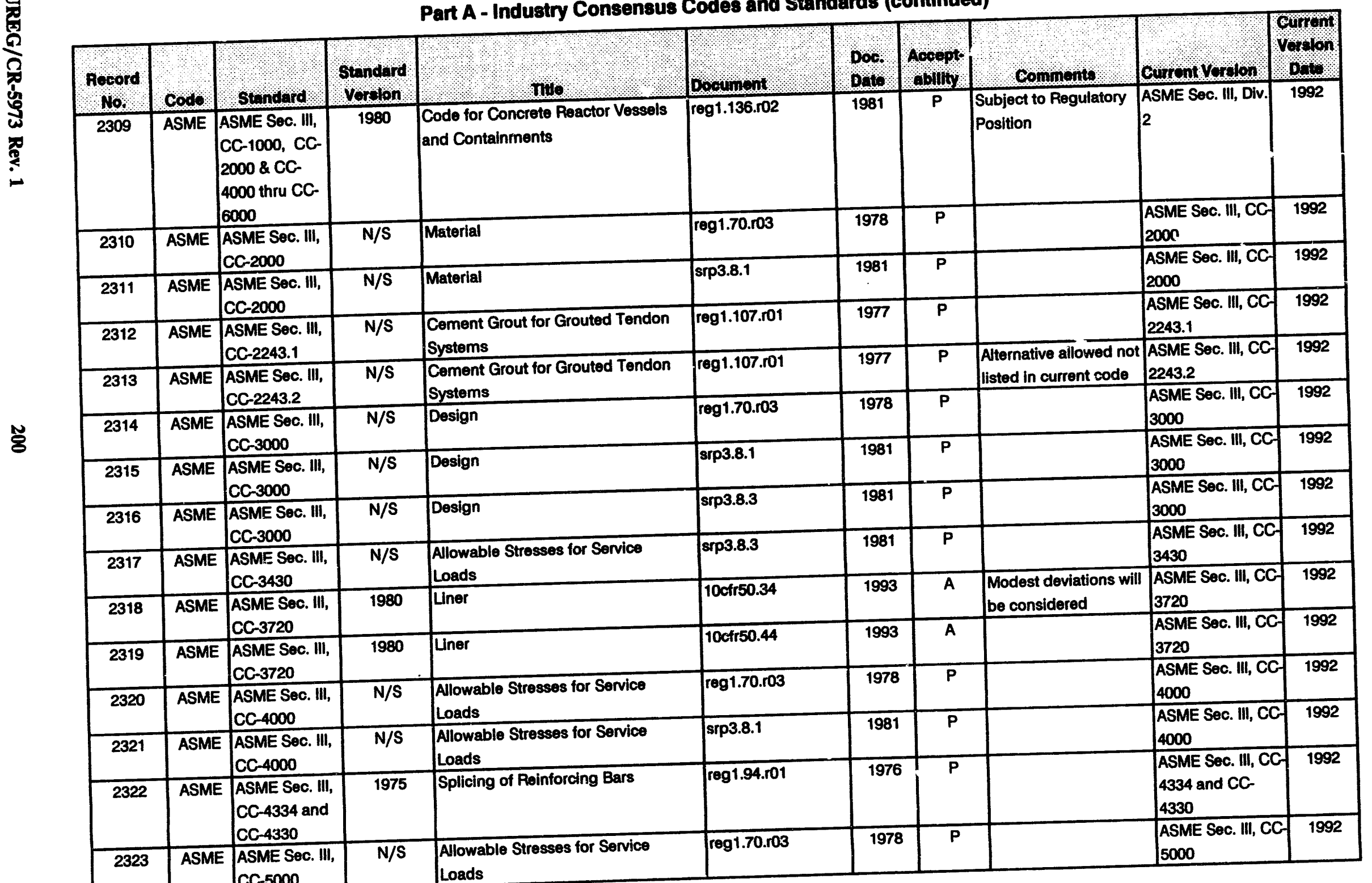


Codes and Standards and Other Guidance Cited in Regulatory Documents

Part A - Industry Consensus Codes and Standards (continued)

\begin{tabular}{|c|c|c|c|c|c|c|c|c|c|c|}
\hline hocord & Codo & Standerd & Standard & (1., & Bocument & Dor. & Acospts & Comments & curront Yorilon & Qurpot \\
\hline 2324 & ASME & $\begin{array}{l}\text { ASME Sec. III, } \\
\text { CC-5000 }\end{array}$ & $\mathrm{N} / \mathrm{S}$ & $\begin{array}{l}\text { Allowable Stresses for Service } \\
\text { Loads }\end{array}$ & srp3.8.1 & 1981 & $\mathbf{P}$ & & $\begin{array}{l}\text { ASME Soc. III, CC- } \\
5000\end{array}$ & 1992 \\
\hline 2325 & ASME & $\begin{array}{l}\text { ASME SeC. III, } \\
\text { CC-6000 }\end{array}$ & $\mathrm{N} / \mathrm{S}$ & $\begin{array}{l}\text { Structural Integrity Test of Concrete } \\
\text { Containments }\end{array}$ & reg1.70.ro3 & 1978 & $\mathbf{P}$ & & $\begin{array}{l}\text { ASME Sec. III, CC- } \\
6000\end{array}$ & 1992 \\
\hline 2326 & ASME & $\begin{array}{l}\text { ASME Sec. III, } \\
\text { CC-9000 }\end{array}$ & $N / S$ & Not found & reg1.70.r03 & 1978 & $\mathbf{P}$ & $\begin{array}{l}\text { Typo in reg1.70-CC- } \\
9000 \text { is not a valid } \\
\text { ASME reference }\end{array}$ & $n / a$ & $n / a$ \\
\hline 2327 & ASME & $\begin{array}{l}\text { ASME Sec. III, } \\
\text { Table CC- } \\
2422-1 \\
\end{array}$ & 1982 & $\begin{array}{l}\text { Analysis Limits of Permanent } \\
\text { Coating Material for CRV } \\
\text { Prestressing }\end{array}$ & reg1.35.r03 & 1990 & $\mathbf{P}$ & & $\begin{array}{l}\text { ASME Sec. III, Div. } \\
\text { 2, Table CC-2422- } \\
1\end{array}$ & 1992 \\
\hline 2328 & ASME & \begin{tabular}{|l} 
ASME Section \\
$\mathrm{v}$
\end{tabular} & $\mathrm{N} / \mathrm{S}$ & Nondestructive Examination & inm49055 & 1982 & $\mathbf{R}$ & & ASME Søc. V & 1992 \\
\hline 2329 & ASME & $\begin{array}{l}\text { ASME Section } \\
\mathrm{v}\end{array}$ & N/S & Nondestructive Examination & inm57050 & 1983 & $\mathbf{R}$ & & ASME Sec. V & 1992 \\
\hline 2330 & ASME & \begin{tabular}{|l|} 
ASME Section \\
$\mathrm{V}$
\end{tabular} & $\mathrm{N} / \mathrm{S}$ & Nondestructive Examination & inm57060 & 1983 & $\mathbf{R}$ & & ASME Sec. V & 1992 \\
\hline 2331 & ASME & $\begin{array}{l}\text { ASME Section } \\
\mathrm{V}\end{array}$ & $\mathrm{N} / \mathrm{S}$ & Nondestructive Examination & inm57070 & 1983 & $\mathbf{R}$ & & ASME Sec. V & 1992 \\
\hline 2332 & ASME & $\begin{array}{l}\text { ASME Section } \\
\mathrm{V}\end{array}$ & $\mathrm{N} / \mathrm{S}$ & Nondestructive Examination & inm57080 & 1983 & $\mathbf{R}$ & & ASME Sec. V & 1992 \\
\hline 2333 & ASME & $\begin{array}{l}\text { ASME Section } \\
\mathrm{V}\end{array}$ & $\mathrm{N} / \mathrm{S}$ & Nondestructive Examination & inm57090 & 1993 & $\mathbf{R}$ & & ASME Sec. V & 1992 \\
\hline 2334 & ASME & $\begin{array}{l}\text { ASME Section } \\
\mathrm{V}\end{array}$ & $\mathrm{N} / \mathrm{S}$ & Nondestructive Examination & inm73052 & 1986 & $\mathbf{R}$ & & ASME Sec. V & 1992 \\
\hline 2335 & ASME & \begin{tabular}{|l|} 
ASME Section \\
$\mathrm{V}$
\end{tabular} & $\mathrm{N} / \mathrm{S}$ & Nondestructive Examination & inm73053B & 1979 & $\mathbf{R}$ & & ASME Sec. V & 1992 \\
\hline 2336 & ASME & $\begin{array}{l}\text { ASME Section } \\
\mathrm{v}\end{array}$ & $\mathrm{N} / \mathrm{S}$ & Nondestructive Examination & nureg0933, 3.67 & 1991 & $\mathbf{R}$ & & ASME Section V & 1992 \\
\hline 2337 & ASME & $\begin{array}{l}\text { ASME Section } \\
\mathrm{V}\end{array}$ & $\mathrm{N} / \mathrm{S}$ & Nondestructive Examination & $\operatorname{sip} 10.2 .3$ & 1981 & $\mathbf{P}$ & & ASME Sec. V & 1992 \\
\hline 2338 & ASME & $\begin{array}{l}\text { ASME Section } \\
\mathrm{V}\end{array}$ & $\mathrm{N} / \mathrm{S}$ & Nondestructive Examination & $\operatorname{srp5.2.1.2}$ & 1981 & $\mathbf{P}$ & & ASME Sec. V & 1992 \\
\hline 2339 & ASME & \begin{tabular}{|l} 
ASME Section \\
$\mathrm{V}$
\end{tabular} & $\mathrm{N} / \mathrm{S}$ & Nondestructive Examination & srp5.3.1 & 1981 & $\mathbf{R}$ & & ASME Sec. V & 1992 \\
\hline
\end{tabular}


Codes and Standards and Other Guidance Cited in Regulatory Documents

Part A - Industry Consensus Codes and Standards (continued)

\begin{tabular}{|c|c|c|c|c|c|c|c|c|c|c|}
\hline & & & & Pan A - Inutusiry Coisentsus & 年 & & & & & \\
\hline Hocord & & & Standord & $\mathrm{nHe}$ & Docoument: & Doo, & Aocopts & commonts & Cunent Yorton & 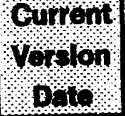 \\
\hline 2340 & ASME & $\begin{array}{l}\text { STIAndara } \\
\text { ASME Section } \\
v\end{array}$ & N/S & Nondestructive Examination & $\operatorname{sip5.3.3}$ & 1981 & $\mathbf{R}$ & & ASME Sec. V & 1992 \\
\hline 2341 & ASME & $\begin{array}{l}\text { ASME Sec. V, } \\
\text { Article } 1 \\
\end{array}$ & 1975 & General Requiremsnts & reg3.27.ro1 & 1977 & $\mathbf{P}$ & & $\begin{array}{l}\text { ASME Sec. V, } \\
\text { Article } 1\end{array}$ & 1992 \\
\hline 2342 & ASME & $\begin{array}{l}\text { ASME Sec. V, } \\
\text { Article } 2\end{array}$ & $\mathrm{~N} / \mathrm{S}$ & Radiographic Examination & inm57090 & 1993 & $\mathbf{R}$ & & $\begin{array}{l}\text { ASME Sec. V, } \\
\text { Articie } 2 \\
\end{array}$ & 1992 \\
\hline 2343 & ASME & $\begin{array}{l}\text { ASME Sec. V, } \\
\text { Article } 4\end{array}$ & 1980 & $\begin{array}{l}\text { Ultrasonic Examination Methods for } \\
\text { Inservice Inspection }\end{array}$ & reg1.147.r10 & 1993 & $\mathbf{P}$ & & $\begin{array}{l}\text { ASME Sec. V, } \\
\text { Article } 4 \\
\end{array}$ & 1992 \\
\hline 2344 & ASME & $\begin{array}{l}\text { ASME Sec. V, } \\
\text { Article } 4\end{array}$ & 1977 & $\begin{array}{l}\text { Ultrasonic Examination Methods for } \\
\text { Inservice Inspection }\end{array}$ & reg1.150.r01 & 1983 & $\mathbf{R}$ & Referred to in text & $\begin{array}{l}\text { ASME Sec. V, } \\
\text { Article } 4\end{array}$ & 1992 \\
\hline 2345 & ASME & $\begin{array}{l}\text { ASME Sec. V, } \\
\text { Article } 4\end{array}$ & 1980 & $\begin{array}{l}\text { Uitrasonic Examination Methods for } \\
\text { Inservice Inspection }\end{array}$ & reg1.150.r01 & 1983 & $\mathbf{R}$ & Referred to in text & $\begin{array}{l}\text { ASME Soc. V, } \\
\text { Article } 4\end{array}$ & 1992 \\
\hline 2346 & ASME & $\begin{array}{l}\text { ASME Sec. V, } \\
\text { Article 4, App. } \\
\text { B }\end{array}$ & 1977 & $\begin{array}{l}\text { General Techniques for Angle } \\
\text { Beam Calibrations }\end{array}$ & reg1.150.r01 & 1983 & $\mathbf{P}$ & & $\begin{array}{l}\text { ASME Sec. V, Art. } \\
\text { 4, Appendix B }\end{array}$ & 1992 \\
\hline 2347 & ASME & $\begin{array}{l}\text { ASME Sec. V, } \\
\text { Article 4, App. I }\end{array}$ & $N / S$ & Screen Height Linearity & reg1.150.ro1 & 1983 & $\mathbf{P}$ & With some exceptions & $\begin{array}{l}\text { ASME Sec. V, Art. } \\
\text { 4, Appendix I }\end{array}$ & 1992 \\
\hline 2348 & ASME & $\begin{array}{l}\text { ASME Sec. V, } \\
\text { Article 4, App. } \\
\Perp\end{array}$ & 1977 & Amplitude Control Linearity & reg1.150.ro1 & 1983 & $\mathbf{P}$ & & $\begin{array}{l}\text { ASME Sec. V, Art. } \\
\text { 4, Appendix II }\end{array}$ & 1992 \\
\hline 2349 & ASME & $\begin{array}{l}\text { ASME Sec. V, } \\
\text { Article 4, } \\
\text { Figure 1-3131 }\end{array}$ & 1977 & $\begin{array}{l}\text { General Techniques for Angle } \\
\text { Beam Calibrations }\end{array}$ & reg1.150.ro1 & 1983 & $\mathbf{P}$ & & $\begin{array}{l}\text { ASME Sec. V, Ant. } \\
\text { 4, Figure } 1-3131\end{array}$ & 1992 \\
\hline 2350 & ASME & $\begin{array}{l}\text { ASME Sec. V, } \\
\text { Article 4, } \\
\text { Figure T-434 }\end{array}$ & $\mathrm{N} / \mathrm{S}$ & Search Unit Requirements & reg1.150.ro1 & 1983 & $\mathbf{R}$ & & $\begin{array}{l}\text { ASME Sec. V, Art. } \\
\text { 4, Figure T-434 }\end{array}$ & 1992 \\
\hline 2351 & ASME & $\begin{array}{l}\text { ASME Sec. V, } \\
\text { Article 4, T- } \\
423.1 .3\end{array}$ & 1977 & Written Procedure Requirements & reg1.150.ro1 & 1983 & $\mathbf{R}$ & $\begin{array}{l}\text { T-423.1.3 is not in } \\
\text { current ASME Code }\end{array}$ & $n / a$ & $n / a$ \\
\hline 2352 & ASME & $\begin{array}{l}\text { ASME Sec. V, } \\
\text { Article 4, T. } \\
432.1 .2\end{array}$ & 1977 & $\begin{array}{l}\text { Calibration Check on Basic } \\
\text { Calibration Block or Similar Check }\end{array}$ & reg1.150.ro1 & 1983 & $\mathbf{R}$ & & $\begin{array}{l}\text { ASME Soc. V, Art. } \\
4, T-432.1 .2\end{array}$ & 1992 \\
\hline 2353 & ASME & $\begin{array}{l}\text { ASME Sec. V, } \\
\text { Article 4, T-433 }\end{array}$ & $\mathbf{N} / \mathbf{S}$ & Calibration Confirmation & reg1.150.ro1 & 1983 & $\bar{P}$ & & $\begin{array}{l}\text { ASME Sec. V, Art. } \\
4, T-433\end{array}$ & 1992 \\
\hline
\end{tabular}


Codes and Standards and Other Guidance Cited in Regulatory Documents

Part A - Industry Consensus Codes and Standards (continued)

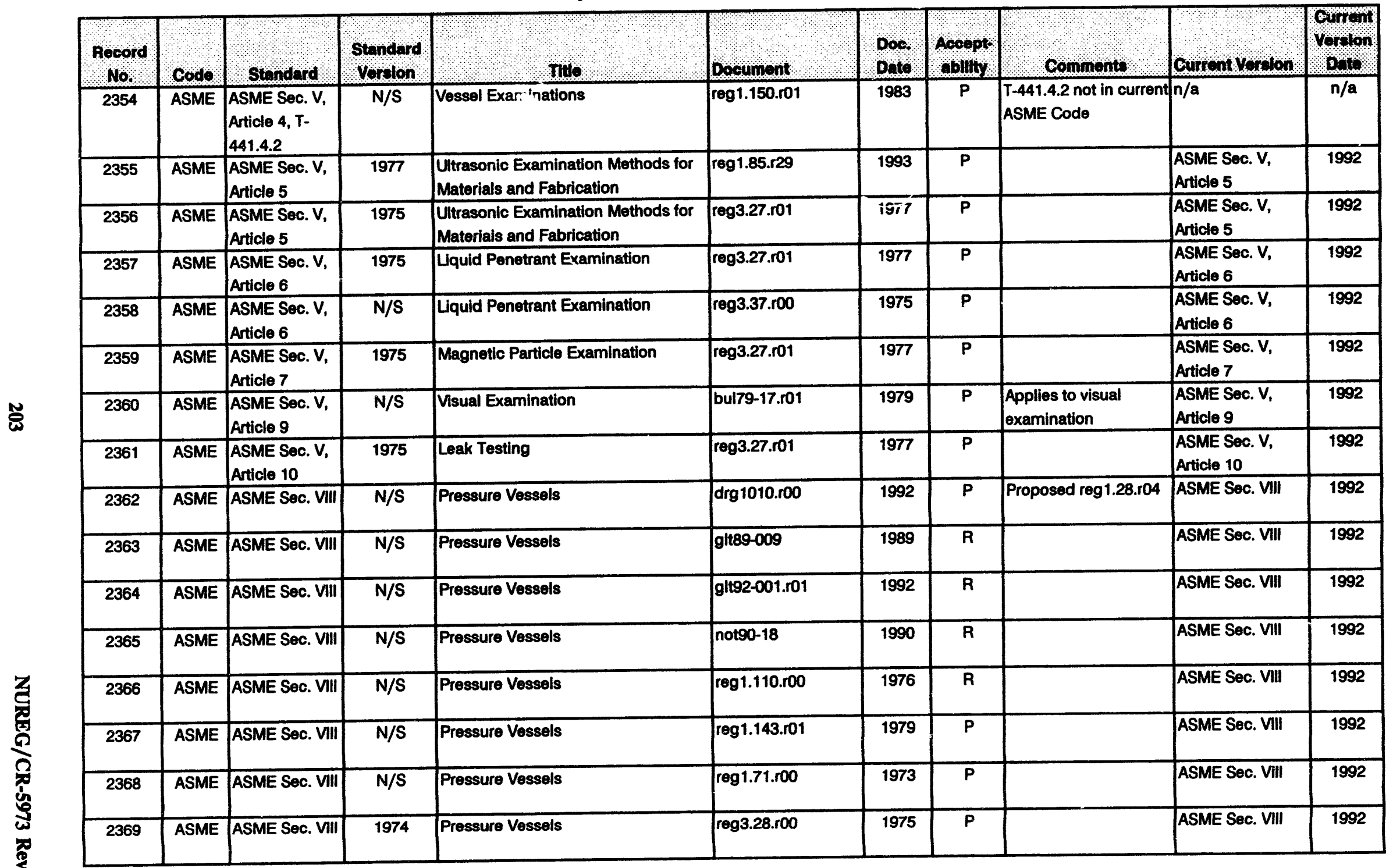


Codes and Standards and Other Guidance Cited in Regulatory Documents

Part A - Industry Consensus Codes and Standards (continued)

\begin{tabular}{|c|c|c|c|c|c|c|c|c|c|c|}
\hline Pocord & codo & Stindord & stmolard & $1,1,0$, no & Dooument & Dop, & $\begin{array}{l}\text { Aorepts. } \\
\text { sollity }\end{array}$ & comanents & currom Vorolon & 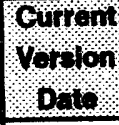 \\
\hline 2370 & ASME & ASME Sec. VIII & 1971 & Pressure Vessels & reg3.29.roo & 1975 & $P$ & & ASME SoC. VIII & 1992 \\
\hline 2371 & ASME & ASME Sec. VIII & $\mathrm{N} / \mathrm{S}$ & Pressure Vessels & reg3.60.r00 & 1987 & $\mathbf{P}$ & & ASME SeC. VIII & 1992 \\
\hline 2372 & ASME & ASME Sec. VIII & 1980 & Pressure Vessels & srp3.2.2 & 1981 & $\mathbf{P}$ & & ASME SOC. VIII & 1992 \\
\hline 2373 & ASME & \begin{tabular}{|l|} 
ASME Soc. VIII, \\
Div. 1
\end{tabular} & $\mathrm{~N} / \mathrm{S}$ & Pressure Vessels & not87-26 & 1987 & $\mathbf{P}$ & & \begin{tabular}{|l} 
ASME Soc. VIII, \\
Div. 1
\end{tabular} & 1992 \\
\hline 2374 & ASME & \begin{tabular}{|l|} 
ASME Sec. VIII, \\
Div. I
\end{tabular} & $N / S$ & Pressure Vessels & not92-58 & 1992 & $\mathbf{R}$ & & $\begin{array}{l}\text { ASME Soc. Vill, } \\
\text { Div. I } \\
\end{array}$ & 1992 \\
\hline 2375 & ASME & \begin{tabular}{|l|} 
ASME Sec. VIII, \\
Div. 1
\end{tabular} & $\mathbf{N} / \mathbf{S}$ & Pressure Vessels & srp3.9.4 & 1984 & A & & $\begin{array}{l}\text { ASME Sec. VIII, } \\
\text { Div. } 1 \\
\end{array}$ & 1992 \\
\hline 2376 & ASME & $\begin{array}{l}\text { ASME Section } \\
\mathrm{x}\end{array}$ & 1986 & Welding and Brazing Qualifications & inm-toc & 1993 & $\mathbf{R}$ & & ASME Sec. IX & 1992 \\
\hline 2377 & ASME & $\begin{array}{l}\text { ASME Section } \\
\text { IX }\end{array}$ & $\mathrm{N} / \mathrm{S}$ & Welding and Brazing Qualifications & inm49055 & 1982 & $\mathbf{R}$ & & ASME Sec. IX & 1992 \\
\hline 2378 & ASME & $\begin{array}{l}\text { ASME Section } \\
\text { DX }\end{array}$ & $\mathrm{N} / \mathrm{S}$ & Wolding and Brazing Qualifications & inm50082B & 1976 & $\mathbf{R}$ & & ASME SOC. $\mathrm{X}$ & 1992 \\
\hline 2379 & ASME & $\begin{array}{l}\text { ASME Section } \\
\mathrm{IX}\end{array}$ & $N / S$ & Wolding and Brazing Qualifications & inm50085B & 1976 & $\mathbf{R}$ & & ASME Sec. $\mathrm{DX}$ & 1992 \\
\hline 2380 & $\overline{A S M E}$ & $\begin{array}{l}\text { ASME Section } \\
\mathrm{IX}\end{array}$ & $\mathbf{N} / \mathrm{S}$ & Welding and Brazing Qualifications & inm55050 & 1983 & $\mathbf{R}$ & & ASME Soc. IX & 1992 \\
\hline 2381 & ASME & $\begin{array}{l}\text { ASME Section } \\
\text { IX }\end{array}$ & $\mathrm{N} / \mathrm{S}$ & Welding and Brazing Qualifications & inm55092B & 1976 & $\bar{R}$ & & ASME Sec. IX & 1992 \\
\hline 2382 & ASME & $\begin{array}{l}\text { ASME Section } \\
\mathrm{IX}\end{array}$ & $\mathrm{N} / \mathrm{S}$ & Welding and Brazing Qualifications & inm55093B & 1977 & $\mathbf{R}$ & & ASME Sec. IX & 1992 \\
\hline 2383 & ASME & $\begin{array}{l}\text { ASME Section } \\
\mathrm{X}\end{array}$ & $\mathrm{N} / \mathrm{S}$ & Welding and Brazing Qualifications & inm9900/ASME IX & 1986 & $\mathbf{R}$ & & ASME SeC. IX & 1992 \\
\hline 2384 & ASME & $\begin{array}{l}\text { ASME Section } \\
\mathrm{DX}\end{array}$ & $\mathrm{N} / \mathrm{S}$ & Welding and Brazing Qualifications & not83-61 & 1983 & $\mathbf{P}$ & & ASME Sec. IX & 1992 \\
\hline 2385 & ASME & $\begin{array}{l}\text { ASME Section } \\
\mathrm{IX}\end{array}$ & $N / S$ & Welding and Brazing Qualifications & not89-75 & 1989 & $\bar{R}$ & & ASME Sec. IX & 1992 \\
\hline 2386 & ASME & $\begin{array}{l}\text { ASME Section } \\
\mathrm{DX}\end{array}$ & $N / S$ & Wolding and Brazing Qualifications & reg1.143.ro1 & 1979 & $\mathbf{P}$ & & ASME Sec. IX & 1992 \\
\hline
\end{tabular}


Codes and Standards and Other Guidance Chted in Regulatory Documents

Part A - Industry Consensus Codes and Standards (continued)

\begin{tabular}{|c|c|c|c|c|c|c|c|c|c|c|}
\hline $\begin{array}{l}\text { Record } \\
\text { No. }\end{array}$ & Code & Sundard & Stenderd & ( & Doovinent & Doo. & Aopopts & Comment: & Cumont Yorolon & 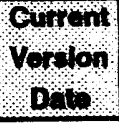 \\
\hline 2387 & ASME & $\begin{array}{l}\text { ASME Section } \\
\mathrm{IX}\end{array}$ & $\mathrm{N} / \mathrm{S}$ & Welding and Brazing Qualifications & reg1.34.ro0 & 1972 & $\mathbf{P}$ & & ASME Sec. IX & 1992 \\
\hline 2388 & ASME & $\begin{array}{l}\text { ASME Section } \\
\mathrm{IX}\end{array}$ & $\mathrm{N} / \mathrm{S}$ & Wolding and Brazing Qualifications & reg1.43.ro0 & 1973 & $\overline{\mathbf{P}}$ & & ASME SOC. IX & 1992 \\
\hline 2389 & ASME & $\begin{array}{l}\text { ASME Section } \\
\mathrm{X}\end{array}$ & N/S & Wolding and Brazing Qualifications & reg1.50.r00 & 1973 & $\bar{P}$ & With supplements & ASME Sec. IX & 1992 \\
\hline 2390 & ASME & $\begin{array}{l}\text { ASME Section } \\
\mathrm{X}\end{array}$ & $\mathrm{N} / \mathrm{S}$ & Wolding and Brazing Qualifications & $\operatorname{reg} 1.71 . r c 0$ & 1973 & $\mathbf{P}$ & & ASME SOC. IX & 1992 \\
\hline 2391 & ASME & $\begin{array}{l}\text { ASME Section } \\
\mathrm{D}\end{array}$ & 1974 & Welding and Brazing Qualifications & reg3.28.ro0 & 1975 & $\mathbf{P}$ & & ASME Sec. IX & 1992 \\
\hline 2392 & ASME & $\begin{array}{l}\text { ASME Section } \\
\mathrm{X}\end{array}$ & 1971 & Welding and Brazing Qualifications & reg3.29.ro0 & 1975 & $\bar{P}$ & & ASME Soc. IX & 1992 \\
\hline 2393 & ASME & $\begin{array}{l}\text { ASME Section } \\
\mathrm{X}\end{array}$ & N/S & Wolding and Brazing Qualifications & reg7.10.101 & 1986 & $\bar{R}$ & & ASME Sec. IX & 1992 \\
\hline 2394 & ASME & $\begin{array}{l}\text { ASME Section } \\
\mathrm{X}\end{array}$ & $\mathrm{N} / \mathrm{S}$ & Welding and Brazing Qualifications & $\operatorname{srp} 10.3 .6$ & 1981 & $\bar{P}$ & & ASME SeC. IX & 1992 \\
\hline 2395 & ASME & $\begin{array}{l}\text { ASME Section } \\
\mathrm{IX}\end{array}$ & $\mathrm{N} / \mathrm{S}$ & Wolding and Brazing Qualifications & srp4.5.2 & 1981 & $\mathbf{R}$ & & ASME Soc. $\mathrm{DX}$ & 1992 \\
\hline 2396 & ASME & $\begin{array}{l}\text { ASME Soction } \\
\mathrm{IX}\end{array}$ & N/S & Wolding and Brazing Qualifications & srp5.2.1.2 & 1981 & $\mathbf{P}$ & & ASME SOC. $\mathbf{X}$ & 1992 \\
\hline 2397 & ASME & $\begin{array}{l}\text { ASME Section } \\
\mathrm{IX}\end{array}$ & N/S & Welding and Brazing Qualifications & $\operatorname{sip5.2.3}$ & 1981 & $\bar{P}$ & & ASME SeC. $\mathrm{XX}$ & 1992 \\
\hline 2398 & ASME & $\begin{array}{l}\text { ASME Section } \\
\mathrm{X}\end{array}$ & $N / S$ & Welding and Brazing Qualifications & $\operatorname{sip5.3.1}$ & 1981 & $\mathbf{P}$ & $\begin{array}{l}\text { Supplemented by Reg. } \\
\text { Guide } 1.50 \text { and } 1.34\end{array}$ & ASME Sec. IX & 1992 \\
\hline 2399 & ASME & $\begin{array}{l}\text { ASME Section } \\
\text { IX }\end{array}$ & $N / S$ & Welding and Brazing Quallifications & $\operatorname{srp5.3.3}$ & 1981 & $\mathbf{R}$ & $\begin{array}{l}\text { Supplemented by Reg. } \\
\text { Guide } 1.50 \text { and } 1.34\end{array}$ & ASME SeC. $\mathrm{DX}$ & 1992 \\
\hline 2400 & ASME & \begin{tabular}{|l|} 
ASME Section \\
$\mathrm{X}$
\end{tabular} & $\mathrm{N} / \mathrm{S}$ & Wolding and Brazing Qualifications & srp5.4.2.1 & 1981 & $\bar{P}$ & & ASME Soc. IX & 1992 \\
\hline 2401 & ASME & $\begin{array}{l}\text { ASME Section } \\
\mathrm{X}\end{array}$ & N/S & Wolding and Brazing Qualifications & srp6.1.1 & 1981 & $\mathbf{P}$ & General endorsements & ASME Soc. IX & 1992 \\
\hline 2402 & ASME & \begin{tabular}{|l|} 
ASME Section \\
$X I$
\end{tabular} & $\mathrm{~N} / \mathrm{S}$ & $\begin{array}{l}\text { Rules for Inservice Inspection of } \\
\text { Nuclear Power Plant Components }\end{array}$ & $10 c f 550$ App. G & 1993 & $\bar{R}$ & & ASME Soc. XI & 1992 \\
\hline 2403 & ASME & $\begin{array}{l}\text { ASME Section } \\
\mathrm{XI}\end{array}$ & $N / S$ & $\begin{array}{l}\text { Rules for Inservice Inspection of } \\
\text { Nuclear Power Plant Components }\end{array}$ & 10 cfr50 App. H & 1993 & $\mathbf{R}$ & & ASME Sec. XI & 1992 \\
\hline
\end{tabular}


Codes and Standards and Other Guidance Cited in Regulatory Documents

Part A - Industry Consensus Codes and Standards (continued)

\begin{tabular}{|c|c|c|c|c|c|c|c|c|c|c|}
\hline $\begin{array}{l}\text { Rocord } \\
\text { No. }\end{array}$ & cods & Standerd & $\begin{array}{l}\text { Standard } \\
\text { Vorlon }\end{array}$ & \& $\quad$ Tllo,$\quad$ & Dowonout & $\begin{array}{l}\text { Dor. } \\
\text { Dove }\end{array}$ & poppls & Cominents & Current Vorolon & 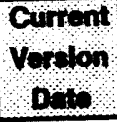 \\
\hline 2404 & ASME & $\begin{array}{l}\text { ASME Section } \\
X I\end{array}$ & 1973 & $\begin{array}{l}\text { Rules for Inservice Inspection of } \\
\text { Nuclear Power Plant Components }\end{array}$ & 10 ctr $50.55 a$ & 1993 & $P$ & & $\begin{array}{l}\text { ASME Sec. XI, } \\
\text { Div. } 1\end{array}$ & 1992 \\
\hline 2405 & ASME & $\begin{array}{l}\text { ASME Soction } \\
X 1\end{array}$ & 1974 & $\begin{array}{l}\text { Rules for Inservice Inspection of } \\
\text { Nuclear Power Plant Components }\end{array}$ & $10 \mathrm{cfr} 50.55 \mathrm{a}$ & 1993 & $\mathbf{P}$ & & $\begin{array}{l}\text { ASME Sec. } X I, \\
\text { Div. } 1\end{array}$ & 1992 \\
\hline 2406 & ASME & $\begin{array}{l}\text { ASME Soction } \\
\mathrm{XI}\end{array}$ & 1983 & $\begin{array}{l}\text { Rules for Inservice Inspection of } \\
\text { Nuclear Power Plant Components }\end{array}$ & $10 \mathrm{ctr} 50.55 \mathrm{a}$ & 1993 & $\mathbf{P}$ & & $\begin{array}{l}\text { ASME Sec. } \mathrm{XI} \text {, } \\
\text { Div. } 1\end{array}$ & 1992 \\
\hline 2407 & ASME & $\begin{array}{l}\text { ASME Section } \\
\mathrm{XI}\end{array}$ & 1974 & $\begin{array}{l}\text { Rules for Inservice Inspection of } \\
\text { Nuclear Power Plant Components }\end{array}$ & bul76-01 & 1976 & $\mathbf{P}$ & for specific hydro test & ASME Sec. X & 1992 \\
\hline 2408 & ASME & $\begin{array}{l}\text { ASME Section } \\
X 1\end{array}$ & 1975 & $\begin{array}{l}\text { Rules for Inservice Inspection of } \\
\text { Nuclear Power Plant Components }\end{array}$ & bul79-17.ro1 & 1979 & $\mathbf{P}$ & & ASME Soc. XI & 1992 \\
\hline 2409 & ASME & $\begin{array}{l}\text { ASME Section } \\
X \mathrm{I}\end{array}$ & $\mathbf{N} / \mathbf{S}$ & $\begin{array}{l}\text { Rules for Inservice Inspection of } \\
\text { Nuclear Power Plant Components }\end{array}$ & bul83-02 & 1983 & $\mathbf{R}$ & & ASME Soc. XI & 1992 \\
\hline 2410 & ASME & $\begin{array}{l}\text { ASME Soction } \\
X 1\end{array}$ & $N / S$ & $\begin{array}{l}\text { Rules for Inservice Inspection of } \\
\text { Nuclear Power Plant Components }\end{array}$ & bul83-03 & 1983 & $\mathbf{P}$ & & ASME Soc. $X$ & 1992 \\
\hline 2411 & ASME & \begin{tabular}{|l|} 
ASME Section \\
$X 1$
\end{tabular} & $\mathbf{N} / \mathrm{S}$ & $\begin{array}{l}\text { Rules for Inservice Inspection of } \\
\text { Nuclear Power Plant Components }\end{array}$ & bul88-08.802 & 1988 & $\mathbf{R}$ & & ASME Soc. XI & 1992 \\
\hline 2412 & ASME & $\begin{array}{l}\text { ASME Soction } \\
X I\end{array}$ & $\mathrm{~N} / \mathrm{S}$ & $\begin{array}{l}\text { Rules for inservice Inspection of } \\
\text { Nuclear Power Plant Components }\end{array}$ & bul88-11 & 1988 & $\mathbf{P}$ & & ASME Soc. XI & 1992 \\
\hline 2413 & ASME & \begin{tabular}{|l|} 
ASME Section \\
$X$
\end{tabular} & N/S & $\begin{array}{l}\text { Rules for Inservice Inspection of } \\
\text { Nuclear Power Plant Components }\end{array}$ & bul89-02 & 1989 & $\mathbf{P}$ & & ASME Sec. XI & 1992 \\
\hline 2414 & ASME & $\begin{array}{l}\text { ASME Soction } \\
X \mathrm{P}\end{array}$ & 1974 & $\begin{array}{l}\text { Rules for Inservice Inspection of } \\
\text { Nuclear Power Plant Components }\end{array}$ & cir76-06 & 1976 & $\mathbf{P}$ & For hydrostatic testing & ASME Soc. XI & 1992 \\
\hline 2415 & ASME & \begin{tabular}{|l|} 
ASME Soction \\
$X$
\end{tabular} & $\mathrm{~N} / \mathrm{S}$ & $\begin{array}{l}\text { Pules for Inservice Inspection of } \\
\text { Nuclear Power Plant Components }\end{array}$ & drg1009.r00 & 1990 & $\mathbf{P}$ & & ASME Sec. X & 1992 \\
\hline 2416 & ASME & $\begin{array}{l}\text { ASME Section } \\
X 1\end{array}$ & N/S & $\begin{array}{l}\text { Rules for Inservice Inspection of } \\
\text { Nuclear Power Plant Components }\end{array}$ & drg1010.r00 & 1992 & $\mathbf{P}$ & Proposed reg1.28.r04 & ASME Sec. XI & 1992 \\
\hline 2417 & ASME & \begin{tabular}{|l|} 
ASME Section \\
$X$
\end{tabular} & 1989 & $\begin{array}{l}\text { Rules for Inservice Inspection of } \\
\text { Nuclear Power Plant Components }\end{array}$ & drg1023.r00 & 1993 & $\mathbf{R}$ & & ASME Section XI & 1992 \\
\hline 2418 & ASME & $\begin{array}{l}\text { ASME Section } \\
X\end{array}$ & $\mathrm{~N} / \mathrm{S}$ & $\begin{array}{l}\text { Rules for Inservice Inspection of } \\
\text { Nuclear Power Plant Components }\end{array}$ & drg7002.100 & 1989 & $\mathbf{R}$ & & ASME SeC. XI & 1992 \\
\hline 2419 & ASME & $\begin{array}{l}\text { ASME Soction } \\
X 1\end{array}$ & 1977 & $\begin{array}{l}\text { Rules for Inservice Inspection of } \\
\text { Nuclear Power Plant Components }\end{array}$ & drgms901-4 & 1981 & $\mathbf{R}$ & & ASME Soc. $X I$ & 1992 \\
\hline 2420 & ASME & $\begin{array}{l}\text { ASME Section } \\
x\end{array}$ & $N / S$ & $\begin{array}{l}\text { Rules for Inservice Inspection of } \\
\text { Nuclear Power Plant Components }\end{array}$ & drgop618-4 & 1982 & $\mathbf{F}$ & Proposed reg8.8r.04 & ASME Sec. XI & 1992 \\
\hline
\end{tabular}


Codes and Standards and Other Guidance Clted in Regulatory Documents

Part A - Industry Consensus Codes and Standards (continued)

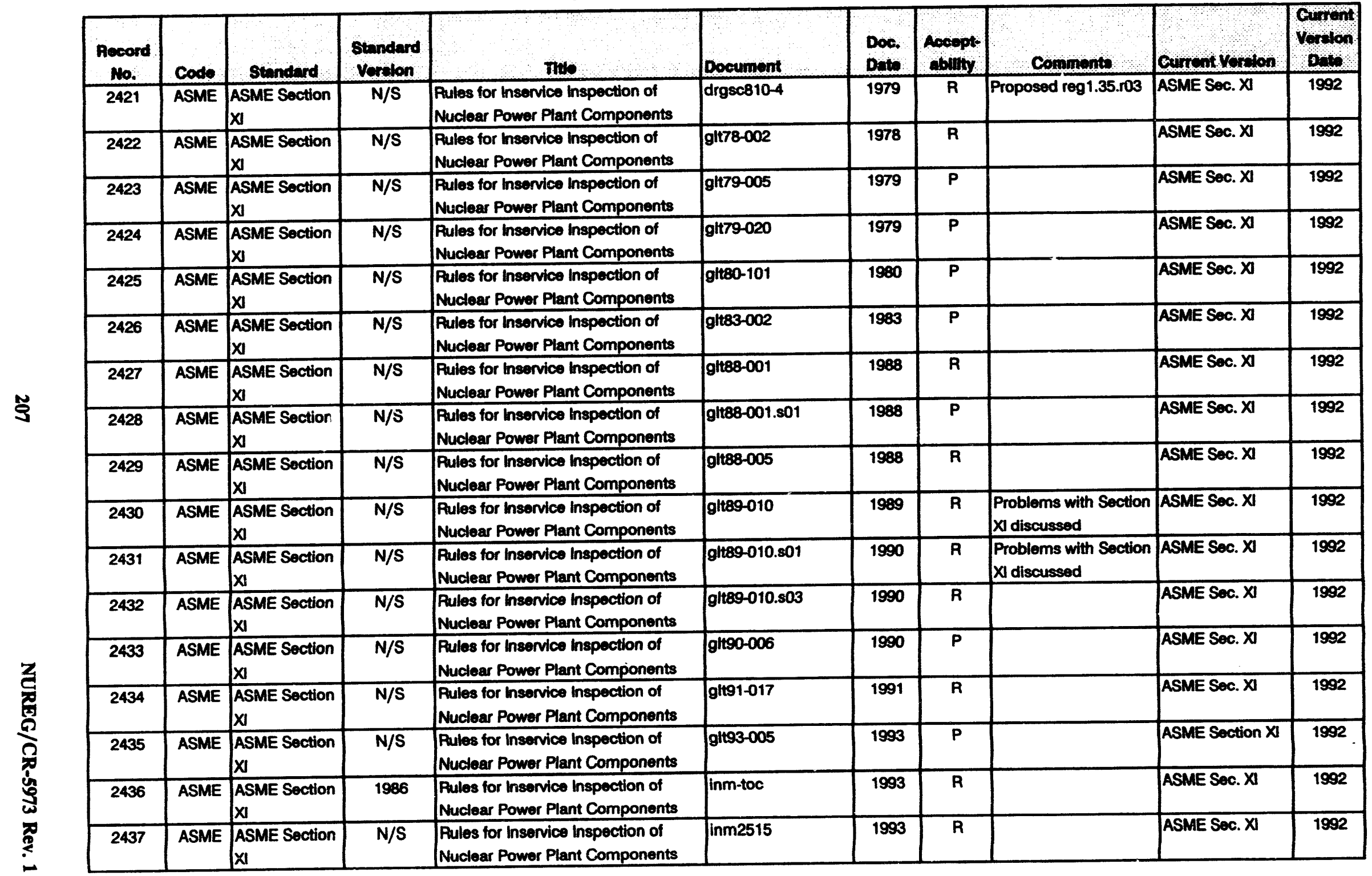


Codes and Standards and Other Guidance Cited in Regulatory Documents

Part A - Industry Consensus Codes and Standards (continued)

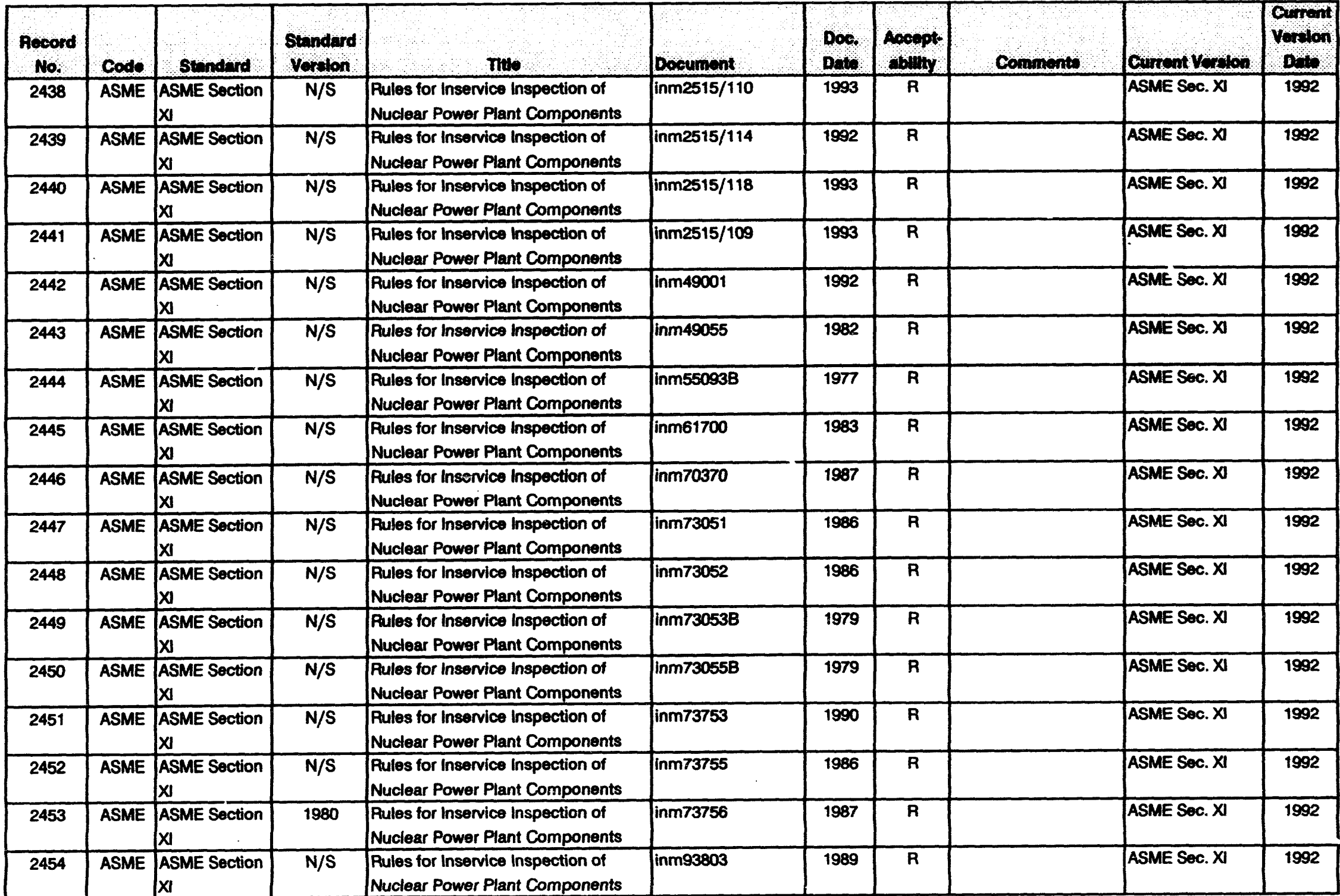


Codes and Standards and Other Guidance Clted in Regulatory Documents

Part A - Industry Consensus Codes and Standards (continued)

\begin{tabular}{|c|c|c|c|c|c|c|c|c|c|c|}
\hline Record & Code & Standard & $\begin{array}{l}\text { Stendard } \\
\text { Vordlon }\end{array}$ & Inte & Document & $\begin{array}{l}\text { Doc. } \\
\text { Dote. }\end{array}$ & $\begin{array}{l}\text { Aocept } \\
\text { sblilty }\end{array}$ & Comments & Gurrent Veralon & $\begin{array}{l}\text { Gurrent } \\
\text { Vorslon } \\
\text { Dalo }\end{array}$ \\
\hline 2455 & ASME & $\begin{array}{l}\text { ASME Section } \\
X \mid\end{array}$ & N/S & $\begin{array}{l}\text { Rules for Inservice Inspection of } \\
\text { Nuclear Power Plant Components }\end{array}$ & inm9900 & 1991 & $R$ & & ASME Sec. XI & 1992 \\
\hline 2456 & ASME & $\begin{array}{l}\text { ASME Section } \\
X 1\end{array}$ & $\mathrm{~N} / \mathrm{S}$ & $\begin{array}{l}\text { Rules for Inservice inspection of } \\
\text { Nuclear Power Plani Components }\end{array}$ & inm9900/4.5.1 & 1986 & $\mathbf{R}$ & & ASME Sec. XI & 1992 \\
\hline 2457 & ASME & \begin{tabular}{|l|} 
ASME Section \\
$X 1$
\end{tabular} & $\mathbf{N} / \mathrm{S}$ & $\begin{array}{l}\text { Rules for Inservice Inspection of } \\
\text { Nuclear Power Plant Components }\end{array}$ & inm9900/4.7.9 & 1975 & $\mathbf{R}$ & & ASME Sec. XI & 1992 \\
\hline 2458 & ASME & \begin{tabular}{|l|} 
ASME Section \\
$X I$
\end{tabular} & 1983 & $\begin{array}{l}\text { Rules for Inservice Inspection of } \\
\text { Nuclear Power Plant Components }\end{array}$ & inm9900/ASME XI & 1986 & $\mathbf{A}$ & & ASME Sec. XI & 1992 \\
\hline 2459 & ASME & \begin{tabular}{|l|} 
ASME Section \\
$\mathrm{X}_{1}$
\end{tabular} & $N / S$ & $\begin{array}{l}\text { Rules for Inservice inspection of } \\
\text { Nuclear Power Plant Components }\end{array}$ & not80-15 & 1980 & $\mathbf{R}$ & & ASME Søc. XI & 1992 \\
\hline 2460 & ASME & \begin{tabular}{|l} 
ASME Section \\
$X I$
\end{tabular} & N/S & $\begin{array}{l}\text { Rules for Inservice Inspection of } \\
\text { Nuclear Power Plant Components }\end{array}$ & not80-27 & 1980 & $\mathbf{R}$ & & ASME Sec. XI & 1992 \\
\hline 2461 & ASME & \begin{tabular}{|l|} 
ASME Section \\
$X I$
\end{tabular} & $N / S$ & $\begin{array}{l}\text { Rules for Inservice Inspection of } \\
\text { Nuclear Power Plant Components }\end{array}$ & not80-38 & 1980 & $\mathbf{R}$ & & ASME Sec. XI & 1992 \\
\hline 2462 & ASME & \begin{tabular}{|l|} 
ASME Soction \\
$X I$
\end{tabular} & 1978 & $\begin{array}{l}\text { Rules for Inservice inspection of } \\
\text { Nuclear Power Plant Components }\end{array}$ & not82-39 & 1982 & $\mathbf{R}$ & & ASME Sec. XI & 1992 \\
\hline 2463 & ASME & \begin{tabular}{|l|} 
ASME Section \\
$\times \mathbf{1}$
\end{tabular} & $\mathrm{N} / \mathrm{S}$ & $\begin{array}{l}\text { Rules for Inservice Inspection of } \\
\text { Nuclear Power Plant Components }\end{array}$ & not86-106.s01 & 1987 & $\mathbf{R}$ & & ASME Sec. XI & 1992 \\
\hline 2464 & ASME & \begin{tabular}{|l|} 
ASME Section \\
$X 1$
\end{tabular} & $\mathrm{~N} / \mathrm{S}$ & $\begin{array}{l}\text { Rules for Inservice Inspection of } \\
\text { Nuclear Power Plant Components }\end{array}$ & not88-70 & 1988 & $\mathbf{P}$ & & ASME Sec. XI & 1992 \\
\hline 2465 & ASME & \begin{tabular}{|l|} 
ASME Section \\
$X I$
\end{tabular} & $N / S$ & $\begin{array}{l}\text { Rules for Inservice Inspection of } \\
\text { Nuclear Power Plant Components }\end{array}$ & not91-32 & 1991 & $\mathbf{R}$ & & ASME Sec. XI & 1992 \\
\hline 2466 & ASME & \begin{tabular}{|l|} 
ASME Section \\
$\times 1$
\end{tabular} & $N / S$ & $\begin{array}{l}\text { Rules for Inservica Inspection of } \\
\text { Nuclear Power Piant Components }\end{array}$ & not92-35 & 1992 & $\mathbf{R}$ & & ASME Sec. XI & 1992 \\
\hline 2467 & ASME & \begin{tabular}{|l|} 
ASME Section \\
$\mathrm{XI}$
\end{tabular} & $N / S$ & $\begin{array}{l}\text { Rules for Inservice Inspection of } \\
\text { Nuclear Power Plant Components }\end{array}$ & not92-85 & 1992 & $\mathbf{R}$ & & ASME Sec. XI & 1992 \\
\hline 2468 & ASME & \begin{tabular}{|l|} 
ASME Section \\
$X I$
\end{tabular} & $N / S$ & $\begin{array}{l}\text { Rules for Inservice Inspection of } \\
\text { Nuclear Power Plant Components }\end{array}$ & not93-20 & 1993 & $\mathbf{R}$ & & ASME Sec. XI & 1992 \\
\hline 2469 & ASME & \begin{tabular}{|l|} 
ASME Section \\
$X I$
\end{tabular} & $\mathrm{~N} / \mathrm{S}$ & $\begin{array}{l}\text { Rules for Inservice Inspection of } \\
\text { Nuclear Power Plant Components }\end{array}$ & not93-21 & 1993 & R. & & ASME Sec. XI & 1992 \\
\hline 2470 & ASME & \begin{tabular}{|l|} 
ASME Section \\
$X \mid$
\end{tabular} & $N / S$ & $\begin{array}{l}\text { Rules for Inservice Inspection of } \\
\text { Nuclear Power Plant Components }\end{array}$ & not93-63 & 1993 & $\mathbf{R}$ & & ASME Sec. XI & 1992 \\
\hline 2471 & ASME & $\begin{array}{l}\text { ASME Section } \\
X I\end{array}$ & 1974 & $\begin{array}{l}\text { Rules for Inservice Inspection of } \\
\text { Nuclear Power Plant Components }\end{array}$ & nureg0933, 2.A-14 & 1983 & $\mathbf{R}$ & & ASME Section XI & 1992 \\
\hline
\end{tabular}


Codes and Standards and Other Guldance Cited in Regulatory Documents

Part A - Industry Consensus Codes and Standards (continued)

\begin{tabular}{|c|c|c|c|c|c|c|c|c|c|c|}
\hline $\begin{array}{l}\text { Rocord } \\
\text { No. }\end{array}$ & & Standard & $\begin{array}{l}\text { Standard } \\
\text { Vorolon }\end{array}$ & \& mus, & Dooumont & $\begin{array}{l}\text { Doo. } \\
\text { Doto }\end{array}$ & $\begin{array}{l}\text { Aocept } \\
\text { sollity }\end{array}$ & comments & Curront Vorolos & 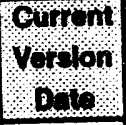 \\
\hline$\frac{100}{2472}$ & Cods & $\begin{array}{l}\text { ASNGF Section } \\
X I\end{array}$ & 1974 & $\begin{array}{l}\text { Rules for Inservice Inspection of } \\
\text { Nuclear Power Plant Components }\end{array}$ & nureg0933, 2.B-26 & 1984 & $R$ & & ASME Section XI & 1992 \\
\hline 2473 & ASME & \begin{tabular}{|l|}
$\mathrm{XI}$ \\
ASME Section \\
$\mathrm{XI}$
\end{tabular} & 1980 & $\begin{array}{l}\text { Rules for Inservice Inspection of } \\
\text { Nuclear Power Plant Components }\end{array}$ & nuregog33, 2.B-47 & 1983 & $\bar{R}$ & & ASME Section XI & 1992 \\
\hline 2474 & ASME & $\begin{array}{l}\text { ASME Section } \\
X I\end{array}$ & 1974 & $\begin{array}{l}\text { Rules for Inservice Inspection of } \\
\text { Nuclear Power Plant Components }\end{array}$ & nureg0933, 3.10 & 1983 & A & & ASME Section XI & 1992 \\
\hline 2475 & ASME & \begin{tabular}{|l|} 
ASME Section \\
$X I$
\end{tabular} & $\mathrm{~N} / \mathrm{S}$ & $\begin{array}{l}\text { Rules for Inservice Inspection of } \\
\text { Nuclear Fower Plant Components }\end{array}$ & nureg0933, 3.111 & 1991 & $\mathbf{R}$ & & ASME Section XI & 1992 \\
\hline 2476 & ASME & $\begin{array}{l}\text { ASME Section } \\
X i\end{array}$ & 1974 & $\begin{array}{l}\text { Rules for Inservice Inspection of } \\
\text { Nuclear Power Plant Components }\end{array}$ & nureg0933, 3.127 & 1987 & $\mathbf{R}$ & & ASME Section XI & 1992 \\
\hline 2477 & ASME & $\begin{array}{l}\text { ASME Section } \\
X I\end{array}$ & $\mathrm{~N} / \mathrm{S}$ & $\begin{array}{l}\text { Pules for Inservice Inspecion of } \\
\text { Nuclear Power Plant Components }\end{array}$ & nureg0933, 3.67 & 1991 & $\mathbf{R}$ & & ASME Section XI & 1992 \\
\hline 2478 & ASME & $\begin{array}{l}\text { ASME Section } \\
x \mid\end{array}$ & N/S & $\begin{array}{l}\text { Rules for Inservice Inspection of } \\
\text { Nuclear Power Plant Components }\end{array}$ & nureg0933, 3.86 & 1988 & R & & ASME Section XI & 1902 \\
\hline 2479 & ASME & $\begin{array}{l}\text { ASME Section } \\
X I\end{array}$ & $\mathbf{N} / \mathrm{S}$ & $\begin{array}{l}\text { Rules for Inservice Inspection of } \\
\text { Nuclear Power Plent Components }\end{array}$ & reg1.116.roo & 1976 & $\mathbf{R}$ & & ASME Sec. XI & 1992 \\
\hline 2480 & ASME & $\begin{array}{l}\text { ASME Soction } \\
X I\end{array}$ & $\mathbf{N} / \mathbf{S}$ & $\begin{array}{l}\text { Rules for Inservice Inspection of } \\
\text { Nuclear Power Plant Components }\end{array}$ & reg1.137.ro1 & 1979 & $\mathbf{P}$ & & ASME Sec. X] & 1992 \\
\hline 2481 & ASME & $\begin{array}{l}\text { ASME Soction } \\
X I\end{array}$ & N/S & $\begin{array}{l}\text { Rules for Inservice Inspection of } \\
\text { Nuclear Power Plant Components }\end{array}$ & reg1.147.r10 & 1993 & $\mathbf{R}$ & & ASME Sec. XI & 1992 \\
\hline 2482 & ASME & $\begin{array}{l}\text { ASME Section } \\
X I\end{array}$ & 1977 & $\begin{array}{l}\text { Rules for Inservice Inspection of } \\
\text { Nuclear Power Plant Components }\end{array}$ & reg1.150.r01 & 1983 & $\mathbf{P}$ & & ASME Sec. XI & 1992 \\
\hline 2483 & ASME & $\begin{array}{l}\text { ASME Section } \\
\mathrm{XI}\end{array}$ & $\mathrm{N} / \mathrm{S}$ & $\begin{array}{l}\text { Rules for Inservice Inspection of } \\
\text { Nuclear Power Plant Components }\end{array}$ & reg1.28.r03 & 1985 & $\mathbf{R}$ & & ASME Sec. XI & 1992 \\
\hline 2484 & ASME & $\begin{array}{l}\text { ASME Section } \\
X I\end{array}$ & $\mathbf{N} / \mathrm{S}$ & $\begin{array}{l}\text { Rules for Inservice Inspection of } \\
\text { Nuclear Power Plant Components }\end{array}$ & reg1.35.r03 & 1990 & $\mathbf{P}$ & Cited for definition & ASME Sec. $X I$ & 1992 \\
\hline 2485 & ASME & \begin{tabular}{|l|} 
ASME Section \\
$x \leq 1$
\end{tabular} & $\mathrm{~N} / \mathrm{S}$ & $\begin{array}{l}\text { Rules for Inservice Inspaction of } \\
\text { Nuclear Power Plant Components }\end{array}$ & reg1.38.r02 & 1977 & $\mathbf{R}$ & & ASME Sec. XI & 1992 \\
\hline 2486 & ASME & \begin{tabular}{|l|} 
ASME Section \\
$X I$
\end{tabular} & $\mathrm{~N} / \mathrm{S}$ & $\begin{array}{l}\text { Rules for Inservice Inspection of } \\
\text { Nuclear Power Plant Components }\end{array}$ & reg1.44.100 & 1973 & $\mathbf{P}$ & & ASME Sec. XI & 1992 \\
\hline 2487 & ASME & $\begin{array}{l}\text { ASME Section } \\
X !\end{array}$ & $\mathrm{N} / \mathrm{S}$ & $\begin{array}{l}\text { Rules for Inservice Inspection of } \\
\text { Nuclear Power Plant Components }\end{array}$ & reg1.65.ro0 & 1973 & $P$ & & ASME Soc. XI & 1992 \\
\hline 2488 & ASME & $\begin{array}{l}\text { ASME Section } \\
X \mid\end{array}$ & N/S & $\begin{array}{l}\text { Rules for Inservice Inspection of } \\
\text { Nuclear Power Plant Components }\end{array}$ & reg1.68.ro2 & 1978 & $\mathbf{P}$ & & ASME Sec. XI & 1992 \\
\hline
\end{tabular}


Codes and Standards and Other Guidance Clted in Regulatory Documents

Part A - Industry Consensus Codes and Standards (continued)

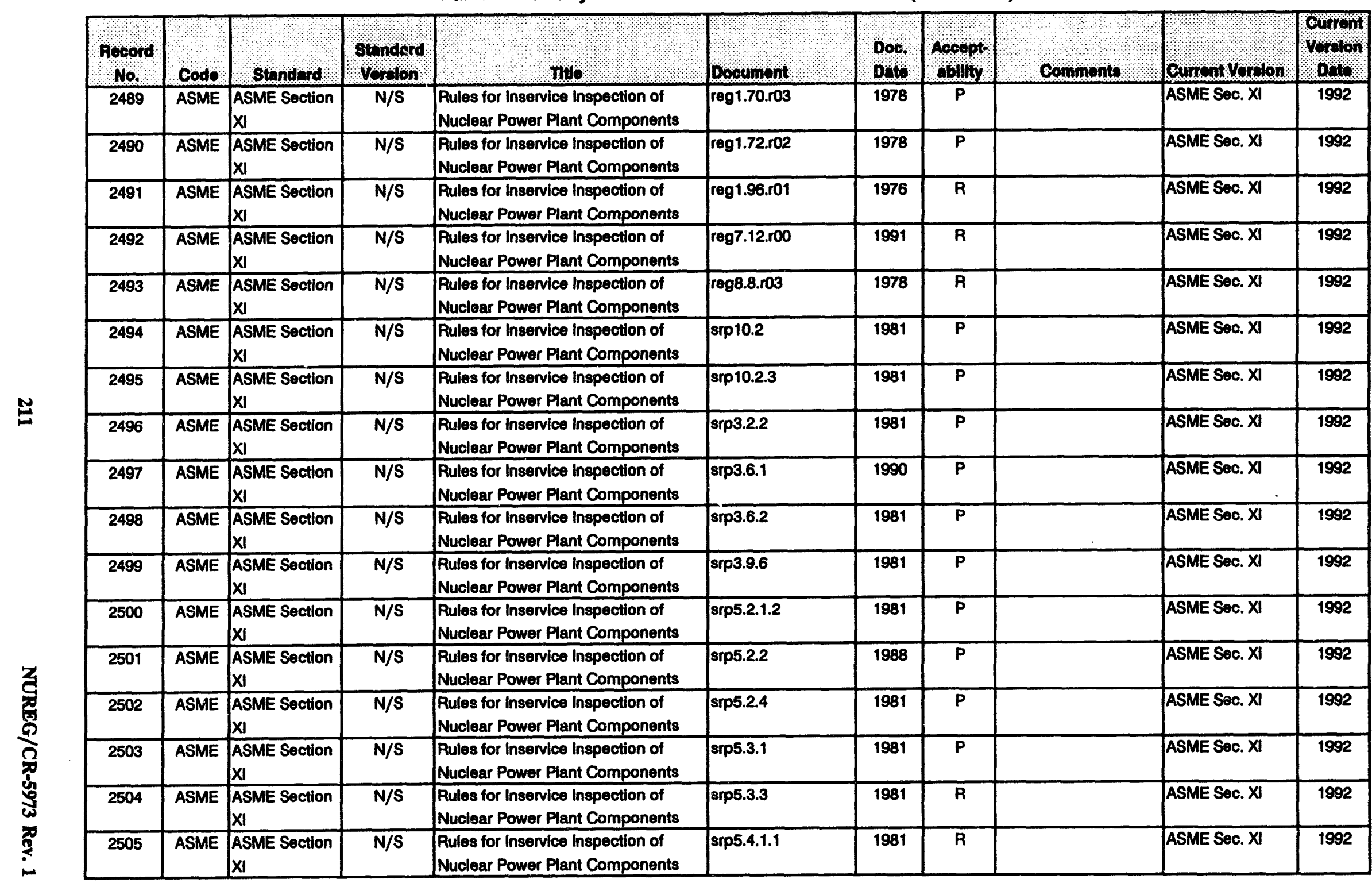


Codes and Standards and Other Guidance Cited in Regulatory Documents

Part A - Industry Consensus Codes and Standards (continued)

\begin{tabular}{|c|c|c|c|c|c|c|c|c|c|c|}
\hline Rocord & & & Strindord & nub & Booument & Doo. & Aocopt & somnentis: & Gulront vorion & Voron \\
\hline 2506 & ASME & $\begin{array}{l}\text { Standind } \\
\text { ASME Soction } \\
X \mathrm{X}\end{array}$ & N/S & $\begin{array}{l}\text { Rules for Inservice Inspection of } \\
\text { Nuclear Power Plant Components }\end{array}$ & srp6.6 & 1981 & $P$ & & ASME Sec. XI & 1992 \\
\hline 2507 & ASME & $\begin{array}{l}\text { ASME Soc. XI, } \\
\text { IWA-4000 }\end{array}$ & $N / S$ & Repair and Replacement & inm73051 & 1986 & $\mathbf{R}$ & & $\begin{array}{l}\text { ASME Sec. XI, } \\
\text { WA-4000 }\end{array}$ & 1992 \\
\hline 2508 & ASME & $\begin{array}{l}\text { ASME Section } \\
X I\end{array}$ & $\mathrm{~N} / \mathrm{S}$ & $\begin{array}{l}\text { Rules for Inservice Inspection of } \\
\text { Nuclear Power Plant Components }\end{array}$ & sip9.1.3 & 1981 & $\bar{R}$ & & ASME Sec. XI & 1992 \\
\hline 2509 & ASME & $\begin{array}{l}\text { ASME Section } \\
X I\end{array}$ & $N / S$ & $\begin{array}{l}\text { Rules for Inservice Inspection of } \\
\text { Nuclear Power Plant Components }\end{array}$ & sts4-ge5.1 & 1991 & $\mathbf{P}$ & & ASME Sec. XI & 1992 \\
\hline 2510 & ASME & $\begin{array}{l}\text { ASME Section } \\
X I\end{array}$ & $\mathrm{~N} / \mathrm{S}$ & $\begin{array}{l}\text { Rules for Inservice Inspection of } \\
\text { Nuclear Power Plant Components }\end{array}$ & sts4-geB3.10.1 & 1991 & $\mathbf{R}$ & & ASME Sec. XI & 1992 \\
\hline 2511 & ASME & $\begin{array}{l}\text { ASME Section } \\
X I\end{array}$ & $\mathrm{~N} / \mathrm{S}$ & $\begin{array}{l}\text { Rules for Inservice Inspection of } \\
\text { Nuclear Power Plant Components }\end{array}$ & sts4-geB3.5.1 & 1991 & $\bar{P}$ & & ASME SeC. XI & 1992 \\
\hline 2512 & ASME & $\begin{array}{l}\text { ASME Section } \\
\mathrm{XI}\end{array}$ & $\mathrm{N} / \mathrm{S}$ & $\begin{array}{l}\text { Rules for Inservice Inspection of } \\
\text { Nuclear Power Plant Components }\end{array}$ & sts4-geB3.6.1.6 & 1991 & $\mathbf{R}$ & & ASME Sec. $X$ & 1992 \\
\hline 2513 & ASME & \begin{tabular}{|l} 
ASME Section \\
$X I$
\end{tabular} & $N / S$ & $\begin{array}{l}\text { Rules for Inservice Inspection of } \\
\text { Nuclear Power Plant Components }\end{array}$ & st84-gøB3.6.2.3 & 1991 & $\bar{R}$ & & ASME Sec. XI & 1992 \\
\hline 2514 & ASME & $\begin{array}{l}\text { ASME Section } \\
X I\end{array}$ & $N / S$ & $\begin{array}{l}\text { Rules for Inservice Inspection of } \\
\text { Nuclear Power Plant Components }\end{array}$ & sts4-geB3.6.2.4 & 1991 & $R$ & & ASME SOC. XI & 1992 \\
\hline 2515 & ASME & $\begin{array}{l}\text { ASME Section } \\
\mathrm{XI}\end{array}$ & $\mathrm{N} / \mathrm{S}$ & $\begin{array}{l}\text { Rules for Inservice Inspection of } \\
\text { Nuclear Power Plant Components }\end{array}$ & sts4-geB3.8.1 & 1991 & $\mathbf{R}$ & & ASME Sec. XI & 1992 \\
\hline 2516 & ASME & ASME Section & $N / S$ & $\begin{array}{l}\text { Rules for Inservice Inspection of } \\
\text { Nuclear Power Plant Components }\end{array}$ & st84-geB3.8.3 & 1991 & $\mathbf{R}$ & & ASME Sec. XI & 1992 \\
\hline 2517 & ASME & $\begin{array}{l}\text { ASME Section } \\
X i\end{array}$ & $\mathrm{~N} / \mathrm{S}$ & $\begin{array}{l}\text { Rules for Inservice Inspection of } \\
\text { Nuclear Power Plant Components }\end{array}$ & sts6-ge5.1 & 1991 & $\mathbf{P}$ & & ASME Sec. XI & 1992 \\
\hline 2518 & ASME & $\begin{array}{l}\text { ASME Section } \\
X I\end{array}$ & $N / S$ & $\begin{array}{l}\text { Rules for Inservice inspection of } \\
\text { Nuclear Power Plant Components }\end{array}$ & sts6-geB3.10.1 & 1991 & $\mathbf{R}$ & & ASME SeC. XI & 1992 \\
\hline 2519 & ASME & $\begin{array}{l}\text { ASME Section } \\
X I\end{array}$ & $\mathrm{~N} / \mathrm{S}$ & $\begin{array}{l}\text { Rules for Inservice Inspection of } \\
\text { Nuclear Power Plant Components }\end{array}$ & sts6-geB3.5.1 & 1991 & $\mathbf{P}$ & & ASME Sec. XI & 1992 \\
\hline 2520 & ASME & $\begin{array}{l}\text { ASME Section } \\
X I\end{array}$ & N/S & $\begin{array}{l}\text { Rules for Inservice Inspection of } \\
\text { Nuclear Power Plant Components }\end{array}$ & sts6-geB3.6.1.6 & 1991 & $\mathbf{R}$ & & ASME Sec. XI & 1992 \\
\hline 2521 & ASME & \begin{tabular}{|l|} 
ASME Section \\
$X I$
\end{tabular} & $N / S$ & $\begin{array}{l}\text { Rules for Inservice inspection of } \\
\text { Nuclear Power Plant Components }\end{array}$ & sts6-geB3.6.1.7 & 1991 & $R$ & & ASME SeC. XI & 1992 \\
\hline 2522 & ASME & $\begin{array}{l}\text { ASME Section } \\
X I\end{array}$ & $\mathrm{~N} / \mathrm{S}$ & $\begin{array}{l}\text { Rules for Inservice inspection of } \\
\text { Nuclear Power Plant Components }\end{array}$ & sts6-geB3.6.2.3 & 1991 & R & & ASME Sec. XI & 1992 \\
\hline
\end{tabular}


Codes and Standards and Other Guidance Cited in Regulatory Documents

Part A - Industry Consensus Codes and Standards (continued)

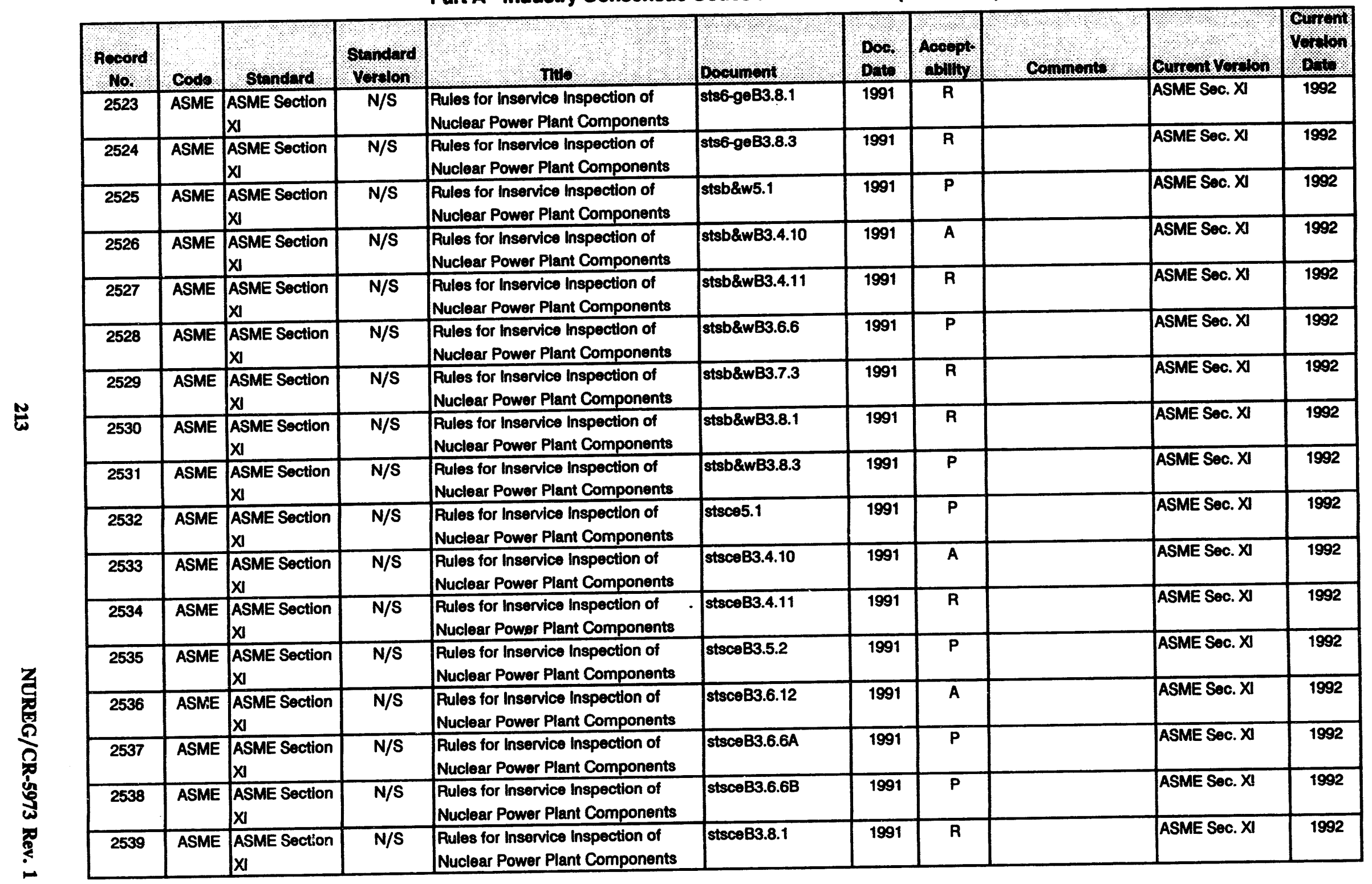


Codes and Standards and Other Guidance Cited in Regulatory Documents

Part A - Industry Consensus Codes and Standards (continued)

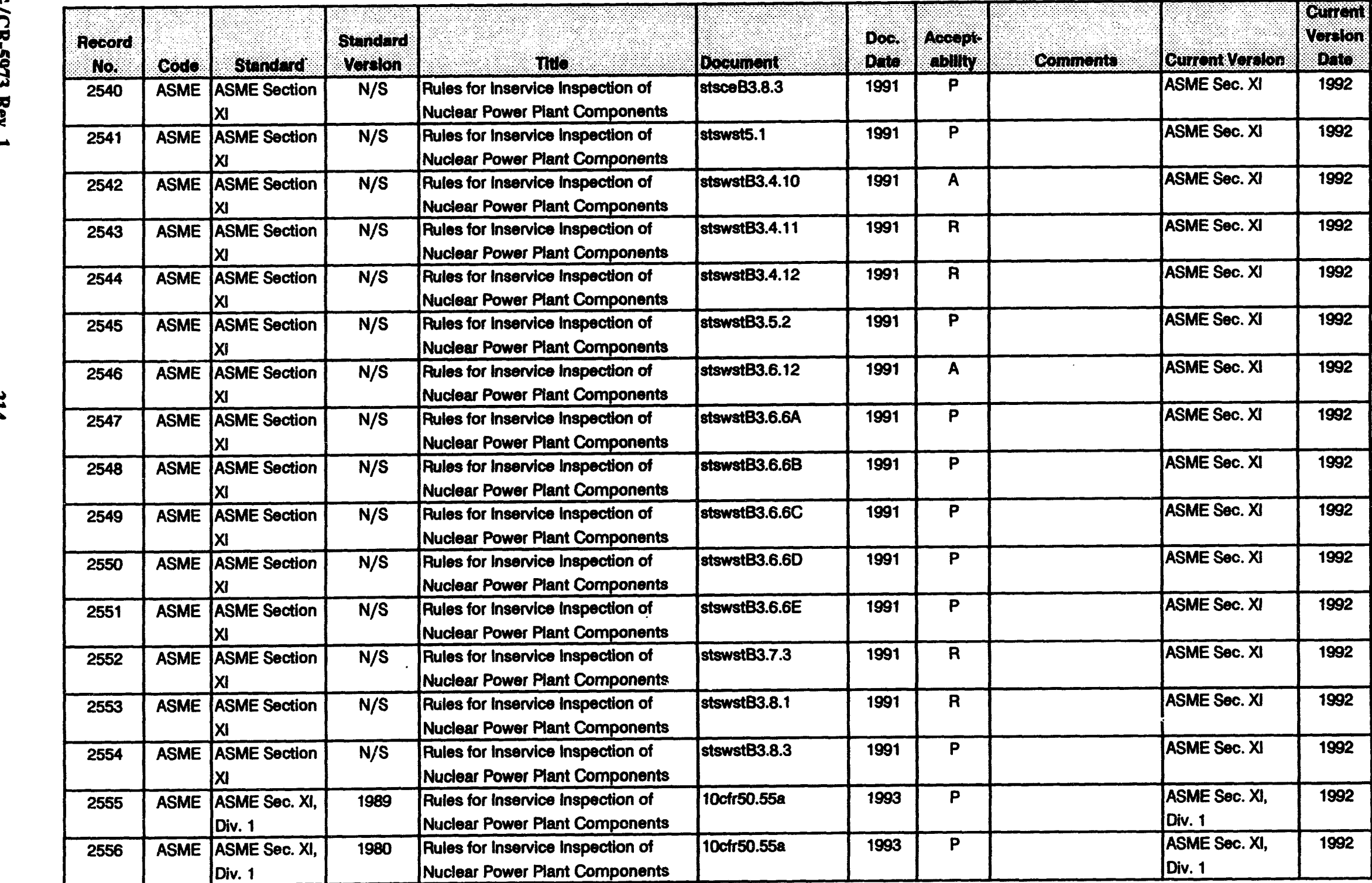


Codes and Standards and Other Guidance Clted in Regulatory Documents

Part A - Industry Consensus Codes and Standards (continued)

\begin{tabular}{|c|c|c|c|c|c|c|c|c|c|c|}
\hline 2557 & ASME & $\begin{array}{l}\text { ASME Sec. XI, } \\
\text { Appendix A }\end{array}$ & N/S & Analysis of Flaws & glt88-001 & 1988 & R & & $\begin{array}{l}\text { ASME Sec. XI, } \\
\text { Appendix A }\end{array}$ & 1992 \\
\hline 2558 & ASME & $\begin{array}{l}\text { ASME Sec. XI, } \\
\text { Appendix E }\end{array}$ & $\mathrm{N} / \mathrm{S}$ & $\begin{array}{l}\text { Evaluation of Unanticipated } \\
\text { Operating Events }\end{array}$ & sts4-geB3.4.10 & 1991 & $\bar{P}$ & & $\begin{array}{l}\text { ASME Sec. XI, } \\
\text { Appendix E }\end{array}$ & 1992 \\
\hline 2559 & ASME & $\begin{array}{l}\text { ASME Sec. XI, } \\
\text { Appendix E }\end{array}$ & $N / S$ & $\begin{array}{l}\text { Evaluation of Unanticipated } \\
\text { Operating Events }\end{array}$ & sts6-geB3.4.11 & 1991 & $\mathbf{P}$ & & $\begin{array}{l}\text { ASME Sec. XI, } \\
\text { Appendix E }\end{array}$ & 1992 \\
\hline 2560 & ASME & $\begin{array}{l}\text { ASME Sec. XI, } \\
\text { Appendix E }\end{array}$ & $N / S$ & $\begin{array}{l}\text { Evaluation of Unanticipated } \\
\text { Operating Events }\end{array}$ & stsb\&wB3.4.3 & 1991 & $\mathbf{P}$ & & $\begin{array}{l}\text { ASME Sec. XI, } \\
\text { Appendix } \mathrm{E}\end{array}$ & 1992 \\
\hline 2561 & ASME & $\begin{array}{l}\text { ASME Sec. XI, } \\
\text { Appendix E }\end{array}$ & $\mathbf{N} / \mathbf{S}$ & $\begin{array}{l}\text { Evaluation of Unanticipated } \\
\text { Operating Events }\end{array}$ & stsceB3.4.3 & 1991 & $\mathbf{P}$ & & $\begin{array}{l}\text { ASME Sec. XI, } \\
\text { Appendix E }\end{array}$ & 1992 \\
\hline 2562 & ASME & $\begin{array}{l}\text { ASME Sec. XI, } \\
\text { Appendix E }\end{array}$ & $\mathbf{N} / \mathrm{S}$ & $\begin{array}{l}\text { Evaluation of Unanticipated } \\
\text { Operating Events }\end{array}$ & stswstB3.4.3 & 1991 & $\mathbf{P}$ & & $\begin{array}{l}\text { ASME Sec. XI, } \\
\text { Appendix E }\end{array}$ & 1992 \\
\hline 2563 & ASME & $\begin{array}{l}\text { ASME Sec. XI, } \\
\text { Appendix I }\end{array}$ & 1974 & Ultrasonic Examinations & bul75-01 & 1975 & $\mathbf{P}$ & & $\begin{array}{l}\text { ASME Sec. XI, } \\
\text { Appendix I }\end{array}$ & 1992 \\
\hline 2564 & ASME & $\begin{array}{l}\text { ASME Sec. XI, } \\
\text { Appendix I }\end{array}$ & $N / S$ & Ultrasonic Examinations & reg1.150.r01 & 1983 & $\mathbf{P}$ & & $\begin{array}{l}\text { ASME Sec. XI, } \\
\text { Appendix I }\end{array}$ & 1992 \\
\hline 2565 & ASME & $\begin{array}{l}\text { ASME Sec. XI, } \\
\text { Appendix I } \\
\text { Article I, I-4420 }\end{array}$ & 1974 & Not found & reg1.150.ro1 & 1983 & $\mathbf{R}$ & $\begin{array}{l}\text { Deleted from ASME } \\
\text { Sec XI prior to } 1980 \\
\text { Edition }\end{array}$ & $n / a$ & $n / a$ \\
\hline 2566 & ASME & \begin{tabular}{|l|} 
ASME Sec. XI, \\
Table IS-251
\end{tabular} & N/S & Not found & bul74-03 & 1974 & R & $\begin{array}{l}\text { Typo in bul74-03 - } \\
\text { Table IS-251 is not a } \\
\text { valid ASME reference }\end{array}$ & $n / a$ & $n / a$ \\
\hline 2567 & ASME & \begin{tabular}{|l|} 
ASME Sec. XI, \\
Article $I, \mid-4410$
\end{tabular} & $N / S$ & $\begin{array}{l}\text { Design Stress Intensity Values, } \\
\text { Allowable Stresses, Material } \\
\text { Properties, and Design Fatigue } \\
\text { Curves }\end{array}$ & reg1.150.r01 & 1983 & $\mathbf{R}$ & & $\begin{array}{l}\text { ASME Sec. XI, } \\
\text { Article I, } 1-4410\end{array}$ & 1992 \\
\hline 2568 & ASME & $\begin{array}{l}\text { ASME Sec. XI, } \\
\text { Article I, l-4510 }\end{array}$ & $N / S$ & $\begin{array}{l}\text { Design Stress Intensity Values, } \\
\text { Allowable Stresses, Material } \\
\text { Properties, and Design Fatigue } \\
\text { Curves }\end{array}$ & reg1.150.ro1 & 1983 & $\mathbf{R}$ & & $\begin{array}{l}\text { ASME Sec. XI, } \\
\text { Article } 1,1-4510\end{array}$ & 1992 \\
\hline 2569 & ASME & \begin{tabular}{|l|} 
ASME Sec. XI, \\
Article $1, \mid-4520$
\end{tabular} & 1974 & $\begin{array}{l}\text { Design Stress Intensity Values, } \\
\text { Allowable Stresses, Material } \\
\text { Properties, and Design Fatigue } \\
\text { Curves }\end{array}$ & reg1.150.ro1 & 1983 & $\mathbf{R}$ & & $\begin{array}{l}\text { ASME Sec. XI, } \\
\text { Article } \quad I, 1-4520\end{array}$ & 1992 \\
\hline
\end{tabular}


Codes and Standards and Other Guidance Clted in Regulatory Documents

Part A - Industry Consensus Codes and Standards (continued)

\begin{tabular}{|c|c|c|c|c|c|c|c|c|c|c|}
\hline Hocord & Code & & Storndard & nut & Dooument & Doo. & Asoppt & Comments & Gurrent Vorlon & Qthot \\
\hline 2570 & ASME & $\begin{array}{l}\text { ASME Sec. XI, } \\
\text { IWA-1400 }\end{array}$ & $N / S$ & Owner's Responsibility & not93-21 & 1993 & $\mathbf{R}$ & & $\begin{array}{l}\text { ASME Sec. XI, } \\
\text { IWA-1400 }\end{array}$ & 1992 \\
\hline 2571 & ASME & $\begin{array}{l}\text { ASME Sec. XI, } \\
\text { IWA-1500 }\end{array}$ & $\mathrm{N} / \mathrm{S}$ & Accessibility & srp5.2.4 & 1981 & $\mathbf{P}$ & & $\begin{array}{l}\text { ASME Sec. XI, } \\
\text { WWA-1500 } \\
\end{array}$ & 1992 \\
\hline 2572 & ASME & $\begin{array}{l}\text { ASME Sec. XI, } \\
\text { IWA-1500 }\end{array}$ & $\mathrm{N} / \mathrm{S}$ & Accessibility & sip6.6 & 1981 & $\mathbf{P}$ & & $\begin{array}{l}\text { ASME Soc. XI, } \\
\text { WWA-1500 }\end{array}$ & 1992 \\
\hline 2573 & ASME & $\begin{array}{l}\text { ASME SOC. XI, } \\
\text { WWA-2000 }\end{array}$ & $\mathrm{N} / \mathrm{S}$ & Examination and Inspection & bul79-17.r01 & 1979 & $\bar{P}$ & & $\begin{array}{l}\text { ASME SeC. XI, } \\
\text { IWA-2000 }\end{array}$ & 1992 \\
\hline 2574 & ASME & $\begin{array}{l}\text { ASME SeC. XI, } \\
\text { IWA-2000 }\end{array}$ & $\mathrm{N} / \mathrm{S}$ & Examination and Inspection & reg1.150.ro1 & 1983 & $\mathbf{P}$ & General endorsements & $\begin{array}{l}\text { ASME Sec. XI, } \\
\text { IWA-2000 }\end{array}$ & 1992 \\
\hline 2575 & ASME & $\begin{array}{l}\text { ASME Sec. XI, } \\
\text { IWA-2000 }\end{array}$ & $\mathrm{N} / \mathrm{S}$ & Examination and Inspection & srp5.2.4 & 1981 & $\bar{P}$ & & $\begin{array}{l}\text { ASME Sec. XI, } \\
\text { IWA-2000 }\end{array}$ & 1992 \\
\hline 2576 & ASME & $\begin{array}{l}\text { ASME Sec. XI, } \\
\text { IWA-2000 }\end{array}$ & $\mathrm{N} / \mathrm{S}$ & Examination and Inspection & sip6.6 & 1981 & $\bar{P}$ & & $\begin{array}{l}\text { ASME Sec. XI, } \\
\text { IWA-2000 }\end{array}$ & $\overline{1992}$ \\
\hline 2577 & ASME & $\begin{array}{l}\text { ASME Sec. XI, } \\
\text { WA-2120 }\end{array}$ & $\mathrm{N} / \mathrm{S}$ & $\begin{array}{l}\text { Qualification of Authorized } \\
\text { Inspection Agencles, Inspectors, } \\
\text { and Supervisors }\end{array}$ & inm73051 & 1986 & $\mathbf{R}$ & & $\begin{array}{l}\text { ASME Sec. XI, } \\
\text { WWA-2120 }\end{array}$ & 1992 \\
\hline 2578 & ASME & $\begin{array}{l}\text { ASME SOC. XI, } \\
\text { IWA-2200 }\end{array}$ & $\mathrm{N} / \mathrm{S}$ & Examination Methods & reg1.14.ro1 & 1975 & $\mathbf{P}$ & & $\begin{array}{l}\text { ASME Sec. XI, } \\
\text { IWA-2200 }\end{array}$ & 1992 \\
\hline 2579 & ASME & $\begin{array}{l}\text { ASME Sec. XI, } \\
\text { WA-2210 }\end{array}$ & 1975 & Visual Examinations & bul79-17 & 1979 & $\mathbf{P}$ & 74 edition or later & $\begin{array}{l}\text { ASME Sec. XI, } \\
\text { WA-2210 } \\
\end{array}$ & 1992 \\
\hline 2580 & ASME & $\begin{array}{l}\text { ASME SeC. XI, } \\
\text { IWA-2210 }\end{array}$ & 1974 & Visual Examination & bul82-02 & 1982 & $\mathbf{P}$ & 74 edition or later & $\begin{array}{l}\text { ASME Sec. } X I, \\
\text { WWA-2210 }\end{array}$ & 1992 \\
\hline 2581 & ASME & $\begin{array}{l}\text { ASME Sec. XI, } \\
\text { WA-2220 }\end{array}$ & 1974 & Surface Examination & bul82-02 & 1982 & $\overline{\mathbf{P}}$ & 74 edition or later & $\begin{array}{l}\text { ASME SөC. XI, } \\
\text { IWA-2220 }\end{array}$ & 1992 \\
\hline 2582 & ASME & $\begin{array}{l}\text { ASME Sec. XI, } \\
\text { IWA-2240 }\end{array}$ & $\mathrm{N} / \mathrm{S}$ & Alternative Examinations & reg1.150.r01 & 1983 & $\bar{P}$ & Implied endorsement & $\begin{array}{l}\text { ASME Sec. XI, } \\
\text { WW-2240 }\end{array}$ & 1992 \\
\hline 2583 & ASME & $\begin{array}{l}\text { ASME SeC. XI, } \\
\text { WA-2400 }\end{array}$ & $\mathrm{N} / \mathrm{S}$ & Inspection Program & glt87-011 & 1987 & $\mathbf{R}$ & & $\begin{array}{l}\text { ASME Sec. XI, } \\
\text { WA-2400 }\end{array}$ & 1992 \\
\hline 2584 & ASME & $\begin{array}{l}\text { ASME SeC. XI, } \\
\text { IWA-2400 }\end{array}$ & $\mathrm{N} / \mathrm{S}$ & Inspection Program & srp3.6.2 & 1981 & $\bar{P}$ & & $\begin{array}{l}\text { ASME Soc. XI, } \\
\text { IWA-2400 }\end{array}$ & 1992 \\
\hline 2585 & ASME & $\begin{array}{l}\text { ASME Sec. XI, } \\
\text { IWA-2430(d) }\end{array}$ & 1989 & Inspection Intervals & $10 \mathrm{cfr} 50.55 \mathrm{a}$ & 1993 & $\mathbf{P}$ & & $\begin{array}{l}\text { ASME Sec. XI, } \\
\text { IWA-2430(d) }\end{array}$ & 1992 \\
\hline
\end{tabular}


Codes and Standards and Other Guidance Cited in Regulatory Documents

Part A - Industry Consensus Codes and Standards (continued)

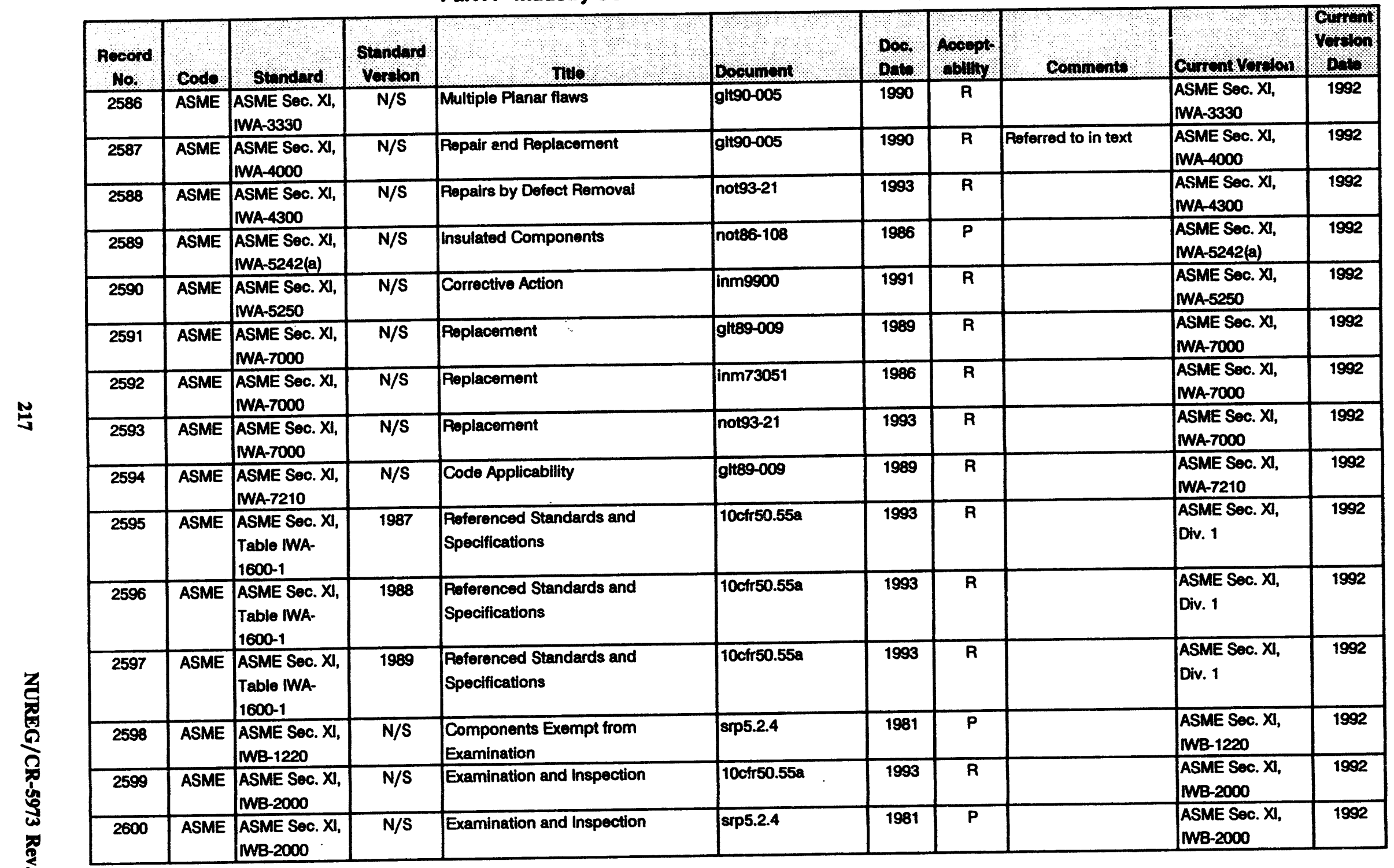




\begin{tabular}{|c|c|c|c|c|c|c|c|c|c|c|c|c|c|c|}
\hline శ్ & 务 & \$ू & & \% & 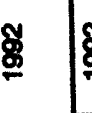 & \$్ & 总 & 8 & \% & รู & \%ू & $\%$ & gू & \% \\
\hline 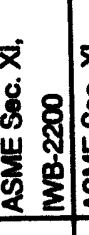 & 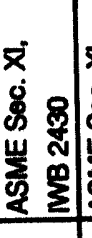 & 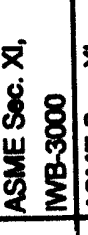 & 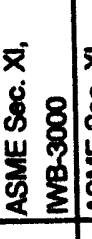 & 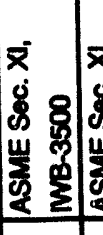 & 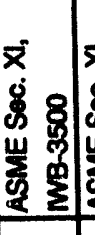 & 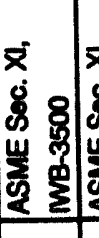 & 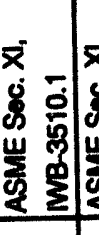 & 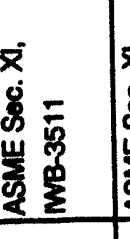 & 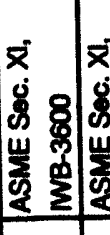 & 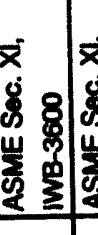 & 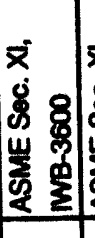 & 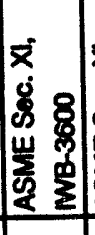 & 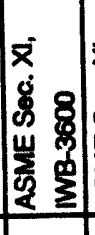 & 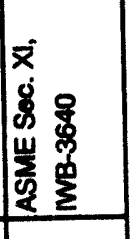 \\
\hline & & & & & & & 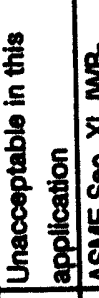 & 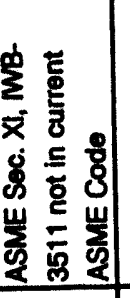 & & & & & 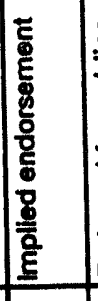 & 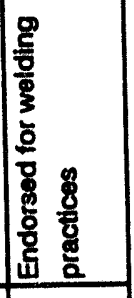 \\
\hline & a & a & a. & $a$ & $\boldsymbol{\alpha}$ & a & 匹 & $a$ & a & $\widetilde{\alpha}$ & $x$ & $\propto$ & $\widetilde{x}$ & a \\
\hline & 思 & $\overline{\$}$ & $\overline{\mathbf{g}}$ & $\not \$$ & $\overline{8}$ & 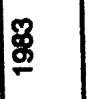 & $\%$ & $\ddot{8}$ & $\stackrel{8}{\%}$ & 8. & $\overline{8}$ & 㫣 & 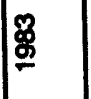 & 荈 \\
\hline مُ & 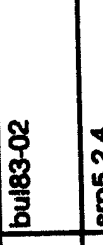 & 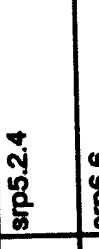 & 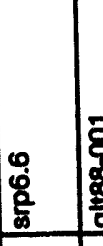 & \begin{tabular}{|}
$\overline{8}$ \\
$\vdots$ \\
兽 \\
\end{tabular} & & 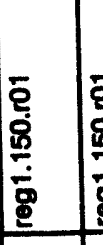 & 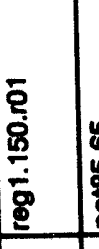 & 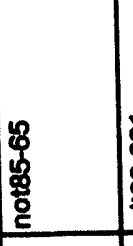 & 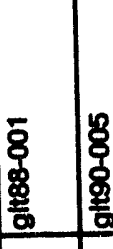 & & $\begin{array}{l}8 \\
8 \\
. \\
E \\
\end{array}$ & 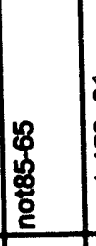 & 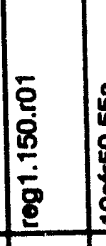 & 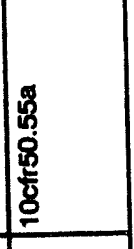 \\
\hline 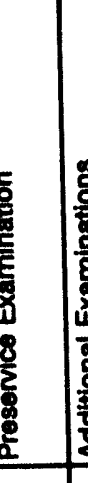 & 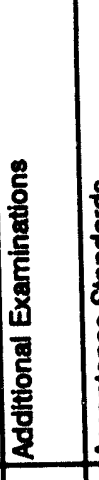 & 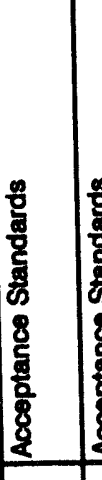 & 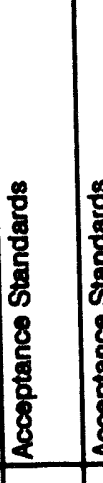 & 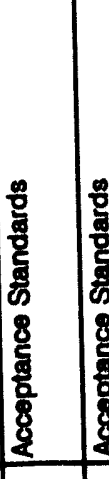 & 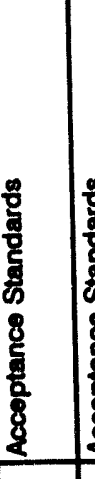 & 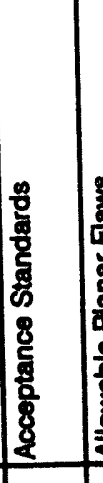 & 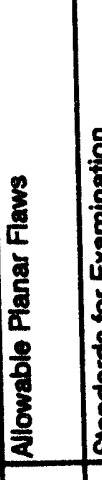 & 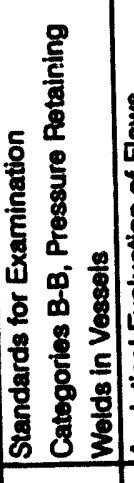 & 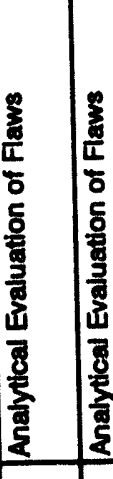 & 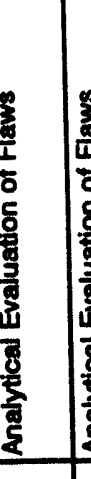 & 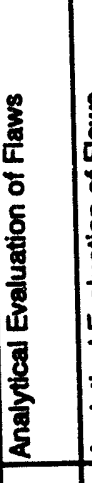 & 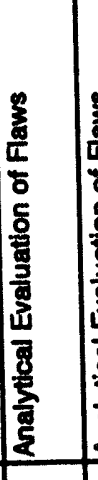 & 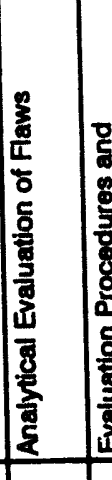 & 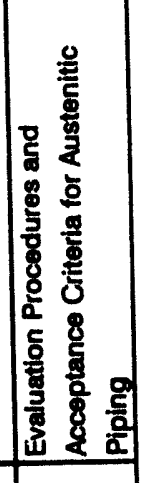 \\
\hline$\sum^{\infty}$ & $\frac{\infty}{z}$ & $\frac{\infty}{z}$ & $\frac{n}{z}$ & $\frac{n}{z}$ & $\frac{n}{z}$ & $\frac{n}{z}$ & $\frac{\infty}{z}$ & $\frac{n}{z}$ & ఖ & $\frac{\rho}{2}$ & $\frac{n}{z}$ & $\frac{\infty}{z}$ & $\frac{\infty}{z}$ & \$ \\
\hline 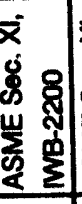 & 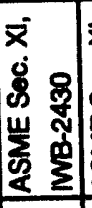 & 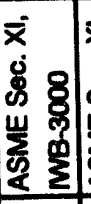 & 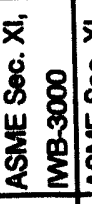 & 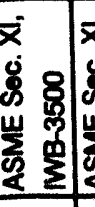 & 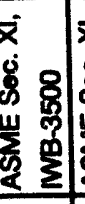 & 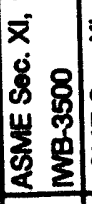 & 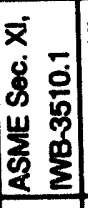 & 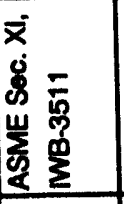 & 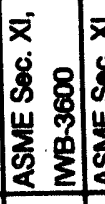 & 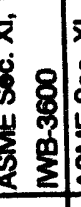 & 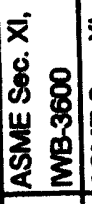 & 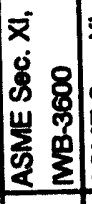 & 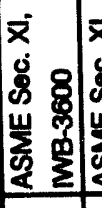 & 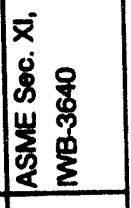 \\
\hline 岁 & 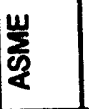 & 㑩 & 岁 & एँ & 岁 & 岁 & 烦 & 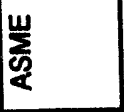 & $\mid$ & 崖 & $\sum_{\frac{W}{Q}}^{\mathbf{W}}$ & 㩿 & 岁 & $\mid \begin{array}{l}\frac{w}{2} \\
\frac{W}{2}\end{array}$ \\
\hline 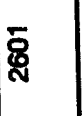 & ర్ర్ర్ & 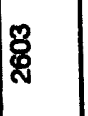 & 若 & 莕 & ष్ర & ఫ్ట్ & ఫ్ర్జ & 悉 & $\frac{0}{\bar{g}}$ & $\overline{\bar{E}}$ & $\frac{N}{\mathscr{N}}$ & $\frac{m}{\frac{m}{2}}$ & $\frac{\pi}{\mathscr{N}}$ & $\frac{n}{2}$ \\
\hline
\end{tabular}


Codes and Standards and Other Guidance Chted in Regulatory Documents

Part A - Industry Consensus Codes and Standards (continued)

\begin{tabular}{|c|c|c|c|c|c|c|c|c|c|c|}
\hline $\begin{array}{l}\text { Reoord } \\
\text { No. }\end{array}$ & Code & Standend & $\begin{array}{l}\text { Stonderd } \\
\text { Yordlon }\end{array}$ & 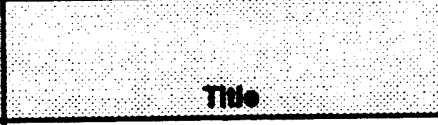 & Docounent & Doos & Aocopt. & Comments: & Cument Vomon & Corront \\
\hline 2616 & ASME & $\begin{array}{l}\text { ASME Sec. XI, } \\
\text { IWB-3640 }\end{array}$ & N/S & $\begin{array}{l}\text { Evaluation Procedures and } \\
\text { Acceptance Criteria for Austenitic } \\
\text { Piping }\end{array}$ & git88-001 & 1988 & $\mathbf{P}$ & & $\begin{array}{l}\text { ASME SeC. X, } \\
\text { WW-3640 }\end{array}$ & 1992 \\
\hline 2617 & ASME & $\begin{array}{l}\text { ASME Sec. XI, } \\
\text { WB-3640 }\end{array}$ & $\mathbf{N} / \mathbf{S}$ & $\begin{array}{l}\text { Evaluation Procedures and } \\
\text { Acceptance Criteria for Austenitic } \\
\text { Piping }\end{array}$ & gite0-005 & 1990 & $\mathbf{R}$ & $\begin{array}{l}\text { For lower-bound } \\
\text { fracture toughness }\end{array}$ & $\begin{array}{l}\text { ASME SeC. XI, } \\
\text { WB-3640 }\end{array}$ & 1992 \\
\hline 2618 & ASME & $\begin{array}{l}\text { ASME Sec. XI, } \\
\text { WB-4000 }\end{array}$ & $\mathbf{N} / \mathbf{S}$ & Repair Procedures & srp5.2.4 & 1981 & $\mathbf{P}$ & & $\begin{array}{l}\text { ASME SeC. XI, } \\
\text { MB-4000 }\end{array}$ & 1992 \\
\hline 2619 & ASME & $\begin{array}{l}\text { ASME Sec. XI, } \\
\text { WB-5000 }\end{array}$ & $\mathbf{N} / \mathbf{S}$ & System Pressure Tests & $\operatorname{srp5.2.4}$ & 1981 & $\mathbf{P}$ & & $\begin{array}{l}\text { ASME Soc. } X \text {, } \\
\text { MB-5000 }\end{array}$ & 1992 \\
\hline 2620 & ASME & $\begin{array}{l}\text { ASME Sec. XI, } \\
\text { Table MB- } \\
2500-1\end{array}$ & 1989 & Examination Categories & $10 \mathrm{ctr} 50.55 \mathrm{a}$ & 1993 & $\mathbf{A}$ & & $\begin{array}{l}\text { ASME Soc. XI, } \\
\text { Div. } 1\end{array}$ & 1992 \\
\hline 2621 & ASME & $\begin{array}{l}\text { ASME Sec. XI, } \\
\text { Table MB- } \\
2500-1 \\
\end{array}$ & $\mathbf{N} / \mathbf{S}$ & Examination Categories & but82-02 & 1982 & $\mathbf{R}$ & & $\begin{array}{l}\text { ASME Sec. XI, } \\
\text { Table WB-2500-1 }\end{array}$ & 1992 \\
\hline 2622 & ASME & $\begin{array}{l}\text { ASME Sec. XI, } \\
\text { Table MB- } \\
2500-1 \\
\end{array}$ & $\mathbf{N} / \mathbf{S}$ & Examination Categories & reg1.147.r10 & 1993 & $\mathbf{R}$ & & $\begin{array}{l}\text { ASME Sec. XI, } \\
\text { Table MB-2500-1 }\end{array}$ & 1992 \\
\hline 2623 & ASME & $\begin{array}{l}\text { ASME Sec. XI, } \\
\text { Table MB- } \\
2600 \\
\end{array}$ & 1974 & Not found & $10 c f r 50.55 a$ & 1993 & $\mathbf{P}$ & $\begin{array}{l}\text { ASME Sec. XI, Table } \\
\text { WB-2600 not in } \\
\text { current ASME Code }\end{array}$ & $n / a$ & $n / a$ \\
\hline 2624 & ASME & $\begin{array}{l}\text { ASME Soc. XI, } \\
\text { Table MB- } \\
3510-1\end{array}$ & $\mathbf{N} / \mathbf{S}$ & Allowable Planar Indications & drg7002.r00 & 1989 & $\mathbf{R}$ & & $\begin{array}{l}\text { ASME Sec. XI, } \\
\text { Table WB-3510-1 }\end{array}$ & 1992 \\
\hline 2625 & ASME & $\begin{array}{l}\text { ASME Sec. XI, } \\
\text { Table MB- } \\
3511-1 \\
\end{array}$ & $\mathbf{N} / \mathbf{S}$ & Allowable Planar Indicationis & not90-04 & 1990 & $\mathbf{R}$ & $\begin{array}{l}\text { ASME Sec. XI, Table } \\
\text { mB-3511-1 not in } \\
\text { current ASME Code }\end{array}$ & $n / a$ & $n / a$ \\
\hline 2626 & ASME & $\begin{array}{l}\text { ASME Sec. XI, } \\
\text { Tables WB- } \\
2500 \text { and MB- } \\
2500-1\end{array}$ & 1975 & Examination Categories & $10 c t r 50.55 a$ & 1993 & $\mathbf{P}$ & & $\begin{array}{l}\text { ASME Sec. XI, } \\
\text { Tables WB-2500 } \\
\text { and MB-2500-1 }\end{array}$ & 1992 \\
\hline 2627 & ASME & $\begin{array}{l}\text { ASME Soc. XI, } \\
\text { WC-1220 }\end{array}$ & 1975 & $\begin{array}{l}\text { Components Exempt from } \\
\text { Examination }\end{array}$ & $10 c t r 50.55 a$ & 1993 & $\mathbf{P}$ & & $\begin{array}{l}\text { ASME Sec. X, } \\
\text { MC-1220 }\end{array}$ & 1992 \\
\hline
\end{tabular}




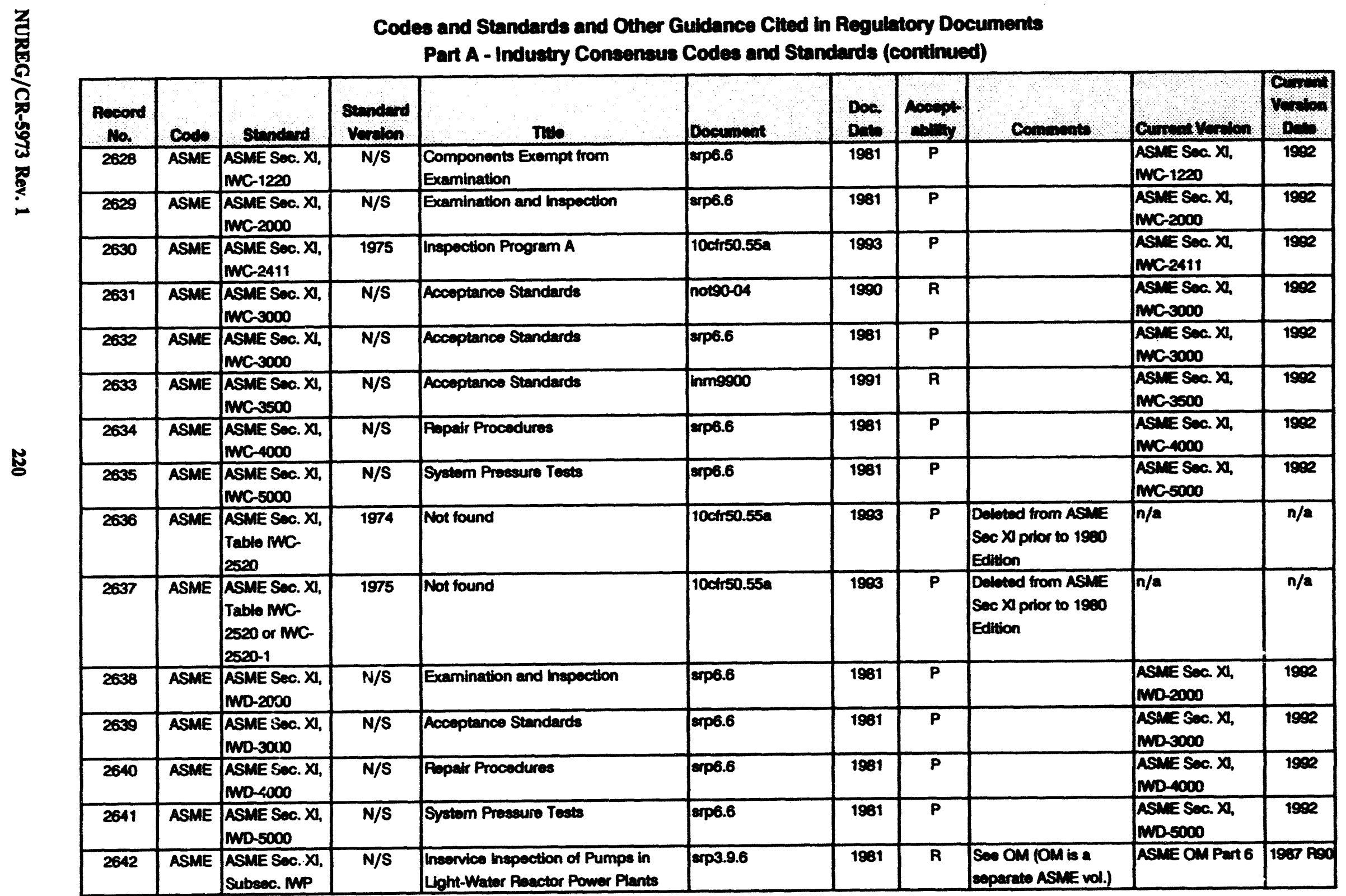




\section{Codes and Standards and Other Guidance Clted in Regulatory Documents}

Part A - Industry Consensus Codes and Standards (continued)

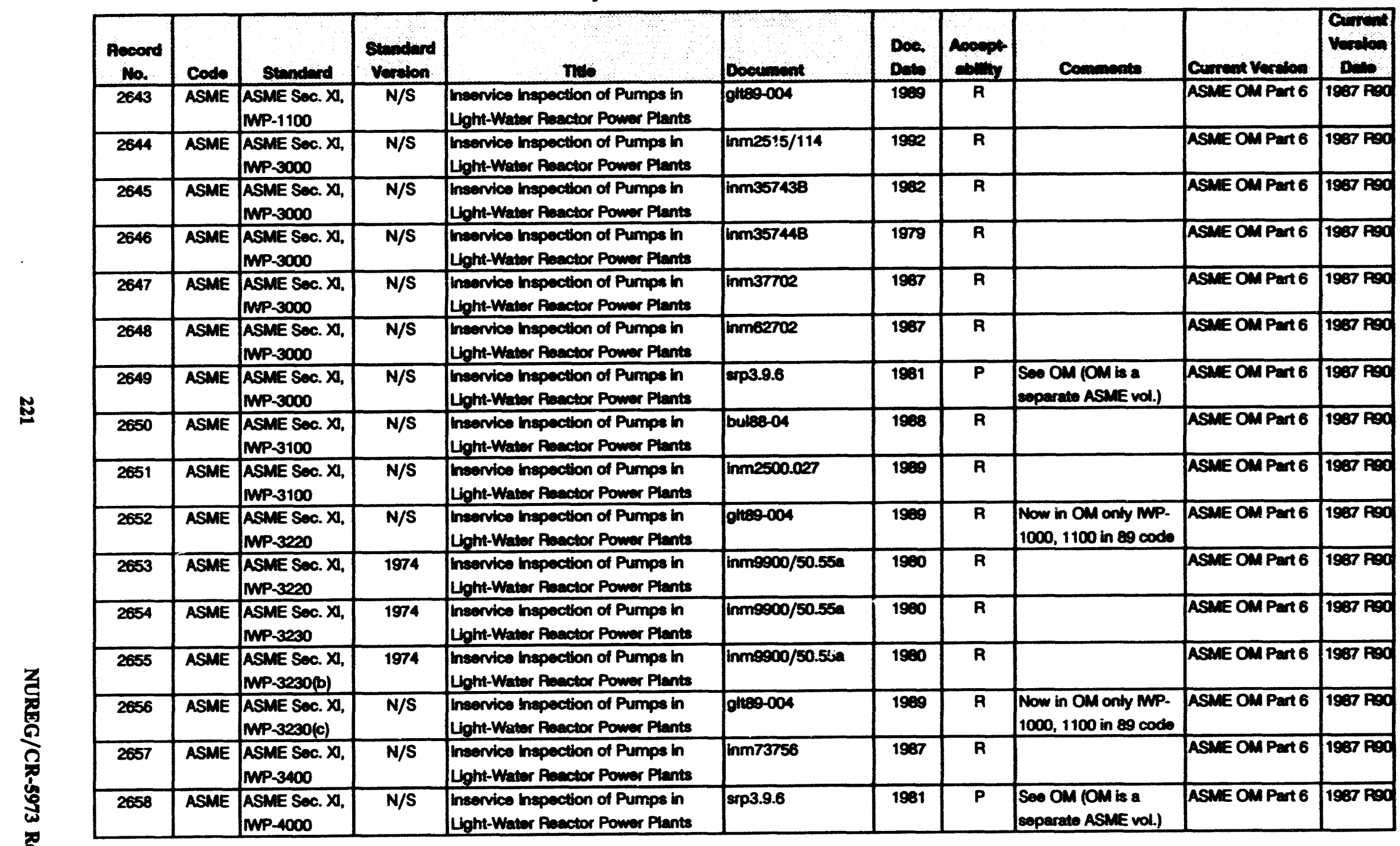


Codes and Standards and Other Guidance CHed in Regulatory Documents

Part A - Industry Consensus Codes and Standards (continued)

\begin{tabular}{|c|c|c|c|c|c|c|c|c|c|c|}
\hline $\begin{array}{l}\text { Aepord } \\
\text { No. }\end{array}$ & Cods & Standand & $\begin{array}{l}\text { Standard } \\
\text { Verclon }\end{array}$ & This & Doevinent & Doe. & $\begin{array}{l}\text { Acopt } \\
\text { cinst }\end{array}$ & Comnents: & Comout $V_{n=10 a}$ & $\begin{array}{l}0,1 \\
v_{n \rightarrow \infty}\end{array}$ \\
\hline 2659 & ASME & $\begin{array}{l}\text { ASME Sec. XI, } \\
\text { IWP-4000 } \\
\text { through }-4400\end{array}$ & $N / S$ & $\begin{array}{l}\text { Inservice Inspection of Pumps in } \\
\text { Light-Water Peactor Power Plants }\end{array}$ & inm73756 & 1987 & $\mathbf{R}$ & & ASME OM Part 6 & 1987 PQO \\
\hline 2660 & ASME & $\begin{array}{l}\text { ASME SOC. XI, } \\
\text { MP-4110 }\end{array}$ & $\mathbf{N} / \mathbf{S}$ & $\begin{array}{l}\text { Inservice Inspection of Pumps in } \\
\text { Light-Water Peactor Power Plants }\end{array}$ & glt89-004 & 1989 & $\mathbf{P}$ & $\begin{array}{l}\text { Now in OM only MP. } \\
1000,1100 \text { listed in } 89 \\
\text { code }\end{array}$ & ASME OM Part 6 & 1987 ROD \\
\hline 2661 & ASME & $\begin{array}{l}\text { ASME SOC. XI, } \\
\text { IWP-5000 }\end{array}$ & $\mathbf{N} / \mathbf{S}$ & $\begin{array}{l}\text { inservice Inspection of Pumps in } \\
\text { Light-Water Poactor Power Plants }\end{array}$ & inm70370 & 1987 & $\mathbf{R}$ & & ASME OM Part 6 & 1987 R90 \\
\hline 2662 & ASME & $\begin{array}{l}\text { ASME Sec. XI, } \\
\text { MP-6000 }\end{array}$ & $\mathbf{N} / \mathbf{S}$ & $\begin{array}{l}\text { Inservice Inspection of Pumps in } \\
\text { Light-Water Peactor Power Plants }\end{array}$ & git89-004 & 1989 & $\mathbf{R}$ & $\begin{array}{l}\text { Now in OM only MP. } \\
1000,1100 \text { in } 89 \text { code }\end{array}$ & ASME OM Part 6 & $1987 \mathrm{RPC}$ \\
\hline 2663 & ASME & $\begin{array}{l}\text { ASME SeC. } X, \\
\text { WP- } 6000\end{array}$ & $N / S$ & $\begin{array}{l}\text { Inservice inspection of Pumps in } \\
\text { Light-Water Peactor Power Plants }\end{array}$ & inm2515/114 & 1992 & $\mathbf{A}$ & & ASME OM Part 6 & 1987 PSO \\
\hline 2664 & ASME & $\begin{array}{l}\text { ASME Sec. XI, } \\
\text { WW-6000 }\end{array}$ & $N / S$ & $\begin{array}{l}\text { Inservice hspection of Pumps in } \\
\text { Uight-Water Reector Power Plants }\end{array}$ & inm73756 & 1987 & $\mathbf{R}$ & & ASME OM Part 6 & $1987 \mathrm{PQO}$ \\
\hline 2665 & ASME & $\begin{array}{l}\text { ASME SeC. XI, } \\
\text { WP-6230 }\end{array}$ & 1974 & $\begin{array}{l}\text { Inservice Inspection of Pumps in } \\
\text { Light-Water Poactor Power Plants }\end{array}$ & inm9900/50.55a & 1980 & $\mathbf{R}$ & & ASME OM Part 6 & $1987 \mathrm{RPO}$ \\
\hline 2666 & ASME & $\begin{array}{l}\text { ASME Sec. XI, } \\
\text { Table MP. } \\
3100-1\end{array}$ & $\mathbf{N} / \mathbf{S}$ & $\begin{array}{l}\text { Inservice inspection of Pumps in } \\
\text { Light-Water Peactor Power Fants }\end{array}$ & glt89-004 & 1989 & $\mathbf{R}$ & & ASME OM Part 6 & 1987 RSO \\
\hline 2667 & ASME & $\begin{array}{l}\text { ASME Sec. XI, } \\
\text { Table MP. } \\
3100-1 \\
\end{array}$ & 1974 & $\begin{array}{l}\text { Inservice Inspection of Pumps in } \\
\text { Light-Water Peactor Power Plants }\end{array}$ & inm9900/50.55a & 1980 & $\mathbf{R}$ & & ASME OM Part 6 & 1987 POO \\
\hline 2668 & ASME & $\begin{array}{l}\text { ASME Sec. XI, } \\
\text { Table MP. } \\
3100-2\end{array}$ & $N / S$ & $\begin{array}{l}\text { Inservice Inspection of Pumps in } \\
\text { Light-Water Peactor Power Plants }\end{array}$ & gtt89-004 & 1989 & $\mathbf{A}$ & $\begin{array}{l}\text { Now in OM only WiP. } \\
1000,1100 \text { in } 89 \text { code }\end{array}$ & ASME OM Part 6 & 1987 PQO \\
\hline 2669 & ASME & $\begin{array}{l}\text { ASME Sec. XI, } \\
\text { Subsec. MW }\end{array}$ & 1989 & $\begin{array}{l}\text { Inservice Testing of Valves in Light- } \\
\text { Water Reactor Power Plants }\end{array}$ & $10 \mathrm{cfr} 50.55 a$ & 1993 & $\mathbf{P}$ & & ASME OM Part 10 & 1987 RQO \\
\hline 2670 & ASME & $\begin{array}{l}\text { ASME Sec. XI, } \\
\text { SubSec. } M W\end{array}$ & 1973 & $\begin{array}{l}\text { Inservice Testing of Valves in Light- } \\
\text { Water Reactor Power Plants }\end{array}$ & drgms926-4 & 1981 & 8 & & ASME OM Part 10 & 1987 POO \\
\hline 2671 & ASME & $\begin{array}{l}\text { ASME Sec. XI, } \\
\text { Table WP. } \\
3100-2\end{array}$ & 1974 & $\begin{array}{l}\text { Inservice Inspection of Pumps in } \\
\text { Light-Water Peactor Power Plants }\end{array}$ & inm $9900 / 50.55 a$ & 1980 & $\mathbf{R}$ & & ASME OM Part 6 & $1987 \mathrm{PQ0}$ \\
\hline 2672 & ASME & $\begin{array}{l}\text { ASME Sec. XI, } \\
\text { Subsec. MW }\end{array}$ & $N / S$ & $\begin{array}{l}\text { Inservice Testing of Valves in Light- } \\
\text { Water Reactor Power Plants }\end{array}$ & gits9-005 & 1990 & $\mathbf{R}$ & & ASME OM Part 10 & 1987 7200 \\
\hline
\end{tabular}


Codes and Standards and Other Guidance Cited in Regulatory Documents

Part A - Industry Consensus Codes and Standards (continued)

\begin{tabular}{|c|c|c|c|c|c|c|c|c|c|c|}
\hline $\begin{array}{c}\text { Resord } \\
\text { No. }\end{array}$ & Code & Standard & $\begin{array}{l}\text { Stindard } \\
\text { Vorston }\end{array}$ & Tho,$\ldots$, & Dooumint & $\begin{array}{l}\text { Dos. } \\
\text { Dots. }\end{array}$ & Aocept & Comments & Curront Votols. & pormon \\
\hline 2673 & ASME & $\begin{array}{l}\text { ASME Sec. XI, } \\
\text { Subsec. } \mathrm{M} \text {, }\end{array}$ & $\mathrm{N} / \mathrm{S}$ & $\begin{array}{l}\text { Inservice Testing of Valves in Light- } \\
\text { Water Reactor Power Plants }\end{array}$ & inm73756 & 1987 & $\mathbf{R}$ & & ASME OM Part 10 & $1987 \mathrm{RSO}$ \\
\hline 2674 & ASME & $\begin{array}{l}\text { ASME Sec. XI, } \\
\text { Subsec. MW }\end{array}$ & $\mathrm{N} / \mathrm{S}$ & $\begin{array}{l}\text { Inservice Testing of Valves in Light- } \\
\text { Water Reactor Power Plants }\end{array}$ & $\operatorname{sip} 3.9 .6$ & 1981 & $\mathbf{R}$ & $\begin{array}{l}\text { Seo OM (OM is a } \\
\text { separate ASME vol.) }\end{array}$ & ASME OM Part 10 & 1987 RSO \\
\hline 2675 & ASME & $\begin{array}{l}\text { ASME Soc. XI, } \\
\text { Subsec. MW }\end{array}$ & $\mathrm{N} / \mathrm{S}$ & $\begin{array}{l}\text { Inservice Testing of Valves in Light- } \\
\text { Water Reactor Power Plants }\end{array}$ & $\operatorname{srp5.4.12}$ & 1981 & $\mathbf{P}$ & & ASME OM Part 10 & $1987 \mathrm{RSO}$ \\
\hline 2676 & ASME & $\begin{array}{l}\text { ASME Sec. XI, } \\
\text { Subsec. MW }\end{array}$ & N/S & $\begin{array}{l}\text { Inservice Testing of Valves in Light- } \\
\text { Water Reactor Power Ptants }\end{array}$ & sts4-geB3.4.5 & 1991 & $R$ & & ASME OM Part 10 & $1987 \mathrm{R90}$ \\
\hline 2677 & ASME & $\begin{array}{l}\text { ASME Sec. XI, } \\
\text { Subsec. MW }\end{array}$ & N/S & $\begin{array}{l}\text { Inservice Testing of Valves in Light- } \\
\text { Water Reactor Power Plants }\end{array}$ & st86-geB3.4.6 & 1991 & $\mathbf{R}$ & & ASME OM Part 10 & 1987 R9O \\
\hline 2678 & ASME & $\begin{array}{l}\text { ASME Sec. XI, } \\
\text { Subsec. MW }\end{array}$ & N/S & $\begin{array}{l}\text { Inservice Testing of Valves in Light- } \\
\text { Water Reactor Power Plants }\end{array}$ & nureg0737 & 1980 & $\mathbf{P}$ & & ASME OM Part 10 & $1987 \mathrm{RSO}$ \\
\hline 2679 & ASME & $\begin{array}{l}\text { ASME Sec. } X, \\
\text { MV-1100 }\end{array}$ & $N / S$ & $\begin{array}{l}\text { Inservice Testing of Valves in Light- } \\
\text { Water Peactor Power Plants }\end{array}$ & ght89-004 & 1989 & $\mathbf{P}$ & General endorsements & ASME OM Part 10 & $1987 \mathrm{RPO}$ \\
\hline 2680 & ASME & $\begin{array}{l}\text { ASME Sec. Xl, } \\
\text { IWV-1110 }\end{array}$ & $\mathrm{N} / \mathrm{S}$ & $\begin{array}{l}\text { Inservice Testing of Valves in Light- } \\
\text { Water Reactor Power Plants }\end{array}$ & $\operatorname{sip} 3.9 .6$ & 1981 & $\mathbf{P}$ & $\begin{array}{l}\text { With additional } \\
\text { requirements }\end{array}$ & ASME OM Part 10 & 1987 RSO \\
\hline 2681 & ASME & $\begin{array}{l}\text { ASME SoC. } X \mathrm{i} \\
M N-1300\end{array}$ & 1974 & $\begin{array}{l}\text { Inservice Testing of Valves in Light- } \\
\text { Water Reactor Power Plants }\end{array}$ & drgms901-4 & 1981 & $\bar{R}$ & & ASME OM Part 10 & 1987 RSO \\
\hline 2682 & ASME & $\begin{array}{l}\text { ASME Sec. XI, } \\
M V-1300 \\
\end{array}$ & 1977 & $\begin{array}{l}\text { Inservice Testing of Valves in Light- } \\
\text { Water Reactor Power Plants }\end{array}$ & drgms901-4 & 1981 & $\mathbf{R}$ & & ASME OM Part 10 & 1987 R90 \\
\hline 2683 & ASME & \begin{tabular}{|l|} 
ASME Sec. $X 1$, \\
$M V-2000$ \\
\end{tabular} & $\mathrm{~N} / \mathrm{S}$ & $\begin{array}{l}\text { Inservice Testing of Valves in Light- } \\
\text { Water Reactor Power Plants }\end{array}$ & drgms901-4 & 1981 & $\mathbf{R}$ & & ASME OM Part 10 & $1987 \mathrm{RSO}$ \\
\hline 2684 & ASME & $\begin{array}{l}\text { ASME Sec. } X I, \\
\text { MW-2000 }\end{array}$ & $\mathrm{N} / \mathrm{S}$ & $\begin{array}{l}\text { Inservice Testing of Valves in Light- } \\
\text { Water Reactor Power Plants }\end{array}$ & srp3.9.6 & 1981 & $P$ & $\begin{array}{l}\text { See OM (OM is a } \\
\text { separate vol. of ASME } \\
\text { code) }\end{array}$ & ASME OM Part 10 & $1987 \mathrm{RSO}$ \\
\hline 2685 & ASME & $\begin{array}{l}\text { ASME Sec. XI, } \\
M N-2100\end{array}$ & N/S & $\begin{array}{l}\text { Inservice Testing of Valves in Light- } \\
\text { Water Reactor Power Plants }\end{array}$ & drgms901-4 & 1981 & $\mathbf{R}$ & & ASME OM Part 10 & $1987 \mathrm{PSO}$ \\
\hline 2686 & ASME & $\begin{array}{l}\text { ASME Soc. XI, } \\
\text { WV-2200 }\end{array}$ & $N / S$ & $\begin{array}{l}\text { Inservice Testing of Valves in Light- } \\
\text { Water Reactor Power Plants }\end{array}$ & not91-56 & 1991 & $P$ & & ASME OM Part 10 & 1987 RSO \\
\hline 2687 & ASME & $\begin{array}{l}\text { ASME SoC. XI, } \\
\text { WW-2200 }\end{array}$ & $\mathbf{N} / \mathbf{S}$ & $\begin{array}{l}\text { Inservice Testing of Valves in Light- } \\
\text { Water Reactor Power Plants }\end{array}$ & reg1.148.roo & 1981 & $\mathbf{R}$ & $\begin{array}{l}\text { See OM (now a } \\
\text { separate ASME } \\
\text { volume) } \\
\end{array}$ & ASME OM Part 10 & 1987 RSO \\
\hline 2688 & ASME & $\begin{array}{l}\text { ASME Soc. XI, } \\
\text { MV-3000 }\end{array}$ & $\mathrm{N} / \mathrm{S}$ & $\begin{array}{l}\text { Inservice Testing of Valves in Light- } \\
\text { Water Reactor Power Plants }\end{array}$ & inm35743B & 1982 & $R$ & & ASME OM Part 10 & $1987 \mathrm{R} 90$ \\
\hline
\end{tabular}


Codes and Standards and Other Guldence Clted in Regulatory Documents

Part A - Industry Consensus Codes and Standards (continued)

\begin{tabular}{|c|c|c|c|c|c|c|c|c|c|c|}
\hline $\begin{array}{l}\text { Aocord } \\
\text { No. }\end{array}$ & Code & Standerd & $\begin{array}{l}\text { Standard } \\
\text { Verelon }\end{array}$ & 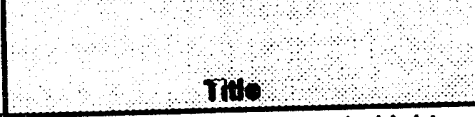 & Booument: & $\begin{array}{l}\text { Doc. } \\
\text { Date }\end{array}$ & $\begin{array}{l}\text { Acoept } \\
\text { ublitity }\end{array}$ & Comments. & Cunous Vorolon & ormon \\
\hline 2689 & ASME & $\begin{array}{l}\text { ASME Sec. XI, } \\
\text { IWV-3000 }\end{array}$ & N/S & $\begin{array}{l}\text { Inservice Testing of Valves in Light- } \\
\text { Water Roactor Power Plants }\end{array}$ & inm357448 & 1979 & $\mathbf{R}$ & & ASME OM Part 10 & 1987 R9O \\
\hline 2690 & ASME & $\begin{array}{l}\text { ASME Sec. } X I, \\
\text { IW }-3000\end{array}$ & N/S & $\begin{array}{l}\text { Inservice Testing of Valves in Light- } \\
\text { Water Roactor Power Plants }\end{array}$ & inm37702 & 1987 & $\mathbf{R}$ & & ASME OM Part 10 & 1987 RSO \\
\hline 2691 & ASME & $\begin{array}{l}\text { ASME Sec. XI, } \\
\text { W }-3000\end{array}$ & $\mathbf{N} / \mathbf{S}$ & $\begin{array}{l}\text { Inservice Testing of Valves in Light- } \\
\text { Water Roactor Power Plants }\end{array}$ & inm62702 & 1987 & $\mathbf{R}$ & & ASME OM Part 10 & 1987 ROO \\
\hline 2692 & ASME & $\begin{array}{l}\text { ASME Soc. } X I, \\
M V-3000\end{array}$ & $N / S$ & $\begin{array}{l}\text { Inservice Testing of Valves in Light- } \\
\text { Water Reactor Power Plants }\end{array}$ & inm9900/ASME XI & 1986 & $\mathbf{R}$ & & ASME OM Part 10 & $1987 \mathrm{RgO}$ \\
\hline 2693 & ASME & $\begin{array}{l}\text { ASME Sec. XI, } \\
\text { IW }-3100\end{array}$ & $\mathbf{N} / \mathbf{S}$ & $\begin{array}{l}\text { Inservice Testing of Valves in Light- } \\
\text { Water Reactor Power Plants }\end{array}$ & inm9900/ASME XI & 1986 & $\mathbf{R}$ & & ASME OM Part 10 & 1987 P90 \\
\hline 2694 & ASME & $\begin{array}{l}\text { ASME Sac. XI, } \\
\text { IW } \mathrm{W}-3300\end{array}$ & $\mathrm{~N} / \mathrm{S}$ & $\begin{array}{l}\text { Inservice Testing of Valves in Light- } \\
\text { Water Reactor Power Plants }\end{array}$ & inm9900/4.5.1 & 1986 & $\mathbf{R}$ & & ASME OM Part 10 & 1987 R90 \\
\hline 2695 & ASME & $\begin{array}{l}\text { ASME Sec. XI, } \\
\text { IWV-3400 }\end{array}$ & $N / S$ & $\begin{array}{l}\text { Inservice Testing of Valves in Light- } \\
\text { Water Reactor Power Plants }\end{array}$ & drgms901-4 & 1981 & $\mathbf{R}$ & & ASME OM Part 10 & 1987 R9O \\
\hline 2696 & ASME & $\begin{array}{l}\text { ASME Sec. } X I, \\
\text { I } W-3400 \\
\end{array}$ & $\mathbf{N} / \mathbf{S}$ & $\begin{array}{l}\text { Inservice Testing of Valves in Light- } \\
\text { Water Reactor Power Plants }\end{array}$ & not89-32 & 1989 & $\mathbf{P}$ & & ASME OM Part 10 & $1987 \mathrm{RgO}$ \\
\hline 2697 & ASME & $\begin{array}{l}\text { ASME Sec. XI, } \\
M V-3400 \\
\end{array}$ & N/S & $\begin{array}{l}\text { Inservice Testing of Valves in Light- } \\
\text { Water Reactor Power Plants }\end{array}$ & stsb\&wB3.5.2 & 1991 & $\mathbf{P}$ & & ASME OM Part 10 & 1987 RSO \\
\hline 2698 & ASME & $\begin{array}{l}\text { ASME Sec. XI, } \\
\text { IW }-3400\end{array}$ & N/S & $\begin{array}{l}\text { Inservice Testing of Valves in Light- } \\
\text { Water Reactor Power Plants }\end{array}$ & stsb\&wB3.7.2 & 1991 & $\mathbf{A}$ & & ASME OM Part 10 & 1987 RSO \\
\hline 2699 & ASME & $\begin{array}{l}\text { ASME Sec. } X I_{1} \\
M N-3400\end{array}$ & $N / S$ & $\begin{array}{l}\text { Inservice Testing of Valves in Light- } \\
\text { Water Reactor Power Plants }\end{array}$ & stsb\&wB3.7.5 & 1991 & $\mathbf{R}$ & & ASME OM Part 10 & 1987 R90 \\
\hline 2700 & ASME & \begin{tabular}{|l|} 
ASME Sec. $X I$, \\
MN-3400 \\
\end{tabular} & N/S & $\begin{array}{l}\text { Inservice Testing of Valves in Light- } \\
\text { Water Reactor Power Plants }\end{array}$ & stsceB3.7.2 & 1991 & $\mathbf{R}$ & & ASME OM Part 10 & 1987 R9O \\
\hline 2701 & ASME & $\begin{array}{l}\text { ASME Sec. XI, } \\
\text { IWV-3400 }\end{array}$ & $\mathbf{N} / \mathbf{S}$ & $\begin{array}{l}\text { inservice Testing of Valves in Light- } \\
\text { Water Reactor Power Plants }\end{array}$ & stsceB3.7.3 & 1991 & $\mathbf{F}$ & & ASME OM Pant 10 & 1987 R90 \\
\hline 2702 & ASME & $\begin{array}{l}\text { ASME Sec. XI, } \\
\text { MV-3400 }\end{array}$ & $\mathrm{N} / \mathrm{S}$ & $\begin{array}{l}\text { Inservice Testing of Valves in Light- } \\
\text { Water Reactor Power Plants }\end{array}$ & stsceB3.7.5 & 1991 & $\mathbf{R}$ & & ASME OM Part 10 & 1987 R90 \\
\hline 2703 & ASME & $\begin{array}{l}\text { ASME Sec. XI, } \\
\text { IW-3400 }\end{array}$ & $\mathrm{N} / \mathrm{S}$ & $\begin{array}{l}\text { Inservice Testing of Valves in Light- } \\
\text { Water Reactor Power Plants }\end{array}$ & stswstB3.7.2 & 1991 & $\mathbf{A}$ & & ASME OM Part 10 & 1987 R90 \\
\hline 2704 & ASME & $\begin{array}{l}\text { ASME Sec. } X I \\
\text { IWV-3400 } \\
\end{array}$ & $N / S$ & $\begin{array}{l}\text { Inservice Testing of Valves in Light- } \\
\text { Water Reactor Power Plants }\end{array}$ & stswstB3.7.5 & 1991 & $\mathbf{R}$ & & ASME OM Part 10 & 1987 R9O \\
\hline 2705 & ASME & $\begin{array}{l}\text { ASME Sec. XI, } \\
\text { IWV-3410 }\end{array}$ & $N / S$ & $\begin{array}{l}\text { Inservice Testing of Valves in Light- } \\
\text { Water Reactor Power Plants }\end{array}$ & drgms901-4 & 1981 & $\mathbf{R}$ & & ASME OM Part 10 & 1987 R90 \\
\hline
\end{tabular}


Codes and Standards and Other Guidance Cited in Regulatory Documents

Part A - Industry Consensus Codes and Standards (continued)

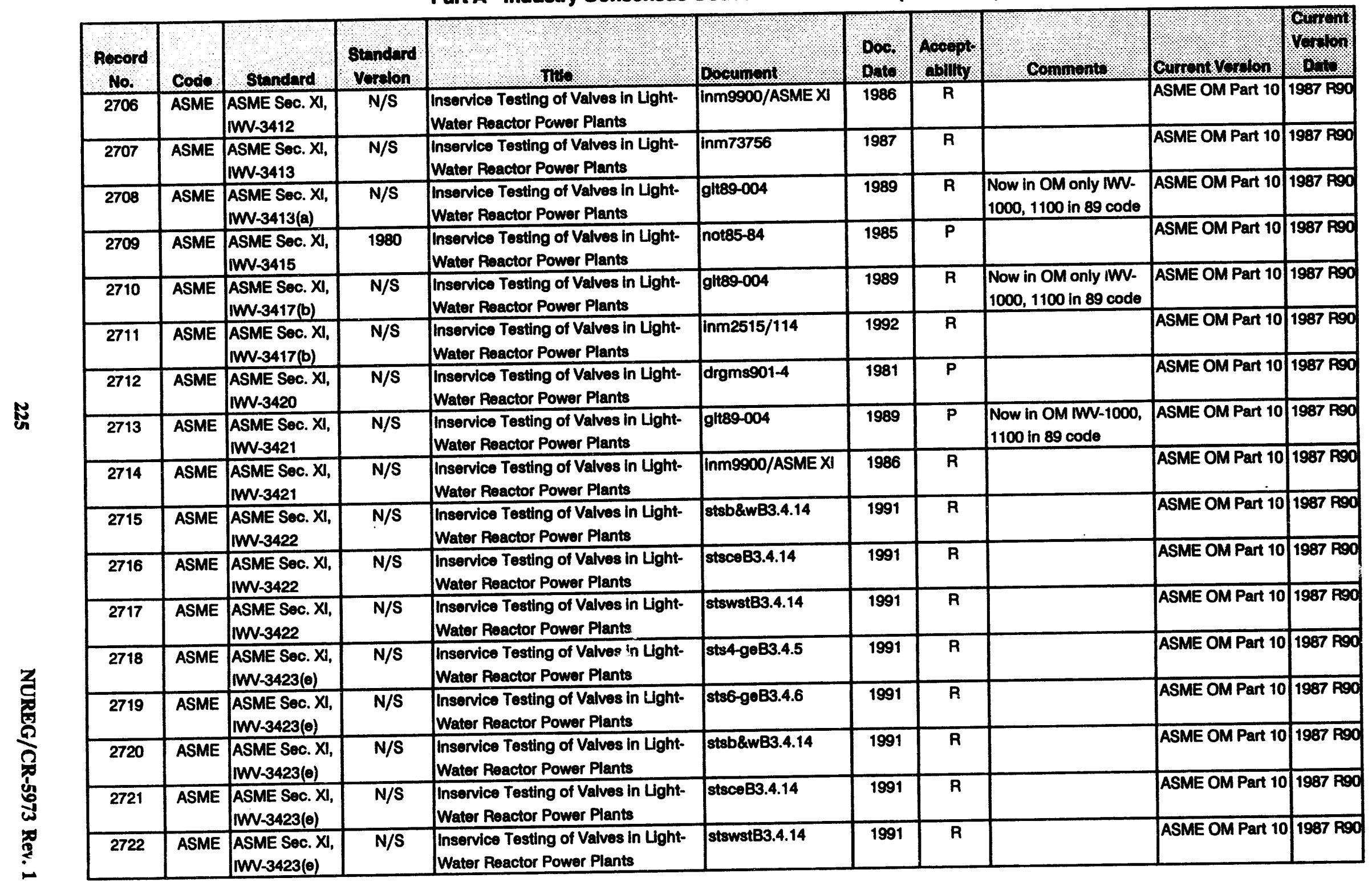


Codes and Standards and Other Guidance Cited in Regulatory Documents

Part A - Industry Consensus Codes and Standards (continued)

Fin

\begin{tabular}{|c|c|c|c|c|c|c|c|c|c|c|}
\hline $\begin{array}{l}\text { Rocord } \\
10 .\end{array}$ & cods & standard & Stridard & (1) & Boodunoul & Dor. & popopts & , comnonto & 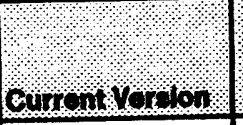 & omo \\
\hline 2723 & ASME & $\begin{array}{l}\text { ASME Sec. XI, } \\
\text { IW-3426 }\end{array}$ & N/S & $\begin{array}{l}\text { Inservice Testing of Valves in Light- } \\
\text { Water Reactor Power Plants }\end{array}$ & glt89-004 & 1989 & $\mathbf{P}$ & $\begin{array}{l}\text { Now in OM only IWV- } \\
1000,1100 \text { in } 89 \text { code }\end{array}$ & ASME OM Part 10 & $1987 \mathrm{ROO}$ \\
\hline 2724 & ASME & $\begin{array}{l}\text { ASME Sec. } X I, \\
\text { i } W-3426\end{array}$ & $\mathbf{N} / \mathbf{S}$ & $\begin{array}{l}\text { Inservice Testing of Valves in Light- } \\
\text { Water Reactor Power Plants }\end{array}$ & inm2515/114 & 1992 & $\mathbf{R}$ & & ASME OM Part 10 & 1987 PSO \\
\hline 2725 & ASME & $\begin{array}{l}\text { ASME Sec. } X I_{1} \\
\text { WW-3426 }\end{array}$ & $\mathrm{N} / \mathrm{S}$ & $\begin{array}{l}\text { Inservice Testing of Valves in Light- } \\
\text { Water Reactor Power Plants }\end{array}$ & inm73756 & 1987 & $\mathbf{R}$ & & ASME OM Part 10 & 1987 ROO \\
\hline 2726 & ASME & $\begin{array}{l}\text { ASME Sec. XI, } \\
\text { WV-3427 }\end{array}$ & $\mathrm{N} / \mathrm{S}$ & $\begin{array}{l}\text { Inservice Testing of Valves in Light- } \\
\text { Water Reactor Power Plants }\end{array}$ & inm73756 & 1987 & $\mathbf{R}$ & & ASME OM Part 10 & 1987 RSO \\
\hline 2727 & ASME & $\begin{array}{l}\text { ASME Sec. XI, } \\
\text { IW }-3427 \text { (a) }\end{array}$ & $\mathrm{N} / \mathrm{S}$ & $\begin{array}{l}\text { Inservice Testing of Valves in Light- } \\
\text { Water Peactor Power Plants }\end{array}$ & glt89-004 & 1989 & $\mathbf{R}$ & $\begin{array}{l}\text { Now in OM only MV- } \\
1000,1100 \text { in } 89 \text { code }\end{array}$ & ASME OM Part 10 & 1987 PQO \\
\hline 2728 & ASME & $\begin{array}{l}\text { ASME Sec. XI, } \\
\text { IWW-3427(a) }\end{array}$ & $\mathbf{N} / \mathbf{S}$ & $\begin{array}{l}\text { Inservice Testing of Valves in Light- } \\
\text { Water Reactor Power Plants }\end{array}$ & inm2515/114 & 1992 & $\mathbf{R}$ & & ASME OM Part 10 & $1987 \mathrm{RgO}$ \\
\hline 2729 & ASME & $\begin{array}{l}\text { ASME Sec. XI, } \\
\text { I }-3427 \text { (b) }\end{array}$ & $\mathrm{N} / \mathrm{S}$ & $\begin{array}{l}\text { Inservice Testing of Valves in Light- } \\
\text { Water Reactor Power Plants }\end{array}$ & glt89-004 & 1989 & $\mathbf{R}$ & $\begin{array}{l}\text { Now in OM only IWV- } \\
1000,1100 \text { in } 89 \text { code }\end{array}$ & ASME OM Part 10 & $1987 \mathrm{RSO}$ \\
\hline 2730 & ASME & $\begin{array}{l}\text { ASME Sec. XI, } \\
\text { IW-3500 }\end{array}$ & $N / S$ & $\begin{array}{l}\text { Inservice Testing of Valves in Light- } \\
\text { Water Reactor Power Plants }\end{array}$ & stsb8wB3.7.1 & 1991 & $\mathbf{P}$ & & ASME OM Part 10 & $1987 \mathrm{RSO}$ \\
\hline 2731 & ASME & \begin{tabular}{|l|} 
ASME Sec. XI, \\
IWV-3500 \\
\end{tabular} & $\mathrm{N} / \mathrm{S}$ & $\begin{array}{l}\text { Inservice Testing of Valves in Light- } \\
\text { Water Reactor Power Plants }\end{array}$ & stsceB3.7.1 & 1991 & $\mathbf{P}$ & & ASME OM Part 10 & 1987 PQO \\
\hline 2732 & ASME & $\begin{array}{l}\text { ASME Sec. XI, } \\
\text { MN-3500 }\end{array}$ & N/S & $\begin{array}{l}\text { Inservice Testing of Valves in Light- } \\
\text { Water Reactor Power Plants }\end{array}$ & stswstB3.7.1 & 1991 & $\mathbf{P}$ & & ASME OM Part 10 & 1987 RQO \\
\hline 2733 & ASME & $\begin{array}{l}\text { ASME Sec. XI, } \\
M W-3510 \\
\text { through }-3513\end{array}$ & $\mathrm{~N} / \mathrm{S}$ & $\begin{array}{l}\text { Inservice Testing of Valves in Light- } \\
\text { Water Reactor Power Plants }\end{array}$ & inm73756 & 1987 & $\mathbf{R}$ & & ASME OM Part 10 & $1987 \mathrm{POO}$ \\
\hline 2734 & ASME & $\begin{array}{l}\text { ASME Sec. XI, } \\
\text { WV-3520 }\end{array}$ & 1983 & $\begin{array}{l}\text { Inservice Testing of Valves in Light- } \\
\text { Water Reactor Power Plants }\end{array}$ & inm9900/ASME XI & 1986 & $\bar{R}$ & & ASME OM Part 10 & 1987 R9O \\
\hline 2735 & ASME & $\begin{array}{l}\text { ASME Sec. XI, } \\
\text { IU }-3522\end{array}$ & $\mathbf{N} / \mathbf{S}$ & $\begin{array}{l}\text { Inservice Testing of Valves in Light- } \\
\text { Water Reactor Power Plants }\end{array}$ & glt89-004 & 1989 & $\bar{P}$ & $\begin{array}{l}\text { Now in OM only IWW- } \\
1000,1100 \text { in } 89 \text { code }\end{array}$ & ASME OM Part 10 & 1987 R9O \\
\hline 2736 & ASME & $\begin{array}{l}\text { ASME Sec. XI, } \\
\text { IW }-3522\end{array}$ & 1983 & $\begin{array}{l}\text { Inservice Testing of Valves in Light- } \\
\text { Water Reactor Power Plants }\end{array}$ & inm9900/ASME XI & 1986 & $\mathbf{R}$ & & ASME OM Part 10 & 1987 R90 \\
\hline 2737 & ASME & $\begin{array}{l}\text { ASME Sec. XI, } \\
\text { MW-6000 }\end{array}$ & N/S & $\begin{array}{l}\text { Inservice Testing of Valves in Light- } \\
\text { Water Reactor Power Plants }\end{array}$ & inm73756 & 1987 & $\mathbf{R}$ & & ASME OM Part 10 & 1987 R9O \\
\hline 2738 & ASME & $\begin{array}{l}\text { ASME Sec. XI, } \\
\text { IWB-5000 }\end{array}$ & $\mathrm{N} / \mathrm{S}$ & System Pressure Tests & stsb\&wB2.1.2 & 1991 & $\mathbf{P}$ & $\begin{array}{l}\text { Typo - IW-5000 for } \\
\text { Class } 1 \text { components }\end{array}$ & $\begin{array}{l}\text { ASME Sec. XI, } \\
\text { WW-5000 }\end{array}$ & 1992 \\
\hline
\end{tabular}


Codes and Standards and Other Guidance Cited in Regulatory Documents

Part A - industry Consensus Codes and Standards (continued)

\begin{tabular}{|c|c|c|c|c|c|c|c|c|c|c|}
\hline $\begin{array}{l}\text { Pooord } \\
\text { No. }\end{array}$ & Code & Standard & $\begin{array}{l}\text { Stundard } \\
\text { Voralon }\end{array}$ & nito & Doodumont & Doc. & Aocopt: & compents & Gun ont $x, r_{10}$ & Qlow \\
\hline 2739 & ASME & $\begin{array}{l}\text { ASME Soc. XI, } \\
\text { WX-5000 }\end{array}$ & N/S & System Pressure Tests & sts4-geB2.1.2 & 1991 & $P$ & & $\begin{array}{l}\text { ASME Sec. XI, } \\
\text { IWX-5000 }\end{array}$ & 1992 \\
\hline 2740 & ASME & $\begin{array}{l}\text { ASME Sec. XI, } \\
\text { WX-5000 }\end{array}$ & $\mathbf{N} / \mathrm{S}$ & System Pressure Tests & sts6-geB2.1.2 & 1991 & $P$ & & $\begin{array}{l}\text { ASME Sec. } X \text {, } \\
\text { IWX-5000 }\end{array}$ & 1992 \\
\hline 2741 & ASME & $\begin{array}{l}\text { ASME Sec. XI, } \\
\text { IWX-5000 }\end{array}$ & $\mathrm{N} / \mathrm{S}$ & System Pressure Tests & stsceB2.1.2(a) & 1991 & $P$ & & $\begin{array}{l}\text { ASME Sec. XI, } \\
W \times-5000\end{array}$ & 1992 \\
\hline 2742 & ASME & $\begin{array}{l}\text { ASME Sec. XI, } \\
\text { WX-5000 }\end{array}$ & N/S & System Pressure Tests & stsceB2.1.2(d) & 1991 & $\mathbf{P}$ & & $\begin{array}{l}\text { ASME Sec. XI, } \\
\text { WX-5000 }\end{array}$ & 1992 \\
\hline 2743 & ASME & $\begin{array}{l}\text { ASME Soc. XI, } \\
\text { IWX-5000 }\end{array}$ & $\mathbf{N} / \mathrm{S}$ & System Pressure Tests & stswstB2.1.2 & 1991 & $\mathbf{P}$ & & $\begin{array}{l}\text { ASME Sec. XI, } \\
\text { IWX-5000 }\end{array}$ & 1992 \\
\hline 2744 & ASME & $\begin{array}{l}\text { ASME Sec. XI, } \\
\text { Subsec. IWP }\end{array}$ & $N / S$ & $\begin{array}{l}\text { Inservice Inspection of Pumps in } \\
\text { Light-Water Reactor Power Plants }\end{array}$ & inm73756 & 1987 & $\mathbf{R}$ & & ASME OM Part 6 & $1987 \mathrm{RSO}$ \\
\hline 2745 & ASME & ASME AG-1 & $\mathbf{N} / \mathrm{S}$ & $\begin{array}{l}\text { Code on Nuclear Air and Gas } \\
\text { Treatment }\end{array}$ & sts4-ge5.1 & 1991 & A & & ASME AG-1 & 1991 \\
\hline 2746 & ASME & ASME AG-1 & $\mathbf{N} / \mathbf{S}$ & $\begin{array}{l}\text { Code on Nuclear Air and Gas } \\
\text { Treatment }\end{array}$ & sts6-ge5.1 & 1991 & A & & ASME AG-1 & 1991 \\
\hline 2747 & ASME & ASME AG-1 & $\mathbf{N} / \mathbf{S}$ & $\begin{array}{l}\text { Code on Nuclear Ar and Gas } \\
\text { Treatment } \\
\end{array}$ & stsb\&w5.1 & 1991 & A & & ASME AG-1 & 1991 \\
\hline 2748 & ASME & ASME AG-1 & $\mathbf{N} / \mathbf{S}$ & $\begin{array}{l}\text { Code on Nuclear Air and Gas } \\
\text { Treatment }\end{array}$ & stswst5.1 & 1991 & A & & ASME AG-1 & 1991 \\
\hline 2749 & ASME & ASME B31.1 & $\mathbf{N} / \mathrm{S}$ & Power Piping & inm49001 & 1992 & $\mathbf{R}$ & & ASME B31.1 & 1992 \\
\hline 2750 & ASME & ASME B31.1 & 1980 & Power Piping & sip3.2.2 & 1981 & $\mathbf{A}$ & & ASME B31.1 & 1992 \\
\hline 2751 & ASME & USAS B31.1 & 1969 & Power Piping & sts4-geB2.1.2 & 1991 & $\mathbf{A}$ & & ASME B31.1 & 1992 \\
\hline 2752 & ASME & USAS B31.1 & 1967 & Power Piping & stsb\&wB2.1.2 & 1991 & $\mathbf{R}$ & & ASME B31.1 & 1992 \\
\hline 2753 & ASME & USAS B31.1 & 1967 & Power Piping & stsceB2.1.2(a) & 1991 & $\mathbf{R}$ & & ASME B31.1 & 1992 \\
\hline 2754 & ASME & USAS B31.1 & 1967 & Power Piping & stsceB2.1.2(d) & 1991 & $\mathbf{R}$ & & ASME B31.1 & 1992 \\
\hline 2755 & ASME & USAS B31.1 & 1969 & Power Piping & stswstB2.1.2 & 1991 & $\mathbf{A}$ & & ASME B31.1 & 1992 \\
\hline 2756 & ASME & ASME N45.2 & 1977 & $\begin{array}{l}\text { Quality Assurance Program } \\
\text { Requirements for Nuclear Facilities }\end{array}$ & drg1010.r00 & 1992 & $\mathbf{P}$ & Proposed reg1.28.ro4 & $\begin{array}{l}\text { ASME N45.2/ } \\
\text { ASME NQA-1 }\end{array}$ & $\begin{array}{c}1977 / \\
1992 \\
\end{array}$ \\
\hline 2757 & ASME & ASME N45.2 & N/S & $\begin{array}{l}\text { Quality Assurance Program } \\
\text { Requirements for Nuclear Facilities }\end{array}$ & inm36100 & 1983 & $\mathbf{R}$ & & $\begin{array}{l}\text { ASME N45.2/ } \\
\text { ASME NQA-1 }\end{array}$ & $\begin{array}{c}1977 / \\
1992 \\
\end{array}$ \\
\hline 2758 & ASME & ASME N45.2 & 1977 & $\begin{array}{l}\text { Quality Assurance Program } \\
\text { Requirements for Nuclear Facilities }\end{array}$ & inm9900/sps & 1991 & $\mathbf{R}$ & Typo was ASME 45.2 & $\begin{array}{l}\text { ASME N45.2/ } \\
\text { ASME NQA-1 }\end{array}$ & $\begin{array}{c}1977 / \\
1992 \\
\end{array}$ \\
\hline
\end{tabular}




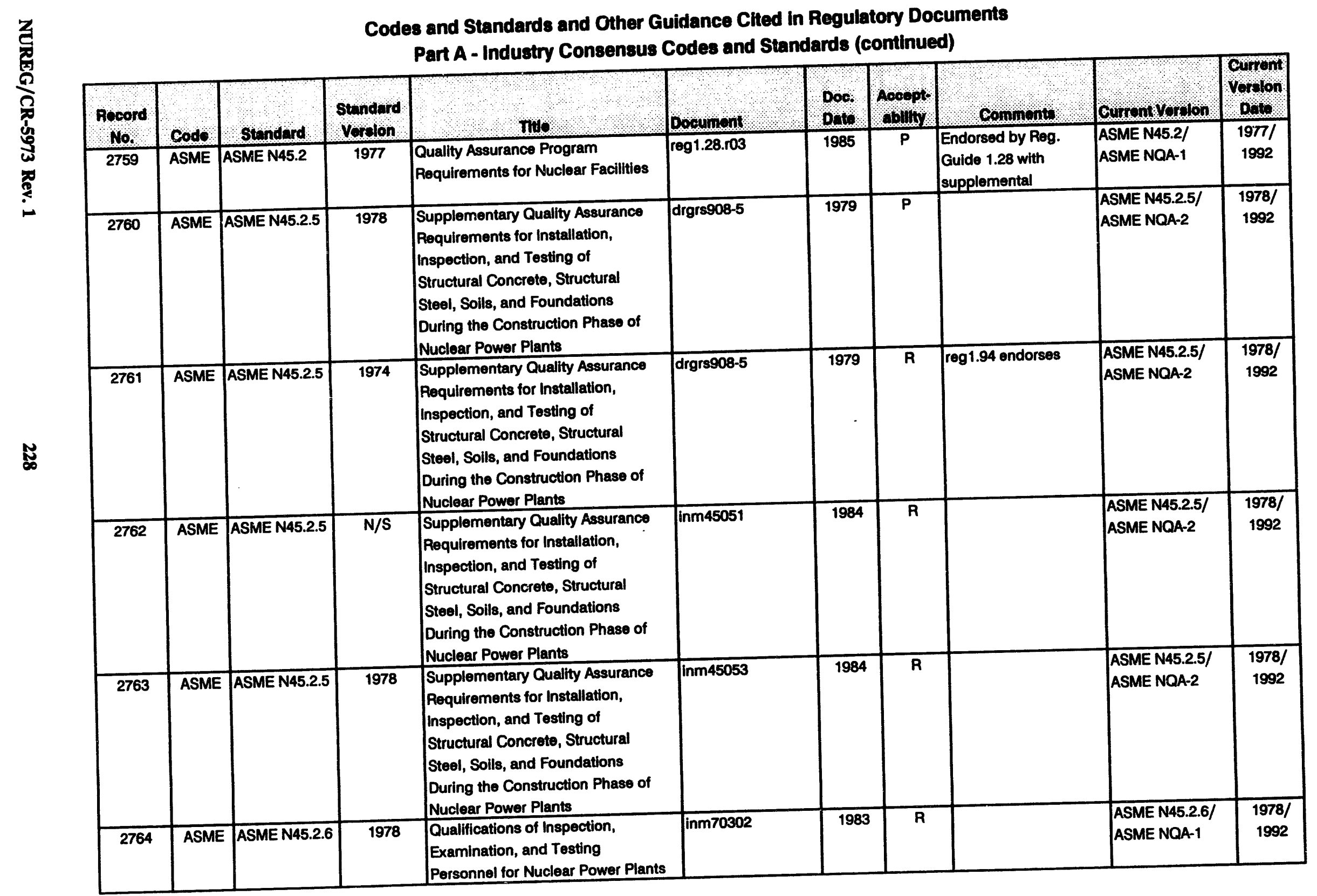


Codes and Standards and Other Guidance Clted in Regulatory Documents

Part A - Industry Consensus Codes and Standards (continued)

\begin{tabular}{|c|c|c|c|c|c|c|c|c|c|c|}
\hline $\begin{array}{l}\text { Rocord } \\
\text { No. }\end{array}$ & Code & Standard & $\begin{array}{l}\text { Standard } \\
\text { Vordon }\end{array}$ & ऽ, & Dooumont & $\begin{array}{l}\text { Dor. } \\
\text { Dote. }\end{array}$ & $\begin{array}{l}\text { Aocopt } \\
\text { eblitity }\end{array}$ & Comments: & Curront Voriton & $0+1$ \\
\hline 2765 & ASME & $\begin{array}{l}\text { ASME } \\
\text { N45.2.12 }\end{array}$ & 1978 & $\begin{array}{l}\text { Requirements for Auditing of } \\
\text { Quality Assurance Programs for } \\
\text { Nuclear Power Plants }\end{array}$ & inm79701 & 1991 & R & & $\begin{array}{l}\text { ASME N45.2.12/ } \\
\text { ASME NQA-1 }\end{array}$ & $\begin{array}{c}1977 / \\
1992\end{array}$ \\
\hline 2766 & ASME & $\begin{array}{l}\text { ASME } \\
\text { N45.2.12 }\end{array}$ & 1977 & $\begin{array}{l}\text { Requirements for Auditing of } \\
\text { Quality Assurance Programs for } \\
\text { Nuclear Power Plants }\end{array}$ & reg1.142.roo & 1978 & $\mathbf{R}$ & & $\begin{array}{l}\text { ASME N45.2.12! } \\
\text { ASME NQA-1 }\end{array}$ & $\begin{array}{c}1977 / \\
1992\end{array}$ \\
\hline 2767 & ASME & \begin{tabular}{|l} 
ASME \\
N45.2.20
\end{tabular} & $N / S$ & $\begin{array}{l}\text { Supplementary Quality Assurance } \\
\text { Requirements for Subsurface } \\
\text { Investigations for Nuclear Power } \\
\text { Plants }\end{array}$ & inm45051 & 1984 & $\mathbf{R}$ & & $\begin{array}{l}\text { ASME N45.2.20/ } \\
\text { ASME NQA-2 }\end{array}$ & $\begin{array}{c}1979 / \\
1992\end{array}$ \\
\hline 2768 & ASME & $\begin{array}{l}\text { ASME } \\
\text { N45.2.20 }\end{array}$ & 1979 & $\begin{array}{l}\text { Supplementary Quality Assurance } \\
\text { Requirements for Subsurface } \\
\text { Investigations for Nuclear Power } \\
\text { Plants }\end{array}$ & inm45053 & 1984 & $\bar{R}$ & & $\begin{array}{l}\text { ASME N45.2.20/ } \\
\text { ASME NQA-2 }\end{array}$ & $\begin{array}{c}1979 / \\
1992\end{array}$ \\
\hline 2769 & ASME & $\begin{array}{l}\text { ASME } \\
\text { N45.2.23 }\end{array}$ & 1978 & $\begin{array}{l}\text { Qualification of Quality Assurance } \\
\text { Program Audit Personnel for } \\
\text { Nuclear Power Plants }\end{array}$ & glt81-001 & 1981 & $\mathbf{P}$ & $\begin{array}{l}\text { Reg. Guide } 1.146 \\
\text { endorses N45.2.23- } \\
1978 \text { with certain } \\
\text { exceptions } \\
\end{array}$ & $\begin{array}{l}\text { ASME N45.2.23/ } \\
\text { ASME NOA-1 }\end{array}$ & $\begin{array}{c}1977 / \\
1992\end{array}$ \\
\hline 2770 & ASME & $\begin{array}{l}\text { ASME } \\
\text { N45.2.23 }\end{array}$ & 1978 & $\begin{array}{l}\text { Qualification of Quality Assurance } \\
\text { Program Audit Personnel for } \\
\text { Nuclear Power Plants }\end{array}$ & inm79502 & 1991 & $\mathbf{R}$ & & $\begin{array}{l}\text { ASME N45.2.23/ } \\
\text { ASME NQA-1 }\end{array}$ & $\begin{array}{c}1977 / \\
1992\end{array}$ \\
\hline 2771 & ASME & $\begin{array}{l}\text { ASME } \\
\text { N45.2.23 }\end{array}$ & 1978 & $\begin{array}{l}\text { Qualification of Quality Assurance } \\
\text { Program Audit Personnel for } \\
\text { Nuclear Power Plants }\end{array}$ & inm80721 & 1991 & $\mathbf{R}$ & & $\begin{array}{l}\text { ASME N45.2.23/ } \\
\text { ASME NQA-1 }\end{array}$ & $\begin{array}{c}1977 / \\
1992\end{array}$ \\
\hline 2772 & ASME & $\begin{array}{l}\text { ASME } \\
\text { N45.2.23 }\end{array}$ & 1978 & $\begin{array}{l}\text { Qualification of Quality Assurance } \\
\text { Program Audit Personnel for } \\
\text { Nuclear Power Plants }\end{array}$ & inm83100 & 1991 & $\mathbf{R}$ & & $\begin{array}{l}\text { ASME N45.2.23/ } \\
\text { ASME NQA-1 }\end{array}$ & $\begin{array}{c}1977 / \\
1992\end{array}$ \\
\hline 2773 & ASME & $\begin{array}{l}\text { ASME } \\
\text { N45.2.23 }\end{array}$ & 1978 & $\begin{array}{l}\text { Qualification of Quality Assurance } \\
\text { Program Audit Personnel for } \\
\text { Nuclear Power Plants }\end{array}$ & inm83522 & 1991 & $\mathbf{R}$ & & $\begin{array}{l}\text { ASME N45.2.23/ } \\
\text { ASME NQA-1 }\end{array}$ & $\begin{array}{c}1977 / \\
1992\end{array}$ \\
\hline 2774 & ASME & $\begin{array}{l}\text { ASME } \\
\text { N45.2.23 }\end{array}$ & 1978 & $\begin{array}{l}\text { Qualification of Quality Assurance } \\
\text { Program Audit Personnel for } \\
\text { Nuclear Power Plants }\end{array}$ & inm83724 & 1991 & $\mathbf{R}$ & & $\begin{array}{l}\text { ASME N45.2.23/ } \\
\text { ASME NQA-1 }\end{array}$ & $\begin{array}{r}1977 / \\
1992\end{array}$ \\
\hline
\end{tabular}




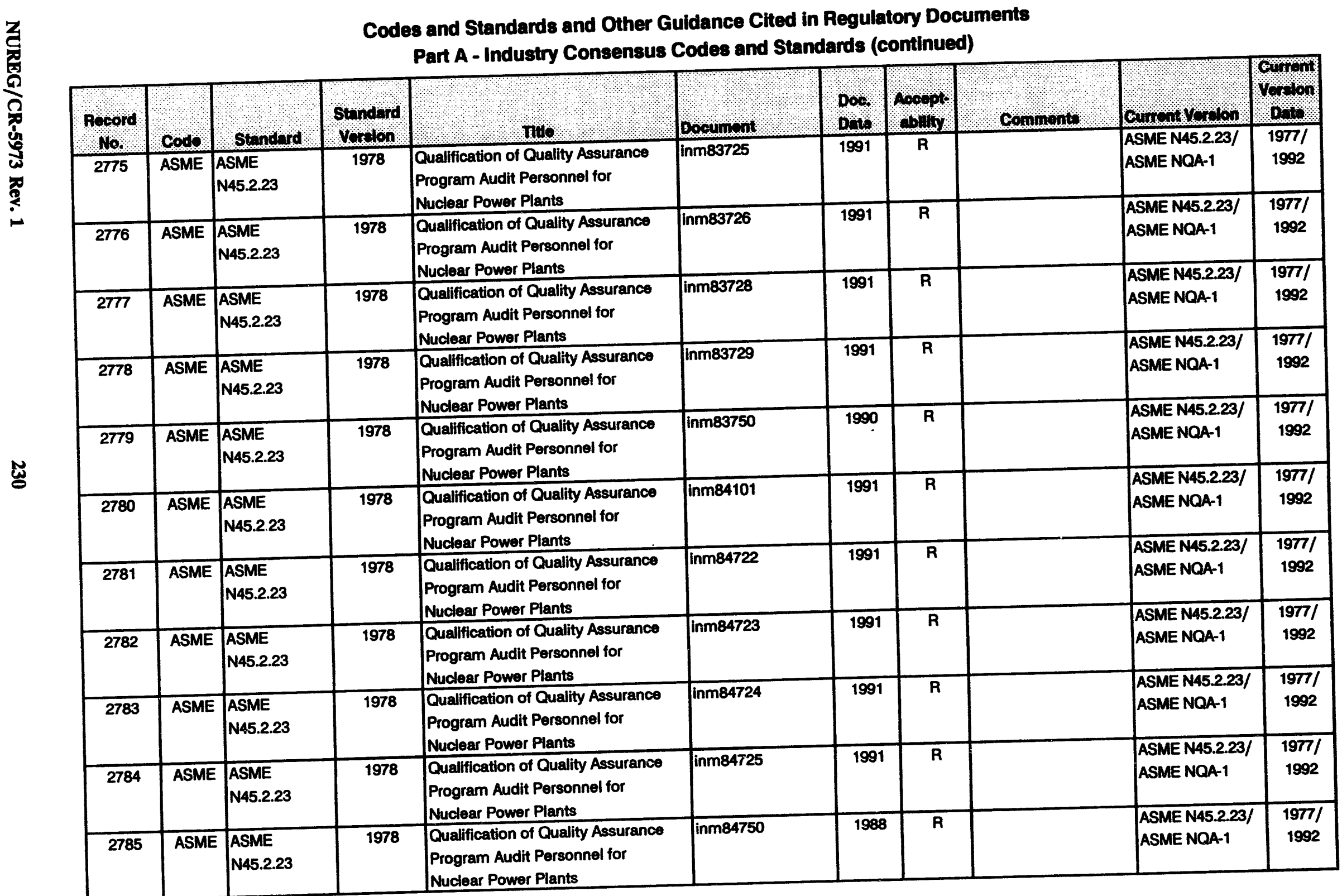

Nuclear Power Plants 
Codes and Standards and Other Guidance Cited in Regulatory Documents

Part A - Industry Consensus Codes and Standards (continued)

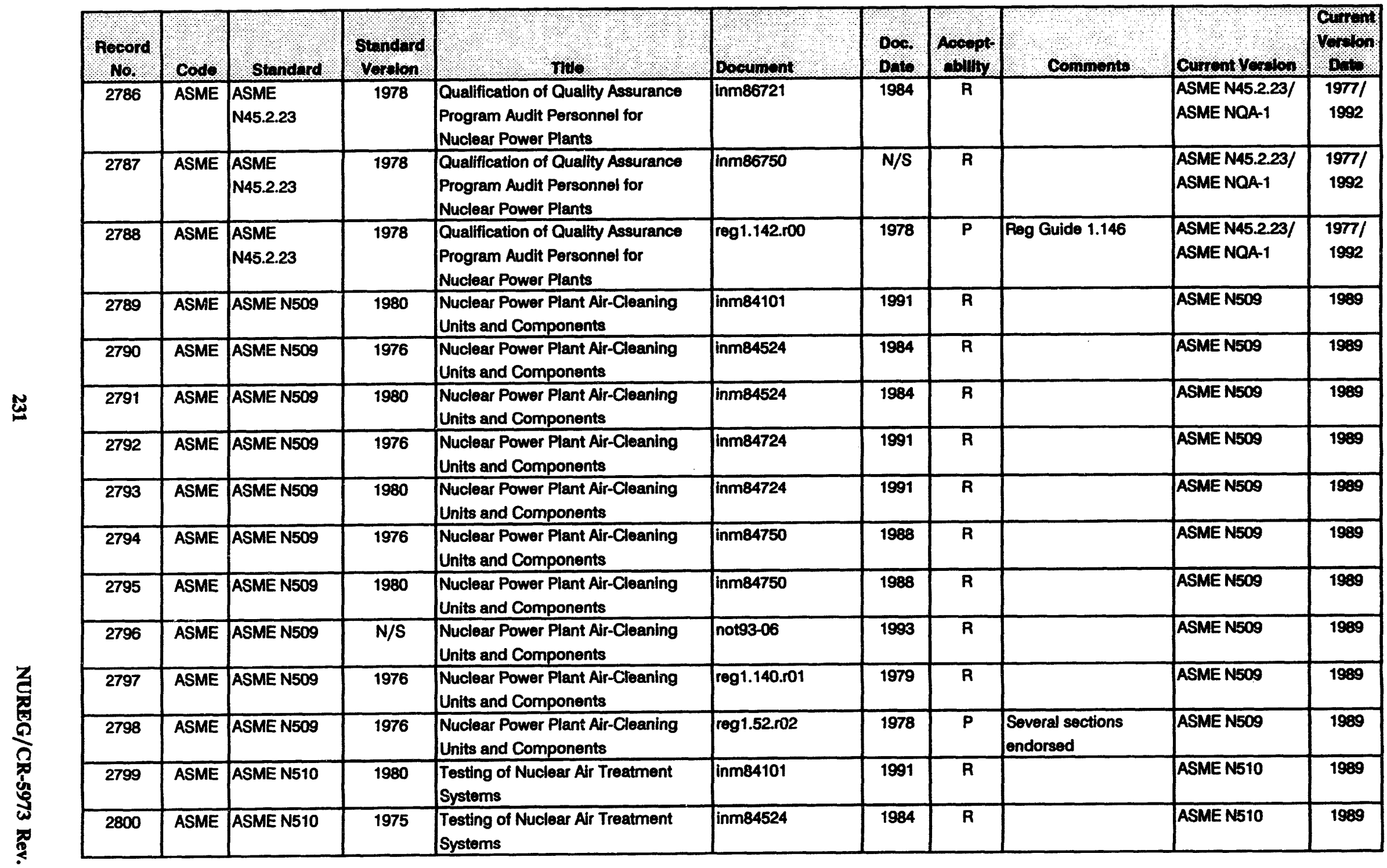




\begin{tabular}{|c|c|c|c|c|c|c|c|c|c|c|}
\hline \multicolumn{11}{|c|}{$\begin{array}{c}\text { Codes and Standards and Other Guidance Clted in Regulatory Documents } \\
\text { Part A - Industry Consensus Codes and Standards (continued) }\end{array}$} \\
\hline $\begin{array}{l}\text { Record } \\
\text { No. }\end{array}$ & Cod & Stobdand & $\begin{array}{l}\text { Standerd } \\
\text { Verolon }\end{array}$ & Tile & Doctureont & Dos. & Aocopt: & conponoms & 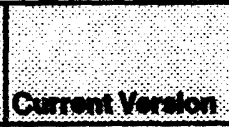 & Geingen \\
\hline 2801 & ASME & ASME N510 & 1980 & $\begin{array}{l}\text { Testing of Nuclear Air Treatment } \\
\text { Systems }\end{array}$ & inm84524 & 1984 & R & & ASME N510 & 1969 \\
\hline 2802 & ASME & ASME N510 & 1975 & $\begin{array}{l}\text { Testing of Nuclear Air Treatment } \\
\text { Systems }\end{array}$ & inm84724 & 1991 & $\mathbf{R}$ & & ASME N510 & 1989 \\
\hline 2803 & ASME & ASME N510 & 1980 & $\begin{array}{l}\text { Testing of Nuclear Air Treatment } \\
\text { Systems }\end{array}$ & inm84724 & 1991 & R & & ASME N510 & 1969 \\
\hline 2804 & ASME & ASME N510 & 1975 & $\begin{array}{l}\text { Testing of Nuclear Air Treatment } \\
\text { Systems }\end{array}$ & inm84750 & 1988 & $\mathbf{R}$ & & ASME N510 & 1989 \\
\hline 2805 & ASME & ASME N510 & 1980 & $\begin{array}{l}\text { Testing of Nuclear Air Treatment } \\
\text { Systems }\end{array}$ & inm84750 & 1988 & $\mathbf{R}$ & & ASWE N510 & 1909 \\
\hline 2806 & ASME & ASME N510 & $N / S$ & $\begin{array}{l}\text { Testing of Nuclear Air Treatment } \\
\text { Systems }\end{array}$ & not92-32 & 1992 & $R$ & & ASME N510 & 1909 \\
\hline 2807 & ASME & ASME N510 & $N / S$ & $\begin{array}{l}\text { Testing of Nuclear Air Treatment } \\
\text { Systems }\end{array}$ & not93-06 & 1993 & $\mathbf{R}$ & & ASME N510 & 1969 \\
\hline 2808 & ASME & ASME N510 & 1989 & $\begin{array}{l}\text { Testing of Nuclear Air Treatment } \\
\text { Systems }\end{array}$ & sts4-ge5.1 & 1991 & A & & ASME N510 & 1989 \\
\hline 2809 & ASME & ASME N510 & 1989 & $\begin{array}{l}\text { Testing of Nuclear Air Treatment } \\
\text { Systems }\end{array}$ & sts6-ge5.1 & 1991 & A & & ASME N510 & 1989 \\
\hline 2810 & ASME & ASME N510 & 1989 & $\begin{array}{l}\text { Testing of Nuclear Air Treatment } \\
\text { Systems }\end{array}$ & stsb\&w5.1 & 1991 & A & & ASME N510 & 1989 \\
\hline 2811 & ASME & ASME N510 & 1989 & $\begin{array}{l}\text { Testing of Nuclear Air Treatment } \\
\text { Systems }\end{array}$ & stsces.1 & 1991 & A & & ASME N510 & 1989 \\
\hline 2812 & ASME & ASME N510 & 1989 & $\begin{array}{l}\text { Testing of Nuclear Air Treatment } \\
\text { Systems }\end{array}$ & stswrst5.1 & 1991 & A & & ASME N510 & 1989 \\
\hline 2813 & ASME & $\begin{array}{l}\text { ASME N551.1 } \\
\text { DRAFT }\end{array}$ & $N / S$ & $\begin{array}{l}\text { Standard for Qualification of ASME } \\
\text { Code Class } 2 \text { and } 3 \text { Pump } \\
\text { Assemblies for Safety Systems } \\
\text { Service General Requirements }\end{array}$ & srp3.10 & 1981 & $\mathbf{R}$ & $\begin{array}{l}\text { Standard under } \\
\text { development - } \\
\text { standard has not been } \\
\text { published }\end{array}$ & $n / a$ & $n / a$ \\
\hline 2814 & ASME & $\begin{array}{l}\text { ASME N551.2 } \\
\text { DRAFT }\end{array}$ & $N / S$ & $\begin{array}{l}\text { Standard for Qualification of ASME } \\
\text { Code Class } 2 \text { and } 3 \text { Pumps for } \\
\text { Safety Systems Service }\end{array}$ & srp3.10 & 1981 & $\mathbf{R}$ & $\begin{array}{l}\text { Standard under } \\
\text { dovelopment - } \\
\text { standard has not been } \\
\text { published }\end{array}$ & $n / a$ & $n / a$ \\
\hline 2815 & ASME & ASME NQA-1 & 1989 & $\begin{array}{l}\text { Quality Assurance Program } \\
\text { Requirements for Nuclear Facilities }\end{array}$ & drg1010.r00 & 1992 & $\bar{R}$ & Proposed reg1.28.ro4 & ASME NQA-1 & 1992 \\
\hline
\end{tabular}




\section{Codes and Standards and Other Guidance Cited in Regulatory Documents}

Part A - Industry Consensus Codes and Standards (continued)

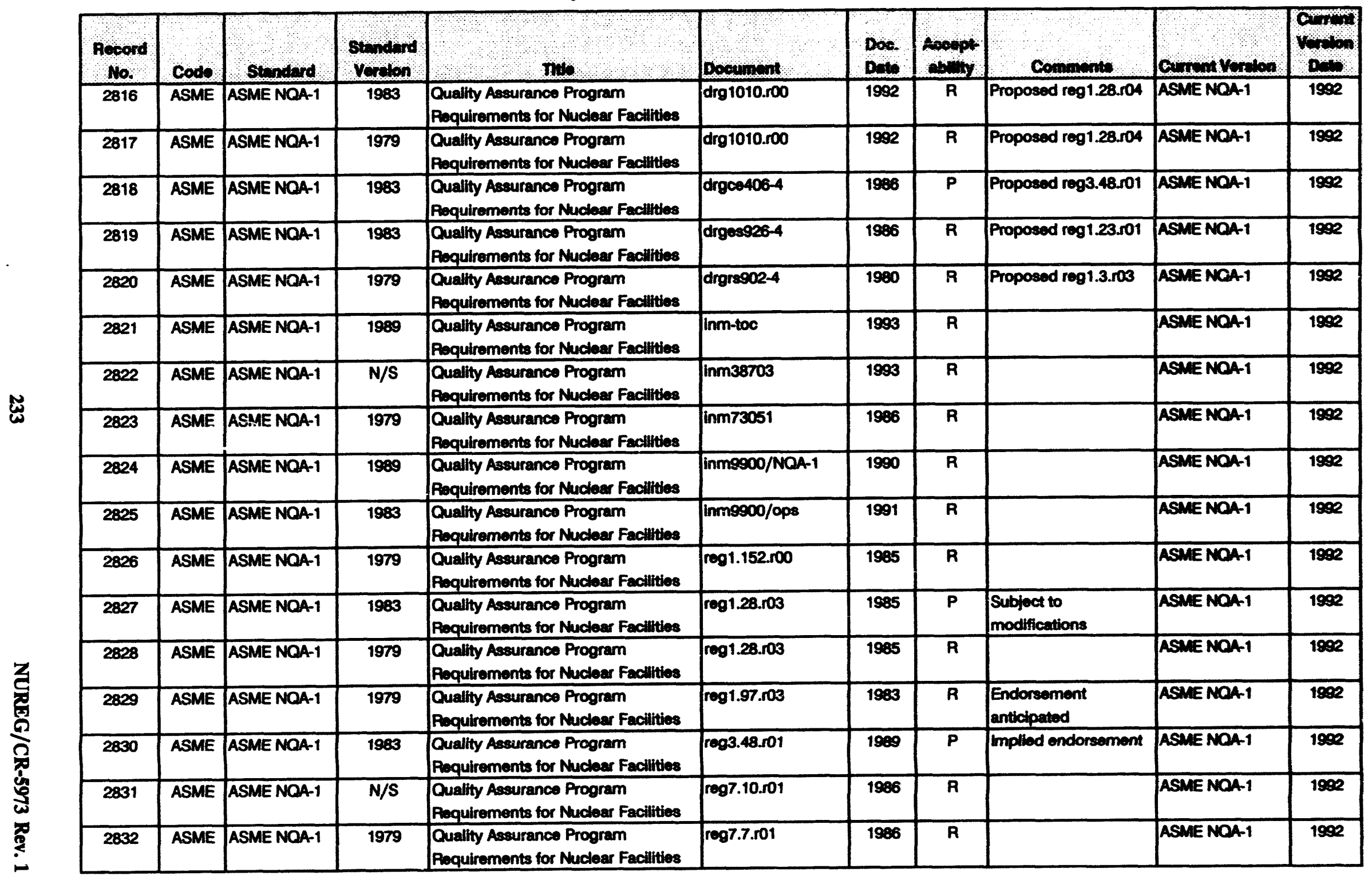




\begin{tabular}{|c|c|c|c|c|c|c|c|c|c|c|}
\hline \multicolumn{11}{|c|}{$\begin{array}{l}\text { Codes and Standards and Other Guldance Cited in Regulatory Documents } \\
\text { Part A - Industry Consensus Codes and Standards (contimued) }\end{array}$} \\
\hline $\begin{array}{l}\text { Hocord } \\
\text { No. }\end{array}$ & coses & Stondend: & $\begin{array}{l}\text { Stondind } \\
\text { Vorclion }\end{array}$ & Ins & Dovornens & $\begin{array}{l}000 \\
0,\end{array}$ & Aooept: & Comenonts & Gunburasa & $\sum_{i=1}^{\infty}$ \\
\hline 2833 & ASME & $\begin{array}{l}\text { ASME NOA-1 } \\
\text { Appendix 17A- } \\
1\end{array}$ & 1983 & $\begin{array}{l}\text { Quality Assurance Program } \\
\text { Pequirements for Nuctear Facilitios }\end{array}$ & ghte8-018 & 1988 & $P$ & $\begin{array}{l}\text { Endorsed by Peg. } \\
\text { Guide } 1.28\end{array}$ & ASME NOA-1 & 1992 \\
\hline 2834 & ASME & ASME NOA-1a & 1983 & $\begin{array}{l}\text { Quallity Assurance Program } \\
\text { Requiroments for Nuctoer Power } \\
\text { Plants }\end{array}$ & reg1.28.003 & 1985 & $\mathbf{P}$ & $\begin{array}{l}\text { Subjoct to } \\
\text { modifications } \\
\text { (Addendum) }\end{array}$ & ASME NOA1 & 1992 \\
\hline 2835 & ASME & ASME NQA-1a & 1981 & $\begin{array}{l}\text { Quality Assuranco Program } \\
\text { Requirements for Nucloar Facllities }\end{array}$ & reg1.28.003 & 1985 & $\mathbf{P}$ & & ASIEE NOA-1 & 1900 \\
\hline 2836 & ASME & ASME NOA-1a & 1983 & $\begin{array}{l}\text { Quality Assurance Program } \\
\text { Requirements for Nuctear Facillites }\end{array}$ & reg1.28.103 & 1905 & $\mathbf{P}$ & & ASWENOA-1 & 1992 \\
\hline 2837 & ASME & ASME NOA-1b & 1981 & $\begin{array}{l}\text { Quality Asourances Program } \\
\text { Roquirements for Nucloer Facillites }\end{array}$ & 1001.28 .103 & 1995 & $\mathbf{P}$ & & ASUENOA-1 & 1992 \\
\hline 2838 & ASME & ASME NOA-1C & 1982 & $\begin{array}{l}\text { Quality Assuranco Program } \\
\text { Requirements for Nuclear Facilitice }\end{array}$ & rog1.28.ro3 & 1985 & $\bar{P}$ & & ASWENOA-1 & 1992 \\
\hline 2839 & ASME & ASME NOA-2 & $N / S$ & $\begin{array}{l}\text { Quality Assurance Prequirements } \\
\text { for Nucloar Faclity Applications }\end{array}$ & inm38703 & 1993 & $\mathbf{R}$ & & ASIEE NOA-2 & 1908 \\
\hline 2840 & ASME & ASME NOA-2 & 1983 & $\begin{array}{l}\text { Quality Assuranco Acquilrements } \\
\text { for Nuclear Faclity Applications }\end{array}$ & inm9so0/ops & 1991 & $\bar{R}$ & & ASMENOA2 & 1992 \\
\hline 2841 & ASME & ASMEOM & 1997 & $\begin{array}{l}\text { Operation and Maintenance of } \\
\text { Nucloear Power Plants }\end{array}$ & ght820010.201 & 1990 & R & Folerred to in text & Asweom & $1937 \mathrm{PBO}$ \\
\hline 2842 & ASME & ASME OM & 1987 & $\begin{array}{l}\text { Operation and Maintenances of } \\
\text { Nucloer Power Plants }\end{array}$ & 9609013 & 1900 & $\overline{\mathbf{R}}$ & $\begin{array}{l}\text { Foformed to as inctuatiny } \\
\text { standard }\end{array}$ & ASWEOA & $1987 \mathrm{~F} 80$ \\
\hline 2843 & ASME & ASME OM & $N / S$ & $\begin{array}{l}\text { Operation and Masintenanco of } \\
\text { Nuctear Power Plants }\end{array}$ & inm2515/110 & 19903 & $\bar{R}$ & & ASTEOOM & $1937 \mathrm{FBO}$ \\
\hline 2844 & ASME & ASME OM & 1990 & $\begin{array}{l}\text { Operation and Maintenencis of } \\
\text { Nuclear Power Plants }\end{array}$ & notegso.201 & 1991 & $\mathbf{R}$ & & ASNEOM & $1987 \mathrm{FBO}$ \\
\hline 2845 & ASME & \begin{tabular}{|l|} 
ASME OM Part \\
1
\end{tabular} & 1987 & 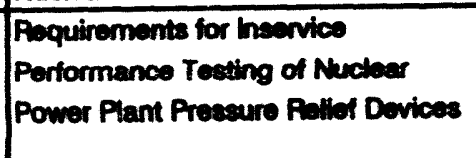 & notsose..01 & 1991 & $\bar{R}$ & & ASIE OM Part 1 & $1967 \mathrm{RPO}$ \\
\hline 2846 & ASME & \begin{tabular}{|l|} 
ASME OM Pant \\
1
\end{tabular} & 1987 & 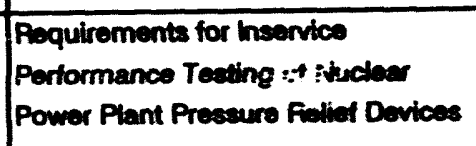 & stab8iw63.7.1 & 1991 & $P$ & & ASME OM Part 1 & $1987 \mathrm{PCO}$ \\
\hline
\end{tabular}


Codes and Standards and Other Guldance Clted in Regulatory Documents

Part A - Industry Consensus Codes and Standards (continued)

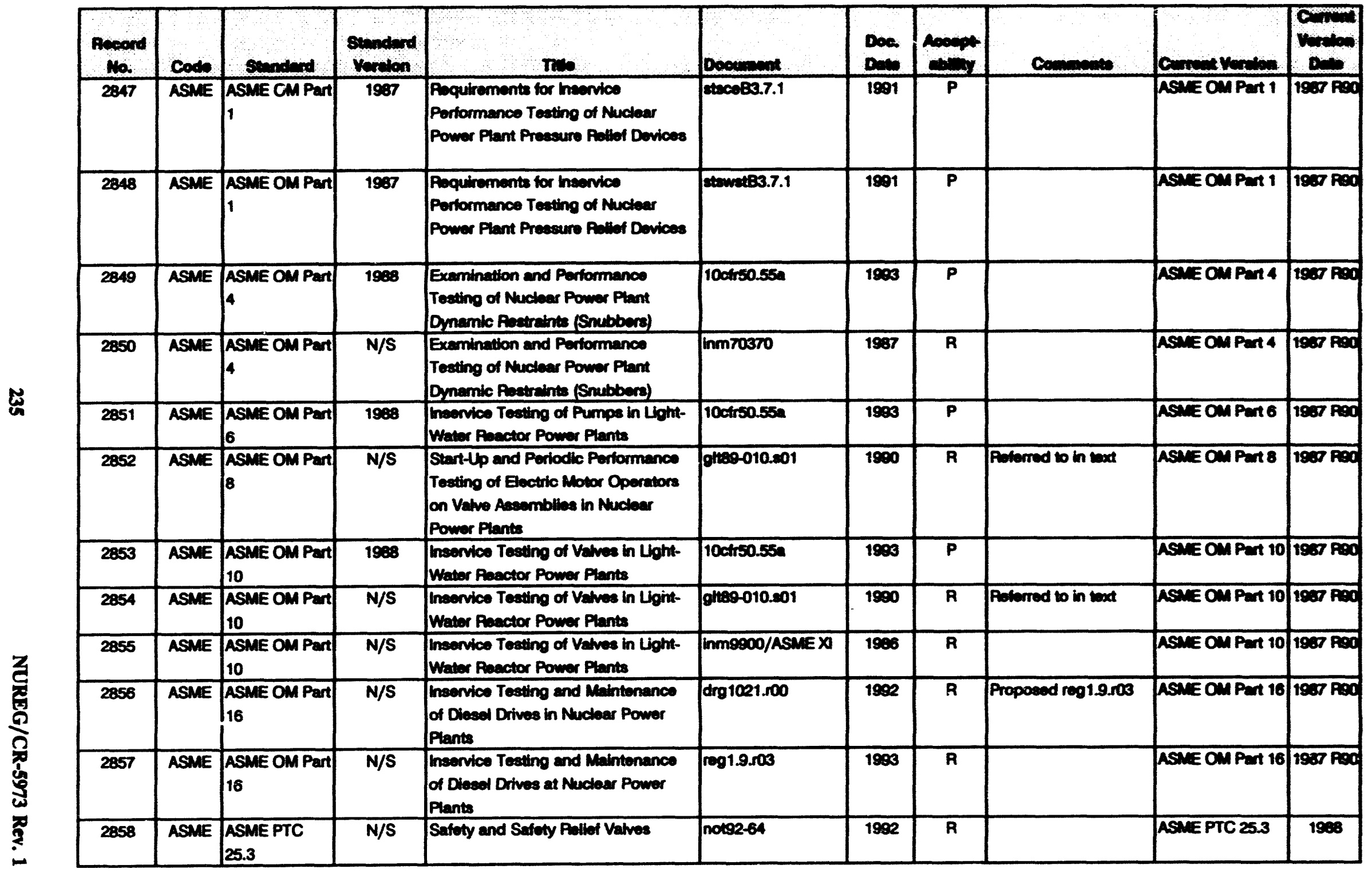


Codes and Standards and Other Guidance Ched in Regulatory Documents

Part A - Industry Consensus Codes and Standards (continued)

\begin{tabular}{|c|c|c|c|c|c|c|c|c|c|c|}
\hline $\begin{array}{l}\text { Record } \\
\text { No. }\end{array}$ & Code & Shandard & $\begin{array}{l}\text { Stand nd } \\
\text { Voroldon }\end{array}$ & $\mathrm{m}=$ & Bownenant & $\begin{array}{l}D \\
D=0\end{array}$ & coopt. & Connenen & Consun ventan & $\begin{array}{l}v_{0}+4 \mathrm{a} \\
\mathrm{D}\end{array}$ \\
\hline 2859 & ASME & $\begin{array}{l}\text { ASME PTC } \\
25.3\end{array}$ & 1976 & Safety and Safety Rollof Valves & reg1.84.r29 & 1993 & $P$ & $\begin{array}{l}\text { Use of } 1977 \text { addenda } \\
\text { of B31.7 }\end{array}$ & ASME PTC 25.3 & 1968 \\
\hline 2860 & ASME & ASME OME-1 & $N / S$ & $\begin{array}{l}\text { Quallification of Active Mochanical } \\
\text { Equipment Used in Nuctear Power } \\
\text { Plants }\end{array}$ & glteg-010.801 & 1990 & $\mathbf{R}$ & $\begin{array}{l}\text { Standard under } \\
\text { developmont - has not } \\
\text { been publlahed }\end{array}$ & $n / a$ & $n / a$ \\
\hline 2861 & ASME & ASME SA350 & $N / S$ & $\begin{array}{l}\text { Specification for Forgings, Carbon } \\
\text { and Low-Alloy Steol, Requiring } \\
\text { Notch Toughness Testing for } \\
\text { Piping Components }\end{array}$ & not89-56.s01 & 1989 & $\mathbf{R}$ & & ASIE SA350 & 1992 \\
\hline 2862 & ASHE & ASME SA370 & $N / S$ & $\begin{array}{l}\text { Specification for Test Mothode and } \\
\text { Definitions for Mochanical Testing } \\
\text { of Steol Products }\end{array}$ & noteg-66.801 & 1989 & $\overline{\mathbf{P}}$ & & ASINE SA370 & 1992 \\
\hline 2863 & ASME & ASME SA-370 & 1970 & $\begin{array}{l}\text { Spectification for Test Mothods and } \\
\text { Definitions for Mechanical Testing } \\
\text { of Stoel Products }\end{array}$ & $\operatorname{rog} 1.65 .100$ & 1973 & $\mathbf{P}$ & & ASNE SA370 & 1992 \\
\hline 2864 & ASME & ASME SA-388 & $N / S$ & $\begin{array}{l}\text { Specification for Ultrasonic } \\
\text { Examination of Hoavy Stoel } \\
\text { Forgings }\end{array}$ & reg1.65.000 & 1973 & $\mathbf{P}$ & & ASME SA-388 & 1992 \\
\hline 2865 & ASNT & $\begin{array}{l}\text { ASNT SNT-TC } \\
\text { IA }\end{array}$ & 1975 & $\begin{array}{l}\text { Pereonnel Qualification and } \\
\text { Certification for Nondestructive } \\
\text { Examination }\end{array}$ & drg1010.100 & 1992 & $\mathbf{P}$ & & ASNT SNT-TC-1A & 1988 \\
\hline 2866 & ASNT & $\begin{array}{l}\text { ASNT SNT-TC- } \\
1 A\end{array}$ & 1980 & $\begin{array}{l}\text { Personnel Qualification and } \\
\text { Certification for Nondestructive } \\
\text { Examination }\end{array}$ & drg1010.000 & 1992 & $\mathbf{P}$ & & ASNT SNT-TC-1A & 1988 \\
\hline 2867 & ASNT & $\begin{array}{l}\text { ASNT SNT-TC- } \\
1 A\end{array}$ & 1988 & $\begin{array}{l}\text { Personnel Qualification and } \\
\text { Certification for Nondestructive } \\
\text { Examination }\end{array}$ & drg1010.r00 & 1992 & $\bar{P}$ & & ASNT SNT-TC-1A & 1988 \\
\hline 2868 & ASNT & $\begin{array}{l}\text { ASNT SNT-TC- } \\
\text { IA }\end{array}$ & 1968 & $\begin{array}{l}\text { Personnel Qualification and } \\
\text { Certification for Nondestructive } \\
\text { Examination }\end{array}$ & inm36301B & 1976 & $\mathbf{R}$ & & ASNT SNT-TC-1A & 1988 \\
\hline 2869 & ASNT & \begin{tabular}{|l|} 
ASNT SNT-TC- \\
$1 A$
\end{tabular} & $N / S$ & $\begin{array}{l}\text { Personnel Qualfication and } \\
\text { Certification for Nondestructive } \\
\text { Examination }\end{array}$ & inm49001 & 1992 & $\bar{A}$ & & ASNT SNT-TC-1A & 1988 \\
\hline
\end{tabular}




\section{Codes and Standards and Other Guidance Ched in Regulatory Documents}

Part A - Industry Consensus Codes and Standards (continued)

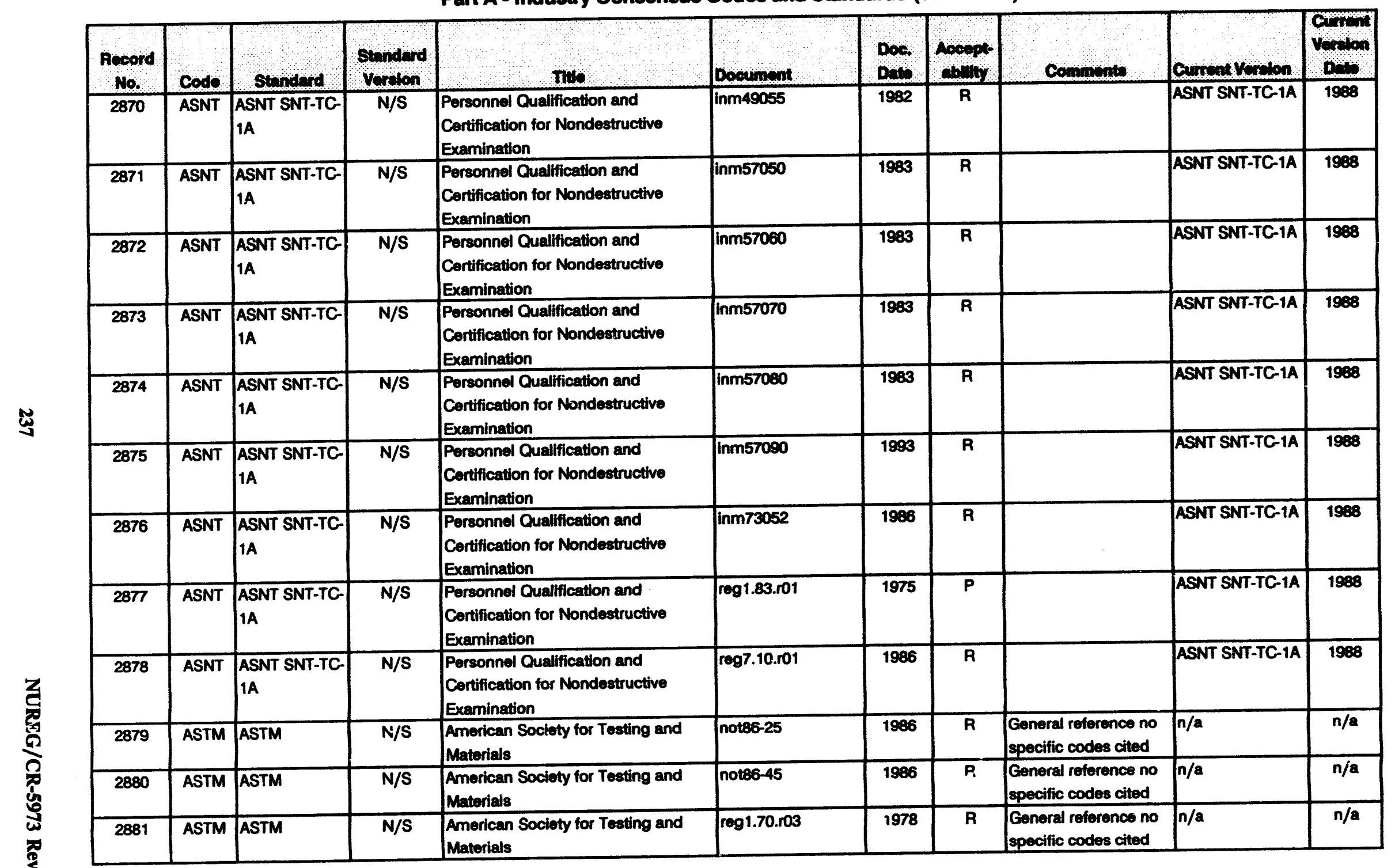




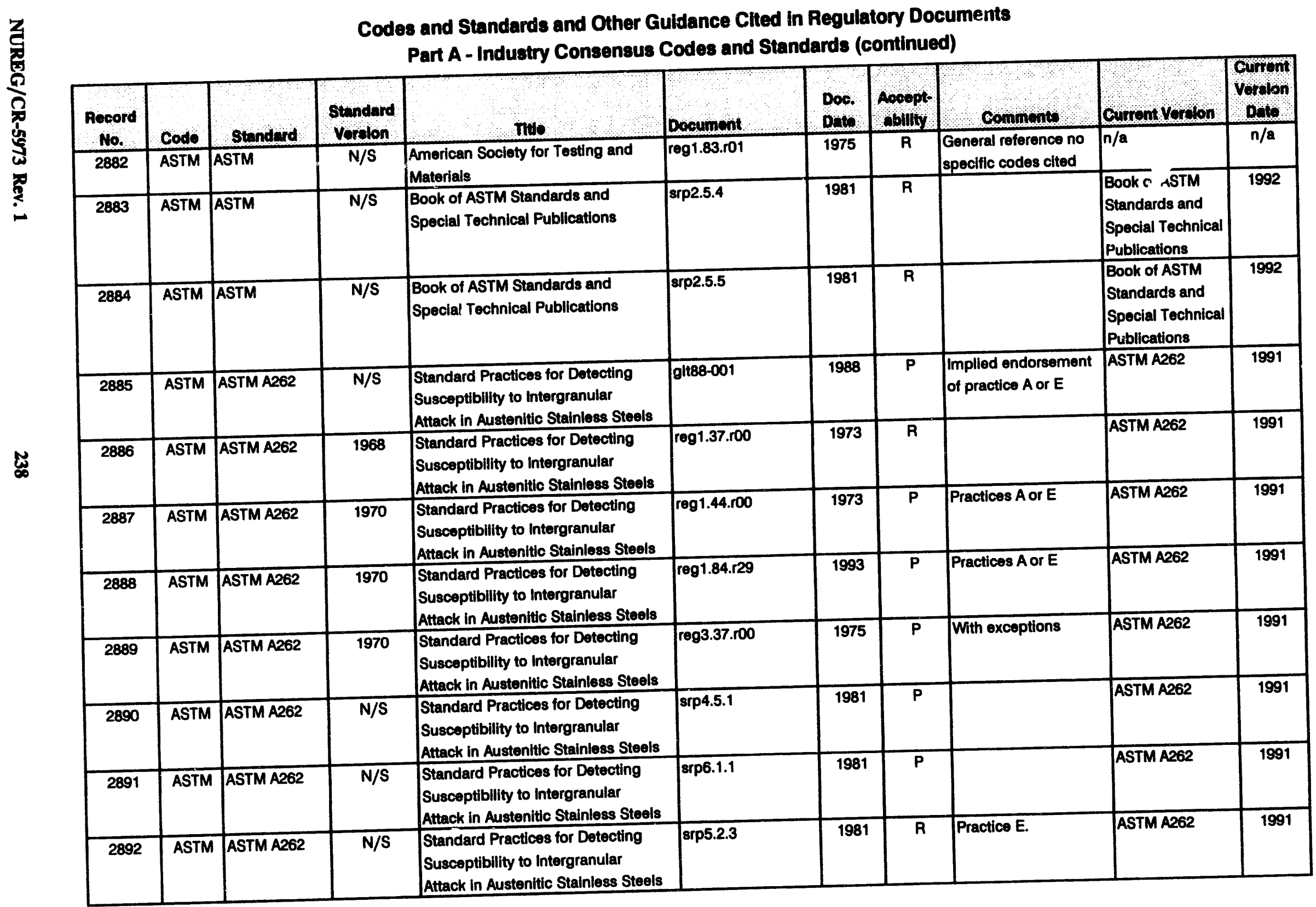




\section{Codes and Standards and Other Guidance Clted in Regulatory Documents}

Part A - Industry Consensus Codes and Standards (continued)

\begin{tabular}{|c|c|c|c|c|c|c|c|c|c|c|}
\hline $\begin{array}{l}\text { Aocord } \\
\text { No. }\end{array}$ & codo & Standard & $\begin{array}{l}\text { stridord } \\
\text { vordon }\end{array}$ & l & Doo, wan, nt: & $\begin{array}{l}\text { Doo. } \\
\text { Doto }\end{array}$ & Aocept & Commonto & Curront Yoroon & Qurow \\
\hline 2893 & ASTM & ASTM A370 & N/S & $\begin{array}{l}\text { Standard Test Mothods and } \\
\text { Definitions for Mechanical Testing } \\
\text { of Steol Products }\end{array}$ & bul80-21 & 1980 & R & & ASTM A370 & 1992 \\
\hline 2894 & ASTM & ASTM A370 & $\mathrm{N} / \mathrm{S}$ & $\begin{array}{l}\text { Standard Test Methods and } \\
\text { Definitions for Mechanical Testing } \\
\text { of Steol Products }\end{array}$ & bul88-05.s02 & 1988 & $\mathbf{R}$ & & ASTM A370 & 1992 \\
\hline 2895 & ASTM & ASTM A370 & $\mathrm{N} / \mathrm{S}$ & $\begin{array}{l}\text { Standard Test Methods and } \\
\text { Definitions for Mechanical Testing } \\
\text { of Steol Products }\end{array}$ & glt80-092 & 1980 & $\mathbf{R}$ & & ASTM A370 & 1992 \\
\hline 2896 & ASTM & ASTM A370 & $N / S$ & $\begin{array}{l}\text { Standard Test Methods and } \\
\text { Definitions for Mechanical Testing } \\
\text { of Steel Products } \\
\end{array}$ & $\operatorname{sip} 10.2 .3$ & 1981 & $\mathbf{P}$ & Implied endorsement & ASTM A370 & 1992 \\
\hline 2897 & ASTM & ASTM A370 & 1972 & $\begin{array}{l}\text { Standard Test Methods and } \\
\text { Definitions for Mechanical Testing } \\
\text { of Steel Products }\end{array}$ & $\operatorname{sip} 5.4 .1 .1$ & 1981 & $\mathbf{P}$ & Implied endorsement & ASTM A370 & 1992 \\
\hline 2898 & ASTM & ASTM A393 & 1963 & $\begin{array}{l}\text { Detection of Intergranular } \\
\text { Precipitation }\end{array}$ & reg1.37.r00 & 1973 & $\mathbf{R}$ & $\begin{array}{l}\text { No longer considered } \\
\text { a valid test }\end{array}$ & Withdrawn & $n / a$ \\
\hline 2899 & ASTM & ASTM A708 & 1974 & $\begin{array}{l}\text { Recommended Practice for } \\
\text { Detection of Susceptibility to } \\
\text { Intergranular Corrosion in Severely } \\
\text { Sensitized Austenitic Stainless Steel }\end{array}$ & reg1.84.r29 & 1993 & $\mathbf{P}$ & Discontinued in 1989 & $n / a$ & $n / a$ \\
\hline 2900 & ASTM & ASTM A708 & $N / S$ & $\begin{array}{l}\text { Recommended Practice for } \\
\text { Detection of Susceptibility to } \\
\text { Intergranular Corrosion in Severely } \\
\text { Sensitized Austenitic Stainless Steel }\end{array}$ & $\operatorname{srp} 4.5 .1$ & 1981 & $\mathbf{P}$ & Discontinued in 1989 & $n / a$ & $n / a$ \\
\hline 2901 & ASTM & ASTM B72 & 1976 & Not found & reg 1.85.r29 & 1993 & $\mathbf{R}$ & Discontinued & $n / a$ & $n / a$ \\
\hline 2902 & ASTM & ASTM C29 & N/S & $\begin{array}{l}\text { Standard Test Mothod for Unit } \\
\text { Weight and Voids in Aggregate } \\
\text { (AASHTO No. T 19/T19M) }\end{array}$ & inm46051 & 1983 & $\mathbf{R}$ & & ASTM C29 & 1991 \\
\hline 2903 & ASTM & ASTM C31 & $N / S$ & $\begin{array}{l}\text { Standard Practice for Making and } \\
\text { Curing Concrete Tost Specimens in } \\
\text { the Field }\end{array}$ & inm46051 & 1983 & $\mathbf{R}$ & & ASTM C31 & 1991 \\
\hline
\end{tabular}


Codes and Standards and Other Guidance Cited in Regulatory Documents

Part A - Industry Consensus Codes and Standards (continued)

\begin{tabular}{|c|c|c|c|c|c|c|c|c|c|c|}
\hline Hocord & 000 & standlord & Stondard & 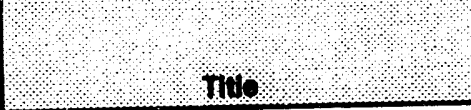 & bocunont & Doo: & \%orpts & Comnento & Cuncul Xolon & profl \\
\hline 2904 & ASTM & ASTM C39 & N/S & $\begin{array}{l}\text { Standard Test Method for } \\
\text { Compressive Strength of } \\
\text { Cylindrical Concrete Specimens } \\
\text { (AASHTO No. T22) }\end{array}$ & inm 46051 & 1983 & R & & ASTM C39 & 1986 \\
\hline 2905 & ASTM & ASTM C40 & $N / S$ & $\begin{array}{l}\text { Standard Test Method for Organic } \\
\text { Impurities in Fine Aggregates for } \\
\text { Concrete (AASHTO No. T21) }\end{array}$ & inm46051 & 1983 & $\mathbf{R}$ & & ASTM C40 & 1992 \\
\hline 2906 & ASTM & ASTM C78 & $\mathrm{N} / \mathrm{S}$ & $\begin{array}{l}\text { Standard Test Method for Fexural } \\
\text { Strength of Concrete (Using Simple } \\
\text { Beam with Third-Point Loading) } \\
\text { (AASHTO No. T97) }\end{array}$ & inm46051 & 1983 & R & & ASTM C78 & 1984 \\
\hline 2907 & ASTM & ASTM C88 & $\mathrm{N} / \mathrm{S}$ & $\begin{array}{l}\text { Standard Test Method for } \\
\text { Soundness of Aggregates by Use of } \\
\text { Sodium Sulfate or Magnesium } \\
\text { Sulfate }\end{array}$ & inm46051 & 1983 & $\mathbf{R}$ & & ASTM C88 & 1990 \\
\hline 2908 & ASTM & ASTM C109 & $\mathbf{N} / \mathrm{S}$ & $\begin{array}{l}\text { Standard Test Method for } \\
\text { Compressive Strength of Hydraulic } \\
\text { Cement Mortars (Using 2-in. or } 50 \\
\text { mm Cube Specimens) }\end{array}$ & inm46051 & 1983 & $\mathbf{A}$ & & ASTM C109 & 1992 \\
\hline 2909 & ASTM & ASTM C109 & 1973 & $\begin{array}{l}\text { Standard Test Method for } \\
\text { Compressive Strength of Hydraulic } \\
\text { Cement Mortars (Uising 2-in. or } 50 \\
\text { mm Cube Specimens) }\end{array}$ & reg1.107.ro1 & 1977 & $\bar{R}$ & & ASTM C109 & 1992 \\
\hline 2910 & ASTM & ASTM C114 & $\mathrm{N} / \mathrm{S}$ & $\begin{array}{l}\text { Standard Test Methods for } \\
\text { Chemical Analysis of Hydraulic } \\
\text { Cement }\end{array}$ & inm46051 & 1983 & $\mathbf{R}$ & & ASTM C114 & 1988 \\
\hline 2911 & ASTM & ASTMC115 & $N / S$ & $\begin{array}{l}\text { Standard Test Method for Fineness } \\
\text { of Portland Cement by the } \\
\text { Turbidimeter }\end{array}$ & inm46051 & 1983 & R & & ASTM C115 & 1993 \\
\hline 2912 & ASTM & ASTM C117 & $\mathrm{N} / \mathrm{S}$ & $\begin{array}{l}\text { Standard Test Method for Materials } \\
\text { Finer Than 75- Micrometer (No. } \\
\text { 200) Sieve in Mineral Aggregates } \\
\text { by Washing }\end{array}$ & inm46051 & 1983 & R & & ASTM C117 & 1990 \\
\hline
\end{tabular}

by Washing 
Codes and Standards and Other Guidance Cited in Regulatory Documents

Part A - Industry Consensus Codes and Standards (continued)

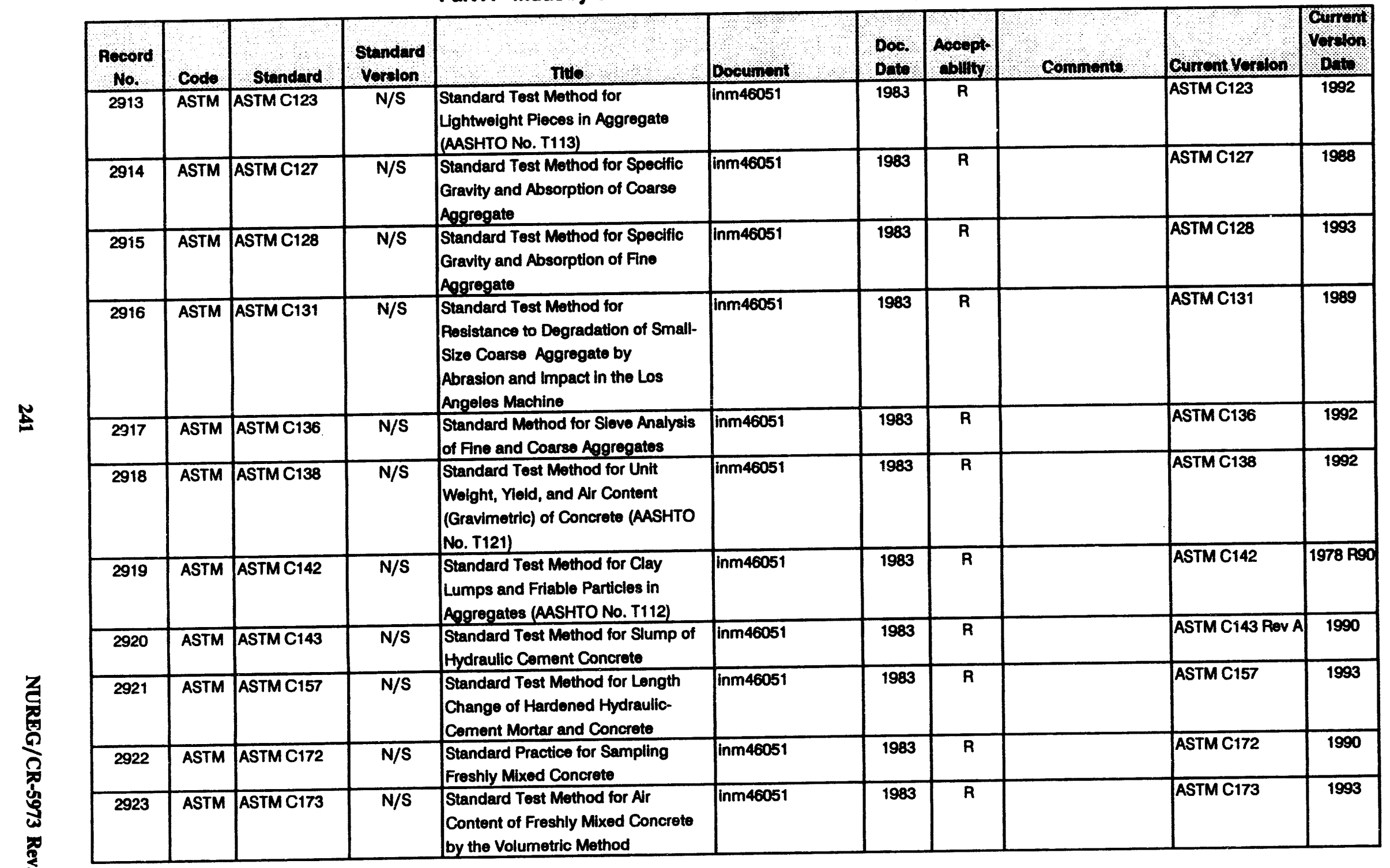




\begin{tabular}{|c|c|c|c|c|c|c|c|c|c|c|}
\hline \multicolumn{11}{|c|}{$\begin{array}{l}\text { Codes and Standards and Other Guidance Cited in Regulatory Documents } \\
\text { Part A - Industry Consensus Codes and Standards (continued) }\end{array}$} \\
\hline $\begin{array}{l}\text { Pocord } \\
\text { No. }\end{array}$ & Code & Standard & $\begin{array}{l}\text { Standard } \\
\text { vorelon. }\end{array}$ & 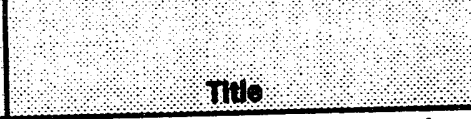 & Bocuments & $\begin{array}{l}\text { Dor: } \\
\text { Dow }\end{array}$ & Acoept: & Commenta & Curront Vorton & vorion \\
\hline 2924 & ASTM & ASTM C191 & N/S & $\begin{array}{l}\text { Standard Test Method for Time of } \\
\text { Setting of Hydraulic Coment by } \\
\text { Vicat Noedle }\end{array}$ & inm46051 & 1983 & $\mathbf{R}$ & & ASTM C191 & 1992 \\
\hline 2925 & ASTM & ASTM C191 & 1974 & $\begin{array}{l}\text { Standard Test Mothod for Time of } \\
\text { Setting of Hydraulic Coment by } \\
\text { Vicat Needle }\end{array}$ & reg1.107.ro1 & 1977 & $\mathbf{P}$ & Implied endorsement & ASTM C191 & 1982 \\
\hline 2926 & ASTM & ASTM C192 & N/S & $\begin{array}{l}\text { Standard Practice for Making and } \\
\text { Curing Concrete Test Specimens in } \\
\text { the Laboratory }\end{array}$ & inm46051 & 1983 & $\bar{R}$ & & ASTM C192 Rev A & 1990 \\
\hline 2927 & ASTM & ASTM C204 & N/S & $\begin{array}{l}\text { Standard Test Method for Fineness } \\
\text { of Portland Cement by Air } \\
\text { Permeability Apparatus (AASHTO } \\
\text { No. T153) }\end{array}$ & inm46051 & 1983 & $\mathbf{R}$ & & ASTM C204 & 1992 \\
\hline 2928 & ASTM & ASTM C231 & N/S & $\begin{array}{l}\text { Standard Test Method for Air } \\
\text { Content of Freshly Mixed Concrete } \\
\text { by the Pressure Method }\end{array}$ & inm46051 & 1983 & $\mathbf{R}$ & & ASTM C231 Rev B & 1991 \\
\hline 2929 & ASTM & ASTM C235 & $N / S$ & Not Found & inm46051 & 1983 & $\mathbf{R}$ & $\begin{array}{l}\text { Replaced by ASTM } \\
\text { C851 which has been } \\
\text { discontinued } \\
\end{array}$ & $n / a$ & $n / 2$ \\
\hline 2930 & ASTM & ASTM C260 & 1974 & $\begin{array}{l}\text { Standard Spectfications for Air } \\
\text { Entraining Admixtures for Concrete }\end{array}$ & reg1.107.ro1 & 1977 & $\bar{R}$ & & ASTM C260 & 1989 \\
\hline 2931 & ASTM & ASTM C266 & $\mathrm{N} / \mathrm{S}$ & $\begin{array}{l}\text { Standard Test Method for Time of } \\
\text { Setting of Hydraulic-Coment Paste } \\
\text { by Gillmore Needles }\end{array}$ & inm46051 & 1983 & $\bar{R}$ & & ASTM C266 & 1989 \\
\hline 2932 & ASTM & ASTM C289 & N/S & $\begin{array}{l}\text { Standard Test Method for Potential } \\
\text { Reactivity of Aggregates (Chemical } \\
\text { Method) }\end{array}$ & inm46051 & 1983 & $\mathbf{R}$ & & ASTM C289 & 1987 \\
\hline 2933 & ASTM & ASTM C311 & $\mathrm{N} / \mathrm{S}$ & $\begin{array}{l}\text { Standard Test Methods for } \\
\text { Sampling and Testing Fly Ash or } \\
\text { Natural Pozzolans for Use as a } \\
\text { Mineral Admixture in Portland- } \\
\text { Cement Concrete }\end{array}$ & $\operatorname{lnm} 46051$ & 1983 & $\bar{R}$ & & ASTM C311 & 1993 \\
\hline
\end{tabular}




\section{Codes and Standards and Other Guidance Cited in Regulatory Documents}

Part A - Industry Consensus Codes and Standards (continued)

\begin{tabular}{|c|c|c|c|c|c|c|c|c|c|c|}
\hline $\begin{array}{l}\text { Pocord } \\
\text { No. }\end{array}$ & Codo & Stondard & standrol & $\left.1, m_{1}\right)_{1}, 1_{1}$ & Docoument & Dor & Aocept & Comnonto & Gunont Yorolon & 16, 100 \\
\hline 2934 & ASTM & ASTM C430 & $\mathrm{N} / \mathrm{S}$ & $\begin{array}{l}\text { Standard Test Method for Fineness } \\
\text { of Hydraulic Cement by the 45- } \\
\text { Micrometer (No. 325) Sieve }\end{array}$ & inm46051 & 1983 & $\mathbf{R}$ & & ASTM C430 & 1992 \\
\hline 2935 & ASTM & ASTM C441 & $\mathrm{N} / \mathrm{S}$ & $\begin{array}{l}\text { Standard Test Method for } \\
\text { Effectiveness of Mineral Admixtures } \\
\text { or Ground Blast-Furnace Slag in } \\
\text { Preventing Excessive Expansion of } \\
\text { Concrete Due to the Alkali-Silica } \\
\text { Reaction }\end{array}$ & inm46051 & 1983 & $\mathbf{R}$ & & ASTM C441 & 1989 \\
\hline 2936 & ASTM & ASTM C469 & $\mathrm{N} / \mathrm{S}$ & $\begin{array}{l}\text { Standard Test Method for Static } \\
\text { Modulus of Elasticity and Poisson's } \\
\text { Ratio of Concrete in Compression }\end{array}$ & inm46051 & 1983 & $\bar{R}$ & & ASTM C469 Rev A & 1987 \\
\hline 2937 & ASTM & ASTM C496 & $\mathbf{N} / \mathbf{S}$ & $\begin{array}{l}\text { Standard Test Method for Splitting } \\
\text { Tensile Strength of Cylindrical } \\
\text { Concrete Specimens }\end{array}$ & inm46051 & 1983 & R & & ASTM C496 & 1990 \\
\hline 2938 & ASTM & ASTM C512 & $N / S$ & $\begin{array}{l}\text { Standard Test Method for Creep of } \\
\text { Concrete in Compression }\end{array}$ & inm46051 & 1983 & $\mathbf{R}$ & & ASTM C512 & $1987 \mathrm{Rg2}$ \\
\hline 2939 & ASTM & ASTM C512 & 1976 & $\begin{array}{l}\text { Standard Test Method for Creep of } \\
\text { Concrete in Compression }\end{array}$ & reg1.35.1.roo & 1990 & $\mathbf{R}$ & & ASTM C512 & 1987 R92 \\
\hline 2940 & ASTM & ASTM C535 & $N / S$ & $\begin{array}{l}\text { Standard Test Method for } \\
\text { Resistance to Degradation of Large- } \\
\text { Size Coarse Aggregate by Abrasion } \\
\text { and Impact in the Los Angeles } \\
\text { Machine }\end{array}$ & inm46051 & 1983 & $\mathbf{R}$ & & ASTM C535 & 1989 \\
\hline 2941 & ASTM & ASTM C566 & $\mathbf{N} / \mathrm{S}$ & $\begin{array}{l}\text { Standard Test Method for Total } \\
\text { Moisture Content of Aggregate by } \\
\text { Drying }\end{array}$ & inm46051 & 1983 & $\mathbf{R}$ & & ASTM C566 & 1989 \\
\hline 2942 & ASTM & ASTM C597 & 1971 & $\begin{array}{l}\text { Standard Test Method for Pulse } \\
\text { Velocity Through Concrete }\end{array}$ & reg1.90.r01 & 1977 & R & & ASTM C597 & 1987 R91 \\
\hline 2943 & ASTM & ASTM C642 & N/S & $\begin{array}{l}\text { Standard Test Method for Specific } \\
\text { Gravity, Absorption, and Voids in } \\
\text { Hardened Concrete }\end{array}$ & inm46051 & 1983 & R & & ASTM C642 & 1990 \\
\hline
\end{tabular}




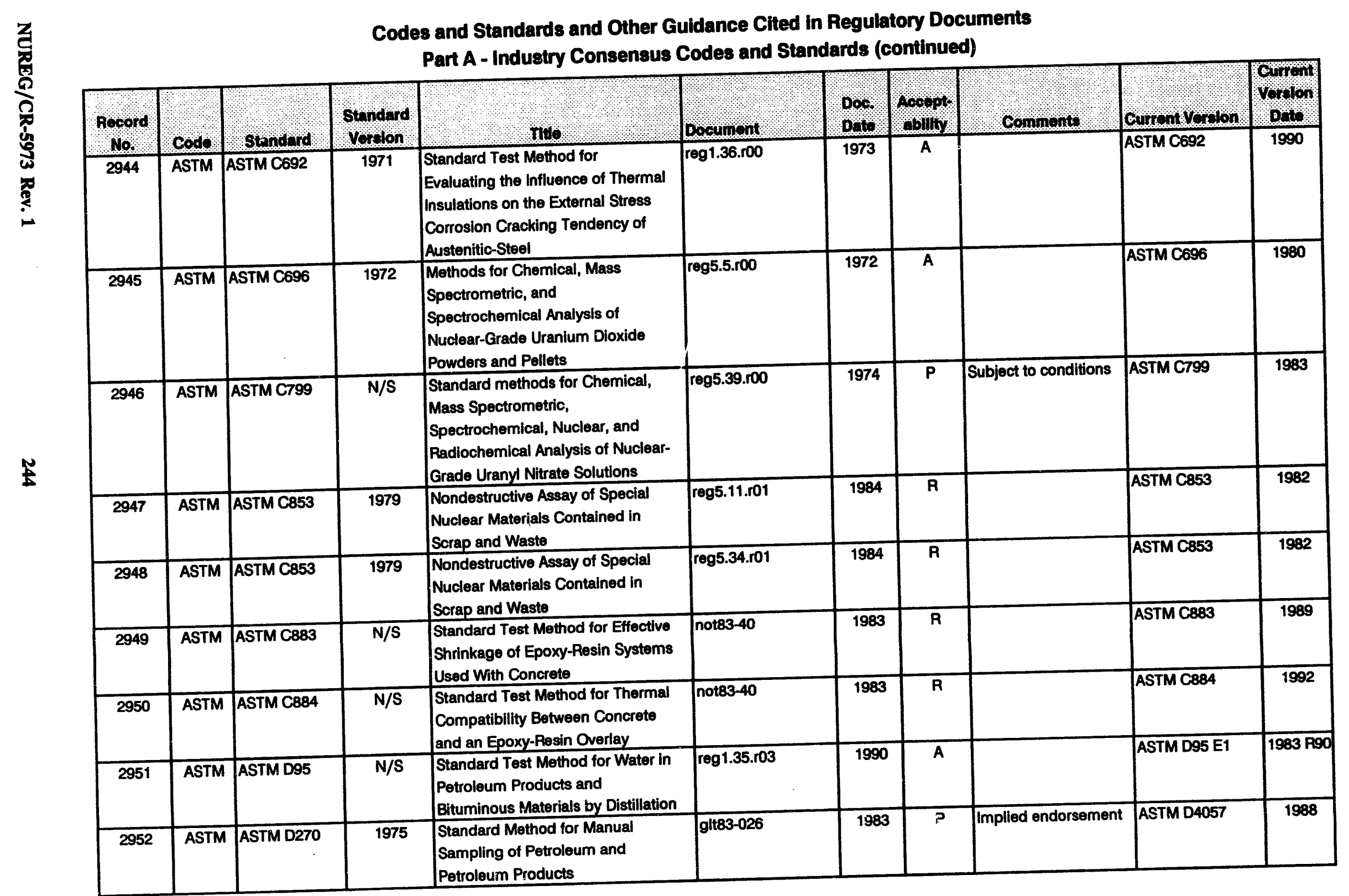


Codes and Standards and Other Guidance Clted in Regulatory Documents

Part A - Industry Consensus Codes and Standards (continued)

\begin{tabular}{|c|c|c|c|c|c|c|c|c|c|c|}
\hline Hocord & Codo & Standard & $\begin{array}{l}\text { Standard } \\
\text { vordon }\end{array}$ & nto & Docunent & Doo & Acoopt & Comments & Cument vorolon & phipto \\
\hline 2953 & ASTM & ASTM D270 & 1975 & $\begin{array}{l}\text { Standard Method for Manual } \\
\text { Sampling of Petroieum and } \\
\text { Petroleum Products }\end{array}$ & reg1.137.ro1 & 1979 & $A$ & & ASTM D4057 & 1988 \\
\hline 2954 & ASTM & ASTM D421 & $\mathrm{N} / \mathrm{S}$ & $\begin{array}{l}\text { Standard Practice for Dry } \\
\text { Preparation of Soil Samples for } \\
\text { Particle-Size Analysis and } \\
\text { Determination of Soll Constants }\end{array}$ & reg1.138.r00 & 1978 & $\mathbf{P}$ & Implied endorsement & ASTM D421 & 1985 \\
\hline 2955 & ASTM & ASTM D422 & $N / S$ & $\begin{array}{l}\text { Standard Test Method for Particle- } \\
\text { Size Analysis of Solls }\end{array}$ & inm45053 & 1984 & $\mathbf{R}$ & & ASTM D422 E1 & $1903 \mathrm{Rg0}$ \\
\hline 2956 & ASTM & ASTM D422 & $N / S$ & $\begin{array}{l}\text { Standard Test Method for Particle- } \\
\text { Size Analysis of Solls }\end{array}$ & reg1.138.ro0 & 1978 & $\mathbf{P}$ & Implied endorsement & ASTM D422 E1 & $1963 \mathrm{R} 90$ \\
\hline 2957 & ASTM & ASTM D423 & $N / S$ & $\begin{array}{l}\text { Standard Test Mothod for Liquid } \\
\text { Limit, Plastic Limit, and Plasticity } \\
\text { Index of Soils }\end{array}$ & inm45053 & 1984 & $\mathbf{R}$ & & ASTM D4318 & 1984 \\
\hline 2958 & ASTM & ASTM D423 & $N / S$ & $\begin{array}{l}\text { Standard Test Method for Liquid } \\
\text { Limit, Plastic Limit, and Plasticity } \\
\text { Index of Soils }\end{array}$ & reg1.138.roo & 1978 & $\mathbf{P}$ & Implied endorsement & ASTM D4318 & 1984 \\
\hline 2959 & ASTM & ASTM D424 & $N / S$ & $\begin{array}{l}\text { Standard Test Method for Liquid } \\
\text { Limit, Plastic Limit, and Plasticity } \\
\text { Index of Solls }\end{array}$ & inm45053 & 1984 & $\mathbf{R}$ & & ASTM D4318 & 1984 \\
\hline 2960 & ASTM & ASTM D424 & $N / S$ & $\begin{array}{l}\text { Standard Test Method for Liquid } \\
\text { Limit, Plastic Limit, and Plasticity } \\
\text { Index of Soils }\end{array}$ & reg1.138.r00 & 1978 & $\mathbf{P}$ & Implied endorsement & ASTM D4318 & 1984 \\
\hline 2961 & ASTM & ASTM D427 & $\mathrm{N} / \mathrm{S}$ & $\begin{array}{l}\text { Standard Test Method for } \\
\text { Shrinkage Factor of Soils by the } \\
\text { Mercury Method }\end{array}$ & reg1.138.roo & 1978 & $\bar{P}$ & Implied endorsement & ASTM D427 E1 & 1992 \\
\hline 2962 & ASTM & ASTM D511 & 1982 & $\begin{array}{l}\text { Standard Test Methods for Calcium } \\
\text { and Magnesium in Water }\end{array}$ & inm79501 & 1989 & $\mathbf{R}$ & & ASTM D511 & 1993 \\
\hline 2963 & ASTM & ASTM D512 & $N / S$ & $\begin{array}{l}\text { Standard Test Methods for Chloride } \\
\text { Ion in Water }\end{array}$ & inm46051 & 1983 & $\mathbf{R}$ & & ASTM D512 & 1989 \\
\hline 2964 & ASTM & ASTM D512 & 1981 & $\begin{array}{l}\text { Standard Test Methods for Chloride } \\
\text { Ion in Water }\end{array}$ & inm79501 & 1989 & $\mathbf{R}$ & & ASTM D512 & 1989 \\
\hline 2965 & ASTM & ASTM D512 & 1967 & $\begin{array}{l}\text { Standard Test Methods for Chloride } \\
\text { Ion in Water }\end{array}$ & reg1.107.r01 & 1977 & $\mathbf{R}$ & & ASTM D512 & 1989 \\
\hline
\end{tabular}




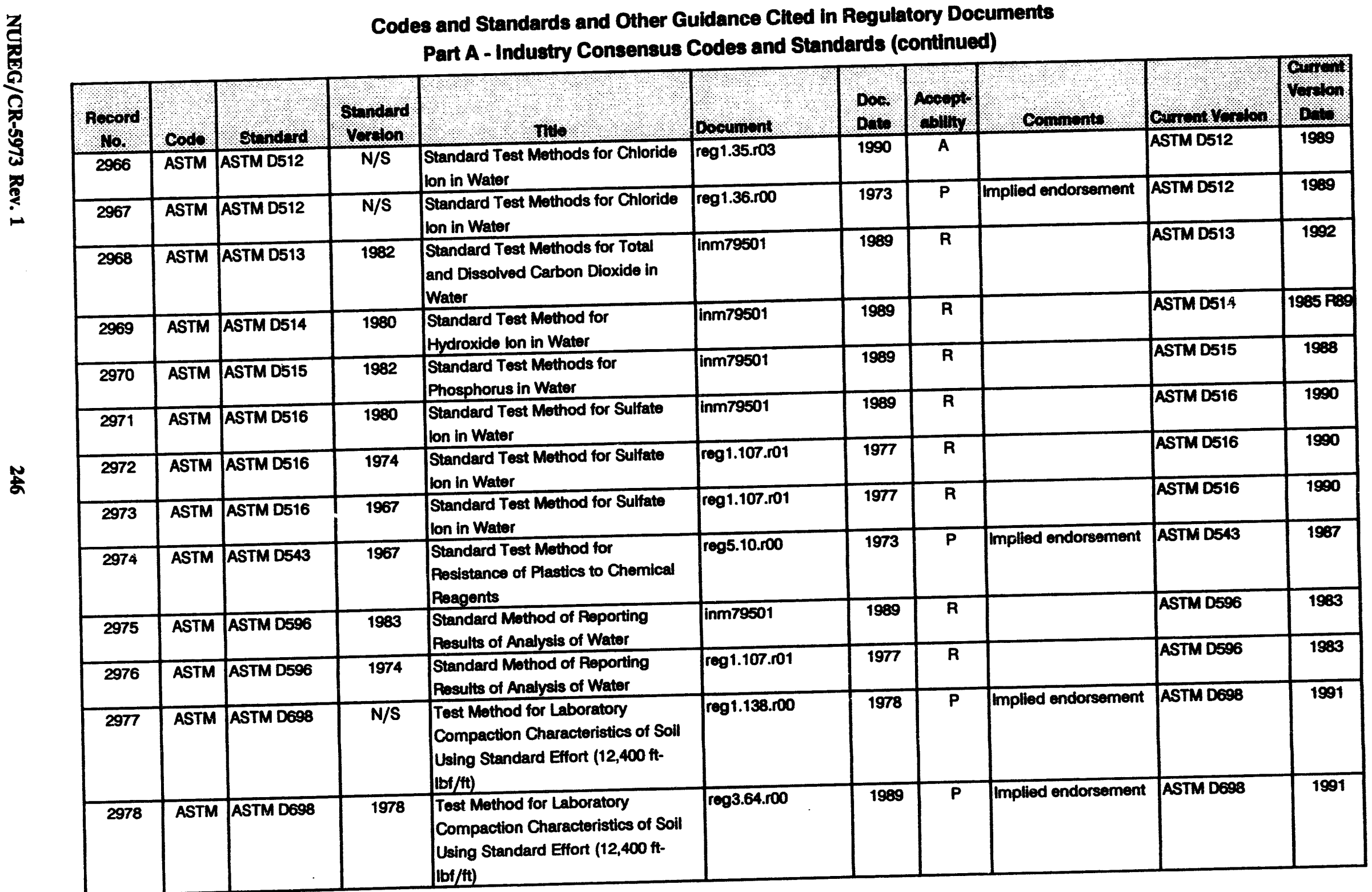


Codes and Standards and Other Guidance Cited in Regulatory Documents

Part A - Industry Consensus Codes and Standards (continued)

\begin{tabular}{|c|c|c|c|c|c|c|c|c|c|c|}
\hline Hocord & Code & Stundord & $\begin{array}{l}\text { Stundard } \\
\text { Verolon }\end{array}$ & 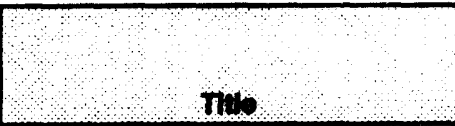 & Document & $\begin{array}{l}\text { Doc. } \\
\text { Dote. }\end{array}$ & Aoospt & Comments. & Con non volon & (6) \\
\hline 2979 & ASTM & ASTM D698 & N/S & $\begin{array}{l}\text { Test Mothod for Laboratory } \\
\text { Compaction Charactoristics of Soil } \\
\text { Using Standard Effort }(12,400 \mathrm{ft}- \\
\text { (bf/ft) }\end{array}$ & inm45053 & 1984 & $\mathbf{R}$ & & ASTM DESB & 1991 \\
\hline 2980 & ASTM & ASTM D854 & N/S & $\begin{array}{l}\text { Standard Test Mothod for Specific } \\
\text { Gravity of Solls }\end{array}$ & reg1.138.r00 & 1978 & $\mathbf{P}$ & Implied endorsement & ASTM DESA E1 & 1992 \\
\hline 2981 & ASTM & ASTM D857 & 1979 & $\begin{array}{l}\text { Standard Test Mothods for } \\
\text { Aluminum in Water }\end{array}$ & inm79501 & 1989 & $\mathbf{R}$ & & ASTM D857 & 1989 \\
\hline 2982 & ASTM & ASTM D859 & 1980 & $\begin{array}{l}\text { Standard Test Mothod for Silica in } \\
\text { Water }\end{array}$ & inm79501 & 1989 & $\mathbf{R}$ & & ASTM D859 & 1988 \\
\hline 2983 & ASTM & ASTM D887 & 1982 & $\begin{array}{l}\text { Standard Practices for Sampling } \\
\text { Water-Formed Deposits }\end{array}$ & inm79501 & 1989 & $\mathbf{R}$ & & ASTM D687 & $1982 \mathrm{Reg}$ \\
\hline 2984 & ASTM & ASTM D888 & 1981 & $\begin{array}{l}\text { Standard Test Mothods for } \\
\text { Dissolved Oxygen in Water }\end{array}$ & inm79501 & 1989 & $\mathbf{R}$ & & ASTM D888 & 1992 \\
\hline 2985 & ASTM & ASTM D974 & $N / S$ & $\begin{array}{l}\text { Standard Test Mothod for Acid and } \\
\text { Base Number by Color-Indicator } \\
\text { Titration }\end{array}$ & reg1.35.ro3 & 1990 & A & & ASTM D974 & 1992 \\
\hline 2986 & ASTM & ASTM D975 & 1977 & Specification for Diesel Fuel Oils & gitt83-026 & 1983 & $\overline{\mathbf{P}}$ & & ASTM D975 & 1992 \\
\hline 2987 & ASTM & ASTM D975 & $N / S$ & Specification for Diesel Fuel Oils & sts4-geB3.8.3 & 1991 & $\mathbf{P}$ & & ASTM D975 & 1992 \\
\hline 2988 & ASTM & ASTM D975 & $\mathrm{N} / \mathrm{S}$ & Specification for Diesel Fuel Oils & sts6-geB3.8.3 & 1991 & $\mathbf{P}$ & & ASTM D975 & 1992 \\
\hline 2989 & ASTM & ASTM D975 & N/S & Specification for Diesel Fuel Oils & stab8wB3.8.3 & 1991 & $\mathbf{P}$ & & ASTM D975 & 1992 \\
\hline 2990 & ASTM & ASTM D975 & $\mathrm{N} / \mathrm{S}$ & Specification for Diesel Fuel Oils & stsce83.8.3 & 1991 & $\mathbf{P}$ & & ASTM 0975 & 1992 \\
\hline 2991 & ASTM & ASTM D975 & $\mathbf{N} / \mathbf{S}$ & Specification for Diesel Fuel Oils & stswst 83.8 .3 & 1991 & $\mathbf{P}$ & & ASTM D975 & 1992 \\
\hline 2992 & ASTM & ASTM D992 & 1971 & $\begin{array}{l}\text { Standard Test Mothods for Nitrite- } \\
\text { Nitrate in Water }\end{array}$ & reg1.107.ro1 & 1977 & $\bar{R}$ & & ASTM D3867 & 1990 \\
\hline 2993 & ASTM & ASTM D992 & $N / S$ & $\begin{array}{l}\text { Standard Test Methods for Nitrite- } \\
\text { Nitrate in Water }\end{array}$ & reg1.35.103 & 1990 & $\bar{R}$ & & ASTM D3867 & 1990 \\
\hline
\end{tabular}


Codes and Standards and Other Guidance Clted in Regulatory Documents Part A - Industry Consensus Codes and Standards (continued)

\begin{tabular}{|c|c|c|c|c|c|c|c|c|c|c|}
\hline $\begin{array}{l}\text { Accord } \\
\text { No. }\end{array}$ & cods & stunderd & $\begin{array}{l}\text { Stunderd } \\
\text { Vercion }\end{array}$ & me & Doounent & Dow & Aconpl & Comments & Cournentronion & Comen \\
\hline 2994 & ASTM & ASTM D1068 & 1982 & $\begin{array}{l}\text { Standard Practico for Sampling } \\
\text { Stoam }\end{array}$ & Inm79501 & 1989 & R & & ASTM D1066 & 1982 A90 \\
\hline 2995 & ASTM & ASTM D1067 & 1982 & $\begin{array}{l}\text { Standard Test Methods for Acldily } \\
\text { or Alkalinity of Water }\end{array}$ & Inm79501 & 1900 & $\mathbf{R}$ & & ASTM D1067 & 1992 \\
\hline 2996 & ASTM & ASTM D1068 & 1984 & $\begin{array}{l}\text { Standard Toest Mothods for ron in } \\
\text { Water }\end{array}$ & inm79501 & 1990 & $\mathbf{R}$ & & ASTM D1068 & 1990 \\
\hline 2997 & ASTM & ASTM D1125 & 1982 & $\begin{array}{l}\text { Standard Teat Mothods for } \\
\text { Eloctrical Conductivity and } \\
\text { Posedstivity of Wator }\end{array}$ & $\operatorname{lnm} 79501$ & 1969 & $\mathbf{R}$ & & ASTM D1125 & 1991 \\
\hline 2998 & ASTM & ASTM D1126 & 1980 & $\begin{array}{l}\text { Standerd Test Mothod for Herdnoss } \\
\text { in Wator }\end{array}$ & Inm79501 & 1909 & $\mathbf{R}$ & & ASTM D1126 & 1996 POE \\
\hline 2999 & ASTM & ASTM D1129 & 1982 & $\begin{array}{l}\text { Standard Torminology Poleteting to } \\
\text { Water }\end{array}$ & Inm79501 & 1909 & $\overline{\mathbf{A}}$ & & ASTM D1120 & 1990 \\
\hline 3000 & ASTM & ASTM D1129 & 1974 & $\begin{array}{l}\text { Standard Torminology Aolating to } \\
\text { Wator }\end{array}$ & reg1.107.ro1 & 197 & $\mathbf{R}$ & & ASTM D1120 & 1990 \\
\hline 3001 & ASTM & ASTM D1140 & N/S & $\begin{array}{l}\text { Test Mothod for Amount of Material } \\
\text { in Soils Finer than tho No. } 200 \text { (75 } \\
\text { micro-m) Siow }\end{array}$ & $\operatorname{Inm} 45053$ & 1984 & $\mathbf{R}$ & & ASTM D1140 E1 & 1992 \\
\hline 3002 & ASTM & ASTM D1140 & $\mathrm{N} / \mathrm{S}$ & $\begin{array}{l}\text { Test Mothod for Amount of Meatorial } \\
\text { in Soils Finor then the No. } 200 \text { (75 } \\
\text { micro-m) Stove }\end{array}$ & $\operatorname{reg} 1.138 .00$ & 1978 & $\mathbf{P}$ & Implied endorsement & ASTM D1140E1 & 1902 \\
\hline 3003 & ASTM & ASTM D1143 & $\mathrm{N} / \mathrm{S}$ & $\begin{array}{l}\text { Standard Toet Mothod for Pilles } \\
\text { Undor Static Axial Comproselve } \\
\text { Load }\end{array}$ & $\operatorname{lnm} 45053$ & 1994 & $\mathbf{R}$ & & ASTM D1143 & $1981 \mathrm{PBO}$ \\
\hline 3004 & ASTM & ASTM D1179 & 1980 & $\begin{array}{l}\text { Standard Test Mothods for Fluoride } \\
\text { Ion in Water }\end{array}$ & Inm79501 & 1900 & $\mathbf{R}$ & & ASTM D1179 & 1968 \\
\hline 3005 & ASTM & ASTM D1179 & N/S & $\begin{array}{l}\text { Standard Test Methods for Fluoride } \\
\text { Ion in Water }\end{array}$ & 1001.36 .500 & 1973 & $\bar{P}$ & Implied endorsement & ASTMD1179 & 1998 \\
\hline 3006 & ASTM & ASTM D1246 & 1982 & $\begin{array}{l}\text { Standard Teet Mothod for Bromido } \\
\text { Ion in Water }\end{array}$ & inm79501 & 1900 & $\mathbf{R}$ & & ASTM D1246 & 1988 \\
\hline 3007 & ASTM & ASTM D1267 & N/S & $\begin{array}{l}\text { Standard Teat Mothod for Vapor } \\
\text { Pressure of Liquefiod Petroloum } \\
\text { (LP) Gases (LP-Gas Mothod) }\end{array}$ & $\operatorname{inm} 45053$ & 1984 & $\mathbf{R}$ & & ASTM D1267 & 1900 \\
\hline
\end{tabular}


Codes and Standands and Other Guidance Chted in Regulatory Documents

Part A - Induetry Consensus Codes and Standards (continued)

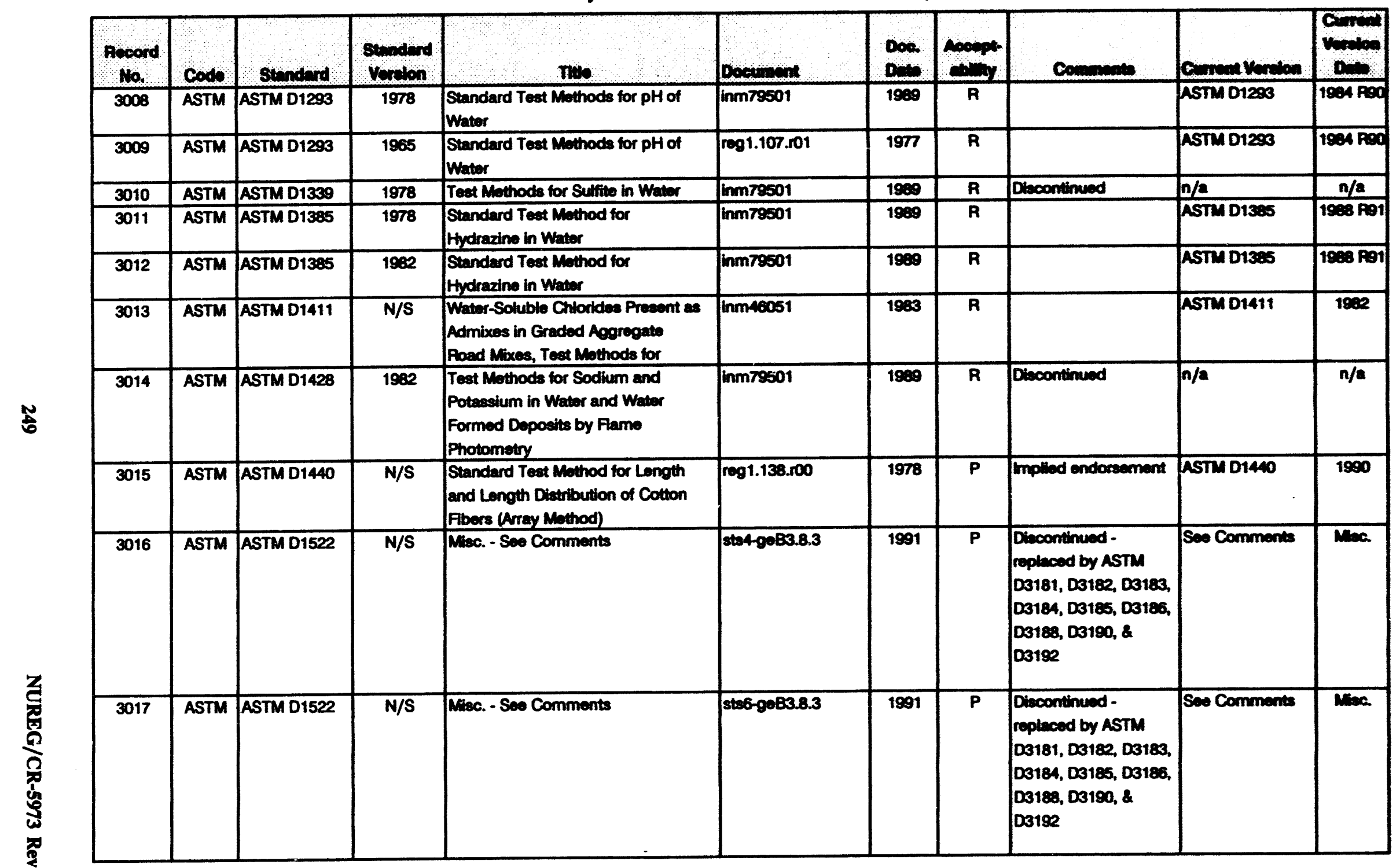




\begin{tabular}{|c|c|c|c|c|c|c|c|c|c|c|}
\hline \multicolumn{11}{|c|}{$\begin{array}{c}\text { Codes and Standards and Other Guidance Ched in Regulatory Documents } \\
\text { Part A - Industry Consensus Codes and Standards (continued) }\end{array}$} \\
\hline $\begin{array}{c}\text { Record } \\
\text { No. }\end{array}$ & Codo & Standard & $\begin{array}{l}\text { Stunderd } \\
\text { Verelon }\end{array}$ & $\quad m_{0}$ & Document & Dow & Alooplop & Connones & Cunnent Voricen & $\begin{array}{l}\text { Coming } \\
\text { Veralon } \\
\text { Ding }\end{array}$ \\
\hline 3018 & ASTM & ASTM D1522 & $N / S$ & Misc. - Soo Comments & stsb\&:wB3.8.3 & 1991 & $P$ & $\begin{array}{l}\text { Discontinued - } \\
\text { replacod by ASTM } \\
\text { D3181, D3182, D3183, } \\
\text { D3184, D3185, D3186. } \\
\text { D3188, D3190, \& } \\
\text { D3192 }\end{array}$ & Soe Comments & Misc. \\
\hline 3019 & ASTM & ASTM D1522 & N/S & Misc. - See Comments & stacoB3.8.3 & 1991 & $\bar{P}$ & $\begin{array}{l}\text { Disconthued - } \\
\text { roplacod by ASTM } \\
\text { D3181, D3182, D3183, } \\
\text { D3184, D3185, D3186, } \\
\text { D3188, D3190, \& } \\
\text { D3192 }\end{array}$ & Seo Comments & Max. \\
\hline 3020 & ASTM & ASTM 01522 & $\mathrm{~N} / \mathrm{S}$ & Misc. - Soe Comments & stowstB3.8.3 & 1991 & $\bar{P}$ & $\begin{array}{l}\text { Discontinued - } \\
\text { replecod by ASTM } \\
\text { D3181, D3182, D3183, } \\
\text { D3184, D3185, D3186, } \\
\text { D3188, D3190, \& } \\
\text { D3192 }\end{array}$ & Soe Comments & Mac. \\
\hline 3021 & ASTM & ASTM D1556 & $\mathrm{N} / \mathrm{S}$ & $\begin{array}{l}\text { Standard Test Method for Density } \\
\text { and Unit Woight of Soll in Place by } \\
\text { the Sand-Cone Mothod }\end{array}$ & inm2620 & 1993 & $\bar{R}$ & & ASTM D1556 E1 & 1990 \\
\hline 3022 & ASTM & ASTM D1556 & $N / S$ & $\begin{array}{l}\text { Standard Test Mothod for Donsity } \\
\text { and Unit Woight of Soll in Place by } \\
\text { the Sand-Cone Mothod }\end{array}$ & $\operatorname{inm} 2620$ & 1993 & $\bar{R}$ & & ASTM D1556 E1 & 1990 \\
\hline 3023 & $\overline{A S T M}$ & ASTM D1557 & $\mathrm{N} / \mathrm{S}$ & $\begin{array}{l}\text { Test Methods for Laboratory } \\
\text { Compaction Characteristics of So:l } \\
\text { Using Modified Effort }(56,000 \mathrm{tt}-\mathrm{bf} \\
(2700 \mathrm{kN}-\mathrm{m} / \mathrm{n}))\end{array}$ & inm45053 & 1984 & $\overline{\mathbf{R}}$ & & ASTM D1557 & 1991 \\
\hline
\end{tabular}




\section{Codes and Standards and Other Guidance Cited in Regulatory Documents}

Part A - Industry Consensus Codes and Standards (continued)

\begin{tabular}{|c|c|c|c|c|c|c|c|c|c|c|}
\hline $\begin{array}{l}\text { Ascord } \\
\text { No. }\end{array}$ & Code & Standard & $\begin{array}{l}\text { Stundard } \\
\text { Voralon }\end{array}$ & Whe & Document & Dos. & Anopis & Comments & Cument Venson & $\begin{array}{l}\text { Comin } \\
\text { Verion } \\
\text { Qnin }\end{array}$ \\
\hline 3024 & ASTM & ASTM D1557 & $\mathbf{N} / \mathbf{S}$ & $\begin{array}{l}\text { Test Methods for Laboratory } \\
\text { Compaction Characteristics of Soll } \\
\text { Using Modified Effort }(56,000 \mathrm{ft}-\mathrm{bf} \\
(2700 \mathrm{kN}-\mathrm{m} / \mathrm{n}))\end{array}$ & reg1.138.r00 & 1978 & $\mathbf{P}$ & Implied endorsement & ASTM D1557 & 1991 \\
\hline 3025 & ASTM & ASTM D1586 & $\mathbf{N} / \mathbf{S}$ & $\begin{array}{l}\text { Standard Test Mothod for } \\
\text { Penetration Test and Split-Barrel } \\
\text { Sampling of Solls }\end{array}$ & inm45053 & 1984 & $\mathbf{R}$ & & ASTM D1586 & 1984 R92 \\
\hline 3026 & ASTM & ASTM D1587 & 1967 & $\begin{array}{l}\text { Standard Test Mothod for Thin- } \\
\text { Walled Tube Sampling of Solls }\end{array}$ & reg1.132.ro1 & 1979 & $\mathbf{P}$ & Implied endorsement & ASTM D1587 & 1983 \\
\hline 3027 & ASTM & ASTMD1588 & 1960 & Not Found & inm79501 & 1989 & $\mathbf{A}$ & Discontinued & $n / a$ & $n / a$ \\
\hline 3028 & ASTM & ASTM D1588 & 1974 & Not Found & inm79501 & 1989 & $\mathbf{R}$ & Discontinued & $n / a$ & $n / a$ \\
\hline 3029 & ASTM & ASTM D1599 & 1974 & $\begin{array}{l}\text { Standard Test Mothod for Short- } \\
\text { Time Hydraulic Failure Pressure of } \\
\text { Plastic Pipe, Tubing, and Fittings }\end{array}$ & reg1.72.02 & 1978 & $\mathbf{P}$ & Implied endorsement & ASTM D1599 E1 & 1988 \\
\hline 3030 & ASTM & ASTM D1687 & 1980 & $\begin{array}{l}\text { Standard Test Mothods for } \\
\text { Chromium in Water }\end{array}$ & inm79501 & 1989 & $\mathbf{A}$ & & ASTM D1687 & 1986 \\
\hline 3031 & ASTM & ASTM D1688 & 1982 & $\begin{array}{l}\text { Standard Teet Mothods for Copper } \\
\text { in Water }\end{array}$ & inm79501 & 1969 & $\mathbf{R}$ & & ASTM D1688 & 1990 \\
\hline 3032 & ASTM & ASTM D1691 & 1977 & $\begin{array}{l}\text { Standard Teat Mothods for Zinc in } \\
\text { Water }\end{array}$ & inm79501 & 1989 & $\mathbf{R}$ & & ASTM D1691 & 1990 \\
\hline 3033 & ASTM & ASTM D1886 & 1984 & $\begin{array}{l}\text { Standard Test Methods for Nickel in } \\
\text { Water }\end{array}$ & inm79501 & 1989 & $\mathbf{R}$ & & ASTM D1886 & 1990 \\
\hline 3034 & ASTM & ASTM D1888 & $N / S$ & $\begin{array}{l}\text { Tests for Particulate and Dissolved } \\
\text { Matter, Solids or Residue in Water }\end{array}$ & inm46051 & 1983 & $\mathbf{R}$ & Discontinued & $n / a$ & $n / a$ \\
\hline 3035 & ASTM & ASTM D1888 & 1978 & $\begin{array}{l}\text { Tests for Particulate and Dissolved } \\
\text { Matter, Solids or Pesidue in Water }\end{array}$ & inm79501 & 1989 & $\mathbf{R}$ & Dissontinued & $n / a$ & $n / a$ \\
\hline 3036 & ASTM & ASTM D1889 & 1981 & $\begin{array}{l}\text { Standard Test Mothods for } \\
\text { Turbidity in Wator }\end{array}$ & inm79501 & 1989 & $\mathbf{R}$ & & ASTM D1889 & 1988 \\
\hline 3037 & ASTM & ASTM D2049 & $N / S$ & $\begin{array}{l}\text { Standard Test Mothods for } \\
\text { Maximum Indox Density and Unit } \\
\text { Weight of Solls Using Vibratory } \\
\text { Tables }\end{array}$ & reg1.138.ro0 & 1978 & $\mathbf{P}$ & $\begin{array}{l}\text { Discontinued - } \\
\text { replaced by ASTM } \\
\text { D4253 \& D4254 }\end{array}$ & $\begin{array}{l}\text { ASTM D4253 \& } \\
\text { D4254 }\end{array}$ & $\begin{array}{c}1993 / \\
1991\end{array}$ \\
\hline
\end{tabular}




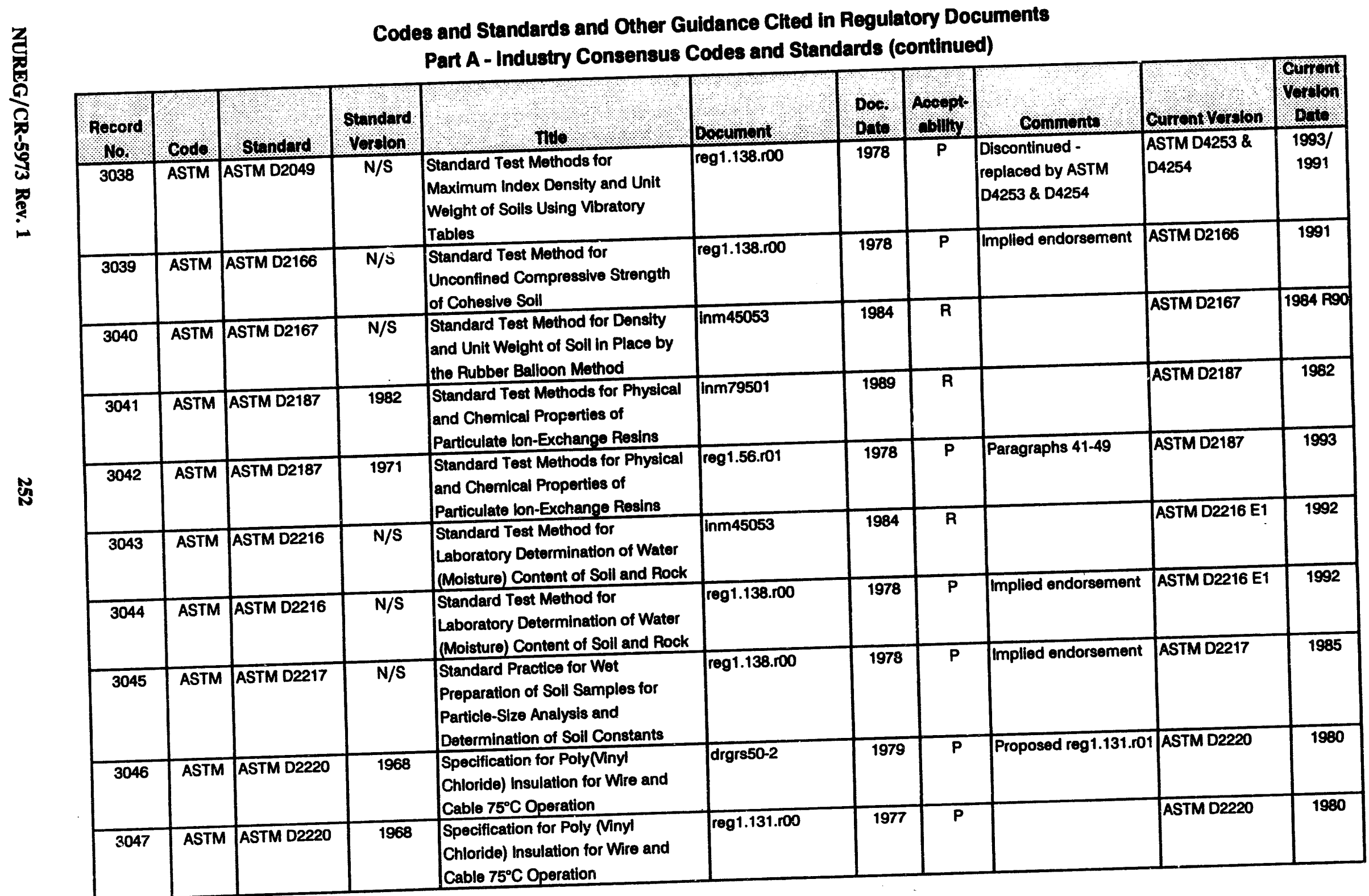


Codes and Standards and Other Guidance Cited in Regulatory Documents

Part A - Industry Consensus Codes and Standards (continued)

\begin{tabular}{|c|c|c|c|c|c|c|c|c|c|c|}
\hline $\begin{array}{l}\text { Pocord } \\
\text { No. }\end{array}$ & Codo & Standard & $\begin{array}{l}\text { Strondard } \\
\text { Vorolon }\end{array}$ & ( & Doosumunt & Doc. & $\begin{array}{l}\text { Acospt } \\
\text { Abllity }\end{array}$ & (_. Commonts & Curront vorolon & Gorront \\
\hline 3048 & ASTM & ASTM D2274 & 1970 & $\begin{array}{l}\text { Standard Test Method for Oxidation } \\
\text { Stability of Distillate Fuel OII } \\
\text { (Accelerated Method) }\end{array}$ & glt83-026 & 1983 & $\mathbf{P}$ & Implied endorsement & ASTM D2274 & 1988 \\
\hline 3049 & ASTM & ASTM D2274 & 1970 & $\begin{array}{l}\text { Standard Test Method for Oxidation } \\
\text { Stability of Distillate Fuel Oil } \\
\text { (Accelerated Method) }\end{array}$ & not91-46 & 1991 & $\mathbf{R}$ & & ASTM D2274 & 1988 \\
\hline 3050 & ASTM & ASTM D2276 & 1978 & $\begin{array}{l}\text { Standard Test Method for } \\
\text { Particulate Contaminant in Aviation } \\
\text { Fuel by Line Sampling }\end{array}$ & not91-46 & 1991 & $\mathbf{P}$ & & ASTM D2276 E1 & 1993 \\
\hline 3051 & ASTM & ASTM D2276 & N/S & $\begin{array}{l}\text { Standard Test Method for } \\
\text { Particulate Contaminant in Aviation } \\
\text { Fuel by Line Sampling }\end{array}$ & sts4-geB3.8.3 & 1991 & $\mathbf{P}$ & & ASTM D2276 E1 & 1993 \\
\hline 3052 & ASTM & ASTM D2276 & $\mathbf{N} / \mathbf{S}$ & $\begin{array}{l}\text { Standard Test Mothod for } \\
\text { Particulate Contaminant in Aviation } \\
\text { Fuel by Line Sampling }\end{array}$ & sts4-geB5.7.2 & 1991 & $\mathbf{P}$ & & ASTM D2276 E1 & 1993 \\
\hline 3053 & ASTM & ASTM D2276 & $\mathrm{N} / \mathrm{S}$ & $\begin{array}{l}\text { Standard Test Method for } \\
\text { Particulate Contaminant in Aviation } \\
\text { Fuel by Line Sampling }\end{array}$ & sts6-geB3.8.3 & 1991 & $\mathbf{P}$ & & ASTM D2276 E1 & 1993 \\
\hline 3054 & ASTM & ASTM D2276 & $\mathrm{N} / \mathrm{S}$ & $\begin{array}{l}\text { Standard Test Method for } \\
\text { Particulate Contaminant in Aviation } \\
\text { Fuel by Line Sampling }\end{array}$ & sts6-geB5.7.2 & 1991 & $\mathbf{P}$ & & ASTM D2276 E1 & 1993 \\
\hline 3055 & ASTM & ASTM D2276 & $N / S$ & $\begin{array}{l}\text { Standard Test Method for } \\
\text { Particulate Contaminant in Aviation } \\
\text { Fuel by Line Sampling }\end{array}$ & stsb\&wB3.8.3 & 1991 & $\mathbf{P}$ & & ASTM D2276 E1 & 1993 \\
\hline 3056 & ASTM & ASTM D2276 & $\mathrm{N} / \mathrm{S}$ & $\begin{array}{l}\text { Standard Test Mothod for } \\
\text { Particulate Contaminant in Aviation } \\
\text { Fuel by Line Sampling } \\
\end{array}$ & stsb\&wB5.7.2 & 1991 & $\mathbf{P}$ & & ASTM D2276 E1 & 1993 \\
\hline 3057 & ASTM & ASTM D2276 & $\mathbf{N} / \mathrm{S}$ & $\begin{array}{l}\text { Standard Test Method for } \\
\text { Particulate Contaminant in Aviation } \\
\text { Fuel by Line Sampling }\end{array}$ & stsceB3.8.3 & 1991 & $\mathbf{P}$ & & ASTM D2276 E1 & 1993 \\
\hline 3058 & ASTM & ASTM D2276 & $N / S$ & $\begin{array}{l}\text { Standard Test Mothod for } \\
\text { Particulate Contaminant in Aviation } \\
\text { Fuel by Line Sampling }\end{array}$ & stsceB5.7.2 & 1991 & $\mathbf{P}$ & & ASTM D2276 E1 & 1993 \\
\hline
\end{tabular}


Codes and Standards and Other Guidance Cited in Regulatory Documents

Part A - Industry Consensus Codes and Standards (continued)

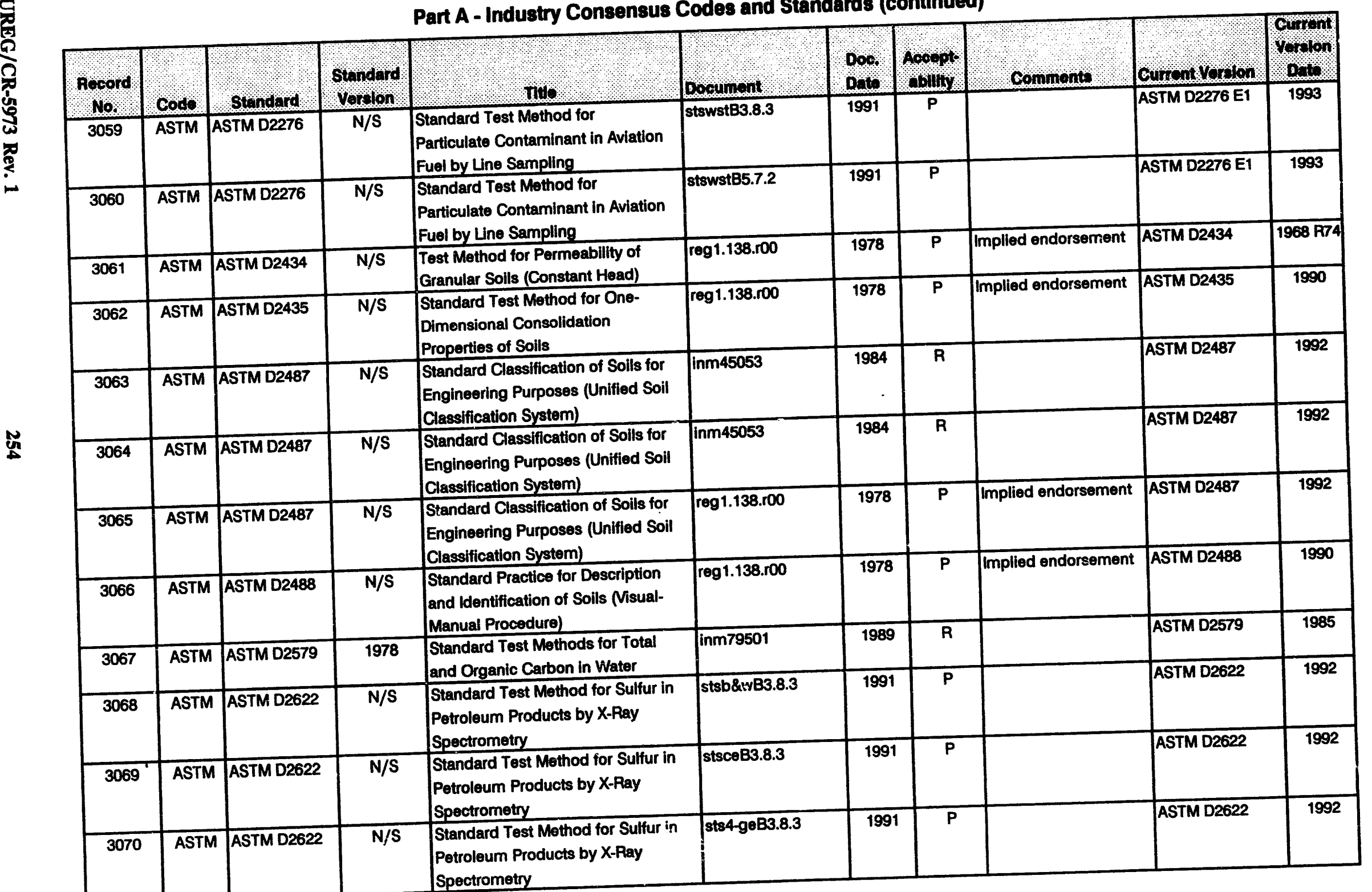


Codes and Standards and Other Guidance Clted in Regulatory Documents

Part A - Industry Consensus Codes and Standards (continued)

\begin{tabular}{|c|c|c|c|c|c|c|c|c|c|c|}
\hline Pooord & Codo & Standnrd & Strondard & (4. & Documont & Doc. & A.01pto & comment & Cunoun 1. & 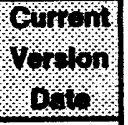 \\
\hline 3071 & ASTM & ASTM D2622 & $N / S$ & $\begin{array}{l}\text { Standard Test Method for Sulfur in } \\
\text { Petroleum Products by X-Rlay } \\
\text { Spectrometry }\end{array}$ & sts6-geB3.8.3 & 1991 & $\mathbf{P}$ & & ASTM D2622 & 1992 \\
\hline 3072 & ASTM & ASTM D2622 & $\mathrm{N} / \mathrm{S}$ & $\begin{array}{l}\text { Standard Test Method for Sulfur in } \\
\text { Petroleum Products by X-Ray } \\
\text { Spectrometry }\end{array}$ & stswstB3.8.3 & 1991 & $\mathbf{P}$ & & ASTM D2622 & 1992 \\
\hline 3073 & ASTM & ASTM D2664 & $\mathrm{N} / \mathrm{S}$ & $\begin{array}{l}\text { Standard Test Method for Triaxial } \\
\text { Compressive Strength of } \\
\text { Undrained Rock Core Specimens } \\
\text { Without Pore Pressure } \\
\text { Measurements }\end{array}$ & reg1.138.r00 & 1978 & $\mathbf{P}$ & Implied endorsement & ASTM D2664 & 1986 \\
\hline 3074 & ASTM & ASTM D2687 & 1984 & $\begin{array}{l}\text { Standard Practices for Sampling } \\
\text { Particulate lon-Exchange Materials }\end{array}$ & inm79501 & 1989 & $\mathbf{R}$ & & ASTM D2687 & 1984 P90 \\
\hline 3075 & ASTM & ASTM D2688 & 1983 & $\begin{array}{l}\text { Standard Test Methods for } \\
\text { Corrosivity of Water in the Absence } \\
\text { of Heat Transfer (Weight Loss } \\
\text { Methods) }\end{array}$ & inm79501 & 1989 & $\bar{R}$ & & ASTM D2688 & 1992 \\
\hline 3076 & ASTM & ASTM D2777 & 1972 & $\begin{array}{l}\text { Standard Practice for Determination } \\
\text { of Precision and Bias of Applicable } \\
\text { Methods of Committee D-19 on } \\
\text { Water }\end{array}$ & drges114-4 & 1983 & $\mathbf{R}$ & & ASTM D2777 & 1986 \\
\hline 3077 & ASTM & ASTM D2777 & 1977 & $\begin{array}{l}\text { Standard Practice for Determination } \\
\text { of Precision and Bias of Applicable } \\
\text { Methods of Committee D-19 on } \\
\text { Water }\end{array}$ & inm79501 & 1989 & $\mathbf{R}$ & & ASTM D2777 & 1986 \\
\hline 3078 & ASTM & ASTM D2791 & 1977 & $\begin{array}{l}\text { Standard Test Method for } \\
\text { Continuous Determination of } \\
\text { Sodium in Water }\end{array}$ & inm79501 & 1989 & $\mathbf{R}$ & & ASTM D2791 & 1987 \\
\hline 3079 & ASTM & ASTM D2845 & $\mathrm{N} / \mathrm{S}$ & $\begin{array}{l}\text { Standard Test Method for } \\
\text { Laboratory Determination of Pulse } \\
\text { Velocities and Ultrasonic Elastic } \\
\text { Constants of Rock }\end{array}$ & reg1.138.ro0 & 1978 & $\bar{P}$ & Implied endorsement & ASTM D2845 & 1990 \\
\hline
\end{tabular}




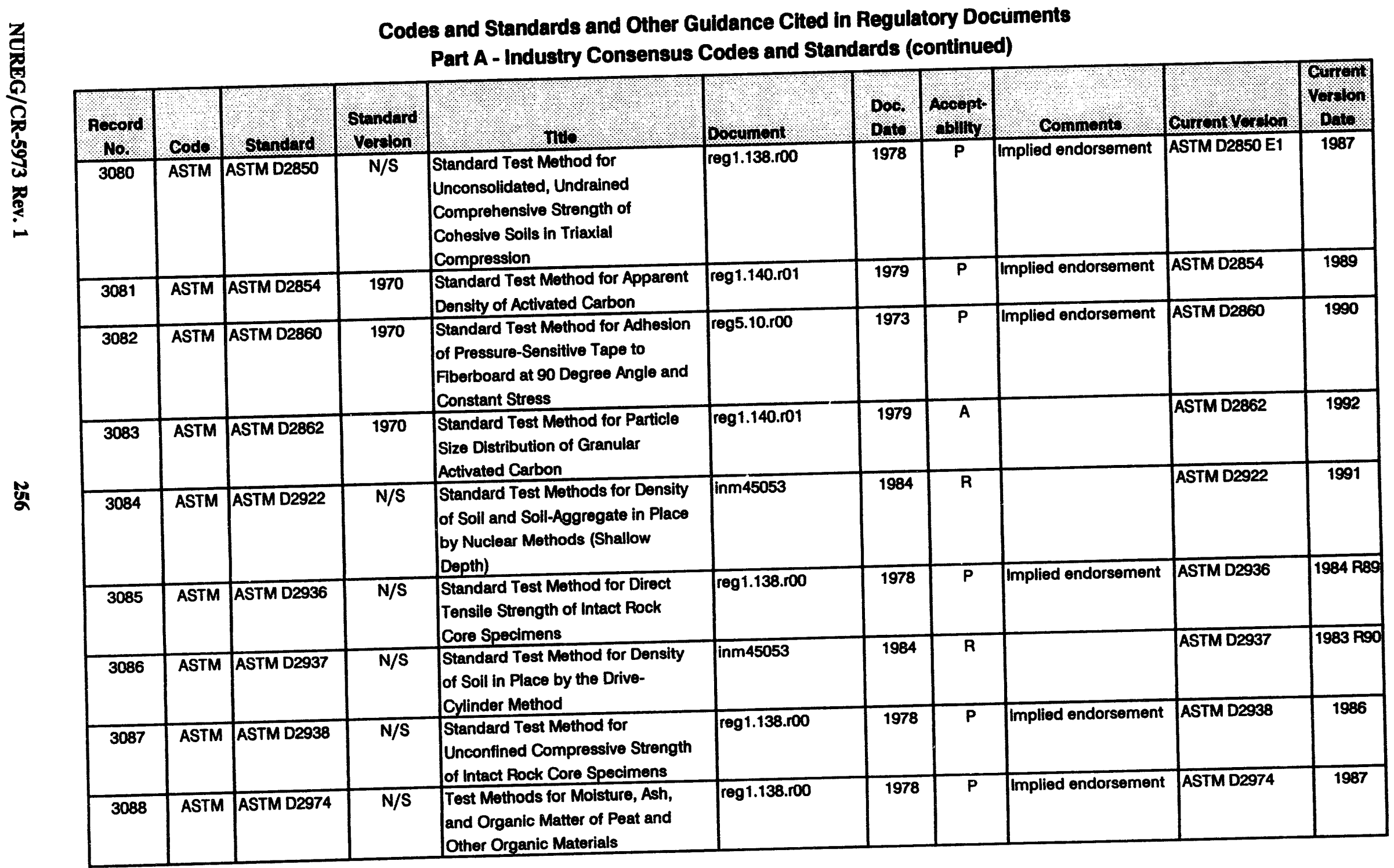


Codes and Standards and Other Guidance Cited in Regulatory Documents

Part A - Industry Consensus Codes and Standards (continued)

\begin{tabular}{|c|c|c|c|c|c|c|c|c|c|c|}
\hline $\begin{array}{l}\text { Record } \\
\text { No. }\end{array}$ & cosos & Standard & $\begin{array}{l}\text { Standard } \\
\text { Vorslon }\end{array}$ & Tho & Dooumont & $\begin{array}{l}\text { Doc. } \\
\text { Date }\end{array}$ & Accopt & Commonts & currom vordon & Voroton \\
\hline 3089 & ASTM & ASTM D2992 & $N / S$ & $\begin{array}{l}\text { Standard Practice for Obtaining } \\
\text { Hydrostatic or Pressure Design } \\
\text { Basis for 'Fiberglass' (Glass-Fiber- } \\
\text { Reinforced-Thermosetting Resin) } \\
\text { Pipe and Fittings }\end{array}$ & reg1.72.ro2 & 1978 & $\mathbf{R}$ & & ASTM D2992 & 1991 \\
\hline 3090 & ASTM & ASTM D3080 & $\mathrm{N} / \mathrm{S}$ & $\begin{array}{l}\text { Standard Test Method for Direct } \\
\text { Shear Test of Solls Under } \\
\text { Consolidated Drained Conditions }\end{array}$ & reg 1.138.r00 & 1978 & $\mathbf{P}$ & Implied endorsement & ASTM D3080 & 1990 \\
\hline 3091 & ASTM & ASTM D3082 & 1979 & $\begin{array}{l}\text { Standard Test Method for Boron in } \\
\text { Water }\end{array}$ & inm79501 & 1989 & $\bar{R}$ & & ASTM D3082 & 1992 \\
\hline 3092 & ASTM & ASTM D3286 & 1973 & $\begin{array}{l}\text { Standard Test Method for Gross } \\
\text { Calorific Value of Coal and Coke by } \\
\text { the lsoperibol Bomb Calorimeter }\end{array}$ & reg1.120.r01 & 1977 & $\mathbf{P}$ & Implied endorsement & ASTM D3286 & 1991 \\
\hline 3093 & ASTM & ASTM D3286 & 1973 & $\begin{array}{l}\text { Standard Test Method for Gross } \\
\text { Calorific Value of Coal and Coke by } \\
\text { the isoperibol Bomb Calorimeter }\end{array}$ & sip9.5.1 & 1981 & $\mathbf{R}$ & & ASTM D3286 & 1991 \\
\hline 3094 & ASTM & ASTM D3370 & 1982 & $\begin{array}{l}\text { Standard Practices for Sampling } \\
\text { Water }\end{array}$ & inm79501 & 1989 & $\bar{R}$ & & ASTM D3370 & 1982 \\
\hline 3095 & ASTM & ASTM D3370 & 1976 & $\begin{array}{l}\text { Standard Practices for Sampling } \\
\text { Water }\end{array}$ & reg4.15.ro1 & 1979 & $\bar{R}$ & & ASTM D3370 & 1982 \\
\hline 3096 & ASTM & ASTM D3558 & 1984 & $\begin{array}{l}\text { Standard Test Methods for Cobalt } \\
\text { in Water }\end{array}$ & inm79501 & 1989 & $\mathbf{R}$ & & ASTM D3558 & 1990 \\
\hline 3097 & ASTM & ASTM D3590 & 1984 & $\begin{array}{l}\text { Standard Test Methods for Total } \\
\text { Kjeldahl Nitrogen in Water }\end{array}$ & inm79501 & 1989 & $\bar{R}$ & & ASTM D3590 & 1989 \\
\hline 3098 & ASTM & ASTM D3685 & 1978 & $\begin{array}{l}\text { Standard Test Methods for } \\
\text { Sampling and Determination of } \\
\text { Particulate Matter in Stack Gases }\end{array}$ & reg4.16.ro1 & 1985 & $\mathbf{R}$ & & ASTM D3685 & 1978 R9O \\
\hline 3099 & ASTM & ASTM D3803 & 1979 & $\begin{array}{l}\text { Standard Test Method for Nuctear- } \\
\text { Grade Activated Carbon }\end{array}$ & not87-32 & 1987 & $\bar{R}$ & & ASTM D3803 & 1989 \\
\hline 3100 & ASTM & ASTM D3803 & 1989 & $\begin{array}{l}\text { Standard Test Method for Nuclear- } \\
\text { Grade Activated Carbon }\end{array}$ & sts4-geB5.7.2 & 1991 & $\mathbf{P}$ & & ASTM D3803 & 1989 \\
\hline 3101 & ASTM & ASTM D3803 & 1989 & $\begin{array}{l}\text { Standard Test Method for Nuclear- } \\
\text { Grade Activated Carbon }\end{array}$ & sts6-geB5.7.2 & 1991 & $\mathbf{P}$ & & ASTM D3803 & 1989 \\
\hline
\end{tabular}




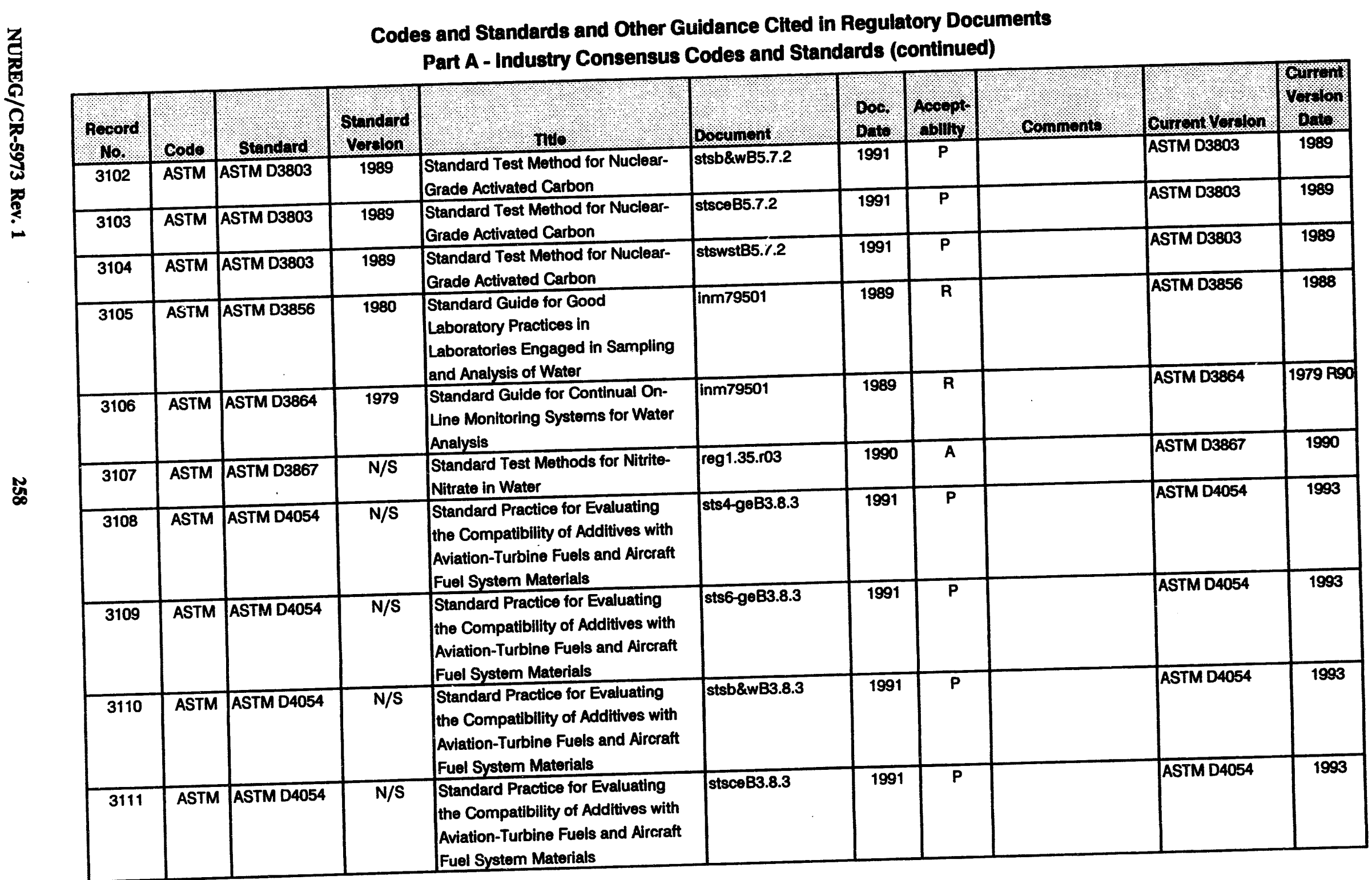


Codes and Standards and Other Guidance Cited in Regulatory Documents

Part A - Industry Consensus Codes and Standards (continued)

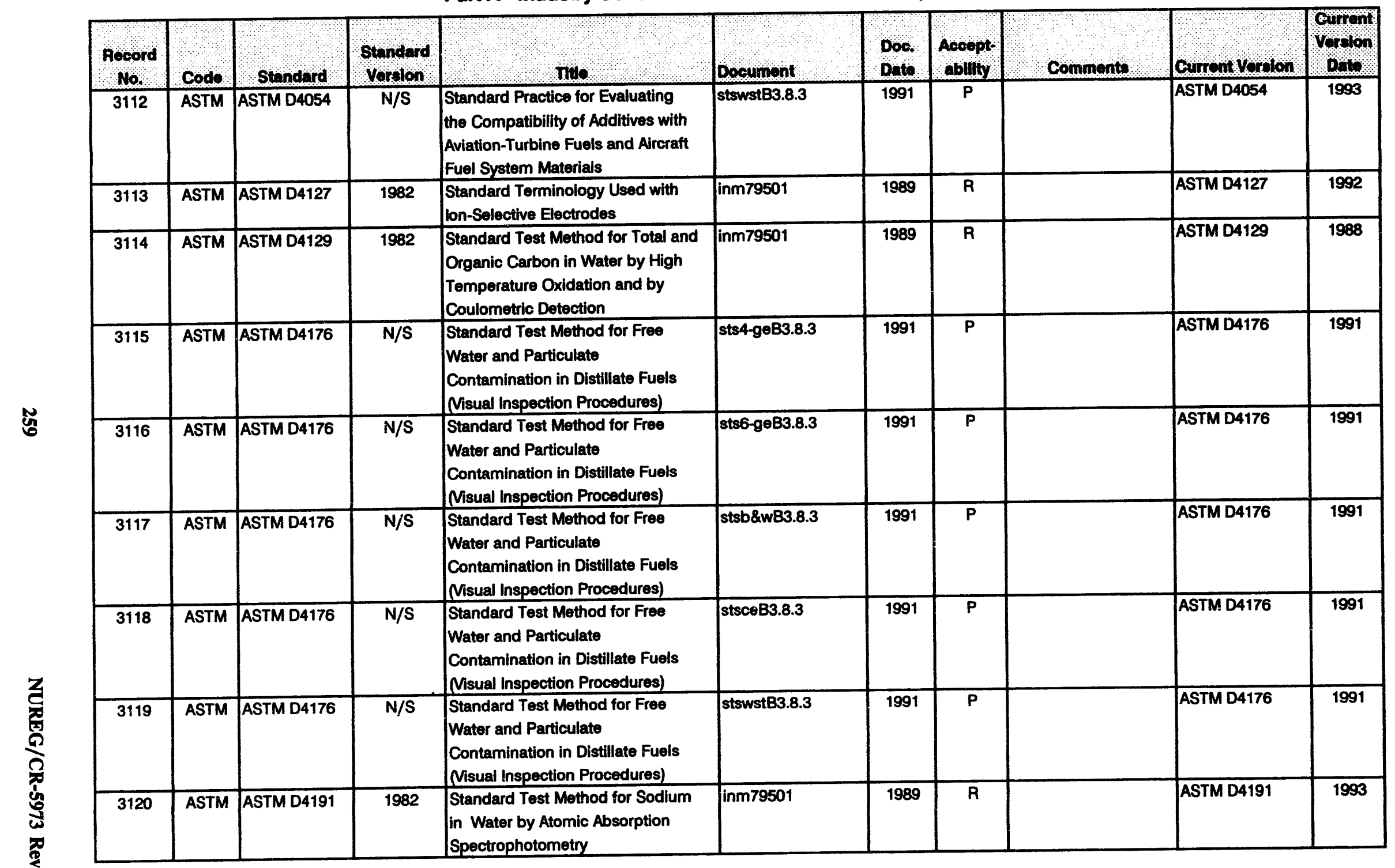




\begin{tabular}{|c|c|c|c|c|c|c|c|c|c|c|}
\hline & & & Code & $\begin{array}{l}\text { es and Standards and Other G } \\
\text { Part A - Industry Consensus }\end{array}$ & $\begin{array}{l}\text { iuidance } \mathrm{Ch} \\
\text { Codes and }\end{array}$ & $\begin{array}{l}\text { Regule } \\
\text { ards (c }\end{array}$ & $\begin{array}{l}\text { itory Do } \\
\text { continue }\end{array}$ & $\begin{array}{l}\text { cuments } \\
\text { dd) }\end{array}$ & & \\
\hline $\begin{array}{l}\text { Pocord } \\
\text { No. }\end{array}$ & Codo & Standard & $\begin{array}{l}\text { Standard } \\
\text { yoralon }\end{array}$ & ntte & Document & $\begin{array}{l}\text { Doc. } \\
\text { D. }\end{array}$ & $\begin{array}{l}\text { Acoeppt } \\
\text { ability }\end{array}$ & Comments. & Carromt Vorolon & Corrent \\
\hline 3121 & ASTM & ASTM D4192 & 1982 & $\begin{array}{l}\text { Standard Test Method for } \\
\text { Potassium in Water by Atomic }\end{array}$ & inm79501 & 1989 & $\mathrm{R}$ & & ASTM D4192 & 1993 \\
\hline 3122 & ASTM & ASTM D4210 & 1982 & $\begin{array}{l}\text { Standard Practice for } \\
\text { Intralaboratory Quality Control } \\
\text { Procedures and a Discussion on } \\
\text { Reporting Low-Level Data }\end{array}$ & inm79501 & 1989 & $\mathbf{R}$ & & ASTM D4210 & 1989 \\
\hline 3123 & ASTM & ASTM D4327 & 1984 & $\begin{array}{l}\text { Standard Test Method for Anions in } \\
\text { Water by Chemically Suppressed } \\
\text { lon Chromatography }\end{array}$ & inm79501 & 1989 & $\mathbf{R}$ & & ASTM D4327 & 1991 \\
\hline 3124 & ASTM & ASTM E23 & $N / S$ & $\begin{array}{l}\text { Standard Test Methods for Notched } \\
\text { Bar impact Testing of Metallic } \\
\text { Materials }\end{array}$ & srp5.2.3 & 1981 & $\mathbf{R}$ & & ASTM E23 & 1993 \\
\hline 3125 & ASTM & ASTM E6O & N/S & $\begin{array}{l}\text { Standard Practice for Photometric } \\
\text { and Spectrophotometric Methods } \\
\text { for Chemical Analysis of Metals }\end{array}$ & rog1.36.ro0 & 1973 & $\mathbf{P}$ & Implied endorsement & ASTM EGO & 1993 \\
\hline 3126 & ASTM & ASTM E62 & $\mathrm{N} / \mathrm{S}$ & $\begin{array}{l}\text { Standard Test Methods for } \\
\text { Chemical Analysis for Copper and } \\
\text { Copper Alloys (Photometric } \\
\text { Methods) }\end{array}$ & reg1.36.roo & 1973 & $\mathbf{P}$ & Implied endorsement & ASTM E62 & 1989 \\
\hline 3127 & ASTM & ASTM E64 & N/S & $\begin{array}{l}\text { Standard Test Mothod for Surface } \\
\text { Burning Characteristics of Building } \\
\text { Material }\end{array}$ & glt86-010 & 1986 & $\mathbf{R}$ & & ASTM E84 Rov. A & 1991 \\
\hline 3128 & ASTM & ASTM E84 & 1976 & $\begin{array}{l}\text { Standard Test Method for Surface } \\
\text { Burning Characteristics of Building } \\
\text { Material }\end{array}$ & inm88055 & 1991 & $\mathbf{R}$ & & ASTM E84 Rov. A & 1991 \\
\hline 3129 & ASTM & ASTM E84 & $N / S$ & $\begin{array}{l}\text { Standard Test Method for Surface } \\
\text { Burning Characteristics of Building } \\
\text { Material }\end{array}$ & not92-82 & 1992 & $\bar{R}$ & & ASTM E84 Rev. A & 1991 \\
\hline 3130 & ASTM & ASTM E84 & 1984 & $\begin{array}{l}\text { Standard Test Method for Surface } \\
\text { Burning Characteristics of Building } \\
\text { Material }\end{array}$ & reg1.120.r01 & 1977 & $\mathbf{P}$ & Implied endorsement & ASTM EB4 Rev. A & 1991 \\
\hline
\end{tabular}




\section{Codes and Standards and Other Guidance Cited in Regulatory Documents}

Part A - Industry Consensus Codes and Standar:s (continued)

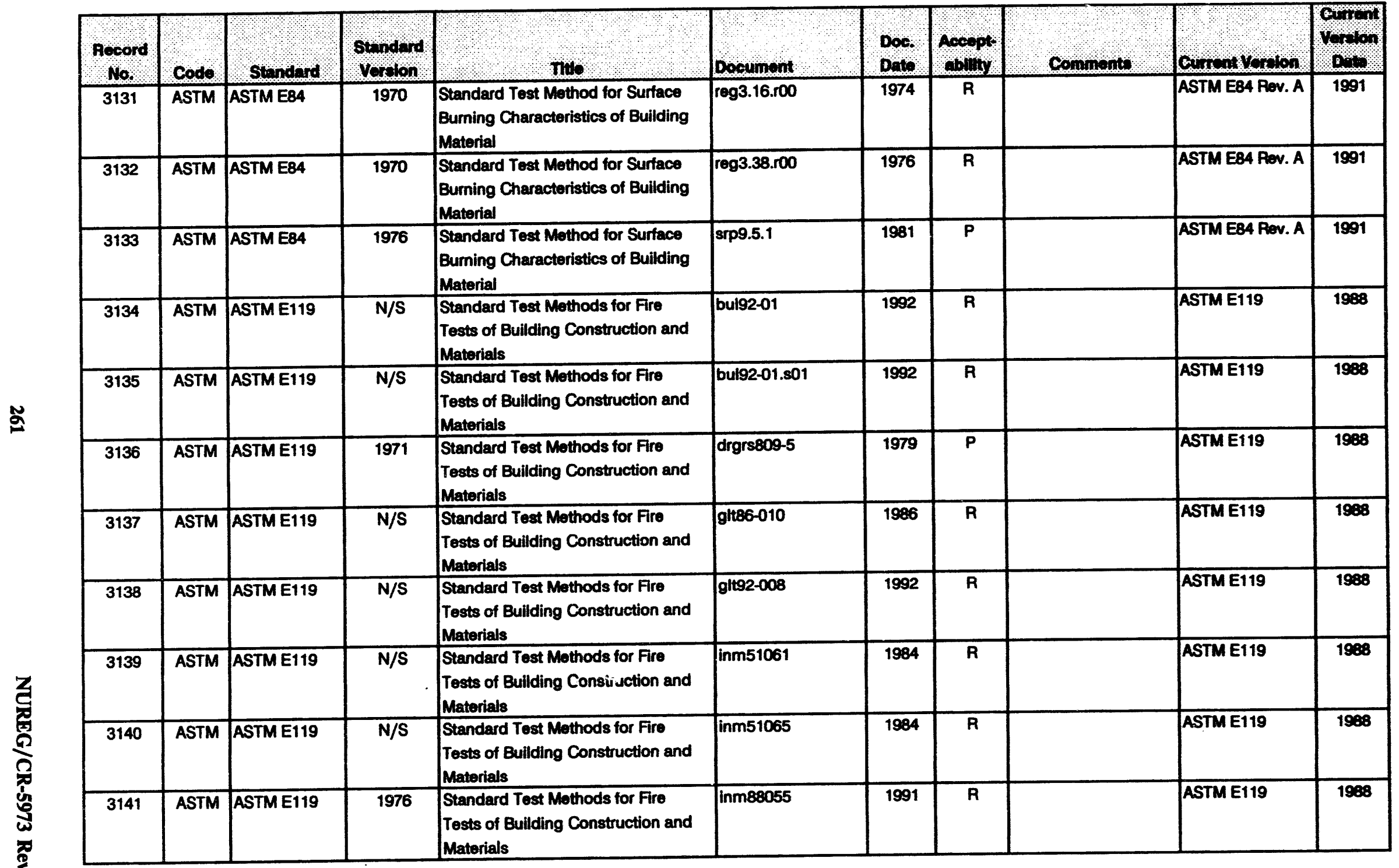




\begin{tabular}{|c|c|c|c|c|c|c|c|c|c|c|}
\hline \multicolumn{11}{|c|}{$\begin{array}{l}\text { Codes and Standards and Other Guidance Chted in Regutatory Documents } \\
\text { Part A - Industry Consensus Codes and Standards (continued) }\end{array}$} \\
\hline $\begin{array}{l}\text { Rocord } \\
\text { No. }\end{array}$ & cods. & standard & $\begin{array}{l}\text { Stundard } \\
\text { Vordion }\end{array}$ & L & Document & Dos & Acospt & comennents & 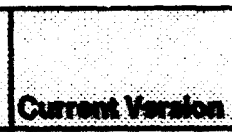 & $\begin{array}{l}C=m \\
\text { lom }\end{array}$ \\
\hline 3142 & $\overline{\text { ASTM }}$ & ASTME119 & $N / S$ & $\begin{array}{l}\text { Standard Test Methods for Fire } \\
\text { Tests of Building Construction and } \\
\text { Materials }\end{array}$ & not88-04 & 1988 & $\mathbf{P}$ & Implied endorsement & ASTM E119 & 1988 \\
\hline 3143 & ASTM & ASTM E119 & $N / S$ & $\begin{array}{l}\text { Standard Test Methods for Fire } \\
\text { Tests of Building Construction and } \\
\text { Materials }\end{array}$ & not91-79 & 1991 & $\mathbf{R}$ & & ASTME119 & 1988 \\
\hline 3144 & ASTM & ASTM E119 & $N / S$ & $\begin{array}{l}\text { Standard Test Mothods for Fire } \\
\text { Tests of Building Construction and } \\
\text { Matorials }\end{array}$ & not92-55 & 1992 & $\bar{R}$ & & ASTM E119 & 1988 \\
\hline 3145 & ASTM & ASTME119 & $\mathrm{N} / \mathrm{S}$ & $\begin{array}{l}\text { Standard Test Methods for Fire } \\
\text { Tests of Building Construction and } \\
\text { Materials }\end{array}$ & not $93-40$ & 1993 & $\bar{R}$ & & ASTM E119 & 1988 \\
\hline 3146 & $\overline{\text { ASTM }}$ & ASTM E119 & $N / S$ & $\begin{array}{l}\text { Standard Test Methods for Fire } \\
\text { Tests of Building Construction and } \\
\text { Materials }\end{array}$ & nureg0933, 3.149 & 1992 & $\bar{R}$ & & ASTME119 & 1988 \\
\hline$\overline{3147}$ & ASTM & ASTME119 & 1976 & $\begin{array}{l}\text { Standard Test Mothods for Firo } \\
\text { Tests of Building Construction and } \\
\text { Materials }\end{array}$ & reg1.120.ro1 & 1977 & $\overline{\mathbf{P}}$ & Implied ondorsement & ASTM E119 & 1908 \\
\hline 3148 & $\overline{\text { ASTM }}$ & ASTM E119 & 1971 & $\begin{array}{l}\text { Standard Test Mothods for Fire } \\
\text { Tests of Building Construction and } \\
\text { Materials }\end{array}$ & reg3.16.ro0 & 1974 & $\overline{\mathbf{R}}$ & & ASTM E119 & 1968 \\
\hline 3149 & $\overline{\text { ASTM }}$ & ASTME119 & 1976 & $\begin{array}{l}\text { Standard Test Methods for Fire } \\
\text { Tests of Building Construction and } \\
\text { Materials }\end{array}$ & $\operatorname{srp9.5.1}$ & 1981 & $\bar{P}$ & $\begin{array}{l}\text { With addifional } \\
\text { acceptance criteria }\end{array}$ & ASTME119 & 1988 \\
\hline 3150 & ASTM & ASTM E120 & $N / S$ & $\begin{array}{l}\text { Standard Test Methods for } \\
\text { Chemical Analysis of Titanium and } \\
\text { Titanium Alloys }\end{array}$ & reg1.36.r00 & 1973 & $\bar{P}$ & Implied Endorsement & ASTM E120 & 1969 \\
\hline 3151 & $\overline{\text { ASTM }}$ & ASTM E136 & $N / S$ & $\begin{array}{l}\text { Standard Test Mothod For Behavior } \\
\text { of Materials in a Vertical Tube } \\
\text { Furnace at } 750^{\circ} \mathrm{C} \text {. }\end{array}$ & not92-82 & $\overline{1992}$ & $\bar{A}$ & & ASTME136 & 1993 \\
\hline 3152 & ASTM & ASTM E146 & $\mathrm{N} / \mathrm{S}$ & $\begin{array}{l}\text { Methods for Chemical Analysis of } \\
\text { Zirconium and Zirconium Alloys }\end{array}$ & reg1.36.r00 & 1973 & $\bar{P}$ & Implied endorsement & ASTM E146 & 1983 \\
\hline 3153 & $\overline{\text { ASTM }}$ & ASTM E178 & 1974 & $\begin{array}{l}\text { Standard Practice for Dealing with } \\
\text { Outlying Observations }\end{array}$ & reg5.36.100 & 1974 & $\bar{P}$ & Subject to condifitions & ASTM E178 & $1960 \mathrm{R} 89$ \\
\hline
\end{tabular}


Codes and Standards and Other Guidance Ched in Regulatory Documents

Part A - Industry Consensus Codes and Standards (continued)

\begin{tabular}{|c|c|c|c|c|c|c|c|c|c|c|}
\hline $\begin{array}{l}\text { Aocord } \\
\text { No. }\end{array}$ & Codo & Stundard & $\begin{array}{l}\text { Standerd } \\
\text { vordion }\end{array}$ & Tho,$\ldots$ & Docannent & Don. & Aosept & Comonents & 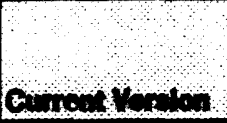 & $p_{1+1,1}$ \\
\hline 3154 & ASTM & ASTM E178 & 1980 & $\begin{array}{l}\text { Standard Practice for Dealing with } \\
\text { Outlying Observations }\end{array}$ & reg5.53.r01 & 1984 & $\mathbf{R}$ & & ASTME178 & $1980 \mathrm{Reg}$ \\
\hline 3155 & ASTM & ASTM E181 & 1982 & $\begin{array}{l}\text { Standard General Mothods for } \\
\text { Detector Callibration and Analysis of } \\
\text { Radionuclides }\end{array}$ & drg1025.100 & 1993 & $\mathbf{P}$ & $\begin{array}{l}\text { Typo in drg 1025.ro0- } \\
\text { Title is for ASTM E181 } \\
\text { vs. E81 }\end{array}$ & ASTME181 & 1993 \\
\hline 3156 & ASTM & ASTME185 & 1979 & $\begin{array}{l}\text { Practice for Conducting } \\
\text { Survelliance Tests for Light-Water } \\
\text { Cooled Nuclear Power Reactor } \\
\text { Vessels }\end{array}$ & 10 cfr50 App. G & 1993 & $\mathbf{R}$ & & ASTME185 & 1982 \\
\hline 3157 & ASTM & ASTM E185 & 1982 & $\begin{array}{l}\text { Practice for Conducting } \\
\text { Survelllance Tests for Light-Water } \\
\text { Cooled Nuclear Power Reactor } \\
\text { Vessels } \\
\end{array}$ & 10 cfr50 App. G & 1993 & $\mathbf{R}$ & & ASTM E185 & 1982 \\
\hline 3158 & ASTM & ASTM E185 & 1973 & $\begin{array}{l}\text { Practice for Conducting } \\
\text { Surveillance Tests for Light-Water } \\
\text { Cooled Nuclear Power Reactor } \\
\text { Vessels }\end{array}$ & $10 c$ fr50 App. H & 1993 & $\mathbf{R}$ & & ASTM E185 & 1982 \\
\hline 3159 & ASTM & ASTM E185 & 1979 & $\begin{array}{l}\text { Practice for Conducting } \\
\text { Surveillance Tests for Light-Water } \\
\text { Cooled Nuclear Power Reactor } \\
\text { Vessels }\end{array}$ & $10 \mathrm{ctr} 50$ App. $H$ & 1993 & $\mathbf{R}$ & & ASTME185 & 1982 \\
\hline 3160 & ASTM & ASTME185 & 1982 & $\begin{array}{l}\text { Practice for Conducting } \\
\text { Survellance Tests for Light-Water } \\
\text { Cooled Nuclear Power Reactor } \\
\text { Vessels }\end{array}$ & 10 ctr50 App. H & 1993 & $\mathbf{R}$ & & ASTM E185 & 1982 \\
\hline 3161 & ASTM & ASTM E185 & 1979 & $\begin{array}{l}\text { Practice for Conducting } \\
\text { Survellance Tests for Light-Water } \\
\text { Cooled Nuctear Power Reactor } \\
\text { Vessels }\end{array}$ & drg1023.100 & 1993 & $\mathbf{R}$ & & ASTME185 & 1982 \\
\hline 3162 & ASTM & ASTM E185 & 1982 & $\begin{array}{l}\text { Practice for Conducting } \\
\text { Surveillance Tests for Light-Water } \\
\text { Cooled Nuclear Power Reactor } \\
\text { Vessels }\end{array}$ & $\operatorname{drg} 1023.100$ & 1993 & $\mathbf{R}$ & & ASTM E185 & 1982 \\
\hline
\end{tabular}




\begin{tabular}{|c|c|c|c|c|c|c|c|c|c|c|}
\hline 2061 & S8Lヨ WLSV & & d & $166 \mathrm{~L}$ & E"tc8e0s48 & 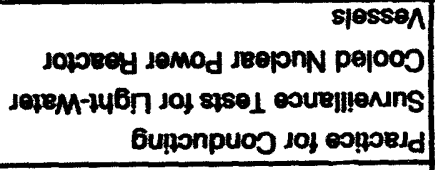 & 2861 & S81 WLSY & WISY & $0<1 \varepsilon$ \\
\hline 2861 & S813 WLSY & & $d$ & 1661 & Ëb'8gmpqäs & 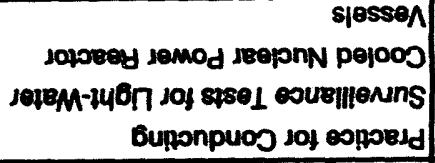 & 2861 & S81ヨ WLSY & WISV & $691 \varepsilon$ \\
\hline 2861 & S813 WISY & pueuresiopue peifdul & $d$ & 1861 & CEgds & 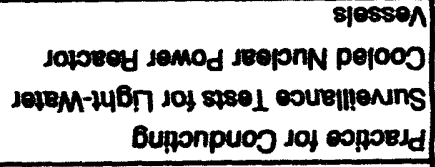 & $\varepsilon<61$ & SBIJ WLSY & WISY & $891 \varepsilon$ \\
\hline 2861 & So13 nusy & $\begin{array}{l}\text { uopdeoxe ve yim } \\
\text { puowessopue peydul }\end{array}$ & $d$ & 1861 & $18 \mathrm{gds}$ & 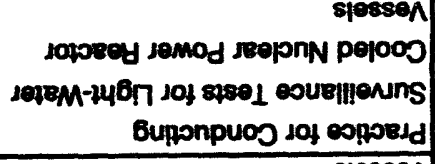 & $S / N$ & S81ヨ WLSV & WISY & $\angle 9 t \varepsilon$ \\
\hline 2061 & SOIJ WISY & & 부 & 8861 & $20066+600$ & 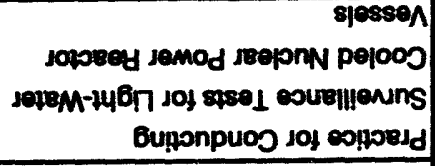 & 2861 & S813 WLSV & WISY & $991 \varepsilon$ \\
\hline 2861 & SOIJ WLSV & & y & 2661 & $10 \times 100-2646$ & 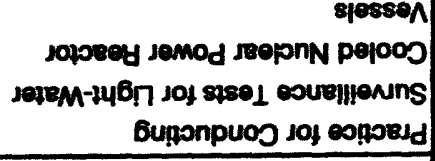 & 2861 & S813 WISH & WISY & s91 \\
\hline 2061 & So13 Misy & & $d$ & $266 \mathrm{~L}$ & $10 \cdot 100-2640$ & 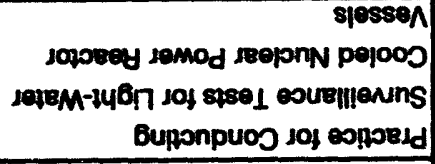 & $6 \angle 61$ & S813 WISY & WISY & t91ع \\
\hline Co6t & So13 wisv & & $\boldsymbol{H}$ & 266: & $10 \times 100-2648$ & 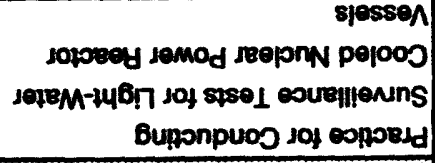 & $\varepsilon \angle 6 t$ & S81ヨWLSY & WISY & ๕9เE \\
\hline $\lim _{\infty}$ & wopher wamp & $90 \times 000$ & Des? & pod & monom & CML & poppls & propuns & $0 \% 0$ & $\begin{array}{l}\text { ON } \\
\text { proper }\end{array}$ \\
\hline
\end{tabular}


Codes and Standards and Other Guidance Cited in Regulatory Documents

Part A - Industry Consensus Codes and Standards (continued)

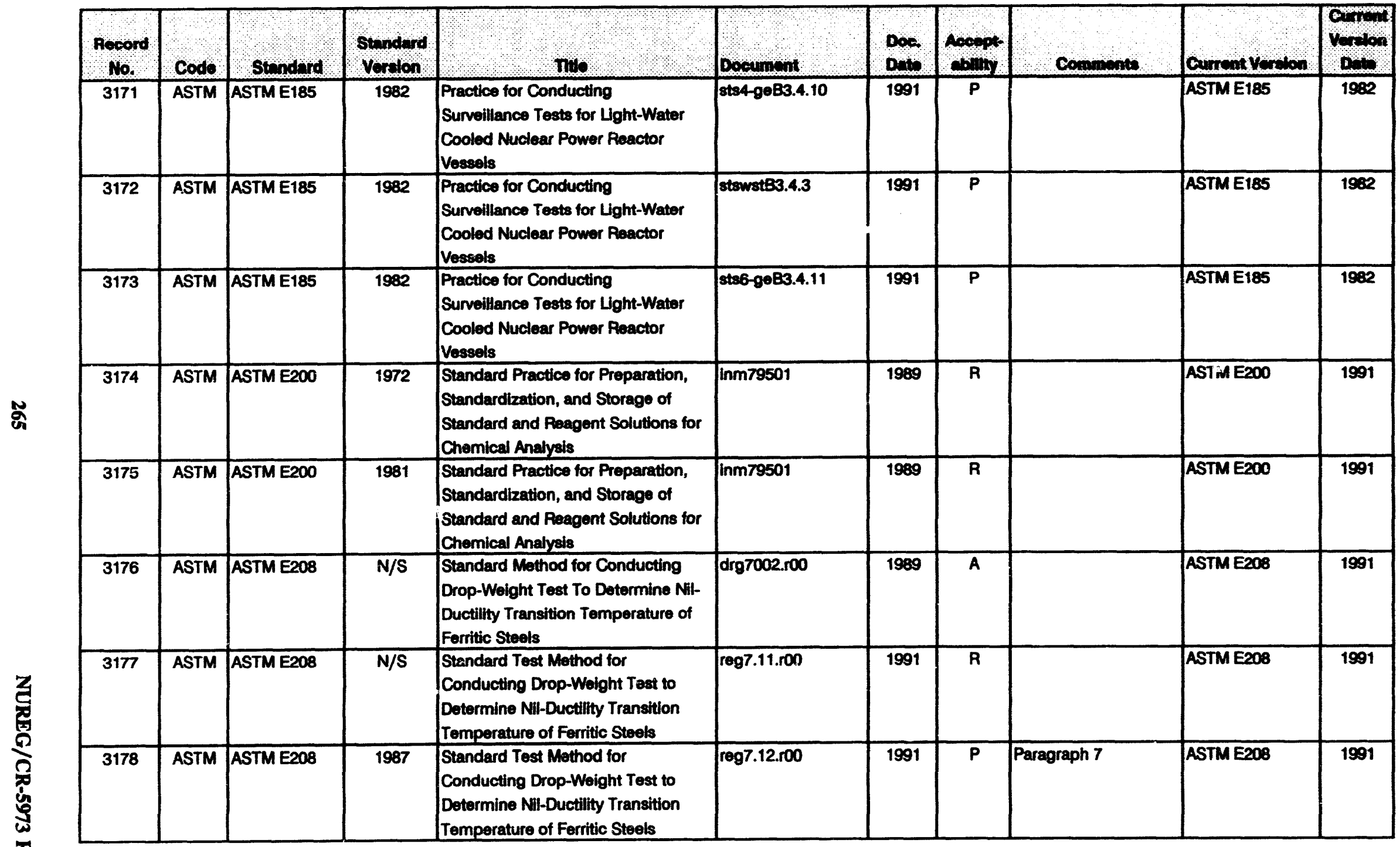


Codes and Standards and Other Guidance Cited in Regulatory Documents

Part A - Industry Consensus Codes and Standards (continued)

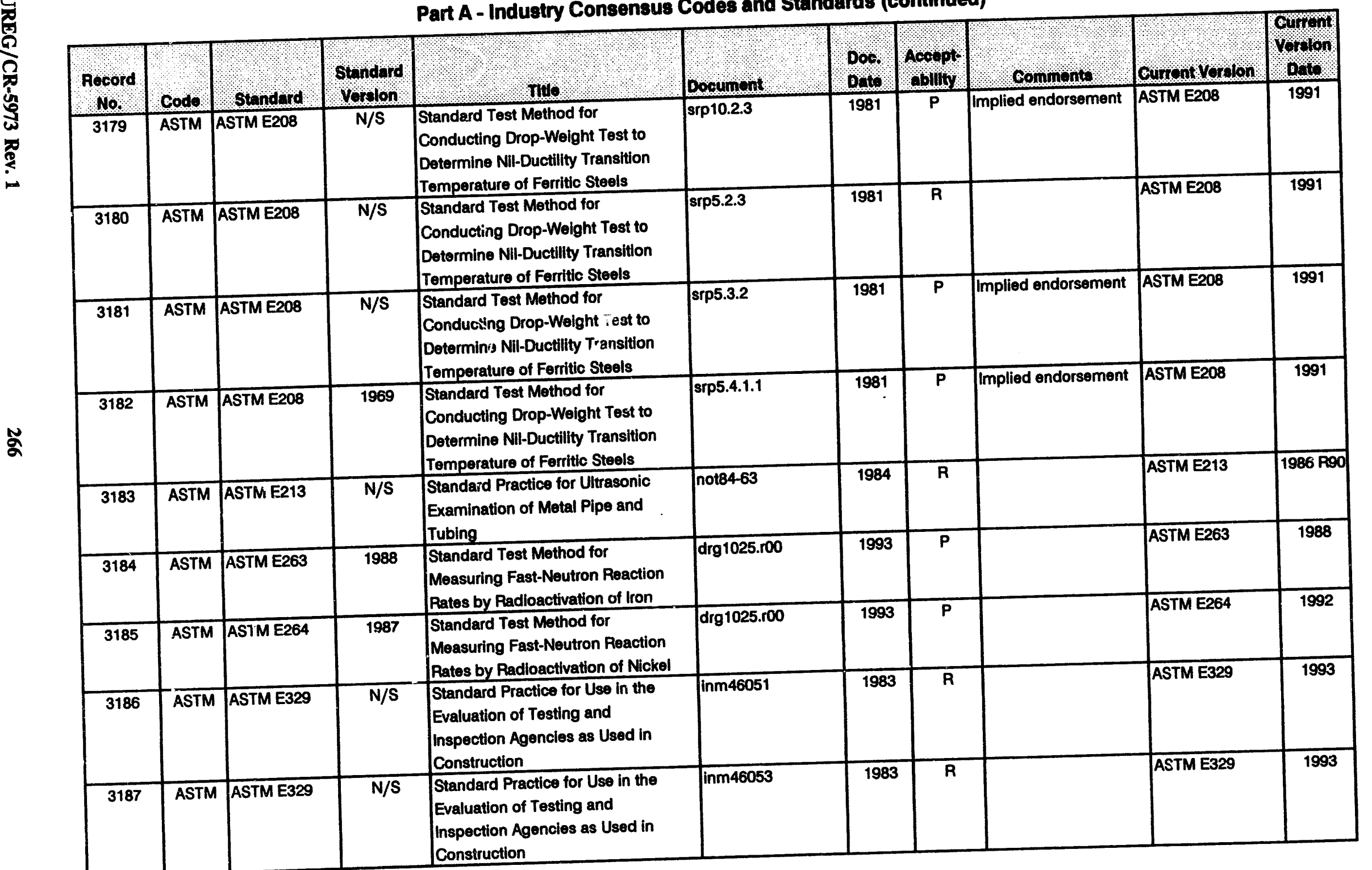


Codes and Standards and Other Guidance Cited in Regulatory Documents

Part A - Industry Consensus Codes and Standards (continued)

\begin{tabular}{|c|c|c|c|c|c|c|c|c|c|c|}
\hline Rocord & codo: & Stendard & stondord & ? & Goovumpol & Doc. & 1000pt) & , commont & c. & oinoling \\
\hline 3188 & ASTM & ASTM E399 & $N / S$ & $\begin{array}{l}\text { Standard Test Methods for Plane- } \\
\text { Strain Fracture Toughness of } \\
\text { Metallic Materials }\end{array}$ & reg1.14.ro1 & 1975 & $P$ & Implied endorsement & ASTM E399 & 1990 \\
\hline 3189 & ASTM & ASTME482 & 1989 & $\begin{array}{l}\text { Standard Guide for Application of } \\
\text { Neutron Transport Methods for } \\
\text { Reactor Vessel Surveillance }\end{array}$ & drg 1025.r00 & 1993 & $\mathbf{R}$ & & ASTM E482 & 1989 \\
\hline 3190 & ASTM & ASTM E523 & 1987 & $\begin{array}{l}\text { Standard Test Method for } \\
\text { Measuring Fast-Neutron Reaction } \\
\text { Rates by Radioactivation of Copper }\end{array}$ & $\operatorname{drg} 1025 . r 00$ & 1993 & $\bar{P}$ & & ASTM E523 & 1992 \\
\hline 3191 & ASTM & ASTM E526 & 1987 & $\begin{array}{l}\text { Standard Test Method for } \\
\text { Measuring Fast-Neutron Reaction } \\
\text { Rates by Radioactivation of } \\
\text { Titanium }\end{array}$ & $\operatorname{drg} 1025 . r 00$ & 1993 & $\mathbf{P}$ & & ASTM E526 & 1992 \\
\hline 3192 & ASTM & ASTM E604 & $\mathrm{N} / \mathrm{S}$ & $\begin{array}{l}\text { Standard Test method for Dynamic } \\
\text { Tear Testing of Metallic Materials }\end{array}$ & reg7.11.r00 & 1991 & $\bar{R}$ & & ASTM E604 & 1983 R88 \\
\hline 3193 & ASTM & ASTM EG93 & 1979 & $\begin{array}{l}\text { Practice for Characterizing Neutron } \\
\text { Exposures in Ferritic Steels in } \\
\text { Terms of Displacements per Atom } \\
\text { DPA }\end{array}$ & reg1.99.ro2 & 1988 & $\mathbf{R}$ & & ASTM E693 & 1979 R85 \\
\hline 3194 & ASTM & ASTM E704 & 1990 & $\begin{array}{l}\text { Standard Test Method for } \\
\text { Measuring Reaction Rates by } \\
\text { Radioactivation of Uranium-238 }\end{array}$ & drg1025.r00 & 1993 & $\bar{P}$ & & ASTM E704 & 1990 \\
\hline 3195 & ASTM & ASTM E705 & 1990 & $\begin{array}{l}\text { Standard Test Method for } \\
\text { Measuring Reaction Rates by } \\
\text { Radioactivation of Neptunium-237 }\end{array}$ & drg1025.r00 & 1993 & $\mathbf{P}$ & & ASTM E705 & 1990 \\
\hline 3196 & ASTM & ASTM E706 & 1984 & $\begin{array}{l}\text { Standard Master Matrix for Light- } \\
\text { Water Reactor Pressure Vesse! } \\
\text { Surveillance Standards }\end{array}$ & drg 1025.r00 & 1993 & $\mathbf{R}$ & & ASTM E706 & 1987 \\
\hline 3197 & ASTM & ASTM E814 & $N / S$ & $\begin{array}{l}\text { Standard Test Method for Fire Tests } \\
\text { of Through-Penetration Fire Stops }\end{array}$ & not88-04 & 1988 & $\mathbf{P}$ & Implied endorsement & ASTM E814 & 1988 \\
\hline 3198 & ASTM & ASTM E844 & 1986 & $\begin{array}{l}\text { Standard Guide for Sensor Set } \\
\text { Design and Irradiation for Reactor } \\
\text { Surveillance }\end{array}$ & $\operatorname{drg} 1025.100$ & 1993 & $\mathbf{P}$ & & ASTM E844 & $1986 \mathrm{R} 91$ \\
\hline
\end{tabular}


Codes and Standards and Other Guidance Cited in Regulatory Documents

Part A - Industry Consensus Codes and Standards (continued)

\begin{tabular}{|c|c|c|c|c|c|c|c|c|c|c|}
\hline Rocord & Codo & swondard & standard & (.) & Documont & Dor) & Acoept: & commonts & Curront Vorolon & onthol \\
\hline 3199 & ASTM & ASTM E854 & 1990 & $\begin{array}{l}\text { Standard Test Method for } \\
\text { Application and Analysis of Solid } \\
\text { State Track Recorder (SSTR) } \\
\text { Monitors for Reactor Surveillance }\end{array}$ & drg1025.r00 & 1993 & $\mathbf{R}$ & & ASTM E854 & 1990 \\
\hline 3200 & ASTM & ASTM E1005 & 1984 & $\begin{array}{l}\text { Standard Test Method for } \\
\text { Application and Analysis of } \\
\text { Radiometric Monitors for Reactor } \\
\text { Vessel Survellance }\end{array}$ & drg1025.ro0 & 1993 & $\mathbf{P}$ & & ASTME1005 & $1984 \mathrm{R} 91$ \\
\hline 3201 & ASTM & ASTM E1152 & 1987 & $\begin{array}{l}\text { Standard Test Method for } \\
\text { Determining J-R Curves }\end{array}$ & drg1023.r00 & 1993 & $\mathbf{P}$ & & ASTME1152 & 1987 \\
\hline 3202 & ASTM & ASTM E1253-B & $\mathrm{N} / \mathrm{S}$ & $\begin{array}{l}\text { Guide for Reconstitution of } \\
\text { Irradiated Charpy Specimens }\end{array}$ & not90-52 & 1990 & $\mathbf{R}$ & $\begin{array}{l}\text { Expected to update in } \\
1991\end{array}$ & ASTM E1253 & 1988 \\
\hline 3203 & ASTM & ASTM E1354 & $\mathrm{N} / \mathrm{S}$ & $\begin{array}{l}\text { Standard Test Method for Heat and } \\
\text { Visible Smoke Release Rates for } \\
\text { Materials and Products Using an } \\
\text { Oxygen Consumption Calorimeter }\end{array}$ & not92-82 & 1992 & $\mathbf{R}$ & & ASTM E1354 & 1992 \\
\hline 3204 & ASTM & ASTM Part 19 & $\mathrm{~N} / \mathrm{S}$ & $\begin{array}{l}\text { Annual Book of American Society } \\
\text { for Testing and Materials Standards }\end{array}$ & reg1.138.ro0 & 1978 & $\mathbf{R}$ & & $\begin{array}{l}\text { Annual Book of } \\
\text { ASTM Standards }\end{array}$ & 1992 \\
\hline 3205 & ASTM & ASTM STP 15D & $\mathrm{N} / \mathrm{S}$ & $\begin{array}{l}\text { Presentation of Data and Control } \\
\text { Chart Analysis }\end{array}$ & inm79501 & 1989 & $\mathbf{R}$ & Out of Print & $n / a$ & $n / a$ \\
\hline 3206 & ASTM & ASTM STP 15D & 1976 & $\begin{array}{l}\text { Presentation of Data and Control } \\
\text { Chart Analysis }\end{array}$ & inm79701 & 1991 & $\bar{R}$ & Out of Print & $n / a$ & $n / a$ \\
\hline 3207 & ASTM & ASTM STP 15D & 1976 & $\begin{array}{l}\text { Presentation of Data and Control } \\
\text { Chart Analysis }\end{array}$ & inm84750 & 1988 & $R$ & Out of Print & $n / a$ & $n / a$ \\
\hline 3208 & ASTM & ASTM STP 477 & 1970 & $\begin{array}{l}\text { Determination of the in Situ } \\
\text { Modulus of Deformation of Rock }\end{array}$ & reg1.132.ro1 & 1979 & $R$ & & ASTM STP 477 & 1970 \\
\hline 3209 & ASTM & ASTM STP 479 & 1969 & $\begin{array}{l}\text { Special Procedures for Testing Soil } \\
\text { and Rock for Engineering Purposes }\end{array}$ & reg1.132.r01 & 1979 & $\bar{R}$ & $\begin{array}{l}\text { Withdrawn - out of } \\
\text { print }\end{array}$ & Withdrawn & $n / a$ \\
\hline 3210 & ASTM & ASTM STP 479 & 1970 & $\begin{array}{l}\text { Special Procedures for Testing Soil } \\
\text { and Rock for Engineering Purposes }\end{array}$ & reg1.138.r00 & 1978 & $\mathbf{R}$ & $\begin{array}{l}\text { Withdrawn - out of } \\
\text { print }\end{array}$ & Withdrawn & $n / a$ \\
\hline 3211 & ASTM & ASTM STP 483 & 1971 & $\begin{array}{l}\text { Use of Chemical Grouts to Sample } \\
\text { Sands, Sampling of Soll and Rock }\end{array}$ & reg1.132.ro1 & 1979 & $\mathbf{R}$ & $\begin{array}{l}\text { Withdrawn - out of } \\
\text { print }\end{array}$ & Withdrawn & $n / a$ \\
\hline
\end{tabular}


Codes and Standards and Other Guidance Cited in Regulatory Documents

Part A - Industry Consensus Codes and Standards (continued)

\begin{tabular}{|c|c|c|c|c|c|c|c|c|c|c|}
\hline R०o, 100 & codos & standard & standard & 1. & Document: & $\begin{array}{l}\text { Doo. } \\
\text { Doto }\end{array}$ & Accept & Comnento & curront vorotorl. & oniplo \\
\hline 3212 & ASTM & ASTM STP 501 & 1972 & $\begin{array}{l}\text { Recent Trends in Underwater Soil } \\
\text { Sampling Methods, Underwater } \\
\text { Soil Sampling Testing and } \\
\text { Construction Control American } \\
\text { Society for Testing and Materials }\end{array}$ & reg1.132.r01 & 1979 & $\mathbf{R}$ & $\begin{array}{l}\text { Withdrawn - out of } \\
\text { print }\end{array}$ & Withdrawn & $n / a$ \\
\hline 3213 & ASTM & ASTM STP 523 & 1973 & $\begin{array}{l}\text { Determination of Relative Density of } \\
\text { Sand Below Groundwater Table }\end{array}$ & reg1.132.ro1 & 1979 & $\mathbf{R}$ & $\begin{array}{l}\text { Withdrawn - out of } \\
\text { print }\end{array}$ & Withdrawn & $n / a$ \\
\hline 3214 & ASTM & ASTM STP 599 & 1976 & $\begin{array}{l}\text { Effects of Storage and Extrusion of } \\
\text { Sample Properties }\end{array}$ & reg1.138.r00 & 1978 & $\mathbf{R}$ & $\begin{array}{l}\text { Withdrawn - out of } \\
\text { print }\end{array}$ & Withdrawn & $n / a$ \\
\hline 3215 & ASTM & ASTM STP 742 & $\mathrm{~N} / \mathrm{S}$ & $\begin{array}{l}\text { Power Plant Instrumentation for } \\
\text { Measurement of High Purity Water } \\
\text { Quality }\end{array}$ & inm79501 & 1989 & $\mathbf{R}$ & & ASTM STP 742 & 1982 \\
\hline 3216 & ASTM & ASTM STP 803 & 1983 & $\begin{array}{l}\text { Engineering Methods for the } \\
\text { Assessment of Ductile Fracture } \\
\text { Margin in Nuclear Power Plant } \\
\text { Piping }\end{array}$ & glt89-010.s03 & 1990 & $\mathbf{R}$ & $\begin{array}{l}\text { Withdrawn - out of } \\
\text { print }\end{array}$ & Withdrawn & $n / a$ \\
\hline 3217 & ASTM & $\begin{array}{l}\text { ASTM W-F- } \\
800 \mathrm{~b}\end{array}$ & 1975 & Federal Oil Specification & reg1.137.ro1 & 1979 & $\mathbf{A}$ & $\begin{array}{l}\text { not a valid ASTM } \\
\text { designation }\end{array}$ & $n / a$ & $n / a$ \\
\hline 3218 & AWS & AWS & $\mathrm{N} / \mathrm{S}$ & American Welding Society & not82-34.ro1 & 1982 & $\mathbf{R}$ & $\begin{array}{l}\text { General reference no } \\
\text { specific codes cited }\end{array}$ & $n / a$ & $n / a$ \\
\hline 3219 & AWS & AWS A4.2 & 1974 & $\begin{array}{l}\text { Standard Procedures for } \\
\text { Calibrating Magnetic Instruments to } \\
\text { Measure the Delta Ferrite Content } \\
\text { of Austenitic Stainless Steel Weld } \\
\text { Metal }\end{array}$ & reg1.31.r03 & 1978 & A & & AWS A4.2 & 1991 \\
\hline 3220 & AWS & AWS D1.1 & $\mathrm{N} / \mathrm{S}$ & Structural Welding Code-Steol & bul88-05.s01 & 1988 & $\mathbf{R}$ & & AWS D1.1 & 1992 \\
\hline 3221 & AWS & AWS D1.1 & $\mathrm{N} / \mathrm{S}$ & Structural Welding Code-Steel & bul88-05.s02 & 1988 & $\mathbf{P}$ & Implied endorsement & AWS D1.1 & 1992 \\
\hline 3222 & AWS & AWS D1.1 & $\mathrm{N} / \mathrm{S}$ & Structural Welding Code-Steel & inm46051 & 1983 & $\mathbf{R}$ & & AWS D1.1 & 1992 \\
\hline 3223 & AWS & AWS D1.1 & $\mathrm{N} / \mathrm{S}$ & Structural Welding Code-Steel & inm48051 & 1986 & $\mathbf{R}$ & & AWS D1.1 & 1992 \\
\hline 3224 & AWS & AWS D1.1 & $\mathrm{N} / \mathrm{S}$ & Structural Welding Code-Steel & inm48053 & 1986 & $\mathbf{R}$ & & AWS D1.1 & 1992 \\
\hline 3225 & AWS & AWS D1.1 & $\mathrm{N} / \mathrm{S}$ & Structural Welding Code-Steol & inm50090 & 1985 & $\mathbf{R}$ & & AWS D1.1 & 1992 \\
\hline 3226 & AWS & AWS D1.1 & $\mathrm{N} / \mathrm{S}$ & Structural Welding Code-Steel & inm51053 & 1984 & $\mathbf{R}$ & & AWS D1.1 & 1992 \\
\hline 3227 & AWS & AWS D1.1 & $\mathrm{N} / \mathrm{S}$ & Structural Welding Code-Steel & inm52053 & 1984 & $\mathbf{R}$ & & AWS D1.1 & 1992 \\
\hline 3228 & AWS & AWS D1.1 & $\mathrm{N} / \mathrm{S}$ & Structural Welding Code-Steel & inm55100 & 1983 & $\mathbf{R}$ & & AWS D1.1 & 1992 \\
\hline
\end{tabular}




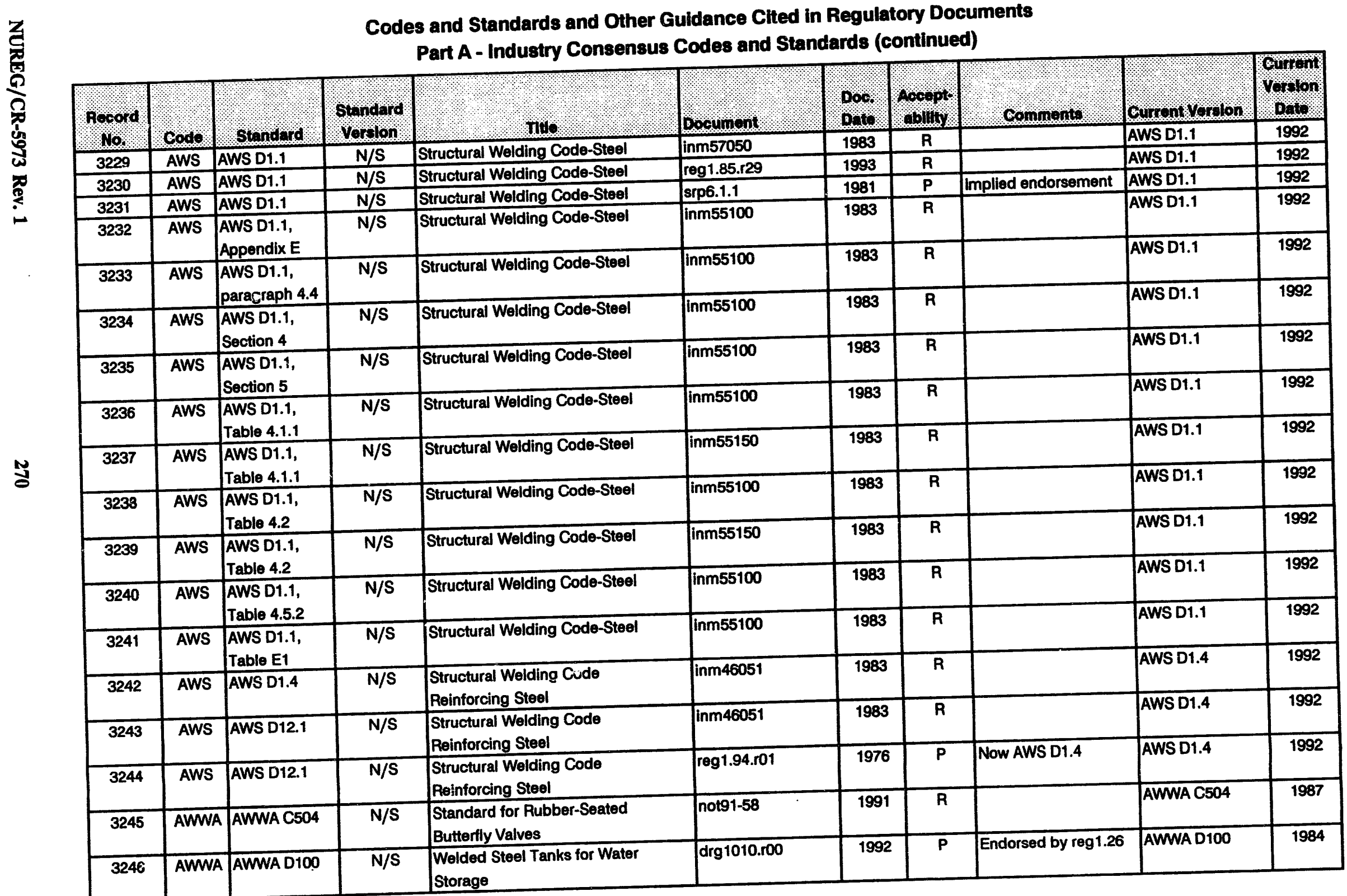


Codes and Standards and Other Guidance Cited in Regulatory Documents

Part A - Industry Consensus Codes and Standards (continued)

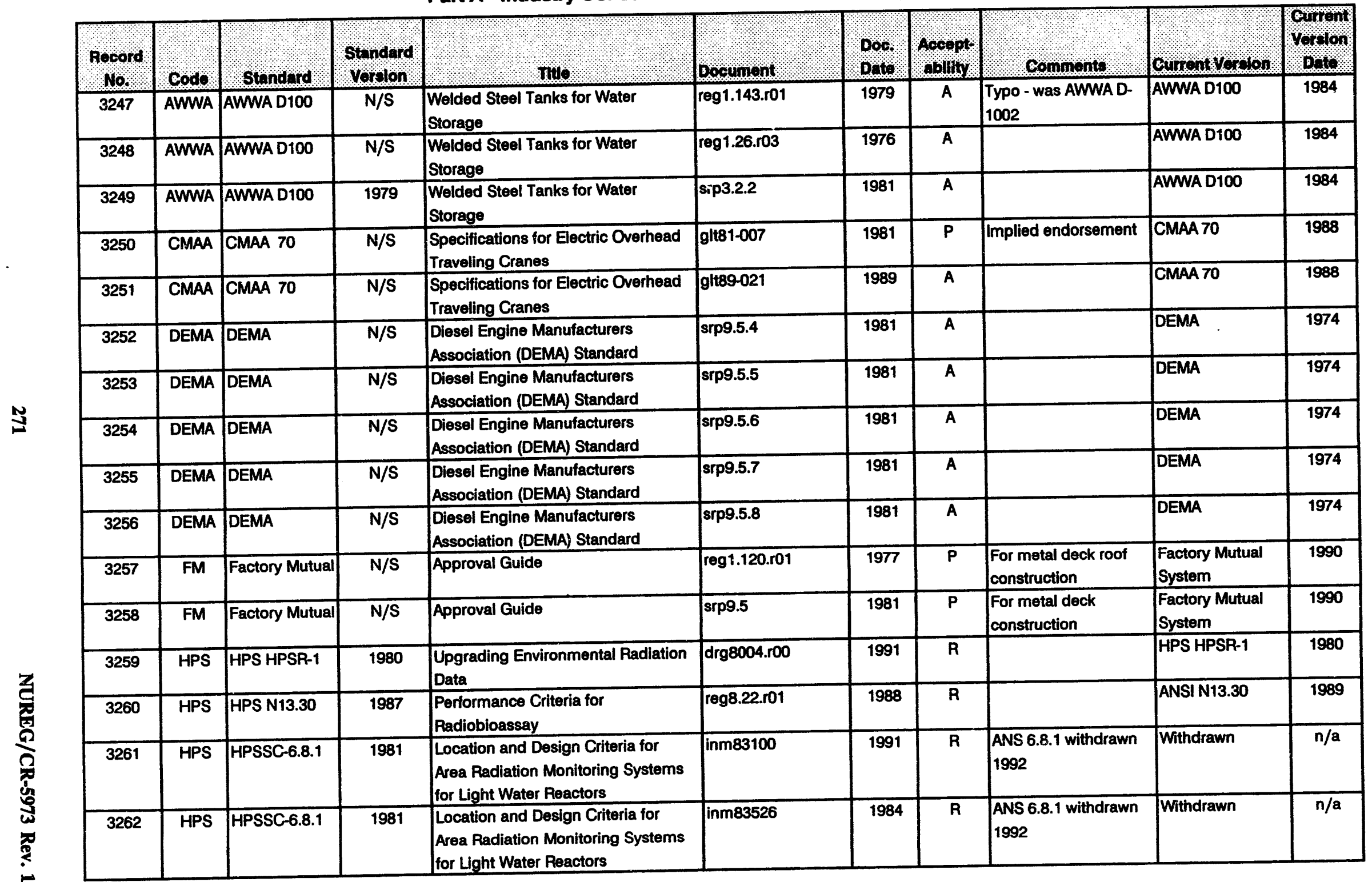




\begin{tabular}{|c|c|c|c|c|c|c|c|c|c|c|}
\hline & & & 80 & $\begin{array}{l}8 \text { and Standards and Uner } \\
\text { Part A - Industry Consensus }\end{array}$ & $\begin{array}{l}\text { idance CI } \\
\text { odes and }\end{array}$ & ards ( & ontinue & ed) & & \\
\hline Hocord & Qodo & Standard & stondard & (1) & Doovenont & $\begin{array}{l}\text { Doo. } \\
\text { Doto }\end{array}$ & Aocept: & $80 \mathrm{mnont}$ & 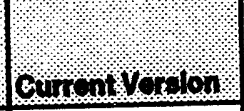 & vornon \\
\hline 3263 & HPS & HPSSC-6.8.1 & 1981 & $\begin{array}{l}\text { Location and Design Criteria for } \\
\text { Area Radiation Monitoring Systems } \\
\text { for Light Water Reactors }\end{array}$ & inm83726 & 1991 & $\mathbf{R}$ & $\begin{array}{l}\text { ANS 6.8.1 withdrawn } \\
1992\end{array}$ & Withdrawn & $n / a$ \\
\hline 3264 & HPS & HPSSC-6.8.1 & 1981 & $\begin{array}{l}\text { Location and Design Criteria for } \\
\text { Area Radiation Monitoring Systems } \\
\text { for Light Water Reactors }\end{array}$ & inm83750 & 1988 & $\mathbf{R}$ & $\begin{array}{l}\text { ANS 6.8.1 withdrawn } \\
1992\end{array}$ & Withdrawn & $n / a$ \\
\hline 3265 & HPS & HPSSC-6.8.1 & $\mathrm{N} / \mathrm{S}$ & $\begin{array}{l}\text { Location and Design Criteria for } \\
\text { Area Radiation Monitoring Systems } \\
\text { for Light Water Reactors }\end{array}$ & reg8.8.r03 & 1978 & $\mathbf{R}$ & $\begin{array}{l}\text { Referenced as } \\
\text { ANS/HPS } 56.8\end{array}$ & ANS 6.8.1 & 1981 \\
\hline 3266 & HPS & HPSSC-6.8.1 & 1981 & $\begin{array}{l}\text { Location and Design Criteria for } \\
\text { Area Radiation Monitoring Systems } \\
\text { for Light Water Reactors }\end{array}$ & srp12.3 & 1981 & $\mathbf{P}$ & $\begin{array}{l}\text { As relates in } \\
\text { Acceptance Criteria }\end{array}$ & ANS 6.8.1 & 1981 \\
\hline 3267 & ICRP & ICRP & 1966 & $\begin{array}{l}\text { Deposition and Retention Models } \\
\text { for Internal Dosimetry of the Human } \\
\text { Respiratory Tract }\end{array}$ & reg3.51.r00 & 1982 & $\mathbf{R}$ & & ICRP & 1966 \\
\hline 3268 & ICRP & $\begin{array}{l}\text { ICRP } \\
\text { Publication } 2 \\
\end{array}$ & 1959 & $\begin{array}{l}\text { Limits for Intake of Radionuclides } \\
\text { ky Workers }\end{array}$ & not82-18 & 1982 & $P$ & $\begin{array}{l}\text { Superseded by ICRP- } \\
30\end{array}$ & $\begin{array}{l}\text { ICRP Publication } \\
30\end{array}$ & 1990 \\
\hline 3269 & ICRP & $\begin{array}{l}\text { ICRP } \\
\text { Publication } 2\end{array}$ & 1959 & $\begin{array}{l}\text { Limits for Intake of Radionuclides } \\
\text { by Workers }\end{array}$ & egg1.24r00 & 1972 & $\mathbf{P}$ & $\begin{array}{l}\text { Implied endorsement. } \\
\text { Superseded by ICRP } \\
30\end{array}$ & $\begin{array}{l}\text { ICRP Publication } \\
30\end{array}$ & 1990 \\
\hline 3270 & ICRP & $\begin{array}{l}\text { ICRP } \\
\text { Publication } 2 \\
\end{array}$ & 1959 & $\begin{array}{l}\text { Limits for intake of Radionuclides } \\
\text { by Workers }\end{array}$ & reg1.25r00 & 1972 & $\mathbf{P}$ & Implied endorsement & \begin{tabular}{|l|} 
ICRP Publication \\
30
\end{tabular} & 1990 \\
\hline 3271 & ICRP & $\begin{array}{l}\text { ICRP } \\
\text { Publication } 2\end{array}$ & 1959 & $\begin{array}{l}\text { Limits for Intake of Radionuclides } \\
\text { by Workers }\end{array}$ & reg1.3.r02 & 1974 & $P$ & & \begin{tabular}{|l|} 
ICRP Publication \\
30
\end{tabular} & 1990 \\
\hline 3272 & ICRP & $\begin{array}{l}\text { ICRP } \\
\text { Publication } 2\end{array}$ & 1959 & $\begin{array}{l}\text { Limits for Intake of Radionuclides } \\
\text { by Workers }\end{array}$ & reg1.4.ro2 & 1974 & $P$ & & $\begin{array}{l}\text { ICRP Publication } \\
30\end{array}$ & 1990 \\
\hline 3273 & ICRP & $\begin{array}{l}\text { ICRP } \\
\text { Publication } 2 \\
\end{array}$ & 1959 & $\begin{array}{l}\text { Limits for Intake of Radionuclides } \\
\text { by Workers }\end{array}$ & reg1.5.ro0 & 1971 & $P$ & $\begin{array}{l}\text { Superseded by ICRP. } \\
30\end{array}$ & $\begin{array}{l}\text { ICRP Publication } \\
30\end{array}$ & 1990 \\
\hline 3274 & ICRP & $\begin{array}{l}\text { ICRP } \\
\text { Publication } 2\end{array}$ & 1959 & $\begin{array}{l}\text { Limits for Intake of Radionuclides } \\
\text { by Workers }\end{array}$ & reg1.77.r00 & 1974 & $P$ & $\begin{array}{l}\text { Superseded by ICRP- } \\
30 .\end{array}$ & $\begin{array}{l}\text { ICRP Publication } \\
30\end{array}$ & 1990 \\
\hline 3275 & ICRP & $\begin{array}{l}\text { ICRP } \\
\text { Publication } 2\end{array}$ & 1959 & $\begin{array}{l}\text { Limits for Intake of Radionuclides } \\
\text { by Workers }\end{array}$ & reg8.9r00 & 1973 & A & & $\begin{array}{l}\text { ICRP Publication } \\
30\end{array}$ & 1990 \\
\hline
\end{tabular}


Codes and Standards and Other Guidance Cited in Regulatory Documents

Part A - Industry Consensus Codes and Standards (continued)

\begin{tabular}{|c|c|c|c|c|c|c|c|c|c|c|}
\hline Nocord & codo & Standard & Standard & 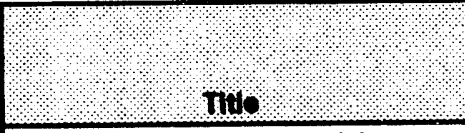 & Documont & $\begin{array}{l}\text { Doo. } \\
\text { Date. }\end{array}$ & Acoppts & Comnent & Curront Yorolon & onont \\
\hline 3276 & ICRP & $\begin{array}{l}\text { ICRP } \\
\text { Publication } 6\end{array}$ & $N / S$ & $\begin{array}{l}1990 \text { Recommendations of the } \\
\text { International Commission on } \\
\text { Radiological Protection }\end{array}$ & not82-18 & 1982 & $\mathbf{P}$ & $\begin{array}{l}\text { Supplements ICRP-2. } \\
\text { Superseded by ICRP. } \\
26\end{array}$ & $\begin{array}{l}\text { ICRP Publication } \\
60\end{array}$ & 1991 \\
\hline 3277 & ICRP & $\begin{array}{l}\text { ICRP } \\
\text { Publication } 6\end{array}$ & 1962 & $\begin{array}{l}1990 \text { Recommendations of the } \\
\text { International Commission on } \\
\text { Radiological Protection }\end{array}$ & reg8.9r00 & 1973 & A & & $\begin{array}{l}\text { ICRP Publication } \\
60\end{array}$ & 1991 \\
\hline 3278 & ICRP & $\begin{array}{l}\text { ICRP } \\
\text { Publication } 9\end{array}$ & $\mathrm{~N} / \mathrm{S}$ & $\begin{array}{l}1989 \text { Recommendations of the } \\
\text { International Commission on } \\
\text { Radiological Protection }\end{array}$ & not82-18 & 1982 & $\mathbf{P}$ & $\begin{array}{l}\text { Supplements ICRP2. } \\
\text { Superseded by ICRP. } \\
26 .\end{array}$ & $\begin{array}{l}\text { ICRP Publication } \\
60\end{array}$ & 1991 \\
\hline 3279 & ICRP & $\begin{array}{l}\text { ICRP } \\
\text { Publication } 9\end{array}$ & 1966 & $\begin{array}{l}1990 \text { Recommendations of the } \\
\text { International Commission on } \\
\text { Radiological Protection }\end{array}$ & reg8.9roo & 1973 & A & & $\begin{array}{l}\text { ICRP Publication } \\
60\end{array}$ & 1991 \\
\hline 3280 & ICRP & \begin{tabular}{|l|} 
ICRP \\
Publicstion 10
\end{tabular} & 1968 & $\begin{array}{l}\text { Individual Monitoring for Intakes of } \\
\text { Radionuclides by Workers: Design } \\
\text { and Interpretation }\end{array}$ & not82-18 & 1982 & $P$ & $\begin{array}{l}\text { Supplements ICRP-2. } \\
\text { Superseded by ICRP. } \\
54 .\end{array}$ & $\begin{array}{l}\text { ICRP Publication } \\
54\end{array}$ & 1989 \\
\hline 3281 & ICRP & $\begin{array}{l}\text { ICRP } \\
\text { Publication } 10\end{array}$ & 1968 & $\begin{array}{l}\text { Individual Monitoring for Intakes of } \\
\text { Radionuclides by Workers: Design } \\
\text { and Interpretation }\end{array}$ & reg8.9ro0 & 1973 & $\bar{A}$ & & $\begin{array}{l}\text { ICRP Publication } \\
54\end{array}$ & 1989 \\
\hline 3282 & ICRP & $\begin{array}{l}\text { ICRP } \\
\text { Publication } \\
\text { 10A } \\
\end{array}$ & 1971 & $\begin{array}{l}\text { Individual Monitoring for Intakes of } \\
\text { Radionuclides by Workers: Design } \\
\text { and Interpretation }\end{array}$ & not82-18 & 1982 & $\mathbf{P}$ & $\begin{array}{l}\text { Supplements ICRP-2. } \\
\text { Superseded by ICRP. } \\
54 .\end{array}$ & $\begin{array}{l}\text { ICRP Publication } \\
54\end{array}$ & 1989 \\
\hline 3283 & ICRP & $\begin{array}{l}\text { ICRP } \\
\text { Publication } \\
10 A \\
\end{array}$ & 1971 & $\begin{array}{l}\text { Individual Monitoring for Intakes of } \\
\text { Radionuclides by Workers: Design } \\
\text { and Interpretation }\end{array}$ & reg3.51.ro0 & 1982 & $\mathbf{R}$ & & $\begin{array}{l}\text { ICRP Publication } \\
54\end{array}$ & 1989 \\
\hline 3284 & ICRP & \begin{tabular}{|l|} 
CRP \\
Publication \\
10A \\
\end{tabular} & 1971 & $\begin{array}{l}\text { Individual Monitoring for Intakes of } \\
\text { Radlonuclides by Workers: Design } \\
\text { and Interpretation }\end{array}$ & reg8.9r00 & 1973 & A & & $\begin{array}{l}\text { ICRP Publication } \\
54\end{array}$ & 1989 \\
\hline 3285 & ICRP & \begin{tabular}{|l|} 
ICRP \\
Publication 12
\end{tabular} & 1969 & $\begin{array}{l}\text { General Principles of Monitoring for } \\
\text { Radiation Protection of Workers }\end{array}$ & not82-18 & 1982 & $\mathbf{P}$ & $\begin{array}{l}\text { Supplements ICRP-2. } \\
\text { Superseded by ICRP. } \\
35 .\end{array}$ & out of print & $n / a$ \\
\hline 3286 & ICRP & $\begin{array}{l}\text { ICRP } \\
\text { Publication } 12\end{array}$ & $N / S$ & $\begin{array}{l}\text { General Principles of Monitoring for } \\
\text { Radiation Protection of Workers }\end{array}$ & reg10.08.r02 & 1987 & $\bar{R}$ & $\begin{array}{l}\text { Supplements ICRP-2. } \\
\text { Superseded by ICRP. } \\
35 .\end{array}$ & out of print & $n / a$ \\
\hline
\end{tabular}




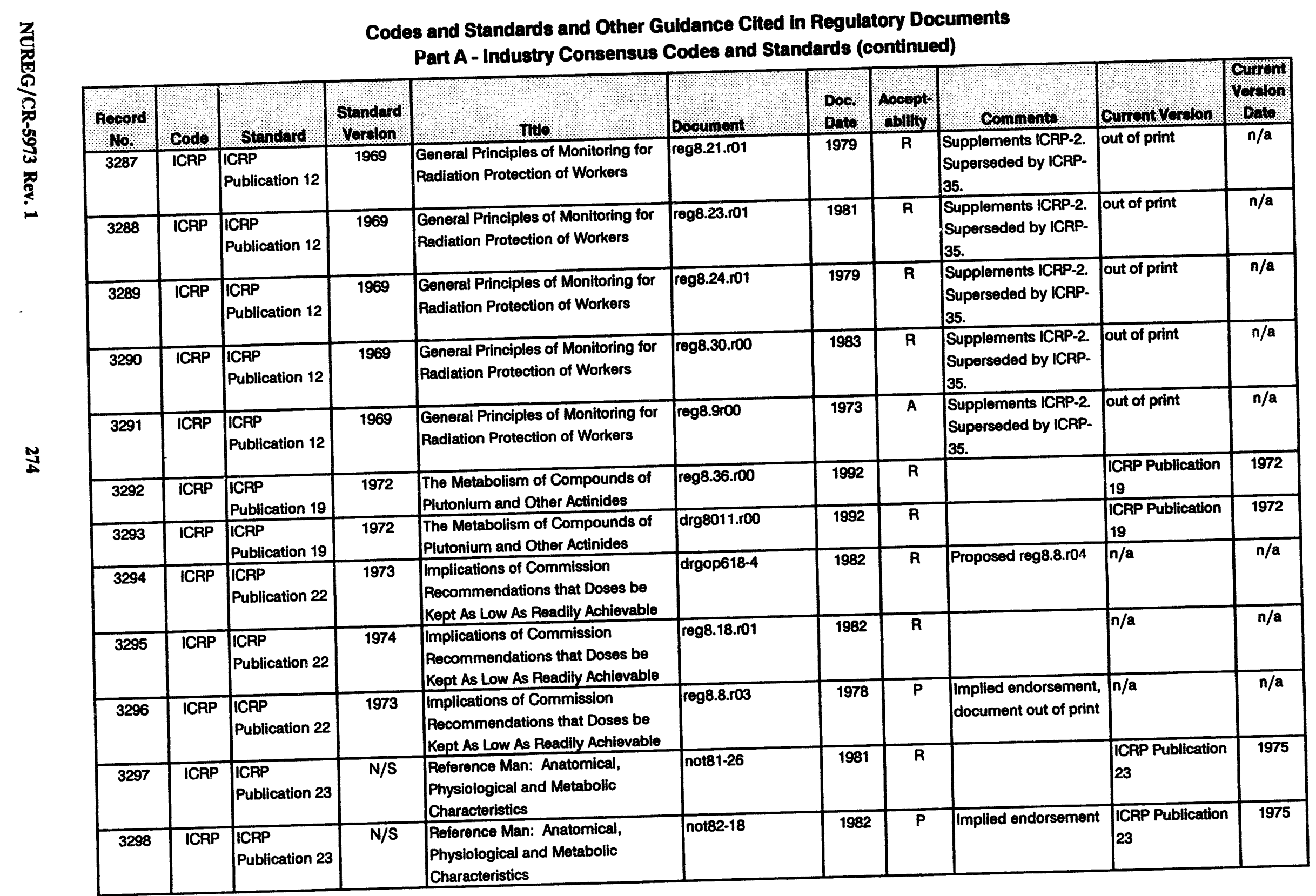


Codes and Standards and Other Guidance Clted in Regulatory Documents

Part A - Industry Consensus Codes and Standards (continued)

\begin{tabular}{|c|c|c|c|c|c|c|c|c|c|c|}
\hline $\begin{array}{l}\text { Rocord } \\
\text { No. }\end{array}$ & codo & Standurd & Standard & 1110 , & Dooument & $\begin{array}{l}\text { Doo. } \\
\text { Doto }\end{array}$ & hocept & Comments & Cunnon Yom on & owo \\
\hline 3299 & ICRP & $\begin{array}{l}\text { ICRP } \\
\text { Publication } 23\end{array}$ & N/S & $\begin{array}{l}\text { Reference Man: Anatomical, } \\
\text { Physiological and Metabolic } \\
\text { Characteristics }\end{array}$ & not83-59 & 1983 & $\mathbf{R}$ & & $\begin{array}{l}\text { ICRP Publication } \\
23\end{array}$ & 1975 \\
\hline 3300 & ICRP & \begin{tabular}{|l|} 
ICRP \\
Publication 23
\end{tabular} & 1975 & $\begin{array}{l}\text { Reference Man: Anatomical, } \\
\text { Physiological and Metabolic } \\
\text { Characteristics }\end{array}$ & reg1.109.rot & 1977 & $\mathbf{P}$ & $\begin{array}{l}\text { Implied endorsement- } \\
\text { No longer in print-1992 } \\
\text { update planned }\end{array}$ & $\begin{array}{l}\text { ICRP Publication } \\
23 \\
\end{array}$ & 1975 \\
\hline 3301 & ICRP & $\begin{array}{l}\text { ICRP } \\
\text { Publication } 26\end{array}$ & $\mathrm{~N} / \mathrm{S}$ & $\begin{array}{l}1990 \text { Recommendations of the } \\
\text { International Commission on } \\
\text { Radiological Protection }\end{array}$ & dirgfc414-4 & 1985 & $\mathbf{R}$ & & $\begin{array}{l}\text { ICAP Publication } \\
60\end{array}$ & 1991 \\
\hline 3302 & ICRP & $\begin{array}{l}\text { ICRP } \\
\text { Publication } 26\end{array}$ & $\mathrm{~N} / \mathrm{S}$ & $\begin{array}{l}1990 \text { Recommendations of the } \\
\text { International Commission on } \\
\text { Radiological Protection } \\
\end{array}$ & drgop212-4 & 1984 & $\mathbf{R}$ & & $\begin{array}{l}\text { ICRP Publication } \\
60\end{array}$ & 1991 \\
\hline 3303 & ICRP & $\begin{array}{l}\text { ICRP } \\
\text { Publication } 26\end{array}$ & 1977 & $\begin{array}{l}1990 \text { Recommendations of the } \\
\text { International Commission on } \\
\text { Radiological Protection }\end{array}$ & not82-18 & 1982 & $\mathbf{P}$ & & $\begin{array}{l}\text { ICRP Publication } \\
60\end{array}$ & 1991 \\
\hline 3304 & ICRP & $\begin{array}{l}\text { ICRP } \\
\text { Publication } 26\end{array}$ & $\mathrm{~N} / \mathrm{S}$ & $\begin{array}{l}1990 \text { Recommendations of the } \\
\text { International Commission on } \\
\text { Radiological Protection }\end{array}$ & not92-34 & 1992 & A & & $\begin{array}{l}\text { ICRP Publication } \\
60\end{array}$ & 1991 \\
\hline 3305 & ICRP & $\begin{array}{l}\text { ICRP } \\
\text { Publication } 26\end{array}$ & 1977 & $\begin{array}{l}1990 \text { Recommendations of the } \\
\text { International Commission on } \\
\text { Radiological Protection }\end{array}$ & pol53FR49886 & 1988 & $\mathbf{R}$ & & $\begin{array}{l}\text { ICRP Publication } \\
60\end{array}$ & 1991 \\
\hline 3306 & ICRP & $\begin{array}{l}\text { ICRP } \\
\text { Publication } 26\end{array}$ & 1977 & $\begin{array}{l}1990 \text { Recommendations of the } \\
\text { International Commission on } \\
\text { Radiological Protection }\end{array}$ & pol55FR27522 & 1990 & $\mathbf{R}$ & & $\begin{array}{l}\text { ICRP Publication } \\
60\end{array}$ & 1991 \\
\hline 3307 & ICRP & $\begin{array}{l}\text { ICRP } \\
\text { Publication } 26\end{array}$ & 1977 & $\begin{array}{l}1990 \text { Recommendations of the } \\
\text { International Commission on } \\
\text { Radiological Protection }\end{array}$ & reg8.27ro0 & 1981 & $\mathbf{R}$ & & $\begin{array}{l}\text { ICRP Publication } \\
60\end{array}$ & 1991 \\
\hline 3308 & ICRP & \begin{tabular}{|l|} 
ICRP \\
Publication 27 \\
\end{tabular} & 1977 & $\begin{array}{l}\text { Problems Involved in Developing } \\
\text { an Index of Harm }\end{array}$ & reg8.27roo & 1981 & $\bar{R}$ & Out of Print & $n / a$ & $n / a$ \\
\hline 3309 & ICRP & \begin{tabular}{|l|} 
ICRP \\
Publication 30 \\
\end{tabular} & 1979 & $\begin{array}{l}\text { limits for Intake of Radionuclides } \\
\text { by Workers }\end{array}$ & drg8005.r00 & 1991 & $P$ & Draft reg guide & $\begin{array}{l}\text { ICRP Publication } \\
30\end{array}$ & 1990 \\
\hline 3310 & ICAP & \begin{tabular}{|l|} 
ICRP \\
Publication 30 \\
\end{tabular} & $N / S$ & $\begin{array}{l}\text { Limits for Intake of Radionuclides } \\
\text { by Workers }\end{array}$ & drg8013.r00 & 1992 & $\mathbf{P}$ & & $\begin{array}{l}\text { ICRP Publication } \\
30\end{array}$ & 1990 \\
\hline
\end{tabular}




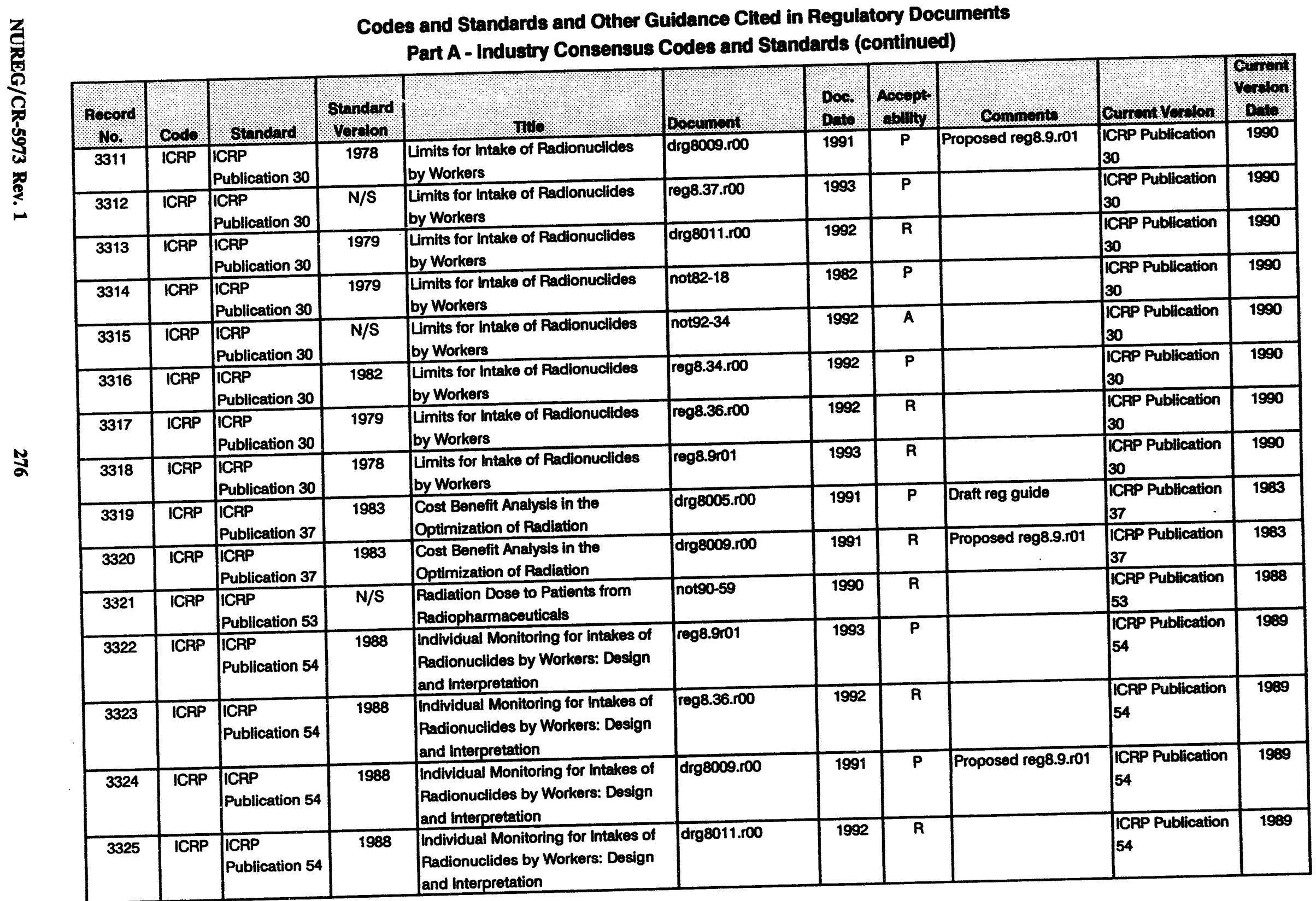


Codes and Standards and Other Guldance Cited in Regulatory Documents

Part A - Industry Consensus Codes and Standards (continued)

\begin{tabular}{|c|c|c|c|c|c|c|c|c|c|c|}
\hline $\begin{array}{l}\text { hocord } \\
\text { No. }\end{array}$ & Qodo & Shandard & Standord & (,, THAO,,, & Docounont & Dor: & $\begin{array}{l}\text { Aocept } \\
\text { ablity }\end{array}$ & commonte & Cum on Vorbon & Yoron \\
\hline 3326 & ICRP & $\begin{array}{l}\text { ICRP } \\
\text { Publication } 56\end{array}$ & 1989 & $\begin{array}{l}\text { Age-Dependent Doses to Members } \\
\text { of the Public from Intake of } \\
\text { Radionuclides: Part } 1\end{array}$ & reg8.36.r00 & 1992 & $\mathbf{R}$ & & $\begin{array}{l}\text { ICRP Publication } \\
56\end{array}$ & 1990 \\
\hline 3327 & ICRP & $\begin{array}{l}\text { ICRP } \\
\text { Publication } 56\end{array}$ & 1989 & $\begin{array}{l}\text { Age-Dependent Doses to Members } \\
\text { of the Public from Intake of } \\
\text { Radionuclides: Part } 1\end{array}$ & drg8011.r00 & 1992 & $\mathbf{R}$ & & $\begin{array}{l}\text { ICRP Publication } \\
56\end{array}$ & 1990 \\
\hline 3328 & IEEE & IEEE 242 & 1986 & $\begin{array}{l}\text { Recommended Practices for } \\
\text { Protection and Coordination of } \\
\text { Industrial and Commercial Power } \\
\text { Systems }\end{array}$ & not88-45 & 1988 & $\mathbf{R}$ & Cited as IEEE 24? & IEEE 242 & $1986 \mathrm{R91}$ \\
\hline 3329 & IEEE & IEEE 242 & 1986 & $\begin{array}{l}\text { Recommended Practices for } \\
\text { Protection and Coordination of } \\
\text { Industrial and Commercial Power } \\
\text { Systems }\end{array}$ & not93-64 & 1993 & $\mathbf{R}$ & & IEEE 242 & $1986 \mathrm{PQ1}$ \\
\hline 3330 & IEEE & IEEE 279 & 1971 & $\begin{array}{l}\text { Siandard Criteria ior Safety } \\
\text { Systems for Nuclear Power } \\
\text { Generating Stations }\end{array}$ & $10 \mathrm{cfr} 50.55 a$ & 1993 & $\mathbf{P}$ & $\begin{array}{l}\text { IEEE } 279 \text { also } \\
\text { designated ANSI N42.7 }\end{array}$ & IEEE 603 & 1991 \\
\hline 3331 & IEEE & IEEE 279 & $\mathbf{N} / \mathrm{S}$ & $\begin{array}{l}\text { Standard Criteria for Safety } \\
\text { Systems for Nuclear Power } \\
\text { Generating Stations } \\
\end{array}$ & bul80-16 & 1980 & $\mathbf{P}$ & $\begin{array}{l}\text { IEEE } 279 \text { also } \\
\text { designated ANSI N42.7 }\end{array}$ & IEEE 603 & 1991 \\
\hline 3332 & IEEE & IEEE 279 & $\mathbf{N} / \mathbf{S}$ & $\begin{array}{l}\text { Standard Criteria for Safety } \\
\text { Systems for Nuclear Power } \\
\text { Generating Stations }\end{array}$ & bul90-01 & 1990 & $\mathbf{P}$ & $\begin{array}{l}\text { IEEE } 279 \text { also } \\
\text { designated ANSI N42.7 }\end{array}$ & IEEE 603 & 1991 \\
\hline 3333 & IEEE & !EEE 279 & $N / S$ & $\begin{array}{l}\text { Standard Criteria for Safety } \\
\text { Systems for Nuclear Power } \\
\text { Generating Stations }\end{array}$ & bul90-1.s01 & 1992 & $R$ & & IEEE 603 & 1991 \\
\hline 3334 & IEEE & IEEE 279 & $N / S$ & $\begin{array}{l}\text { Standard Criteria for Safety } \\
\text { Systems for Nuclear Power } \\
\text { Generating Stations }\end{array}$ & bul93-03 & 1993 & $\mathbf{R}$ & & IEEE 603 & 1991 \\
\hline 3335 & IEEE & IEEE 279 & N/S & $\begin{array}{l}\text { Standard Criteria for Safety } \\
\text { Systems tor Nuclear Power } \\
\text { Generating Stations }\end{array}$ & cir78-19 & 1978 & A & $\begin{array}{l}\text { IEEE } 279 \text { also } \\
\text { designated ANSI N42.7 }\end{array}$ & FEEE 603 & 1991 \\
\hline
\end{tabular}


Codes and Standards and Other Guidance Cited in Regulatory Documents

Part A - Industry Consensus Codes and Standards (continued)

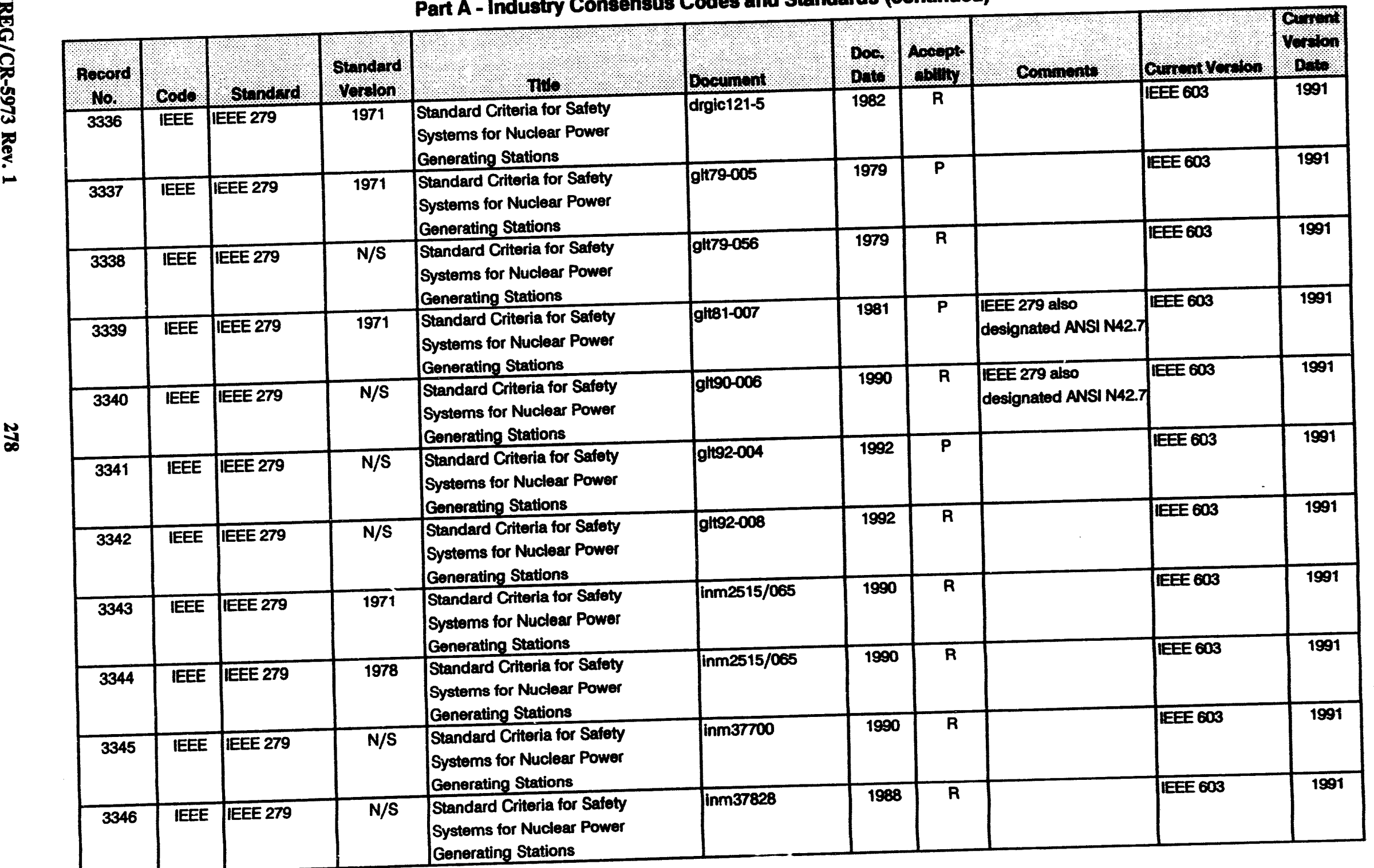

Generating Stations 
Codes and Standards and Other Guidence Cited in Regulatory Documents

Part A - Industry Consensus Codes and Standards (continued)

\begin{tabular}{|c|c|c|c|c|c|c|c|c|c|c|}
\hline $\begin{array}{l}\text { Roeord } \\
\text { No. }\end{array}$ & Code & Stenderd & $\begin{array}{l}\text { Standerd } \\
\text { Vorslon }\end{array}$ & mos & Document & $\begin{array}{l}\text { Doc. } \\
\text { Dote }\end{array}$ & $\begin{array}{l}\text { Aocopt } \\
\text { ebluly }\end{array}$ & Componts & comal $V_{0}=100$ & Yorom \\
\hline 3347 & IEEE & IEEE 279 & $N / S$ & $\begin{array}{l}\text { Standard Criteria for Safety } \\
\text { Systems for Nuclear Power } \\
\text { Generating Stations }\end{array}$ & inm51051 & 1984 & $\mathbf{R}$ & & IEEE 603 & 1991 \\
\hline 3348 & IEEE & IEEE 279 & $N / S$ & $\begin{array}{l}\text { Standard Criteria for Safety } \\
\text { Systems for Nuclear Power } \\
\text { Generating Stations }\end{array}$ & inm51053 & 1984 & $\mathbf{R}$ & & IEEE 603 & 1991 \\
\hline 3349 & IEEE & |EEE 279 & $N / S$ & $\begin{array}{l}\text { Standard Criteria for Safety } \\
\text { Systems for Nuclear Power } \\
\text { Generating Stations }\end{array}$ & inm51055 & 1984 & $\mathbf{R}$ & & IEEE 603 & 1991 \\
\hline 3350 & IEEE & IEEE 279 & $\mathrm{~N} / \mathrm{S}$ & $\begin{array}{l}\text { Standard Criteria for Safety } \\
\text { Systems for Nuclear Power } \\
\text { Generating Stations }\end{array}$ & inm51061 & 1984 & $\mathbf{R}$ & & IEEE 603 & 1991 \\
\hline 3351 & IEEE & IEEE 279 & N/S & $\begin{array}{l}\text { Standard Criteria for Safety } \\
\text { Systems for Nuclear Power } \\
\text { Generating Siations } \\
\end{array}$ & inm51063 & 1985 & $\mathbf{R}$ & & IEEE 603 & 1991 \\
\hline 3352 & IEEE & IEEE 279 & $N / S$ & $\begin{array}{l}\text { Standard Criteria for Safety } \\
\text { Systems for Nuclear Power } \\
\text { Generating Stations }\end{array}$ & inm51065 & 1984 & $\mathbf{R}$ & & IEEE 603 & 1991 \\
\hline 3353 & $\begin{array}{c}\text { IEEE } \\
!\end{array}$ & IEEE 279 & $N / S$ & $\begin{array}{l}\text { Standard Criteria for Safety } \\
\text { Systems for Nuclear Power } \\
\text { Generating Stations }\end{array}$ & inm52051 & 1984 & $\mathbf{R}$ & & FEEE 603 & 1991 \\
\hline 3354 & IEEE & IEEE 279 & N/S & $\begin{array}{l}\text { Standard Criteria for Safety } \\
\text { Systems for Nuclear Power } \\
\text { Generating Stations }\end{array}$ & inm52053 & 1984 & $\mathbf{R}$ & & IEEE 603 & 1991 \\
\hline 3355 & IEEE & IEEE 279 & $N / S$ & $\begin{array}{l}\text { Standard Criteria for Safety } \\
\text { Systems for Nuclear Power } \\
\text { Generating Stations }\end{array}$ & inm52055 & 1984 & $\mathbf{R}$ & & IEEE 6003 & 1991 \\
\hline 3356 & IEEE & IEEE 279 & $N / S$ & $\begin{array}{l}\text { Standard Criteria for Safety } \\
\text { Systems for Nuclear Power } \\
\text { Generating Stations } \\
\end{array}$ & inm9900/STS & 1986 & $\mathbf{R}$ & & IEEE 603 & 1991 \\
\hline 3357 & IEEE & IEEE 279 & 1971 & $\begin{array}{l}\text { Standard Criteria for Safety } \\
\text { Systems for Nuclear Power } \\
\text { Generating Stations }\end{array}$ & nureg0737 & 1980 & $\mathbf{P}$ & & IEEE 603 & 1991 \\
\hline
\end{tabular}



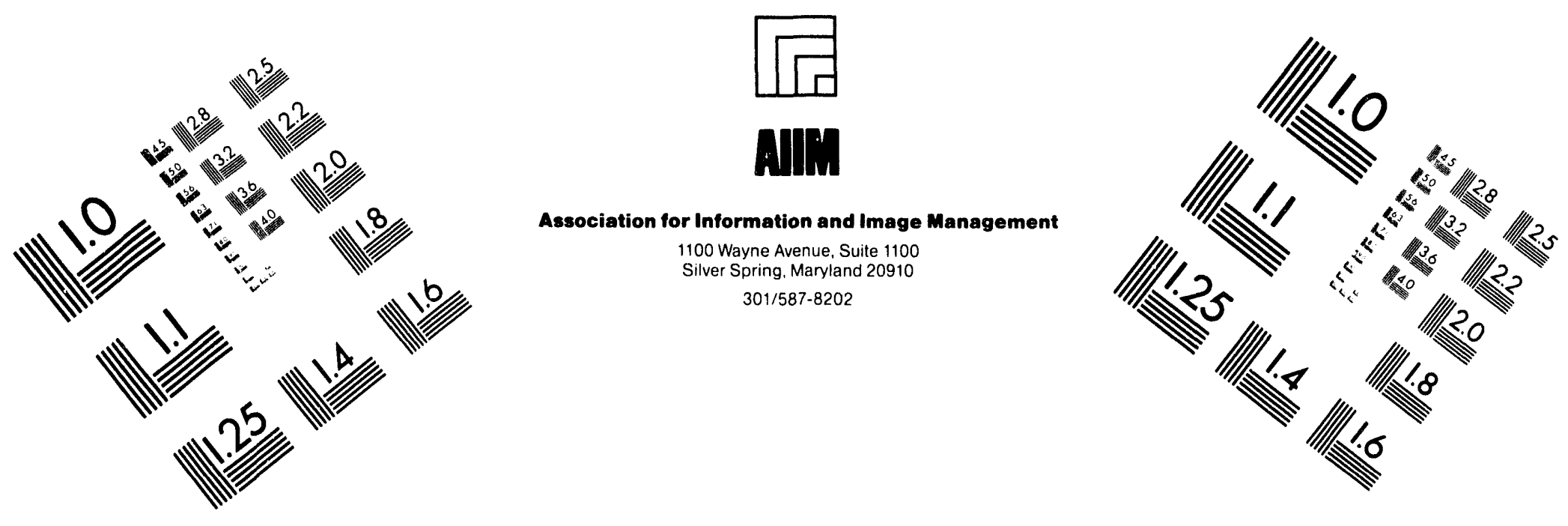

\section{Centimeter}

| Inches
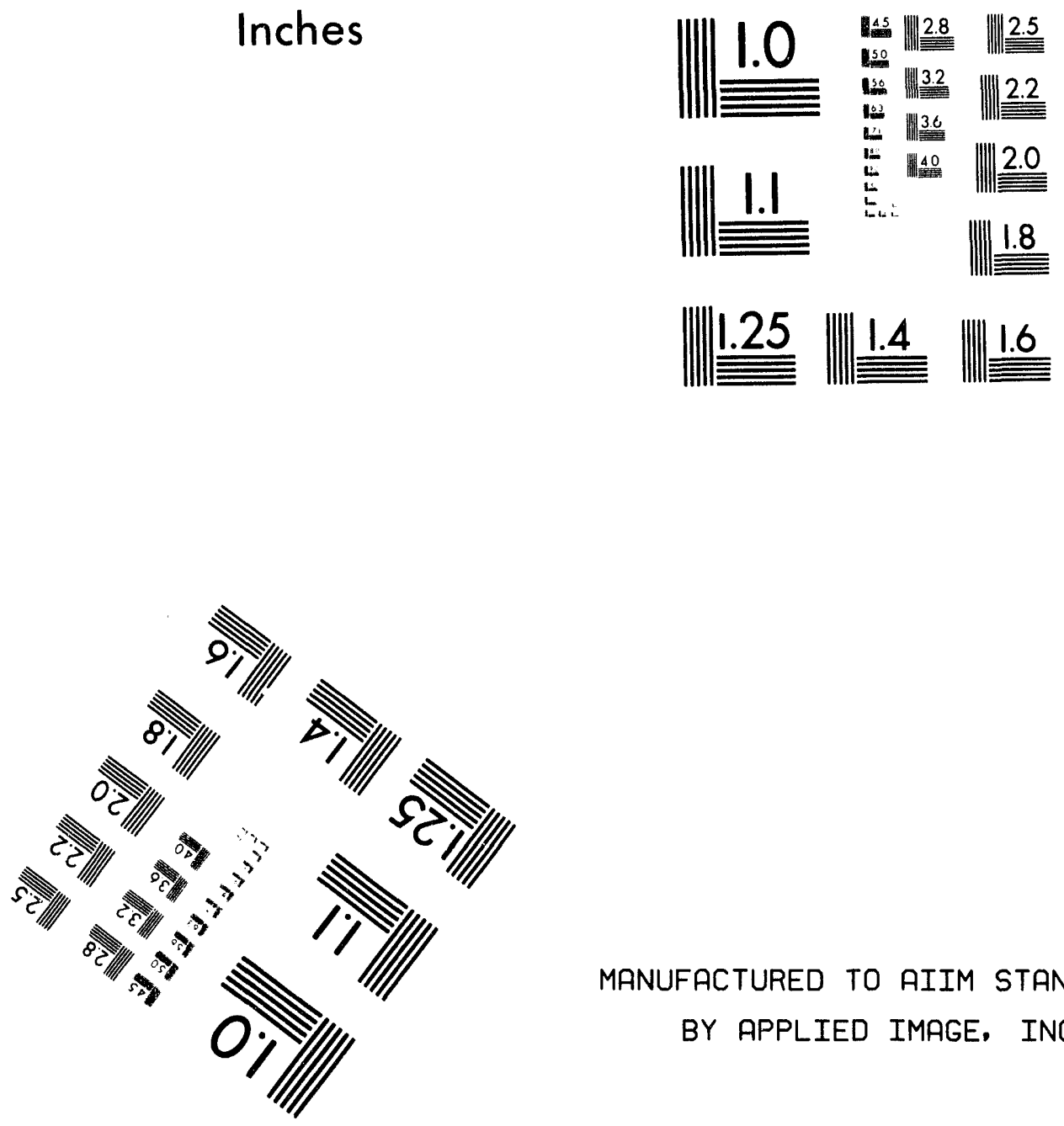

MANUFACTURED TO AIIM STANDAPÚS

BY APPLIED IMAGE, INC.

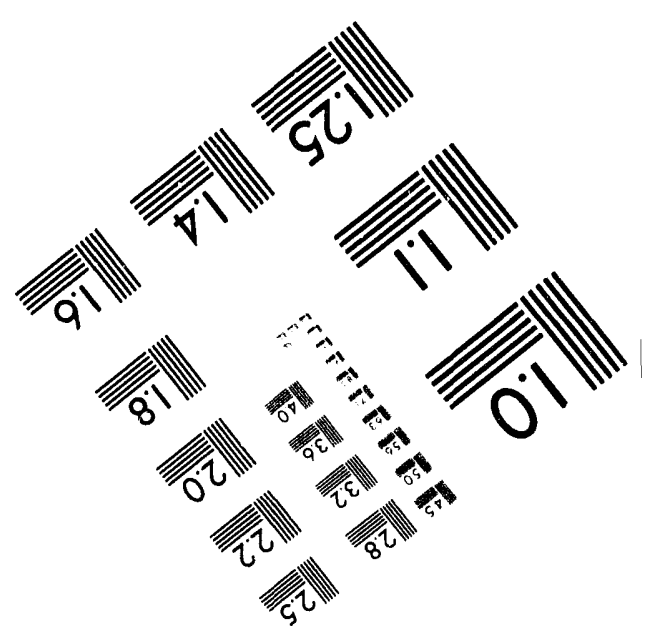



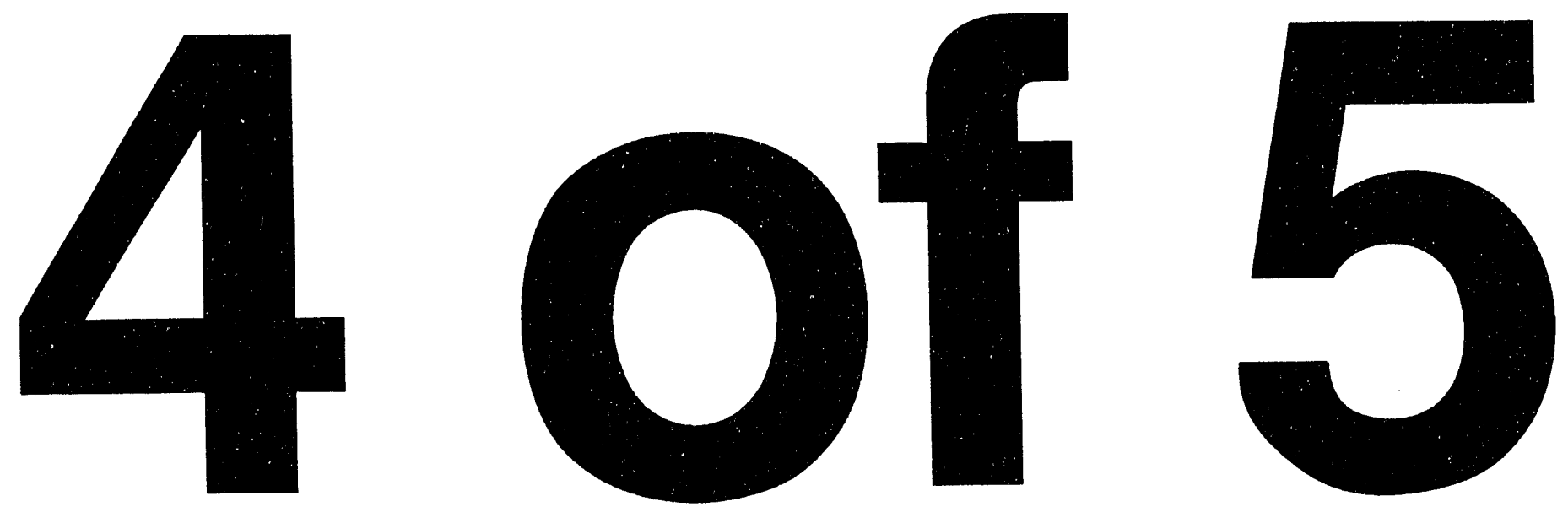
Codes and Standards and Other Guidance Cited in Regulatory Documents

Part A - Industry Consensus Codes and Standards (continued)

\begin{tabular}{|c|c|c|c|c|c|c|c|c|c|c|}
\hline 3358 & IEEE & IEEE 279 & N/S & $\begin{array}{l}\text { Standard Criteria for Safety } \\
\text { Systems for Nuclear Power } \\
\text { Generating Stations }\end{array}$ & nureg0933, 3.110 & 1992 & $\mathbf{R}$ & & IEEE 603 & 1991 \\
\hline 3359 & IEEE & IEEE 279 & 1971 & $\begin{array}{l}\text { Standard Criteria for Safety } \\
\text { Systems for Nuclear Power } \\
\text { Generating Stations }\end{array}$ & nureg0933, 3.156 & 1992 & $\mathbf{R}$ & & IEEE 603 & 1991 \\
\hline 3360 & IEEE & IEEE 279 & 1979 & $\begin{array}{l}\text { Standard Criteria for Safety } \\
\text { Systems for Nuclear Power } \\
\text { Generating Stations }\end{array}$ & nureg0933, 3.50 & 1984 & $\mathbf{R}$ & & IEEE 603 & 1991 \\
\hline 3361 & IEEE & IEEE 279 & 1971 & $\begin{array}{l}\text { Standard Criteria for Safety } \\
\text { Systems for Nuclear Power } \\
\text { Generating Stations }\end{array}$ & nureg0933, 3.64 & 1983 & $\mathbf{R}$ & & IEEE 603 & 1991 \\
\hline 3362 & IEEE & IEEE 279 & N/S & $\begin{array}{l}\text { Standard Criteria for Safety } \\
\text { Systems for Nuclear Power } \\
\text { Generating Stations }\end{array}$ & nureg0935, 3.70 & 1991 & $\mathbf{R}$ & & IEEE 603 & 1991 \\
\hline 3363 & IEEE & IEEE 279 & 1971 & $\begin{array}{l}\text { Standard Criteria for Safety } \\
\text { Systems for Nuclear Power } \\
\text { Generating Stations }\end{array}$ & reg1.106.ro1 & 1977 & $\mathbf{P}$ & $\begin{array}{l}\text { EEE } 279 \text { also } \\
\text { designated ANSI N42.7 }\end{array}$ & IEEE 603 & 1991 \\
\hline 3364 & IEEE & IEEE 279 & 1971 & $\begin{array}{l}\text { Standard Criteria for Safety } \\
\text { Systems for Nuclear Power } \\
\text { Generating Stations }\end{array}$ & reg1.118.r02 & 1978 & $\bar{P}$ & $\begin{array}{l}\text { IEEE } 279 \text { also } \\
\text { designated ANSI N42.7 }\end{array}$ & IEEE 603 & 1991 \\
\hline 3365 & IEEE & IEEE 279 & 1971 & $\begin{array}{l}\text { Standard Criteria for Safety } \\
\text { Systems for Nuclear Power } \\
\text { Generating Stations }\end{array}$ & reg1.151.r00 & 1983 & $\mathbf{P}$ & $\begin{array}{l}\text { IEEE } 279 \text { also } \\
\text { designated ANSI N42.7 }\end{array}$ & IEEE 603 & 1991 \\
\hline 3366 & IEEE & IEEE 279 & 1971 & $\begin{array}{l}\text { Standard Criteria for Safety } \\
\text { Systems for Nuclear Power } \\
\text { Generating Stations }\end{array}$ & reg1.152.ro0 & 1985 & $\mathbf{P}$ & \begin{tabular}{|l|} 
IEEE 279 also \\
designated ANSI N42.7
\end{tabular} & IEEE 603 & 1991 \\
\hline 3367 & IEEE & IEEE 279 & 1971 & $\begin{array}{l}\text { Standard Criteria for Safety } \\
\text { Systems for Nuclear Power } \\
\text { Generating Stations }\end{array}$ & reg1.153.r00 & 1985 & $\mathbf{P}$ & $\begin{array}{l}\text { IEEE } 279 \text { also } \\
\text { designated ANSI N42.7 }\end{array}$ & IEEE 603 & 1991 \\
\hline 3368 & IEEE & IEEE 279 & 1971 & $\begin{array}{l}\text { Standard Criteria for Safety } \\
\text { Systems for Nuclear Power } \\
\text { Generating Stations }\end{array}$ & reg1.22.ro0 & 1972 & $\mathbf{R}$ & $\begin{array}{l}\text { IEEE } 279 \text { also } \\
\text { designated ANSI N42.7] }\end{array}$ & IEEE 603 & 1991 \\
\hline
\end{tabular}


Codes and Standards and Other Guidance Cited in Regulatory Documents

Part A - Industry Consensus Codes and Standards (continued)

\begin{tabular}{|c|c|c|c|c|c|c|c|c|c|c|}
\hline Ronord & Codo & Sthndara & $\begin{array}{l}\text { Standard } \\
\text { Vorelon }\end{array}$ & nito & Doouliont & Doo. & $\begin{array}{l}\text { Aooppts } \\
\text { obillty }\end{array}$ & Commonts & Curront Verdon & Curront \\
\hline 3369 & IEEE & IEEE 279 & 1971 & $\begin{array}{l}\text { Standard Criteria for Safety } \\
\text { Systems for Nuclear Power } \\
\text { Generating Stations }\end{array}$ & reg1.45.ro0 & 1973 & $\mathbf{P}$ & $\begin{array}{l}\text { IEEE } 279 \text { also } \\
\text { designated ANSI N42.7 }\end{array}$ & IEEE 603 & 1991 \\
\hline 3370 & IEEE & IEEE 279 & 1971 & $\begin{array}{l}\text { Standard Criteria for Safety } \\
\text { Systems for Nuclear Power } \\
\text { Generating Stations }\end{array}$ & reg1.47.ro0 & 1973 & $\mathbf{P}$ & $\begin{array}{l}\text { IEEE } 279 \text { also } \\
\text { designated ANSI N42.7 }\end{array}$ & IEEE 603 & 1991 \\
\hline 3371 & IEEE & IEEE 279 & $N / S$ & $\begin{array}{l}\text { Standard Friteria for Safety } \\
\text { Systems for Nuclear Power } \\
\text { Generating Stations }\end{array}$ & $\operatorname{reg} 1.52 .102$ & 1978 & A & $\begin{array}{l}\text { IEEE } 279 \text { also } \\
\text { designated ANSI N42.7 }\end{array}$ & IEEE 603 & 1991 \\
\hline 3372 & IEEE & IEEE 279 & $N / S$ & $\begin{array}{l}\text { Standard Criteria for Safety } \\
\text { Systems for Nuclear Power } \\
\text { Generating Stations }\end{array}$ & reg1.53.r00 & 1973 & $\mathbf{P}$ & General endorsement & IEEE 603 & 1991 \\
\hline 3373 & IEEE & IEEE 279 & 1971 & $\begin{array}{l}\text { Standard Criteria for Safety } \\
\text { Systems for Nuclear Power } \\
\text { Generating Stations }\end{array}$ & reg1.62.ro0 & 1973 & $P$ & \begin{tabular}{|l|} 
IEEE 279 also \\
designated ANSI N42.7
\end{tabular} & IEEE 603 & 1991 \\
\hline 3374 & IEEE & IEEE 279 & 1971 & $\begin{array}{l}\text { Standard Criteria for Safety } \\
\text { Systems for Nuclear Po. } \\
\text { Generating Stations }\end{array}$ & reg1.70.r03 & 1978 & $\mathbf{P}$ & \begin{tabular}{|l|} 
IEEE 279 also \\
designated ANSI N42.7
\end{tabular} & IEEE 603 & 1991 \\
\hline 3375 & IEEE & IEEE 279 & 1971 & $\begin{array}{l}\text { Standard Criteria for Safety } \\
\text { Systems for Nuclear Power } \\
\text { Generating Stations }\end{array}$ & reg1.75.r02 & 1978 & $\mathbf{P}$ & $\begin{array}{l}\text { IEEE } 279 \text { also } \\
\text { designated ANSI Ni2.7 }\end{array}$ & IEEE 603 & 1991 \\
\hline 3376 & IEEE & IEEE 279 & 1971 & $\begin{array}{l}\text { Standard Criteria for Safety } \\
\text { Systems for Nuclear Power } \\
\text { Generating Stations }\end{array}$ & reg1.9.ro3 & 1993 & A & \begin{tabular}{|l|} 
IEEE 279 also \\
designated ANSI N42.7
\end{tabular} & IEEE 603 & 1991 \\
\hline 3377 & IEEE & IEEE 279 & 1971 & $\begin{array}{l}\text { Standard Criteria for Safety } \\
\text { Systems for Nuclear Power } \\
\text { Generating Stations }\end{array}$ & reg1.90.ro1 & 1977 & $P$ & \begin{tabular}{|l|}
$\begin{array}{l}\text { With additional } \\
\text { requirements } \\
\text { stipulated }\end{array}$ \\
\end{tabular} & IEEE 603 & 1991 \\
\hline 3378 & IEEE & IEEE 279 & $N / S$ & $\begin{array}{l}\text { Standard Criteria for Safety } \\
\text { Systems for Nuclear Power } \\
\text { Generating Stations }\end{array}$ & reg1.95.r01 & 1977 & A & $\begin{array}{l}\text { IEEE } 279 \text { also } \\
\text { designated ANSI N42.7 }\end{array}$ & IEEE 603 & 1991 \\
\hline 3379 & IEEE & IEEE 279 & 1971 & $\begin{array}{l}\text { Standard Criteria for Safety } \\
\text { Systems for Nuclear Power } \\
\text { Generating Stations }\end{array}$ & reg1.97.ro3 & 1983 & $\mathbf{P}$ & $\begin{array}{l}\text { IEEE } 279 \text { also } \\
\text { designated ANSI N42.7 }\end{array}$ & IEEE 603 & 1991 \\
\hline
\end{tabular}


Codes and Standards and Other Guidance Cited in Regulatory Documents

Part A - Industry Consensus Codes and Standards (continued)

\begin{tabular}{|c|c|c|c|c|c|c|c|c|c|c|}
\hline Forord & Cods & stondurd & stridard & (1. & boedmont & Doo. & Aopepts & Commente, & Curront Yorolon & ouron \\
\hline 3380 & IEEE & IEEE 279 & $N / S$ & $\begin{array}{l}\text { Standard Criteria for Safety } \\
\text { Systems for Nuclear Power } \\
\text { Generating Stations }\end{array}$ & reg2.2.ro0 & 1973 & A & $\begin{array}{l}\text { IEEE } 279 \text { also } \\
\text { rusignated ANSI N42.7 }\end{array}$ & IEEE 603 & 1991 \\
\hline 3381 & IEEE & IEEE 279 & 1971 & $\begin{array}{l}\text { Standard Criteria for Safety } \\
\text { Systems for Nuclear Power } \\
\text { Generating Stations }\end{array}$ & reg5.30.r00 & 1974 & $\bar{R}$ & $\begin{array}{l}\text { IEEE } 279 \text { also } \\
\text { designated ANSI N42.7 }\end{array}$ & IEEE 603 & 1991 \\
\hline 3382 & IEEE & IEEE 279 & $\mathrm{~N} / \mathrm{S}$ & $\begin{array}{l}\text { Standard Criteria for Safety } \\
\text { Systems for Nuclear Power } \\
\text { Generating Stations }\end{array}$ & $\operatorname{sip} 3.11$ & 1981 & $\mathbf{R}$ & $\begin{array}{l}\text { IEEE } 279 \text { also } \\
\text { designated ANSI N42.7 }\end{array}$ & IEEE 603 & 1991 \\
\hline 3383 & IEEE & IEEE 279 & N/S & $\begin{array}{l}\text { Standard Criteria for Safety } \\
\text { Systems for Nuclear Power } \\
\text { Generating Stations }\end{array}$ & sip3.6.1 & 1990 & $\mathbf{R}$ & \begin{tabular}{|l|} 
IEEE 279 also \\
designated ANSI N42.7
\end{tabular} & IEEE 603 & 1991 \\
\hline 3384 & IEEE & IEEE 279 & $N / S$ & $\begin{array}{l}\text { Standard Criteria for Safety } \\
\text { Systems for Nuclear Power } \\
\text { Generating Stations }\end{array}$ & srp5.2.2 & 1988 & $P$ & $\begin{array}{l}\text { IEEE } 279 \text { also } \\
\text { designated ANSI N42.7 }\end{array}$ & IEEE 603 & 1991 \\
\hline 3385 & IEEE & IEEE 279 & $N / S$ & $\begin{array}{l}\text { Standard Criteria for Safety } \\
\text { Systems for Nuclear Power } \\
\text { Generating Stations }\end{array}$ & $\operatorname{sip} 7.1$ & 1984 & $\bar{A}$ & & IEEE 603 & 1991 \\
\hline 3386 & IEEE & IEEE 279 & $N / S$ & $\begin{array}{l}\text { Standard Criteria for Safety } \\
\text { Systems for Nuclear Power } \\
\text { Generating Stations }\end{array}$ & $\operatorname{sip} 7.2$ & 1981 & $\bar{A}$ & \begin{tabular}{|l|} 
IEEE 279 also \\
designated ANSI N42.7
\end{tabular} & IEEE 603 & 1991 \\
\hline 3387 & IEEE & IEEE 279 & $\mathrm{~N} / \mathrm{S}$ & $\begin{array}{l}\text { Standard Criteria for Safety } \\
\text { Systems for Nuclear Power } \\
\text { Generating Stations }\end{array}$ & srp7.3 & 1981 & $\bar{A}$ & \begin{tabular}{|l|} 
IEEE 279 also \\
designated ANSI N42.7
\end{tabular} & IEEE 603 & 1991 \\
\hline 3388 & IEEE & IEEE 279 & N/S & $\begin{array}{l}\text { Standard Criteria for Safety } \\
\text { Systems for Nuclear Power } \\
\text { Generating Stations }\end{array}$ & $\operatorname{sip7.6}$ & 1981 & $\mathbf{P}$ & $\begin{array}{l}\text { IEEE } 279 \text { also } \\
\text { designated ANSI N42.7 }\end{array}$ & IEEE 603 & 1991 \\
\hline 3389 & IEEE & IEEE 279 & $\mathrm{~N} / \mathrm{S}$ & $\begin{array}{l}\text { Standard Criteria for Safety } \\
\text { Systems for Nuclear Power } \\
\text { Generating Stations }\end{array}$ & $\operatorname{sip} 7.7$ & 1984 & $P$ & \begin{tabular}{|l|} 
IEEE 279 also \\
designated ANSI N42.7
\end{tabular} & IEEE 603 & 1991 \\
\hline 3390 & IEEE & IEEE 279 & 1971 & $\begin{array}{l}\text { Standard Criteria for Safety } \\
\text { Systems for Nuclear Power } \\
\text { Generating Stations }\end{array}$ & sip8A & 1981 & $\bar{P}$ & $\begin{array}{l}\text { IEEE } 279 \text { also } \\
\text { designated ANSI N42.7 }\end{array}$ & IEEE 603 & 1991 \\
\hline
\end{tabular}

enerating Stations 
Codes and Standards and Other Guidance Cited in Regulatory Dccuments

Part A - Industry Consensus Codes and Standards (continued)

\begin{tabular}{|c|c|c|c|c|c|c|c|c|c|c|}
\hline $\begin{array}{l}\text { Rocord } \\
10 .\end{array}$ & Code & Stundard & $\begin{array}{l}\text { Standard } \\
\text { Verolon }\end{array}$ & V. & Dooument & Doc. & Aooept & Comments, & Current Vorrolon & $\begin{array}{l}\text { ourtent } \\
\text { votuph } \\
\text { pollo }\end{array}$ \\
\hline 3391 & IEEE & IEEE 279 & $\mathrm{~N} / \mathrm{S}$ & $\begin{array}{l}\text { Standard Criteria for Safety } \\
\text { Systems for Nuclear Power } \\
\text { Generating Stations }\end{array}$ & srp9.2.2 & 1986 & $P$ & $\begin{array}{l}\text { IEEE } 279 \text { also } \\
\text { designated ANSI N42.7 }\end{array}$ & IEEE 603 & 1991 \\
\hline 3392 & IEEE & IEEE 279 & 1971 & $\begin{array}{l}\text { Standard Criteria for Safety } \\
\text { Systems for Nuclear Power } \\
\text { Generating Stations }\end{array}$ & stsb\&wB3.3.11 & 1991 & $\mathbf{R}$ & & IEEE 603 & 1991 \\
\hline 3393 & IEEE & IEEE 279 & 1971 & $\begin{array}{l}\text { Standard Criteria for Safety } \\
\text { Systems for Nuclear Power } \\
\text { Generating Stations }\end{array}$ & stsb\&wB3.3.12 & 1991 & $\bar{R}$ & & IEEE 603 & 1991 \\
\hline 3394 & IEEE & IEEE 279 & 1971 & $\begin{array}{l}\text { Standard Criteria for Safety } \\
\text { Systems for Nuclear Power } \\
\text { Generating Stations }\end{array}$ & stsb\&wB3.3.8 & 1991 & $\mathbf{R}$ & & IEEE 603 & 1991 \\
\hline 3395 & IEEE & IEEE 279 & 1971 & $\begin{array}{l}\text { Standard Criteria for Safety } \\
\text { Systems for Nuclear Power } \\
\text { Generating Stations }\end{array}$ & stsb\&wB3.5.1 & 1991 & $\mathbf{P}$ & & IEEE 603 & 1991 \\
\hline 3396 & IEEE & IEEE 279 & 1971 & $\begin{array}{l}\text { Standard Criteria for Safety } \\
\text { Systems for Nuclear Power } \\
\text { Generating Stations }\end{array}$ & stsceB3.3.1(A) & 1991 & $\mathbf{P}$ & . & IEEE 603 & 1991 \\
\hline 3397 & IEEE & IEEE 279 & 1971 & $\begin{array}{l}\text { Standard Criteria for Safety } \\
\text { Systems for Nuclear Power } \\
\text { Generating Stations }\end{array}$ & stsceB3.3.1(D) & 1991 & $\mathbf{P}$ & & IEEE 603 & 1991 \\
\hline 3398 & IEEE & IEEE 279 & 1971 & $\begin{array}{l}\text { Standard Criteria for Safety } \\
\text { Systems for Nuclear Power } \\
\text { Generating Stations }\end{array}$ & stsceB3.3.4 & 1991 & $\mathbf{P}$ & & IEEE 603 & 1991 \\
\hline 3399 & IEEE & IEEE 279 & 1971 & $\begin{array}{l}\text { Standard Criteria for Safety } \\
\text { Systems for Nuclear Power } \\
\text { Generating Stations }\end{array}$ & stsceB3.3.5 & 1991 & $\mathbf{P}$ & & IEEE 603 & 1991 \\
\hline 3400 & IEEE & IEEE 279 & 1971 & $\begin{array}{l}\text { Standard Criteria for Safety } \\
\text { Systems for Nuclear Power } \\
\text { Generating Stations }\end{array}$ & stsceB3.3.6 & 1991 & $\mathbf{P}$ & & IEEE 603 & 1991 \\
\hline 3401 & IEEE & IEEE 279 & 1971 & $\begin{array}{l}\text { Standard Criteria for Safety } \\
\text { Systems for Nuclear Power } \\
\text { Generating Stations }\end{array}$ & stsceB3.3.7 & 1991 & $P$ & & IEEE 603 & 1991 \\
\hline
\end{tabular}




\begin{tabular}{|c|c|c|c|c|c|c|c|c|c|c|}
\hline & & & Code & $\begin{array}{l}\text { es and Standards and Other G } \\
\text { Part A - Industry Consensus }\end{array}$ & $\begin{array}{l}\text { iuidance } \mathrm{Cl} \\
\text { Codes and }\end{array}$ & $\begin{array}{l}\text { Regule } \\
\text { ards ( }\end{array}$ & $\begin{array}{l}\text { tory Do } \\
\text { ontinue }\end{array}$ & $\begin{array}{l}\text { cuments } \\
\text { d) }\end{array}$ & & \\
\hline $\begin{array}{l}\text { Pocord } \\
\text { No. }\end{array}$ & Code & Standard & $\begin{array}{l}\text { Standard } \\
\text { Vordon. }\end{array}$ & nue & Document & $\begin{array}{l}\text { Doc. } \\
\text { Date }\end{array}$ & $\begin{array}{c}\text { Acceppt } \\
\text { abilly, }\end{array}$ & Comments: & Curront Version & Vorron \\
\hline 3402 & IEEE & IEEE 279 & 1971 & $\begin{array}{l}\text { Standard Criteria for Safety } \\
\text { Systems for Nuclear Power } \\
\text { Generating Stations } \\
\end{array}$ & stsceB3.5.1 & 1991 & $P$ & & IEEE 603 & 1991 \\
\hline 3403 & IEEE & IEEE 279 & 1971 & $\begin{array}{l}\text { Standard Criteria for Safety } \\
\text { Systems for Nuclear Power } \\
\text { Generating Stations }\end{array}$ & stswstB3.3.1 & 1991 & $\bar{P}$ & & IEEE 603 & 1991 \\
\hline 3404 & IEEE & IEEE 279 & 1971 & $\begin{array}{l}\text { Standard Criteria for Safety } \\
\text { Systems for Nuclear Power } \\
\text { Generating Stations } \\
\end{array}$ & stswstB3.3.2 & 1991 & $\mathbf{P}$ & & IEEE 603 & 1991 \\
\hline 3405 & IEEE & IEEE 279 & 1971 & $\begin{array}{l}\text { Standard Criteria for Safety } \\
\text { Systems for Nuclear Power } \\
\text { Generating Stations }\end{array}$ & stswstB3.5.1 & 1991 & $\bar{P}$ & & IEEE 603 & 1991 \\
\hline 3406 & IEEE & IEEE 301 & $N / S$ & $\begin{array}{l}\text { Standard Test Procedures for } \\
\text { Amplifiers and Pre-Amplifiers Used } \\
\text { With Detectors for lonizing } \\
\text { Radiation }\end{array}$ & reg5.9.102 & 1983 & $\bar{R}$ & & IEEE 301 & 1988 \\
\hline 3407 & IEEE & IEEE 308 & 1974 & $\begin{array}{l}\text { Standard Criteria for Class IE Power } \\
\text { Systems for Nuclear Power } \\
\text { Generating Stations }\end{array}$ & $\operatorname{drg} 1021.100$ & 1992 & $\mathbf{R}$ & Proposed reg1.9.ro3 & |IEEE 308 & 1991 \\
\hline 3408 & IEEE & IEEE 308 & 1974 & $\begin{array}{l}\text { Standard Criteria for Class IE Power } \\
\text { Systems for Nuclear Power } \\
\text { Generating Stations }\end{array}$ & drgrs802-5 & 1988 & $\mathbf{P}$ & Proposed reg1.9.ro3 & IEEE 308 & 1991 \\
\hline 3409 & IEEE & IEEE 308 & $\mathrm{~N} / \mathrm{S}$ & $\begin{array}{l}\text { Standard Criteria for Class IE Power } \\
\text { Systems for Nuclear Power } \\
\text { Generating Stations }\end{array}$ & inm51051 & 1984 & $\mathbf{R}$ & & IEEE 308 & 1991 \\
\hline 3410 & IEEE & IEEE 308 & $\mathrm{~N} / \mathrm{S}$ & $\begin{array}{l}\text { Standard Criteria for Class IE Power } \\
\text { Systems for Nuclear Power } \\
\text { Generating Stations }\end{array}$ & inm51053 & 1984 & $\mathbf{R}$ & & IEEE 308 & 1991 \\
\hline 3411 & IEEE & IEEE 308 & $N / S$ & $\begin{array}{l}\text { Standard Criteria for Class IE Power } \\
\text { Systems for Nuclear Power } \\
\text { Generating Stations }\end{array}$ & inm51061 & 1984 & $\bar{R}$ & & IEEE 308 & 1991 \\
\hline 3412 & IEEE & IEEE 308 & $\mathrm{~N} / \mathrm{S}$ & $\begin{array}{l}\text { Standard Criteria for Class IE Power } \\
\text { Systems for Nuclear Power } \\
\text { Generating Stations }\end{array}$ & inm51063 & 1985 & $\bar{R}$ & & IEEE 308 & 1991 \\
\hline
\end{tabular}




\section{Codes and Standards and Other Guidance Cited in Regulatory Documents}

Part A - Industry Consensus Codes and Standards (continued)

\begin{tabular}{|c|c|c|c|c|c|c|c|c|c|c|}
\hline $\begin{array}{l}\text { Record } \\
\text { No. }\end{array}$ & Code & Standard & $\begin{array}{l}\text { Standard } \\
\text { Verton }\end{array}$ & ( & Document & $\begin{array}{l}\text { Doo. } \\
\text { Dote }\end{array}$ & $\begin{array}{l}\text { Aocept: } \\
\text { Ablitity }\end{array}$ & Comments & Curront Vordon & $\begin{array}{l}\text { cortont } \\
\text { vorotont }\end{array}$ \\
\hline 3413 & IEEE & IEEE 308 & $N / S$ & $\begin{array}{l}\text { Standard Criteria for Class IE Power } \\
\text { Systems for Nuclear Power } \\
\text { Generating Stations }\end{array}$ & inm51065 & 1984 & R & & IEEE 308 & 1991 \\
\hline 3414 & IEEE & IEEE 308 & $\mathrm{~N} / \mathrm{S}$ & $\begin{array}{l}\text { Standard Criteria for Class IE Power } \\
\text { Systems for Nuclear Power } \\
\text { Generating Stations }\end{array}$ & inm52051 & 1984 & $\mathbf{R}$ & & IEEE 308 & 1991 \\
\hline 3415 & IEEE & IEEE 308 & $\mathrm{~N} / \mathrm{S}$ & $\begin{array}{l}\text { Standard Criteria for Class IE Power } \\
\text { Systems for Nuclear Power } \\
\text { Generating Stations }\end{array}$ & inm52055 & 1984 & $\mathbf{R}$ & & IEEE 308 & 1991 \\
\hline 3416 & IEEE & IEEE 308 & $\mathrm{~N} / \mathrm{S}$ & $\begin{array}{l}\text { Standard Criteria for Class IE Power } \\
\text { Systems for Nuclear Power } \\
\text { Generating Stations }\end{array}$ & inm70340B & 1981 & $\mathbf{R}$ & & IEEE 308 & 1991 \\
\hline 3417 & IEEE & IEEE 308 & 1974 & $\begin{array}{l}\text { Standard Criteria for Class IE Power } \\
\text { Systems for Nuclear Power } \\
\text { Generating Stations }\end{array}$ & not84-02 & 1984 & $\mathbf{R}$ & Cited for definitions & IEEE 308 & 1991 \\
\hline 3418 & IEEE & IEEE 308 & 1974 & $\begin{array}{l}\text { Standard Criteria for Class IE Power } \\
\text { Systems for Nuclear Power } \\
\text { Generating Stations }\end{array}$ & not93-64 & 1993 & $\mathbf{A}$ & & IEEE 308 & 1991 \\
\hline 3419 & IEEE & IEEE 308 & $\mathrm{~N} / \mathrm{S}$ & $\begin{array}{l}\text { Standard Criteria for Class IE Power } \\
\text { Systems for Nuclea: Power } \\
\text { Generating Stations }\end{array}$ & nureg0933, 3.128 & 1991 & $\mathbf{R}$ & & IEEE 308 & 1991 \\
\hline 3420 & IEEE & IEEE 308 & 1974 & $\begin{array}{l}\text { Standard Criteria for Class IE Power } \\
\text { Systems for Nuclear Power } \\
\text { Generating Stations }\end{array}$ & reg1.118.r02 & 1978 & $P$ & Implied endorsement & IEEE 308 & 1991 \\
\hline 3421 & IEEE & IEEE 308 & $\mathrm{~N} / \mathrm{S}$ & $\begin{array}{l}\text { Standard Criteria for Class IE Power } \\
\text { Systems for Nuclear Power } \\
\text { Generating Stations }\end{array}$ & reg1.153.roo & 1985 & $\mathbf{R}$ & & IEEE 308 & 1991 \\
\hline 3422 & IEEE & IEEE 308 & 1971 & $\begin{array}{l}\text { Standard Criteria for Class IE Power } \\
\text { Systems for Nuclear Power } \\
\text { Ganerating Stations }\end{array}$ & reg1.32.r02 & 1977 & $\mathbf{R}$ & & IEEE 308 & 1991 \\
\hline 3423 & IEEE & IEEE 308 & 1974 & $\begin{array}{l}\text { Standard Criteria for Class IE Power } \\
\text { Systems for Nuclear Power } \\
\text { Generating Stations }\end{array}$ & reg1.32.r02 & 1977 & $\mathbf{P}$ & With exceptions & IEEE 308 & 1991 \\
\hline
\end{tabular}




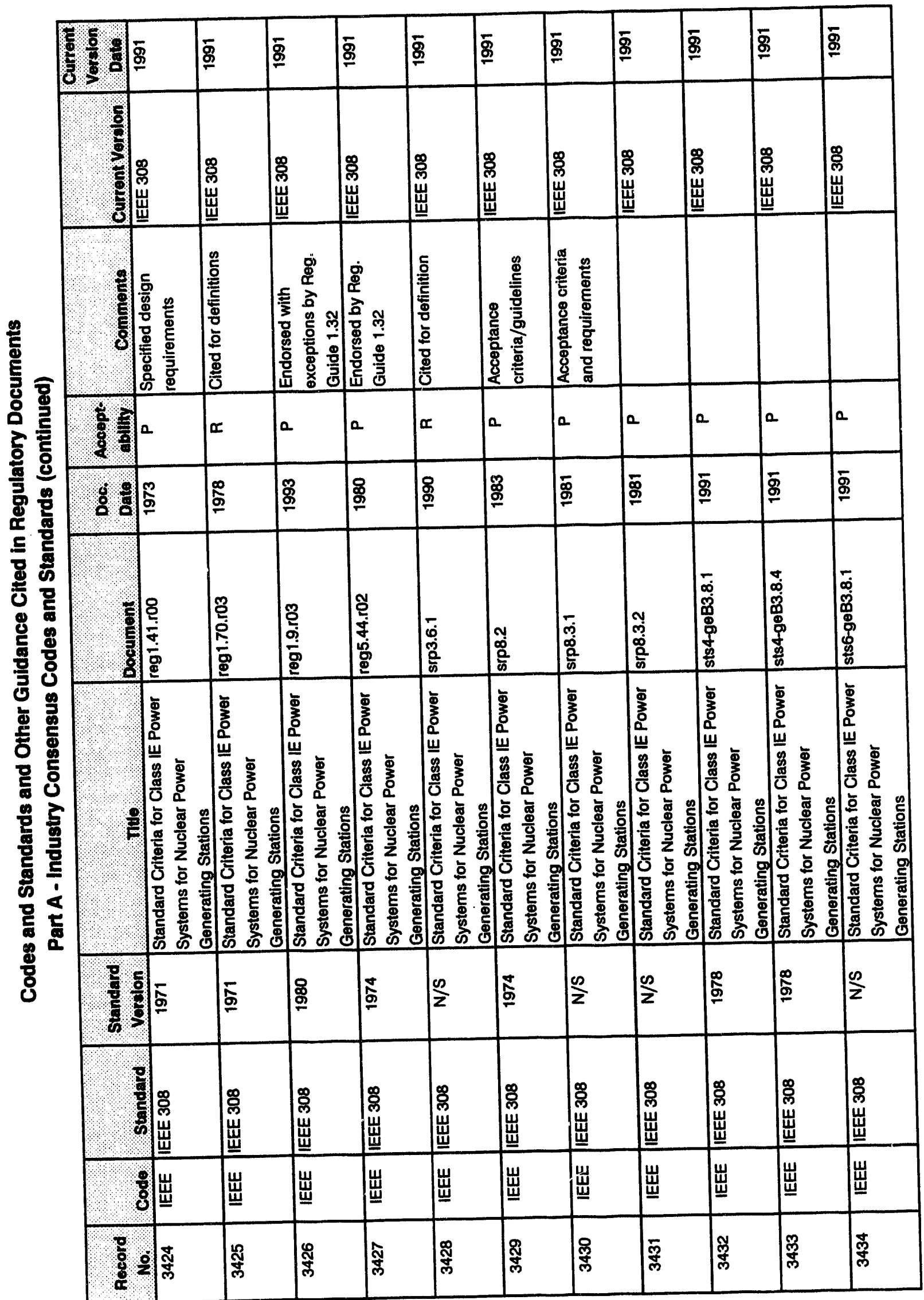


Codes and Standards and Other Guidance Cited in Regulatory Documents

Part A - Industry Consensus Codes and Standards (continued)

\begin{tabular}{|c|c|c|c|c|c|c|c|c|c|c|}
\hline $\begin{array}{l}\text { Record } \\
\text { No. }\end{array}$ & code. & Standard & $\begin{array}{l}\text { Standard } \\
\text { Vorelon }\end{array}$ & (ै। & Dooument & $\begin{array}{l}\text { Doo. } \\
\text { Date }\end{array}$ & Accept & Comments & Current Vorsion & $\begin{array}{l}\text { current } \\
\text { Verton }\end{array}$ \\
\hline 3435 & IEEE & IEEE 308 & 1978 & $\begin{array}{l}\text { Standard Criteria for Class IE Power } \\
\text { Systems for Nuclear Power } \\
\text { Generating Stations }\end{array}$ & sts6-geB3.8.4 & 1991 & $\mathbf{P}$ & & IEEE 308 & 1991 \\
\hline 3436 & IEEE & IEEE 308 & $\mathrm{~N} / \mathrm{S}$ & $\begin{array}{l}\text { Standard Criteria for Class IE Power } \\
\text { Systems for Nuclear Power } \\
\text { Generating Stations }\end{array}$ & stsb\&wB3.8.1 & 1991 & $\bar{R}$ & & IEEE 308 & 1991 \\
\hline 3437 & IEEE & IEEE 308 & 1978 & $\begin{array}{l}\text { Standard Criteria for Class IE Power } \\
\text { Systems for Nuclear Power } \\
\text { Generating Stations }\end{array}$ & stsb\&wB3.8.4 & 1991 & $\overline{\mathbf{P}}$ & & IEEE 308 & 1991 \\
\hline 3438 & IEEE & IEEE 308 & 1978 & $\begin{array}{l}\text { Standard Criteria for Class IE Power } \\
\text { Systems for Nuclear Power } \\
\text { Generating Stations }\end{array}$ & stsceB3.8.1 & 1991 & $\mathbf{P}$ & & IEEE 308 & 1991 \\
\hline 3439 & IEEE & IEEE 308 & 1578 & $\begin{array}{l}\text { Standard Criteria for Class IE Power } \\
\text { Systems for Nuclear Power } \\
\text { Generating Stations }\end{array}$ & stsceB3.8.4 & 1991 & $\mathbf{P}$ & & IEEE 308 & 1991 \\
\hline 3440 & IEEE & IEEE 308 & 1978 & $\begin{array}{l}\text { Stanciard Criteria for Class IE Power } \\
\text { Systems for Nuclear Power } \\
\text { Generating Stations }\end{array}$ & stswstB3.8.1 & 1991 & $\mathbf{P}$ & & IEEE 308 & 1991 \\
\hline 3441 & IEEE & IEEE 308 & 1978 & $\begin{array}{l}\text { Standard Criteria for Class IE Power } \\
\text { Systems for Nuclear Power } \\
\text { Generating Stations }\end{array}$ & stswstB3.8.4 & 1991 & $P$ & . & IEEE 308 & 1991 \\
\hline 3442 & IEEE & IEEE 317 & $N / S$ & $\begin{array}{l}\text { Standard for Electric Penetration } \\
\text { Assemblies in Containment } \\
\text { Structures for Nuclear Power } \\
\text { Generating Stations }\end{array}$ & inm51051 & 1984 & R & & IEEE 317 & $1983 \mathrm{R} 92$ \\
\hline 3443 & IEEE & IEEE 317 & $N / S$ & $\begin{array}{l}\text { Standard for Electric Penetration } \\
\text { Assemblies in Containment } \\
\text { Structures for Nuclear Power } \\
\text { Generating Stations }\end{array}$ & inm51053 & 1984 & $R$ & & IEEE 317 & $1983 \mathrm{R} 92$ \\
\hline 3444 & IEEE & IEEE 317 & $\mathrm{~N} / \mathrm{S}$ & $\begin{array}{l}\text { Standard for Electric Penetration } \\
\text { Assemblies in Containment } \\
\text { Structures for Nuclear Power } \\
\text { Generating Stations }\end{array}$ & inm51055 & 1984 & $\mathbf{R}$ & & IEEE 317 & $1983 \mathrm{Rg} 2$ \\
\hline
\end{tabular}


Codes and Standards and Other Guidance Cited in Regulatory Documents

Part A - Industry Consensus Codes and Standards (continued)

\begin{tabular}{|c|c|c|c|c|c|c|c|c|c|c|}
\hline & & & & Pan A - Industry Consensu & . & & & & & \\
\hline $\begin{array}{l}\text { Recond } \\
\text { No. }\end{array}$ & Codo: & Standard & $\begin{array}{l}\text { Sundard } \\
\text { Verolon }\end{array}$ & (.) & Document & $\begin{array}{l}\text { Dor. } \\
\text { Date }\end{array}$ & $\begin{array}{l}\text { Aocepot } \\
\text { ablithy }\end{array}$ & Comments & Current Vordon & $\begin{array}{l}\text { Curtent } \\
\text { Verolon }\end{array}$ \\
\hline$\frac{\text { No. }}{3445}$ & $\frac{\operatorname{Codo}}{\text { IEEE }}$ & IEEE 317 & $\frac{N / S}{N}$ & $\begin{array}{l}\text { Standard for Electric Penetration } \\
\text { Assemblies in Containment } \\
\text { Structures for Nuclear Power } \\
\text { Generating Stations }\end{array}$ & inm52051 & 1984 & R & & IEEE 317 & 1983 R92 \\
\hline 3446 & IEEE & IEEE 317 & $N / S$ & $\begin{array}{l}\text { Standard for Electric Penetration } \\
\text { Assemblies in Containment } \\
\text { Struc } \quad \text { Nuclear Power } \\
\text { Generain. } \\
\end{array}$ & inm52053 & 1984 & $\mathbf{R}$ & & IEEE 317 & $1983 \mathrm{Rg2}$ \\
\hline 3447 & IEEE & IEEE 317 & $\mathrm{~N} / \mathrm{S}$ & $\begin{array}{l}\text { Standard . Electric Penetration } \\
\text { Assembli } 3 \text { in Containment } \\
\text { Stuuctures for Nuclear Power } \\
\text { Generating Stations }\end{array}$ & inm52055 & 1984 & $\mathbf{R}$ & & IEEE 317 & $1983 \mathrm{R92}$ \\
\hline 3448 & IEEE & IEEE 317 & 1976 & $\begin{array}{l}\text { Standard ror Electric Penetration } \\
\text { Assemblies in Containment } \\
\text { Structures for Nuclear Power } \\
\text { Generating Stations }\end{array}$ & nureg0933, 2.B-9 & 1983 & $\mathbf{R}$ & & IEEE 317 & $1983 \mathrm{R92}$ \\
\hline 3449 & IEEE & IEEE 317 & 1983 & $\begin{array}{l}\text { Standard for Electric Penetration } \\
\text { Assemblies in Containment } \\
\text { Structures for Nuclear Power } \\
\text { Generating Stations }\end{array}$ & reg1.63.r03 & 1987 & $\mathbf{P}$ & $\begin{array}{l}\text { Provides a method } \\
\text { acceptable to the NRC }\end{array}$ & IEEE 317 & $1983 \mathrm{R92}$ \\
\hline 3450 & IEEE & IEEE 317 & 1976 & $\begin{array}{l}\text { Standard for Electric Penetration } \\
\text { Assemblies in Containment } \\
\text { Structures for Nuclear Power } \\
\text { Generating Stations }\end{array}$ & reg1.63.r03 & 1987 & $\mathbf{P}$ & $\begin{array}{l}\text { Reg. Guide 1.63 Rev } 2 \\
\text { endorsed 317-1976 } \\
\text { with seven except }\end{array}$ & IEEE 317 & $1983 \mathrm{R92}$ \\
\hline 3451 & IEEE & IEEE 317 & $\mathrm{~N} / \mathrm{S}$ & $\begin{array}{l}\text { Standard for Electric Penetration } \\
\text { Assemblies in Containment } \\
\text { Structures for Nuclear Power } \\
\text { Generating Stations }\end{array}$ & $\operatorname{sip3.11}$ & 1981 & $\bar{P}$ & $\begin{array}{l}\text { Augmented by Reg. } \\
\text { Guide } 1.63 \text { and } \\
\text { NUREG-0588 }\end{array}$ & IEEE 317 & $1983 \mathrm{R92}$ \\
\hline 3452 & IEEE & IEEE 317 & $N / S$ & $\begin{array}{l}\text { Standard for Electric Penetration } \\
\text { Assemblies in Containment } \\
\text { Structures for Nuclear Power } \\
\text { Generating Stations }\end{array}$ & srp8.1 & 1981 & $\mathbf{R}$ & Table 8-1 & IEEE 317 & $1983 \mathrm{R92}$ \\
\hline
\end{tabular}


Codes and Standards and Other Guidance Cited in Regulatory Documents

Part A - Industry Consensus Codes and Standards (continued)

\begin{tabular}{|c|c|c|c|c|c|c|c|c|c|c|}
\hline $\begin{array}{l}\text { Rocord } \\
\text { No. }\end{array}$ & Code & Standard & $\begin{array}{l}\text { Standard } \\
\text { Vorroton }\end{array}$ & Tos & Bocumont & $\begin{array}{l}\text { Doo. } \\
\text { Date. }\end{array}$ & $\begin{array}{l}\text { Acoept } \\
\text { abliliy }\end{array}$ & Commonts & Current Vorslon & corrof \\
\hline 3453 & IEEE & IEEE 317 & N/S & $\begin{array}{l}\text { Standard for Electric Penetration } \\
\text { Assemblies in Containment } \\
\text { Structures for Nuclear Power } \\
\text { Generating Stations }\end{array}$ & srp8.3.1 & 1981 & $\mathbf{P}$ & $\begin{array}{l}\text { Acceptance } \\
\text { criterion/guidelines }\end{array}$ & IEEE 317 & $1983 \mathrm{R} 92$ \\
\hline 3454 & IEEE & IEEE 317 & $N / S$ & $\begin{array}{l}\text { Standard for Electric Penetration } \\
\text { Assemblies in Containment } \\
\text { Structures for Nuclear Power } \\
\text { Generating Stations }\end{array}$ & srp8.3.2 & 1981 & $\mathbf{P}$ & & IEEE 317 & 1983 R92 \\
\hline 3455 & IEEE & IEEE 323 & 1974 & $\begin{array}{l}\text { Standard for Qualifying Class IE } \\
\text { Equipment for Nuclear Power } \\
\text { Generating Stations }\end{array}$ & $10 \operatorname{cfr} 50.49$ & 1993 & $R$ & & IEEE 323 & 1983 R90 \\
\hline 3456 & IEEE & IEEE 323 & 1974 & $\begin{array}{l}\text { Standard for Qualifying Class IE } \\
\text { Equipment for Nuclear Power } \\
\text { Generating Stations }\end{array}$ & bul77-05 & 1977 & $\mathbf{R}$ & Referred to in text & IEEE 323 & 1983 R90 \\
\hline 3457 & IEEE & IEEE 323 & 1971 & $\begin{array}{l}\text { Standard for Qualifying Class IE } \\
\text { Equipment for Nuclear Power } \\
\text { Generating Stations }\end{array}$ & bul79-01B & 1980 & $R$ & Referenced in text & IEEE 323 & $1983 \mathrm{R} 90$ \\
\hline 3458 & IEEE & IEEE 323 & 1974 & $\begin{array}{l}\text { Standard for Qualifying Class IE } \\
\text { Equipment for Nuclear Power } \\
\text { Generating Stations }\end{array}$ & bul79-01B & 1980 & $\mathbf{P}$ & $\begin{array}{l}\text { Cited as a current } \\
\text { industry standard }\end{array}$ & IEEE 323 & $1983 \mathrm{R} 90$ \\
\hline 3459 & IEEE & IEEE 323 & 1974 & $\begin{array}{l}\text { Standard for Qualifying Class IE } \\
\text { Equipment for Nuclear Power } \\
\text { Generating Stations }\end{array}$ & bul79-01b.s01 & 1980 & $\mathbf{P}$ & $\begin{array}{l}\text { Approved } \\
\text { requirements for } \\
\text { equipment }\end{array}$ & IEEE 323 & $1983 \mathrm{R} 90$ \\
\hline 3460 & IEEE & IEEE 323 & 1971 & $\begin{array}{l}\text { Standard for Qualifying Class IE } \\
\text { Equipment for Nuclear Power } \\
\text { Generating Stations }\end{array}$ & bui79-01b.s02 & 1980 & $R$ & Referenced in text & IEEE 323 & 1983 R90 \\
\hline 3461 & IEEE & IEEE 323 & 1974 & $\begin{array}{l}\text { Standard for Qualifying Class IE } \\
\text { Equipment for Nuclear Power } \\
\text { Generating Stations }\end{array}$ & bul79-01b.s02 & 1980 & $\mathbf{P}$ & $\begin{array}{l}\text { Licensees reviewed } \\
\text { against IEEE 323-1974 }\end{array}$ & IEEE 323 & 1983 R90 \\
\hline 3462 & IEEE & IEEE 323 & 1974 & $\begin{array}{l}\text { Standard for Qualifying Class IE } \\
\text { Equipment for Nuclear Power } \\
\text { Generating Stations }\end{array}$ & bul80-23 & 1980 & $\mathbf{P}$ & $\begin{array}{l}\text { Standard to be used } \\
\text { per applicable } \\
\text { requirements }\end{array}$ & IEEE 323 & 1983 R90 \\
\hline
\end{tabular}




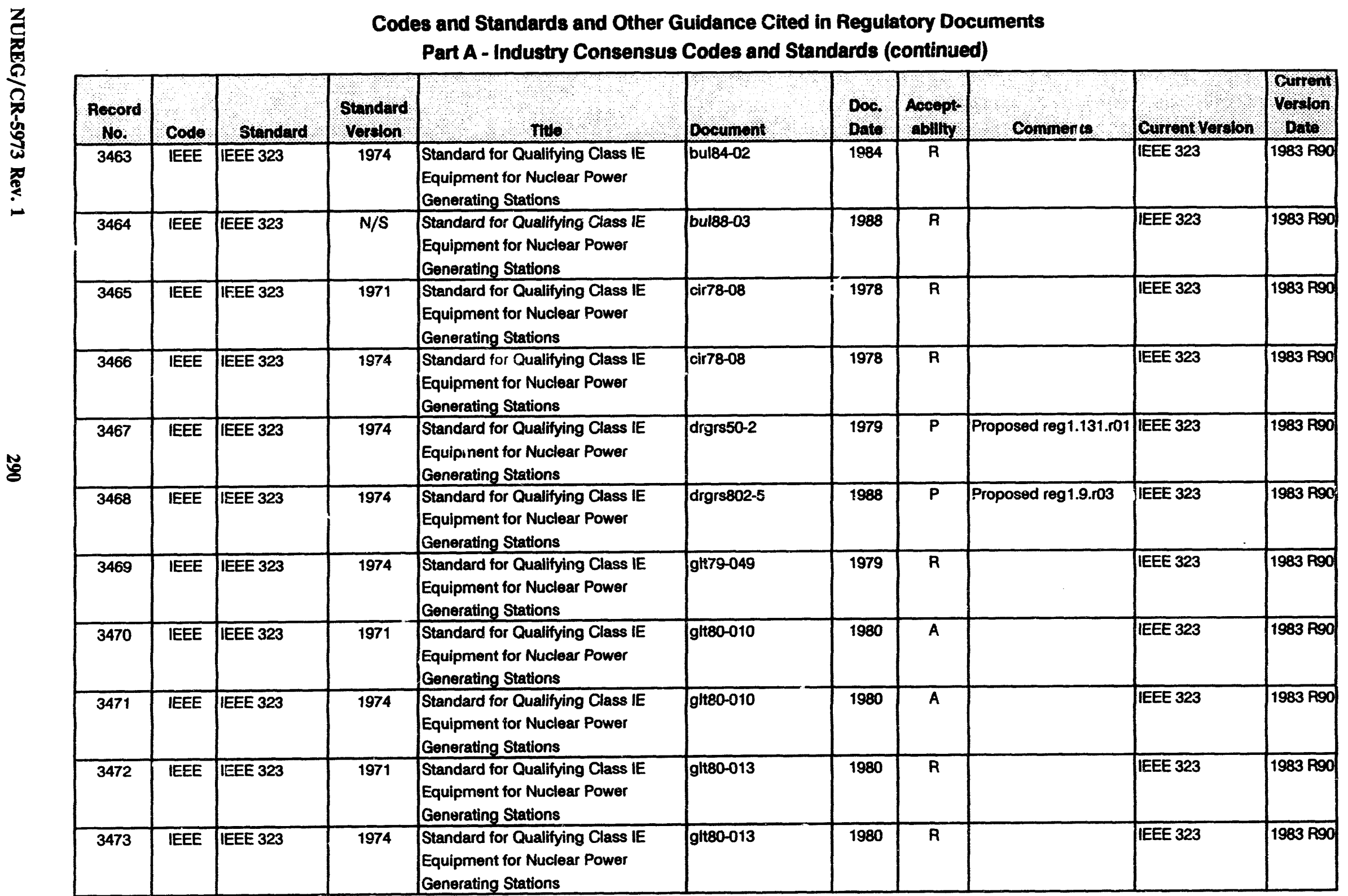




\section{Codes and Standards and Other Guidance Cited in Regulatory Documents}

Part A - Industry Consensus Codes and Standards (continued)

\begin{tabular}{|c|c|c|c|c|c|c|c|c|c|c|}
\hline $\begin{array}{l}\text { Rocord } \\
\text { No. }\end{array}$ & codo & Stondord & standord & \} $&{\text { Dooument }} &{000} &{\text { 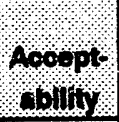 }} &{\text { Comments }} &{\text { curcont voroton }} &{\text { Q10 }} \\
{\hline 3474} &{\text { IEEE }} &{\text { IEEE } 323} &{1974} &{\begin{array}{l}\text { Standard for Qualifying Class IE } \\
\text { Equipment for Nuclear Power } \\
\text { Generating Stations }\end{array}} &{\text { glt80-097 }} &{1980} &{\mathbf{R}} &{ } &{\text { IEEE 325 }} &{1983 \mathrm{R} 90} \\
{\hline 3475} &{\text { IEEE }} &{\text { IEEE } 323} &{1974} &{\begin{array}{l}\text { Standard for Qualitying Class IE } \\
\text { Equipment for Nuclear Power } \\
\text { Generating :suitions }\end{array}} &{\text { glt82-009 }} &{1982} &{\mathbf{P}} &{\begin{array}{l}\text { Requirement for } \\
\text { equipment }\end{array}} &{\text { IEEE } 323} &{1983 \text { R90 }} \\
{\hline 3476} &{\text { IEEE }} &{\text { IEEE } 323} &{1971} &{\begin{array}{l}\text { Standard fi-i Qualifying Class IE } \\
\text { Equipment for Nuclear Power } \\
\text { Generating Stations }\end{array}} &{\text { glt89-021 }} &{1989} &{\mathbf{R}} &{\text { Referenced in text }} &{\text { IEEE } 323} &{1983 \text { R90 }} \\
{\hline 3477} &{\text { IEEE }} &{\text { IEEE } 323} &{1974} &{\begin{array}{l}\text { Standard for Quallfying Class IE } \\
\text { Equipment for Nuclear Power } \\
\text { Generating Stations }\end{array}} &{\text { git89-021 }} &{1989} &{\mathbf{R}} &{\text { Referenced in text }} &{\text { IEEE } 323} &{1983 \text { R90 }} \\
{\hline 3478} &{\text { IEEE }} &{\text { IEEE } 323} &{1974} &{\begin{array}{l}\text { Standará for Qualifying Class IE } \\
\text { Equipment for Nuclear Power } \\
\text { Generating Stations }\end{array}} &{\text { inm2515/076 }} &{1986} &{\mathbf{R}} &{ } &{\text { IEEE } 323} &{1983 \text { R9O }} \\
{\hline 3479} &{\text { IELEE }} &{\text { HEEE } 323} &{N / S} &{\begin{array}{l}\text { Standard for Qualifying Class 'E } \\
\text { Equipment for Nuclear Power } \\
\text { Generating Stations }\end{array}} &{\text { inm35061 }} &{1985} &{\mathbf{R}} &{ } &{\text { IEEE } 323} &{1983 \text { R90 }} \\
{\hline 3480} &{\text { IEEE }} &{\text { IEEE } 323} &{\mathrm{~N} / \mathrm{S}} &{\begin{array}{l}\text { Standard for Qualifying Class IE } \\
\text { Equipment for Nuclear Power } \\
\text { Generating Stations }\end{array}} &{\text { inm38703 }} &{1993} &{\mathbf{R}} &{ } &{\text { IEEE } 323} &{1983 \text { R9O }} \\
{\hline 3481} &{\text { IEEE }} &{\text { IEEE } 323} &{1974} &{\begin{array}{l}\text { Standard for Qualifying Class IE } \\
\text { Equipment for Nuclear Power } \\
\text { Generating Stations }\end{array}} &{\text { inm50100 }} &{1985} &{\mathbf{R}} &{ } &{\text { IEEE } 323} &{1983 \text { P90 }} \\
{\hline 3482} &{\text { IEEE }} &{\text { IEEE } 323} &{\text { N/S }} &{\begin{array}{l}\text { Standard for Qualifying Class IE } \\
\text { Equipment for Nuclear Power } \\
\text { Generating Stations }\end{array}} &{\text { inm51051 }} &{1984} &{\mathbf{R}} &{ } &{\text { IEEE } 323} &{1983 \text { R90 }} \\
{\hline 3483} &{\text { IEEE }} &{\text { IEEE } 323} &{\mathrm{~N} / \mathrm{S}} &{\begin{array}{l}\text { Standard for Qualifying Class IE } \\
\text { Equipment for Nuclear Power } \\
\text { Generating Stations }\end{array}} &{\text { inm51053 }} &{1984} &{\mathbf{R}} &{ } &{\text { IEEE } 323} &{1983 R 90} \\
{\hline 3484} &{\text { IEEE }} &{\text { IEEE } 323} &{\mathrm{~N} / \mathrm{S}} &{\begin{array}{l}\text { Standard for Qualifying Class IE } \\
\text { Equipment for Nuclear Power } \\
\text { Generating Stations }\end{array}} &{\text { inm51055 }} &{1984} &{\mathbf{R}} &{ } &{\text { IEEE } 323} &{1983 \mathrm{R} 90} \\
$\hline
\end{tabular}


Codes and Standards and Other Guldance Clted in Regulatory Documents

Part A - Industry Consensus Codes and Standards (continued)

\begin{tabular}{|c|c|c|c|c|c|c|c|c|c|c|}
\hline Rocord & Codo & Standard & standard & 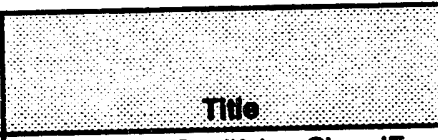 & Booponos & $\begin{array}{l}\text { Doo. } \\
\text { Doto }\end{array}$ & Aooppt & comments & culcont Voroton & owon \\
\hline 3485 & IEEE & IEEE 323 & N/S & $\begin{array}{l}\text { Standard for Qualifying Class IE } \\
\text { Equipment for Nuclear Power } \\
\text { Generating Stations }\end{array}$ & inm5:761 & 1984 & $\mathbf{R}$ & & IEEE 323 & $1983 \mathrm{R} 90$ \\
\hline 3486 & IEEE & IEEE 323 & $\mathrm{~N} / \mathrm{S}$ & $\begin{array}{l}\text { Standard for Qualifying Class IE } \\
\text { Equipment for Nuclear Power } \\
\text { Generating Stations }\end{array}$ & inm51063 & 1985 & $\mathbf{R}$ & & IEEE 323 & 1983 R9O \\
\hline 3487 & IEEE & IEEE 323 & $\mathrm{~N} / \mathrm{S}$ & $\begin{array}{l}\text { Standard for Qualifying Class IE } \\
\text { Equipment for Nuclear Power } \\
\text { Generating Stations }\end{array}$ & inm51065 & 1984 & $\bar{R}$ & & IEEE 323 & 1983 R90 \\
\hline 3488 & IEEE & IEEE 323 & $\mathrm{~N} / \mathrm{S}$ & $\begin{array}{l}\text { Standard for Qualifying Class IE } \\
\text { Equipment for Nuclear Power } \\
\text { Generating Stations }\end{array}$ & inm52051 & 1984 & $\mathbf{R}$ & & IEEE 323 & 1983 R90 \\
\hline 3489 & IEEE & IEEE 323 & N/S & $\begin{array}{l}\text { Standard for Qualifying Class IE } \\
\text { Equipment for Nuclear Power } \\
\text { Generating Stations }\end{array}$ & inm52053 & 1984 & $\overline{\mathbf{R}}$ & & IEEE 323 & 1983 R90 \\
\hline 3490 & IEEE & IEEE 323 & $\mathrm{~N} / \mathrm{S}$ & $\begin{array}{l}\text { Standard for Qualifying Class IE } \\
\text { Equipment for Nuclear Power } \\
\text { Generating Stations }\end{array}$ & inm52055 & 1984 & $\bar{R}$ & & IEEE 323 & 1983 R9O \\
\hline 3491 & IEEE & IEEE 323 & 1971 & $\begin{array}{l}\text { Standard for Qualifying Class IE } \\
\text { Equipment for Nuclear Power } \\
\text { Generating Stations }\end{array}$ & not81-29 & 1981 & $\mathbf{R}$ & $\begin{array}{l}\text { Referenced under } \\
\text { generic implication }\end{array}$ & IEEE 323 & 1983 R90 \\
\hline 3492 & IEEE & IEEE 323 & 1974 & $\begin{array}{l}\text { Standard for Qualifying Class IE } \\
\text { Equipment for Nuclear Power } \\
\text { Generating Stations }\end{array}$ & not81-29 & 1981 & $\bar{R}$ & $\begin{array}{l}\text { Referenced under Test } \\
\text { Summary Report No. } 1\end{array}$ & IEEE 323 & 1983 R90 \\
\hline 3493 & IEEE & IEEE 323 & $\mathrm{~N} / \mathrm{S}$ & $\begin{array}{l}\text { Standard for Qualifying Class IE } \\
\text { Equipment for Nuclear Power } \\
\text { Generating Stations }\end{array}$ & not82-04 & 1982 & $\bar{R}$ & Referenced in text & IEEE 323 & 1983 R90 \\
\hline 3494 & IEEE & IEEE 323 & 1971 & $\begin{array}{l}\text { Standard for Qualifying Class IE } \\
\text { Equipment for Nuclear Power } \\
\text { Generating Stations }\end{array}$ & not82-52 & 1982 & $\mathbf{R}$ & $\begin{array}{l}\text { Referenced under } \\
\text { generic implication }\end{array}$ & IEEE 323 & $1983 \mathrm{RgO}$ \\
\hline 3495 & IEEE & IEEE 323 & 1974 & $\begin{array}{l}\text { Standard for Qualifying Class IE } \\
\text { Equipment for Nuclear Power } \\
\text { Generating Stations }\end{array}$ & not82-52 & 1982 & $\mathbf{R}$ & $\begin{array}{l}\text { Referenced in test } \\
\text { report section }\end{array}$ & IEEE 323 & 1983 R90 \\
\hline
\end{tabular}


Codes and Standards and Other Guidance Clted in Regulatory Documents

Part A - Industry Consensus Codes and Standards (continued)

\begin{tabular}{|c|c|c|c|c|c|c|c|c|c|c|}
\hline Hocord & $80 d 0$ & stondard & Standard & mino & bocument & Doo: & Aocept: & commonts & 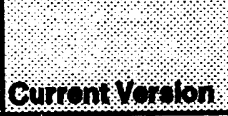 & owoly \\
\hline 3496 & IEEE & IEEE 323 & $N / S$ & $\begin{array}{l}\text { Standard for Qualifying Class IE } \\
\text { Equipment for Nuclear Power } \\
\text { Generating Stations }\end{array}$ & not83-72 & 1983 & $\mathbf{R}$ & $\begin{array}{l}\text { Referred to in types of } \\
\text { test }\end{array}$ & IEEE 323 & $1983 \mathrm{R} 90$ \\
\hline 3497 & IEEE & IEEE 323 & 1974 & $\begin{array}{l}\text { Standard for Qualifying Class IE } \\
\text { Equipment for Nuclear Power } \\
\text { Generating Stations }\end{array}$ & not84-23 & 1984 & $\bar{R}$ & \begin{tabular}{|l|} 
Test program \\
patterned after 323 \\
(Referenced) \\
\end{tabular} & IEEE 323 & 1983 RPO \\
\hline 3498 & IEEE & IEEE 323 & 1974 & $\begin{array}{l}\text { Standard for Qualifying Class IE } \\
\text { Equipment for Nuclear Power } \\
\text { Generating Stations } \\
\end{array}$ & not84-47 & 1984 & F & $\begin{array}{l}\text { Roferences test } \\
\text { conditions in Appendix } \\
\text { A of IEEE } 323\end{array}$ & IEEE 323 & $1983 \mathrm{R} 90$ \\
\hline 3499 & IEEE & IEEE 323 & 1971 & $\begin{array}{l}\text { Standard for Qualifying Class IE } \\
\text { Equipment for Nuclear Power } \\
\text { Generating Stations }\end{array}$ & not85-39 & 1985 & $\mathbf{R}$ & $\begin{array}{l}\text { Defines auditable data } \\
\text { for EQ }\end{array}$ & IEEE 323 & 1983 R90 \\
\hline 3500 & IEEE & IEEE 323 & 1974 & $\begin{array}{l}\text { Standard for Quallifying Class IE } \\
\text { Equipment for Nuclear Power } \\
\text { Generating Stations }\end{array}$ & not85-39 & 1985 & $\mathbf{R}$ & $\begin{array}{l}\text { Defines auditable data } \\
\text { for EQ }\end{array}$ & IEEE 323 & 1983 R9O \\
\hline 3501 & IEEE & IECEE 323 & 1974 & $\begin{array}{l}\text { Standard for Qualifying Class IE } \\
\text { Equipment for Nuclear Power } \\
\text { Generating Stations }\end{array}$ & not85-40 & 1985 & $\mathbf{P}$ & $\begin{array}{l}\text { Reg. Guide } 1.89 \\
\text { endorses IEEE 323- } \\
1974 \\
\end{array}$ & IEEE 323 & 1983 R90 \\
\hline 3502 & IEEE & IEEE 323 & 1983 & $\begin{array}{l}\text { Standard for Qualifying Class IE } \\
\text { Equipment for Nuclear Power } \\
\text { Generating Stations }\end{array}$ & not85-40 & 1985 & $\mathbf{R}$ & Referenced in text & IEEE 323 & 1983 PLO \\
\hline 3503 & IEEE & IEEE 323 & 1974 & $\begin{array}{l}\text { Standard for Qualifying Class IE } \\
\text { Equipment for Nuclear Power } \\
\text { Generating Stations }\end{array}$ & not88-29 & 1988 & $\bar{R}$ & & IEEE 323 & 1983 R90 \\
\hline 3504 & IEEE & IEEE 323 & 1974 & $\begin{array}{l}\text { Standard for Qualifying Class IE } \\
\text { Equipment for Nuclear Power } \\
\text { Generating Stations }\end{array}$ & not92-81 & 1992 & $\mathbf{R}$ & & IEEE 323 & 1983 R90 \\
\hline 3505 & IEEE & IEEE 323 & 1974 & $\begin{array}{l}\text { Standard for Qualifying Class IE } \\
\text { Equipment for Nuclear Power } \\
\text { Generating Stations }\end{array}$ & not93-33 & 1993 & $\mathbf{R}$ & & IEEE 323 & $1983 \mathrm{RSO}$ \\
\hline 3506 & IEEE & IEEE 323 & 1974 & $\begin{array}{l}\text { Standard for Quallfying Class IE } \\
\text { Equipment for Nuclear Power } \\
\text { Generating Stations }\end{array}$ & nureg0933, 2.A-24 & 1985 & $\mathrm{R}$ & & IEEE 323 & 1983 R9O \\
\hline
\end{tabular}




\begin{tabular}{|c|c|c|c|c|c|c|c|c|c|c|}
\hline & & & Cod & $\begin{array}{l}38 \text { and Standards and Other } \\
\text { Part A - Industry Consensus }\end{array}$ & idance Cite & $\begin{array}{l}\text { Regule } \\
\text { ards (c }\end{array}$ & $\begin{array}{l}\text { tory Do } \\
\text { ontinue }\end{array}$ & $\begin{array}{l}\text { cuments } \\
\text { ed) }\end{array}$ & & \\
\hline focord & Code & Stondord & $\begin{array}{l}\text { Slandard } \\
\text { Vorston }\end{array}$ & ritus & Document & Doo. & Aooppl: & Com & Currontrorolon & Girront \\
\hline 3507 & IEEE & IEEE 323 & 1974 & $\begin{array}{l}\text { Standard for Qualifying Class IE } \\
\text { Equipment for Nuclear Power } \\
\text { Generating Stations }\end{array}$ & nureg0933, 3.4 & 1983 & $\mathbf{R}$ & & IEEE 323 & 1983 A90 \\
\hline 3508 & IEEE & IEEE 323 & 1974 & $\begin{array}{l}\text { Standa:'i for Qualifying Class IE } \\
\text { Equipment for Nuclear Power } \\
\text { Generating Stations }\end{array}$ & reg1.131.r00 & 1977 & $\bar{P}$ & With exceptions & IEEE 323 & $1983 \mathrm{R} 90$ \\
\hline 3509 & IEEE & IEEE 323 & 1974 & $\begin{array}{l}\text { Standard for Qualitying Class IE } \\
\text { Equipment for Nuclear Power } \\
\text { Generating Stations }\end{array}$ & reg1.89.ro1 & 1984 & $\bar{P}$ & & IEEE 323 & $1983 \mathrm{R} 90$ \\
\hline 3510 & IEEE & IEEE 323 & 1974 & $\begin{array}{l}\text { Standard for Qualifying Class IE } \\
\text { Equipment for Nuclear Power } \\
\text { Generating Stations }\end{array}$ & $\operatorname{reg} 1.9 .503$ & 1993 & $\mathbf{P}$ & \begin{tabular}{|l|} 
Subject to Regulatory \\
position of Reg. Guide \\
1.89
\end{tabular} & IEEE 323 & 1983 R90 \\
\hline 3511 & IEEE & IEEE 323 & 1974 & $\begin{array}{l}\text { Standard for Qualifying Class IE } \\
\text { Equipment for Nuclear Power } \\
\text { Generating Stations }\end{array}$ & sip3.10 & 1981 & $\bar{P}$ & \begin{tabular}{|l|} 
Endorses Section 6 of \\
IEEE 323-1974
\end{tabular} & IEEE $\overline{323}$ & 1983 R90 \\
\hline 3512 & IEEE & IEEE 323 & 1974 & $\begin{array}{l}\text { Standard for Qualitying Class IE } \\
\text { Equipment for Nuclear Power } \\
\text { Generating Stations }\end{array}$ & sip3.11 & 1981 & $\mathbf{P}$ & $\begin{array}{l}\text { Provides acceptance } \\
\text { criteria }\end{array}$ & IEEE 323 & 1983 R90 \\
\hline 3513 & IEEE & IEEE 325 & 1971 & $\begin{array}{l}\text { Standard Test Procedures for } \\
\text { Germanium Gamma-Ray Detectors }\end{array}$ & reg5.9.r02 & 1983 & $\bar{A}$ & & |IEEE 325 & 1986 \\
\hline 3514 & IEEE & IEEE 325 & 1977 & $\begin{array}{l}\text { Standard Test Procedures for } \\
\text { Germanium Gamma-Ray Detectors }\end{array}$ & reg5.9.ro2 & 1983 & $\mathbf{R}$ & Supplements IEEE 325 & IEEE 325 & 1986 \\
\hline 3515 & IEEE & IEEE 334 & $\mathrm{~N} / \mathrm{S}$ & $\begin{array}{l}\text { Standard for Type Tests of } \\
\text { Continuous Duty Class IE Motors } \\
\text { for Nuclear Power Generating } \\
\text { Stations }\end{array}$ & inm51051 & 1984 & $\bar{R}$ & $\begin{array}{l}\text { IEEE } 334 \text { withdrawn } \\
1993\end{array}$ & Withdrawn & $n / 2$ \\
\hline 3516 & IEEE & IEEE 334 & 1971 & $\begin{array}{l}\text { Standard for Type Tests of } \\
\text { Continuous Duty Class IE Motors } \\
\text { for Nuclear Power Generating } \\
\text { Stations }\end{array}$ & reg1.40.r00 & 1973 & $\mathbf{P}$ & \begin{tabular}{|l} 
IEEE 334 withdrawn \\
1993
\end{tabular} & Withdrawn & $n / a$ \\
\hline 3517 & IEEE & IEEE 334 & 1974 & $\begin{array}{l}\text { Standard for Type Tests of } \\
\text { Continuous Duty Class IE Motors } \\
\text { for Nuclear Power Generating } \\
\text { Stations }\end{array}$ & reg1.52.ro2 & 1978 & $\bar{P}$ & $\begin{array}{l}\text { IEEE } 334 \text { withdrawn } \\
1993\end{array}$ & Withdrawn & $n / a$ \\
\hline
\end{tabular}




\section{Codes and Standards and Other Guidance Clted in Regulatory Documents}

Part A - Industry Consensus Codes and Standards (continued)

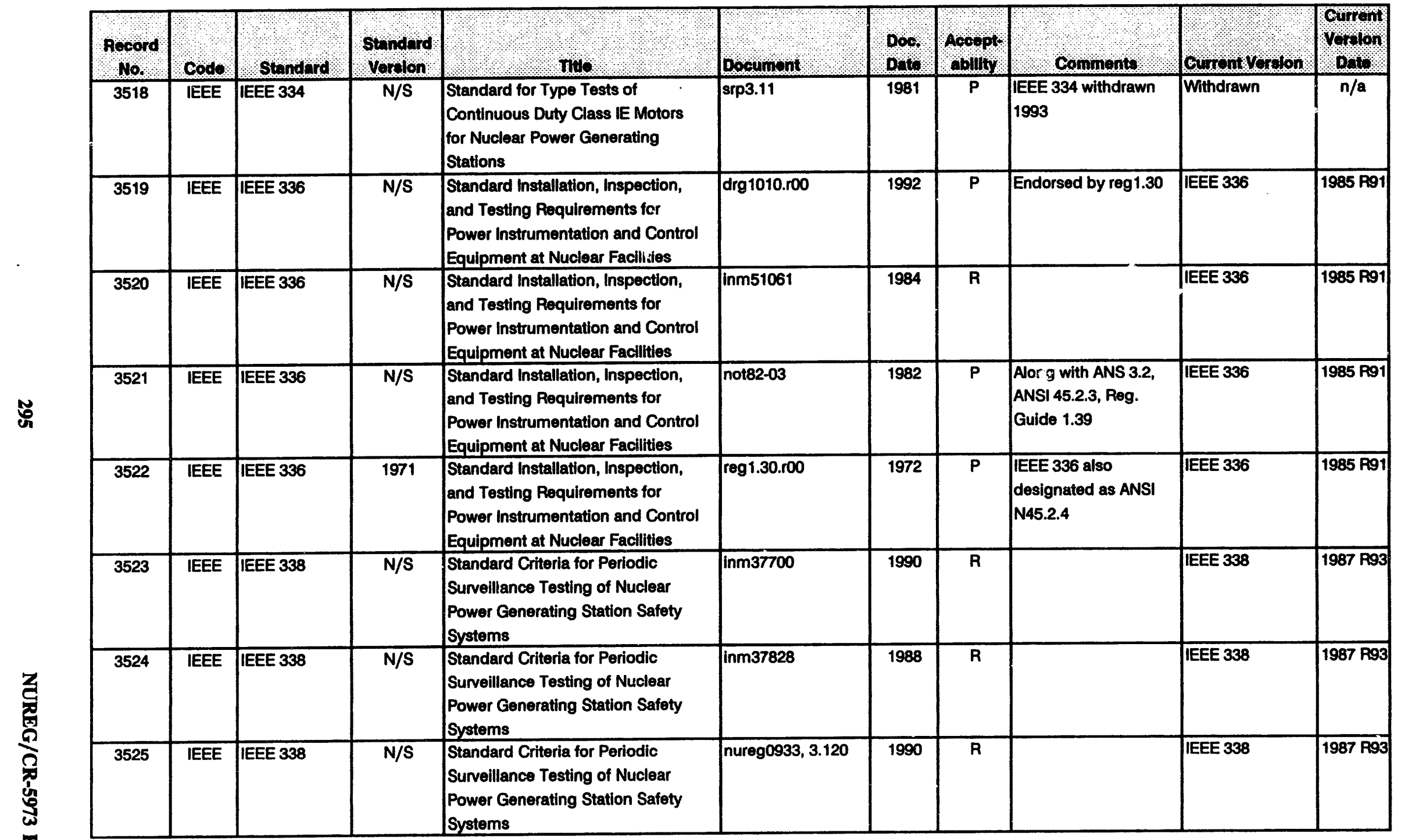




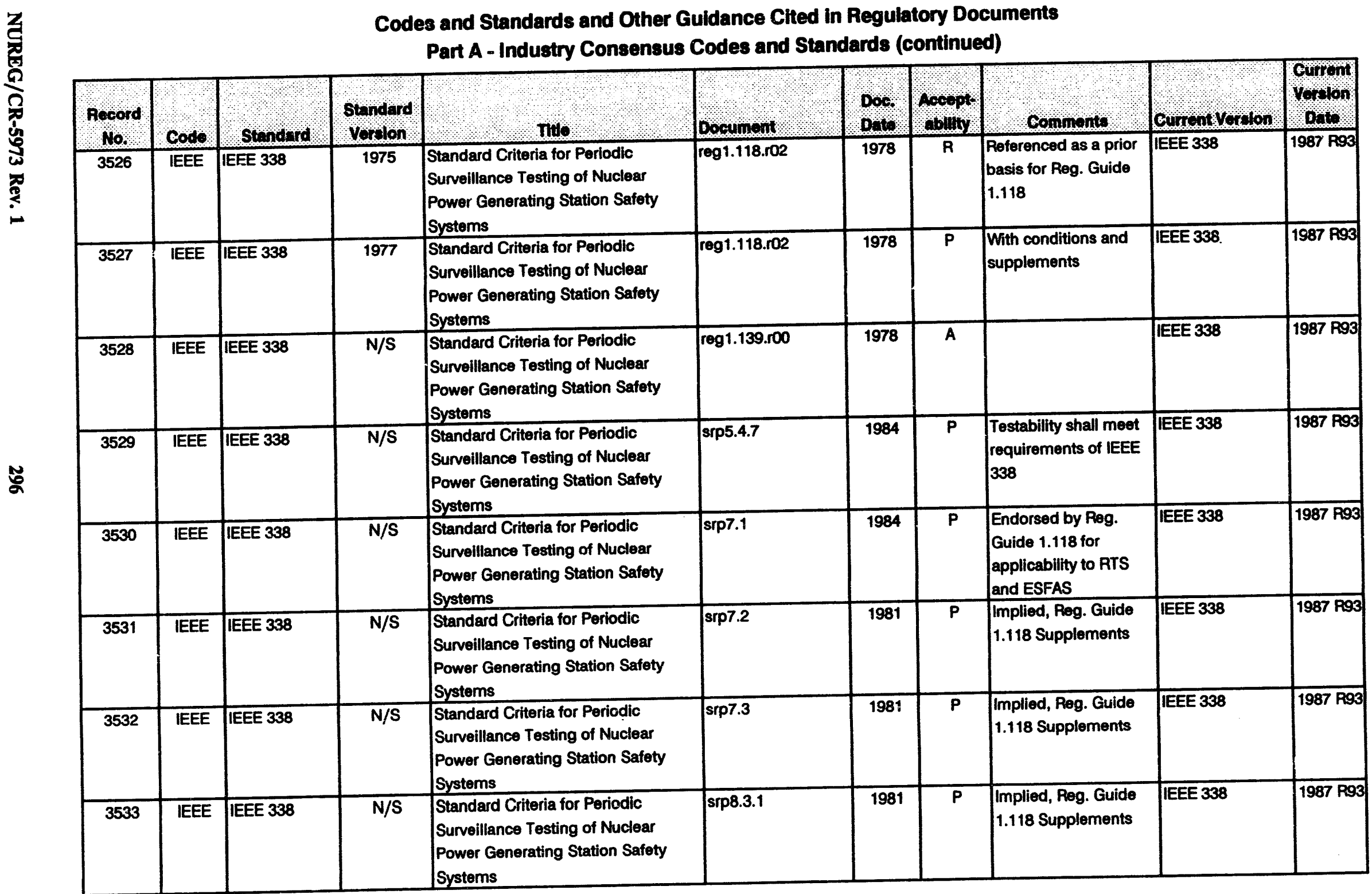




\section{Codes and Standards and Other Guidance Cited in Regulatory Documents}

Part A - Industry Consensus Codes and Standards (continued)

\begin{tabular}{|c|c|c|c|c|c|c|c|c|c|c|}
\hline Hocord & Codo & Standard & Standard & ( & Documont. & Dos. & Acoppts & Comments & Curront verolon & 6urfort \\
\hline 3534 & IEEE & IEEE 344 & 1975 & $\begin{array}{l}\text { Recommended Practice for } \\
\text { Seismic Qualification of Class IE } \\
\text { Equipment for Nuclear Power } \\
\text { Generating Stations }\end{array}$ & bul80-23 & 1980 & $P$ & $\begin{array}{l}\text { Per applicable } \\
\text { requirements }\end{array}$ & IEEE 344 & 1987 \\
\hline 3535 & IEEE & IEEE 344 & 1975 & $\begin{array}{l}\text { Recommended Practice for } \\
\text { Selsmic Quallification of Class IE } \\
\text { Equipment for Nuclear Power } \\
\text { Generating Stations }\end{array}$ & bul84-02 & 1984 & $\mathbf{R}$ & & IEEE 344 & 1987 \\
\hline 3536 & IEEE & IEEE 344 & $N / S$ & $\begin{array}{l}\text { Recommended Practice for } \\
\text { Seismic Qualification of Class IE } \\
\text { Equipment for fivuclear Power } \\
\text { Generating Stations }\end{array}$ & bul88-03 & 1988 & $\mathbf{R}$ & & IEEE 344 & 1987 \\
\hline 3537 & IEEE & IEEE 344 & 1975 & $\begin{array}{l}\text { Recommended Practice for } \\
\text { Seismic Qualification of Class IE } \\
\text { Equipment for Nuclear Power } \\
\text { Generating Stations }\end{array}$ & drgrs802-5 & 1988 & $P$ & Proposed reg1.9.r03 & IEEE 344 & 1987 \\
\hline 3538 & IEEE & IEEE 344 & 1987 & $\begin{array}{l}\text { Recommended Practice for } \\
\text { Seismic Qualification of Class IE } \\
\text { Equipment for Nuclear Power } \\
\text { Generating Stations }\end{array}$ & drgrs802-5 & 1988 & $\mathbf{P}$ & Proposed reg1.9.r03 & IEEE 344 & 1987 \\
\hline 3539 & IEEE & IEEE 344 & 1975 & $\begin{array}{l}\text { Recommended Practice for } \\
\text { Seismic Qualification of Class IE } \\
\text { Equipment for Nuclear Power } \\
\text { Generating Stations }\end{array}$ & glt80-097 & 1980 & A & & IEEE 344 & 1987 \\
\hline 3540 & IEEE & IEEE 344 & 1975 & $\begin{array}{l}\text { Recommended Practice for } \\
\text { Seismic Qualification of Class IE } \\
\text { Equipment for Nuclear Power } \\
\text { Generating Stations }\end{array}$ & glt87-002.s01 & 1992 & $\mathbf{R}$ & & IEEE 344 & 1987 \\
\hline 3541 & IEEE & IEEE 344 & 1975 & $\begin{array}{l}\text { Recommended Practice for } \\
\text { Seismic Qualification of Class IE } \\
\text { Equipment for Nuclear Power } \\
\text { Generating Stations }\end{array}$ & glt87-003 & 1987 & $\overline{\mathbf{R}}$ & & IEEE 344 & 1987 \\
\hline
\end{tabular}


Codes and Standards and Other Guidance Clted in Regulatory Documents

Part A - Industry Consensus Codes and Standards (continued)

\begin{tabular}{|c|c|c|c|c|c|c|c|c|c|c|}
\hline $\begin{array}{l}\text { Rocord } \\
\text { No. }\end{array}$ & Cods: & Standard. & $\begin{array}{l}\text { Standard } \\
\text { Vordolon }\end{array}$ & nut & Documont & $\begin{array}{l}\text { Do. } \\
\text { D.t. }\end{array}$ & $\begin{array}{l}\text { Accopt } \\
\text { oblinty }\end{array}$ & Comments & Currom Vorolon: & $\begin{array}{l}\text { Currom } \\
\text { Vorron } \\
\text { Deto }\end{array}$ \\
\hline 3542 & IEEE & IEEE 344 & $\mathrm{~N} / \mathrm{S}$ & $\begin{array}{l}\text { Recommended Practice for } \\
\text { Seismic Qualification of Class IE } \\
\text { Equipment for Nuclear Power } \\
\text { Generating Stations } \\
\end{array}$ & inm38703 & 1993 & $R$ & & IEEE 344 & 1987 \\
\hline 3543 & IEEE & IEEE 344 & 1975 & $\begin{array}{l}\text { Recommendec Practice for } \\
\text { Seismic Quaurication of Class IE } \\
\text { Equipment for Nuclear Power } \\
\text { Generating Statlons } \\
\end{array}$ & inms'J100 & 1985 & $\mathbf{R}$ & & IEEE 344 & 1987 \\
\hline 3544 & IEEE & IEEE 344 & $\mathrm{~N} / \mathrm{S}$ & $\begin{array}{l}\text { Recommended Practice for } \\
\text { Seismic Qualification of Class IE } \\
\text { Equipment for Nuclear Power } \\
\text { Generating Stations } \\
\end{array}$ & inm51051 & 1984 & $\mathbf{R}$ & & IEEE 344 & 1987 \\
\hline 3545 & IEEE & IEEE 344 & N/S & $\begin{array}{l}\text { Recommended Practice for } \\
\text { Seismic Qualification of Class IE } \\
\text { Equipment for Nuclear Power } \\
\text { Generating Stations } \\
\end{array}$ & inm51053 & 1984 & $\mathbf{R}$ & & IEEE 344 & 1987 \\
\hline 3546 & IEEE & IEEE 344 & $N / S$ & $\begin{array}{l}\text { Recommended Practice for } \\
\text { Seismic Qualification of Class IE } \\
\text { Equipment for Nuclear Power } \\
\text { Generating Stations }\end{array}$ & inm52051 & 1984 & R & & IEEE 344 & 1987 \\
\hline 3547 & IEEE & IEEE 344 & N/S & $\begin{array}{l}\text { Recommended Practice for } \\
\text { Seismic Qualification of Class IE } \\
\text { Equipment for Nuclear Power } \\
\text { Generating Stations } \\
\end{array}$ & inm52053 & 1984 & $\bar{R}$ & & IEEE 344 & 1987 \\
\hline 3548 & IEEE & IEEE 344 & $\mathrm{~N} / \mathrm{S}$ & $\begin{array}{l}\text { Recommended Practice for } \\
\text { Seismic Qualification of Class IE } \\
\text { Equipment for Nuclear Power } \\
\text { Generating Stations }\end{array}$ & inm52055 & 1984 & $\bar{R}$ & & IEEE 344 & 1987 \\
\hline 3549 & IEEE & IEEE 344 & 1971 & $\begin{array}{l}\text { Recommended Practice for } \\
\text { Seismic Qualification of Class IE } \\
\text { Equipment for Nuclear Power } \\
\text { Generating Stations } \\
\end{array}$ & not80-21 & 1980 & $\mathbf{R}$ & & IEEE 344 & 1987 \\
\hline
\end{tabular}


Codes and Standards and Other Guidance Clted in Regulia.ory Documents

Part A - Industry Consensus Codes and Standards (continued)

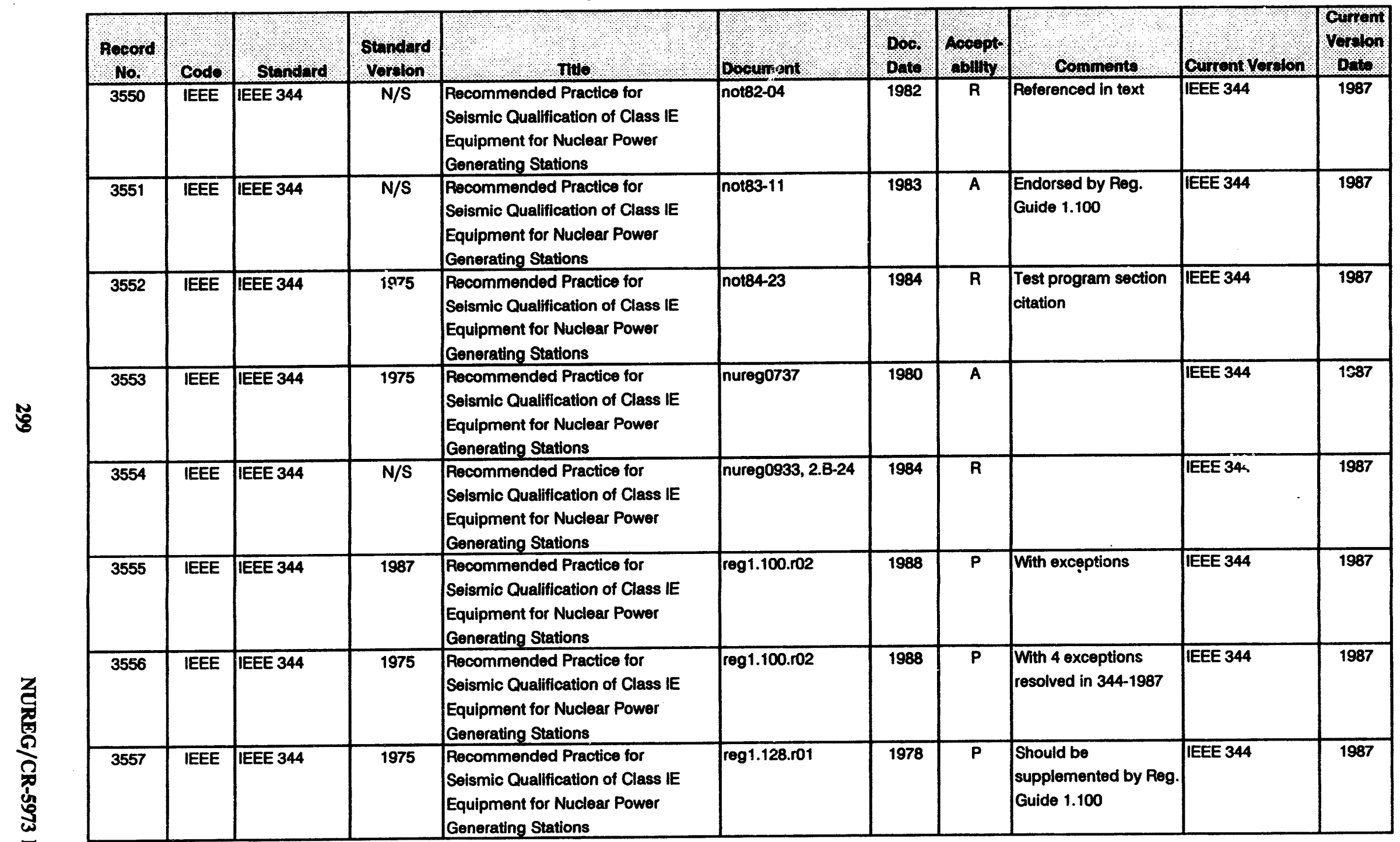


Codes and Standards and Other Guidance Cited in Regulatory Documents

Part A - Industry Consensus Codes and Standards (continued)

\begin{tabular}{|c|c|c|c|c|c|c|c|c|c|c|}
\hline $\begin{array}{l}\text { Record } \\
\text { No. }\end{array}$ & Code & Standard & $\begin{array}{l}\text { Standard } \\
\text { Vortlon }\end{array}$ & L & Dooument & Dos. & nooppt. & Commente & Gurront Verelon & Qurent \\
\hline 3558 & IEEE & IEEE 344 & 1975 & $\begin{array}{l}\text { Recommended Practice for } \\
\text { Seismic Qualification of Class IE } \\
\text { Equipment for Nuclear Power } \\
\text { Generating Stations }\end{array}$ & reg1.9.ro3 & 1993 & $P$ & $\begin{array}{l}\text { Subject to regulatory } \\
\text { position of Reg. Guide } \\
1.100\end{array}$ & IEEE 344 & 1987 \\
\hline 3559 & IEEE & IEEE 344 & 1975 & $\begin{array}{l}\text { Recommended Practice for } \\
\text { Seismic Qualification of Class IE } \\
\text { Equipment for Nuclear Power } \\
\text { Generating Stations }\end{array}$ & $\operatorname{sip} 3.10$ & 1981 & $\mathbf{P}$ & $\begin{array}{l}\text { References } \\
\text { endorsement by Reg } \\
\text { Guide } 1.100\end{array}$ & IEEE 344 & 1987 \\
\hline 3560 & IEEE & IEEE 344 & 1971 & $\begin{array}{l}\text { Recommended Practice for } \\
\text { Seismic Qualification of Class IE } \\
\text { Equipment for Nuclear Power } \\
\text { Generating Stations }\end{array}$ & $\operatorname{srp3.10}$ & 1981 & $P$ & $\begin{array}{l}\text { Listed as minimum } \\
\text { requirement }\end{array}$ & IEEE 344 & 1987 \\
\hline 3561 & IEEE & IEEE 344 & $N / S$ & $\begin{array}{l}\text { Recommended Practice for } \\
\text { Selsmic Qualification of Class IE } \\
\text { Equipment for Nuclear Power } \\
\text { Generating Stations } \\
\end{array}$ & srp3.11 & 1981 & $P$ & & IEEE 344 & 1987 \\
\hline 3562 & IEEE & IEEE 344 & 1975 & $\begin{array}{l}\text { Pecommended Practice for } \\
\text { Seismic Qualification of Class IE } \\
\text { Equipment for Nuclear Power } \\
\text { Generating Stations }\end{array}$ & $\operatorname{srp5.4.12}$ & 1981 & $\overline{\mathbf{P}}$ & \begin{tabular}{|l|} 
With Reg. Guide 1.100 \\
1.92, SRP $3.92,3.43$, \\
3.10 Supplementing
\end{tabular} & IEEE 344 & 1987 \\
\hline 3563 & IEEE & IEEE 352 & 1976 & $\begin{array}{l}\text { Guide for General Principles of } \\
\text { Reliability Analysis of Nuclear } \\
\text { Power Generating Station Safety } \\
\text { Systems }\end{array}$ & nureg0933, Ref & 1991 & $\mathbf{R}$ & $\begin{array}{l}\text { IEEE } 352 \text { withdrawn } \\
1993\end{array}$ & Withdrawn & $n / a$ \\
\hline 3564 & IEEE & IEEE 378 & $N / S$ & Standard on Network Analyzers & $\operatorname{sip8.3.2}$ & 1981 & $R$ & $\begin{array}{l}\text { Referenced with Reg. } \\
\text { Guide } 1.118 \text { (1 }\end{array}$ & IEEE 378 & 1986 \\
\hline 3565 & IEEE & IEEE 379 & 1977 & $\begin{array}{l}\text { Standard Application of the Single } \\
\text { Failure Criterion to Nuclear Power } \\
\text { Generating Station Safety Systems }\end{array}$ & glt81-018 & 1981 & R & & IEEE 379 & 1988 \\
\hline 3566 & IEEE & IEEE 379 & $N / S$ & $\begin{array}{l}\text { Standard Application of the Single } \\
\text { Failure Criterion to Nuclear Power } \\
\text { Generating Station Ssfety Systems }\end{array}$ & inm51051 & 1984 & R & & IEEE 379 & 1988 \\
\hline
\end{tabular}


Codes and Standards and Other Guidance Cited in Regulatory Documents

Part A - Industry Consensus Codes and Standards (continued)

\begin{tabular}{|c|c|c|c|c|c|c|c|c|c|c|}
\hline $\begin{array}{l}\text { Record } \\
\text { No. }\end{array}$ & Code & Standard & $\begin{array}{l}\text { Standard } \\
\text { verslon }\end{array}$ & Tho, & Document & $\begin{array}{l}\text { Doc. } \\
\text { Dote. }\end{array}$ & $\begin{array}{l}\text { Aocept: } \\
\text { oblity }\end{array}$ & Comments & Current Vorolon & Gorion \\
\hline 3567 & IEEE & IEEE 379 & $N / S$ & $\begin{array}{l}\text { Standard Application of the Single } \\
\text { Failure Criterion to Nuclear Power } \\
\text { Generating Station Safety Systems }\end{array}$ & inm51053 & 1984 & $\mathbf{R}$ & & IEEE 379 & 1988 \\
\hline 3568 & IEEE & IEEE 379 & $\mathrm{~N} / \mathrm{S}$ & $\begin{array}{l}\text { Standard Application of the Single } \\
\text { Failure Criterion to Nuclear Power } \\
\text { Generating Station Safety Systems }\end{array}$ & inm51055 & 1984 & $\mathbf{R}$ & & IEEE 379 & 1988 \\
\hline 3569 & IEEE & IEEE 379 & $\mathrm{~N} / \mathrm{S}$ & $\begin{array}{l}\text { Standard Application of the Single } \\
\text { Failure Criterion to Nuclear Power } \\
\text { Generating Station Safety Systems }\end{array}$ & inm51061 & 1984 & R & & IEEE 379 & 1988 \\
\hline 3570 & IEEE & IEEE 379 & $\mathrm{~N} / \mathrm{S}$ & $\begin{array}{l}\text { Standard Application of the Single } \\
\text { Failure Criterion to Nuclear Power } \\
\text { Generating Station Safety Systems }\end{array}$ & inm51063 & 1985 & $\mathbf{R}$ & & IEEE 379 & 1988 \\
\hline 3571 & IEEE & IEEE 379 & $N / S$ & $\begin{array}{l}\text { Standard Application of the Single } \\
\text { Fallure Criterion to Nuclear Power } \\
\text { Generating Station Safety Systems }\end{array}$ & inm51065 & 1984 & $\mathbf{R}$ & & IEEE 379 & 1988 \\
\hline 3572 & IEEE & IEEE 379 & $N / S$ & $\begin{array}{l}\text { Standard Application of the Single } \\
\text { Failure Criterion to Nuclear Power } \\
\text { Generating Station Safety Systems }\end{array}$ & inm52051 & 1984 & $\mathbf{R}$ & & IEEE 379 & 1988 \\
\hline 3573 & IEEE & IEEE 379 & $N / S$ & $\begin{array}{l}\text { Standard Application of the Single } \\
\text { Failure Criterion to Nuclear Power } \\
\text { Generating Station Safety Systems }\end{array}$ & inm52053 & 1984 & $\mathbf{R}$ & & IEEE 379 & 1988 \\
\hline 3574 & IEEE & IEEE 379 & $N / S$ & $\begin{array}{l}\text { Standard Application of the Single } \\
\text { Failure Criterion to Nuclear Power } \\
\text { Generating Station Safety Systems }\end{array}$ & inm52055 & 1984 & $\mathbf{R}$ & & IEEE 379 & 1988 \\
\hline 3575 & IEEE & IEEE 379 & $N / S$ & $\begin{array}{l}\text { Standard Application of the Single } \\
\text { Failure Criterion to Nuclear Power } \\
\text { Generating Station Safety Systems }\end{array}$ & reg1.153.r00 & 1985 & $\bar{R}$ & & IEEE 379 & 1988 \\
\hline 3576 & IEEE & IEEE 379 & 1972 & $\begin{array}{l}\text { Standard Application of the Single } \\
\text { Fallure Criterion to Nuclear Power } \\
\text { Generating Station Safety Systems }\end{array}$ & reg1.53.ro0 & 1973 & $\mathbf{P}$ & Generally acceptable & IEEE 379 & 1988 \\
\hline 3577 & IEEE & IEEE 379 & $N / S$ & $\begin{array}{l}\text { Standard Application of the Single } \\
\text { Failure Criterion to Nuclear Power } \\
\text { Generating Station Safety Systems }\end{array}$ & srp7.1 & 1984 & $\mathbf{P}$ & & IEEE 379 & 1988 \\
\hline
\end{tabular}




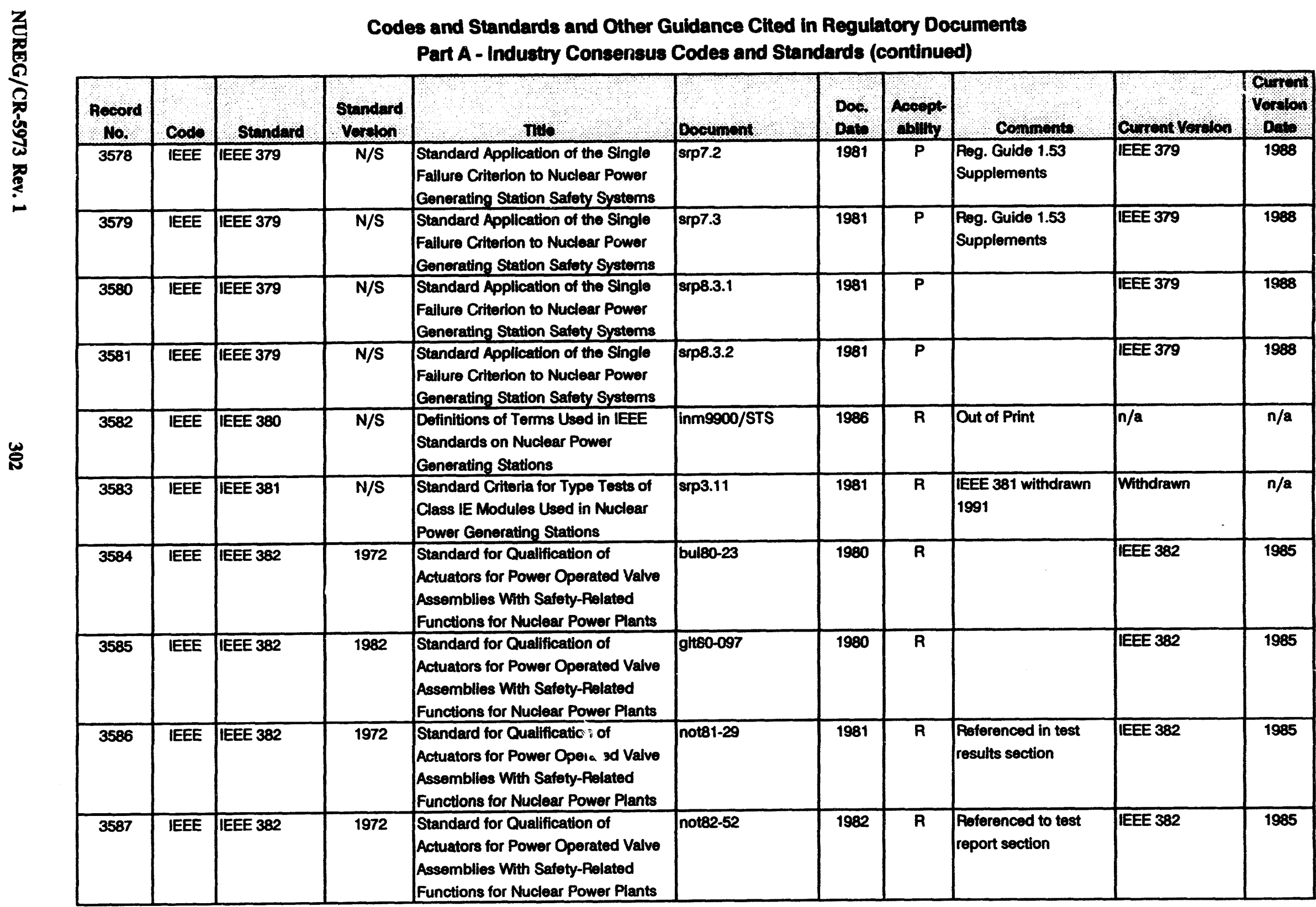


Codes and Standards and Other Guidance Cited in Regulatory Documents

Part A - Industry Consensus Codes and Standards (continued)

\begin{tabular}{|c|c|c|c|c|c|c|c|c|c|c|}
\hline $\begin{array}{l}\text { Record } \\
\text { No, }\end{array}$ & Code & Standard & $\begin{array}{l}\text { Shndard } \\
\text { Vorelon }\end{array}$ & Thlo , & Document: & Doc. & Aocopts & Commonti & Current verolon & entront \\
\hline 3588 & IEEE & IEEE 382 & 1972 & $\begin{array}{l}\text { Standard for Qualification of } \\
\text { Actuators for Power Operated Valve } \\
\text { Assemblies With Safety-Related } \\
\text { Functions for Nuclear Power Plants }\end{array}$ & not83-72 & 1983 & $\mathbf{R}$ & $\begin{array}{l}\text { Referred to in problem } \\
\text { discussion }\end{array}$ & IEEE 382 & 1985 \\
\hline 3589 & IEEE & IEEE 382 & 1980 & $\begin{array}{l}\text { Standard for Qualification of } \\
\text { Actuators for Power Operated Valve } \\
\text { Assemblies With Safety-Prelated } \\
\text { Functions for Nuclear Power Plants }\end{array}$ & not84-23 & 1984 & $\mathbf{R}$ & $\begin{array}{l}\text { Referred to in } \\
\text { discussion of test } \\
\text { program }\end{array}$ & IEEE 382 & 1985 \\
\hline 3590 & IEEE & IEEE 382 & 1972 & $\begin{array}{l}\text { Standard for Qualification of } \\
\text { Actuators for Power Operated Valve } \\
\text { Assemblies With Safety-Prelated } \\
\text { Functions for Nuclear Power Plants }\end{array}$ & reg1.73.roD & 1974 & $\mathbf{P}$ & & IEEE 382 & 1985 \\
\hline 3591 & IEEE & IEEE 382 & $N / S$ & $\begin{array}{l}\text { Standard for Qualification of } \\
\text { Actuators for Power Operated Valve } \\
\text { Assemblies With Safety-Pelated } \\
\text { Functions for Nuclear Power Plants }\end{array}$ & $\operatorname{srp3.11}$ & 1981 & $\mathbf{P}$ & $\begin{array}{l}\text { Implied, } \\
\text { Supplemented by Reg. } \\
\text { Guide } 1.73\end{array}$ & IEEE 382 & 1985 \\
\hline 3592 & IEEE & IEEE 383 & $\mathrm{~N} / \mathrm{S}$ & $\begin{array}{l}\text { Standard for Type Test of Class IE } \\
\text { Electric Cables, Field Splices, and } \\
\text { Connections for Nuclear Power } \\
\text { Generating Stations }\end{array}$ & glt83-033 & 1983 & $\mathbf{R}$ & & IEEE 383 & $1974 \mathrm{Rg2}$ \\
\hline 3593 & IEEE & IEEE 383 & $\mathrm{~N} / \mathrm{S}$ & $\begin{array}{l}\text { Standard for Type Test of Class IE } \\
\text { Electric Cables, Field Splices, and } \\
\text { Connections for Nuclear Power } \\
\text { Generating Stations }\end{array}$ & glt86-010 & 1986 & $\bar{P}$ & & IEEE 383 & 1974 R92 \\
\hline 3594 & IEEE & IEEE 383 & $N / S$ & $\begin{array}{l}\text { Standard for Type Test of Class IE } \\
\text { Electric Cables, Field Splices, and } \\
\text { Connections for Nuclear Power } \\
\text { Generating Stations }\end{array}$ & drgrs50-2 & 1979 & $\mathbf{P}$ & Proposed reg1.131.ro1 & IEEE 383 & $1974 \mathrm{Rg2}$ \\
\hline 3595 & IEEE & IEEE 383 & $\mathbf{N} / \mathrm{S}$ & $\begin{array}{l}\text { Standard for Type Test of Class IE } \\
\text { Electric Cables, Field Splices, and } \\
\text { Connections for Nuclear F'ower } \\
\text { Generating Stations }\end{array}$ & inm51061 & 1984 & $\mathbf{R}$ & & IEEE 383 & $1974 \mathrm{R92}$ \\
\hline
\end{tabular}




\begin{tabular}{|c|c|c|c|c|c|c|c|c|c|c|}
\hline & & & Cod & $\begin{array}{l}8 \text { and Standards and Other } \\
\text { Part A - Industry Consensus }\end{array}$ & $\begin{array}{l}\text { uidance Cit } \\
\text { Codes and }\end{array}$ & ards (c & $\begin{array}{l}\text { tory Do } \\
\text { tontinue }\end{array}$ & $\begin{array}{l}\text { Suments } \\
\text { d) }\end{array}$ & & \\
\hline $\begin{array}{l}\text { Hocord } \\
\text { No. }\end{array}$ & Codes & Standard & $\begin{array}{l}\text { Standard } \\
\text { Vorelon }\end{array}$ & $\mathrm{T}=\mathrm{Tw}$ & Document & $\begin{array}{l}\text { Doo: } \\
\text { Date: }\end{array}$ & $\begin{array}{l}\text { Accopt? } \\
\text { abilly' }\end{array}$ & Comments & Current Vorton & $\begin{array}{l}\text { Curremt } \\
\text { Vorlon } \\
\text { potsol }\end{array}$ \\
\hline 3596 & IEEE & IEEE 383 & N/S & $\begin{array}{l}\text { Standard for Type Test of Class IE } \\
\text { Electric Cables, Field Splices, and } \\
\text { Connections for Nuclear Power } \\
\text { Generating Stations }\end{array}$ & inm51063 & 1985 & $\mathbf{R}$ & & IEEE 383 & 1974 R92 \\
\hline 3597 & IEEE & IEEE 383 & N/S & $\begin{array}{l}\text { Standard for Type Test of Class IE } \\
\text { Electric Cables, Feld Splices, and } \\
\text { Connections for Nuclear Power } \\
\text { Generating Stations }\end{array}$ & inm51065 & 1984 & $\bar{R}$ & & IEEE 383 & 1974 R92 \\
\hline 3598 & IEEE & IEEE 383 & N/S & $\begin{array}{l}\text { Standard for Type Test of Class IE } \\
\text { Electric Cables, Field Splices, and } \\
\text { Connections for Nuclear Power } \\
\text { Generating Stations }\end{array}$ & not84-09 & 1984 & $\bar{R}$ & & IEEE 383 & 1974 R92 \\
\hline 3599 & IEEE & IEEE 383 & $N / S$ & $\begin{array}{l}\text { Standard for Type Test of Class IE } \\
\text { Electric Cables, Field Splices, and } \\
\text { Connections for Nuclear Power } \\
\text { Generating Stations }\end{array}$ & notg2-28 & 1992 & $\mathbf{R}$ & & IEEE 383 & 1974 R92 \\
\hline 3600 & IEEE & IEEE 383 & 1974 & $\begin{array}{l}\text { Standard for Type Test of Class IE } \\
\text { Electric Cables, Field Splices, and } \\
\text { Connections for Nuclear Power } \\
\text { Generating Stations }\end{array}$ & not93-33 & 1993 & $\mathbf{R}$ & & IEEE 383 & 1974 R92 \\
\hline 3601 & IEEE & IEEE 383 & 1974 & $\begin{array}{l}\text { Standard for Type Test of Class IE } \\
\text { Electric Cables, Field Splices, and } \\
\text { Connections for Nuclear Power } \\
\text { Generating Stations }\end{array}$ & reg1.120.ro1 & 1977 & $\mathbf{P}$ & & IEEE 383 & 1974 R92 \\
\hline 3602 & IEEE & IEEE 383 & 1974 & $\begin{array}{l}\text { Standard for Type Test of Class IE } \\
\text { Electric Cables, Field Splices, and } \\
\text { Connections for Nuclear Power } \\
\text { Generating Stations }\end{array}$ & reg1.131.roo & 1977 & $\mathbf{P}$ & With conditions & IEEE 383 & 1974 R92 \\
\hline 3603 & IEEE & IEEE 383 & N/S & $\begin{array}{l}\text { Standard for Type Test of Class IE } \\
\text { Electric Cables, Fieid Splices, and } \\
\text { Connections for Nuclear Power } \\
\text { Generating Stations }\end{array}$ & reg1.70.ro3 & 1978 & $\mathbf{P}$ & Minimum requirement & IEEE 383 & 1974 R92 \\
\hline
\end{tabular}


Codes and Standards and Other Guidance Cited in Regulatory Documents

Part A - Industry Consensus Codes and Standards (sontinued)

\begin{tabular}{|c|c|c|c|c|c|c|c|c|c|c|}
\hline Pocord & Cods & Stondard & standard & (1) & Dovon, on, & Doo. & (poppts & 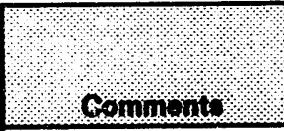 & 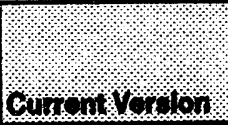 & 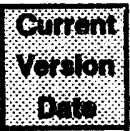 \\
\hline 3604 & IEEE & |EEE 383 & $N / S$ & $\begin{array}{l}\text { Standard for Type Test of Class IE } \\
\text { Electric Cables, Field Splices, and } \\
\text { Connections for Nuclear Power } \\
\text { Generating Stations }\end{array}$ & $\operatorname{sip3.11}$ & 1981 & $\mathbf{P}$ & $\begin{array}{l}\text { Implied: Reg. Guide } \\
\text { 1.131 NUREG-0588 } \\
\text { supplement }\end{array}$ & IEEE 383 & 1974 R92 \\
\hline 3605 & IEEE & IEEE 383 & 1974 & $\begin{array}{l}\text { Standard for Type Test of Class IE } \\
\text { Electric Cables, Fleld Splices, and } \\
\text { Connections for Nuclear Power } \\
\text { Generating Stations }\end{array}$ & sip9.5.1 & 1981 & $\mathbf{P}$ & & IEEE 383 & 1974 R92 \\
\hline 3606 & IEEF: & IEEE 384 & 1974 & $\begin{array}{l}\text { Standard Criteria for Independence } \\
\text { of Class IE Equipment and Circuits }\end{array}$ & 10 cfr50 App. R & 1988 & $\mathbf{P}$ & $\begin{array}{l}\text { Alternate method: } \\
\text { Reg. Guide } 1.75 \text { and } \\
\text { Sec. } 4.5\end{array}$ & IEEE 384 & 1992 \\
\hline 3607 & IEEE & IEEE 384 & 1974 & $\begin{array}{l}\text { Standard Criteria for Independence } \\
\text { of Class IE Equipment and Circults }\end{array}$ & 10cir50 App. R & 1993 & $\mathbf{P}$ & & IEEE 384 & 1992 \\
\hline 3608 & IEEE & IEEE 384 & $\mathrm{~N} / \mathrm{S}$ & $\begin{array}{l}\text { Standard Criteria for Independence } \\
\text { of Class IE Equipment and Circults }\end{array}$ & inm50100 & 1985 & $\mathbf{R}$ & & IEEE 384 & 1992 \\
\hline 3609 & IEEE & IEEE 384 & $N / S$ & $\begin{array}{l}\text { Standard Criteria for Independence } \\
\text { of Class IE Equipment and Circuits }\end{array}$ & inm51051 & 1984 & $\mathbf{R}$ & & IEEE 364 & 1992 \\
\hline 3610 & IEEE & IEEE 384 & $N / S$ & $\begin{array}{l}\text { Standard Criteria for Independence } \\
\text { of Class IE Equipment and Circults }\end{array}$ & inm51053 & 1984 & $\mathbf{R}$ & & IEEE 384 & 1992 \\
\hline 3611 & IEEE & IEEE 384 & $N / S$ & $\begin{array}{l}\text { Standard Criteria for Independence } \\
\text { of Class IE Equipment and Clrcults }\end{array}$ & inm51061 & 1984 & $\mathbf{R}$ & & IEEE 384 & 1992 \\
\hline 3612 & IEEE & IEEE 384 & $\mathrm{~N} / \mathrm{S}$ & $\begin{array}{l}\text { Standard Criteria for independence } \\
\text { of Class IE Equipment and Circults }\end{array}$ & inm51063 & 1985 & $\mathbf{R}$ & & IEEE 384 & 1992 \\
\hline 3613 & IEEE & IEEE 384 & $N / S$ & $\begin{array}{l}\text { Standard Criteria for Independence } \\
\text { of Class IE Equipment and Circults }\end{array}$ & inm51065 & 1984 & $\mathbf{R}$ & & IEEE 384 & 1992 \\
\hline 3614 & IEEE & IEEE 384 & $\mathrm{~N} / \mathrm{S}$ & $\begin{array}{l}\text { Standard Criteria for Independence } \\
\text { of Class IE Equipment and Circuits }\end{array}$ & inm52051 & 1984 & $\bar{R}$ & & IEEE 384 & 1992 \\
\hline 3615 & IEEE & IEEE 384 & $N / S$ & $\begin{array}{l}\text { Standard Criteria for Independence } \\
\text { of Class IE Equipment and Circults }\end{array}$ & inm52053 & 1984 & $\mathbf{R}$ & & IEEE 384 & 1992 \\
\hline 3616 & IEEE & IEEE 384 & $\mathrm{~N} / \mathrm{S}$ & $\begin{array}{l}\text { Standard Criteria for Independence } \\
\text { of Class IE Equipment and Circuits }\end{array}$ & inm52055 & 1984 & $\mathbf{R}$ & & IEEE 384 & 1992 \\
\hline 3617 & IEEE & IEEE 384 & 1974 & $\begin{array}{l}\text { Standard Criteria for Independencs } \\
\text { of Class IE Equipment and Circuits }\end{array}$ & not85-11 & 1985 & $\mathbf{P}$ & Implied endorsement & IEEE 384 & 1992 \\
\hline
\end{tabular}


Codes and Standards and Other Guidance Ched in Regulatory Documents

Part A - Industry Consensus Codes and Standards (continued)

\begin{tabular}{|c|c|c|c|c|c|c|c|c|c|c|}
\hline Rocord & $\operatorname{cod} \theta$ & Sthndard & Stindard & ২ै। & 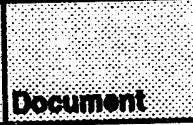 & 000 & poppls & Qommonte & currom Yoralom & ontrot \\
\hline 3618 & IEEE & IEEE 384 & N/S & $\begin{array}{l}\text { Standard Criteria for Indeperidence } \\
\text { of Class IE Equipment and Circuits }\end{array}$ & reg1.153.100 & 1985 & $\mathbf{A}$ & $\begin{array}{l}\text { Endorsed by Reg. } \\
\text { Guide } 1.75\end{array}$ & IEEE 384 & 1992 \\
\hline 3619 & IEEE & IEEE 384 & 1973 & $\begin{array}{l}\text { Standard Criteria for Independence } \\
\text { of Class IE Equipment and Circuits }\end{array}$ & reg1.75.r02 & 1978 & $\bar{R}$ & $\begin{array}{l}\text { Basis for IEEE } 384 \\
\text { 1974. Draft Std. }\end{array}$ & IEEE 384 & 1992 \\
\hline 3620 & IEEE & IEEE 384 & 1974 & $\begin{array}{l}\text { Standard Criteria for Independence } \\
\text { of Class IE Equipment and Circuits }\end{array}$ & reg1.75.ro2 & 1978 & $\mathbf{P}$ & $\begin{array}{l}\text { leee } 384 \text { also } \\
\text { designated ANSI } \\
\text { N41.14 }\end{array}$ & IEEE 384 & 1992 \\
\hline 3621 & IEEE & IEEE 384 & N/S & $\begin{array}{l}\text { Standard Criteria for independence } \\
\text { of Class IE Equipment and Circuits }\end{array}$ & sip7.1 & 1984 & $\mathbf{P}$ & $\begin{array}{l}\text { Endorsed by Reg. } \\
\text { Guide } 1.75\end{array}$ & IEEE 384 & 1992 \\
\hline 3622 & IEEE & IEEE 384 & $\mathrm{~N} / \mathrm{S}$ & $\begin{array}{l}\text { Standard Criteria for Independence } \\
\text { of Class IE Equipment and Circuits }\end{array}$ & $\operatorname{sip} 7.2$ & 1981 & $\bar{P}$ & $\begin{array}{l}\text { Implied, Reg. Guide } \\
1.75 \text { Supplements } \\
\end{array}$ & IEEE 384 & 1992 \\
\hline 3623 & IEEE & IEEE 384 & $\mathrm{~N} / \mathrm{S}$ & $\begin{array}{l}\text { Standard Criteria for Independence } \\
\text { of Class IE Equipment and Circuits }\end{array}$ & $\operatorname{sip} 7.3$ & 1981 & $\mathbf{P}$ & \begin{tabular}{|l|} 
Implied, with Reg. \\
Guide 1.75 \\
Supplements \\
\end{tabular} & IEEE 384 & 1992 \\
\hline 3624 & IEEE & IEEE 384 & $\mathrm{~N} / \mathrm{S}$ & $\begin{array}{l}\text { Standard Criteria for Independence } \\
\text { of Class IE Equipment and Circults }\end{array}$ & srp8.3.1 & 1981 & $\mathbf{P}$ & \begin{tabular}{|l|} 
Review considers IEEE \\
384 augmented by \\
Reg. Guide 1.75
\end{tabular} & IEEE 384 & 1992 \\
\hline 3625 & IEEE & IEEE 384 & $N / S$ & $\begin{array}{l}\text { Standard Criteria for Independence } \\
\text { of Class IE Equipment and Circuits }\end{array}$ & srp8.3.2 & 1981 & $\mathbf{P}$ & $\begin{array}{l}\text { Acceptance criteria, } \\
\text { augmented by Reg. } \\
\text { Guide } 1.75\end{array}$ & IEEE 384 & 1992 \\
\hline 3626 & IEEE & IEEE 387 & 1984 & $\begin{array}{l}\text { Standard Criteria for Diesel- } \\
\text { Generator Units Applied as Standby } \\
\text { Power Supplies for Nuclear Power } \\
\text { Generating Stations }\end{array}$ & drgrs802-5 & 1988 & $\mathbf{P}$ & $\begin{array}{l}\text { IEEE } 387 \text { withdrawn } \\
1993\end{array}$ & Withdrawn & $n / a$ \\
\hline 3627 & IEEE & IEEE 387 & 1977 & $\begin{array}{l}\text { Standard Criteria for Diesel- } \\
\text { Generator Units Applied as Standby } \\
\text { Power Supplies for Nuclear Power } \\
\text { Generating Stations }\end{array}$ & drgrs802-5 & 1988 & $\mathbf{R}$ & $\begin{array}{l}\text { IEEE } 387 \text { withdrawn } \\
1993\end{array}$ & Withdrawn & $n / a$ \\
\hline 3628 & IEEE & IEEE 387 & 1984 & $\begin{array}{l}\text { Standard Criteria for Diesel- } \\
\text { Generator Units Applied as Standby } \\
\text { Power Supplies for Nuclear Power } \\
\text { Generating Stations }\end{array}$ & $\operatorname{drg} 1021.000$ & 1992 & $\mathbf{P}$ & $\begin{array}{l}\text { IEEE } 387 \text { withdrawn } \\
1993\end{array}$ & Withdrawn & $n / a$ \\
\hline
\end{tabular}

Generating Stations 


\section{Codes and Standards and Other Guldance Clted in Regulatory Documents}

Part A - Industry Consensus Codes and Standards (continued)

\begin{tabular}{|c|c|c|c|c|c|c|c|c|c|c|}
\hline Hocord & Codo & stundurd & Standard & Into & booumont & Doo. & $\begin{array}{l}\text { Acoept } \\
\text { obilly }\end{array}$ & 1, Comments & Contont 1, & 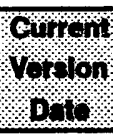 \\
\hline 3629 & IEEE & IEEE 387 & 1984 & $\begin{array}{l}\text { Standard Criteria for Diesel- } \\
\text { Generator Units Applied as Standby } \\
\text { Power Supplies for Nuclear Power } \\
\text { Generating Stations }\end{array}$ & reg1.155.r00 & 1988 & $R$ & $\begin{array}{l}\text { IEEE } 387 \text { withdrawn } \\
1993\end{array}$ & Withdrawn & $n / a$ \\
\hline 3630 & IEEE & IEEE 387 & 1972 & $\begin{array}{l}\text { Standard Critoria for Diesel- } \\
\text { Generator Units Applied as Standby } \\
\text { Power Supplies for Nuctear Power } \\
\text { Generating Stations }\end{array}$ & reg1.70.r02 & 1975 & $\bar{P}$ & $\begin{array}{l}\text { IEEE } 387 \text { withdrawn } \\
1993\end{array}$ & Withdrawn & $n / a$ \\
\hline 3631 & IEEE & IEEE 387 & $N / S$ & $\begin{array}{l}\text { Standard Criteria for Diesel- } \\
\text { Generator Units Applied as Standby } \\
\text { Power Supplies for Nuclear Power } \\
\text { Generating Stations }\end{array}$ & reg1.70.r03 & 1978 & $\mathbf{P}$ & $\begin{array}{l}\text { IEEE } 387 \text { withdrawn } \\
1993\end{array}$ & Withdrawn & $n / a$ \\
\hline 3632 & IEEE & IEEE 387 & 1977 & $\begin{array}{l}\text { Standard Criteria for Diesel- } \\
\text { Generator Units Applied as Standby } \\
\text { Power Supplies for Nuclear Power } \\
\text { Generating Stations }\end{array}$ & reg1.9.r03 & 1993 & $\mathbf{R}$ & $\begin{array}{l}\text { IEEE } 387 \text { withdrawn } \\
1993\end{array}$ & Withdrawn & $n / a$ \\
\hline 3633 & IEEE & IEEE 387 & 1984 & $\begin{array}{l}\text { Standard Criteria for Dlesel- } \\
\text { Generator Units Applied as Standby } \\
\text { Power Supplies for Nuclear Power } \\
\text { Generating Stations }\end{array}$ & reg1.9.r03 & 1993 & $\bar{P}$ & $\begin{array}{l}\text { IEEE } 387 \text { withdrawn } \\
1993\end{array}$ & Withdrawn & $n / a$ \\
\hline 3634 & IEEE & IEEE 387 & $N / S$ & $\begin{array}{l}\text { Standard Criteria for Diesel- } \\
\text { Generator Units Applied as Standby } \\
\text { Power Supplies for Nuclear Power } \\
\text { Generating Stations }\end{array}$ & srp8.3.1 & 1981 & $\mathbf{P}$ & $\begin{array}{l}\text { IEEE } 387 \text { withdrawn } \\
1993\end{array}$ & Withdrawn & $n / a$ \\
\hline 3635 & IEEE & IEEE 387 & $N / S$ & $\begin{array}{l}\text { Standard Criteria for Diesel- } \\
\text { Generator Units Applied as Standby } \\
\text { Power Supplies for Nuclear Power } \\
\text { Generating Stations }\end{array}$ & srp8A & 1981 & $\mathbf{P}$ & $\begin{array}{l}\text { IEEE } 387 \text { withdrawn } \\
1993\end{array}$ & Withdrawn & $n / a$ \\
\hline 3636 & IEEE & IEEE 387 & $N / S$ & $\begin{array}{l}\text { Standard Criteria for Diesel- } \\
\text { Generator Units Applied as Standby } \\
\text { Power Supplies for Nuclear Power } \\
\text { Generating Stations }\end{array}$ & srp9.5.4 & 1981 & $\bar{P}$ & $\begin{array}{l}\text { IEEE } 387 \text { withdrawn } \\
1993\end{array}$ & Withdrawn & $n / a$ \\
\hline
\end{tabular}


Codes and Standards and Other Guidance Cited in Regulatory Documents

Part A - Industry Consensus Codes and Standards (continued)

\begin{tabular}{|c|c|c|c|c|c|c|c|c|c|c|}
\hline $\begin{array}{l}\text { Rosord } \\
\text { No. }\end{array}$ & Codo & stom dord & Stridard & 1. & Doovum & 000 & Aocopt: & commonto & Courront Vorolon & Voron \\
\hline 3637 & IEEE & IEEE 387 & $N / S$ & $\begin{array}{l}\text { Standard Criteria for Diesel- } \\
\text { Generator Units Applied as Standby } \\
\text { Power Supplies for Nuclear Power } \\
\text { Generating Stations }\end{array}$ & sip9.5.5 & 1981 & $\mathbf{P}$ & $\begin{array}{l}\text { IEEE } 387 \text { withdrawn } \\
1993\end{array}$ & Withdrawn & $n / a$ \\
\hline 3638 & IEEE & IEEE 387 & N/S & $\begin{array}{l}\text { Standard Criteria for Diesel- } \\
\text { Generator Units Applied as Standby } \\
\text { Power Supplies for Nuclear Power } \\
\text { Generating Stations }\end{array}$ & sip9.5.6 & 1981 & $\mathbf{P}$ & $\begin{array}{l}\text { IEEE } 387 \text { withdrawn } \\
1993\end{array}$ & Withdrawn & $n / a$ \\
\hline 3639 & IEEE & IEEE 387 & $N / S$ & $\begin{array}{l}\text { Standard Criteria for Diesel- } \\
\text { Generator Units Applied as Standby } \\
\text { Power Supplies for Nuclear Power } \\
\text { Generating Stations }\end{array}$ & $\operatorname{sip9.5.7}$ & 1981 & $\mathbf{P}$ & $\begin{array}{l}\text { IEEE } 387 \text { withdrawn } \\
1993\end{array}$ & Withdrawn & $n / a$ \\
\hline 3640 & IEEE & IEEE 387 & $\mathrm{~N} / \mathrm{S}$ & $\begin{array}{l}\text { Standard Criteria for Diesel- } \\
\text { Generator Units Applied as Standby } \\
\text { Power Supplies for Nuclear Power } \\
\text { Generating Stations } \\
\end{array}$ & srp9.5.8 & 1981 & $\mathbf{P}$ & $\begin{array}{l}\text { IEEE } 387 \text { withdrawn } \\
1993\end{array}$ & Withdrawn & $n / a$ \\
\hline 3641 & IEEE & IEEE 450 & 1975 & $\begin{array}{l}\text { Recommended Practice for } \\
\text { Maintenance, Testing, and } \\
\text { Replacement of Large Lead } \\
\text { Storage Batteries for Generating } \\
\text { Stations and Substations }\end{array}$ & inm-toc & 1993 & $\mathbf{R}$ & & IEEE 450 & 1987 \\
\hline 3642 & IEEE & IEEE 450 & $N / S$ & $\begin{array}{l}\text { Recommended Practice for } \\
\text { Maintenance, Testing, and } \\
\text { Replacement of Large Lead } \\
\text { Storage Batteries for Generating } \\
\text { Stations and Substations }\end{array}$ & inm70340B & 1981 & $\mathbf{R}$ & & IEEE 450 & 1987 \\
\hline 3643 & IEEE & IEEE 450 & 1975 & $\begin{array}{l}\text { Recommended Practice for } \\
\text { Maintenance, Testing, and } \\
\text { Replacement of Large Lead } \\
\text { Storage Batterles for Generating } \\
\text { Stations and Substations }\end{array}$ & inm70440B & 1979 & $\bar{R}$ & & IEEE 450 & 1987 \\
\hline
\end{tabular}


Codes and Standards and Other Guidance Clted in Regulatory Documents

Part A - Industry Consensus Codes and Standards (continued)

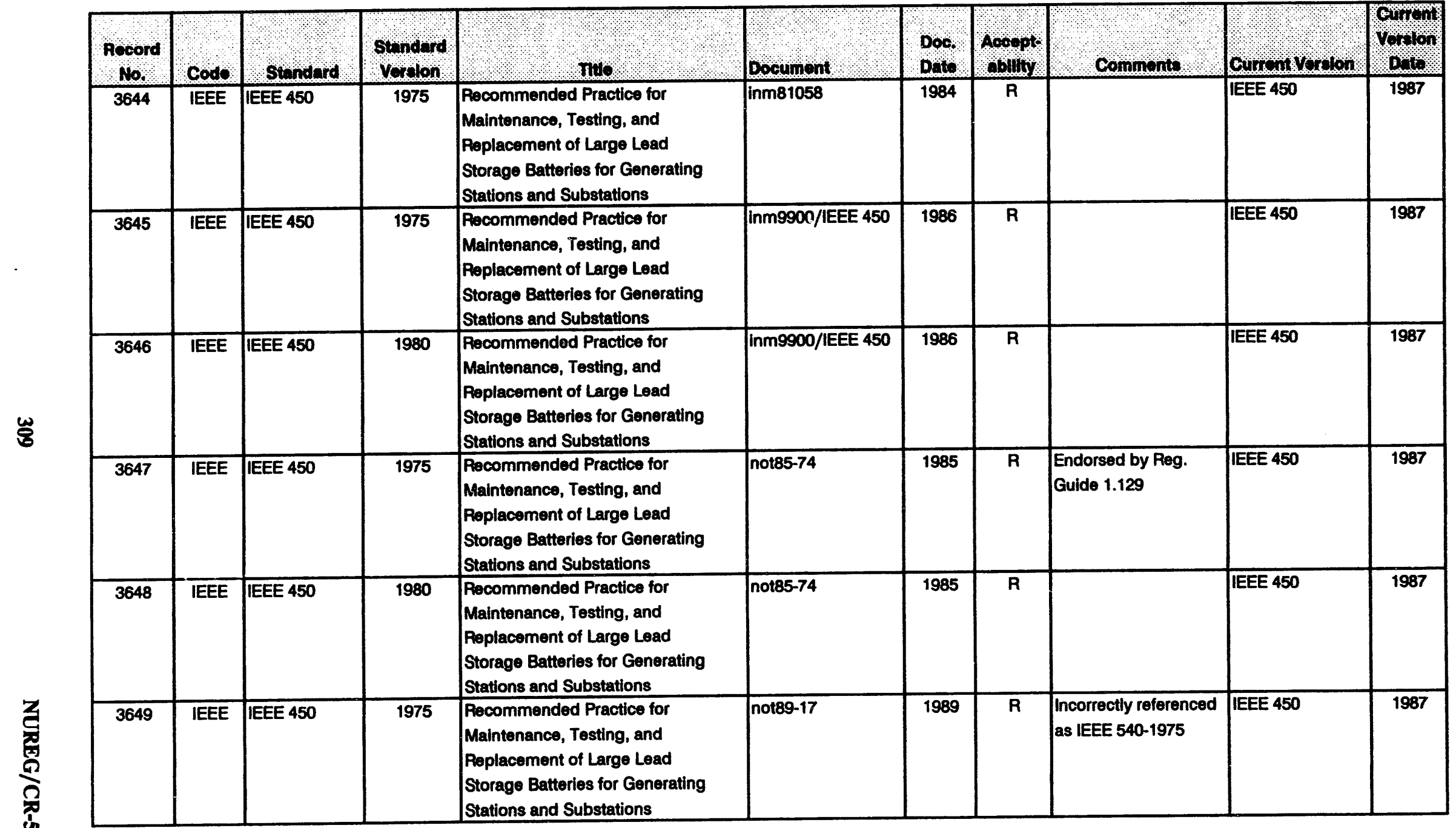


Codes and Standards and Other Guidance Cited in Regulatory Documents

Part A - Industry Consensus Codes and Standards (continued)

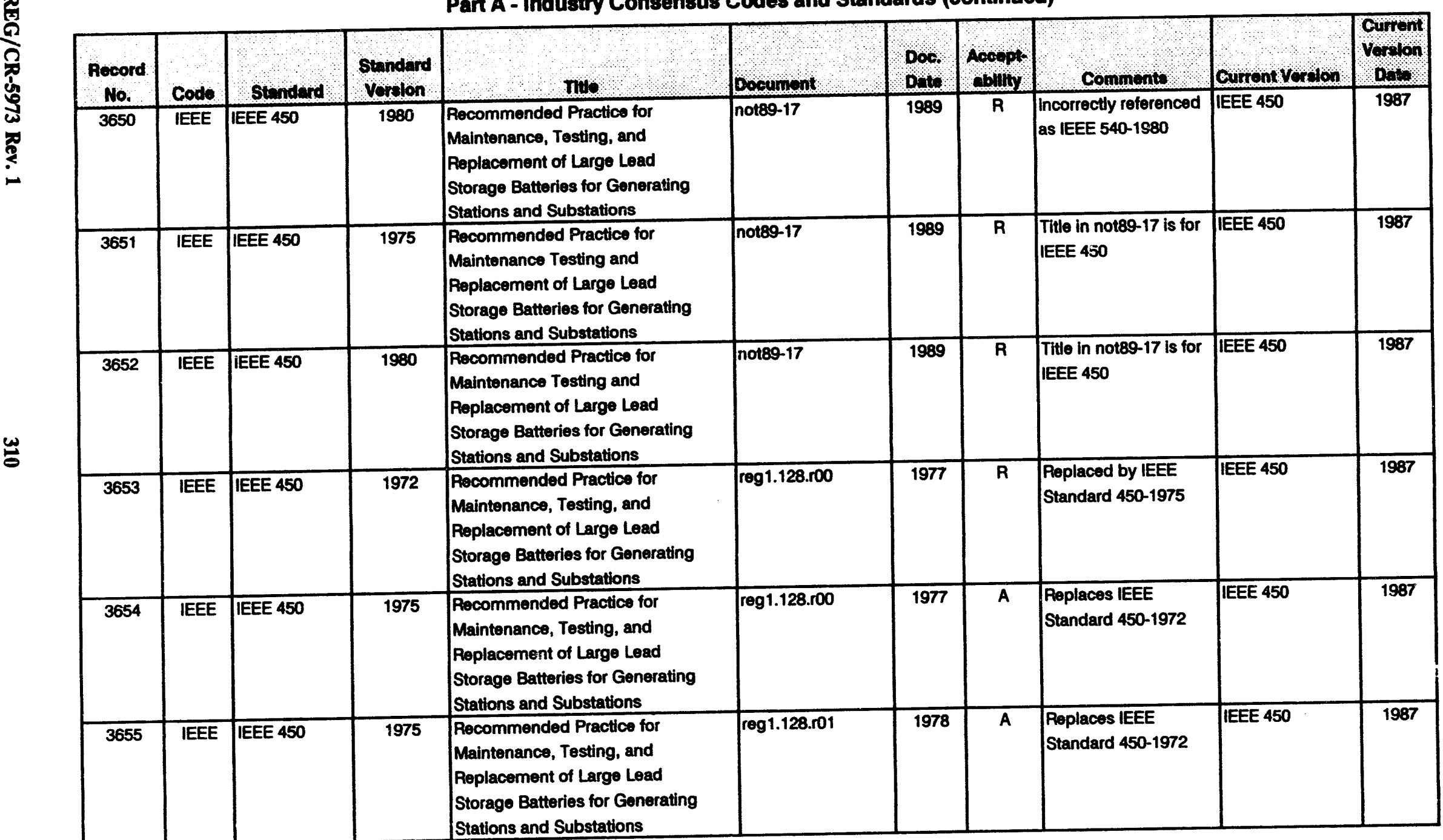


Codes and Standards and Other Guidance Cited in Regulatory Documents

Part A - Industry Consensus Codes and Standards (continued)

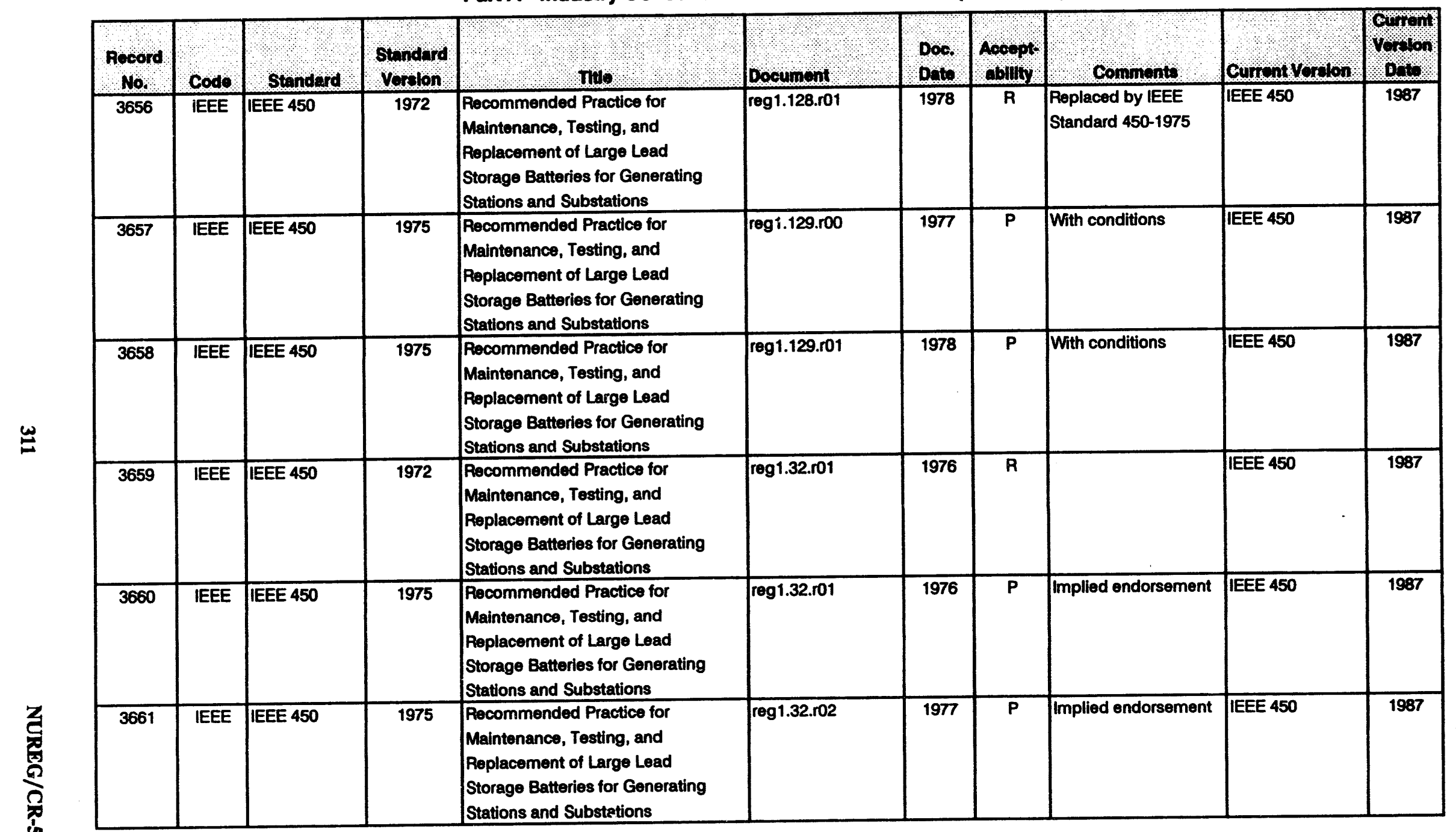




\begin{tabular}{|c|c|c|c|c|c|c|c|c|c|c|}
\hline \multicolumn{11}{|c|}{$\begin{array}{l}\text { Codes and Standards and Other Guidance Clted in Regulatory Documents } \\
\text { Part A - Industry Consensus Codes and Standards (continued) }\end{array}$} \\
\hline $\begin{array}{l}\text { Pocord } \\
\text { No. }\end{array}$ & code & Standerd & $\begin{array}{l}\text { Stundard: } \\
\text { Vorelon }\end{array}$ & s mo & Document & Doc. & $\begin{array}{l}\text { Aocopt: } \\
\text { abilty }\end{array}$ & Comments & Curront Vorelon & Gorron \\
\hline 3662 & IEEE & IEEE 450 & 1972 & \begin{tabular}{|l|} 
Recommended Practice for \\
Maintenance, Tosting, and \\
Replacement of Large Lead \\
Storage Batteries for Generating \\
Stations and Substations
\end{tabular} & reg1.32.ro2 & 1977 & $P$ & & IEEE 450 & 1987 \\
\hline 3663 & IEEE & IEEE 450 & 1975 & $\begin{array}{l}\text { Recommended Practice for } \\
\text { Maintenance, Testing, and } \\
\text { Replacement of Large Lead } \\
\text { Storage Batteries for Generating } \\
\text { Stations and Substations }\end{array}$ & reg5.44.102 & 1980 & $\mathbf{R}$ & $\begin{array}{l}\text { Endorsed by Reg. } \\
\text { Guide } 1.129\end{array}$ & IEEE 450 & 1987 \\
\hline 3664 & IEEE & IEEE 450 & N/S & $\begin{array}{l}\text { Recommended Practice for } \\
\text { Maintenance, Testing, and } \\
\text { Replacoment of Large Lead } \\
\text { Storage Batteries for Generating } \\
\text { Stations and Substations }\end{array}$ & srp8.3.2 & 1981 & $\overline{\mathbf{P}}$ & $\begin{array}{l}\text { Implied with Reg. } \\
\text { Guide } 1.129\end{array}$ & IEEE 450 & 1987 \\
\hline 3665 & IEEE & IEEE 450 & 1987 & $\begin{array}{l}\text { Recommended Practice for } \\
\text { Maintenance, Testing, and } \\
\text { Replacement of Large Lead } \\
\text { Storage Batteries for Generating } \\
\text { Stations and Substations }\end{array}$ & stsb\&wB3.8.4 & 1991 & $\mathbf{P}$ & & IEEE 450 & 1987 \\
\hline 3606 & IEEE & IEEE 450 & 1980 & $\begin{array}{l}\text { Recommended Practice for } \\
\text { Maintenance, Tosting, and } \\
\text { Replacement of Large Lead } \\
\text { Storage Batteries for Generating } \\
\text { Stations and Substations } \\
\end{array}$ & stsb\&wB3.8.6 & 1991 & $\bar{P}$ & & IEEE 450 & 1987 \\
\hline 3667 & IEEE & IEEE 450 & 1987 & $\begin{array}{l}\text { Recommended Practice for } \\
\text { Maintenance, Testing, and } \\
\text { Replacement of Large Lead } \\
\text { Storage Batteries for Generating } \\
\text { Stations and Substations }\end{array}$ & stsceB3.8.4 & 1991 & $\bar{P}$ & & IEEE 450 & 1987 \\
\hline
\end{tabular}


Codes and Standards and Other Guidance Cited in Regulatory Documents

Part A - Industry Consensus Codes and Standards (continued)

\begin{tabular}{|c|c|c|c|c|c|c|c|c|c|c|}
\hline $\begin{array}{c}\text { Aecord } \\
\text { No. }\end{array}$ & Code & Standard & $\begin{array}{l}\text { Sthndard } \\
\text { Vordion }\end{array}$ & THo. & bocumont & $\begin{array}{l}\text { Doc: } \\
\text { Duts: }\end{array}$ & $\begin{array}{l}\text { Acoeppt. } \\
\text { ablitity }\end{array}$ & Comments & curront Vorton & $\begin{array}{l}\text { Curront } \\
\text { Vorton } \\
\text { powo }\end{array}$ \\
\hline 3668 & IEEE & IEEE 450 & 1980 & $\begin{array}{l}\text { Recommended Practice for } \\
\text { Maintenance, Testing, and } \\
\text { Replacement of Large Lead } \\
\text { Storage Batterles for Generating } \\
\text { Stations and Substations }\end{array}$ & stsceB3.8.6 & 1991 & $P$ & & IEEE 450 & 1987 \\
\hline 3669 & IEEE & IEEE 450 & N/S & \begin{tabular}{|l|} 
Recommended Practice for \\
Maintenance, Testing, and \\
Replacement of Large Lead \\
Storage Batteries for Generating \\
Stations and Substations \\
\end{tabular} & sts4-geB3.8.4 & 1991 & $\mathbf{P}$ & & IEEE 450 & 1987 \\
\hline 3670 & IEEE & IEEE 450 & 1987 & $\begin{array}{l}\text { Recommended Practice for } \\
\text { Maintenance, Testing, and } \\
\text { Replacement of Large Lead } \\
\text { Storage Batteries for Generating } \\
\text { Stations and Substations }\end{array}$ & sts4-geB3.8.6 & 1991 & $\mathbf{P}$ & & $\mid$ IEEE 450 & 1987 \\
\hline 3671 & IEEE & IEEE 450 & 1987 & $\begin{array}{l}\text { Recommended Practice for } \\
\text { Maintenance, Testing, and } \\
\text { Replacement of Large Lead } \\
\text { Storage Batteries for Generating } \\
\text { Stations and Substations }\end{array}$ & sts4-geB5.5 & 1991 & $\mathbf{P}$ & & $\begin{array}{l}\text { IEEE } 450 \\
\end{array}$ & 1987 \\
\hline 3672 & IEEE & IEEE 450 & 1987 & $\begin{array}{l}\text { Recommended Practice for } \\
\text { Maintenance, Testing, and } \\
\text { Replacement of Large Lead } \\
\text { Storage Batteries for Generating } \\
\text { Stations and Substations }\end{array}$ & sts6-geB3.8.4 & 1991 & $\bar{P}$ & & IEEE 450 & 1987 \\
\hline 3673 & IEEE & IEEE 450 & 1987 & $\begin{array}{l}\text { Recommended Practice for } \\
\text { Maintenance, Testing, and } \\
\text { Replacement of Large Lead } \\
\text { Storage Batteries for Generating } \\
\text { Stations and Substations }\end{array}$ & sts6-geB3.8.6 & 1991 & $P$ & & IEEE 450 & 1987 \\
\hline
\end{tabular}




\begin{tabular}{|c|c|c|c|c|c|c|c|c|c|c|}
\hline \multicolumn{11}{|c|}{$\begin{array}{l}\text { Codes and Standards and Other Guidance Cited in Regulatory Documents } \\
\text { Part A - Industry Consensus Codes and Standards (continued) }\end{array}$} \\
\hline $\begin{array}{c}\text { Pocord } \\
\text { no. }\end{array}$ & Code & Standard & $\begin{array}{l}\text { Standard } \\
\text { Voraton }\end{array}$ & ness. & Document & $\begin{array}{l}\text { Doo } \\
\text { Det }\end{array}$ & Accepts & Commonts & Cunsut Vorton: & Vorion \\
\hline 3674 & IEEE & IEEE 450 & 1987 & $\begin{array}{l}\text { Recommended Practice for } \\
\text { Maintenance, Testing, and } \\
\text { Replacement of Large Lead } \\
\text { Storage Batteries for Generating } \\
\text { Stations and Substations } \\
\end{array}$ & stswstB3.8.4 & 1991 & $P$ & & IEEE 450 & 1987 \\
\hline 3675 & IEEE & IEEE 450 & 1980 & $\begin{array}{l}\text { Recommended Practice for } \\
\text { Maintenance, Testing, and } \\
\text { Replacement of Large Lead } \\
\text { Storage Batteries for Generating } \\
\text { Stations and Substations }\end{array}$ & stswstB3.8.6 & 1991 & $\mathbf{P}$ & & IEEE 450 & 1987 \\
\hline 3676 & IEEE & IEEE 467 & 1980 & $\begin{array}{l}\text { Standard Quality Assurance } \\
\text { Program Requirements for the } \\
\text { Design and Manufacture of Class IE } \\
\text { Instrumentation and Electric } \\
\text { Equipment for Nuclear Power } \\
\text { Gonerating Stations } \\
\end{array}$ & reg1.152.ro0 & 1985 & $\mathbf{R}$ & & IEEE 467 & 1980 \\
\hline 3677 & IEEE & IEEE 484 & N/S & $\begin{array}{l}\text { Recommended Practico for } \\
\text { Installation Design and Installation } \\
\text { of Large Lead Storage Batteries for } \\
\text { Generating Stations and } \\
\text { Substations } \\
\end{array}$ & inm51051 & 1984 & $\mathbf{R}$ & & IEEE 484 & 1987 \\
\hline 3678 & IEEE & IEEE 484 & $\mathrm{~N} / \mathrm{S}$ & $\begin{array}{l}\text { Recommended Practice for } \\
\text { Installation Design and Installation } \\
\text { of Large Lead Storage Batteries for } \\
\text { Generating Stations and } \\
\text { Substations } \\
\end{array}$ & inm51053 & 1984 & $\mathbf{R}$ & & IEEE 484 & 1987 \\
\hline 3679 & IEEE & IEEE 484 & N/S & $\begin{array}{l}\text { Precommended Practice for } \\
\text { Installation Design and Installation } \\
\text { of Large Lead Storage Batteries for } \\
\text { Generating Stations and } \\
\text { Substations }\end{array}$ & inm51055 & 1984 & $\mathbf{R}$ & & IEEE 484 & 1987 \\
\hline
\end{tabular}


Codes and Standards and Other Guidance Clted in Regulatory Documents

Part A - Industry Consensus Codes and Standards (continued)

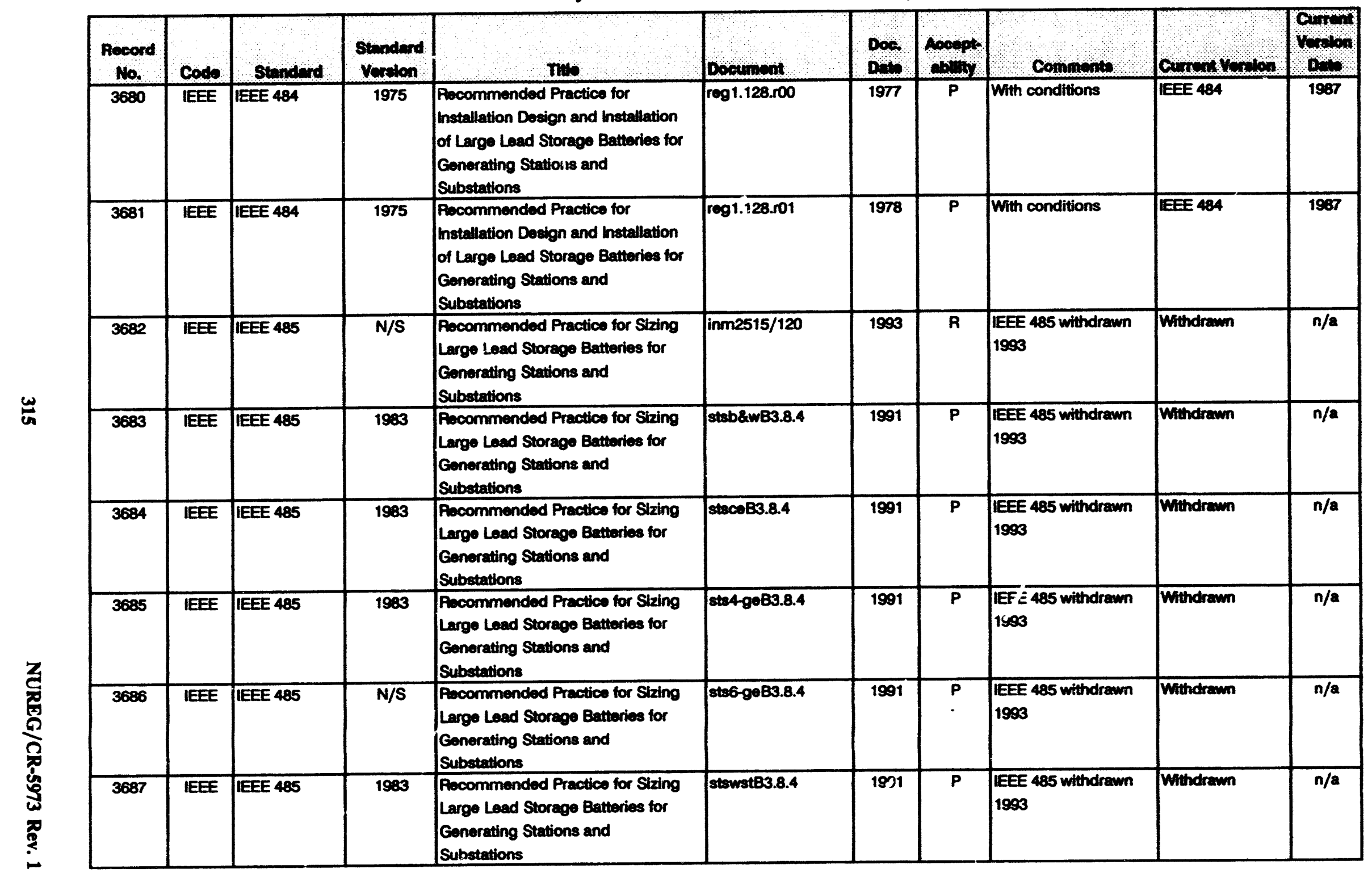


Codes and Standards and Other Guidance Cited in Regubatory Documents

Part A - Indusiry Consensus Codes and Standards (continued)

\begin{tabular}{|c|c|c|c|c|c|c|c|c|c|c|}
\hline $\begin{array}{l}\text { Aecord } \\
\text { No. }\end{array}$ & Code & sindend & $\begin{array}{l}\text { Standerd } \\
\text { Vercion }\end{array}$ & mo & Docoursent & $\begin{array}{l}\text { Doe. } \\
\text { Dens }\end{array}$ & Alocept & Commenes & Consene Veriton & 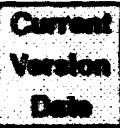 \\
\hline 3688 & IEEE & EEEE 497 & 1981 & $\begin{array}{l}\text { Standard Criteria for Accident } \\
\text { Monitoring Instrumentation for } \\
\text { Nuclear Power Gonereting Stations }\end{array}$ & inm82102 & 1993 & $\mathrm{R}$ & $\begin{array}{l}\text { EEE } 497 \text { withdrawn } \\
1993\end{array}$ & Withorawen & $n / 2$ \\
\hline 3689 & IEEE & IEEE 497 & 1977 & $\begin{array}{l}\text { Standard Criteria for Accidient } \\
\text { Monitoring Instrumentation for } \\
\text { Nuclear Power Genorating Stations }\end{array}$ & 1091.153 .500 & 1985 & $\mathbf{R}$ & $\begin{array}{l}\text { EEEE } 497 \text { withdrawn } \\
1993\end{array}$ & Withotramen & $\mathbf{n} / \mathbf{a}$ \\
\hline 3690 & IEEE & IEEE 497 & 1977 & $\begin{array}{l}\text { Standard Criteria for Accident } \\
\text { Mloniboring Instrumentation for } \\
\text { Nuclear Power Generating Stations }\end{array}$ & reg1.97.103 & 1983 & $\bar{R}$ & $\begin{array}{l}\text { IEEE } 497 \text { withdrawn } \\
1993\end{array}$ & Withdrawn & $n / \mathbf{a}$ \\
\hline 3691 & IEEE & IEFE 498 & 1980 & $\begin{array}{l}\text { Standard Fequirements for the } \\
\text { Calloration and Control of } \\
\text { Moasuring and Teat Equipment } \\
\text { Ubed in Nuclear Fecillties }\end{array}$ & $\operatorname{lnm} 35750$ & 1992 & $\bar{A}$ & & EEE 498 & 1990 \\
\hline 3092 & IEEE & IEEE 500 & 197 & 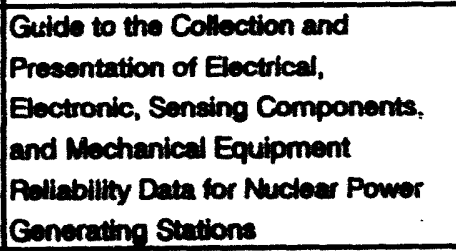 & bul180-19.101 & 1900 & $\mathbf{R}$ & & FEx 500 & 1994 PO1 \\
\hline 3693 & IEEE & IEEE 500 & 1984 & $\begin{array}{l}\text { Guide to tho Collection and } \\
\text { Prosentation of Electrical, } \\
\text { Electronic, Sensing Components, } \\
\text { and Mochanical Equipment } \\
\text { Prollability Data for Nuclear Power } \\
\text { Generating Stations }\end{array}$ & not93-64 & 1993 & $\bar{R}$ & & IEEX 500 & $1934 \mathrm{FP1}$ \\
\hline 3604 & IEEE & IEEE 500 & 1977 & $\begin{array}{l}\text { Gulde to the Collection and } \\
\text { Presentation of Eloctrical, } \\
\text { Electronic, Sensing Components, } \\
\text { and Mochanical Equipment } \\
\text { Poliabilly Data for Nuclear Power } \\
\text { Gonerating Stations }\end{array}$ & nurego933, 3.2 & 1992 & $\overline{\mathbf{R}}$ & & TEEE 500 & 1994 R91 \\
\hline 3695 & IEEE & IEEE 535 & 1979 & $\begin{array}{l}\text { Standard for Qualification of Class } \\
\text { IE Load Storage Batteries for } \\
\text { Nuclear Powor Generating Stations }\end{array}$ & not:33-11 & 1983 & $\overline{\mathbf{R}}$ & Referred to in toxt & IEEE 535 & 1986 \\
\hline
\end{tabular}


Codes and Standards and Other Guidance Ched in Regulatory Documents

Part A - Industry Consensus Codes and Standards (continued)

\begin{tabular}{|c|c|c|c|c|c|c|c|c|c|c|}
\hline $\begin{array}{l}\text { Pecord } \\
\text { No. }\end{array}$ & Cods & Standend & $\begin{array}{l}\text { Sonnderd } \\
\text { Voulon }\end{array}$ & 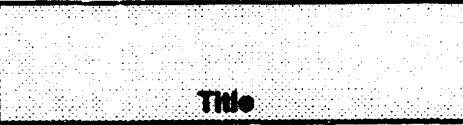 & Dovewnont & Doe. & Aocept & Comenents & 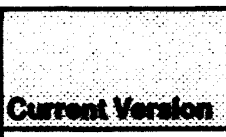 & Cownt \\
\hline 3696 & IEEE & IEEE 535 & 1986 & $\begin{array}{l}\text { Standard for Cualification of Class } \\
\text { IE Load Storage Batteries for } \\
\text { Nuclear Power Generating Stations }\end{array}$ & reg1.158.r00 & 1989 & $A$ & & IEEE 535 & 1986 \\
\hline 3697 & IEEE & IEEE 535 & 1979 & $\begin{array}{l}\text { Standard for Qualification of Class } \\
\text { IE Lead Storage Batteries for } \\
\text { Nuclear Power Generating Stations }\end{array}$ & reg1.158.roo & 1989 & $\bar{R}$ & & IEEE 535 & 1966 \\
\hline 3698 & IEEE & IEEE 535 & $N / S$ & $\begin{array}{l}\text { Standard for Qualification of Class } \\
\text { IE Lead Storage Batteries for } \\
\text { Nuclear Power Generating Stations }\end{array}$ & $\operatorname{sep} 3.11$ & 1981 & $\mathbf{R}$ & $\begin{array}{l}\text { May be used for } \\
\text { guidance }\end{array}$ & IEEE 535 & 1986 \\
\hline 3699 & IEEE & IEEE 572 & 1985 & $\begin{array}{l}\text { Standard for Quallification of Class } \\
\text { IE Connection Assemblles for } \\
\text { Nuclear Power Generating Stations }\end{array}$ & reg1.156.100 & 1987 & $\bar{A}$ & $\begin{array}{l}\text { Endorsed without } \\
\text { exceptions }\end{array}$ & IEEE 572 & 1985 \\
\hline 3700 & IEEE & IEEE 603 & 1977 & $\begin{array}{l}\text { Standard Criteria for Safety } \\
\text { Systems for Nuclear Power } \\
\text { Generating Stations }\end{array}$ & nureg0933, 1.II.F & 1989 & $\overline{\mathbf{R}}$ & & IEEE 603 & 1991 \\
\hline 3701 & IEEE & IEEE 603 & $\mathrm{~N} / \mathrm{S}$ & $\begin{array}{l}\text { Standard Criteria for Safety } \\
\text { Systems for Nuclear Power } \\
\text { Generating Stations }\end{array}$ & nureg0933, 3.110 & 1992 & $\mathbf{R}$ & & IEEE 603 & 1991 \\
\hline 3702 & IEEE & IEEE 603 & $N / S$ & $\begin{array}{l}\text { Standard Criteria for Safety } \\
\text { Systems for Nuclear Power } \\
\text { Genorating Stations }\end{array}$ & nureg0933, 3.128 & 1991 & $\mathbf{R}$ & & IEEE 603 & 1991 \\
\hline 3703 & IEEE & IEEE 603 & 1980 & $\begin{array}{l}\text { Standard Criteria for Safety } \\
\text { Systems for Nuclear Power } \\
\text { Generating Stations }\end{array}$ & $\operatorname{reg} 1.152 .00$ & 1985 & $\bar{R}$ & & IEEE 603 & 1991 \\
\hline 3704 & IEEE & IEEE 603 & 1980 & $\begin{array}{l}\text { Standard Criteria for Safety } \\
\text { Systems for Nuclear Power } \\
\text { Generating Stations }\end{array}$ & reg1.153.r00 & 1985 & $\bar{P}$ & $\begin{array}{l}\text { With modifications and } \\
\text { supplements }\end{array}$ & IEEE 603 & 1991 \\
\hline 3705 & IEEE & IEEE 627 & $N / S$ & $\begin{array}{l}\text { Standard for Design Qualification of } \\
\text { Safety Systems Equipment Used in } \\
\text { Nuclear Power Generating Stations }\end{array}$ & $\operatorname{sip} 3.11$ & 1981 & $\mathbf{R}$ & $\begin{array}{l}\text { May be used for } \\
\text { guidance }\end{array}$ & IEEE 627 & 1980 \\
\hline 3706 & IEEE & IEEE 634 & 1978 & $\begin{array}{l}\text { Standard Cable Penetration Fire } \\
\text { Stop Qualification Test }\end{array}$ & drgrs809-5 & 1979 & $P$ & $\begin{array}{l}\text { IEEE } 634 \text { withdrawn } \\
1990\end{array}$ & Withdrawn & $n / a$ \\
\hline 3707 & IEEE & IEEE 634 & $N / S$ & $\begin{array}{l}\text { Standard Cable Penetration Fire } \\
\text { Stop Qualification Test }\end{array}$ & inm51061 & 1984 & $\bar{R}$ & $\begin{array}{l}\text { IEEE } 634 \text { withdrawn } \\
1990\end{array}$ & Withdrawn & $n / a$ \\
\hline
\end{tabular}




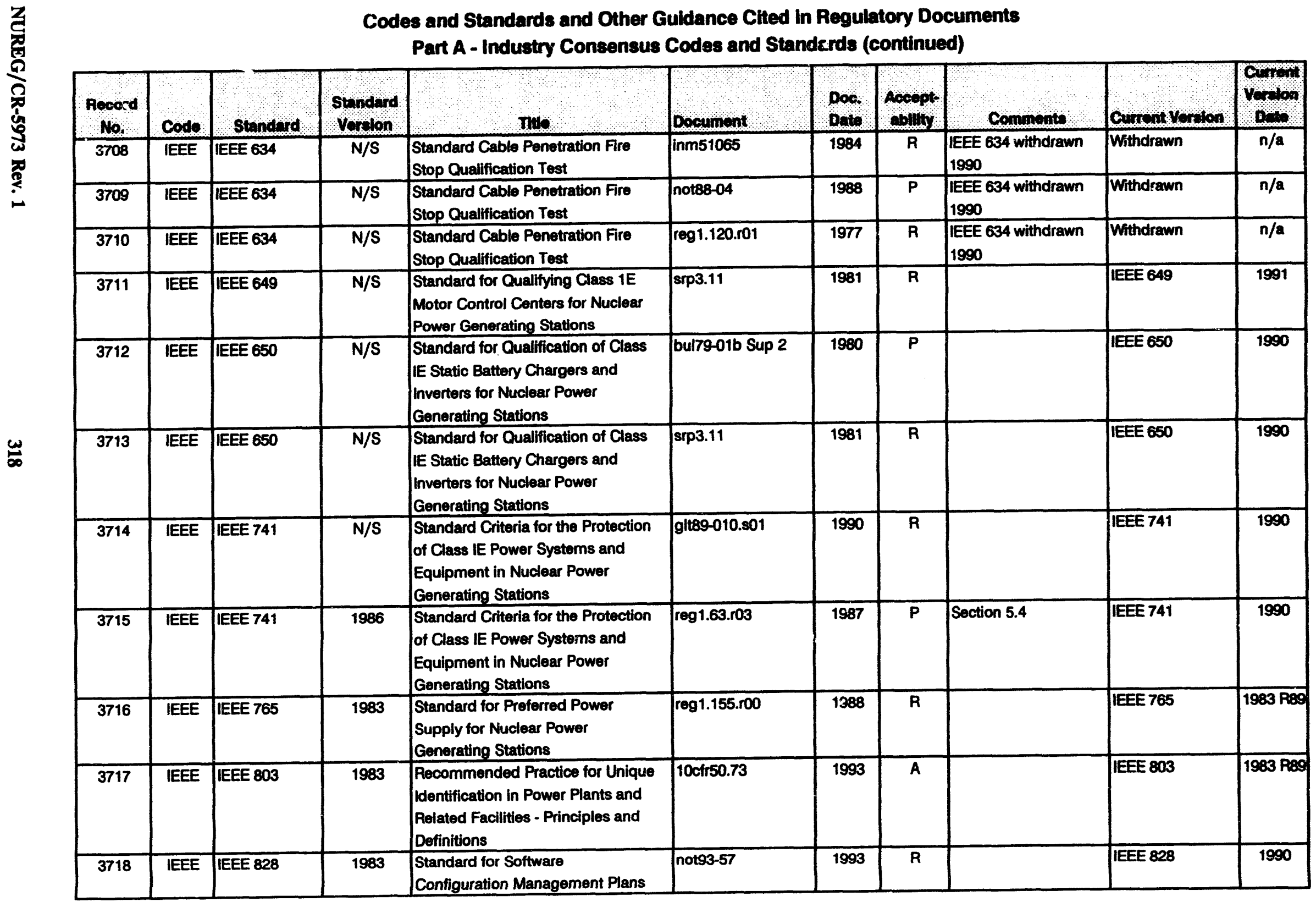


Codes and Standards and Other Guldance Chted in Regulatory Documents

Part A - Industry Consensus Codes and Standards (continued)

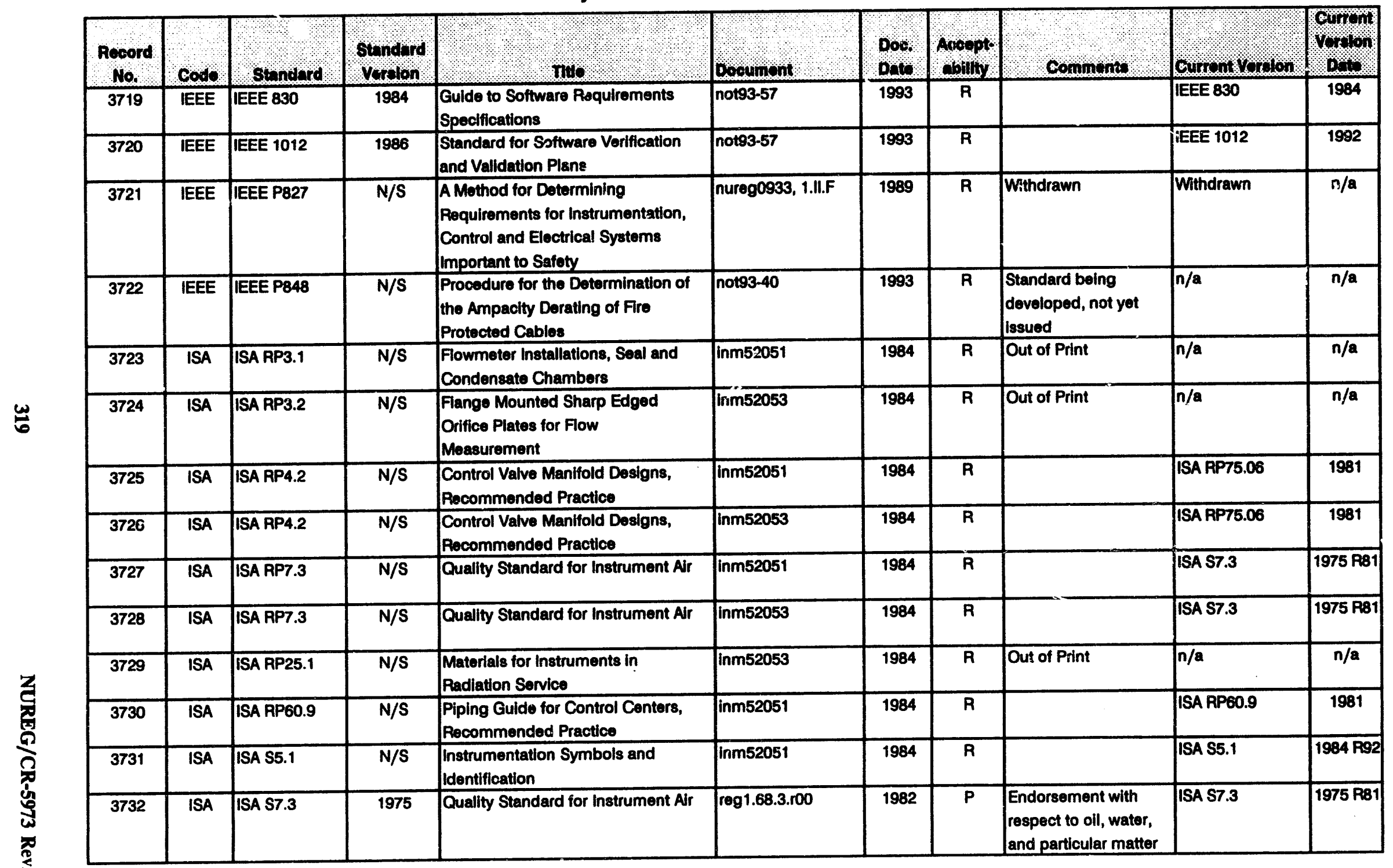


Codes and Standards and Other Guidance Cited in Regulatory Documents

Part A - Industry Consensus Codes and Standards (continued)

\begin{tabular}{|c|c|c|c|c|c|c|c|c|c|c|}
\hline $\begin{array}{l}\text { Pocord } \\
\text { No. }\end{array}$ & codo & Standind & $\begin{array}{l}\text { Standard } \\
\text { volon }\end{array}$ & 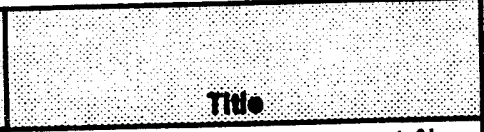 & Boowunoll & Doo. & pooppts & comnonts & 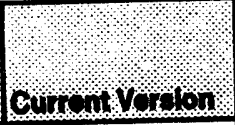 & $0,+1$ \\
\hline 3733 & ISA & ISA S7.3 & 1975 & Quality Standard for Instrument Air & reg1.68.r03 & 1987 & $\mathbf{P}$ & & ISA S7.3 & 1975 RB1 \\
\hline 3734 & ISA & ISA S7.3 & $N / S$ & Quality Standard for Instrument Air & $\operatorname{srp9.3.1}$ & 1981 & $\mathbf{P}$ & Implied endorsement & ISA S7.3 & 1975 RB1 \\
\hline 3735 & ISA & ISA S7.4 & $\mathrm{N} / \mathrm{S}$ & $\begin{array}{l}\text { Air Pressures for Pneumatic } \\
\text { Controllers, Transmitters, and } \\
\text { Transmission Systems }\end{array}$ & inm52053 & 1984 & $\mathbf{R}$ & & 1 ISA S7.4 & 1981 \\
\hline 3736 & ISA & ISA S26 & $\mathrm{N} / \mathrm{S}$ & $\begin{array}{l}\text { Dynamic Response Testing of } \\
\text { Process Control Instrumentation }\end{array}$ & inm52051 & 1984 & $\mathbf{R}$ & & ISA S26 & 1968 \\
\hline 3737 & ISA & ISA S37.1 & $\mathrm{N} / \mathrm{S}$ & $\begin{array}{l}\text { Electrical Transducer Nomenclature } \\
\text { and Terminology }\end{array}$ & inm52051 & 1984 & $\mathbf{A}$ & & ISA S37.1 & 1975 R82 \\
\hline 3738 & ISA & ISA S50.1 & $\mathrm{N} / \mathrm{S}$ & $\begin{array}{l}\text { Compatibility of Analog Signals for } \\
\text { Electronic Industrial Process } \\
\text { Instruments }\end{array}$ & inm52051 & 1984 & $\mathbf{R}$ & & ISA S50.1 & $1982 \mathrm{Rg} 2$ \\
\hline 3739 & ISA & ISA S51.1 & $N / S$ & $\begin{array}{l}\text { Process instrumentation } \\
\text { Terminology }\end{array}$ & inm52051 & 1984 & $\bar{R}$ & & ISA S51.1 & 1979 \\
\hline 3740 & ISA & ISA S67.01 & 1979 & $\begin{array}{l}\text { Transducer and Transmitter } \\
\text { Installation For Nuclear Safety } \\
\text { Applications }\end{array}$ & drglc131-5 & 1982 & $\mathbf{P}$ & & ISA S67.01 & 1979 PB7 \\
\hline 3741 & ISA & ISA S67.01 & $\mathrm{N} / \mathrm{S}$ & $\begin{array}{l}\text { Transducer and Transmitter } \\
\text { Installation For Nuclear Safety } \\
\text { Applications }\end{array}$ & inm52051 & 1984 & $\mathbf{R}$ & & ISA S67.01 & 1979 R87 \\
\hline 3742 & ISA & ISA S67.01 & $N / S$ & $\begin{array}{l}\text { Transducer and Transmitter } \\
\text { Installation For Nuclear Safety } \\
\text { Applications }\end{array}$ & inm52053 & 1984 & $\mathbf{R}$ & & ISA S67.01 & 1979 P87 \\
\hline 3743 & ISA & ISA S67.02 & $\mathbf{N} / \mathbf{S}$ & $\begin{array}{l}\text { Nuclear-Safety-Related Instrument } \\
\text { Sensing Line Piping and Tubing } \\
\text { Standards for Use in Nuclear Power } \\
\text { Plants }\end{array}$ & inm52051 & 1984 & $\mathbf{R}$ & & ISA S67.02 & 1980 \\
\hline 3744 & ISA & ISA S67.02 & $N / S$ & $\begin{array}{l}\text { Nuclear-Safety-Relater Instrument } \\
\text { Sensing Line Piping and Tubing } \\
\text { Standards for Use in Nuclear Power } \\
\text { Plants }\end{array}$ & inm52053 & 1984 & R & & ISA S67.02 & 1980 \\
\hline
\end{tabular}

Plants 
Codes and Standards and Other Guidance Chted in Regulatory Documents

Part A - Industry Consensus Codes and Standards (continued)

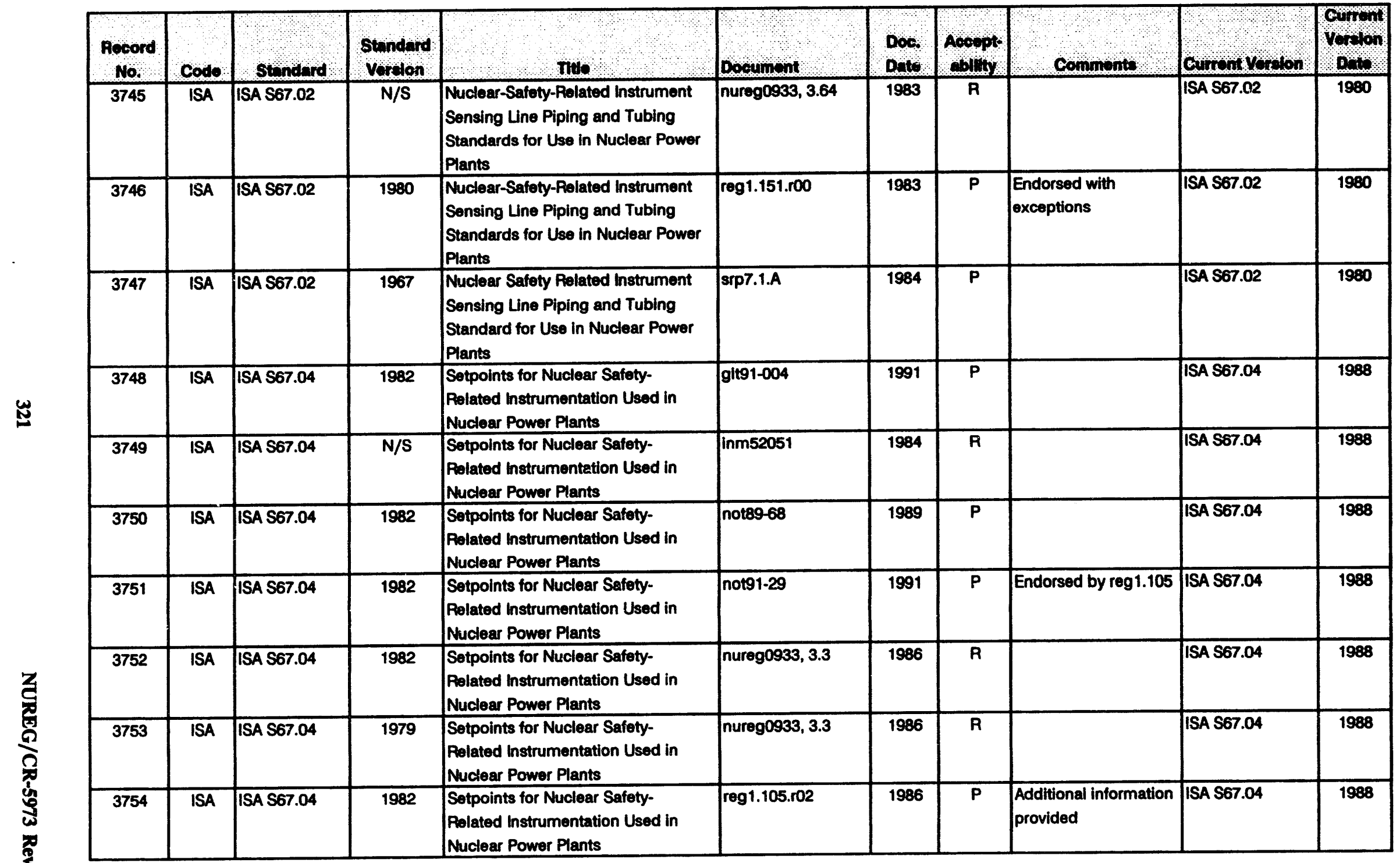




\begin{tabular}{|c|c|c|c|c|c|c|c|c|c|c|}
\hline \multicolumn{11}{|c|}{$\begin{array}{l}\text { Codes and Standards and Other Guidance Clted in Regulatory Documents } \\
\text { Part A - Industry Consensus Codes and Standards (continued) }\end{array}$} \\
\hline $\begin{array}{l}\text { Rocord } \\
\text { No. }\end{array}$ & Codo: & Standard & $\begin{array}{l}\text { Stundard } \\
\text { Voralon }\end{array}$ & 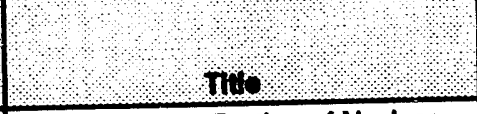 & Bocoumont & Doo. & $\begin{array}{l}\text { Acoopt: } \\
\text { abilly }\end{array}$ & Comments: & Cuncent Vordon. & vorion \\
\hline 3755 & ISA & ISA-dS67.06 & 1980 & $\begin{array}{l}\text { Response Time Testing of Nuclear } \\
\text { Safety-Related Instrument } \\
\text { Channels in Nuclear Power Plants }\end{array}$ & drgic121-5 & 1982 & $P$ & & ISA S67.06 & 1984 \\
\hline 3756 & ISO & 150389 & 1975 & $\begin{array}{l}\text { Acoustics Standard Peference Zero } \\
\text { for the Calibration of Pure-tone Alr } \\
\text { Conduction Audiometers }\end{array}$ & $10 \mathrm{ctr73}$ & 1993 & $\mathbf{R}$ & & 150389 & 1991 \\
\hline 3757 & ISO & ISO 1496 & 1978 & $\begin{array}{l}\text { Series } 1 \text { Frelght Containers - } \\
\text { Specification and Testing: General } \\
\text { Cargo Containers for General } \\
\text { Purposes }\end{array}$ & $10 \mathrm{ctr73}$ & 1993 & $\mathbf{R}$ & & ISO 1496 & 1993 \\
\hline 3758 & ISO & ISO 1496 & $\mathrm{~N} / \mathrm{S}$ & $\begin{array}{l}\text { Series } 1 \text { Freight Containers - } \\
\text { Specification and Testing: General } \\
\text { Cargo Containers for General } \\
\text { Purposes }\end{array}$ & inm81370 & 1986 & $\bar{R}$ & & ISO 1496 & 1993 \\
\hline 3759 & ISO & 1502889 & 1975 & $\begin{array}{l}\text { Genoral Principles for Sampling } \\
\text { Arborne Radioactivo Materials }\end{array}$ & reg4.15.ro1 & 1979 & $\mathbf{R}$ & & ISO 2889 & 1975 \\
\hline 3760 & ISO & ISO 2919 & 1980 & $\begin{array}{l}\text { Classification of Sealed Radioactive } \\
\text { Sources }\end{array}$ & reg10.11.r00 & 1987 & $\mathbf{R}$ & \begin{tabular}{|l|} 
ANSI N43.6 also \\
designatod as NBS \\
Handbook 126 \\
\end{tabular} & ANSI N43.6 & 1977 P89 \\
\hline 3761 & MSS & MSS-SP-25 & N/S & $\begin{array}{l}\text { Standard Marking System for } \\
\text { Valves, Fittings, Flanges, and } \\
\text { Unions }\end{array}$ & srp3.9.4 & 1984. & A & & MSS-SP-25 & 1993 \\
\hline 3762 & NBS & $\begin{array}{l}\text { NBS GCR 80- } \\
296\end{array}$ & 1981 & $\begin{array}{l}\text { The Directory of Commercial } \\
\text { Calibration Services for lonizing } \\
\text { Radiation Survey Instruments }\end{array}$ & drgop32-5 & 1984 & $\mathbf{R}$ & & NBS GCR 80-296 & 1981 \\
\hline 3763 & NBS & $\begin{array}{l}\text { NBS } \\
\text { Handbook } 59 \\
\end{array}$ & 1966 & $\begin{array}{l}\text { Permissible Dose From External } \\
\text { Sources of lonizing Radiation }\end{array}$ & not86-23 & 1986 & $\bar{A}$ & & $\begin{array}{l}\text { NBS Hand Book } \\
59\end{array}$ & 1966 \\
\hline 3764 & NBS & $\begin{array}{l}\text { NBS } \\
\text { jHandbook } 59\end{array}$ & 1954 & \begin{tabular}{|l|} 
Permissible Dose From External \\
Sources of lonizing Radiation \\
\end{tabular} & reg8.18.ro1 & 1982 & $\bar{R}$ & & $\begin{array}{l}\text { NBS Hand Book } \\
59\end{array}$ & 1966 \\
\hline 3765 & NBS & $\begin{array}{l}\text { NBS } \\
\text { Handbook } 59\end{array}$ & $\mathrm{~N} / \mathrm{S}$ & \begin{tabular}{|l|} 
Permissible Dose From External \\
Sources of lonizing Radiation \\
\end{tabular} & inm9900/20 & 1970 & $\mathbf{R}$ & & $\begin{array}{l}\text { NBS Hand Book } \\
59\end{array}$ & 1966 \\
\hline
\end{tabular}




\section{Codes and Standards and Other Guidance Clted in Regulatory Documents}

Part A - Industry Consensus Codes and Standards (continued)

\begin{tabular}{|c|c|c|c|c|c|c|c|c|c|c|}
\hline Nocond & Codo & Stand hrd & $\begin{array}{l}\text { Sundard } \\
\text { Vortlon }\end{array}$ & ২ै। & Dooumont & Doof & hoopply & Commont. & counontrorowon & onin \\
\hline 3766 & NBS & $\begin{array}{l}\text { NBS } \\
\text { Handbook } 69\end{array}$ & 1959 & $\begin{array}{l}\text { Maximum Permissible Body } \\
\text { Burdens and Maximum Permissible } \\
\text { Concentrations of Radionuclides in } \\
\text { Air and in Water for Occupational } \\
\text { Exposure }\end{array}$ & 10 cfr 100 & 1993 & $\mathbf{R}$ & & NCRP 22 & 1959 \\
\hline 3767 & NBS & $\begin{array}{l}\text { NBS } \\
\text { Handbook } 69\end{array}$ & 1959 & $\begin{array}{l}\text { Maximum Permissible Body } \\
\text { Burdens and Maximum Permissible } \\
\text { Concentrations of Radionuclides in } \\
\text { Air and in Water for Occupational } \\
\text { Exposure }\end{array}$ & not84-40 & 1984 & $\mathbf{R}$ & & NCRP 22 & 1959 \\
\hline 3768 & NBS & $\begin{array}{l}\text { NBS } \\
\text { Handbook } 80\end{array}$ & $\mathrm{~N} / \mathrm{S}$ & $\begin{array}{l}\text { A Handbook of Radioactivity } \\
\text { Measurements }\end{array}$ & reg4.15.ro1 & 1979 & $\bar{R}$ & & NCRP Report 58 & 1985 \\
\hline 3769 & NBS & $\begin{array}{l}\text { NBS } \\
\text { Handbook 116 }\end{array}$ & 1975 & $\begin{array}{l}\text { Classification of Radioactive Self- } \\
\text { Luminous Light Sources }\end{array}$ & reg6.4.ro2 & 1980 & $\bar{R}$ & $\begin{array}{l}\text { ANSI N43.4 also } \\
\text { designated as NBS } \\
\text { Handbook } 116\end{array}$ & ANSI N43.4 & $1975 \mathrm{R} 89$ \\
\hline 3770 & NBS & \begin{tabular}{|l|} 
NBS \\
Handbook 126
\end{tabular} & 1977 & $\begin{array}{l}\text { Classification of Sealed Radioactive } \\
\text { Sources }\end{array}$ & reg6.4.r02 & 1980 & $\mathbf{R}$ & $\begin{array}{l}\text { ANSI N43.6 also } \\
\text { designated as NBS } \\
\text { Handbook } 126 \\
\end{array}$ & ANSI N43.6 & 1977 R89 \\
\hline 3771 & NBS & $\begin{array}{l}\text { NBS } \\
\text { Handbook 129 }\end{array}$ & 1979 & $\begin{array}{l}\text { Classification of Industrial lonizing } \\
\text { Radiation Gauging Devices }\end{array}$ & drgtp102-5 & 1980 & A & $\begin{array}{l}\text { ANSI N43.8 also } \\
\text { designated as NBS } \\
\text { Handbook } 129\end{array}$ & ANSI N43.8 & 1979 R88 \\
\hline 3772 & NBS & \begin{tabular}{|l|} 
NBS \\
Handbook 136
\end{tabular} & 1982 & $\begin{array}{l}\text { Gamma Radiography - } \\
\text { Specifications for Design and Test } \\
\text { of Apparatus }\end{array}$ & 10 cfr34 & 1993 & $\bar{A}$ & $\begin{array}{l}\text { ANSI N43.9 also } \\
\text { designated as NBS } \\
\text { Handbook } 136\end{array}$ & ANSI N43.9 & 1991 \\
\hline 3773 & NBS & $\begin{array}{l}\text { NBS Special } \\
\text { Publication } \\
510\end{array}$ & 1978 & $\begin{array}{l}\text { Tentative Provisions for the } \\
\text { Development of Seismic } \\
\text { Regulations for Buildings }\end{array}$ & drgce406-4 & 1986 & $\overline{\mathbf{R}}$ & Proposed reg3.48.ro1 & ATC 3-06 & 1984 \\
\hline 3774 & NBS & $\begin{array}{l}\text { NBS Special } \\
\text { Publication } \\
603 \\
\end{array}$ & 1981 & $\begin{array}{l}\text { Requirements for an Effective } \\
\text { National lonizing Radiation } \\
\text { Measurements Program }\end{array}$ & drgop32-5 & 1984 & $\bar{R}$ & & $\begin{array}{l}\text { NBS Special } \\
\text { Publication } 603\end{array}$ & 1981 \\
\hline 3775 & NBS & $\begin{array}{l}\text { NBS Special } \\
\text { Publication } \\
603\end{array}$ & 1981 & $\begin{array}{l}\text { Requirements for an Effective } \\
\text { National lonizing Radiation } \\
\text { Measurements Program }\end{array}$ & drgop212-4 & 1984 & $\bar{R}$ & & $\begin{array}{l}\text { NBS Special } \\
\text { Publication } 603\end{array}$ & 1981 \\
\hline
\end{tabular}




\begin{tabular}{|c|c|c|c|c|c|c|c|c|c|c|}
\hline \multicolumn{11}{|c|}{$\begin{array}{l}\text { Codes and Standards and Other Guidance Cited in Regulatory Documents } \\
\text { Part A - Industry Consensus Codes and Standards (continued) }\end{array}$} \\
\hline $\begin{array}{l}\text { Record } \\
\text { No. }\end{array}$ & Code. & Stunderd & $\begin{array}{l}\text { Standard } \\
\text { Vereton }\end{array}$ & Jillo & Document & $\begin{array}{l}\text { Doc. } \\
\text { Datos }\end{array}$ & Acoopt & Comments & Cursont Voston & vorion \\
\hline 3776 & NBS & $\begin{array}{l}\text { NBS Special } \\
\text { Publication } \\
633 \\
\end{array}$ & 1982 & $\begin{array}{l}\text { Procedures for Calibrating Noutron } \\
\text { Personnel Dosimeters }\end{array}$ & drgop32-5 & 1984 & $\mathbf{R}$ & & $\begin{array}{l}\text { NBS Special } \\
\text { Publication } 633\end{array}$ & 1982 \\
\hline 377 & NBS & NBSIR 73-107 & 1973 & $\begin{array}{l}\text { Improved Point Karnels for Electron } \\
\text { and Beta-Pay Dosimetry }\end{array}$ & reg1.109.ro1 & 1977 & $\bar{R}$ & & NBSIR 73-107 & 1973 \\
\hline 3778 & NBS & NBSIR 76-1050 & 1976 & Tornado-Borne Missile Speeds & srp3.5.1.4 & 1981 & $\mathbf{P}$ & & NBSIR 76-1050 & 1976 \\
\hline 3779 & NCMA & NCMA TR75B & 1979 & $\begin{array}{l}\text { Specification for the Design and } \\
\text { Construction of Load-Bearing } \\
\text { Concrete Masonry }\end{array}$ & srp3.8.4 & 1981 & $\bar{R}$ & & NCMA TR75B & 1987 \\
\hline 3780 & NCRP & $\begin{array}{l}\text { NCRP } \\
\text { Commentary } \\
\text { No. } 3 \\
\end{array}$ & 1989 & $\begin{array}{l}\text { Screoning Tochniques for } \\
\text { Determining Complianco with } \\
\text { Environmental Standards } \\
\end{array}$ & $\operatorname{drg} 8013.500$ & 1992 & A & & $\begin{array}{l}\text { NCRP } \\
\text { Commentary No. } \\
\end{array}$ & 1989 \\
\hline 3781 & NCRP & \begin{tabular}{|l|l} 
NCRP \\
Commentary \\
No.3
\end{tabular} & 1989 & $\begin{array}{l}\text { Screening Techniques for } \\
\text { Determining Complianco with } \\
\text { Environmental Standards }\end{array}$ & reg8.37.ro0 & 1993 & $\mathbf{P}$ & & $\begin{array}{l}\text { NCRP } \\
\text { Commentary No. } \\
3\end{array}$ & 1989 \\
\hline 3782 & NCRP & NCRP Report 8 & 1951 & $\begin{array}{l}\text { Control and Pemoval of } \\
\text { Radioactive Contamination in } \\
\text { Laboratories }\end{array}$ & reg8.21.ro1 & 1979 & $\mathbf{R}$ & & NCRP Peport 8 & 1951 \\
\hline 3783 & NCRP & NCRP Roport 8 & 1951 & $\begin{array}{l}\text { Control and Promoval of } \\
\text { Radioactive Contamination in } \\
\text { Laboratories }\end{array}$ & reg8.23.101 & 1981 & $\bar{R}$ & & NCRP Report 8 & 1951 \\
\hline 3784 & NCRP & NCRP Report \& & 1951 & $\begin{array}{l}\text { Control and Removal of } \\
\text { Radioactive Contamination in } \\
\text { Laboratories }\end{array}$ & reg8.24.ro1 & 1979 & $\bar{R}$ & & NCAP Report 8 & 1951 \\
\hline 3785 & NCRP & NCRP Peport 9 & 1951 & \begin{tabular}{|l|} 
Recommendations for Wasto \\
Disposal of Phosphorus-32 and \\
lodine-131 for Modical Users \\
\end{tabular} & reg8.21.ro1 & 1979 & R & Out of Print & $n / a$ & $n / a$ \\
\hline 3786 & NCRP & NCRP Report of & 1951 & $\begin{array}{l}\text { Pocommendations for Waste } \\
\text { Disposal of Phosphorus } 32 \text { and } \\
\text { lodine-131 for Medical Users }\end{array}$ & reg8.23.ro1 & 1981 & $\bar{R}$ & Out of Print & $n / a$ & $n / a$ \\
\hline 3787 & NCRP & $\begin{array}{l}\text { NCRP Report } \\
10\end{array}$ & N/S & \begin{tabular}{|l|} 
Instrumentation and Monitoring \\
Mothods for Radiation Protection \\
\end{tabular} & reg10.7.ro1 & 1979 & $\bar{R}$ & & NCRP Report 57 & 1978 \\
\hline 3788 & NCRP & $\begin{array}{l}\text { NCRP Report } \\
10\end{array}$ & 1952 & $\begin{array}{l}\text { Instrumentation and Monitoring } \\
\text { Mothods for Radiation Protection }\end{array}$ & reg8.21.ro1 & 1979 & $\overline{\mathbf{R}}$ & & NCRP Report 57 & 1978 \\
\hline
\end{tabular}




\section{Codes and Standards and Other Guidance Clted in Regulatory Documents}

Part A - Industry Consensus Codes and Standards (continued)

\begin{tabular}{|c|c|c|c|c|c|c|c|c|c|c|}
\hline $\begin{array}{l}\text { Record } \\
\text { No. }\end{array}$ & Code & Standard & Stundard & Tho & Dosument & Don. & apollty & Commente & Curront Vorolon & owtol, \\
\hline 3789 & NCRP & $\begin{array}{l}\text { NCRP Report } \\
10\end{array}$ & 1978 & $\begin{array}{l}\text { Instrumentation and Monitoring } \\
\text { Methods for Radiation Protection }\end{array}$ & reg8.23.ro1 & 1981 & R & & NCRP Report 57 & 1978 \\
\hline 3790 & NCRP & $\begin{array}{l}\text { NCRP Report } \\
17\end{array}$ & 1954 & $\begin{array}{l}\text { Recommendations on Limits for } \\
\text { Exposure to lonizing Radiation }\end{array}$ & reg8.18.ro1 & 1982 & $\mathbf{R}$ & & NCRP Report 91 & 1987 \\
\hline 3791 & NCRP & $\begin{array}{l}\text { NCRP Report } \\
17\end{array}$ & 1954 & $\begin{array}{l}\text { Recommendations on Limits for } \\
\text { Exposure to lonizing Radiation }\end{array}$ & reg8.31 & 1983 & $\mathbf{R}$ & & NCRP Report 91 & 1987 \\
\hline 3792 & NCRP & $\begin{array}{l}\text { NCRP Report } \\
28 \\
\end{array}$ & 1961 & $\begin{array}{l}\text { A Handbook of Radioactivity } \\
\text { Measurements }\end{array}$ & reg4.15.ro1 & 1979 & $\mathbf{R}$ & & NCRP Report 58 & 1985 \\
\hline 3793 & NCRP & $\begin{array}{l}\text { NCRP Report } \\
30\end{array}$ & 1964 & $\begin{array}{l}\text { Safe Handling of Radioactive } \\
\text { Materials }\end{array}$ & reg 10.8.ro2 & 1987 & $\mathbf{A}$ & & NCRP Report 30 & 1964 \\
\hline 3794 & NCRP & $\begin{array}{l}\text { NCRP Report } \\
32\end{array}$ & 1966 & $\begin{array}{l}\text { Radiation Protection in Educational } \\
\text { Institutions }\end{array}$ & drgop212-4 & 1984 & $\mathbf{R}$ & & NCRP Report 32 & 1966 \\
\hline 3795 & NCRP & $\begin{array}{l}\text { NCRP Report } \\
33\end{array}$ & $N / S$ & $\begin{array}{l}\text { Medical X-Ray, Electron Beam and } \\
\text { Gamma-Ray Protection for } \\
\text { Energies up to } 50 \mathrm{MeV} \text { (Equipment } \\
\text { Design, Performance and Use) }\end{array}$ & drgfc414-4 & 1985 & $\mathbf{P}$ & & NCRP Report 102 & 1989 \\
\hline 3796 & NCRP & $\begin{array}{l}\text { NCRP Report } \\
33\end{array}$ & $\mathrm{~N} / \mathrm{S}$ & $\begin{array}{l}\text { Medical X-Ray, Electron Beam and } \\
\text { Gamma-Ray Protection for } \\
\text { Energies up to } 50 \text { MeV (Equipment } \\
\text { Design, Performance and Use) }\end{array}$ & reg10.10.roo & 1987 & $\mathbf{R}$ & & NCRP Report 102 & 1989 \\
\hline 3797 & NCAP & $\begin{array}{l}\text { NCRP Report } \\
33\end{array}$ & $N / S$ & $\begin{array}{l}\text { Medical X-Ray, Electron Beam and } \\
\text { Gamma-Ray Protection for } \\
\text { Energies up to } 50 \mathrm{MeV} \text { (Equipment } \\
\text { Design, Performance and Use) }\end{array}$ & reg10.11.r00 & 1987 & $\mathbf{R}$ & & NCRP Peport 102 & 1989 \\
\hline 3798 & NCRP & $\begin{array}{l}\text { NCRP Report } \\
34\end{array}$ & 1970 & $\begin{array}{l}\text { Structural Shielding Design and } \\
\text { Evaluation for Medical Use of X- } \\
\text { Rays and Gamma Rays of Energies } \\
\text { up to } 10 \mathrm{MeV}\end{array}$ & reg8.18.ro1 & 1982 & $\mathbf{R}$ & $\begin{array}{l}\text { Reissued as NCRP } \\
\text { Report } 49\end{array}$ & NCRP Report 49 & 1976 \\
\hline 3799 & NCRP & $\begin{array}{l}\text { NCRP Report } \\
37\end{array}$ & 1970 & $\begin{array}{l}\text { Precautions in the Management of } \\
\text { Patients Who Have Received } \\
\text { Therapeutic Amounts of } \\
\text { Radionuclides }\end{array}$ & drgop212-4 & 1984 & $\mathbf{R}$ & & NCRP Report 37 & 1970 \\
\hline
\end{tabular}


Codes and Standards and Other Guidance Cited in Regulatory Documents

Part A - Industry Consensus Codes and Standards (continued)

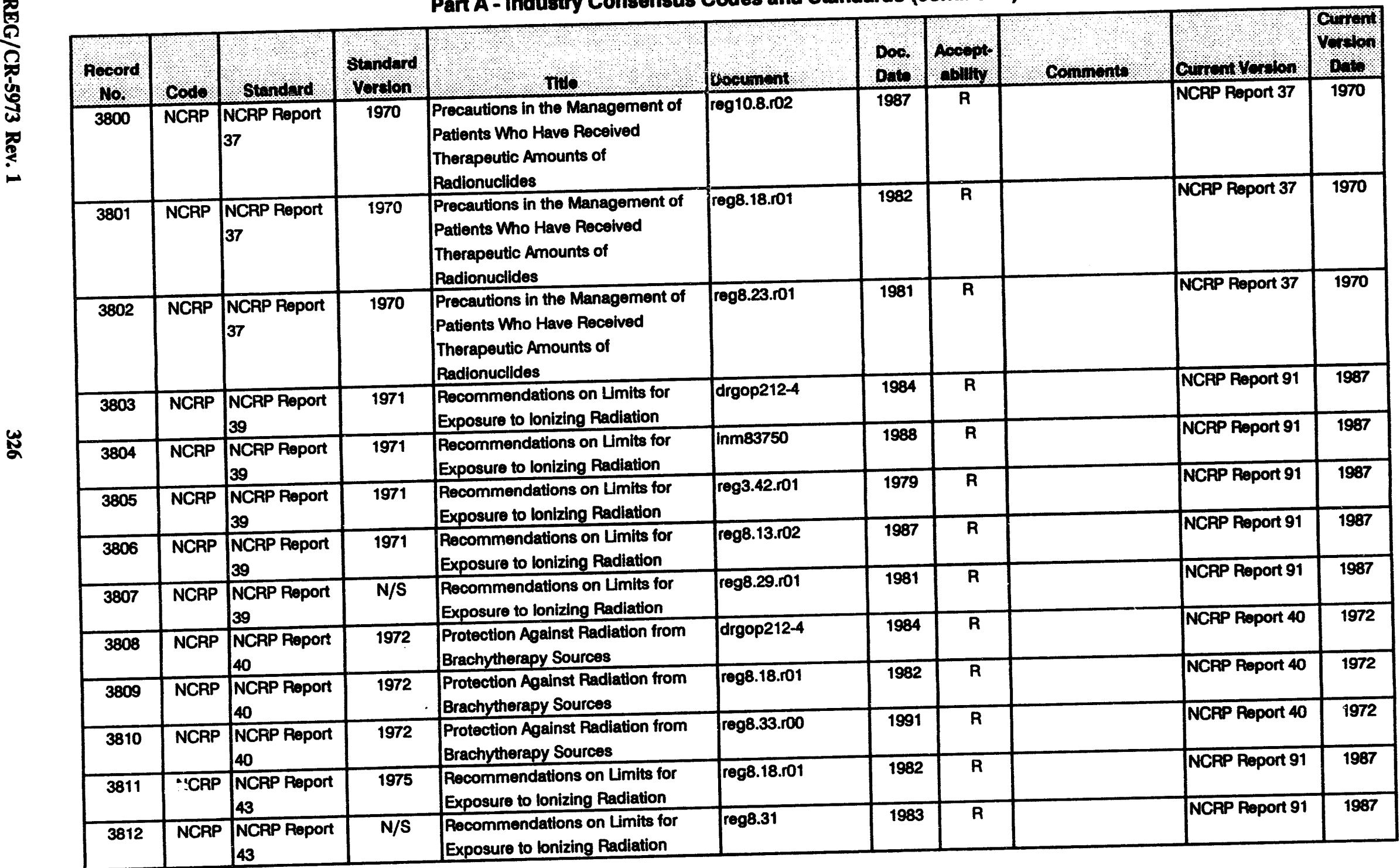


Codes and Standards and Other Guidance Clted in Regulatory Documents

Part A - Industry Consensus Codes and Standards (continued)

\begin{tabular}{|c|c|c|c|c|c|c|c|c|c|c|}
\hline Rocord & Code. & Standind & $\begin{array}{l}\text { Stundard } \\
\text { Verdon }\end{array}$ & THo & ( & Doos. & Aocopt. & Commonts & Gurront voroson & Oron. \\
\hline 3813 & NCRP & $\begin{array}{l}\text { NCRP Report } \\
45\end{array}$ & N/S & $\begin{array}{l}\text { Exposure of the Population in the } \\
\text { United States and Canada from } \\
\text { Natural Background Radiation }\end{array}$ & drgop212-4 & 1984 & $\mathbf{R}$ & & NCRP Report 94 & 1987 \\
\hline 3814 & NCRP & $\begin{array}{l}\text { NCRP Report } \\
45\end{array}$ & $\mathrm{~N} / \mathrm{S}$ & $\begin{array}{l}\text { Exposure of the Population in the } \\
\text { United States and Canada from } \\
\text { Natural Background Radiation }\end{array}$ & reg4.2.ro2 & 1976 & $\mathbf{R}$ & & NCRP Report 94 & 1987 \\
\hline 3815 & NCRP & $\begin{array}{l}\text { NCAP Report } \\
45\end{array}$ & 1975 & $\begin{array}{l}\text { Exposure of the Population in the } \\
\text { United States and Canada from } \\
\text { Natural Background Radiation }\end{array}$ & reg8.30.ro0 & 1983 & $\mathbf{R}$ & & NCRP Report 94 & 1987 \\
\hline 3816 & NCRP & $\begin{array}{l}\text { NCAP Report } \\
47\end{array}$ & 1976 & Tritium Measurement Techniques & reg4.15.r01 & 1979 & $\mathbf{R}$ & & NCRP Report 47 & 1976 \\
\hline 3817 & NCRP & $\begin{array}{l}\text { NCAP Report } \\
48\end{array}$ & 1976 & $\begin{array}{l}\text { Radiation Protection for Medical } \\
\text { and Allied Health Personnel }\end{array}$ & drgop212-4 & 1984 & $\mathbf{R}$ & & NCRP Report 105 & 1989 \\
\hline 3818 & NCRP & $\begin{array}{l}\text { NCRP Report } \\
48\end{array}$ & 1976 & $\begin{array}{l}\text { Radiation Protection for Medical } \\
\text { and Allied Health Personnel }\end{array}$ & reg10.8.r02 & 1987 & $\mathbf{R}$ & & NCRP Report 105 & 1989 \\
\hline 3819 & NCRP & $\begin{array}{l}\text { NCRP Report } \\
48\end{array}$ & 1976 & $\begin{array}{l}\text { Radiation Protection for Medical } \\
\text { and Allied Health Personnel }\end{array}$ & reg8.18.r01 & 1982 & $\mathbf{R}$ & & NCRP Report 105 & 1989 \\
\hline 3820 & NCRP & $\begin{array}{l}\text { NCRP Report } \\
48\end{array}$ & 1976 & $\begin{array}{l}\text { Padiation Protection for Medical } \\
\text { and Allied Health Personnel }\end{array}$ & reg8.23.r01 & 1981 & $\mathbf{R}$ & & NCRP Report 105 & 1989 \\
\hline 3821 & NCRP & $\begin{array}{l}\text { NCRP Report } \\
49\end{array}$ & $N / S$ & $\begin{array}{l}\text { Structural Shielding Design and } \\
\text { Evaluation for Medical Use of X- } \\
\text { Rays and Gamma Rays of Energies } \\
\text { up to } 10 \mathrm{MeV}\end{array}$ & drgfc414-4 & 1985 & $\mathbf{R}$ & & NCRP Report 49 & 1976 \\
\hline 3822 & NCRP & $\begin{array}{l}\text { NCRP Report } \\
49\end{array}$ & 1976 & $\begin{array}{l}\text { Structural Shielding Design and } \\
\text { Evaluation for Medical Use of X- } \\
\text { Rays and Gamma Rays of Energies } \\
\text { up to } 10 \mathrm{MeV}\end{array}$ & reg8.18.r01 & 1982 & $\mathbf{R}$ & & NCRP Report 49 & 1976 \\
\hline 3823 & NCRP & $\begin{array}{l}\text { NCRP Report } \\
50\end{array}$ & 1976 & $\begin{array}{l}\text { Environmental Radiation } \\
\text { Measurements }\end{array}$ & reg4.15.ro1 & 1979 & $\mathbf{R}$ & & NCRP Report 50 & 1976 \\
\hline 3824 & NCRP & $\begin{array}{l}\text { NCRP Report } \\
56\end{array}$ & 1977 & $\begin{array}{l}\text { Radiation Exposure of the U.S. } \\
\text { Population from Consumer } \\
\text { Products and Miscellaneous }\end{array}$ & drgop212-4 & 1984 & $\mathbf{R}$ & & NCRP Report 95 & 1987 \\
\hline 3825 & NCRP & $\begin{array}{l}\text { NCRP Report } \\
57\end{array}$ & 1978 & $\begin{array}{l}\text { Instrumentation and Monitoring } \\
\text { Methods for Radiation Protection }\end{array}$ & inm83525 & 1984 & $\mathbf{R}$ & & NCRP Report 57 & 1978 \\
\hline
\end{tabular}


Codes and Standards and Other Guidance Cited in Regulatory Documents

Part A - Industry Consensus Codes and Standards (continued)

\begin{tabular}{|c|c|c|c|c|c|c|c|c|c|c|}
\hline Hocord & Code & Standerd & $\begin{array}{l}\text { Strondard } \\
\text { Verolon }\end{array}$ & L, nos & Document & Doc. & Aocept & Commente & Cunsemt Verion & $\begin{array}{l}\text { Currom } \\
\text { Vorion } \\
\text { Dewe }\end{array}$ \\
\hline 3826 & NCRP & $\begin{array}{l}\text { NCRP Report } \\
57\end{array}$ & 1978 & \begin{tabular}{|l} 
Instrumentation and Monitoring \\
Mothods for Radiation Protoction
\end{tabular} & inm83526 & 1984 & A & & NCPP Report 57 & 1978 \\
\hline 3827 & NCRP & $\begin{array}{l}\text { NCRP Report } \\
57\end{array}$ & 1978 & $\begin{array}{l}\text { Instrumentation and Monitoring } \\
\text { Methods for Radiation Protection }\end{array}$ & inm83726 & 1991 & $\mathbf{R}$ & & NCAP Report 57 & 1978 \\
\hline 3828 & NCRP & $\begin{array}{l}\text { NCRP Report } \\
57\end{array}$ & 1978 & $\begin{array}{l}\text { Instrumentation and Monitoring } \\
\text { Mothods for Radiation Protection }\end{array}$ & inm83750 & 1988 & $\mathbf{R}$ & & NCPP Roport 57 & 1978 \\
\hline 3829 & NCRP & $\begin{array}{l}\text { NCRP Roport } \\
57\end{array}$ & 1978 & $\begin{array}{l}\text { Instrumentation and Monitoring } \\
\text { Methods for Radiation Protoction }\end{array}$ & $\operatorname{rog} 10.8 .102$ & 1987 & $\mathbf{A}$ & & NCPP Repont 57 & 1978 \\
\hline 3830 & NCRP & $\begin{array}{l}\text { NCRP Report } \\
57\end{array}$ & 1978 & $\begin{array}{l}\text { instrumentation and Monitoring } \\
\text { Methods for Padiation Protection }\end{array}$ & reg8.21.ro1 & 1979 & $\bar{R}$ & & NCRP Report 57 & 1978 \\
\hline 3831 & NCRP & $\begin{array}{l}\text { NCRP Report } \\
57\end{array}$ & 1978 & $\begin{array}{l}\text { Instrumentation and Monitoring } \\
\text { Mothods for Padiation Protoction }\end{array}$ & reg8.23.ro1 & 1981 & $\mathbf{R}$ & & NCRP Report 57 & 1978 \\
\hline 3832 & NCRP & $\begin{array}{l}\text { NCRP Report } \\
57\end{array}$ & 1978 & $\begin{array}{l}\text { Instrumentation and Monitoring } \\
\text { Methods for Paadiation Protection }\end{array}$ & reg8.24.101 & 1979 & $\bar{R}$ & & NCRP Report 57 & 1978 \\
\hline 3833 & NCRP & $\begin{array}{l}\text { NCRP Report } \\
58\end{array}$ & 1985 & $\begin{array}{l}\text { A Handbook of Radioactivity } \\
\text { Moasurements }\end{array}$ & reg10.8.ro2 & 1987 & $\mathbf{R}$ & & NCPP Roport 58 & 1965 \\
\hline 3834 & NCRP & $\begin{array}{l}\text { NCRP Report } \\
58\end{array}$ & 1978 & $\begin{array}{l}\text { A Handbook of Radioactivity } \\
\text { Measurements }\end{array}$ & reg4.15.ro1 & 1979 & $\bar{P}$ & & NCRP Roport 58 & 1985 \\
\hline 3835 & NCRP & $\begin{array}{l}\text { NCRP Report } \\
58\end{array}$ & 1978 & $\begin{array}{l}\text { A Handbook of Radioactivity } \\
\text { Measurements }\end{array}$ & reg4.16.ro1 & 1985 & $\bar{R}$ & & NCRP Roport 58 & 1995 \\
\hline 3836 & NCRP & $\begin{array}{l}\text { NCRI? Report } \\
58\end{array}$ & 1978 & $\begin{array}{l}\text { A Handbook of Radioactivity } \\
\text { Measurements }\end{array}$ & reg8.21.101 & 1979 & $\bar{R}$ & & NCPP Roport 58 & 1965 \\
\hline 3837 & NCRP & $\begin{array}{l}\text { NCRP Report } \\
58\end{array}$ & 1978 & $\begin{array}{l}\text { A Handbook of Radioactivity } \\
\text { Measurements }\end{array}$ & reg8.23.ro1 & 1981 & $\bar{R}$ & & NCRP Report 58 & 1985 \\
\hline 3838 & NCRP & $\begin{array}{l}\text { NCPP Report } \\
58\end{array}$ & 1978 & $\begin{array}{l}\text { A Handbook of Radioactivity } \\
\text { Moasurements }\end{array}$ & reg8.24.101 & 1979 & $\bar{R}$ & & NCRP Report 58 & 1985 \\
\hline 3839 & NCRP & $\begin{array}{l}\text { NCRP Roport } \\
59\end{array}$ & 1978 & $\begin{array}{l}\text { Operational Radiation Safety } \\
\text { Programs }\end{array}$ & reg8.23.r01 & 1981 & $\mathbf{A}$ & & NCRP Report 59 & 1978 \\
\hline 3840 & NCRP & $\begin{array}{l}\text { NCRP Peport } \\
65\end{array}$ & 1980 & $\begin{array}{l}\text { Management of Persons } \\
\text { Accidentally Contaminated with } \\
\text { Radionuclides }\end{array}$ & inm83526 & 1984 & $\bar{A}$ & & MCAP Roport 65 & 1960 \\
\hline 3841 & NCRP & $\begin{array}{l}\text { NCRP Roport } \\
65\end{array}$ & 1980 & $\begin{array}{l}\text { Management of Porsons } \\
\text { Accidentally Contaminated with } \\
\text { Radionuclides }\end{array}$ & inm83726 & 1991 & $\mathbf{R}$ & & NCRP Peport 65 & 1980 \\
\hline
\end{tabular}


Codes and Standards and Other Guidance Clted in Regulatory Documents

Part A - Industry Consensus Codes and Standards (continued)

\begin{tabular}{|c|c|c|c|c|c|c|c|c|c|c|}
\hline $\begin{array}{l}\text { Aocord } \\
\text { No. }\end{array}$ & Cods: & Stondad & $\begin{array}{l}\text { Standerd } \\
\text { Vorelon }\end{array}$ & $(1, \quad$ mo & Docanoned & Dos. & Aospt & Conmens & 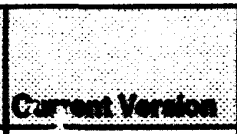 & 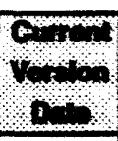 \\
\hline 3842 & NCRP & $\begin{array}{l}\text { NCRP Roport } \\
65\end{array}$ & 1980 & $\begin{array}{l}\text { Managomont of Porsons } \\
\text { Accidentally Contaminated with } \\
\text { Pradionuclides }\end{array}$ & inm83750 & 1988 & $\mathbf{A}$ & & NCTP Report 65 & 1900 \\
\hline 3843 & NCRP & $\begin{array}{l}\text { NCRP Roport } \\
71\end{array}$ & 1983 & $\begin{array}{l}\text { Operational Padiation Safoty- } \\
\text { Training }\end{array}$ & drgfo414-4 & 1985 & $\mathbf{A}$ & & NCPP Report 71 & 1983 \\
\hline 3844 & NCRP & $\begin{array}{l}\text { NCRP Poport } \\
71\end{array}$ & 1983 & $\begin{array}{l}\text { Operational Radiation Safoty- } \\
\text { Training }\end{array}$ & $\operatorname{lnm} 83523$ & 1994 & $\mathbf{A}$ & & NCAP Report 71 & 1983 \\
\hline 3845 & NCRP & $\begin{array}{l}\text { NCRP Report } \\
71\end{array}$ & 1983 & $\begin{array}{l}\text { Operational Radiation Safoty- } \\
\text { Training }\end{array}$ & inm83750 & 1988 & $\mathbf{R}$ & & MCFP Roport 71 & 1983 \\
\hline 3846 & NCRP & $\begin{array}{l}\text { NCPP Poport } \\
71\end{array}$ & 1983 & $\begin{array}{l}\text { Operattonal Radiation Safoty } \\
\text { Training }\end{array}$ & $\log 10.8 .102$ & 1987 & $\mathbf{R}$ & & NCPP Report 71 & 1983 \\
\hline 3847 & NCRP & $\begin{array}{l}\text { NCRP Report } \\
73\end{array}$ & 1983 & $\begin{array}{l}\text { Protection in Nuclear Modicino and } \\
\text { Utrasound Diagnostic Procedures } \\
\text { in Children }\end{array}$ & reg8.13.102 & 1987 & $\bar{R}$ & & NCFP Report 73 & 1983 \\
\hline 3848 & NCRP & $\begin{array}{l}\text { NCRP Report } \\
87\end{array}$ & 1987 & $\begin{array}{l}\text { Use of Bloassay Procedures for } \\
\text { Assessment of Internal } \\
\text { Padionuclide Doposition }\end{array}$ & reg8.201 & 1993 & $\overline{\mathbf{P}}$ & & NCRP Roport 87 & 1937 \\
\hline 3849 & NCRP & $\begin{array}{l}\text { NCRP Report } \\
88\end{array}$ & 1986 & $\begin{array}{l}\text { Paadiation Alarms and Accoss } \\
\text { Control Systoms }\end{array}$ & drg3003.r00 & 1990 & $\bar{A}$ & & NCPP Report 88 & 1987 \\
\hline 3850 & NCRP & $\begin{array}{l}\text { NCRP Aoport } \\
91\end{array}$ & 1987 & $\begin{array}{l}\text { Pocommendations on Limits for } \\
\text { Exposure to lonizing Padiation }\end{array}$ & drge011.000 & 1992 & $\bar{R}$ & & NCPP Report 91 & 1987 \\
\hline 3851 & NCRP & $\begin{array}{l}\text { NCRP Peport } \\
91\end{array}$ & 1987 & $\begin{array}{l}\text { Rocommendations on Limits for } \\
\text { Exposure to lonizing Radiation }\end{array}$ & Pol55FP27522 & 1990 & $\overline{\mathbf{R}}$ & & NCPP Report 91 & 1987 \\
\hline 3852 & NCPP & $\begin{array}{l}\text { NCPP Peport } \\
93\end{array}$ & N/S & $\begin{array}{l}\text { Ionizing Padiation Exposure of the } \\
\text { Population of the Unitod States }\end{array}$ & pol55FFE27522 & 1990 & $\bar{R}$ & & NCRP Report 93 & 1987 \\
\hline 3853 & NCPP & $\begin{array}{l}\text { NCRP Report } \\
95\end{array}$ & $N / S$ & $\begin{array}{l}\text { Padiation Exposurn of tho U.S. } \\
\text { Population from Consumer } \\
\text { Products and Miscollaneous }\end{array}$ & Pol55FP27522 & 1990 & $\overline{\mathbf{R}}$ & & NCPP Roport 95 & 1987 \\
\hline 3854 & NCFP & $\begin{array}{l}\text { NCRP Report } \\
106\end{array}$ & 1989 & $\begin{array}{l}\text { Limits for Exposure to 'Hot } \\
\text { Particles' on the Skin }\end{array}$ & notsa-48 & 1990 & A & & MCFP Report 106 & 1900 \\
\hline 3855 & NCPP & $\begin{array}{l}\text { NCRP Report } \\
107\end{array}$ & 1990 & $\begin{array}{l}\text { Implementation of the Principle of } \\
\text { As Low As is Pleasonably } \\
\text { Achlovable (ALARA) for Medical and } \\
\text { Dontal Personnel }\end{array}$ & drg0002.r00 & 1991 & $\bar{A}$ & & MCFP Report 107 & 1990 \\
\hline
\end{tabular}




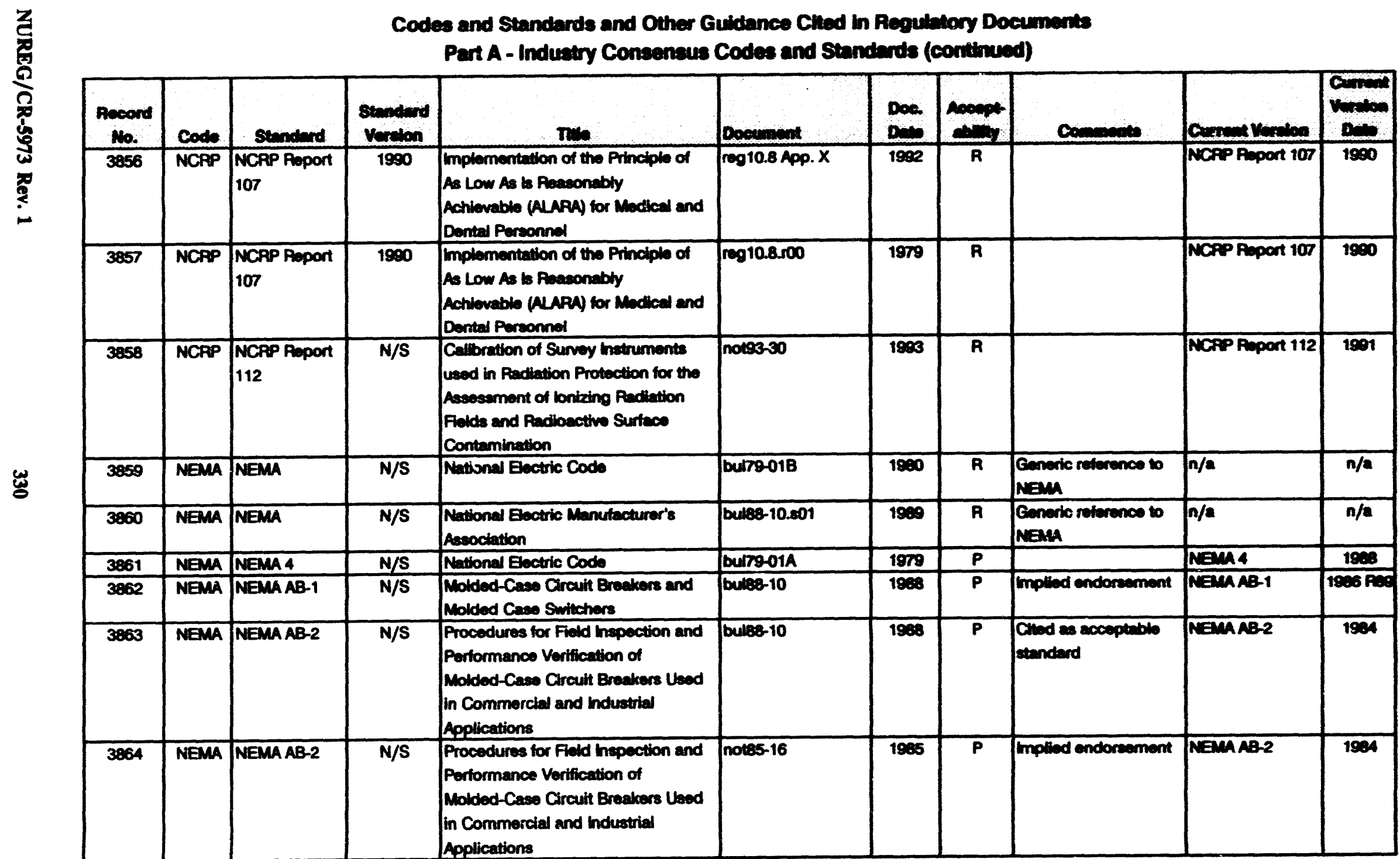


Codes and Standards and Other Guidance Clled in Regulatory Da zuments

Part A - Industry Consensus Codes and Standards (continued)

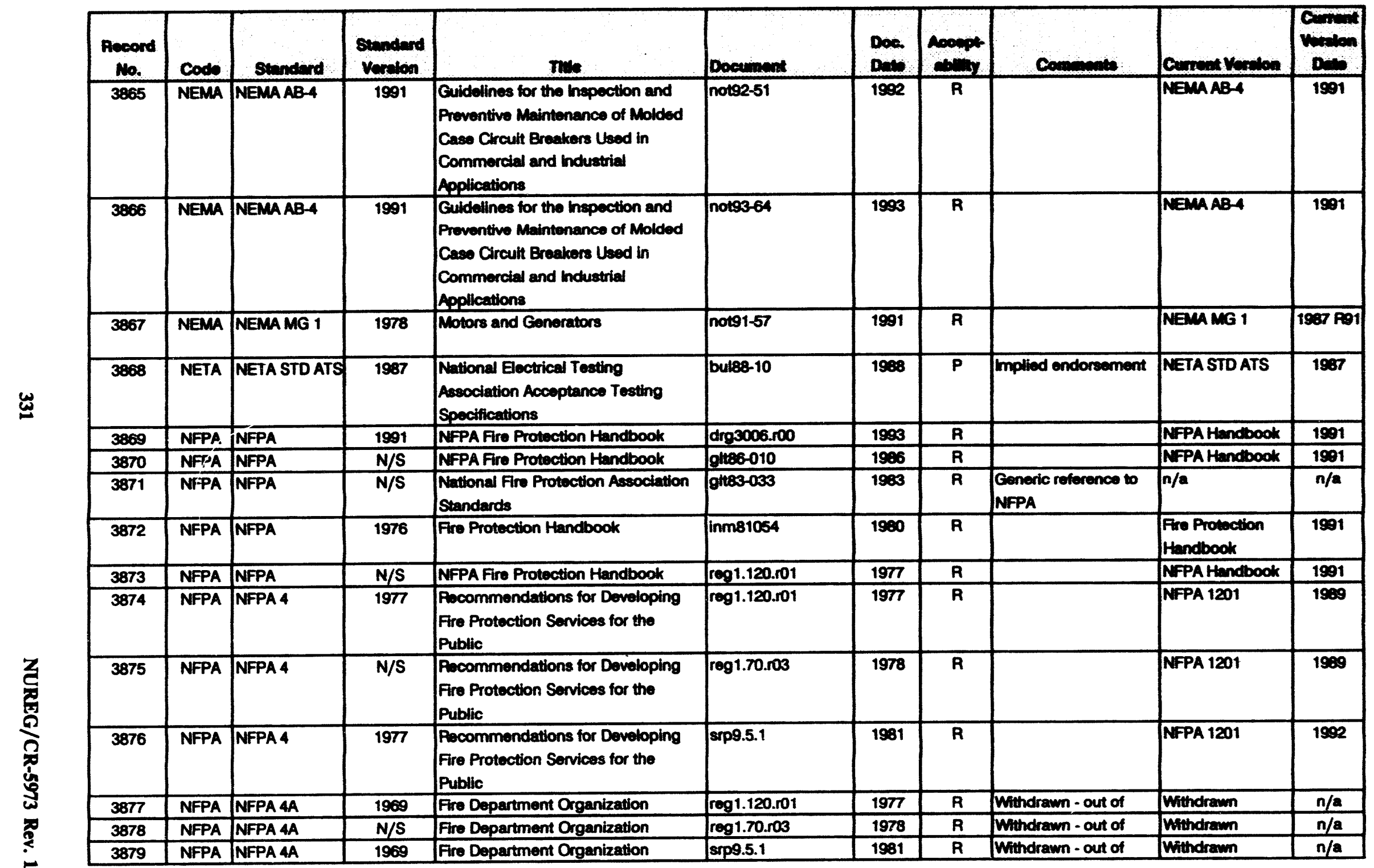


Codes and Standards and Other Guidance Cited in Regulatory Documents

Part A - Industry Consensus Codes and Standards (continued)

\begin{tabular}{|c|c|c|c|c|c|c|c|c|c|c|}
\hline $\begin{array}{c}\text { Aecord } \\
\text { No. }\end{array}$ & Code & Standind & $\begin{array}{l}\text { Standerd } \\
\text { Vortan }\end{array}$ & Inim & Dooument & Dow & Arones. & Componto & 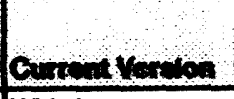 & Yorton \\
\hline 3880 & NFPA & NFPA 6 & 1974 & $\begin{array}{l}\text { Precommendations for Organization } \\
\text { of industrial Fire Loss Prevention }\end{array}$ & reg1.120.ro1 & 1977 & R & $\begin{array}{l}\text { Withdrawn - out of } \\
\text { print }\end{array}$ & Withdrawn & $n / a$ \\
\hline 3881 & NFPA & NFPA 6 & $N / S$ & $\begin{array}{l}\text { Recommendations for Organization } \\
\text { of Industrial Fire Loss Prevention }\end{array}$ & $\mathrm{reg} 1.70 .103$ & 1978 & $\mathbf{R}$ & $\begin{array}{l}\text { Withdranm - out of } \\
\text { print }\end{array}$ & Withdrawen & $n / a$ \\
\hline 3882 & NFPA & NFPA 6 & 1974 & $\begin{array}{l}\text { Recommendations for Organization } \\
\text { of Industrial Fire Loss Provention }\end{array}$ & srp9.5.1 & 1981 & $\mathbf{P}$ & $\begin{array}{l}\text { Withdrawn - out of } \\
\text { print }\end{array}$ & Withdrawn & $n / a$ \\
\hline 3883 & NFPA & NFPA 7 & 1974 & Manegement of Fire Emergencies & reg1.120.101 & 197 & $\mathbf{R}$ & Withdramn - out of & Withdrawn & $n / a$ \\
\hline 3884 & NFPA & NFPA 7 & $\mathbf{N} / \mathbf{S}$ & Management of Fire Emergencies & reg1.70.003 & 1978 & $\mathbf{R}$ & Windramn - out of & Whindrawn & $n / a$ \\
\hline 3885 & NFPA & NFPA7 & 1974 & Management of Fire Emergencies & $\operatorname{sip} 9.5 .1$ & 1981 & $\mathbf{R}$ & Withdrawn - out of & Withdrann & $n / a$ \\
\hline 3886 & NFPA & NFPA 8 & 1974 & $\begin{array}{l}\text { Eflects of Fire on Operations } \\
\text { Management Posponsibility }\end{array}$ & reg1.120.ro1 & 1977 & $\bar{R}$ & $\begin{array}{l}\text { Withdramen - out of } \\
\text { print }\end{array}$ & Withdrawn & $n / a$ \\
\hline 3887 & NFPA & NFPA 8 & $N / S$ & $\begin{array}{l}\text { Effects of Fire on Operations } \\
\text { Management Responsibility }\end{array}$ & reg1.70.ros & 1978 & $\mathbf{R}$ & $\begin{array}{l}\text { Withdrawn - out of } \\
\text { print }\end{array}$ & Mithdrawn & $n / a$ \\
\hline 3888 & NFPA & NFPA B & 1974 & $\begin{array}{l}\text { Effects of Fire on Operations } \\
\text { Management Respossibility }\end{array}$ & srp9.5.1 & 1981 & $\mathbf{R}$ & $\begin{array}{l}\text { Whindram - out of } \\
\text { print }\end{array}$ & Withdrawn & $n / a$ \\
\hline 3889 & NFPA & NFPA 10 & 1984 & $\begin{array}{l}\text { Standard for Portable Fro } \\
\text { Extinguiahers }\end{array}$ & inm64704 & 1986 & $\mathbf{R}$ & & NFPA 10 & 1990 \\
\hline 3890 & NFPA & NFPA 10 & $N / S$ & $\begin{array}{l}\text { Standard for Portable Fire } \\
\text { Extinguishers }\end{array}$ & inm88055 & 1991 & $\bar{R}$ & & NFPA 10 & 1990 \\
\hline 3891 & NFPA & NFPA 10 & 1975 & $\begin{array}{l}\text { Standard for Portable Fire } \\
\text { Extinguishers }\end{array}$ & reg1.120.ro1 & 1977 & $\mathbf{P}$ & & NFPA 10 & 1990 \\
\hline 3892 & NFPA & NFPA 10 & 1977 & $\begin{array}{l}\text { Standard for Portable Fire } \\
\text { Extinguishers }\end{array}$ & $\operatorname{srp9.5.1}$ & 1981 & $\mathbf{P}$ & & NFPA 10 & 1990 \\
\hline 3893 & NFPA & NFPA 11 & $N / S$ & $\begin{array}{l}\text { Standard for Low Expansion Foam } \\
\text { and Combined Agent Syatems }\end{array}$ & inm88055 & 1991 & $\mathbf{A}$ & & NFPA 11 & 1988 \\
\hline 3894 & NFPA & NFPA 11 & 1975 & $\begin{array}{l}\text { Standard for Low Expansion Foam } \\
\text { and Combined Agent Systerns }\end{array}$ & reg1.120.101 & 1977 & $\mathbf{P}$ & & NFPA 11 & 1988 \\
\hline 3895 & NFPA & NFPA 11 & 1977 & $\begin{array}{l}\text { Standard for Low Expansion Foam } \\
\text { and Combined Agent Systems }\end{array}$ & $\operatorname{srp9.5.1}$ & 1981 & $P$ & & NFPA 11 & 1988 \\
\hline 3896 & NFPA & NFPA 11A & N/S & $\begin{array}{l}\text { Standard for Modium- and High- } \\
\text { Expansion Foam Systems }\end{array}$ & inm88055 & 1991 & R & & NFPA 11A & 1988 \\
\hline 3897 & NFPA & NFPA 11A & 1970 & $\begin{array}{l}\text { Standard for Modium-and High- } \\
\text { Expansion Foam Systerns }\end{array}$ & reg1.120.101 & 1977 & $P$ & & NFPA 11A & 1988 \\
\hline
\end{tabular}




\section{Codes and Standards and Other Guldance Ched in Regulatory Documents}

Part A - Industry Consensus Codes and Standards (continued)

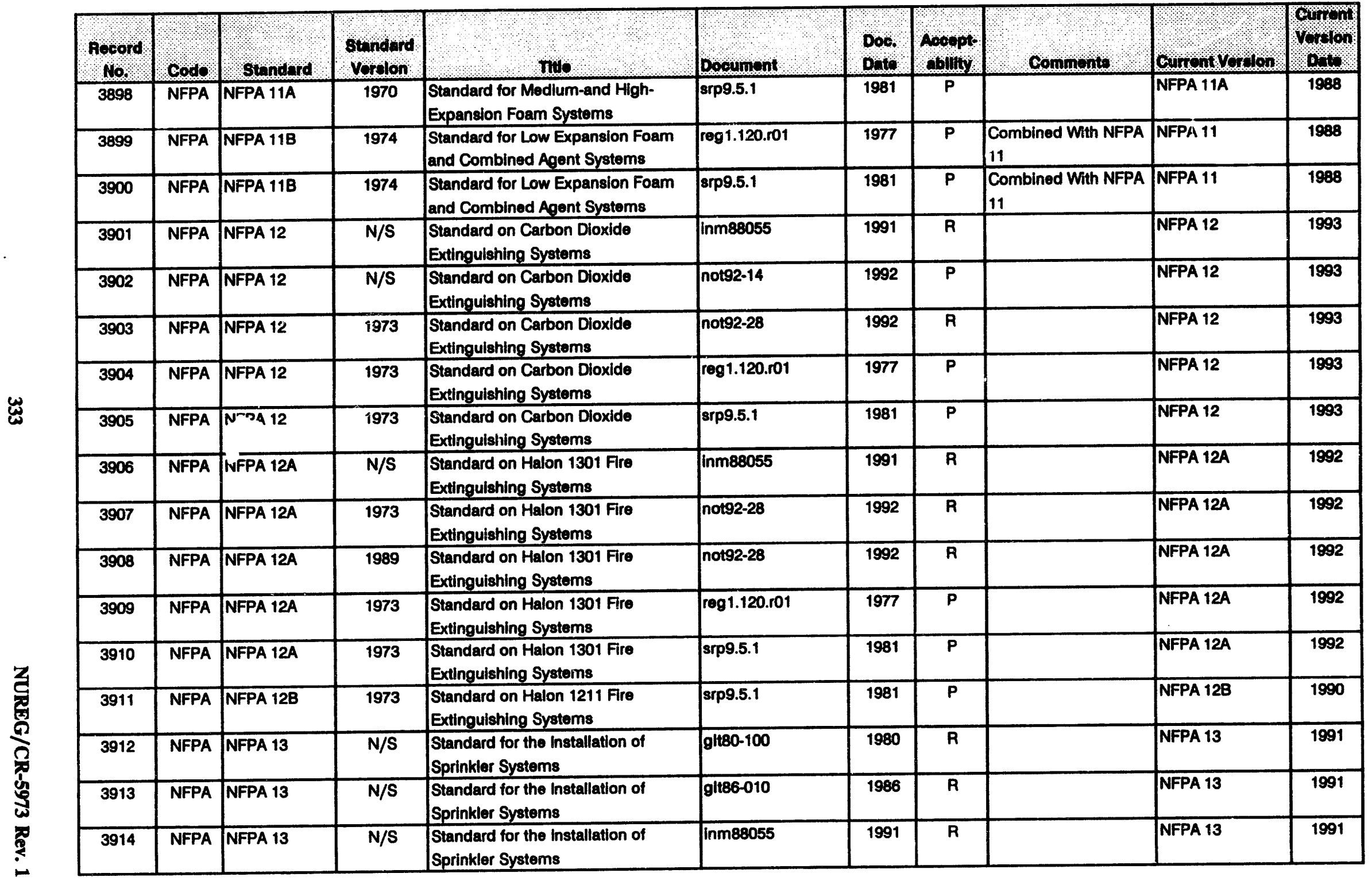




\begin{tabular}{|c|c|c|c|c|c|c|c|c|c|c|}
\hline & & & Cod & $\begin{array}{l}8 \text { and Standards and Other } \\
\text { Part A - Industry Consensu }\end{array}$ & $\begin{array}{l}\text { uidance Clt } \\
\text { Codes and }\end{array}$ & $\begin{array}{l}\text { Regule } \\
\text { ards ( }\end{array}$ & tory Do & ents & & \\
\hline $\begin{array}{l}\text { Pocord } \\
\text { No. }\end{array}$ & Code & Standard & $\begin{array}{l}\text { Standard } \\
\text { Voriton }\end{array}$ & nute & Document: & $\begin{array}{l}\text { Doc. } \\
\text { Dote. }\end{array}$ & $\begin{array}{l}\text { Aoceppts } \\
\text { obility }\end{array}$ & Comments: & Cunnonvorelon. & voringin \\
\hline 3915 & NFPA & NFPA 13 & $N / S$ & $\begin{array}{l}\text { Standard for the Installation of } \\
\text { Sprinkler Systems }\end{array}$ & not84-09 & 1984 & $P$ & & NFPA 13 & 1991 \\
\hline 3916 & NFPA & NFPA 13 & 1973 & $\begin{array}{l}\text { Standard for the Installation of } \\
\text { Sprinkler Systems }\end{array}$ & reg1.120.ro1 & 1977 & $\bar{P}$ & & NFPA 13 & 1991 \\
\hline 3917 & NFPA & NFPA 13 & 1976 & $\begin{array}{l}\text { Standard for the Installation of } \\
\text { Sprinkler Systems }\end{array}$ & sip9.5.1 & 1981 & $\bar{P}$ & & NFPA 13 & 1991 \\
\hline 3918 & NFPA & NFPA 14 & 1983 & $\begin{array}{l}\text { Standard for the Installiation of } \\
\text { Standpipe and Hose Systems }\end{array}$ & inm64704 & 1986 & $\bar{R}$ & & NFPA 14 & 1993 \\
\hline 3919 & NFPA & NFPA 14 & $\mathrm{~N} / \mathrm{S}$ & $\begin{array}{l}\text { Standard for the Installation of } \\
\text { Standpipe and Hose Systems }\end{array}$ & inm88055 & 1991 & $\mathbf{R}$ & & $\overline{\text { NFPA } 14}$ & 1993 \\
\hline 3920 & NFPA & NFPA 14 & 1974 & $\begin{array}{l}\text { Standard for the Installation of } \\
\text { Standpipe and Hose Systerns }\end{array}$ & reg1.120.001 & 1977 & $\mathbf{P}$ & & NFPA 14 & 1993 \\
\hline 3921 & NFPA & NFPA 14 & 1974 & $\begin{array}{l}\text { Standard for the Installation of } \\
\text { Standpipe and Hose Systems }\end{array}$ & sip9.5.1 & 1981 & $\bar{P}$ & & NFPA 14 & 1993 \\
\hline$\overline{3922}$ & NFPA & NFPA 15 & $\mathrm{~N} / \mathrm{S}$ & $\begin{array}{l}\text { Standard for Water Spray Fixed } \\
\text { Systems for Fire Protoction }\end{array}$ & glt86-010 & 1986 & $\mathbf{P}$ & & NFPA 15 & 1990 \\
\hline 3923 & NFPA & NFPA 15 & $\mathrm{~N} / \mathrm{S}$ & $\begin{array}{l}\text { Standard for Water Spray Fixed } \\
\text { Systems for Fire Protection }\end{array}$ & inm88055 & 1991 & $\overline{\mathbf{R}}$ & & $\widehat{\text { NFPA } 15}$ & 1990 \\
\hline 3924 & NFPA & NFPA 15 & $\overline{1973}$ & $\begin{array}{l}\text { Standard for Water Spray Fixed } \\
\text { Systems for Fire Protection }\end{array}$ & reg1.120.101 & 1977 & $\mathbf{P}$ & & NFPA 15 & 1990 \\
\hline 3925 & NFPA & NFPA 15 & 1973 & $\begin{array}{l}\text { Standard for Water Spray Fixed } \\
\text { Systems for Fire Protection }\end{array}$ & srp9.5.1 & 1981 & $\bar{P}$ & & NFPA 15 & 1990 \\
\hline 3926 & NFPA & NFPA 16 & $N / S$ & $\begin{array}{l}\text { Standard on Deluge Foam-Water } \\
\text { Sprinkler and Foam-Water Spray } \\
\text { Systems }\end{array}$ & inm88055 & 1991 & $\overline{\mathbf{A}}$ & & NFPA 16 & 1991 \\
\hline 3927 & NFPA & NFPA 16 & 1973 & $\begin{array}{l}\text { Standard on Deluge Foam-Water } \\
\text { Sprinkler and Foam-Water Spray } \\
\text { Systems }\end{array}$ & sip9.5.1 & 1981 & $\bar{P}$ & & NFPA 16 & 1991 \\
\hline 3928 & NFPA & NFPA 20 & $\mathrm{~N} / \mathrm{S}$ & $\begin{array}{l}\text { Standard for the Installation of } \\
\text { Centrifugal Fire Pumps }\end{array}$ & glt $80-100$ & 1980 & $\overline{\mathbf{R}}$ & & NFPA 20 & 1993 \\
\hline 3929 & NFPA & NFPA 20 & N/S & $\begin{array}{l}\text { Standard for the Installation of } \\
\text { Contrifugal Fire Pumps }\end{array}$ & inm64053B & 1979 & $\bar{R}$ & & NFPA 20 & 1993 \\
\hline 3930 & NFPA & NFPA 20 & $\mathrm{N} / \mathrm{S}$ & $\begin{array}{l}\text { Standard for the Installation of } \\
\text { Centrifugal Fire Pumps }\end{array}$ & inm88055 & 1991 & $\mathbf{R}$ & & NFPA 20 & 1993 \\
\hline
\end{tabular}


Cocles and Standards and Other Guldance Chted in Regulatory Documents

Part A - Industry Consensus Codes and Standards (continued)

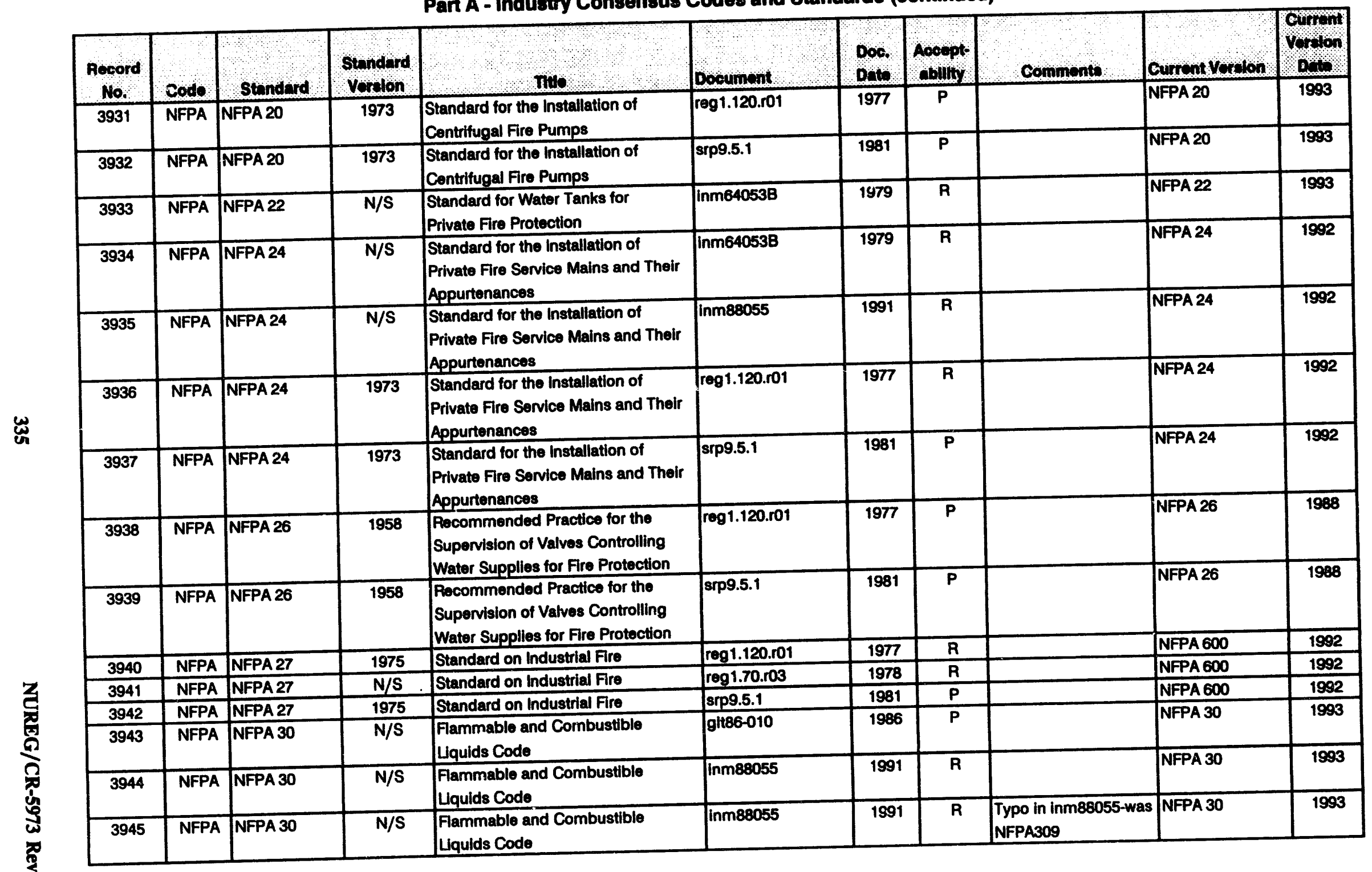


Codes and Standards and Other Guidance Chted in Regulatory Documents

Part A - Industry Consensus Codes and Standards (continued)

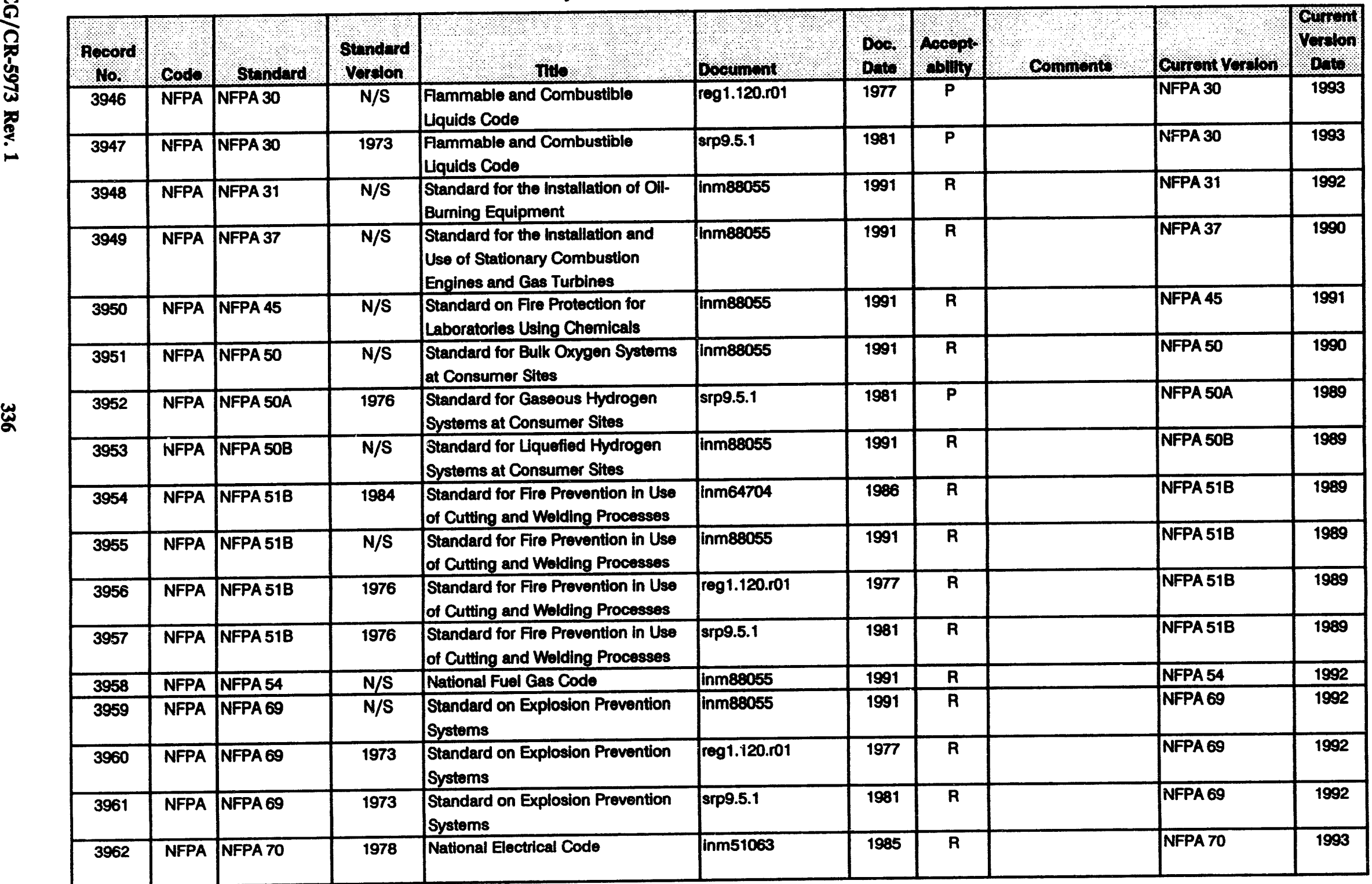


Codes and Standards and Other Guidance Cited in Regulatory Documents

Part A - Industry Consensus Codes and Standards (continued)

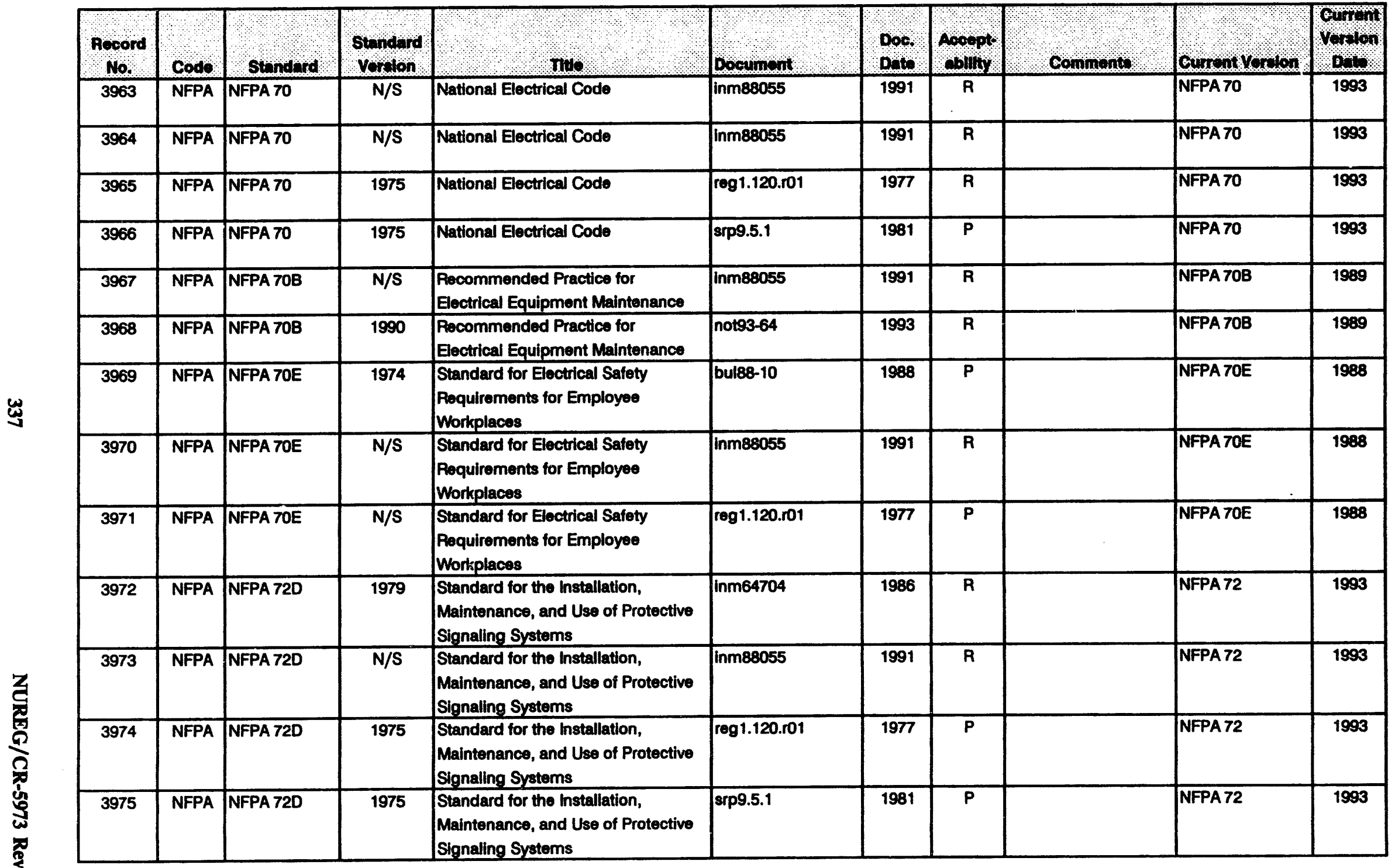




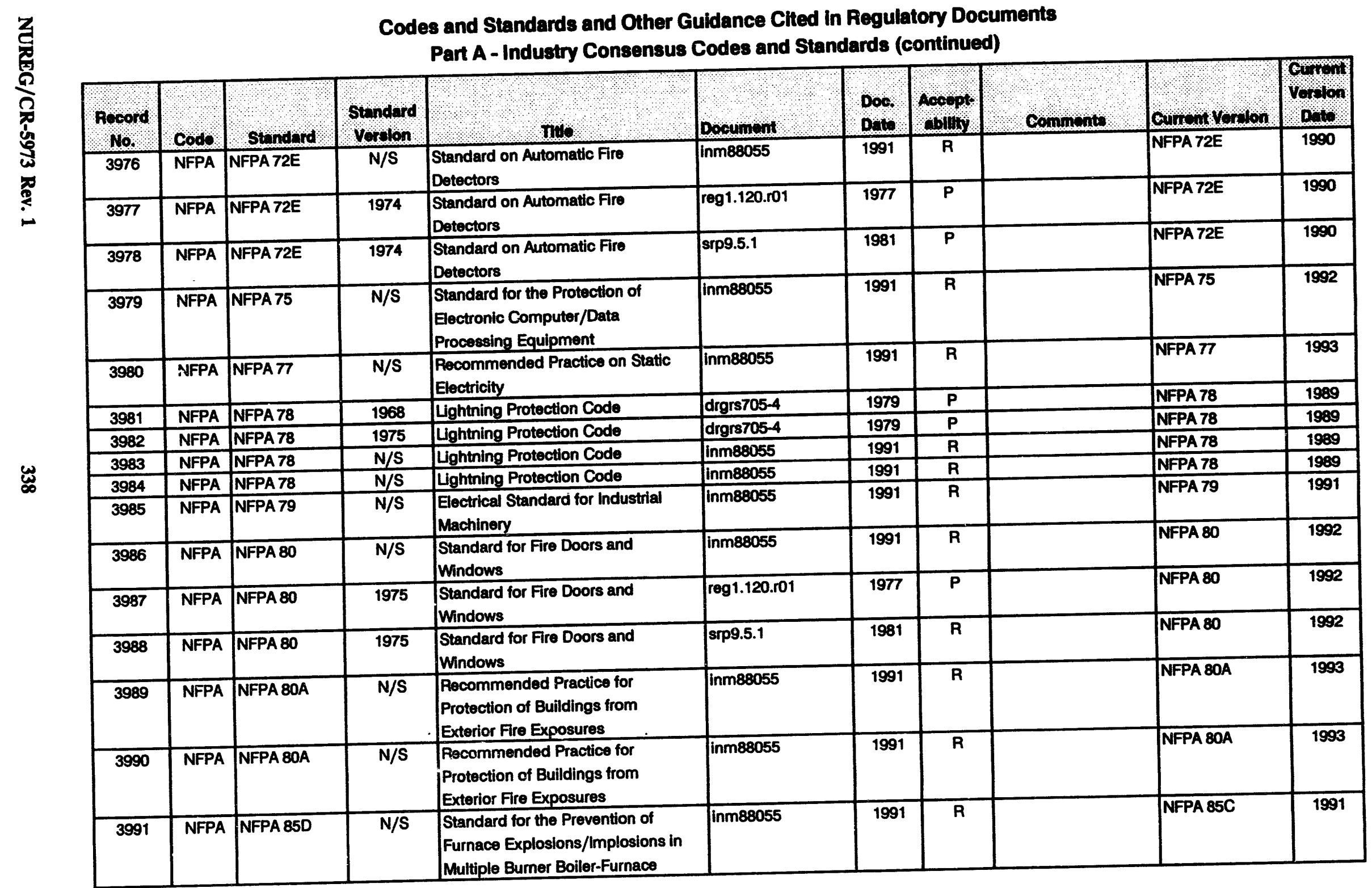


Codes and Standards and Other Guidance Cited in Regulatory Documents

Part A - Industry Consensus Codes and Standards (continued)

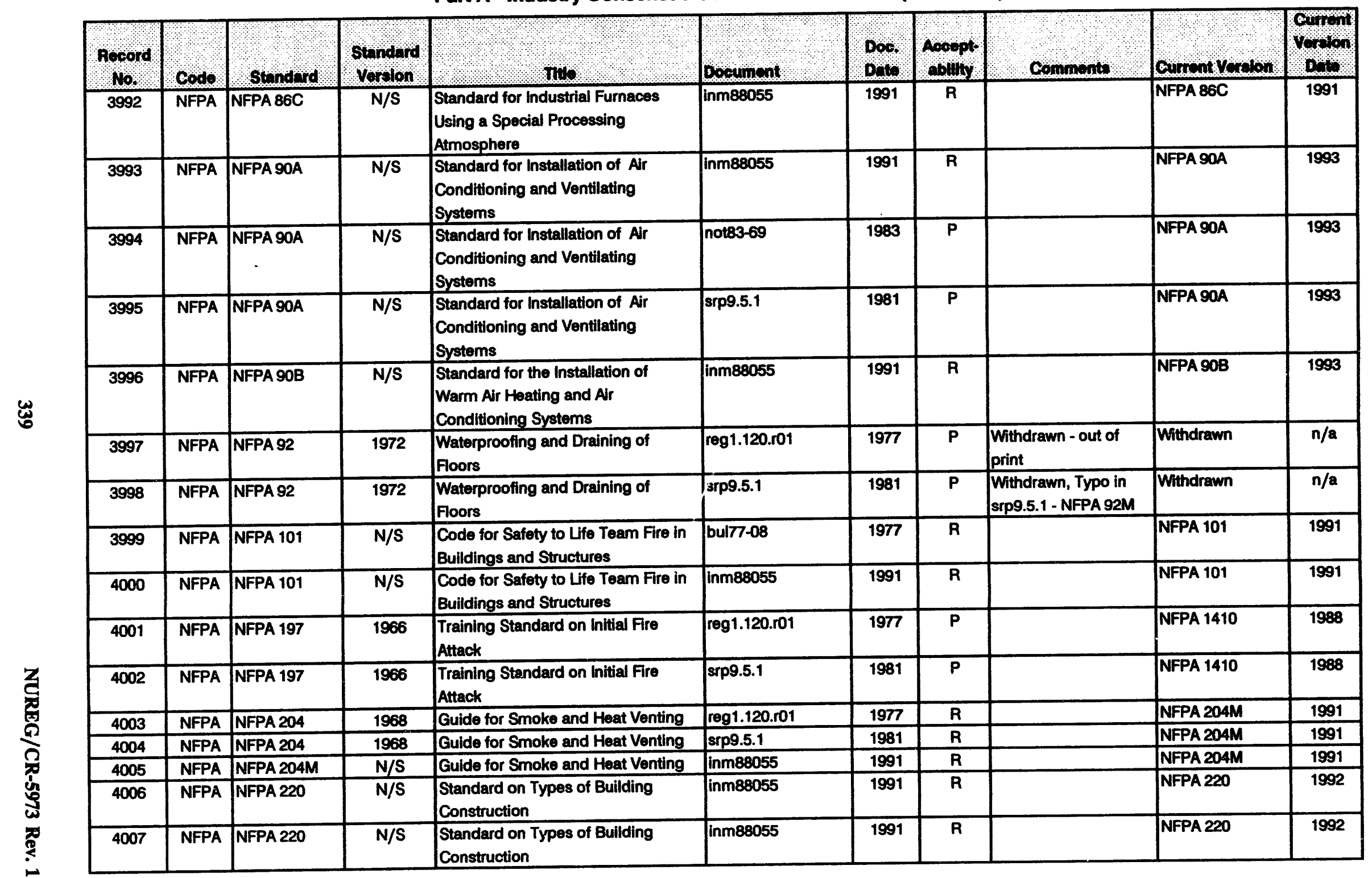


Codes and Standards and Other Guidance Cited in Regulatory Documents

Part A - Industry Consensus Codes and Standards (continued)

\begin{tabular}{|c|c|c|c|c|c|c|c|c|c|c|}
\hline Hocord & Codo & Stondind & $\begin{array}{l}\text { Standard } \\
\text { Vorlon }\end{array}$ & THol, & Dovountont: & Dor. & poppls & comments & Cunsent Vorolon & Corront \\
\hline 4008 & NFPA & NFPA 220 & 1975 & $\begin{array}{l}\text { Standard on Types of Building } \\
\text { Construction }\end{array}$ & $\operatorname{rog} 1.120 .101$ & 1977 & $\mathbf{R}$ & & NFPA 220 & 1992 \\
\hline 4009 & NFPA & NFPA 220 & $\mathrm{~N} / \mathrm{S}$ & $\begin{array}{l}\text { Standard on Types of Building } \\
\text { Construction }\end{array}$ & $\operatorname{srp} 9.5 .1$ & 1981 & $\mathbf{P}$ & & NFPA 220 & 1992 \\
\hline 4010 & NFPA & NFPA 232 & 1975 & $\begin{array}{l}\text { Standard for the Protection of } \\
\text { Records }\end{array}$ & inm39701 & 1992 & $\mathbf{R}$ & & NFPA 232 & 1991 \\
\hline 4011 & NFPA & NFPA 232 & $\mathrm{~N} / \mathrm{S}$ & $\begin{array}{l}\text { Standard for the Protection of } \\
\text { Records }\end{array}$ & inm64704 & 1986 & $\mathbf{R}$ & & NFPA 232 & 1991 \\
\hline 4012 & NFPA & NFPA 232 & $\mathrm{~N} / \mathrm{S}$ & $\begin{array}{l}\text { Standard for the Protection of } \\
\text { Records }\end{array}$ & $\operatorname{sip} 17.1$ & 1981 & $\mathbf{P}$ & & NFPA 232 & 1991 \\
\hline 4013 & NFPA & NFPA 251 & $\mathrm{~N} / \mathrm{S}$ & $\begin{array}{l}\text { Standard Methods of Fire Tests of } \\
\text { Building Construction and Materials }\end{array}$ & gtt86-010 & 1986 & $\mathbf{R}$ & & NFPA 251 & 1990 \\
\hline 4014 & NFPA & NFPA 251 & $\mathrm{~N} / \mathrm{S}$ & $\begin{array}{l}\text { Standard Methods of Fire Tests of } \\
\text { Building Construction and Materials }\end{array}$ & glt92-008 & 1992 & A & & NFPA 251 & 1990 \\
\hline 4015 & NFPA & NFPA 251 & $\mathrm{~N} / \mathrm{S}$ & $\begin{array}{l}\text { Standard Mothods of Fire Tests of } \\
\text { Building Construction and Materials }\end{array}$ & inm88055 & 1991 & $\mathbf{R}$ & & NFPA 251 & 1990 \\
\hline 4016 & NFPA & NFPA 251 & $N / S$ & $\begin{array}{l}\text { Standard Methods of Fire Tests of } \\
\text { Building Construction and Materials }\end{array}$ & not92-55 & 1992 & $\mathbf{R}$ & & NFPA 251 & 1990 \\
\hline 4017 & NFPA & NFPA 251 & $\mathrm{~N} / \mathrm{S}$ & $\begin{array}{l}\text { Standard Methods of Fire Tests of } \\
\text { Building Construction and Materials }\end{array}$ & nureg0933, 3.149 & 1992 & $\mathbf{R}$ & & NFPA 251 & 1990 \\
\hline 4018 & NFPA & NFPA 251 & 1975 & $\begin{array}{l}\text { Standard Methods of Fire Tests of } \\
\text { Building Construction and Materials }\end{array}$ & reg1.120.ro1 & 1977 & $\mathbf{R}$ & & NFPA 251 & 1990 \\
\hline 4019 & NFPA & NFPA 251 & 1975 & $\begin{array}{l}\text { Standard Methods of Fire Tests of } \\
\text { Building Construction and Materials }\end{array}$ & srp9.5.1 & 1981 & $P$ & & NFPA 251 & 1990 \\
\hline 4020 & NFPA & NFPA 252 & $N / S$ & $\begin{array}{l}\text { Standard Methods of Fire Tests of } \\
\text { Door Assemblies }\end{array}$ & nureg0933, 3.149 & 1992 & A & & NFPA 252 & 1990 \\
\hline 4021 & NFPA & NFPA 259 & 1976 & $\begin{array}{l}\text { Standard Test Method for Potential } \\
\text { Heat of Building Materials }\end{array}$ & reg1.120.ro1 & 1977 & $\mathbf{P}$ & & NFPA 259 & 1993 \\
\hline 4022 & NFPA & NFPA 259 & 1976 & $\begin{array}{l}\text { Standard Test Method for Potential } \\
\text { Heat of Building Materials }\end{array}$ & sip9.5.1 & 1981 & $R$ & & NFPA 259 & 1993 \\
\hline 4023 & NFPA & NFPA 312 & $N / S$ & $\begin{array}{l}\text { Standard for Fire Protection of } \\
\text { Vessels During Construction, } \\
\text { Repair, and Lay-Up }\end{array}$ & inm88055 & 1991 & $\mathbf{R}$ & & NFPA 312 & 1990 \\
\hline
\end{tabular}


Codes and Standards and Other Guidance Cited in Regulatory Documents

Part A - Industry Consensus Codes and Standards (continued)

\begin{tabular}{|c|c|c|c|c|c|c|c|c|c|c|}
\hline Bocord & Code & Standerd & $\begin{array}{l}\text { Standard } \\
\text { Vorslon }\end{array}$ & (n) & Document & $\begin{array}{l}\text { Doe. } \\
\text { Date. }\end{array}$ & $\begin{array}{l}\text { Accept: } \\
\text { ablility }\end{array}$ & Commente & Current Voraton & $\begin{array}{l}\text { Cowrom } \\
\text { Vorion } \\
\text { Dowle }\end{array}$ \\
\hline 4024 & NFPA & NFPA 321 & $\mathrm{~N} / \mathrm{S}$ & $\begin{array}{l}\text { Standard on Basic Classification of } \\
\text { Flammable and Combustible }\end{array}$ & inm88055 & 1991 & $\mathbf{R}$ & & NFPA 321 & 1991 \\
\hline 4025 & NFPA & NFPA 482 & N/S & $\begin{array}{l}\text { Standard for the Production, } \\
\text { Processing, Handling, and Storage } \\
\text { of Zrconium }\end{array}$ & inm88055 & 1991 & $\mathbf{R}$ & & NFPA 482 & 1987 \\
\hline 4026 & NFPA & NFPA 600 & $\mathrm{~N} / \mathrm{S}$ & $\begin{array}{l}\text { Standard on Industrial Fire } \\
\text { Brigades }\end{array}$ & inm88055 & 1991 & $\mathbf{R}$ & & NFPA 600 & 1992 \\
\hline 4027 & NFPA & NFPA 801 & N/S & $\begin{array}{l}\text { Recommended Fire Protection } \\
\text { Practice for Facilitios Handling } \\
\text { Radioactive Materials }\end{array}$ & inm88055 & 1991 & $\mathbf{R}$ & & NFPA 801 & 1991 \\
\hline 4028 & NFPA & NFPA 802 & 1974 & $\begin{array}{l}\text { Recommended Fire Protection } \\
\text { Practice for Nuclear Research and } \\
\text { Production Reactors }\end{array}$ & reg1.120.ro1 & 1977 & $\mathbf{R}$ & & NFPA 802 & 1993 \\
\hline 4029 & NFPA & NFPA 802 & $\mathrm{~N} / \mathrm{S}$ & $\begin{array}{l}\text { Recommended Fire Protection } \\
\text { Practice for Nuclear Research and } \\
\text { Production Reactors }\end{array}$ & $\operatorname{rog} 1.70 .103$ & 1978 & $\mathbf{R}$ & & NFPA 802 & 1993 \\
\hline 4030 & NFPA & NFPA 802 & 1974 & $\begin{array}{l}\text { Recommended Fire Protection } \\
\text { Practice for Nuclear Research and } \\
\text { Production Reactors }\end{array}$ & srp9.5.1 & 1981 & $\bar{A}$ & & NFPA 80\% & 1993 \\
\hline 4031 & NFPA & NFPA 803 & 1983 & $\begin{array}{l}\text { Standard for Fire Protection for } \\
\text { Light Water Nuclear Power Plants }\end{array}$ & inm64704 & 1986 & $\bar{R}$ & & NFPA 803 & 1993 \\
\hline 4032 & NFPA & $\longdiv { \text { NFPA } 8 0 3 }$ & $\mathrm{N} / \mathrm{S}$ & $\begin{array}{l}\text { Standard for Fire Protectlon for } \\
\text { Light Wator Nuclear Power Plants }\end{array}$ & inm88055 & 1991 & $\mathbf{R}$ & & NFPA 803 & $19: 3$ \\
\hline 4033 & NFPA & NFPA 1962 & 1962 & $\begin{array}{l}\text { Standard for the Care, Use, and } \\
\text { Service Testing of Fire Hose } \\
\text { Including Couplings and Nozzles }\end{array}$ & srp9.5.1 & 1981 & $\mathbf{P}$ & & NFPA 1962 & 1993 \\
\hline 4034 & NRA & NRA & 1976 & High Power Pifile Aules Book & $10 \mathrm{ctr} 73$ & 1993 & $\mathbf{P}$ & & $\begin{array}{l}\text { High Power Rifle } \\
\text { Rules Book }\end{array}$ & 1993 \\
\hline 4035 & NRA & NRA & 1976 & High Power Rifle Rules Book & inm81501 & 1984 & $\mathbf{A}$ & & $\begin{array}{l}\text { High Power Rifle } \\
\text { Rules Book }\end{array}$ & 1993 \\
\hline 4036 & NRA & NRA & $\mathrm{N} / \mathrm{S}$ & NRA Target Manufacturers Index & reg5.20.roo & 1974 & $\bar{A}$ & & \begin{tabular}{|l} 
NRA Target \\
Manufacturers \\
index
\end{tabular} & 1992 \\
\hline
\end{tabular}




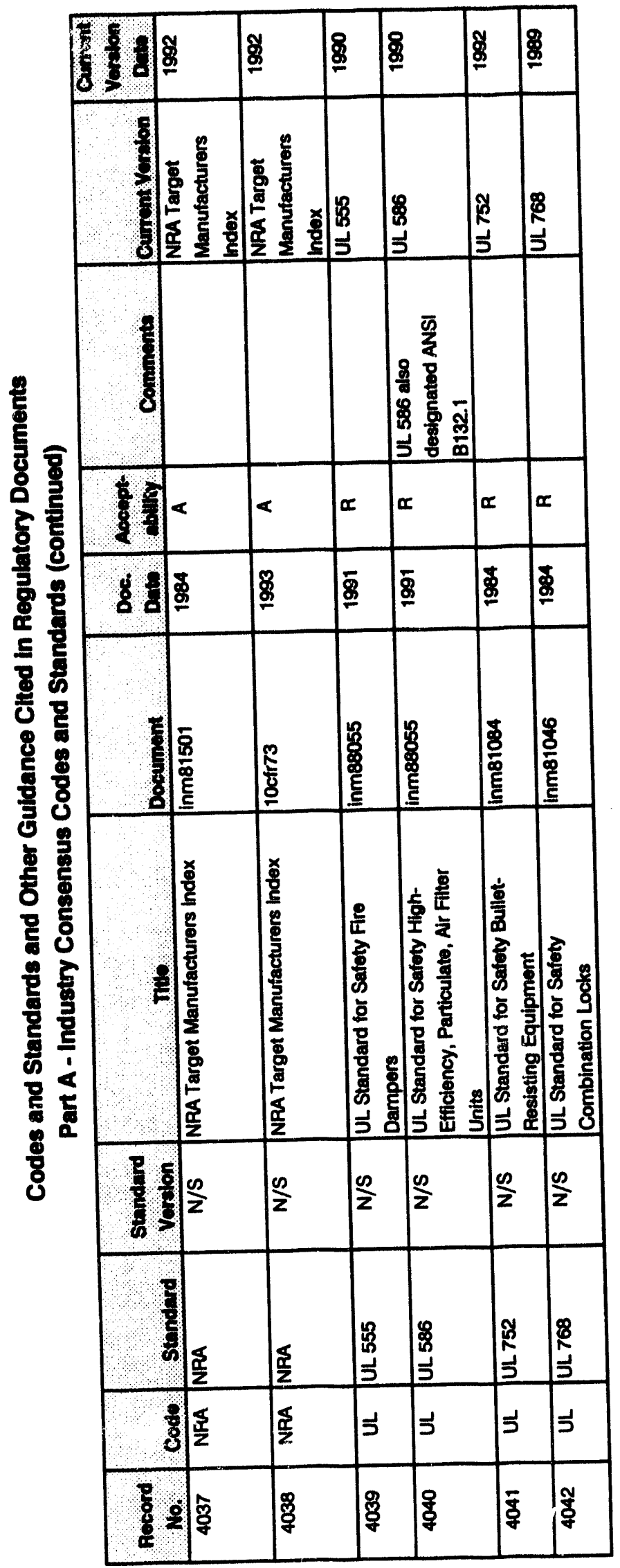


Part B

Other Guidance 
Codes and Standards and Other Guidance Ched in Regulatory Documents

Part B - Other Guidance

\begin{tabular}{|c|c|c|c|c|c|c|c|c|c|c|}
\hline $\begin{array}{c}\text { Record } \\
\text { No. }\end{array}$ & Code & Standard & $\begin{array}{l}\text { Stunderd } \\
\text { Voraton }\end{array}$ & The & Doonment & $\begin{array}{l}\text { Doe. } \\
\text { Dene }\end{array}$ & noospt: & Comanom & Conent verien & 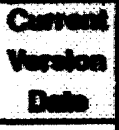 \\
\hline 4043 & $\mathrm{ACl}$ & ACl Journal & 1960 & $\begin{array}{l}\text { Corrosion of Prestressed Wire in } \\
\text { Concrete }\end{array}$ & reg1.107.01 & 197 & $\mathbf{R}$ & & ACT Journed & 1900 \\
\hline 4044 & $\overline{\mathrm{ACI}}$ & ACI Journal & 1969 & $\begin{array}{l}\text { Corrosion and Corrosion Protection } \\
\text { of Tendons in Prostressed Concreto } \\
\text { Bridges }\end{array}$ & reg1.107.501 & 197 & $\mathbf{R}$ & Paper & ACI Joumal & 1900 \\
\hline 4045 & $\mathbf{A C I}$ & ACI Journal & 1906 & $\begin{array}{l}\text { Influence of Stze and Shape of } \\
\text { Momber on the Shrinkage and } \\
\text { Creep of Concrote }\end{array}$ & reg1.35.1.100 & 1990 & $\mathbf{R}$ & & Act Joume & 1900 \\
\hline 4046 & $\overline{\mathrm{ACI}}$ & ACI Journal & 1975 & $\begin{array}{l}\text { Concreto Quality Control } \\
\text { Instruments }\end{array}$ & reg1.90.ro1 & 197 & $\mathbf{A}$ & & AC Journed & 1975 \\
\hline 4047 & $\mathrm{ACl}$ & ACl Journal & 1949 & $\begin{array}{l}\text { An Ultrasonic Method of Studying } \\
\text { Doterioration and Cracking in } \\
\text { Concrete Structures }\end{array}$ & reg1.90.501 & 1977 & $\mathbf{R}$ & & ACI Jound & 1940 \\
\hline 4048 & $\overline{\mathrm{ACI}}$ & \begin{tabular}{|l|}
$\mathrm{ACl}$ \\
Monograph No \\
2
\end{tabular} & N/S & $\begin{array}{l}\text { Evaluation of Concrote Properties } \\
\text { trom Sonic Tests }\end{array}$ & reg1.90.ro1 & 197 & $\overline{\mathbf{R}}$ & & $\begin{array}{l}\text { ACG Monograph } \\
\text { No } 2\end{array}$ & N/S \\
\hline 4049 & $\overline{\mathrm{ACT}}$ & $\begin{array}{l}\text { ACI Paper } 31 \\
\text { of ACI SP } 34\end{array}$ & 1972 & $\begin{array}{l}\text { Effect of Temperature on Creep of } \\
\text { Concrete, A Literature Reviow }\end{array}$ & $\operatorname{reg} 1.35 .1 .100$ & 1990 & $\mathbf{R}$ & & $\begin{array}{l}\text { Aa Peper } 31 \text { of } \\
\text { ACT SP } 34\end{array}$ & 1972 \\
\hline 4050 & AEC & 28 F.R. 9678 & 1963 & $\begin{array}{l}\text { Notice Pegarding Need for } \\
\text { Establishment of Film Dosimetry } \\
\text { Callbration Laboratory }\end{array}$ & reg8.3.100 & 1973 & $\mathbf{R}$ & & 28 F.R. 9678 & 1963 \\
\hline 4051 & AEC & \begin{tabular}{|l} 
AEC Air \\
Cleaning \\
Conference
\end{tabular} & 1974 & $\begin{array}{l}\text { Nuclear Power Plant Control Foom } \\
\text { Ventilation Systom Destgn for } \\
\text { Moeting General Deaign Critorion } \\
19\end{array}$ & nureg0737 & 1980 & $\mathbf{R}$ & & $\begin{array}{l}\text { AEC Air Cleening } \\
\text { Conforence }\end{array}$ & 1974 \\
\hline 4052 & AEC & \begin{tabular}{|l|} 
AEC Contract \\
W-7405-ong-26
\end{tabular} & 1972 & $\begin{array}{l}\text { Soil Bohavior Under Earthquake } \\
\text { Loading Conditions - State-of-the- } \\
\text { Art Evaluation of Characteristics for } \\
\text { Soismic Posponses Analyees }\end{array}$ & $\operatorname{sip} 2.5 .5$ & 1981 & $\mathbf{R}$ & & $\begin{array}{l}\text { AEC Contrect W } \\
7405 \text {-eng-26 }\end{array}$ & 1972 \\
\hline 4053 & AEC & AEC Paper & 1967 & Your Body and Radiation & droop212-4 & 1984 & A & & AEC Paper & 1967 \\
\hline 4054 & AEC & $\begin{array}{l}\text { AEC RED } \\
\text { Report GEAP } \\
4616\end{array}$ & 1964 & $\begin{array}{l}\text { Two-Phase Pressure Drop in } \\
\text { Straight Pipes and Channets: } \\
\text { Water-Steam Mixtures at } 600 \text { to } \\
1400 \text { psia }\end{array}$ & 1091.157 .000 & 1969 & $\bar{R}$ & & $\begin{array}{l}\text { AEC RED Peport } \\
\text { GEAP } 4616\end{array}$ & 1964 \\
\hline
\end{tabular}




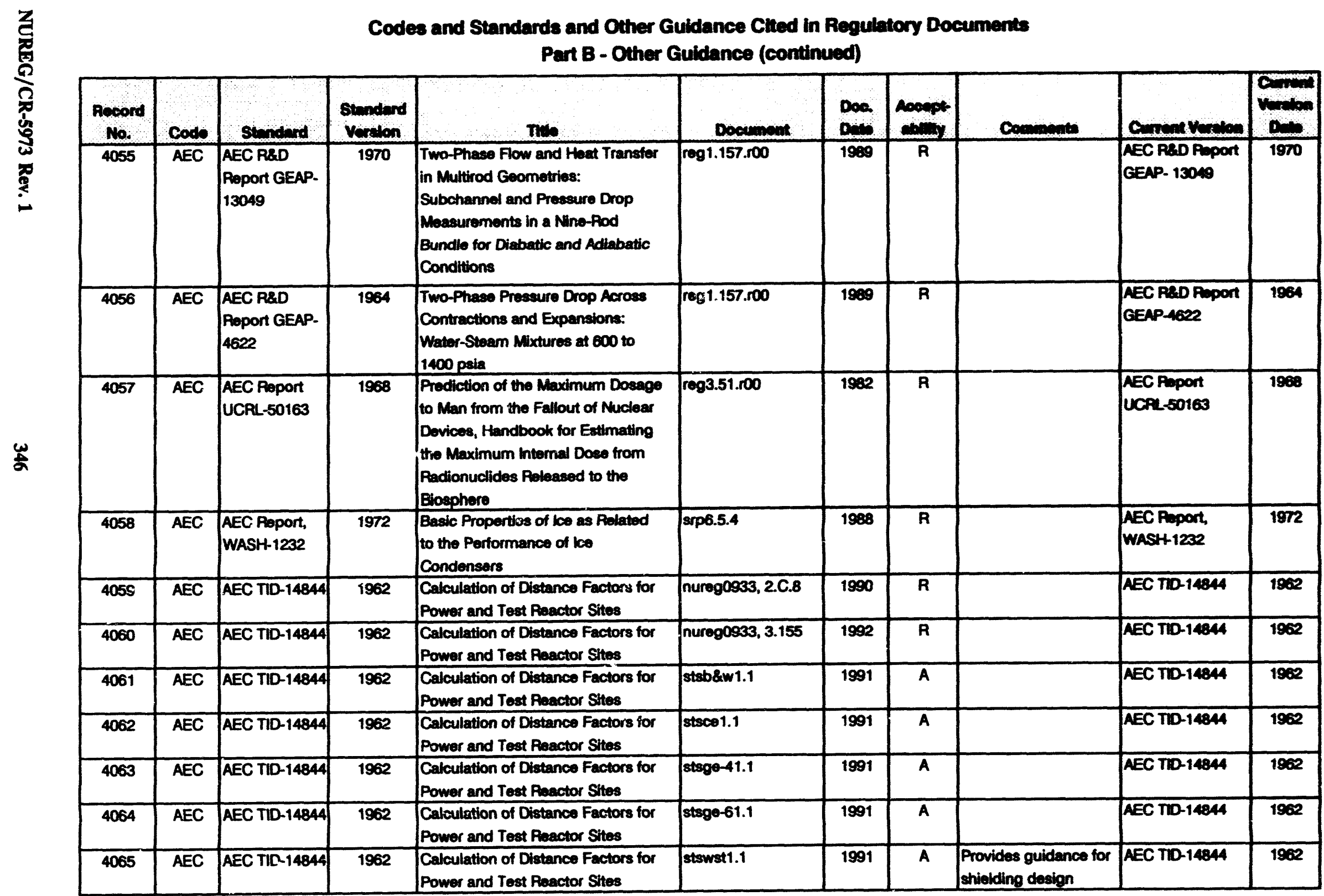


Codes and Standards and Other Guidance Clted in Regulatory Documents

Part B - Other Guidance (continued)

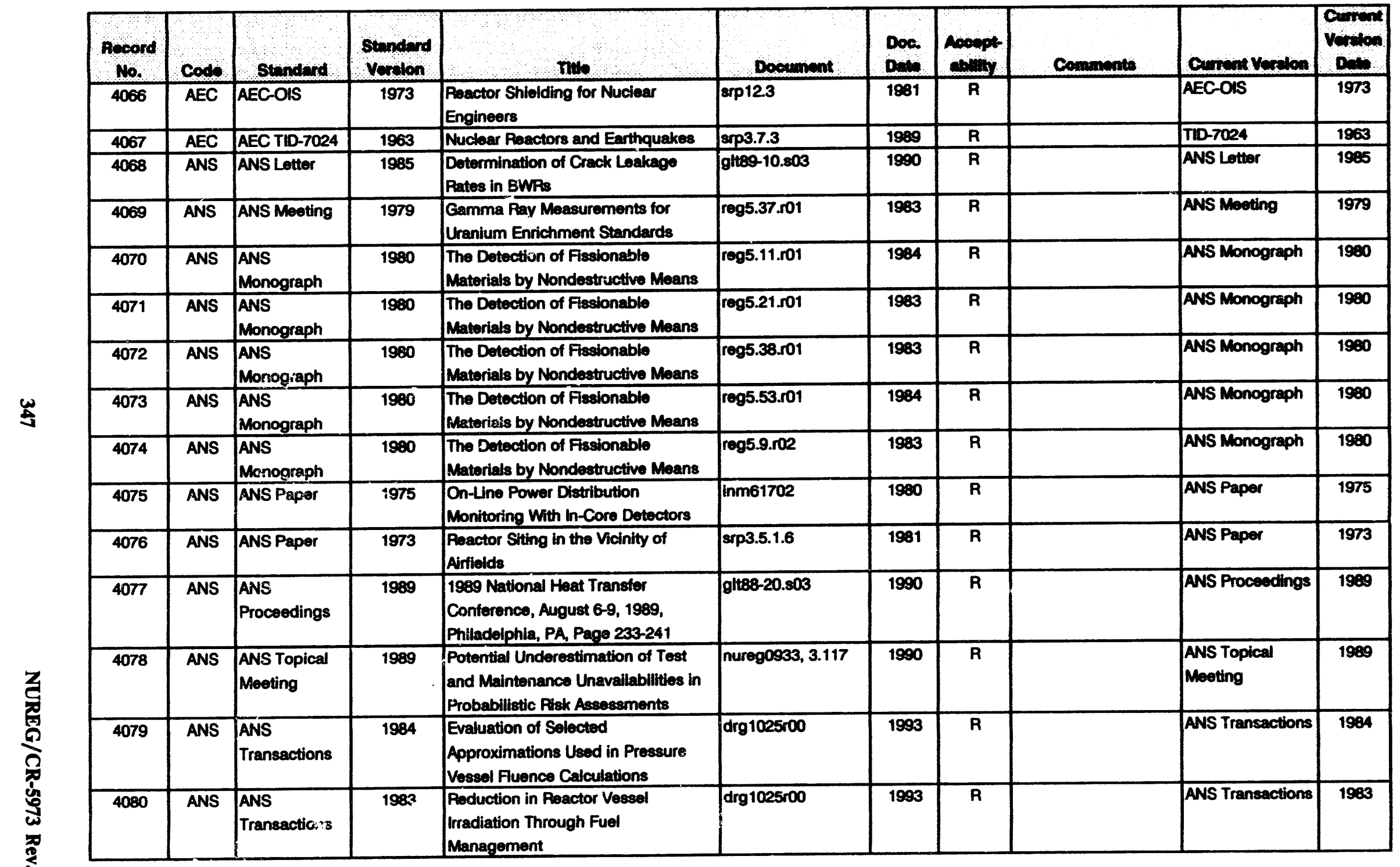


Codes and Standards and Other Guidance Cited in Regulatory Documents Part B - Other Guidance (continued)

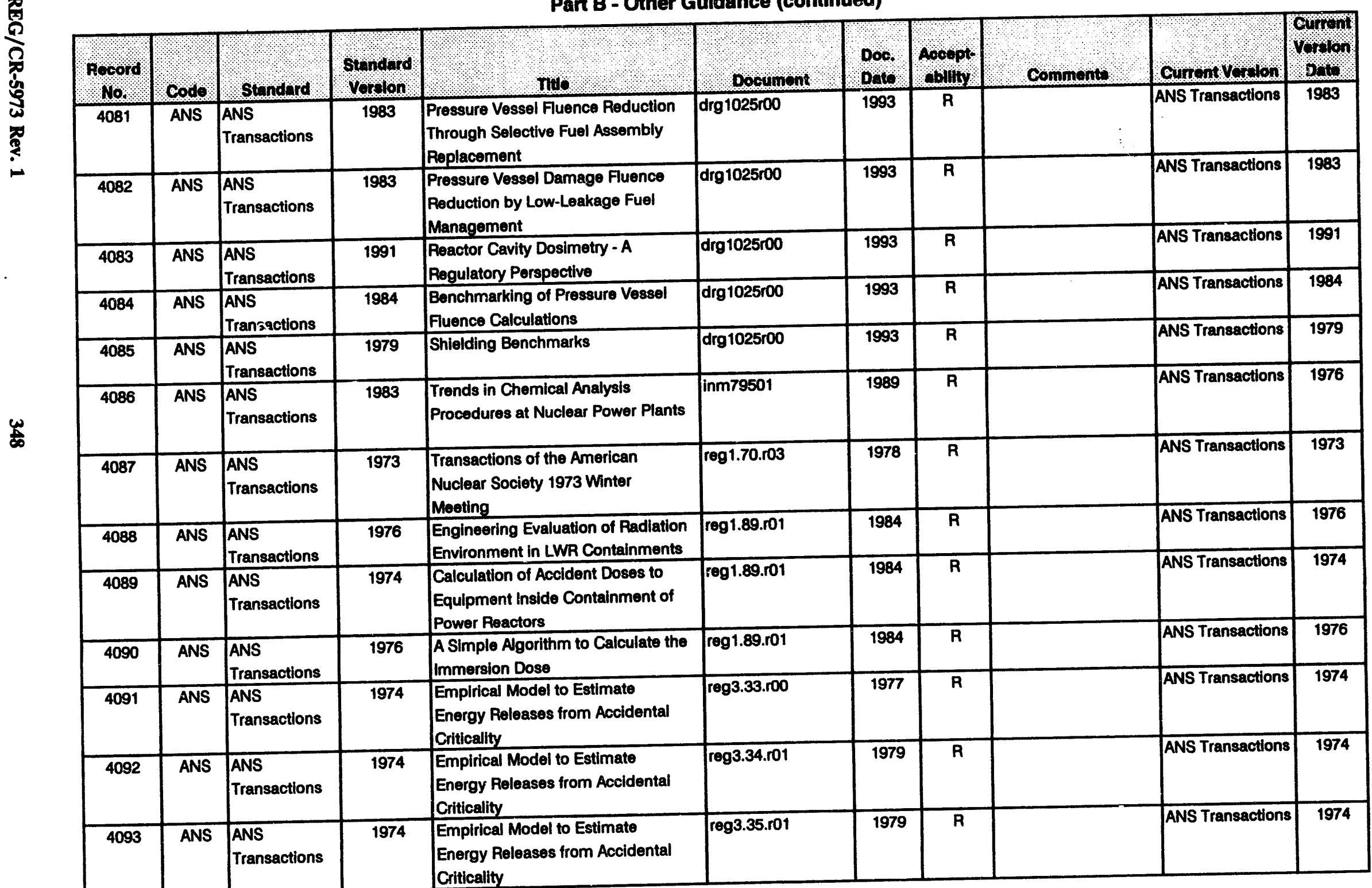


Codes and Standards and Other Guldance Clted in Regulatory Documents

Part B - Other Guldance (continued)

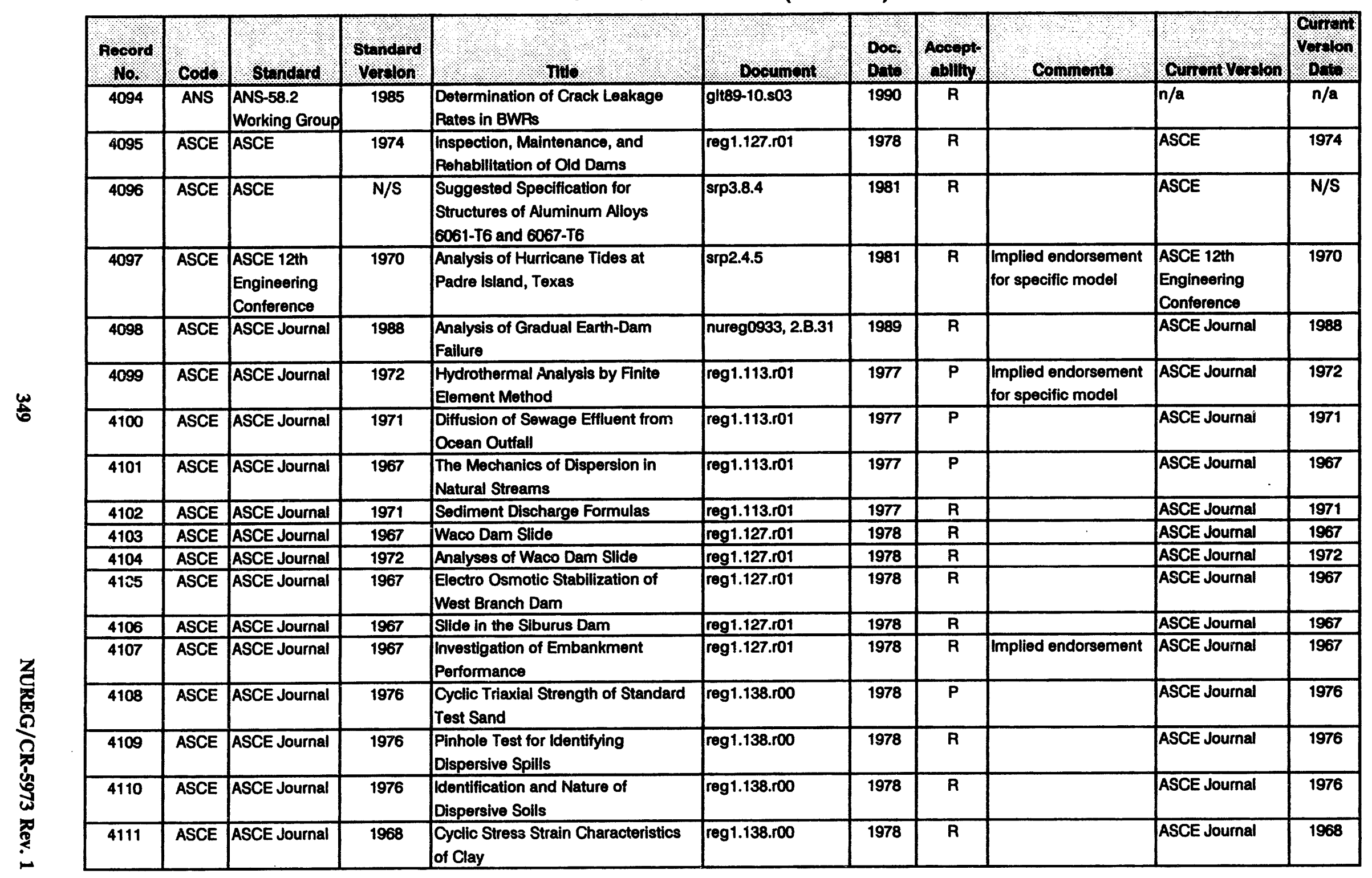


Codes and Standards and Other Guidance Cited in Regulatory Documents Part B - Other Guldance (continued)

\begin{tabular}{|c|c|c|c|c|c|c|c|c|c|c|}
\hline & & & & & & & & & & \\
\hline Rocord & 00.0 & & Standard & Tito & Doormont & 000 . & Acoopts & Comnents & Cun ont vor, lon & Gorron \\
\hline 4112 & ASCE & $\frac{\text { Standard }}{\text { ASCE Journal }}$ & 1971 & $\begin{array}{l}\text { Deformation Characteristics of } \\
\text { Sand Under Cyclic Loading }\end{array}$ & reg1.138.100 & 1978 & $\mathbf{R}$ & & ASCE Journal & 1971 \\
\hline 4113 & ASCE & ASCE Journal & 1971 & $\begin{array}{l}\text { Sand Liquefaction in Triaxial and } \\
\text { Simple Shear Tests }\end{array}$ & reg1.138.roo & 1978 & $R$ & & ASCE Journal & 1971 \\
\hline 4114 & ASCE & ASCE Journal & 1975 & $\begin{array}{l}\text { Testing Procedure Effects on } \\
\text { Dynamic Soll Behavior }\end{array}$ & reg1.138.r00 & 1978 & 9 & & ASCE Journal & 1975 \\
\hline 4115 & ASCE & ASCE Journal & 1977 & $\begin{array}{l}\text { Creep of Concrete A Consistent } \\
\text { Engineering Approach }\end{array}$ & reg1.35.1.r00 & 1990 & $\mathbf{P}$ & $\begin{array}{l}\text { Implied endorsement } \\
\text { as acceptable } \\
\text { scientific metiod }\end{array}$ & ASCE Journal & 1977 \\
\hline 4116 & ASCE & ASCE Journal & 1977 & $\begin{array}{l}\text { Stochastic Process for } \\
\text { Extrapolating Concrete Creep }\end{array}$ & reg1.35.1.r00 & 1990 & $\bar{P}$ & & ASCE Journal & 1977 \\
\hline 4117 & ASCE & ASCE Journal & 1975 & \begin{tabular}{|l|} 
Definition of Statistically \\
Independent Time Histories
\end{tabular} & reg1.92.ro1 & 1976 & $\mathbf{R}$ & & ASCE Journal & 1975 \\
\hline 4118 & ASCE & ASCE Journal & 1967 & $\begin{array}{l}\text { Seismic Failures of Chilean Tailings } \\
\text { Dams }\end{array}$ & reg3.11.ro2 & 1977 & $\mathbf{R}$ & & ASCE Journal & 1967 \\
\hline 4119 & ASCE & ASCE Journal & 1974 & $\begin{array}{l}\text { Inspection, Maintenance, and } \\
\text { Rehabilitation of Old Dams }\end{array}$ & reg3.11.1.r01 & 1980 & $\mathbf{R}$ & & ASCE Journal & 1974 \\
\hline 4120 & ASCE & ASCE Journal & 1967 & $\begin{array}{l}\text { Seismic Fallures of Chilean Tailings } \\
\text { Dams }\end{array}$ & reg3.11.1.ro1 & 1980 & $\mathbf{R}$ & & ASCE Journal & 1967 \\
\hline 4121 & ASCE & ASCE Journal & 1967 & Stability Analysis of Embankments & reg3.11.r02 & 1977 & $\mathbf{R}$ & & ASCE Journal & 1967 \\
\hline 4122 & ASCE & ASCE Journal & 1967 & $\begin{array}{l}\text { Use of Computers for Slope } \\
\text { Stability Analysis }\end{array}$ & reg3.11.r02 & 1977 & $\mathbf{R}$ & & ASCE Journal & 1967 \\
\hline 4123 & ASCE & ASCE Journal & 1965 & $\begin{array}{l}\text { Earthquake Induced Displacenient } \\
\text { in Sand Embankments }\end{array}$ & reg3.11.ro2 & 1977 & $\mathbf{R}$ & & ASCE Journal & 1965 \\
\hline 4124 & ASCE & ASCE Journal & 1971 & $\begin{array}{l}\text { The Slides in the San Fernando } \\
\text { Dam During the Earthquake of } \\
\text { February } 9,1971\end{array}$ & reg3.11.ro2 & 1977 & $R$ & & ASCE Journal & 1971 \\
\hline 4125 & ASCE & ASCE Journal & 1967 & Slope Stability During Earthquakes & reg3.11.ro2 & 1977 & $\mathbf{R}$ & & ASCE Journal & 1967 \\
\hline 4126 & ASCE & ASCE Journal & 1971 & $\begin{array}{l}\text { Dynamic Analysis of the Slide in the } \\
\text { Lower San }\end{array}$ & reg3.11.r02 & 1977 & $\mathbf{R}$ & & ASCE Journal & 1971 \\
\hline 4127 & ASCE & ASCE Journal & 1982 & $\begin{array}{l}\text { Estimating Soil Water Retention } \\
\text { From Soil Properties }\end{array}$ & reg3.64.r01 & 1989 & $\bar{R}$ & & ASCE Journal & 1982 \\
\hline 4128 & ASCE & ASCE Journal & 1972 & $\begin{array}{l}\text { Hydrothermal Analysis by the Finite } \\
\text { Element Method }\end{array}$ & reg4.4.ro0 & 1975 & $\mathbf{R}$ & & ASCE Journal & 1972 \\
\hline
\end{tabular}


Codes and Standards and Other Guidance Cited in Regulatory Documents

Part B - Other Guidance (continued)

\begin{tabular}{|c|c|c|c|c|c|c|c|c|c|c|}
\hline Hocord & Codo & Standard & strond & 1 & Ooconont & Doo: & poopplof &., 60 mmonts & Curront vorilon & portos \\
\hline 4129 & ASCE & ASCE Journal & 1968 & New Distribution of Extreme Winds & srp2.3.1 & 1981 & $\mathbf{R}$ & $\begin{array}{l}\text { Endorsed on a case by } \\
\text { case basis }\end{array}$ & ASCE Journal & 1968 \\
\hline 4130 & ASCE & ASCE Journal & 1967 & $\begin{array}{l}\text { Darcy's Flow Solutions with a Free } \\
\text { Surface }\end{array}$ & $\operatorname{sip2.4.13}$ & 1981 & $\mathbf{P}$ & & ASCE Journal & 1967 \\
\hline 4131 & ASCE & ASCE Journal & 1968 & $\begin{array}{l}\text { New Distribution of Extreme Winds } \\
\text { in the United States }\end{array}$ & srp2.4.3 & 1989 & $\mathbf{R}$ & & ASCE Journal & 1968 \\
\hline 4132 & ASCE & ASCE Journal & $\mathrm{N} / \mathrm{S}$ & $\begin{array}{l}\text { Journal of the Geotechnical } \\
\text { Engineering Division }\end{array}$ & srp2.5.4 & 1981 & $\mathbf{R}$ & $\begin{array}{l}\text { Acceptable for three } \\
\text { dimensional } \\
\text { earthquake effects }\end{array}$ & ASCE Journal & N/S \\
\hline 4133 & ASCE & ASCE Journal & 1973 & \begin{tabular}{|l} 
Design Response Spectra for \\
Nuclear Power Plants \\
\end{tabular} & srp3.9.2 & 1981 & A & & ASCE Journal & 1973 \\
\hline 4134 & ASCE & ASCE Journal & 1975 & $\begin{array}{l}\text { Definition of Statistically } \\
\text { Independent Time Histories }\end{array}$ & sip3.9.2 & 1981 & $\mathbf{R}$ & Acceptable model & ASCE Journal & 1975 \\
\hline 4135 & ASCE & ASCE Paper & 1971 & $\begin{array}{l}\text { Regional Water Supply Systems } \\
\text { Analysis for a Megalopolis }\end{array}$ & reg1.113.rot & 1977 & $\mathbf{P}$ & & ASCE Paper & 1971 \\
\hline 4136 & ASCE & ASCE Paper & 1981 & $\begin{array}{l}\text { Consequences of Earthquakes in } \\
\text { the Mississippi Valley }\end{array}$ & srp2.5.2 & 1989 & $\mathbf{R}$ & & ASCE Paper & 1981 \\
\hline 4137 & ASCE & ASCE Paper & 1974 & $\begin{array}{l}\text { Soll Motion Computation by } \\
\text { Characteristics Methods }\end{array}$ & $\operatorname{sip} 2.5 .2$ & 1989 & $\mathbf{R}$ & $\begin{array}{l}\text { Implied endorsement } \\
\text { on a case by case } \\
\text { basis }\end{array}$ & ASCE Paper & 1974 \\
\hline 4138 & ASCE & $\begin{array}{l}\text { ASCE Paper } \\
3269\end{array}$ & 1961 & Wind Forces on Structures & srp3.3.1 & 1981 & $\mathbf{P}$ & $\begin{array}{l}\text { Implied endorsement } \\
\text { on a case by case } \\
\text { basis }\end{array}$ & ASCE Paper 3269 & 1961 \\
\hline 4139 & ASCE & $\begin{array}{l}\text { ASCE Paper } \\
3269 \\
\end{array}$ & 1961 & Wind Forces on Structures & sip3.3.2 & 1984 & $\mathbf{P}$ & & ASCE Paper 3269 & 1961 \\
\hline 4140 & ASCE & \begin{tabular}{|l|} 
ASCE Power \\
Division \\
\end{tabular} & 1971 & $\begin{array}{l}\text { Testing for Large Curved } \\
\text { Prestressing Tendons }\end{array}$ & reg1.107.r01 & 1977 & $\mathbf{R}$ & & $\begin{array}{l}\text { ASCE Power } \\
\text { Division }\end{array}$ & 1971 \\
\hline 4141 & ASCE & \begin{tabular}{|l} 
ASCE Soil \\
Mechanics \\
Foundation \\
Division \\
\end{tabular} & 1972 & $\begin{array}{l}\text { Investigation for Design and } \\
\text { Construction of Foundations of } \\
\text { Buildings }\end{array}$ & reg1.132.ro1 & 1979 & $\mathbf{R}$ & & $\begin{array}{l}\text { ASCE Soil } \\
\text { Mechanics } \\
\text { Foundation } \\
\text { Division } \\
\end{array}$ & 1972 \\
\hline
\end{tabular}


Codes and Standards and Other Guidance Ched in Regulatory Documents

Part B - Other Guidance (continued)

\begin{tabular}{|c|c|c|c|c|c|c|c|c|c|c|}
\hline 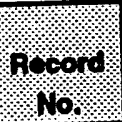 & $0 \% \cdot 0$ & 5.1 .010 & Shorolon & V. & 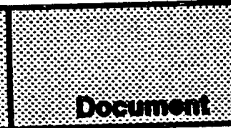 & $0.1 \%$ & \%ocon & , & 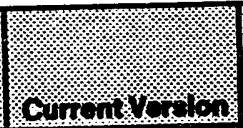 & $\operatorname{lom}_{0.1}$ \\
\hline 4142 & ASCE & $\begin{array}{l}\text { ASCE Soll } \\
\text { Mochanics } \\
\text { Foundation } \\
\text { Division } \\
\end{array}$ & 1967 & $\begin{array}{l}\text { ASCE Soll Mochanics and } \\
\text { Foundation Division Conference on } \\
\text { Stability and Performance of } \\
\text { Slopes and Embankments }\end{array}$ & srp2.5.5 & 1981 & $\mathbf{R}$ & Accoptable model & $\begin{array}{l}\text { ASCE Soll } \\
\text { Mochanics } \\
\text { Foundation } \\
\text { Division } \\
\end{array}$ & 1967 \\
\hline 4143 & ASCE & $\begin{array}{l}\text { ASCE } \\
\text { Specialty } \\
\text { Conference } \\
\end{array}$ & 1969 & Dolaware River Basin Modeling & reg1.113.ro1 & 1977 & $\mathbf{P}$ & Implied endorsement & $\begin{array}{l}\text { ASCE Specialty } \\
\text { Conference }\end{array}$ & 1909 \\
\hline 4144 & ASCE & $\begin{array}{l}\text { ASCE } \\
\text { Specialty } \\
\text { Conference } \\
\end{array}$ & 1975 & $\begin{array}{l}\text { Soismio Field Mothods for In Situ } \\
\text { Modull }\end{array}$ & reg1.132.101 & 1979 & $\mathbf{P}$ & ASCE paper & $\begin{array}{l}\text { ASCE Specialty } \\
\text { Conference }\end{array}$ & 1975 \\
\hline 4145 & ASCE & $\begin{array}{l}\text { ASCE } \\
\text { Specialty } \\
\text { Conference } \\
\end{array}$ & 1970 & $\begin{array}{l}\text { Technique for Study of Granular } \\
\text { Materials }\end{array}$ & reg1.132.ro1 & 1979 & $\mathbf{R}$ & Implied endorsement & $\begin{array}{l}\text { ASCE Speciaity } \\
\text { Conference }\end{array}$ & 1970 \\
\hline 4146 & ASCE & \begin{tabular}{|l} 
ASCE \\
Specialty \\
Conference \\
\end{tabular} & 1973 & $\begin{array}{l}\text { Generation of Seiemic Floor } \\
\text { Response Spectra for Equipment } \\
\text { Design }\end{array}$ & reg1.143.ro1 & 1979 & $\mathbf{P}$ & & $\begin{array}{l}\text { ASCE Specialty } \\
\text { Conference }\end{array}$ & 1973 \\
\hline 4147 & ASCE & $\begin{array}{l}\text { ASCE } \\
\text { Specialty } \\
\text { Conference }\end{array}$ & 1972 & $\begin{array}{l}\text { A Method for Analyzing Effects of } \\
\text { Dam Failures in Design Studies }\end{array}$ & $\operatorname{srp2.4.4}$ & 1981 & $\mathbf{R}$ & & $\begin{array}{l}\text { ASCE Specialty } \\
\text { Conference }\end{array}$ & 1972 \\
\hline 4148 & ASCE & $\begin{array}{l}\text { ASCE } \\
\text { Specialty } \\
\text { Conference } \\
\end{array}$ & 1973 & $\begin{array}{l}\text { Dynamic Analysis of Structural } \\
\text { Systems Excited at Multiple } \\
\text { Support Locations }\end{array}$ & srp3.9.2 & 1981 & $\mathbf{R}$ & & $\begin{array}{l}\text { ASCE Specialty } \\
\text { Conference }\end{array}$ & 1973 \\
\hline 4149 & ASCE & $\begin{array}{l}\text { ASCE } \\
\text { Specialty } \\
\text { Conference } \\
\end{array}$ & 1973 & $\begin{array}{l}\text { Influence of Closely Spaced Modes } \\
\text { in Response Spectrum Method of } \\
\text { Analysis }\end{array}$ & sip3.9.2 & 1981 & $\mathbf{R}$ & Implied endorsement & $\begin{array}{l}\text { ASCE Specialty } \\
\text { Conference }\end{array}$ & 1973 \\
\hline 4150 & ASCE & $\begin{array}{l}\text { ASCE } \\
\text { Structural } \\
\text { Engineering } \\
\text { Moeting } \\
\end{array}$ & 1973 & $\begin{array}{l}\text { Design Response Spectra for } \\
\text { Nuclear Power Plants }\end{array}$ & reg1.61.ro0 & 1973 & $\bar{P}$ & Implied endorsement & $\begin{array}{l}\text { ASCE Structural } \\
\text { Engineoring } \\
\text { Moeting }\end{array}$ & 1973 \\
\hline 4151 & ASCE & \begin{tabular}{|l} 
ASCE \\
Structural \\
Engineering \\
Meeting
\end{tabular} & 1973 & $\begin{array}{l}\text { Design Response Spectra for } \\
\text { Nuclear Power Plants }\end{array}$ & reg1.60.ro1 & 1973 & $\mathbf{P}$ & & $\begin{array}{l}\text { ASCE Structural } \\
\text { Engineoring } \\
\text { Moeting }\end{array}$ & 1973 \\
\hline
\end{tabular}


Codes and Standards and Other Guidance Clted in Regulatory Documents

Part B - Other Guidance (continued)

\begin{tabular}{|c|c|c|c|c|c|c|c|c|c|c|}
\hline $\begin{array}{l}\text { Rosord } \\
\text { No. }\end{array}$ & codo & Sthnderd & Strndard & Illo & Documont & $\begin{array}{l}\text { Doo. } \\
\text { Dote. }\end{array}$ & $\begin{array}{l}\text { Acoopt: } \\
\text { ablilly }\end{array}$ & Comments & cunent Vorton & Qnoptont \\
\hline 4152 & ASME & ASME & 1969 & $\begin{array}{l}\text { Correlation of Critical Heat Flux in a } \\
\text { Bundle Cooled by Pressurized } \\
\text { Water }\end{array}$ & 10 cfr50 App. K & 1991 & A & & ASME Paper & 1969 \\
\hline 4153 & ASME & $\begin{array}{l}\text { ASME Paper } \\
\text { HT-31 }\end{array}$ & 1969 & $\begin{array}{l}\text { Prediction of Blowdown and Jet } \\
\text { Thrust Forces }\end{array}$ & $\operatorname{srp3.6.2}$ & 1981 & R & & $\begin{array}{l}\text { ASME Paper HT- } \\
31\end{array}$ & 1969 \\
\hline 4154 & ASME & $\begin{array}{l}\text { Journal of } \\
\text { Heat Transfer }\end{array}$ & 1965 & $\begin{array}{l}\text { Maximum Flow Rate of a Single } \\
\text { Component, Two-Phase Mixture }\end{array}$ & 10cfr50 App. K & 1991 & A & & \begin{tabular}{|l|} 
Journal of Heat \\
Transfer
\end{tabular} & 1965 \\
\hline 4155 & ASME & $\begin{array}{l}\text { Nuclear } \\
\text { Engineering } \\
\text { and Design }\end{array}$ & 1991 & $\begin{array}{l}\text { A Review of the ASME Low Upper } \\
\text { Shelf Toughness Evaluation } \\
\text { Procedures for Nuclear Reactor } \\
\text { Pressure Vessels }\end{array}$ & drg1023.r00 & 1993 & $\mathbf{R}$ & & $\begin{array}{l}\text { Nuclear } \\
\text { Engineering and } \\
\text { Design }\end{array}$ & 1991 \\
\hline 4156 & ASME & $\begin{array}{l}\text { Transactions } \\
\text { of ASME, } 695- \\
702\end{array}$ & 1948 & $\begin{array}{l}\text { Prediction of Pressure Drop During } \\
\text { Forced Circulation Boiling of Water }\end{array}$ & $10 \mathrm{cfr} 50$ App. K & 1991 & A & $\begin{array}{l}\text { certification required } \\
\text { by ASNT-IRRSP }\end{array}$ & $\begin{array}{l}\text { Transactions of } \\
\text { ASME, } 695-702\end{array}$ & 1948 \\
\hline 4157 & ASNT & ASNT-IRRSP & $N / S$ & $\begin{array}{l}\text { American Society for } \\
\text { Nondestructive Testing }\end{array}$ & 10 cfr34 & 1991 & $\mathbf{A}$ & & $n / a$ & $n / a$ \\
\hline 4158 & ASTM & ASTM Bullotin & 1957 & $\begin{array}{l}\text { Stress Corrosion Cracking of } \\
\text { insulated Austenitic Stainless Steol }\end{array}$ & reg1.36.roo & 1973 & $\mathbf{R}$ & & ASTM Bulletin & 1957 \\
\hline 4159 & ASTM & $\begin{array}{l}\text { ASTM Fifth } \\
\text { International } \\
\text { Conference } \\
\end{array}$ & 1980 & $\begin{array}{l}\text { Burst Criterion of Zircaloy Fuel } \\
\text { Claddings in a LOCA }\end{array}$ & $\operatorname{sip} 4.2$ & 1981 & $\mathbf{R}$ & $\begin{array}{l}\text { Current version and } \\
\text { date not found }\end{array}$ & $\begin{array}{l}\text { ASTM Fifth } \\
\text { International } \\
\text { Conference } \\
\end{array}$ & 1980 \\
\hline 4160 & ASTM & $\begin{array}{l}\text { ASTM- } \\
\text { EURATOM } \\
\text { Symposium }\end{array}$ & 1985 & $\begin{array}{l}\text { Solid-State Track Recorder Neutron } \\
\text { Dosimetry in Light-Water Reactor } \\
\text { Pressure Vessel Surveillance } \\
\text { Mockups in Reactor Dosimetry }\end{array}$ & drg10025.r00 & 1993 & $\mathbf{R}$ & & $\begin{array}{l}\text { ASTM-EURATOM } \\
\text { Symposium }\end{array}$ & 1985 \\
\hline 4161 & ASTM & $\begin{array}{l}\text { ASTM- } \\
\text { EURATOM } \\
\text { Symposium } \\
\end{array}$ & 1990 & $\begin{array}{l}\text { Contributions of the Venus- } \\
\text { Engineering Mock-Up Experiments } \\
\text { to the LWR-PV Surveillance }\end{array}$ & drg10025.r00 & 1993 & $\mathbf{R}$ & & $\begin{array}{l}\text { ASTM-EURATOM } \\
\text { Symposium }\end{array}$ & 1990 \\
\hline 4162 & AWWA & AWWA & $N / S$ & $\begin{array}{l}\text { Standard Methods for the } \\
\text { Examination of Water and } \\
\text { Wastewater }\end{array}$ & reg1.137.ro0 & 1978 & $\mathbf{R}$ & & $n / a$ & $n / a$ \\
\hline 4163 & AWWA & AWWA Journal & 1965 & $\begin{array}{l}\text { Corrosion Protection Properties of } \\
\text { Portland Cement Concrete }\end{array}$ & reg1.107.ro1 & 1977 & $\mathbf{R}$ & $\begin{array}{l}\text { Current version and } \\
\text { date not found }\end{array}$ & AWWA Journal & 1965 \\
\hline
\end{tabular}


Codes and Standards and Other Guidance Cited in Regulatory Documents

Part B - Other Guidance (continued)

\begin{tabular}{|c|c|c|c|c|c|c|c|c|c|c|}
\hline \multicolumn{9}{|c|}{ 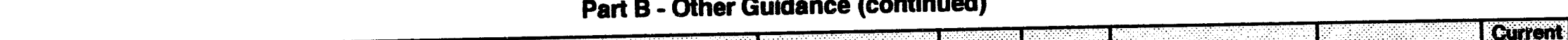 } & & \\
\hline Reord & codo & Standind & Stondard & (n) no, & Dosumont & 000 & ofollity & Comments & ourrontrow & Qhonton \\
\hline 4164 & DOE & DOE Bulletin & $\mathrm{N} / \mathrm{S}$ & $\begin{array}{l}\text { Filter Unit Inspection and Testing } \\
\text { Service }\end{array}$ & reg1.52.r02 & 1978 & R & & $n / a$ & $n / a$ \\
\hline 4165 & DOE & $\begin{array}{l}\text { DOE Order } \\
5632.1 \\
\end{array}$ & $\mathrm{~N} / \mathrm{S}$ & Protection Program Operations & $10 \operatorname{cfr} 73$ & 1992 & $\mathbf{R}$ & & $\begin{array}{l}\text { DOE Order } \\
5632.1 \mathrm{~B}\end{array}$ & 1992 \\
\hline 4166 & DOE & $\begin{array}{l}\text { DOE Order } \\
5632.2\end{array}$ & $\mathrm{~N} / \mathrm{S}$ & $\begin{array}{l}\text { Physical Protection of Special } \\
\text { Nuclear Material and Vital } \\
\text { Equipment }\end{array}$ & $10 \mathrm{cfr} 73$ & 1993 & $\mathbf{R}$ & & $\begin{array}{l}\text { DOE Order } \\
5632.2 A\end{array}$ & 1989 \\
\hline 4167 & DOE & $\begin{array}{l}\text { DOE ORO-651 } \\
\text { Rev } 5\end{array}$ & $\mathrm{~N} / \mathrm{S}$ & $\begin{array}{l}\text { Uranium Hexafluoride: Handling } \\
\text { Procedures and Container } \\
\text { Descriptions }\end{array}$ & not80-27 & 1990 & $\mathbf{R}$ & & ANSI N14.1 & 1990 \\
\hline 4168 & DOE & \begin{tabular}{|l|} 
DOE/DO/0053 \\
$-H 1$ (MLM- \\
$3245)$ \\
\end{tabular} & $N / S$ & $\begin{array}{l}\text { DOE Evaluation Document for DOT } \\
\text { 7A-Type A Packaging }\end{array}$ & not90-35 & 1990 & $\bar{R}$ & & $\begin{array}{l}\text { DOE/DO/0053- } \\
\text { H1 (MLM-3245) }\end{array}$ & 1987 \\
\hline 4169 & DOE & $\begin{array}{l}\text { DOE/EV/1830- } \\
\text { T5 }\end{array}$ & 1980 & $\begin{array}{l}\text { A Guide to Reducing Radiation } \\
\text { Exposure to As Low As Reasonably } \\
\text { Achievable (ALARA) }\end{array}$ & drg618-4.100 & 1982 & $\mathbf{R}$ & & DOE/EV/1830-T5 & 1980 \\
\hline 4170 & DOE & $\begin{array}{l}\text { UMTRA- } \\
\text { DOE/ALO } 165\end{array}$ & 1981 & $\begin{array}{l}\text { Radon Attenuation Effectiveness } \\
\text { and Cost Optimization for Uranium } \\
\text { Mill Tailings and Composite Covers }\end{array}$ & reg3.64.r00 & 1989 & $\mathbf{R}$ & & $\begin{array}{l}\text { UMTRA-DOE/ALO } \\
165\end{array}$ & 1981 \\
\hline 4171 & $\overline{D O E}$ & \begin{tabular}{|l|} 
UMTRA- \\
DOE/ALO-193
\end{tabular} & 1982 & $\begin{array}{l}\text { Engineering Guides for Estimating } \\
\text { Cover Material Thickness and } \\
\text { Volume }\end{array}$ & reg3.64.r00 & 1989 & $\mathbf{R}$ & & $\begin{array}{l}\text { UMTRA- } \\
\text { DOE/ALO-193 }\end{array}$ & 1982 \\
\hline 4172 & DOT & 10 CFR 71.3 & $\mathrm{~N} / \mathrm{S}$ & $\begin{array}{l}\text { Packaging and Transportation of } \\
\text { Radioactive Material }\end{array}$ & glt80-09 & 1980 & $\mathbf{R}$ & & 10 CFR 71 & 1993 \\
\hline 4173 & DOT & 10 CFR 71.5(b) & $N / S$ & $\begin{array}{l}\text { Packaging and Transportation of } \\
\text { Radioactive Material }\end{array}$ & reg7.7ror & 1977 & $\bar{R}$ & & 10 CFR 71 & 1993 \\
\hline 4174 & DOT & DOT & 1983 & $\begin{array}{l}\text { A Review of the DOT Regulations } \\
\text { for Transportation of Radioactive } \\
\text { Material }\end{array}$ & not84-14 & 1984 & $\mathbf{R}$ & $\begin{array}{l}\text { Packages not } \\
\text { authorized after } \\
6 / 30 / 85 \text {. }\end{array}$ & 49 CFR & 1993 \\
\hline 4175 & DOT & DOT & $N / S$ & DOT Specification 55 & not87-47 & 1987 & $\mathbf{P}$ & $\begin{array}{l}\text { Deals with Medical } \\
\text { Waste }\end{array}$ & 49 CFR & 1993 \\
\hline 4176 & DOT & $\begin{array}{l}49 \text { CFR } 100- \\
177\end{array}$ & $\mathrm{~N} / \mathrm{S}$ & Hazardous Materials Regulations & inm86740 & 1992 & $\mathbf{R}$ & & 49 CFR $100-177$ & 1993 \\
\hline 4177 & DOT & DOT & $N / S$ & DOT $17 \mathrm{H}$ Steol Drums & not89-85 & 1989 & $\mathbf{R}$ & & 49 CFR & 1993 \\
\hline
\end{tabular}


Codes and Standards and Other Guidance Cited in Regulatory Documents Part B - Other Guidance (continued)

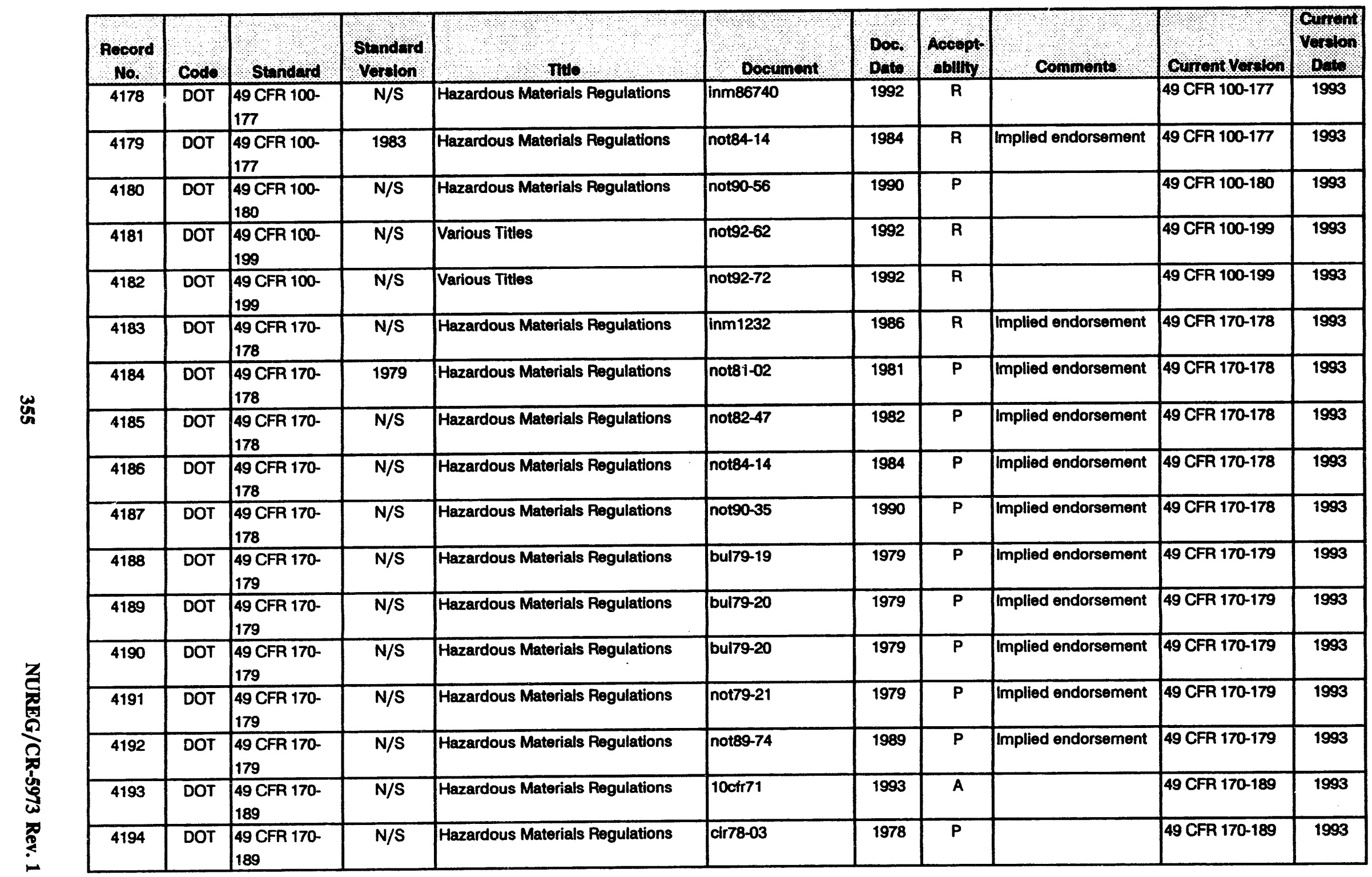




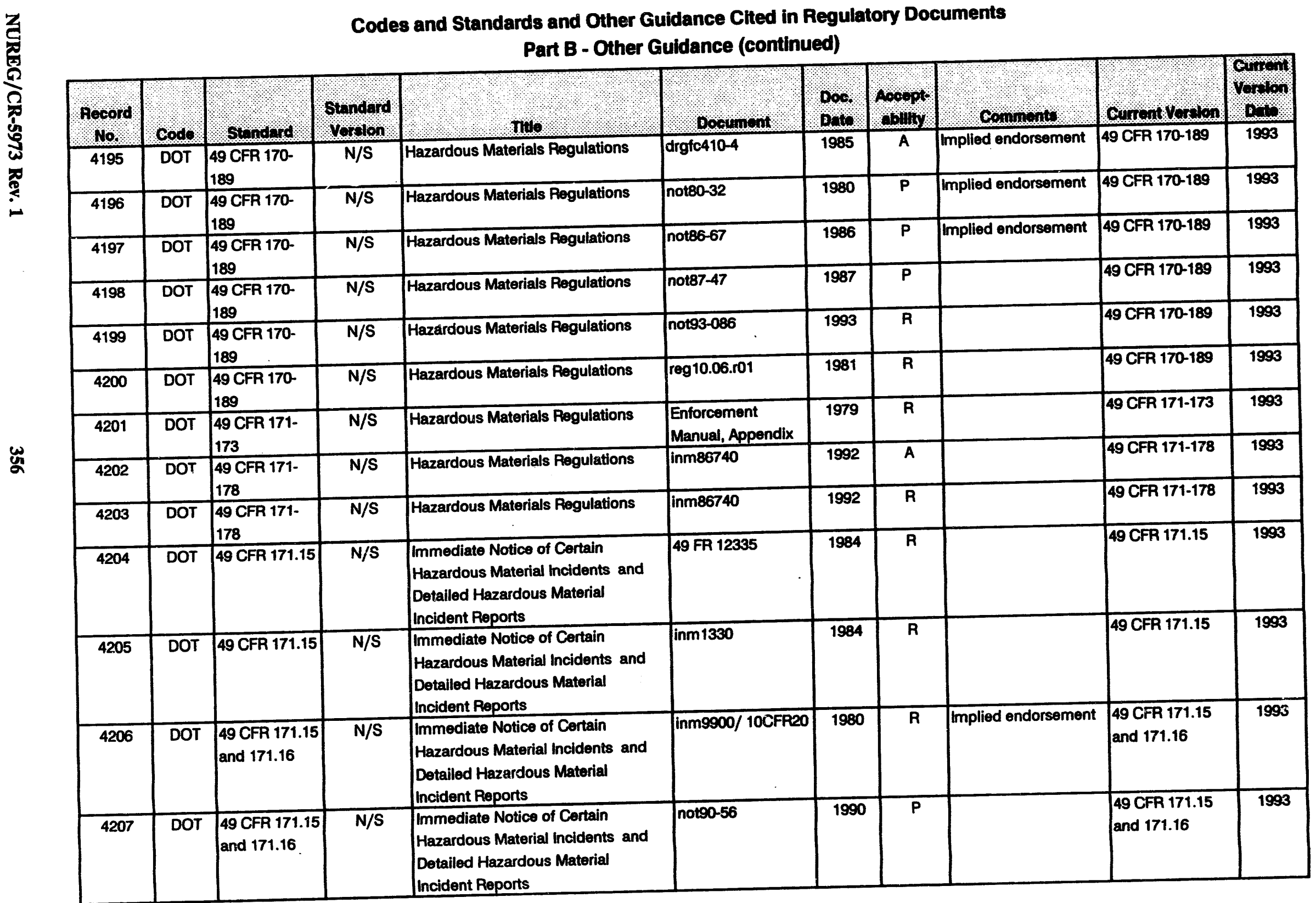


Codes and Standards and Other Guidance Clted in Regulatory Documents

Part B - Other Guidance (continued)

\begin{tabular}{|c|c|c|c|c|c|c|c|c|c|c|}
\hline $\begin{array}{l}\text { Rocord } \\
\text { No. }\end{array}$ & Code & Standerd & $\begin{array}{l}\text { Standard } \\
\text { Vorblon }\end{array}$ & The & Dooument & Don. & Accopt & Comments & Contem Vorton & amplon \\
\hline 4208 & DOT & 49 CFR 171.16 & $\mathrm{~N} / \mathrm{S}$ & $\begin{array}{l}\text { Detailed Hazardous Material } \\
\text { Incident Reports }\end{array}$ & $\begin{array}{l}\text { Enforcement } \\
\text { Manual, Appendix }\end{array}$ & 1979 & $\mathbf{R}$ & & 49 CFR 171.16 & 1993 \\
\hline 4209 & DOT & 49 CFR 172 & $N / S$ & $\begin{array}{l}\text { Hazardous Materials Tables, } \\
\text { Hazardous Materials } \\
\text { Communications Requirements } \\
\text { and Emergency Response } \\
\text { Information Requirements }\end{array}$ & drgfc407-4 & 1985 & $\mathbf{R}$ & Implied endorsement & 49 CFR 172 & 1993 \\
\hline 4210 & DOT & $\begin{array}{l}49 \text { CFR } \\
172.101 \\
\end{array}$ & 1979 & $\begin{array}{l}\text { Purpose and Use of Hazardous } \\
\text { Material Table }\end{array}$ & not82-47 & 1982 & $\mathbf{P}$ & Implied endorsement & 49 CFR 172.101 & 1993 \\
\hline 4211 & DOT & $\begin{array}{l}49 \text { CFR } \\
172.101 \text { and } \\
173.21(\mathrm{~b}) \\
\end{array}$ & 1979 & Various Titles & not80-25 & 1980 & $\mathbf{P}$ & & $\begin{array}{l}49 \text { CFR } 172.101 \\
\text { and } 173.21(\mathrm{~b})\end{array}$ & 1993 \\
\hline 4212 & DOT & $\begin{array}{l}49 \text { CFR } \\
172.202 \\
\end{array}$ & $N / S$ & $\begin{array}{l}\text { Description of Hazardous Material } \\
\text { on Shipping Papers }\end{array}$ & $10 \mathrm{cfr} 73$ & 1993 & A & & 49 CFR 172.202 & 1993 \\
\hline 4213 & DOT & $\begin{array}{l}49 \text { CFR } \\
172.400\end{array}$ & $\mathrm{~N} / \mathrm{S}$ & $\begin{array}{l}\text { Hazardous Materials Tables, } \\
\text { Hazardous Materials } \\
\text { Communications Pequirements } \\
\text { and Emergency Response } \\
\text { Information Requirements }\end{array}$ & not91-35 & 1991 & A & Implied endorsement & 49 CFR 172.400 & 1993 \\
\hline 4214 & DOT & \begin{tabular}{|l|}
49 CFR \\
$172.436-440$ \\
\end{tabular} & $\mathrm{~N} / \mathrm{S}$ & Radioactive Labeling & nots0-35 & 1990 & $\mathbf{P}$ & Implied endorsement & $\begin{array}{l}49 \text { CFA 172.436- } \\
440\end{array}$ & 1993 \\
\hline 4215 & DOT & \begin{tabular}{|l|}
49 CFR \\
$172.500-519,8$ \\
172.556
\end{tabular} & $N / S$ & Various Titles & not87-47 & 1987 & $\mathbf{P}$ & Implied endorsement & $\begin{array}{l}49 \text { CFA } 172.500- \\
519, \& 172.556\end{array}$ & 1993 \\
\hline 4216 & DOT & \begin{tabular}{|l|}
49 CFR \\
172.506 and \\
172.508 \\
\end{tabular} & 1979 & Vohicle Placards & not82-47 & 1982 & $\mathbf{P}$ & & $\begin{array}{l}49 \text { CFR } 172.506 \\
\text { and } 172.508\end{array}$ & 1993 \\
\hline 4217 & DOT & 49 CFR 173 & $\mathrm{~N} / \mathrm{S}$ & $\begin{array}{l}\text { Shippers General Requirements for } \\
\text { Shipments and Packaging }\end{array}$ & $10 \mathrm{cfr} 71$ & 1993 & A & Implied endorsement & 49 CFR 173 & 1993 \\
\hline 4218 & DOT & 49 CFR 173 & $\mathrm{~N} / \mathrm{S}$ & $\begin{array}{l}\text { Shippers General Pequirements for } \\
\text { Shipments and Packaging }\end{array}$ & 55 FR 27522 & 1990 & $\mathbf{R}$ & & 49 CFR 173 & 1993 \\
\hline 4219 & DOT & 49 CFR 173 & $\mathrm{~N} / \mathrm{S}$ & $\begin{array}{l}\text { Shippers General Requirements for } \\
\text { Shipments and Packaging }\end{array}$ & glt80-09 & 1980 & $\mathbf{R}$ & & 49 CFR 173 & 1993 \\
\hline
\end{tabular}


Codes and Standards and Other Guidance Cited in Regulatory Documents Part B - Other Guidance (continued)

\begin{tabular}{|c|c|c|c|c|c|c|c|c|c|c|}
\hline \multicolumn{11}{|c|}{ Pant B - otner Gulaance (Contanued) } \\
\hline $\begin{array}{l}\text { Aocord } \\
\text { No. }\end{array}$ & Cods: & Standurd & $\begin{array}{l}\text { Standard } \\
\text { Vorsion }\end{array}$ & $\perp \quad$ nwo & Doosument & $\begin{array}{l}\text { Doc. } \\
\text { Dots }\end{array}$ & Aooept & Comments & Current Voralom & 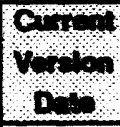 \\
\hline 4220 & DOT & $\begin{array}{l}49 \text { CFR } \\
173.1(\mathrm{~b}) \\
\end{array}$ & $\mathrm{N} / \mathrm{S}$ & $\begin{array}{l}\text { Shippers General Requirements for } \\
\text { Shipments and Packaging }\end{array}$ & inm83723 & 1991 & $R$ & Implied endorsemen? & 49 CFR 173.1(D) & 1993 \\
\hline 4221 & DOT & $\begin{array}{l}49 \mathrm{CFR} \\
173.389(0)\end{array}$ & 1979 & $\begin{array}{l}\text { Requirements for Exclusive Use } \\
\text { Shipments }\end{array}$ & not $80-32$ & 1980 & $\mathbf{P}$ & Implied endorsement & $\begin{array}{l}49 \mathrm{CFP} \\
173.389(0) \\
\end{array}$ & 1993 \\
\hline 4222 & DOT & \begin{tabular}{|l|}
49 CFR \\
$173.393(0) \&(0)$ \\
\end{tabular} & N/S & $\begin{array}{l}\text { Shippers - General Pequirements } \\
\text { for Shipments and Packages }\end{array}$ & not80-52.101 & 1982 & $\mathbf{P}$ & Implied endorsement & $\begin{array}{l}49 \text { CFR } 173.393 \\
\text { (i) \& (0) }\end{array}$ & $n / a$ \\
\hline 4223 & DOT & \begin{tabular}{|l|}
49 CFR \\
173.394 (a) (2) \\
and 178.250 \\
\end{tabular} & 1979 & DOT Specification 55 & not81-02 & 1981 & $\bar{P}$ & Implised ondorsement & $\begin{array}{l}49 \text { CFA } 173.394 \\
\text { (a) (2) and } \\
178.250 \\
\end{array}$ & 1993 \\
\hline 4224 & DOT & $\begin{array}{l}49 \mathrm{CFR} \\
173.397 \\
\end{array}$ & $\mathrm{~N} / \mathrm{S}$ & $\begin{array}{l}\text { Shippers - General Requirements } \\
\text { for Shipments and Packages }\end{array}$ & $9 \operatorname{gtt81-38}$ & 1981 & $\bar{P}$ & & 49 CFR 173.397 & $\mathbf{n} / \mathbf{a}$ \\
\hline 4225 & DOT & \begin{tabular}{|l|}
49 CFR \\
$173.394(a)(2)$ \\
and 178.250
\end{tabular} & 1979 & DOT Spocification 55 & not82-47 & 1982 & $\bar{P}$ & & $\begin{array}{l}49 \text { CFR } 173.394 \\
\text { (a) (2) and } \\
178.250 \\
\end{array}$ & 1993 \\
\hline 4226 & DOT & $\begin{array}{l}49 \text { CFR } \\
173.403(m) \\
\text { and }(w)\end{array}$ & $\mathrm{N} / \mathrm{S}$ & $\begin{array}{l}\text { Shippers - General Requirements } \\
\text { for Shipments and Packages }\end{array}$ & $\begin{array}{l}10 c t r 20 \text { Subparts A- } \\
0\end{array}$ & 1993 & $\mathbf{R}$ & Implied endorsement & $\begin{array}{l}49 \text { CFP } 173.403 \\
(m) \text { and }(w)\end{array}$ & 1993 \\
\hline 4227 & DOT & \begin{tabular}{|l|}
49 CFR \\
$173.394(a)(1)$ \\
and 178.350 \\
\end{tabular} & 1979 & $\begin{array}{l}\text { Specification 7A, General } \\
\text { Packaging, Type A }\end{array}$ & not81-02 & 1981 & $\overline{\mathbf{P}}$ & \begin{tabular}{|l|} 
Packages not \\
authorized aftor \\
$6 / 30 / 85$. \\
\end{tabular} & \begin{tabular}{|l|}
49 CFR \\
$173.394(a)$ (1) and \\
178.350 \\
\end{tabular} & 1993 \\
\hline 4228 & DOT & \begin{tabular}{|l|}
49 CFR \\
$173.415(b)$ and \\
173.416 (a) \\
\end{tabular} & 1983 & $\begin{array}{l}\text { Authorized Type A and Type B } \\
\text { Fackages }\end{array}$ & not84-14 & 1984 & $\mathbf{R}$ & Implied endorsement & \begin{tabular}{|l|}
$49 \mathrm{CFR}$ \\
$173.415(b)$ and \\
$173.416(a)$ \\
\end{tabular} & 1993 \\
\hline 4229 & DOT & \begin{tabular}{|l|}
49 CFR \\
$173.415 a$ \\
\end{tabular} & N/S & Authorized Type A Packages & not90-35 & 1990 & $\bar{P}$ & Implied endorsement & 49 CFR $173.415 a$ & 1993 \\
\hline 4230 & DOT & $\begin{array}{l}49 \text { CFR } \\
173.417 \\
\end{array}$ & $\mathrm{~N} / \mathrm{S}$ & $\begin{array}{l}\text { Authorized Packaging - Fissile } \\
\text { Materials }\end{array}$ & not $86-86$ & 1986 & $\mathbf{P}$ & Implied endorsement & 49 CFA 173.417 & 1993 \\
\hline 4231 & $\overline{\text { DOT }}$ & $\begin{array}{l}49 \text { CFR } \\
173.420\end{array}$ & 1989 & $\begin{array}{l}\text { UF6 Cylinder Packing } \\
\text { Requirements }\end{array}$ & not90-27 & 1990 & $P$ & & 49 CFR 173.420 & 1993 \\
\hline 4232 & DOT & $\begin{array}{l}49 \text { CFR } \\
173.420\end{array}$ & $\mathrm{~N} / \mathrm{S}$ & $\begin{array}{l}\text { Shippers - General Requirements } \\
\text { for Shipments and Packages }\end{array}$ & not92-58 & 1992 & $\overline{\mathbf{A}}$ & & 49 CFR 173 & 1993 \\
\hline 4233 & $\overline{D O T}$ & $\begin{array}{l}49 \mathrm{CFF} \\
173.421-424\end{array}$ & $N / S$ & $\begin{array}{l}\text { Shippers - General Pequirements } \\
\text { for Shipments and Packages }\end{array}$ & $\begin{array}{l}10 c f r 20 \text { Subparts A- } \\
0\end{array}$ & 1993 & $\mathbf{R}$ & Implied endorsement & $\begin{array}{l}49 \text { CFR } 173.421 \text { - } \\
424\end{array}$ & 1993 \\
\hline
\end{tabular}


Codes and Standards and Other Guidance Cited in Regulatory Documents Part B - Other Guidance (continued)

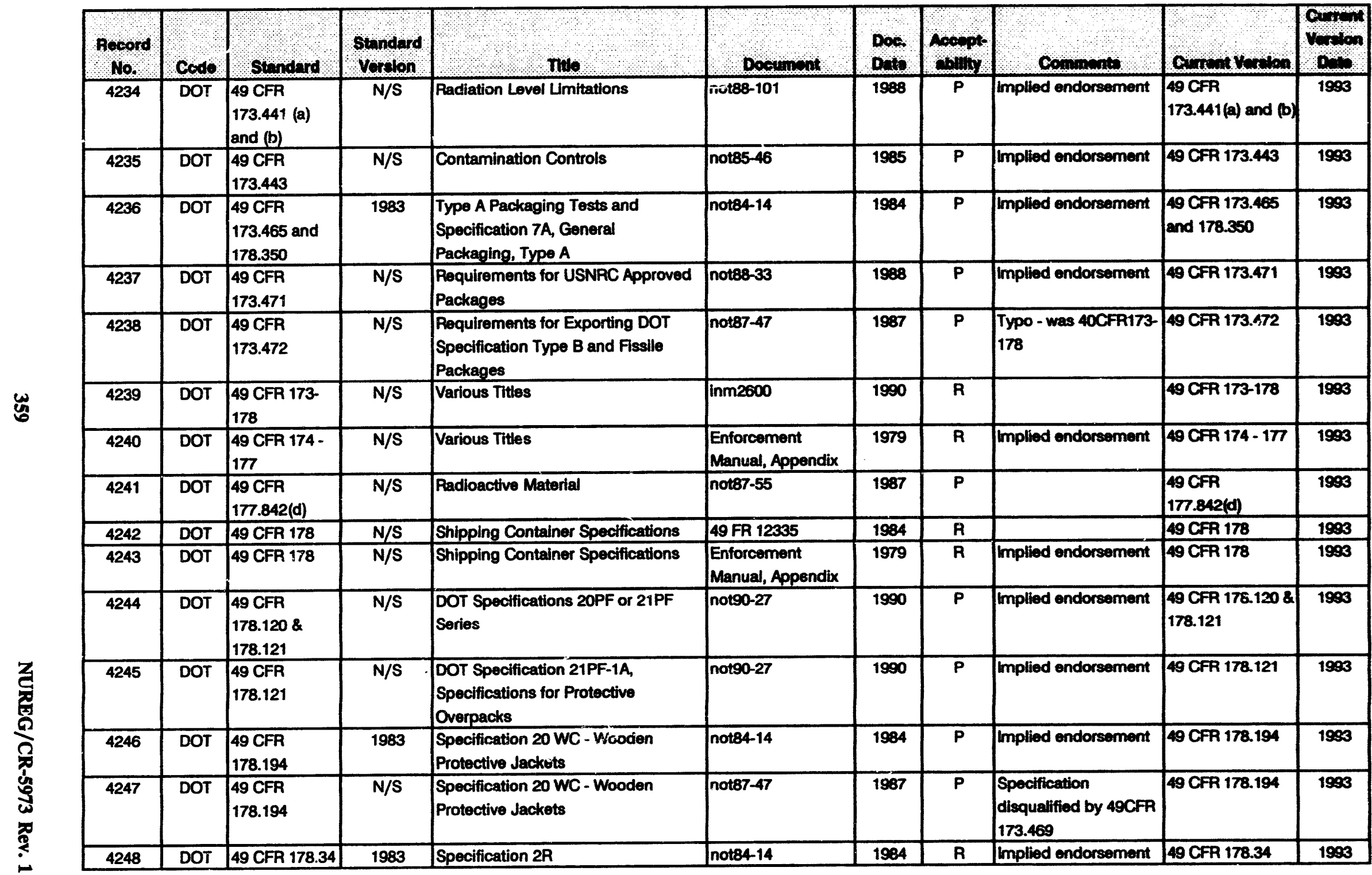


Codes and Standards and Other Guidance Cited in Regulatory Documents Part B - Other Guidance (continued)

\begin{tabular}{|c|c|c|c|c|c|c|c|c|c|c|}
\hline $\begin{array}{l}\text { Pocord } \\
\text { No. }\end{array}$ & codo & Sthond & Stonderd & mo & Dowonont & $\begin{array}{l}\text { Doe. } \\
\text { Dote }\end{array}$ & Acospt & comersmo & Coment voraton & $\begin{array}{l}\infty \\
\text { Whind } \\
\text { n. }\end{array}$ \\
\hline 4249 & DOT & $\begin{array}{l}49 \text { CFR } \\
178.350\end{array}$ & $N / S$ & $\begin{array}{l}\text { DOT Specification 7A, Type A } \\
\text { Packaging }\end{array}$ & not85-46 & 1995 & $P$ & Implied endorsement & 49 CFR 178.350 & 1993 \\
\hline 4250 & DOT & $\begin{array}{l}49 \text { CFR } \\
178.350\end{array}$ & $\mathrm{~N} / \mathrm{S}$ & $\begin{array}{l}\text { DOT Specification 7A, Type A } \\
\text { Packaging }\end{array}$ & not87-47 & 1987 & $\mathbf{P}$ & Implied endorsement & 49 CFR 178.350 & 1993 \\
\hline 4251 & DOT & $\begin{array}{l}49 \text { CFR } \\
178.350\end{array}$ & $\mathrm{~N} / \mathrm{S}$ & $\begin{array}{l}\text { DOT Specification 7A, Typo A } \\
\text { Packaging }\end{array}$ & notsa-27 & 1990 & $\mathbf{P}$ & Implied endorsement & 49 CFR 178.350 & 1993 \\
\hline 4252 & DOT & $\begin{array}{l}49 \text { CFR } \\
178.350 \& \\
173.395 \text { (a) (1) }\end{array}$ & 1979 & DOT Specification $7 A$ & not82-47 & 1982 & $\mathbf{P}$ & & $\begin{array}{l}49 \text { CFA } 178.350 \\
8173.395 \text { (a) (1) }\end{array}$ & 1993 \\
\hline 4253 & DOT & $\begin{array}{l}49 \text { CFR } 390 \\
397\end{array}$ & $\mathrm{~N} / \mathrm{S}$ & Various Titles & $\begin{array}{l}\text { Enforcement } \\
\text { Manual, Appendix }\end{array}$ & 1979 & $\bar{R}$ & & 49 CFR $390-397$ & 1903 \\
\hline 4254 & DOT & $\begin{array}{l}49 \text { CFR } 390- \\
397\end{array}$ & $N / S$ & Various Titles & reg10.06.101 & 1981 & $\mathbf{R}$ & Implied endorsement & 49 CFR $390-397$ & 1993 \\
\hline 4255 & DOT & $\begin{array}{l}49 \text { CFR } \\
393.100 \\
393.102 \\
393.104\end{array}$ & $N / S$ & Tie Down of Radioactive Packages & not87-31 & 1987 & $\overline{\mathbf{P}}$ & & $\begin{array}{l}9 \text { CFR 393.100, } \\
393.102,393.104\end{array}$ & 1993 \\
\hline 4256 & DOT & DOT & 1990 & $\begin{array}{l}\text { 19s0 Emergency Response } \\
\text { Guidebook }\end{array}$ & inm86740 & 1992 & $\mathbf{R}$ & & 49 CFR & 1993 \\
\hline 4257 & DOT & DOT 7A & $N / S$ & Authorized Type A Packages & $\begin{array}{l}\text { 10efroo2, Appendix } \\
\text { C }\end{array}$ & 1990 & A & & 49 CFR & 1993 \\
\hline 4258 & DOT & DOT 7A & $\mathrm{N} / \mathrm{S}$ & Authorized Type A Packages & inm86740 & 1992 & A & Cited as a reference & 49 CFA & 1993 \\
\hline 4259 & DOT & $\begin{array}{l}\text { DOT Class } \\
\text { 106A }\end{array}$ & 1989 & $\begin{array}{l}\text { Specifications for Multi-Unit Tank } \\
\text { Cars }\end{array}$ & not $90-27$ & 1990 & $\bar{R}$ & Modical Usage & 49 CFA & 1903 \\
\hline 4260 & DOT & DOT E 7060 & $N / S$ & DOT Exemption DOT E 7060 & not $89-74$ & 1989 & $\mathbf{R}$ & Arr Cylinder Exemption & 49CFA & 1993 \\
\hline 4261 & DOT & DOTE 7235 & N/S & DOT Exemption DOT E 7235 & not84-34 & 1984 & $R$ & Air Oylinder Exemption & 49 CFA & 1993 \\
\hline 4262 & DOT & DOTE 7235 & N/S & DOT Exemption DOT E 7235 & not85-48 & 1985 & $R$ & Air Cylinder Exemption & 49 CFA & 1993 \\
\hline 4263 & DOT & DOTE 7235 & $\mathrm{~N} / \mathrm{S}$ & DOT Exemption DOT E 7235 & nov66-24 & 1986 & $\mathbf{R}$ & Air Oylinder Exemption & 49 CFA & 1993 \\
\hline 4264 & DOT & DOTE 8059 & $N / S$ & DOT Exemption DOT E 8059 & not $86-24$ & 1986 & $\mathbf{R}$ & Modical Usage & 49 CFR & 1993 \\
\hline 4265 & DOT & DOT E 8308 & $\mathrm{~N} / \mathrm{S}$ & DOT Exemption DOT E 8308 & not $89-74$ & 1989 & $\mathbf{R}$ & implied endorsement & 49 CFA & 1908 \\
\hline$\frac{4200}{4266}$ & DOT & DOT P5200.8 & 1973 & $\begin{array}{l}\text { Truck Top Markings for Visual } \\
\text { Identification }\end{array}$ & reg5.17.100 & 1974 & $P$ & & 49 CFR & 1993 \\
\hline
\end{tabular}


Codes and Standards and Other Guidance CHed in Regulatory Documents

Part B - Other Guidance (continued)

\begin{tabular}{|c|c|c|c|c|c|c|c|c|c|c|}
\hline $\begin{array}{l}\text { Record } \\
\text { No. }\end{array}$ & Code & Standard & $\begin{array}{l}\text { Stundard } \\
\text { Voraton }\end{array}$ & nis & Doesument & Don. & Aconpt- & Comments & Current Veralon & 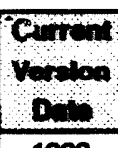 \\
\hline 4267 & $\overline{\text { DOT }}$ & DOT Pamphlet & 1983 & $\begin{array}{l}\text { A Review of the DOT Regulations } \\
\text { for Transportation of Radioactive } \\
\text { Materials }\end{array}$ & inm86740 & 1992 & $\mathbf{R}$ & & DOT Pamphlot & 1983 \\
\hline 4268 & DOT & $\begin{array}{l}\text { DOT } \\
\text { Publication }\end{array}$ & 1983 & $\begin{array}{l}\text { A Review of the DOT Regulations } \\
\text { for Transportation of Radioactive } \\
\text { Matorials }\end{array}$ & inm86740 & 1992 & $\mathbf{R}$ & & DOT Publication & 1983 \\
\hline 4269 & DOT & \begin{tabular}{|l|} 
DOT \\
Publication \\
\end{tabular} & 1990 & $\begin{array}{l}1990 \text { Emergency Posponse } \\
\text { Guidebook }\end{array}$ & inm86740 & 1992 & $\mathbf{R}$ & & DOT Publication & 1990 \\
\hline $4 \angle 70$ & DOT & \begin{tabular}{|l|} 
DOT \\
Publication \\
DOT/RSPA/M \\
TB-81-4 \\
\end{tabular} & N/S & $\begin{array}{l}\text { Radioactivo Materials } \\
\text { Transportation Information and } \\
\text { Incident Guidance }\end{array}$ & inm86740 & $19 \%$ & $\mathbf{R}$ & & $\begin{array}{l}\text { DOT Publication } \\
\text { DOT/RSPA/MTB- } \\
81-4\end{array}$ & 1981 \\
\hline 4271 & EPA & 38 FR 24936 & 1973 & $\begin{array}{l}\text { Momorandum of Understanding } \\
\text { between the Environmental } \\
\text { Protection Agency and the Atomic } \\
\text { Energy Commission }\end{array}$ & $10 c t r 20$ & 1989 & A & & 10CFP2O & 1993 \\
\hline 4272 & EPA & 40 CFR 190 & $\mathrm{~N} / \mathrm{S}$ & $\begin{array}{l}\text { Environmental Padiation Protection } \\
\text { Standards for Nuclear Power } \\
\text { Operations }\end{array}$ & 10ctro02 App. C & 1992 & $\mathbf{R}$ & & $40 \mathrm{CFR}$ & 1993 \\
\hline 4273 & EPA & 40 CFR 190 & N/S & $\begin{array}{l}\text { Environmental Radiation Protection } \\
\text { Standards for Nuclear Power } \\
\text { Operations }\end{array}$ & git79-41 & 1979 & $\bar{R}$ & & 40 CFA & 1993 \\
\hline 4274 & EPA & 40 CFR 190 & $N / S$ & $\begin{array}{l}\text { Environmental Padiation Protaction } \\
\text { Standards for Nuclear Power } \\
\text { Operations }\end{array}$ & $\operatorname{rog} 3.51 .000$ & 1982 & $\mathbf{R}$ & & $40 \mathrm{CFR}$ & 1993 \\
\hline 4275 & EPA & 40 CFR 191 & $\mathrm{~N} / \mathrm{S}$ & $\begin{array}{l}\text { Environmental Padiation Protection } \\
\text { Standards for Management and } \\
\text { Disposal of Spent Nuclear Fuel, } \\
\text { High-level and Transuranic } \\
\text { Radioactive Wastes } \\
\end{array}$ & drg3003.100 & 1990 & $\bar{R}$ & & $40 \mathrm{CFR}$ & 1993 \\
\hline 4276 & EPA & 40 CFR 192 & $N / S$ & $\begin{array}{l}\text { Health and Environmental } \\
\text { Protection Standards for Uranium } \\
\text { and Thorium Mill Tallings }\end{array}$ & reg3.64.roo & 1989 & $\mathbf{R}$ & & 40 CFR & 1993 \\
\hline
\end{tabular}




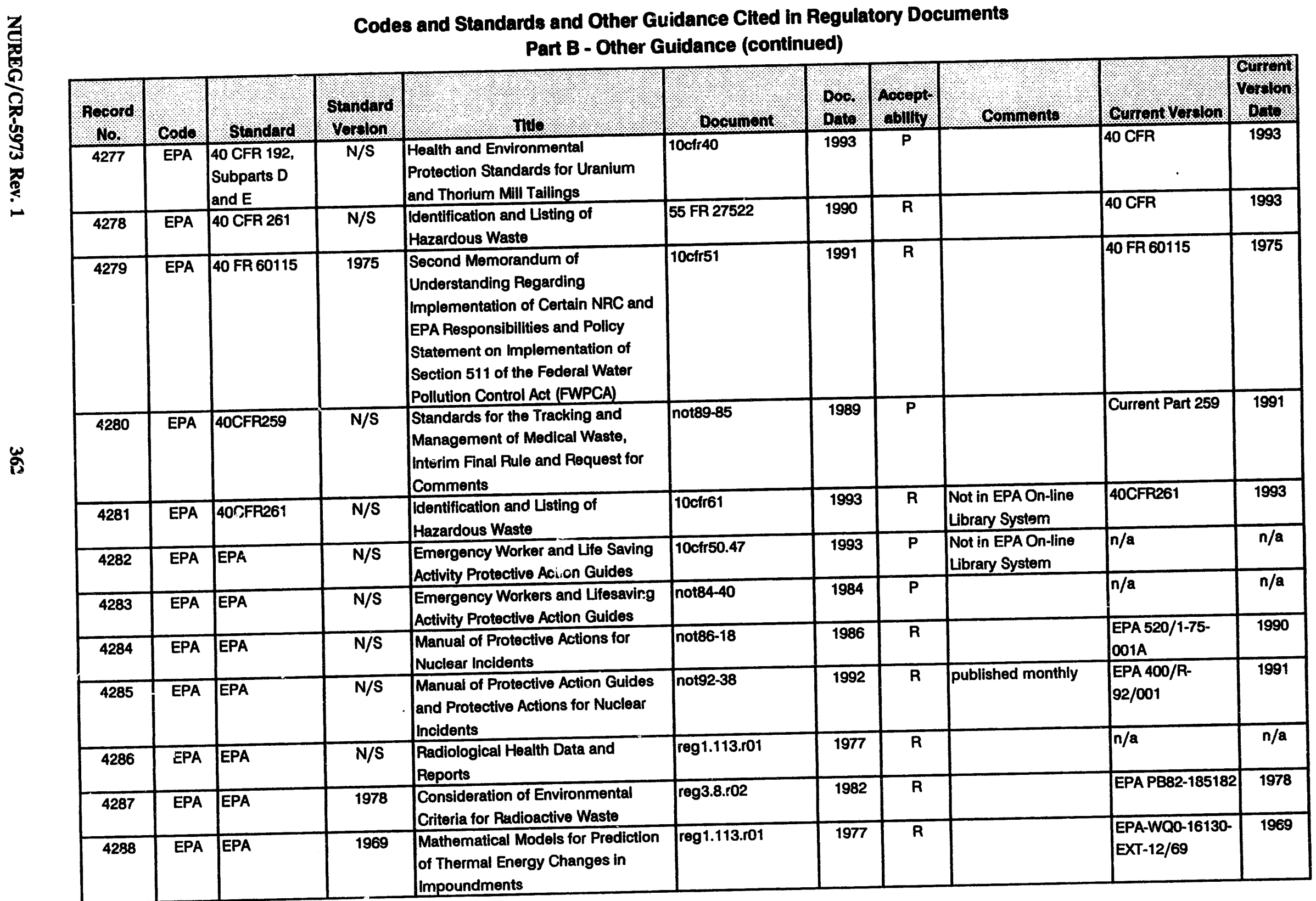


Codes and Standards and Other Guidance Cited in Regulatory Documents

Part B - Other Guidance (continued)

\begin{tabular}{|c|c|c|c|c|c|c|c|c|c|c|}
\hline Hocord & 0000 & standard & Standard & ntio & (v) & Doo: & Acoopls & Comnenta & Ginont Vor $10 n$ & porton \\
\hline 4289 & EPA & $\begin{array}{l}\text { EPA 520/5-84- } \\
006\end{array}$ & 1984 & $\begin{array}{l}\text { Eastern Environmental Radiation } \\
\text { Facility Radiochemistry Procedures } \\
\text { Mar,ual }\end{array}$ & inm84725 & 1991 & R & & EPA 520/5-84-006 & 1984 \\
\hline 4290 & EPA & $\begin{array}{l}\text { EPA Contracts } \\
68-02-0006\end{array}$ & 1971 & $\begin{array}{l}\text { A Particle in Cell Method for } \\
\text { Numerical Solution of the } \\
\text { Atmospheric Diffusion Equation } \\
\text { and Applications to Air Pollution } \\
\text { Problems }\end{array}$ & reg1.111.r01 & 1977 & $\bar{R}$ & & EPA APTD-0952 & 1971 \\
\hline 4291 & EPA & \begin{tabular}{|l|} 
EPA Project \\
No. 160700Z V, \\
Contract 14- \\
$12-551$
\end{tabular} & 1971 & Estuarine Modeling, An Assessment & reg1.113.ro1 & 1977 & $\bar{R}$ & & $\begin{array}{l}\text { EPA-WQO-16070- } \\
\text { DZV-02/71 }\end{array}$ & 1971 \\
\hline 4292 & EPA & $\begin{array}{l}\text { EPA-68-01- } \\
5971\end{array}$ & 1980 & $\begin{array}{l}\text { Development of Procedures and } \\
\text { Costs for Proper Abandonment and } \\
\text { Plugging of Injection Wells }\end{array}$ & drges114-4 & 1983 & $\mathbf{R}$ & $\begin{array}{l}\text { Not listed in EPA On- } \\
\text { line Library System }\end{array}$ & EPA-68-01-5971 & 1980 \\
\hline 4293 & EPA & $\begin{array}{l}\text { EPA Report } \\
\text { 16070DGW }\end{array}$ & 1971 & $\begin{array}{l}\text { One-Dimensional Real Time Model } \\
\text { for Estuarine Water Quality } \\
\text { Prediction }\end{array}$ & reg1.113.ro1 & 1977 & $\mathbf{R}$ & & $n / a$ & $n / a$ \\
\hline 4294 & EPA & $\begin{array}{l}\text { EPA-69-03- } \\
3089\end{array}$ & 1982 & $\begin{array}{l}\text { MINTEQ: A Computer Program for } \\
\text { Calculating Aqueous Geochemical } \\
\text { Equillbria }\end{array}$ & drges115-4 & 1983 & $\mathbf{R}$ & & EPA-69-03-3089 & 1982 \\
\hline 4295 & EPA & $\begin{array}{l}\text { EPA-440/9-73- } \\
003\end{array}$ & 1973 & Auto-Qual Modeling System & reg1.113.ro1 & 1977 & $\mathbf{R}$ & & EPA TR-54 & 1973 \\
\hline 4296 & EPA & $\begin{array}{l}\mathrm{EPA}-520 / 1-84- \\
022-1\end{array}$ & $N / S$ & $\begin{array}{l}\text { Radionuclides: Background } \\
\text { Information Document for Final } \\
\text { Rules. Volume } 1\end{array}$ & reg8.13.r02 & 1987 & R & & $\begin{array}{l}\text { EPA 520/1- } \\
84 / 022-1\end{array}$ & 1984 \\
\hline 4297 & EPA & $\begin{array}{l}\text { EPA-520/1-75- } \\
001\end{array}$ & $N / S$ & $\begin{array}{l}\text { Manual of Protective Actions for } \\
\text { Nuclear Incidents }\end{array}$ & not91-72 & 1991 & R & & $\begin{array}{l}\text { EPA 520/1-75- } \\
\text { O01A }\end{array}$ & 1990 \\
\hline 4298 & EPA & $\begin{array}{l}\text { EPA-520/1-75- } \\
001\end{array}$ & $N / S$ & $\begin{array}{l}\text { Manual of Protective Actions for } \\
\text { Nuclear Incidents }\end{array}$ & not92-08 & 1992 & $\mathbf{P}$ & & $\begin{array}{l}\text { EPA 520/1-75- } \\
001 A\end{array}$ & 1990 \\
\hline 4299 & EPA & $\begin{array}{l}\text { EPA-520/1-75- } \\
001\end{array}$ & 1975 & $\begin{array}{l}\text { Manual of Protective Actions for } \\
\text { Nuclear Incidents }\end{array}$ & reg3.42.r01 & 1979 & $\mathbf{R}$ & & $\begin{array}{l}\text { EPA 520/1-75- } \\
001 A\end{array}$ & 1990 \\
\hline
\end{tabular}


Codes and Standards and Other Guidance Chted in Regulatory Documents

Part B - Other Guidance (continued)

\begin{tabular}{|c|c|c|c|c|c|c|c|c|c|c|}
\hline Porord & codo & Stundurd & shondord & 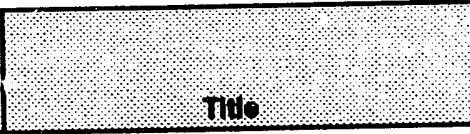 & अै & popto & $600+1$ & Commontr & ounonoworion & 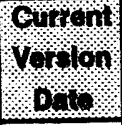 \\
\hline 4300 & EPA & $\begin{array}{l}\text { EPA-520/1-75- } \\
\text { OO1-A }\end{array}$ & 1990 & $\begin{array}{l}\text { Manual of Protective Actions for } \\
\text { Nuclear Incidents }\end{array}$ & inm82202 & 1993 & $\mathbf{R}$ & & $\begin{array}{l}\text { EPA 520/1-75- } \\
\text { O01A }\end{array}$ & 1990 \\
\hline 4301 & EPA & $\begin{array}{l}\text { EPA-520/1-75- } \\
001-A\end{array}$ & 1990 & $\begin{array}{l}\text { Manual of Protective Actions for } \\
\text { Nuclear Incidents }\end{array}$ & inm82206 & 1993 & $\mathbf{R}$ & & $\begin{array}{l}\text { EPA 520/1-75- } \\
\text { O01A } \\
\end{array}$ & 1990 \\
\hline 4302 & EPA & $\begin{array}{l}\text { EPA-520/1-75- } \\
001-A\end{array}$ & 1990 & $\begin{array}{l}\text { Manual of Protective Actions for } \\
\text { Nuclear Incidents }\end{array}$ & inm82206 & 1993 & $\mathbf{R}$ & & $\begin{array}{l}\text { EPA 520/1-75- } \\
\text { O01A }\end{array}$ & 1990 \\
\hline 4303 & EPA & $\begin{array}{l}\text { EPA-520/1-75- } \\
\text { OO1-A }\end{array}$ & 1990 & $\begin{array}{l}\text { Manual of Protective Actions for } \\
\text { Nuclear Incidents }\end{array}$ & inm82207 & 1993 & $\mathbf{R}$ & & $\begin{array}{l}\text { EPA 520/1-75- } \\
001 A\end{array}$ & 1990 \\
\hline 4304 & EPA & \begin{tabular}{|l} 
EPA-520/1-75- \\
$001-A$
\end{tabular} & 1990 & $\begin{array}{l}\text { Manual of Protective Actions for } \\
\text { Nuctear Incidents }\end{array}$ & inm822202 & 1993 & $\mathbf{R}$ & & $\begin{array}{l}\text { EPA 520/1-75- } \\
001 A\end{array}$ & 1990 \\
\hline 4305 & EPA & $\begin{array}{l}\text { EPA-520/1-75- } \\
001-A\end{array}$ & 1990 & $\begin{array}{l}\text { Manual of Protective Actions for } \\
\text { Nuclear Incidents }\end{array}$ & inm82301 & 1993 & $\mathbf{R}$ & & $\begin{array}{l}\text { EPA 520/1-75- } \\
\text { OO1A }\end{array}$ & 1990 \\
\hline 4306 & EPA & \begin{tabular}{|l|} 
EPA-520/1-75- \\
$001-A$
\end{tabular} & 1990 & $\begin{array}{l}\text { Manual of Protective Actions for } \\
\text { Nuclear Incidents }\end{array}$ & inm82301 & 1993 & $\mathbf{R}$ & & $\begin{array}{l}\text { EPA 520/1-75- } \\
\text { O01A }\end{array}$ & 1990 \\
\hline 4307 & EPA & $\begin{array}{l}\text { EPA-520/1-75- } \\
001-A\end{array}$ & 1990 & $\begin{array}{l}\text { Manual of Protective Actions for } \\
\text { Nuclear Incidents }\end{array}$ & inm82701 & 1993 & $\mathbf{R}$ & & $\begin{array}{l}\text { EPA 520/1-75- } \\
001 A \\
\end{array}$ & 1990 \\
\hline 4308 & EPA & $\begin{array}{l}\text { EPA-520/1-75- } \\
\text { O01-A }\end{array}$ & 1990 & $\begin{array}{l}\text { Manual of Protective Actions for } \\
\text { Nuclear Incidents }\end{array}$ & reg3.67.ro0 & 1992 & $\mathbf{R}$ & & $\begin{array}{l}\text { EPA 520/1-75- } \\
001 A\end{array}$ & 1990 \\
\hline 4309 & EPA & $\begin{array}{l}\text { EPA-520/1-76- } \\
001\end{array}$ & 1976 & $\begin{array}{l}\text { Potential Radiological Impact of } \\
\text { Airborne Releases and Direct } \\
\text { Gamma Radiation to Individuals } \\
\text { Living Near Inactive Uranium Mill } \\
\text { Tailings Piles }\end{array}$ & reg3.51.r00 & 1982 & R & & EPA-520/1-76-001 & 1976 \\
\hline 4310 & EPA & $\begin{array}{l}\text { EPA-520/1-76- } \\
001\end{array}$ & 1976 & $\begin{array}{l}\text { Poteritial Radiological Impact of } \\
\text { Airborne Releases and Direct } \\
\text { Gamma Radiation to Individuals } \\
\text { Living Near Inactive Uranium Mill } \\
\text { Tailings Piles }\end{array}$ & reg3.51.r01 & 1982 & $\bar{R}$ & & EPA 520/1-76-001 & 1976 \\
\hline 4311 & EPA & \begin{tabular}{|l|} 
EPA-520/1-78- \\
016
\end{tabular} & 1978 & $\begin{array}{l}\text { Planning Basis for the Development } \\
\text { of State and Local Government } \\
\text { Radiological Emergency Response } \\
\text { Plans in Support of Light-Water } \\
\text { Nuclear Power Plants }\end{array}$ & 10 fr50 App. E & 1993 & $\bar{R}$ & & EPA 520/1-78-016 & 1978 \\
\hline
\end{tabular}


Codes and Standards and Other Guidance Cited in Regulatory Documents

Part B - Other Guidance (continued)

\begin{tabular}{|c|c|c|c|c|c|c|c|c|c|c|}
\hline $\begin{array}{l}\text { Ropord } \\
\text { Non }\end{array}$ & Cods & Standard & $\begin{array}{l}\text { Shandard } \\
\text { Yorton }\end{array}$ & (1, & 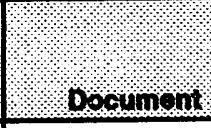 & Dooto & nopopts & connonto & curront Vor tom & Gorron \\
\hline 4312 & EPA & $\begin{array}{l}\text { EPA-520/1-78- } \\
016\end{array}$ & 1978 & $\begin{array}{l}\text { Planning Basis for the Development } \\
\text { of State and Local Government } \\
\text { Radiological Emergency Response } \\
\text { Plans in Support of Light-Water } \\
\text { Nuclear Power Plants }\end{array}$ & $10 \mathrm{cfr} 50.33$ & 1993 & $\mathbf{R}$ & & EFA 520/1-78-016 & 1978 \\
\hline 4313 & EPA & $\begin{array}{l}\text { EPA-520/1-78- } \\
016\end{array}$ & 1978 & $\begin{array}{l}\text { Planning Basis for the Development } \\
\text { of State and Local Government } \\
\text { Radiological Emergency Response } \\
\text { Plans in Support of Light-Water } \\
\text { Nuclear Power Plants }\end{array}$ & $10 \mathrm{cfr} 50.54$ & 1993 & $\mathbf{R}$ & & EPA 520/1-78-016 & 1978 \\
\hline 4314 & EPA & $\begin{array}{l}\text { EPA-520/1-78- } \\
016\end{array}$ & 1978 & $\begin{array}{l}\text { Planning Basis for the Development } \\
\text { of State and Local Government } \\
\text { Radiological Emergency Response } \\
\text { Plans in Support of Light Water } \\
\text { Nuclear Power Plants }\end{array}$ & 44 FR61123 & 1979 & $\mathbf{R}$ & & EPA 520/1-78-016 & 1978 \\
\hline 4315 & EPA & $\begin{array}{l}\text { EPA-520/1-78- } \\
016\end{array}$ & 1978 & $\begin{array}{l}\text { Planning Basis for the Development } \\
\text { of State and Local Government } \\
\text { Radiological Emergency Response } \\
\text { Plans in Support of Light Water } \\
\text { Nuclear Power Plants }\end{array}$ & 45 FR 2893 & 1980 & $\mathbf{R}$ & & EPA 520/1-78-016 & 1978 \\
\hline 4316 & EPA & $\begin{array}{l}\text { EPA-520/1-80- } \\
012\end{array}$ & 1980 & $\begin{array}{l}\text { Upgrading Environmental Radiation } \\
\text { Data: Health Physics Society } \\
\text { Committee Report HPSR-1 }\end{array}$ & reg8004-draft & 1991 & $\mathbf{R}$ & & EPA 520/1-80-012 & 1980 \\
\hline 4317 & EPA & $\begin{array}{l}\text { EPA-520/1-80- } \\
012(P B-81- \\
100364)\end{array}$ & 1980 & $\begin{array}{l}\text { Upgrading Environmental Radiation } \\
\text { Data: Health Physics Society } \\
\text { Committee Report HPSR-1 }\end{array}$ & drg8004.r00 & 1991 & $\mathbf{R}$ & & EPA 520/1-80-012 & 1980 \\
\hline 4318 & EPA & $\begin{array}{l}\text { EPA-520/1-88- } \\
020\end{array}$ & 1988 & $\begin{array}{l}\text { Limiting Values of Radionuclide } \\
\text { intake and Air Concentration and } \\
\text { Dose Conversion Factors for } \\
\text { Inhalation, Submersion, and } \\
\text { Ingestion }\end{array}$ & drg8010.r00 & 1992 & $\mathbf{R}$ & & EPA-520/1-88-020 & 1988 \\
\hline
\end{tabular}


Codes and Standards and Other Guidance Cited in Regulatory Documents

Part B - Other Guidance (continued)

\begin{tabular}{|c|c|c|c|c|c|c|c|c|c|c|}
\hline $\begin{array}{l}\text { Pecord } \\
\text { No. }\end{array}$ & Codo & Standigrd & $\begin{array}{l}\text { Standard } \\
\text { Vordon? }\end{array}$ & 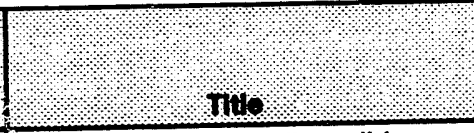 &. .8 oorunont: & $\begin{array}{l}\text { Dor } \\
\text { Dots }\end{array}$ & Aocopt & Comnenta & courion Vor ilon & Gorron \\
\hline 4319 & EPA & \begin{tabular}{|l|}
$\mathrm{EPA}-520 / 1-88-$ \\
020
\end{tabular} & 1988 & $\begin{array}{l}\text { Imiting Values of Radionuclide } \\
\text { Intake and Air Concentration and } \\
\text { Dose Conversion Factors for } \\
\text { Inhalation, Submersion, and } \\
\text { Ingestion }\end{array}$ & drg8011.r00 & 1992 & $R$ & & EPA-520/1-88-020 & 1988 \\
\hline 4320 & EPA & $\begin{array}{l}\text { EPA-520/1-88- } \\
020\end{array}$ & 1988 & $\begin{array}{l}\text { Limiting Values of Radionuclide } \\
\text { Intake and Air Concentration and } \\
\text { Dose Conversion Factors for } \\
\text { Inhalation, Submersion, and } \\
\text { Ingestion } \\
\end{array}$ & reg8.34.r00 & 1992 & $\mathbf{R}$ & & EPA 520/1-88-020 & 1988 \\
\hline 4321 & EPA & $\begin{array}{l}\text { EPA-520/1-88- } \\
020\end{array}$ & 1988 & $\begin{array}{l}\text { Limiting Values of Radionuclide } \\
\text { Intake and Air Concentration and } \\
\text { Dose Conversion Factors for } \\
\text { Inhalation, Submersion, and } \\
\text { Ingestion }\end{array}$ & reg8.36.roo & 1992 & $\mathbf{R}$ & $\begin{array}{l}\text { Typo in inm82207 in } \\
\text { Standard }\end{array}$ & EPA-520/1-88-020 & 1988 \\
\hline 4322 & EPA & $\begin{array}{l}\text { EPA-520/1-95- } \\
\text { O01-A }\end{array}$ & 1990 & $\begin{array}{l}\text { Manual of Protective Action Guides } \\
\text { and Protective Actions for Nuclear } \\
\text { Incidents }\end{array}$ & inm82207 & 1993 & $\mathbf{P}$ & & $\begin{array}{l}\text { EPA 400/R- } \\
92 / 001\end{array}$ & 1991 \\
\hline 4323 & EPA & $\begin{array}{l}\text { EPA-520/3-74- } \\
009\end{array}$ & 1974 & $\begin{array}{l}\text { Storage of Low-Level Radioactive } \\
\text { Wastes in the Ground: } \\
\text { Hydrogeologic and Hydrochemical } \\
\text { Factors }\end{array}$ & drges 115-4 & 1983 & $\mathbf{R}$ & & $\begin{array}{l}\text { EPA-520/3-74 } \\
009\end{array}$ & 1974 \\
\hline 4324 & EPA & $\begin{array}{l}\text { EPA-520/4-73- } \\
002\end{array}$ & 1974 & $\begin{array}{l}\text { Environmental Radiation Dose } \\
\text { Commiltment: An Application to the } \\
\text { Nuclear Power Industry }\end{array}$ & reg3.51.roo & 1982 & $\bar{R}$ & $\begin{array}{l}\text { Not listed in EPA On- } \\
\text { line Library System }\end{array}$ & EPA-520/4-73-002 & 1974 \\
\hline 4325 & EPA & $\begin{array}{l}\text { EPA-520/4-73- } \\
002\end{array}$ & 1974 & $\begin{array}{l}\text { Environmental Radiation Dose } \\
\text { Committrent: An Application to the } \\
\text { Nuclear Power Industry }\end{array}$ & reg3.51.r01 & 1982 & $\mathbf{R}$ & & $n / a$ & $n / a$ \\
\hline 4326 & EPA & $\begin{array}{l}\text { EPA-520/4-80- } \\
001\end{array}$ & 1980 & $\begin{array}{l}\text { Occupational Exposure to lonizing } \\
\text { Radiation in the United States: A } \\
\text { Compsehensive Summary for } 1975\end{array}$ & reg8.29.ro0 & 1981 & $\bar{R}$ & & EPA 520/4-80-001 & 1980 \\
\hline 4327 & EPA & \begin{tabular}{|l|} 
EPA-520/5-84- \\
006
\end{tabular} & 1984 & $\begin{array}{l}\text { Eastern Environmental Radiation } \\
\text { Facillty Radiochemistry Procedures } \\
\text { Manual }\end{array}$ & inm84850 & 1988 & $\mathbf{R}$ & & EPA 520/5-84-006 & 1984 \\
\hline
\end{tabular}


Codes and Standards and Other Guidance Cited in Regulatory Documents

Part B - Other Guidance (continued)

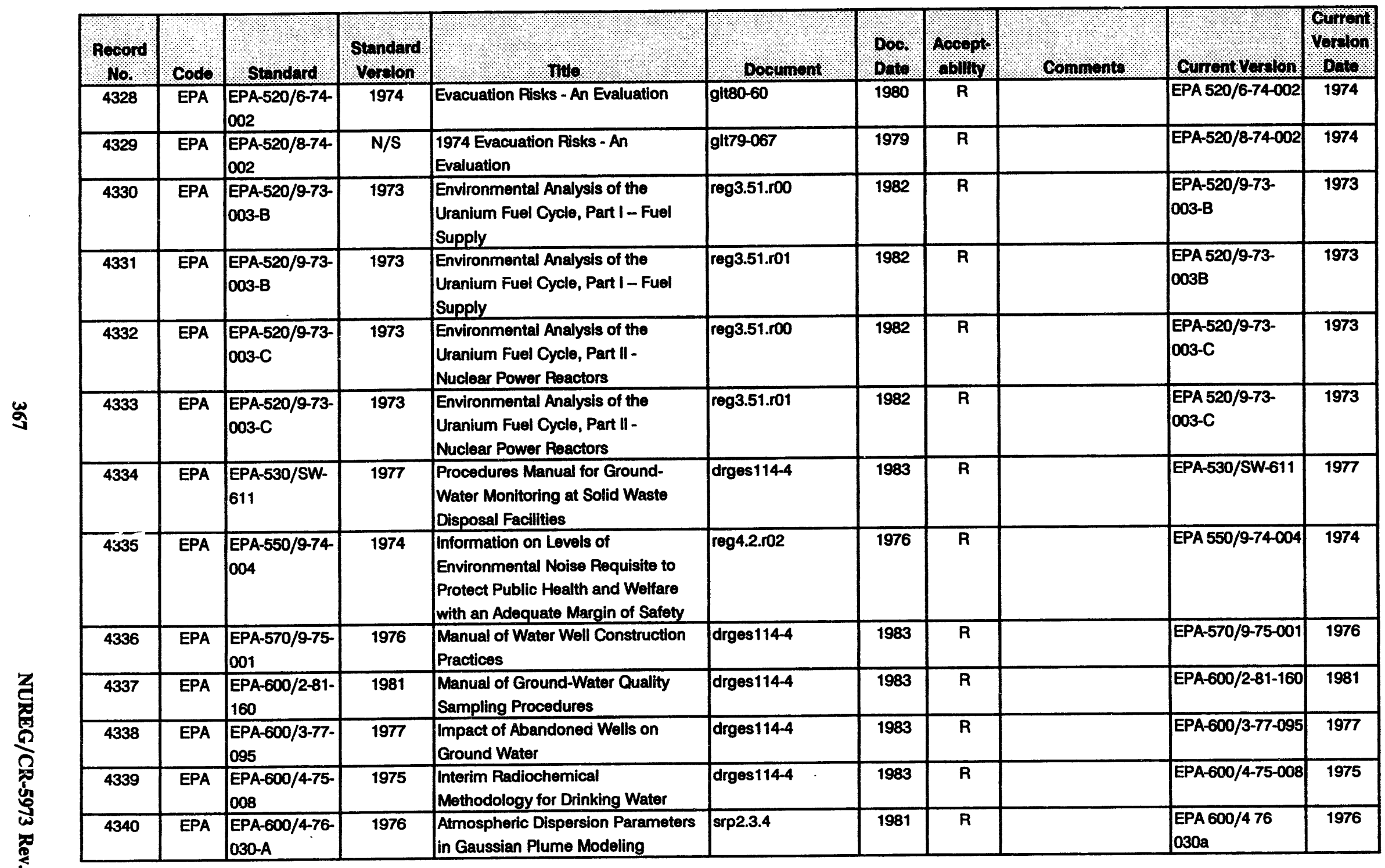




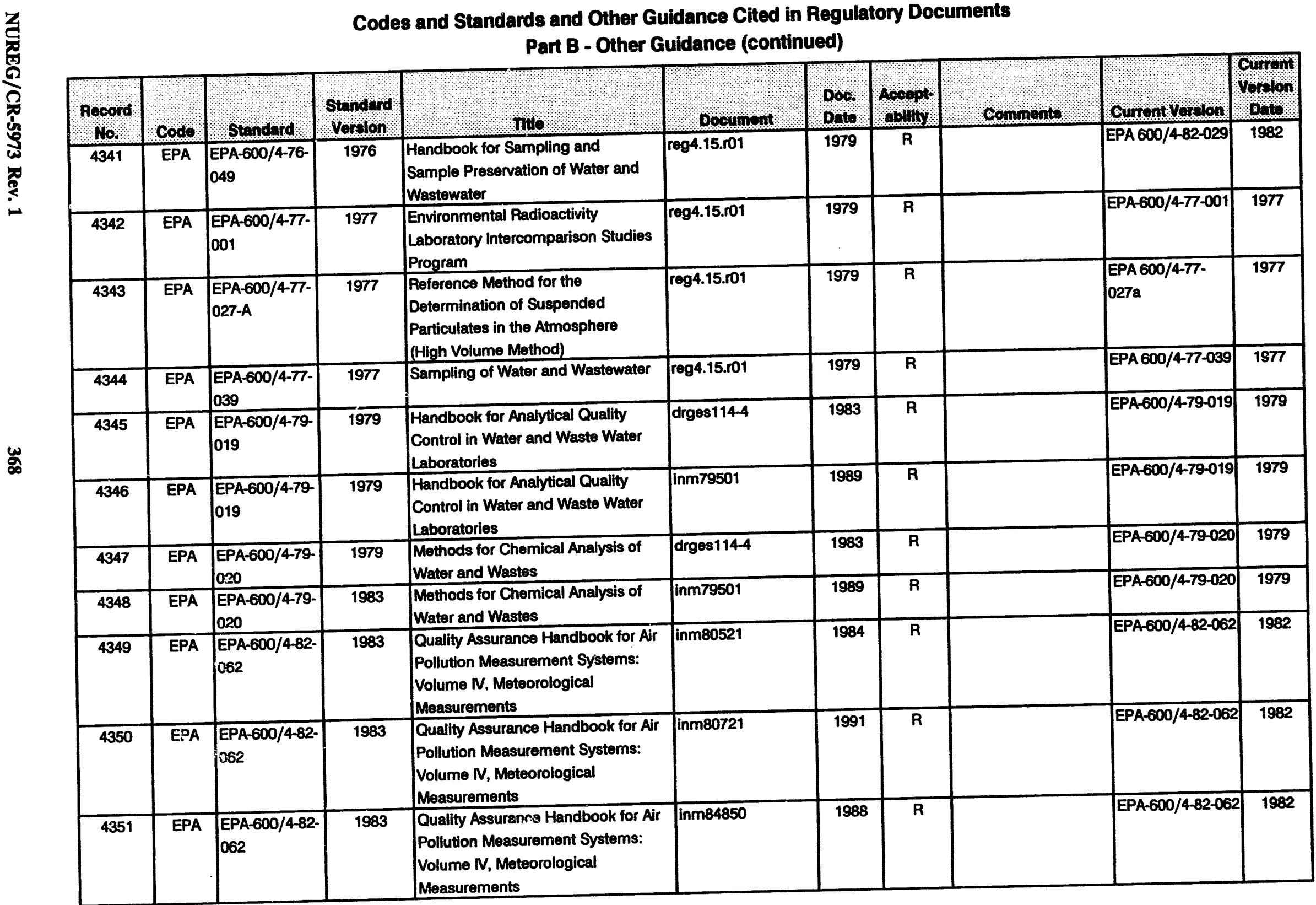


Codes and Standards and Other Guidance Cited in Regulatory Documents Part B - Other Guidance (continued)

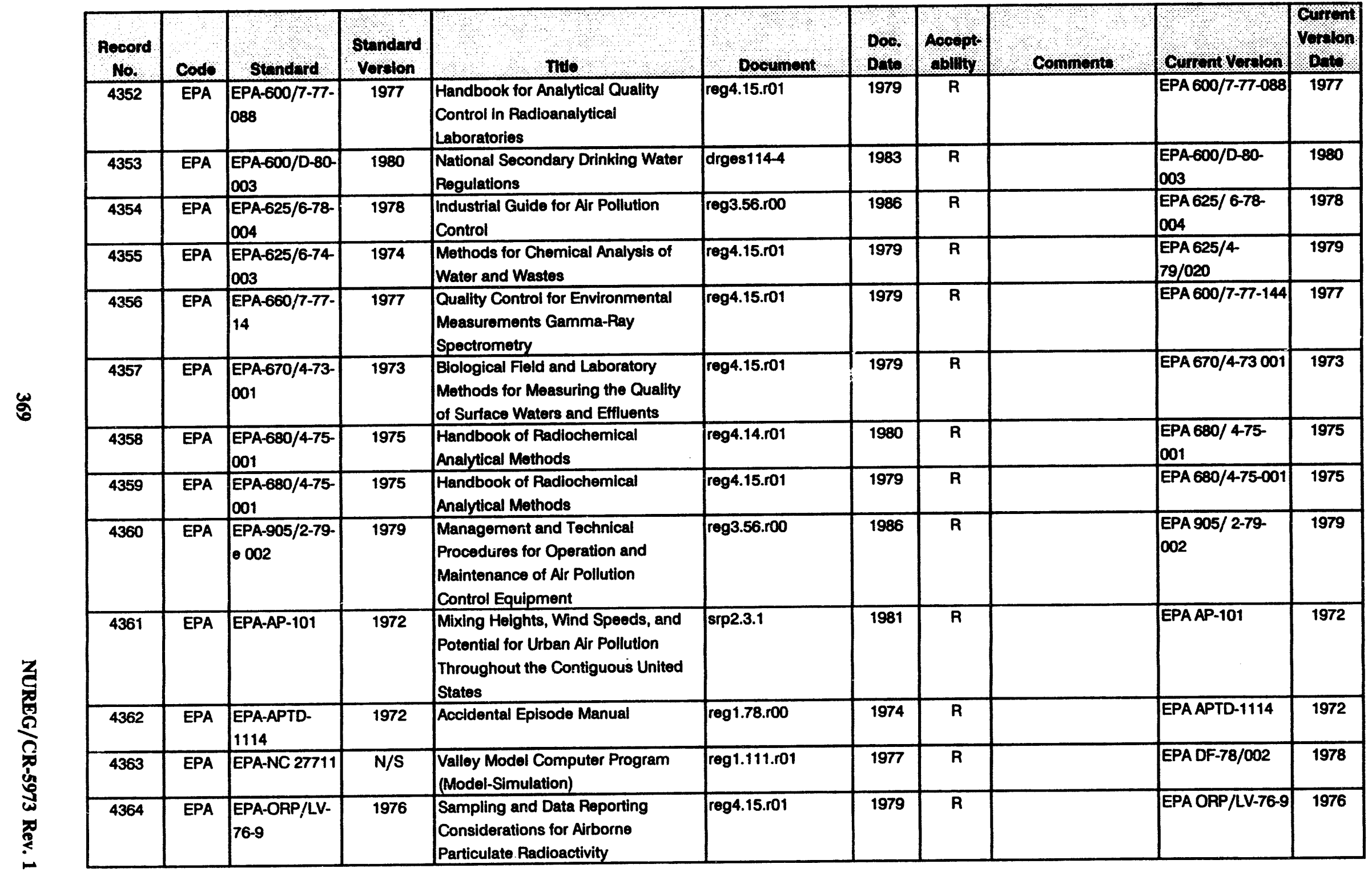


Codes and Standards and Other Guidance Cited in Regulatory Documents

Part B - Other Guidance (continued)

\begin{tabular}{|c|c|c|c|c|c|c|c|c|c|c|}
\hline \multicolumn{11}{|c|}{ Part B - Other Gurabince (Conmulu) } \\
\hline 4365 & EPA & $\begin{array}{l}\text { EPA-ORP/LV- } \\
76-9\end{array}$ & 1976 & $\begin{array}{l}\text { Sampling and Data Reporting } \\
\text { Considerations for Airborne } \\
\text { Particulate Radioactivity }\end{array}$ & reg4.16.ro1 & 1985 & $\mathbf{R}$ & & EPA ORP/LV-76-9 & 1976 \\
\hline 4366 & EPA & $\begin{array}{l}\text { EPA-ORP/SID } \\
72-2\end{array}$ & 1972 & Analytical Quality Control Methods & reg4.15.ro1 & 1979 & $\mathbf{R}$ & $\begin{array}{l}\text { Not listed in EPA On- } \\
\text { line Library System }\end{array}$ & $\begin{array}{l}\text { EPA ORP/SID 72- } \\
2\end{array}$ & 1972 \\
\hline 4367 & EPA & $\begin{array}{l}\text { EPA-ORP/SID } \\
72-2\end{array}$ & 1972 & Analytical Quality Control Methods & reg4.15.r01 & 1979 & $\mathbf{R}$ & & $\begin{array}{l}\text { EPA ORP/SID-72- } \\
2\end{array}$ & 1972 \\
\hline 4368 & EPA & $\begin{array}{l}\text { EPA-ORP/SID } \\
72-2\end{array}$ & 1972 & Analytical Quality Control Methods & reg4.14.ro1 & 1980 & $\mathbf{R}$ & & $\begin{array}{l}\text { EPA ORP/SID-72- } \\
2\end{array}$ & 1972 \\
\hline 4369 & EPA & EPA-PAGs & N/A & $\begin{array}{l}\text { EPA Protective Action Guidelines } \\
\text { (PAGs) }\end{array}$ & inm82206 & 1993 & $\bar{R}$ & $\begin{array}{l}\text { Not listed in EPA On- } \\
\text { line Library System }\end{array}$ & $n / a$ & $n / a$ \\
\hline 4370 & EPA & $\begin{array}{l}\text { EPA-PB-275- } \\
525\end{array}$ & 1977 & $\begin{array}{l}\text { Compilation of Air Pollutant } \\
\text { Emission Factors }\end{array}$ & reg3.59.r00 & 1987 & $\overline{\mathbf{R}}$ & & $n / a$ & $n / a$ \\
\hline 4371 & EPA & $\begin{array}{l}\text { EPA-R2-72- } \\
005 b\end{array}$ & 1974 & $\begin{array}{l}\text { Workbook on Thermal Plume } \\
\text { Prediction Vol. 2-Surface Discharge }\end{array}$ & reg1.113.ro1 & 1977 & $\bar{R}$ & & EPA PB 235841 & 1974 \\
\hline 4372 & EPA & $\begin{array}{l}\text { EPA-RD No. } \\
71-1\end{array}$ & 1971 & $\begin{array}{l}\text { Radiological Surveillance Studies at } \\
\text { a Pressurized Water Nuclear Power } \\
\text { Reactor }\end{array}$ & reg1.113.ro1 & 1977 & $\mathbf{R}$ & & EPA-RD-71-1 & 1971 \\
\hline 4373 & EPA & EPA/NRC & $\mathrm{N} / \mathrm{S}$ & $\begin{array}{l}\text { Joint EPA/NRC Guidance on the } \\
\text { Definition and Identification of } \\
\text { Commercial Mixed Low-Level } \\
\text { Radioactive and Hazardous Waste } \\
\text { and Answers to Anticipated } \\
\text { Questions }\end{array}$ & not90-31 & 1990 & $\mathbf{R}$ & & $\begin{array}{l}\text { EPA OSWER } \\
: 943200-2\end{array}$ & 1998 \\
\hline 4374 & EPA & NEPA & 1969 & $\begin{array}{l}\text { National Environmental Policy Act } \\
\text { of } 1969\end{array}$ & reg4.7.ro1 & 1975 & $\mathbf{R}$ & & NEPA & 1969 \\
\hline 4375 & EPA & NPDES & N/S & $\begin{array}{l}\text { National Pollutant Discharge } \\
\text { Elimination System }\end{array}$ & ght89-13 & 1989 & $\mathbf{R}$ & Computer Code & Clean Water Act & 1977 \\
\hline 4574 & ERDA & ERDA 76-21 & 1976 & Nuclear Air Cleaning Handbook & reg1.140.ro1 & 1979 & $\mathbf{P}$ & & ERDA 76-21 & 1976 \\
\hline 4575 & ERDA & ERDA 76-21 & 1976 & Nuclear Air Cleaning Handbook & reg1.52.r02 & 1978 & $\mathbf{P}$ & & ERDA 70-21 & 1976 \\
\hline 4576 & ERDA & ERDA 76-21 & 1976 & Nuclear Air Cleaning Handbook & srp6.5.1 & 1981 & $\mathbf{P}$ & & ERDA 76-21 & 1976 \\
\hline 4577 & ERDA & ERDA $77-24$ & 1977 & $\begin{array}{l}\text { A Guide for Environmental } \\
\text { Radiological Surveillance at ERDA } \\
\text { Installations }\end{array}$ & reg4.14.ro1 & 1980 & $\mathbf{R}$ & & ERDA $77-24$ & 1977 \\
\hline
\end{tabular}




\section{Codes and Standards and Other Guidance Cited in Regulatory Documents}

Part B - Other Guidance (continued)

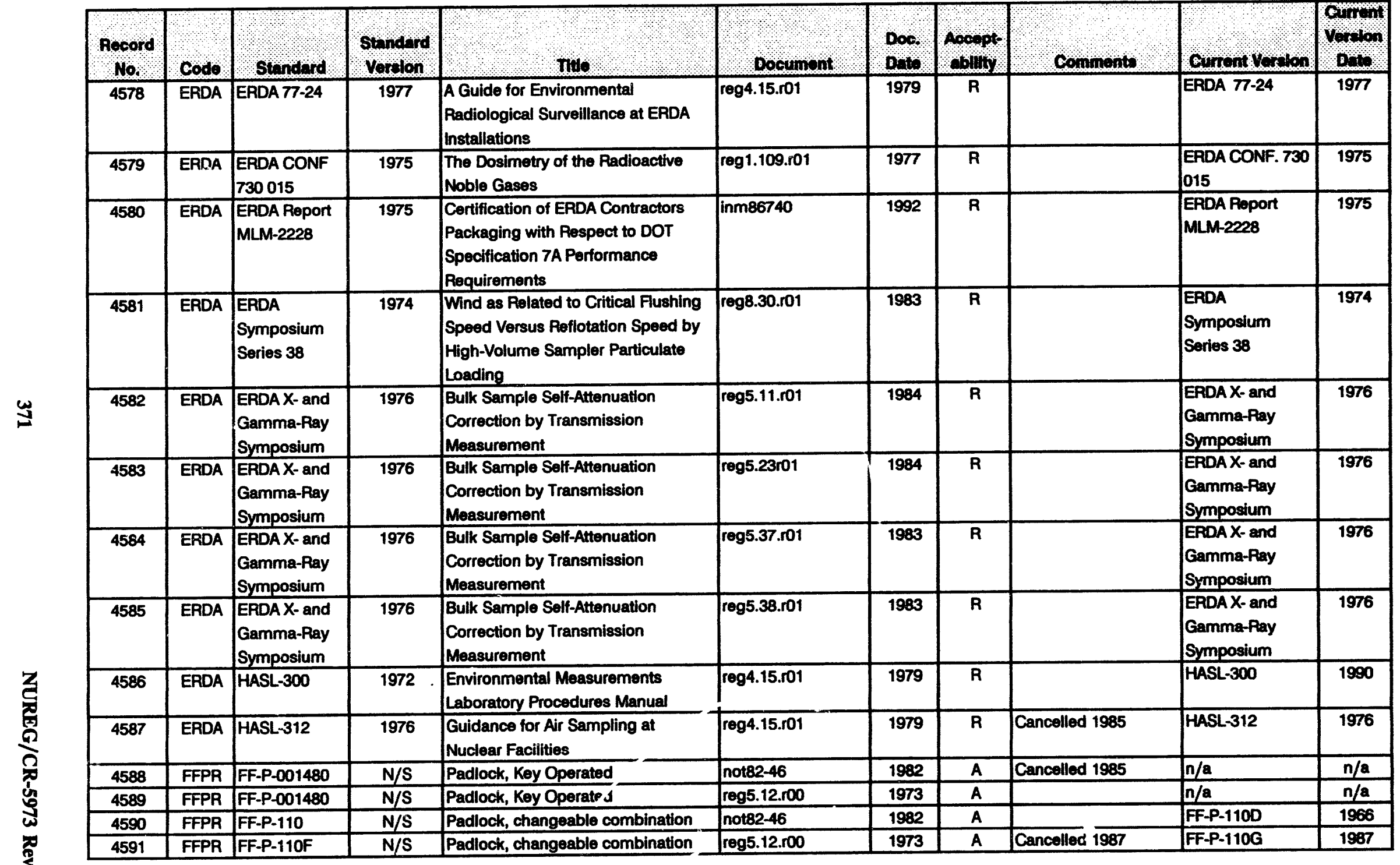


Codes and Standards and Other Guidance Cited in Regulatory Documents

Part B - Other Guldance (continued)

\begin{tabular}{|c|c|c|c|c|c|c|c|c|c|c|}
\hline $\begin{array}{l}\text { Hocord } \\
\text { No. }\end{array}$ & Codo & standard & $\begin{array}{l}\text { Standard } \\
\text { Vot lon }\end{array}$ & Tho & Document & Dow & $\begin{array}{l}\text { Acoppt } \\
\text { ablity }\end{array}$ & Comments & O.ront Voralon & omplon \\
\hline 4592 & FFPR & MIL-L 29151 & N/S & $\begin{array}{l}\text { Locks and Lock Sets, Exterior, } \\
\text { Ordinance, High Security }\end{array}$ & not82-46 & 1982 & A & & $n / a$ & $n / a$ \\
\hline 4593 & FFPR & MIL-P-43607 & $N / S$ & $\begin{array}{l}\text { Padlock, Key Operated, High } \\
\text { Security, Shrouded Shackle }\end{array}$ & not82-46 & 1982 & A & & MiLL-P-43607G(3) & 1986 \\
\hline 4594 & FFPR & W-A-00450 A & $\mathrm{N} / \mathrm{S}$ & $\begin{array}{l}\text { Components for Interior Alarm } \\
\text { Systems }\end{array}$ & reg1.17.ro1 &.$\overline{973}$ & A & & W-A-00450B & 1973 \\
\hline 4595 & FFPR & W-A-00450 B & $\mathbf{N} / \mathrm{S}$ & $\begin{array}{l}\text { Components for Interior Alarm } \\
\text { Systems }\end{array}$ & $10 \mathrm{cft} 73$ & 1993 & A & & W-A-O0150B & 197 's \\
\hline 4596 & FFPR & W-A-00450 B & 1973 & $\begin{array}{l}\text { Components for Interior Alarm } \\
\text { Systems }\end{array}$ & reg1.70.r03 & 1978 & A & & W-A-OO450B & 1973 \\
\hline 4597 & FM & $F M$ & $\mathbf{N} / \mathrm{S}$ & $\begin{array}{l}\text { Factory Mutual System Approval } \\
\text { Guide }\end{array}$ & inm88055 & 1991 & $\mathbf{R}$ & & $\begin{array}{l}\text { FM Approval } \\
\text { Guide }\end{array}$ & 1993 \\
\hline 4598 & $F M$ & $F M$ & $N / S$ & $\begin{array}{l}\text { Factory Mutual System Approval } \\
\text { Guide }\end{array}$ & reg1.120.r01 & 1977 & $\mathbf{P}$ & & $\begin{array}{l}\text { FM Approval } \\
\text { Guide }\end{array}$ & 1993 \\
\hline 4599 & FM & $F M$ & $\mathrm{~N} / \mathrm{S}$ & $\begin{array}{l}\text { Factory Mutual System Approval } \\
\text { Guide }\end{array}$ & srp9.5.1 & 1977 & $\mathbf{P}$ & & $\begin{array}{l}\text { FM Approval } \\
\text { Guide }\end{array}$ & 1993 \\
\hline 4600 & FWPCA & AN,CA & $N / S$ & Federal Water Pollution Control Act & 10 ctr51 & 1991 & $\mathbf{R}$ & & $\mathrm{n} / \mathrm{a}$ & $n / a$ \\
\hline 4601 & HPS & $\begin{array}{l}\text { Publication } \\
\text { No. (FDA) 73- }\end{array}$ & 1972 & Health Physics in the Healing Ants & reg8.18.ro1 & 1982 & $\mathbf{R}$ & & $\begin{array}{l}\text { Publication No. } \\
\text { (FDA) 73-8029 }\end{array}$ & 1972 \\
\hline 4602 & IEEE & $\begin{array}{l}\text { IEEE Catalog } \\
\text { No. TH0073-7 }\end{array}$ & 1980 & $\begin{array}{l}\text { Record of the Working Conference } \\
\text { on Advanced Electrotechnology } \\
\text { Applications to Nuclear Power } \\
\text { Plants }\end{array}$ & nureg0933, 1.E & 1989 & $\mathbf{R}$ & & $\begin{array}{l}\text { IEEE Catalog No. } \\
\text { THOOT3-7 }\end{array}$ & 1980 \\
\hline 4603 & IEEE & $\begin{array}{l}\text { IEEE No. } \\
\text { FT7017-7 }\end{array}$ & 1978 & $\begin{array}{l}\text { Joint Frequency Distribution of } \\
\text { Stroke Current Pise and Crest } \\
\text { Magnitude to Transmission Lines }\end{array}$ & drgrs705-4 & 1979 & $\mathbf{R}$ & & IEEE No. F77017- & 1978 \\
\hline 4604 & IEEE & \begin{tabular}{|l|} 
IEEE Power \\
Engineering \\
Committee \\
Vol. 88, No. 5 \\
\end{tabular} & 1969 & $\begin{array}{l}\text { Comparison of Polish Versus } \\
\text { American Lightning Fiecords }\end{array}$ & drgrs705-4 & 1979 & $\mathbf{R}$ & & $\begin{array}{l}\text { IEEE Power } \\
\text { Engineering } \\
\text { Committee Vol. } \\
88, \text { No. } 5 \\
\end{array}$ & 1969 \\
\hline 4605 & IEEE & \begin{tabular}{|l|} 
IEEE Power \\
Engineering \\
Committee \\
Vol. 97, No. 1 \\
\end{tabular} & 1978 & $\begin{array}{l}\text { Transient Lightning Overvoltage } \\
\text { Protection Requirements for a } 500 \\
\text { kV Gas-Insulated Substation }\end{array}$ & drgr8705-4 & 1979 & $\mathbf{R}$ & & \begin{tabular}{|l} 
IEEE Power \\
Engineering \\
Committee Vol. \\
97, No. 1 \\
\end{tabular} & 1978 \\
\hline
\end{tabular}


Codes and Standards and Other Guidance Cited in Regulatory Documents

Part B - Other Guidance (continued)

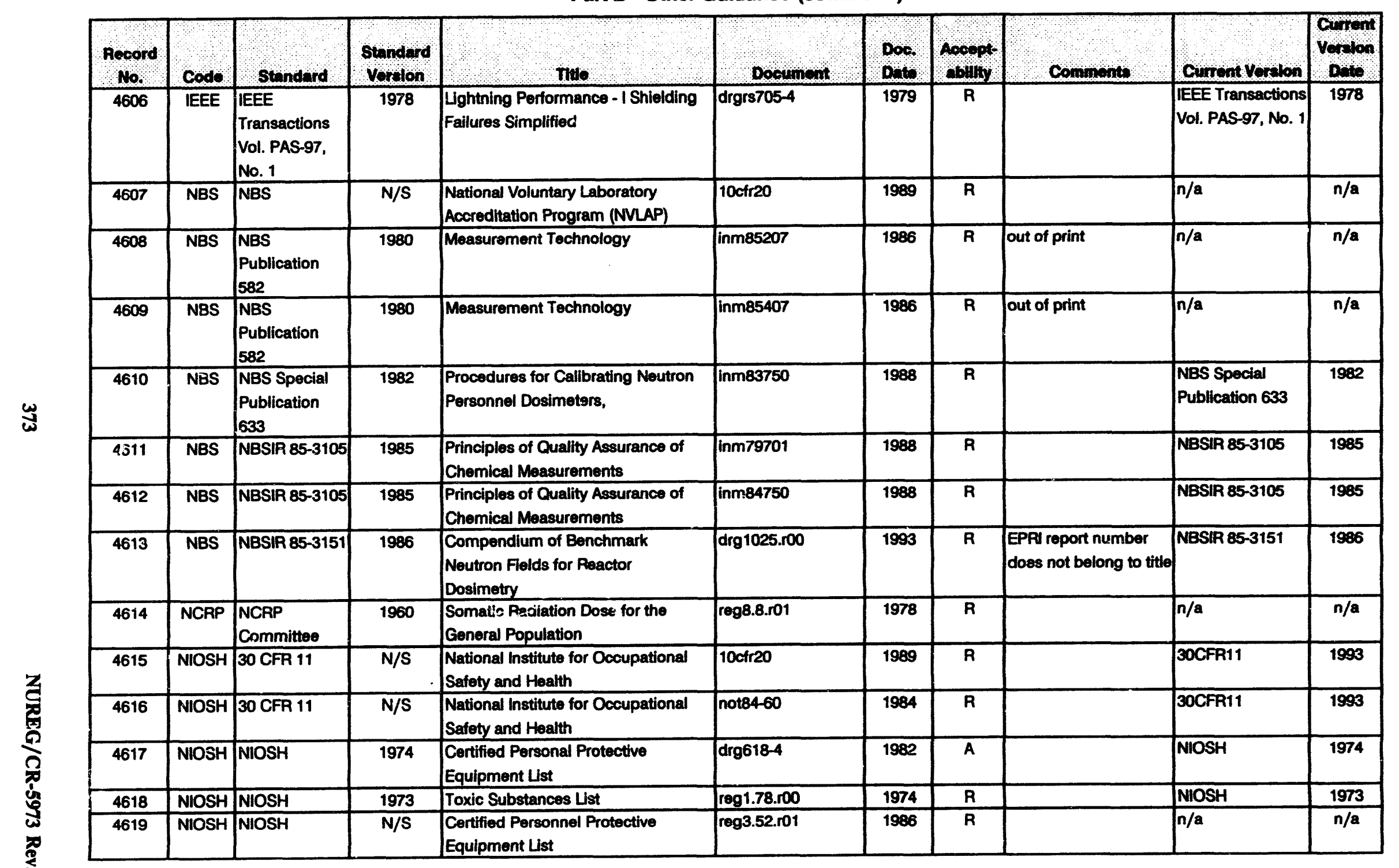




\begin{tabular}{|c|c|c|c|c|c|c|c|c|c|c|}
\hline \multicolumn{11}{|c|}{$\begin{array}{l}\text { Codes and Standards and Other Guidance Cited in Regulatory Documents } \\
\text { Part B - Other Guidance (continued) }\end{array}$} \\
\hline $\begin{array}{c}\text { Record } \\
\text { No. }\end{array}$ & Code & stindond & $\begin{array}{l}\text { Standard } \\
\text { Vortion }\end{array}$ & . $\mathbf{T H}_{\mathbf{0}}$ & Document & $\begin{array}{l}\text { Doe } \\
\text { Doth }\end{array}$ & $\begin{array}{l}\text { Acospt- } \\
\text { obluty }\end{array}$ & comments & curremt vorsion & Comion \\
\hline 4620 & NIOSH & NIOSH & $N / S$ & $\begin{array}{l}\text { Certified Personnel Protective } \\
\text { Equipment List }\end{array}$ & reg3.55.roo & 1985 & $R$ & & $n / a$ & $n / a$ \\
\hline 4621 & NIOSH & NIOSH & 1974 & $\begin{array}{l}\text { Certified Parsonnel Protoctive } \\
\text { Equipment List }\end{array}$ & reg8.8.r03 & 1978 & $\bar{R}$ & & $n / a$ & $n / \mathbf{a}$ \\
\hline 4622 & NIOSH & NIOSH 80-106 & 1979 & $\begin{array}{l}\text { Crittria for a Pecommended } \\
\text { Standard...Working in Confined } \\
\text { Spaces }\end{array}$ & not81-26 & 1981 & $\bar{R}$ & & NIOSH 80-106 & 1979 \\
\hline 4623 & NIOSH & NIOSH 80-106 & 1979 & $\begin{array}{l}\text { Critoria for a Rocommended } \\
\text { Standard... Working in Confined } \\
\text { Spaces }\end{array}$ & not81-26Part4 & 1981 & $\bar{R}$ & & NIOSH 80-106 & 1912 \\
\hline 4624 & $\mathrm{NIOSH}$ & NIOSH 82-106 & 1981 & $\begin{array}{l}\text { Supplement to the NIOSH Cortified } \\
\text { Equipment Lst }\end{array}$ & inm83100 & 1991 & $\bar{R}$ & & NIOSH 92-101 & 1991 \\
\hline 4625 & NIOSH & NIOSH 82-106 & 1981 & $\begin{array}{l}\text { Supplement to the NIOSH Certified } \\
\text { Equipment List }\end{array}$ & inm83525 & 1984 & $\bar{R}$ & & NIOSH 92-101 & 1991 \\
\hline 4626 & $\mathrm{NIOSH}$ & NIOSH 82-106 & 1981 & $\begin{array}{l}\text { Supplernent to the NIOSH Certified } \\
\text { Equipment List }\end{array}$ & inm83725 & 1991 & $\bar{R}$ & & NIOSH 92-101 & 1991 \\
\hline 4627 & $\mathrm{NIOSH}$ & NIOSH 82-106 & 1981 & $\begin{array}{l}\text { Supplement to the NIOSH Cortified } \\
\text { Equipment List }\end{array}$ & inm83750 & 1988 & A & & NIOSH 92-101 & 1991 \\
\hline 4628 & NIST & NIST & $\mathrm{N} / \mathrm{S}$ & $\begin{array}{l}\text { National Voluntary Laboratory } \\
\text { Accreditation Program (NMLAP) }\end{array}$ & $\begin{array}{l}100 \text { fr20 Subparts A- } \\
0\end{array}$ & 1993 & $\bar{A}$ & & $n / a$ & $n / a$ \\
\hline 4629 & $\overline{U L}$ & $\begin{array}{l}\text { UL Outline of } \\
\text { Investigation } \\
17724\end{array}$ & 1984 & $\begin{array}{l}\text { Fire Tests for Electrical Circuit } \\
\text { Protective Systems }\end{array}$ & not93-40 & 1993 & $\bar{R}$ & & \begin{tabular}{|l|} 
UL Outtine of \\
Investigation 1724
\end{tabular} & 1984 \\
\hline
\end{tabular}




\section{Appendix A}

Summation of Codes and Standards Citations

in Regulatory Documents (Part A) 

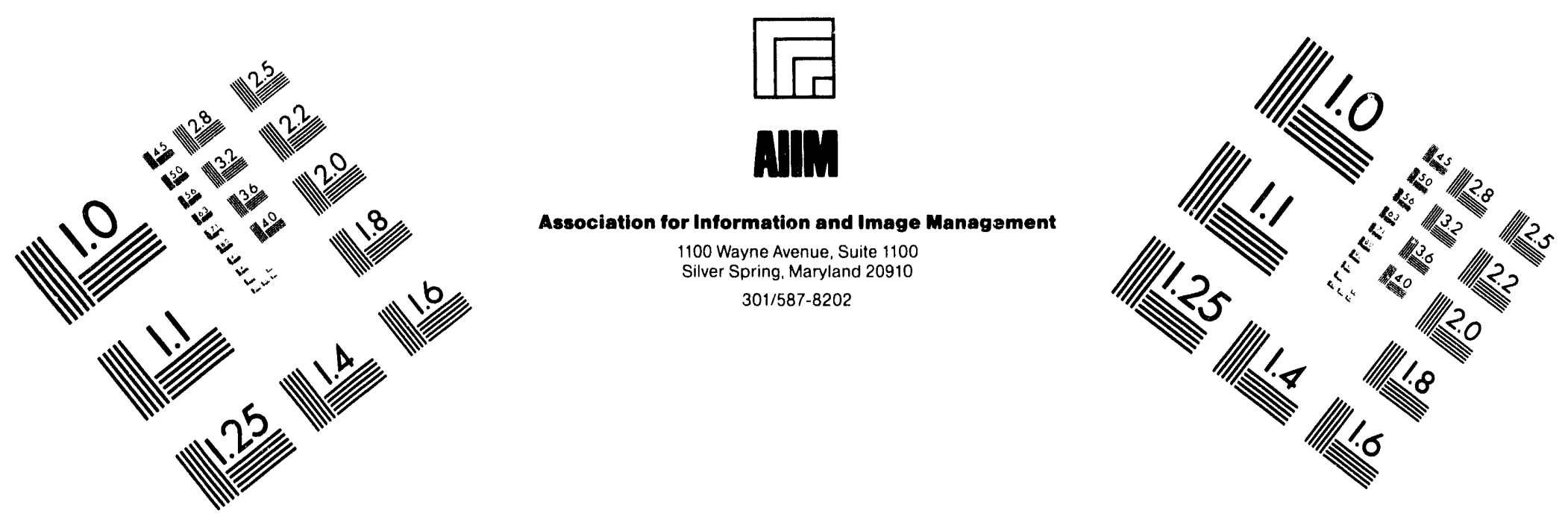

\section{Centimeter}

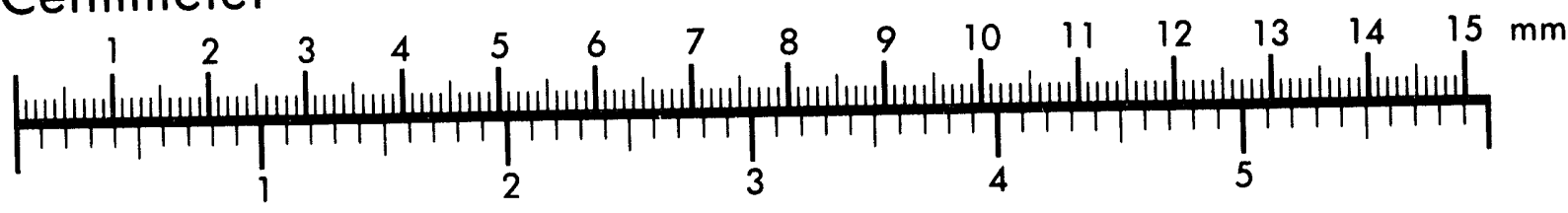
Inches
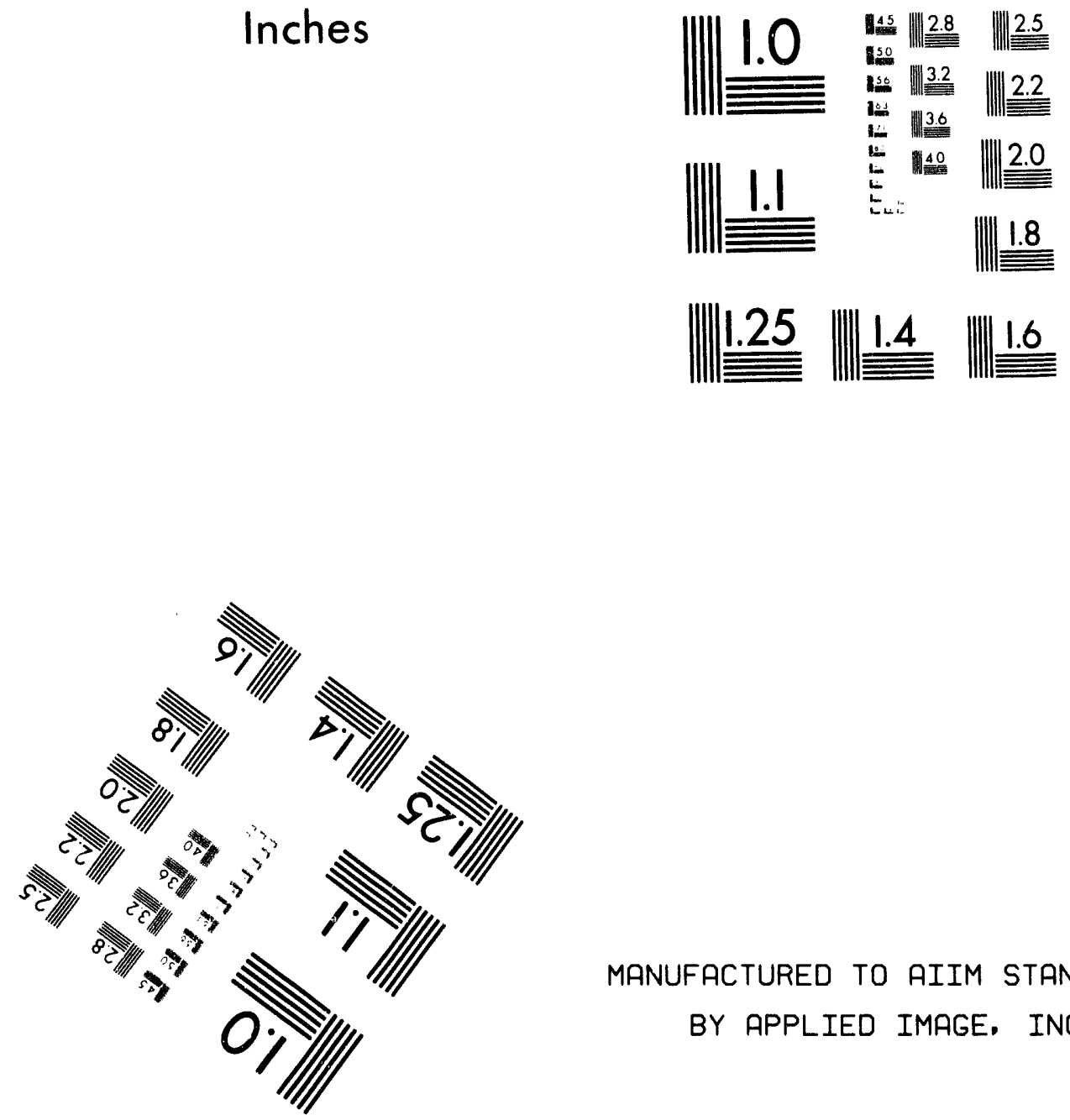

MANUFACTURED TO AIIM STANDARDS

BY APPLIED IMAGE, INC.

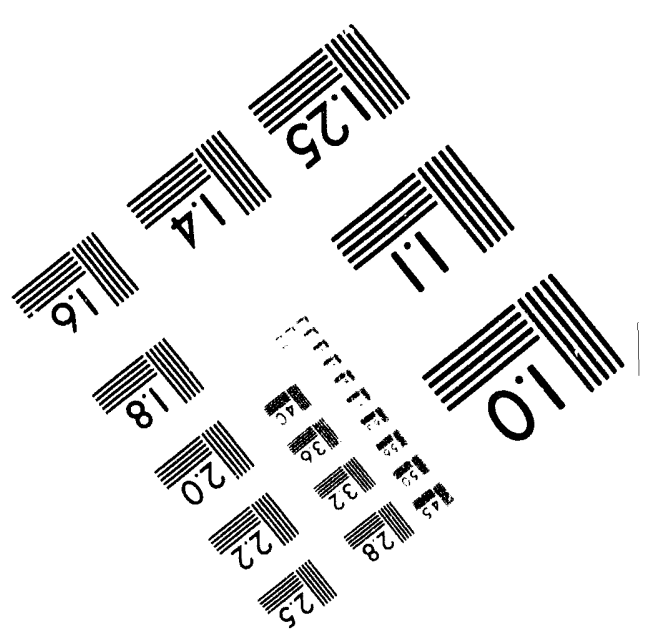



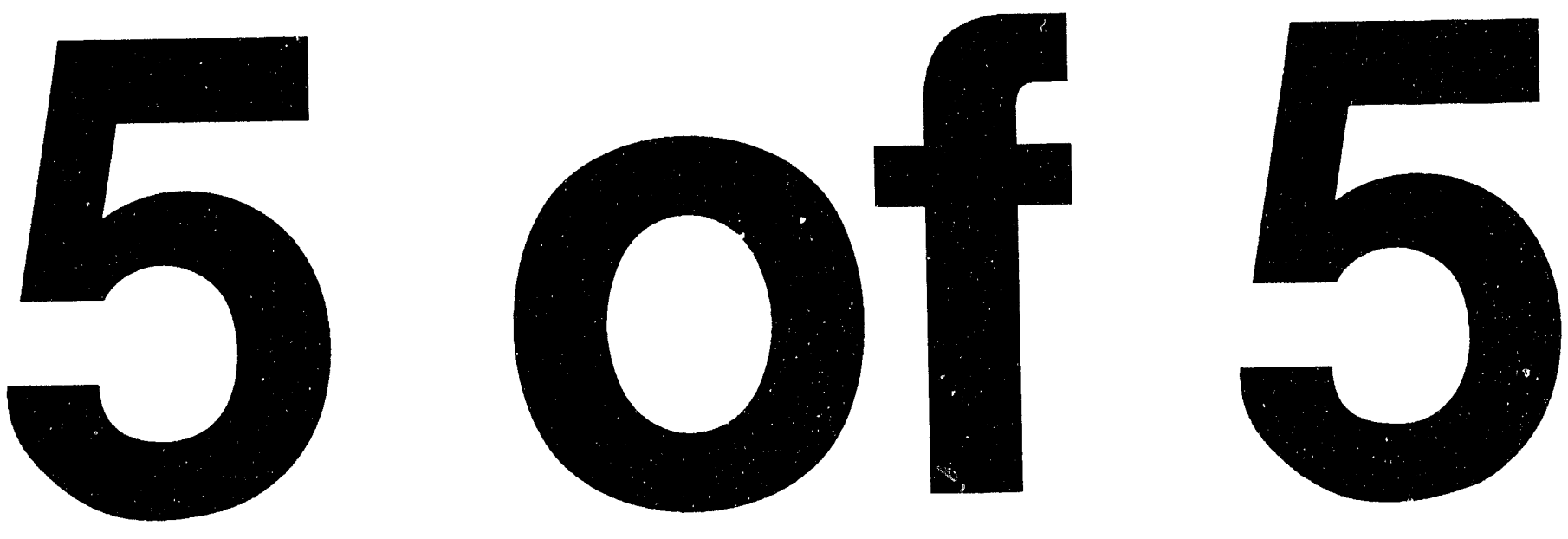


\section{Appendix A}

\section{Summation of Codes and Standards Citations}

in Regulatory Documents (Part A)

American Concrete Institute

Citations

American Institute of Steel Construction

American Nuclear Soriety

American National Standards Institute

234

American Petroleum Institute

906

Acoustical Society of America

American Society of Civil Engineers

American Society of Mechanical Engineers

American Society for Nondestructive Testing

1617

American Society for Testing and Materials

American Welding Society

American Water Works Association

Crane Manufacturers Association of America

Diesel Engine Manufacturers Association

Factory Mutual

Health Physics Society

International Commission on Radiological Protection

Institute of Electrical and Electronics Engineers

Instrument Society of America

International Standards organization

Manufacturers Standards Society

National Bureau of Standards

National Concrete and Masonry Association

National Council on Radiation Protection

National Electrical Manufacturers Association $\quad 9$

National Electrical Testing Association

National Fire Protection Association

National Rifle Association

Underwriters Laboratory 


\section{Appendix B}

Summation of Other Government and Industry Guidance Citations in Regulatory Documents (Part B) 


\section{Appendix B}

Summatiun of Other Government and Industry Guidance Citations in Regulatory Documents (Part B)

American Concrete Institute

Citations

Atomic Energy Commission

American Nuclear Society

American Society of Civil Engineers

American Society of Mechanical Engineers

American Society for Nondestructive Testing

American Society for Testing and Materials

American Water Works Association

Department of Energy

Department of Transportation

Environmental Protection Agency

Energy Research and Development Administration

Federal Specification

9

Federal Water Pollution Control Act

Factory Mutual

Health Physics Society

Institute of Electrical and Electronics Engineers 5

National Bureau of Standards

National Council on Radiation Protection 1

National Institute for Occupational Health and Safety 13

Underwriters Laboratory

Total 388 
NUREG/CR-5973

PNL-8462

\section{DISTRIBUTION}

No. of

Copies

\section{OFFSITE}

U.S. Nuclear Regulatory Commission

5 Mr. Gene Suh

Office of Nuclear Reactor Regulation

Mailstop 0-12 E4

Washington, DC 20555

\section{ONSITE}

26 Pacific Northwest Laboratory

Mr. E. J. Cleary (20)

Publishing Coordination (1)

Technical Report Files (5) 

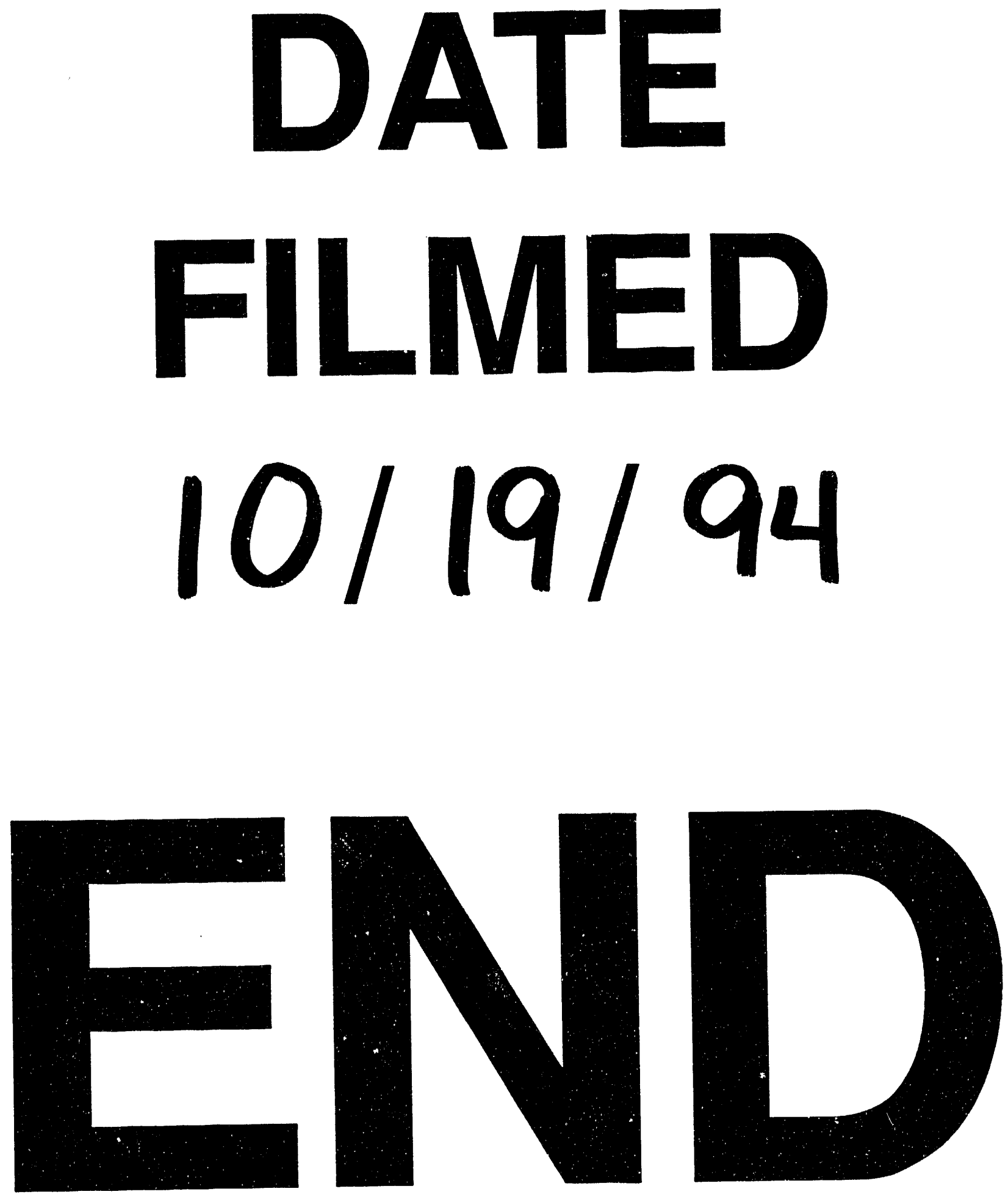


$$
\text { (20) }
$$

